


\section{PIRLS 2006 \\ International Report}

IEA's Progress in International Reading Literacy Study in Primary Schools in 40 Countries

Ina V.S. Mullis

Michael O. Martin

Ann M. Kennedy

Pierre Foy 


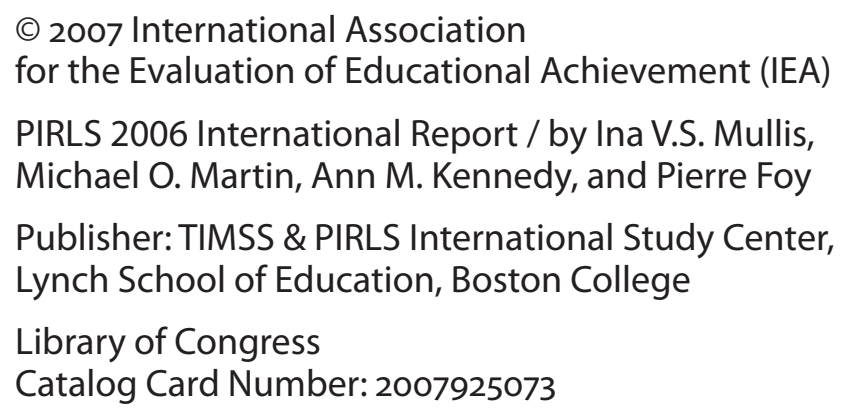

Boston College is an equal opportunity, affirmative action employer.

Printed and bound in the United States. 


\section{Contents}

Executive Summary ................... 1

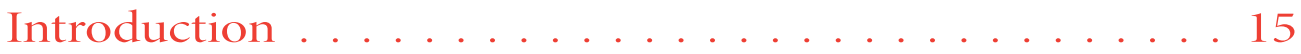

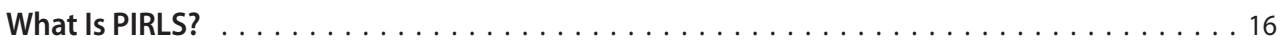

What Was the Nature of the PIRLS 2006 Test of Reading Comprehension? . . . . . . . . . . 17

What Background Information Is Available About the Contexts for Students

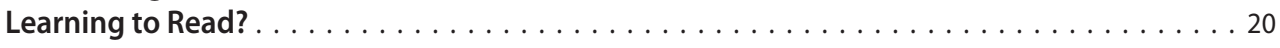

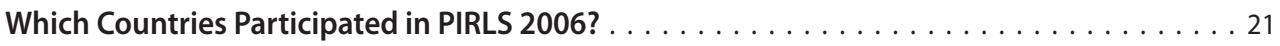

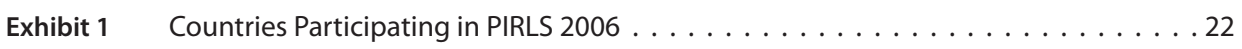

Exhibit 2 Selected Characteristics of PIRLS 2006 Countries . . . . . . . . . . . . 26

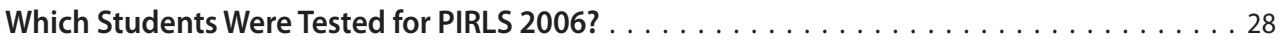

Exhibit 3 Information about the Students Tested for PIRLS $2006 \ldots \ldots \ldots \ldots \ldots$

Chapter $1 \ldots \ldots . \ldots . \ldots . \ldots 35$

International Student Achievement in Reading

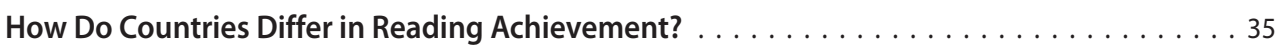

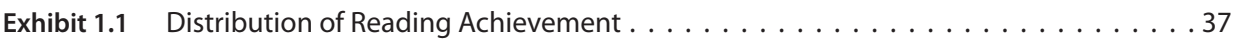

Exhibit 1.2 Multiple Comparisons of Average Reading Achievement. . . . . . . . . . . 40

How Has Achievement in Reading Comprehension Changed Between PIRLS 2001 and

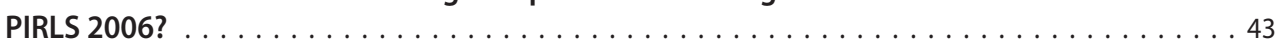

Exhibit 1.3 Trends in Reading Achievement. . . . . . . . . . . . . . . . . . . 44

What Are the Gender Differences in Reading Achievement? . . . . . . . . . . . . . 46

How Does Achievement Differ Across Countries for Reading Literacy Purposes? . . . . . . . 47

Exhibit 1.4 Differences in Average Reading Achievement by Gender. . . . . . . . . . . . . . . . 48

Exhibit 1.5 Trends in Average Reading Achievement by Gender. . . . . . . . . . . . . . . . . . 49

Exhibit 1.6 Relative Difference in Performance Between Literary and Informational Purposes. . . 51

Exhibit 1.7 Trends in Reading Achievement for Literary Purposes. . . . . . . . . . . . . . . . 53

Exhibit 1.8 Trends in Reading Achievement for Informational Purposes. . . . . . . . . . . . . . 54

What Are the Gender Differences in Achievement for the Reading Purposes? . . . . . . . . 55

How Does Achievement Differ Across Countries for Reading Comprehension Processes? . . 55

Exhibit 1.9 Average Achievement in Reading for Literary and Informational Purposes

by Gender. . . . . . . . . . . . . . . . . . . . . . . . . . . 56

Exhibit 1.10 Relative Differences in Performance Between Reading Comprehension Processes . . 59

Exhibit 1.11 Trends in Reading Achievement for Retrieving and Straightforward Inferencing Processes . . . . . . . . . . . . . . . . . . . . . . . . 61

Exhibit 1.12 Trends in Reading Achievement for Interpreting, Integrating, and Evaluating

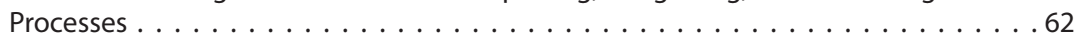

What Are the Gender Differences in Achievement for the Reading Processes? . . . . . . . 63

Exhibit 1.13 Average Achievement in Reading Processes of Comprehension by Gender . . . . . 64 
Chapter $2 \ldots \ldots . \ldots . \ldots 67$

Performance at the PIRLS 2006 International Benchmarks

How Does Performance in Countries Compare with the PIRLS 2006

International Benchmarks?

Exhibit 2.1 Percentages of Students Reaching the PIRLS 2006 International Benchmarks

of Reading Achievement . . . . . . . . . . . . . . . . . . . . . . . . . . . . . . . 69

Exhibit 2.2 Trends in Percentages of Students Reaching the PIRLS 2006 International Benchmarks of Reading Achievement in 2001 and 2006 . . . . . . . . . . . . 72

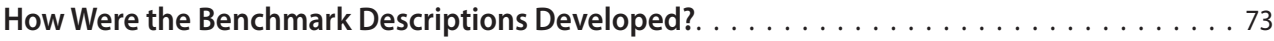

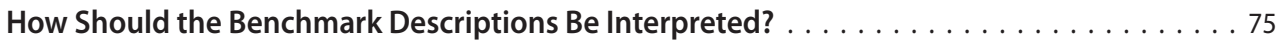

Exhibit 2.3 Description of Texts in PIRLS $2006 \ldots \ldots \ldots \ldots \ldots \ldots$

Achievement at the PIRLS 2006 Low International Benchmark . . . . . . . . . . . . . 78

Exhibit 2.4 Description of the PIRLS 2006 Low International Benchmark of Reading

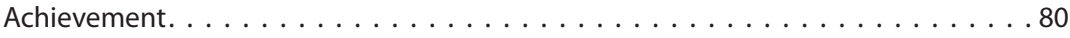

Exhibit 2.5 PIRLS 2006 Low International Benchmark - Item 1 - Literary Example . . . . . . . . 81

Exhibit 2.6 PIRLS 2006 Low International Benchmark - Item 2 - Literary Example . . . . . . . . 82

Exhibit 2.7 PIRLS 2006 Low International Benchmark - Item 3 - Informational Example . . . . 83

Exhibit 2.8 PIRLS 2006 Low International Benchmark - Item 4 - Informational Example . . . . . 84

Achievement at the PIRLS 2006 Intermediate International Benchmark . . . . . . . . . 85

Exhibit 2.9 Description of the PIRLS 2006 Intermediate International Benchmark of Reading

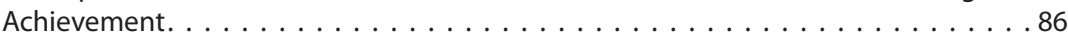

Exhibit 2.10 PIRLS 2006 Intermediate International Benchmark - Item 5 - Literary Example. . . . 87

Exhibit 2.11 PIRLS 2006 Intermediate International Benchmark - Item 6 - Literary Example. . . . 88

Exhibit 2.12 PIRLS 2006 Intermediate International Benchmark - Item 7 - Informational

Example. . . . . . . . . . . . . . . . . . . . . . . . 89

Exhibit 2.13 PIRLS 2006 Intermediate International Benchmark - Item 8 - Informational

Example. . . . . . . . . . . . . . . . . . . . . . . . 90

Achievement at the PIRLS 2006 High International Benchmark. . . . . . . . . . . . . . . 91

Exhibit 2.14 Description of the PIRLS 2006 High International Benchmark of Reading

Achievement. . . . . . . . . . . . . . . . . . . . . . . . .92

Exhibit 2.15 PIRLS 2006 High International Benchmark - Item 9 - Literary Example . . . . . . . . 93

Exhibit 2.16 PIRLS 2006 High International Benchmark - Item 10 - Literary Example . . . . . . . 94

Exhibit 2.17 PIRLS 2006 High International Benchmark - Item 11 - Informational Example . . . . 95

Exhibit 2.18 PIRLS 2006 High International Benchmark - Item 12 - Informational Example . . . . 96

Achievement at the PIRLS 2006 Advanced International Benchmark. . . . . . . . . . . . . . . 97

Exhibit 2.19 Description of the PIRLS 2006 Advanced International Benchmark of Reading

Achievement. . . . . . . . . . . . . . . . . . . . . . . . . 99

Exhibit 2.20 PIRLS 2006 Advanced International Benchmark - Item 13 - Literary Example. . . . 100

Exhibit 2.21 PIRLS 2006 Advanced International Benchmark - Item 14 - Literary Example. . . . 101

Exhibit 2.22 PIRLS 2006 Advanced International Benchmark - Item 15 - Informational Example. . . . . . . . . . . . . . . . . . . . . . . . . . . . . . . . 102

Exhibit 2.23 PIRLS 2006 Advanced International Benchmark - Item 16 - Informational Example. . . . . . . . . . . . . . . . . . . . . . . . . . . . . . . 103 
Chapter $3 \ldots \ldots \ldots . \ldots . \ldots . \ldots 105$

Literacy-related Activities in the Home

What Activities Fostering Literacy Did Parents Engage in with Their Child? . . . . . . . 106

Exhibit 3.1 Index of Early Home Literacy Activities (EHLA) with Trends. . . . . . . . . . . . . . 109

What Literacy Resources Do Students Have in Their Homes? . . . . . . . . . . . . . . . 110

Exhibit 3.2 Index of Home Educational Resources (HER) with Trends . . . . . . . . . . . . 111

Exhibit 3.3 Parents' Reports of Children's Books in the Home . . . . . . . . . . . . . . . . . 114

Exhibit 3.4 Trends in Parents' Reports of Children's Books in the Home . . . . . . . . . . . . 116

Exhibit 3.5 Highest Level of Education of Either Parent . . . . . . . . . . . . . . . . . . 120

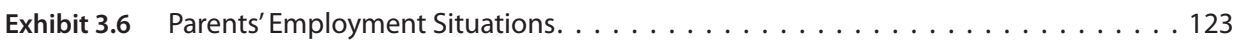

Exhibit 3.7 Parents' Occupational Level . . . . . . . . . . . . . . . . . . . . . . . . 124

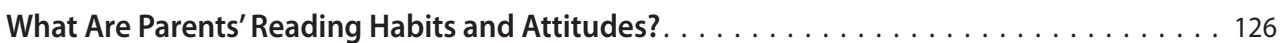

Exhibit 3.8 Parents Reading* at Home with Trends . . . . . . . . . . . . . . . . . . . . . 129

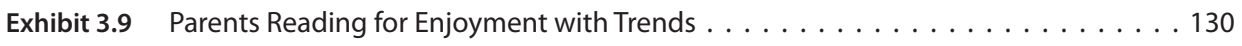

Exhibit 3.10 Index of Parents' Attitudes Toward Reading (PATR) with Trends . . . . . . . . . . . 131

What Languages Are Spoken at Home? . . . . . . . . . . . . . . . . . . . 132

Exhibit 3.11 Students and Parents Speak Language of the Test at Home . . . . . . . . . . . 135

Exhibit 3.12 Students' Parents Born in Country with Trends . . . . . . . . . . . . . . . 136

Chapter 4 ....................... . . 139

Students'Reading Attitudes, Self-Concept, and Out-of-School Activities

What Are Students' Attitudes Toward Reading? . . . . . . . . . . . . . . . . 139

Exhibit 4.1 Index of Students' Attitudes Toward Reading (SATR) with Trends . . . . . . . . . . . 141

What Are Students' Perceptions of Their Reading Ability? . . . . . . . . . . . . . . 142

Exhibit 4.2 Index of Students' Reading Self-Concept (SRSC) with Trends . . . . . . . . . . . . 144

How Often Do Children Engage in Literacy Activities Outside of School? . . . . . . . . . . 145

Exhibit 4.3 Students Reading Stories or Novels Outside of School with Trends . . . . . . . . . 146

Exhibit 4.4 Students Reading for Information Outside of School with Trends . . . . . . . . . 150

Exhibit 4.5 Students Read Stories or Articles Outside of School . . . . . . . . . . . . . . . 152

Exhibit 4.6 Students Reading for Fun Outside of School with Trends . . . . . . . . . . . . . 155

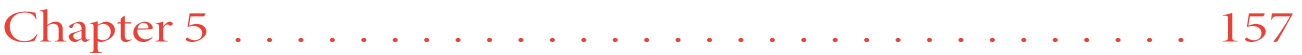

School Curriculum and Organization for Teaching Reading

How Well Prepared Are Students to Learn to Read? . . . . . . . . . . . . . . . . . 158

Exhibit 5.1 Number of Years Children Attended Preprimary Education (Preschool,

Kindergarten, and Other Similar Programs) . . . . . . . . . . . . . . . . . 162

Exhibit 5.2 Age Students Began Primary School. . . . . . . . . . . . . . . . . . 163

Exhibit 5.3 How Well Students Could Perform Beginning Literacy Activities When They Entered School with Trends . . . . . . . . . . . . . . . . . . . . . . . . . . . . 164

Exhibit 5.4 Principals' Estimates of the Percentage of Students Entering School Able to Perform Beginning Literacy Skills with Trends . . . . . . . . . . . . . . . . . 166

How Much Is Reading Emphasized in the School Curriculum? . . . . . . . . . . . . . . 167

Exhibit 5.5 Structural Characteristics of Reading Curricula for Primary/Elementary Grades. . . . 169 
Exhibit 5.6 Emphasis on Reading Curriculum in the Schools . . . . . . . . . . . . . . 170

Exhibit 5.7 Emphasis on Purposes for Reading in the Reading Curriculum . . . . . . . . . . 171

Exhibit 5.8 Emphasis on Processes of Comprehension in the Reading Curriculum . . . . . . . 174

Exhibit 5.9 Schools' Grade-by-Grade Emphases on Reading Comprehension Skills

or Strategies . . . . . . . . . . . . . . . . . . 176

How Much Instructional Time Is Devoted to Reading? . . . . . . . . . . . . . . 178

Exhibit 5.10 Intended and Implemented Weekly Instructional Time for Language and Reading (Formal and Integrated) . . . . . . . . . . . . . . . . . . . . . . . . 181

Exhibit 5.11 Number of Hours Reading Is Taught Weekly (Formal and Integrated) with Trends . . 182

Exhibit 5.12 Teachers Spend Time for Formal Reading Instruction . . . . . . . . . . . . 183

Exhibit 5.13 Frequency of Reading Instruction During the Week . . . . . . . . . . . . . . 184

Exhibit 5.14 Teachers'Time Spent on Classroom Activities . . . . . . . . . . . . . . . . 185

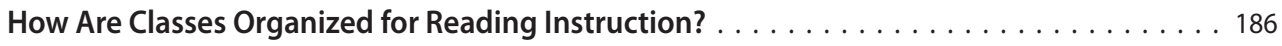

Exhibit 5.15 Organization of Students for Reading Instruction . . . . . . . . . . . . 187

Exhibit 5.16 Class Size for Reading and Language Instruction with Trends* $\ldots \ldots$. . . . . 188

How Do Schools Help Students with Reading Difficulties? . . . . . . . . . . . . . . . . . 191

Exhibit 5.17 Students In Class Needing Special Instruction . . . . . . . . . . . . . . . . . 192

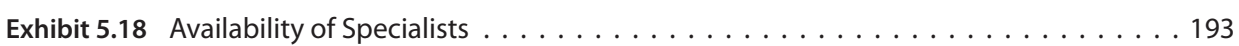

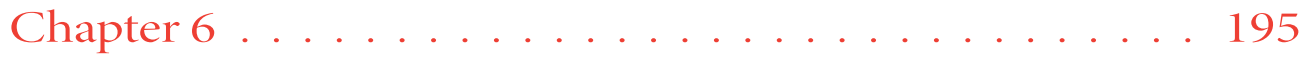

Teachers and Reading Instruction

What Education and Training Do Teachers Have for Teaching Reading? . . . . . . . . . 195

Exhibit 6.1 Reading Teachers'Formal Education . . . . . . . . . . . . . . . 198

Exhibit 6.2 Areas of Emphasis in Teachers' Formal Education and Training . . . . . . . . . . 199

What Are the Background Characteristics and Responsibilities of Reading Teachers? . . . 200

Exhibit 6.3 Teachers' Gender, Age, and Number of Years Teaching . . . . . . . . . . . . 201

Exhibit 6.4 Teachers'Teaching Responsibilities. . . . . . . . . . . . . . . . . . . . . 202

What Instructional Resources Do Teachers Use? . . . . . . . . . . . . . . . . . . . . . . 204

Exhibit 6.5 Materials Schools Used for Their Reading Instructional Programs . . . . . . . . . . 206

Exhibit 6.6 Materials Used by Teachers for Reading Instruction . . . . . . . . . . . . . . 208

Exhibit 6.7 Teachers' Use of Literary Texts for Reading Instruction. . . . . . . . . . . . . . . . 211

Exhibit 6.8 Teachers' Use of Informational Texts for Reading Instruction. . . . . . . . . . . . . 212

Exhibit 6.9 Trends in Teachers' Use of Literary and Informational Texts for Reading

Instruction . . . . . . . . . . . . . . . . . . . . . . 213

How Often Do Students Read Aloud and Independently in Class? . . . . . . . . . . . . . . 214

Exhibit 6.10 Emphasis on Decoding and Vocabulary. . . . . . . . . . . . . . . . 216

Exhibit 6.11 Teachers Ask Students to Do Classroom Activities at Least Weekly to Develop Reading Comprehension Skills or Strategies . . . . . . . . . . . . . . . . . 217

Exhibit 6.12 Teachers' Reports About Reading Aloud or Silently in Class . . . . . . . . . . . . 218

Exhibit 6.13 Students' Reports About Students Reading Aloud in Class . . . . . . . . . . . . . 220

Exhibit 6.14 Students' Reports About Independent Reading. . . . . . . . . . . . . . . . . 221

What Activities Do Students Do in Response to Class Reading? . . . . . . . . . . . . . . . . 222

Exhibit 6.15 Teachers' Reports About Reading Comprehension Activities After Instruction with Trends. . . . . . . . . . . . . . . . . . . . . . . . . . . . . 224 
Exhibit 6.16 Students Answer Questions Aloud About Class Reading . . . . . . . . . . . . . 225

Exhibit 6.17 Students Answer Questions in Workbooks or Worksheets About Class Reading . . . 226

Exhibit 6.18 Students Write Something About Class Reading . . . . . . . . . . . . . . 227

Exhibit 6.19 Teachers' Reports on Giving a Written Quiz or Test After Students Read. . . . . . . . 228

What Library Resources Are Available and How Are They Used? . . . . . . . . . . . . . . . . . 229

Exhibit 6.20 Access to School and Classroom Libraries . . . . . . . . . . . . . . . . . . . . . . . 230

Exhibit 6.21 Students Visit and Borrow Books from School or Local Library . . . . . . . . . . 231

How Are Computers Used for Reading Instruction? . . . . . . . . . . . . . . . . . . 232

Exhibit 6.22 Computer Availability and Instructional Use with Trends . . . . . . . . . . . 233

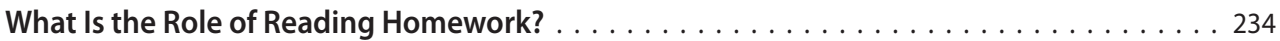

How Do Teachers Assess Reading Progress? . . . . . . . . . . . . . . . . . . . 235

Exhibit 6.23 Index of Reading for Homework (RFH) with Trends. . . . . . . . . . . . . . 236

Exhibit 6.24 Emphasis on Sources to Monitor Students' Progress in Reading with Trends. . . . . 238

Exhibit 6.25 Approaches to Assessing Students' Performance in Reading with Trends. . . . . . . 241

Exhibit 6.26 Teachers' Reports on Uses to Which Classroom Assessment of Reading Is Put. . . . . 242

Chapter $7 \ldots \ldots \ldots \ldots \ldots \ldots \ldots \ldots$

School Contexts

What Are the Schools' Demographic Characteristics? . . . . . . . . . . . . . . . . . 245

Exhibit 7.1 Principals' Reports on Their Schools' Locations with Trends . . . . . . . . . . . . . . 249

Exhibit 7.2 Principals' Reports on Their Primary Grade Students Coming from Economically Disadvantaged Homes with Trends . . . . . . . . . . . . . . . . . . . 250

Exhibit 7.3 Principals' Reports on Primary Grade Students Not Speaking the Language of the Test as Their First Language with Trends . . . . . . . . . . . . . . . 252

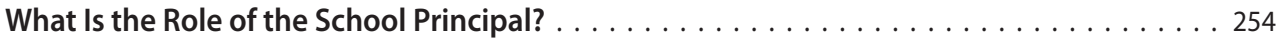

What Shortages of School Resources Affect Reading Instruction? . . . . . . . . . . . . . . 254

Exhibit 7.4 Principals'Time Spent on Various School-related Activities . . . . . . . . . . . . . 256

Exhibit 7.5 Index of Availability of School Resources (ASR) with Trends . . . . . . . . . . . . . 259

Exhibit 7.6 Workspace for Teachers Provided by Schools . . . . . . . . . . . . . . . . 260

Exhibit 7.7 Schools' Reports of Availability of Computers for Instructional Purposes with Trends. . . . . . . . . . . . . . . . . . . . . . . . . 261

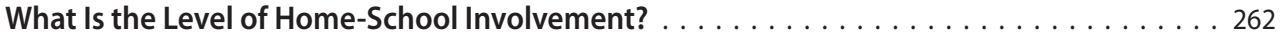

Exhibit 7.8 Index of Home-School Involvement (HSI) with Trends . . . . . . . . . . . . . . 264

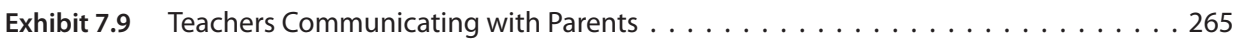

What Are the School Attendance Levels and Climate Characteristics? . . . . . . . . . 266

Exhibit 7.10 Parents Discuss Their Child's Classroom Reading Work with Him or Her. . . . . . . . 267

Exhibit 7.11 Seriousness of Absenteeism in Schools with Trends . . . . . . . . . . . . . . . . . 268

Exhibit 7.12 Index of Principal's Perception of School Climate (PPSC) with Trends . . . . . . . 271

Exhibit 7.13 Index of Teacher Career Satisfaction (TCS) $\ldots \ldots \ldots \ldots$. . . . . . . . . . . . 273

How Safe Are Schools? . . . . . . . . . . . . . . . . . . . . . . . . . . . . . . . . . 275

Exhibit 7.14 Index of Parents' Perceptions of School Environment (PPSE) . . . . . . . . . . . . . 276

Exhibit 7.15 Index of Student Safety in School (SSS) . . . . . . . . . . . . . . . . . . . . 277

Exhibit 7.16 Index of Principals' Perception of School Safety (PPSS) with Trends . . . . . . . . 279 
Appendix A

Supplementary Information About PIRLS 2006 Procedures

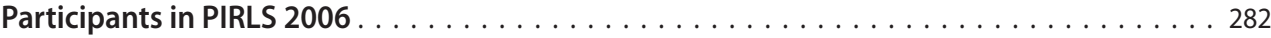

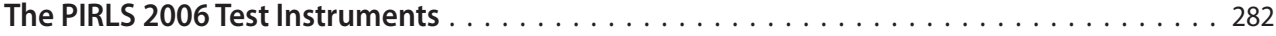

Exhibit A.1 Countries Participating in PIRLS 2006 and 2001 . . . . . . . . . . . . . . . . 283

Exhibit A.2 Distribution of Items by Reading Purpose and Process Category . . . . . . . . . . 285

PIRLS Testing Time and Booklet Design $\ldots \ldots \ldots \ldots \ldots \ldots \ldots \ldots \ldots \ldots \ldots \ldots \ldots$

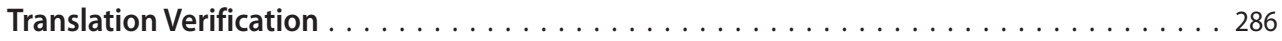

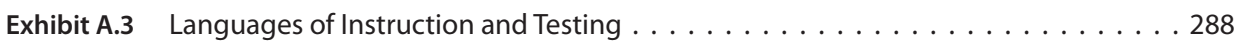

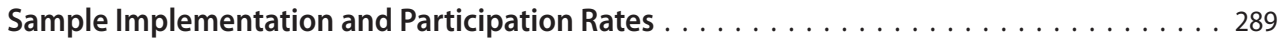

Exhibit A.4 Coverage of PIRLS Target Population. . . . . . . . . . . . . . . . . . . 292

Exhibit A.5 School Sample Sizes . . . . . . . . . . . . . . . . . . . . . . . . . . 293

Exhibit A.6 Student Sample Sizes . . . . . . . . . . . . . . . . . . . . . . . . . . . . . 294

Exhibit A.7 Participation Rates (Weighted) . . . . . . . . . . . . . . . . . . . . . . . . . 295

Exhibit A.8 Trends in Student Populations. . . . . . . . . . . . . . . . . . . . . . . . . . . . . . 296

Exhibit A.9 Percentage of Students with Any Available Student, Parent, Teacher, and

Principal Questionnaire Data. . . . . . . . . . . . . . . . . . . . . . . . . . 297

Exhibit A.10 Percentage of Students with One or More Teachers . . . . . . . . . . . . . . . . 298

Data Collection . . . . . . . . . . . . . . . . . . . . . . . . . . . . . . . . 299

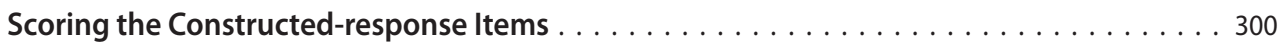

Exhibit A.11 PIRLS Within-country Constructed-response Scoring Reliability Data . . . . . . . . 302

Exhibit A.12 PIRLS 2006 Trend Scoring Reliability (2001-2006) for the Constructed-response

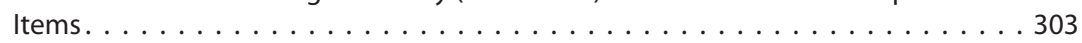

Exhibit A.13 PIRLS Cross-country Constructed-response Scoring Reliability . . . . . . . . . . . 304

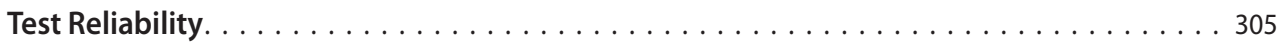

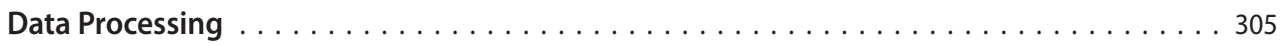

Exhibit A.14 Cronbach's Alpha Reliability Coefficient - Overall Reading . . . . . . . . . . . 306

IRT Scaling and Data Analysis . . . . . . . . . . . . . . . . . . 307

Exhibit A.15 Correlation Between Two Scales for Purposes and Two Scales for Processes for Reading. . . . . . . . . . . . . . . . . . . . . . . . . . . 309

Exhibit A.16 Average Percent Correct by PIRLS 2006 Scale . . . . . . . . . . . . . . . 311

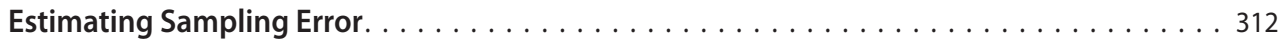

Reporting Student Achievement at the PIRLS 2006 International Benchmarks . . . . . . 312

Appendix B . . . . . . . . . . . . . . 317 Multiple Comparisons of Average Achievement in Reading Purposes and Processes of Comprehension

Exhibit B.1 Multiple Comparisons of Average Reading Achievement for Literary Purposes. . . . 318

Exhibit B.2 Multiple Comparisons of Average Reading Achievement for Informational Purposes . . . . . . . . . . . . . . . . . . . . . . . . . 320

Exhibit B.3 Multiple Comparisons of Average Reading Achievement for Retrieving and Straightforward Inferencing Processes . . . . . . . . . . . . . . . . . 322

Exhibit B.4 Multiple Comparisons of Average Reading Achievement for Interpreting, Integrating, and Evaluating Processes . . . . . . . . . . . . . . . . . . . . 324 
Appendix C. . . . . . . . . . . . . . . . . 327

Percentiles and Standard Deviations of Reading Achievement

Exhibit C.1 Percentiles of Achievement in Reading . . . . . . . . . . . . . . . . . . . . 328

Exhibit C.2 Standard Deviations of Achievement in Reading . . . . . . . . . . . . . . . . . 329

Exhibit C.3 Percentiles of Achievement in Literary Reading . . . . . . . . . . . . . . . 330

Exhibit C.4 Standard Deviations of Achievement in Literary Reading. . . . . . . . . . . . . . 331

Exhibit C.5 Percentiles of Achievement in Informational Reading . . . . . . . . . . . . . . . 332

Exhibit C.6 Standard Deviations of Achievement in Informational Reading . . . . . . . . . . . . 333

Exhibit C.7 Percentiles of Achievement in Reading for Retrieval and Straightforward

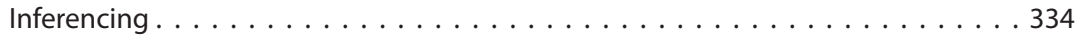

Exhibit C.8 Standard Deviations of Achievement in Reading for Retrieval and Straightforward Inferencing . . . . . . . . . . . . . . . . 335

Exhibit C.9 Percentiles of Achievement in Reading for Interpreting, Integrating, and

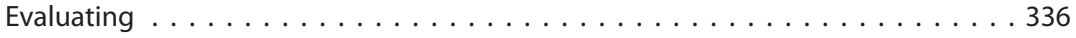

Exhibit C.10 Standard Deviations of Achievement in Reading for Interpreting, Integrating, and Evaluating. . . . . . . . . . . . . . . . . . . . . . . . . . . 337

Appendix D. . . . . . . . . . . . . . . . 339

Sample Passages, Questions, and Scoring Guides

Appendix E . . . . . . . . . . . . . . 381

Item Descriptions Developed During the PIRLS 2006 Benchmarking

Appendix F . . . . . . . . . . . . . . . . 393

Iceland and Norway - Fifth Grade Reading Achievement

Exhibit F.1 Iceland-Selected Reading Achievement Results for the Fifth Grade . . . . . . . . . 394

Exhibit F.2 Norway_-Selected Reading Achievement Results for the Fifth Grade . . . . . . . . 395

Appendix G. . . . . . . . . . . . . . . . . 397

Organizations and Individuals Responsible for PIRLS 2006 



\section{Executive Summary}

With PIRLS 2006, the second in the PIRLS (Progress in International Reading Literacy Study) series of studies, the IEA (International Association for the Evaluation of Educational Achievement) continues its mission of conducting comparative studies of student achievement in school subjects to inform educational policies and practices around the world. For almost 50 years, the IEA has carried out studies on a wide range of topics and subjects including mathematics, science, civics, and technology, as well as reading. By providing a cross-national perspective on education systems and on school organizational and instructional practices, and by measuring trends in student achievement, IEA studies have made significant contributions to increasing understanding of the educational process.

Inaugurated in 2001 and conducted every 5 years, PIRLS is IEA's assessment of students' reading achievement at fourth grade. Administered in 35 countries in 2001, PIRLS in 2006 was implemented in 40 countries, including Belgium with 2 educational systems and Canada with 5 provinces (45 participants in total). The success of PIRLs depends on a collaborative effort among the research centers in each country responsible for data collection and implementing the key aspects of the project, as well as on the network of international centers responsible for managing the across-country tasks, such as training country representatives in standardized procedures, selecting comparable samples of schools and students, developing instruments, and conducting the various steps required for data processing and analysis. 
Building on the PIRLS 2001 framework, PIRLS 2006 assessed a range of reading comprehension processes within two major reading purposesliterary and informational. The assessment included a variety of passages drawn from materials that students encounter in their everyday experiences inside and outside school. More than half the questions were in the constructed-response format, requiring students to generate and write their answers. Example passages, questions, and scoring guides can be found in Appendix D and the pocket at the back of this report.

To provide national contexts for understanding the reading achievement results, PIRLS 2006 collected a broad array of background information about students' home and school experiences in learning to read. Countries contributed chapters to the PIRLS 2006 Encyclopedia and completed questionnaires describing their education systems, reading curricula, and resources for teaching reading. Students' parents, teachers, and school principals, as well as the students themselves completed questionnaires covering various aspects of home literacy support, school environment, and classroom instruction.

All aspects of PIRLS 2006 were conducted with concerted attention to quality. Countries met rigorous standards for sampling designed to prevent bias and ensure comparability. Translating the tests and questionnaires involved a detailed iterative review process, and numerous training sessions were held in data collection and scoring procedures. Prior to analysis, each country's data were subjected to exhaustive checks for consistency and comparability across countries.

\section{Fourth-grade Students' Reading Achievement in PIRLS 2006}

- The Russian Federation, Hong Kong SAR, and Singapore were the three top-performing countries in PIRLS 2006. Luxembourg, Italy, Hungary, Sweden, Germany, the Netherlands, Belgium (Flemish), Bulgaria, and Denmark also had higher achievement than the majority of other participants. Three Canadian provinces (Alberta, British Columbia, and Ontario) were also among the highest achieving participants. 
- Girls had significantly higher reading achievement than boys in all except two countries, Luxembourg and Spain, where average achievement was equivalent between the sexes.

- For about half the PIRLS 2006 participants, almost all (95\% or more) of their students demonstrated at least some basic reading competencies, by achieving at or above the Low International Benchmark (e.g., could locate explicitly stated information in the texts and make some straightforward inferences). Six participants had 99 percent of their students reaching this level, including Luxembourg, Hong Kong SAR, Belgium (Flemish), the Netherlands, and Lithuania, as well as the Canadian province of Alberta.

- In general, about half the participants had three fourths of their students reach the Intermediate International Benchmark (e.g., could locate different parts of the texts and identify sequences), and about two fifths reach the High International Benchmark (e.g., could distinguish embedded details and provide explanations for overall messages or ideas).

- Singapore and the Russian Federation had the greatest percentages of high-achieving students, with nearly one fifth of students (19\%) reaching the Advanced International Benchmark (e.g., could provide and support interpretations, integrate information across texts, and understand literary and organizational features). However, about half of the participants had 7 percent or fewer of their students reaching the highest benchmark.

- The PIRLS 2006 participants with the highest average achievement overall, also tended to have the highest average achievement when the results were examined separately for literary and informational reading and for the comprehension processes.

- Despite their level of reading achievement overall, however, most of the PIRLS 2006 participants had relative strengths and weaknesses: relatively higher achievement in reading for literary purposes compared to informational purposes or the reverse (relatively higher achievement in informational reading than literary reading), and relatively higher achievement either in the retrieving and straightforward inferencing processes or in the interpreting, integrating, and evaluating processes. 


\section{Changes in Reading Achievement: PIRLS 2001 to PIRLS 2006}

- Of the 26 countries and 2 Canadian provinces that also participated in PIRLS 2001, eight countries showed significant gains in average reading achievement in PIRLS 2006, including the Russian Federation, Hong Kong SAR, Singapore, Slovenia, the Slovak Republic, Italy, Germany, and Hungary. Countries with significant decreases since 2001 were Lithuania, the Netherlands, Sweden, England, Romania, and Morocco.

- Typically, in the countries with higher average achievement in 2006 than in 2001, achievement in 2006 was higher for both girls and boys, and, in the countries with lower average achievement, achievement was lower for both. Exceptions were Germany and Hungary where boys but not girls had significant gains, and the Netherlands where the decrease seemed to be primarily attributable to girls' lower achievement.

- In general, higher average achievement in 2006 than 2001 involved increases spanning the performance distributions. Singapore, Hong Kong SAR, and Slovenia had significant improvement across the four PIRLS 2006 International Benchmarks (low, intermediate, high, and advanced). The Russian Federation, the Slovak Republic, and Germany had improvement at all except the low benchmark, and Italy had improvements at all except the advanced benchmark. Hungary showed improvement at the advanced level.

- For countries with decreases since PIRLS 2001, Lithuania and the Netherlands had decreases at the two highest benchmarks, England and Sweden had decreases at all except the low benchmark, and Romania had decreases across the distribution.

- Countries with higher average reading achievement in 2006 than 2001 tended to have higher average achievement in both the reading purposes and processes of comprehension, and, similarly, those with lower reading achievement in 2006 than 2001 tended to have lower achievement in both the reading purposes and processes. For example, the eight countries with overall increases in average reading achievement between PIRLS 2001 and PIRLS 2006 all improved in literary reading and the majority improved in informational reading (Hong Kong SAR, 
the Russian Federation, Singapore, Slovenia, Germany, and Italy). Most improved in retrieving and straightforward inferencing processes (except Italy and Hungary) and all improved in interpreting, integrating, and evaluating processes.

\section{Home Activities Fostering Reading Literacy}

- In both PIRLS 2001 and PIRLS 2006, there was a positive relationship between students' reading achievement at the fourth grade and parents having engaged their children in early literacy activities before starting school (e.g., reading books, telling stories, singing songs, playing with alphabet toys, and playing word games). Although parents generally reported a fairly high level of engagement in PIRLS 2001, it was encouraging that parents in 14 countries reported increases in PIRLS 2006.

- The presence of children's books in the home also continued to show a strong positive relationship with reading achievement. The average reading achievement difference between students from homes with many children's books (more than 100) and those from homes with few children's books (10 or fewer) was very large ( 91 score points, almost 1 standard deviation). On average across countries, there was a slight decrease in parents' reports of the number of children's books in the home, perhaps reflecting increased access to Internet-based literacy media.

- As was the case in PIRLS 2001, average reading achievement in PIRLS 2006 was highest among students whose parents were frequent readers. However, 13 of the participants in both assessments had decreases in the percentages of students whose parents reported reading for more than 5 hours a week, and none had increases. In PIRLS 2006, on average across countries, 37 percent of the fourth-grade students had parents who read more than 5 hours a week, 43 percent for 1 to 5 hours, and 20 percent for less than 1 hour a week.

- In both PIRLS 2001 and PIRLS 2006, reading achievement was highest for students whose parents had favorable attitudes toward reading. In PIRLS 2006 , on average across countries, the majority of students (52\%) had parents with favorable attitudes. Decreases for six participants in the 
percentages of students whose parents had favorable attitudes towards reading were balanced by increases for seven participants.

\section{Students' Attitudes and Reading Habits}

- In PIRLS 2001, and again in PIRLS 2006, students with the most positive attitudes toward reading had the highest reading achievement. In PIRLS 2006, internationally, about half the students (49\%), on average, agreed with five statements about enjoying reading and appreciating books. However, this reflected decreases in nine countries compared to four countries with increases. (There also was a decrease in the Canadian province of Ontario.)

- There was a good deal of agreement between students' perceptions of themselves as good or poor readers and their reading achievement. Internationally, about the half the students, on average, had "high" self-concepts of themselves as readers, agreeing with four statements about their reading ability. Ten participants showed increased percentages of students with positive self-concepts in 2006 compared with 2001, and eight participants (including both Canadian provinces) had decreased percentages.

- In PIRLs 2006, on average across countries, students reported reading stories and novels outside of school more frequently than informational materials (e.g., magazines, catalogs, and instructions), with more decreases than increases in daily reading for both the literary and informational categories. On average, nearly one third of students (32\%) reported reading stories or novels outside of school every day or almost every day (reflecting decreases for 12 countries and increases for eight), and an additional one third (31\%) reading them at least once a week (reflecting three decreases and four increases). In contrast, 16 percent of the students, on average, reported reading informational materials on a daily basis (reflecting seven decreases compared to two increases), and 43 percent reading such materials on a weekly basis (one decrease and three increases). (The Canadian province of Quebec increased in daily literary reading, but decreased in both daily and weekly informational reading, and Ontario decreased in daily informational reading.) 
- On average across countries, in PIRLS 2006, students reported spending more time in a typical day reading stories and articles in books or magazines than on the Internet (1.4 hours vs. 1.o hours). On average, girls reported more time than boys reading from books or magazines (1.5 hours vs. 1.3 hours) and this difference was found for almost every participant. In comparison, on average, boys reported more time than girls reading on the Internet (1.o hours vs. 0.9 hours), a pattern found for approximately half the participants.

- In PIRLS 2006, on average across countries, 40 percent of the students reported reading for fun on a daily basis, and 28 percent at least weekly. However, almost one third of students (32\%) internationally reported reading for fun only twice a month or less. Although there were decreases for four participants, unfortunately, seven countries had increases in the percentages of students who reported reading for fun only twice a month or less.

\section{School Curriculum and Organization for Teaching Reading}

- Internationally, in PIRLS 2006, there was a positive relationship between fourth-grade students' reading achievement, on average, and the amount of time spent in preprimary education. According to parents' reports, 81 percent of the students, on average, had attended more than 1 year of preprimary education, although there was considerable variation from country to country.

- In both PIRLS 2001 and PIRLS 2006, parents' assessments of their children's early literacy skills corresponded well with reading achievement at the fourth grade. According to their parents, nearly one third of the students in PIRLS 2006, on average across countries, entered school able to perform early literacy activities very well (i.e., recognize most of the alphabet, read some words, read sentences, write letters of the alphabet, and write some words).

- This represented increased percentages for 17 participants since PIRLS 2001, and no decreases (except for the Canadian province of Ontario). 
- Principals generally agreed with parents, but were a little less positive about these same early literacy skills for students entering their schools, and reported somewhat less improvement. Internationally, principals reported that 20 percent of the students, on average, were in schools where most children (more than 75\%) entered school with these skills. There were increases for five participants together with a decrease in Slovenia (and the two Canadian provinces). At the other end of the continuum, principals reported that 44 percent of the students, on average, were in schools where relatively few children (less than 25\%) entered school able to perform these literacy skills. There were decreases for nine participants compared to three increases.

- Internationally, schools placed considerable emphasis on the reading curriculum and instruction. In PIRLS 2006, four fifths of students, on average across countries, were in schools that had informal initiatives to encourage students to read, and half in schools with programs to help teachers improve instruction and with guidelines for coordinating instruction across grades. Almost three fourths of students, on average, were in schools that placed more emphasis on reading than on other school subject areas.

- In PIRLS 2006, 78 percent of students, on average, were taught reading by teachers who reported frequent use of a variety of organizational approaches. Among the various approaches, however, the most popular one was teaching reading as a whole-class activity-used always or almost always, on average across countries, for 35 percent of the students.

- Across the PIRLS 2006 countries, the average class size for fourth-grade reading instruction was 24 students. The range in average class size varied from 17 students in Luxembourg to 42 in South Africa. Among the participants in both PIRLS 2001 and PIRLS 2006, more than half had a reduction in average class size of between one and three students since 2001.

- In PIRLS 2006, teachers reported that 17 percent of their students, on average across countries, were in need of remedial reading instruction. In nearly every country, the percentage of students needing remedial 
reading instruction exceeded the percentage who received remedial instruction. On average across countries, 60 percent of the fourthgrade students were attending schools without access to a remedial reading specialist.

\section{Teachers and Reading Instruction}

- In PIRLS 2006, the majority of students (57\%), on average across countries, were taught reading by teachers whose studies emphasized a combination of pedagogy, language, and literature. Another 19 percent, on average, had teachers whose studies emphasized language and/or literature, and 7 percent an emphasis on pedagogy.

- Across countries in PIRLS 2006, the students' teachers had been teaching for an average of 17 years. Most of the students (91\%), on average, were taught reading by full-time teachers.

- In PIRLS 2006, both principals and teachers reported that textbooks were the foundation of reading instruction, supplemented by other materials. Internationally, 90 percent of the students, on average, had teachers who used textbooks at least once or twice a week. Workbooks and worksheets also were used weekly (for $82 \%$ of students, on average) as were reading series ( $60 \%$ of students) and children's books (55\% of students).

- In PIRLS 2006, on average across countries, the percentage of students whose teachers asked them to read literary texts (e.g., short stories and chapter books) at least once a week was greater than the percentage asked to read informational texts (e.g., descriptions and explanations) that frequently ( $84 \%$ vs. $58 \%$ ). Across countries, teachers reported that 70 percent of students, on average, were asked to read short stories at least weekly and 36 percent were asked to read chapter books.

- Across the countries participating in PIRLS 2006, teachers reported asking students to use a variety of reading skills and strategies at least once or twice a week, including identifying main ideas (90\% of students, on average), supporting understanding with evidence from the text ( $91 \%$ of students), comparing reading with their own experiences ( $72 \%$ 
of students), and making generalizations and drawing inferences ( $71 \%$ of students).

- In PIRLs 2006, both teachers and students agreed that independent silent reading was a frequent classroom activity. Internationally, 65 percent of fourth-grade students, on average, reported reading silently on their own as a daily activity, and a further 27 percent reading silently at least weekly. Across countries, students reported reading aloud less frequently, with 20 percent, on average, reporting reading aloud daily and 34 percent weekly.

- In PIRLS 2006, teachers reported using various techniques to query students about their reading. About three fourths of the students, on average, were asked to write answers in workbooks or worksheets at least weekly, and 62 percent were asked to answer questions aloud. More than half (57\%), on average, wrote responses to what they read at least weekly. Only about one fourth were given a weekly written quiz or text about what they had read.

- Internationally, almost all students (89\%), on average, attended schools with libraries, and 69 percent had access to classroom libraries. In PIRLS 2006, teachers reported that about half the students, on average, could borrow books from their classroom library to take home, and that 50 percent of the students were given opportunities to visit a library other than the classroom library at least weekly. On average, 40 percent of the students reported borrowing library books on a weekly basis, and another 28 percent reported borrowing library books once or twice a month.

- On average across the countries in PIRLS 2006,65 percent of the students had access to computers in school, and 57 percent were in schools with Internet access. Since PIRLS 2001, there was a substantial increase, primarily in the Eastern European countries, in the percentages of students having access to computers and the Internet. However, the percentage of fourth-grade students with computer access differed greatly across countries. 
- In PIRLS 2006, teachers reported that 30 percent of the students, on average across countries, had used instructional software to develop their reading skills (reflecting increases in 14 countries), and 39\% had read stories or other texts on the computer (reflecting increases in 17 countries).

\section{School Contexts}

- Internationally, the reading achievement of students in schools with few disadvantaged students (no more than 10\%) was much higher (56 scale score points, more than half a standard deviation) than for students with a high percentage of disadvantaged classmates (more than 50\% disadvantaged economically). According to school principals, in PIRLS 2006 about two fifths of students (39\%), on average across countries, were in schools with few students from disadvantaged homes. This average percentage reflected an increase in seven countries and one decrease since PIRLS 2001. On average, 18 percent of students were in schools with a high percentage of disadvantaged students, reflecting a decrease in four countries, but also an increase in three countries.

- In PIRLS 2006, principals reported that about half the students (52\%), on average across countries, were attending schools that were not hampered by resource shortages. However, 15 percent, on average, were attending schools where principals reported that resource shortages greatly affected the provision of reading instruction. Although the situation varied from country to country, on average, there was a positive relationship between an absence of school resource shortages and average reading achievement.

- Internationally, according to principals' reports in PIRLS 2006, nearly half the students (48\%), on average, attended schools emphasizing home-school involvement, whereas about one fourth $(27 \%)$ attended schools with little communication between the school and the home and little parental participation in the life of the school. There was a modest positive relationship between the level of home-school involvement and average reading achievement. 
- On average across countries, in PIRLs 2006 most students were in schools where principals reported that absenteeism either was not a problem (37\% of students) or was a minor problem $(40 \%)$. As was the case in PIRLS 2001, students in these schools had higher average reading achievement than students attending schools where absenteeism was a serious problem ( $9 \%$ of students on average). Across the categories, absenteeism was less of a problem in the Russian Federation, Iceland, and Macedonia in PIRLS 2006 than PIRLS 2001. On the other hand, the attendance problem appears to have grown worse in France, Latvia, Romania, and Morocco.

- Internationally, the majority of teachers had a positive view of the teaching profession and their career as a teacher. In PIRLS 2006, on average, 64 percent of students had teachers who agreed with five positive statements about their careers and teaching. Interestingly, there was a correspondence with parents' views. On average, 60 percent of the students had parents who reported a good deal of satisfaction with their child's school, agreeing with four positive statements about the quality of education and attention provided by the school.

- In PIRLS 2006, students who agreed that they felt safe in their schools and reported minimal, if any, incidents of stealing, bullying, and injury had higher reading achievement than those who did not agree that they felt safe and reported at least several such incidents involving themselves and their classmates. Across countries, 47 percent of the students, on average, reported an atmosphere of safety, 50 percent a moderately safe school, and only a small percentage $(3 \%)$ reported being in unsafe schools.

- Complementing students' perceptions, on average across countries, principals reported a fairly high level of school safety. In PIRLS 2006, principals reported that 60 percent of students were in schools where safety was not a problem, and 32 percent were in schools where it was a minor problem at most. On average, and, in many countries, there was a positive relationship between principals' perception of school safety and average reading achievement. 




\section{Introduction}

In today's information society, the ability to read is essential for maximizing success in the endeavors of daily life, continuing intellectual growth, and realizing personal potential. Similarly, a literate citizenry is vital to a nation's social growth and economic prosperity. To help countries make informed decisions about reading education, IEA's Progress in International Reading Literacy Study (PIRLS) provides internationally comparative data about students' reading achievement in primary school (the fourth grade in most participating countries). The fourth grade is an important transition point in children's development as readers, because most of them should have learned to read, and are now reading to learn. 
The IEA (International Association for the Evaluation of Educational Achievement) is an independent international cooperative of national research institutions and governmental agencies with a permanent secretariat based in Amsterdam, the Netherlands. For the past 50 years, IEA has been conducting large-scale comparative studies of educational achievement to gain a deeper understanding of the effects of policies and practices within and across systems of education internationally.

\section{What Is PIRLS?}

PIRLS 2006 continues IEA's series of highly significant international studies in reading literacy. As an important event in its 50-year history of educational research, IEA marked the beginning of the $21^{\text {st }}$ century by inaugurating PIRLS to monitor international trends in primary school reading achievement on a 5-year cycle. PIRLS 2001 was conducted in 35 countries around the world on the tenth anniversary of IEA's 1991 Reading Literacy Study. ${ }^{1}$ This provided participants an opportunity to obtain 10-year change measures linking back to $1991^{2}$ and to lay the foundation for measuring trends into the future.

All the countries, institutions, and agencies involved in PIRLS 2001 worked collaboratively to design and implement the most innovative and comprehensive measure of reading achievement possible within the constraints of a large-scale international assessment. ${ }^{3}$ As such, PIRLs 2001 was based on a newly developed framework, describing the interaction between two major reading purposes (literary and informative) and a range of four comprehension processes. The assessment itself was based on a variety of "authentic" texts taken from children's reading materials, and included a special PIRLs Reader printed in color. About half the questions asked students to write out their answers.

Conducted in 40 countries, including Belgium with 2 education systems and Canada with 5 provinces (45 participants in total), PIRLS 2006 continued the collaborative effort among participants to improve PIRLs' primary purpose of providing policy and instructionally relevant information about reading achievement in primary schools. Building on PIRLS 2001, every effort was made to use state-of-the-art methods in constructing the reading

Elley, W.B. (Ed). (1994). The IEA study of reading literacy: Achievement and instruction in thirty-two school systems. Oxford, England: Elsevier Science Ltd.

2 Martin, M.O., Mullis I.V.S., Gonzalez, E.J., \& Kennedy, A.M. (2003). Trends in children's reading literacy achievement 1991-2001: IEA's repeat in nine countries of the 1991 Reading Literacy Study. Chestnut Hill, MA: Boston College.

3 Mullis, I.V.S., Martin, M.O., Gonzalez, E.J., \& Kennedy, A.M. (2003). PIRLS 2001 international report: IEA's study of reading literacy achievement in primary schools in 35 countries. Chestnut Hill, MA: Boston College. 
assessment, and to collect a full array of contextual information about home and school environments for learning to read. Most notably, parents and caregivers in almost all countries provided information about students' early literacy activities and environments. In addition, to advance its mission of improving the teaching and learning of reading, PIRLS 2006 collected information about classrooms and schools via a full range of student, teacher, and principal questionnaires. This enables PIRLS 2006 to provide information about students' achievement in relation to the different types of curricula, instructional practices, and school environments found in countries around the world. The variation across the participating countries provides a unique opportunity to study different approaches to educational practice and how these can improve achievement.

In addition to this report, the PIRLS 2006 Assessment Framework and Specifications ${ }^{4}$ describes the conceptual framework and design of the study. The PIRLS 2006 Encyclopedia: A Guide to Reading Education in the Forty PIRLS 2006 Countries $^{5}$ is intended to complement the achievement results by providing a sense of the educational settings in each country, including the national and regional contexts for reading instruction. The PIRLS 2006 Technical Report ${ }^{6}$ describes the methods and procedures used for instruments development, sampling, data collection, and analysis. The full set of PIRLS 2006 reports can be obtained from the TIMSS \& PIRLS International Study Center (web: http://isc.bc.edu).

\section{What Was the Nature of the PIRLS 2006 Test of Reading Comprehension?}

As described in the PIRLS 2006 Assessment Framework and Specifications, purposes for reading and processes of comprehension are the foundation of the PIRLS 2006 assessment of reading comprehension. The two purposes for reading are: 1) For literary experience and 2) To acquire and use information. The four processes of comprehension are: 1) Focus on and retrieve explicitly stated information, 2) Make straightforward inferences, 3) Interpret and integrate ideas and information, and 4) Examine and evaluate content, language, and textual elements. The four processes were assessed within each of the two major purposes for reading.

Mullis, I.V.S., Kennedy, A.M., Martin, M.O., \& Sainsbury, M. (2006). PIRLS 2006 assessment framework and specifications (2nd ed.). Chestnut Hill, MA: Boston College.

5 Kennedy, A.M., Mullis, I.V.S., Martin, M.O., \& Trong, K.L. (Eds.). (2007). PIRLS 2006 encyclopedia: A guide to reading education in the forty PIRLS 2006 countries. Chestnut Hill, MA: Boston College.

6 Martin, M.O., Mullis, I.V.S., \& Kennedy, A.M. (Eds.). (2007). PIRLS 2006 technical report. Chestnut Hill, MA: Boston College. 
An important innovation in PIRLS 2006 is the ability to report the achievement results according to reading comprehension processes, in addition to reading purposes. In the PIRLS 2001 International Report, the achievement results were reported for reading comprehension overall and by literary and informational purposes. Research by several countries and by the TIMSS \& PIRLS International Study Center indicated that the results also could be reported by comprehension process, especially if the total assessment was increased from 8 passages and item sets to 10 passages and item sets. ${ }^{7,8}$

In PIRLS 2006, the reading purposes and comprehension processes were assessed based on 10 passages, 5 for the literary purpose and 5 for the informational purpose. Altogether, the assessment consisted of 126 items (see Appendix A for details). Each passage was accompanied by approximately 12 questions (test items), with about half in the multiple-choice format and half in the constructed-response format, requiring students to write their own answers. Four of the 10 passages and item sets ( 2 literary and 2 informational) were retained from PIRLS 2001 to provide a foundation for measuring trends in reading achievement; the remaining 6 were developed specifically for the 2006 assessment. That is, PIRLS 2006 included three newly developed literary passages and item sets, and three newly developed informational passages and item sets.

Developing the instruments for the PIRLS 2006 assessment was a cooperative venture, involving the National Research Coordinators (NRCs) from the participating countries throughout the entire process. Identifying prospective passages began even before the first NRC meeting for PIRLS 2006, so that initial review could take place and a consensus be established about characteristics of desirable texts. Primarily, with the aim of motivating students as much as possible, there was agreement about searching for texts that would interest fourth-grade students in general, and, in particular, boys as well as girls. In PIRLS 2001, girls had significantly higher achievement than boys in every country so efforts were made to make the passages equally interesting to both genders. More than 100 texts were submitted, reviewed, and, mostly, discovered to not be suitable for PIRLs due to various concerns.

7 Bos, W., Lankes, E. M., Prenzel, M., Schwippert, K., Walther, G., \& Valtin, R. (Hrsg.). (2003). Ergebnisse aus IGLU: Schülerleistungen am Ende der vierten Jahrgangsstufe im internationalen Vergleich. New York: Waxmann.

8 Mullis, I.V.S., Martin, M.O., Gonzalez, E.J. (2004). PIRLS international achievement in the processes of reading comprehension: Results from PIRLS 2001 in 35 countries. Chestnut Hill, MA : Boston College. 
However, eventually the NRCs selected six literary and six informational text passages for field testing.

To develop the items based on the text passages identified for the field test, the TIMSS \& PIRLS International Study Center conducted an itemwriting workshop for NRCs and their colleagues. The items were drafted at the workshop, reviewed extensively by reading and measurement specialists, and produced in booklets for the field test, with extensive translation and layout verification along the way. Please see Appendix A for information about the translation and verification process. Participating countries field tested the items with representative samples of students, and all of the potential new items were reviewed by the PIRLS 2006 Reading Development Group of internationally recognized experts. On the basis of the field-test data and the recommendations of the PIRLS 2006 Reading Development Group, the NRCs selected three literary and three informational passages and the related item sets for inclusion in the PIRLS 2006 assessment.

In PIRLS 2006, the 10 passages and item sets were distributed across 13 test booklets, each consisting of two 40-minute sections, with each section containing a passage with its item set. Each student completed one test booklet. Eight of the passages were paired in different combinations throughout 12 of the booklets according to a plan that enabled linking the booklets. Appendix A contains further detail about the PIRLS 2006 design and testing time.

To present at least some of the assessment in a more natural, authentic setting, two passages (one literary and one informational) were presented in color in a magazine format with the questions in a separate booklet. A copy of this booklet, referred to as the PIRLS 2006 Reader, is found in the back pocket of this report. Appendix D contains the question/answer booklet for the reader, two other PIRLS 2006 passages and item sets (one literary and one informational) being released to the public, and the scoring guides for the released constructed-response items. 


\section{What Background Information Is Available About the Contexts for Students Learning to Read?}

Primarily, fourth-grade students develop reading literacy skills, behaviors, and attitudes at home and in school. However, the experiences and instruction that students have at school and home often are affected by the community and the country in which students live and attend school. Cultural, social, and economic factors can all influence the success a country has in educating its children. Thus, PIRLS 2006 incorporated several approaches to collecting background information.

To provide information about the national and regional contexts for reading education, the PIRLS 2006 Encyclopedia, ${ }^{9}$ consisting of a chapter prepared by each country, provides an important resource for interpreting the achievement results. The encyclopedia provides a perspective on the structure and organization of the education system in each country, and describes the policies and reading curriculum pertaining to the educational level and grade in school of the students that were assessed (typically, the primary-school curriculum pertaining to students in the fourth year of schooling). In addition, each chapter describes teacher education and training, instructional resources and materials used in teaching reading, availability of specialists, and assessment practices. To collect some basic information, each country was also responsible for completing the online administration of the PIRLS 2006 Reading Curriculum Questionnaire.

The PIRLS 2006 Learning to Read Survey was completed by the parents or caregivers of the students who participated in the assessment. This questionnaire included questions about children's early literacy activities, parents' estimates of their children's early literacy skills, home resources supporting literacy, parents' attitudes and habits regarding reading, and parents' occupation.

Each student was asked to complete a background questionnaire. The PIRLS 2006 Student Questionnaire was the vehicle for collecting information about the students' reading behaviors and attitudes. Students also were asked about their classroom instruction. Each student's reading teacher was asked to

9 Kennedy, A.M., Mullis, I.V.S., Martin, M.O., \& Trong, K. L. (2007). PIRLS 2006 encyclopedia: A guide to reading education in the forty PIRLS 2006 countries. Chestnut Hill, MA: Boston College. 
complete the PIRLS 2006 Teacher Questionnaire. The questionnaire collected information about the classroom organization and instructional approaches used to teach reading, the resources used, and assessment strategies, as well as information about teachers' educational training. The PIRLS 2006 School Questionnaire, completed by the principal or school head, was designed to collect information about overall school policies and resources, as well as the role of the principal in the school.

\section{Which Countries Participated in PIRLS 2006?}

The decision to participate in an IEA study is coordinated through the IEA Secretariat in Amsterdam and made solely by each member country according to its own data needs and resources. Exhibit 1 shows the 40 countries that participated in PIRLS 2006. More specifically, as part of IEA's long history, some practices have become established across the decades. That is, with distinct education systems of their own, England and Scotland have always participated separately in IEA studies, as has Hong Kong, so in the report these entities are treated as countries. Traditionally, the two major geographic and cultural regions of Belgium, the French-speaking part and the Dutchspeaking part (Flanders), have separate education systems and participate separately. Canada currently participates in IEA as a country, however, education is primarily a provincial matter and several provinces were early members of IEA. For PIRLS 2006, the Canadian provinces worked with IEA procedurally and financially so that they could be reported separately but not collectively as a country, even though they represent 88 percent of the student population in Canada.

Of the participants in PIRLS 2006, Exhibit 1 shows that 26 countries and 2 provinces also participated in PIRLS 2001 (displayed in orange). For these participants, the report includes data about changes between the two assessments. The PIRLs community also was extremely pleased to welcome 13 new countries (including both separate education systems in Belgium) and 3 new provinces to the study (displayed in red). Altogether, there were 45 participants. (For a complete listing of the participants in PIRLS 2001 as well as those in PIRLS 2006, please see Appendix A.) 


\title{
2006 and 2001
}

\author{
Bulgaria \\ Canada, Ontario \\ Canada, Quebec \\ England \\ France \\ Germany \\ Hong Kong SAR \\ Hungary \\ Iceland \\ Iran, Islamic Rep. of \\ Israel \\ Italy \\ Latvia \\ Lithuania \\ Macedonia, Rep. of \\ Moldova, Rep. of \\ Morocco \\ Netherlands \\ New Zealand \\ Norway \\ Romania \\ Russian Federation \\ Scotland \\ Singapore \\ Slovak Republic \\ Slovenia \\ Sweden \\ United States
}

\section{6}

Austria

Belgium (Flemish)

Belgium (French)

Canada, Alberta

Canada, British Columbia

Canada, Nova Scotia

Chinese Taipei

Denmark

Georgia

Indonesia

Kuwait

Luxembourg

Poland

Qatar

South Africa

Spain

Trinidad and Tobago 


\section{Exhibit 1 Countries Participating in PIRLS 2006 (Continued)}

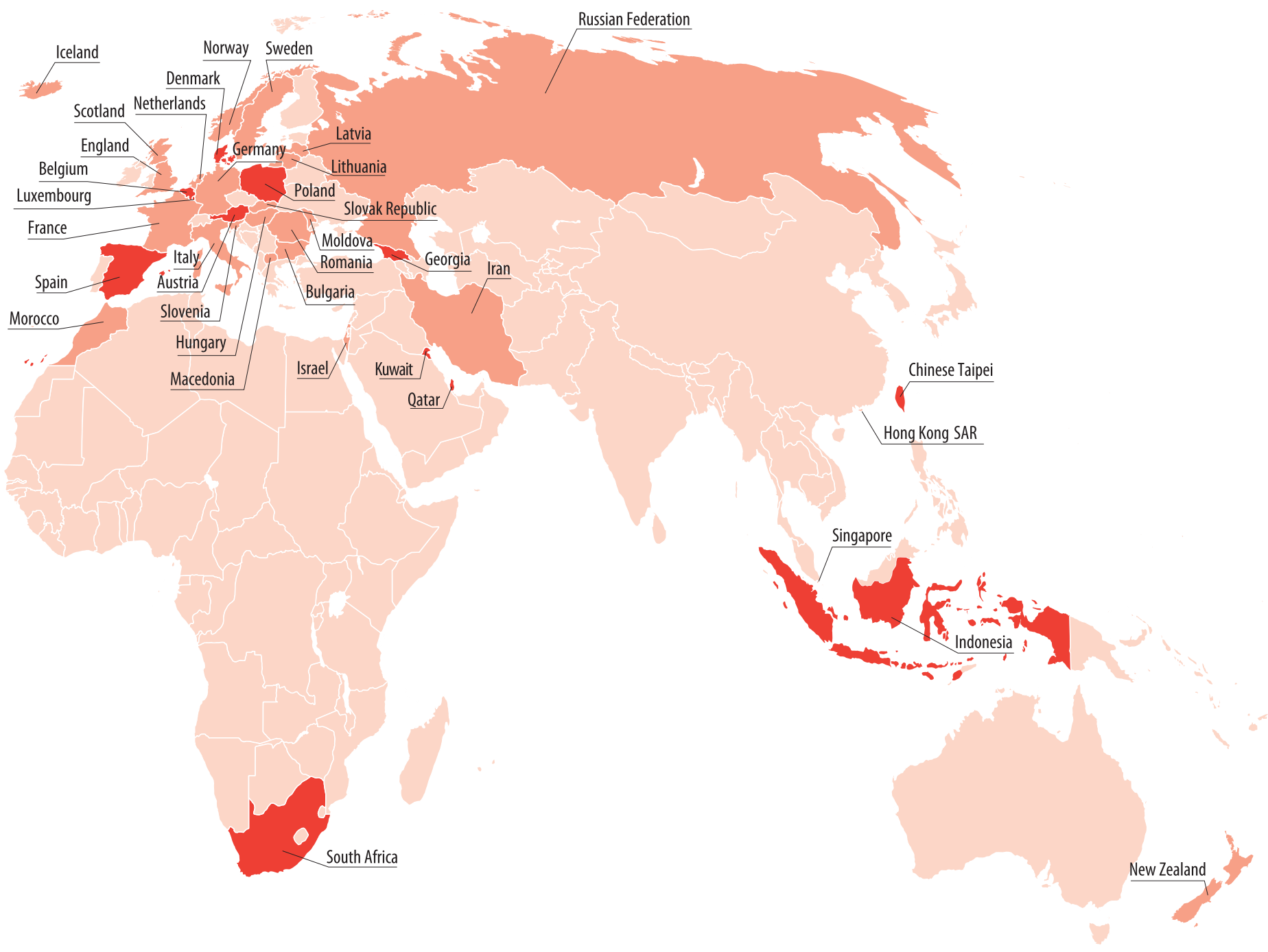


For the sake of comparability across participants, testing was conducted at the end of the school year. Thus, almost all of the countries tested in April through June on a Northern Hemisphere school schedule. The three countries on a Southern Hemisphere school schedule (New Zealand, Singapore, and South Africa) tested in October through December of 2005. It is important to note, however, that in PIRLS 2001 the Southern Hemisphere testing also was in October through December, but in calendar year 2001 (after the Northern Hemisphere testing instead of before it). Thus, for the two Southern Hemisphere countries that participated in both PIRLS 2001 and PIRLS 2006-New Zealand and Singapore-the changes in the report are over a 4-year period rather than a 5-year period.

PIRLS 2006 provides valuable comparative information across countries about students' reading achievement, reading curriculum, instructional practices, and school resources. However, it is important to consider the results in light of country-wide demographic and economic factors. Some selected demographic characteristics of the PIRLS 2006 countries and provinces are presented in Exhibit 2. As can be seen, the countries and education systems that participated in PIRLS 2006 vary widely in population size and geographic area. The participants also vary widely on indicators of health, such as life expectancy at birth and infant mortality rate. Most of the participants had a life expectancy of 75 to 81 years and a low infant mortality rate. However, several had a relatively lower life expectancy of 66 to 69 years and relatively high infant mortality rates, including Indonesia, Iran, Moldova, Morocco, and the Russian Federation. South Africa had a life expectancy of 46 years and the highest infant mortality rate. 
The economic indicators in Exhibit 2, such as gross national income per capita, reveal great disparity in the economic resources available, and also that different policies exist about the percentage of funds that are spent on education. Economically, the PIRLS 2006 countries ranged from Luxembourg and Norway at the high end to Georgia, Indonesia, and Moldova at the low end. Although many of the PIRLS 2006 participants had 99 to 100 percent of their fourth-grade (or grade assessed) students in school, there were differences in enrollment rates. Finally, pupil-teacher ratios ranged from 10 to 12 for a number of participants to 28 in Morocco and 35 in South Africa. 
Exhibit 2 Selected Characteristics of PIRLS 2006 Countries

PIRLS 2006

4th Grade

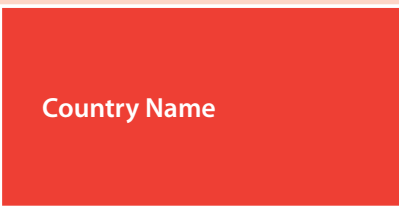

Population
Size
(in Millions) $^{1}$

Area of
Country
$(1000$ Square
Kilometers $)^{2}$

Population
Density
(People per
Square
Kilometer)

Urban
Population
${\text { (\% of Total })^{4}}^{4}$

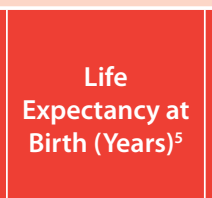

Infant Mortality

\begin{tabular}{|c|c|c|c|c|c|c|}
\hline Austria & 8.1 & 84 & 98 & 68 & 79 & 5 \\
\hline Belgium (French and Flemish) & 10.4 & 31 & 343 & 98 & 78 & 4 \\
\hline Bulgaria & 7.8 & 111 & 71 & 68 & 72 & 12 \\
\hline${ }^{15}$ Canada, Alberta & 3.4 & 662 & 5 & 81 & 80 & 6 \\
\hline${ }^{15}$ Canada, British Columbia & 4.3 & 945 & 4 & 85 & 81 & 4 \\
\hline${ }^{15}$ Canada, Nova Scotia & 0.9 & 55 & 17 & 56 & 79 & 5 \\
\hline${ }^{15}$ Canada, Ontario & 12.5 & 1076 & 13 & 85 & 80 & 6 \\
\hline${ }^{15}$ Canada, Quebec & 7.6 & 1542 & 6 & 80 & 79 & 5 \\
\hline${ }^{17}$ Chinese Taipei & 22.8 & 4 & 633 & 79 & 79 & 5 \\
\hline Denmark & 5.4 & 43 & 127 & 85 & 77 & 4 \\
\hline 17 England & 50.0 & 130 & 380 & 90 & 79 & 5 \\
\hline France & 59.8 & 552 & 109 & 76 & 79 & 4 \\
\hline Georgia & 5.1 & 70 & 74 & 57 & 74 & 41 \\
\hline${ }^{10}$ Germany & 82.5 & 357 & 237 & 88 & 78 & 4 \\
\hline Hong Kong SAR & 6.8 & 1 & 6541 & 100 & 80 & 3 \\
\hline Hungary & 10.1 & 93 & 110 & 65 & 73 & 8 \\
\hline Iceland & 0.3 & 103 & 3 & 93 & 80 & 3 \\
\hline Indonesia & 214.7 & 1905 & 119 & 44 & 67 & 31 \\
\hline Iran, Islamic Rep. of & 66.4 & 1648 & 41 & 66 & 69 & 33 \\
\hline Israel & 6.7 & 22 & 308 & 92 & 79 & 5 \\
\hline${ }^{2}$ Italy & 57.6 & 301 & 196 & 67 & 80 & 4 \\
\hline Kuwait & 2.4 & 18 & 135 & 96 & 77 & 8 \\
\hline Latvia & 2.3 & 65 & 37 & 60 & 71 & 10 \\
\hline Lithuania & 3.5 & 65 & 55 & 69 & 72 & 8 \\
\hline Luxembourg & 0.4 & 3 & 173 & 93 & 78 & 5 \\
\hline Macedonia, Rep. of & 2.1 & 26 & 81 & 60 & 74 & 10 \\
\hline Moldova, Rep. of & 4.2 & 34 & 129 & 42 & 67 & 26 \\
\hline Morocco & 30.1 & 447 & 68 & 57 & 69 & 36 \\
\hline${ }^{16}$ Netherlands & 16.2 & 42 & 479 & 90 & 79 & 5 \\
\hline New Zealand & 4.0 & 271 & 15 & 86 & 79 & 5 \\
\hline Norway & 4.6 & 324 & 15 & 76 & 79 & 3 \\
\hline Poland & 38.2 & 313 & 125 & 63 & 75 & 6 \\
\hline${ }^{13}$ Qatar & 0.8 & 11 & 72 & 93 & 75 & 11 \\
\hline Romania & 21.7 & 238 & 95 & 56 & 70 & 18 \\
\hline Russian Federation & 143.4 & 17075 & 9 & 73 & 66 & 16 \\
\hline${ }^{7}$ Scotland & 5.1 & 78 & 66 & 81 & 77 & 5 \\
\hline${ }^{4}$ Singapore & 4.3 & 1 & 6343 & 100 & 78 & 3 \\
\hline Slovak Republic & 5.4 & 49 & 111 & 58 & 73 & 7 \\
\hline 2 Slovenia & 2.0 & 20 & 99 & 49 & 76 & 4 \\
\hline South Africa & 45.8 & 1219 & 38 & 59 & 46 & 53 \\
\hline Spain & 41.1 & 506 & 82 & 78 & 80 & 4 \\
\hline Sweden & 9.0 & 450 & 22 & 83 & 80 & 3 \\
\hline Trinidad and Tobago & 1.3 & 5 & 256 & 75 & 72 & 17 \\
\hline United States & 299.0 & 9629 & 32 & 78 & 77 & 7 \\
\hline
\end{tabular}

All data taken from the World Bank's World Development Indicators Online, retrieved April 19, 2007, unless otherwise noted.

Data are from most recent year available.

A dash (-) indicates that data are not available.

NOTE: Data provided for Belgium (French and Flemish) are for the entire country of Belgium.

1 Includes all residents regardless of legal status or citizenship except refugees not permanently settled in the country of asylum as they are generally considered to be part of their country of origin. Data for Qatar provided by NRC.

2 Area is the total surface area in square kilometers, comprising all land area, inland bodies of water, and some coastal water way.
3 Midyear population divided by land area in square kilometers. Data for Qatar provided by NRC.

4 Urban population is the midyear population of areas defined as urban in each country and reported to the United Nations. It is measured here as the percentage of the total population.

5 Number of years a newborn infant would live if prevailing patterns of mortality at its birth were to stay the same throughout its life.

6 Infant mortality rate is the number of infants who die before reaching one year of age, per 1,000 live births in a given year.

7 GNI per capita in U.S. dollars is converted using the World Bank Atlas method.

8 An international dollar has the same purchasing power over GNI as a U.S. dollar in the United States. 
Exhibit 2 Selected Characteristics of PIRLS 2006 Countries (Continued)

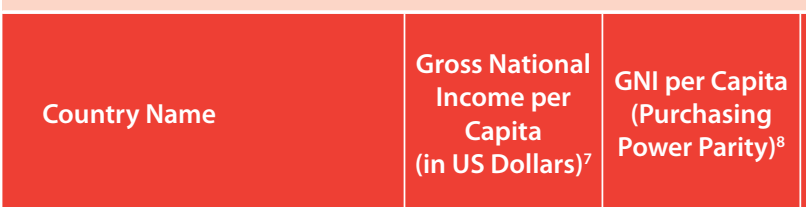

\begin{tabular}{c|c|}
$\begin{array}{c}\text { Public } \\
\text { Expenditure } \\
\text { on Education } \\
(\% \text { of GDP })^{9}\end{array}$ & $\begin{array}{c}\text { Net Enrollment } \\
\text { Ratio in Primary } \\
\text { Education } \\
\left(\begin{array}{c}\% \text { of relevant } \\
\text { group })^{10}\end{array}\right.\end{array}$
\end{tabular}

Primary
Pupil-Teacher
Ratio

Austria
Belgium (French and Flemish)
Bulgaria

\begin{tabular}{|r|r|}
\hline 26810 & 29740 \\
\hline 25760 & 28920 \\
\hline 2130 & 7540 \\
\hline
\end{tabular}

${ }^{15}$ Canada, Alberta

${ }^{15}$ Canada, British Columbia

38628

${ }^{15}$ Canada, Nova Scotia

${ }^{15}$ Canada, Ontario

${ }^{15}$ Canada, Quebec

35518

17 Chinese Taipei

$$
42812
$$

Denmark

${ }^{17}$ England

France

$$
\text { Georgia }
$$

${ }^{10}$ Germany

Hong Kong SAR

Hungary

Iceland

Indonesia

Iran, Islamic Rep. of

Israel

12 Italy

Kuwait

Latvia

Lithuania

Luxembourg

29856

13970

33570

-

24730

770
25270

\begin{tabular}{l}
6.0 \\
6.0 \\
4.0 \\
\hline
\end{tabular}

\begin{tabular}{r|r|}
99 \\
100 \\
90
\end{tabular}

\begin{tabular}{l|l}
5.0 & 100
\end{tabular}

5.0

-

6.0
7.0
5.0

35534
28940

14030

31050

8.0
4.0

100
100

13
12

100
100
100

$+\frac{1}{17}$

17

Macedonia, Rep. of 457

Moldova, Rep. of 590

\begin{tabular}{|r} 
Morocco \\
\hline
\end{tabular}

${ }^{16}$ Netherlands $\quad 26230$

\begin{tabular}{|l|l|}
\hline New Zealand & 15530 \\
\hline
\end{tabular}

$$
\text { Norway }
$$

Poland

${ }^{13}$ Qatar

Romania

Russian Federation

27640

27640

4.0
9.0

27610

25860

6350
30910

28680

13840

30570

9.0
6.0
6.0

100

6.0
2.0

2.0
5.0

\begin{tabular}{|r|r|l|}
\hline 5.0 & 100 & 14 \\
\hline 4.0 & 98 & 20 \\
\hline 5.0 & 91 & 10 \\
\hline 6.0 & 100 & 11 \\
\hline
\end{tabular}

\begin{tabular}{|r|r|l|}
\hline 5.0 & 100 & 14 \\
\hline 4.0 & 98 & 20 \\
\hline 5.0 & 91 & 10 \\
\hline 6.0 & 100 & 11 \\
\hline
\end{tabular}

\begin{tabular}{r|r}
810 & 3210 \\
\hline
\end{tabular}

810
2010
16240

\begin{tabular}{l|l}
3210 & 1.0 \\
7000 & 5.0 \\
\hline
\end{tabular}

\begin{tabular}{|r|r|l|}
\hline 6.0 & 100 & 11 \\
\hline 1.0 & 92 & 21 \\
\hline 5.0 & 87 & 24 \\
\hline 7.0 & 100 & 12 \\
\hline
\end{tabular}

${ }^{17}$ Scotland

14 Singapore

Slovak Republic

${ }^{12}$ Slovenia

South Africa

Spain

Sweden

Trinidad and Tobago

United States

21570

19440

26830

19480
10210

11390

55500

7.0
5.0

7.0
5.0

4400

4500

$1980 \quad 6750$

$590 \quad 1760$

6750
1760

3940

$$
28560
$$

21350

21350
37910

43400
5280

11210

$\begin{array}{rr}5280 & 29607 \\ - & 7140\end{array}$

8.0
6.0

6.0
6.0
4.0

4.0

4.0
5.0

5.0
7.0

7.0
5.0
7.0

5.0
7.0
7.0

7.0
6.0
4.0

3.0
3.0

7140
8950

2260
2610

2610
21230

4940

11920

2750

17040

28910

28910
7790

37870

-
24180
13440
19100
10130
22150
26710
10390
37750

3.0
3.0
6.0

6.0
4.0

4.0
4.0
6.0

\begin{tabular}{|c|c|}
\hline 100 & 12 \\
\hline 99 & 11 \\
\hline 83 & 13 \\
\hline 88 & 14 \\
\hline 94 & 16 \\
\hline 96 & 12 \\
\hline 92 & 21 \\
\hline 79 & 19 \\
\hline 90 & 28 \\
\hline 99 & 14 \\
\hline 100 & 18 \\
\hline 100 & 10 \\
\hline 98 & 11 \\
\hline 95 & 12 \\
\hline 88 & 17 \\
\hline 99 & 17 \\
\hline 100 & 16 \\
\hline 96 & 24 \\
\hline 87 & 19 \\
\hline 100 & 13 \\
\hline 89 & 35 \\
\hline 100 & 14 \\
\hline 100 & 12 \\
\hline 91 & 19 \\
\hline 93 & 15 \\
\hline
\end{tabular}

4.0
6.0
5.0

5.0
4.0
7.0
4.0

4.0
6.0

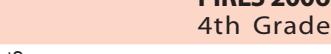




\section{Which Students Were Tested for PIRLS 2006?}

Exhibit 3 contains information about the grade tested in each country, together with information about the age at which students begin school and promotion policies. The last column shows the average age of the students assessed. Because PIRLs studies the effectiveness of curriculum and instruction on students' learning, it is designed to assess reading achievement at the same point in schooling across countries. In particular, the target grade should be the grade that represents 4 years of schooling, counting from the first year of ISCED Level 1. ISCED stands for the International Standard Classification of Education developed by the UNESCo Institute for Statistics. ${ }^{10}$ Level 1 corresponds to primary education or the first stage of basic education. The first year of Level 1 should mark the beginning of "systematic apprenticeship of reading, writing and mathematics". However, IEA has a policy that children should be at least 9 years old before being asked to participate in a paper-and-pencil assessment such as PIRLS. Thus, as a policy, PIRLS also tries to ensure that, at the time of testing, students do not fall under the minimum average age of 9.5 years old.

Exhibit 3 reveals that, with few exceptions, the grade tested in each country represented the fourth year of formal schooling. Thus, solely for convenience, the report usually refers to the grade tested as the fourth grade. In addition to the information listed in Exhibit 3, Iceland and Norway assessed smaller samples of students in the fifth grade. Selected information about these students is provided in Appendix F.

Exhibit 3 also shows that countries have different policies and practices about the age of entry to primary school. To provide additional information about actual practices, parents were asked at what age their child started school, and, considering issues such as immigration, there was agreement with the country reports (see Chapter 5). More than half of the PIRLS 2006 participants reported that policy and actual practice was for children to begin school at age 6. Depending on such aspects as whether or not the policy is according to calendar year, this would make students assessed at the end of their fourth year of schooling approximately 10.5 years old, and this was 
the case for most countries. However, in England, New Zealand, Scotland, and Trinidad and Tobago children begin school at age 5. Although these countries assessed students in the fifth grade according to the PIRLS policy, their students were still among the youngest (9.9 to 10.3 years old).

In most of the Eastern European countries as well as Sweden and Denmark, as a matter of policy and, especially, practice, children begin school at age 7 , and students in these countries were among the oldest (10.6 to 11.0). Finally, because of challenges presented by multiple native languages and languages of instruction in South Africa and in Luxembourg, these two countries tested the fifth grade even though it meant students were older. In an attempt to conduct the assessment in each student's language of instruction, South Africa tested in 11 different languages. In Luxembourg, the assessment was conducted in German, which is the language of reading instruction, but usually is either the student's second or a foreign language. Please see Exhibits 3.11 and A.3 for more information about the languages spoken in the home, the languages of instruction, and the languages of testing. Also, for each participant, the PIRLS 2006 Encyclopedia describes the languages spoken, and the languages of instruction.

Policies on promotion and retention also can affect how old students are when they reach a particular grade. Promotion in primary schools was automatic for approximately half of the PIRLS 2006 countries, but in the others, promotion depended on academic achievement. Because the lower achievers are the most likely to be retained and, consequently, be older for their grade, in these countries, the older students often have lower achievement.

Because of the many policies and practices involved, the interaction between grade and age in school can be extremely complicated. The variations in policies and practices across the countries resulted in a range in the average age of students assessed. Although students averaged between 10 and 11 years old in most of the countries, because grade and age are fundamental factors in considering the achievement results, this information is reproduced in conjunction with the achievement results in Exhibit 1.1. 
Exhibit 3 Information about the Students Tested for PIRLS 2006

\begin{tabular}{|c|c|c|c|}
\hline Country Name & $\begin{array}{l}\text { Country's Name } \\
\text { for Grade Tested }\end{array}$ & $\begin{array}{l}\text { Policy on Age of Entry } \\
\text { to Primary School }\end{array}$ & $\begin{array}{l}\text { Practice on Age of Entry } \\
\text { to Primary School }\end{array}$ \\
\hline Austria & Grade 4 & 6 & 6 \\
\hline Belgium (Flemish) & Grade 4 & 6 & 6 \\
\hline Belgium (French) & Grade 4 & 6 & 6 \\
\hline Bulgaria & Grade 4 & 7 & 6 or 7 \\
\hline Canada, Alberta & Grade 4 & 6 & 5 \\
\hline Canada, British Columbia & Grade 4 & 5 & 5 \\
\hline Canada, Nova Scotia & Grade 4 & 5 & 5 \\
\hline Canada, Ontario & Grade 4 & 6 & Between 5 and 6 \\
\hline Canada, Quebec & Second year of elementary cycle 2 & 6 & 6 \\
\hline Chinese Taipei & Grade 4 & 6 & Between 6 and 7 \\
\hline Denmark & Grade 4 or 4 th form & 7 & 7 \\
\hline England & Year 5 (Y5) & 5 & Between 4 and 5 \\
\hline France & $\begin{array}{c}\text { CM1 = Mean Course 1st year, or Second year } \\
\text { of the 3rd Cycle - (Deepenings Cycle) }\end{array}$ & 6 & 6 \\
\hline Georgia & Grade 4 & 6.5 & 6.5 \\
\hline Germany & Grade 4 & 6 & 6 \\
\hline Hong Kong SAR & Primary 4 & 6 & 6 \\
\hline Hungary & Grade 4 & Between 6 and 8 & 7 \\
\hline Iceland & Grade 4 & 6 & 6 \\
\hline Indonesia & Grade 4 & 7 & 6 \\
\hline Iran, Islamic Rep. of & Grade 4 & 6 & 6 \\
\hline Israel & Grade 4 & 6 & 6 \\
\hline Italy & Primary school - fourth class & 5 & 6 \\
\hline Kuwait & Grade 4 & 6 & 6 \\
\hline Latvia & Grade 4 & 7 & 7 \\
\hline Lithuania & Grade 4 & 6 & 7 \\
\hline Luxembourg & 5th year of primary studies & 6 & 6 \\
\hline Macedonia, Rep. of & Grade 4 & Between 6 and 7 & Between 6 and 7 \\
\hline Moldova, Rep. of & Grade 4 & 6 & Between 6 and 7 \\
\hline Morocco & - & - & - \\
\hline Netherlands & Group 6 & 6 & 6 \\
\hline New Zealand & Year 5 & 6 & $\begin{array}{c}\text { Continuous entry into school; children begin } \\
\text { on or soon after 5th birthday }\end{array}$ \\
\hline Norway & Grade 4 & 6 & 6 \\
\hline Poland & Grade 3 of primary school & 6 & 6 \\
\hline Qatar & Grade 4 & 6 & 6 \\
\hline Romania & Grade 4 & 7 & Between 6 and 7 \\
\hline Russian Federation & Grade 4 & Between 6.5 and 7 & 7 \\
\hline Scotland & Primary 5 / P5 & 5 & Between 4.5 and 5.5 \\
\hline Singapore & Primary 4 & 6 & 6 \\
\hline Slovak Republic & Grade 4 & 6 & 6 \\
\hline Slovenia & $\begin{array}{l}\text { Grade } 4 \text { of 9-year elementary school; } \\
\text { Grade } 3 \text { of 8-year elementary school }\end{array}$ & $\begin{array}{l}6 \text { for } 9 \text {-year elementary school; } \\
7 \text { for } 8 \text {-year elementary school }\end{array}$ & $\begin{array}{l}6 \text { for } 9 \text {-year elementary school; } \\
7 \text { for } 8 \text {-year elementary school }\end{array}$ \\
\hline South Africa & Grade 5 & Year students turn 7 & 6 \\
\hline Spain & Grade 4 & 6 & 6 \\
\hline Sweden & Grade 4 & 7 & 7 \\
\hline Trinidad and Tobago & Standard Three (3) & 5 & 5 \\
\hline United States & Grade 4 & Varies by state; typically 6 & 6 \\
\hline
\end{tabular}


Exhibit 3 Information about the Students Tested for PIRLS 2006 (Continued)

\begin{tabular}{|c|c|c|}
\hline Country Name & Policy on Promotion / Retention & $\begin{array}{l}\text { Average Age } \\
\text { at Time of } \\
\text { Testing }\end{array}$ \\
\hline
\end{tabular}

Austria
Belgium (Flemish)
Belgium (French)
Bulgaria
Canada, Alberta
Canada, British Columbia
Canada, Nova Scotia
Canada, Ontario
Canada, Quebec
Chinese Taipei
Denmark
England

Depends on results of teacher assessments throughout the year

10.3
Automatic, though students may decide to repeat a grade

Student may not be retained in the same grade more than twice

Automatic

Varies by school board

Automatic for grades 1-4; 0ther grades are decided by teacher, principal, and parents

France Varies by school board

Varies by school board

Automatic for most students

Automatic

Automatic

Automatic for most students

Georgia
Germany
Hong Kong SAR
Hungary
Iceland
Indonesia
Iran, Islamic Rep. of
Israel
Italy
Kuwait
Latvia
Lithuania

Luxembourg

Students must meet competencies, as decided by teacher

Automatic

Varies by federal state

Automatic

10.5

Automatic in grades 1-3; Dependent on academic progress in grades 4-8 10.7

$\begin{array}{lr}\text { Automatic } & 9.8\end{array}$

None

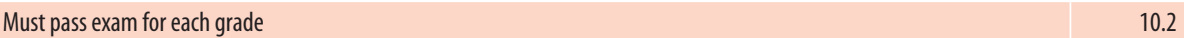

Automatic for most students $\quad 10.1$

\begin{tabular}{l|l} 
Essentially automatic, though students must make satisfactory progress & 9.7
\end{tabular}

Students must pass school-developed tests at each grade $\quad 9.8$

Depends on satisfactory performance in final assessments, as well recommendations by teacher and parents $\quad 11.0$

Depends on academic progress, and is discussed with parents 10.7

Depends on academic performance; Students can be retained by teachers if results are unsatisfactory in 2 of 3 main subjects $\quad 11.4$

(German, French, Mathematics)

Macedonia, Rep. of

Automatic for grades 1-4; Dependent on academic progress for grades 5-8

Moldova, Rep. of Automatic

Automatic for most students

New Zealand

Normally automatic, subject to parent/principal decisions

Automatic

9.8

Poland

Automatic for the preparatory grade; 0ther grades are decided by teaching staff, though retention in grades $1-3$ is rare

Qatar Students must pass Arabic exam each year

Russian Federation

Dependent on academic progress

Scotland Automatic for most students

Singapore

Automatic for grades 1-3; Dependent on academic progress for grades 4-6

Slovak Republic

Dependent on academic progress; students can repeat the same grade only once

Slovenia None

\section{South Africa}

Spain

Sweden

Trinidad and Tobago

United States
Students can repeat a grade once per phase, after which promotion is automatic Dependent on achievement of basic competencies; students in grades 1-6 can repeat a grade only once Automatic Dependent on academic progress Varies by state
11.9

9.9


The student sampling for PIRLS 2006 was conducted with careful attention to quality and comparability. Staff from Statistics Canada worked with the participants on all phases of the sampling activities. The Statistics Canada sampling experts provided training and, in conjunction with the PIRLS 2006 sampling referee (Keith Rust, Westat, Inc.), reviewed national sampling plans, sampling data, sampling frames, and sample selections. The sampling documentation was used by the TIMSS \& PIRLS International Study Center to evaluate the quality of the samples. As presented in the "Sample Implementation and Participation Rates" section of Appendix A, country coverage was typically good, with most countries sampling about 150 schools and approximately 4,00o students (see Exhibits A.4 to A.6). The participation rates were generally high (see Exhibit A.7), but in a few cases the PIRLS 2006 sampling guidelines were not met, and there are annotations to this effect in Exhibit 1.1 and subsequent tables.

PIRLS made every effort to attend to the quality and comparability of the data through careful planning and documentation, cooperation among participating countries, standardized procedures, and rigorous attention to quality control throughout. For example, an extensive series of verification checks were conducted to ensure the comparability of the test translations, and detailed documentation was required to satisfy adherence to the sampling standards. Appendix A contains further descriptions of the procedures used, and more detailed information is provided in the PIRLS 2006 Technical Report. Appendix G describes and lists the organizations and individuals responsible for implementing PIRLS 2006.

\section{$\diamond \diamond \diamond$}

This report benefited from extensive reviews by National Research Coordinators and their staff, and by members of IEA's Publications and Editorial Committee: David F. Robitaille (Chair), Robert A. Garden, and Nancy Law. 




\section{Chapter 1}

\section{International Student Achievement in Reading}

Chapter 1 presents the reading comprehension achievement results for students in their fourth year of formal schooling for the 40 countries, including Belgium with 2 education systems and Canada with 5 provinces, that participated in PIRLS 2006 (45 participants in total). The chapter begins with a discussion of students' achievement in PIRLS 2006, and then presents changes in achievement over the past 5-year period for those countries that also participated in PIRLS 2001. The reading comprehension achievement results for 2006 and changes from 2001 also are provided by gender. Next, the chapter presents the corresponding achievement results for the reading purposes and processes of reading comprehension described in the PIRLS 2006 Assessment Framework and Specifications. ${ }^{1}$ The two reading purposes are Literary and Informational. Achievement in the processes of reading comprehension is reported for two categories: (1) Retrieving and straightforward inferencing and (2) Interpreting, integrating, and evaluating.

\section{How Do Countries Differ in Reading Achievement?}

Exhibit 1.1 displays the distributions of achievement for PIRLS 2006 for 40 participating countries, including Belgium with 2 education systems and Canada with 5 provinces. Historically, because they have separately administered education systems, England and Scotland, as well as Hong Kong, prior to becoming a Special Administrative Region (SAR) of the People's Republic of China, have participated separately in IEA studies and 
this practice continues in TIMSS and PIRLS to monitor trends and have comparability between the two studies. For the purposes of this report, these three education systems are treated as countries. Since Belgium has two education systems, one administered by the French-speaking community and the other by the Dutch-speaking community, the two education systems traditionally have participated separately in IEA studies, so again, this practice has been kept and two sets of data are reported for Belgium. The five Canadian provinces represent 88 percent of the student population in Canada, but preferred to participate in PIRLS 2006 separately but not collectively as a country. Thus, as a compromise, their results are reported in italics together with the other participants. Altogether, then, the tables in the PIRLS 2006 International Report typically contain results for the 45 participants in PIRLS 2006. For their own purposes as an additional effort, Iceland and Norway administered PIRLS 2006 to small samples of their fifthgrade students, and these results are presented in Appendix F.

In Exhibit 1.1, the 45 participants are shown in descending order of average reading achievement. Each participant's average score on the PIRLS achievement scale (with its $95 \%$ confidence interval) is shown graphically on the participant's achievement distribution, and listed (together with its standard error) in the first column in the table. Because there often are relatively small differences between participants in average achievement, Exhibit 1.2 shows whether or not the differences in average achievement are statistically significant.

The highest achieving participants in PIRLS 2006 represent different regions of the world geographically, including Eastern Europe (the Russian Federation, Hungary, and Bulgaria), Asia (Hong Kong saR and Singapore), Canada (Alberta, British Columbia, and Ontario), Italy in Southern Europe, Western Europe (Luxembourg, Germany, the Netherlands, and Belgium (Flemish)), and Scandinavia (Sweden and Denmark). The PIRLS reading achievement scale was established in PIRLS 2001 to have a mean of 500 and a standard deviation of $100,{ }^{2}$ and was designed to remain constant from assessment to assessment. There is an indication by a participant's average

2 PIRLS uses item response theory scaling (IRT) methods to summarize achievement results on a scale with a mean of 500 and a standard deviation of 100. For more information, see the "IRT Scaling and Data Analysis" section of Appendix A. 
Exhibit 1.1 Distribution of Reading Achievement

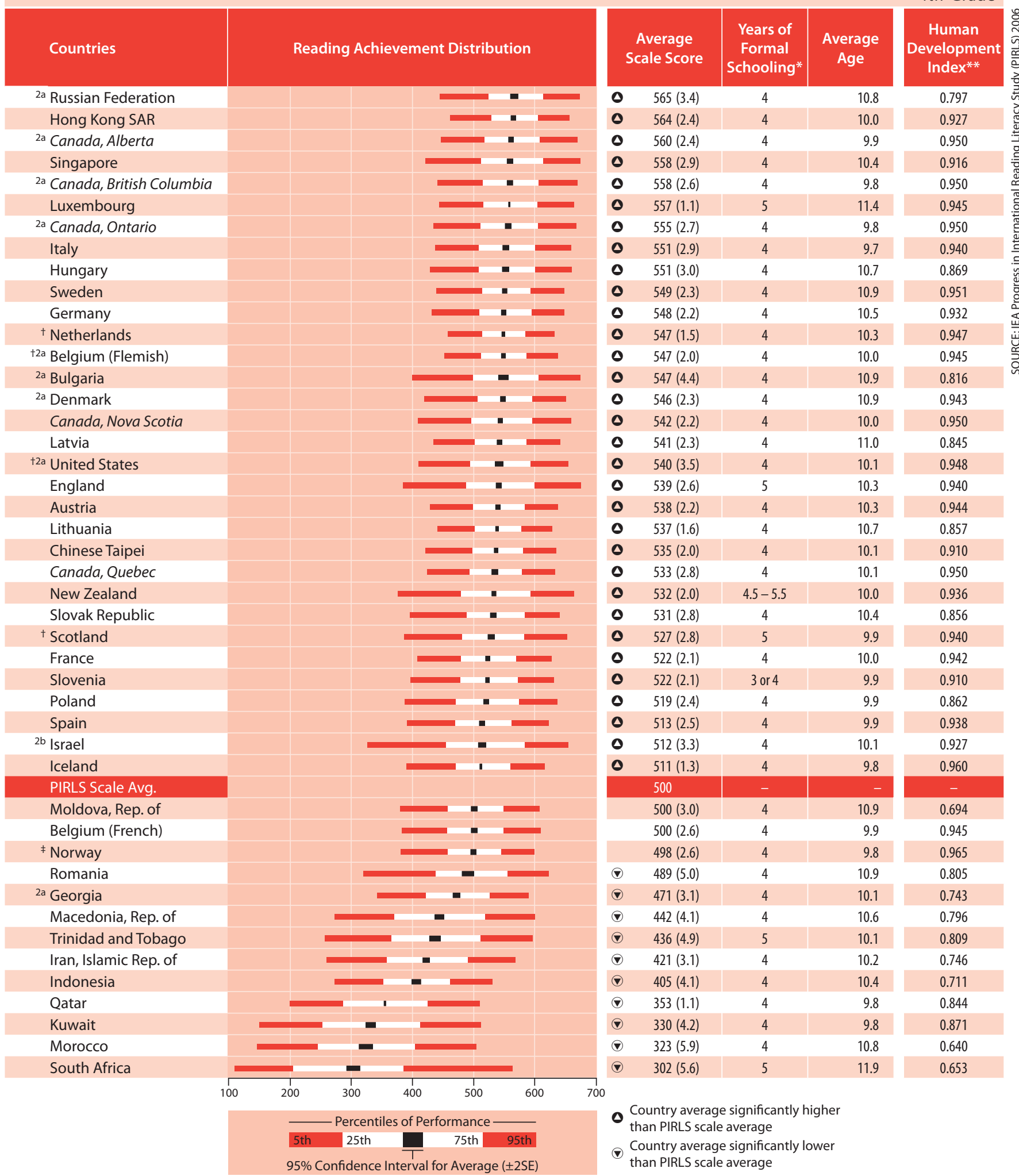

PIRLS 2006

4th Grade

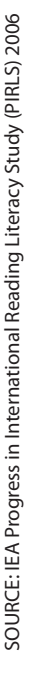


scale score, if the average achievement is significantly higher (up arrow) or lower (down arrow) than the scale average of 500.

The graph of the achievement distributions indicates the ranges in performance for the middle half of students $\left(25^{\text {th }}\right.$ to $75^{\text {th }}$ percentiles) and the extremes $\left(5^{\text {th }}\right.$ and $95^{\text {th }}$ percentiles). Although it was to a differing degree from country to country, by the fourth year of schooling, every PIRLS 2006 participant had some percentage of students who were good readers and some percentage of students who demonstrated difficulties. The majority of countries had approximately a 250-point difference between the $5^{\text {th }}$ and $95^{\text {th }}$ percentiles of achievement, although some had larger differences and others had more homogeneity in performance. It is important to note that the range in achievement in most countries is comparable to the difference in average achievement ( 263 points) between the highest performing country, the Russian Federation, and lowest performing country, South Africa.

PIRLS devoted considerable energy to maximizing comparability across the grades and ages tested, but this is difficult considering the variation internationally in many educational policies, such as school entry ages and the number of languages of instruction. Exhibit 1.1 shows that, in accordance with the PIRLs guidelines, most countries assessed students in their fourth year of formal schooling. Thus, for convenience in this report, the students will be referred to as fourth-grade students even though several countries did not assess students in the fourth grade. In consultation with the PIRLS sampling specialists, Slovenia included some students in third grade because the country is in transition toward having students start school at a younger age so they will have 4 years of primary schooling instead of 3 years, but the transition is not complete. Also, in accordance with PIRLs guidelines, since their students start school at a very early age and otherwise would have been very young, four countries (England, New Zealand, Scotland, and Trinidad and Tobago) tested the fifth year of schooling. Two other countries also tested the fifth year because of challenges concerning the language(s) of instruction (Luxembourg and South Africa), which resulted in their students being older, on average, than the rest of the students tested. 
Given that students are in their fourth year of schooling and the majority begin school at age 6, as presented in Exhibit 3, they are expected to be approximately 10 years old. Typically, as was the case in most of the countries (and all provinces), students would average from 9.7 to 10.6 years old, depending on whether they started school at age 6 or 7 and when during the calendar year they started school (January, the beginning of the school year, or some other time). In a few countries, primarily in Eastern Europe, students do not start school until age 7 and consequently were a little older (10.7 to 11 years old). As would be anticipated in PIRLS with a wide range of cultural and economic diversity, higher performing countries included those with younger and older students, on average, and lower performing countries also had students averaging from the youngest to the oldest.

To provide some context about the economic and educational development of the PIRLS 2006 participants, Exhibit 1.1 also includes each one's value on the Human Development Index ${ }^{3}$ provided by the United Nations Development Programme. The index has a minimum value of 0.0 and a maximum of 1.o. Countries with high values on the index have a long life expectancy, high levels of school enrollment and adult literacy, and a good standard of living, as measured by per capita Gross Domestic Product. The majority of the PIRLS 2006 participants had index values greater than 0.9, and most with values this high performed above the 500 scale average, except Belgium (French) and Norway that were approximately at the average. The countries with averages significantly below 500 all had values lower than 0.9 (.653 to .871) and the two lowest performing countries, Morocco and South Africa, had the lowest values (.640 and .653). However, it is important to note that some countries with reading achievement significantly above the 500 average had indices ranging from .797 to .869 , including the top-achieving Russian Federation, as well as Hungary, Latvia, the Slovak Republic, and Poland.

Exhibit 1.2 depicts whether or not the differences in average achievement between pairs of countries and/or Canadian provinces are statistically significant. Selecting a PIRLS 2006 participant of interest and reading across the table, a circle with a triangle pointing up indicates significantly higher performance than the comparison country listed across the top. Absence of 
Instructions: Read across the row for a country to compare performance with the countries listed along the top of the chart. The symbols indicate whether the average achievement of the country in the row is significantly lower than that of the comparison country, significantly higher than that of the comparison country, or if there is no statistically significant difference between the average achievement of the two countries.

\begin{tabular}{|c|c|c|c|c|c|c|c|c|c|c|c|c|c|c|c|c|c|c|c|c|c|c|c|c|c|c|c|c|c|c|}
\hline Countries & 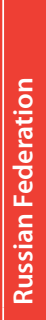 & 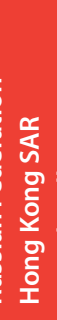 & $\begin{array}{l}5 \\
\frac{5}{2} \\
\frac{1}{\square} \\
\frac{5}{0} \\
\frac{0}{5} \\
5\end{array}$ & & 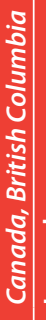 & 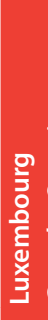 & & & & & & & & & & 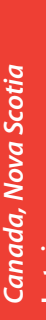 & & & & & & . & & & $\begin{array}{l}\frac{\text { 능 }}{3} \\
\frac{0}{2} \\
\frac{2}{0}\end{array}$ & & & & & 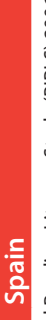 \\
\hline Russian Federation & & & & & & 0 & & 0 & 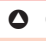 & $\theta$ & 0 & $\boldsymbol{\theta}$ & 0 & 0 & $\theta$ & $\theta$ & 0 & 0 & 0 & $\Delta$ & $\boldsymbol{\theta}$ & $\boldsymbol{\theta}$ & 0 & $\theta$ & 0 & $\Delta$ & Q & $\boldsymbol{0}$ & 00 & $\boldsymbol{\theta}$ \\
\hline Hong Kong SAR & & & & & & 0 & 0 & 0 & & 0 & 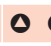 & 0 & 0 & 0 & 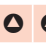 & 0 & $\theta$ & $\theta$ & 0 & 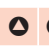 & 0 & 0 & 0 & 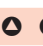 & 0 & $\Delta$ & 0 & $\Delta$ & & 0 \\
\hline Canada, Alberta & & & & & & & & 0 & 0 & 0 & 0 & 0 & 0 & 0 & 0 & 0 & 0 & 0 & 0 & 0 & 0 & 0 & $\Delta$ & 0 & 0 & $\Delta$ & 0 & $\Delta$ & & 0 \\
\hline Singapore & & & & & & & & & & $\theta$ & $\Delta$ & 0 & 0 & $\Delta$ & 0 & 0 & 0 & $\boldsymbol{\theta}$ & 0 & Q & 0 & 0 & $\theta$ & $\boldsymbol{\theta}$ & 0 & $\Delta$ & 0 & $\theta$ & 00 & $\boldsymbol{\theta}$ \\
\hline Canada, British Columbia & & & & & & & & & & 0 & 0 & 0 & 0 & 0 & 0 & 0 & 0 & 0 & 0 & 0 & 0 & 0 & 0 & $\boldsymbol{\theta}$ & $\boldsymbol{\theta}$ & $\Delta$ & 0 & $\Delta$ & & 0 \\
\hline Luxembourg & $(\nabla$ & $(1)$ & & & & & & & 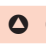 & 0 & $\Delta$ & 0 & 0 & 0 & 0 & 0 & 0 & 0 & 0 & Q & $\boldsymbol{0}$ & 0 & 0 & $\boldsymbol{\theta}$ & Q & $\Delta$ & 0 & $\Delta$ & 00 & $\boldsymbol{\theta}$ \\
\hline Canada, Ontario & 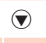 & & & & & & & & & & $\Delta$ & 0 & 0 & & 0 & 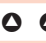 & $\boldsymbol{\theta}$ & $\boldsymbol{0}$ & 0 & $\mathbf{0}$ & 0 & 0 & 0 & $\boldsymbol{\theta}$ & 0 & $\Delta$ & 0 & $\Delta$ & 00 & $\boldsymbol{\theta}$ \\
\hline Italy & & $(-)$ & & & & & & & & & & & & & & 0 & $\theta$ & 0 & 0 & $\mathbf{0}$ & 0 & 0 & $\boldsymbol{\theta}$ & $\boldsymbol{\theta}$ & Q & $\Delta$ & 0 & 0 & & 0 \\
\hline Hungary & 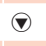 & $(\nabla$ & & & & ( & & & & & & & & & & 0 & 0 & 0 & 0 & $\Delta$ & 0 & 0 & 0 & 0 & $\Delta$ & $\Delta$ & 0 & $\Delta$ & 00 & 0 \\
\hline Sweden & ( & $(-)$ & $(\nabla$ & $(\nabla$ & ( ) & ( & & & & & & & & & & 0 & $\Delta$ & 0 & 0 & 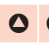 & 0 & 0 & $\theta$ & $\Delta$ & 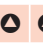 & $\Delta$ & 0 & $\Delta$ & 00 & $\theta$ \\
\hline Gern & $\nabla$ & $(\nabla$ & 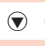 & ( & ( & ( & ( & & & & & & & & & & Q & & 0 & 0 & 0 & 0 & 0 & 0 & 0 & $\Delta$ & 0 & $\boldsymbol{\Delta}$ & 00 & 0 \\
\hline Netherlands & ( & 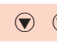 & $\uparrow$ & ( & ( ) & ( ) & ( & & & & & & & & & 0 & 0 & & 0 & 0 & 0 & 0 & 0 & 0 & 0 & 0 & 0 & 0 & 00 & 0 \\
\hline Belgium & $(\nabla$ & $(>$ & $(\nabla$ & $(\nabla$ & $(\nabla)$ & ( & $(\nabla$ & & & & & & & & & & 0 & & 0 & $\Delta$ & 0 & 0 & $\Delta$ & $\Delta$ & $\Delta$ & $\Delta$ & 0 & $\Delta$ & 00 & $\Delta$ \\
\hline Bulgaria & ( & $(-)$ & $\uparrow$ & ( & ( ) & ( & & & & & & & & & & & & & & & 0 & 0 & 0 & 0 & 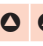 & $\Delta$ & 0 & $\Delta$ & 00 & 0 \\
\hline Denmark & 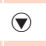 & $(\nabla$ & $\uparrow$ & ( & ( ) & ( & ( & & & & & & & & & & & & 0 & 0 & 0 & 0 & 0 & 0 & 0 & $\Delta$ & 0 & 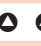 & 00 & 0 \\
\hline Canada, Nova Scotia & $(\nabla$ & 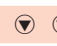 & $(\nabla$ & $(7$ & ( ) & 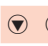 & $(7)$ & ( & $(\nabla$ & ( & & $(\nabla$ & & & & & & & & & & 0 & $\boldsymbol{\theta}$ & $\boldsymbol{0}$ & Q & $\Delta$ & 0 & 0 & 00 & 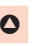 \\
\hline Latvia & 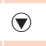 & 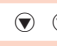 & $\uparrow$ & 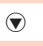 & $(\nabla$ & $(\nabla$ & $(\nabla$ & ( & $\uparrow$ & ( & 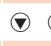 & 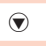 & ( & & & & & & & & & & 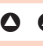 & $\boldsymbol{\theta}$ & $\boldsymbol{\theta}$ & $\Delta$ & 0 & $\boldsymbol{\Delta}$ & 00 & $\mathbf{0}$ \\
\hline United States & 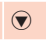 & 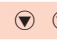 & ( & ( & ( & $(\nabla$ & $(\nabla$ & 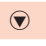 & 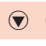 & ( & & & & & & & & & & & & & & $\theta$ & 0 & $\Delta$ & 0 & $\Delta$ & 00 & 0 \\
\hline Engla & $(\nabla$ & $(-)$ & $(\nabla$ & $(\nabla$ & $(\nabla)$ & $(\nabla$ & $(\nabla$ & $(\nabla$ & $(\nabla$ & ( & (1) & $(\nabla$ & 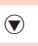 & & $(\nabla)$ & & & & & & & & & 0 & 0 & $\Delta$ & 0 & 0 & 00 & $\boldsymbol{\theta}$ \\
\hline Austria & 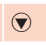 & 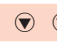 & 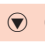 & $\uparrow$ & ( ) & $(\nabla$ & ( & ( & 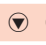 & ( & 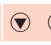 & 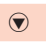 & $($ ) & & (1) & & & & & & & & & $\boldsymbol{\theta}$ & Q & $\Delta$ & 0 & $\Delta$ & 00 & 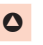 \\
\hline Lithuania & $\nabla$ & 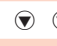 & 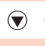 & 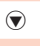 & 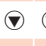 & ( & 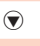 & $\odot$ & 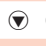 & 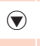 & ( & 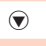 & 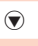 & $(\nabla$ & $(\nabla$ & & & & & & & & & 0 & & $\Delta$ & 0 & $\Delta$ & 00 & 0 \\
\hline Chinese Taipei & 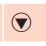 & $(\nabla$ & 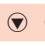 & 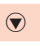 & ( $)$ & $\uparrow$ & $(\nabla$ & ( & 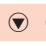 & ( & 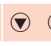 & 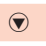 & ( & ( & (i) & (1) & & & & & & & & & & $\Delta$ & $\Delta$ & $\Delta$ & 00 & 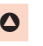 \\
\hline Canada, Quebec & 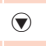 & $(-$ & $\uparrow$ & 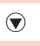 & $(\nabla$ & 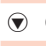 & $(\nabla$ & ( & $(\nabla$ & ( & $(>$ & 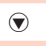 & ( & 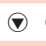 & $(-)$ & 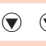 & ( & & & & & & & & & & $\Delta$ & $\Delta$ & 00 & 0 \\
\hline New Zealand & ( & $(\nabla$ & $(\uparrow$ & 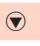 & ( & 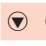 & 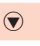 & $(\nabla$ & 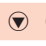 & ( & ( & 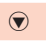 & ( & 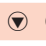 & (i) & (i) & $(>$ & ( & ( & ( & 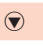 & & & & & & $\Delta$ & $\Delta$ & 00 & 0 \\
\hline Slova & 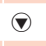 & $(\nabla$ & 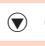 & $\uparrow$ & ( ) & $\uparrow$ & $(\nabla$ & ( & $(\nabla$ & ( & $(>$ & 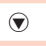 & ( & 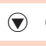 & (i) & $(>$ & $(>$ & ( & 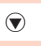 & 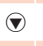 & & & & & & & $\Delta$ & $\Delta$ & 00 & 0 \\
\hline Scotland & ( & $(-)$ & $(\nabla$ & ( & ( ) & $(\nabla$ & ( & ( & $(\nabla$ & ( & $(1)$ & $(\nabla$ & $(1)$ & 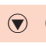 & (1) & $(1)$ & $(1)$ & $(7$ & $(\nabla$ & ( & 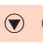 & $(1)$ & & & & & & & 00 & $\theta$ \\
\hline France & $\nabla$ & $(\nabla$ & 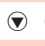 & ( & ( & 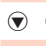 & ( & ( & 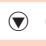 & ( & $(\nabla$ & 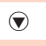 & ( & 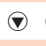 & $(\nabla$ & $(\nabla$ & $(\nabla$ & ( & 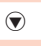 & ( & 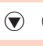 & ( & $(>$ & ( & ( & & & & & 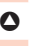 \\
\hline Slove & $(7$ & 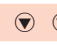 & $\uparrow$ & $\uparrow$ & ( ) & $(\nabla$ & ( & ( & $(\nabla$ & ( & $(1$ & 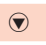 & (1) & $(\nabla$ & (7) & (1) & $(\nabla$ & ( & $\uparrow$ & ( & 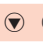 & $(1)$ & ( ) & ( & (†) & & & & & 0 \\
\hline Poland & $(\nabla$ & $(>$ & ( & $(\nabla$ & $(\nabla)$ & ( & 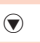 & ( $)$ & $(\nabla$ & ( & $(>$ & ( & $(>$ & ( & $(-)$ & $(\nabla$ & (1) & ( & ( & $(\nabla$ & 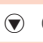 & ( & $(\nabla)$ & ( & (1) & $\nabla$ & & & & $\Delta$ \\
\hline Spain & ( & $(-)$ & $\uparrow$ & $\uparrow$ & ( ) & 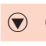 & ( & ( & $\uparrow$ & ( & 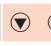 & $(>$ & (1) & $\uparrow$ & (i) & (1) & $(\nabla$ & ( & ( & ( ) & ( & (1) & $(-$ & ( & (1) & ( ) & $\nabla$ & (7) & (1) & \\
\hline Israel & $\nabla$ & 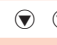 & $(\nabla$ & ( & 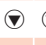 & ( & ( & $\odot$ & 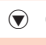 & $\nabla$ & $(-$ & 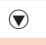 & 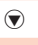 & ( & $(\nabla$ & $(\nabla)$ & $(-)$ & $(\nabla$ & $(\nabla$ & $(\nabla$ & 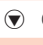 & (†) & 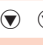 & ( & $(\nabla$ & $\nabla$ & $\nabla$ & ( & & \\
\hline Iceland & $(\nabla$ & $\theta$ & $(\nabla$ & (1) & ( ) & $(\nabla$ & $(7)$ & ( & ( & ( & $(1)$ & $(\nabla$ & (1) & $(1)$ & $(\nabla)$ & $(1)$ & $(1)$ & (1) & (1) & (1) & $(1)$ & ( & $(\uparrow$ & ( & ( & $(>$ & $\nabla$ & 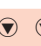 & $(\uparrow$ & \\
\hline Moldova & 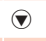 & ( & ( & 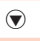 & ( & 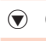 & 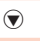 & ( & 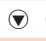 & $\boldsymbol{\nabla}$ & ( & ( & ( & 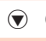 & $(\nabla$ & ( & ( & ( & ( & 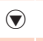 & ( & $(\nabla$ & $(\nabla$ & ( & ( & $(>$ & $\nabla 0$ & $($ () & (i) & ( \\
\hline Belgium & 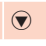 & 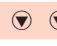 & ( & ( & ( & ( & ( & 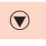 & $(\nabla$ & ( & 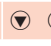 & 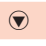 & ( & ( & ( ) & (i) & ( & ( & ( & ( & ( & ( & $(\nabla$ & ( & ( ) & $(\bullet$ & $\nabla$ & ( ) & (i) & $(\nabla$ \\
\hline Norway & $(\nabla$ & $(\nabla)$ & $(\nabla$ & ( & $(\nabla)$ & $(\nabla$ & (†) & $(\nabla$ & $(\nabla$ & ( & (1) & ( & $(7)$ & $(\nabla$ & $(\nabla)$ & $(1)$ & $(-)$ & $(\nabla$ & (1) & $(\nabla$ & (1) & ( & $(-)$ & $(\nabla$ & $(-)$ & $(7$ & $\nabla$ & $(\nabla)$ & (1) & (1) \\
\hline Romania & $(\nabla$ & ( $)$ & 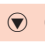 & $\uparrow$ & ( ) & $(\nabla$ & ( & ( & $(\nabla$ & ( & ( & $(>$ & 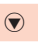 & $(\nabla$ & (1) & (1) & $(\nabla$ & ( & (1) & ( ) & ( & $(1)$ & 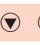 & ( & (1) & ( & $\nabla$ & $(-$ & (i) & ( \\
\hline Geo & $\nabla$ & $(\nabla$ & 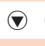 & 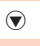 & 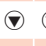 & $(\nabla$ & 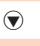 & $\odot$ & 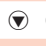 & 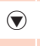 & ( & 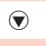 & 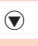 & $(\nabla$ & $(\nabla$ & $(\nabla$ & 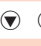 & 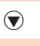 & 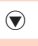 & $(\nabla$ & 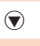 & $(\nabla$ & ( & 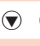 & 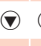 & ( & $\theta$ & $(>$ & $\oplus$ & $(\nabla$ \\
\hline Macedonia & $(\nabla$ & (1) & $(1)$ & $(\nabla$ & ( ) & $(-$ & $(\nabla$ & ( & $(\nabla$ & ( & (1) & 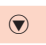 & (1) & $(\nabla$ & (i) & $(-)$ & (1) & $(\nabla$ & (1) & (1) & $(7$ & (1) & 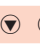 & (1) & (1) & $(7$ & $\nabla$ & $(7)$ & (1) & (1) \\
\hline Trinidad and Tobago & 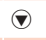 & 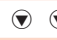 & ( & $(\nabla$ & ( & 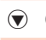 & ๑ & ( & ( & ( & ( & 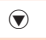 & ( & 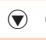 & $(-)$ & $(\nabla$ & $(\nabla$ & ( & (7) & ๑ & ( & (†) & $(\nabla$ & ( & $(\nabla$ & $(>$ & $\nabla$ & 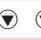 & (i) & ( \\
\hline Iran, Islamic & ( & ( $)$ & 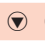 & 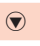 & ( & 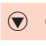 & 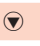 & 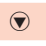 & 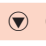 & ( & ( & 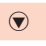 & ( & 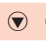 & (i) & (i) & ( & ( & ( & ( & ( & $(\nabla$ & ( & ( & ( & $(\nabla$ & $\nabla$ & ( $)$ & (i) & ( \\
\hline Indonesia & $(\nabla$ & $(1)$ & $(\nabla$ & ( & $(\nabla)$ & $(\nabla$ & $(\nabla$ & ( & $(\nabla$ & ( & (1) & $(\nabla$ & $(7)$ & $(\nabla$ & $(\nabla)$ & $(-)$ & 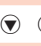 & ( & (1) & $(\nabla$ & (1) & $(\nabla)$ & $(\nabla)$ & (1) & (1) & $(>$ & $\nabla$ & $(\nabla$ & $(1)$ & $(1)$ \\
\hline Qatar & $(\nabla$ & $(7)$ & $(\nabla$ & (†) & ( & $(\nabla$ & $\nabla$ & ( & ( & ( ) & $(1)$ & $(\nabla$ & ( & $(\nabla$ & (1) & (1) & (1) & ( & ( & ( & ( & (1) & ( & (1) & (1) & $(\nabla$ & ( & ( & () & ( \\
\hline Kuwait & $\nabla$ & $(\nabla$ & 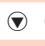 & ( & ( & 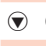 & ( & ( & 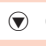 & ( & $(\nabla$ & 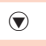 & ( & 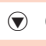 & $(\nabla$ & ( & ( & ( & ( & ( & ( & ( & ( & ( & $(\nabla$ & $(\nabla$ & $\nabla$ & $(\nabla$ & ( $)$ & ( \\
\hline Morocco & $(7$ & ( $)$ & $\uparrow$ & $\uparrow$ & ( ) & $(\nabla$ & ( & ( & $(\nabla$ & ( & $(1$ & 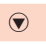 & (1) & $(\nabla$ & (7) & (1) & $($ ) & ( & ( ) & ( & ( & (1) & $(\nabla$ & (1) & (†) & $(\bullet$ & $\nabla$ & $(>$ & (1) & ( \\
\hline outh Africa & $(\nabla$ & $(\nabla)$ & $(\nabla$ & $(\nabla$ & $(\nabla)$ & $(\nabla$ & $(\nabla$ & ( $)$ & $(\nabla$ & ( & $(\nabla$ & ( & ( & ( & $(-)$ & 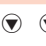 & (1) & ( & (1) & ( & ( & (†) & (†) & (1) & (1) & $(\nabla$ & $(7$ & ( & (1) & (1) \\
\hline
\end{tabular}




\section{Exhibit 1.2 Multiple Comparisons of Average Reading Achievement (Continued)}

Instructions: Read across the row for a country to compare performance with the countries listed along the top of the chart. The symbols indicate whether the average achievement of the country in the row is significantly lower than that of the comparison country, significantly higher than that of the comparison country, or if there is no statistically significant difference between the average achievement of the two countries.

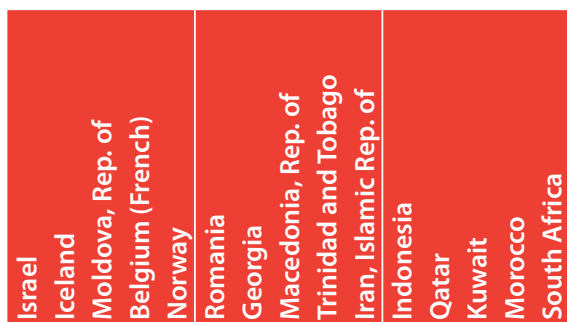

000000000000000 000000000000000 000000000000000 $00 \Delta 000000000000$ 000000000000000 000000000000000 000000000000000 000000000000000 $\begin{array}{lllllllllllllll}0 & 0 & 0 & 0 & 0 & 0 & 0 & 0 & 0 & 0 & 0 & 0 & 0 & 0\end{array}$ 0000000000000000 000000000000000 000000000000000 0000000000000000 000000000000000 000000000000000

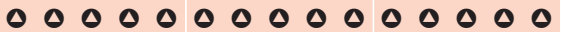
000000000000000 000000000000000 00000000000000000 000000000000000 00000000000000000 000000000000000 000000000000000 000000000000000 0000000000000000 000000000000000

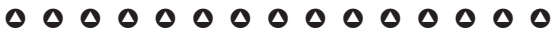
000000000000000 00000000000000 0000000000000 0000000000000 0000000000000 (1) 10000000000 (1) 1000000000 (1) 10000000000 - $10 \quad 000000000$

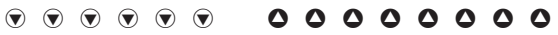

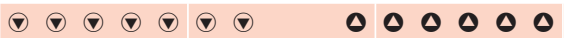

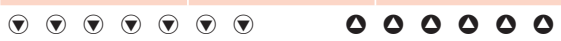
(1) (1) (1) (1) (1) (1) (1) 10000000

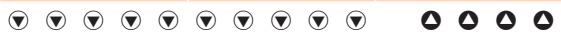

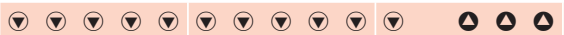

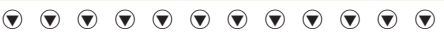

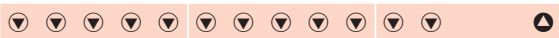

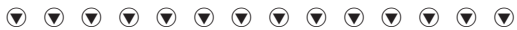

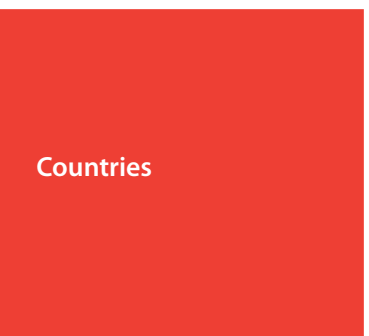

Russian Federation Hong Kong SAR

Canada, Alberta

Singapore

Canada, British Columbia

Luxembourg

Canada, Ontario

Italy

Hungary

Sweden

Germany

Netherlands

Belgium (Flemish)

Bulgaria

Denmark

Canada, Nova Scotia

Latvia

United States

England

Austria

Lithuania

Chinese Taipei

Canada, Quebec New Zealand

Slovak Republic

Scotland

France

Slovenia

Poland

Spain

Israel

Iceland

Moldova, Rep. of

Belgium (French)

Norway

Romania

Georgia

Macedonia, Rep. of

Trinidad and Tobago

Iran, Islamic Rep. of

Indonesia

Qatar

Kuwait

Morocco

South Africa
Average achievement significantly
higher than comparison country

- Average achievement significantly

lower than comparison country 
a symbol indicates no significant difference in performance, and a circle with a triangle pointing down indicates significantly lower performance than the comparison country or Canadian province.

The results in Exhibit 1.2 help interpret the typically small differences in achievement among the PIRLS 2006 participants shown close to each other in Exhibit 1.1. The Russian Federation, Hong Kong SAR, and Singapore were the three top-performing countries, and fourth-grade students in the Canadian provinces of Alberta and British Colombia had similar average achievement. Considering the five participants with the highest achievement, the Russian Federation and Hong Kong SAR had significantly higher average achievement than all of the remaining participants except the other three in the top five, while the Canadian province of Alberta also performed similarly to Luxembourg and the province of Ontario. In turn, Singapore and the Canadian province of British Columbia showed no significant difference compared to two additional countries-Italy and Hungary. Luxembourg, the Canadian province of Ontario, Italy, and Hungary also performed very well. Luxembourg and the Canadian province of Ontario were outperformed only by the Russian Federation and Hong Kong SAR, Italy by those two and the Canadian province of Alberta, and Hungary also by Luxembourg. Next, although outperformed by the highest achieving countries and provinces, Sweden, Germany, the Netherlands, Belgium (Flemish), Bulgaria, and Denmark achieved at comparable levels, and had higher achievement than the majority of other participants.

Looking at the other end of the achievement continuum in Exhibit 1.2, the lowest performing countries were each, in turn, outperformed by one or two additional countries. That is, South Africa had lower achievement than all the other countries, while Kuwait and Morocco had higher achievement than South Africa (but no other countries). In turn, Qatar had higher achievement than the previous 3 countries, Indonesia than the previous 4 countries, Iran than the previous 5 countries, Trinidad and Tobago together with Macedonia than the previous 6 countries, and Georgia had higher achievement than the previous 8 countries. The next cluster of countries after Georgia all had similar achievement-Romania, Norway, Belgium (French), and Moldova. 
Fourth-grade students in these four countries outperformed those in the previously mentioned nine lowest-performing countries, but had significantly lower average achievement than the rest of the participants.

\section{How Has Achievement in Reading Comprehension Changed Between PIRLS 2001 and PIRLS 2006?}

Exhibit 1.3 displays changes in average achievement between 2001 and 2006 for the 26 countries and 2 Canadian provinces that participated in both assessments. ${ }^{4}$ The participants are shown in order of the most improvement to largest decline. A red bar indicates that the difference is statistically significant. The Russian Federation, Hong Kong SAR, Singapore, Slovenia, the Slovak Republic, Italy, Germany, and Hungary all showed significant gains in average reading achievement between 2001 and 2006. The gain in Moldova was not significant statistically due to a comparatively larger standard error, even though the increase ( 8 points) was comparable to that in Hungary. Countries with significant decreases in reading achievement since PIRLS 2001 were Lithuania, the Netherlands, Sweden, England, Romania, and Morocco. (As a trend note, for the Canadian province of Ontario, only public schools participated in PIRLS 2001. To be comparable to PIRLS 2001, the PIRLS 2006 data for Ontario used in the trend analyses included only public schools and differs slightly from Exhibit 1.1.)

It is interesting to consider the PIRLS 2006 achievement results in light of the information countries provided in the PIRLS 2006 Encyclopedia. For example, the trend results illustrate how PIRLS data can be used to monitor the impact of structural and curricular changes in education systems. Although the education systems in the PIRLs trend countries and provinces have been relatively stable in most respects between 2001 and 2006, several have undergone fundamental changes. Table A.8 in Appendix A documents the grades, average ages, and percentages of exclusions in 2001 and 2006 for the trend participants.

According to ongoing reforms described in the PIRLS 2006 Encyclopedia, improvement in the Russian Federation and Slovenia may have been anticipated. These two countries have been undergoing structural changes

4 For PIRLS 2006, New Zealand and Singapore tested on the Southern Hemisphere schedule of October through December 2005 For PIRLS 2001, the Southern Hemisphere testing was scheduled after the Northern Hemisphere (instead of before it) in October through December of 2001. Thus, the changes for New Zealand and Singapore are over a 4-year period rather than a 5-year period. 
Exhibit 1.3 Trends in Reading Achievement

\begin{tabular}{|c|c|c|c|}
\hline Countries & $\begin{array}{c}\text { PIRLS } 2006 \\
\text { Average } \\
\text { Scale Score }\end{array}$ & $\begin{array}{c}\text { PIRLS } 2001 \\
\text { Average } \\
\text { Scale Score }\end{array}$ & $\begin{array}{c}\text { Difference } \\
\text { Between } \\
2001 \text { and } 2006 \\
\text { Scores }\end{array}$ \\
\hline 2a Russian Federation & $565(3.4)$ & $528(4.4)$ & $37(5.6)$ \\
\hline Hong Kong SAR & $564(2.4)$ & $528(3.1)$ & $36(3.9)$ \\
\hline Singapore & $558(2.9)$ & $528(5.2)$ & $30(5.9)$ \\
\hline Slovenia & $522(2.1)$ & $502(2.0)$ & $20(2.9)$ \\
\hline Slovak Republic & $531(2.8)$ & $518(2.8)$ & $13(4.0)$ \\
\hline Italy & $551(2.9)$ & $541(2.4)$ & $11(3.8)$ \\
\hline Germany & $548(2.2)$ & $539(1.9)$ & $9(2.9)$ \\
\hline Moldova, Rep. of & $500(3.0)$ & $492(4.0)$ & $8(5.0)$ \\
\hline Hungary & $551(3.0)$ & $543(2.2)$ & $8(3.7)$ \\
\hline Iran, Islamic Rep. of & $421(3.1)$ & $414(4.2)$ & $7(5.2)$ \\
\hline 2a Canada, Ontario & $554(2.8)$ & $548(3.3)$ & $6(4.4)$ \\
\hline $2 \mathrm{~b}$ Israel & $512(3.3)$ & $509(2.8)$ & $4(4.4)$ \\
\hline New Zealand & $532(2.0)$ & $529(3.6)$ & $3(4.1)$ \\
\hline Macedonia, Rep. of & $442(4.1)$ & $442(4.6)$ & $1(6.2)$ \\
\hline + Scotland & $527(2.8)$ & $528(3.6)$ & $-1(4.6)$ \\
\hline ‡ Norway & $498(2.6)$ & $499(2.9)$ & $-1(3.9)$ \\
\hline Iceland & $511(1.3)$ & $512(1.2)$ & $-2(1.8)$ \\
\hline †2a United States & $540(3.5)$ & $542(3.8)$ & $-2(5.2)$ \\
\hline 2a Bulgaria & $547(4.4)$ & $550(3.8)$ & $-3(5.8)$ \\
\hline France & $522(2.1)$ & $525(2.4)$ & $-4(3.1)$ \\
\hline Latvia & $541(2.3)$ & $545(2.3)$ & $-4(3.3)$ \\
\hline Canada, Quebec & $533(2.8)$ & $537(3.0)$ & $-4(4.1)$ \\
\hline Lithuania & $537(1.6)$ & $543(2.6)$ & $-6(3.1)$ \\
\hline${ }^{\dagger}$ Netherlands & $547(1.5)$ & $554(2.5)$ & $-7(2.9)$ \\
\hline Sweden & $549(2.3)$ & $561(2.2)$ & $-12(3.2)$ \\
\hline England & $539(2.6)$ & $553(3.4)$ & $-13(4.3)$ \\
\hline Romania & $489(5.0)$ & $512(4.6)$ & $-22(6.8)$ \\
\hline Morocco & $323(5.9)$ & $350(9.6)$ & $-27(11.3)$ \\
\hline
\end{tabular}

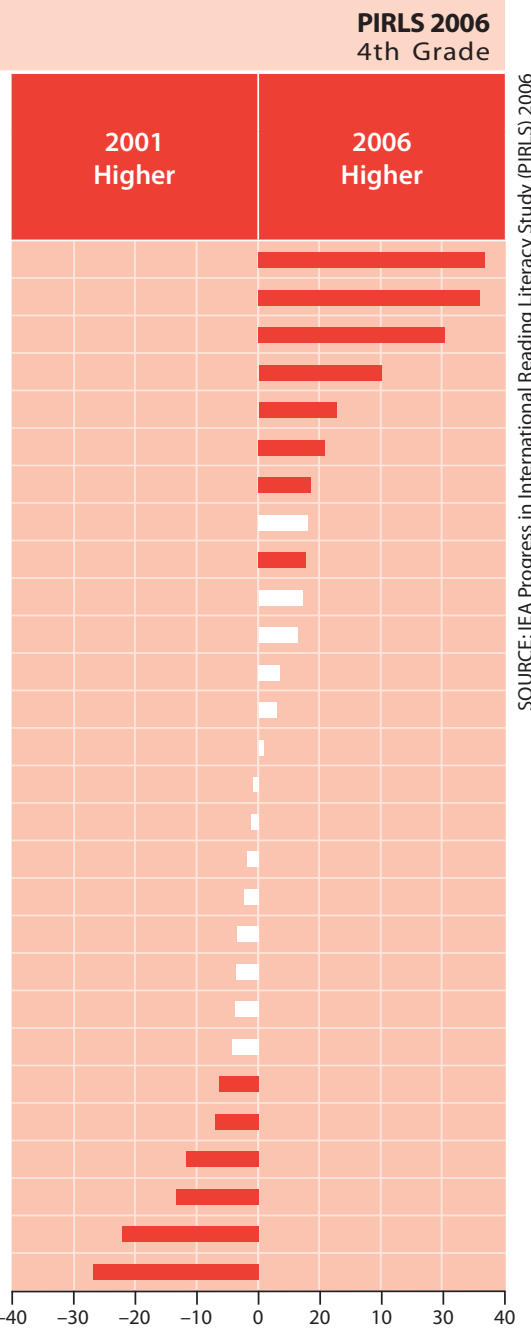

Difference statistically significant Not statistically significant $\dagger$ Met guidelines for sample participation rates only after replacement schools were included (see Exhibit A.7).

₹ Nearly satisfying guidelines for sample participation rates after replacement schools were included (see Exhibit A.7).

2a National Defined Population covers less than $95 \%$ of National Desired Population (see Exhibit A.4) 2b National Defined Population covers less than $80 \%$ of National Desired Population (see Exhibit A.4).

( ) Standard errors appear in parentheses. Because results are rounded to the nearest whole number, some totals may appear inconsistent.

Trend Note: The primary education systems of the Russian Federation and Slovenia underwent structural changes. Data for Canada, Ontario include only public schools. 
in their primary system that involve adding one more year of schooling at the primary level, as well as associated curricular and instructional reforms. In the Russian Federation, the primary level of the education system has been undergoing a transition from 3 years to 4 years of schooling. In PIRLS 2001, more than half of the Russian students were still in the 3-year system, whereas by 2006 the transition essentially was complete to the 4 -year system. When the transition was conceived, the idea was to have students start school a year younger at age 6, but in actuality, parents are still sending their children to school at age 7. Thus, in 2006, about half of the students in the Russian Federation had an extra year of school, and the average age increased from 10.3 to 10.8. Slovenia is in the middle of a similar transition; so, in anticipation of this reform, they tested students in their third year of schooling in 2001. By 2006, about half of the students had attended school for 4 years. However, in Slovenia, students having attended school for 4 years started school younger, so the average age has not changed (9.8 to 9.9). According to the Slovenian chapter in the PIRLS 2006 Encyclopedia, one of the reasons for changing from an 8- to a 9-year elementary school system was to improve literacy.

In Hong Kong SAR, as described in the PIRLS 2006 Encyclopedia, curriculum reform since 2000 has involved the government doing extensive work to promote reading and enable all children to read with comprehension in both official languages of Chinese and English. In 2000, the Curriculum Development Council established clear reading goals for schools giving them the responsibility for promoting reading and building a culture of reading. The Curriculum Development Council gave schools the power to adjust the curriculum and schedule to meet the literacy needs of students, and suggested that teachers expand the range of teaching materials used in lessons. Schools ensure that students are given opportunities to develop reading fluency, and many have trained "Reading Mothers" to help students read stories. There also has been considerable community involvement. For example, the Reading Ambassador project has trained 2,500 parents, university students including prospective teachers, older secondary school students, and community leaders to read and share their perspectives with students in schools. 
According to the Singaporean chapter in the PIRLS 2006 Encyclopedia, Singapore implemented a new English language syllabus in 2001 that was significantly different from the previous one. Organized loosely around three areas: language for information, language for literary response and expression, and language for social interaction, the new syllabus focuses on language use through study of a wide range of text types. It is supported by new instructional materials, and learning outcomes specified in the syllabus give teachers more explicit information on teaching reading skills and strategies at various levels. During 2000 and 2001, nationwide training workshops were conducted to prepare all English language teachers to teach the new syllabus. Children in Singapore also have been exposed to more opportunities to learn English (the language of the test). The number of students whose predominant home language is English increased from 37 percent in 2001 to 42 percent in 2005. The 2-year Learning Support Programme provides early intervention and support for students who enter primary school with weak English language skills.

\section{What Are the Gender Differences in Reading Achievement?}

Exhibit 1.4 shows differences in fourth-grade students' reading achievement between girls and boys. For each of the PIRLs 2006 participants, the percentage of girls and boys is shown with their respective average achievement. The countries and provinces are shown in increasing order of the gender difference. Because girls had higher average achievement than boys in every country and province, the ordering is according to the extent of the difference favoring girls from the least to the most difference (shown in the last column). Except in the two countries with the most equitable results, Luxembourg and Spain, the differences were statistically significant.

For the first time in this chapter, Exhibit 1.4 provides an "international average" based on averaging the results across countries. The five Canadian provinces were not included in the computations. As point of reference, the results were averaged separately for boys and for girls to examine the extent of the gender difference, on average internationally. On average internationally, 
the average scale score for girls was 509 compared to that of 492 for boys, a difference of 17 scale score points on average.

Exhibit 1.5 presents, in alphabetical order, changes in average reading achievement for girls and boys between PIRLS 2001 and PIRLS 2006. The changes for Hong Kong sar, Italy, the Russian Federation, Singapore, the Slovak Republic, and Slovenia mirror their overall gains, with increases for both girls and boys. Interestingly, only boys showed improvement in Germany and Hungary (as well as in Moldova). The boys in the Canadian province of Ontario and Iran also showed improvement in average achievement between PIRLS 2001 and PIRLS 2006. For countries showing declines overall, both girls and boys showed decreases in achievement in England, Morocco, Romania, and Sweden. In the Netherlands, the decline overall seemed to be primarily attributable to the decrease in girls' average reading achievement.

\section{How Does Achievement Differ Across Countries for Reading Literacy Purposes?}

In both PIRLS 2001 and 2006, the assessment framework included two overarching purposes for reading:

- Reading for literary experience, and

- Reading to acquire and use information.

The PIRLS 2006 assessment included five literary passages and five informational passages, so that half of the assessment time was devoted to each purpose. Comprehension processes were assessed within each purpose (see next section). The literary texts were fictional stories where students could engage with the events, characters' actions and feelings, the setting, and ideas, as well as the language itself. The informational passages dealt with aspects of the real universe, and covered a variety of content and organizational structures. In addition to prose, each one involved some variety in format, by including features such as photographs, illustrations, text boxes, maps, and diagrams. More information about the passages can be found in Chapter 2, and two of the literary and informational passages are 
Exhibit 1.4 Differences in Average Reading Achievement by Gender

PIRLS 2006
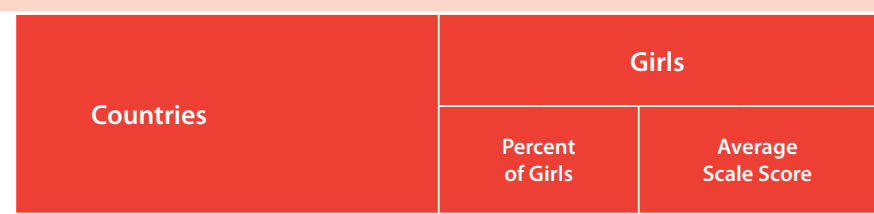

\begin{tabular}{|c|c|}
\hline \multicolumn{2}{|c|}{ Boys } \\
$\begin{array}{c}\text { Percent } \\
\text { of Students }\end{array}$ & $\begin{array}{c}\text { Average } \\
\text { Scale Score }\end{array}$ \\
\hline
\end{tabular}

4th Grade

\begin{tabular}{|l|}
\hline Luxembourg \\
\hline Spain \\
\hline Belgium (French) \\
Hungary \\
\hline +2a Belgium (Flemish) \\
\hline Italy \\
\hline Netherlands \\
\hline Germany
\end{tabular}

$49(0.7)$

$559(1.3)$

$49(1.1) \quad 515(2.6)$

$50(0.7)$

$502(2.8)$

$50(0.9) \quad 554(3.6)$

$50(0.9)$

$550(2.3)$

$48(0.8) \quad 555(3.3)$

$51(0.8)$

$551(2.0)$

2a Canada, Alberta

$49(0.7) \quad 551(2.5)$

2a Canada, British Columbia

$48(0.8)$

$564(2.4)$

Austria

$50(0.8)$

t2a United States

$49(0.7)$

$562(2.9)$

Hong Kong SAR

$51(0.7)$

$543(2.3)$

France

49 (1.3)

545 (3.3)

Slovak Republic

$48(0.7)$

$569(2.5)$

Canada, Quebec

$49(0.8)$

527 (2.4)

2a Canada, Ontario

49 (1.0)

Chinese Taipei

49 (1.1)

537 (2.7)

539 (2.7)

2a Denmark

$48(0.5)$

$562(3.3)$

251

Moldova, Rep. o

Iran, Islamic Rep. of

$52(0.9)$

542 (2.2)

$553(2.8)$

Romania

$2 \mathrm{~b}$ Israel

$46(1.1)$

$507(3.1)$

$429(5.3)$

$48(1.0) \quad 497(5.0)$

2a Russian Federation $\quad 48(1.2)$

Singapore

$520(4.1)$

$51(0.9) \quad 572(3.9)$

Poland

$48(0.6) \quad 567(3.1)$

2a Georgia $\quad 48(1.0)$

$51(0.8)$

528 (2.6)

$47(1.0)$

Sweden

48 (1.1)

Lithuania

$49(0.9)$

celand

$50(0.9)$

* Norway

49 (1.1)

England

$50(0.9)$

Slovenia

$48(0.7)$

Indonesia

$49(0.9)$

2a Bulgaria

49 (1.0)

Canada, Nova Scotia

$49(0.7)$

Macedonia, Rep. of

$49(0.7)$

+ Scotland

$51(0.9)$

48 (1.0)

New Zealand

$49(0.9)$

49 (1.7)

Trinidad and Tobago

$52(0.6)$

$50(0.2)$

Qatar

$50(2.0)$

\section{$480(3.3)$}

0

$51(0.7)$

$556(1.6)$

Difference

Girls Higher

Average

Achievement

Than Boys

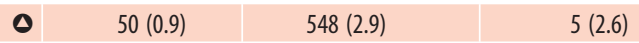

- $50(0.9)$

- $52(0.8)$

- $49(0.8)$

- $51(0.7)$

- $52(0.8)$

- $50(0.8)$

- $51(0.7)$

- $49(0.7)$

- $51(1.3)$

- $52(0.7)$

- $51(0.8)$

- 51 (1.0)

- $51(1.1)$

- $52(0.5)$

- $48(0.9)$

- $50(1.0)$

- $54(1.1)$

- $52(1.0)$

- $52(1.2)$

- $49(0.9)$

- $52(0.6)$

- $49(0.8)$

- $52(1.0)$

- $53(1.0)$

559 (2.6)

$546(2.0)$

$520(1.7)$

- $52(1.1)$

\begin{tabular}{ll} 
- & $51(0.9)$ \\
\hline & $50(0.9)$
\end{tabular}

- 51 (1.1)

$508(2.8)$

549 (3.0)

532 (2.1)

- $50(0.9)$

- $52(0.7)$

- $51(0.9)$

$415(4.2)$

- 51 (1.0)

$3(2.0)$

497 (2.9)

$548(2.9)$

$548(3.3)$

$543(1.6)$

$544(2.5)$

$556(2.7)$

554 (3.1)

$533(2.6)$

$533(2.6)$
$535(4.4)$

$559(2.8)$

$516(2.4)$

525 (3.3)

$525(3.3)$

$549(3.3)$

$529(2.3)$

$539(2.7)$

$493(3.5)$
$414(3.8)$

\begin{tabular}{l}
$483(5.7)$ \\
\hline $506(3.7)$
\end{tabular}

557 (3.4)

$550(3.3)$

$550(3.3)$
$511(2.7)$

$511(2.7)$
$463(3.8)$

$463(3.8)$
$314(6.6)$

$$
541(2.6)
$$

$541(2.6)$
$528(2.0)$

$501(1.9)$

$489(3.1)$

$530(2.8)$
$512(2.7)$

$512(2.7)$
$395(4.6)$
$537(5.0)$

$553(2.5)$

$453(4.4)$

- $51(0.7)$

- $51(0.7)$

$531(2.8)$
$432(4.4)$

$516(3.1)$
$530(2.6)$

$553(2.7)$

- $49(0.9)$

\begin{tabular}{ll}
0 & $52(1.0)$ \\
\hline & $51(0.9)$
\end{tabular}

$544(2.2)$

\section{$451(4.9)$}

- $51(1.7)$

$520(2.9)$

$420(6.0)$

$283(5.5)$

$372(1.7)$

Kuwait $49(0.2)$

$509(0.6)$

0

$48(0.6)$

$335(1.7)$
$297(6.2)$

International Avg.

Average significantly higher than other gender

+ Met guidelines for sample participation rates only after replacement schools were included (see Exhibit A.7).

‡ Nearly satisfying guidelines for sample participation rates after replacement schools were included (see Exhibit A.7)

2a National Defined Population covers less than $95 \%$ of National Desired Population (see Exhibit A.4). 2b National Defined Population covers less than $80 \%$ of National Desired Population (see Exhibit A.4).

() Standard errors appear in parentheses. Because results are rounded to the nearest whole number, some totals may appear inconsistent.

NOTE: The International Average does not include the results from the Canadian provinces. 


\begin{tabular}{|c|c|c|c|c|c|c|}
\hline \multirow{2}{*}{ Countries } & \multicolumn{3}{|c|}{ Girls } & \multicolumn{3}{|c|}{ Boys } \\
\hline & $\begin{array}{l}2006 \text { Average } \\
\text { Scale Score }\end{array}$ & $\begin{array}{c}2001 \text { to } 2006 \\
\text { Difference }\end{array}$ & & $\begin{array}{l}2006 \text { Average } \\
\text { Scale Score }\end{array}$ & $\begin{array}{c}2001 \text { to } 2006 \\
\text { Difference }\end{array}$ & \\
\hline Bulgaria & $558(4.4)$ & $-5(5.7)$ & & $537(5.0)$ & $-1(6.8)$ & \\
\hline Canada, Ontario & $560(3.3)$ & $2(5.0)$ & & $548(3.3)$ & $10(4.8)$ & $\theta$ \\
\hline Canada, Quebec & $539(2.7)$ & $-5(4.3)$ & & $527(3.5)$ & $-3(4.7)$ & \\
\hline England & $549(3.0)$ & $-14(4.9)$ & ( & $530(2.8)$ & $-11(4.7)$ & ( \\
\hline France & $527(2.4)$ & $-3(3.6)$ & & $516(2.4)$ & $-4(3.9)$ & \\
\hline Germany & $551(2.5)$ & $6(3.3)$ & & $544(2.5)$ & $11(3.5)$ & $\theta$ \\
\hline Hong Kong SAR & $569(2.5)$ & $32(3.9)$ & $\theta$ & $559(2.8)$ & $40(4.5)$ & $\theta$ \\
\hline Hungary & $554(3.6)$ & $3(4.3)$ & & $548(2.9)$ & $12(3.8)$ & $\theta$ \\
\hline Iceland & $520(1.7)$ & $-2(2.5)$ & & $501(1.9)$ & $-2(2.4)$ & \\
\hline Iran, Islamic Rep. of & $429(5.3)$ & $2(7.8)$ & & $414(3.8)$ & $15(6.8)$ & $\theta$ \\
\hline Israel & $520(4.1)$ & $1(5.3)$ & & $506(3.7)$ & $8(5.2)$ & \\
\hline Italy & $555(3.3)$ & $10(4.2)$ & $\theta$ & $548(3.3)$ & $11(4.2)$ & $\theta$ \\
\hline Latvia & $553(2.7)$ & $-3(4.1)$ & & $530(2.6)$ & $-4(3.6)$ & \\
\hline Lithuania & $546(2.0)$ & $-6(3.5)$ & & $528(2.0)$ & $-7(3.4)$ & \\
\hline Macedonia, Rep. of & $453(4.4)$ & $1(6.8)$ & & $432(4.4)$ & $1(6.5)$ & \\
\hline Moldova, Rep. of & $507(3.1)$ & $3(5.6)$ & & $493(3.5)$ & $14(5.3)$ & $\theta$ \\
\hline Morocco & $332(6.6)$ & $-29(11.6)$ & $(\nabla)$ & $314(6.6)$ & $-27(12.8)$ & ( \\
\hline Netherlands & $551(2.0)$ & $-11(3.4)$ & ( & $543(1.6)$ & $-4(3.2)$ & \\
\hline New Zealand & $544(2.2)$ & $2(5.2)$ & & $520(2.9)$ & $4(5.1)$ & \\
\hline Norway & $508(2.8)$ & $-3(4.5)$ & & $489(3.1)$ & $0(4.6)$ & \\
\hline Romania & $497(5.0)$ & $-22(6.6)$ & ( & $483(5.7)$ & $-22(8.1)$ & ( \\
\hline Russian Federation & $572(3.9)$ & $38(5.8)$ & $\theta$ & $557(3.4)$ & $35(5.9)$ & $\theta$ \\
\hline Scotland & $538(3.6)$ & $2(5.3)$ & & $516(3.1)$ & $-3(5.2)$ & \\
\hline Singapore & $567(3.1)$ & $27(6.1)$ & 0 & $550(3.3)$ & $34(6.6)$ & $\theta$ \\
\hline Slovak Republic & $537(2.7)$ & $10(4.0)$ & 0 & $525(3.3)$ & $15(4.7)$ & $\theta$ \\
\hline Slovenia & $532(2.1)$ & $19(3.3)$ & $\theta$ & $512(2.7)$ & $22(3.6)$ & $\theta$ \\
\hline Sweden & $559(2.6)$ & $-14(3.7)$ & $(\nabla)$ & $541(2.6)$ & $-10(3.6)$ & ( \\
\hline United States & $545(3.3)$ & $-6(5.0)$ & & $535(4.4)$ & $2(6.6)$ & \\
\hline International Avg. & $526(0.7)$ & $1(1.0)$ & & $510(0.7)$ & $5(1.1)$ & $\theta$ \\
\hline
\end{tabular}

Met guidelines for sample participation rates only after replacement schools were included (see Exhibit A.7).

₹ Nearly satisfying guidelines for sample participation rates after replacement schools were included (see Exhibit A.7).

2a National Defined Population covers less than $95 \%$ of National Desired Population (see Exhibit A.4)

2b National Defined Population covers less than $80 \%$ of National Desired Population (see Exhibit A.4).
() Standard errors appear in parentheses. Because results are rounded to the nearest whole number, some totals may appear inconsistent.

NOTE: The International Average does not include the results from the Canadian provinces. Trend Note: The primary education systems of the Russian Federation and Slovenia underwent structural changes. Data for Canada, Ontario include only public schools. 
reproduced in the Appendix D and in the PIRLS 2006 Reader in the pocket at the end of this report.

For each country and Canadian province, Exhibit 1.6 presents the average achievement for fourth-grade students in reading for literary purposes and in reading for informational purposes. The two numerical scale scores are not directly comparable, since they represent different constructs, and the assessments were of slightly different difficulties. As shown in Exhibit A.16 containing the average percent correct across the items on the PIRLS 2006 scales, on average internationally, the informational scale was slightly more difficult than the literary scale, 52 percent correct on average compared to 55 percent correct. This pattern held for most but not all of the PIRLS 2006 participants. However, to allow comparison of the relative performance of each PIRLS 2006 participant for each purpose, the international average for each purpose was scaled to be 500, the same as the overall PIRLS scale average. This makes it possible to examine relative strengths and weaknesses of countries by comparing the relative positions of the participants on the two scales. To assist in the relative comparisons, the graph displays the differences.

The first two columns in Exhibit 1.6 present the average achievement for the literary purpose and the average achievement for the informational purpose. Generally, the PIRLS 2006 participants with the highest achievement overall also had the highest achievement in both literary and informational reading. Exhibit B.1 for literary reading and Exhibit B.2 for informational reading, respectively, present the PIRLS 2006 participants in order of average achievement and show whether or not the differences in average achievement are statistically significant.

The results in Exhibit 1.6 reveal, however, that many countries performed relatively better or worse in one reading purpose compared to the other (the red bar indicates that the difference is statistically significant). The countries with relatively better performance in informational reading are shown in the upper portion of the exhibit, and those with relatively better performance in literary reading are shown in the lower portion. Except for a consistent pattern among countries where Chinese is one of the major languages (Singapore, 
Exhibit 1.6 Relative Difference in Performance Between Literary and Informational Purposes
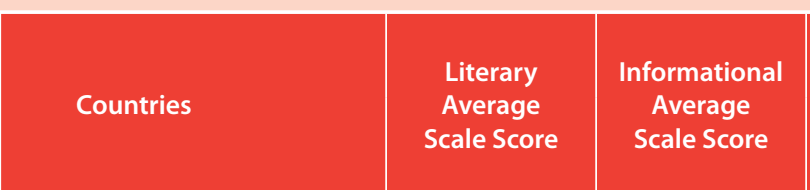

Indonesia

Morocco

South Africa

Moldova, Rep. of

Singapore

Hong Kong SAR

Macedonia, Rep. of

France

Chinese Taipei

2a Bulgaria

New Zealand

Trinidad and Tobago

Canada, Quebec

Slovenia

t2a Belgium (Flemish)

${ }^{\dagger}$ Netherlands

${ }^{2 a}$ Russian Federation

Sweden

Luxembourg

Latvia

+ Scotland

Austria

Belgium (French)

England

Qatar

Italy

${ }^{2 a}$ Canada, Ontario

†2a United States

Canada, Nova Scotia

Germany

2a Canada, Alberta

2a Canada, British Columbia

2a Denmark

Romania

Iran, Islamic Rep. of

Slovak Republic

₹ Norway

Poland

Spain

${ }^{2 b}$ Israel

Iceland

2a Georgia

Lithuania

Kuwait

Hungary

\begin{abstract}
$397(3.9)$
\end{abstract}
$317(6.5)$

299 (5.2)

$492(2.8)$

$552(2.9)$

557 (2.6)

439 (3.7)

516 (2.4)

$530(2.0)$

$542(4.5)$

$527(2.1)$

$434(4.6)$

$529(2.8)$

$519(2.0)$

544 (1.9)

$545(1.8)$

561 (3.3)

$546(2.3)$

$555(1.0)$

$539(2.4)$

527 (2.6)

$537(2.1)$

499 (2.4)

539 (2.6)

358 (1.3)

551 (3.3)

555 (3.0)

541 (3.6)

$543(2.4)$

$549(2.2)$

$561(2.7)$

$559(2.7)$

$547(2.6)$

$493(4.8)$

$426(3.1)$

$533(2.9)$

501 (2.5)

$523(2.5)$

$516(2.7)$

$516(3.4)$

$514(1.7)$

476 (3.2)

$542(1.9)$

340 (3.7)

557 (2.9)

$\begin{array}{r}-418 \\ -\quad 335 \\ \hline\end{array}$

$418(4.2)$

$418(4.2)$

$316(5.1)$

508 (3.0)

$563(2.8)$

$568(2.3)$

$450(4.2)$

$526(2.1)$

$538(1.8)$

550 (4.4)

$534(2.2)$

$440(4.6)$

$533(2.7)$

$523(2.4)$

$547(2.0)$

$548(1.6)$

$564(3.3)$

549 (2.4)

$557(1.0)$

540 (2.4)

$527(2.6)$

$536(2.3)$

$498(2.8)$

$537(2.5)$

356 (1.6)

$549(2.9)$

$552(3.0)$

537 (3.4)

$539(2.4)$

544 (2.3)

$556(2.4)$

$554(2.7)$

$542(2.4)$

487 (4.9)

$420(3.1)$

527 (2.6)

$494(2.8)$

$515(2.2)$

508 (2.9)

507 (3.6)

$505(1.4)$

465 (3.6)

$530(1.6)$

$327(4.3)$

541 (3.1)

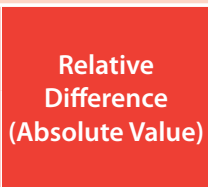

$20(1.3)$

$17(2.8)$

$16(1.2)$

$16(1.5)$

$12(1.1)$

11 (1.1)

$11(1.3)$

$10(2.1)$

$8(1.1)$

$8(1.2)$

$6(0.7)$

$6(1.5)$

$4(1.3)$

$4(1.4)$

$3(1.3)$

$3(1.3)$
$3(1.7)$

$3(1.3)$

$3(1.3)$

2 (1.1)

1 (1.4)

$0(1.3)$

1 (1.2)

2 (1.1)

$2(1.6)$

$2(1.8)$

$3(1.7)$

$3(1.6)$

$3(0.9)$

$4(1.6)$

$4(1.5)$

$5(1.8)$

$6(1.2)$

$6(2.1)$

$6(1.5)$

$6(1.6)$

$7(1.6)$

$7(1.4)$

8 (1.6)

$8(1.9)$

$9(1.0)$

9 (1.6)

11 (2.4)

12 (1.1)

$14(1.9)$

$16(1.2)$

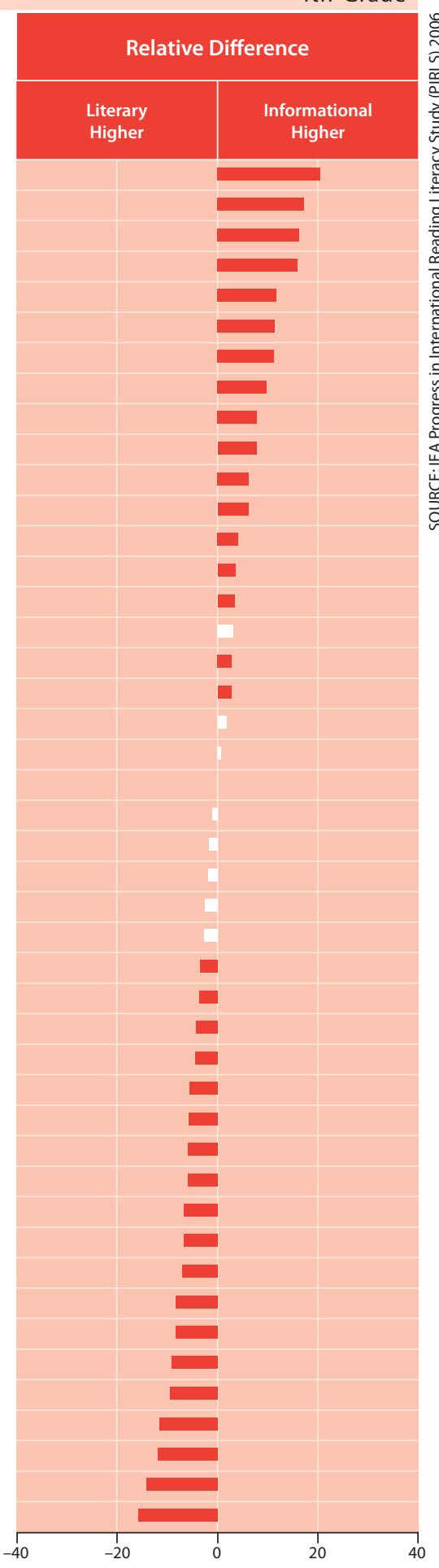

Difference statistically significant

Not statistically significant

† Met guidelines for sample participation rates only after replacement schools were included (see Exhibit A.7).

‡ Nearly satisfying guidelines for sample participation rates after replacement schools were included (see Exhibit A.7).

2a National Defined Population covers less than $95 \%$ of National Desired Population (see Exhibit A.4). 2b National Defined Population covers less than $80 \%$ of National Desired Population (see Exhibit A.4).

() Standard errors appear in parentheses. Because results are rounded to the nearest whole number, some totals may appear inconsistent. 
Hong Kong SAR, and Chinese Taipei), there is considerable diversity among the countries achieving relatively higher in informational reading. Similarly, there is considerable diversity among countries with a relative strength in literary reading, except perhaps for English-speaking North America (the United States as well as the English-speaking Canadian provinces), which achieved somewhat higher in literary reading.

Exhibit 1.7 shows changes in average achievement in reading for the literary purpose. Again, the countries are shown according to the amount of their increase between PIRLS 2001 and PIRLS 2006 (with the bar colored if the change is statistically significant). Consistent with their improvement overall, Hong Kong SAR, the Russian Federation, Singapore, the Slovak Republic, Slovenia, Moldova, Germany, Italy, and Hungary all showed improvement in reading for literary purposes. Also, consistent with their overall decreases, Morocco, England, Romania, Sweden, and the Netherlands declined in average achievement. Iceland, which showed essentially no change overall, had a decrease in reading for literary purposes.

The changes in average achievement in reading for the informational purpose are presented in Exhibit 1.8. Similar to the results for reading for the literary purpose, many countries with improvement overall also improved in informational reading, including Singapore, the Russian Federation, Hong Kong SAR, Slovenia, Italy, and Germany. Interestingly, even though the Slovak Republic, Moldova, and Hungary improved in reading for the literary purpose, they showed little, if any, change in achievement in informational reading. Iran and the Canadian province of Ontario showed improvement in informational reading. (Also, New Zealand had a 9-point increase that did not meet the criteria used for statistical significance.) The decreases in Romania, Sweden, Lithuania, and England as well as in Morocco were consistent with their overall declines. Average achievement in informational reading also declined in Latvia and France. (The 7-point decrease in the Canadian province of Quebec was accompanied by a slightly larger standard error, so the difference was not statistically significant.) 
Exhibit 1.7 Trends in Reading Achievement for Literary Purposes

PIRLS 2006

\begin{tabular}{|c|c|c|c|}
\hline Countries & $\begin{array}{c}\text { PIRLS } 2006 \\
\text { Average } \\
\text { Scale Score }\end{array}$ & $\begin{array}{c}\text { PIRLS } 2001 \\
\text { Average } \\
\text { Scale Score }\end{array}$ & $\begin{array}{c}\text { Difference } \\
\text { Between } \\
2001 \text { and } 2006 \\
\text { Scores }\end{array}$ \\
\hline Hong Kong SAR & $557(2.6)$ & $518(3.1)$ & $39(4.0)$ \\
\hline 2a Russian Federation & $561(3.3)$ & $523(3.9)$ & $38(5.1)$ \\
\hline Singapore & $552(2.9)$ & $528(5.6)$ & $23(6.3)$ \\
\hline Slovak Republic & $533(2.9)$ & $512(2.6)$ & $21(3.9)$ \\
\hline Slovenia & $519(2.0)$ & $499(1.8)$ & $20(2.7)$ \\
\hline Moldova, Rep. of & $492(2.8)$ & $480(3.7)$ & $12(4.7)$ \\
\hline Germany & $549(2.2)$ & $537(1.9)$ & $12(2.9)$ \\
\hline Italy & $551(3.3)$ & $543(2.7)$ & $8(4.2)$ \\
\hline Hungary & $557(2.9)$ & $548(2.0)$ & $8(3.6)$ \\
\hline 2b Israel & $516(3.4)$ & $510(2.6)$ & $6(4.3)$ \\
\hline Iran, Islamic Rep. of & $426(3.1)$ & $421(4.5)$ & $5(5.5)$ \\
\hline 2a Canada, Ontario & $554(3.1)$ & $551(3.3)$ & $3(4.5)$ \\
\hline Latvia & $539(2.4)$ & $537(2.2)$ & $2(3.2)$ \\
\hline France & $516(2.4)$ & $518(2.6)$ & $-2(3.6)$ \\
\hline + Scotland & $527(2.6)$ & $529(3.5)$ & $-2(4.4)$ \\
\hline Macedonia, Rep. of & $439(3.7)$ & $441(4.5)$ & $-3(5.8)$ \\
\hline Lithuania & $542(1.9)$ & $546(3.1)$ & $-4(3.6)$ \\
\hline New Zealand & $527(2.1)$ & $531(3.9)$ & $-4(4.4)$ \\
\hline Canada, Quebec & $529(2.8)$ & $534(3.0)$ & $-4(4.1)$ \\
\hline ₹ Norway & $501(2.5)$ & $506(2.7)$ & $-5(3.7)$ \\
\hline Iceland & $514(1.7)$ & $520(1.3)$ & $-6(2.1)$ \\
\hline 2a Bulgaria & $542(4.5)$ & $550(3.9)$ & $-7(5.9)$ \\
\hline${ }^{\dagger}$ Netherlands & $545(1.8)$ & $552(2.5)$ & $-8(3.1)$ \\
\hline †2a United States & $541(3.6)$ & $550(3.8)$ & $-10(5.2)$ \\
\hline Sweden & $546(2.3)$ & $559(2.4)$ & $-13(3.3)$ \\
\hline Romania & $493(4.8)$ & $512(4.7)$ & $-19(6.8)$ \\
\hline England & $539(2.6)$ & $559(3.9)$ & $-20(4.7)$ \\
\hline Morocco & $317(6.5)$ & $347(8.4)$ & $-30(10.6)$ \\
\hline
\end{tabular}

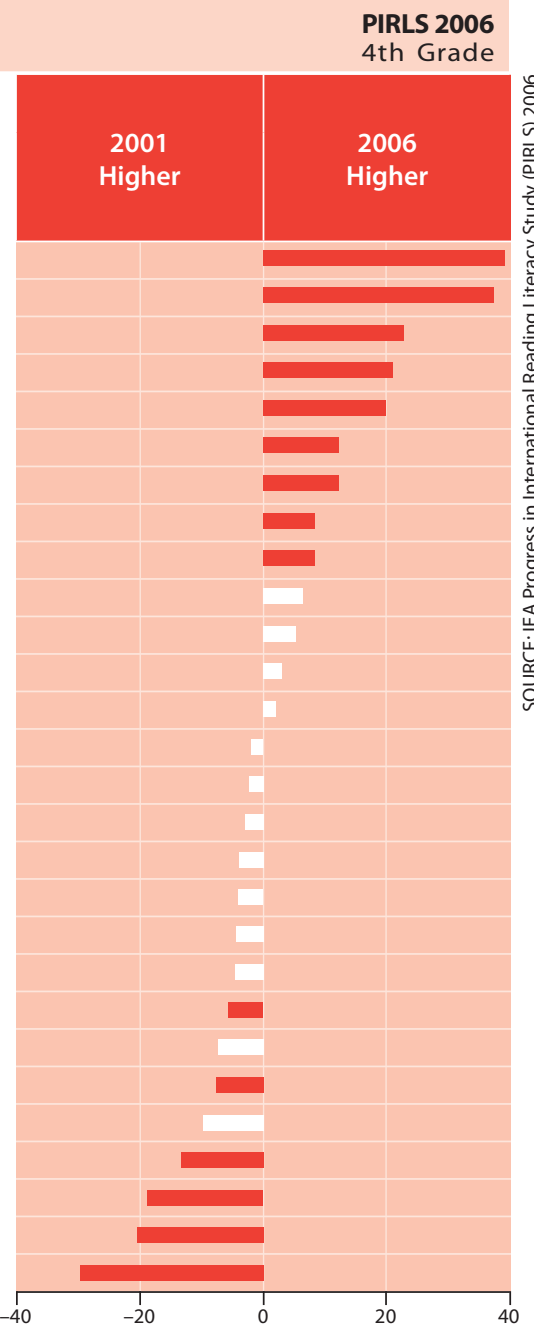

Difference statistically significant Not statistically significant

$+\quad$ Met guidelines for sample participation rates only after replacement schools were included (see Exhibit A.7).

₹ Nearly satisfying guidelines for sample participation rates after replacement schools were included (see Exhibit A.7).

2a National Defined Population covers less than $95 \%$ of National Desired Population (see Exhibit A.4) 2b National Defined Population covers less than $80 \%$ of National Desired Population (see Exhibit A.4).

() Standard errors appear in parentheses. Because results are rounded to the nearest whole number, some totals may appear inconsistent.

Trend Note: The primary education systems of the Russian Federation and Slovenia underwent structural changes. Data for Canada, Ontario include only public schools. 
Exhibit 1.8 Trends in Reading Achievement for Informational Purposes

PIRLS 2006

\begin{tabular}{|c|c|c|c|}
\hline Countries & $\begin{array}{c}\text { PIRLS 2006 } \\
\text { Average } \\
\text { Scale Score }\end{array}$ & $\begin{array}{c}\text { PIRLS 2001 } \\
\text { Average } \\
\text { Scale Score }\end{array}$ & $\begin{array}{c}\text { Difference } \\
\text { Between } \\
\text { 2001 and 2006 } \\
\text { Scores }\end{array}$ \\
\hline Singapore & $563(2.8)$ & $527(4.8)$ & $36(5.6)$ \\
\hline 2a Russian Federation & $564(3.3)$ & $531(4.3)$ & $32(5.5)$ \\
\hline Hong Kong SAR & $568(2.3)$ & $537(2.9)$ & $31(3.7)$ \\
\hline Slovenia & $523(2.4)$ & $503(1.9)$ & $20(3.1)$ \\
\hline Italy & $549(2.9)$ & $536(2.4)$ & $13(3.8)$ \\
\hline Iran, Islamic Rep. of & $420(3.1)$ & $408(4.6)$ & $11(5.6)$ \\
\hline 2a Canada, Ontario & $551(3.1)$ & $542(3.2)$ & $10(4.4)$ \\
\hline New Zealand & $534(2.2)$ & $525(3.8)$ & $9(4.4)$ \\
\hline Germany & $544(2.3)$ & $538(1.9)$ & $6(3.0)$ \\
\hline Slovak Republic & $527(2.6)$ & $522(2.7)$ & $5(3.8)$ \\
\hline Macedonia, Rep. of & $450(4.2)$ & $445(5.2)$ & $5(6.7)$ \\
\hline Hungary & $541(3.1)$ & $537(2.2)$ & $4(3.8)$ \\
\hline +2a United States & $537(3.4)$ & $533(3.7)$ & $4(5.0)$ \\
\hline Moldova, Rep. of & $508(3.0)$ & $505(4.7)$ & $3(5.6)$ \\
\hline ‡ Norway & $494(2.8)$ & $492(2.8)$ & $2(4.0)$ \\
\hline Iceland & $505(1.4)$ & $504(1.5)$ & $1(2.0)$ \\
\hline 2b Israel & $507(3.6)$ & $507(2.9)$ & $1(4.6)$ \\
\hline${ }^{+}$Scotland & $527(2.6)$ & $527(3.6)$ & $0(4.4)$ \\
\hline 2a Bulgaria & $550(4.4)$ & $551(3.6)$ & $-1(5.6)$ \\
\hline${ }^{+}$Netherlands & $548(1.6)$ & $553(2.6)$ & $-5(3.1)$ \\
\hline Latvia & $540(2.4)$ & $547(2.3)$ & $-7(3.3)$ \\
\hline France & $526(2.1)$ & $533(2.5)$ & $-7(3.3)$ \\
\hline Canada, Quebec & $533(2.7)$ & $541(2.9)$ & $-7(4.0)$ \\
\hline England & $537(2.5)$ & $546(3.6)$ & $-9(4.4)$ \\
\hline Lithuania & $530(1.6)$ & $540(2.7)$ & $-10(3.1)$ \\
\hline Sweden & $549(2.4)$ & $559(2.2)$ & $-10(3.2)$ \\
\hline Morocco & $335(6.0)$ & $358(10.9)$ & $-24(12.4)$ \\
\hline Romania & $487(4.9)$ & $512(4.6)$ & $-25(6.8)$ \\
\hline & & & \\
\hline & & & \\
\hline
\end{tabular}

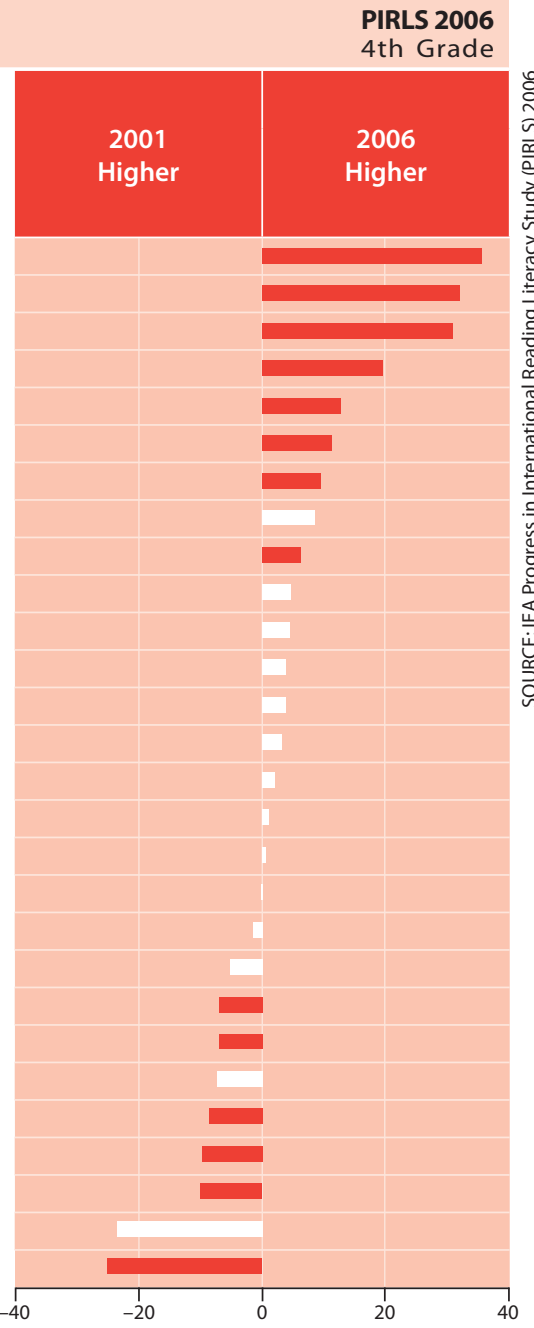

Difference statistically significant Not statistically significant

$\dagger$ Met guidelines for sample participation rates only after replacement schools were included (see Exhibit A.7).

‡ Nearly satisfying guidelines for sample participation rates after replacement schools were included (see Exhibit A.7).

2a National Defined Population covers less than $95 \%$ of National Desired Population see Exhibit A.4). 2b National Defined Population covers less than $80 \%$ of National Desired Population (see Exhibit A.4).

() Standard errors appear in parentheses. Because results are rounded to the nearest whole number, some totals may appear inconsistent.

Trend Note: The primary education systems of the Russian Federation and Slovenia underwent structural changes. Data for Canada, Ontario include only public schools. 


\section{What Are the Gender Differences in Achievement for the Reading Purposes?}

Exhibit 1.9 presents the PIRLS 2006 gender differences in average achievement for literary and informational reading. For the literary reading purpose, girls had significantly higher average achievement than boys in every participating entity (except Iran where the 11-point difference was not statistically significant). In contrast, some European countries had little if any gender difference in informational reading, including Belgium (French), Hungary, Italy, Luxembourg, and Spain.

To provide an international context for the differences, Exhibit 1.9 presents the international average for girls and boys for each of the purposes. The international average is the mean of the average scale score of the PIRLS 2006 countries, excluding the five Canadian provinces. Across all the participating countries (excluding the provinces), the average differences were similar for the two purposes -17 points for literary and 16 points for informational.

\section{How Does Achievement Differ Across Countries for Reading Comprehension Processes?}

Within reading for literary and informational purposes, the test questions or items were designed to measure four major processes of reading comprehension described in the framework. Briefly, the four major reading comprehension processes addressed by PIRLS 2006 are:

- Focus on and retrieve explicitly stated information,

- Make straightforward inferences,

- Interpret and integrate ideas and information, and

- Examine and evaluate content, language, and textual elements.

Given the number of items in the assessment, it was not possible to create four separate achievement scales for the reading processes. Based on research conducted by Germany ${ }^{5}$ and at the TIMSS \& PIRLS International Study Center ${ }^{6}$ examining PIRLS 2001, PIRLS 2006 was designed to support the

5 Bos, W., Lankes, E. M., Prenzel, M., Schwippert, K., Walther, G., \& Valtin, R. (Hrsg.). (2003). Ergebnisse aus IGLU: Schülerleistungen am Ende der vierten Jahrgangsstufe im internationalen Vergleich. New York: Waxmann.

6 Mullis, I.V.S., Martin, M.O., and Gonzalez, E.J. (2004). PIRLS international achievement in the processes of reading comprehension: Results from PIRLS 2001 in 35 countries. Chestnut Hill, MA: Boston College. 
Exhibit 1.9 Average Achievement in Reading for Literary and Informational Purposes by Gender

PIRLS 2006

4th Grade

\begin{tabular}{|c|c|c|c|c|c|c|c|c|}
\hline \multirow[b]{2}{*}{ Countries } & \multicolumn{4}{|c|}{ Literary } & \multicolumn{4}{|c|}{ Informational } \\
\hline & $\begin{array}{c}\text { Girls } \\
\text { Average } \\
\text { Scale Score }\end{array}$ & & $\begin{array}{l}\text { Boys } \\
\text { Average } \\
\text { Scale Score }\end{array}$ & $\begin{array}{l}\text { Girls Higher } \\
\text { Average } \\
\text { Achievement }\end{array}$ & $\begin{array}{c}\text { Girls } \\
\text { Average } \\
\text { Scale Score }\end{array}$ & & $\begin{array}{c}\text { Boys } \\
\text { Average } \\
\text { Scale Score }\end{array}$ & $\begin{array}{l}\text { Girls Higher } \\
\text { Average } \\
\text { Achievement }\end{array}$ \\
\hline Austria & $543(2.6)$ & $\mathbf{0}$ & $531(2.4)$ & $11(2.7)$ & $540(2.7)$ & 0 & $533(2.6)$ & $7(2.6)$ \\
\hline †2a Belgium (Flemish) & $547(2.2)$ & 0 & $541(2.3)$ & $6(2.4)$ & $550(2.4)$ & 0 & $545(2.2)$ & $5(2.1)$ \\
\hline Belgium (French) & $504(2.6)$ & 0 & $495(2.8)$ & $9(2.5)$ & $499(3.3)$ & & $497(3.0)$ & $1(3.0)$ \\
\hline 2a Bulgaria & $553(4.6)$ & $\boldsymbol{0}$ & $532(5.4)$ & $21(4.7)$ & $558(4.4)$ & $\boldsymbol{\theta}$ & $542(5.2)$ & $16(4.3)$ \\
\hline 2a Canada, Alberta & $567(2.9)$ & 0 & $556(3.0)$ & $11(2.2)$ & $559(2.5)$ & 0 & $553(2.8)$ & $7(2.1)$ \\
\hline 2a Canada, British Columbia & $565(3.0)$ & $\boldsymbol{\theta}$ & $553(3.2)$ & $12(3.2)$ & $556(3.3)$ & 0 & $551(2.8)$ & $6(3.0)$ \\
\hline Canada, Nova Scotia & $552(3.4)$ & 0 & $534(2.6)$ & $18(3.7)$ & $549(2.8)$ & 0 & $529(3.0)$ & $20(3.3)$ \\
\hline 2a Canada, Ontario & $562(3.5)$ & 0 & $549(3.3)$ & $12(3.5)$ & $558(3.3)$ & 0 & $547(3.9)$ & $11(4.0)$ \\
\hline Canada, Quebec & $536(3.1)$ & 0 & $523(3.4)$ & $12(3.5)$ & $539(2.7)$ & 0 & $528(3.6)$ & $11(3.3)$ \\
\hline Chinese Taipei & $538(2.2)$ & 0 & $523(2.2)$ & $15(1.8)$ & $543(1.8)$ & 0 & $534(2.3)$ & $8(2.0)$ \\
\hline 2a Denmark & $554(3.0)$ & 0 & $541(3.1)$ & $13(3.2)$ & $547(2.8)$ & 0 & $536(3.1)$ & $11(3.4)$ \\
\hline England & $550(3.1)$ & 0 & $528(2.7)$ & $22(2.7)$ & $545(2.8)$ & 0 & $529(2.9)$ & $16(2.6)$ \\
\hline France & $523(2.6)$ & 0 & $510(2.7)$ & $12(2.4)$ & $531(2.7)$ & 0 & $521(2.3)$ & $10(2.8)$ \\
\hline 2a Georgia & $484(3.7)$ & 0 & $470(3.6)$ & $14(3.3)$ & $474(3.7)$ & 0 & $457(4.4)$ & $17(3.8)$ \\
\hline Germany & $554(2.4)$ & 0 & $544(2.6)$ & $9(2.5)$ & $547(2.4)$ & 0 & $542(2.7)$ & $6(2.4)$ \\
\hline Hong Kong SAR & $564(2.6)$ & 0 & $551(3.3)$ & $13(2.8)$ & $572(2.2)$ & 0 & $564(2.8)$ & $8(2.2)$ \\
\hline Hungary & $560(3.6)$ & 0 & $553(2.9)$ & $7(2.9)$ & $543(3.7)$ & & $539(3.1)$ & $4(2.8)$ \\
\hline Iceland & $525(2.4)$ & 0 & $504(1.9)$ & $20(2.9)$ & $514(1.9)$ & 0 & $497(2.1)$ & $17(2.9)$ \\
\hline Indonesia & $408(4.0)$ & 0 & $387(4.4)$ & $20(3.3)$ & $427(4.6)$ & 0 & $409(5.0)$ & $18(4.8)$ \\
\hline Iran, Islamic Rep. of & $432(5.3)$ & & $421(4.0)$ & $11(6.8)$ & $429(4.9)$ & $\boldsymbol{\Delta}$ & $412(3.8)$ & $17(6.1)$ \\
\hline${ }^{2 b}$ Israel & $524(4.0)$ & 0 & $509(3.8)$ & $15(3.8)$ & $513(4.5)$ & 0 & $502(4.1)$ & $11(4.8)$ \\
\hline Italy & $556(3.6)$ & $\boldsymbol{0}$ & $548(3.6)$ & $8(3.0)$ & $551(3.1)$ & & $547(3.4)$ & $5(2.9)$ \\
\hline Kuwait & $372(4.5)$ & 0 & $310(5.2)$ & $62(6.8)$ & $361(6.3)$ & 0 & $292(6.0)$ & $68(9.2)$ \\
\hline Latvia & $550(3.0)$ & 0 & $529(2.7)$ & $21(3.1)$ & $553(2.7)$ & 0 & $527(2.7)$ & $26(2.8)$ \\
\hline Lithuania & $550(2.4)$ & 0 & $533(2.0)$ & $17(2.2)$ & $539(2.2)$ & 0 & $521(2.0)$ & $17(2.6)$ \\
\hline Luxembourg & $557(1.4)$ & 0 & $552(1.4)$ & $5(2.2)$ & $557(1.2)$ & & $556(1.5)$ & $1(1.9)$ \\
\hline Macedonia, Rep. of & $449(4.3)$ & 0 & $429(4.0)$ & $20(3.7)$ & $460(4.6)$ & 0 & $440(4.4)$ & $21(3.4)$ \\
\hline Moldova, Rep. of & $499(3.3)$ & $\boldsymbol{\theta}$ & $486(3.0)$ & $13(2.9)$ & $514(3.2)$ & 0 & $502(3.5)$ & $13(2.6)$ \\
\hline Morocco & $326(6.9)$ & 0 & $310(7.4)$ & $17(6.3)$ & $344(6.1)$ & 0 & $326(6.9)$ & $19(5.1)$ \\
\hline${ }^{\dagger}$ Netherlands & $548(2.2)$ & $\boldsymbol{\theta}$ & $541(2.3)$ & $6(2.7)$ & $552(1.8)$ & $\boldsymbol{0}$ & $543(1.9)$ & $9(2.0)$ \\
\hline New Zealand & $539(2.3)$ & 0 & $516(2.9)$ & $23(3.1)$ & $545(2.3)$ & 0 & $522(3.0)$ & $23(2.9)$ \\
\hline${ }^{\ddagger}$ Norway & $512(2.8)$ & 0 & $491(2.7)$ & $21(2.6)$ & $502(3.4)$ & 0 & $486(2.8)$ & $16(3.0)$ \\
\hline Poland & $532(2.8)$ & 0 & $514(3.0)$ & $18(3.0)$ & $523(2.3)$ & 0 & $507(2.8)$ & $16(2.6)$ \\
\hline Qatar & $376(1.8)$ & 0 & $341(2.3)$ & $36(3.3)$ & $374(2.3)$ & 0 & $339(2.3)$ & $35(3.2)$ \\
\hline Romania & $501(4.9)$ & 0 & $485(5.6)$ & $16(4.2)$ & $494(5.2)$ & 0 & $481(5.4)$ & $13(3.8)$ \\
\hline${ }^{2 a}$ Russian Federation & $568(3.8)$ & $\boldsymbol{0}$ & $554(3.3)$ & $15(2.5)$ & $572(3.5)$ & $\boldsymbol{\theta}$ & $555(3.6)$ & $17(2.7)$ \\
\hline † Scotland & $538(3.4)$ & 0 & $515(3.0)$ & $23(3.9)$ & $537(3.6)$ & 0 & $517(2.8)$ & $20(3.9)$ \\
\hline Singapore & $560(3.2)$ & 0 & $544(3.4)$ & $16(3.2)$ & $572(2.9)$ & 0 & $555(3.3)$ & $16(2.7)$ \\
\hline Slovak Republic & $539(2.9)$ & 0 & $527(3.5)$ & $12(3.1)$ & $532(2.5)$ & 0 & $522(3.3)$ & $10(2.7)$ \\
\hline Slovenia & $529(2.3)$ & 0 & $511(2.6)$ & $18(2.7)$ & $533(2.4)$ & 0 & $514(3.2)$ & $18(3.2)$ \\
\hline South Africa & $318(6.0)$ & 0 & $281(5.3)$ & $38(4.3)$ & $332(5.8)$ & 0 & $299(5.4)$ & $33(4.5)$ \\
\hline Spain & $520(3.1)$ & 0 & $513(3.1)$ & $7(3.0)$ & $508(3.2)$ & & $508(3.2)$ & $0(2.7)$ \\
\hline Sweden & $557(2.7)$ & 0 & $536(2.6)$ & $20(2.8)$ & $557(2.9)$ & 0 & $541(2.6)$ & $15(3.0)$ \\
\hline Trinidad and Tobago & $450(4.9)$ & $\boldsymbol{0}$ & $419(5.6)$ & $31(5.4)$ & $455(5.0)$ & $\boldsymbol{0}$ & $426(5.5)$ & $28(5.4)$ \\
\hline †2a United States & $547(3.6)$ & 0 & $534(4.1)$ & $12(2.8)$ & $542(3.1)$ & 0 & $532(4.4)$ & $9(3.3)$ \\
\hline International Avg. & $509(0.6)$ & 0 & $491(0.6)$ & $17(0.5)$ & $509(0.7)$ & 0 & $493(0.6)$ & $16(0.7)$ \\
\hline
\end{tabular}

$\dagger$ Met guidelines for sample participation rates only after replacement schools were included (see Exhibit A.7).

‡ Nearly satisfying guidelines for sample participation rates after replacement schools were included (see Exhibit A.7).

2a National Defined Population covers less than $95 \%$ of National Desired Population (see Exhibit A.4). 2b National Defined Population covers less than $80 \%$ of National Desired Population (see Exhibit A.4).

() Standard errors appear in parentheses. Because results are rounded to the nearest whole number, some totals may appear inconsistent.

NOTE: The International Average does not include the results from the Canadian provinces. 
creation of two reading achievement scales for the reading processes. One scale combines the retrieval and straightforward inferencing processes and is called the retrieving and straightforward inferencing scale. The second scale combines the interpreting and integrating processes with the examining and evaluating processes and is called the interpreting, integrating, and evaluating scale. This combination makes sense conceptually, because it combines the two text-based processes and the two processes that require more reasoning. It also works well analytically, because it allocates about half of the assessment items to each scale.

In the PIRLS 2006 Assessment Framework and Specifications, retrieving information was allocated 20 percent of the assessment and straightforward inferencing 30 percent. These two comprehension processes involve locating explicitly stated information, as well as filling in the "gaps" in information contained in the text. Straightforward inferences are very much text based. Although not explicitly stated in the text, the meaning remains relatively clear. Interpreting and integrating ideas and information was given a weight of 30 percent and examining and evaluating 20 percent. Interpreting and integrating ideas can be at a global level or require relating details and information in the text to an overall idea. Readers are making connections that are not only implicit, but that may be open to some interpretation based on their own perspective. Evaluation focuses on considering the text itself, including the author's purpose, claims made in the text, and the structure and genre, as well as language conventions.

Exhibit 1.10 presents average achievement for the two achievement scales for the reading processes. Just as was the case with the scales for the reading purposes, the scales for the processes are not directly comparable, since they represent different constructs, and, as shown by average percent correct in Exhibit A.16, items on the interpreting, integrating, and evaluating scale were more difficult than those on the retrieving and straightforward inferencing scale. Internationally on average, for the retrieving and straightforward inferencing scale, the average percent correct was 64 percent. In contrast the average percent correct was 20 percentage points lower for the interpreting, 
integrating, and evaluating scale (44\%). This pattern held to some degree for each of the PIRLS 2006 participants.

To allow for relative comparisons between the processes for the PIRLS 2006 participants, again just as was done for the purposes, the international average of each process was scaled to be 500 (the same as the overall PIRLS scale average). However, as can be seen in Exhibit A.16, the items in the interpreting, integrating, and evaluating scale were very difficult, less than 20 percent correct on average, for students in Kuwait, Morocco, Qatar, and South Africa. This low performance created a floor effect in the scaling process that made it difficult to obtain accurate achievement scale estimates for these countries (please see Appendix A for further information). Therefore, achievement results for the interpreting, integrating, and evaluating scale were not produced for these four countries.

As would be anticipated, the countries and Canadian provinces with high achievement overall tended to have the highest achievement in the reading processes (as they did in the reading purposes). Exhibits B.3 and B.4 provide the multiple-comparison information for the two achievement scales for the reading processes.

Exhibit 1.10 also displays the differences between average achievement in the retrieving and straightforward inferencing processes compared to the interpreting, integrating, and evaluating processes. There were some interesting results in relative performance, with about half the PIRLS 2006 participants performing relatively better in the reasoning processes, and most of the others performing relatively better in the text-based processes (the red bar indicates that the difference is statistically significant).

Countries and provinces with higher relative performance in the interpreting, integrating, and evaluating processes included Moldova, Bulgaria, New Zealand, the United States, Italy, Latvia, Hungary, England, Lithuania, Israel, Hong Kong SAR, Spain, Poland, Slovenia, Scotland, Belgium (Flemish), and the Slovak Republic as well as the Canadian provinces of Ontario, Nova Scotia, British Columbia, and Alberta. Interestingly, all of the participants with English as the predominant language had relatively higher 
Exhibit 1.10 Relative Differences in Performance Between Reading Comprehension Processes

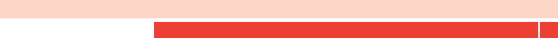

\begin{tabular}{|c|c|c|c|}
\hline Countries & $\begin{array}{l}\text { Retrieving and } \\
\text { Straightforward } \\
\text { Inferencing } \\
\text { Average Scale } \\
\text { Score }\end{array}$ & $\begin{array}{l}\text { Interpreting, } \\
\text { Integrating and } \\
\text { Evaluating } \\
\text { Average Scale } \\
\text { Score }\end{array}$ & $\begin{array}{c}\text { Relative } \\
\text { Difference } \\
\text { (Absolute Value) }\end{array}$ \\
\hline Moldova, Rep. of & $486(2.9)$ & $515(2.9)$ & $29(1.7)$ \\
\hline 2a Canada, Ontario & $543(3.1)$ & $563(2.9)$ & $19(1.6)$ \\
\hline 2a Bulgaria & $538(4.2)$ & $553(4.4)$ & $15(1.5)$ \\
\hline Canada, Nova Scotia & $533(2.2)$ & $548(2.0)$ & $15(0.8)$ \\
\hline New Zealand & $524(2.3)$ & $538(2.2)$ & $14(1.3)$ \\
\hline †2a United States & $532(3.3)$ & $546(3.3)$ & $14(0.9)$ \\
\hline Italy & $544(2.8)$ & $556(2.9)$ & $12(1.1)$ \\
\hline 2a Canada, British Columbia & $551(2.8)$ & $562(2.5)$ & $11(1.4)$ \\
\hline Latvia & $534(2.5)$ & $545(1.9)$ & $11(1.2)$ \\
\hline 2a Canada, Alberta & $553(2.6)$ & $564(2.3)$ & $11(1.2)$ \\
\hline Hungary & $544(2.8)$ & $554(3.0)$ & $10(1.9)$ \\
\hline England & $533(2.8)$ & $543(2.4)$ & $10(1.1)$ \\
\hline Lithuania & $531(1.9)$ & $540(1.6)$ & $9(1.2)$ \\
\hline 2b Israel & 507 (3.2) & $516(3.6)$ & $9(1.4)$ \\
\hline Hong Kong SAR & $558(2.5)$ & $566(2.4)$ & $8(1.3)$ \\
\hline Spain & $508(2.5)$ & $515(2.6)$ & $7(1.1)$ \\
\hline Poland & $516(2.4)$ & $522(2.3)$ & $6(1.6)$ \\
\hline Slovenia & $519(2.1)$ & $523(2.0)$ & $5(0.8)$ \\
\hline † Scotland & $525(2.8)$ & $528(2.6)$ & $4(1.9)$ \\
\hline †2a Belgium (Flemish) & $545(1.9)$ & $547(1.8)$ & $3(1.2)$ \\
\hline Slovak Republic & $529(2.8)$ & $531(2.8)$ & $2(0.8)$ \\
\hline Romania & $489(5.2)$ & $490(5.3)$ & $1(1.2)$ \\
\hline 2a Russian Federation & $562(3.4)$ & $563(3.2)$ & $0(1.7)$ \\
\hline Canada, Quebec & $533(2.7)$ & $531(2.7)$ & $2(1.1)$ \\
\hline Trinidad and Tobago & $438(4.7)$ & $437(5.0)$ & $2(1.9)$ \\
\hline Sweden & $550(2.4)$ & $546(2.2)$ & $4(1.0)$ \\
\hline Belgium (French) & $501(2.6)$ & $497(2.5)$ & $4(1.2)$ \\
\hline Singapore & $560(3.3)$ & $556(2.7)$ & $5(1.1)$ \\
\hline Indonesia & $409(3.9)$ & $404(4.1)$ & $5(1.5)$ \\
\hline France & $523(2.1)$ & $518(2.3)$ & $6(1.1)$ \\
\hline Macedonia, Rep. of & $446(3.8)$ & $439(4.0)$ & $7(1.6)$ \\
\hline ‡ Norway & $502(2.3)$ & $495(2.4)$ & $7(1.2)$ \\
\hline 2a Denmark & $551(2.7)$ & $542(2.3)$ & $9(1.9)$ \\
\hline${ }^{+}$Netherlands & $551(2.0)$ & $542(1.5)$ & $9(1.6)$ \\
\hline Iran, Islamic Rep. of & $428(3.3)$ & $418(3.3)$ & $10(1.5)$ \\
\hline Chinese Taipei & $541(2.0)$ & $530(1.9)$ & $11(0.7)$ \\
\hline Iceland & $516(1.2)$ & $503(1.3)$ & $13(1.2)$ \\
\hline Austria & $544(2.1)$ & $530(2.2)$ & $14(0.9)$ \\
\hline Germany & $555(2.6)$ & $540(2.2)$ & $14(1.5)$ \\
\hline 2a Georgia & $478(3.3)$ & $461(3.5)$ & $17(1.3)$ \\
\hline Luxembourg & $565(1.2)$ & $548(0.9)$ & $17(1.0)$ \\
\hline Kuwait & $337(3.9)$ & ++ & ++ \\
\hline Morocco & $336(6.2)$ & ++ & ++ \\
\hline Qatar & 361 (1.2) & ++ & ++ \\
\hline South Africa & $307(5.3)$ & ++ & ++ \\
\hline
\end{tabular}

Difference statistically significant Not statistically significant

$\dagger \quad$ Met guidelines for sample participation rates only after replacement schools were included (see Exhibit A.7).

‡ Nearly satisfying guidelines for sample participation rates after replacement schools were included (see Exhibit A.7).

2a National Defined Population covers less than $95 \%$ of National Desired Population (see Exhibit A.4) 2b National Defined Population covers less than $80 \%$ of National Desired Population (see Exhibit A.4).

() Standard errors appear in parentheses. Because results are rounded to the nearest whole number, some totals may appear inconsistent.

A plus (+) sign indicates average achievement could not be accurately estimated on the interpreting, integrating, and evaluating scale. 
achievement in the reasoning processes than in the text-based processes. Countries with higher relative performance in retrieving and straightforward inferencing processes included Luxembourg, Georgia, Germany, Austria, Iceland, Chinese Taipei, Iran, the Netherlands, Denmark, Norway, Macedonia, France, Indonesia, Singapore, Belgium (French), and Sweden. This set of countries included the German- and French-speaking countries (except the Canadian province of Quebec) as well as the Scandinavian countries.

Exhibit 1.11 presents changes between PIRLS 2001 and PIRLS 2006 in average achievement for the retrieving and straightforward inferencing processes. Many, but not all, of the countries with improved achievement overall also showed improvement in retrieving and straightforward inferencing (red bar), including Hong Kong sAR, the Russian Federation, Singapore, Slovenia, Germany, and the Slovak Republic. Also, almost all of the countries showing declines overall (except the Netherlands) also had decreases in these text-based comprehension processes, including Romania, Morocco (large but not significant), Sweden, England, and Lithuania. In addition, Bulgaria and Latvia had decreases in average achievement in retrieving and straightforward inferencing.

Exhibit 1.12 presents changes in average achievement between PIRLS 2001 and PIRLS 2006 for the interpreting, integrating, and evaluating processes. The same countries that showed improvement overall, also had higher average achievement in 2006 in interpreting, integrating, and evaluatingthe Russian Federation, Hong Kong sar, Singapore, Slovenia, Moldova, the Slovak Republic, Italy, Hungary, and Germany. In addition, Iran improved in the interpreting, integrating, and evaluating processes. (The 8-point increase in the Canadian province of Ontario was not statistically significant.) Many of the countries that had declines overall also had decreases in average achievement in interpreting, integrating, and evaluating, including Romania, England, Sweden, and the Netherlands. In addition, the Canadian province of Quebec and Iceland declined in this area. 
Exhibit 1.11 Trends in Reading Achievement for Retrieving and Straightforward

PIRLS 2006 Inferencing Processes

\begin{tabular}{|c|c|c|c|}
\hline Countries & $\begin{array}{c}\text { PIRLS } 2006 \\
\text { Average } \\
\text { Scale Score }\end{array}$ & $\begin{array}{c}\text { PIRLS } 2001 \\
\text { Average } \\
\text { Scale Score }\end{array}$ & $\begin{array}{c}\text { Difference } \\
\text { Between } \\
2001 \text { and } 2006 \\
\text { Scores }\end{array}$ \\
\hline Hong Kong SAR & $558(2.5)$ & $522(3.2)$ & $35(4.1)$ \\
\hline 2a Russian Federation & $562(3.4)$ & $529(4.0)$ & $33(5.3)$ \\
\hline Singapore & $560(3.3)$ & $531(5.6)$ & $29(6.5)$ \\
\hline Slovenia & $519(2.1)$ & $503(2.3)$ & $16(3.1)$ \\
\hline Germany & $555(2.6)$ & $543(1.9)$ & $11(3.2)$ \\
\hline Slovak Republic & $529(2.8)$ & $521(2.7)$ & $8(3.8)$ \\
\hline Iran, Islamic Rep. of & $428(3.3)$ & $422(4.4)$ & $6(5.5)$ \\
\hline Italy & $544(2.8)$ & $538(2.4)$ & $6(3.7)$ \\
\hline Macedonia, Rep. of & $446(3.8)$ & $441(4.6)$ & $5(6.0)$ \\
\hline 2b Israel & $507(3.2)$ & $503(2.9)$ & $4(4.3)$ \\
\hline Hungary & $544(2.8)$ & $540(2.1)$ & $4(3.5)$ \\
\hline 2a Canada, Ontario & $542(3.2)$ & $538(3.3)$ & $3(4.6)$ \\
\hline Iceland & $516(1.2)$ & $513(1.3)$ & $3(1.8)$ \\
\hline New Zealand & $524(2.3)$ & $522(3.7)$ & $2(4.3)$ \\
\hline Canada, Quebec & $533(2.7)$ & $534(3.0)$ & $-2(4.0)$ \\
\hline ₹ Norway & $502(2.3)$ & $505(2.9)$ & $-3(3.7)$ \\
\hline France & $523(2.1)$ & $526(2.7)$ & $-3(3.4)$ \\
\hline +2a United States & $532(3.3)$ & $535(3.9)$ & $-3(5.1)$ \\
\hline † Scotland & $525(2.8)$ & $529(3.7)$ & $-4(4.6)$ \\
\hline Moldova, Rep. of & $486(2.9)$ & $491(4.1)$ & $-5(5.0)$ \\
\hline${ }^{\dagger}$ Netherlands & $551(2.0)$ & $556(2.5)$ & $-5(3.2)$ \\
\hline Latvia & $534(2.5)$ & $543(2.2)$ & $-9(3.3)$ \\
\hline Lithuania & $531(1.9)$ & $541(2.9)$ & $-10(3.4)$ \\
\hline 2a Bulgaria & $538(4.2)$ & $550(4.0)$ & $-12(5.8)$ \\
\hline England & $533(2.8)$ & $546(3.3)$ & $-13(4.4)$ \\
\hline Sweden & $550(2.4)$ & $563(2.3)$ & $-13(3.3)$ \\
\hline Morocco & $336(6.2)$ & $353(8.9)$ & $-17(10.8)$ \\
\hline Romania & $489(5.2)$ & $509(5.2)$ & $-20(7.4)$ \\
\hline
\end{tabular}

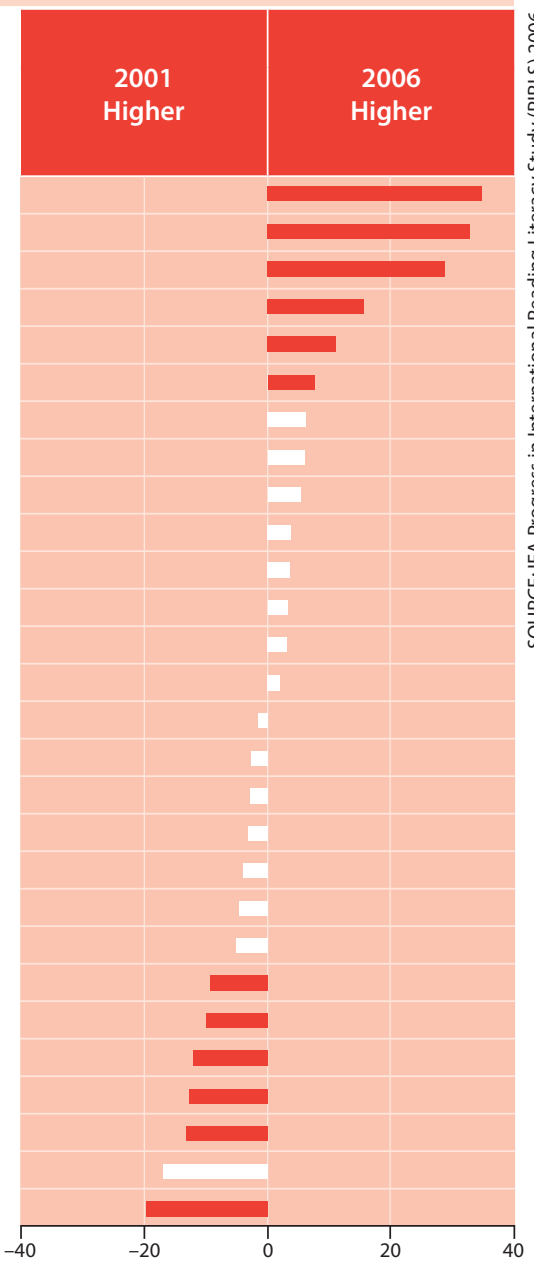

Difference statistically significant Not statistically significant

Met guidelines for sample participation rates only after replacement schools were included (see Exhibit A.7).

‡ Nearly satisfying guidelines for sample participation rates after replacement schools were included (see Exhibit A.7).

2a National Defined Population covers less than $95 \%$ of National Desired Population (see Exhibit A.4). 2b National Defined Population covers less than $80 \%$ of National Desired Population (see Exhibit A.4).

() Standard errors appear in parentheses. Because results are rounded to the nearest whole number, some totals may appear inconsistent.

Trend Note: The primary education systems of the Russian Federation and Slovenia underwent structural changes. Data for Canada, Ontario include only public schools. 
Exhibit 1.12 Trends in Reading Achievement for Interpreting, Integrating, and Evaluating Processes PIRLS 2006

\begin{tabular}{|c|c|c|c|}
\hline Countries & $\begin{array}{c}\text { PIRLS 2006 } \\
\text { Average } \\
\text { Scale Score }\end{array}$ & $\begin{array}{c}\text { PIRLS 2001 } \\
\text { Average } \\
\text { Scale Score }\end{array}$ & $\begin{array}{c}\text { Difference } \\
\text { Between } \\
2001 \text { and 2006 } \\
\text { Scores }\end{array}$ \\
\hline 2a Russian Federation & $563(3.2)$ & $525(4.5)$ & $37(5.5)$ \\
\hline Hong Kong SAR & $566(2.4)$ & $533(3.2)$ & $33(4.0)$ \\
\hline Singapore & $556(2.7)$ & $527(4.9)$ & $29(5.6)$ \\
\hline Slovenia & $523(2.0)$ & $501(2.2)$ & $22(2.9)$ \\
\hline Moldova, Rep. of & $515(2.9)$ & $494(4.0)$ & $22(4.9)$ \\
\hline Slovak Republic & $531(2.8)$ & $513(3.0)$ & $18(4.1)$ \\
\hline Italy & $556(2.9)$ & $541(2.5)$ & $14(3.8)$ \\
\hline Iran, Islamic Rep. of & $418(3.3)$ & $405(5.0)$ & $12(6.0)$ \\
\hline Hungary & $554(3.0)$ & $545(1.9)$ & $9(3.6)$ \\
\hline 2a Canada, Ontario & $561(2.9)$ & $554(2.9)$ & $8(4.1)$ \\
\hline Germany & $540(2.2)$ & $535(1.9)$ & $6(2.8)$ \\
\hline New Zealand & $538(2.2)$ & $535(3.8)$ & $3(4.4)$ \\
\hline 2b Israel & $516(3.6)$ & $513(2.9)$ & $3(4.6)$ \\
\hline 2a Bulgaria & $553(4.4)$ & $550(3.6)$ & $2(5.7)$ \\
\hline${ }^{\dagger}$ Scotland & $528(2.6)$ & $528(3.7)$ & $1(4.5)$ \\
\hline Latvia & $545(1.9)$ & $545(2.1)$ & $0(2.8)$ \\
\hline † Norway & $495(2.4)$ & $495(2.8)$ & $0(3.7)$ \\
\hline +2a United States & $546(3.3)$ & $548(3.2)$ & $-2(4.6)$ \\
\hline Lithuania & $540(1.6)$ & $545(2.6)$ & $-5(3.1)$ \\
\hline France & $518(2.3)$ & $524(2.4)$ & $-6(3.3)$ \\
\hline Macedonia, Rep. of & $439(4.0)$ & $446(4.8)$ & $-7(6.3)$ \\
\hline Iceland & $503(1.3)$ & $512(1.3)$ & $-9(1.8)$ \\
\hline${ }^{\dagger}$ Netherlands & $542(1.5)$ & $552(2.4)$ & $-10(2.8)$ \\
\hline Canada, Quebec & $531(2.7)$ & $541(2.9)$ & $-10(3.9)$ \\
\hline Sweden & $546(2.2)$ & $558(2.2)$ & $-12(3.1)$ \\
\hline England & $543(2.4)$ & $556(3.2)$ & $-13(4.1)$ \\
\hline Romania & $490(5.3)$ & $515(4.5)$ & $-25(7.0)$ \\
\hline Morocco & ++ & ++ & ++ \\
\hline
\end{tabular}

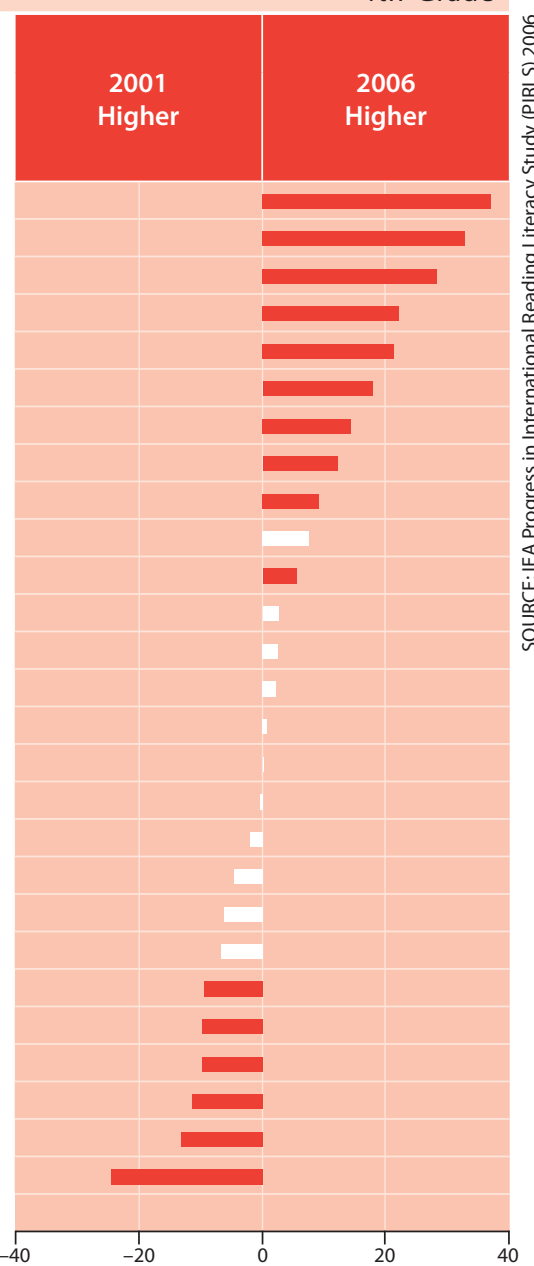

Difference statistically significant

Not statistically significant

+ Met guidelines for sample participation rates only after replacement schools were included (see Exhibit A.7).

₹ Nearly satisfying guidelines for sample participation rates after replacement schools were included (see Exhibit A.7).

2a National Defined Population covers less than $95 \%$ of National Desired Population (see Exhibit A.4).

2b National Defined Population covers less than $80 \%$ of National Desired Population see Exhibit A.4).
() Standard errors appear in parentheses. Because results are rounded to the nearest whole number, some totals may appear inconsistent.

A plus (+) sign indicates average achievement could not be accurately estimated on the interpreting, integrating, and evaluating scale.

Trend Note: The primary education systems of the Russian Federation and Slovenia underwent structural changes. Data for Canada, Ontario include only public schools. 


\section{What Are the Gender Differences in Achievement for the Reading Processes?}

Exhibit 1.13 shows (in alphabetical order) average achievement for girls and boys for the retrieval and straightforward inferencing processes and the interpreting, integrating, and evaluating processes. Mirroring the results overall and for the literary and informational purposes, girls typically had higher achievement than boys. Notably, several countries did not have statistically significant differences for the text-based processes, including, Hungary, Iran, Italy, Luxembourg, the Netherlands, and Spain. However, in the remaining countries and provinces, girls had significantly higher average achievement than boys. For interpreting, integrating, and evaluating, girls had higher achievement than boys in all the participating countries and provinces except Hungary and Iran.

To indicate the pattern internationally, Exhibit 1.13 provides the international average for girls and boys for the two processes, based on the average achievement across the PIRLS 2006 countries, excluding the five Canadian provinces. On average, the advantage for girls was 15 points for the retrieving and straightforward inferencing processes, compared to 17 points for interpreting, integrating, and evaluating. 
Exhibit 1.13 Average Achievement in Reading Processes of Comprehension by Gender

RLS 2006

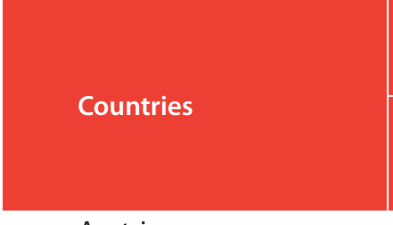

Retrieving and Straightforward Inferencing Processes

Austria

t2a Belgium (Flemish)

Belgium (French)

\begin{tabular}{c|cc} 
Boys \\
Average \\
Scale Score
\end{tabular}

2a Bulgaria

2a Canada, Alberta

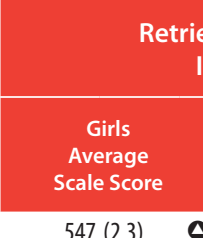

${ }^{2 a}$ Canada, British Columbia

Canada, Nova Scotia

$547(2.3) \quad 0$

$541(2.5)$

2a Canada, Ontario Canada, Quebec

$548(2.3)$

$504(2.8)$

- $542(2.3)$

$6(2.4)$

Girls Higher Achievement

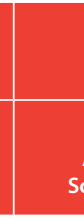

Chinese Taipei

544 (4.3)

$556(2.7)$

- $498(3.0)$

$554(3.0)$

$542(3.1)$

- $531(5.0)$

$550(3.1)$

$6(2.6)$

$6(2.6)$
$6(2.5)$

$13(4.1)$

$6(2.5)$

$548(3.8)$

- 547 (3.2)

$7(2.8)$

- $525(2.9)$

$17(4.1)$

$537(2.8)$

2a Denmark

$546(2.1)$

England

$558(3.1)$

France

$543(3.5)$

2a Georgia

$529(2.5)$

Germany

486 (3.5)

$559(2.8)$

Hong Kong SAR

$562(2.5)$

Hungary

$545(3.5)$

Iceland

$525(1.7)$

Indonesia

$418(4.0)$

Iran, Islamic Rep. of

$435(5.4)$

${ }^{2 b}$ Israel

Italy $\quad 546(2.9)$

Kuwait

Latvia

$546(2.7)$

Lithuania

$541(2.2)$

Luxembourg

$567(1.9)$

Macedonia, Rep. of

$456(4.1)$

Moldova, Rep. of

$491(3.0)$

Morocco

$345(7.2)$

${ }^{+}$Netherlands

$553(2.7)$

New Zealand

$535(2.4)$

₹ Norway

Poland

$510(3.1)$

Oatar

$525(2.6)$

Romania

$377(2.0)$

2a Russian Federation

$495(5.2)$

+ Scotland

$570(3.9)$

$537(3.8)$

Singapore

Slovak Republic

Slovenia

South Africa

$570(3.6)$

$534(2.8)$

$527(2.0)$

$322(6.0)$

Spain

$509(2.8)$

Sweden

Trinidad and Tobago

$558(2.5)$

$453(5.0)$

t2a United States

$537(3.2)$

International Avg.

$508(0.6)$

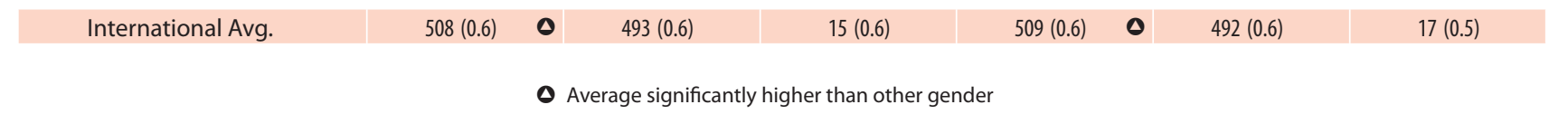

$11(3.8)$

$9(3.0)$

$10(2.2)$

$15(3.3)$

$20(2.8)$

$11(2.7)$

$15(3.3)$

$8(2.7)$

$8(2.3)$

$553(3.0)$

$542(2.8)$

- $508(1.9)$

401 (4.4)

$422(4.0)$

- $502(3.7)$

$542(3.4)$

- $306(5.2)$

- $523(3.0)$

- $521(2.4)$

$564(1.5)$

- $\quad 437$ (4.2)

- $481(3.4)$

- $329(6.5)$

$549(2.3)$

- $513(3.1)$

- 494 (3.1)

- $507(2.8)$

- $344(1.6)$

- $483(5.9)$

- 554 (3.4)

- $512(3.0)$

- $552(3.9)$

- 524 (3.6)

- $511(2.8)$

- $291(5.4)$

508 (2.7)

- $\quad 544(2.9)$

- 424 (5.6)

$4(3.1)$

$17(2.7)$

$17(3.1)$

$13(6.7)$

$11(4.0)$

$4(2.8)$

$62(6.6)$

$23(3.2)$

$20(2.5)$

$3(2.3)$

$19(3.2)$

$10(2.8)$

$16(5.8)$

$4(3.0)$

$22(3.1)$

$16(4.2)$

$16(4.2)$

$33(2.7)$

$13(4.1)$

$16(2.5)$

$24(3.8)$

$18(3.6)$

$10(3.3)$

$16(2.6)$

$31(4.4)$

$1(2.4)$

$14(2.7)$

$29(5.4)$

$10(3.1)$

Interpreting, Integrating,

and Evaluating Processes

4th Grade

$\dagger$ Met guidelines for sample participation rates only after replacement schools were included (see Exhibit A.7).

\# Nearly satisfying guidelines for sample participation rates after replacement schools were included (see Exhibit A.7).

2a National Defined Population covers less than $95 \%$ of National Desired Population (see Exhibit A.4).

2b National Defined Population covers less than $80 \%$ of National Desired Population (see Exhibit A.4).
( ) Standard errors appear in parentheses. Because results are rounded to the nearest whole number, some totals may appear inconsistent.

A plus (+) sign indicates average achievement could not be accurately estimated on the interpreting, integrating, and evaluating scale.

NOTE: The International Average does not include the results from the Canadian provinces. 




\section{Chapter 2}

\section{Performance at the PIRLS 2006 International Benchmarks}

The PIRLS achievement scale summarizes fourth-grade students' performance in reading a variety of literary and informational texts. Students' achievement was based on their responses to test questions designed to assess a range of comprehension processes (e.g., retrieval, inferencing, integration, and evaluation). To provide descriptions of achievement on the scale in relation to performance on the questions asked, PIRLS uses four points on the scale as international benchmarks. The benchmarks represent the range of performance shown by students internationally (and complement the TIMss International Benchmarks). For PIRLS 2006, the Advanced International Benchmark is 625 , the High International Benchmark is 550, the Intermediate International Benchmark is 475 , and the Low International Benchmark is 400 . The TIMSS \& PIRLS International Study Center worked with the PIRLS Reading Development Group ${ }^{1}$ to conduct a detailed scale anchoring analysis to describe reading comprehension at these benchmarks. This chapter describes the types of reading skills and strategies demonstrated by fourth-grade students at each of the international benchmarks together with illustrative items and examples of the answers typically provided by students.

\section{How Does Performance in Countries Compare with the PIRLS 2006 International Benchmarks?}

Exhibit 2.1 displays the percentage of students in each participating country and province that reached each international benchmark. The results are presented in descending order according to the percentage of students 
reaching the Advanced International Benchmark (indicated by the red dots, and shown in the column labeled "Advanced"). Although Exhibit 2.1 is organized to draw particular attention to the percentage of high-achieving students in each country and province, it also conveys information about the distribution of middle and low performers. Since students reaching a particular benchmark also reached lower benchmarks, the percentages illustrated graphically, and shown in the table, are cumulative.

In general, the PIRLS 2006 countries with the highest average achievement had greater percentages of students reaching each benchmark, and lower achieving countries had smaller percentages. Among the countries with the highest average achievement, Singapore and the Russian Federation had nearly one fifth of their students (19\%) reaching the Advanced International Benchmark, about three fifths (58-61\%) reaching the High International Benchmark, 86 to 90 percent reaching the Intermediate International Benchmark, and nearly all (97-98\%) reaching the Low International Benchmark.

Bulgaria and England as well as three of the Canadian provinces (Alberta, British Columbia, and Ontario) had similar percentages of students (15-17\%) reaching the Advanced International Benchmark, but slightly fewer students reaching one or another of the lower benchmarks. Luxembourg, Hong Kong sar, Italy, and Hungary had somewhat smaller percentages of students reaching the Advanced International Benchmark (14-15\%), but substantial percentages reaching all of the rest of the benchmarks.

As a point of reference, Exhibit 2.1 provides the median for each of the international benchmarks. By definition, half the countries (not including the Canadian provinces) will have a percentage above the median percentage and half below. The median percentage of students reaching the Advanced International Benchmark was 7 percent. For students reaching the high benchmark, the median was 41 percent and for the intermediate benchmark, it was 76 percent. Quite impressively, the median for the low benchmark was 94 percent. That is, half the countries ( 23 after rounding) had more than 94 percent of their students reaching the low level (indicated graphically by 


\begin{tabular}{|c|c|}
\hline Exhibit 2.1 & $\begin{array}{l}\text { Percentag } \\
\text { of Reading }\end{array}$ \\
\hline \multicolumn{2}{|l|}{ Countries } \\
\hline \multicolumn{2}{|l|}{ Singapore } \\
\hline \multicolumn{2}{|c|}{ 2a Russian Federation } \\
\hline \multicolumn{2}{|c|}{ 2a Canada, Alberta } \\
\hline \multicolumn{2}{|l|}{ 2a Bulgaria } \\
\hline \multicolumn{2}{|c|}{ 2a Canada, British Columbia } \\
\hline \multicolumn{2}{|c|}{ 2a Canada, Ontario } \\
\hline \multicolumn{2}{|l|}{ England } \\
\hline \multicolumn{2}{|c|}{ Luxembourg } \\
\hline \multicolumn{2}{|c|}{ Hong Kong SAR } \\
\hline \multicolumn{2}{|l|}{ Hungary } \\
\hline \multicolumn{2}{|l|}{ Italy } \\
\hline \multicolumn{2}{|c|}{ New Zealand } \\
\hline \multicolumn{2}{|c|}{ Canada, Nova Scotia } \\
\hline \multicolumn{2}{|c|}{ †2a United States } \\
\hline \multicolumn{2}{|c|}{ 2a Denmark } \\
\hline \multicolumn{2}{|l|}{ Germany } \\
\hline \multicolumn{2}{|l|}{ Sweden } \\
\hline \multicolumn{2}{|l|}{$2 \mathrm{~b}$ Israel } \\
\hline \multicolumn{2}{|l|}{${ }^{\dagger}$ Scotland } \\
\hline \multicolumn{2}{|l|}{ Latvia } \\
\hline \multicolumn{2}{|c|}{ Slovak Republic } \\
\hline \multicolumn{2}{|c|}{ Austria } \\
\hline \multicolumn{2}{|c|}{ †2a Belgium (Flemish) } \\
\hline Chinese $\mathrm{T}$ & pei \\
\hline Poland & \\
\hline${ }^{\dagger}$ Netherlan & \\
\hline Canada, $Q$ & ebec \\
\hline Slovenia & \\
\hline Lithuania & \\
\hline France & \\
\hline Spain & \\
\hline Romania & \\
\hline Iceland & \\
\hline Belgium ( & ench) \\
\hline Moldova, & ep. of \\
\hline Macedoni & Rep. of \\
\hline Trinidad a & d Tobago \\
\hline${ }^{\ddagger}$ Norway & \\
\hline South Afri & \\
\hline 2a Georgia & \\
\hline Iran, Islam & Rep. of \\
\hline Indonesia & \\
\hline Morocco & \\
\hline Kuwait & \\
\hline Qatar & \\
\hline Internatio & al Median \\
\hline
\end{tabular}

\section{Achievement}
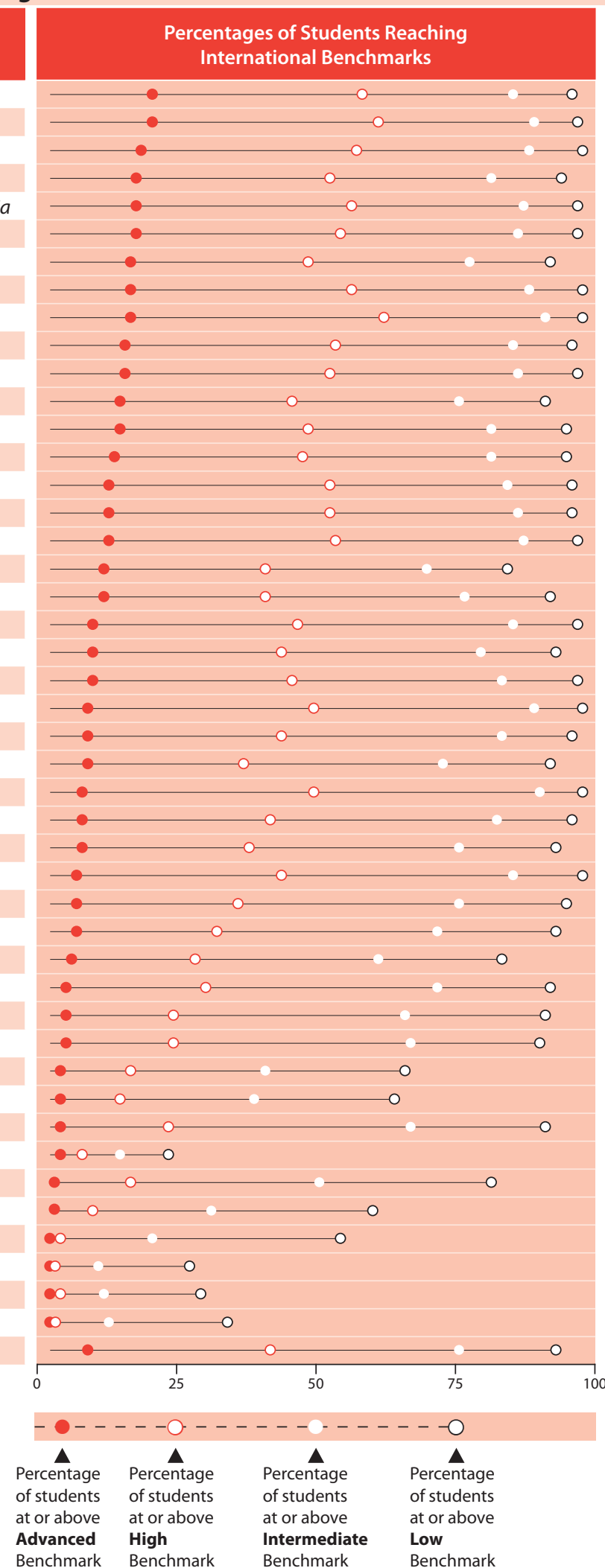

PIRLS 2006

4th Grade

\begin{tabular}{|c|c|c|c|}
\hline $\begin{array}{l}\text { Advanced } \\
\text { (625) }\end{array}$ & $\begin{array}{l}\text { High } \\
(550)\end{array}$ & $\begin{array}{c}\text { Intermediate } \\
\text { (475) }\end{array}$ & $\begin{array}{l}\text { Low } \\
(400)\end{array}$ \\
\hline $19(1.4)$ & $58(1.7)$ & $86(1.0)$ & $97(0.4)$ \\
\hline $19(1.5)$ & $61(2.0)$ & $90(1.1)$ & $98(0.5)$ \\
\hline $17(1.1)$ & $57(1.6)$ & $89(0.8)$ & $99(0.2)$ \\
\hline $16(1.4)$ & $52(2.3)$ & $82(1.8)$ & $95(1.0)$ \\
\hline $16(1.3)$ & $56(1.6)$ & $88(1.0)$ & $98(0.3)$ \\
\hline 16 (1.1) & $54(1.9)$ & $87(1.1)$ & $98(0.5)$ \\
\hline $15(0.9)$ & $48(1.3)$ & $78(1.1)$ & $93(0.7)$ \\
\hline $15(0.6)$ & $56(0.8)$ & $89(0.5)$ & $99(0.2)$ \\
\hline $15(1.0)$ & $62(1.6)$ & $92(0.8)$ & $99(0.2)$ \\
\hline $14(0.9)$ & $53(1.8)$ & $86(1.4)$ & $97(0.5)$ \\
\hline $14(1.4)$ & $52(1.8)$ & $87(1.3)$ & $98(0.4)$ \\
\hline $13(0.7)$ & $45(1.0)$ & $76(1.0)$ & $92(0.6)$ \\
\hline $13(1.0)$ & $48(1.2)$ & $82(1.0)$ & $96(0.4)$ \\
\hline $12(1.2)$ & $47(2.0)$ & $82(1.4)$ & $96(0.6)$ \\
\hline
\end{tabular}

\begin{tabular}{l|l|l|l}
$11(0.9)$ & $52(1.6)$ & $87(0.8)$ & $97(0.3)$
\end{tabular}

$11(0.9) \quad 53(1.5) \quad 88(1.0) \quad 98(0.4)$

\begin{tabular}{l|l|l|l|}
\hline $10(1.0)$ & $40(1.3)$ & $70(1.3)$ & $85(1.2)$ \\
\hline
\end{tabular}

$10(0.8) \quad 40(1.4) \quad 77(1.4) \quad 93(0.8)$

\begin{tabular}{ll|l|l}
$8(0.8)$ & $46(1.5)$ & $86(1.2)$ & $98(0.4)$
\end{tabular}

$8(0.6) \quad 43(1.5) \quad 80(1.3) \quad 94(0.9)$

\begin{tabular}{l|l|l|l}
$8(0.9)$ & $45(1.5)$ & $84(1.1)$ & $98(0.4)$
\end{tabular}

$7(0.6) \quad 49(1.5) \quad 90(0.9) \quad 99(0.2)$

\begin{tabular}{l|l|l|l|}
\hline $7(0.7)$ & $43(1.3)$ & $84(1.0)$ & $97(0.4)$
\end{tabular}

$7(0.5) \quad 36(1.2) \quad 73(1.1) \quad 93(0.7)$

\begin{tabular}{l|l|r|r}
$6(0.5)$ & $49(1.2)$ & $91(0.8)$ & $99(0.2)$
\end{tabular}

$6(0.8) \quad 41(1.9) \quad 83(1.3) \quad 97(0.4)$

\begin{tabular}{ll|l|l}
$6(0.6)$ & $37(1.2)$ & $76(1.1)$ & $94(0.5)$
\end{tabular}

$5(0.8) \quad 43(1.3) \quad 86(0.9) \quad 99(0.3)$

\begin{tabular}{l|l|l|l|}
$5(0.6)$ & $35(1.2)$ & $76(1.2)$ & $96(0.4)$
\end{tabular}

$5(0.5) \quad 31(1.3) \quad 72(1.3) \quad 94(0.8)$

\begin{tabular}{l|l|l|l}
$4(0.5)$ & $27(1.6)$ & $61(2.2)$ & $84(1.8)$
\end{tabular}

$3(0.4) \quad 29(1.1) \quad 72(0.8) \quad 93(0.8)$

\begin{tabular}{l|l|l|l|}
$3(0.4)$ & $23(1.3)$ & $66(1.6)$ & $92(0.7)$
\end{tabular}

$3(0.4) \quad 23(1.5) \quad 67(1.9) \quad 91(0.9)$

\begin{tabular}{l|l|l|l|}
\hline $2(0.4)$ & $15(1.1)$ & $40(1.7)$ & $66(1.6)$
\end{tabular}

$2(0.5) \quad 13(1.2) \quad 38(1.7) \quad 64(2.0)$

\begin{tabular}{l|l|l|l}
$2(0.3)$ & $22(1.1)$ & $67(1.6)$ & $92(0.8)$
\end{tabular}

$2(0.4) \quad 6(0.9) \quad 13(1.4) \quad 22(1.6)$

\begin{tabular}{l|l|l|l|}
$1(0.4)$ & $15(1.3)$ & $50(1.8)$ & $82(1.3)$
\end{tabular}

$1(0.2) \quad 8(0.7) \quad 30(1.3) \quad 60(1.6)$

\begin{tabular}{ll|l|l}
$0(0.1)$ & $2(0.3)$ & $19(1.6)$ & $54(2.1)$
\end{tabular}

$\begin{array}{llll}0(0.0) & 1(0.4) & 9(1.2) & 26(2.0)\end{array}$

\begin{tabular}{l|l|l|l|}
\hline $0(0.1)$ & $2(0.3)$ & $10(0.8)$ & $28(1.2)$ \\
\hline
\end{tabular}

$0(0.0) \quad 1(0.2) \quad 11(0.4) \quad 33(0.7)$

\begin{tabular}{|l|l|l|l}
\hline 7 & 41 & 76 & 94 \\
\hline
\end{tabular}

Advanced Benchmark $=625$

High Benchmark $=550$

Intermediate Benchmark $=475$

Low Benchmark $=400$

$\dagger$ Met guidelines for sample participation rates only after replacement schools were included (see Exhibit A.7).

‡ Nearly satisfying guidelines for sample participation rates after replacement schools were included (see Exhibit A.7).

2a National Defined Population covers less than $95 \%$ of National Desired Population (see Exhibit A.4) 2b National Defined Population covers less than $80 \%$ of National Desired Population (see Exhibit A.4).

() Standard errors appear in parentheses. Because results are rounded to the nearest whole number, some totals may appear inconsistent.

NOTE: The International Median does not include the results from the Canadian provinces. 
the circle to the far right), including five countries with 99 percent, including Luxembourg, Hong Kong SAR, Belgium (Flemish), the Netherlands, and Lithuania. Also, all five of the Canadian provinces were above the median for the Low International Benchmark. Unfortunately, however, several countries had less than half of their students reaching the low benchmark, including South Africa, Morocco, Kuwait, and Qatar.

Considering the countries with average performance significantly below the PIRLS scale average of 500, four of them-Indonesia, Morocco, Kuwait, and Qatar-had very few, if any, students reaching the Advanced International Benchmark, and about 1 to 2 percent reaching the High International Benchmark. Of these four, Indonesia had 19 percent reaching the Intermediate International Benchmark and more than half (54\%) reaching the Low International Benchmark. For the other three, however, only about 10 percent reached the Intermediate International Benchmark and from 26-33 percent reached the Low International Benchmark, indicating that from three fourths to two thirds of the fourth-grade students have minimal comprehension skills.

Five other countries with averages lower than 500-Iran, Georgia, South Africa, Trinidad and Tobago, and Macedonia-had from 1 to 2 percent reaching the Advanced International Benchmark, but differed in the percentages reaching the High International Benchmark, with 6 to 8 percent for Iran and South Africa contrasted with 13 to 15 percent for Georgia, Trinidad and Tobago, and Macedonia. The results for these five countries at the Intermediate and Low International Benchmarks reflect differences in their average achievement overall, with Georgia having the highest performance among the five and South Africa the lowest. Half the Georgian fourth-grade students reached the Intermediate International Benchmark and 82 percent reached the Low International Benchmark, whereas the South African results were 13 and 22 percent, respectively.

Exhibit 2.2 presents changes between PIRLS 2001 and PIRLS 2006 in the percentages of fourth-grade students reaching the benchmarks. ${ }^{2}$ At the advanced and high levels, there were the same number of increases

2 For PIRLS 2006, the procedure for identifying International Benchmarks was changed from the PIRLS 2001 method of using percentiles to using points that will not change from cycle to cycle. For comparability in this report, the 2001 data were analyzed using the new method (See Appendix A). 
and decreases (seven each). At the lower levels, however, there were more increases than decreases indicating some improvement in basic literacy levels over the past 5 years. More specifically, including the two Canadian provinces, at the intermediate level, nine participants had an increase and three a decrease. Seven participants had an increase in the percentage of students reaching the low benchmark and one had a decrease.

Consistent with significant improvements in their overall average achievement between PIRLS 2001 and PIRLS 2006, fourth-grade students in Singapore, Hong Kong SAR, and Slovenia showed significant increases at all four benchmarks. The Russian Federation, Germany, and the Slovak Republic had increases at all except the Low International Benchmark, whereas Italy had increase at all except the Advanced International Benchmark. The improvement in Hungary was primarily at the Advanced International Benchmark. The Canadian province of Ontario had increases at the two lowest benchmarks, Moldova an increase at the intermediate benchmark, and the United States an increase at the low benchmark. Norway also had an increase at the low benchmark, but this was balanced by decreases at the two highest benchmarks.

For countries with declines overall, Romania had decreases at all four benchmarks. England and Sweden had smaller percentages of students reaching the three highest benchmarks. The Netherlands and Lithuania had decreases at the two highest benchmarks as did Iceland (although it did not have a change in average achievement). 


\begin{tabular}{|c|c|c|c|c|c|c|c|c|c|c|c|c|}
\hline \multirow[t]{3}{*}{ Exhibit 2.2} & \multicolumn{11}{|c|}{$\begin{array}{l}\text { Trends in Percentages of Students Reaching the PIRLS } 2006 \text { International Benchmarks } \\
\text { of Reading Achievement in } 2001 \text { and } 2006\end{array}$} & \multirow[t]{2}{*}{$\begin{array}{l}\text { PIRLS } 2006 \\
\text { 4th Grade }\end{array}$} \\
\hline & \multicolumn{3}{|c|}{$\begin{array}{l}\text { Advanced International } \\
\text { Benchmark (625) }\end{array}$} & \multicolumn{3}{|c|}{$\begin{array}{l}\text { High International } \\
\text { Benchmark (550) }\end{array}$} & \multicolumn{3}{|c|}{$\begin{array}{l}\text { Intermediate International } \\
\text { Benchmark (475) }\end{array}$} & \multicolumn{2}{|c|}{$\begin{array}{l}\text { Low International } \\
\text { Benchmark (400) }\end{array}$} & \\
\hline & $\begin{array}{l}2006 \\
\text { Percent } \\
\text { of Students }\end{array}$ & & $\begin{array}{l}2001 \\
\text { Percent } \\
\text { of Students }\end{array}$ & $\begin{array}{l}2006 \\
\text { Percent } \\
\text { of Students }\end{array}$ & & $\begin{array}{l}2001 \\
\text { Percent } \\
\text { of Students }\end{array}$ & $\begin{array}{c}2006 \\
\text { Percent } \\
\text { of Students }\end{array}$ & & $\begin{array}{l}2001 \\
\text { Percent } \\
\text { of Students }\end{array}$ & $\begin{array}{l}2006 \\
\text { Percent } \\
\text { of Students }\end{array}$ & & $\begin{array}{l}2001 \\
\text { Percent } \\
\text { of Students }\end{array}$ \\
\hline Singapore & $19(1.4)$ & 0 & $12(1.4)$ & $58(1.7)$ & 0 & $45(2.4)$ & $86(1.0)$ & 0 & $76(2.0)$ & $97(0.4)$ & 0 & $90(1.4)$ \\
\hline${ }^{2 a}$ Russian Federation & $19(1.5)$ & 0 & $5(0.9)$ & $61(2.0)$ & 0 & $39(2.3)$ & $90(1.1)$ & 0 & $80(1.9)$ & $98(0.5)$ & & $96(1.2)$ \\
\hline 2a Bulgaria & $16(1.4)$ & & $17(1.2)$ & $52(2.3)$ & & $54(1.9)$ & $82(1.8)$ & & $83(1.6)$ & $95(1.0)$ & & $95(0.9)$ \\
\hline England & $15(0.9)$ & (7) & $20(1.4)$ & $48(1.3)$ & (7) & $54(1.7)$ & $78(1.1)$ & ( & $82(1.2)$ & $93(0.7)$ & & $94(0.7)$ \\
\hline 2a Canada, Ontario & $15(1.2)$ & & $15(1.2)$ & $54(2.0)$ & & $50(1.8)$ & $87(1.1)$ & 0 & $84(1.3)$ & $98(0.5)$ & 0 & $96(0.6)$ \\
\hline Hong Kong SAR & $15(1.0)$ & 0 & $5(0.6)$ & $62(1.6)$ & 0 & $39(1.9)$ & $92(0.8)$ & 0 & $81(1.5)$ & $99(0.2)$ & 0 & $97(0.6)$ \\
\hline Hungary & $14(0.9)$ & 0 & $10(0.9)$ & $53(1.8)$ & & $49(1.8)$ & $86(1.4)$ & & $85(1.0)$ & $97(0.5)$ & & $98(0.3)$ \\
\hline Italy & $14(1.4)$ & & $11(0.9)$ & $52(1.8)$ & 0 & $48(1.4)$ & $87(1.3)$ & 0 & $83(1.2)$ & $98(0.4)$ & 0 & $97(0.6)$ \\
\hline New Zealand & $13(0.7)$ & & $14(1.2)$ & $45(1.0)$ & & $45(1.6)$ & $76(1.0)$ & & $74(1.4)$ & $92(0.6)$ & & $90(1.0)$ \\
\hline †2a United States & $12(1.2)$ & & $15(1.1)$ & $47(2.0)$ & & $50(2.0)$ & $82(1.4)$ & & $80(1.7)$ & $96(0.6)$ & 0 & $94(0.7)$ \\
\hline Germany & $11(0.9)$ & 0 & $9(0.6)$ & $52(1.6)$ & 0 & $47(1.3)$ & $87(0.8)$ & 0 & $83(0.9)$ & $97(0.3)$ & & $97(0.4)$ \\
\hline Sweden & $11(0.9)$ & ( & $15(1.0)$ & $53(1.5)$ & ( & $59(1.4)$ & $88(1.0)$ & 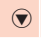 & $90(0.8)$ & $98(0.4)$ & & $98(0.3)$ \\
\hline${ }^{2 b}$ Israel & $10(1.0)$ & & $9(0.7)$ & $40(1.3)$ & & $36(1.2)$ & $70(1.3)$ & & $67(1.2)$ & $85(1.2)$ & & $87(1.0)$ \\
\hline${ }^{+}$Scotland & $10(0.8)$ & & $11(0.9)$ & $40(1.4)$ & & $42(1.9)$ & $77(1.4)$ & & $75(1.5)$ & $93(0.8)$ & & $92(0.9)$ \\
\hline Latvia & $8(0.8)$ & & $9(0.9)$ & $46(1.5)$ & & $49(2.0)$ & $86(1.2)$ & & $87(0.9)$ & $98(0.4)$ & & $99(0.4)$ \\
\hline Slovak Republic & $8(0.6)$ & 0 & $5(0.8)$ & $43(1.5)$ & 0 & $34(1.7)$ & $80(1.3)$ & 0 & $76(1.5)$ & $94(0.9)$ & & $94(0.8)$ \\
\hline${ }^{\dagger}$ Netherlands & $6(0.5)$ & 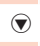 & $10(0.9)$ & $49(1.2)$ & ( & $54(1.8)$ & $91(0.8)$ & & $92(1.0)$ & $99(0.2)$ & & $99(0.3)$ \\
\hline Canada, Quebec & $6(0.8)$ & & $8(0.7)$ & $41(1.9)$ & & $43(2.0)$ & $83(1.3)$ & & $84(1.5)$ & $97(0.4)$ & & $98(0.4)$ \\
\hline Slovenia & $6(0.6)$ & 0 & $3(0.4)$ & $37(1.2)$ & 0 & $25(1.1)$ & $76(1.1)$ & 0 & $67(1.2)$ & $94(0.5)$ & 0 & $91(0.6)$ \\
\hline Lithuania & $5(0.8)$ & (1) & $9(1.0)$ & $43(1.3)$ & (1) & $48(1.8)$ & $86(0.9)$ & & $85(1.2)$ & $99(0.3)$ & & $98(0.4)$ \\
\hline France & $5(0.6)$ & & $7(0.8)$ & $35(1.2)$ & & $37(1.4)$ & $76(1.2)$ & & $77(1.2)$ & $96(0.4)$ & & $95(0.6)$ \\
\hline Romania & $4(0.5)$ & ( & $9(1.2)$ & $27(1.6)$ & ( & $35(2.2)$ & $61(2.2)$ & ( & $69(2.0)$ & $84(1.8)$ & ( & $88(1.3)$ \\
\hline Iceland & $3(0.4)$ & ( & $6(0.5)$ & $29(1.1)$ & ( & $32(0.9)$ & $72(0.8)$ & & $71(1.1)$ & $93(0.8)$ & & $92(0.6)$ \\
\hline Moldova, Rep. of & $3(0.4)$ & & $3(0.7)$ & $23(1.5)$ & & $22(2.1)$ & $67(1.9)$ & 0 & $61(2.1)$ & $91(0.9)$ & & $88(1.2)$ \\
\hline Macedonia, Rep. of & $2(0.4)$ & & $2(0.3)$ & $15(1.1)$ & & $15(1.1)$ & $40(1.7)$ & & $41(1.9)$ & $66(1.6)$ & & $67(2.1)$ \\
\hline ‡ Norway & $2(0.3)$ & ( & $4(0.8)$ & $22(1.1)$ & ( & $28(1.5)$ & $67(1.6)$ & & $65(1.6)$ & $92(0.8)$ & 0 & $88(0.9)$ \\
\hline Iran, Islamic Rep. of & $1(0.2)$ & & $0(0.2)$ & $8(0.7)$ & & $7(0.8)$ & $30(1.3)$ & & $28(1.8)$ & $60(1.6)$ & & $56(2.0)$ \\
\hline Morocco & $0(0.0)$ & & $1(0.9)$ & $1(0.4)$ & & $4(1.6)$ & $9(1.2)$ & & $14(2.6)$ & $26(2.0)$ & & $33(3.4)$ \\
\hline International Avg. & $9(0.2)$ & 0 & $8(0.2)$ & $40(0.3)$ & 0 & $38(0.3)$ & $74(0.3)$ & $\theta$ & $72(0.3)$ & $90(0.2)$ & 0 & $89(0.2)$ \\
\hline
\end{tabular}

+ Met guidelines for sample participation rates only after replacement schools were included (see Exhibit A.7).

₹ Nearly satisfying guidelines for sample participation rates after replacement schools were included (see Exhibit A.7).

2a National Defined Population covers less than $95 \%$ of National Desired Population (see Exhibit A.4).

2b National Defined Population covers less than $80 \%$ of National Desired Population (see Exhibit A.4)
() Standard errors appear in parentheses. Because results are rounded to the nearest whole number, some totals may appear inconsistent.

NOTE: The International Average does not include the results from the Canadian provinces. Trend Note: The primary education systems of the Russian Federation and Slovenia underwent structural changes. Data for Canada, Ontario include only public schools. 


\section{How Were the Benchmark Descriptions Developed?}

The TIMSS \& PIRLS International Study Center conducted a scale anchoring analysis to develop descriptions of achievement at the PIRLS 2006 international benchmarks. The scale anchoring data provided a basis for describing students' performance at different points on the reading achievement scale in terms of the types of texts they were asked to read, the types of items they were able to answer successfully, and the quality of their answers (for multipoint constructed-response questions). In addition to the data analysis component to identify items that discriminated between successive points on the scale, the process also involved a judgmental component in which the PIRLS 2006 committee of reading experts examined the content of the texts and items and generalized to describe students' comprehension skills and strategies.

For the scale anchoring data analysis, the students' achievement results from all the participating countries and provinces were pooled, so that the benchmark descriptions refer to all students achieving at that level. Thus, in determining performance in relation to the benchmarks, it does not matter what country or province a student is from, only how he or she performed on the test. Considering students' reading achievement scale scores, criteria were applied to identify the sets of items that students reaching each international benchmark were likely to answer correctly and that those at the next lower benchmark were unlikely to answer correctly.

For example, a multiple-choice item anchored at the Advanced International Benchmark if at least 65 percent of students scoring at 625 answered the item correctly and fewer than 50 percent of students scoring at the High International Benchmark (550) answered correctly. Similarly, a multiple-choice item anchored at the High International Benchmark if at least 65 percent of students scoring at 550 answered the item correctly and fewer than 50 percent of students scoring at the Intermediate International Benchmark answered it correctly; and so on, for each successively lower benchmark. Since constructed-response questions nearly eliminate guessing, the criterion for the constructed-response items was simply 50 percent at the 
particular benchmark, and, for multipoint items, the analysis differentiated between partial-credit and full-credit responses.

There were 126 items in the assessment, about half (64) assessing "literary experience" and half (62) assessing "acquire and use information". Please see Appendix A for the distribution of items by reading purpose and process category.

About half the PIRLS 2006 items required students to construct their own answers to the questions (with no help from those administering the assessment). ${ }^{3}$ The constructed-response questions took three different forms:

- For 1-point items, responses were scored as acceptable if they included all elements required by the questions and were determined to be accurate based on ideas and information in the text.

- For 2-point items, responses that were given full credit demonstrated complete comprehension by providing appropriate inferences and interpretations consistent with the text and adequate textually-based support if required. Responses were given partial credit (1 point), if they included only some of the information or demonstrated only a literal understanding when an inference or interpretation was required.

- For 3-point items, responses were given full credit if they demonstrated extensive comprehension by presenting relatively complex, abstract ideas or by providing substantial textual support for inferences and interpretations. Responses were considered satisfactory and given 2 points if they contained all the required elements but did not provide complex or abstract ideas, were more literal than interpretive, or were weak in textually-based support. Minimal responses (1 point) contained some but not all of the required elements.

For students to demonstrate achievement in the reading comprehension process being assessed by multipoint items, usually the response needed to receive full credit. That is, a more literal response to an item requiring interpretation, integration, or evaluation of ideas in the text did provide text-

3 To ensure reliable scoring, PIRLS developed scoring guides for each constructed-response item and conducted training in how to apply the guides. To monitor reliability within countries, across countries, and between the 2001 and 2006 assessments, subsamples of students' systematic responses were scored independently by more than one reader (see Appendix A). 
based information, demonstrating that the student could locate and retrieve information. However, this type of response did not demonstrate that the student was able to interpret, integrate, or evaluate the information in the text. So, even though students providing such literal responses received partial credit, the partial credit responses did not necessarily reflect competence in the comprehension process being assessed.

The sets of items identified by the scale anchoring analysis represented the accomplishments of students reaching each successively higher benchmark, and were used by the PIRLS 2006 Reading Development Group (RDG) to develop the benchmark descriptions. For each benchmark, the work of the RDG involved developing a short description for each anchor item that characterized the reading skills and strategies demonstrated by students answering it successfully (and for multipoint constructed-response questions, according to whether students answered partially or fully). These item-byitem descriptions are found in Appendix E. Then, the RDG summarized students' reading comprehension skills and strategies across the set of items for each benchmark to provide more general statements of achievement.

\section{How Should the Benchmark Descriptions Be Interpreted?}

In thinking about the reading demands underlying any assessment question, there is, of course, a substantial interaction between the sophistication of the comprehension required by the question, the length and complexity of the text, and the likelihood of the students' familiarity with the reading content and structure. Although the PIRLS 2006 texts were constrained by the assessment situation, they still varied in features such as length, syntactic complexity, vocabulary, abstractness of ideas, and organizational structure. In particular, because of the differences between the literary and informational texts, the benchmark descriptions are presented separately for the two reading purposes. It also should be kept in mind that the descriptions of reading skills and strategies at the PIRLS 2006 benchmarks were developed on the basis of these texts, and are intended to explain differences in achievement on the 
PIRLS 2006 assessment. The descriptions do not purport to encompass all reading situations encountered by fourth-grade students.

The PIRLS 2006 assessment was based on 10 different texts, 5 for the literary purpose and 5 for the informational purpose. Exhibit 2.3 summarizes the characteristics of the literary and informational texts. To support the variety of questions necessary to cover the range of comprehension processes (e.g., locating and retrieving, integrating, evaluating, etc.), the passages averaged 760 words in length, with a range from 495 to 872 words. Considering the assessment context, PIRLS 2006 included a variety of text types within the two purposes. Considerable effort was expended by the participating countries to identify a wide selection of appropriate passages, and there was considerable variety in the informational texts. These included a brochure, a biography, a "how to" article about science projects, as well as descriptive articles within the areas of geography and biology.

In reflecting on the reading comprehension processes assessed by PIRLS 2006, it might seem that locating and extracting explicitly stated information would be less difficult than, for example, making interpretations and integrating ideas across a whole text. Also, students with higher performance on the PIRLS reading achievement scale were more likely than those at lower levels to successfully complete questions requiring interpretation and integration of information. All texts are not equal, however, and because the PIRLS 2006 texts needed to conform to the assessment situation, they represent a limited view of the universe of texts available to fourth-grade students. It is not the case that interpretive reading tasks are always more difficult than tasks requiring retrieval of explicit information.

For some items, the comprehension processes necessary to answer successfully may vary according to students' experiences. Understanding vocabulary use may be explicit for one student and require interpretation for another. Nevertheless, the descriptions are based on what the panel believed to be the way the great majority of students would approach the item. Finally, some students scoring below a benchmark may very well know or understand some of the concepts that characterize a higher level. It is 


\section{Literary Texts}

The literary texts were complete short stories or episodes accompanied by supportive illustrations. The five stories covered a variety of settings, with each having essentially two main characters and a plot with one or two central events.

Informational Texts

The five informational texts covered a variety of content, including scientific, geographical, biographical, and procedural material. The texts were structured sequentially or by topic. As well as prose, each text included organizational and presentational features such as diagrams, maps, illustrations, photographs, text boxes, lists, or tables. 
important to consider performance on the individual items and clusters of items in developing a profile of student achievement in each country.

The remainder of this chapter describes fourth-grade students' reading achievement at each of the four benchmarks, beginning with the Low International Benchmark and working up the scale to the Advanced International Benchmark. The description of achievement at each higher benchmark is cumulative, building on the description of achievement demonstrated by students at the next lower benchmark. Students reaching a particular benchmark demonstrated the comprehension skills and strategies characterizing that benchmark as well as the competencies of students at any lower benchmarks. For each benchmark, there is a description of the comprehension skills and strategies demonstrated by the students on PIRLS 2006, as well as four example items (two literary and two informational) with results from each participating country or province.

\section{Achievement at the PIRLS 2006 Low International Benchmark}

Exhibit 2.4 describes the reading skills and strategies demonstrated by fourth-grade students reaching level 400 on the PIRLS reading achievement scale. Essentially, these students displayed basic reading skills. They were able to recognize, locate, and reproduce explicitly stated details from the informational texts, particularly if the details were close to the beginning of the text. Students reaching the Low International Benchmark also demonstrated success with some items requiring straightforward inferences.

Exhibits 2.5 and 2.6 present two literary examples, both from the "Unbelievable Night" story found in the PIRLs Reader (in the back pocket of the report). Both questions are in the multiple-choice format. The first question, Item 1, about the crocodile's tail breaking the bedroom door required recognizing an important event in the story. This question was relatively easy for students, with 77 percent answering correctly, on average, across the participating countries. (The Canadian provinces were not included in calculating the international average.) More than 90 percent of students answered correctly in the Russian Federation, Hong Kong SAR, and Chinese 
Taipei. The second question, Item 2, directed students to the end of the story and required a straightforward inference. Thus, it was somewhat more difficult, but still answered correctly by 69 percent of the students, on average.

Exhibits 2.7 and 2.8 present two informational examples, both from the article about Antarctica found in Appendix D. The first question, Item 3, asked students to write their answer to the question about where Antarctica could be found on the globe. The information was explicitly stated in the first paragraph (the bottom) or could be found on the map (South Pole). In many participating countries and all the provinces, 9o percent or more of the students answered correctly. The second example, Item 4, also in the constructed-response format, was more demanding, although students at the low level provided only one text-based reason (of two required) for not visiting Antarctica between April and September. As can be seen from Exhibit 2.8, on average internationally, just over half the students (55\%) provided only one reason, far fewer than provided at least one reason (those providing one reason plus those providing two reasons). Internationally on average, 78 percent of the students provided at least one reason. 
Exhibit 2.4 Description of the PIRLS 2006 Low International Benchmark of Reading Achievement

\section{Informational}

When reading information texts, students can:

- Locate and reproduce explicitly stated information that is readily accessible, for example, at the beginning of the text or in a clearly defined section

- Begin to provide a straightforward inference clearly suggested by the text 


\section{Exhibit 2.5 PIRLS 2006 Low International Benchmark - Item 1 - Literary Example}
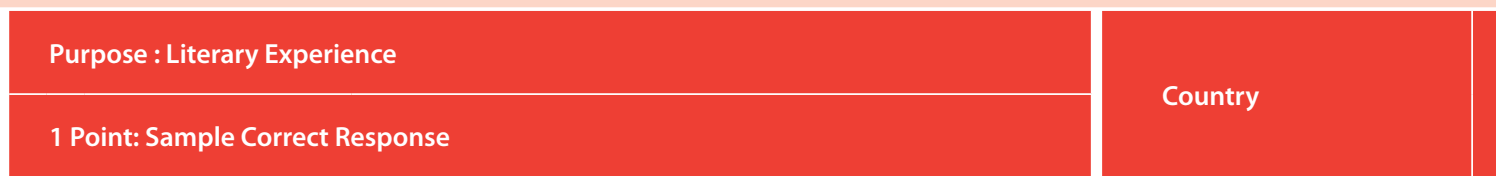

4th Grade

7. How did the bedroom door get broken?

\begin{tabular}{|l|}
\hline 2a Russian Federation \\
Hong Kong SAR \\
Chinese Taipei \\
2a Bulgaria \\
\hline Slovenia \\
\hline 2a Denmark \\
\hline Sweden \\
\hline Lithuania \\
\hline France
\end{tabular}

Percent

Correct

The crocodile's tail pushed through it.

(B) The big vase cracked against it.

(C) The flamingo's sharp beak crashed into it.

D The bed smashed against it.

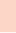

${ }^{+}$Netherlar

Singapore

2a Canada, Alberta $84(1.4)$ ○

Germany $83(1.2)$ ○

t2a United States $\quad 83(1.3) \quad 0$

Belgium (French) $\quad 82(1.2) \quad 0$

Hungary $\quad 82(1.6) \quad 0$

2a Georgia $82(1.4) \quad 0$

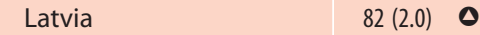

Poland $82(1.4) \quad 0$

\begin{tabular}{l|l} 
Spain & $82(1.5) \quad 0$
\end{tabular}

Canada, Quebec $81(1.8)$ ○

2a Canada, British Columbia 81 (1.3) 0

Iceland $81(1.5) \quad 0$

\begin{tabular}{l|l} 
t2a Belgium (Flemish) & $81(1.6) \quad 0$
\end{tabular}

Austria $79(1.5)$

\begin{tabular}{l|l} 
₹ Norway & $79(2.2)$ \\
\hline
\end{tabular}

England $\quad 78(1.6)$

\begin{tabular}{l|l} 
Romania & $78(2.2)$ \\
\hline
\end{tabular}

Luxembourg $\quad 78(1.2)$

\begin{tabular}{l|l}
\hline † Scotland & $77(2.0)$ \\
\hline
\end{tabular}

\begin{tabular}{l|l} 
International Avg. & $77(0.3)$ \\
\hline
\end{tabular}

Canada, Nova Scotia $\quad 77(1.7)$

Moldova, Rep. of $75(1.9)$

2a Canada, Ontario $75(2.3)$

Slovak Republic $\quad 75(1.5)$

\begin{tabular}{l|l}
${ }^{2 b}$ Israel & $75(1.6)$
\end{tabular}

Italy $74(1.8)$

\begin{tabular}{l|l} 
New Zealand & $73(1.5) \quad(1)$
\end{tabular}

Macedonia, Rep. of $67(2.3)$

\begin{tabular}{l|l} 
Trinidad and Tobago & $63(1.8) \quad$ (7)
\end{tabular}

Qatar $62(1.4)$

Iran, Islamic Rep. of $60(2.3)$

Indonesia $\quad 60(2.1)$

\begin{tabular}{l|r} 
Kuwait & $49(2.0) \quad 0$
\end{tabular}

Morocco $48(2.6)$

\begin{tabular}{l||l} 
South Africa & $40(1.1)$ \\
\hline
\end{tabular}

Percentage of students answering correctly significantly higher than international average

Percentage of students answering correctly significantly lower than international average

Met guidelines for sample participation rates only after replacement schools were included (see Exhibit A.7).

‡ Nearly satisfying guidelines for sample participation rates after replacement schools were included (see Exhibit A.7).

2a National Defined Population covers less than $95 \%$ of National Desired Population (see Exhibit A.4) 2b National Defined Population covers less than $80 \%$ of National Desired Population (see Exhibit A.4).

() Standard errors appear in parentheses. Because results are rounded to the neares whole number, some totals may appear inconsistent.

NOTE: The International Average does not include the results from the Canadian provinces. 
Exhibit 2.6 PIRLS 2006 Low International Benchmark - Item 2 - Literary Example

PIRLS 2006

4th Grade

\section{Purpose : Literary Experience}

1 Point: Sample Correct Response

9. At the end of the story, how did Anina feel toward the flamingos?

(A) guilty

(B) cautious

grateful

(D) annoyed

(ans

Country

Percent

Correct

Chinese Taipei

$90(1.2) \quad \mathbf{0}$

Latvia

Luxembourg

$88(1.5) \quad 0$

2a Russian Federation

$88(0.9) \quad 0$

Sweden

2a Bulgaria

$87(1.4) \quad 0$

Hong Kong SAR 85 (1.5)

\begin{tabular}{l|l}
\hline Lithuania & $84(1.9) \quad 0$
\end{tabular}

Germany

$84(1.3) \bigcirc$

Iceland

Slovenia

$83(1.4) \bigcirc$

Poland $82(1.8)$ ○

\begin{tabular}{l|r} 
Austria & $81(1.4) \quad 0$
\end{tabular}

2a Denmark $81(1.5) \quad \mathbf{0}$

\begin{tabular}{l|r} 
Spain & $80(1.7) \quad 0$
\end{tabular}

${ }^{\dagger}$ Netherlands $\quad 79(2.0) \quad \mathbf{0}$

Singapore $79(1.3) \quad 0$

Indonesia $79(1.6) \quad 0$

Macedonia, Rep. of $75(1.8) \quad 0$

Romania $74(2.2) \quad 0$

+2a Belgium (Flemish) $\quad 74(1.7) \quad 0$

${ }^{2 b}$ Israel $\quad 72(2.0)$

\begin{tabular}{l|l} 
Moldova, Rep. of & $72(1.8)$
\end{tabular}

2a Georgia $70(2.6)$

Italy $70(2.3)$

\begin{tabular}{|l|l}
\hline International Avg. & $69(0.3)$ \\
\hline
\end{tabular}

Hungary $65(1.8)$

2a Canada, Alberta $63(1.7)$

2a Canada, British Columbia $63(2.0)$

England $61(1.9)$

\begin{tabular}{l|l} 
t2a United States & $61(1.8) \quad \nabla$
\end{tabular}

Canada, Quebec $\quad 60(2.3)$

${ }^{2 a}$ Canada, Ontario $59(2.5)$

New Zealand $58(1.5)$

Canada, Nova Scotia $58(1.8)$

Iran, Islamic Rep. of $55(1.9)$

‡Norway $55(2.4)$

+ Scotland $54(1.9)$

\begin{tabular}{l|l} 
Trinidad and Tobago & $47(1.9)$
\end{tabular}

Belgium (French) $\quad 41(1.7)$

\begin{tabular}{l|l} 
Qatar & $37(1.5) \quad \odot$
\end{tabular}

France $37(1.8)$

Morocco $34(2.3)$

South Africa $31(1.2)$

Kuwait

$31(2.0)$

Percentage of students answering correctly significantly higher than international average

Percentage of students answering correctly significantly lower than international average

$\dagger$ Met guidelines for sample participation rates only after replacement schools were included (see Exhibit A.7).

‡ Nearly satisfying guidelines for sample participation rates after replacement schools were included (see Exhibit A.7)

2a National Defined Population covers less than $95 \%$ of National Desired Population (see Exhibit A.4) 2b National Defined Population covers less than $80 \%$ of National Desired Population (see Exhibit A.4).

() Standard errors appear in parentheses. Because results are rounded to the nearest whole number, some totals may appear inconsistent.

NOTE: The International Average does not include the results from the Canadian provinces. 


\section{Exhibit 2.7 PIRLS 2006 Low International Benchmark - Item 3 - Informational Example}

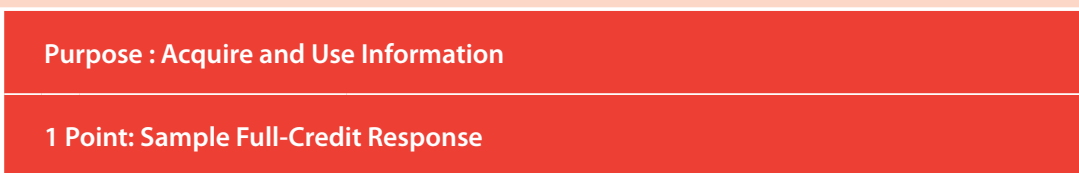

1. Where can you find Antarctica on a globe?

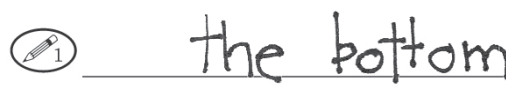

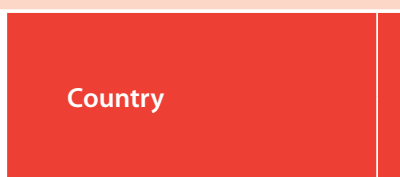

Luxembourg

+2a United States

France

Singapore

+ Scotland

England

2a Canada, Alberta

Canada, Quebec

t2a Belgium (Flemish)

Austria

Germany

Slovenia

2a Canada, Ontario

Poland

Canada, Nova Scotia

New Zealand

2a Russian Federation

${ }^{\dagger}$ Netherlands

Lithuania

${ }^{2 a}$ Canada, British Columbia

Italy

Slovak Republic

Sweden

Hong Kong SAR

* Norway

Hungary

Latvia

Chinese Taipei

2a Bulgaria

Iceland

${ }^{2 b}$ Israel

2a Georgia

2a Denmark

Spain

Moldova, Rep. of

International Avg.

Belgium (French)

Romania

Trinidad and Tobago

Macedonia, Rep. of

Qatar

Indonesia

Morocco

Kuwait

Iran, Islamic Rep. of

South Africa
Met guidelines for sample participation rates only after replacement schools were included (see Exhibit A.7).

₹ Nearly satisfying guidelines for sample participation rates after replacement schools were included (see Exhibit A.7).

2a National Defined Population covers less than $95 \%$ of National Desired Population (see Exhibit A.4) 2b National Defined Population covers less than $80 \%$ of National Desired Population (see Exhibit A.4).

( ) Standard errors appear in parentheses. Because results are rounded to the nearest whole number, some totals may appear inconsistent.

NOTE: The International Average does not include the results from the Canadian provinces. 
Exhibit 2.8 PIRLS 2006 Low International Benchmark - Item 4 - Informational Example

\section{1 out of 2 Points: Sample Partial-Credit Response}

4. Think about what the article says about Antarctica. Give two reasons why most people who visit Antarctica choose not to go there between April and September.

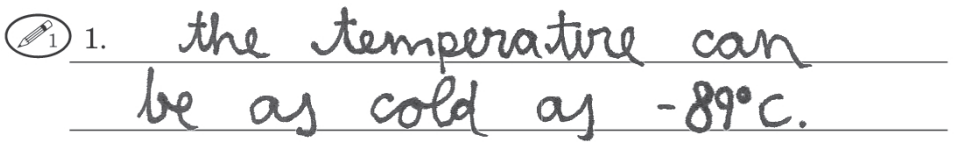
2.

\begin{tabular}{|c|c|c|c|}
\hline Country & $\begin{array}{l}\text { Percent } \\
\text { at Least } \\
1 \text { Point }\end{array}$ & & $\begin{array}{c}\text { Percent } \\
\text { Only } \\
1 \text { Point }\end{array}$ \\
\hline Luxembourg & $95(0.6)$ & 0 & $53(1.2)$ \\
\hline 2a Canada, Alberta & $93(1.1)$ & $\theta$ & $62(1.9)$ \\
\hline 2a Canada, Ontario & $92(1.2)$ & $\boldsymbol{\theta}$ & $62(2.3)$ \\
\hline Singapore & $92(0.8)$ & 0 & $59(1.5)$ \\
\hline †2a Belgium (Flemish) & $92(1.1)$ & 0 & $70(1.7)$ \\
\hline Chinese Taipei & $92(1.0)$ & 0 & $65(1.7)$ \\
\hline †2a United States & $92(1.0)$ & $\boldsymbol{0}$ & $68(1.8)$ \\
\hline Hong Kong SAR & $92(0.9)$ & 0 & $52(1.9)$ \\
\hline 2a Canada, British Columbia & $92(1.1)$ & 0 & $66(2.0)$ \\
\hline${ }^{\dagger}$ Netherlands & $92(1.4)$ & 0 & $63(2.0)$ \\
\hline Canada, Quebec & $91(1.3)$ & 0 & $62(2.7)$ \\
\hline Sweden & $90(1.3)$ & 0 & $58(2.0)$ \\
\hline Canada, Nova Scotia & $90(1.2)$ & 0 & $61(1.8)$ \\
\hline Germany & $89(1.2)$ & 0 & $61(1.7)$ \\
\hline England & $89(1.2)$ & 0 & $61(2.0)$ \\
\hline Austria & $89(1.3)$ & 0 & $58(1.7)$ \\
\hline Italy & $89(1.4)$ & 0 & $53(2.2)$ \\
\hline New Zealand & $88(1.0)$ & 0 & $59(1.6)$ \\
\hline Latvia & $87(1.5)$ & 0 & $57(2.1)$ \\
\hline † Scotland & $87(1.6)$ & 0 & $62(1.9)$ \\
\hline${ }^{2 a}$ Denmark & $86(1.4)$ & 0 & $63(2.0)$ \\
\hline France & $85(1.4)$ & 0 & $64(1.8)$ \\
\hline Lithuania & $85(1.3)$ & 0 & $63(1.7)$ \\
\hline Iceland & $85(1.3)$ & 0 & $65(1.5)$ \\
\hline Slovak Republic & $84(1.2)$ & 0 & $64(1.9)$ \\
\hline Belgium (French) & $84(1.4)$ & 0 & $67(1.9)$ \\
\hline 2a Bulgaria & $83(1.9)$ & 0 & $46(2.2)$ \\
\hline Hungary & $83(1.8)$ & 0 & $63(1.9)$ \\
\hline Moldova, Rep. of & $79(1.6)$ & & $59(2.0)$ \\
\hline Spain & $79(1.8)$ & & $55(1.9)$ \\
\hline${ }^{2 a}$ Russian Federation & $79(2.0)$ & & $49(2.4)$ \\
\hline International Avg. & $78(0.3)$ & & $55(0.3)$ \\
\hline Poland & $78(1.6)$ & & $55(1.8)$ \\
\hline${ }^{\ddagger}$ Norway & $77(2.1)$ & & $56(2.0)$ \\
\hline${ }^{2 b}$ Israel & $76(1.7)$ & & $52(1.9)$ \\
\hline Romania & $76(2.1)$ & & $49(2.4)$ \\
\hline Macedonia, Rep. of & $73(1.7)$ & ( & $56(2.2)$ \\
\hline 2a Georgia & $73(1.9)$ & ( & $55(2.4)$ \\
\hline Slovenia & $70(1.5)$ & $(\nabla$ & $53(1.5)$ \\
\hline Trinidad and Tobago & $68(1.9)$ & ( & $55(1.9)$ \\
\hline Indonesia & $59(1.9)$ & ( & $44(1.6)$ \\
\hline Iran, Islamic Rep. of & $54(2.4)$ & ( & $44(2.1)$ \\
\hline Qatar & $46(1.4)$ & (1) & $40(1.4)$ \\
\hline Morocco & $44(2.5)$ & ( & $39(2.4)$ \\
\hline South Africa & $41(1.7)$ & ( & $34(1.4)$ \\
\hline Kuwait & $33(2.2)$ & (i) & $26(2.0)$ \\
\hline
\end{tabular}

$\dagger$ Met guidelines for sample participation rates only after replacement schools were included (see Exhibit A.7).

‡ Nearly satisfying guidelines for sample participation rates after replacement schools were included (see Exhibit A.7).

2a National Defined Population covers less than $95 \%$ of National Desired Population (see Exhibit A.4). 2b National Defined Population covers less than $80 \%$ of National Desired Population (see Exhibit A.4).

() Standard errors appear in parentheses. Because results are rounded to the neares whole number, some totals may appear inconsistent.

NOTE: The International Average does not include the results from the Canadian provinces. 


\section{Achievement at the PIRLS 2006 Intermediate International Benchmark}

As described in Exhibit 2.9, students at the Intermediate International Benchmark demonstrated some reading proficiency, especially with the stories. They were able to understand the plots at a literal level, and also to make some inferences and connections across the texts. In the informational texts, they were able to use text organizers (headings, illustrations, etc.) to find information beyond the initial parts of the texts, and to provide two pieces of information in answering a question.

Exhibit 2.10 and 2.11 present the two literary examples, Items 5 and 6, respectively. Both are in the constructed-response format. The first literary example is from the "Little Lump of Clay" story found in Appendix D. On average, about two thirds of the fourth-grade students internationally were able to make an inference and briefly describe the aims/reactions of the lump of clay. Similarly, about two thirds were able to order the sequence of events in the "Unbelievable Night" story.

The first informational example is from the "Searching for Food" text in the PIRLs Reader, which was a three-part text about conducting science projects. Shown in Exhibit 2.12 and labeled Item 7, the question about the reason for putting the apple by the ants' nest was in the multiple-choice format. However, it did require students to make an inference based on several pieces of information provided in the text. Austrian and German fourth-grade students had the highest percentages of correct responses $(89 \%)$, and the average across countries was 72 percent. Presented in Exhibit 2.13 , the second informational example, Item 8 , is another question based on the "Antarctica" article. Students at the intermediate level made the necessary inferences and provided two pieces of information (of three required) about how penguins keep warm. On average internationally, only 22 percent of students provided two (of three) reasons. However, this item was relatively easy for students in some countries. For example, in the Russian Federation and Hong Kong SAR, 91 to 92 percent of the students provided twoor three-reasons. 
Exhibit 2.9 Description of the PIRLS 2006 Intermediate International Benchmark

\section{Literary}

When reading literary texts, students can:

- Identify central events, plot sequences, and relevant story details

- Make straightforward inferences about the attributes, feelings, and motivations of main characters

- Begin to make connections across parts of the text

\section{Informational}

When reading information texts, students can:

- Locate and reproduce one or two pieces of information from within the text

- Make straightforward inferences to provide information from a single part of the text

- Use subheadings, textboxes, and illustrations to locate parts of the text 
Exhibit 2.10 PIRLS 2006 Intermediate International Benchmark - Item 5 - Literary Example

PIRLS 2006

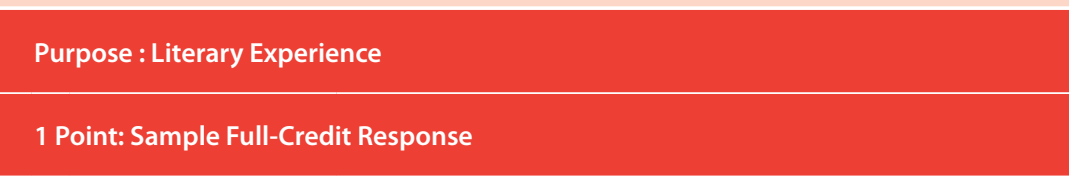

Country

th Grade

3. At the beginning of the story, what did the lump of clay wish for?

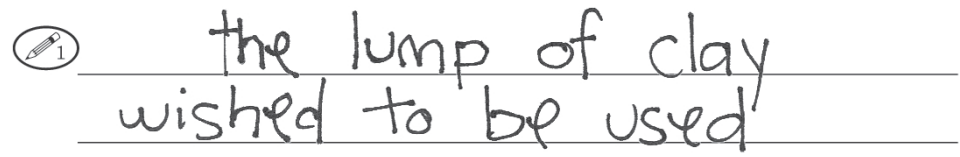

2a Canada,

Percent

Full

Credit

Canada, British Columbia

89 (1.4) 0

Denmark $87(1.5)$ ○

Germany $87(1.4) \quad 0$

${ }^{\dagger}$ Netherlands $\quad 86(1.4) \quad 0$

\begin{tabular}{l|l} 
Luxembourg & $86(1.0)$ \\
\hline
\end{tabular}

Chinese Taipei $\quad 86(1.2) \quad 0$

2a Canada, Alberta 85 (1.4)

Sweden

$85(1.7) \quad \mathbf{0}$

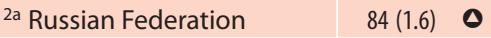

Lithuania $84(1.6) \quad 0$

2a Canada, Ontario $\quad 84(1.7) \quad 0$

Hong Kong SAR $83(1.5) \quad 0$ ㄴ

+2a Belgium (Flemish) $83(1.4) \quad 0$

Canada, Nova Scotia $83(1.3)$ ○

Italy $83(1.6) \quad 0$

Austria $82(1.5)$ 0

Singapore $82(1.3) \quad 0$

2a Bulgaria $\quad 81(2.1) \quad 0$

t2a United States $79(1.4) \quad 0$

+ Scotland $79(1.9) \quad 0$

Iceland $\quad 78(1.5) \quad 0$

Slovak Republic $\quad 78(1.8) \quad 0$

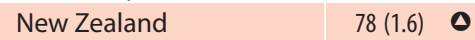

England $\quad 78(1.9) \quad 0$

\begin{tabular}{l|l} 
Canada, Quebec & $77(1.9) \quad 0$
\end{tabular}

Latvia $\quad 76(2.2) \quad 0$

France $\quad 76(1.6) \quad 0$

Slovenia $\quad 76(1.3) \quad \mathbf{0}$

\begin{tabular}{l|l} 
Poland & $75(1.9) \quad 0$
\end{tabular}

Belgium (French) $\quad 74(1.8) \quad 0$

‡ Norway $71(2.4)$

Romania $\quad 70(2.4)$

\begin{tabular}{l|l} 
International Avg. & $68(0.3)$ \\
\hline
\end{tabular}

Spain $65(1.8)$

2a Georgia $64(2.6)$

${ }^{2 b}$ Israel $\quad 63(1.8)$

Moldova, Rep. of $56(1.9)$

Macedonia, Rep. of 48 (1.9) (

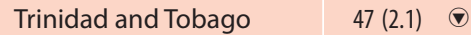

Iran, Islamic Rep. of $34(1.7)$

Qatar $28(1.2)$

Indonesia $26(1.6) \oplus$

Kuwait $19(1.5) \quad \nabla$

South Africa $16(1.6)$

Morocco

$6(1.0)$

Percentage of students receiving full credit significantly higher than international average

Percentage of students receiving full credit significantly lower than international average

$+\quad$ Met guidelines for sample participation rates only after replacement schools were included (see Exhibit A.7).

‡ Nearly satisfying guidelines for sample participation rates after replacement schools were included (see Exhibit A.7).

2a National Defined Population covers less than $95 \%$ of National Desired Population (see Exhibit A.4). 2b National Defined Population covers less than $80 \%$ of National Desired Population (see Exhibit A.4).

( ) Standard errors appear in parentheses. Because results are rounded to the nearest whole number, some totals may appear inconsistent.

NOTE: The International Average does not include the results from the Canadian provinces. 


\section{Exhibit 2.11 PIRLS 2006 Intermediate International Benchmark - Item 6 - Literary Example}

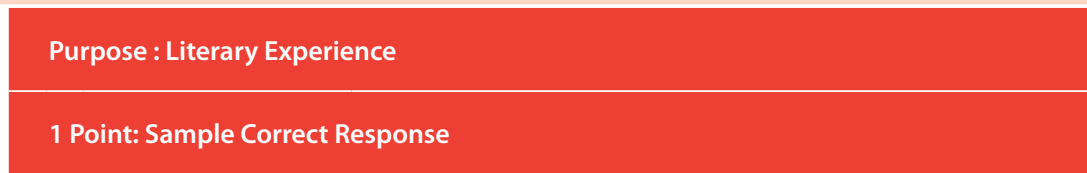

5. Put the following sentences in the order in which they happened in the story.

The first one has been done for you.

2 Anina saw the crocodile

$\frac{4}{5}$ The crocodile ate two flamingos.

Anina tried to explain the broken door to her parents.

$\frac{1}{3}$ Anina started to walk to the bathroom.
Anina ran to the bedroom and slammed the door.

\section{西}

Country

2a Russian Federation

Luxembourg

Hungary

2a Denmark Latvia

2a Canada, British Columbia Hong Kong SAR

2a Bulgaria Germany

2a Canada, Alberta

2a Canada, Ontario Canada, Quebec

${ }^{\dagger}$ Netherlands

+2a United States

Austria

Slovak Republic

Italy

Sweden

Spain

Lithuania

t2a Belgium (Flemish)

Canada, Nova Scotia

Chinese Taipei

Moldova, Rep. of

Poland

England

France

Singapore

Iceland

+ Scotland

Romania

Belgium (French)

New Zealand

Slovenia

2b Israel

International Avg.

‡ Norway

2a Georgia

Macedonia, Rep. of

Trinidad and Tobago

Iran, Islamic Rep. of

Qatar

Indonesia

Kuwait

Morocco

South Africa $\dagger \quad$ Met guidelines for sample participation rates only after replacement schools were included (see Exhibit A.7).

‡ Nearly satisfying guidelines for sample participation rates after replacement schools were included (see Exhibit A.7).

2a National Defined Population covers less than $95 \%$ of National Desired Population (see Exhibit A.4). 2b National Defined Population covers less than $80 \%$ of National Desired Population (see Exhibit A.4).

() Standard errors appear in parentheses. Because results are rounded to the nearest whole number, some totals may appear inconsistent.

NOTE: The International Average does not include the results from the Canadian provinces. 


\section{Exhibit 2.12 PIRLS 2006 Intermediate International Benchmark - Item 7 - Informational Example}

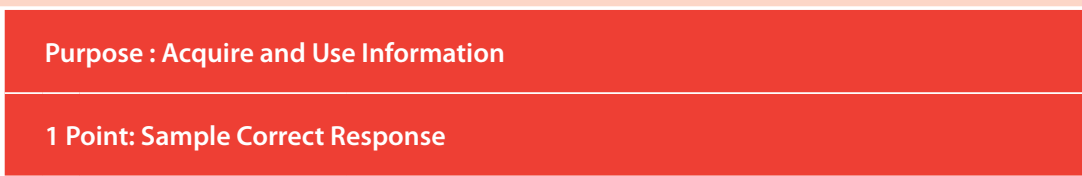

3. Why do you put the apple by the ants' nest?

A to block the ants' trail

so the ants will make a trail

(C) to confuse the ants

(D) so the ants will scurry around

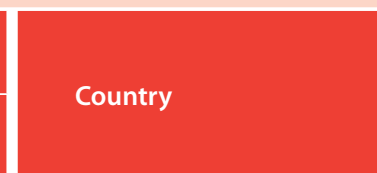

Austria

Germany

†2a Belgium (Flemish)

Hong Kong SAR

Sweden

Luxembourg

Hungary

Latvia

2a Denmark

${ }^{+}$Netherlands

2a Russian Federation Chinese Taipei

2a Canada, Alberta England

2a Canada, Ontario

${ }^{\ddagger}$ Norway

2a Canada, British Columbia New Zealand

Lithuania

Canada, Nova Scotia

Iceland

+2a United States Singapore

2a Bulgaria

Belgium (French)

Slovak Republic

+ Scotland

Canada, Quebec

Slovenia

France

Italy

Poland

Spain

Romania

International Avg.

Moldova, Rep. of

2a Georgia

${ }^{2 b}$ Israel

Macedonia, Rep. of

Trinidad and Tobago

Iran, Islamic Rep. of

Indonesia

Kuwait

Morocco

South Africa

Qatar

Percentage of students answering correctly significantly higher than international average

Percentage of students answering correctly significantly lower than international average
Met guidelines for sample participation rates only after replacement schools were included (see Exhibit A.7).

‡ Nearly satisfying guidelines for sample participation rates after replacement schools were included (see Exhibit A.7).

2a National Defined Population covers less than $95 \%$ of National Desired Population (see Exhibit A.4). 2b National Defined Population covers less than $80 \%$ of National Desired Population (see Exhibit A.4).

() Standard errors appear in parentheses. Because results are rounded to the nearest whole number, some totals may appear inconsistent.

NOTE: The International Average does not include the results from the Canadian provinces. 


\section{Exhibit 2.13 PIRLS 2006 Intermediate International Benchmark - Item 8 - Informational Example}

\section{Purpose : Acquire and Use Information}

2 of 3 Points: Sample Partial-Credit Response

7. Give three ways penguins are able to keep warm in Antarctica.

\section{They have many feathers which overlap} 2. and a thick layer of fat 3.

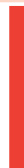$$
\text { . }
$$

\begin{tabular}{|c|c|c|c|}
\hline 2a Russian Federation & $92(1.2)$ & $\theta$ & $11(1.3)$ \\
\hline Hong Kong SAR & $91(1.1)$ & $\theta$ & $9(1.0)$ \\
\hline Singapore & $86(0.9)$ & 0 & $18(1.3)$ \\
\hline Chinese Taipei & $84(1.3)$ & $\theta$ & $10(1.1)$ \\
\hline 2a Canada, Alberta & $84(1.4)$ & 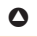 & $23(1.4)$ \\
\hline Sweden & $84(1.4)$ & $\theta$ & $29(1.9)$ \\
\hline Latvia & $84(1.9)$ & $\theta$ & $17(1.6)$ \\
\hline t2a Belgium (Flemish) & $83(1.4)$ & 0 & $29(1.6)$ \\
\hline 2a Canada, Ontario & $83(1.7)$ & $\theta$ & $22(2.3)$ \\
\hline 2a Canada, British Columbia & $83(1.6)$ & 0 & $22(1.8)$ \\
\hline${ }^{\dagger}$ Netherlands & $82(1.9)$ & $\boldsymbol{\theta}$ & $37(1.7)$ \\
\hline 2a Bulgaria & $81(2.3)$ & 0 & $19(1.8)$ \\
\hline Luxembourg & $81(1.1)$ & 0 & $33(1.5)$ \\
\hline
\end{tabular}

\begin{tabular}{|l|lll|}
\hline Luxembourg & $81(1.1)$ & $\boldsymbol{0}$ & $33(1.5)$ \\
\hline Canada, Nova Scotia & $80(1.4)$ & $\boldsymbol{0}$ & $22(1.5)$ \\
\hline Lithuania & $79(1.7)$ & $\boldsymbol{0}$ & $30(1.9)$ \\
\hline Slovak Republic & $78(1.6)$ & $\boldsymbol{0}$ & $22(1.3)$ \\
\hline
\end{tabular}

\begin{tabular}{|c|c|c|c|}
\hline Slovak Republic & $78(1.6)$ & $\boldsymbol{0}$ & $22(1.3)$ \\
\hline t2a United States & $76(1.8)$ & $\boldsymbol{0}$ & $24(1.5)$ \\
\hline Hungary & $75(2.0)$ & $\boldsymbol{0}$ & $26(1.6)$ \\
\hline Poland & $75(1.9)$ & $\boldsymbol{0}$ & $15(1.5)$ \\
\hline Germany & $75(1.7)$ & $\boldsymbol{0}$ & $35(1.7)$ \\
\hline
\end{tabular}

Canada, Quebec $75(2.1)$ ○ $28(2.0)$

\begin{tabular}{l|l|l|l|}
\hline Slovenia & $74(1.7)$ & 0 & $24(1.5)$
\end{tabular}

Italy $74(2.1) \quad$ ○ $19(1.6)$

\begin{tabular}{l|ll|l} 
England & $74(1.9)$ & 0 & $24(1.6)$
\end{tabular}

+ Scotland $73(2.4)$ ○ $23(1.6)$

New Zealand $73(1.3)$ ○ $22(1.3)$

Austria $73(1.8) \quad$ ○ $27(1.7)$

$71(1.8)-0$

France 70 (2.2)

\begin{tabular}{l|l|l}
\hline Spain & $69(2.0)$ & $33(1.6)$ \\
\hline
\end{tabular}

\begin{tabular}{|l|l|l}
\hline International Avg. & $67(0.3)$ & $22(0.2)$ \\
\hline
\end{tabular}

\begin{tabular}{|l|l|l|}
\hline Moldova, Rep. of & $67(2.4)$ & $33(2.0)$
\end{tabular}

2a Denmark $65(1.9) \quad 28(1.8)$

\begin{tabular}{l|l|l} 
Iceland & $64(1.5)$ & $16(1.4)$
\end{tabular}

Romania $63(2.5) \quad 21(1.8)$

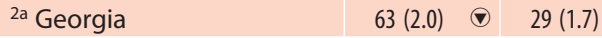

Belgium (French) $\quad 62(2.2) \quad \nabla \quad 26(1.6)$

Macedonia, Rep. of $\quad 60(2.1) \quad \nabla \quad 21(1.5)$

* Norway $59(1.8) \quad \nabla \quad 27(2.3)$

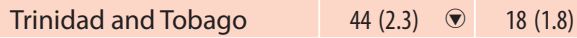

Indonesia $39(2.1) \quad \nabla \quad 19(1.6)$

Iran, Islamic Rep. of $\quad 38(1.8) \quad \nabla \quad 17(1.5)$

Qatar $33(1.3) \quad \nabla \quad 13(0.9)$

\begin{tabular}{l|l|l}
\hline Kuwait & $25(1.7) \quad \odot \quad 9(1.0)$
\end{tabular}

Morocco $21(2.0) \quad \nabla \quad 7(1.2)$

\begin{tabular}{l|l|l} 
South Africa & $20(1.5) \quad \nabla$ & $7(0.7)$
\end{tabular}

Percentage of students receiving at least 2 points significantly

higher than international average

Percentage of students receiving at least 2 points significantly lower than international average

$\dagger$ Met guidelines for sample participation rates only after replacement schools were included (see Exhibit A.7).

‡ Nearly satisfying guidelines for sample participation rates after replacement schools were included (see Exhibit A.7).

2a National Defined Population covers less than $95 \%$ of National Desired Population (see Exhibit A.4) 2b National Defined Population covers less than $80 \%$ of National Desired Population (see Exhibit A.4).

() Standard errors appear in parentheses. Because results are rounded to the nearest whole number, some totals may appear inconsistent.

NOTE: The International Average does not include the results from the Canadian provinces. 


\section{Achievement at the PIRLS 2006 High International Benchmark}

Exhibit 2.14 describes performance at the High International Benchmark. Students reaching this level were competent readers. For example, based on the literary texts included in PIRLS 2006, they could retrieve significant details embedded across the text and provide text-based support for inferences. They could use organizational features to navigate through the informational texts, and make inferences and connections. At this level, students recognized main ideas, some textual features and elements, and were beginning to integrate ideas and information across texts.

Exhibit 2.15 contains Item 9, a literary example from the "Little Lump of Clay" story. This item demonstrates that students at this level were able to recognize the idea of personification. Internationally on average, 55 percent of the students answered correctly. The fourth-grade students in the Netherlands performed the best, with 84 percent answering correctly. Exhibit 2.16, Item 10, is from the "Unbelievable Night" story. This question about two ways the magazine helped Anina illustrates students' achievement at the high level, in providing two pieces of text-based support (of two required). This question was difficult for students in general, with only 41 percent giving two ways, on average internationally.

Exhibit 2.17 contains Example Item 11, which is based on the "Antarctica" article. The results indicate that students at the high level were able to use organizational features such as section headings. On average, 57 percent of the students internationally were able to locate the section with the information about how thick the ice is. The high achievers on this question were the Russian Federation, Sweden, and Luxembourg with 83 to 84 percent of the students answering correctly. Example Item 12 in Exhibit 2.18 is from the "Searching for Food" science projects. It required students to integrate information within the section about the pill bugs project, make an inference about the point of the experiment, and write their answer. Similar to the results for other items exemplifying this level, 55 percent of the students, on average internationally, provided answers receiving full credit. The Singaporean fourth-grade students were by far the highest achievers on this question with 83 percent receiving full credit. 
Exhibit 2.14 Description of the PIRLS 2006 High International Benchmark of Reading Achievement

\section{Literary}

When reading literary texts, students can:

- Locate relevant episodes and distinguish significant details embedded across the text

- Make inferences to explain relationships between intentions, actions, events, and feelings, and give text-based support

- Recognize the use of some textual features (e.g., figurative language, an abstract message)

- Begin to interpret and integrate story events and character actions across the text

\section{Informational}

When reading information texts, students can:

- Recognize and use a variety of organizational features to locate and distinguish relevant information

- Make inferences based on abstract or embedded information

- Integrate information across a text to recognize main ideas and provide explanations

- Compare and evaluate parts of a text to give a preference and a reason for it

- Begin to understand textual elements, such as simple metaphors and author's point of view 
Exhibit 2.15 PIRLS 2006 High International Benchmark - Item 9 - Literary Example

PIRLS 2006
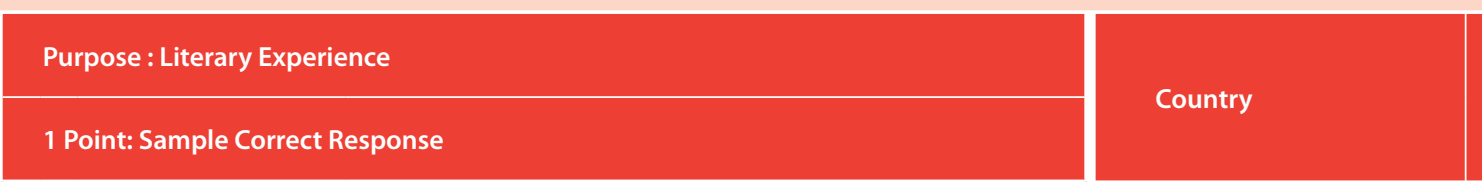

12. The author of the story writes about the lump of clay as if it were a person. What is the author trying to make you imagine?

(A) what it is like in the rain

how a lump of clay might feel

(C) what it is like to work with clay

D how it feels to make something

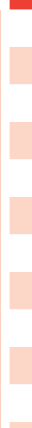

${ }^{\dagger}$ Netherlands
${ }^{+2 a}$ Belgium (Flemish)

Percent

Correct

England

2a Canada, British Columbia

2a Canada, Alberta

2a Canada, Ontario

Hungary

Canada, Nova Scotia

t2a United States

Lithuania

+ Scotland

Singapore

84 (1.3) 0

Sweden

80 (1.4) 0

New Zealand

Slovak Republic

77 (1.5) 0

Poland

$77(1.7) \bullet$

Latvia 76 (1.6) 0

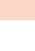

Italy

$75(1.8) \otimes$

$75(1.9)$

‡ Norway

74 (1.7) 0

Chinese Taip

$71(1.8) 0$

uxembourg

${ }^{2 a}$ Russian Federation

$71(1.8) \quad 0$

France

$70(2.1) \quad 0$

2a Denmark

$70(1.6) \bigcirc$

${ }^{2 b}$ Is

Belgium

$69(2.0) \quad 0$

$68(1.6) \bullet$

$66(1.8) \quad 0$

$66(1.5) \quad 0$

many

$63(2.4) \quad 0$

$62(2.0) \quad 0$

$62(2.0) \quad 0$

\begin{tabular}{l|l} 
Hong Kong SAR & $57(1.7)$
\end{tabular}

Iceland $57(1.9)$

\begin{tabular}{l|l} 
Canada, Quebec & $56(2.2)$
\end{tabular}

Slovenia $\quad 56(1.7)$

\begin{tabular}{l|l} 
International Avg. & $55(0.3)$
\end{tabular}

Romania

$54(1.9)$

Moldova, Rep. of $51(2.5)$

Spain

$48(2.0)$

Austria

2a Bulgaria

$47(1.7)$

2a Georgia

$22(2.5)$

Trinidad ar $39(2.9)$

$38(1.9)$

Macedonia, Rep. of

$36(1.8) \odot$

Iran, Islamic Rep. of

$34(1.7) \odot$

South Africa

$24(1.4)$

Qatar

$24(1.3)$

Morocco

$20(1.7) \odot$

Kuwait

$20(1.6) \odot$

Indonesia

$17(1.5) \odot$

Percentage of students answering correctly significantly higher than international average

Percentage of students answering correctly significantly lower than international average

Met guidelines for sample participation rates only after replacement schools were included (see Exhibit A.7).

‡ Nearly satisfying guidelines for sample participation rates after replacement schools were included (see Exhibit A.7).

2a National Defined Population covers less than $95 \%$ of National Desired Population (see Exhibit A.4) 2b National Defined Population covers less than $80 \%$ of National Desired Population (see Exhibit A.4).

() Standard errors appear in parentheses. Because results are rounded to the nearest whole number, some totals may appear inconsistent.

NOTE: The International Average does not include the results from the Canadian provinces. 
Exhibit 2.16 PIRLS 2006 High International Benchmark - Item 10 - Literary Example

PIRLS 2006

2 Points: Sample Full-Credit Response

Country

4th Grade

8. How did the magazine help Anina? Write two ways.

(1) The magazine help Anina to tell where the crocodile came from. 2. It help Anina to know what the crocodile would do when it was going to attack.

\begin{tabular}{|c|c|c|}
\hline 2a Canada, Ontario & $66(2.2)$ & 0 \\
\hline Hong Kong SAR & $66(2.1)$ & 0 \\
\hline 2a Canada, British Columbia & $63(2.0)$ & 0 \\
\hline 2a Canada, Alberta & $63(1.9)$ & 0 \\
\hline Hungary & $60(2.3)$ & 0 \\
\hline Canada, Quebec & $59(2.4)$ & 0 \\
\hline Canada, Nova Scotia & $57(2.0)$ & 0 \\
\hline${ }^{\dagger}$ Netherlands & $56(2.0)$ & 0 \\
\hline Germany & $56(2.0)$ & 0 \\
\hline Italy & $56(2.1)$ & 0 \\
\hline Luxembourg & $55(1.7)$ & 0 \\
\hline t2a United States & $54(2.1)$ & 0 \\
\hline 2a Russian Federation & $53(2.3)$ & 0 \\
\hline 2a Denmark & $53(2.4)$ & 0 \\
\hline Lithuania & $53(2.0)$ & 0 \\
\hline Sweden & $53(1.8)$ & 0 \\
\hline England & $50(1.8)$ & 0 \\
\hline Spain & $49(1.8)$ & 0 \\
\hline Chinese Taipei & $49(2.0)$ & 0 \\
\hline t2a Belgium (Flemish) & $48(1.8)$ & 0 \\
\hline Austria & $48(2.2)$ & 0 \\
\hline Singapore & $47(1.7)$ & 0 \\
\hline${ }^{2 b}$ Israel & $47(2.3)$ & 0 \\
\hline 2a Bulgaria & $45(2.7)$ & \\
\hline Belgium (French) & $45(2.0)$ & 0 \\
\hline Poland & $45(2.2)$ & \\
\hline Latvia & $43(2.1)$ & \\
\hline New Zealand & $43(1.8)$ & \\
\hline Slovenia & $43(1.6)$ & \\
\hline France & $43(1.9)$ & \\
\hline + Scotland & $42(2.1)$ & \\
\hline Slovak Republic & $41(2.4)$ & \\
\hline Romania & $41(2.6)$ & \\
\hline International Avg. & $41(0.3)$ & \\
\hline Moldova, Rep. of & $41(2.5)$ & \\
\hline Iceland & $35(1.8)$ & 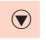 \\
\hline ₹ Norway & $34(2.3)$ & $\odot$ \\
\hline 2a Georgia & $34(1.9)$ & $(\nabla$ \\
\hline Trinidad and Tobago & $24(2.2)$ & 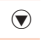 \\
\hline Macedonia, Rep. of & $22(2.0)$ & ( \\
\hline Iran, Islamic Rep. of & $20(1.6)$ & 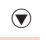 \\
\hline Kuwait & $12(1.4)$ & ( \\
\hline South Africa & $8(0.9)$ & 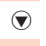 \\
\hline Indonesia & $6(0.9)$ & 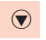 \\
\hline Qatar & $6(0.7)$ & 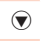 \\
\hline Morocco & $5(1.0)$ & ( \\
\hline
\end{tabular}

Percentage of students receiving full credit significantly higher than international average

$\dagger$ Met guidelines for sample participation rates only after replacement schools were included (see Exhibit A.7).

‡ Nearly satisfying guidelines for sample participation rates after replacement schools were included (see Exhibit A.7).

2a National Defined Population covers less than $95 \%$ of National Desired Population (see Exhibit A.4).

\footnotetext{
2b National Defined Population covers less than $80 \%$ of National Desired Population (see Exhibit A.4).

( ) Standard errors appear in parentheses. Because results are rounded to the nearest whole number, some totals may appear inconsistent.

NOTE: The International Average does not include the results from the Canadian provinces.
} 
Exhibit 2.17 PIRLS 2006 High International Benchmark - Item 11 - Informational Example

PIRLS 2006

4th Grade

\section{Purpose : Acquire and Use Information}

1 Point: Sample Correct Response

10. Which section of the article tells you how thick the ice is in Antarctica?

What is Antarctica?

(B) The Weather in Antarctica

(C) Penguins in Antarctica

(D) A Letter from Antarctica
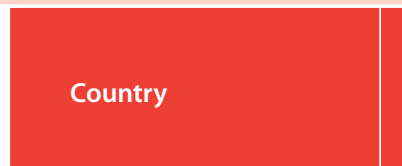

Percent

Correct

2a Russian Federation

$84(1.6) \quad 0$

Luxembourg

$84(1.6) \quad 0$

\begin{tabular}{l|l}
${ }^{+}$Netherlands & $81(1.6) \quad 0$
\end{tabular}

Germany $\quad 80(1.4) \quad 0$

+2a Belgium (Flemish) 79 (1.4) 0

2a Bulgaria $78(2.1) \quad 0$

\begin{tabular}{l|l}
\hline Latvia & $77(1.8) \quad 0$
\end{tabular}

2a Denmark $76(1.9) \quad 0$

Lithuania 75 (1.4) 0

Italy $74(2.0) \quad 0$

Austria $74(1.5) \quad 0$

Slovak Republic $73(1.7)$ ○

${ }^{2 a}$ Canada, British Columbia 70 (2.2) ๑

Hungary $70(2.4)$

\begin{tabular}{l|l} 
Hong Kong SAR & $70(1.8) \quad 0$
\end{tabular}

2a Canada, Alberta $68(2.0)$ 0

Canada, Nova Scotia $67(1.5)$ ○

2a Canada, Ontario $66(2.2) \quad 0$

New Zealand $65(1.6)$ ○

Singapore $\quad 65(1.6) \quad 0$

Canada, Quebec $\quad 65(2.3)$ ○

England $64(1.9) \quad 0$

\begin{tabular}{l|l} 
Poland & $63(2.0) \quad 0$
\end{tabular}

$+{ }^{+}$Scotland $63(2.2) 0$

France $\quad 61(1.8) \quad 0$

t2a United States $60(1.7)$

\begin{tabular}{l|r} 
Slovenia & $60(1.9)$ \\
\hline
\end{tabular}

Iceland $\quad 58(1.6)$

\begin{tabular}{l|l} 
International Avg. & $57(0.3)$
\end{tabular}

Moldova, Rep. of $\quad 56(2.7)$

Chinese Taipei $\quad 54(1.7)$

Romania $53(2.4)$

${ }^{2 \mathrm{~b}}$ Israel $52(1.9)$

Belgium (French) $\quad 51(2.2) \quad(7)$

Macedonia, Rep. of $50(2.5)$

2a Georgia $\quad 47(2.7)$

‡ Norway $\quad 43(2.4)$

Spain $39(2.2)$

Iran, Islamic Rep. of 32 (1.6)

Trinidad and Tobago $24(2.0)$

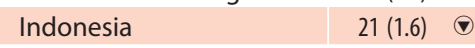

South Africa $15(1.2) \oplus$

Qatar $15(0.9)$

Kuwait $14(1.3)$

\begin{tabular}{l|l} 
Morocco & $12(1.5)$ \\
\hline
\end{tabular}

Percentage of students answering correctly significantly higher than international average

Percentage of students answering correctly significantly lower than international average

Met guidelines for sample participation rates only after replacement schools were included (see Exhibit A.7).

‡ Nearly satisfying guidelines for sample participation rates after replacement schools were included (see Exhibit A.7).

2a National Defined Population covers less than $95 \%$ of National Desired Population (see Exhibit A.4). 2b National Defined Population covers less than $80 \%$ of National Desired Population (see Exhibit A.4).

() Standard errors appear in parentheses. Because results are rounded to the nearest whole number, some totals may appear inconsistent.

NOTE: The International Average does not include the results from the Canadian provinces. 
Exhibit 2.18 PIRLS 2006 High International Benchmark - Item 12 - Informational Example

PIRLS 2006

4th Grade

Purpose : Acquire and Use Information
1 Point: Sample Full-Credit Response
9. In Step 3 of the pill bugs project, what do you think will happen if
you move the damp leaves to the left corner of the box?

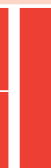

Country

Percent

Full

Credit

Singapore

$83(1.3) \quad 0$

Italy

2a Russian Federation

$74(1.9) \quad 0$

\begin{tabular}{l|l}
${ }^{+}$Netherlands & $72(2.1) \quad 0$
\end{tabular}

2a Canada, Ontario $71(1.9) \quad 0$

2a Canada, British Columbia $71(2.0) \quad 0$

+2a Belgium (Flemish) $69(1.4) \quad 0$

2a Canada, Alberta $\quad 69(1.8) \quad 0$

Sweden

Latvia

Lithuania

$69(1.8)$

$68(2.1)$

\begin{tabular}{l|l} 
Hong Kong SAR & $67(1.7) \quad 0$ \\
\hline
\end{tabular}

Austria $67(1.9) \circlearrowright$

Germany $65(1.9) \quad 0$

Luxembourg $\quad 64(1.5) \quad \mathbf{0}$

\begin{tabular}{l|l} 
t2a United States & $63(1.8) \quad 0$
\end{tabular}

Chinese Taipei $\quad 63(1.5) \quad 0$

Hungary $63(1.9) \quad 0$

2a Bulgaria $63(2.8) \quad \mathbf{0}$

Canada, Nova Scotia 61 (1.9)

England $\quad 61(1.9) \quad 0$

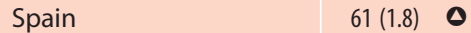

Slovenia $\quad 60(1.7) \quad 0$

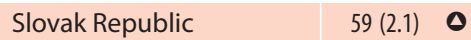

2a Denmark $59(2.4)$

\begin{tabular}{l|l} 
Iceland & $58(1.7) \quad 0$
\end{tabular}

France $\quad 58(2.1)$

\begin{tabular}{l|l} 
New Zealand & $57(1.4)$
\end{tabular}

2b Israel 57 (2.1)

Canada, Quebec $\quad 55(2.2)$

+ Scotland $\quad 55(2.1)$

\begin{tabular}{l|l} 
International Avg. & $55(0.3)$
\end{tabular}

Romania $\quad 55$ (2.4)

\begin{tabular}{l|l} 
Moldova, Rep. of & $54(2.7)$
\end{tabular}

* Norway $\quad 52(2.2)$

Poland $50(1.9)$

Belgium (French) $48(2.0)$

\begin{tabular}{l|l} 
2a Georgia & $47(2.5) \quad \odot$
\end{tabular}

Macedonia, Rep. of $\quad 40(2.1)$

\begin{tabular}{l|l} 
Trinidad and Tobago & $40(2.1)$
\end{tabular}

Iran, Islamic Rep. of $39(2.1)$

\begin{tabular}{l|l} 
Indonesia & $25(1.8) \quad(1)$ \\
\hline
\end{tabular}

Kuwait $16(1.7) \odot$

Qatar $15(1.0) \quad(7)$

Morocco $15(1.8)$

\begin{tabular}{l||c|} 
South Africa & $12(1.0) \quad(1)$
\end{tabular}

Percentage of students receiving full credit significantly higher than international average

Percentage of students receiving full credit significantly lower than international average $\dagger \quad$ Met guidelines for sample participation rates only after replacement schools were included (see Exhibit A.7).

‡ Nearly satisfying guidelines for sample participation rates after replacement schools were included (see Exhibit A.7).

2a National Defined Population covers less than $95 \%$ of National Desired Population (see Exhibit A.4).

\footnotetext{
2b National Defined Population covers less than $80 \%$ of National Desired Population (see Exhibit A.4).

() Standard errors appear in parentheses. Because results are rounded to the nearest whole number, some totals may appear inconsistent.
}

NOTE: The International Average does not include the results from the Canadian provinces. 


\section{Achievement at the PIRLS 2006 Advanced International Benchmark}

The description provided in Exhibit 2.19 reveals that students performing at the Advanced level responded fully to the PIRLS 2006 assessment. Students could make interpretations of figurative language and demonstrated that they understood the function of organizational features. They could integrate information across the texts, and provide full text-based support. The four example items illustrate how students performing at the Advanced International Benchmark demonstrated their ability to comprehend, interpret, and integrate details across the relatively challenging texts included in PIRLS 2006.

Exhibit 2.20 shows Item 13, a literary example from the "Little Lump of Clay" story. In this multiple-choice question, students were asked about the meaning of figurative language. The highest achievement was in the Russian Federation and Hungary with 65 percent of students responding correctly. However, on average internationally, only 42 percent of students gave the correct answer. Exhibit 2.21 presents Item 14, another example from the "Clay" story. To receive full credit on this 3-point question, students had to respond to all of the elements as demonstrated in the response shown in the exhibit. The task was very difficult for students, with less than half receiving full credit in any of the participating countries or provinces. With 47 percent receiving full credit, the Swedish fourth-grade students had the highest achievement. The international average was 27 percent.

Exhibits 2.22 and 2.23 contain Items 15 and 16, both informational examples from the "Searching for Food" science projects. The first item asked students to explain the function of the presentational device of the picture included with the pill bugs experiment, and all participants had difficulty with this item. Less than half the students in any country or Canadian province received full credit, with the highest achievement, 48 percent, occurring in the province of Ontario. The second item asked students to integrate information across several parts of the text to make an inference and provide an explanation about the overall purpose of the wormery project. 
Bulgarian fourth-grade students outdistanced those in other countries on the wormery item, with 63 percent receiving full credit ( 22 percentage points higher than the next best country). These two items, illustrating the skills and strategies demonstrated by the students reaching the Advanced International Benchmark, were extremely difficult for fourth-grade students in general. The international averages were 21 and 26 percent, respectively. 
Exhibit 2.19 Description of the PIRLS 2006 Advanced International Benchmark of Reading Achievement

\section{Literary}

When reading literary texts, students can:

- Integrate ideas across a text to provide interpretations of a character's traits, intentions, and feelings, and provide full text-based support

- Interpret figurative language

- Begin to examine and evaluate story structure

\section{Informational}

When reading information texts, students can:

- Distinguish and interpret complex information from different parts of text, and provide full text-based support

- Understand the function of organizational features

- Integrate information across a text to sequence activities and fully justify preferences 
Exhibit 2.20 PIRLS 2006 Advanced International Benchmark - Item 13 - Literary Example

PIRLS 2006

4th Grade

\section{Purpose : Literary Experience}

1 Point: Sample Correct Response

9. Which words in the story show that the little girl knew what she wanted to make?

A 'her fingers felt heavenly.'

B 'The little girl saw the lump of clay.'

(C) 'The little girl holds him gently.'

'her hands moved with purpose.'

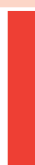

Country

Percent

Correct

${ }^{2 a}$ Russian Federation

$65(1.9) \quad 0$

Hungary

$65(1.9) \quad 0$

Hong Kong SAR $59(1.7) \quad 0$

2a Denmark $59(1.8) \quad 0$

${ }^{\dagger}$ Netherlands $59(1.9) \quad 0$

\begin{tabular}{l|l} 
2a Bulgaria & $59(2.8) \quad 0$
\end{tabular}

Chinese Taipei $\quad 57(1.8) \quad 0$

\begin{tabular}{l|l|}
\hline Italy & $55(2.2) \quad 0$ \\
\hline
\end{tabular}

${ }^{2 b}$ Israel $\quad 55(1.7)$ ○

Singapore $\quad 53(1.9) \quad 0$

Luxembourg $52(1.5) 0$

\begin{tabular}{l|l}
\hline Lithuania & $51(1.9) \quad 0$ \\
\hline
\end{tabular}

Poland $50(2.1) \quad 0$

\begin{tabular}{l|l} 
†2a Belgium (Flemish) & $48(1.6) \quad 0$
\end{tabular}

Slovak Republic $\quad 48(2.2) \quad 0$

\begin{tabular}{l|r} 
t2a United States & $47(1.9) \quad 0$
\end{tabular}

Latvia $\quad 47(2.1)$

\begin{tabular}{l|l} 
Romania & $46(2.3)$
\end{tabular}

2a Canada, British Columbia 45 (1.9)

2a Canada, Alberta 44 (2.3)

2a Canada, Ontario 44 (2.1)

\begin{tabular}{l|l} 
2a Georgia & $43(2.7)$
\end{tabular}

\begin{tabular}{|l|l}
\hline International Avg. & $42(0.3)$
\end{tabular}

Canada, Quebec $\quad 41(2.8)$

Sweden $\quad 41(2.0)$

France $\quad 40(1.6)$

₹ Norway $\quad 40$ (2.4)

\begin{tabular}{|l|l|} 
Moldova, Rep. of & $39(2.1)$
\end{tabular}

+ Scotland $38(2.1)$

\begin{tabular}{l|l} 
Austria & $37(1.9) \quad(7)$
\end{tabular}

Canada, Nova Scotia $37(1.7)$

\begin{tabular}{l|l} 
England & $35(2.0)$
\end{tabular}

Germany $35(1.6)$

\begin{tabular}{l|l} 
New Zealand & $34(1.5) \quad(7)$
\end{tabular}

Iceland $33(1.8)$

Iran, Islamic Rep. of $33(1.6)$

Indonesia $31(1.6)$ (

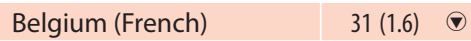

Trinidad and Tobago $\quad 30(2.2)$

Spain $28(1.6) \quad(7)$

Qatar $25(1.3)$

\begin{tabular}{l|l} 
Slovenia & $24(1.4) \quad(1)$
\end{tabular}

Macedonia, Rep. of $23(2.0) \circledast$

Morocco $22(2.1) \quad \odot$

Kuwait $20(1.6)$

\begin{tabular}{l|l} 
South Africa & $17(1.1)$ \\
\hline
\end{tabular}

Percentage of students answering correctly significantly higher than international average

Percentage of students answering correctly significantly lower than international average

+ Met guidelines for sample participation rates only after replacement schools were included (see Exhibit A.7).

‡ Nearly satisfying guidelines for sample participation rates after replacement schools were included (see Exhibit A.7).

2a National Defined Population covers less than $95 \%$ of National Desired Population (see Exhibit A.4). 2b National Defined Population covers less than $80 \%$ of National Desired Population (see Exhibit A.4).

( ) Standard errors appear in parentheses. Because results are rounded to the nearest whole number, some totals may appear inconsistent.

NOTE: The International Average does not include the results from the Canadian provinces. 


\section{Exhibit 2.21 PIRLS 2006 Advanced International Benchmark - Item 14 - Literary Example}

\section{Purpose : Literary Experience}

3 Points: Sample Full-Credit Response

10. Describe the different feelings the clay had at the beginning and the end of the story. Explain why his feelings changed.

\section{in the beginning, the lump of clay felt lost} hope, as nobody has picked him up from the bin. At the end the lump of lay was proud as it was made into a cup.
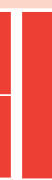

$$
\text { 2a Slo }
$$

New Zealand $\quad 40(1.7) \quad 0$

Hungary $\quad 40(2.2) \quad 0$

Luxembourg $39(1.6) \quad 0$

\begin{tabular}{l|l} 
†2a United States & $39(2.1) \quad \mathbf{0}$
\end{tabular}

Hong Kong SAR 38 (1.9) 0

Chinese Taipei $38(1.8) \quad 0$

†2a Belgium (Flemish) $38(2.0) \quad \mathbf{0}$

2a Bulgaria $38(2.3) \quad 0$

${ }^{2 a}$ Canada, British Columbia $37(2.0) \quad 0$ Canada, Nova Scotia $37(2.1)$ ○

2a Denmark $37(1.6) \quad 0$

\begin{tabular}{l|l} 
England & $36(2.4)$
\end{tabular}

Italy $36(2.1)$ 0

Germany $36(1.7) 0$

2a Canada, Alberta $36(2.3) \quad 0$

Slovak Republic $36(1.9)$ ○

+ Scotland $35(2.5)$ 0

Canada, Quebec $34(2.1) \quad 0$

Poland $32(1.6) \quad 0$

\begin{tabular}{l|l} 
Austria & $32(1.7) \quad 0$
\end{tabular}

2a Russian Federation $31(2.0)$

\begin{tabular}{l|l} 
Romania & $30(2.0)$
\end{tabular}

Latvia $29(2.1)$

Lithuania $\quad 28(1.7)$

$2 \mathrm{~b}$ Israel $\quad 28(1.6)$

\begin{tabular}{|l|l}
\hline & \\
\hline
\end{tabular}

\begin{tabular}{|l|l}
\hline International Avg. & $27(0.3)$ \\
\hline
\end{tabular}

Moldova, Rep. of 27 (1.9)

Iceland $\quad 26(1.6)$

France $22(1.6)$

Belgium (French) $22(1.9) \quad($

Spain $21(1.6)$

* Norway $19(1.9)$

Macedonia, Rep. of $15(1.3)$

Trinidad and Tobago $13(1.3)$

2a Georgia $\quad 9(1.3)$

Iran, Islamic Rep. of $\quad 7(0.8)$

\begin{tabular}{l|l} 
South Africa & $5(0.9) \quad(1)$ \\
\hline
\end{tabular}

Indonesia $\quad 5(0.8)$

Kuwait $4(0.8)$

Morocco $4(0.8)$

Qatar $3(0.5)$
† Met guidelines for sample participation rates only after replacement schools were included (see Exhibit A.7).

\# Nearly satisfying guidelines for sample participation rates after replacement schools were included (see Exhibit A.7).

2a National Defined Population covers less than $95 \%$ of National Desired Population (see Exhibit A.4) 2b National Defined Population covers less than $80 \%$ of National Desired Population (see Exhibit A.4).

( ) Standard errors appear in parentheses. Because results are rounded to the nearest whole number, some totals may appear inconsistent.

NOTE: The International Average does not include the results from the Canadian provinces. 
Exhibit 2.22 PIRLS 2006 Advanced International Benchmark - Item 15 - Informational Example PIRLS 2006

Purpose : Acquire and Use Information

2 Points: Sample Full-Credit Response

7. Look at the picture for Study Pill Bugs. How does the picture help you to know what to do in the experiment?

it helps you understand because it has arrows and theres lines in different places to tell you where to put everything

.

2a Canada, Ontario

2a Russian Federation

2a Canada, British Columbia

2a Canada, Alberta

t2a United States

England

Latvia

Canada, Nova Scotia

New Zealand

t Scotland

Italy

Hungary

Singapore

2a Denmark

t2a Belgium (Flemish)

Luxembourg

† Netherlands

₹ Norway

Slovak Republic

Canada, Quebec

2a Bulgaria

Hong Kong SAR

International Avg.

Spain

Poland

2b Israel

Romania

France

Lithuania

Sweden

Germany

Trinidad and Tobago

Austria

Moldova, Rep. of

Iceland

Slovenia

Macedonia, Rep. of

2a Georgia

Belgium (French)

South Africa

Chinese Taipei

Iran, Islamic Rep. of

Indonesia

Kuwait

Qatar

Morocco
+ Met guidelines for sample participation rates only after replacement schools were included (see Exhibit A.7).

‡ Nearly satisfying guidelines for sample participation rates after replacement schools were included (see Exhibit A.7).

2a National Defined Population covers less than $95 \%$ of National Desired Population (see Exhibit A.4).

\footnotetext{
2b National Defined Population covers less than $80 \%$ of National Desired Population (see Exhibit A.4).

() Standard errors appear in parentheses. Because results are rounded to the nearest whole number, some totals may appear inconsistent.

NOTE: The International Average does not include the results from the Canadian provinces.
} 


\section{Exhibit 2.23 PIRLS 2006 Advanced International Benchmark - Item 16 - Informational Example}

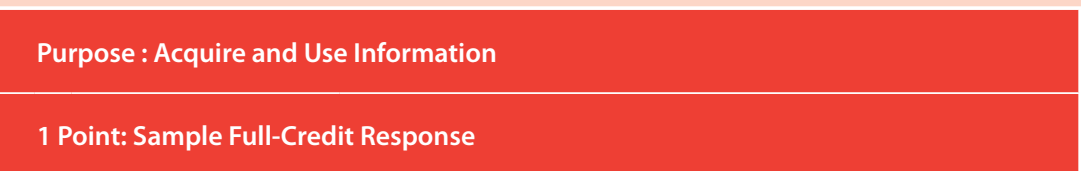

12. Explain why it is important to put layers of soil and sand in the bottle.

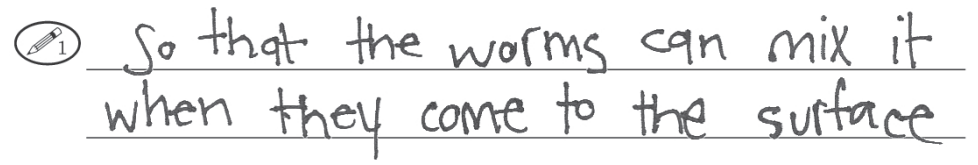

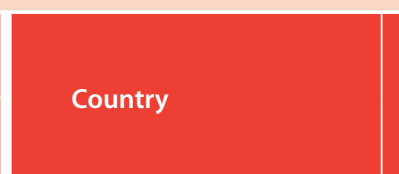

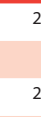

2a Bulgaria

Austria

2a Russian Federation

Italy

Slovak Republic

Germany

Sweden

Latvia

Luxembourg

t2a Belgium (Flemish)

Spain

Hong Kong SAR

2a Canada, British Columbia

2a Denmark

Chinese Taipei

Lithuania

${ }^{\dagger}$ Netherlands

Poland

Slovenia

2a Canada, Alberta

Romania

France

International Avg.

Belgium (French)

2a Canada, Ontario

Hungary

Canada, Nova Scotia

New Zealand

England

Moldova, Rep. of

Canada, Quebec

+ Scotland

t2a United States

Singapore

2a Georgia

${ }^{2 b}$ Israel

Indonesia

‡ Norway

Macedonia, Rep. of

Trinidad and Tobago

Iceland

Iran, Islamic Rep. of

Kuwait

Qatar

Morocco

South Africa
Met guidelines for sample participation rates only after replacement schools were included (see Exhibit A.7).

‡ Nearly satisfying guidelines for sample participation rates after replacement schools were included (see Exhibit A.7).

2a National Defined Population covers less than $95 \%$ of National Desired Population (see Exhibit A.4) 2b National Defined Population covers less than $80 \%$ of National Desired Population (see Exhibit A.4).

( ) Standard errors appear in parentheses. Because results are rounded to the nearest whole number, some totals may appear inconsistent.

NOTE: The International Average does not include the results from the Canadian provinces. 



\section{Chapter 3}

\section{Literacy-related Activities in the Home}

Abundant research evidence has established that early exposure to literacy activities is a key element of later reading achievement. To expand on the information available from PIRLS 2001 and to measure changes during the intervening 5-year period, PIRLS 2006 collected information from parents about their child's experiences in learning to read, literacy resources in the home, their own reading, and the language(s) spoken in the home. PIRLS 2006 also asked the fourth-grade students themselves about their reading activities at home and at school. Chapter 3 presents parents' and students' responses to a subset of these questions.

The parents' data were collected using the Learning to Read Survey, in which PIRLS 2006 asked the parents or primary caregivers of each child participating in the study to provide information about their child's experiences in learning to read. When information from the parents' questionnaires is being reported, it is always presented as an attribute of the student, so that the student is the unit of analysis. That is, the data are shown as the percentages of students whose parents reported on various activities or events. Using the student as the unit of analysis makes it possible to describe students' early literacy experiences and is consistent with the PIRLS goal of providing information about the educational contexts and performances of students.

Although, for reporting purposes, the information provided by parents was linked directly to students, parents sometimes did not complete the questionnaire given to them. When more than 15 percent of the students 
were missing parents' questionnaire data, the exhibits in this chapter have special notations. For a country where parent responses were available for 70 to 84 percent of students, an " $r$ " is displayed next to its data. Where parent responses were available for 50 to 69 percent of students, an " $\mathrm{s}$ " is displayed. Where parent responses were available for less than 50 percent, an " $x$ " replaces the data. ${ }^{1}$

To summarize questionnaire data concisely and focus attention on educationally relevant support and practice, PIRLS sometimes has combined information from individual questions to form an index that is more valid and reliable than the component questions (e.g., early home literacy activities). According to their responses, students were placed in a "high," "medium," or "low" category. Cutoff points were established so that the high level of an index corresponds to conditions or activities generally associated with good educational practice and high reading achievement. For each index, the percentages of students in each category are presented in relation to their average reading achievement. Wherever possible, the change since 2001 in percentage of students in each category also is presented.

\section{What Activities Fostering Literacy Did Parents Engage in with Their Child?}

To examine early literacy experiences, PIRLS 2006 asked if parents (or someone else in the home) engaged in a range of activities with their child before the child began primary school. The Index of Early Home Literacy Activities summarizes parents' responses to six of these activities:

- Read books

- Tell stories

- Sing songs

- Play with alphabet toys (e.g., blocks with letters of the alphabet)

- Play word games

- Read aloud signs and labels. 
Responses about each activity were on a 3-point scale-often, sometimes, and never or almost never. To construct the index, parents' responses were averaged across the six activities and then students were assigned to 1 of 3 categories (high, medium, or low) on the basis of their parents' average responses. Students in the high category had parents who reported often engaging in the six activities, on average, whereas parents of those in the low category never or almost never did so. Students in the medium category had parents reporting between these two extremes.

Exhibit 3.1 presents the percentage of students in 2006 at each level of the index for each country, together with average reading achievement for those students. Standard errors also are shown. The international average across all countries (not including the Canadian provinces) is shown at the bottom of the columns for the 2006 results. Countries are ordered by the percentage of students at the high level of the index in 2006. In addition, for countries that participated in PIRLS 2001, the exhibit presents the difference in the percent of students from 2001 at each level, with an indication of whether or not that difference was statistically significant.

As was the case in 2001, in most countries, parents of the PIRLS 2006 students reported a fairly high level of engagement with their children in preschool literacy activities, with more than half $(54 \%)$ of students in the high category of the index, on average, across all countries. The highest level of engagement was reported in Scotland, where 85\% of students had parents reporting often reading books, telling stories, singing songs, playing with alphabet toys, playing word games, and reading aloud signs and labels with their child before the child began school. High levels of engagement also were reported in the Russian Federation, New Zealand, Israel, and the Canadian provinces of Nova Scotia, Ontario, British Columbia, and Alberta, where 70 percent or more of students were in the high category. Among the countries where parents reported lower levels of engagement were Iran and Morocco, with 37 and 56 percent of students, respectively, in the low category, where parents reported never or almost never engaging in the activities with their children before they began school. 
Of the participants shown in Exhibit 3.1 with data from 2006, 24 had comparable data from 2001. More than half of these (14 of the 24), had an increase in the percentage of students in the high category, accompanied in 12 of the 14 by a decrease in the percentage of students in the medium or low category (or both). Among the countries with the greatest increase in the high category were the Russian Federation (9\%), the Netherlands (9\%), Germany (13\%), and Hong Kong SAR (10\%). Interestingly, three of these countries, the Russian Federation, Hong Kong SAR, and Germany, showed an increase in average student achievement in 2006 (see Exhibit 1.3). However, the Netherlands had a decrease in average achievement.

Although the countries with the highest average reading achievement were not necessarily those with the highest percentage of students in the high category of the Index of Early Home Literacy Activities (the Russian Federation, the highest performing country, did have 75\%, but Hong Kong SAR, the next highest, had just $26 \%$ ), there was a positive relationship between engaging in early literacy activities and performance on the PIRLS 2006 reading assessment in every country. On average internationally, students in the high category of the index had about a 20-point advantage in reading achievement over students in the medium category, who in turn scored 20 points above students in the low category. Countries where the students in the high category had the greatest advantage over those in the medium category (3o points or more) included New Zealand, Trinidad and Tobago, Romania, and South Africa. 


\section{What Literacy Resources Do Students Have in Their Homes?}

IEA studies have shown that students from homes with extensive literacy resources have higher achievement in reading and other subjects than those from less-advantaged backgrounds. For example, IEA's 1991 study of reading literacy in 32 countries found a clear-cut relationship between the number of books students reported in their homes and their reading achievement levels. ${ }^{2}$ TIMSs, IEA's international study of trends in student achievement in mathematics and science, has consistently shown that students with large numbers of books in the home have higher achievement in mathematics and science at both fourth and eighth grades. ${ }^{3}$ Building on this work, PIRLS in 2001 developed an Index of Home Educational Resources based on parents' and students' reports of the number of books, the number of children's books, and the presence of four educational aids (computer, study desk for own use, books of their own, and access to a daily newspaper) in the home and on parents' education.

Students assigned to the high level of this index came from homes with more than 100 books, more than 25 children's books, at least 3 of the 4 educational aids, and where at least one parent completed university. Students assigned to the low level had 25 or fewer books in the home, 25 or fewer children's books, no more than two of the four educational aids, and parents that had not completed secondary education. The remaining students were assigned to the medium level. Exhibit 3.2 presents the percentage of students in 2006 at each level of the index for each country, together with the average reading achievement for these students. The international average across all countries is shown at the bottom of these columns. The exhibit also presents the difference from 2001 in the percentage of students at each level of the index, together with an indication of the statistical significance of this difference. Participants are ordered by the percentage of students at the high level of the index in 2006.

On average across countries, there were 11 percent of students at the high level of the index, 80 percent at the medium level, and 9 percent at the low level, although the distribution varied considerably from country to country.

2 Elley, W.B. (1992). How in the world do students read? The Hague: International Association for the Evaluation of Educational Achievement (IEA).

3 See, for example, Mullis, I.V.S., Martin, M.O., Gonzalez, E.G., \& Chrostowski, S. J. (2004). TIMSS 2003 international mathematics report: Findings from IEA's Trends in International Mathematics and Science Study at the fourth and eighth grades. Chestnut Hill, MA: Boston College. 
Exhibit 3.2 Index of Home Educational Resources (HER) with Trends

PIRLS 2006

Countries

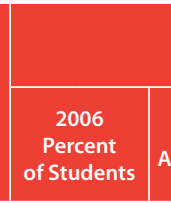

High HER

Medium HER

Norway

$26(1.2) \quad 531(2.5)$

\begin{tabular}{c|c} 
Average & \\
Achievement & t \\
\hline
\end{tabular}

Difference 2006

(

Denmark

in Percent

Percent
Students

Average

$-7(1.8) @ \quad 74(1.2)$

Achievement

Difference
in Percent
from 2001

$\begin{array}{ll}-7(1.8) & 74(1.2) \\ \Delta & 75(1.3)\end{array}$

$493(2.9)$

Iceland

$24(0.8)$

576 (3.4)

Sweden

$22(1.4)$

$550(2.9)$

$4(1.1) \quad 0 \quad 76(0.8)$

$540(2.3)$

7 (1.8)

$78(1.4)$

$507(1.6)$

Scotland

$21(1.6)$

$578(3.5)$

$-3(1.9)$

$4(2.1)$

Canada, British Columbia

Netherlands

$20(1.2)$

$589(6.7)$

○ $77(1.6)$

$546(2.1)$

$\Delta 0$

79 (1.3)

Canada, Alberta

$20(1.1)$

$584(2.9)$

$13(1.3) \quad 0 \quad 79(1.1)$

$531(3.1)$

Canada, Nova Scotia

$19(1.1)$

$598(3.9)$

$\Delta 0$

556 (2.9)

550 (1.4)

$590(3.5)$

$\Delta \Delta$

$80(1.2)$

559 (2.6)

Canada, Ontario

$18(1.5)$

New Zealand

$591(4.2)$

$0(2.1)$

$81(1.1)$

Israel

Hungary

$18(1.0)$

$591(3.6)$

$-1(1.5)$

$16(1.3)$

$587(4.9)$

$\mathrm{xx}$

France

5 (1.1)

$607(2.9)$

$\begin{array}{ll}-1 & 82(1.4)\end{array}$

$539(1.8)$

Luxembourg

$15(1.1)$

$570(3.1)$

$-5(1.7) \quad 8 \quad 80(1.3)$

$14(0.5)$

$601(2.6)$

$\Delta \Delta$

$571(4.8)$

$\Delta \diamond \quad 82(0.5)$

$550(2.6)$

$541(2.0)$

526 (3.8)

$548(2.8)$

Canada, Quebec

$14(1.0)$

$580(2.5)$

$0(1.5) \quad 84(0.9)$

$520(1.9)$

$555(1.1)$

$13(1.3)$

Germany

$12(0.9)$

$560(4.4)$

$84(1.0)$

534 (2.6)

$592(2.9)$

$\Delta \diamond \quad 82(1.2)$

$545(1.8)$

$-4(1.2) \bigcirc \quad 85(0.9)$

$520(2.4)$

$553(2.1)$

Singapore

$12(1.0)$

$610(4.8)$

$4(1.2) \quad 0 \quad 86(1.0)$

$560(3.4)$

$11(1.1)$

Bulgaria

$11(0.7)$

$0(1.0) \quad 86(0.5)$

$556(2.8)$

$\begin{array}{ll}0(1.4) & 74(1.8) \\ 0(1.3) & 88(0.8)\end{array}$

Lithuania

$11(0.7) \quad 577(2.9)$

Slovak Republic

(0.7) $\quad 585(3.1)$

$1(1.3) \quad 86(1.0)$

$549(4.0)$

$533(1.6)$

Chinese Taipei

$10(0.8) \quad 585(3.9)$

$\Delta \diamond \quad 86(0.8)$

$533(2.2)$

$534(1.9)$

\begin{tabular}{ll|rrr}
$9(0.8)$ & $571(3.9)$ & $-5(1.2)$ & $\bigcirc$ & $90(0.8)$ \\
$9(0.9)$ & $589(2.9)$ & $5(1.0)$ & $\bullet$ & $85(0.7)$
\end{tabular}

Hong Kong SAR

Georgia

$9(0.9)$

$59(2.9)$

$\Delta \diamond \quad 87(1.1)$

$540(2.4)$

565 (2.2)

470 (3.1)

$578(3.4)$

$-2(0.9) \quad 9 \quad 90(0.6)$

$519(2.0)$

$9(0.5)$

Poland

$9(0.7)$

$583(4.5)$

$\Delta 0 \quad 82(0.8)$

$522(2.2)$

$553(2.9)$

Oata

$8(0.7)$

$598(4.4)$

$1(0.9)$

$84(1.0)$

\begin{tabular}{ll} 
Austria & $7(0.7)$ \\
\hline
\end{tabular}

Trinidad and Tobago $\quad r \quad 5(0.5)$

$402(5.4) \quad \Delta \nabla \quad 85(0.4)$

$592(4.2)$

$\Delta \diamond \quad 92(0.7)$

$363(1.7)$

$538(1.8)$

\begin{tabular}{l|l|l|l}
$510(8.3)$ & $\diamond \diamond$ & $89(0.9)$ & $443(4.8)$ \\
\hline
\end{tabular}

$502(2.5)$

$553(5.8) \quad \Delta \diamond \quad 91(0.6)$

Macedonia, Rep. of $\quad s \quad 5(0.5)$

Kuwait

$523(9.0)$

$3(0.6) \quad 0 \quad 85(1.1)$

$457(3.7)$

$348(4.4)$

$401(12.4) \quad \diamond \diamond \quad 90(0.6)$

$348(4.4)$

$-4(1.1) \quad 1(0.2)$

$3(1.9)$

$-4(2.1)-1(0.4)$

$\Delta 0 \quad 1(0.4)$

$-12(1.3) \quad \nabla \quad 1(0.3)$

$\Delta \diamond \quad 0(0.1)$

$\Delta \diamond \quad 0(0.1)$

\begin{tabular}{l|l}
$0(2.0)$ & $1(0.2)$
\end{tabular}

$2(1.6) \quad 1(0.1)$

\begin{tabular}{l|l}
$x x$ & $2(0.4)$ \\
4 &
\end{tabular}

$\begin{array}{lll}x x & 2(0.4) \\ 4(1.7) \quad 0 & 5(0.9)\end{array}$

$1(1.5) \quad 4(0.4)$

$\Delta \Delta$

$-1(1.5)$

$3(0.3)$

$-1(1.5) \quad 2(0.3)$

$\infty 0 \quad 2(0.3)$

$\Delta \diamond \quad 5(0.7)$

$3(1.2) \quad 0 \quad 3(0.3)$

$3(0.3)$

$-4(1.2)$

$0(1.0)$

$2(0.3)$

$-2(2.4)$

$-1(1.3) \quad 1(0.2)$

$2(0.3)$

$-1(1.6) \quad 4(0.8)$

$00 \quad 3(0.3)$

$6(1.2) \quad 0 \quad 1(0.2)$

$3(1.1) \quad 0 \quad 6(0.5)$

$\begin{array}{ll}\Delta & 4(0.9)\end{array}$

$3(0.9) \quad 0 \quad 2(0.2)$

$\begin{array}{ll}\diamond \diamond & 9(0.7) \\ -2(1.3) \quad \odot \quad 8(0.9)\end{array}$

$-2(1.3)$

$8(0.9)$

$\Delta \Delta \quad 8(0.3)$

$\diamond \diamond \quad 1(0.2)$

$7(0.2)$

\begin{tabular}{l|l}
$\Delta \diamond$ & $7(0.8)$ \\
$\diamond \diamond$ & $4(0.5)$
\end{tabular}

\begin{tabular}{l|r|}
$\diamond \diamond$ & $4(0.5)$ \\
$3(1.7)$ & $11(1.1)$
\end{tabular}

$\begin{array}{lr}\Delta \diamond & 6(0.5) \\ -2(2.0) & 19(1.7)\end{array}$

$6(0.5)$

$506(2.9)-16(1.6) \quad \odot \quad 22(1.1)$

Moldova, Rep. of

$4(0.6)$

$578(5.7)$

$-1(1.1)$
$0(0.9)$

$74(1.1)$

South Africa

$3(0.5)$

$\Delta 0$

$506(2.9)$

$-16(1.6)$

$457(2.8)$

00

$537(7.7) \quad 1(0.4) \quad 47(1.7)$

Morocco

Indonesia

$1(0.3)$

$\sim$

$--\quad 38(1.8)$

$-5(2.7)$

$26(1.1)$

$62(1.7)$

$418(4.1)$

$\Delta \diamond$

$51(1.9)$

$\begin{array}{cc}\mathrm{xx} & \mathrm{xx} \\ -\end{array}$

England

United States

$\begin{array}{ll}\mathrm{xx} & \mathrm{xx}\end{array}$

$\mathrm{xx} \quad \mathrm{xx}$

$\begin{array}{cc}\mathrm{xx} & \mathrm{xx}\end{array}$

International Avg

$11(0.1)$

$563(1.0)$

$--$

$80(0.2) \quad 503(0.5)$

XX

$61(1.9)$

Low HER

4th Grade

( Percent in 2006 significantly higher

$9(0.1) \quad 426(1.9)$
P Percent in 2006 significantly lower

Based on students' responses to two questions about home educational resources: number of books in the home, and educational aids in the home (computer, study desk/ table for own use, books of their own, access to a daily newspaper); and parents' responses to two questions: number of children's books in the home, and parents' education. High level indicates more than 100 books in the home; more than 25 children's books; at least 3 of 4 educational aids; and at least one parent finished university. Low level indicates 25 or fewer books in the home; 25 or fewer children's books; no more than 2 educational aids; and parents that have not completed secondary education. Medium level includes all other combinations of responses.

() Standard errors appear in parentheses. Because results are rounded to the nearest whole number, some totals may appear inconsistent. An " $r$ " indicates data are available for $70-84 \%$ of the students. An " $s$ " indicates data are
available for $50-69 \%$ of the students. An " $x$ " indicates data are available for less than $50 \%$ of the students.

A dash $(-)$ indicates comparable data are not available. A tilde $(\sim)$ indicates insufficient data to report achievement.

A diamond $(\diamond)$ indicates the country did not participate in the 2001 assessment.

NOTE: The International Average does not include the results from the Canadian provinces. 
While most countries had from 70 to 90 percent of students at the middle level, the most striking differences were at the high and low levels. Participants with relatively high proportions of students from well-resourced homes (20\% or more of students at the high level of the index) included Norway, Denmark, Iceland, Sweden, Scotland, the Netherlands, and the Canadian province of British Columbia. All except Norway had average reading achievement above the average on the PIRLs international reading scale (as shown in Exhibit 1.1). At the other extreme, Moldova, South Africa, Iran, Morocco, and Indonesia had 20 percent or more of their students at the low level of the index and very few at the high level. Except for Moldova, these countries had average achievement below the PIRLs international scale average.

Although there was some correspondence between the percentage of students at the high level of the index of home educational resources and a country's average reading achievement, more significant were the differences within each country between students at the three levels of the index for every country. The difference between the international average for those at the high level (563) and those at the low level (426) amounted to 137 score points, about twice the difference between the highest performing country (the Russian Federation) and the PIRLs scale average.

Of the countries that also participated in PIRLS 2001, Iceland, Scotland, the Netherlands, the Russian Federation, Hong Kong SAR, and Macedonia had increased percentages of students in the high category of the index, while Norway, Hungary, Germany, Latvia, and Slovenia had decreased percentages.

Because books, and children's books, in particular, are probably the most important literacy resource, Exhibits 3.3 and 3.4 provide more detail about the number of children's books in the home. Parents' reports on the number of children's books in the home (Exhibit 3.3) indicated a fairly high level of book ownership. On average across countries, the majority of students (57\%) were from homes with more than 25 children's books. According to parents, the highest percentages of students with many children's books were in the Scandinavian countries (Denmark, Iceland, Norway, and Sweden), 
New Zealand, Scotland, and the Canadian provinces of Alberta, British Columbia, Nova Scotia, and Ontario, where 25 percent or more of students had parents reporting more than 100 children's books in the home. However, there were also countries with relatively few children's books in the home. In Bulgaria, Georgia, Hong Kong SAR, Macedonia, Moldova, Qatar, and Romania, more than 25 percent of students were from homes with no more than 10 children's books, and in Indonesia, Iran, Kuwait, Morocco, and South Africa, the situation was even worse, with the majority of students in homes with 10 or fewer children's books.

The average reading achievement difference between students from homes with many children's books and those from homes with few children's books was very large. Students from homes with more than 100 children's books had an average achievement score of 553 score points, whereas those from homes with 10 books or fewer had an average of just 462 pointsa difference of 91 score points (almost 1 standard deviation on the PIRLS reading scale). There were large average achievement differences between students from homes with many children's books and those from homes with few books in every country.

Because of the association between number of children's books in the home and average reading achievement at fourth grade, Exhibit 3.4 presents information on trends in parents' reports on children's book ownership from 2001. More specifically, for those countries that also participated in PIRLS 2001, the exhibit shows the percentage of students in each category of book ownership in 2006 and the change in the percentage since 2001. The average across all countries is shown at the foot of each column.

On average across the countries, there was a slight decrease in parents' reports of the number of children's books in the home, perhaps reflecting increased access to other literacy media, primarily Internet based. For the countries shown in Exhibit 3.4 (a subset of the entire group of PIRLS 2006 participants), 14 percent of students, on average, had parents reporting more than 100 children's books in the home in 2006, a decrease of 2 percentage points from 2001. There also was a slight decrease in the percentage of 
Exhibit 3.3 Parents' Reports of Children's Books in the Home

PIRLS 2006

4th Grade

\begin{tabular}{|c|c|c|c|c|c|c|c|}
\hline \multirow{2}{*}{ Countries } & & \multicolumn{2}{|c|}{ More than 100 Books } & \multicolumn{2}{|c|}{ 51-100 Books } & \multicolumn{2}{|c|}{ 26-50 Books } \\
\hline & & $\begin{array}{l}\text { Percent of } \\
\text { Students }\end{array}$ & $\begin{array}{c}\text { Average } \\
\text { Achievement }\end{array}$ & $\begin{array}{l}\text { Percent of } \\
\text { Students }\end{array}$ & $\begin{array}{c}\text { Average } \\
\text { Achievement }\end{array}$ & $\begin{array}{l}\text { Percent of } \\
\text { Students }\end{array}$ & $\begin{array}{c}\text { Average } \\
\text { Achievement }\end{array}$ \\
\hline Austria & & $14(0.8)$ & $579(2.8)$ & $24(0.8)$ & $561(2.9)$ & $34(0.7)$ & $537(2.2)$ \\
\hline Belgium (Flemish) & & $15(0.8)$ & $571(2.8)$ & $24(0.9)$ & $559(2.5)$ & $32(0.7)$ & $550(2.6)$ \\
\hline Belgium (French) & & $20(1.3)$ & $536(3.1)$ & $26(1.0)$ & $518(2.7)$ & $27(0.9)$ & $498(2.9)$ \\
\hline Bulgaria & & $5(0.5)$ & $606(5.6)$ & $11(0.8)$ & $590(6.0)$ & $24(1.3)$ & $571(3.9)$ \\
\hline Canada, Alberta & $r$ & $39(1.4)$ & $578(3.0)$ & $29(1.1)$ & $566(2.8)$ & $21(1.0)$ & $556(3.3)$ \\
\hline Canada, British Columbia & $r$ & $36(1.5)$ & $582(3.2)$ & $29(1.0)$ & $562(3.5)$ & $22(1.0)$ & $549(4.1)$ \\
\hline Canada, Nova Scotia & & $42(1.0)$ & $561(2.6)$ & $32(0.8)$ & $546(2.8)$ & $19(0.8)$ & $524(3.1)$ \\
\hline Canada, Ontario & & $30(1.5)$ & $577(3.7)$ & $29(0.9)$ & $559(3.5)$ & $25(1.2)$ & $549(3.0)$ \\
\hline Canada, Quebec & & $17(1.0)$ & $561(3.9)$ & $28(1.1)$ & $544(3.5)$ & $31(0.9)$ & $529(3.9)$ \\
\hline Chinese Taipei & & $21(0.9)$ & $566(2.7)$ & $18(0.7)$ & $550(2.9)$ & $23(0.7)$ & $537(2.6)$ \\
\hline Denmark & & $25(0.9)$ & $568(3.7)$ & $32(1.1)$ & $552(2.6)$ & $26(0.8)$ & $540(3.3)$ \\
\hline England & & $x \times$ & $x \times$ & $\mathrm{xx}$ & $x x$ & $\mathrm{xx}$ & $x x$ \\
\hline France & & $19(1.0)$ & $560(2.6)$ & $28(0.9)$ & $538(2.7)$ & $28(0.8)$ & $518(2.4)$ \\
\hline Georgia & & $4(0.4)$ & $510(6.6)$ & $8(0.6)$ & $500(4.9)$ & $21(1.1)$ & $489(3.9)$ \\
\hline Germany & & $19(1.1)$ & $587(2.5)$ & $28(1.0)$ & $567(3.3)$ & $31(1.0)$ & $547(2.1)$ \\
\hline Hong Kong SAR & & $9(0.8)$ & $582(3.3)$ & $13(0.8)$ & $579(3.3)$ & $25(0.9)$ & $570(2.4)$ \\
\hline Hungary & & $15(0.8)$ & $596(3.6)$ & $24(1.1)$ & $578(3.3)$ & $31(1.2)$ & $550(3.0)$ \\
\hline Iceland & $r$ & $28(0.7)$ & $537(2.3)$ & $41(0.8)$ & $517(2.1)$ & $26(0.8)$ & $501(2.9)$ \\
\hline Indonesia & & $1(0.2)$ & $\sim \sim$ & $1(0.2)$ & $\sim \sim$ & $6(0.6)$ & $430(7.6)$ \\
\hline Iran, Islamic Rep. of & & $2(0.2)$ & $\sim \sim$ & $4(0.4)$ & $507(6.8)$ & $11(0.7)$ & $476(5.0)$ \\
\hline Israel & $s$ & $13(1.0)$ & $571(6.5)$ & $22(1.1)$ & $559(4.3)$ & $29(1.2)$ & $536(4.0)$ \\
\hline Italy & & $5(0.4)$ & $593(4.9)$ & $15(0.7)$ & $574(4.1)$ & $29(0.8)$ & $562(3.1)$ \\
\hline Kuwait & $r$ & $2(0.3)$ & $\sim \sim$ & $3(0.4)$ & $369(12.6)$ & $15(0.8)$ & $370(6.8)$ \\
\hline Latvia & & $13(0.8)$ & $571(3.5)$ & $21(0.8)$ & $559(3.4)$ & $31(0.9)$ & $544(2.9)$ \\
\hline Lithuania & & $5(0.4)$ & $570(3.8)$ & $14(0.7)$ & $564(3.4)$ & $28(0.8)$ & $546(2.1)$ \\
\hline Luxembourg & & $20(0.5)$ & $601(2.0)$ & $24(0.6)$ & $576(1.9)$ & $25(0.6)$ & $556(2.3)$ \\
\hline Macedonia, Rep. of & & $4(0.4)$ & $464(10.9)$ & $9(0.6)$ & $472(7.7)$ & $27(1.0)$ & $478(5.1)$ \\
\hline Moldova, Rep. of & & $2(0.3)$ & $\sim \sim$ & $5(0.6)$ & $529(8.4)$ & $16(1.0)$ & $523(4.4)$ \\
\hline Morocco & & $1(0.2)$ & $\sim \sim$ & $1(0.2)$ & $\sim \sim$ & $6(0.6)$ & $368(13.3)$ \\
\hline Netherlands & $s$ & $17(1.1)$ & $577(3.7)$ & $29(1.2)$ & $563(2.2)$ & $31(1.1)$ & $552(2.0)$ \\
\hline New Zealand & $s$ & $36(1.0)$ & $575(3.1)$ & $29(1.0)$ & $550(3.1)$ & $23(0.8)$ & $527(3.8)$ \\
\hline Norway & & $29(1.0)$ & $518(3.4)$ & $36(1.0)$ & $505(3.0)$ & $24(0.9)$ & $491(3.1)$ \\
\hline Poland & & $6(0.5)$ & $570(6.3)$ & $16(0.6)$ & $546(3.5)$ & $35(0.8)$ & $526(3.2)$ \\
\hline Qatar & $r$ & $5(0.3)$ & $387(6.5)$ & $8(0.4)$ & $376(5.9)$ & $18(0.5)$ & $372(3.9)$ \\
\hline Romania & & $3(0.3)$ & $559(9.0)$ & $9(0.7)$ & $548(5.9)$ & $20(1.2)$ & $531(4.4)$ \\
\hline Russian Federation & & $11(0.8)$ & $596(4.1)$ & $19(0.8)$ & $584(4.1)$ & $32(1.1)$ & $572(3.3)$ \\
\hline Scotland & $s$ & $32(1.4)$ & $573(5.2)$ & $29(1.2)$ & $548(5.5)$ & $26(1.0)$ & $520(4.5)$ \\
\hline Singapore & & $19(0.6)$ & $597(3.5)$ & $23(0.5)$ & $574(3.2)$ & $32(0.6)$ & $555(3.0)$ \\
\hline Slovak Republic & & $5(0.3)$ & $581(4.0)$ & $18(0.8)$ & $567(3.5)$ & $37(1.0)$ & $543(2.3)$ \\
\hline Slovenia & & $9(0.6)$ & $567(3.7)$ & $19(0.8)$ & $548(2.4)$ & $35(0.8)$ & $525(2.5)$ \\
\hline South Africa & $r$ & $4(0.3)$ & $367(19.2)$ & $6(0.5)$ & $360(16.4)$ & $13(0.6)$ & $344(12.3)$ \\
\hline Spain & $s$ & $12(1.1)$ & $557(4.4)$ & $23(1.0)$ & $540(3.8)$ & $32(1.1)$ & $521(3.2)$ \\
\hline Sweden & & $28(1.1)$ & $572(3.0)$ & $32(0.9)$ & $553(2.3)$ & $25(0.8)$ & $544(3.2)$ \\
\hline Trinidad and Tobago & & $9(0.7)$ & $489(10.5)$ & $18(0.8)$ & $466(6.7)$ & $29(0.9)$ & $448(5.6)$ \\
\hline United States & & -- & -- & -- & -- & -- & -- \\
\hline International Avg. & & $13(0.1)$ & $553(1.0)$ & $19(0.1)$ & $532(0.9)$ & $25(0.1)$ & $510(0.8)$ \\
\hline
\end{tabular}

Background data provided by parents.

() Standard errors appear in parentheses. Because results are rounded to the nearest whole number, some totals may appear inconsistent.

An " $r$ " indicates data are available for $70-84 \%$ of the students. An "s" indicates data are available for $50-69 \%$ of the students. An " $x$ " indicates data are available for less than $50 \%$ of the students.
A dash (-) indicates comparable data are not available. A tilde $(\sim)$ indicates insufficient data to report achievement.

NOTE: The International Average does not include the results from the Canadian provinces. 
Exhibit 3.3 Parents' Reports of Children's Books in the Home (Continued)

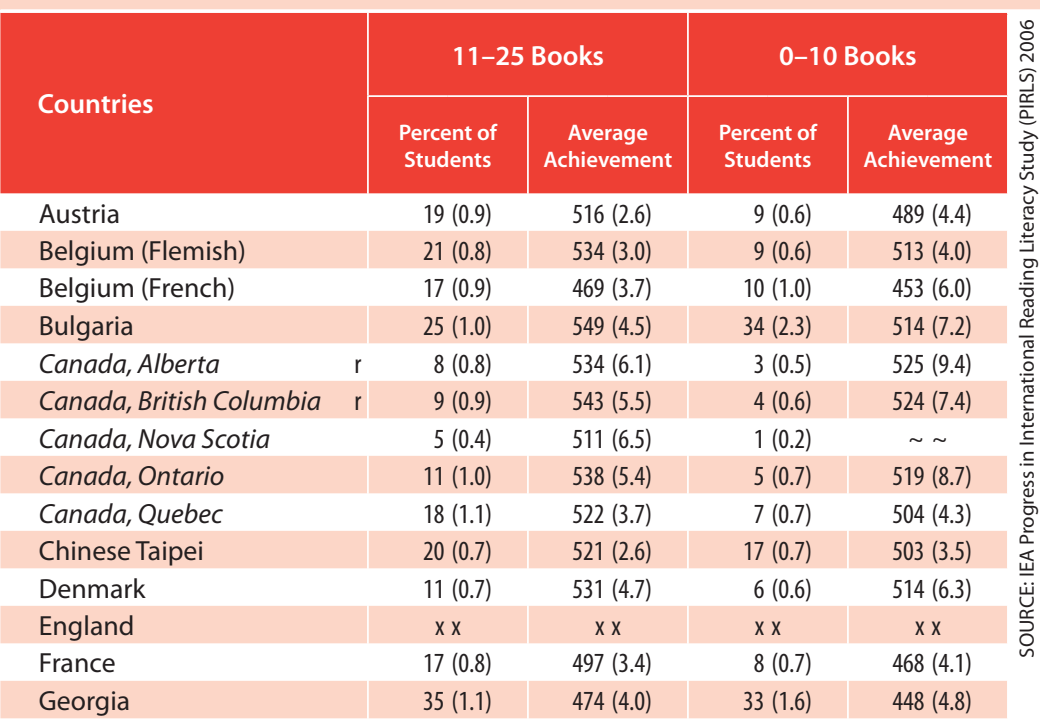

\begin{tabular}{|l|l|l|r|r|}
\hline Germany & $16(0.7)$ & $519(3.6)$ & $6(0.5)$ & $487(4.7)$ \\
\hline Hong Kong SAR & $26(0.8)$ & $561(2.7)$ & $26(1.2)$ & $551(3.7)$ \\
\hline Hungary & $19(0.9)$ & $529(3.2)$ & $11(1.3)$ & $488(5.5)$ \\
\hline
\end{tabular}

\begin{tabular}{|c|c|c|c|c|c|}
\hline Hungary & & $19(0.9)$ & $529(3.2)$ & $11(1.3)$ & $488(5.5)$ \\
\hline Iceland & $r$ & $5(0.4)$ & $481(5.9)$ & $1(0.1)$ & $\sim \sim$ \\
\hline Indonesia & & $23(1.4)$ & $410(7.1)$ & $69(1.6)$ & $401(4.0)$ \\
\hline Iran, Islamic Rep. of & & $21(0.9)$ & $444(3.6)$ & $62(1.6)$ & $398(3.4)$ \\
\hline Israel & $s$ & $20(1.0)$ & $502(5.7)$ & $16(1.3)$ & $467(8.3)$ \\
\hline
\end{tabular}

\begin{tabular}{|c|c|c|c|c|c|}
\hline & & & & & \\
\hline Italy & & $29(1.0)$ & $541(3.6)$ & $22(0.9)$ & $534(5.7)$ \\
\hline Kuwait & $r$ & $27(0.8)$ & $353(5.5)$ & $53(1.2)$ & $318(5.8)$ \\
\hline Latvia & & $24(0.9)$ & $527(3.1)$ & $11(0.8)$ & $501(5.0)$ \\
\hline Lithuania & & $33(0.8)$ & $532(1.6)$ & $21(1.0)$ & $510(2.8)$ \\
\hline Luxembourg & & $19(0.5)$ & $531(2.3)$ & $11(0.4)$ & $506(2.7)$ \\
\hline Macedonia, Rep. of & & $31(0.9)$ & $444(4.4)$ & $28(1.3)$ & $406(5.1)$ \\
\hline Moldova, Rep. of & & $29(1.1)$ & $505(3.4)$ & $47(1.6)$ & $486(4.0)$ \\
\hline Morocco & & $16(1.0)$ & $349(9.5)$ & $76(1.2)$ & $315(6.7)$ \\
\hline Netherlands & $s$ & $17(1.0)$ & $542(2.9)$ & $6(0.7)$ & $517(7.2)$ \\
\hline New Zealand & s & $8(0.6)$ & $517(4.9)$ & $4(0.4)$ & $489(7.3)$ \\
\hline Norway & & $8(0.5)$ & $465(6.8)$ & $2(0.3)$ & $\sim \sim$ \\
\hline Poland & & $30(0.8)$ & $506(3.0)$ & $12(0.8)$ & $481(5.3)$ \\
\hline Qatar & $r$ & $26(0.7)$ & $365(2.9)$ & $43(0.7)$ & $340(2.2)$ \\
\hline Romania & & $27(1.1)$ & $504(3.4)$ & $41(2.1)$ & $445(7.8)$ \\
\hline Russian Federation & & $26(0.9)$ & $549(4.2)$ & $12(0.8)$ & $522(5.6)$ \\
\hline Scotland & s & $10(0.8)$ & $510(5.7)$ & $3(0.5)$ & $468(13.7)$ \\
\hline Singapore & & $17(0.5)$ & $537(4.0)$ & $9(0.5)$ & $499(5.5)$ \\
\hline Slovak Republic & & $26(1.0)$ & $522(3.1)$ & $14(1.1)$ & $460(7.1)$ \\
\hline Slovenia & & $26(0.8)$ & $505(2.7)$ & $11(0.7)$ & $480(4.3)$ \\
\hline South Africa & $r$ & $23(0.5)$ & $305(6.0)$ & $54(1.1)$ & $286(4.2)$ \\
\hline Spain & $s$ & $22(1.0)$ & $501(3.8)$ & $11(1.1)$ & $476(5.9)$ \\
\hline Sweden & & $11(0.8)$ & $529(3.8)$ & $4(0.6)$ & $506(7.1)$ \\
\hline Trinidad and Tobago & & $27(1.0)$ & $425(5.9)$ & $17(1.0)$ & $392(7.7)$ \\
\hline United States & & -- & -- & -- & -- \\
\hline International Avg. & & $21(0.1)$ & $489(0.7)$ & $22(0.2)$ & $462(1.0)$ \\
\hline
\end{tabular}


Exhibit 3.4 Trends in Parents' Reports of Children's Books in the Home

PIRLS 2006

4th Grade

\begin{tabular}{|c|c|c|c|c|c|c|c|c|c|c|}
\hline \multirow{2}{*}{ Countries } & \multicolumn{4}{|c|}{ More than 100 Books } & \multicolumn{3}{|c|}{ 51-100 Books } & \multicolumn{3}{|c|}{ 26-50 Books } \\
\hline & & $\begin{array}{c}2006 \\
\text { Percent } \\
\text { of Students }\end{array}$ & $\begin{array}{l}\text { Difference } \\
\text { in Percent } \\
\text { from } 2001\end{array}$ & & $\begin{array}{c}2006 \\
\text { Percent } \\
\text { of Students }\end{array}$ & $\begin{array}{l}\text { Difference } \\
\text { in Percent } \\
\text { from 2001 }\end{array}$ & & $\begin{array}{c}2006 \\
\text { Percent } \\
\text { of Students }\end{array}$ & $\begin{array}{l}\text { Difference } \\
\text { in Percent } \\
\text { from } 2001\end{array}$ & \\
\hline Bulgaria & & $5(0.5)$ & $-3(0.9)$ & $\nabla$ & $11(0.8)$ & $-4(1.2)$ & $\nabla$ & $24(1.3)$ & $-4(1.7)$ & $(\nabla$ \\
\hline Canada, Ontario & $r$ & $30(1.6)$ & $-7(2.3)$ & ( & $29(0.9)$ & $-2(1.4)$ & & $25(1.2)$ & $3(1.7)$ & \\
\hline Canada, Quebec & $r$ & $17(1.0)$ & $-2(1.5)$ & & $28(1.1)$ & $0(1.4)$ & & $31(0.9)$ & $-1(1.5)$ & \\
\hline England & & $x \times$ & $x x$ & & $x x$ & $x x$ & & $x x$ & $x x$ & \\
\hline France & & $19(1.0)$ & $0(1.5)$ & & $28(0.9)$ & $2(1.3)$ & & $28(0.8)$ & $-2(1.3)$ & \\
\hline Germany & & $19(1.1)$ & $3(1.4)$ & $\theta$ & $28(1.0)$ & $2(1.3)$ & & $31(1.0)$ & $-2(1.3)$ & $(\nabla$ \\
\hline Hong Kong SAR & & $9(0.8)$ & $6(0.9)$ & $\theta$ & $13(0.8)$ & $6(0.9)$ & 0 & $25(0.9)$ & $7(1.2)$ & 0 \\
\hline Hungary & & $15(0.8)$ & $-3(1.3)$ & ( & $24(1.1)$ & $-3(1.4)$ & & $31(1.2)$ & $2(1.4)$ & \\
\hline Iceland & $r$ & $28(0.7)$ & $-5(1.1)$ & ( & $41(0.8)$ & $1(1.2)$ & & $26(0.8)$ & $3(1.2)$ & $\theta$ \\
\hline Iran, Islamic Rep. of & & $2(0.2)$ & $0(0.4)$ & & $4(0.4)$ & $1(0.6)$ & & $11(0.7)$ & $0(1.0)$ & \\
\hline Israel & & $13(1.0)$ & $x x$ & & $22(1.1)$ & $x x$ & & $29(1.2)$ & $x x$ & \\
\hline Italy & & $5(0.4)$ & $0(0.6)$ & & $15(0.7)$ & $2(1.0)$ & $\theta$ & $29(0.8)$ & $2(1.2)$ & \\
\hline Latvia & & $13(0.8)$ & $-2(1.2)$ & & $21(0.8)$ & $-2(1.2)$ & & $31(0.9)$ & $-1(1.5)$ & \\
\hline Lithuania & & $5(0.4)$ & $-1(0.7)$ & & $14(0.7)$ & $0(1.2)$ & & $28(0.8)$ & $0(1.2)$ & \\
\hline Macedonia, Rep. of & $r$ & $4(0.4)$ & $0(0.6)$ & & $9(0.6)$ & $1(0.9)$ & & $27(1.0)$ & $1(1.6)$ & \\
\hline Moldova, Rep. of & & $2(0.3)$ & $0(0.5)$ & & $5(0.6)$ & $0(0.9)$ & & $16(1.0)$ & $1(1.7)$ & \\
\hline Morocco & & $1(0.2)$ & -- & & $1(0.2)$ & -- & & $6(0.6)$ & -- & \\
\hline Netherlands & $s$ & $17(1.1)$ & $-1(1.4)$ & & $29(1.2)$ & $-2(1.6)$ & & $31(1.1)$ & $1(1.6)$ & \\
\hline New Zealand & $s$ & $36(1.0)$ & $2(1.7)$ & & $29(1.0)$ & $-1(1.6)$ & & $23(0.8)$ & $0(1.4)$ & \\
\hline Norway & & $29(1.0)$ & $-1(1.6)$ & & $36(1.0)$ & $0(1.4)$ & & $24(0.9)$ & $0(1.2)$ & \\
\hline Romania & & $3(0.3)$ & $0(0.5)$ & & $9(0.7)$ & $-1(1.0)$ & & $20(1.2)$ & $-1(1.6)$ & \\
\hline Russian Federation & & $11(0.8)$ & $-1(1.2)$ & & $19(0.8)$ & $0(1.1)$ & & $32(1.1)$ & $3(1.5)$ & \\
\hline Scotland & $s$ & $32(1.4)$ & $4(2.0)$ & $\theta$ & $29(1.2)$ & $-3(1.9)$ & & $26(1.0)$ & $-1(2.0)$ & \\
\hline Singapore & & $19(0.6)$ & $-1(1.2)$ & & $23(0.5)$ & $-1(0.9)$ & & $32(0.6)$ & $1(0.9)$ & \\
\hline Slovak Republic & & $5(0.3)$ & $-2(0.7)$ & ( & $18(0.8)$ & $-1(1.2)$ & & $37(1.0)$ & $-1(1.3)$ & \\
\hline Slovenia & & $9(0.6)$ & $3(0.8)$ & $\theta$ & $19(0.8)$ & $4(1.2)$ & 0 & $35(0.8)$ & $3(1.2)$ & $\theta$ \\
\hline Sweden & & $28(1.1)$ & $-4(1.6)$ & $\nabla$ & $32(0.9)$ & $-2(1.2)$ & 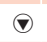 & $25(0.8)$ & $3(1.1)$ & $\theta$ \\
\hline United States & & -- & -- & & -- & -- & & -- & -- & \\
\hline International Avg. & & $14(0.2)$ & $-2(0.2)$ & ( & $20(0.2)$ & $-1(0.3)$ & & $26(0.2)$ & $0(0.3)$ & \\
\hline
\end{tabular}

Based on data provided by parents.

() Standard errors appear in parentheses. Because results are rounded to the nearest whole number, some totals may appear inconsistent.

An " $r$ " indicates data are available for $70-84 \%$ of the students. An " $s$ " indicates data are available for $50-69 \%$ of the students. An " $x$ " indicates data are available for less than $50 \%$ of the students.
A dash (-) indicates comparable data are not available.

NOTE: The International Average does not include the results from the Canadian provinces. Trend Note: The primary education systems of the Russian Federation and Slovenia underwent structural changes. Data for Canada, Ontario include only public schools. 
Exhibit 3.4 Trends in Parents' Reports of Children's Books in the Home (Continued)

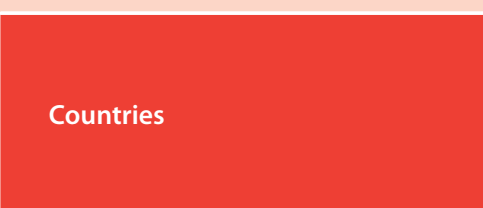

\begin{tabular}{|c|}
\hline \multicolumn{2}{|c|}{$11-25$ B } \\
\hline $\begin{array}{c}2006 \\
\text { Percent } \\
\text { of Students }\end{array}$ \\
\hline
\end{tabular}

Bulgaria

$25(1.0)$

Canada, Ontario

$11(1.1)$

Canada, Quebec

$r \quad 18(1.1)$

England

France

$\mathrm{xx}$

\section{0-10 Books}

Germany

$17(0.8)$

$16(0.7)$

Hong Kong SAR

$26(0.8)$

Hungary

$19(0.9)$

Iceland

$5(0.4)$

Iran, Islamic Rep. of

$21(0.9)$

Israel

$20(1.0)$

$29(1.0)$

$24(0.9)$

Latvia

$33(0.8)$

Lithuania

$31(0.9)$

Macedonia, Rep. of

$29(1.1)$

$16(1.0)$

Morocco

Netherlands

New Zealand

$17(1.0)$

Norway

$\mathrm{s} \quad 8(0.6)$

Romania

$8(0.5)$

$27(1.1)$

$26(0.9)$

Russian Federation

$10(0.8)$

Scotland

$17(0.5)$

$26(1.0)$

$26(0.8)$

Slovenia

$11(0.8)$

United States

$--$

International Avg.

$20(0.2)$

$1(0.3)$

$20(0.2)$

$2(0.3)$

\begin{tabular}{ll|l|l|l}
$3(1.4)$ & $\bullet$ & $5(0.7)$ & $3(0.8)$ & 0 \\
\hline
\end{tabular}

$2(1.5)$

$-1(1.1)$

$x(0.7)$

$x x$

$8(0.7)$

$1(0.9)$

$-2(1.1)$

$6(0.5)$

$26(1.2)$

$2(1.2)$

$11(1.3)$

$1(0.1)$

1 (1.4)

$62(1.6)$

$x(1.3)=-16(1.3)$

$16(1.3)$

$-3(1.3)$

$11(0.8)$

$1(1.6)$

$1(1.3)$

$0(1.5)$

$2(1.5)$

$--$

$47(1.6)=-3(2.4)$

$76(1.2) \quad--$

$6(0.7) \quad 1(1.0)$
$-4(0.4)$

$-1(1.0)$

$1(0.8)$

$4(0.4) \quad 0(0.7)$

$2(0.3) \quad 0(0.5)$

$41(2.1) \quad 2(2.9)$

$3(1.3)$

$0(1.1)$

$0(0.9)$

$1(1.5)$

$-4(1.2)$

$3(0.9)$

$--$

- Percent in 2006 significantly higher

๑ Percent in 2006 significantly lower 
students in the next highest category, 51-100 children's books. The percentage of students in homes with more than 100 children's books decreased in Bulgaria, Hungary, Iceland, the Slovak Republic, Sweden, and the Canadian province of Ontario. However, there also were countries where the percentage of students in this category increased-Germany, Hong Kong SAR, Scotland, and Slovenia.

Accompanying the decrease in percentages of students in homes with many children's books was an increase in the percentages in homes with few books. For example, according to parents, 20 percent of students in 2006 were from homes with no more than 10 children's books, an increase of 2 percentage points from 2001. However, three countries showed a decrease in the percentage of students in homes with very few books-Hong Kong SAR, the Russian Federation, and Slovenia. These are three of the four countries with the greatest increase in average reading achievement from 2001, as shown in Exhibit 1.3.

Another important component of the Index of Home Educational Resources was the parents' highest level of education. As shown in Exhibit 3.5, parental education varied greatly, both within and across the PIRLS countries. On average across countries, 25 percent of students came from homes where parents reported that one or both of them had finished university, 21 percent where one or both had finished post-secondary school but not university, 31 percent where one or both had finished upper-secondary education, 15 percent where one or both had finished lower-secondary school, and 8 percent where neither parent had finished lower-secondary education. 
Participants with the highest percentages (40\% or more) of universityeducated parents included Denmark, Georgia, Iceland, Israel, the Netherlands, Norway, Qatar, and the Canadian provinces of British Columbia, Ontario, and Quebec. In contrast, countries with relatively low levels of parental education (more than $25 \%$ of students whose parents did not complete lowersecondary education) included Indonesia (46\%), Iran (35\%), Morocco (59\%), and South Africa (26\%).

The PIRLs data highlight the powerful association between parental education and children's reading achievement. Higher levels of parental education were associated with higher average fourth-grade reading achievement in almost every country. At 543 score points, the average reading achievement of students with at least one university-educated parent was 120 points (more than 1 standard deviation) greater than the average of those whose parents did not complete lower-secondary education (423 points). 


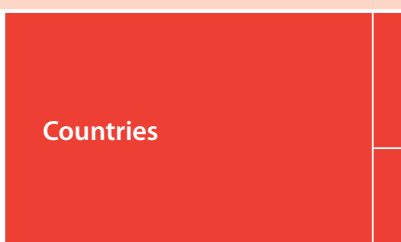

Austria

Belgium (Flemish)

Belgium (French)

Bulgaria

Canada, Alberta

Canada, British Columbia

Canada, Nova Scotia

Canada, Ontario

Canada, Quebec

Chinese Taipei

Denmark

England

France

Georgia

Germany

Hong Kong SAR

Hungary

Iceland

Indonesia

Iran, Islamic Rep. of

Israel

Italy

Kuwait

Latvia

Lithuania

Finished University or Higher
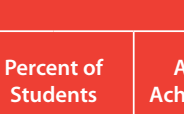

Students

Average
Achievement

\begin{tabular}{|c|c|}
\hline \multicolumn{2}{|c|}{$\begin{array}{c}\text { Finished Post-Secondary } \\
\text { Education but Not } \\
\text { University }\end{array}$} \\
$\begin{array}{c}\text { Percent of } \\
\text { Students }\end{array}$ & $\begin{array}{c}\text { Average } \\
\text { Achievement }\end{array}$ \\
\hline
\end{tabular}

Finished

Upper- Secondary School

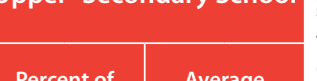

Students Achievement Students

$22(0.8) \quad 548(2.7)$

\begin{tabular}{|l|l|l|l|}
\hline $31(1.2)$ & $572(2.3)$ & $27(0.9)$ & $559(1.9)$ \\
\hline
\end{tabular}

$10(0.7)$

539 (4.8)

$25(1.7) \quad 594(5.2)$

$39(1.6) \quad 582(3.7)$

$$
48 \text { (1.3) }
$$

$559(1.9)$

$6(0.4) \quad 564(7.0)$

$39(1.2) \quad 560(2.6)$

580 (3.8)

41 (1.8)

35 (1.4)

$571(2.9)$

$41(2.0) \quad 575(3.3)$

$\begin{array}{ll}42(2.0) & 556(3.6) \\ 21(1.2) & 573(2.6)\end{array}$

49 (1.7)

565 (2.8)

$\mathrm{x}$

$\begin{array}{ll}\mathrm{xx} & \mathrm{xx}\end{array}$

$26(1.6)$

48 (1.8)

561 (2.5)

$17(1.3)$

19 (1.4)

498 (3.0)

$589(2.6)$

$580(2.3)$

25 (1.5)

598 (2.7)

\begin{tabular}{ll|l}
$r$ & $45(0.9)$ & $536(2.3)$
\end{tabular}

$476(6.8)$

$9(0.9) \quad 508(5.4)$

$508(5.4)$

40 (1.8)

$16(1.1)$

$586(4.0)$

$37(1.3)$

$372(5.6)$

$18(1.1) \quad 568(3.1)$

Luxembourg

$27(1.3)$

$568(3.1)$

Macedonia, Rep. of

r $19(0.6)$

$594(2.2)$

Moldova, Rep. of

Morocco

$20(1.3)$

$6(0.9)$

$529(5.3)$

Netherlands

$47(1.7) \quad 574(2.0)$

New Zealand

32 (1.4)

$577(3.0)$

Norway

Poland

$49(1.5$

19 (1.1)

Qata

Romania

$45(0.7)$

9 (1.1)

$519(2.7)$
$564(3.7)$

Russian Federation

$385(2.0)$

Scotland

Singapore

Slovak Republic

Slovenia

South Africa

Spain

Sweden

$38(1$
$\times x$

590 (3.5)

$23(0.7)$

$19(1.0)$

$24(1.2)$

$\mathrm{x} \mathrm{X}$

603 (3.4)

$577(2.5)$

$577(2.5)$

$39(1.3)$

$43(1.2)$

$37(1.3) \quad 555(2.9)$

38 (1.4) $\quad 529$ (3.2)

$22(0.8) \quad 550(2.2)$

$30(1.3) \quad 547(3.5)$

\begin{tabular}{c|c}
$x x$ & $x x$
\end{tabular}

$16(0.7) \quad 542(3.9)$

$24(1.2) \quad 459(4.6)$

$10(0.5) \quad 580(2.6)$

$12(0.6) \quad 575(3.1)$

$20(1.0) \quad 572(3.4)$

$22(0.7) \quad 508(2.8)$

$4(0.5)$

$4(0.5)$

$19(0.9)$

$8(0.5)$

$18(0.9)$

63 (1.2)

$39(0.9)$

$16(0.6)$

$11(0.7)$

$35(1.0)$

$8(0.6)$

$3(0.4)$

38 (1.3)

$29(1.0)$

$9(0.6)$

$10(0.4)$

$13(0.9)$

$49(1.1)$

$\begin{array}{cc}x \times & x x\end{array}$

$444(6.8)$

$480(5.7)$

$541(5.0)$

$554(5.6)$

$348(5.5)$

$543(2.4)$

$536(1.8)$

$583(2.2)$

$500(6.7)$

$505(3.3)$
$364(10.0)$

$558(6.6)$

$550(2.9)$

493 (3.0)

$541(3.4)$

375 (5.7)

508 (6.1)

556 (3.7)

$26(0.8) \quad 574(2.8)$

$11(0.5) \quad 544(3.3)$

$15(0.6)$

$533(3.8)$

$7(0.4)$

450 (14.3)

$551(2.9)$

$12(0.8)$

$12(0.8)$

$366(10.6)$

$529(5.6)$

$552(2.3)$

$28(1.4) \quad 471(6.1)$

- - - -

$--$

United States

International Avg.

$25(0.2) \quad 543(0.8)$

$21(0.1)$

$515(0.8)$

$31(0.2)$

$496(0.7)$

Background data provided by parents.

() Standard errors appear in parentheses. Because results are rounded to the nearest whole number, some totals may appear inconsistent.

An " $r$ " indicates data are available for $70-84 \%$ of the students. An " $s$ " indicates data are available for $50-69 \%$ of the students. An " $x$ " indicates data are available for less than $50 \%$ of the students.
A dash (-) indicates comparable data are not available. A tilde $(\sim)$ indicates insufficient data to report achievement.

NOTE: The International Average does not include the results from the Canadian provinces. 
Exhibit 3.5 Highest Level of Education of Either Parent (Continued)
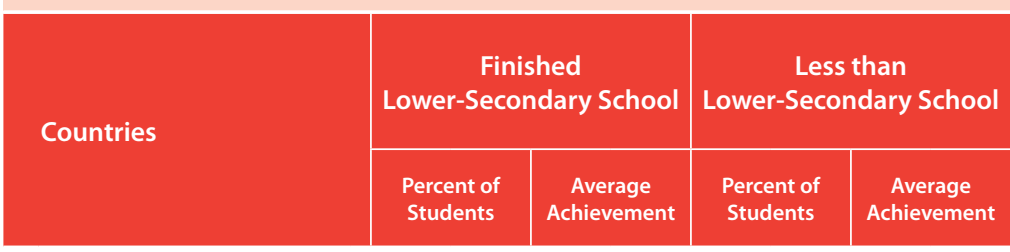

\begin{tabular}{|c|c|c|c|c|c|}
\hline Austria & & $4(0.4)$ & $488(5.9)$ & $0(0.1)$ & $\sim \sim$ \\
\hline Belgium (Flemish) & & $10(0.7)$ & $512(3.4)$ & $2(0.3)$ & $\sim \sim$ \\
\hline Belgium (French) & $r$ & $14(0.9)$ & $467(3.8)$ & $5(0.6)$ & $456(6.7)$ \\
\hline Bulgaria & & $14(1.4)$ & $517(10.8)$ & $7(1.1)$ & $513(14.4)$ \\
\hline Canada, Alberta & $r$ & $4(0.5)$ & $538(7.1)$ & $2(0.3)$ & $\sim \sim$ \\
\hline Canada, British Columbia & $r$ & $2(0.3)$ & $\sim \sim$ & $2(0.3)$ & $\sim \sim$ \\
\hline Canada, Nova Scotia & & $4(0.4)$ & $508(6.9)$ & $2(0.3)$ & $\sim \sim$ \\
\hline Canada, Ontario & & $2(0.4)$ & $\sim \sim$ & $2(0.5)$ & $\sim \sim$ \\
\hline Canada, Quebec & $r$ & $4(0.6)$ & $500(8.2)$ & $2(0.4)$ & $\sim \sim$ \\
\hline Chinese Taipei & & $11(0.7)$ & $504(3.8)$ & $2(0.3)$ & $\sim \sim$ \\
\hline Denmark & $r$ & $7(0.7)$ & $516(6.1)$ & $4(0.4)$ & $496(10.6)$ \\
\hline
\end{tabular}

\begin{tabular}{|c|c|c|c|c|c|}
\hline Denmark & 1 & $7(0.7)$ & $516(6.1)$ & $4(0.4)$ & 496 (10.6) \\
\hline England & & $\mathrm{xx}$ & $\mathrm{xx}$ & $\mathrm{xx}$ & $\mathrm{xx}$ \\
\hline France & & $9(0.7)$ & $490(4.5)$ & $4(0.4)$ & $481(5.4)$ \\
\hline Georgia & & $3(0.5)$ & $431(11.8)$ & $1(0.7)$ & $\sim \sim$ \\
\hline Germany & r & $33(1.3)$ & $530(2.3)$ & $2(0.3)$ & $\sim \sim$ \\
\hline Hong Kong SAR & & $22(1.1)$ & $560(3.4)$ & $12(0.8)$ & $544(5.5)$ \\
\hline Hungary & & $12(1.4)$ & $487(5.4)$ & $1(0.3)$ & $\sim \sim$ \\
\hline Iceland & r & $10(0.5)$ & $483(3.6)$ & $0(0.1)$ & $\sim \sim$ \\
\hline Indonesia & & $20(0.9)$ & $405(5.2)$ & $46(2.2)$ & $382(4.7)$ \\
\hline Iran, Islamic Rep. of & & $32(1.1)$ & $420(3.6)$ & $35(1.7)$ & $375(4.2)$ \\
\hline Israel & s & $7(1.0)$ & $431(10.5)$ & $4(0.5)$ & 457 (11.2) \\
\hline Italy & & $32(1.2)$ & $536(4.5)$ & $2(0.5)$ & $\sim \sim$ \\
\hline Kuwait & r & $15(0.9)$ & $305(7.1)$ & $5(0.4)$ & $289(13.0)$ \\
\hline Latvia & & $4(0.6)$ & $506(8.5)$ & $0(0.1)$ & $\sim \sim$ \\
\hline Lithuania & & $3(0.3)$ & $509(5.6)$ & $0(0.1)$ & $\sim \sim$ \\
\hline Luxembourg & r & $35(0.7)$ & $539(2.2)$ & $8(0.4)$ & $522(3.2)$ \\
\hline Macedonia, Rep. of & & $19(1.1)$ & $388(5.5)$ & $5(0.5)$ & $352(11.0)$ \\
\hline Moldova, Rep. of & & $33(1.1)$ & $487(3.9)$ & $11(1.0)$ & $478(8.0)$ \\
\hline Morocco & & $20(1.2)$ & $329(7.6)$ & $59(1.8)$ & $307(9.4)$ \\
\hline Netherlands & s & $15(1.0)$ & $537(3.3)$ & $1(0.2)$ & $\sim \sim$ \\
\hline New Zealand & $s$ & $8(0.6)$ & $497(5.9)$ & $2(0.2)$ & $\sim \sim$ \\
\hline Norway & & $4(0.4)$ & $456(8.2)$ & $0(0.1)$ & $\sim \sim$ \\
\hline Poland & & $35(1.2)$ & $490(3.3)$ & $4(0.4)$ & $467(7.1)$ \\
\hline Qatar & $s$ & $11(0.5)$ & $318(5.3)$ & $12(0.3)$ & $322(3.7)$ \\
\hline Romania & & $22(1.8)$ & $451(7.6)$ & $7(1.3)$ & $388(16.7)$ \\
\hline Russian Federation & & $4(0.5)$ & $502(8.7)$ & $0(0.1)$ & $\sim \sim$ \\
\hline Scotland & & $x x$ & $x x$ & $x x$ & $x x$ \\
\hline Singapore & & $12(0.5)$ & $524(3.9)$ & $7(0.4)$ & $497(5.3)$ \\
\hline Slovak Republic & & $5(0.7)$ & $440(10.6)$ & $1(0.2)$ & $\sim \sim$ \\
\hline Slovenia & & $5(0.4)$ & $467(5.5)$ & $1(0.1)$ & $\sim \sim$ \\
\hline South Africa & $s$ & $15(0.7)$ & $277(4.8)$ & $26(1.2)$ & $260(5.2)$ \\
\hline Spain & $s$ & $26(1.3)$ & $496(3.9)$ & $5(0.6)$ & $478(8.5)$ \\
\hline Sweden & $r$ & $7(0.7)$ & $513(5.7)$ & $0(0.1)$ & $\sim \sim$ \\
\hline Trinidad and Tobago & $r$ & $11(0.6)$ & $399(9.3)$ & $15(1.0)$ & $391(8.8)$ \\
\hline United States & & -- & -- & -- & -- \\
\hline International Avg. & & $15(0.1)$ & $465(1.1)$ & $8(0.1)$ & $423(2.0)$ \\
\hline
\end{tabular}


Because literacy resources are dependent to a large extent on economic considerations, Exhibits 3.6 and 3.7 present information on parents' employment situations and occupational level. As shown in Exhibit 3.6, about one third of students ( $36 \%)$, on average across countries, were from homes where both parents were working full time for pay, about half $(47 \%)$ from homes where one (but not both) was working full time, and just 7 percent from homes where both parents were working less than full time. Almost one tenth ( $9 \%)$ were from homes with other situations. Internationally on average, reading achievement was highest among students from homes where both parents were working full time for pay and lowest where both were working less than full time. However, for a number of participants, average achievement was similar between both or either parent working full time for pay, or even higher for only one parent working (Luxembourg, the Netherlands, Germany, and the Canadian province of Alberta).

As shown in Exhibit 3.7, parents' responses to a question about the kinds of work they did for their main job were grouped into seven categories: professional, small business owner, clerical, skilled worker, general laborer, never worked outside the home for pay, and not applicable. Students were assigned to the highest occupational category of either parent, taking professional as the highest category and never worked outside the home for pay as the lowest. On average across countries, 35 percent of students had at least one parent in a professional occupation, although the percentage varied widely from country to country. Highest percentages (50\% or more) were reported in Denmark, Iceland, Kuwait, New Zealand, Norway, Scotland, Sweden, and the Canadian provinces (Alberta, British Columbia, Nova Scotia, Ontario, and Quebec). Average student reading achievement was highest for students with a parent in a professional occupation (533 points) and lowest for students whose parents reported never working outside the home for pay (409 points). 
Exhibit 3.6 Parents' Employment Situations

PIRLS 2006

4th Grade

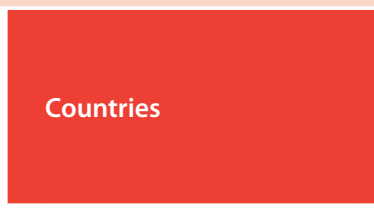

Austria

Belgium (Flemish)

Belgium (French)

Bulgaria

Canada, Alberta

Canada, British Columbia $r$

Canada, Nova Scotia

Canada, Ontario

Canada, Quebec

Chinese Taipei

Denmark

England

France

Georgia

Germany

Hong Kong SAR

Hungary

Iceland

Indonesia

Iran, Islamic Rep. of

$$
\text { Israel }
$$

$$
\text { Italy }
$$

$$
\text { Kuwait }
$$

Latvia

Lithuania

Luxembourg

Macedonia, Rep.

Moldova, Rep. of

Morocco

Netherlands

New Zealand

Norway

Poland

Qatar

Romania

Russian Federation

Scotland

Singapore

Slovak Republic

Slovenia

South Africa

Spain

\section{Sweden}

Trinidad and Tobago

United States

International Avg.

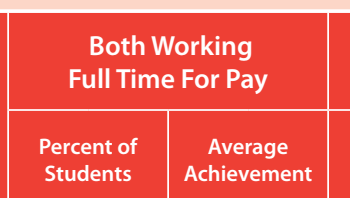

$23(0.8)$

$23(0.8) \quad 543(2.8)$

$36(1.2)$

$34(1.1)$

50 (1.6)

551 (2.1)

$518(2.7)$

$569(3.6)$

$40(1.3)$

$39(1.0)$

$48(0.9)$

$51(1.3)$

$54(1.4)$

$49(0.8)$

$60(1.2)$

$\mathrm{x} x$

$40(1.2)$

$560(2.8)$

$560(3.4)$

$550(2.6)$

$560(3.0)$

539 (3.4)

$546(2.2)$

552 (2.6)

$\mathrm{xx}$

$13(0.9)$

$12(0.6)$

$38(1.1)$

$52(1.3)$

$53(0.9)$

$19(1.5)$

$8(0.8)$

$44(1.6)$

$31(0.9)$

$27(1.1)$

$51(1.0)$

$49(1.1)$

$21(0.6)$

$24(1.1)$

$21(1.1)$

$537(2.1)$

$494(5.3)$

547 (3.6)

570 (2.4)

$567(3.0)$

$521(1.9)$

$413(6.0)$

$460(9.0)$

$562(3.4)$

$564(3.6)$

362 (4.8)

549 (2.6)

$548(2.0)$

$548(2.4)$

496 (5.6)

$514(4.4)$

$11(1.2)$

$5(0.4)$

$363(16.0)$

547 (5.1)

$549(2.9)$

$505(2.9)$

51 (1.2)

$48(1.3)$

$535(2.8)$

$335(2.8)$

$20(0.6)$

$38(1.8)$

$523(3.9)$

\begin{tabular}{ll|l}
$48(1.0)$ & $575(3.2)$
\end{tabular}

$\mathrm{xx}$

$41(0.7)$

$\mathrm{xx}$

$62(1.1)$

$571(3.0)$

$547(2.4)$

\begin{tabular}{l|l}
$75(0.8)$ & $530(2.2)$ \\
\hline
\end{tabular}

$\mathrm{xx}$

$530(2.2)$

532 (3.1)

$556(3.0)$

467 (5.3)

- -

$520(0.8)$

$36(0.2)$

$47(0.2)$

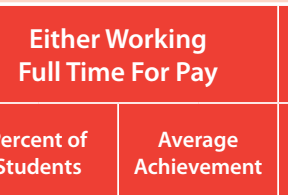

\begin{tabular}{|c|c|}
\hline \multicolumn{2}{|c|}{$\begin{array}{c}\text { Both Less than } \\
\text { Full Time For Pay }\end{array}$} \\
\hline $\begin{array}{c}\text { Percent of } \\
\text { Students }\end{array}$ & $\begin{array}{c}\text { Average } \\
\text { Achievement }\end{array}$ \\
\hline $2(0.3)$ & $\sim \sim$ \\
\hline
\end{tabular}

Other Situations

$71(1.0) \quad 543(22)$

$54(1.2)$

$553(2.2)$

$54(1.1) \quad 504(2.8)$

$30(1.1) \quad 541(5.1)$

55 (1.3)

$541(5.1)$

$54(0.9)$

$568(3.0)$

$46(0.9)$

$547(2.8)$

$44(1.3) \quad 561(3.6)$

$40(1.2)$

$537(3.2)$

$34(0.8) \quad 539(2.9)$

$33(1.1) \quad 549(2.9)$

$1(0.2)$

$\sim$

$5(0.4)$

$\sim \sim$

$15(1.4)$

$1(0.2)$

$472(6.9)$

$514(8.3)$

$2(0.3)$

$2(0.2)$

$1(0.2)$

$2(0.3)$

$\sim$

$\sim \sim$

$\sim \quad 5(0.5)$

$4(0.5)$

$x \mathrm{xx}$

$5(0.3)$

$4(0.5)$

$521(9.6)$

$51(1.0) \quad 525(2.4)$

$1(0.1)$

$37(1.5)$

$484(4.0)$

$77(1.0) \quad 559(2.6)$

$50(1.0) \quad 565(2.6)$

$38(1.1) \quad 555(3.3)$

\begin{tabular}{l}
$44(0.9) \quad 513(2.0)$ \\
\hline $50(1.8)$
\end{tabular}

50 (1.8)

$513(2.0)$

$53(1.9) \quad 436(4.1)$

$42(1.4) \quad 527(4.5)$

$56(1.0) \quad 553(3.1)$

$56(1.0)$

$553(3.1)$

$39(0.9) \quad 541(3.1)$

$35(0.8)$

$537(2.3)$

\begin{tabular}{l|l}
$70(0.7)$ & $568(1.6)$
\end{tabular}

$38(1.0) \quad 451(5.0)$

$30(1.2) \quad 507(4.8)$

$\mathrm{xx}$

$\mathrm{X} \quad \mathrm{x}$

$60(1.7) \quad 341(5.4)$

$82(1.0) \quad 557(1.7)$

$62(1.1) \quad 555(2.3)$

$3(0.3)$

$\mathrm{x} \mathrm{x}$

$36(1.7)$

$36(1.7)$

$498(8.5)$

$4(0.4) \quad 535(6.4)$

$4(0.4)$

$4(0.6)$

$550(5.6)$

$1(0.2)$

$20(1.8)$

493 (10.7)

$12(0.6)$

$\begin{array}{cc}6(0.5) & 524(6.3) \\ \mathrm{xx} & \mathrm{xx}\end{array}$

$9(1.0)$

$\sim \sim \sim$

$6(0.5) \quad 499(6.4)$

\begin{tabular}{l|l|l|l|}
\hline $9(1.0)$ & $415(13.1)$ & $29(1.5)$ & $406(5.3)$ \\
\hline
\end{tabular}

$\begin{array}{llll}5(0.6) & 466(9.7) & 9(0.7) & 482(9.4)\end{array}$

\begin{tabular}{|l|l|l|l|}
\hline $4(0.5)$ & $549(7.2)$ & $10(0.7)$ & $552(4.8)$ \\
\hline
\end{tabular}

\begin{tabular}{ll|l|l}
$4(0.5)$ & $549(7.2)$ & $10(0.7)$ & $552(4.8)$ \\
$7(0.6)$ & $352(12.4)$ & $17(0.8)$ & $323(7.0)$
\end{tabular}

$\begin{array}{llll}7(0.6) & 352(12.4) & 17(0.8) & 323(7.0)\end{array}$

\begin{tabular}{r|r|r|r|}
\hline $3(0.5)$ & $522(15.1)$ & $8(0.5)$ & $534(5.5)$ \\
\hline $4(0.3)$ & $515(5.4)$ & $13(0.7)$ & $523(4.2)$
\end{tabular}

\begin{tabular}{l|l|l|r|}
\hline $2(0.2)$ & $\sim \sim$ & $6(0.3)$ & $551(3.9)$
\end{tabular}

\begin{tabular}{r|r|r|r}
$29(1.2)$ & $445(5.3)$ & $9(0.7)$ & $444(8.0)$ \\
\hline $36(1.6)$ & $487(4.5)$ & $13(1.1)$ & $504(5.2)$
\end{tabular}

\begin{tabular}{|l|l|l|l|}
\hline $36(1.6)$ & $487(4.5)$ & $13(1.1)$ & $504(5.2)$ \\
\hline
\end{tabular}

$\begin{array}{llll}5(1.1) & 260(15.9) & 24(1.4) & 315(10.0)\end{array}$

$6(0.7)$

$6(0.7)$

$571(5.9)$

\begin{tabular}{|c|c|c|c|}
\hline $2(0.2)$ & $\sim \sim$ & $7(0.5)$ & $527(8.3)$ \\
\hline $1(0.2)$ & $\sim \sim$ & $4(0.5)$ & $465(8.8)$ \\
\hline $7(0.5)$ & $501(5.4)$ & $4(0.4)$ & $490(7.8)$ \\
\hline
\end{tabular}

$42(1.1) \quad 515(3.0)$

\begin{tabular}{l|c|c|c}
\hline $2(0.2)$ & $\sim \sim$ & $7(0.5)$ & $527(8.3)$ \\
\hline $1(0.2)$ & $\sim \sim$ & $4(0.5)$ & $465(8.8)$ \\
\hline $7(0.5)$ & $501(5.4)$ & $4(0.4)$ & $490(7.8)$
\end{tabular}

$8(0.6) \quad 545(5.4)$

\begin{tabular}{|r|r|r|l|r|r|}
\hline $49(0.8)$ & $365(2.6)$ & $14(0.5)$ & $357(4.0)$ & $16(0.6)$ & $337(3.7)$ \\
\hline $33(1.3)$ & $496(5.2)$ & $9(1.2)$ & $445(12.7)$ & $20(1.7)$ & $460(8.1)$
\end{tabular}

\begin{tabular}{|r|r|r|r|r|r|}
\hline $44(1.4)$ & $499(3.9)$ & $1(0.2)$ & $\sim \sim$ & $4(0.5)$ & $465(8.8)$ \\
\hline $42(1.1)$ & $515(3.0)$ & $7(0.5)$ & $501(5.4)$ & $4(0.4)$ & $490(7.8)$ \\
\hline $49(0.8)$ & $365(2.6)$ & $14(0.5)$ & $357(4.0)$ & $16(0.6)$ & $337(3.7)$ \\
\hline $33(1.3)$ & $496(5.2)$ & $9(1.2)$ & $445(12.7)$ & $20(1.7)$ & $460(8.1)$ \\
\hline
\end{tabular}

\begin{tabular}{|c|c|c|c|c|c|}
\hline $33(1.3)$ & $496(5.2)$ & $9(1.2)$ & $445(12.7)$ & $20(1.7)$ & $460(8.1)$ \\
\hline $40(0.9)$ & $565(4.0)$ & $5(0.6)$ & $533(12.2)$ & $6(0.5)$ & $543(6.2)$ \\
\hline$x x$ & $x x$ & $x x$ & $x x$ & $x x$ & $x x$ \\
\hline
\end{tabular}

$\begin{array}{ccc}\mathrm{xx} & \mathrm{xx} & \mathrm{xx}\end{array}$

\begin{tabular}{|c|c|c|c|c|c|}
\hline $\mathrm{x} \mathrm{x}$ & $\mathrm{x} \mathrm{x}$ & $\mathrm{x} \mathrm{x}$ & $\mathrm{xx}$ & $\mathrm{x} \mathrm{x}$ & $\mathrm{x} \mathrm{x}$ \\
\hline $51(0.8)$ & $559(3.2)$ & $2(0.2)$ & $\sim \sim$ & $6(0.3)$ & $545(5.2)$ \\
\hline $29(1.0)$ & $535(2.6)$ & $5(0.6)$ & $446(8.7)$ & $3(0.3)$ & $514(9.1)$
\end{tabular}

\begin{tabular}{|c|c|c|c|c|c|}
\hline $51(0.8)$ & $559(3.2)$ & $2(0.2)$ & $\sim \sim$ & $6(0.3)$ & $545(5.2)$ \\
\hline $29(1.0)$ & $535(2.6)$ & $5(0.6)$ & $446(8.7)$ & $3(0.3)$ & $514(9.1)$ \\
\hline $23(0.7)$ & $508(3.3)$ & $2(0.2)$ & $\sim \sim$ & $1(0.2)$ & $\sim \sim$ \\
\hline
\end{tabular}

\begin{tabular}{|c|c|c|c|c|c|}
\hline $23(0.7)$ & $508(3.3)$ & $2(0.2)$ & $\sim \sim$ & $1(0.2)$ & $\sim \sim$ \\
\hline $\mathrm{x} \mathrm{x}$ & $\mathrm{x} \mathrm{x}$ & $\mathrm{x} \mathrm{x}$ & $\mathrm{x} \mathrm{x}$ & $\mathrm{x} \mathrm{x}$ & $\mathrm{x} \mathrm{x}$ \\
\hline $50(1.4)$ & $523(2.9)$ & $3(0.5)$ & $488(9.0)$ & $10(0.7)$ & $520(5.4)$ \\
\hline $51(0.9)$ & $551(2.3)$ & $3(0.4)$ & $537(7.1)$ & $2(0.3)$ & $\sim \sim$ \\
\hline
\end{tabular}

\begin{tabular}{|l|l|l|l|r|r|}
\hline $50(1.4)$ & $523(2.9)$ & $3(0.5)$ & $488(9.0)$ & $10(0.7)$ & $520(5.4)$ \\
\hline $51(0.9)$ & $551(2.3)$ & $3(0.4)$ & $537(7.1)$ & $2(0.3)$ & $\sim$ \\
\hline
\end{tabular}

\begin{tabular}{c|c|c|c|c|c|}
\hline $51(0.9)$ & $551(2.3)$ & $3(0.4)$ & $537(7.1)$ & $2(0.3)$ & $\sim \sim$ \\
$49(1.2)$ & $432(5.8)$ & $5(0.6)$ & $394(10.7)$ & $6(0.5)$ & $445(9.3)$ \\
\hline-- & -- & -- & -- & -- & -- \\
\hline
\end{tabular}

$\begin{array}{cccc}5(0.6) & 394(10.7) & 6(0.5) & 445(9.3) \\ - & -- & -- & --\end{array}$

$47(0.2) \quad 508(0.6)$

$$
\begin{array}{l|l}
7(0.1) & 472(2.1)
\end{array}
$$

$9(0.1)$

$488(1.5)$

Background data provided by parents.

() Standard errors appear in parentheses. Because results are rounded to the nearest whole number, some totals may appear inconsistent.

An " $r$ " indicates data are available for $70-84 \%$ of the students. An " $s$ " indicates data are available for $50-69 \%$ of the students. An " $\mathrm{x}$ " indicates data are available for less than $50 \%$ of the students. 
Exhibit 3.7 Parents' Occupational Level

PIRLS 2006

4th Grade

\begin{tabular}{|c|c|c|c|c|c|c|c|c|c|}
\hline \multirow{2}{*}{ Countries } & \multicolumn{3}{|c|}{ Professional } & \multicolumn{2}{|c|}{ Small Business Owner } & \multicolumn{2}{|c|}{ Clerical } & \multicolumn{2}{|c|}{ Skilled Worker } \\
\hline & & $\begin{array}{l}\text { Percent of } \\
\text { Students }\end{array}$ & $\begin{array}{c}\text { Average } \\
\text { Achievement }\end{array}$ & $\begin{array}{l}\text { Percent of } \\
\text { Students }\end{array}$ & $\begin{array}{c}\text { Average } \\
\text { Achievement }\end{array}$ & $\begin{array}{l}\text { Percent of } \\
\text { Students }\end{array}$ & $\begin{array}{c}\text { Average } \\
\text { Achievement }\end{array}$ & $\begin{array}{l}\text { Percent of } \\
\text { Students }\end{array}$ & $\begin{array}{c}\text { Average } \\
\text { Achievement }\end{array}$ \\
\hline Austria & & $19(1.1)$ & $570(2.9)$ & $14(0.6)$ & $545(3.3)$ & $45(1.0)$ & $542(1.9)$ & $15(1.1)$ & $521(4.0)$ \\
\hline Belgium (Flemish) & & $43(1.2)$ & $567(2.0)$ & $14(0.6)$ & $548(2.8)$ & $24(0.8)$ & $543(2.7)$ & $12(0.7)$ & $523(3.2)$ \\
\hline Belgium (French) & r & $37(1.5)$ & $533(2.5)$ & $9(0.5)$ & $500(4.5)$ & $28(1.1)$ & $499(3.0)$ & $13(0.8)$ & $473(3.5)$ \\
\hline Bulgaria & & $28(1.6)$ & $585(5.8)$ & $10(0.7)$ & $561(5.1)$ & $19(1.1)$ & $555(4.4)$ & $17(1.1)$ & $529(6.4)$ \\
\hline Canada, Alberta & $r$ & $56(1.6)$ & $576(2.9)$ & $15(0.7)$ & $562(4.5)$ & $16(0.9)$ & $554(3.3)$ & $7(0.7)$ & $543(5.8)$ \\
\hline Canada, British Columbia & $r$ & $51(1.6)$ & $578(3.2)$ & $16(0.7)$ & $557(3.7)$ & $19(0.8)$ & $547(4.0)$ & $8(0.7)$ & $548(6.6)$ \\
\hline Canada, Nova Scotia & & $52(1.2)$ & $563(2.7)$ & $9(0.6)$ & $536(5.1)$ & $23(0.9)$ & $532(2.7)$ & $9(0.6)$ & $525(4.9)$ \\
\hline Canada, Ontario & & $53(2.0)$ & $573(3.2)$ & $12(1.0)$ & $543(4.3)$ & $18(1.0)$ & $547(3.5)$ & $10(1.0)$ & $540(5.3)$ \\
\hline Canada, Quebec & & $55(1.9)$ & $551(3.4)$ & $10(0.6)$ & $520(4.2)$ & $19(1.1)$ & $520(3.4)$ & $9(0.9)$ & $525(6.2)$ \\
\hline Chinese Taipei & & $34(1.3)$ & $560(2.5)$ & $17(0.6)$ & $532(2.5)$ & $19(0.7)$ & $537(2.6)$ & $18(1.0)$ & $511(2.8)$ \\
\hline Denmark & & $53(1.6)$ & $565(2.5)$ & $10(0.7)$ & $535(4.7)$ & $23(0.9)$ & $538(3.5)$ & $9(0.7)$ & $517(5.9)$ \\
\hline England & & $x x$ & $x x$ & $x x$ & $x x$ & $x x$ & $x x$ & $x x$ & $x x$ \\
\hline France & & $39(1.6)$ & $552(2.2)$ & $11(0.7)$ & $525(4.4)$ & $23(0.9)$ & $521(2.7)$ & $16(1.0)$ & $496(3.2)$ \\
\hline Georgia & & $29(1.3)$ & $502(3.4)$ & $10(0.8)$ & $477(5.4)$ & $14(0.8)$ & $458(5.1)$ & $17(1.2)$ & $460(5.5)$ \\
\hline Germany & $r$ & $28(1.1)$ & $582(2.3)$ & $12(0.7)$ & $554(3.1)$ & $40(1.2)$ & $552(2.4)$ & $12(0.9)$ & $524(4.4)$ \\
\hline Hong Kong SAR & & $31(1.6)$ & $573(2.2)$ & $12(0.5)$ & $561(3.9)$ & $28(0.9)$ & $565(2.9)$ & $16(0.9)$ & $561(4.1)$ \\
\hline Hungary & & $26(1.5)$ & $594(2.7)$ & $12(0.7)$ & $563(4.6)$ & $28(1.0)$ & $551(2.6)$ & $18(1.0)$ & $534(4.1)$ \\
\hline Iceland & $r$ & $52(0.9)$ & $531(2.0)$ & $12(0.6)$ & $505(3.7)$ & $21(0.7)$ & $503(3.2)$ & $9(0.7)$ & $496(3.8)$ \\
\hline Indonesia & r & $5(0.6)$ & $462(8.6)$ & $9(0.8)$ & $422(7.1)$ & $13(1.0)$ & $447(5.1)$ & $37(2.0)$ & $393(5.9)$ \\
\hline Iran, Islamic Rep. of & & $11(1.1)$ & $499(4.6)$ & $16(0.7)$ & $428(4.4)$ & $15(0.9)$ & $448(4.4)$ & $21(1.2)$ & $412(4.3)$ \\
\hline Israel & s & $49(1.7)$ & $568(3.8)$ & $8(0.6)$ & $535(6.9)$ & $15(1.1)$ & $526(6.3)$ & $13(0.8)$ & $486(6.9)$ \\
\hline Italy & & $25(1.2)$ & $575(3.2)$ & $14(0.8)$ & $556(3.9)$ & $26(0.9)$ & $558(3.9)$ & $28(1.1)$ & $538(3.6)$ \\
\hline Kuwait & $s$ & $53(1.4)$ & $360(4.8)$ & $3(0.3)$ & $342(17.5)$ & $15(0.8)$ & $320(7.8)$ & $1(0.3)$ & $\sim \sim$ \\
\hline Latvia & & $35(1.2)$ & $562(2.6)$ & $8(0.5)$ & $561(6.0)$ & $26(0.9)$ & $539(2.4)$ & $16(0.9)$ & $523(4.2)$ \\
\hline Lithuania & & $29(1.1)$ & $563(2.3)$ & $7(0.5)$ & $542(4.3)$ & $31(1.0)$ & $536(2.4)$ & $17(0.8)$ & $521(2.9)$ \\
\hline Luxembourg & & $29(0.7)$ & $592(2.1)$ & $8(0.4)$ & $557(3.6)$ & $35(0.7)$ & $563(1.8)$ & $17(0.5)$ & $528(2.4)$ \\
\hline Macedonia, Rep. of & r & $18(1.0)$ & $505(5.8)$ & $14(0.6)$ & $465(5.9)$ & $22(0.9)$ & $480(5.0)$ & $21(0.8)$ & $432(5.5)$ \\
\hline Moldova, Rep. of & & $22(1.0)$ & $518(5.4)$ & $7(0.7)$ & $509(8.3)$ & $19(0.9)$ & $508(3.5)$ & $22(1.1)$ & $489(3.3)$ \\
\hline Morocco & & $21(1.8)$ & $354(8.6)$ & $16(1.1)$ & $331(7.9)$ & $11(0.8)$ & $358(7.6)$ & $34(2.0)$ & $299(10.7)$ \\
\hline Netherlands & s & $49(1.7)$ & $569(1.7)$ & $14(0.8)$ & $549(4.4)$ & $25(1.0)$ & $547(2.6)$ & $8(0.7)$ & $528(4.6)$ \\
\hline New Zealand & s & $50(1.3)$ & $569(2.6)$ & $14(0.7)$ & $553(5.0)$ & $19(0.8)$ & $536(3.2)$ & $11(0.8)$ & $523(7.1)$ \\
\hline Norway & & $57(1.3)$ & $515(2.3)$ & $10(0.6)$ & $478(5.3)$ & $18(1.0)$ & $491(5.0)$ & $10(0.7)$ & $470(6.4)$ \\
\hline Poland & & $34(1.4)$ & $545(3.8)$ & $11(0.6)$ & $521(4.1)$ & $13(0.8)$ & $519(4.1)$ & $34(1.6)$ & $505(2.8)$ \\
\hline Qatar & s & $43(0.7)$ & $381(2.5)$ & $4(0.3)$ & $347(9.3)$ & $13(0.5)$ & $362(4.0)$ & $4(0.3)$ & $339(7.8)$ \\
\hline Romania & & $12(1.2)$ & $556(4.4)$ & $7(0.6)$ & $530(6.8)$ & $18(0.9)$ & $516(4.4)$ & $34(1.6)$ & $492(5.5)$ \\
\hline Russian Federation & & $38(1.2)$ & $583(3.4)$ & $5(0.4)$ & $573(5.5)$ & $33(0.9)$ & $565(3.4)$ & $14(0.8)$ & $543(5.1)$ \\
\hline Scotland & s & $56(1.9)$ & $565(3.5)$ & $7(0.7)$ & $530(6.6)$ & $22(1.3)$ & $525(4.6)$ & $8(1.1)$ & $502(9.1)$ \\
\hline Singapore & & $44(0.8)$ & $588(2.8)$ & $13(0.5)$ & $554(4.0)$ & $22(0.6)$ & $545(3.3)$ & $11(0.5)$ & $523(4.8)$ \\
\hline Slovak Republic & & $30(1.1)$ & $566(2.4)$ & $12(0.7)$ & $542(3.3)$ & $26(0.9)$ & $534(3.1)$ & $20(0.9)$ & $519(3.4)$ \\
\hline Slovenia & & $38(1.1)$ & $553(2.3)$ & $8(0.5)$ & $522(4.1)$ & $31(0.8)$ & $518(2.5)$ & $16(0.9)$ & $490(3.2)$ \\
\hline South Africa & s & $28(1.6)$ & $399(12.1)$ & $11(0.5)$ & $353(12.0)$ & $17(0.6)$ & $330(7.2)$ & $14(0.6)$ & $289(6.7)$ \\
\hline Spain & $s$ & $37(1.8)$ & $546(2.9)$ & $13(0.8)$ & $523(4.6)$ & $20(1.0)$ & $520(3.9)$ & $18(1.2)$ & $507(4.1)$ \\
\hline Sweden & & $57(1.6)$ & $566(2.4)$ & $11(0.7)$ & $537(4.7)$ & $23(1.2)$ & $537(2.5)$ & $7(0.6)$ & $524(5.0)$ \\
\hline Trinidad and Tobago & $r$ & $27(1.4)$ & $486(5.9)$ & $12(0.6)$ & $448(8.5)$ & $25(1.1)$ & $448(5.6)$ & $20(1.0)$ & $419(7.1)$ \\
\hline United States & & -- & -- & -- & -- & -- & -- & -- & -- \\
\hline International Avg. & & $35(0.2)$ & $533(0.7)$ & $11(0.1)$ & $506(1.0)$ & $23(0.1)$ & $504(0.8)$ & $17(0.2)$ & $485(0.9)$ \\
\hline
\end{tabular}

Based on parents' response to the following question: What kind of work do the child's father (or stepfather or male guardian) and mother (or stepmother or female guardian) do for their main jobs? Has never worked outside the home for pay (a); Small business owner (b); Clerk (c); Service or sales worker (d); Skilled agricultural or fishery worker (e); Craft or trade worker (f); Plant or machine operator (g); General laborer (h); Corporate manager or senior official (i); Professional (j); Technician or associate professional (k); Not applicable (I). Some categories were combined so that Professional includes option i through $\mathrm{k}$, Clerical includes options $\mathrm{c}$ and $\mathrm{d}$, and Skilled Worker includes options $\mathrm{e}$ through g. Students were categorized according to the highest occupational category of either parent, taking Professional as the highest category and Never Worked Outside the Home for Pay as the lowest.
() Standard errors appear in parentheses. Because results are rounded to the nearest whole number, some totals may appear inconsistent.

An " $r$ " indicates data are available for $70-84 \%$ of the students. An " $s$ " indicates data are available for $50-69 \%$ of the students. An " $x$ " indicates data are available for less than $50 \%$ of the students.

A dash (-) indicates comparable data are not available. A tilde ( ) indicates insufficient data to report achievement.

NOTE: The International Average does not include the results from the Canadian provinces. 
Exhibit 3.7 Parents' Occupational Level (Continued)

\begin{tabular}{|c|c|c|c|c|c|c|c|}
\hline Austria & & $4(0.3)$ & $504(5.5)$ & $1(0.1)$ & $\sim \sim$ & $3(0.3)$ & $516(8.5)$ \\
\hline Belgium (Flemish) & & $4(0.4)$ & $513(5.7)$ & $1(0.1)$ & $\sim \sim$ & $2(0.3)$ & $\sim \sim$ \\
\hline Belgium (French) & r & $4(0.4)$ & $462(8.1)$ & $3(0.4)$ & $455(7.4)$ & $6(0.5)$ & $472(6.1)$ \\
\hline Bulgaria & & $15(1.7)$ & $519(10.0)$ & $3(0.6)$ & $533(14.2)$ & $8(0.7)$ & $520(9.4)$ \\
\hline Canada, Alberta & $\mathrm{r}$ & $2(0.4)$ & $\sim \sim$ & $1(0.2)$ & $\sim \sim$ & $2(0.3)$ & $\sim \sim$ \\
\hline Canada, British Columbia & $r$ & $3(0.4)$ & $540(8.5)$ & $1(0.1)$ & $\sim \sim$ & $2(0.3)$ & $\sim \sim$ \\
\hline Canada, Nova Scotia & & $3(0.3)$ & $506(7.8)$ & $0(0.1)$ & $\sim \sim$ & $2(0.3)$ & $\sim \sim$ \\
\hline Canada, Ontario & & $3(0.4)$ & $529(7.5)$ & $0(0.1)$ & $\sim \sim$ & $3(0.5)$ & $512(12.0)$ \\
\hline Canada, Quebec & & $2(0.4)$ & $\sim \sim$ & $1(0.2)$ & $\sim \sim$ & $4(0.5)$ & $507(6.8)$ \\
\hline Chinese Taipei & & $4(0.3)$ & $514(5.5)$ & $1(0.2)$ & $\sim \sim$ & $6(0.4)$ & $529(5.0)$ \\
\hline Denmark & & $2(0.3)$ & $\sim \sim$ & $0(0.1)$ & $\sim \sim$ & $2(0.3)$ & $\sim \sim$ \\
\hline England & & $x x$ & $x x$ & $x x$ & $x x$ & $x x$ & $x x$ \\
\hline
\end{tabular}

\begin{tabular}{|c|c|c|c|c|c|c|c|}
\hline France & & $4(0.4)$ & $476(4.7)$ & $1(0.3)$ & $\sim \sim$ & $6(0.6)$ & $498(6.7)$ \\
\hline Georgia & & $4(0.5)$ & $457(12.9)$ & $17(1.2)$ & $448(5.1)$ & $10(0.9)$ & $471(7.4)$ \\
\hline Germany & $r$ & $2(0.3)$ & $\sim \sim$ & $1(0.1)$ & $\sim \sim$ & $5(0.4)$ & $519(5.7)$ \\
\hline Hong Kong SAR & & $6(0.5)$ & $554(5.2)$ & $3(0.3)$ & $556(5.7)$ & $4(0.3)$ & $561(6.1)$ \\
\hline Hungary & & $8(0.9)$ & $502(6.1)$ & $1(0.3)$ & $\sim \sim$ & $6(0.6)$ & $534(7.1)$ \\
\hline Iceland & $r$ & $4(0.4)$ & $479(7.9)$ & $0(0.0)$ & $\sim \sim$ & $2(0.3)$ & $\sim \sim$ \\
\hline Indonesia & $r$ & $26(1.9)$ & $394(5.0)$ & $7(0.6)$ & $398(6.8)$ & $3(0.5)$ & $415(10.5)$ \\
\hline Iran, Islamic Rep. of & & $22(1.6)$ & $391(6.2)$ & $10(0.6)$ & $391(7.3)$ & $5(0.5)$ & $422(8.6)$ \\
\hline Israel & $s$ & $3(0.4)$ & $478(13.0)$ & $8(0.7)$ & $438(9.8)$ & $4(0.6)$ & $496(13.1)$ \\
\hline Italy & & $3(0.4)$ & $526(11.0)$ & $2(0.4)$ & $\sim \sim$ & $3(0.4)$ & $544(10.7)$ \\
\hline Kuwait & $s$ & $1(0.2)$ & $\sim \sim$ & $14(0.9)$ & $309(7.6)$ & $12(0.9)$ & $318(9.3)$ \\
\hline Latvia & & $8(0.7)$ & $516(6.7)$ & $0(0.2)$ & $\sim \sim$ & $7(0.6)$ & $527(5.6)$ \\
\hline Lithuania & & $8(0.5)$ & $507(4.8)$ & $1(0.2)$ & $\sim \sim$ & $6(0.5)$ & $523(4.1)$ \\
\hline Luxembourg & & $4(0.3)$ & $519(4.9)$ & $2(0.2)$ & $\sim \sim$ & $5(0.3)$ & $550(5.0)$ \\
\hline Macedonia, Rep. of & $r$ & $11(0.8)$ & $415(6.7)$ & $11(0.9)$ & $394(6.2)$ & $3(0.4)$ & 449 (10.5) \\
\hline Moldova, Rep. of & & $13(1.5)$ & $486(7.0)$ & $4(0.5)$ & $467(8.5)$ & $12(0.9)$ & $500(5.7)$ \\
\hline Morocco & & $5(0.6)$ & $296(13.5)$ & $6(0.6)$ & $311(14.1)$ & $7(0.7)$ & $316(11.1)$ \\
\hline Netherlands & $s$ & $1(0.3)$ & $\sim \sim$ & $0(0.2)$ & $\sim \sim$ & $2(0.4)$ & $\sim \sim$ \\
\hline New Zealand & $s$ & $3(0.3)$ & $500(8.9)$ & $1(0.2)$ & $\sim \sim$ & $3(0.4)$ & 499 (11.6) \\
\hline Norway & & $2(0.3)$ & $\sim \sim$ & $1(0.2)$ & $\sim \sim$ & $2(0.2)$ & $\sim \sim$ \\
\hline Poland & & $3(0.4)$ & $490(8.0)$ & $2(0.3)$ & $\sim \sim$ & $3(0.4)$ & $506(10.6)$ \\
\hline Qatar & $s$ & $1(0.1)$ & $\sim \sim$ & $16(0.6)$ & $341(3.6)$ & $18(0.6)$ & $338(3.5)$ \\
\hline Romania & & $10(1.7)$ & $456(9.0)$ & $10(1.0)$ & $422(12.5)$ & $9(1.2)$ & $435(9.1)$ \\
\hline Russian Federation & & $5(0.6)$ & $530(10.8)$ & $1(0.2)$ & $\sim \sim$ & $5(0.7)$ & $532(10.7)$ \\
\hline Scotland & s & $4(0.8)$ & $496(8.5)$ & $1(0.3)$ & $\sim \sim$ & $2(0.3)$ & $\sim \sim$ \\
\hline Singapore & & $2(0.2)$ & $\sim \sim$ & $2(0.2)$ & $\sim \sim$ & $5(0.3)$ & $535(5.2)$ \\
\hline Slovak Republic & & $4(0.6)$ & $462(12.3)$ & $2(0.3)$ & $\sim \sim$ & $7(0.8)$ & $489(16.9)$ \\
\hline Slovenia & & $3(0.3)$ & $481(7.5)$ & $1(0.1)$ & $\sim \sim$ & $4(0.3)$ & $498(6.0)$ \\
\hline South Africa & s & $10(0.8)$ & $282(8.8)$ & $12(0.7)$ & $261(6.4)$ & $8(0.4)$ & $294(7.1)$ \\
\hline Spain & $s$ & $8(0.8)$ & $488(5.4)$ & $2(0.3)$ & $\sim \sim$ & $3(0.5)$ & $493(11.7)$ \\
\hline Sweden & & $1(0.2)$ & $\sim \sim$ & $1(0.2)$ & $\sim \sim$ & $1(0.3)$ & $\sim \sim$ \\
\hline Trinidad and Tobago & $r$ & $11(0.8)$ & $387(10.2)$ & $3(0.4)$ & $408(11.6)$ & $3(0.4)$ & $425(15.5)$ \\
\hline United States & & -- & -- & -- & -- & -- & -- \\
\hline International Avg. & & $6(0.1)$ & $469(1.6)$ & $4(0.1)$ & $409(2.3)$ & $5(0.1)$ & $475(1.7)$ \\
\hline
\end{tabular}




\section{What Are Parents' Reading Habits and Attitudes?}

In addition to engaging in early literacy activities with their children and providing extensive literacy materials in the home, PIRLS 2001 found that students who had high reading achievement at the fourth grade often had parents who read a lot themselves and had favorable attitudes to reading.

To measure trends in the amount of time parents reported reading at home, PIRLS 2006 asked them how much time they spent reading for themselves at home, including books, magazines, newspapers, and materials for work. Exhibit 3.8 summarizes parents' responses, together with average student reading achievement and changes since 2001.

On average across countries, 37 percent of students had parents who reported reading for more than 5 hours each week, 43 percent of students had parents reading $1-5$ hours each week, and 20 percent less than 1 hour per week. Countries where the majority of students had parents reporting reading more than 5 hours each week included Norway, Scotland, Iceland, Germany, and Sweden. Lowest levels of parental reading were reported in Bulgaria, Kuwait, Romania, Qatar, Morocco, Indonesia, and Iran, where more than 30 percent of students had parents reading for less than 1 hour per week.

Based on parents' reports, there appears to have been a decrease since 2001 in the amount of time parents spend reading. Of the 24 countries with data from 2001, 13 had a decrease in the percentage of students with a parent reporting reading for more than 5 hours a week, and almost all of these countries had a corresponding increase in the percentage of students with parents in either the 1-5 hours a week category or the less-than-1-hour-aweek category or both. Average reading achievement was highest among students with parents reporting reading for more than 5 hours a week (516 points), next highest among students with parents reading for $1-5$ hours (502 points), and lowest for students with parents reading for less than 1 hour a week (477 points).

Regardless of the total amount of weekly reading by parents, almost half the students $(47 \%)$, on average, had parents reporting reading for 
enjoyment every day or almost every day, as shown in Exhibit 3.9. About one third (34\%) had parents reading once or twice a week, and 18 percent reading twice a month or less. Most frequent reading for enjoyment was reported in Norway, Sweden, Scotland, Iceland, and New Zealand, where 60 percent or more of students had parents reading for enjoyment every day or almost every day. Compared with 2001, eight countries-Norway, New Zealand, Germany, Singapore, Macedonia, Slovenia, Hong Kong sar, and Romania-showed an increase in the percentage of students in the most frequent parental reading category, while Sweden, the Russian Federation, and Bulgaria had decreased percentages.

Average reading achievement was highest among students whose parents frequently read for enjoyment- 512 points for students with parents who read every day or almost every day, 498 for students with parents reading once or twice a week, and 487 for students with parents reading twice a month or less.

To monitor trends in parents' attitudes to reading, PIRLS 2006 employed an Index of Parents' Attitudes Toward Reading based on parents' agreement with five statements about reading:

- I read only if I have to (reverse coded).

- I like talking about books with other people.

- I like to spend my spare time reading.

- I read only if I need information (reverse coded).

- Reading is an important activity in my home.

Parents were asked if they agree a lot, agree a little, disagree a little, or disagree a lot with each of the statements. To construct the index, parents' responses were assigned a numerical code that was averaged across the five statements, and then students were assigned to one of three categories, high, medium, or low, on the basis of their parents' average response. Students in the high category had parents that reported agreeing a little or a lot with the five statements, on average, whereas those in the low category, on average, 
disagreed a lot. Students in the medium category had parents reporting in between these extremes.

Exhibit 3.10 presents the percentage of students in 2006 at each level of the index for each country, together with average student achievement for those students. International averages are shown at the foot of the column for each level. Also shown is the change from 2001 in the percentage of students at each level of the index for countries that participated in PIRLS 2001, together with an indication of the statistical significance of the change. Participants are ordered by the percentage of students in 2006 at the high level of the index.

Parents generally reported very favorable attitudes toward reading, with more than half the students (52\%), on average, at the high level of the index and 41 percent at the medium level. Just 7 percent were at the low level. Countries with the greatest percentages of students with parents having favorable attitudes toward reading included the Scandinavian countries (Sweden, Norway, and Denmark) and Scotland, where 70 percent or more of students were at the high level of the index. Countries where fewer parents expressed favorable attitudes included Hong Kong SAR (one of the countries with the highest average student reading achievement) and Indonesia, each of which had less than 30 percent of students at the high level. In comparison with 2001, seven participants showed an increase in the percentage of students with parents holding favorable attitudes toward reading - the Netherlands, Germany, Latvia, Macedonia, Lithuania, Moldova, and the Canadian province of Quebec. On average internationally, students at the high level of the index had higher average reading achievement (518 points) than students at the medium (488 points) or low level (475 points). 
Exhibit 3.8 Parents Reading* at Home with Trends

PIRLS 2006

Countries

Norway

Scotland

Iceland

Germany

Sweden

New Zealand

Canada, British Columbia

Canada, Alberta

Canada, Nova Scotia

Canada, Ontario

Macedonia, Rep. of

Netherlands

Trinidad and Tobago

Hong Kong SAR

Georgia

Singapore

Austria

Denmark

Luxembourg

Spain Hungary

Canada, Quebec

Lithuania

Latvia

South Africa

Israel

Slovak Republic

Belgium (French)

Chinese Taipei

Belgium (Flemish)

Slovenia

Russian Federation

Italy

Poland

Bulgaria

Moldova, Rep. of

France

Kuwait

Romania

Qatar

Morocco

Indonesia

Iran, Islamic Rep. of

England

United States

International Avg.
More than 5 Hours a Week

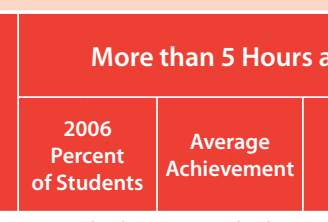

Difference
in Percent
from 2001

$61(1.0)$

s $54(1.7)$

$53(0.9)$

51 (1.1)

$51(1.3)$

49 (1.1)

49 (1.2)

$47(1.1)$

$45(0.8)$

$43(1.1)$

$43(1.1)$

S $43(1.3)$

$42(1.0)$

$42(0.9)$

42 (1.1)

$41(0.7)$

$40(1.0)$

40 (1.1)

$40(0.7)$

$\mathrm{s} \quad 40(1.2)$

$39(1.3)$

$507(2.5)$

2 (1.5)

$526(2.1)$

$566(1.9)$

$560(2.7)$

$557(2.5)$

570 (3.3)

$571(3.0)$

$555(2.5)$

567 (3.6)

$461(4.7)$

$565(2.2)$

455 (5.1)

$569(2.3)$

486 (3.4)

576 (3.1)

$555(2.7)$

$556(2.5)$

581 (1.5)

$536(3.3)$

$576(2.7)$

r 38 (1.4)

$38(1.0)$

37 (1.1)

$543(3.7)$

$548(2.1)$

$36(0.7)$

$550(3.0)$

$314(7.8)$

$35(1.2) \quad 549(5.0)$

$35(1.0)$

$551(2.4)$

$34(0.9) \quad 552(2.7)$

$34(0.8) \quad 559(2.4)$

$33(1.1) \quad 540(2.5)$

$32(0.9) \quad 574(4.1)$

$31(1.0) \quad 569(3.3)$

$30(0.9) \quad 539(3.2)$

$30(1.5) \quad 572(4.4)$

$28(1.0)$

$26(0.9)$

$24(1.4)$

$21(0.6)$

$21(1.2)$

$20(1.2)$

$17(0.9)$

$545(2.6)$

$348(6.1)$

$521(5.5)$

376 (3.2)

338 (7.1)

$415(6.1)$

$445(4.6)$

$\mathrm{xx} \quad \mathrm{x} \mathrm{x}$

$--$

$37(0.2)$

$0(1.5)$

00

$2(1.4)$

$\mathrm{xx}$

$\mathrm{xX}$
--
$35(1.2) \quad 519(3.1)$

$30(1.1) \quad 512(3.6)$

$-7(2.1)$

(- $39(1.4)$

$41(0.9)$

$-8(1.7) \quad \nabla \quad 41(1.2)$

$-3(1.6) \quad 40(1.0)$

$\diamond \diamond \quad 41(0.9)$

$\Delta \diamond \quad 44(1.0)$

$-10(1.8) \quad \square \quad 45(1.0)$

$-2(1.8) \quad 42(1.0)$

$-6(1.7) \quad 45(1.1)$

$\Delta \diamond \quad 40(1.0)$

$30-38(10)$

$-6(1.2) \odot \quad 41(0.7)$

$\begin{array}{ll}-6(1.2) & 41(0.7) \\ \Delta \diamond & 48(0.9)\end{array}$

$\Delta \diamond \quad 51(1.0)$

$\Delta \diamond \quad 42(0.7)$

$\Delta \diamond \quad 46(1.3)$

$-7(1.6) \quad \quad \gg \quad 46(1.0)$

$1(1.9) \quad 49(1.2)$

$0(1.6) \quad 46(0.8)$

$-8(1.4) \circlearrowright 47(0.9)$

$\Delta \diamond \quad 35(0.6)$

$\begin{array}{ll}x \times & 47(1.2)\end{array}$

$-8(1.5) \circlearrowright \quad \odot 8(1.1)$

$\diamond 0 \quad 47(0.9)$

$\Delta \diamond \quad 41(0.8)$

$\Delta 0 \quad 46(0.8)$

$-4(1.6) \quad 52(0.9)$

$-3(1.4) \odot 45(0.8)$

$-1(1.3) \quad 45(1.2)$

$\Delta 0 \quad 53(0.8)$

$-17(2.1) \quad 38(1.0)$

$-2(1.7) \quad 42(1.3)$

$-3(1.5) \odot 53(0.8)$

$00 \quad 43(1.0)$

$-3(2.0) \quad 42(1.3)$

$00 \quad 43(0.7)$

- $\quad 33(1.4)$

$00 \quad 33(1.4)$

$-6(1.5) \oslash 34(1.0)$

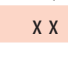

$--$

$43(0.2) \quad 502(0.6)$

Percent in 2006 significantly higher

(7) Percent in 2006 significantly lower

Background data provided by parents.

* Includes books, magazines, newspapers, and materials for work.

() Standard errors appear in parentheses. Because results are rounded to the nearest whole number, some totals may appear inconsistent.

An " $r$ " indicates data are available for $70-84 \%$ of the students. An " $s$ " indicates data are available for $50-69 \%$ of the students. An " $x$ " indicates data are available for less than $50 \%$ of the students

A dash (-) indicates comparable data are not available.

A diamond $(\diamond)$ indicates the country did not participate in the 2001 assessment.

NOTE: The International Average does not include the results from the Canadian provinces.

Trend Note: The primary education systems of the Russian Federation and Slovenia

underwent structural changes. Data for Canada, Ontario include only public schools.

th Grade 
Exhibit 3.9 Parents Reading for Enjoyment with Trends

PIRLS 2006

4th Grade

\begin{tabular}{|c|c|c|c|c|c|c|c|c|c|c|c|c|c|}
\hline \multirow{2}{*}{ Countries } & \multicolumn{5}{|c|}{ Every Day or Almost Every Day } & \multicolumn{4}{|c|}{ Once or Twice a Week } & \multicolumn{4}{|c|}{ Twice a Month or Less } \\
\hline & & $\begin{array}{c}2006 \\
\text { Percent } \\
\text { of Students }\end{array}$ & $\begin{array}{c}\text { Average } \\
\text { Achievement }\end{array}$ & $\begin{array}{l}\text { Difference } \\
\text { in Percent } \\
\text { from } 2001\end{array}$ & & $\begin{array}{c}2006 \\
\text { Percent } \\
\text { of Students }\end{array}$ & $\begin{array}{c}\text { Average } \\
\text { Achievement }\end{array}$ & $\begin{array}{l}\text { Difference } \\
\text { in Percent } \\
\text { from } 2001\end{array}$ & & $\begin{array}{c}2006 \\
\text { Percent } \\
\text { of Students }\end{array}$ & $\begin{array}{c}\text { Average } \\
\text { Achievement }\end{array}$ & $\begin{array}{l}\text { Difference } \\
\text { in Percent } \\
\text { from } 2001\end{array}$ & \\
\hline Norway & & $66(1.0)$ & $505(2.5)$ & $7(1.6)$ & 0 & $23(0.9)$ & $493(4.2)$ & $-4(1.4)$ & ( & $11(0.7)$ & $489(6.5)$ & $-3(0.9)$ & $\nabla$ \\
\hline Sweden & & $64(0.9)$ & $556(2.4)$ & $-3(1.3)$ & ( & $21(0.8)$ & $546(2.9)$ & $-1(1.1)$ & & $15(0.7)$ & $540(4.6)$ & $5(0.9)$ & 0 \\
\hline Scotland & $s$ & $63(1.3)$ & $550(4.0)$ & $1(1.8)$ & & $23(0.9)$ & $531(5.3)$ & $-3(1.5)$ & & $14(1.1)$ & $525(7.0)$ & $2(1.4)$ & \\
\hline Iceland & $r$ & $61(0.9)$ & $523(1.9)$ & $-1(1.2)$ & & $21(0.8)$ & $513(2.9)$ & $-4(1.1)$ & ( & $18(0.6)$ & $498(3.3)$ & $5(0.8)$ & $\theta$ \\
\hline New Zealand & $s$ & $60(1.1)$ & $557(2.4)$ & $4(1.6)$ & 0 & $25(0.9)$ & $539(3.6)$ & $-4(1.4)$ & ( & $15(0.7)$ & $528(4.1)$ & $0(1.1)$ & \\
\hline Netherlands & s & $59(1.2)$ & $562(1.8)$ & $-1(1.8)$ & & $25(0.9)$ & $548(2.9)$ & $-3(1.3)$ & ( & $16(0.9)$ & $543(2.6)$ & $4(1.2)$ & $\theta$ \\
\hline Trinidad and Tobago & & $59(0.9)$ & $441(5.1)$ & $\Delta \diamond$ & & $31(1.0)$ & $436(6.1)$ & $\Delta \diamond$ & & $10(0.7)$ & $434(9.2)$ & $\Delta \diamond$ & \\
\hline Germany & $r$ & $58(0.9)$ & $561(2.4)$ & $7(1.3)$ & $\theta$ & $28(0.7)$ & $544(2.9)$ & $-3(1.0)$ & ( & $14(0.6)$ & $535(4.5)$ & $-3(0.9)$ & $\nabla$ \\
\hline Denmark & & $57(1.0)$ & $554(2.6)$ & $\Delta \Delta$ & & $27(0.9)$ & $544(3.0)$ & $\Delta \Delta$ & & $16(0.7)$ & $534(5.0)$ & $\Delta \Delta$ & \\
\hline Latvia & & $57(1.3)$ & $547(2.7)$ & $3(2.0)$ & & $32(1.1)$ & $537(3.4)$ & $-3(1.5)$ & & $11(0.6)$ & $532(4.2)$ & $0(1.2)$ & \\
\hline Canada, British Columbia & $r$ & $57(1.1)$ & $569(3.2)$ & $\Delta \Delta$ & & $29(1.1)$ & $560(3.5)$ & $\Delta \Delta$ & & $15(0.7)$ & $547(4.7)$ & $\Delta \Delta$ & \\
\hline Canada, Alberta & $r$ & $55(1.2)$ & $571(2.8)$ & $\Delta \Delta$ & & $29(1.0)$ & $561(3.5)$ & $\Delta \Delta$ & & $17(0.7)$ & $554(3.7)$ & $\Delta \Delta$ & \\
\hline Luxembourg & & $54(0.7)$ & $573(1.3)$ & $\Delta \Delta$ & & $29(0.7)$ & $544(2.4)$ & $\Delta \Delta$ & & $18(0.6)$ & $543(2.4)$ & $\Delta \Delta$ & \\
\hline Canada, Nova Scotia & & $53(0.9)$ & $556(2.1)$ & $\Delta \Delta$ & & $27(0.8)$ & $539(2.8)$ & $\Delta \Delta$ & & $19(0.7)$ & $528(3.2)$ & $\Delta \Delta$ & \\
\hline Austria & & $53(1.1)$ & $549(2.3)$ & $\Delta \Delta$ & & $30(0.9)$ & $533(3.2)$ & $\Delta \Delta$ & & $17(0.7)$ & $524(2.9)$ & $\Delta \Delta$ & \\
\hline Lithuania & & $51(1.1)$ & $542(2.0)$ & $-1(1.5)$ & & $36(1.0)$ & $532(2.1)$ & $-1(1.4)$ & & $12(0.6)$ & $534(2.6)$ & $2(0.9)$ & \\
\hline Belgium (French) & & $51(1.3)$ & $512(2.9)$ & $\Delta \diamond$ & & $32(0.9)$ & $496(3.3)$ & $\Delta \diamond$ & & $17(0.8)$ & $483(4.4)$ & $\Delta \Delta$ & \\
\hline Canada, Ontario & $r$ & $51(1.0)$ & $563(3.4)$ & $-2(1.6)$ & & $32(1.1)$ & $552(3.0)$ & $1(1.6)$ & & $18(0.9)$ & $547(4.5)$ & $1(1.3)$ & \\
\hline France & & $50(1.0)$ & $535(2.5)$ & $-1(1.4)$ & & $32(0.8)$ & $518(2.6)$ & $-2(1.2)$ & & $19(0.8)$ & $505(3.0)$ & $3(1.1)$ & $\theta$ \\
\hline Spain & $s$ & $50(1.2)$ & $531(3.0)$ & $\Delta \Delta$ & & $33(1.0)$ & $512(3.6)$ & $\Delta \Delta$ & & $18(0.7)$ & $507(4.5)$ & $\Delta \Delta$ & \\
\hline Singapore & & $50(0.7)$ & $569(3.0)$ & $14(1.1)$ & $\theta$ & $33(0.7)$ & $552(3.1)$ & $-3(0.9)$ & ( & $17(0.5)$ & $546(4.3)$ & $-11(0.9)$ & ( \\
\hline Canada, Quebec & $r$ & $49(1.4)$ & $542(3.2)$ & $1(1.9)$ & & $32(1.2)$ & $536(3.5)$ & $-2(1.7)$ & & $18(0.9)$ & $518(4.1)$ & $1(1.4)$ & \\
\hline Italy & & $49(1.0)$ & $564(3.0)$ & $2(1.4)$ & & $32(1.0)$ & $547(3.7)$ & $-4(1.2)$ & ( & $19(0.9)$ & $535(4.2)$ & $2(1.2)$ & \\
\hline Hungary & & $49(1.0)$ & $561(3.2)$ & $-2(1.4)$ & & $37(0.8)$ & $546(3.7)$ & $1(1.2)$ & & $14(0.6)$ & $544(4.4)$ & $1(0.9)$ & \\
\hline Macedonia, Rep. of & $r$ & $48(1.1)$ & $459(4.6)$ & $7(1.7)$ & $\theta$ & $41(0.9)$ & $441(4.5)$ & $0(1.4)$ & & $11(0.9)$ & $423(8.4)$ & $-7(1.5)$ & ( \\
\hline South Africa & $r$ & $48(0.7)$ & $322(7.5)$ & $\Delta \Delta$ & & $36(0.5)$ & $301(5.2)$ & $\Delta \Delta$ & & $16(0.5)$ & $276(6.3)$ & $\Delta \diamond$ & \\
\hline Israel & & $47(1.4)$ & $538(5.0)$ & $x x$ & & $39(1.2)$ & $520(4.4)$ & $x x$ & & $15(0.7)$ & $508(6.5)$ & $x x$ & \\
\hline Slovak Republic & & $46(1.0)$ & $544(2.4)$ & $-2(1.5)$ & & $39(1.0)$ & $529(3.2)$ & $0(1.4)$ & & $14(0.8)$ & $504(7.7)$ & $3(1.0)$ & $\theta$ \\
\hline Slovenia & & $45(0.8)$ & $530(2.4)$ & $4(1.3)$ & $\theta$ & $36(0.8)$ & $520(2.6)$ & $-6(1.2)$ & $(\nabla$ & $18(0.6)$ & $510(3.3)$ & $2(0.9)$ & $\boldsymbol{\theta}$ \\
\hline Poland & & $45(0.9)$ & $529(3.0)$ & $\Delta \Delta$ & & $41(0.8)$ & $517(2.8)$ & $\Delta \Delta$ & & $14(0.6)$ & $504(4.5)$ & $\Delta \Delta$ & \\
\hline Qatar & $r$ & $44(0.7)$ & $361(2.3)$ & $\Delta \Delta$ & & $39(0.7)$ & $357(2.3)$ & $\Delta \Delta$ & & $17(0.5)$ & $348(3.8)$ & $\Delta \Delta$ & \\
\hline Kuwait & $r$ & $44(1.2)$ & $339(5.4)$ & $\Delta \Delta$ & & $36(0.9)$ & $337(5.4)$ & $\Delta \Delta$ & & $20(0.9)$ & $335(5.9)$ & $\Delta \Delta$ & \\
\hline Russian Federation & & $42(1.0)$ & $573(3.7)$ & $-7(1.6)$ & $\nabla$ & $41(0.8)$ & $560(3.9)$ & $6(1.4)$ & 0 & $17(0.6)$ & $557(3.6)$ & $1(1.1)$ & \\
\hline Belgium (Flemish) & & $40(0.9)$ & $558(2.2)$ & $\Delta \Delta$ & & $33(0.8)$ & $545(2.4)$ & $\Delta \Delta$ & & $27(1.0)$ & $538(2.4)$ & $\Delta \Delta$ & \\
\hline Georgia & & $39(1.3)$ & $486(3.4)$ & $\Delta \Delta$ & & $44(1.0)$ & $467(3.2)$ & $\Delta \Delta$ & & $17(1.2)$ & $450(7.6)$ & $\Delta \Delta$ & \\
\hline Bulgaria & & $39(1.4)$ & $565(4.2)$ & $-14(2.1)$ & ( & $34(1.1)$ & $547(4.7)$ & $6(1.6)$ & 0 & $27(2.0)$ & $532(7.6)$ & $8(2.6)$ & $\theta$ \\
\hline Hong Kong SAR & & $36(0.8)$ & $574(2.3)$ & $6(1.4)$ & $\theta$ & $39(0.7)$ & $561(2.6)$ & $10(1.0)$ & 0 & $25(0.6)$ & $558(3.4)$ & $-15(1.3)$ & $\diamond$ \\
\hline Chinese Taipei & & $35(0.9)$ & $547(2.5)$ & $\Delta \Delta$ & & $42(0.7)$ & $536(2.5)$ & $\Delta \diamond$ & & $23(0.7)$ & $520(2.4)$ & $\Delta \Delta$ & \\
\hline Moldova, Rep. of & & $35(1.1)$ & $509(3.3)$ & $-1(1.9)$ & & $46(1.1)$ & $498(3.5)$ & $6(1.7)$ & $\theta$ & $20(1.5)$ & $491(5.9)$ & $-5(1.9)$ & 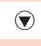 \\
\hline Indonesia & & $31(1.2)$ & $413(4.6)$ & $\Delta \Delta$ & & $44(1.3)$ & $405(4.5)$ & $\Delta \Delta$ & & $25(1.3)$ & $395(4.9)$ & $\Delta \Delta$ & \\
\hline Romania & & $27(1.1)$ & $514(4.7)$ & $11(1.6)$ & $\theta$ & $43(1.0)$ & $497(4.8)$ & $11(1.7)$ & $\boldsymbol{\theta}$ & $30(1.4)$ & $459(8.0)$ & $-22(2.4)$ & 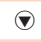 \\
\hline Morocco & & $24(1.1)$ & $346(6.4)$ & -- & & $34(1.4)$ & $329(5.3)$ & -- & & $42(1.9)$ & $308(10.9)$ & -- & \\
\hline Iran, Islamic Rep. of & & $24(1.1)$ & $441(4.9)$ & $2(1.5)$ & & $41(1.1)$ & $426(3.4)$ & $6(1.8)$ & 0 & $35(1.6)$ & $402(4.3)$ & $-8(2.2)$ & 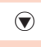 \\
\hline England & & $x x$ & $x x$ & $x x$ & & $x \times$ & $\mathrm{xx}$ & $x x$ & & $x x$ & $x x$ & $x x$ & \\
\hline United States & & -- & -- & -- & & -- & -- & -- & & -- & -- & -- & \\
\hline International Avg. & & $47(0.2)$ & $512(0.6)$ & & & $34(0.2)$ & $498(0.6)$ & & & $18(0.2)$ & $487(0.9)$ & & \\
\hline
\end{tabular}

- Percent in 2006 significantly higher

( Percent in 2006 significantly lower

Background data provided by parents.

() Standard errors appear in parentheses. Because results are rounded to the nearest whole number, some totals may appear inconsistent.

An " $r$ " indicates data are available for $70-84 \%$ of the students. An "s" indicates data are available for $50-69 \%$ of the students. An " $x$ " indicates data are available for less than $50 \%$ of the students.
A dash (-) indicates comparable data are not available.

A diamond $(0)$ indicates the country did not participate in the 2001 assessment. NOTE: The International Average does not include the results from the Canadian provinces. Trend Note: The primary education systems of the Russian Federation and Slovenia underwent structural changes. Data for Canada, Ontario include only public schools. 
Exhibit 3.10 Index of Parents' Attitudes Toward Reading (PATR) with Trends

PIRLS 2006

Countries

\begin{tabular}{|c|c|}
\multicolumn{2}{c}{ High PATR } \\
\hline $\begin{array}{c}2006 \\
\text { Percent } \\
\text { of Students }\end{array}$ & $\begin{array}{c}\text { Average } \\
\text { Achievement }\end{array}$ \\
\hline
\end{tabular}

Sweden

$71(0.9)$

$559(2.3)$

Norway

$71(1.0)$

Scotland

$70(1.4)$

Denmark

$70(1.0)$

$508(2.6)$

$552(3.9)$

Hungary

$68(1.3)$

$557(2.4)$

$568(2.6)$

erlands

$68(1.3)$

Canada, Alberta

$67(1.2)$

$563(1.8)$

Canada, Nova Scotia

$67(0.9) \quad 558(2.3)$

New Zealand s $66(1.1)$

Canada, British Columbia $\quad r \quad 66(1.4)$

Canada, Quebec

$66(1.4)$

Trinidad and Tobago

$65(1.2)$

Iceland

$64(1.0)$

Slovak Republic

$62(1.1)$

Canada, Ontario

$61(1.4)$

Spain

Germany

$61(1.4)$

Latvia

Poland

$60(1.3)$

$562(2.5)$

$572(2.7)$

\begin{tabular}{l|l} 
& \\
\hline Difference \\
in Percent \\
from 2001
\end{tabular}

2006
Percent
of Students
$24(0.8)$

Medium PATR

4th Grade

Austria 56

Israel

$57(1.2)$

$545(3.1)$

$0(1.2)$

$24(0.8)$

\begin{tabular}{|c|} 
Average \\
Achievement
\end{tabular}

$454(5.0)$

$526(1.9)$

$551(2.2)$

$-2(1.5)$

$1(1.9) \quad 24(1.2)$

Difference
in Percent
from 2001

\begin{tabular}{|c|}
\hline $\begin{array}{c}2006 \\
\text { Percent } \\
\text { of Students }\end{array}$ \\
$5(0.5)$ \\
\hline
\end{tabular}

Low PATR

Italy

Slovenia

$56(1.1) \quad 536(2.7)$

$\Delta \Delta \quad 25(0.9)$

$535(3.3)$

0 (1.1)

$-6(1.7) \quad 28(1.2)$

$525(4.7)$

1 (1.4)

$5(0.5)$

531 (3.5)

$-1(1.7)$

$6(0.8)$

$528(3.9)$

00

$6(0.5)$

$5(1.9) \quad 0 \quad 25(1.2)$

$542(2.7)$

$5(1.5)$

$\Delta \diamond \quad 27(1.1)$

$551(4.4)$

$-7(1.6)$

$7(0.5)$

$00 \quad 27(0.9)$

$524(3.0)$

00

$5(0.4)$

$1(1.6) \quad 28(1.0)$

$526(3.3)$

$\Delta \diamond \quad 7(0.5)$

$6(0.5)$

$547(4.3)$

$-2(1.5)$

$5(0.4)$

$7(1.8) \quad 0 \quad 27(1.2)$

$523(3.6)$

$-11(1.8) \odot 8(0.5)$

$00 \quad 31(1.1)$

419 (5.2)

$-3(1.3) \quad 31(0.9)$

$503(2.3)$

$\Delta \diamond \quad 4(0.4)$

$5(0.4)$

$565(2.9)$

$-4(1.7)-33(1.0)$

$533(2.5)$

$-6(1.9) \circlearrowright \quad \gg 32(1.5)$

$516(3.2)$

$2(1.2)$

$544(3.8)$

$1(1.6)$

$5(0.8)$

$34(1.2)$

$506(4.0)$

$3(2.0)$

$6(0.6)$

$537(2.7)$

00

$5(0.6)$

$7(0.5)$

$9(1.8) \quad 0 \quad 37(1.1)$

$535(3.0)$

$-3(1.5)$

$39(1.1)$

$508(3.1)$

$-13(1.8)$

$6(0.5)$

$4(0.4)$

$\begin{array}{rr}\Delta \diamond & 10(0.5) \\ \times x & 6(0.6)\end{array}$

$502(5.5)$

$55(1.4)$

$549(3.9)$

$\begin{array}{ll}x x & 35(0.8) \\ -1(1.6) & 39(1.3)\end{array}$

\begin{tabular}{|l|l|l|l|}
$55(1.1)$ & $570(2.7)$ & $-1(1.6)$ & $36(0.9)$
\end{tabular}

$540(4.3) \quad-1(1.4)$

$6(0.6)$

(T)

Luxembourg
Belgium (French)

$54(1.2)$

$538(2.4)$

$-6(1.5) \bigcirc 41(1.0)$

$509(2.5)$

$9(0.6)$

Average Achievement

Difference

in Percent

from 2001

$521(6.3) \quad 0(0.6)$

$471(7.6)$

$1(0.7)$

$1(0.7)$

$505(9.8) \quad 0(1.0)$

$524(7.4)$

$1(0.6)$

$530(4.0)$

$2(0.8)$

$539(4.6) \quad \diamond \diamond$

$510(8.3)$

$1(0.7)$

$538(7.3)$

$509(4.9)$

407 (13.4)

$486(6.2)$

$463(17.8)$

$542(6.8)$

$494(7.2)$

$517(4.4)$

$524(8.4)$

$486(7.5) \quad \diamond \diamond$

$517(4.2) \quad \Delta 0$

$512(8.1)$

$529(5.8) \quad 2(0.8) \quad 0$

$479(4.0) \quad 6(2.3) \quad 0 \quad 42(1.1)$

$419(4.4) \quad-6(2.1) \quad \odot \quad 3(0.4)$

$508(5.5)$

$2(0.4)$

$53(0.7) \quad 577(1.3) \quad \diamond \diamond \quad 38(0.7)$

$543(1.9)$

$\Delta \diamond$

$3(0.4)$

$405(12.5)$

$0(0.6)$

Lithuania

$53(1.3)$

$518(2.7)$

$\Delta \diamond \quad 41(1.1)$

$489(2.9)$

$\Delta \diamond \quad 7(0.6)$

$405(12.5)$

$\Delta \Delta$

France

Russian Federation

$51(0.9)$

$549(1.9)$

$9(1.5) \quad 0 \quad 43(0.9)$

$529(1.9)$

$\begin{array}{ll}00 & 7(0.6) \\ -9(1.5) \rightarrow 6(0.4)\end{array}$

$474(6.1)$

$524(5.0)$

$\Delta \Delta$

Belgium (Flemish)

\begin{tabular}{l}
$541(2.5) \quad-2(1.6)$ \\
\hline
\end{tabular}

$44(1.1)$

$512(2.4)$

$1(1.6)$

$5(0.4)$

$494(6.1)$

$0(0.6)$

$50(1.3) \quad 577(3.9) \quad 0(1.8) \quad 43(1.1)$

$558(3.2)$

$-2(1.7)$

$7(0.4)$

$\quad 1(0.6)$

Bulgari:

Iran, Islamic Rep. of $43(1.4)$

$45(0.9) \quad 353(5.0)$

$\Delta \Delta \quad 38(0.9)$

$49(0.9)$

$538(2.5)$

$00 \quad 13(0.8)$

$529(2.9)$

$2(0.6)$

$53(5.0)$

$-10(2.5) \odot \quad 37(1.3)$

$330(5.4)$

$\Delta \diamond \quad 6(0.5)$

$529(2.9) \quad \Delta 0$

Qatar

Georgia

$43(0.7)$

$441(4.4)$

$0(2.0) \quad 52(1.4)$

$535(5.2)$

\begin{tabular}{l|l}
$0(1.9)$ & $18(2.0)$
\end{tabular}

$327(12.6)$

$\Delta \Delta$

Singapore

Chinese Taipei

$41(1.4) \quad 488(3.2)$

$\Delta 0 \quad 50(0.7)$

$410(3.4)$

$-1(1.9)$

$5(0.5)$

$535(9.7) \quad 10(2.3)$

Romania

Morocco

$(0.8)$

$575(2.9)$

$\begin{array}{cc}\diamond \diamond & 54(1.4) \\ -1(1.2) & 55(0.7)\end{array}$

$347(2.0)$

$\Delta 0 \quad 6(0.3)$

$6(0.3)$

$377(11.3) \quad 0(1.0)$

$466(3.8)$

$\diamond \diamond$

$5(1.0)$

$\begin{array}{ll}346(5.4) & \Delta \diamond \\ 432(20.4) & \diamond \diamond\end{array}$

$\diamond \diamond \quad 58(0.8)$

$531(2.3)$

1 (1.2)

$6(0.3)$

$556(4.6) \quad 0(0.4)$

$38(1.7) \quad 528(3.8)$

$\begin{array}{ll}4(2.4) & 48(1.4) \\ -- & 53(1.9)\end{array}$

$531(2.3)$

$\Delta \diamond \quad 3(0.3)$

$520(6.7)$

$0(0.4)$

South Africa

$37(1.7) \quad 340(6.5)$

$479(5.8)$

$-7(2.0) \quad 14(1.5)$

$428(12.7)$

3 (1.9)

Moldova, Rep of

$37(1.1) \quad 358(9.1)$

$\Delta \Delta \quad 60(1.1)$

$276(4.2)$

$--\quad 10(1.3)$

$316(12.7)$ 


\section{What Languages Are Spoken at Home?}

Although speaking more than one language has advantages, PIRLS 2001 has shown that countries with large proportions of students from homes where the language of the test is not often spoken had lower average reading achievement at the fourth grade than those who spoke it more often. For PIRLS 2006, Exhibit 3.11 presents students' reports of how frequently they speak the language of the PIRLs test at home, together with average student reading achievement. To complement the students' reports, the exhibit also presents the percentage of students whose parents reported that the language of the test is the language they speak most often at home.

About two thirds of the students (66\%), on average internationally, reported always speaking the language of the test at home, but there were significant percentages that reported sometimes ( $29 \%$ on average) or never ( $5 \%$ on average) speaking it. Only about half the students in Iran, Israel, and Morocco reported always speaking the language of the PIRLS test at home, and even lower percentages in Indonesia (38\%), Chinese Taipei (36\%), Kuwait (26\%), Singapore (21\%), and Luxembourg (3\%).

According to parents' reports, in almost all countries, a large majority of students $(87 \%)$, on average, were from homes where at least one parent spoke the language of the PIRLS test at home most of the time. Notable exceptions were Luxembourg, where almost all parents spoke Luxembourgish most of the time, and Kuwait, where the Kuwaiti dialect, a variant of the classical Arabic taught in school, is widely spoken at home.

Although in many countries there was a positive relationship between frequency of students speaking the language of the PIRLS test at home and performance on the PIRLS reading test, the relationship was by no means universal, and in about one third of the countries, average achievement among students reporting sometimes speaking the language of the PIRLS test at home was greater than for those reporting always speaking the language. In all countries the students who reported never speaking the language of the PIRLS test at home had lower average reading achievement than those speaking it more frequently. 
Many of the PIRLS 2006 participants tested in two languages in order to cover their whole population. These included the five Canadian provinces (English and French), Israel (Hebrew and Arabic), Latvia (Latvian and Russian), Macedonia (Macedonian and Albanian), Moldova (Romanian and Russian), New Zealand (English and Māori), Norway (Bokmål and Nynorsk), Romania (Romanian and Hungarian), and the Slovak Republic (Slovak and Hungarian). Spain tested in five languages (Castilian, Catalonian, Galician, Basque, and Valencian) and South Africa in 11 languages (Afrikaans, English, isiZulu, isiXhosa, Sepedi, Sesotho, Setswana, isiNdebele, Siswati, Tshivenda, and Xitsonga).

Related to the question of the language spoken in the home is the size of the immigrant population. Exhibit 3.12 presents students' reports of where their parents were born. The exhibit shows the percentage of students in 2006 reporting both parents, one parent only, or neither parent born in the country, together with their average achievement. Also shown is the change in percentage of students in each category since 2001. In most countries, a large majority of students reported that both parents were born in the country -76 percent on average. However, there were substantial percentages of students, on average internationally, reporting only one parent (14\%) or neither parent (10\%) born in the country. Some countries, such as Bulgaria, Georgia, Hungary, Iran, Poland, Romania, and the Slovak Republic, had very little immigration, with more than 90 percent of students reporting that both parents were born in the country. In contrast, participants with the greatest percentages of students ( $20 \%$ or more) reporting both parents born abroad included Hong Kong sar, Israel, Latvia, Luxembourg, New Zealand, Qatar, and the Canadian provinces of Alberta, British Columbia, and Ontario. Participants showing increases since 2001 in the percentage of students with both parents born abroad included Italy, Latvia, Singapore, and the Canadian provinces of Ontario and Quebec.

On average internationally, and in most countries, students with both parents born in the country had the highest average reading achievement (508 points), followed by students with one parent born in the country 
(492 points). Students with both parents born abroad had the lowest average achievement (477 points). Although generally students with one or both parents born in the country had an advantage in terms of reading achievement, Israel, New Zealand, Singapore, and British Columbia showed little difference in achievement among the three categories of students. In Hong Kong SAR, Qatar, and Trinidad and Tobago, students with both parents born abroad had the highest achievement, while students in this category in Kuwait and Latvia had higher achievement than students with one but not both parents born in the country. 
Exhibit 3.11 Students and Parents Speak Language of the Test at Home

\begin{tabular}{|c|c|c|c|c|c|c|c|c|}
\hline \multirow{3}{*}{ Countries } & \multicolumn{6}{|c|}{ Students Speak Language of the Test at Home } & \multirow{2}{*}{\multicolumn{2}{|c|}{$\begin{array}{l}\text { Percentage of Student } \\
\text { Whose Parents Most } \\
\text { Often Speak Language } \\
\text { of the Test at Home }\end{array}$}} \\
\hline & \multicolumn{2}{|c|}{ Always } & \multicolumn{2}{|c|}{ Sometimes } & \multicolumn{2}{|c|}{ Never } & & \\
\hline & $\begin{array}{l}\text { Percent } \\
\text { of Students }\end{array}$ & $\begin{array}{c}\text { Average } \\
\text { Achievement }\end{array}$ & $\begin{array}{l}\text { Percent } \\
\text { of Students }\end{array}$ & \begin{tabular}{c|} 
Average \\
Achievement
\end{tabular} & $\begin{array}{l}\text { Percent } \\
\text { of Students }\end{array}$ & $\begin{array}{c}\text { Average } \\
\text { Achievement }\end{array}$ & $\begin{array}{l}\text { Either } \\
\text { Parent }\end{array}$ & $\begin{array}{l}\text { Both } \\
\text { Parents }\end{array}$ \\
\hline Austria & $74(1.2)$ & $551(1.9)$ & $24(1.1)$ & $520(3.5)$ & $2(0.5)$ & $\sim \sim$ & $90(0.8)$ & $80(1.1)$ \\
\hline Belgium (Flemish) & $77(1.3)$ & $555(1.7)$ & $21(1.1)$ & $526(3.5)$ & $2(0.3)$ & $\sim \sim$ & $94(0.8)$ & $85(1.2)$ \\
\hline Belgium (French) & $66(1.3)$ & $507(2.3)$ & $32(1.2)$ & $493(4.0)$ & $2(0.2)$ & $\sim \sim$ & $93(0.9)$ & $79(1.2)$ \\
\hline Bulgaria & $70(2.2)$ & $558(3.9)$ & $27(2.0)$ & $537(7.2)$ & $3(0.7)$ & $494(16.0)$ & $83(2.0)$ & $72(2.1)$ \\
\hline Canada, Alberta & $70(1.5)$ & $566(2.2)$ & $29(1.4)$ & $554(3.9)$ & $1(0.2)$ & $\sim \sim$ & $92(1.1)$ & $84(1.5)$ \\
\hline Canada, British Columbia & $64(1.8)$ & $563(2.6)$ & $35(1.8)$ & $556(3.5)$ & $1(0.2)$ & $\sim \sim$ & $84(1.6)$ & $73(2.3)$ \\
\hline Canada, Nova Scotia & $72(0.9)$ & $548(2.3)$ & $28(0.9)$ & $544(3.0)$ & $1(0.1)$ & $\sim \sim$ & $98(0.3)$ & $89(0.8)$ \\
\hline Canada, Ontario & $61(1.8)$ & $562(2.8)$ & $38(1.8)$ & $550(3.5)$ & $1(0.2)$ & $\sim \sim$ & $85(1.6)$ & $75(2.2)$ \\
\hline Canada, Quebec & $64(1.4)$ & $540(3.4)$ & $35(1.4)$ & $532(2.6)$ & $2(0.3)$ & $\sim \sim$ & $93(1.0)$ & $78(1.5)$ \\
\hline Chinese Taipei & $36(0.8)$ & $528(2.6)$ & $63(0.8)$ & $544(2.0)$ & $1(0.2)$ & $\sim \sim$ & $82(1.0)$ & $54(1.3)$ \\
\hline Denmark & $81(1.1)$ & $551(2.3)$ & $18(1.0)$ & $535(4.3)$ & $1(0.2)$ & $\sim \sim$ & $96(0.5)$ & $88(1.0)$ \\
\hline England & $76(1.3)$ & $546(2.7)$ & $23(1.2)$ & $532(4.5)$ & $1(0.2)$ & $\sim \sim$ & $x x$ & $x x$ \\
\hline France & $66(1.2)$ & $531(2.1)$ & $34(1.2)$ & $512(2.7)$ & $1(0.1)$ & $\sim \sim$ & $96(0.6)$ & $85(1.2)$ \\
\hline Georgia & $85(1.5)$ & $475(3.4)$ & $14(1.2)$ & $486(6.3)$ & $1(0.8)$ & $\sim \sim$ & $97(1.2)$ & $73(1.9)$ \\
\hline Germany & $73(1.0)$ & $564(2.1)$ & $26(0.9)$ & $536(3.3)$ & $1(0.2)$ & $\sim \sim$ & $93(0.6)$ & $81(1.1)$ \\
\hline Hong Kong SAR & $65(0.8)$ & $562(2.4)$ & $33(0.8)$ & $571(2.5)$ & $2(0.3)$ & $\sim \sim$ & $98(0.4)$ & $80(0.8)$ \\
\hline Hungary & $75(1.2)$ & $553(2.9)$ & $24(1.2)$ & $550(4.4)$ & $1(0.2)$ & $\sim \sim$ & $99(0.2)$ & $89(0.8)$ \\
\hline Iceland & $64(0.8)$ & $512(1.5)$ & $35(0.8)$ & $513(1.9)$ & $1(0.1)$ & $\sim \sim$ & $99(0.2)$ & $92(0.5)$ \\
\hline Indonesia & $38(2.0)$ & $403(5.7)$ & $50(1.8)$ & $424(4.3)$ & $11(1.0)$ & $406(7.9)$ & -- & -- \\
\hline Iran, Islamic Rep. of & $53(2.2)$ & $447(3.5)$ & $28(1.5)$ & $423(5.1)$ & $19(1.6)$ & $364(7.4)$ & $62(2.0)$ & $52(2.1)$ \\
\hline Israel & $57(1.1)$ & $524(4.1)$ & $39(1.1)$ & $539(2.9)$ & $4(0.4)$ & $468(14.4)$ & $90(1.3)$ & $76(1.5)$ \\
\hline Italy & $71(1.2)$ & $558(3.1)$ & $27(1.1)$ & $549(4.1)$ & $3(0.4)$ & $521(13.4)$ & $97(0.4)$ & $89(0.7)$ \\
\hline Kuwait & $26(1.5)$ & $330(8.2)$ & $43(1.6)$ & $367(5.5)$ & $32(2.1)$ & $336(6.3)$ & $5(0.5)$ & $1(0.3)$ \\
\hline Latvia & $69(1.4)$ & $546(2.4)$ & $28(1.2)$ & $538(3.6)$ & $3(0.5)$ & $525(13.0)$ & $94(0.8)$ & $77(1.4)$ \\
\hline Lithuania & $79(1.0)$ & $539(1.7)$ & $21(1.0)$ & $538(2.8)$ & $0(0.1)$ & $\sim \sim$ & $99(0.3)$ & $86(0.7)$ \\
\hline Luxembourg & $3(0.2)$ & $568(7.1)$ & $44(0.6)$ & $563(1.4)$ & $53(0.6)$ & $553(1.6)$ & $4(0.3)$ & $1(0.1)$ \\
\hline Macedonia, Rep. of & $83(1.2)$ & $450(4.0)$ & $15(1.0)$ & $489(6.8)$ & $2(0.5)$ & $\sim \sim$ & $95(1.2)$ & $84(1.4)$ \\
\hline Moldova, Rep. of & $74(1.1)$ & $498(3.3)$ & $25(1.1)$ & $511(3.9)$ & $1(0.2)$ & $\sim \sim$ & $96(0.6)$ & $80(1.1)$ \\
\hline Morocco & $50(2.9)$ & $337(6.7)$ & $24(1.7)$ & $337(8.1)$ & $26(2.8)$ & $303(15.0)$ & $71(3.1)$ & $51(2.8)$ \\
\hline Netherlands & $76(1.2)$ & $553(1.6)$ & $23(1.2)$ & $533(2.6)$ & $1(0.3)$ & $\sim \sim$ & $95(1.1)$ & $87(1.5)$ \\
\hline New Zealand & $73(1.0)$ & $542(2.1)$ & $26(0.9)$ & $519(3.1)$ & $1(0.2)$ & $\sim \sim$ & $92(0.8)$ & $78(1.1)$ \\
\hline Norway & $80(0.9)$ & $505(2.2)$ & $20(0.9)$ & $494(4.0)$ & $1(0.1)$ & $\sim \sim$ & $100(0.1)$ & $93(0.7)$ \\
\hline Poland & $85(0.6)$ & $519(2.3)$ & $14(0.6)$ & $533(4.1)$ & $0(0.1)$ & $\sim \sim$ & $100(0.1)$ & $90(0.5)$ \\
\hline Qatar & $61(0.7)$ & $360(1.9)$ & $35(0.7)$ & $369(2.0)$ & $4(0.3)$ & $309(8.6)$ & $96(0.3)$ & $78(0.6)$ \\
\hline Romania & $81(1.6)$ & $495(4.0)$ & $17(1.2)$ & $515(8.8)$ & $2(0.6)$ & $\sim \sim$ & $92(1.8)$ & $82(1.9)$ \\
\hline Russian Federation & $82(1.1)$ & $568(3.4)$ & $16(1.0)$ & $568(6.3)$ & $2(0.4)$ & $\sim \sim$ & $93(1.0)$ & $76(1.2)$ \\
\hline Scotland & $80(1.0)$ & $528(3.0)$ & $19(1.0)$ & $536(4.4)$ & $1(0.2)$ & $\sim \sim$ & $99(0.3)$ & $90(0.8)$ \\
\hline Singapore & $21(0.7)$ & $571(3.5)$ & $73(0.8)$ & $559(3.0)$ & $5(0.3)$ & $516(5.2)$ & $56(0.8)$ & $26(0.9)$ \\
\hline Slovak Republic & $71(1.6)$ & $537(2.3)$ & $26(1.4)$ & $531(5.1)$ & $3(0.7)$ & $439(31.6)$ & $95(1.2)$ & $84(1.3)$ \\
\hline Slovenia & -- & -- & -- & -- & -- & -- & $97(0.6)$ & $89(0.9)$ \\
\hline South Africa & $62(1.2)$ & $306(6.6)$ & $30(1.0)$ & $359(8.0)$ & $8(0.5)$ & $270(8.1)$ & $79(1.4)$ & $43(1.4)$ \\
\hline Spain & $60(1.3)$ & $519(2.5)$ & $32(1.1)$ & $517(3.0)$ & $8(0.7)$ & $498(6.0)$ & $84(1.5)$ & $69(1.7)$ \\
\hline Sweden & $75(1.2)$ & $555(2.3)$ & $24(1.1)$ & $538(3.4)$ & $1(0.2)$ & $\sim \sim$ & $93(0.8)$ & $84(1.0)$ \\
\hline Trinidad and Tobago & $77(1.3)$ & $439(5.0)$ & $20(1.1)$ & $455(7.5)$ & $3(0.4)$ & $351(13.2)$ & $99(0.3)$ & $84(0.9)$ \\
\hline United States & $72(1.4)$ & $547(3.4)$ & $27(1.4)$ & $534(4.7)$ & $1(0.2)$ & $\sim \sim$ & -- & -- \\
\hline International Avg. & $66(0.2)$ & $506(0.7)$ & $29(0.2)$ & $505(0.8)$ & $5(0.1)$ & $424(3.4)$ & $87(0.2)$ & $73(0.2)$ \\
\hline
\end{tabular}

Background data provided by students and parents

() Standard errors appear in parentheses. Because results are rounded to the nearest whole number, some totals may appear inconsistent.

An " $\mathrm{r}$ " indicates data are available for $70-84 \%$ of the students. An " $\mathrm{s}$ " indicates data are available for $50-69 \%$ of the students. An " $x$ " indicates data are available for less than $50 \%$ of the students.
A dash (-) indicates comparable data are not available. A tilde ( ) indicates insufficient data to report achievement.

NOTE: The International Average does not include the results from the Canadian provinces. 
Exhibit 3.12 Students' Parents Born in Country with Trends

PIRLS 2006

4th Grade

\begin{tabular}{|c|c|c|c|c|c|c|c|c|c|c|c|c|c|}
\hline \multirow[b]{2}{*}{ Countries } & \multicolumn{5}{|c|}{ Both Parents Born in Country } & \multicolumn{4}{|c|}{ Only One Parent Born in Country } & \multicolumn{4}{|c|}{ Neither Parent Born in Country } \\
\hline & & $\begin{array}{c}2006 \\
\begin{array}{c}\text { Percent } \\
\text { of Students }\end{array}\end{array}$ & $\begin{array}{c}\text { Average } \\
\text { Achievement }\end{array}$ & $\begin{array}{l}\text { Difference } \\
\text { in Percent } \\
\text { from } 2001\end{array}$ & & $\begin{array}{c}2006 \\
\text { Percent } \\
\text { of Students }\end{array}$ & $\begin{array}{c}\text { Average } \\
\text { Achievement }\end{array}$ & $\begin{array}{l}\text { Difference } \\
\text { in Percent } \\
\text { from } 2001\end{array}$ & & $\begin{array}{c}2006 \\
\text { Percent } \\
\text { of Students }\end{array}$ & $\begin{array}{c}\text { Average } \\
\text { Achievement }\end{array}$ & $\begin{array}{l}\text { Difference } \\
\text { in Percent } \\
\text { from } 2001\end{array}$ & \\
\hline Austria & & $72(1.3)$ & $548(2.0)$ & $\Delta \diamond$ & & $11(0.6)$ & $540(3.9)$ & $\Delta \Delta$ & & $17(1.1)$ & $501(3.6)$ & $\Delta \diamond$ & \\
\hline Belgium (Flemish) & & $79(1.4)$ & $554(1.8)$ & $\Delta 0$ & & $13(0.7)$ & $530(3.6)$ & 00 & & $8(0.9)$ & $511(5.7)$ & 00 & \\
\hline Belgium (French) & & $56(1.7)$ & $511(2.4)$ & $\Delta \Delta$ & & $25(1.0)$ & $498(4.3)$ & $\Delta \Delta$ & & $19(1.5)$ & $479(5.1)$ & $\Delta \Delta$ & \\
\hline Bulgaria & & $95(0.6)$ & $552(3.9)$ & $0(0.7)$ & & $4(0.5)$ & $504(15.5)$ & $0(0.6)$ & & $1(0.2)$ & $\sim \sim$ & $1(0.2)$ & \\
\hline Canada, Alberta & & $59(1.8)$ & $568(2.5)$ & $\Delta 0_{0}-1-1-1$ & & $21(0.8)$ & $560(3.6)$ & $\Delta \Delta$ & & $21(1.8)$ & $553(4.8)$ & $\Delta \Delta$ & \\
\hline Canada, British Columbia & & $44(2.0)$ & $563(3.0)$ & $\Delta 0$ & & $23(1.0)$ & $562(3.8)$ & $\Delta 0$ & & $33(2.3)$ & $559(3.8)$ & $\Delta 0$ & \\
\hline Canada, Nova Scotia & & $82(0.9)$ & $549(2.3)$ & $\Delta \diamond$ & & $14(0.7)$ & $546(3.6)$ & $\Delta \diamond$ & & $5(0.5)$ & $537(6.1)$ & $\Delta \diamond$ & \\
\hline Canada, Ontario & & $45(2.8)$ & $564(3.3)$ & $-8(3.7)$ & ( & $18(1.0)$ & $557(4.0)$ & $-2(1.4)$ & & $37(3.5)$ & $548(4.0)$ & $10(4.3)$ & 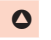 \\
\hline Canada, Quebec & & $73(2.2)$ & $544(3.0)$ & $-1(2.8)$ & & $12(0.7)$ & $534(4.9)$ & $-4(1.1)$ & $(7)$ & $15(2.0)$ & $517(4.1)$ & $5(2.4)$ & 0 \\
\hline Chinese Taipei & & $86(0.5)$ & $544(1.9)$ & $\Delta \diamond$ & & $11(0.5)$ & $519(4.1)$ & $\Delta \Delta$ & & $3(0.3)$ & $481(7.5)$ & $\Delta \Delta$ & \\
\hline Denmark & & $79(1.3)$ & $551(2.3)$ & $\Delta \Delta$ & & $12(0.7)$ & $546(4.1)$ & $\Delta \Delta$ & & $9(1.0)$ & $511(5.4)$ & $\Delta \Delta$ & \\
\hline England & & $71(1.7)$ & $552(2.5)$ & $4(2.5)$ & & $17(0.8)$ & $539(4.8)$ & $-4(1.2)$ & (7) & $12(1.5)$ & $502(6.4)$ & $0(2.1)$ & \\
\hline France & & $67(1.7)$ & $531(2.2)$ & $-2(2.4)$ & & $19(0.8)$ & $518(3.0)$ & $3(1.1)$ & 0 & $14(1.3)$ & $496(3.9)$ & $-1(1.8)$ & \\
\hline Georgia & & $92(0.6)$ & $476(3.1)$ & $\Delta \diamond$ & & $6(0.5)$ & $448(6.4)$ & $\Delta \Delta$ & & $2(0.3)$ & $\sim \sim$ & $\Delta \Delta$ & \\
\hline Germany & & $71(1.2)$ & $564(2.1)$ & $-4(1.7)$ & 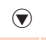 & $14(0.7)$ & $543(3.9)$ & $3(0.8)$ & 0 & $16(1.0)$ & $515(3.4)$ & $1(1.5)$ & \\
\hline Hong Kong SAR & $r$ & $44(1.5)$ & $562(2.9)$ & $7(2.4)$ & 0 & $26(0.9)$ & $562(3.1)$ & $4(1.1)$ & 0 & $29(1.4)$ & $572(3.0)$ & $-11(2.3)$ & ( \\
\hline Hungary & & $93(0.5)$ & $553(3.0)$ & $0(0.7)$ & & $4(0.5)$ & $541(6.8)$ & $0(0.6)$ & & $2(0.3)$ & $\sim \sim$ & $0(0.4)$ & \\
\hline Iceland & & $85(0.6)$ & $516(1.5)$ & $-2(0.9)$ & ( & $13(0.6)$ & $504(3.8)$ & $2(0.8)$ & 0 & $3(0.3)$ & $462(8.9)$ & $1(0.4)$ & \\
\hline Indonesia & $r$ & $84(1.1)$ & $415(4.3)$ & $\Delta \diamond$ & & $9(0.7)$ & $374(6.0)$ & $\Delta \Delta$ & & $7(0.6)$ & $374(7.0)$ & $\Delta \Delta$ & \\
\hline Iran, Islamic Rep. of & & $93(0.9)$ & $425(3.5)$ & $1(1.2)$ & & $3(0.4)$ & $383(11.7)$ & $-2(0.6)$ & ( & $4(0.7)$ & $430(7.7)$ & $0(0.8)$ & \\
\hline Israel & & $62(1.4)$ & $521(3.6)$ & $5(2.0)$ & 0 & $17(0.7)$ & $524(5.8)$ & $-3(1.1)$ & ( & $20(1.3)$ & $519(5.0)$ & $-2(1.8)$ & \\
\hline Italy & & $86(0.7)$ & $555(3.0)$ & $-3(1.0)$ & ( & $8(0.5)$ & $538(4.6)$ & $0(0.7)$ & & $6(0.6)$ & $524(7.0)$ & $2(0.7)$ & 0 \\
\hline Kuwait & $r$ & $67(1.4)$ & $344(5.0)$ & $\Delta \diamond$ & & $24(1.2)$ & $315(7.0)$ & $\Delta \diamond$ & & $9(0.7)$ & $350(12.0)$ & $\Delta \diamond$ & \\
\hline Latvia & & $58(1.3)$ & $543(2.7)$ & $-8(1.9)$ & ( & $21(0.9)$ & $537(4.3)$ & $-4(1.3)$ & ( & $21(1.1)$ & $547(3.7)$ & $11(1.5)$ & 0 \\
\hline Lithuania & & $89(0.7)$ & $540(1.7)$ & $1(1.1)$ & & $9(0.7)$ & $525(4.3)$ & $-1(1.0)$ & & $2(0.2)$ & $\sim \sim$ & $0(0.4)$ & \\
\hline Luxembourg & & $40(0.6)$ & $583(1.4)$ & $\Delta \Delta$ & & $20(0.5)$ & $568(2.1)$ & $\Delta \Delta$ & & $40(0.5)$ & $528(1.6)$ & $\Delta \Delta$ & \\
\hline Macedonia, Rep. of & & $86(0.8)$ & $453(3.9)$ & $-2(1.4)$ & & $11(0.7)$ & $427(8.0)$ & $2(1.1)$ & & $3(0.3)$ & $399(9.7)$ & $0(0.5)$ & \\
\hline Moldova, Rep. of & & $82(1.1)$ & $501(3.1)$ & $3(1.7)$ & & $13(0.8)$ & $504(4.7)$ & $-3(1.3)$ & ( & $5(0.4)$ & $497(8.8)$ & $0(0.7)$ & \\
\hline Morocco & & $83(1.2)$ & $335(6.2)$ & $0(1.7)$ & & $13(1.0)$ & $287(10.2)$ & $0(1.4)$ & & $4(0.5)$ & $286(11.9)$ & $0(0.7)$ & \\
\hline Netherlands & & $77(1.3)$ & $553(1.7)$ & $-2(1.9)$ & & $11(0.6)$ & $547(2.3)$ & $0(0.9)$ & & $12(1.3)$ & $513(3.1)$ & $2(1.8)$ & \\
\hline New Zealand & & $56(1.0)$ & $536(2.6)$ & $-2(1.9)$ & & $23(0.7)$ & $536(3.7)$ & $1(1.3)$ & & $20(1.1)$ & $536(3.9)$ & $1(1.8)$ & \\
\hline Norway & & $82(0.9)$ & $504(2.4)$ & $0(1.5)$ & & $12(0.7)$ & $500(3.8)$ & $0(1.1)$ & & $6(0.6)$ & $446(6.2)$ & $0(0.9)$ & \\
\hline Poland & & $97(0.3)$ & $522(2.3)$ & $\Delta \diamond$ & & $3(0.3)$ & $498(9.8)$ & $\Delta \Delta$ & & $0(0.1)$ & $\sim \sim$ & $\Delta \diamond$ & \\
\hline Qatar & & $54(0.6)$ & $344(1.8)$ & $\Delta 0$ & & $18(0.5)$ & $339(3.2)$ & $\Delta 0$ & & $28(0.5)$ & $391(2.8)$ & $\Delta 0$ & \\
\hline Romania & & $96(0.4)$ & $495(4.6)$ & $0(0.6)$ & & $3(0.4)$ & $452(13.5)$ & $0(0.5)$ & & $1(0.2)$ & $\sim \sim$ & $0(0.2)$ & \\
\hline Russian Federation & & $79(0.9)$ & $566(3.5)$ & $3(1.8)$ & & $14(0.6)$ & $568(4.5)$ & $-1(1.2)$ & & $7(0.5)$ & $557(6.0)$ & $-2(1.1)$ & \\
\hline Scotland & & $79(0.8)$ & $537(2.9)$ & $-1(1.3)$ & & $15(0.6)$ & $525(5.7)$ & $0(1.0)$ & & $6(0.6)$ & $484(7.8)$ & $1(0.7)$ & \\
\hline Singapore & & $60(0.6)$ & $559(3.0)$ & $-6(1.1)$ & ( & $25(0.6)$ & $559(3.6)$ & $3(0.9)$ & 0 & $15(0.5)$ & $566(4.3)$ & $3(0.8)$ & 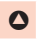 \\
\hline Slovak Republic & & $91(0.5)$ & $533(2.9)$ & $3(0.9)$ & 0 & $8(0.5)$ & $521(5.8)$ & $-1(0.8)$ & & $1(0.1)$ & $\sim \sim$ & $-1(0.5)$ & \\
\hline Slovenia & & $81(1.2)$ & $527(2.2)$ & $4(1.8)$ & 0 & $12(0.6)$ & $517(3.9)$ & $1(0.9)$ & & $8(0.8)$ & $488(4.7)$ & $-4(1.3)$ & (1) \\
\hline South Africa & $r$ & $69(1.0)$ & $326(6.5)$ & $\Delta \diamond$ & & $20(0.7)$ & $294(8.4)$ & $\Delta \Delta$ & & $11(0.5)$ & $266(9.2)$ & $\Delta \Delta$ & \\
\hline Spain & & $81(1.1)$ & $521(2.2)$ & $\Delta \Delta$ & & $8(0.5)$ & $509(5.2)$ & $\Delta \Delta$ & & $10(1.0)$ & $481(4.9)$ & $\Delta \Delta$ & \\
\hline Sweden & & $73(1.6)$ & $557(2.3)$ & $-3(2.3)$ & & $15(0.7)$ & $547(3.9)$ & $2(1.0)$ & & $13(1.2)$ & $520(5.0)$ & $1(2.0)$ & \\
\hline Trinidad and Tobago & & $81(0.9)$ & $438(4.9)$ & $\Delta \diamond$ & & $16(0.7)$ & $435(8.1)$ & $\Delta \Delta$ & & $4(0.4)$ & $448(11.2)$ & $\Delta \Delta$ & \\
\hline United States & & $66(1.8)$ & $552(3.9)$ & $-2(2.5)$ & & $16(0.7)$ & $533(5.1)$ & $1(1.0)$ & & $18(1.5)$ & $522(4.7)$ & $1(2.3)$ & \\
\hline International Avg. & & $76(0.2)$ & $508(0.5)$ & & & $14(0.1)$ & $491(1.0)$ & & & $10(0.1)$ & $476(1.2)$ & & \\
\hline
\end{tabular}

- Percent in 2006 significantly higher

(7) Percent in 2006 significantly lower

Background data provided by students.

() Standard errors appear in parentheses. Because results are rounded to the nearest whole number, some totals may appear inconsistent.

An " $r$ " indicates data are available for $70-84 \%$ of the students. An "s" indicates data are available for $50-69 \%$ of the students. An " $x$ " indicates data are available for less than $50 \%$ of the students.
A tilde $(\sim)$ indicates insufficient data to report achievement.

A diamond $(0)$ indicates the country did not participate in the 2001 assessment. NOTE: The International Average does not include the results from the Canadian provinces. Trend Note: The primary education systems of the Russian Federation and Slovenia underwent structural changes. Data for Canada, Ontario include only public schools. 




\section{Chapter 4}

\section{Students' Reading Attitudes,}

Self-Concept, and Out-of-School Activities

Positive student attitudes toward reading and a healthy reading self-concept are major objectives of the reading curriculum in most countries. Students who enjoy reading and who perceive themselves to be good readers usually read more frequently and more widely, which in turn broadens their reading experience and improves their comprehension skills. This chapter examines trends in students' attitudes toward reading and in their self-concept as readers, as well as the frequency with which they read stories, novels, and informational texts outside of school.

\section{What Are Students' Attitudes Toward Reading?}

The PIRLs Index of Students' Attitudes Toward Reading summarizes students' views on reading for enjoyment and appreciating books. The index was based on students' agreement with the following statements related to reading:

- I read only if I have to (reverse coded).

- I like talking about books with other people.

- I would be happy if someone gave me a book as a present.

- I think reading is boring (reverse coded).

- I enjoy reading. 
Students were asked to indicate their degree of agreement with each statement on a 4-point scale, as follows: agree a lot, agree a little, disagree a little, and disagree a lot. Responses to each statement were averaged for each student. Students who, on average, agreed a lot or agreed a little with the statements were assigned to the high category of the index. Students in the low category disagreed a lot or disagreed a little, on average, with the statements. Students in the medium category had other combinations of responses.

Exhibit 4.1 presents, for each country, the percentage of students in 2006 at each level of the index, together with their average reading achievement. To measure change in student attitudes since the previous PIRLS, the exhibit shows the difference from 2001 in the percentage of students at each level of the index, together with an indication of its statistical significance.

As was the case in 2001, fourth-grade students in 2006 generally had positive attitudes toward reading. On average internationally, almost half the students were at the high level of the index and more than 90 percent were at either the high or medium level. Only 8 percent of students, on average, had unfavorable attitudes toward reading (i.e., they were at the low level of the index). Countries with the greatest percentages of students with favorable attitudes toward reading $(60 \%$ or more at the high level of the index) included Iran, Italy, Macedonia, and Romania. Countries with increased percentages of students at the high level of the index in comparison with 2001 included Iran, Italy, Germany, and Hong Kong SAR, each of which also had increased average student achievement over that period (although the difference for Iran was not statistically significant). It may be a matter for concern that a greater number of participants had decreased percentages of students at the high level, including Moldova, Slovenia, Singapore, Sweden, Iceland, Scotland, England, the Netherlands, Latvia, and the Canadian province of Ontario.

On average internationally, and in every country, students at the high level of the index of positive attitudes toward reading had substantially higher average reading achievement than those at the medium or low levels. 
Exhibit 4.1 Index of Students' Attitudes Toward Reading (SATR) with Trends

PIRLS 2006

Countries

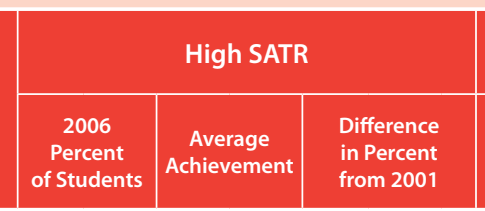

\begin{tabular}{|c|c|c|}
\hline \multicolumn{3}{|c|}{ Medium SATR } \\
\hline $\begin{array}{c}2006 \\
\text { Percent } \\
\text { of Students }\end{array}$ & $\begin{array}{c}\text { Average } \\
\text { Achievement }\end{array}$ & $\begin{array}{l}\text { Difference } \\
\text { in Percent } \\
\text { from } 2001\end{array}$ \\
\hline $21(1.2)$ & $382(5.6)$ & $-6(2.2)$ \\
\hline
\end{tabular}

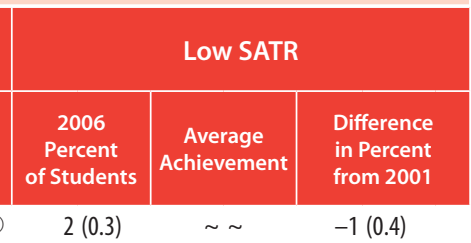

Iran,

$77(1.3)$

$437(3.0)$

$6(2.3)$

$64(1.4)$

$565(3.0)$

Macedonia, Rep. of

$63(1.5)$

$471(4.1)$

$8(1.9)$

Romania

$60(1.3)$

$513(5.0)$

$1(2.1)$

$58(1.5)$

$553(2.8)$

$0(2.1)$

- $31(1.2$

$531(3.4)$

$-6(2.2)$

$35(1.5)$

Canada, Quebec

$407(5.0)$

$-2(2.1)$

$5(0.5)$

$520(6.1)$

$-1(0.4)$

\begin{tabular}{|l|l|r|}
$58(1.1)$ & $569(2.5)$ & $8(1.4)$ \\
\hline
\end{tabular}

Moldova, Rep. of

$58(1.6)$

$514(3.3)$

France

Bulgaria

$57(0.9)$

542 (2.3)

Spain

$57(1.4)$

$567(4.3)$

$0(1.5)$

$462(6.2)$

$-2(2.1)$

$2(0.2)$

$512(2.6)$

$0(1.8)$

$4(0.4)$

$466(9.2)$

$1(0.3)$

Hong Kong SAR

56 (1.1)

Trinidad and Tobago

$55(1.1)$

$528(2.1)$

$-3(2.1)$

$41(1.6)$

$533(2.5)$

$-6(1.2)$

Morocco

$54(1.3)$

$579(2.3)$

$\diamond 0$

$38(0.8)$

484 (4.2)

$7(2.5)$

Slovenia

$53(2.0)$

$466(5.0)$

6 (1.6)

$37(1.2)$

498 (2.2)

$0(1.4)$

$8(0.4)$

$503(6.7)$

$3(0.5)$

$3(0.5)$
$-1(0.9)$

Chinese Taipei

52 (1.1)

351 (5.8)

00

$40(1.0)$

$527(5.2)$

$1(1.9)$

- $2(0.3)$

$516(4.1)$

Belgium (French)

$52(1.1)$

$542(2.0)$

$5(3.0)$

$41(1.0)$

$497(3.1)$

00

$4(0.4)$

485

$485(5.9) \quad 0(0.4)$

Russian Federation

$52(1.0)$

$553(2.1)$

$5(3.0)$

$41(1.2)$

$546(2.9)$

$-6(1.5)$

$4(0.4)$

$509(9.4)$

$400(5.4)$

$298(9.3)$

Georgia

$50(1.1)$

$521(2.9)$

$\diamond \diamond$

$40(0.9)$

Austria

$50(1.9)$

581 (3.6)

00

$44(1.0)$

$501(2.7)$

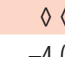

$\Delta>$
$-4(2.9)$

$4(0.3)$

$492(6.7)$

$539(5.0)$

$4(0.4)$

426 (9.7)

$\sim \sim \quad-1(1.0)$

Hungary

$50(1.2)$

$493(3.4)$

$-4(2.1)$

$42(0.9)$

$518(2.5)$

$\diamond \diamond$

$557(2.7)$

00

$45(1.0)$

$479(2.9)$

$\Delta 0$

$8(0.5)$

$493(4.4)$

$4(0.4)$
$6(0.4)$

$520(6.0) \quad \Delta \diamond$

$571(2.9)$

$\Delta \Delta$

$47(1.8)$

$550(3.7)$

3 (1.9)

$5(0.4)$

475 (5.1)

$540(5.0)$

$\Delta 0$

$49(1.0)$

$583(2.5)$

$0(1.8)$

$524(2.7)$

$\diamond \diamond \quad 3(0.4)$

Indonesia

New Zealand

$49(1.2)$

$432(3.8)$

$0(1.8)$

$39(1.2)$

$524(2.7)$

$\Delta 0$

$42(0.9)$

$532(4.3)$

$\Delta \diamond$
$-1(1.6)$

$10(0.7)$

$445(9.6)$

$1(0.6)$

Canada, Alberta

$48(1.0)$

$563(2.3)$

00

$50(1.2)$

Lithuania

$48(1.1)$

$584(2.4)$

$-3(1.7)$

$383(4.6)$

$\Delta \diamond$

$11(0.7)$

$510(3.8) \quad \diamond \diamond$

Norway

$47(1.1)$

$551(2.1)$

$\diamond \Delta$

$507(2.6)$

$\Delta \diamond \quad 1(0.1)$

$531(3.1) \quad 1(1.0)$

Singapore

$47(1.3)$

$519(2.4)$

$41(0.9)$

$542(2.7)$

$4(1.6) \quad 0 \quad 7(0.4)$

$524(4.3)$

$\Delta \Delta$

Canada, Ontario

$47(1.0)$

$1(1.8)$

$46(1.0)$

$525(2.0)$

$\diamond \diamond \quad 11(0.6)$

$493(47)$

$\Delta 0$

Canada, Nova Scotia

Slovak Republic

$46(1.4)$

$582(3.1)$

$3(1.7) \quad 45(1.2)$

$487(2.9)$

$-2(1.7)$

$11(0.6)$
$7(0.4)$

$531(3.6)$

$520(4.2) \quad 0(0.7)$

Sweden

$541(3.1)$

$-3(1.5)$

$8(0.8)$

$469(5.8) \quad-1(1.0)$

$-6(1.9)-42(1.0)$

\begin{tabular}{l|l|l|l|}
\hline $46(1.0)$ & $569(2.7)$ & $\diamond \diamond$ & $41(0.9)$ \\
\hline $46(1.3)$ & $553(2.7)$ & $2(2.0)$ & $46(1.2)$
\end{tabular}

$538(3.4)$

$3(1.5) \quad 0 \quad 8(0.4)$

$527(4.0)$

$(1.0)$

$526(2.5)$

$3(1.7)$

$12(0.9)$

$529(5.2)$

$3(1.0)$

$45(1.2) \quad 571(2.9) \quad-9(1.6) \quad \circledast \quad 44(1.0)$

$\begin{array}{lll}514(3.5) & -4(1.7) \quad \odot \quad 9(0.6)\end{array}$

$513(3.9) \quad \Delta 0$

Iceland 44

Israe

Scotland

$45(1.3)$

$\diamond \diamond \quad 44(1.0)$

$535(2.3)$

$44(0.8) \quad 533(1.6) \quad-4(1.3) \quad \odot \quad 49(0.8)$

$503(2.7)$

$5(1.4) \quad 0 \quad 10(0.6)$

$511(5.6) \quad 2(0.8) \quad 0$

$-00(0.7)$

$496(2.0) \quad 3(1.3) \quad 0 \quad 7(0.4)$

$519(3.5)$

$3(0.8)$

Kuwait $\quad r \quad 411$

England

$42(1.4)$

$547(3.7)$

$-2(1.8)$

$49(1.0)$

$495(3.9)$

$511(3.1)$

$1(1.6) \quad 9(0.7)$

$494(4.1)$

$\diamond \diamond$

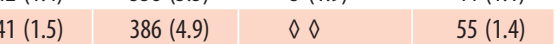

$313(5.4)$

$40(1.4) \quad 576(3.4) \quad-4(2.0) \quad \odot \quad 45(1.1)$

\begin{tabular}{|l|l|l|l|}
\hline $40(1.3)$ & $566(3.4)$ & $-3(1.7)$ & $46(1.1)$
\end{tabular}

$520(2.7)$

$2(1.6) \quad 14(1.0)$

$484(4.4)$

$1(0.6)$

United States

$40(0.6)$

Luxembourg

$39(1.3)$


At least for reading at this grade level, it seems that positive attitudes and high achievement in reading go hand in hand.

\section{What Are Students' Perceptions of Their Reading Ability?}

As was shown in PIRLS 2001, by the fourth grade, students in all countries can give an indication of their perception of themselves as good or poor readers, with a good deal of agreement between students' self-reports and average reading achievement. In PIRLS 2006, students' self-concept in reading was assessed based on their agreement with four statements about their ability to read:

- Reading is very easy for me.

- I do not read as well as other students in my class (reverse coded).

- When I am reading by myself, I understand almost everything I read.

- I read slower than other students in my class (reverse coded).

Response options were on a 4-point scale, as follows: agree a lot, agree a little, disagree a little, and disagree a lot. PIRLs combined students' responses to these four statements to construct an Index of Students' Reading SelfConcept. Students in the high category agreed (a lot or a little), on average, with the four statements about their reading ability. Students in the low category disagreed (a lot or a little), on average, with the statements. The medium level of the index includes all other response combinations. Exhibit 4.2 summarizes the results for this index.

Around the world, fourth-grade students generally seem to view themselves as good, or at least moderately good, readers. Almost half the students (49\%), on average internationally, were at the high level of the index of reading self-concept, and almost half (48\%) were at the medium level. Just 3 percent were at the low level of the index. Countries with the greatest percentages of high self-concept students (60\% or more) included Israel, Austria, Sweden, Poland, Norway, the Netherlands, and Denmark. Interestingly, although these countries all have average reading achievement above the PIRLS international scale average, there are other countries with 
higher average achievement. Countries with lesser percentages of students at the high level (less than 40\%) included Kuwait, Morocco, Belgium (French), New Zealand, France, Indonesia, Moldova, and South Africa.

Ten countries showed increased percentages of students at the high level in 2006 compared with $2001,{ }^{1}$ including Norway, the Netherlands, Iceland, Germany, Italy, the Russian Federation, Hong Kong sar, Latvia, Lithuania, and France. However, eight participants had decreased percentages at the high level-Macedonia, the United States, Romania, Morocco, New Zealand, Moldova, and the Canadian provinces of Ontario and Quebec.

As in PIRLS 2001, students' characterizations of themselves in terms of the levels of the reading self-concept index were broadly aligned with their average reading achievement. Students at the high level had average achievement 50 points higher than students at the medium level, and those at the medium level had average achievement more than 40 points higher than students at the low level. As noted above, however, there were few students in the low group in each country. 
Exhibit 4.2 Index of Students' Reading Self-Concept (SRSC) with Trends

PIRLS 2006

4th Grade

\begin{tabular}{|c|c|c|c|c|c|c|c|c|c|c|c|c|}
\hline \multirow[b]{2}{*}{ Countries } & \multicolumn{4}{|c|}{ High SRSC } & \multicolumn{4}{|c|}{ Medium SRSC } & \multicolumn{4}{|c|}{ Low SRSC } \\
\hline & $\begin{array}{c}2006 \\
\text { Percent } \\
\text { of Students }\end{array}$ & $\begin{array}{c}\text { Average } \\
\text { Achievement }\end{array}$ & $\begin{array}{l}\text { Difference } \\
\text { in Percent } \\
\text { from } 2001\end{array}$ & & $\begin{array}{c}2006 \\
\text { Percent } \\
\text { of Students }\end{array}$ & $\begin{array}{c}\text { Average } \\
\text { Achievement }\end{array}$ & $\begin{array}{l}\text { Difference } \\
\text { in Percen } \\
\text { from } 200\end{array}$ & & $\begin{array}{c}2006 \\
\text { Percent } \\
\text { of Students }\end{array}$ & $\begin{array}{c}\text { Average } \\
\text { Achievement }\end{array}$ & $\begin{array}{l}\text { Difference } \\
\text { in Percent } \\
\text { from } 2001\end{array}$ & \\
\hline Israel & $63(0.9)$ & $544(2.8)$ & $2(1.3)$ & & $36(0.9)$ & $477(4.6)$ & $-2(1.3)$ & & $1(0.2)$ & $\sim \sim$ & $0(0.3)$ & \\
\hline Austria & $62(0.9)$ & $553(2.4)$ & $\Delta \Delta$ & & $36(0.9)$ & $517(2.7)$ & $\Delta 0$ & & $2(0.3)$ & $\sim \sim$ & $\Delta 0$ & \\
\hline Sweden & $62(0.9)$ & $569(2.2)$ & $-2(1.3)$ & & $37(0.9)$ & $523(3.0)$ & $1(1.3)$ & & $2(0.2)$ & $\sim \sim$ & $1(0.3)$ & \\
\hline Poland & $61(0.9)$ & $547(2.2)$ & 00 & & $36(0.8)$ & $483(3.3)$ & $\Delta 0$ & & $2(0.3)$ & $\sim \sim$ & $\Delta 0$ & \\
\hline Norway & $61(1.2)$ & $518(2.4)$ & $5(1.6)$ & 0 & $37(1.2)$ & $477(3.5)$ & $-5(1.6)$ & (i) & $2(0.3)$ & $\sim \sim$ & $0(0.4)$ & \\
\hline Netherlands & $60(0.9)$ & $560(1.8)$ & $4(1.3)$ & 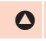 & $36(0.9)$ & $531(2.2)$ & $-7(1.2)$ & ( & $4(0.4)$ & $508(5.8)$ & $2(0.5)$ & 0 \\
\hline Denmark & $60(0.9)$ & $574(2.2)$ & $\Delta \diamond$ & & $38(0.9)$ & $511(3.1)$ & $\Delta \diamond$ & & $3(0.3)$ & $442(8.9)$ & $\Delta \diamond$ & \\
\hline Slovenia & $58(1.0)$ & $545(2.1)$ & $0(1.5)$ & & $40(1.0)$ & $491(2.4)$ & $-1(1.5)$ & & $2(0.2)$ & $\sim \sim$ & $1(0.4)$ & \\
\hline Iceland & $58(0.8)$ & $534(1.5)$ & $3(1.2)$ & 0 & $40(0.9)$ & $484(2.1)$ & $-3(1.2)$ & (i) & $2(0.3)$ & $\sim \sim$ & $1(0.4)$ & \\
\hline Germany & $58(0.9)$ & $571(2.4)$ & $5(1.2)$ & 0 & $40(0.9)$ & $529(2.2)$ & $-6(1.2)$ & $\nabla$ & $2(0.2)$ & $\sim \sim$ & $1(0.3)$ & \\
\hline Bulgaria & $58(1.4)$ & $570(3.9)$ & $0(2.0)$ & & $38(1.3)$ & $523(5.6)$ & $-3(1.9)$ & & $4(0.6)$ & $482(14.2)$ & $3(0.7)$ & 0 \\
\hline Italy & $56(1.1)$ & $569(3.2)$ & $6(1.7)$ & 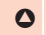 & $41(1.1)$ & $534(2.9)$ & $-7(1.7)$ & (i) & $3(0.3)$ & $496(9.2)$ & $1(0.4)$ & \\
\hline Macedonia, Rep. of & $55(1.3)$ & $486(3.9)$ & $-9(1.8)$ & 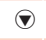 & $44(1.2)$ & $401(4.6)$ & $8(1.7)$ & 0 & $1(0.2)$ & $\sim \sim$ & $0(0.3)$ & \\
\hline Iran, Islamic Rep. of & $54(1.2)$ & $458(3.3)$ & $3(2.1)$ & & $44(1.2)$ & $383(3.8)$ & $-3(2.1)$ & & $2(0.2)$ & $\sim \sim$ & $0(0.3)$ & \\
\hline Canada, British Columbia & $54(0.9)$ & $584(2.5)$ & $\Delta 0$ & & $43(0.9)$ & $533(3.0)$ & $\Delta \Delta$ & & $2(0.3)$ & $\sim \sim$ & $\Delta \Delta$ & \\
\hline Qatar & $54(0.6)$ & $400(1.7)$ & $\Delta \Delta$ & & $43(0.6)$ & $309(1.7)$ & $\Delta \Delta$ & & $3(0.2)$ & $279(9.6)$ & $\Delta \Delta$ & \\
\hline Canada, Alberta & $53(0.9)$ & $584(2.8)$ & $\Delta \diamond$ & & $44(0.9)$ & $538(2.5)$ & $\Delta \diamond$ & & $3(0.3)$ & $505(6.6)$ & $\Delta \diamond$ & \\
\hline Russian Federation & $53(1.1)$ & $584(3.5)$ & $15(1.7)$ & 0 & $45(0.9)$ & $546(3.8)$ & $-15(1.5)$ & 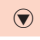 & $2(0.3)$ & $\sim \sim$ & $0(0.4)$ & \\
\hline Canada, Nova Scotia & $52(0.9)$ & $572(2.4)$ & $\Delta \Delta$ & & $45(0.9)$ & $518(2.3)$ & $\Delta \diamond$ & & $3(0.3)$ & $469(7.5)$ & $\Delta \diamond$ & \\
\hline United States & $51(0.8)$ & $566(3.5)$ & $-6(1.5)$ & 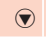 & $44(0.8)$ & $518(3.9)$ & $5(1.4)$ & 0 & $4(0.3)$ & $495(5.9)$ & $1(0.6)$ & \\
\hline Belgium (Flemish) & $51(1.0)$ & $565(2.0)$ & $\Delta \diamond$ & & $44(1.0)$ & $532(2.3)$ & $\Delta \diamond$ & & $5(0.4)$ & $502(4.3)$ & $\Delta \diamond$ & \\
\hline Georgia & $51(1.3)$ & $498(3.2)$ & $\Delta 0$ & & $46(1.2)$ & $450(3.7)$ & $\Delta \Delta$ & & $4(0.6)$ & $428(14.8)$ & 00 & \\
\hline Canada, Ontario & $51(1.0)$ & $579(2.8)$ & $-5(1.5)$ & 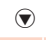 & $46(1.0)$ & $533(3.5)$ & $4(1.5)$ & 0 & $3(0.3)$ & $494(7.8)$ & $1(0.5)$ & \\
\hline Luxembourg & $50(0.7)$ & $582(1.3)$ & $\Delta \diamond$ & & $46(0.7)$ & $535(1.5)$ & $\Delta \Delta$ & & $4(0.3)$ & $497(4.5)$ & $\Delta \Delta$ & \\
\hline Romania & $50(1.4)$ & $524(4.2)$ & $-4(1.9)$ & $\odot$ & $45(1.3)$ & $467(5.6)$ & $0(1.8)$ & & $5(0.7)$ & $416(10.6)$ & $4(0.8)$ & 0 \\
\hline Canada, Quebec & $50(1.3)$ & $560(2.5)$ & $-9(1.8)$ & 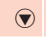 & $46(1.2)$ & $515(3.1)$ & $6(1.7)$ & 0 & $4(0.5)$ & $463(6.8)$ & $2(0.6)$ & $\boldsymbol{0}$ \\
\hline Trinidad and Tobago & $49(1.4)$ & $482(3.7)$ & $\Delta \diamond$ & & $47(1.3)$ & $399(5.8)$ & $\Delta \diamond$ & & $4(0.6)$ & $338(16.4)$ & $\Delta \Delta$ & \\
\hline Spain & $48(1.0)$ & $535(2.6)$ & $\Delta \Delta$ & & $50(1.0)$ & $495(2.7)$ & $\Delta \Delta$ & & $2(0.2)$ & $\sim \sim$ & $\Delta \Delta$ & \\
\hline Hong Kong SAR & $48(0.9)$ & $585(1.9)$ & $9(1.3)$ & 0 & $50(0.9)$ & $545(2.7)$ & $-7(1.3)$ & (1) & $2(0.2)$ & $\sim \sim$ & $-1(0.4)$ & \\
\hline Hungary & $45(1.3)$ & $579(2.7)$ & $-2(1.6)$ & & $51(1.1)$ & $531(3.3)$ & $1(1.4)$ & & $4(0.4)$ & $495(7.7)$ & $2(0.5)$ & 0 \\
\hline Chinese Taipei & $45(0.9)$ & $560(2.1)$ & $\Delta \diamond$ & & $51(0.9)$ & $518(2.2)$ & $\Delta \diamond$ & & $4(0.3)$ & $494(5.9)$ & $\Delta \diamond$ & \\
\hline Singapore & $45(0.9)$ & $583(2.9)$ & $-2(1.4)$ & & $52(0.8)$ & $542(3.0)$ & $2(1.3)$ & & $3(0.2)$ & $489(6.4)$ & $0(0.4)$ & \\
\hline Latvia & $43(1.2)$ & $567(2.6)$ & $9(1.5)$ & 0 & $53(1.2)$ & $523(3.0)$ & $-9(1.6)$ & $\nabla$ & $3(0.4)$ & $498(7.0)$ & $1(0.5)$ & \\
\hline Slovak Republic & $43(0.9)$ & $562(2.4)$ & $0(1.6)$ & & $54(0.9)$ & $512(3.3)$ & $-2(1.5)$ & & $4(0.3)$ & $459(7.0)$ & $2(0.5)$ & $\theta$ \\
\hline Scotland & $43(1.1)$ & $556(3.7)$ & $-1(1.7)$ & & $52(1.1)$ & $512(2.9)$ & $-1(1.6)$ & & $6(0.5)$ & $457(5.1)$ & $2(0.6)$ & 0 \\
\hline England & $42(1.1)$ & $578(3.5)$ & $-3(1.6)$ & & $51(1.1)$ & $519(2.7)$ & $0(1.6)$ & & $7(0.5)$ & $468(7.0)$ & $3(0.7)$ & 0 \\
\hline Lithuania & $40(0.9)$ & $561(2.1)$ & $5(1.4)$ & 0 & $57(0.8)$ & $523(1.9)$ & $-6(1.3)$ & $\odot$ & $3(0.3)$ & $493(6.8)$ & $1(0.5)$ & \\
\hline Kuwait & $38(1.3)$ & $384(5.2)$ & $\Delta 0$ & & $59(1.2)$ & $318(5.0)$ & $\Delta \Delta$ & & $3(0.3)$ & $247(11.6)$ & 00 & \\
\hline Morocco & $38(1.6)$ & $354(5.8)$ & $-8(2.4)$ & 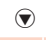 & $59(1.6)$ & $309(7.6)$ & $9(2.3)$ & 0 & $3(0.5)$ & $276(22.0)$ & $-1(1.1)$ & \\
\hline Belgium (French) & $38(0.9)$ & $526(3.1)$ & $\Delta \Delta$ & & $58(0.8)$ & $487(2.7)$ & $\Delta \Delta$ & & $4(0.5)$ & $454(6.1)$ & $\Delta \Delta$ & \\
\hline New Zealand & $36(0.8)$ & $574(2.2)$ & $-9(1.3)$ & $\odot$ & $60(0.8)$ & $513(2.4)$ & $8(1.3)$ & 0 & $4(0.3)$ & $459(6.1)$ & $1(0.5)$ & \\
\hline France & $36(0.8)$ & $549(3.1)$ & $3(1.3)$ & 0 & $60(0.9)$ & $510(1.9)$ & $-4(1.3)$ & $\nabla$ & $4(0.3)$ & $472(5.1)$ & $1(0.4)$ & \\
\hline Indonesia & $34(1.3)$ & $426(3.8)$ & $\Delta \Delta$ & & $65(1.2)$ & $398(4.7)$ & $\Delta \Delta$ & & $1(0.3)$ & $\sim \sim$ & $\Delta \diamond$ & \\
\hline Moldova, Rep. of & $32(1.3)$ & $525(3.6)$ & $-11(2.1)$ & 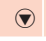 & $65(1.3)$ & $490(3.4)$ & $11(2.1)$ & 0 & $3(0.4)$ & $469(10.7)$ & $-1(0.7)$ & \\
\hline South Africa & $31(0.8)$ & $369(6.6)$ & $\Delta \diamond$ & & $64(0.8)$ & $282(5.3)$ & $\Delta \diamond$ & & $4(0.3)$ & $232(6.6)$ & $\Delta \diamond$ & \\
\hline International Avg. & $49(0.2)$ & $529(0.5)$ & & & $48(0.2)$ & $479(0.6)$ & & & $3(0.1)$ & $436(1.9)$ & & \\
\hline
\end{tabular}

Based on students' responses to the following: reading is very easy for me, I do not read as well as other students in my class, when I am reading by myself I understand almost everything I read, and I read slower than other students in my class. Average is computed on a 4-point scale: Disagree a lot $=1$, Disagree a little $=2$, Agree a little $=3$, and Agree a lot $=4$. Responses for negative statements were reverse-coded. High indicates an average of greater than 3 through 4. Medium indicates an average of 2 through 3. Low indicates an average of 1 to less than 2 .

Please note that "I read slower than other students in my class" is a new variable added to the index in PIRLS 2006, and is not a part of the PIRLS 2001 index calculations.

() Standard errors appear in parentheses. Because results are rounded to the nearest whole number, some totals may appear inconsistent.
An " $r$ " indicates data are available for $70-84 \%$ of the students. An " $s$ " indicates data are available for $50-69 \%$ of the students. An " $x$ " indicates data are available for less than $50 \%$ of the students.

A tilde $(\sim)$ indicates insufficient data to report achievement.

A diamond $(\diamond)$ indicates the country did not participate in the 2001 assessment.

NOTE: The International Average does not include the results from the Canadian provinces. Trend Note: The primary education systems of the Russian Federation and Slovenia underwent structural changes. Data for Canada, Ontario include only public schools. 


\section{How Often Do Children Engage in Literacy Activities Outside of School?}

Exhibit 4.3 presents trends in the frequency with which students read stories or novels outside of school. The exhibit shows the percentage of students in each country in 2006 that reported reading stories or novels, as follows: every day or almost every day, once or twice a week, once or twice a month, and never or almost never, as well as the average reading achievement of those students. Also presented for those countries that participated in both cycles of PIRLs is the change in the percentage of students in each of these categories since 2001.

On average across countries, nearly one third of students (32\%) reported reading stories or novels outside of school every day or almost every day, and an additional one third (31\%) at least once a week. PIRLS 2006 participants with the highest percentages of frequent readers of novels and stories (at least $50 \%$ of students reading every day or almost every day) included four of the Canadian provinces (British Columbia, Nova Scotia, Alberta, and Ontario) and the Russian Federation. Although most countries had a majority of students reading novels and stories at least weekly, many countries also had a significant percentage of nonreaders. Countries where the percentage of students reporting never or almost never reading a novel or short story exceeded 25\% included Germany, Spain, Belgium (French), Luxembourg, Norway, Austria, Bulgaria, Italy, Slovenia, Latvia, and the Slovak Republic.

A number of participants showed an increase in the percentage of students reading stories or novels every day or almost every day, including Israel, Hungary, Hong Kong sar, Germany, Morocco, Romania, Norway, Italy, and the Canadian province of Quebec. The greatest increases were found in Israel and Hong Kong SAR (16\%). Countries with a decrease in the percentage of students in this frequent reader category included the Russian Federation, the Netherlands, Singapore, Macedonia, Iceland, Scotland, England, Sweden, Bulgaria, Slovenia, Moldova, and Latvia. Countries with increased percentages in the never or almost never category included Scotland, England, Sweden, Bulgaria, Moldova, and Latvia. 
Exhibit 4.3 Students Reading Stories or Novels Outside of School with Trends

PIRLS 2006

4th Grade

\begin{tabular}{|c|c|c|c|c|c|c|c|c|}
\hline \multirow[b]{2}{*}{ Countries } & \multicolumn{4}{|c|}{ Every Day or Almost Every Day } & \multicolumn{4}{|c|}{ Once or Twice a Week } \\
\hline & $\begin{array}{c}2006 \\
\text { Percent } \\
\text { of Students }\end{array}$ & $\begin{array}{c}\text { Average } \\
\text { Achievement }\end{array}$ & $\begin{array}{l}\text { Difference } \\
\text { in Percent } \\
\text { from } 2001\end{array}$ & & $\begin{array}{c}2006 \\
\text { Percent } \\
\text { of Students }\end{array}$ & $\begin{array}{c}\text { Average } \\
\text { Achievement }\end{array}$ & $\begin{array}{l}\text { Difference } \\
\text { in Percent } \\
\text { from } 2001\end{array}$ & \\
\hline Canada, British Columbia & $57(1.2)$ & $572(2.5)$ & $\Delta \Delta$ & & $26(0.7)$ & $552(3.2)$ & $\Delta \Delta$ & \\
\hline Canada, Nova Scotia & $54(1.0)$ & $557(2.4)$ & $\Delta \Delta$ & & $26(0.8)$ & $534(2.9)$ & $\Delta \Delta$ & \\
\hline Canada, Alberta & $53(1.2)$ & $577(2.7)$ & $\Delta \Delta$ & & $27(0.9)$ & $549(3.5)$ & $\Delta \diamond$ & \\
\hline Canada, Ontario & $50(1.7)$ & $566(3.2)$ & $0(2.0)$ & & $28(1.4)$ & $548(3.0)$ & $0(1.7)$ & \\
\hline Russian Federation & $50(1.1)$ & $568(3.6)$ & $-6(1.9)$ & $(\uparrow$ & $32(0.7)$ & $565(3.6)$ & $3(1.3)$ & $\theta$ \\
\hline Trinidad and Tobago & $47(1.4)$ & $452(5.8)$ & $\Delta \diamond$ & & $31(1.3)$ & $431(6.6)$ & $\Delta \diamond$ & \\
\hline New Zealand & $46(1.1)$ & $556(2.3)$ & $0(1.9)$ & & $29(0.8)$ & $526(2.6)$ & $-1(1.4)$ & \\
\hline Israel & $46(1.0)$ & $532(4.2)$ & $16(1.5)$ & $\theta$ & $32(0.8)$ & $513(3.8)$ & $6(1.3)$ & $\theta$ \\
\hline Netherlands & $45(1.0)$ & $558(2.0)$ & $-3(1.5)$ & ( & $28(0.7)$ & $542(1.8)$ & $2(1.1)$ & $\theta$ \\
\hline Singapore & $44(1.1)$ & $579(3.0)$ & $-9(1.6)$ & ( & $33(0.8)$ & $555(3.1)$ & $6(1.1)$ & $\theta$ \\
\hline Macedonia, Rep. of & $44(1.5)$ & $442(5.1)$ & $-4(2.1)$ & $(\nabla$ & $35(1.3)$ & $449(5.0)$ & $1(1.8)$ & \\
\hline Canada, Quebec & $44(1.4)$ & $546(3.3)$ & $5(1.9)$ & $\theta$ & $28(0.8)$ & $530(3.1)$ & $-1(1.3)$ & \\
\hline Iceland & $42(0.9)$ & $522(2.4)$ & $-2(1.1)$ & ( & $27(0.8)$ & $512(2.3)$ & $1(1.0)$ & \\
\hline South Africa & $39(1.0)$ & $308(7.0)$ & $\Delta \Delta$ & & $33(0.6)$ & $310(6.7)$ & $\Delta \Delta$ & \\
\hline Qatar & $37(0.6)$ & $361(2.1)$ & $\Delta \Delta$ & & $37(0.6)$ & $359(2.0)$ & $\Delta \Delta$ & \\
\hline United States & $36(1.3)$ & $558(4.6)$ & $2(2.0)$ & & $28(0.8)$ & $541(3.6)$ & $-1(1.3)$ & \\
\hline Hungary & $36(1.2)$ & $554(4.6)$ & $3(1.5)$ & $\theta$ & $34(0.9)$ & $553(3.5)$ & $1(1.3)$ & \\
\hline Hong Kong SAR & $36(0.9)$ & $575(2.4)$ & $16(1.3)$ & 0 & $40(0.8)$ & $568(2.6)$ & $-5(1.2)$ & ( \\
\hline Scotland & $35(1.3)$ & $555(4.2)$ & $-5(1.8)$ & ( & $29(0.9)$ & $525(3.3)$ & $-2(1.4)$ & \\
\hline Kuwait & $33(1.2)$ & $351(5.2)$ & $\Delta \nu$ & & $40(1.0)$ & $337(4.9)$ & $\Delta \Delta$ & \\
\hline England & $33(1.2)$ & $573(3.9)$ & $-5(1.8)$ & $(\nabla$ & $30(1.0)$ & $535(3.2)$ & $-1(1.4)$ & \\
\hline Germany & $32(0.7)$ & $566(3.0)$ & $5(1.0)$ & 0 & $21(0.8)$ & $547(3.7)$ & $0(1.1)$ & \\
\hline France & $32(1.0)$ & $533(2.9)$ & $2(1.5)$ & & $30(0.8)$ & $524(2.3)$ & $1(1.2)$ & \\
\hline Georgia & $32(1.5)$ & $467(3.5)$ & $\Delta \Delta$ & & $32(1.3)$ & $479(3.5)$ & $\Delta \Delta$ & \\
\hline Belgium (Flemish) & $31(0.9)$ & $557(2.8)$ & $\Delta \Delta$ & & $34(0.9)$ & $546(2.4)$ & $\Delta \Delta$ & \\
\hline Spain & $31(1.1)$ & $511(3.7)$ & $\Delta \Delta$ & & $24(0.8)$ & $515(3.0)$ & $\Delta \Delta$ & \\
\hline Poland & $31(1.1)$ & $524(3.3)$ & $\Delta \Delta$ & & $37(1.2)$ & $524(3.1)$ & $\Delta \Delta$ & \\
\hline Sweden & $30(0.9)$ & $564(3.2)$ & $-3(1.3)$ & ( & $33(0.9)$ & $549(3.4)$ & $-1(1.2)$ & \\
\hline Chinese Taipei & $30(0.8)$ & $549(2.7)$ & $\Delta \diamond$ & & $36(0.9)$ & $541(2.4)$ & $\Delta \Delta$ & \\
\hline Denmark & $29(1.1)$ & $558(3.2)$ & $\Delta \Delta$ & & $26(0.8)$ & $548(3.2)$ & $\Delta \Delta$ & \\
\hline Belgium (French) & $28(0.9)$ & $509(3.7)$ & $\Delta \Delta$ & & $27(0.8)$ & $498(3.5)$ & $\Delta \Delta$ & \\
\hline Lithuania & $28(1.0)$ & $542(2.5)$ & $1(1.6)$ & & $30(0.9)$ & $538(2.5)$ & $-1(1.3)$ & \\
\hline Morocco & $27(1.2)$ & $334(6.2)$ & $9(1.6)$ & $\theta$ & $36(1.5)$ & $334(6.3)$ & $2(2.2)$ & \\
\hline Romania & $27(1.4)$ & $491(6.1)$ & $7(1.9)$ & $\theta$ & $34(1.3)$ & $497(5.8)$ & $-4(1.9)$ & ( \\
\hline Iran, Islamic Rep. of & $25(0.9)$ & $435(5.8)$ & $2(1.3)$ & & $43(1.3)$ & $429(3.6)$ & $1(1.6)$ & \\
\hline Luxembourg & $24(0.6)$ & $587(2.6)$ & $\Delta \Delta$ & & $24(0.6)$ & $554(2.6)$ & $\Delta \Delta$ & \\
\hline Norway & $23(1.1)$ & $509(3.2)$ & $5(1.4)$ & $\theta$ & $24(0.9)$ & $501(3.5)$ & $-1(1.2)$ & \\
\hline Austria & $23(0.9)$ & $554(3.0)$ & $\Delta \Delta$ & & $23(0.8)$ & $541(3.0)$ & $\Delta 0$ & \\
\hline Indonesia & $22(1.0)$ & $399(4.8)$ & $\Delta \Delta$ & & $36(1.1)$ & $413(4.4)$ & $\Delta \diamond$ & \\
\hline Bulgaria & $22(1.0)$ & $558(6.4)$ & $-6(1.6)$ & ( & $26(1.0)$ & $555(4.9)$ & $-2(1.5)$ & \\
\hline Italy & $22(1.2)$ & $573(4.4)$ & 7 (1.4) & $\theta$ & $20(1.0)$ & $556(3.3)$ & $2(1.2)$ & \\
\hline Slovenia & $21(0.8)$ & $536(3.0)$ & $-5(1.4)$ & ( & $29(0.8)$ & $520(3.0)$ & $2(1.3)$ & \\
\hline Moldova, Rep. of & $19(1.0)$ & $499(4.4)$ & $-4(1.8)$ & 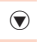 & $37(1.2)$ & $503(3.7)$ & $-7(2.2)$ & $\ominus$ \\
\hline Latvia & $19(0.9)$ & $558(4.0)$ & $-4(1.3)$ & ( & $25(1.1)$ & $548(3.6)$ & $-2(1.4)$ & \\
\hline Slovak Republic & $15(0.7)$ & $540(4.5)$ & $0(1.1)$ & & $21(0.9)$ & $536(3.5)$ & $0(1.3)$ & \\
\hline International Avg. & $32(0.2)$ & $512(0.6)$ & & & $31(0.2)$ & $503(0.6)$ & & \\
\hline
\end{tabular}

Background data provided by students.

() Standard errors appear in parentheses. Because results are rounded to the nearest whole number, some totals may appear inconsistent.

A diamond $(\diamond)$ indicates the country did not participate in the 2001 assessment.
NOTE: The International Average does not include the results from the Canadian provinces. Trend Note: The primary education systems of the Russian Federation and Slovenia underwent structural changes. Data for Canada, Ontario include only public schools. 


\section{Exhibit 4.3 Students Reading Stories or Novels Outside of School with Trends (Continued)}

\begin{tabular}{|c|c|c|c|c|c|c|c|c|}
\hline \multirow[b]{2}{*}{ Countries } & \multicolumn{4}{|c|}{ Once or Twice a Month } & \multicolumn{4}{|c|}{ Never or Almost Never } \\
\hline & $\begin{array}{c}2006 \\
\text { Percent } \\
\text { of Students }\end{array}$ & $\begin{array}{c}\text { Average } \\
\text { Achievement }\end{array}$ & $\begin{array}{l}\text { Difference } \\
\text { in Percent } \\
\text { from } 2001\end{array}$ & & $\begin{array}{c}2006 \\
\text { Percent } \\
\text { of Students }\end{array}$ & $\begin{array}{c}\text { Average } \\
\text { Achievement }\end{array}$ & $\begin{array}{l}\text { Difference } \\
\text { in Percent } \\
\text { from } 2001\end{array}$ & \\
\hline Canada, British Columbia & $11(0.7)$ & $539(4.1)$ & $\Delta \Delta$ & & $6(0.6)$ & $511(7.0)$ & $\Delta \Delta$ & \\
\hline Canada, Nova Scotia & $12(0.5)$ & $531(3.9)$ & $\Delta \Delta$ & & $8(0.5)$ & $497(6.3)$ & $\Delta \Delta$ & \\
\hline Canada, Alberta & $12(0.7)$ & $544(3.4)$ & $\Delta \diamond$ & & $8(0.6)$ & $523(4.9)$ & $\Delta \diamond$ & \\
\hline Canada, Ontario & $14(0.9)$ & $544(5.1)$ & $0(1.1)$ & & $7(0.7)$ & $520(7.5)$ & $1(1.0)$ & \\
\hline Russian Federation & $11(0.6)$ & $563(4.9)$ & $2(0.9)$ & $\boldsymbol{\theta}$ & $7(0.6)$ & $548(5.4)$ & $1(0.8)$ & \\
\hline Trinidad and Tobago & $10(0.6)$ & $420(7.1)$ & $\Delta \diamond$ & & $11(1.0)$ & $408(7.2)$ & $\Delta \diamond$ & \\
\hline New Zealand & $14(0.6)$ & $513(3.9)$ & $1(1.0)$ & & $10(0.6)$ & $473(4.2)$ & $0(1.0)$ & \\
\hline Israel & $15(0.6)$ & $495(4.8)$ & $1(0.9)$ & & $7(0.5)$ & $481(7.2)$ & $-22(1.4)$ & ( \\
\hline Netherlands & $13(0.6)$ & $540(2.2)$ & $0(0.9)$ & & $14(0.7)$ & $531(3.2)$ & $0(1.1)$ & \\
\hline Singapore & $15(0.5)$ & $534(3.5)$ & $4(0.7)$ & 0 & $8(0.4)$ & $505(4.6)$ & $-1(0.7)$ & \\
\hline Macedonia, Rep. of & $16(1.1)$ & $452(7.1)$ & $2(1.6)$ & & $5(0.4)$ & $445(10.1)$ & $1(0.6)$ & \\
\hline Canada, Quebec & $16(0.9)$ & $528(3.4)$ & $-1(1.3)$ & & $12(0.9)$ & $502(5.1)$ & $-4(1.6)$ & ( \\
\hline Iceland & $16(0.6)$ & $508(2.8)$ & $2(0.8)$ & $\boldsymbol{\theta}$ & $15(0.6)$ & $486(2.9)$ & $-1(0.8)$ & \\
\hline South Africa & $14(0.5)$ & $302(6.6)$ & $\Delta \Delta$ & & $13(0.7)$ & $294(7.2)$ & $\Delta \Delta$ & \\
\hline Qatar & $15(0.4)$ & $347(3.4)$ & $\Delta \diamond$ & & $10(0.4)$ & $342(4.4)$ & $\Delta \diamond$ & \\
\hline United States & $18(0.7)$ & $539(3.6)$ & $1(1.0)$ & & $18(0.9)$ & $509(3.2)$ & $-3(1.3)$ & \\
\hline Hungary & $20(0.9)$ & $553(2.9)$ & $-2(1.2)$ & $(\nabla$ & $10(0.7)$ & $535(6.7)$ & $-2(1.0)$ & \\
\hline Hong Kong SAR & $17(0.7)$ & $550(3.3)$ & $-6(1.0)$ & ( & $7(0.5)$ & $518(5.0)$ & $-5(0.8)$ & ( \\
\hline Scotland & $18(0.9)$ & $521(3.8)$ & $3(1.2)$ & 0 & $17(1.2)$ & $484(4.8)$ & $5(1.5)$ & 0 \\
\hline Kuwait & $19(0.8)$ & $326(5.8)$ & 00 & & $8(0.7)$ & $312(8.9)$ & $\Delta \diamond$ & \\
\hline England & $20(0.7)$ & $536(4.2)$ & $3(1.0)$ & 0 & $17(0.8)$ & $492(3.5)$ & $3(1.2)$ & 0 \\
\hline Germany & $16(0.5)$ & $550(3.6)$ & $1(0.8)$ & & $31(0.9)$ & $535(2.6)$ & $-6(1.4)$ & ( \\
\hline France & $19(0.6)$ & $522(2.8)$ & $-1(0.9)$ & & $19(0.9)$ & $501(2.9)$ & $-2(1.3)$ & \\
\hline Georgia & $20(1.4)$ & $484(6.8)$ & $\Delta \Delta$ & & $17(1.2)$ & $459(5.6)$ & $\Delta \Delta$ & \\
\hline Belgium (Flemish) & $21(0.8)$ & $545(3.0)$ & $\Delta \Delta$ & & $14(0.8)$ & $530(3.3)$ & $\Delta \diamond$ & \\
\hline Spain & $15(0.6)$ & $523(3.7)$ & $\Delta 0$ & & $29(1.0)$ & $508(3.2)$ & $\Delta 0$ & \\
\hline Poland & $21(0.8)$ & $516(3.7)$ & $\Delta \diamond$ & & $11(0.8)$ & $504(4.6)$ & $\Delta \diamond$ & \\
\hline Sweden & $22(0.8)$ & $546(2.7)$ & $0(1.0)$ & & $15(0.8)$ & $529(3.4)$ & $3(1.0)$ & 0 \\
\hline Chinese Taipei & $20(0.7)$ & $531(3.2)$ & $\Delta \Delta$ & & $15(0.7)$ & $505(3.1)$ & $\Delta \diamond$ & \\
\hline Denmark & $20(0.8)$ & $551(3.0)$ & $\Delta \Delta$ & & $25(1.1)$ & $529(3.0)$ & $\Delta \Delta$ & \\
\hline Belgium (French) & $18(0.7)$ & $507(3.3)$ & $\Delta \diamond$ & & $27(0.9)$ & $488(3.0)$ & $\Delta \diamond$ & \\
\hline Lithuania & $19(0.7)$ & $540(2.6)$ & $-1(1.2)$ & & $23(1.0)$ & $528(2.4)$ & $1(1.5)$ & \\
\hline Morocco & $21(1.5)$ & $327(10.2)$ & $-4(1.9)$ & & $16(2.5)$ & $282(16.8)$ & $-8(3.5)$ & $\nabla$ \\
\hline Romania & $23(1.3)$ & $503(5.8)$ & $-4(1.8)$ & ( & $16(1.1)$ & $463(9.8)$ & $2(1.7)$ & \\
\hline Iran, Islamic Rep. of & $22(0.9)$ & $423(4.3)$ & $1(1.3)$ & & $10(1.0)$ & $356(9.3)$ & $-5(1.6)$ & 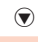 \\
\hline Luxembourg & $21(0.5)$ & $553(2.4)$ & $\Delta \Delta$ & & $31(0.6)$ & $539(1.8)$ & $\Delta \diamond$ & \\
\hline Norway & $21(0.8)$ & $503(5.1)$ & $1(1.2)$ & & $32(1.2)$ & $488(3.5)$ & $-5(1.8)$ & $\nabla$ \\
\hline Austria & $18(0.7)$ & $537(3.8)$ & $\Delta \Delta$ & & $37(1.1)$ & $528(2.6)$ & $\Delta \Delta$ & \\
\hline Indonesia & $16(0.7)$ & $411(5.7)$ & $\Delta \diamond$ & & $25(1.2)$ & $406(6.0)$ & $\Delta \Delta$ & \\
\hline Bulgaria & $23(1.0)$ & $555(6.0)$ & $2(1.4)$ & & $29(1.3)$ & $529(5.2)$ & $7(2.0)$ & 0 \\
\hline Italy & $15(0.8)$ & $554(4.1)$ & $-1(1.0)$ & & $43(1.3)$ & $540(3.3)$ & $-7(1.7)$ & ( \\
\hline Slovenia & $23(0.8)$ & $522(3.0)$ & $4(1.3)$ & 0 & $28(0.9)$ & $513(2.5)$ & $0(1.7)$ & \\
\hline Moldova, Rep. of & $23(0.9)$ & $504(4.1)$ & $0(1.8)$ & & $21(1.1)$ & $493(4.4)$ & $11(1.6)$ & $\theta$ \\
\hline Latvia & $21(0.9)$ & $544(3.6)$ & $1(1.2)$ & & $36(1.4)$ & $526(2.5)$ & $6(1.7)$ & 0 \\
\hline Slovak Republic & $21(0.7)$ & $540(3.7)$ & $-1(1.1)$ & & $43(1.1)$ & $522(3.9)$ & $1(1.7)$ & \\
\hline International Avg. & $18(0.1)$ & $500(0.7)$ & & & $19(0.2)$ & $479(0.9)$ & & \\
\hline
\end{tabular}

- Percent in 2006 significantly higher

( Percent in 2006 significantly lower 
On average internationally, and in most countries, students who reported reading novels and short stories most frequently had higher average achievement than those who read less frequently. Students who never or almost never did this kind of reading had the lowest achievement in most countries. The average achievement difference between students who read novels or short stories daily or almost daily and those who never or almost never read them was 33 points on the PIRLS reading scale.

Although literary texts such as short stories and novels make up a large part of the reading material of fourth-grade students outside of school, there are also many kinds of informational texts that children may read, such as magazines, newspapers, books that explain things, directions, or instructions. Exhibit 4.4 presents students' reports of how often they read such materials, together with average student reading achievement and changes since 2001. The PIRLS Reading for Information Scale was based on student responses to six questions/statements, as follows:

How often do you do these things outside of school?

- I read to find out about things I want to learn.

How often do you read these things after school?

- I read books that explain things.

- I read magazines.

- I read newspapers.

- I read directions or instructions.

- I read brochures or catalogs.

Student responses were recorded on a 4-point scale, as follows: every day or almost every day, once or twice a week, once or twice a month, and never or almost never, and students were assigned to one of the four categories of reading frequency for information on the basis of their average response (see Exhibit 4.4).

Compared to literary reading, which for many students was a daily or at least weekly occurrence, student reading of informational texts was 
more intermittent. Fewer students, on average internationally, reported daily informational reading (16\%) than literary reading (32\%), and relatively more reported reading for information once or twice a week or once or twice a month (72\% informational vs. $49 \%$ literary). Countries with the greatest percentages of students reporting frequent informational reading (30\% or more) included South Africa, Macedonia, Qatar, and Kuwait. Only two countries, Hong Kong SAR and Sweden, had greater percentages of students in 2006 than in 2001 reporting frequent reading for information. In contrast, several participants (Slovenia, Lithuania, Bulgaria, Singapore, Latvia, the United States, Iran, and the provinces of Ontario and Quebec) showed a decrease from 2001 to 2006 in the percentage of students reading informational texts daily or almost daily.

Not only was student reading for information more intermittent than literary reading, it also had a less clear-cut relationship with student reading achievement. Unlike literary reading, where students who read most frequently (every day or almost every day) had higher average achievement, average achievement for informational reading was highest among students who reported reading once or twice a week or once or twice a month.

In view of the widespread access to the Internet in today's world, and the reading opportunities that the Internet provides over and above those provided by print media, PIRLS 2006 asked students how much time they spent reading stories or articles on the Internet and how much time they spent reading them in books or magazines. Exhibit 4.5 presents the average number of hours on a typical day students in each country reported reading on the Internet and reading from books and magazines, separately for girls and boys and for all students together.

As might be expected, on average across countries, students reported spending more time on a typical day reading stories and articles in books or magazines than on the Internet (1.4 hours vs. 1.0 hours). Students in Kuwait, Qatar, and South Africa reported spending the most time reading on the Internet (more than 2 hours, on average, on a typical day), whereas in Iran, Luxembourg, the Netherlands, the Russian Federation, and Sweden, 
Exhibit 4.4 Students Reading for Information Outside of School with Trends

PIRLS 2006

4th Grade

\begin{tabular}{|c|c|c|c|c|c|c|c|c|}
\hline \multirow[b]{2}{*}{ Countries } & \multicolumn{4}{|c|}{ Every Day or Almost Every Day } & \multicolumn{4}{|c|}{ Once or Twice a Week } \\
\hline & $\begin{array}{c}2006 \\
\text { Percent } \\
\text { of Students }\end{array}$ & $\begin{array}{c}\text { Average } \\
\text { Achievement }\end{array}$ & $\begin{array}{l}\text { Difference } \\
\text { in Percent } \\
\text { from } 2001\end{array}$ & & $\begin{array}{c}2006 \\
\text { Percent } \\
\text { of Students }\end{array}$ & $\begin{array}{c}\text { Average } \\
\text { Achievement }\end{array}$ & $\begin{array}{l}\text { Difference } \\
\text { in Percent } \\
\text { from } 2001\end{array}$ & \\
\hline South Africa & $36(1.1)$ & $302(6.0)$ & $\Delta \Delta$ & & $45(0.7)$ & $307(5.8)$ & $\Delta \Delta$ & \\
\hline Macedonia, Rep. of & $33(1.3)$ & $440(5.4)$ & $-3(1.9)$ & & $48(1.1)$ & $453(4.4)$ & $1(1.7)$ & \\
\hline Qatar & $33(0.6)$ & $359(1.9)$ & $\Delta \Delta$ & & $49(0.6)$ & $355(1.7)$ & $\Delta \Delta$ & \\
\hline Kuwait & $30(1.4)$ & $342(5.6)$ & $\Delta \Delta$ & & $50(1.1)$ & $343(4.5)$ & $\Delta \Delta$ & \\
\hline Trinidad and Tobago & $29(1.4)$ & $440(6.2)$ & $\Delta \Delta$ & & $48(1.2)$ & $435(5.9)$ & $\Delta \Delta$ & \\
\hline Israel & $21(0.8)$ & $499(5.4)$ & $-1(1.3)$ & & $44(1.0)$ & $510(4.0)$ & $1(1.4)$ & \\
\hline Poland & $21(0.8)$ & $523(2.9)$ & $\Delta \Delta$ & & $46(0.9)$ & $522(3.3)$ & $\Delta \Delta$ & \\
\hline Slovak Republic & $20(1.0)$ & $527(4.2)$ & $1(1.3)$ & & $50(0.9)$ & $539(2.5)$ & $0(1.4)$ & \\
\hline Moldova, Rep. of & $19(1.0)$ & $497(4.0)$ & $1(1.8)$ & & $50(1.3)$ & $503(3.9)$ & $5(2.3)$ & \\
\hline Indonesia & $19(0.9)$ & $409(5.4)$ & $\Delta \Delta$ & & $50(1.1)$ & $409(4.4)$ & $\Delta \Delta$ & \\
\hline Hungary & $19(0.9)$ & $533(4.6)$ & $0(1.2)$ & & $50(0.9)$ & $552(3.5)$ & $4(1.3)$ & $\theta$ \\
\hline Russian Federation & $18(0.9)$ & $555(4.2)$ & $-2(1.4)$ & & $47(0.7)$ & $564(3.7)$ & $-1(1.4)$ & \\
\hline Slovenia & $18(0.6)$ & $510(3.3)$ & $-5(1.3)$ & ( & $49(0.9)$ & $519(2.6)$ & $2(1.4)$ & \\
\hline Lithuania & $18(0.8)$ & $530(3.3)$ & $-4(1.3)$ & $(\nabla$ & $53(0.9)$ & $538(1.9)$ & $1(1.3)$ & \\
\hline Bulgaria & $17(1.1)$ & $544(6.7)$ & $-10(1.6)$ & ( & $47(1.5)$ & $556(4.5)$ & $2(1.9)$ & \\
\hline Romania & $16(1.0)$ & $493(6.1)$ & $-3(1.7)$ & & $49(1.4)$ & $500(5.4)$ & $1(1.9)$ & \\
\hline Singapore & $16(0.5)$ & $558(3.5)$ & $-8(0.9)$ & ( & $47(0.8)$ & $561(3.2)$ & $0(1.0)$ & \\
\hline Latvia & $16(0.8)$ & $530(4.8)$ & $-3(1.3)$ & $(\nabla$ & $48(0.9)$ & $541(2.9)$ & $-3(1.7)$ & 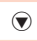 \\
\hline Georgia & $16(1.3)$ & $465(4.5)$ & $\Delta \Delta$ & & $43(1.3)$ & $480(3.0)$ & $\Delta \diamond$ & \\
\hline Germany & $15(0.6)$ & $536(3.3)$ & $1(0.9)$ & & $40(0.8)$ & $551(3.1)$ & $2(1.2)$ & $\theta$ \\
\hline Austria & $15(0.7)$ & $526(3.3)$ & $\Delta \Delta$ & & $43(1.0)$ & $540(2.7)$ & $\Delta \Delta$ & \\
\hline Spain & $14(0.8)$ & $501(3.6)$ & $\Delta \diamond$ & & $45(1.1)$ & $513(3.0)$ & $\Delta \Delta$ & \\
\hline New Zealand & $14(0.6)$ & $514(4.5)$ & $-1(1.1)$ & & $43(0.8)$ & $534(2.2)$ & $1(1.3)$ & \\
\hline United States & $14(0.6)$ & $519(4.5)$ & $-4(1.1)$ & ( & $43(0.9)$ & $538(3.5)$ & $-1(1.3)$ & \\
\hline Scotland & $13(0.8)$ & $506(5.2)$ & $-1(1.1)$ & & $42(1.0)$ & $527(3.6)$ & $-1(1.5)$ & \\
\hline Morocco & $13(1.2)$ & $324(7.5)$ & $2(1.7)$ & & $45(1.6)$ & $326(6.8)$ & $5(2.4)$ & $\boldsymbol{\theta}$ \\
\hline Belgium (French) & $13(0.7)$ & $480(4.6)$ & $\Delta \Delta$ & & $40(0.8)$ & $498(2.9)$ & $\Delta \Delta$ & \\
\hline France & $12(0.7)$ & $506(4.0)$ & $1(0.9)$ & & $40(0.8)$ & $520(2.2)$ & $1(1.2)$ & \\
\hline Hong Kong SAR & $12(0.5)$ & $554(4.0)$ & $4(0.7)$ & $\theta$ & $43(0.8)$ & $569(2.6)$ & $0(1.2)$ & \\
\hline Canada, Nova Scotia & $12(0.6)$ & $523(4.5)$ & $\Delta \Delta$ & & $42(1.0)$ & $545(2.6)$ & $\Delta \Delta$ & \\
\hline Iran, Islamic Rep. of & $11(0.8)$ & $435(6.7)$ & $-2(1.1)$ & ( & $40(1.5)$ & $436(3.8)$ & $1(2.1)$ & \\
\hline Norway & $11(0.7)$ & $485(4.7)$ & $0(1.0)$ & & $38(0.9)$ & $499(2.7)$ & $0(1.4)$ & \\
\hline Italy & $11(0.8)$ & $539(6.3)$ & $-1(1.1)$ & & $40(0.8)$ & $554(3.1)$ & $1(1.2)$ & \\
\hline Canada, Ontario & $11(0.9)$ & $532(6.2)$ & $-3(1.2)$ & ( & $40(1.5)$ & $554(3.1)$ & $-1(1.8)$ & \\
\hline Canada, Alberta & $10(0.6)$ & $543(4.2)$ & $\Delta \Delta$ & & $43(0.9)$ & $560(2.7)$ & $\Delta \Delta$ & \\
\hline England & $10(0.7)$ & $502(5.5)$ & $-2(1.0)$ & & $44(1.0)$ & $537(2.8)$ & $0(1.5)$ & \\
\hline Canada, Quebec & $10(0.6)$ & $520(3.9)$ & $-2(1.0)$ & ( & $37(1.1)$ & $532(3.3)$ & $-6(1.5)$ & ( \\
\hline Sweden & $9(0.7)$ & $539(6.0)$ & $2(0.8)$ & $\theta$ & $33(1.0)$ & $550(2.8)$ & $2(1.3)$ & \\
\hline Canada, British Columbia & $9(0.6)$ & $541(5.3)$ & $\Delta \Delta$ & & $39(1.0)$ & $557(3.3)$ & $\Delta \diamond$ & \\
\hline Chinese Taipei & $8(0.4)$ & $538(3.6)$ & $\Delta \diamond$ & & $38(0.8)$ & $538(2.2)$ & $\Delta \Delta$ & \\
\hline Iceland & $8(0.4)$ & $496(4.9)$ & $-1(0.7)$ & & $33(0.8)$ & $511(2.0)$ & $0(1.1)$ & \\
\hline Denmark & $6(0.5)$ & $526(5.7)$ & $\Delta \diamond$ & & $30(1.0)$ & $539(3.1)$ & $\Delta \diamond$ & \\
\hline Luxembourg & $6(0.3)$ & $542(4.1)$ & $\Delta \Delta$ & & $33(0.6)$ & $555(2.0)$ & $\Delta \Delta$ & \\
\hline Belgium (Flemish) & $4(0.4)$ & $532(5.7)$ & $\Delta \Delta$ & & $29(1.0)$ & $544(2.8)$ & $\Delta \Delta$ & \\
\hline Netherlands & $4(0.4)$ & $528(6.0)$ & $0(0.6)$ & & $25(1.0)$ & $542(2.3)$ & $1(1.2)$ & \\
\hline International Avg. & $16(0.1)$ & $492(0.8)$ & & & $43(0.2)$ & $503(0.6)$ & & \\
\hline
\end{tabular}

Based on students' responses on how often they read to find out about things they want to learn and how often they read the following things outside of school: books that explain things, magazines, newspapers, directions or instructions, and brochures and catalogs. Average is computed on a 4-point scale: Never or almost never $=1$, Once or twice a month $=2$, Once or twice a week $=3$, and Every day or almost every day $=4$. Every day or almost every day indicates an average of greater than 3.25 through 4 . Once or twice a week indicates an average of greater than 2.5 through 3.25 . Once or twice a month indicates an average of greater than 1.75 through 2.5. Never or almost never indicates an average of 1 to less than 1.75 .

Please note that "I read brochures and catalogs" is a new item added to the index in 2006, and is not included in the 2001 index calculations.

() Standard errors appear in parentheses. Because results are rounded to the nearest whole number, some totals may appear inconsistent. 
Exhibit 4.4 Students Reading for Information Outside of School with Trends (Continued)
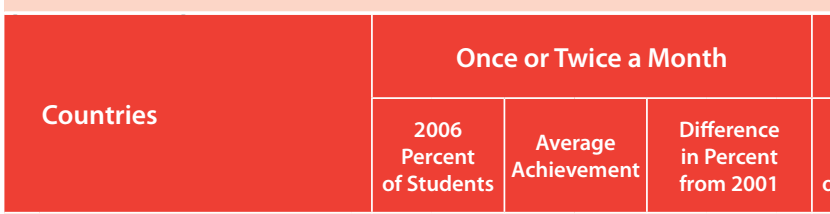

\begin{tabular}{|c|c|c|}
\hline \multicolumn{3}{|c}{ Never or Almost Never } \\
$\begin{array}{c}2006 \\
\text { Percent } \\
\text { of Students }\end{array}$ & $\begin{array}{c}\text { Average } \\
\text { Achievement }\end{array}$ & $\begin{array}{c}\text { Difference } \\
\text { in Percent } \\
\text { from } 2001\end{array}$ \\
\hline $3(0.5)$ & $304(19.7)$ & 00
\end{tabular}

South Africa
Macedonia, Rep. of
Qatar

$16(0.8) \quad 317(10.1)$

$16(1.2) \quad 447(6.3) \quad 2(1.5) \quad 2(0.3)$

$447(6.3)$

$\Delta \diamond$

$3(0.5)$

Kuwait

$15(0.4)$

Trinidad and Tobago

$16(0.9)$

359 (3.5)

$\Delta \diamond \quad 3(0.2)$

Israel

$329(6.4)$

$\begin{array}{cl}304(19.7) & \diamond \diamond \\ \sim \sim & 0(0.6)\end{array}$

Poland

$25(1.0)$

$444(5.8)$

$\Delta \diamond \quad 3(0.6)$

$345(7.6) \quad \diamond \diamond$

Slovak Republic

Moldova, Rep. of

$27(0.7)$

$532(3.9)$

$\Delta \diamond$

$4(0.5)$

$295(15.3)$

$-1(1.3) \quad 10(0.6)$

$414(13.6) \quad 00$

\begin{tabular}{|l|l|l|}
$518(3.4)$ & $\diamond \diamond$ & $6(0.5)$ \\
\hline
\end{tabular}

Indonesia

$24(0.8)$

$530(4.3)$

$-2(1.2)$

$500(4.3)$

$6(0.6)$

Hungary

$25(1.0)$

$-5(2.1)$

$7(0.9)$

$25(0.8)$

$562(3.4)-3(1.3)-6(0.9)$

Russian Federation

$28(0.9)$

Slovenia

Lithuania

$26(0.7)$

$562(3.4)$

$-3(1.3)$

$572(3.5)$

$1(1.3)$

$7(0.5)$

$25(0.8)$

$533(2.9) \quad 1(1.2)$

$8(0.5)$

$549(5.1)$

$508(6.8) \quad \diamond \diamond$

Bulgaria

25 (1.2)

$541(2.4)$

0 (1.4)

$4(0.4)$

$492(15.3) \quad 1(0.8)$

$26(1.1)$

Singapore

$550(4.3)$

4 (1.6)

$490(5.5) \quad-3(1.7)$

Latvia

Georgia

$561(3.4) \quad 4(0.9)$

- $11(1.3)$

$492(8.7)$

$391(8.0) \quad 0(1.4)$

$558(6.4) \quad 0(0.7)$

$558(6.4)$

$570(5.6) \quad 2(0.9)$

\begin{tabular}{ll}
$524(4.9) \quad 2(0.7) \quad 0$ \\
\hline
\end{tabular}

Germany

$29(0.9)$

$9(1.0)$

$533(4.7)$

$3(0.4)$

Austria

$30(1.5)$

- $8(0.3)$

$475(4.7)-4(1.7) \quad 0 \quad 7(0.6)$

\begin{tabular}{llll}
$475(4.7)$ & $\diamond \diamond$ & $11(1.0)$ \\
\hline $555(2.6)$ & $-4(1.0)$ & $\nabla$ & $13(0.7)$
\end{tabular}

$32(0.7)$

Spain

New Zealand

$31(0.8)$

$-4(1.0)$

$13(0.7)$
$12(0.7)$

$521(9.6) \quad 4(1.6) \quad 0$

United States

$30(0.9)$

$\Delta 0$

$518(2.7) \quad \diamond \diamond \quad 10(0.6)$

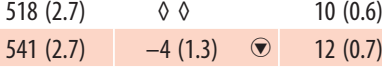

$31(0.6)$

$12(0.7)$
$10(0.6)$

$444(12.7)$

$538(4.4) \quad 4(0.4)$

Scotland

$34(1.0)$

$553(4.0) \quad 2(1.4)$

$10(0.6)$

Morocco

$28(1.4)$

Belgium (French)

$538(3.0) \quad-1(1.6)$

$11(1.0)$

$545(5.1)$

$450(10.3) \quad 0$

$547(3.7) \quad 0(1.0)$

$543(4.3) \quad \Delta \diamond$

$514(5.4) \quad \Delta \diamond$

$\begin{array}{lll}531(5.2) & 3(0.9) \quad 0\end{array}$

$\begin{array}{lll}546(5.3) & 3(0.9) & 0\end{array}$

France

Hong Kong SAR

Canada, Nova Scotia

$31(0.7)$

$331(9.1) \quad-5(2.4)$

$14(1.8)$

\begin{tabular}{l|l|l}
$506(3.1)$ & $\Delta \diamond$ & $16(0.8)$
\end{tabular}

$522(6.7)$

$3(1.2) \quad \boldsymbol{\theta}$

Iran, Islamic Rep. of

$34(0.9)$

$527(3.0) \quad-5(1.4) \quad \nabla \quad 14(0.6)$

$310(12.6) \quad-3(3.4)$

Norway

$33(0.8)$

$567(2.5) \quad-8(1.1) \quad \nabla$

$13(0.6)$

$552(2.7) \quad \Delta \diamond \quad 13(0.6)$

$510(3.6) \quad \diamond \diamond$

$530(3.8) \quad 3(0.8) \quad 0$

$33(0.8)$

35 (1.2)

$421(4.0) \quad-3(1.8)$

$14(1.2)$

$550(3.5)$

$3(0.8)$

Italy

Canada, Ontario

$34(0.9)$

$503(3.7)-4(1.5) \quad \odot \quad 17(0.8)$

$533(4.0) \quad \Delta \diamond$

Canada, Alberta

$36(1.0)$

$554(3.7) \quad-2(1.4)$

$14(0.8)$

$474(9.4) \quad 4(1.5) \quad 0$

$497(3.9) \quad 3(1.3) \quad 0$

Orta

England

$34(0.8)$

$558(3.3) \quad-1(1.6)$

$14(1.0)$

$552(4.8)$

$552(4.8) \quad 2(1.0) \quad 0$

Canada, Quebec

Sweden

Canada, British Columbia

Chinese Taipei

Iceland

Denmark

Luxembourg

Belgium (Flemish)

Netherlands

$35(1.1)$

\begin{tabular}{l|l|l}
$566(2.8)$ & $\Delta \diamond$ & $13(0.6)$
\end{tabular}

$564(4.5)$

4 (1.1) $\boldsymbol{\theta}$

$\begin{array}{llllll}556(3.2) & -2(1.6) & 11(0.7) & 537(5.8) & 3(0.9) & \boldsymbol{\Delta}\end{array}$

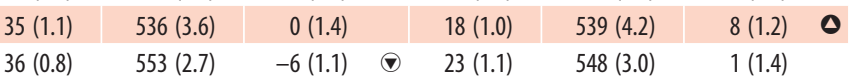

\begin{tabular}{|l|l|l|l|l|l|}
$38(0.8)$ & $564(3.1)$ & $\diamond \diamond$ & $14(0.7)$ & $561(4.2)$ & $\diamond \diamond$
\end{tabular}

$38(0.8) \quad 536(2.8) \quad \Delta \diamond \quad 16(0.7) \quad 529(3.5) \quad \Delta \diamond$

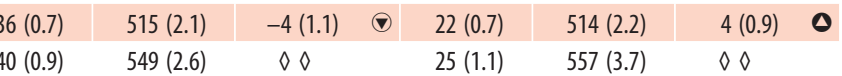

\begin{tabular}{|c|c|c|}
\hline $11(0$. & & \\
\hline
\end{tabular}

$42(0.8) \quad 549(2.3) \quad \Delta \diamond \quad 25(0.8) \quad 550(2.1) \quad \diamond$

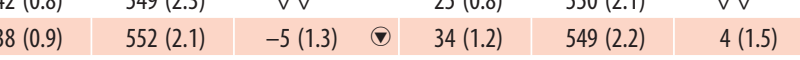

International Avg.

$29(0.1) \quad 506(0.7)$

$12(0.1) \quad 496(1.3)$

ه Percent in 2006 significantly higher

( Percent in 2006 significantly lower

A tilde $(\sim)$ indicates insufficient data to report achievement.

A diamond $(\diamond)$ indicates the country did not participate in the 2001 assessment.

NOTE: The International Average does not include the results from the Canadian provinces.

Trend Note: The primary education systems of the Russian Federation and Slovenia

underwent structural changes. Data for Canada, Ontario include only public schools. 
Exhibit 4.5 Students Read Stories or Articles Outside of School

PIRLS 2006

4th Grade

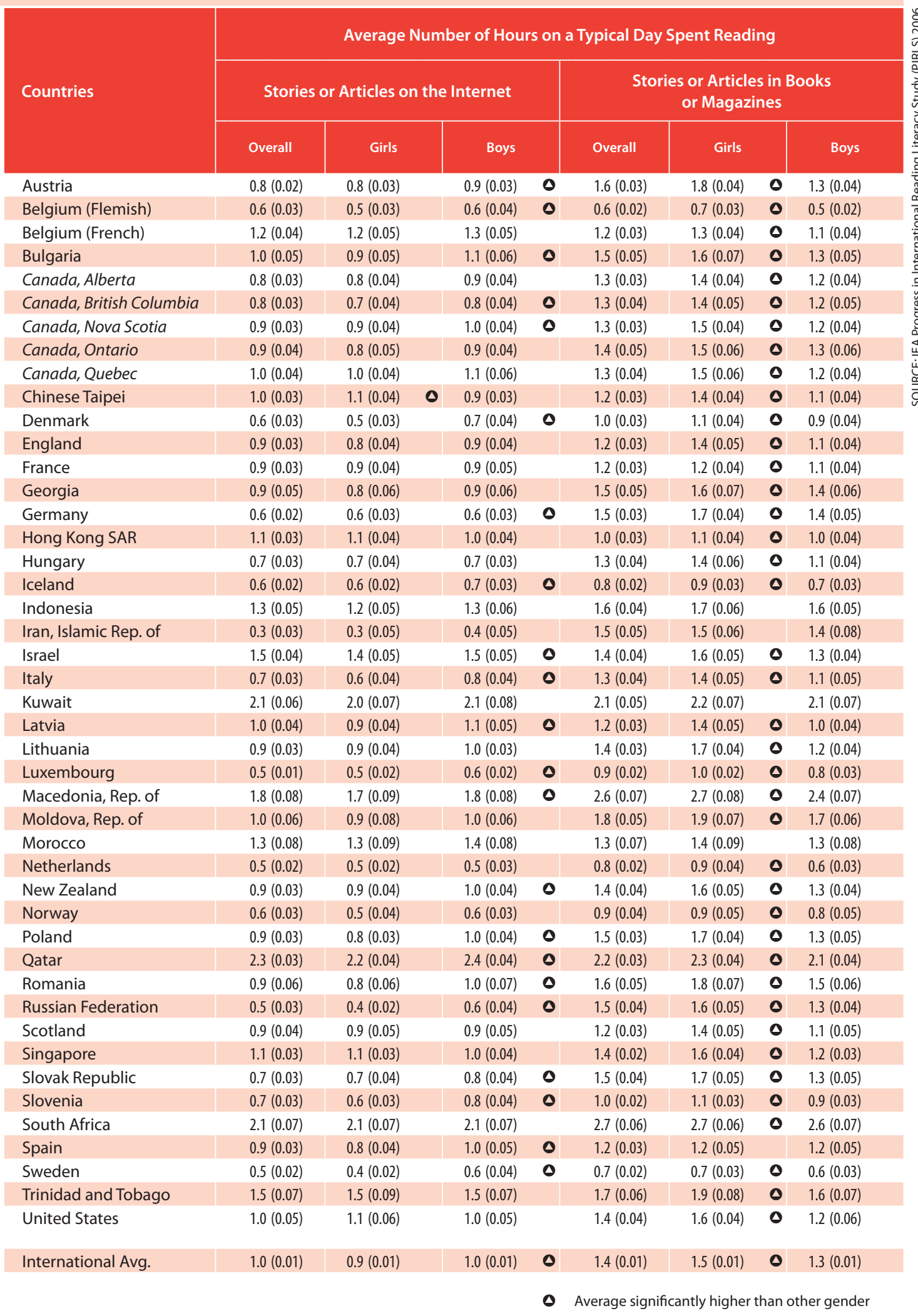

Background data provided by students.

() Standard errors appear in parentheses. Because results are rounded to the nearest

whole number, some totals may appear inconsistent.

NOTE: The International Average does not include the results from the Canadian provinces. 
students reported half an hour or less spent reading daily on the Internet. Countries where students reported the most time spent reading stories or articles in books or magazines included Kuwait, Macedonia, Qatar, and South Africa (more than 2 hours on a typical day, on average). Students in Belgium (Flemish), Iceland, Luxembourg, the Netherlands, Norway, and Sweden reported reading from books or magazines for less than one hour, on average, on a typical day. In almost every country, girls reported more time than boys reading from books or magazines (1.5 hours vs. 1.3 hours) and in many countries, boys reported more time than girls reading stories or articles on the Internet (1.0 hours vs. 0.9 hours). Only in Chinese Taipei did girls report more time reading on the Internet than boys.

Exhibit 4.6 presents trends in students' reports of how frequently they read for fun outside of school, regardless of the source of their reading material. In general, students around the world reported a good deal of reading for fun, with 40 percent, on average internationally, reading every day or almost every day, and a further 28 percent once or twice a week. However, almost one third of students (32\%, on average) reported reading for fun no more than twice a month. Reading for fun was reported most frequently in the Russian Federation, Germany, Lithuania, Moldova, France, and the Canadian provinces of Alberta and British Columbia, where the majority of students reported reading for fun daily or almost daily. In contrast, less than 30 percent of students in Georgia, Morocco, Singapore, Romania, and Chinese Taipei reported daily reading for fun. Of the PIRLS 2001 participants, five had increased percentages of students in 2006 reporting reading for fun daily or almost daily-Germany, Hungary, Italy, Hong Kong SAR, and Ontario province. Seven countries had fewer students reporting daily reading for fun in 2006-Iceland, Israel, Slovenia, Sweden, Latvia, Norway, and Singapore.

There was a positive association between the frequency of reading for fun and average student reading achievement, on average, across countries, and in most countries. Across all countries, students who reported reading 
for fun every day or almost every day had average reading achievement of 516 points, compared to 503 points for those reading for fun once or twice a week, and 484 points for those reading for fun twice a month or less. 
Exhibit 4.6 Students Reading for Fun Outside of School with Trends

PIRLS 2006

\begin{tabular}{|c|c|c|c|c|c|c|c|c|c|c|c|c|}
\hline \multirow[b]{2}{*}{ Countries } & \multicolumn{4}{|c|}{ Every Day or Almost Every Day } & \multicolumn{4}{|c|}{ Once or Twice a Week } & \multicolumn{4}{|c|}{ Twice a Month or Less } \\
\hline & $\begin{array}{c}2006 \\
\text { Percent } \\
\text { of Students }\end{array}$ & $\begin{array}{c}\text { Average } \\
\text { Achievement }\end{array}$ & $\begin{array}{l}\text { Difference } \\
\text { in Percent } \\
\text { from } 2001\end{array}$ & & $\begin{array}{c}2006 \\
\text { Percent } \\
\text { of Students }\end{array}$ & $\begin{array}{c}\text { Average } \\
\text { Achievement }\end{array}$ & $\begin{array}{l}\text { Difference } \\
\text { in Percent } \\
\text { from } 2001\end{array}$ & & $\begin{array}{c}2006 \\
\text { Percent } \\
\text { of Students }\end{array}$ & $\begin{array}{c}\text { Average } \\
\text { Achievement }\end{array}$ & $\begin{array}{l}\text { Difference } \\
\text { in Percent } \\
\text { from } 2001\end{array}$ & \\
\hline Russian Federation & $58(1.1)$ & $570(3.8)$ & $0(1.8)$ & & $28(0.8)$ & $559(3.9)$ & $-1(1.3)$ & & $14(0.8)$ & $556(3.9)$ & $2(1.3)$ & \\
\hline Canada, Alberta & $53(0.9)$ & $575(2.5)$ & $\Delta 0$ & & $23(0.8)$ & $555(3.5)$ & $\Delta 0$ & & $23(0.9)$ & $537(2.7)$ & $\Delta 0$ & \\
\hline Canada, British Columbia & $53(1.0)$ & $573(2.9)$ & $\Delta \Delta$ & & $26(0.8)$ & $554(2.5)$ & $\Delta \diamond$ & & $21(0.9)$ & $531(4.1)$ & $\Delta \diamond$ & \\
\hline Germany & $53(0.9)$ & $563(2.7)$ & $5(1.2)$ & 0 & $24(0.6)$ & $545(3.0)$ & $0(0.9)$ & & $24(0.8)$ & $525(2.5)$ & $-5(1.2)$ & (1) \\
\hline Lithuania & $52(1.2)$ & $545(2.1)$ & $-1(1.9)$ & & $30(1.0)$ & $533(2.2)$ & $-1(1.6)$ & & $17(0.8)$ & $520(2.8)$ & $2(1.2)$ & \\
\hline Moldova, Rep. of & $52(1.4)$ & $507(3.2)$ & $2(2.6)$ & & $34(1.1)$ & $498(4.2)$ & $0(1.9)$ & & $14(1.0)$ & $484(5.1)$ & $-2(1.6)$ & \\
\hline France & $51(1.0)$ & $540(2.5)$ & $2(1.6)$ & & $24(0.8)$ & $517(2.3)$ & $-2(1.2)$ & & $25(0.9)$ & $491(2.7)$ & $0(1.3)$ & \\
\hline Canada, Ontario & $49(1.4)$ & $567(3.2)$ & $14(2.0)$ & 0 & $25(1.1)$ & $552(3.6)$ & $2(1.4)$ & & $26(1.1)$ & $534(4.1)$ & $-16(1.9)$ & $\odot$ \\
\hline Belgium (French) & $49(1.1)$ & $517(3.0)$ & $\Delta \Delta$ & & $26(0.7)$ & $495(2.9)$ & $\Delta \Delta$ & & $25(0.9)$ & $473(3.1)$ & $\Delta \diamond$ & \\
\hline Iceland & $49(0.9)$ & $527(1.9)$ & $-3(1.2)$ & ( & $23(0.7)$ & $511(2.6)$ & $2(1.0)$ & & $28(0.7)$ & $485(2.3)$ & $1(1.0)$ & \\
\hline Denmark & $49(1.1)$ & $559(2.9)$ & $\Delta \diamond$ & & $30(0.8)$ & $540(2.7)$ & $\Delta \Delta$ & & $21(0.9)$ & $528(3.2)$ & $\Delta \diamond$ & \\
\hline Canada, Nova Scotia & $48(0.9)$ & $560(2.6)$ & $\Delta 0$ & & $25(0.8)$ & $541(2.7)$ & $\Delta \diamond$ & & $27(0.8)$ & $515(3.2)$ & $\Delta \Delta$ & \\
\hline Bulgaria & $47(1.6)$ & $561(4.4)$ & $-4(2.3)$ & & $27(1.0)$ & $555(5.0)$ & $-2(1.5)$ & & $26(1.6)$ & $520(6.5)$ & $5(2.2)$ & 0 \\
\hline Canada, Quebec & $47(1.3)$ & $549(3.0)$ & $1(1.9)$ & & $26(1.0)$ & $530(3.8)$ & $1(1.4)$ & & $27(1.2)$ & $509(3.2)$ & $-3(1.7)$ & \\
\hline South Africa & $45(0.9)$ & $303(6.4)$ & $\Delta \Delta$ & & $26(0.5)$ & $314(6.4)$ & $\Delta \Delta$ & & $28(0.8)$ & $307(6.4)$ & $\Delta \Delta$ & \\
\hline Austria & $45(1.1)$ & $555(3.0)$ & $\Delta 0_{0}$ & & $25(0.8)$ & $535(2.4)$ & $\Delta \Delta$ & & $29(1.0)$ & $516(2.9)$ & $\Delta 0$ & \\
\hline Spain & $45(1.1)$ & $525(2.9)$ & $\Delta \diamond$ & & $27(0.7)$ & $515(2.5)$ & $\Delta \Delta$ & & $28(1.0)$ & $494(3.7)$ & $\Delta \Delta$ & \\
\hline Hungary & $44(1.2)$ & $565(3.7)$ & $4(1.7)$ & 0 & $30(0.9)$ & $547(3.4)$ & $-2(1.4)$ & & $26(1.1)$ & $532(4.2)$ & $-2(1.5)$ & \\
\hline Indonesia & $44(1.4)$ & $405(4.7)$ & $\Delta \diamond$ & & $31(1.1)$ & $414(4.6)$ & $\Delta \diamond$ & & $25(1.2)$ & $403(4.8)$ & $\Delta \diamond$ & \\
\hline Macedonia, Rep. of & $43(1.2)$ & $453(5.7)$ & $-3(1.9)$ & & $31(1.0)$ & $451(4.6)$ & $3(1.4)$ & $\boldsymbol{\theta}$ & $25(1.1)$ & $435(5.1)$ & $0(1.8)$ & \\
\hline Poland & $43(1.3)$ & $538(2.5)$ & $\Delta \diamond$ & & $29(1.0)$ & $518(3.2)$ & $\diamond \diamond$ & & $27(1.0)$ & $495(3.0)$ & $\Delta 0$ & \\
\hline New Zealand & $42(1.1)$ & $562(2.4)$ & $-1(1.8)$ & & $24(0.7)$ & $531(2.5)$ & $0(1.1)$ & & $34(1.0)$ & $500(3.0)$ & $1(1.6)$ & \\
\hline Belgium (Flemish) & $40(1.1)$ & $563(2.1)$ & $\Delta \Delta$ & & $29(0.8)$ & $545(2.9)$ & $\Delta \Delta$ & & $31(1.2)$ & $529(2.3)$ & $\Delta \diamond$ & \\
\hline Slovak Republic & $39(1.0)$ & $545(2.9)$ & $0(1.5)$ & & $33(0.9)$ & $535(3.2)$ & $0(1.3)$ & & $27(1.1)$ & $507(5.4)$ & $0(1.6)$ & \\
\hline Italy & $38(1.3)$ & $573(3.3)$ & $7(1.7)$ & 0 & $25(0.7)$ & $554(3.2)$ & $1(1.0)$ & & $37(1.3)$ & $529(3.8)$ & $-7(1.7)$ & $\nabla$ \\
\hline Luxembourg & $38(0.6)$ & $581(1.8)$ & $\Delta \Delta$ & & $27(0.7)$ & $551(2.0)$ & $\Delta 0$ & & $35(0.5)$ & $537(1.5)$ & $\Delta \Delta$ & \\
\hline Israel & $38(1.2)$ & $538(4.2)$ & $-6(1.6)$ & $(7)$ & $28(0.9)$ & $518(4.4)$ & $1(1.2)$ & & $35(1.1)$ & $497(4.1)$ & $5(1.5)$ & 0 \\
\hline Slovenia & $37(0.9)$ & $543(2.5)$ & $-8(1.6)$ & ( & $33(0.7)$ & $519(3.0)$ & $4(1.1)$ & 0 & $30(0.9)$ & $500(2.6)$ & $4(1.5)$ & 0 \\
\hline Netherlands & $36(1.1)$ & $566(2.1)$ & $0(1.6)$ & & $22(0.7)$ & $550(1.8)$ & $2(1.1)$ & 0 & $42(1.1)$ & $530(1.8)$ & $-2(1.6)$ & \\
\hline Sweden & $36(1.0)$ & $569(2.8)$ & $-8(1.3)$ & (1) & $31(0.9)$ & $549(3.2)$ & $-1(1.2)$ & & $33(1.0)$ & $530(2.6)$ & $9(1.3)$ & 0 \\
\hline United States & $35(1.3)$ & $561(4.3)$ & $1(1.8)$ & & $22(0.7)$ & $550(3.3)$ & $0(1.3)$ & & $43(1.4)$ & $521(3.3)$ & $-1(1.8)$ & \\
\hline Latvia & $35(1.2)$ & $556(3.0)$ & $-8(1.7)$ & ( & $31(0.8)$ & $543(2.8)$ & $-2(1.2)$ & & $34(1.2)$ & $524(2.6)$ & $10(1.7)$ & 0 \\
\hline Hong Kong SAR & $35(1.0)$ & $575(2.6)$ & $14(1.3)$ & 0 & $33(0.9)$ & $567(2.7)$ & $-5(1.2)$ & $\odot$ & $32(1.0)$ & $549(2.8)$ & $-8(1.4)$ & 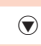 \\
\hline Iran, Islamic Rep. of & $33(1.2)$ & $428(4.2)$ & $-1(1.7)$ & & $41(1.2)$ & $429(3.9)$ & $2(1.9)$ & & $26(1.0)$ & $406(5.2)$ & $0(1.5)$ & \\
\hline Norway & $33(1.1)$ & $514(3.4)$ & $-5(1.5)$ & $(7)$ & $30(1.0)$ & $505(3.2)$ & $2(1.3)$ & & $37(1.2)$ & $481(3.1)$ & $4(1.7)$ & 0 \\
\hline Qatar & $33(0.6)$ & $357(2.2)$ & $\Delta \Delta$ & & $28(0.5)$ & $367(2.5)$ & $\Delta \Delta$ & & $39(0.6)$ & $352(2.2)$ & $\Delta \Delta$ & \\
\hline Scotland & $33(1.1)$ & $555(4.4)$ & $2(1.6)$ & & $24(1.0)$ & $533(3.1)$ & $0(1.3)$ & & $44(1.5)$ & $505(2.7)$ & $-2(2.2)$ & \\
\hline England & $33(1.2)$ & $575(4.0)$ & $0(1.8)$ & & $25(0.8)$ & $537(3.5)$ & $-1(1.2)$ & & $42(1.3)$ & $517(2.9)$ & $1(2.0)$ & \\
\hline Kuwait & $32(1.1)$ & $338(5.5)$ & $\Delta \Delta$ & & $32(1.0)$ & $342(5.5)$ & $\Delta \Delta$ & & $36(1.2)$ & $332(5.4)$ & $\Delta \diamond$ & \\
\hline Trinidad and Tobago & $32(1.2)$ & $450(6.7)$ & 00 & & $25(1.0)$ & $442(5.9)$ & 00 & & $43(1.4)$ & $427(5.6)$ & 00 & \\
\hline Georgia & $29(1.4)$ & $479(4.5)$ & $\Delta \diamond$ & & $29(1.2)$ & $484(4.0)$ & $\Delta \diamond$ & & $41(1.4)$ & $461(4.3)$ & $\Delta \Delta$ & \\
\hline Morocco & $29(1.3)$ & $317(8.2)$ & $-3(2.3)$ & & $34(1.4)$ & $326(6.9)$ & $-3(2.0)$ & & $37(1.9)$ & $331(7.1)$ & $6(3.1)$ & \\
\hline Singapore & $27(0.9)$ & $587(3.9)$ & $-3(1.5)$ & $(>$ & $26(0.6)$ & $564(3.1)$ & $3(0.9)$ & 0 & $47(1.0)$ & $540(2.7)$ & $0(1.5)$ & \\
\hline Romania & $25(1.3)$ & $510(5.5)$ & $-3(2.2)$ & & $26(1.1)$ & $502(5.5)$ & $-4(1.6)$ & $\odot$ & $50(1.6)$ & $478(5.5)$ & $7(2.3)$ & 0 \\
\hline Chinese Taipei & $24(0.7)$ & $553(2.6)$ & $\Delta \Delta$ & & $31(0.8)$ & $539(2.6)$ & $\Delta \Delta$ & & $45(1.0)$ & $525(2.2)$ & $\Delta \diamond$ & \\
\hline International Avg. & $40(0.2)$ & $516(0.6)$ & & & $28(0.1)$ & $503(0.6)$ & & & $32(0.2)$ & $484(0.6)$ & & \\
\hline
\end{tabular}

- Percent in 2006 significantly higher

(1) Percent in 2006 significantly lower

Background data provided by students.

() Standard errors appear in parentheses. Because results are rounded to the nearest whole number, some totals may appear inconsistent.

A diamond $(\diamond)$ indicates the country did not participate in the 2001 assessment.
NOTE: The International Average does not include the results from the Canadian provinces. Trend Note: The primary education systems of the Russian Federation and Slovenia underwent structural changes. Data for Canada, Ontario include only public schools. 



\section{Chapter 4}

\section{Students' Reading Attitudes,}

Self-Concept, and Out-of-School Activities

Positive student attitudes toward reading and a healthy reading self-concept are major objectives of the reading curriculum in most countries. Students who enjoy reading and who perceive themselves to be good readers usually read more frequently and more widely, which in turn broadens their reading experience and improves their comprehension skills. This chapter examines trends in students' attitudes toward reading and in their self-concept as readers, as well as the frequency with which they read stories, novels, and informational texts outside of school.

\section{What Are Students' Attitudes Toward Reading?}

The PIRLs Index of Students' Attitudes Toward Reading summarizes students' views on reading for enjoyment and appreciating books. The index was based on students' agreement with the following statements related to reading:

- I read only if I have to (reverse coded).

- I like talking about books with other people.

- I would be happy if someone gave me a book as a present.

- I think reading is boring (reverse coded).

- I enjoy reading. 
Students were asked to indicate their degree of agreement with each statement on a 4-point scale, as follows: agree a lot, agree a little, disagree a little, and disagree a lot. Responses to each statement were averaged for each student. Students who, on average, agreed a lot or agreed a little with the statements were assigned to the high category of the index. Students in the low category disagreed a lot or disagreed a little, on average, with the statements. Students in the medium category had other combinations of responses.

Exhibit 4.1 presents, for each country, the percentage of students in 2006 at each level of the index, together with their average reading achievement. To measure change in student attitudes since the previous PIRLS, the exhibit shows the difference from 2001 in the percentage of students at each level of the index, together with an indication of its statistical significance.

As was the case in 2001, fourth-grade students in 2006 generally had positive attitudes toward reading. On average internationally, almost half the students were at the high level of the index and more than 90 percent were at either the high or medium level. Only 8 percent of students, on average, had unfavorable attitudes toward reading (i.e., they were at the low level of the index). Countries with the greatest percentages of students with favorable attitudes toward reading $(60 \%$ or more at the high level of the index) included Iran, Italy, Macedonia, and Romania. Countries with increased percentages of students at the high level of the index in comparison with 2001 included Iran, Italy, Germany, and Hong Kong SAR, each of which also had increased average student achievement over that period (although the difference for Iran was not statistically significant). It may be a matter for concern that a greater number of participants had decreased percentages of students at the high level, including Moldova, Slovenia, Singapore, Sweden, Iceland, Scotland, England, the Netherlands, Latvia, and the Canadian province of Ontario.

On average internationally, and in every country, students at the high level of the index of positive attitudes toward reading had substantially higher average reading achievement than those at the medium or low levels. 
Exhibit 4.1 Index of Students' Attitudes Toward Reading (SATR) with Trends

PIRLS 2006

Countries

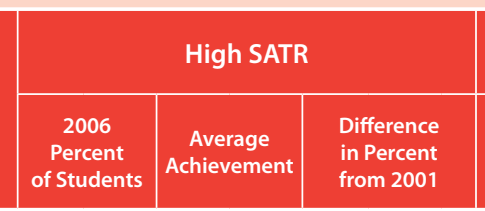

\begin{tabular}{|c|c|c|}
\hline \multicolumn{3}{|c|}{ Medium SATR } \\
\hline $\begin{array}{c}2006 \\
\text { Percent } \\
\text { of Students }\end{array}$ & $\begin{array}{c}\text { Average } \\
\text { Achievement }\end{array}$ & $\begin{array}{l}\text { Difference } \\
\text { in Percent } \\
\text { from } 2001\end{array}$ \\
\hline $21(1.2)$ & $382(5.6)$ & $-6(2.2)$ \\
\hline
\end{tabular}

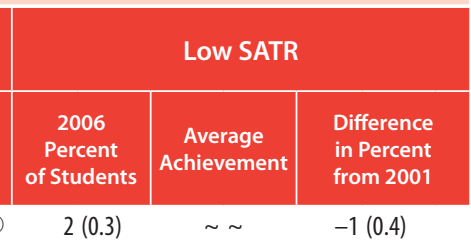

Iran,

$77(1.3)$

$437(3.0)$

$6(2.3)$

$64(1.4)$

$565(3.0)$

Macedonia, Rep. of

$63(1.5)$

$471(4.1)$

$8(1.9)$

Romania

$60(1.3)$

$513(5.0)$

$1(2.1)$

$58(1.5)$

$553(2.8)$

$0(2.1)$

- $31(1.2$

$531(3.4)$

$-6(2.2)$

$35(1.5)$

Canada, Quebec

$407(5.0)$

$-2(2.1)$

$5(0.5)$

$520(6.1)$

$-1(0.4)$

\begin{tabular}{|l|l|r|}
$58(1.1)$ & $569(2.5)$ & $8(1.4)$ \\
\hline
\end{tabular}

Moldova, Rep. of

$58(1.6)$

$514(3.3)$

France

Bulgaria

$57(0.9)$

542 (2.3)

Spain

$57(1.4)$

$567(4.3)$

$0(1.5)$

$462(6.2)$

$-2(2.1)$

$2(0.2)$

$512(2.6)$

$0(1.8)$

$4(0.4)$

$466(9.2)$

$1(0.3)$

Hong Kong SAR

56 (1.1)

Trinidad and Tobago

$55(1.1)$

$528(2.1)$

$-3(2.1)$

$41(1.6)$

$533(2.5)$

$-6(1.2)$

Morocco

$54(1.3)$

$579(2.3)$

$\diamond 0$

$38(0.8)$

484 (4.2)

$7(2.5)$

Slovenia

$53(2.0)$

$466(5.0)$

6 (1.6)

$37(1.2)$

498 (2.2)

$0(1.4)$

$8(0.4)$

$503(6.7)$

$3(0.5)$

$3(0.5)$
$-1(0.9)$

Chinese Taipei

52 (1.1)

351 (5.8)

00

$40(1.0)$

$527(5.2)$

$1(1.9)$

- $2(0.3)$

$516(4.1)$

Belgium (French)

$52(1.1)$

$542(2.0)$

$5(3.0)$

$41(1.0)$

$497(3.1)$

00

$4(0.4)$

485

$485(5.9) \quad 0(0.4)$

Russian Federation

$52(1.0)$

$553(2.1)$

$5(3.0)$

$41(1.2)$

$546(2.9)$

$-6(1.5)$

$4(0.4)$

$509(9.4)$

$400(5.4)$

$298(9.3)$

Georgia

$50(1.1)$

$521(2.9)$

$\diamond \diamond$

$40(0.9)$

Austria

$50(1.9)$

581 (3.6)

00

$44(1.0)$

$501(2.7)$

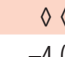

$\Delta>$
$-4(2.9)$

$4(0.3)$

$492(6.7)$

$539(5.0)$

$4(0.4)$

426 (9.7)

$\sim \sim \quad-1(1.0)$

Hungary

$50(1.2)$

$493(3.4)$

$-4(2.1)$

$42(0.9)$

$518(2.5)$

$\diamond \diamond$

$557(2.7)$

00

$45(1.0)$

$479(2.9)$

$\Delta 0$

$8(0.5)$

$493(4.4)$

$4(0.4)$
$6(0.4)$

$520(6.0) \quad \Delta \diamond$

$571(2.9)$

$\Delta \Delta$

$47(1.8)$

$550(3.7)$

3 (1.9)

$5(0.4)$

475 (5.1)

$540(5.0)$

$\Delta 0$

$49(1.0)$

$583(2.5)$

$0(1.8)$

$524(2.7)$

$\diamond \diamond \quad 3(0.4)$

Indonesia

New Zealand

$49(1.2)$

$432(3.8)$

$0(1.8)$

$39(1.2)$

$524(2.7)$

$\Delta 0$

$42(0.9)$

$532(4.3)$

$\Delta \diamond$
$-1(1.6)$

$10(0.7)$

$445(9.6)$

$1(0.6)$

Canada, Alberta

$48(1.0)$

$563(2.3)$

00

$50(1.2)$

Lithuania

$48(1.1)$

$584(2.4)$

$-3(1.7)$

$383(4.6)$

$\Delta \diamond$

$11(0.7)$

$510(3.8) \quad \diamond \diamond$

Norway

$47(1.1)$

$551(2.1)$

$\diamond \Delta$

$507(2.6)$

$\Delta \diamond \quad 1(0.1)$

$531(3.1) \quad 1(1.0)$

Singapore

$47(1.3)$

$519(2.4)$

$41(0.9)$

$542(2.7)$

$4(1.6) \quad 0 \quad 7(0.4)$

$524(4.3)$

$\Delta \Delta$

Canada, Ontario

$47(1.0)$

$1(1.8)$

$46(1.0)$

$525(2.0)$

$\diamond \diamond \quad 11(0.6)$

$493(47)$

$\Delta 0$

Canada, Nova Scotia

Slovak Republic

$46(1.4)$

$582(3.1)$

$3(1.7) \quad 45(1.2)$

$487(2.9)$

$-2(1.7)$

$11(0.6)$
$7(0.4)$

$531(3.6)$

$520(4.2) \quad 0(0.7)$

Sweden

$541(3.1)$

$-3(1.5)$

$8(0.8)$

$469(5.8) \quad-1(1.0)$

$-6(1.9)-42(1.0)$

\begin{tabular}{l|l|l|l|}
\hline $46(1.0)$ & $569(2.7)$ & $\diamond \diamond$ & $41(0.9)$ \\
\hline $46(1.3)$ & $553(2.7)$ & $2(2.0)$ & $46(1.2)$
\end{tabular}

$538(3.4)$

$3(1.5) \quad 0 \quad 8(0.4)$

$527(4.0)$

$(1.0)$

$526(2.5)$

$3(1.7)$

$12(0.9)$

$529(5.2)$

$3(1.0)$

$45(1.2) \quad 571(2.9) \quad-9(1.6) \quad \circledast \quad 44(1.0)$

$\begin{array}{lll}514(3.5) & -4(1.7) \quad \odot \quad 9(0.6)\end{array}$

$513(3.9) \quad \Delta 0$

Iceland 44

Israe

Scotland

$45(1.3)$

$\diamond \diamond \quad 44(1.0)$

$535(2.3)$

$44(0.8) \quad 533(1.6) \quad-4(1.3) \quad \odot \quad 49(0.8)$

$503(2.7)$

$5(1.4) \quad 0 \quad 10(0.6)$

$511(5.6) \quad 2(0.8) \quad 0$

$-00(0.7)$

$496(2.0) \quad 3(1.3) \quad 0 \quad 7(0.4)$

$519(3.5)$

$3(0.8)$

Kuwait $\quad r \quad 411$

England

$42(1.4)$

$547(3.7)$

$-2(1.8)$

$49(1.0)$

$495(3.9)$

$511(3.1)$

$1(1.6) \quad 9(0.7)$

$494(4.1)$

$\diamond \diamond$

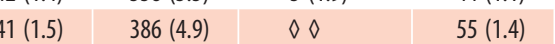

$313(5.4)$

$40(1.4) \quad 576(3.4) \quad-4(2.0) \quad \odot \quad 45(1.1)$

\begin{tabular}{|l|l|l|l|}
\hline $40(1.3)$ & $566(3.4)$ & $-3(1.7)$ & $46(1.1)$
\end{tabular}

$520(2.7)$

$2(1.6) \quad 14(1.0)$

$484(4.4)$

$1(0.6)$

United States

$40(0.6)$

Luxembourg

$39(1.3)$


At least for reading at this grade level, it seems that positive attitudes and high achievement in reading go hand in hand.

\section{What Are Students' Perceptions of Their Reading Ability?}

As was shown in PIRLS 2001, by the fourth grade, students in all countries can give an indication of their perception of themselves as good or poor readers, with a good deal of agreement between students' self-reports and average reading achievement. In PIRLS 2006, students' self-concept in reading was assessed based on their agreement with four statements about their ability to read:

- Reading is very easy for me.

- I do not read as well as other students in my class (reverse coded).

- When I am reading by myself, I understand almost everything I read.

- I read slower than other students in my class (reverse coded).

Response options were on a 4-point scale, as follows: agree a lot, agree a little, disagree a little, and disagree a lot. PIRLs combined students' responses to these four statements to construct an Index of Students' Reading SelfConcept. Students in the high category agreed (a lot or a little), on average, with the four statements about their reading ability. Students in the low category disagreed (a lot or a little), on average, with the statements. The medium level of the index includes all other response combinations. Exhibit 4.2 summarizes the results for this index.

Around the world, fourth-grade students generally seem to view themselves as good, or at least moderately good, readers. Almost half the students (49\%), on average internationally, were at the high level of the index of reading self-concept, and almost half (48\%) were at the medium level. Just 3 percent were at the low level of the index. Countries with the greatest percentages of high self-concept students (60\% or more) included Israel, Austria, Sweden, Poland, Norway, the Netherlands, and Denmark. Interestingly, although these countries all have average reading achievement above the PIRLS international scale average, there are other countries with 
higher average achievement. Countries with lesser percentages of students at the high level (less than 40\%) included Kuwait, Morocco, Belgium (French), New Zealand, France, Indonesia, Moldova, and South Africa.

Ten countries showed increased percentages of students at the high level in 2006 compared with $2001,{ }^{1}$ including Norway, the Netherlands, Iceland, Germany, Italy, the Russian Federation, Hong Kong sar, Latvia, Lithuania, and France. However, eight participants had decreased percentages at the high level-Macedonia, the United States, Romania, Morocco, New Zealand, Moldova, and the Canadian provinces of Ontario and Quebec.

As in PIRLS 2001, students' characterizations of themselves in terms of the levels of the reading self-concept index were broadly aligned with their average reading achievement. Students at the high level had average achievement 50 points higher than students at the medium level, and those at the medium level had average achievement more than 40 points higher than students at the low level. As noted above, however, there were few students in the low group in each country. 
Exhibit 4.2 Index of Students' Reading Self-Concept (SRSC) with Trends

PIRLS 2006

4th Grade

\begin{tabular}{|c|c|c|c|c|c|c|c|c|c|c|c|c|}
\hline \multirow[b]{2}{*}{ Countries } & \multicolumn{4}{|c|}{ High SRSC } & \multicolumn{4}{|c|}{ Medium SRSC } & \multicolumn{4}{|c|}{ Low SRSC } \\
\hline & $\begin{array}{c}2006 \\
\text { Percent } \\
\text { of Students }\end{array}$ & $\begin{array}{c}\text { Average } \\
\text { Achievement }\end{array}$ & $\begin{array}{l}\text { Difference } \\
\text { in Percent } \\
\text { from } 2001\end{array}$ & & $\begin{array}{c}2006 \\
\text { Percent } \\
\text { of Students }\end{array}$ & $\begin{array}{c}\text { Average } \\
\text { Achievement }\end{array}$ & $\begin{array}{l}\text { Difference } \\
\text { in Percen } \\
\text { from } 200\end{array}$ & & $\begin{array}{c}2006 \\
\text { Percent } \\
\text { of Students }\end{array}$ & $\begin{array}{c}\text { Average } \\
\text { Achievement }\end{array}$ & $\begin{array}{l}\text { Difference } \\
\text { in Percent } \\
\text { from } 2001\end{array}$ & \\
\hline Israel & $63(0.9)$ & $544(2.8)$ & $2(1.3)$ & & $36(0.9)$ & $477(4.6)$ & $-2(1.3)$ & & $1(0.2)$ & $\sim \sim$ & $0(0.3)$ & \\
\hline Austria & $62(0.9)$ & $553(2.4)$ & $\Delta \Delta$ & & $36(0.9)$ & $517(2.7)$ & $\Delta 0$ & & $2(0.3)$ & $\sim \sim$ & $\Delta 0$ & \\
\hline Sweden & $62(0.9)$ & $569(2.2)$ & $-2(1.3)$ & & $37(0.9)$ & $523(3.0)$ & $1(1.3)$ & & $2(0.2)$ & $\sim \sim$ & $1(0.3)$ & \\
\hline Poland & $61(0.9)$ & $547(2.2)$ & 00 & & $36(0.8)$ & $483(3.3)$ & $\Delta 0$ & & $2(0.3)$ & $\sim \sim$ & $\Delta 0$ & \\
\hline Norway & $61(1.2)$ & $518(2.4)$ & $5(1.6)$ & 0 & $37(1.2)$ & $477(3.5)$ & $-5(1.6)$ & (i) & $2(0.3)$ & $\sim \sim$ & $0(0.4)$ & \\
\hline Netherlands & $60(0.9)$ & $560(1.8)$ & $4(1.3)$ & 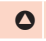 & $36(0.9)$ & $531(2.2)$ & $-7(1.2)$ & ( & $4(0.4)$ & $508(5.8)$ & $2(0.5)$ & 0 \\
\hline Denmark & $60(0.9)$ & $574(2.2)$ & $\Delta \diamond$ & & $38(0.9)$ & $511(3.1)$ & $\Delta \diamond$ & & $3(0.3)$ & $442(8.9)$ & $\Delta \diamond$ & \\
\hline Slovenia & $58(1.0)$ & $545(2.1)$ & $0(1.5)$ & & $40(1.0)$ & $491(2.4)$ & $-1(1.5)$ & & $2(0.2)$ & $\sim \sim$ & $1(0.4)$ & \\
\hline Iceland & $58(0.8)$ & $534(1.5)$ & $3(1.2)$ & 0 & $40(0.9)$ & $484(2.1)$ & $-3(1.2)$ & (i) & $2(0.3)$ & $\sim \sim$ & $1(0.4)$ & \\
\hline Germany & $58(0.9)$ & $571(2.4)$ & $5(1.2)$ & 0 & $40(0.9)$ & $529(2.2)$ & $-6(1.2)$ & $\nabla$ & $2(0.2)$ & $\sim \sim$ & $1(0.3)$ & \\
\hline Bulgaria & $58(1.4)$ & $570(3.9)$ & $0(2.0)$ & & $38(1.3)$ & $523(5.6)$ & $-3(1.9)$ & & $4(0.6)$ & $482(14.2)$ & $3(0.7)$ & 0 \\
\hline Italy & $56(1.1)$ & $569(3.2)$ & $6(1.7)$ & 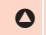 & $41(1.1)$ & $534(2.9)$ & $-7(1.7)$ & (i) & $3(0.3)$ & $496(9.2)$ & $1(0.4)$ & \\
\hline Macedonia, Rep. of & $55(1.3)$ & $486(3.9)$ & $-9(1.8)$ & 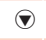 & $44(1.2)$ & $401(4.6)$ & $8(1.7)$ & 0 & $1(0.2)$ & $\sim \sim$ & $0(0.3)$ & \\
\hline Iran, Islamic Rep. of & $54(1.2)$ & $458(3.3)$ & $3(2.1)$ & & $44(1.2)$ & $383(3.8)$ & $-3(2.1)$ & & $2(0.2)$ & $\sim \sim$ & $0(0.3)$ & \\
\hline Canada, British Columbia & $54(0.9)$ & $584(2.5)$ & $\Delta 0$ & & $43(0.9)$ & $533(3.0)$ & $\Delta \Delta$ & & $2(0.3)$ & $\sim \sim$ & $\Delta \Delta$ & \\
\hline Qatar & $54(0.6)$ & $400(1.7)$ & $\Delta \Delta$ & & $43(0.6)$ & $309(1.7)$ & $\Delta \Delta$ & & $3(0.2)$ & $279(9.6)$ & $\Delta \Delta$ & \\
\hline Canada, Alberta & $53(0.9)$ & $584(2.8)$ & $\Delta \diamond$ & & $44(0.9)$ & $538(2.5)$ & $\Delta \diamond$ & & $3(0.3)$ & $505(6.6)$ & $\Delta \diamond$ & \\
\hline Russian Federation & $53(1.1)$ & $584(3.5)$ & $15(1.7)$ & 0 & $45(0.9)$ & $546(3.8)$ & $-15(1.5)$ & 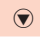 & $2(0.3)$ & $\sim \sim$ & $0(0.4)$ & \\
\hline Canada, Nova Scotia & $52(0.9)$ & $572(2.4)$ & $\Delta \Delta$ & & $45(0.9)$ & $518(2.3)$ & $\Delta \diamond$ & & $3(0.3)$ & $469(7.5)$ & $\Delta \diamond$ & \\
\hline United States & $51(0.8)$ & $566(3.5)$ & $-6(1.5)$ & 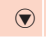 & $44(0.8)$ & $518(3.9)$ & $5(1.4)$ & 0 & $4(0.3)$ & $495(5.9)$ & $1(0.6)$ & \\
\hline Belgium (Flemish) & $51(1.0)$ & $565(2.0)$ & $\Delta \diamond$ & & $44(1.0)$ & $532(2.3)$ & $\Delta \diamond$ & & $5(0.4)$ & $502(4.3)$ & $\Delta \diamond$ & \\
\hline Georgia & $51(1.3)$ & $498(3.2)$ & $\Delta 0$ & & $46(1.2)$ & $450(3.7)$ & $\Delta \Delta$ & & $4(0.6)$ & $428(14.8)$ & 00 & \\
\hline Canada, Ontario & $51(1.0)$ & $579(2.8)$ & $-5(1.5)$ & 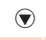 & $46(1.0)$ & $533(3.5)$ & $4(1.5)$ & 0 & $3(0.3)$ & $494(7.8)$ & $1(0.5)$ & \\
\hline Luxembourg & $50(0.7)$ & $582(1.3)$ & $\Delta \diamond$ & & $46(0.7)$ & $535(1.5)$ & $\Delta \Delta$ & & $4(0.3)$ & $497(4.5)$ & $\Delta \Delta$ & \\
\hline Romania & $50(1.4)$ & $524(4.2)$ & $-4(1.9)$ & $\odot$ & $45(1.3)$ & $467(5.6)$ & $0(1.8)$ & & $5(0.7)$ & $416(10.6)$ & $4(0.8)$ & 0 \\
\hline Canada, Quebec & $50(1.3)$ & $560(2.5)$ & $-9(1.8)$ & 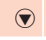 & $46(1.2)$ & $515(3.1)$ & $6(1.7)$ & 0 & $4(0.5)$ & $463(6.8)$ & $2(0.6)$ & $\boldsymbol{0}$ \\
\hline Trinidad and Tobago & $49(1.4)$ & $482(3.7)$ & $\Delta \diamond$ & & $47(1.3)$ & $399(5.8)$ & $\Delta \diamond$ & & $4(0.6)$ & $338(16.4)$ & $\Delta \Delta$ & \\
\hline Spain & $48(1.0)$ & $535(2.6)$ & $\Delta \Delta$ & & $50(1.0)$ & $495(2.7)$ & $\Delta \Delta$ & & $2(0.2)$ & $\sim \sim$ & $\Delta \Delta$ & \\
\hline Hong Kong SAR & $48(0.9)$ & $585(1.9)$ & $9(1.3)$ & 0 & $50(0.9)$ & $545(2.7)$ & $-7(1.3)$ & (1) & $2(0.2)$ & $\sim \sim$ & $-1(0.4)$ & \\
\hline Hungary & $45(1.3)$ & $579(2.7)$ & $-2(1.6)$ & & $51(1.1)$ & $531(3.3)$ & $1(1.4)$ & & $4(0.4)$ & $495(7.7)$ & $2(0.5)$ & 0 \\
\hline Chinese Taipei & $45(0.9)$ & $560(2.1)$ & $\Delta \diamond$ & & $51(0.9)$ & $518(2.2)$ & $\Delta \diamond$ & & $4(0.3)$ & $494(5.9)$ & $\Delta \diamond$ & \\
\hline Singapore & $45(0.9)$ & $583(2.9)$ & $-2(1.4)$ & & $52(0.8)$ & $542(3.0)$ & $2(1.3)$ & & $3(0.2)$ & $489(6.4)$ & $0(0.4)$ & \\
\hline Latvia & $43(1.2)$ & $567(2.6)$ & $9(1.5)$ & 0 & $53(1.2)$ & $523(3.0)$ & $-9(1.6)$ & $\nabla$ & $3(0.4)$ & $498(7.0)$ & $1(0.5)$ & \\
\hline Slovak Republic & $43(0.9)$ & $562(2.4)$ & $0(1.6)$ & & $54(0.9)$ & $512(3.3)$ & $-2(1.5)$ & & $4(0.3)$ & $459(7.0)$ & $2(0.5)$ & $\theta$ \\
\hline Scotland & $43(1.1)$ & $556(3.7)$ & $-1(1.7)$ & & $52(1.1)$ & $512(2.9)$ & $-1(1.6)$ & & $6(0.5)$ & $457(5.1)$ & $2(0.6)$ & 0 \\
\hline England & $42(1.1)$ & $578(3.5)$ & $-3(1.6)$ & & $51(1.1)$ & $519(2.7)$ & $0(1.6)$ & & $7(0.5)$ & $468(7.0)$ & $3(0.7)$ & 0 \\
\hline Lithuania & $40(0.9)$ & $561(2.1)$ & $5(1.4)$ & 0 & $57(0.8)$ & $523(1.9)$ & $-6(1.3)$ & $\odot$ & $3(0.3)$ & $493(6.8)$ & $1(0.5)$ & \\
\hline Kuwait & $38(1.3)$ & $384(5.2)$ & $\Delta 0$ & & $59(1.2)$ & $318(5.0)$ & $\Delta \Delta$ & & $3(0.3)$ & $247(11.6)$ & 00 & \\
\hline Morocco & $38(1.6)$ & $354(5.8)$ & $-8(2.4)$ & 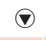 & $59(1.6)$ & $309(7.6)$ & $9(2.3)$ & 0 & $3(0.5)$ & $276(22.0)$ & $-1(1.1)$ & \\
\hline Belgium (French) & $38(0.9)$ & $526(3.1)$ & $\Delta \Delta$ & & $58(0.8)$ & $487(2.7)$ & $\Delta \Delta$ & & $4(0.5)$ & $454(6.1)$ & $\Delta \Delta$ & \\
\hline New Zealand & $36(0.8)$ & $574(2.2)$ & $-9(1.3)$ & $\odot$ & $60(0.8)$ & $513(2.4)$ & $8(1.3)$ & 0 & $4(0.3)$ & $459(6.1)$ & $1(0.5)$ & \\
\hline France & $36(0.8)$ & $549(3.1)$ & $3(1.3)$ & 0 & $60(0.9)$ & $510(1.9)$ & $-4(1.3)$ & $\nabla$ & $4(0.3)$ & $472(5.1)$ & $1(0.4)$ & \\
\hline Indonesia & $34(1.3)$ & $426(3.8)$ & $\Delta \Delta$ & & $65(1.2)$ & $398(4.7)$ & $\Delta \Delta$ & & $1(0.3)$ & $\sim \sim$ & $\Delta \diamond$ & \\
\hline Moldova, Rep. of & $32(1.3)$ & $525(3.6)$ & $-11(2.1)$ & 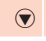 & $65(1.3)$ & $490(3.4)$ & $11(2.1)$ & 0 & $3(0.4)$ & $469(10.7)$ & $-1(0.7)$ & \\
\hline South Africa & $31(0.8)$ & $369(6.6)$ & $\Delta \diamond$ & & $64(0.8)$ & $282(5.3)$ & $\Delta \diamond$ & & $4(0.3)$ & $232(6.6)$ & $\Delta \diamond$ & \\
\hline International Avg. & $49(0.2)$ & $529(0.5)$ & & & $48(0.2)$ & $479(0.6)$ & & & $3(0.1)$ & $436(1.9)$ & & \\
\hline
\end{tabular}

Based on students' responses to the following: reading is very easy for me, I do not read as well as other students in my class, when I am reading by myself I understand almost everything I read, and I read slower than other students in my class. Average is computed on a 4-point scale: Disagree a lot $=1$, Disagree a little $=2$, Agree a little $=3$, and Agree a lot $=4$. Responses for negative statements were reverse-coded. High indicates an average of greater than 3 through 4. Medium indicates an average of 2 through 3. Low indicates an average of 1 to less than 2 .

Please note that "I read slower than other students in my class" is a new variable added to the index in PIRLS 2006, and is not a part of the PIRLS 2001 index calculations.

() Standard errors appear in parentheses. Because results are rounded to the nearest whole number, some totals may appear inconsistent.
An " $r$ " indicates data are available for $70-84 \%$ of the students. An " $s$ " indicates data are available for $50-69 \%$ of the students. An " $x$ " indicates data are available for less than $50 \%$ of the students.

A tilde $(\sim)$ indicates insufficient data to report achievement.

A diamond $(\diamond)$ indicates the country did not participate in the 2001 assessment.

NOTE: The International Average does not include the results from the Canadian provinces. Trend Note: The primary education systems of the Russian Federation and Slovenia underwent structural changes. Data for Canada, Ontario include only public schools. 


\section{How Often Do Children Engage in Literacy Activities Outside of School?}

Exhibit 4.3 presents trends in the frequency with which students read stories or novels outside of school. The exhibit shows the percentage of students in each country in 2006 that reported reading stories or novels, as follows: every day or almost every day, once or twice a week, once or twice a month, and never or almost never, as well as the average reading achievement of those students. Also presented for those countries that participated in both cycles of PIRLs is the change in the percentage of students in each of these categories since 2001.

On average across countries, nearly one third of students (32\%) reported reading stories or novels outside of school every day or almost every day, and an additional one third (31\%) at least once a week. PIRLS 2006 participants with the highest percentages of frequent readers of novels and stories (at least $50 \%$ of students reading every day or almost every day) included four of the Canadian provinces (British Columbia, Nova Scotia, Alberta, and Ontario) and the Russian Federation. Although most countries had a majority of students reading novels and stories at least weekly, many countries also had a significant percentage of nonreaders. Countries where the percentage of students reporting never or almost never reading a novel or short story exceeded 25\% included Germany, Spain, Belgium (French), Luxembourg, Norway, Austria, Bulgaria, Italy, Slovenia, Latvia, and the Slovak Republic.

A number of participants showed an increase in the percentage of students reading stories or novels every day or almost every day, including Israel, Hungary, Hong Kong sar, Germany, Morocco, Romania, Norway, Italy, and the Canadian province of Quebec. The greatest increases were found in Israel and Hong Kong SAR (16\%). Countries with a decrease in the percentage of students in this frequent reader category included the Russian Federation, the Netherlands, Singapore, Macedonia, Iceland, Scotland, England, Sweden, Bulgaria, Slovenia, Moldova, and Latvia. Countries with increased percentages in the never or almost never category included Scotland, England, Sweden, Bulgaria, Moldova, and Latvia. 
Exhibit 4.3 Students Reading Stories or Novels Outside of School with Trends

PIRLS 2006

4th Grade

\begin{tabular}{|c|c|c|c|c|c|c|c|c|}
\hline \multirow[b]{2}{*}{ Countries } & \multicolumn{4}{|c|}{ Every Day or Almost Every Day } & \multicolumn{4}{|c|}{ Once or Twice a Week } \\
\hline & $\begin{array}{c}2006 \\
\text { Percent } \\
\text { of Students }\end{array}$ & $\begin{array}{c}\text { Average } \\
\text { Achievement }\end{array}$ & $\begin{array}{l}\text { Difference } \\
\text { in Percent } \\
\text { from } 2001\end{array}$ & & $\begin{array}{c}2006 \\
\text { Percent } \\
\text { of Students }\end{array}$ & $\begin{array}{c}\text { Average } \\
\text { Achievement }\end{array}$ & $\begin{array}{l}\text { Difference } \\
\text { in Percent } \\
\text { from } 2001\end{array}$ & \\
\hline Canada, British Columbia & $57(1.2)$ & $572(2.5)$ & $\Delta \Delta$ & & $26(0.7)$ & $552(3.2)$ & $\Delta \Delta$ & \\
\hline Canada, Nova Scotia & $54(1.0)$ & $557(2.4)$ & $\Delta \Delta$ & & $26(0.8)$ & $534(2.9)$ & $\Delta \Delta$ & \\
\hline Canada, Alberta & $53(1.2)$ & $577(2.7)$ & $\Delta \Delta$ & & $27(0.9)$ & $549(3.5)$ & $\Delta \diamond$ & \\
\hline Canada, Ontario & $50(1.7)$ & $566(3.2)$ & $0(2.0)$ & & $28(1.4)$ & $548(3.0)$ & $0(1.7)$ & \\
\hline Russian Federation & $50(1.1)$ & $568(3.6)$ & $-6(1.9)$ & $(\uparrow$ & $32(0.7)$ & $565(3.6)$ & $3(1.3)$ & $\theta$ \\
\hline Trinidad and Tobago & $47(1.4)$ & $452(5.8)$ & $\Delta \diamond$ & & $31(1.3)$ & $431(6.6)$ & $\Delta \diamond$ & \\
\hline New Zealand & $46(1.1)$ & $556(2.3)$ & $0(1.9)$ & & $29(0.8)$ & $526(2.6)$ & $-1(1.4)$ & \\
\hline Israel & $46(1.0)$ & $532(4.2)$ & $16(1.5)$ & $\theta$ & $32(0.8)$ & $513(3.8)$ & $6(1.3)$ & $\theta$ \\
\hline Netherlands & $45(1.0)$ & $558(2.0)$ & $-3(1.5)$ & ( & $28(0.7)$ & $542(1.8)$ & $2(1.1)$ & $\theta$ \\
\hline Singapore & $44(1.1)$ & $579(3.0)$ & $-9(1.6)$ & ( & $33(0.8)$ & $555(3.1)$ & $6(1.1)$ & $\theta$ \\
\hline Macedonia, Rep. of & $44(1.5)$ & $442(5.1)$ & $-4(2.1)$ & $(\nabla$ & $35(1.3)$ & $449(5.0)$ & $1(1.8)$ & \\
\hline Canada, Quebec & $44(1.4)$ & $546(3.3)$ & $5(1.9)$ & $\theta$ & $28(0.8)$ & $530(3.1)$ & $-1(1.3)$ & \\
\hline Iceland & $42(0.9)$ & $522(2.4)$ & $-2(1.1)$ & ( & $27(0.8)$ & $512(2.3)$ & $1(1.0)$ & \\
\hline South Africa & $39(1.0)$ & $308(7.0)$ & $\Delta \Delta$ & & $33(0.6)$ & $310(6.7)$ & $\Delta \Delta$ & \\
\hline Qatar & $37(0.6)$ & $361(2.1)$ & $\Delta \Delta$ & & $37(0.6)$ & $359(2.0)$ & $\Delta \Delta$ & \\
\hline United States & $36(1.3)$ & $558(4.6)$ & $2(2.0)$ & & $28(0.8)$ & $541(3.6)$ & $-1(1.3)$ & \\
\hline Hungary & $36(1.2)$ & $554(4.6)$ & $3(1.5)$ & $\theta$ & $34(0.9)$ & $553(3.5)$ & $1(1.3)$ & \\
\hline Hong Kong SAR & $36(0.9)$ & $575(2.4)$ & $16(1.3)$ & 0 & $40(0.8)$ & $568(2.6)$ & $-5(1.2)$ & ( \\
\hline Scotland & $35(1.3)$ & $555(4.2)$ & $-5(1.8)$ & ( & $29(0.9)$ & $525(3.3)$ & $-2(1.4)$ & \\
\hline Kuwait & $33(1.2)$ & $351(5.2)$ & $\Delta \nu$ & & $40(1.0)$ & $337(4.9)$ & $\Delta \Delta$ & \\
\hline England & $33(1.2)$ & $573(3.9)$ & $-5(1.8)$ & $(\nabla$ & $30(1.0)$ & $535(3.2)$ & $-1(1.4)$ & \\
\hline Germany & $32(0.7)$ & $566(3.0)$ & $5(1.0)$ & 0 & $21(0.8)$ & $547(3.7)$ & $0(1.1)$ & \\
\hline France & $32(1.0)$ & $533(2.9)$ & $2(1.5)$ & & $30(0.8)$ & $524(2.3)$ & $1(1.2)$ & \\
\hline Georgia & $32(1.5)$ & $467(3.5)$ & $\Delta \Delta$ & & $32(1.3)$ & $479(3.5)$ & $\Delta \Delta$ & \\
\hline Belgium (Flemish) & $31(0.9)$ & $557(2.8)$ & $\Delta \Delta$ & & $34(0.9)$ & $546(2.4)$ & $\Delta \Delta$ & \\
\hline Spain & $31(1.1)$ & $511(3.7)$ & $\Delta \Delta$ & & $24(0.8)$ & $515(3.0)$ & $\Delta \Delta$ & \\
\hline Poland & $31(1.1)$ & $524(3.3)$ & $\Delta \Delta$ & & $37(1.2)$ & $524(3.1)$ & $\Delta \Delta$ & \\
\hline Sweden & $30(0.9)$ & $564(3.2)$ & $-3(1.3)$ & ( & $33(0.9)$ & $549(3.4)$ & $-1(1.2)$ & \\
\hline Chinese Taipei & $30(0.8)$ & $549(2.7)$ & $\Delta \diamond$ & & $36(0.9)$ & $541(2.4)$ & $\Delta \Delta$ & \\
\hline Denmark & $29(1.1)$ & $558(3.2)$ & $\Delta \Delta$ & & $26(0.8)$ & $548(3.2)$ & $\Delta \Delta$ & \\
\hline Belgium (French) & $28(0.9)$ & $509(3.7)$ & $\Delta \Delta$ & & $27(0.8)$ & $498(3.5)$ & $\Delta \Delta$ & \\
\hline Lithuania & $28(1.0)$ & $542(2.5)$ & $1(1.6)$ & & $30(0.9)$ & $538(2.5)$ & $-1(1.3)$ & \\
\hline Morocco & $27(1.2)$ & $334(6.2)$ & $9(1.6)$ & $\theta$ & $36(1.5)$ & $334(6.3)$ & $2(2.2)$ & \\
\hline Romania & $27(1.4)$ & $491(6.1)$ & $7(1.9)$ & $\theta$ & $34(1.3)$ & $497(5.8)$ & $-4(1.9)$ & ( \\
\hline Iran, Islamic Rep. of & $25(0.9)$ & $435(5.8)$ & $2(1.3)$ & & $43(1.3)$ & $429(3.6)$ & $1(1.6)$ & \\
\hline Luxembourg & $24(0.6)$ & $587(2.6)$ & $\Delta \Delta$ & & $24(0.6)$ & $554(2.6)$ & $\Delta \Delta$ & \\
\hline Norway & $23(1.1)$ & $509(3.2)$ & $5(1.4)$ & $\theta$ & $24(0.9)$ & $501(3.5)$ & $-1(1.2)$ & \\
\hline Austria & $23(0.9)$ & $554(3.0)$ & $\Delta \Delta$ & & $23(0.8)$ & $541(3.0)$ & $\Delta 0$ & \\
\hline Indonesia & $22(1.0)$ & $399(4.8)$ & $\Delta \Delta$ & & $36(1.1)$ & $413(4.4)$ & $\Delta \diamond$ & \\
\hline Bulgaria & $22(1.0)$ & $558(6.4)$ & $-6(1.6)$ & ( & $26(1.0)$ & $555(4.9)$ & $-2(1.5)$ & \\
\hline Italy & $22(1.2)$ & $573(4.4)$ & 7 (1.4) & $\theta$ & $20(1.0)$ & $556(3.3)$ & $2(1.2)$ & \\
\hline Slovenia & $21(0.8)$ & $536(3.0)$ & $-5(1.4)$ & ( & $29(0.8)$ & $520(3.0)$ & $2(1.3)$ & \\
\hline Moldova, Rep. of & $19(1.0)$ & $499(4.4)$ & $-4(1.8)$ & 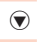 & $37(1.2)$ & $503(3.7)$ & $-7(2.2)$ & $\ominus$ \\
\hline Latvia & $19(0.9)$ & $558(4.0)$ & $-4(1.3)$ & ( & $25(1.1)$ & $548(3.6)$ & $-2(1.4)$ & \\
\hline Slovak Republic & $15(0.7)$ & $540(4.5)$ & $0(1.1)$ & & $21(0.9)$ & $536(3.5)$ & $0(1.3)$ & \\
\hline International Avg. & $32(0.2)$ & $512(0.6)$ & & & $31(0.2)$ & $503(0.6)$ & & \\
\hline
\end{tabular}

Background data provided by students.

() Standard errors appear in parentheses. Because results are rounded to the nearest whole number, some totals may appear inconsistent.

A diamond $(\diamond)$ indicates the country did not participate in the 2001 assessment.
NOTE: The International Average does not include the results from the Canadian provinces. Trend Note: The primary education systems of the Russian Federation and Slovenia underwent structural changes. Data for Canada, Ontario include only public schools. 


\section{Exhibit 4.3 Students Reading Stories or Novels Outside of School with Trends (Continued)}

\begin{tabular}{|c|c|c|c|c|c|c|c|c|}
\hline \multirow[b]{2}{*}{ Countries } & \multicolumn{4}{|c|}{ Once or Twice a Month } & \multicolumn{4}{|c|}{ Never or Almost Never } \\
\hline & $\begin{array}{c}2006 \\
\text { Percent } \\
\text { of Students }\end{array}$ & $\begin{array}{c}\text { Average } \\
\text { Achievement }\end{array}$ & $\begin{array}{l}\text { Difference } \\
\text { in Percent } \\
\text { from } 2001\end{array}$ & & $\begin{array}{c}2006 \\
\text { Percent } \\
\text { of Students }\end{array}$ & $\begin{array}{c}\text { Average } \\
\text { Achievement }\end{array}$ & $\begin{array}{l}\text { Difference } \\
\text { in Percent } \\
\text { from } 2001\end{array}$ & \\
\hline Canada, British Columbia & $11(0.7)$ & $539(4.1)$ & $\Delta \Delta$ & & $6(0.6)$ & $511(7.0)$ & $\Delta \Delta$ & \\
\hline Canada, Nova Scotia & $12(0.5)$ & $531(3.9)$ & $\Delta \Delta$ & & $8(0.5)$ & $497(6.3)$ & $\Delta \Delta$ & \\
\hline Canada, Alberta & $12(0.7)$ & $544(3.4)$ & $\Delta \diamond$ & & $8(0.6)$ & $523(4.9)$ & $\Delta \diamond$ & \\
\hline Canada, Ontario & $14(0.9)$ & $544(5.1)$ & $0(1.1)$ & & $7(0.7)$ & $520(7.5)$ & $1(1.0)$ & \\
\hline Russian Federation & $11(0.6)$ & $563(4.9)$ & $2(0.9)$ & $\boldsymbol{\theta}$ & $7(0.6)$ & $548(5.4)$ & $1(0.8)$ & \\
\hline Trinidad and Tobago & $10(0.6)$ & $420(7.1)$ & $\Delta \diamond$ & & $11(1.0)$ & $408(7.2)$ & $\Delta \diamond$ & \\
\hline New Zealand & $14(0.6)$ & $513(3.9)$ & $1(1.0)$ & & $10(0.6)$ & $473(4.2)$ & $0(1.0)$ & \\
\hline Israel & $15(0.6)$ & $495(4.8)$ & $1(0.9)$ & & $7(0.5)$ & $481(7.2)$ & $-22(1.4)$ & ( \\
\hline Netherlands & $13(0.6)$ & $540(2.2)$ & $0(0.9)$ & & $14(0.7)$ & $531(3.2)$ & $0(1.1)$ & \\
\hline Singapore & $15(0.5)$ & $534(3.5)$ & $4(0.7)$ & 0 & $8(0.4)$ & $505(4.6)$ & $-1(0.7)$ & \\
\hline Macedonia, Rep. of & $16(1.1)$ & $452(7.1)$ & $2(1.6)$ & & $5(0.4)$ & $445(10.1)$ & $1(0.6)$ & \\
\hline Canada, Quebec & $16(0.9)$ & $528(3.4)$ & $-1(1.3)$ & & $12(0.9)$ & $502(5.1)$ & $-4(1.6)$ & ( \\
\hline Iceland & $16(0.6)$ & $508(2.8)$ & $2(0.8)$ & $\boldsymbol{\theta}$ & $15(0.6)$ & $486(2.9)$ & $-1(0.8)$ & \\
\hline South Africa & $14(0.5)$ & $302(6.6)$ & $\Delta \Delta$ & & $13(0.7)$ & $294(7.2)$ & $\Delta \Delta$ & \\
\hline Qatar & $15(0.4)$ & $347(3.4)$ & $\Delta \diamond$ & & $10(0.4)$ & $342(4.4)$ & $\Delta \diamond$ & \\
\hline United States & $18(0.7)$ & $539(3.6)$ & $1(1.0)$ & & $18(0.9)$ & $509(3.2)$ & $-3(1.3)$ & \\
\hline Hungary & $20(0.9)$ & $553(2.9)$ & $-2(1.2)$ & $(\nabla$ & $10(0.7)$ & $535(6.7)$ & $-2(1.0)$ & \\
\hline Hong Kong SAR & $17(0.7)$ & $550(3.3)$ & $-6(1.0)$ & ( & $7(0.5)$ & $518(5.0)$ & $-5(0.8)$ & ( \\
\hline Scotland & $18(0.9)$ & $521(3.8)$ & $3(1.2)$ & 0 & $17(1.2)$ & $484(4.8)$ & $5(1.5)$ & 0 \\
\hline Kuwait & $19(0.8)$ & $326(5.8)$ & 00 & & $8(0.7)$ & $312(8.9)$ & $\Delta \diamond$ & \\
\hline England & $20(0.7)$ & $536(4.2)$ & $3(1.0)$ & 0 & $17(0.8)$ & $492(3.5)$ & $3(1.2)$ & 0 \\
\hline Germany & $16(0.5)$ & $550(3.6)$ & $1(0.8)$ & & $31(0.9)$ & $535(2.6)$ & $-6(1.4)$ & ( \\
\hline France & $19(0.6)$ & $522(2.8)$ & $-1(0.9)$ & & $19(0.9)$ & $501(2.9)$ & $-2(1.3)$ & \\
\hline Georgia & $20(1.4)$ & $484(6.8)$ & $\Delta \Delta$ & & $17(1.2)$ & $459(5.6)$ & $\Delta \Delta$ & \\
\hline Belgium (Flemish) & $21(0.8)$ & $545(3.0)$ & $\Delta \Delta$ & & $14(0.8)$ & $530(3.3)$ & $\Delta \diamond$ & \\
\hline Spain & $15(0.6)$ & $523(3.7)$ & $\Delta 0$ & & $29(1.0)$ & $508(3.2)$ & $\Delta 0$ & \\
\hline Poland & $21(0.8)$ & $516(3.7)$ & $\Delta \diamond$ & & $11(0.8)$ & $504(4.6)$ & $\Delta \diamond$ & \\
\hline Sweden & $22(0.8)$ & $546(2.7)$ & $0(1.0)$ & & $15(0.8)$ & $529(3.4)$ & $3(1.0)$ & 0 \\
\hline Chinese Taipei & $20(0.7)$ & $531(3.2)$ & $\Delta \Delta$ & & $15(0.7)$ & $505(3.1)$ & $\Delta \diamond$ & \\
\hline Denmark & $20(0.8)$ & $551(3.0)$ & $\Delta \Delta$ & & $25(1.1)$ & $529(3.0)$ & $\Delta \Delta$ & \\
\hline Belgium (French) & $18(0.7)$ & $507(3.3)$ & $\Delta \diamond$ & & $27(0.9)$ & $488(3.0)$ & $\Delta \diamond$ & \\
\hline Lithuania & $19(0.7)$ & $540(2.6)$ & $-1(1.2)$ & & $23(1.0)$ & $528(2.4)$ & $1(1.5)$ & \\
\hline Morocco & $21(1.5)$ & $327(10.2)$ & $-4(1.9)$ & & $16(2.5)$ & $282(16.8)$ & $-8(3.5)$ & $\nabla$ \\
\hline Romania & $23(1.3)$ & $503(5.8)$ & $-4(1.8)$ & ( & $16(1.1)$ & $463(9.8)$ & $2(1.7)$ & \\
\hline Iran, Islamic Rep. of & $22(0.9)$ & $423(4.3)$ & $1(1.3)$ & & $10(1.0)$ & $356(9.3)$ & $-5(1.6)$ & 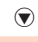 \\
\hline Luxembourg & $21(0.5)$ & $553(2.4)$ & $\Delta \Delta$ & & $31(0.6)$ & $539(1.8)$ & $\Delta \diamond$ & \\
\hline Norway & $21(0.8)$ & $503(5.1)$ & $1(1.2)$ & & $32(1.2)$ & $488(3.5)$ & $-5(1.8)$ & $\nabla$ \\
\hline Austria & $18(0.7)$ & $537(3.8)$ & $\Delta \Delta$ & & $37(1.1)$ & $528(2.6)$ & $\Delta \Delta$ & \\
\hline Indonesia & $16(0.7)$ & $411(5.7)$ & $\Delta \diamond$ & & $25(1.2)$ & $406(6.0)$ & $\Delta \Delta$ & \\
\hline Bulgaria & $23(1.0)$ & $555(6.0)$ & $2(1.4)$ & & $29(1.3)$ & $529(5.2)$ & $7(2.0)$ & 0 \\
\hline Italy & $15(0.8)$ & $554(4.1)$ & $-1(1.0)$ & & $43(1.3)$ & $540(3.3)$ & $-7(1.7)$ & ( \\
\hline Slovenia & $23(0.8)$ & $522(3.0)$ & $4(1.3)$ & 0 & $28(0.9)$ & $513(2.5)$ & $0(1.7)$ & \\
\hline Moldova, Rep. of & $23(0.9)$ & $504(4.1)$ & $0(1.8)$ & & $21(1.1)$ & $493(4.4)$ & $11(1.6)$ & $\theta$ \\
\hline Latvia & $21(0.9)$ & $544(3.6)$ & $1(1.2)$ & & $36(1.4)$ & $526(2.5)$ & $6(1.7)$ & 0 \\
\hline Slovak Republic & $21(0.7)$ & $540(3.7)$ & $-1(1.1)$ & & $43(1.1)$ & $522(3.9)$ & $1(1.7)$ & \\
\hline International Avg. & $18(0.1)$ & $500(0.7)$ & & & $19(0.2)$ & $479(0.9)$ & & \\
\hline
\end{tabular}

- Percent in 2006 significantly higher

( Percent in 2006 significantly lower 
On average internationally, and in most countries, students who reported reading novels and short stories most frequently had higher average achievement than those who read less frequently. Students who never or almost never did this kind of reading had the lowest achievement in most countries. The average achievement difference between students who read novels or short stories daily or almost daily and those who never or almost never read them was 33 points on the PIRLS reading scale.

Although literary texts such as short stories and novels make up a large part of the reading material of fourth-grade students outside of school, there are also many kinds of informational texts that children may read, such as magazines, newspapers, books that explain things, directions, or instructions. Exhibit 4.4 presents students' reports of how often they read such materials, together with average student reading achievement and changes since 2001. The PIRLS Reading for Information Scale was based on student responses to six questions/statements, as follows:

How often do you do these things outside of school?

- I read to find out about things I want to learn.

How often do you read these things after school?

- I read books that explain things.

- I read magazines.

- I read newspapers.

- I read directions or instructions.

- I read brochures or catalogs.

Student responses were recorded on a 4-point scale, as follows: every day or almost every day, once or twice a week, once or twice a month, and never or almost never, and students were assigned to one of the four categories of reading frequency for information on the basis of their average response (see Exhibit 4.4).

Compared to literary reading, which for many students was a daily or at least weekly occurrence, student reading of informational texts was 
more intermittent. Fewer students, on average internationally, reported daily informational reading (16\%) than literary reading (32\%), and relatively more reported reading for information once or twice a week or once or twice a month (72\% informational vs. $49 \%$ literary). Countries with the greatest percentages of students reporting frequent informational reading (30\% or more) included South Africa, Macedonia, Qatar, and Kuwait. Only two countries, Hong Kong SAR and Sweden, had greater percentages of students in 2006 than in 2001 reporting frequent reading for information. In contrast, several participants (Slovenia, Lithuania, Bulgaria, Singapore, Latvia, the United States, Iran, and the provinces of Ontario and Quebec) showed a decrease from 2001 to 2006 in the percentage of students reading informational texts daily or almost daily.

Not only was student reading for information more intermittent than literary reading, it also had a less clear-cut relationship with student reading achievement. Unlike literary reading, where students who read most frequently (every day or almost every day) had higher average achievement, average achievement for informational reading was highest among students who reported reading once or twice a week or once or twice a month.

In view of the widespread access to the Internet in today's world, and the reading opportunities that the Internet provides over and above those provided by print media, PIRLS 2006 asked students how much time they spent reading stories or articles on the Internet and how much time they spent reading them in books or magazines. Exhibit 4.5 presents the average number of hours on a typical day students in each country reported reading on the Internet and reading from books and magazines, separately for girls and boys and for all students together.

As might be expected, on average across countries, students reported spending more time on a typical day reading stories and articles in books or magazines than on the Internet (1.4 hours vs. 1.0 hours). Students in Kuwait, Qatar, and South Africa reported spending the most time reading on the Internet (more than 2 hours, on average, on a typical day), whereas in Iran, Luxembourg, the Netherlands, the Russian Federation, and Sweden, 
Exhibit 4.4 Students Reading for Information Outside of School with Trends

PIRLS 2006

4th Grade

\begin{tabular}{|c|c|c|c|c|c|c|c|c|}
\hline \multirow[b]{2}{*}{ Countries } & \multicolumn{4}{|c|}{ Every Day or Almost Every Day } & \multicolumn{4}{|c|}{ Once or Twice a Week } \\
\hline & $\begin{array}{c}2006 \\
\text { Percent } \\
\text { of Students }\end{array}$ & $\begin{array}{c}\text { Average } \\
\text { Achievement }\end{array}$ & $\begin{array}{l}\text { Difference } \\
\text { in Percent } \\
\text { from } 2001\end{array}$ & & $\begin{array}{c}2006 \\
\text { Percent } \\
\text { of Students }\end{array}$ & $\begin{array}{c}\text { Average } \\
\text { Achievement }\end{array}$ & $\begin{array}{l}\text { Difference } \\
\text { in Percent } \\
\text { from } 2001\end{array}$ & \\
\hline South Africa & $36(1.1)$ & $302(6.0)$ & $\Delta \Delta$ & & $45(0.7)$ & $307(5.8)$ & $\Delta \Delta$ & \\
\hline Macedonia, Rep. of & $33(1.3)$ & $440(5.4)$ & $-3(1.9)$ & & $48(1.1)$ & $453(4.4)$ & $1(1.7)$ & \\
\hline Qatar & $33(0.6)$ & $359(1.9)$ & $\Delta \Delta$ & & $49(0.6)$ & $355(1.7)$ & $\Delta \Delta$ & \\
\hline Kuwait & $30(1.4)$ & $342(5.6)$ & $\Delta \Delta$ & & $50(1.1)$ & $343(4.5)$ & $\Delta \Delta$ & \\
\hline Trinidad and Tobago & $29(1.4)$ & $440(6.2)$ & $\Delta \Delta$ & & $48(1.2)$ & $435(5.9)$ & $\Delta \Delta$ & \\
\hline Israel & $21(0.8)$ & $499(5.4)$ & $-1(1.3)$ & & $44(1.0)$ & $510(4.0)$ & $1(1.4)$ & \\
\hline Poland & $21(0.8)$ & $523(2.9)$ & $\Delta \Delta$ & & $46(0.9)$ & $522(3.3)$ & $\Delta \Delta$ & \\
\hline Slovak Republic & $20(1.0)$ & $527(4.2)$ & $1(1.3)$ & & $50(0.9)$ & $539(2.5)$ & $0(1.4)$ & \\
\hline Moldova, Rep. of & $19(1.0)$ & $497(4.0)$ & $1(1.8)$ & & $50(1.3)$ & $503(3.9)$ & $5(2.3)$ & \\
\hline Indonesia & $19(0.9)$ & $409(5.4)$ & $\Delta \Delta$ & & $50(1.1)$ & $409(4.4)$ & $\Delta \Delta$ & \\
\hline Hungary & $19(0.9)$ & $533(4.6)$ & $0(1.2)$ & & $50(0.9)$ & $552(3.5)$ & $4(1.3)$ & $\theta$ \\
\hline Russian Federation & $18(0.9)$ & $555(4.2)$ & $-2(1.4)$ & & $47(0.7)$ & $564(3.7)$ & $-1(1.4)$ & \\
\hline Slovenia & $18(0.6)$ & $510(3.3)$ & $-5(1.3)$ & ( & $49(0.9)$ & $519(2.6)$ & $2(1.4)$ & \\
\hline Lithuania & $18(0.8)$ & $530(3.3)$ & $-4(1.3)$ & $(\nabla$ & $53(0.9)$ & $538(1.9)$ & $1(1.3)$ & \\
\hline Bulgaria & $17(1.1)$ & $544(6.7)$ & $-10(1.6)$ & ( & $47(1.5)$ & $556(4.5)$ & $2(1.9)$ & \\
\hline Romania & $16(1.0)$ & $493(6.1)$ & $-3(1.7)$ & & $49(1.4)$ & $500(5.4)$ & $1(1.9)$ & \\
\hline Singapore & $16(0.5)$ & $558(3.5)$ & $-8(0.9)$ & ( & $47(0.8)$ & $561(3.2)$ & $0(1.0)$ & \\
\hline Latvia & $16(0.8)$ & $530(4.8)$ & $-3(1.3)$ & $(\nabla$ & $48(0.9)$ & $541(2.9)$ & $-3(1.7)$ & 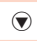 \\
\hline Georgia & $16(1.3)$ & $465(4.5)$ & $\Delta \Delta$ & & $43(1.3)$ & $480(3.0)$ & $\Delta \diamond$ & \\
\hline Germany & $15(0.6)$ & $536(3.3)$ & $1(0.9)$ & & $40(0.8)$ & $551(3.1)$ & $2(1.2)$ & $\theta$ \\
\hline Austria & $15(0.7)$ & $526(3.3)$ & $\Delta \Delta$ & & $43(1.0)$ & $540(2.7)$ & $\Delta \Delta$ & \\
\hline Spain & $14(0.8)$ & $501(3.6)$ & $\Delta \diamond$ & & $45(1.1)$ & $513(3.0)$ & $\Delta \Delta$ & \\
\hline New Zealand & $14(0.6)$ & $514(4.5)$ & $-1(1.1)$ & & $43(0.8)$ & $534(2.2)$ & $1(1.3)$ & \\
\hline United States & $14(0.6)$ & $519(4.5)$ & $-4(1.1)$ & ( & $43(0.9)$ & $538(3.5)$ & $-1(1.3)$ & \\
\hline Scotland & $13(0.8)$ & $506(5.2)$ & $-1(1.1)$ & & $42(1.0)$ & $527(3.6)$ & $-1(1.5)$ & \\
\hline Morocco & $13(1.2)$ & $324(7.5)$ & $2(1.7)$ & & $45(1.6)$ & $326(6.8)$ & $5(2.4)$ & $\boldsymbol{\theta}$ \\
\hline Belgium (French) & $13(0.7)$ & $480(4.6)$ & $\Delta \Delta$ & & $40(0.8)$ & $498(2.9)$ & $\Delta \Delta$ & \\
\hline France & $12(0.7)$ & $506(4.0)$ & $1(0.9)$ & & $40(0.8)$ & $520(2.2)$ & $1(1.2)$ & \\
\hline Hong Kong SAR & $12(0.5)$ & $554(4.0)$ & $4(0.7)$ & $\theta$ & $43(0.8)$ & $569(2.6)$ & $0(1.2)$ & \\
\hline Canada, Nova Scotia & $12(0.6)$ & $523(4.5)$ & $\Delta \Delta$ & & $42(1.0)$ & $545(2.6)$ & $\Delta \Delta$ & \\
\hline Iran, Islamic Rep. of & $11(0.8)$ & $435(6.7)$ & $-2(1.1)$ & ( & $40(1.5)$ & $436(3.8)$ & $1(2.1)$ & \\
\hline Norway & $11(0.7)$ & $485(4.7)$ & $0(1.0)$ & & $38(0.9)$ & $499(2.7)$ & $0(1.4)$ & \\
\hline Italy & $11(0.8)$ & $539(6.3)$ & $-1(1.1)$ & & $40(0.8)$ & $554(3.1)$ & $1(1.2)$ & \\
\hline Canada, Ontario & $11(0.9)$ & $532(6.2)$ & $-3(1.2)$ & ( & $40(1.5)$ & $554(3.1)$ & $-1(1.8)$ & \\
\hline Canada, Alberta & $10(0.6)$ & $543(4.2)$ & $\Delta \Delta$ & & $43(0.9)$ & $560(2.7)$ & $\Delta \Delta$ & \\
\hline England & $10(0.7)$ & $502(5.5)$ & $-2(1.0)$ & & $44(1.0)$ & $537(2.8)$ & $0(1.5)$ & \\
\hline Canada, Quebec & $10(0.6)$ & $520(3.9)$ & $-2(1.0)$ & ( & $37(1.1)$ & $532(3.3)$ & $-6(1.5)$ & ( \\
\hline Sweden & $9(0.7)$ & $539(6.0)$ & $2(0.8)$ & $\theta$ & $33(1.0)$ & $550(2.8)$ & $2(1.3)$ & \\
\hline Canada, British Columbia & $9(0.6)$ & $541(5.3)$ & $\Delta \Delta$ & & $39(1.0)$ & $557(3.3)$ & $\Delta \diamond$ & \\
\hline Chinese Taipei & $8(0.4)$ & $538(3.6)$ & $\Delta \diamond$ & & $38(0.8)$ & $538(2.2)$ & $\Delta \Delta$ & \\
\hline Iceland & $8(0.4)$ & $496(4.9)$ & $-1(0.7)$ & & $33(0.8)$ & $511(2.0)$ & $0(1.1)$ & \\
\hline Denmark & $6(0.5)$ & $526(5.7)$ & $\Delta \diamond$ & & $30(1.0)$ & $539(3.1)$ & $\Delta \diamond$ & \\
\hline Luxembourg & $6(0.3)$ & $542(4.1)$ & $\Delta \Delta$ & & $33(0.6)$ & $555(2.0)$ & $\Delta \Delta$ & \\
\hline Belgium (Flemish) & $4(0.4)$ & $532(5.7)$ & $\Delta \Delta$ & & $29(1.0)$ & $544(2.8)$ & $\Delta \Delta$ & \\
\hline Netherlands & $4(0.4)$ & $528(6.0)$ & $0(0.6)$ & & $25(1.0)$ & $542(2.3)$ & $1(1.2)$ & \\
\hline International Avg. & $16(0.1)$ & $492(0.8)$ & & & $43(0.2)$ & $503(0.6)$ & & \\
\hline
\end{tabular}

Based on students' responses on how often they read to find out about things they want to learn and how often they read the following things outside of school: books that explain things, magazines, newspapers, directions or instructions, and brochures and catalogs. Average is computed on a 4-point scale: Never or almost never $=1$, Once or twice a month $=2$, Once or twice a week $=3$, and Every day or almost every day $=4$. Every day or almost every day indicates an average of greater than 3.25 through 4 . Once or twice a week indicates an average of greater than 2.5 through 3.25 . Once or twice a month indicates an average of greater than 1.75 through 2.5. Never or almost never indicates an average of 1 to less than 1.75 .

Please note that "I read brochures and catalogs" is a new item added to the index in 2006, and is not included in the 2001 index calculations.

() Standard errors appear in parentheses. Because results are rounded to the nearest whole number, some totals may appear inconsistent. 
Exhibit 4.4 Students Reading for Information Outside of School with Trends (Continued)
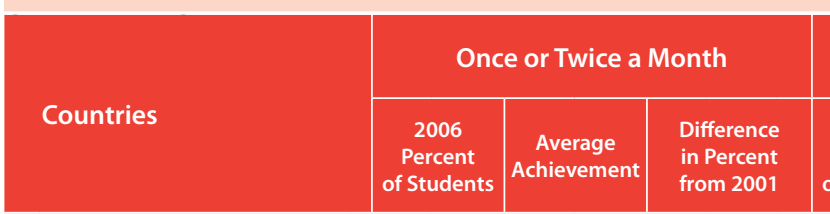

\begin{tabular}{|c|c|c|}
\hline \multicolumn{3}{|c}{ Never or Almost Never } \\
$\begin{array}{c}2006 \\
\text { Percent } \\
\text { of Students }\end{array}$ & $\begin{array}{c}\text { Average } \\
\text { Achievement }\end{array}$ & $\begin{array}{c}\text { Difference } \\
\text { in Percent } \\
\text { from } 2001\end{array}$ \\
\hline $3(0.5)$ & $304(19.7)$ & 00
\end{tabular}

South Africa
Macedonia, Rep. of
Qatar

$16(0.8) \quad 317(10.1)$

$16(1.2) \quad 447(6.3) \quad 2(1.5) \quad 2(0.3)$

$447(6.3)$

$\Delta \diamond$

$3(0.5)$

Kuwait

$15(0.4)$

Trinidad and Tobago

$16(0.9)$

359 (3.5)

$\Delta \diamond \quad 3(0.2)$

Israel

$329(6.4)$

$\begin{array}{cl}304(19.7) & \diamond \diamond \\ \sim \sim & 0(0.6)\end{array}$

Poland

$25(1.0)$

$444(5.8)$

$\Delta \diamond \quad 3(0.6)$

$345(7.6) \quad \diamond \diamond$

Slovak Republic

Moldova, Rep. of

$27(0.7)$

$532(3.9)$

$\Delta \diamond$

$4(0.5)$

$295(15.3)$

$-1(1.3) \quad 10(0.6)$

$414(13.6) \quad 00$

\begin{tabular}{|l|l|l|}
$518(3.4)$ & $\diamond \diamond$ & $6(0.5)$ \\
\hline
\end{tabular}

Indonesia

$24(0.8)$

$530(4.3)$

$-2(1.2)$

$500(4.3)$

$6(0.6)$

Hungary

$25(1.0)$

$-5(2.1)$

$7(0.9)$

$25(0.8)$

$562(3.4)-3(1.3)-6(0.9)$

Russian Federation

$28(0.9)$

Slovenia

Lithuania

$26(0.7)$

$562(3.4)$

$-3(1.3)$

$572(3.5)$

$1(1.3)$

$7(0.5)$

$25(0.8)$

$533(2.9) \quad 1(1.2)$

$8(0.5)$

$549(5.1)$

$508(6.8) \quad \diamond \diamond$

Bulgaria

25 (1.2)

$541(2.4)$

0 (1.4)

$4(0.4)$

$492(15.3) \quad 1(0.8)$

$26(1.1)$

Singapore

$550(4.3)$

4 (1.6)

$490(5.5) \quad-3(1.7)$

Latvia

Georgia

$561(3.4) \quad 4(0.9)$

- $11(1.3)$

$492(8.7)$

$391(8.0) \quad 0(1.4)$

$558(6.4) \quad 0(0.7)$

$558(6.4)$

$570(5.6) \quad 2(0.9)$

\begin{tabular}{ll}
$524(4.9) \quad 2(0.7) \quad 0$ \\
\hline
\end{tabular}

Germany

$29(0.9)$

$9(1.0)$

$533(4.7)$

$3(0.4)$

Austria

$30(1.5)$

- $8(0.3)$

$475(4.7)-4(1.7) \quad 0 \quad 7(0.6)$

\begin{tabular}{llll}
$475(4.7)$ & $\diamond \diamond$ & $11(1.0)$ \\
\hline $555(2.6)$ & $-4(1.0)$ & $\nabla$ & $13(0.7)$
\end{tabular}

$32(0.7)$

Spain

New Zealand

$31(0.8)$

$-4(1.0)$

$13(0.7)$
$12(0.7)$

$521(9.6) \quad 4(1.6) \quad 0$

United States

$30(0.9)$

$\Delta 0$

$518(2.7) \quad \diamond \diamond \quad 10(0.6)$

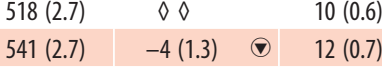

$31(0.6)$

$12(0.7)$
$10(0.6)$

$444(12.7)$

$538(4.4) \quad 4(0.4)$

Scotland

$34(1.0)$

$553(4.0) \quad 2(1.4)$

$10(0.6)$

Morocco

$28(1.4)$

Belgium (French)

$538(3.0) \quad-1(1.6)$

$11(1.0)$

$545(5.1)$

$450(10.3) \quad 0$

$547(3.7) \quad 0(1.0)$

$543(4.3) \quad \Delta \diamond$

$514(5.4) \quad \Delta \diamond$

$\begin{array}{lll}531(5.2) & 3(0.9) \quad 0\end{array}$

$\begin{array}{lll}546(5.3) & 3(0.9) & 0\end{array}$

France

Hong Kong SAR

Canada, Nova Scotia

$31(0.7)$

$331(9.1) \quad-5(2.4)$

$14(1.8)$

\begin{tabular}{l|l|l}
$506(3.1)$ & $\Delta \diamond$ & $16(0.8)$
\end{tabular}

$522(6.7)$

$3(1.2) \quad \boldsymbol{\theta}$

Iran, Islamic Rep. of

$34(0.9)$

$527(3.0) \quad-5(1.4) \quad \nabla \quad 14(0.6)$

$310(12.6) \quad-3(3.4)$

Norway

$33(0.8)$

$567(2.5) \quad-8(1.1) \quad \nabla$

$13(0.6)$

$552(2.7) \quad \Delta \diamond \quad 13(0.6)$

$510(3.6) \quad \diamond \diamond$

$530(3.8) \quad 3(0.8) \quad 0$

$33(0.8)$

35 (1.2)

$421(4.0) \quad-3(1.8)$

$14(1.2)$

$550(3.5)$

$3(0.8)$

Italy

Canada, Ontario

$34(0.9)$

$503(3.7)-4(1.5) \quad \odot \quad 17(0.8)$

$533(4.0) \quad \Delta \diamond$

Canada, Alberta

$36(1.0)$

$554(3.7) \quad-2(1.4)$

$14(0.8)$

$474(9.4) \quad 4(1.5) \quad 0$

$497(3.9) \quad 3(1.3) \quad 0$

Orta

England

$34(0.8)$

$558(3.3) \quad-1(1.6)$

$14(1.0)$

$552(4.8)$

$552(4.8) \quad 2(1.0) \quad 0$

Canada, Quebec

Sweden

Canada, British Columbia

Chinese Taipei

Iceland

Denmark

Luxembourg

Belgium (Flemish)

Netherlands

$35(1.1)$

\begin{tabular}{l|l|l}
$566(2.8)$ & $\Delta \diamond$ & $13(0.6)$
\end{tabular}

$564(4.5)$

4 (1.1) $\boldsymbol{\theta}$

$\begin{array}{llllll}556(3.2) & -2(1.6) & 11(0.7) & 537(5.8) & 3(0.9) & \boldsymbol{\Delta}\end{array}$

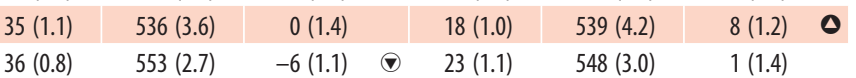

\begin{tabular}{|l|l|l|l|l|l|}
$38(0.8)$ & $564(3.1)$ & $\diamond \diamond$ & $14(0.7)$ & $561(4.2)$ & $\diamond \diamond$
\end{tabular}

$38(0.8) \quad 536(2.8) \quad \Delta \diamond \quad 16(0.7) \quad 529(3.5) \quad \Delta \diamond$

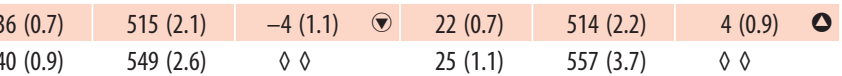

\begin{tabular}{|c|c|c|}
\hline $11(0$. & & \\
\hline
\end{tabular}

$42(0.8) \quad 549(2.3) \quad \Delta \diamond \quad 25(0.8) \quad 550(2.1) \quad \diamond$

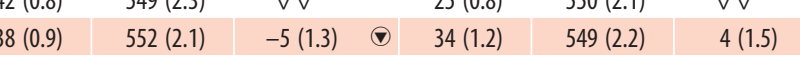

International Avg.

$29(0.1) \quad 506(0.7)$

$12(0.1) \quad 496(1.3)$

ه Percent in 2006 significantly higher

( Percent in 2006 significantly lower

A tilde $(\sim)$ indicates insufficient data to report achievement.

A diamond $(\diamond)$ indicates the country did not participate in the 2001 assessment.

NOTE: The International Average does not include the results from the Canadian provinces.

Trend Note: The primary education systems of the Russian Federation and Slovenia

underwent structural changes. Data for Canada, Ontario include only public schools. 
Exhibit 4.5 Students Read Stories or Articles Outside of School

PIRLS 2006

4th Grade

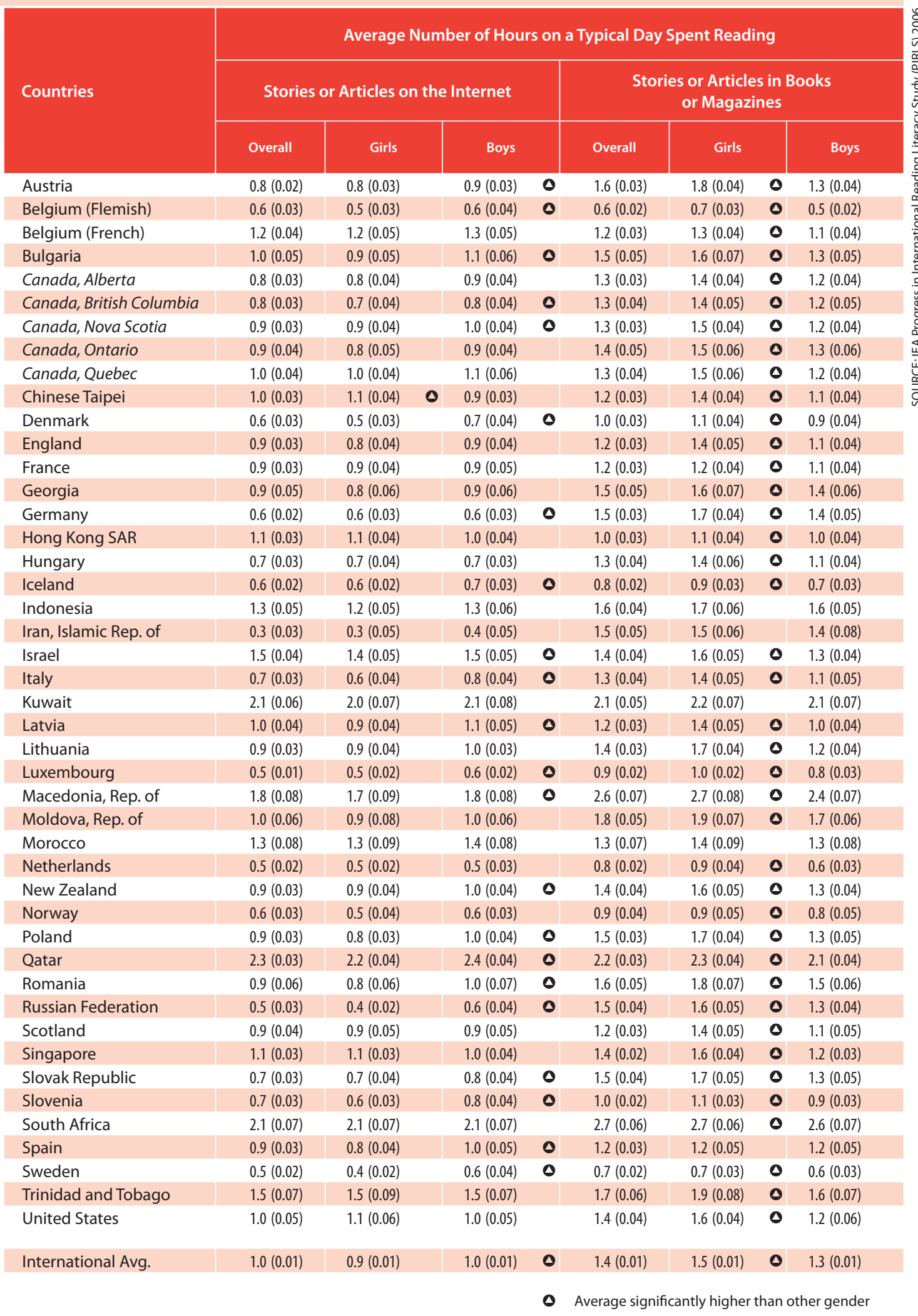

Background data provided by students.

() Standard errors appear in parentheses. Because results are rounded to the nearest

whole number, some totals may appear inconsistent.

NOTE: The International Average does not include the results from the Canadian provinces. 
students reported half an hour or less spent reading daily on the Internet. Countries where students reported the most time spent reading stories or articles in books or magazines included Kuwait, Macedonia, Qatar, and South Africa (more than 2 hours on a typical day, on average). Students in Belgium (Flemish), Iceland, Luxembourg, the Netherlands, Norway, and Sweden reported reading from books or magazines for less than one hour, on average, on a typical day. In almost every country, girls reported more time than boys reading from books or magazines (1.5 hours vs. 1.3 hours) and in many countries, boys reported more time than girls reading stories or articles on the Internet (1.0 hours vs. 0.9 hours). Only in Chinese Taipei did girls report more time reading on the Internet than boys.

Exhibit 4.6 presents trends in students' reports of how frequently they read for fun outside of school, regardless of the source of their reading material. In general, students around the world reported a good deal of reading for fun, with 40 percent, on average internationally, reading every day or almost every day, and a further 28 percent once or twice a week. However, almost one third of students (32\%, on average) reported reading for fun no more than twice a month. Reading for fun was reported most frequently in the Russian Federation, Germany, Lithuania, Moldova, France, and the Canadian provinces of Alberta and British Columbia, where the majority of students reported reading for fun daily or almost daily. In contrast, less than 30 percent of students in Georgia, Morocco, Singapore, Romania, and Chinese Taipei reported daily reading for fun. Of the PIRLS 2001 participants, five had increased percentages of students in 2006 reporting reading for fun daily or almost daily-Germany, Hungary, Italy, Hong Kong SAR, and Ontario province. Seven countries had fewer students reporting daily reading for fun in 2006-Iceland, Israel, Slovenia, Sweden, Latvia, Norway, and Singapore.

There was a positive association between the frequency of reading for fun and average student reading achievement, on average, across countries, and in most countries. Across all countries, students who reported reading 
for fun every day or almost every day had average reading achievement of 516 points, compared to 503 points for those reading for fun once or twice a week, and 484 points for those reading for fun twice a month or less. 
Exhibit 4.6 Students Reading for Fun Outside of School with Trends

PIRLS 2006

\begin{tabular}{|c|c|c|c|c|c|c|c|c|c|c|c|c|}
\hline \multirow[b]{2}{*}{ Countries } & \multicolumn{4}{|c|}{ Every Day or Almost Every Day } & \multicolumn{4}{|c|}{ Once or Twice a Week } & \multicolumn{4}{|c|}{ Twice a Month or Less } \\
\hline & $\begin{array}{c}2006 \\
\text { Percent } \\
\text { of Students }\end{array}$ & $\begin{array}{c}\text { Average } \\
\text { Achievement }\end{array}$ & $\begin{array}{l}\text { Difference } \\
\text { in Percent } \\
\text { from } 2001\end{array}$ & & $\begin{array}{c}2006 \\
\text { Percent } \\
\text { of Students }\end{array}$ & $\begin{array}{c}\text { Average } \\
\text { Achievement }\end{array}$ & $\begin{array}{l}\text { Difference } \\
\text { in Percent } \\
\text { from } 2001\end{array}$ & & $\begin{array}{c}2006 \\
\text { Percent } \\
\text { of Students }\end{array}$ & $\begin{array}{c}\text { Average } \\
\text { Achievement }\end{array}$ & $\begin{array}{l}\text { Difference } \\
\text { in Percent } \\
\text { from } 2001\end{array}$ & \\
\hline Russian Federation & $58(1.1)$ & $570(3.8)$ & $0(1.8)$ & & $28(0.8)$ & $559(3.9)$ & $-1(1.3)$ & & $14(0.8)$ & $556(3.9)$ & $2(1.3)$ & \\
\hline Canada, Alberta & $53(0.9)$ & $575(2.5)$ & $\Delta 0$ & & $23(0.8)$ & $555(3.5)$ & $\Delta 0$ & & $23(0.9)$ & $537(2.7)$ & $\Delta 0$ & \\
\hline Canada, British Columbia & $53(1.0)$ & $573(2.9)$ & $\Delta \Delta$ & & $26(0.8)$ & $554(2.5)$ & $\Delta \diamond$ & & $21(0.9)$ & $531(4.1)$ & $\Delta \diamond$ & \\
\hline Germany & $53(0.9)$ & $563(2.7)$ & $5(1.2)$ & 0 & $24(0.6)$ & $545(3.0)$ & $0(0.9)$ & & $24(0.8)$ & $525(2.5)$ & $-5(1.2)$ & (1) \\
\hline Lithuania & $52(1.2)$ & $545(2.1)$ & $-1(1.9)$ & & $30(1.0)$ & $533(2.2)$ & $-1(1.6)$ & & $17(0.8)$ & $520(2.8)$ & $2(1.2)$ & \\
\hline Moldova, Rep. of & $52(1.4)$ & $507(3.2)$ & $2(2.6)$ & & $34(1.1)$ & $498(4.2)$ & $0(1.9)$ & & $14(1.0)$ & $484(5.1)$ & $-2(1.6)$ & \\
\hline France & $51(1.0)$ & $540(2.5)$ & $2(1.6)$ & & $24(0.8)$ & $517(2.3)$ & $-2(1.2)$ & & $25(0.9)$ & $491(2.7)$ & $0(1.3)$ & \\
\hline Canada, Ontario & $49(1.4)$ & $567(3.2)$ & $14(2.0)$ & 0 & $25(1.1)$ & $552(3.6)$ & $2(1.4)$ & & $26(1.1)$ & $534(4.1)$ & $-16(1.9)$ & $\odot$ \\
\hline Belgium (French) & $49(1.1)$ & $517(3.0)$ & $\Delta \Delta$ & & $26(0.7)$ & $495(2.9)$ & $\Delta \Delta$ & & $25(0.9)$ & $473(3.1)$ & $\Delta \diamond$ & \\
\hline Iceland & $49(0.9)$ & $527(1.9)$ & $-3(1.2)$ & ( & $23(0.7)$ & $511(2.6)$ & $2(1.0)$ & & $28(0.7)$ & $485(2.3)$ & $1(1.0)$ & \\
\hline Denmark & $49(1.1)$ & $559(2.9)$ & $\Delta \diamond$ & & $30(0.8)$ & $540(2.7)$ & $\Delta \Delta$ & & $21(0.9)$ & $528(3.2)$ & $\Delta \diamond$ & \\
\hline Canada, Nova Scotia & $48(0.9)$ & $560(2.6)$ & $\Delta 0$ & & $25(0.8)$ & $541(2.7)$ & $\Delta \diamond$ & & $27(0.8)$ & $515(3.2)$ & $\Delta \Delta$ & \\
\hline Bulgaria & $47(1.6)$ & $561(4.4)$ & $-4(2.3)$ & & $27(1.0)$ & $555(5.0)$ & $-2(1.5)$ & & $26(1.6)$ & $520(6.5)$ & $5(2.2)$ & 0 \\
\hline Canada, Quebec & $47(1.3)$ & $549(3.0)$ & $1(1.9)$ & & $26(1.0)$ & $530(3.8)$ & $1(1.4)$ & & $27(1.2)$ & $509(3.2)$ & $-3(1.7)$ & \\
\hline South Africa & $45(0.9)$ & $303(6.4)$ & $\Delta \Delta$ & & $26(0.5)$ & $314(6.4)$ & $\Delta \Delta$ & & $28(0.8)$ & $307(6.4)$ & $\Delta \Delta$ & \\
\hline Austria & $45(1.1)$ & $555(3.0)$ & $\Delta 0_{0}$ & & $25(0.8)$ & $535(2.4)$ & $\Delta \Delta$ & & $29(1.0)$ & $516(2.9)$ & $\Delta 0$ & \\
\hline Spain & $45(1.1)$ & $525(2.9)$ & $\Delta \diamond$ & & $27(0.7)$ & $515(2.5)$ & $\Delta \Delta$ & & $28(1.0)$ & $494(3.7)$ & $\Delta \Delta$ & \\
\hline Hungary & $44(1.2)$ & $565(3.7)$ & $4(1.7)$ & 0 & $30(0.9)$ & $547(3.4)$ & $-2(1.4)$ & & $26(1.1)$ & $532(4.2)$ & $-2(1.5)$ & \\
\hline Indonesia & $44(1.4)$ & $405(4.7)$ & $\Delta \diamond$ & & $31(1.1)$ & $414(4.6)$ & $\Delta \diamond$ & & $25(1.2)$ & $403(4.8)$ & $\Delta \diamond$ & \\
\hline Macedonia, Rep. of & $43(1.2)$ & $453(5.7)$ & $-3(1.9)$ & & $31(1.0)$ & $451(4.6)$ & $3(1.4)$ & $\boldsymbol{\theta}$ & $25(1.1)$ & $435(5.1)$ & $0(1.8)$ & \\
\hline Poland & $43(1.3)$ & $538(2.5)$ & $\Delta \diamond$ & & $29(1.0)$ & $518(3.2)$ & $\diamond \diamond$ & & $27(1.0)$ & $495(3.0)$ & $\Delta 0$ & \\
\hline New Zealand & $42(1.1)$ & $562(2.4)$ & $-1(1.8)$ & & $24(0.7)$ & $531(2.5)$ & $0(1.1)$ & & $34(1.0)$ & $500(3.0)$ & $1(1.6)$ & \\
\hline Belgium (Flemish) & $40(1.1)$ & $563(2.1)$ & $\Delta \Delta$ & & $29(0.8)$ & $545(2.9)$ & $\Delta \Delta$ & & $31(1.2)$ & $529(2.3)$ & $\Delta \diamond$ & \\
\hline Slovak Republic & $39(1.0)$ & $545(2.9)$ & $0(1.5)$ & & $33(0.9)$ & $535(3.2)$ & $0(1.3)$ & & $27(1.1)$ & $507(5.4)$ & $0(1.6)$ & \\
\hline Italy & $38(1.3)$ & $573(3.3)$ & $7(1.7)$ & 0 & $25(0.7)$ & $554(3.2)$ & $1(1.0)$ & & $37(1.3)$ & $529(3.8)$ & $-7(1.7)$ & $\nabla$ \\
\hline Luxembourg & $38(0.6)$ & $581(1.8)$ & $\Delta \Delta$ & & $27(0.7)$ & $551(2.0)$ & $\Delta 0$ & & $35(0.5)$ & $537(1.5)$ & $\Delta \Delta$ & \\
\hline Israel & $38(1.2)$ & $538(4.2)$ & $-6(1.6)$ & $(7)$ & $28(0.9)$ & $518(4.4)$ & $1(1.2)$ & & $35(1.1)$ & $497(4.1)$ & $5(1.5)$ & 0 \\
\hline Slovenia & $37(0.9)$ & $543(2.5)$ & $-8(1.6)$ & ( & $33(0.7)$ & $519(3.0)$ & $4(1.1)$ & 0 & $30(0.9)$ & $500(2.6)$ & $4(1.5)$ & 0 \\
\hline Netherlands & $36(1.1)$ & $566(2.1)$ & $0(1.6)$ & & $22(0.7)$ & $550(1.8)$ & $2(1.1)$ & 0 & $42(1.1)$ & $530(1.8)$ & $-2(1.6)$ & \\
\hline Sweden & $36(1.0)$ & $569(2.8)$ & $-8(1.3)$ & (1) & $31(0.9)$ & $549(3.2)$ & $-1(1.2)$ & & $33(1.0)$ & $530(2.6)$ & $9(1.3)$ & 0 \\
\hline United States & $35(1.3)$ & $561(4.3)$ & $1(1.8)$ & & $22(0.7)$ & $550(3.3)$ & $0(1.3)$ & & $43(1.4)$ & $521(3.3)$ & $-1(1.8)$ & \\
\hline Latvia & $35(1.2)$ & $556(3.0)$ & $-8(1.7)$ & ( & $31(0.8)$ & $543(2.8)$ & $-2(1.2)$ & & $34(1.2)$ & $524(2.6)$ & $10(1.7)$ & 0 \\
\hline Hong Kong SAR & $35(1.0)$ & $575(2.6)$ & $14(1.3)$ & 0 & $33(0.9)$ & $567(2.7)$ & $-5(1.2)$ & $\odot$ & $32(1.0)$ & $549(2.8)$ & $-8(1.4)$ & 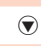 \\
\hline Iran, Islamic Rep. of & $33(1.2)$ & $428(4.2)$ & $-1(1.7)$ & & $41(1.2)$ & $429(3.9)$ & $2(1.9)$ & & $26(1.0)$ & $406(5.2)$ & $0(1.5)$ & \\
\hline Norway & $33(1.1)$ & $514(3.4)$ & $-5(1.5)$ & $(7)$ & $30(1.0)$ & $505(3.2)$ & $2(1.3)$ & & $37(1.2)$ & $481(3.1)$ & $4(1.7)$ & 0 \\
\hline Qatar & $33(0.6)$ & $357(2.2)$ & $\Delta \Delta$ & & $28(0.5)$ & $367(2.5)$ & $\Delta \Delta$ & & $39(0.6)$ & $352(2.2)$ & $\Delta \Delta$ & \\
\hline Scotland & $33(1.1)$ & $555(4.4)$ & $2(1.6)$ & & $24(1.0)$ & $533(3.1)$ & $0(1.3)$ & & $44(1.5)$ & $505(2.7)$ & $-2(2.2)$ & \\
\hline England & $33(1.2)$ & $575(4.0)$ & $0(1.8)$ & & $25(0.8)$ & $537(3.5)$ & $-1(1.2)$ & & $42(1.3)$ & $517(2.9)$ & $1(2.0)$ & \\
\hline Kuwait & $32(1.1)$ & $338(5.5)$ & $\Delta \Delta$ & & $32(1.0)$ & $342(5.5)$ & $\Delta \Delta$ & & $36(1.2)$ & $332(5.4)$ & $\Delta \diamond$ & \\
\hline Trinidad and Tobago & $32(1.2)$ & $450(6.7)$ & 00 & & $25(1.0)$ & $442(5.9)$ & 00 & & $43(1.4)$ & $427(5.6)$ & 00 & \\
\hline Georgia & $29(1.4)$ & $479(4.5)$ & $\Delta \diamond$ & & $29(1.2)$ & $484(4.0)$ & $\Delta \diamond$ & & $41(1.4)$ & $461(4.3)$ & $\Delta \Delta$ & \\
\hline Morocco & $29(1.3)$ & $317(8.2)$ & $-3(2.3)$ & & $34(1.4)$ & $326(6.9)$ & $-3(2.0)$ & & $37(1.9)$ & $331(7.1)$ & $6(3.1)$ & \\
\hline Singapore & $27(0.9)$ & $587(3.9)$ & $-3(1.5)$ & $(>$ & $26(0.6)$ & $564(3.1)$ & $3(0.9)$ & 0 & $47(1.0)$ & $540(2.7)$ & $0(1.5)$ & \\
\hline Romania & $25(1.3)$ & $510(5.5)$ & $-3(2.2)$ & & $26(1.1)$ & $502(5.5)$ & $-4(1.6)$ & $\odot$ & $50(1.6)$ & $478(5.5)$ & $7(2.3)$ & 0 \\
\hline Chinese Taipei & $24(0.7)$ & $553(2.6)$ & $\Delta \Delta$ & & $31(0.8)$ & $539(2.6)$ & $\Delta \Delta$ & & $45(1.0)$ & $525(2.2)$ & $\Delta \diamond$ & \\
\hline International Avg. & $40(0.2)$ & $516(0.6)$ & & & $28(0.1)$ & $503(0.6)$ & & & $32(0.2)$ & $484(0.6)$ & & \\
\hline
\end{tabular}

- Percent in 2006 significantly higher

(1) Percent in 2006 significantly lower

Background data provided by students.

() Standard errors appear in parentheses. Because results are rounded to the nearest whole number, some totals may appear inconsistent.

A diamond $(\diamond)$ indicates the country did not participate in the 2001 assessment.
NOTE: The International Average does not include the results from the Canadian provinces. Trend Note: The primary education systems of the Russian Federation and Slovenia underwent structural changes. Data for Canada, Ontario include only public schools. 



\section{Chapter 5}

\section{School Curriculum and}

\section{Organization for Teaching Reading}

The school curriculum for reading instruction is affected by many factors within a country, including the degree of centralization, availability of resources, and the structure of primary schools within the education system. The PIRLS 2006 Encyclopedia summarizes the structure of the education system for each of the PIRLS 2006 countries and Canadian provinces and specifically describes the reading curriculum for the fourth year of schooling. This chapter combines reports of parents, reading teachers, and school principals that describe students' reading readiness for school, the structural characteristics of fourth-grade reading curricula, school policies that support the curricula, and the organization of time and classrooms for teaching reading.

Because students were the basis for sampling, the student remains the unit of analysis, regardless of the source of information from the questionnaires. That is, data shown in the tables in this chapter are the percentages of students whose parents, teachers, or school principals reported on a particular activity or characteristic. When a parent, teacher, or principal did not complete the assigned questionnaire, the background data were not available for those students. If the percentage of students with background data fell below 85 percent, a special notation was made in the tables. An " $r$ " is included next to data where responses are available for 70 to 84 percent of students, an " $\mathrm{s}$ " where responses are available for 50 to 69 percent of students, and an " $\mathrm{x}$ " is included where responses are available for less than 50 percent. 
In Luxembourg, because school administration is shared between the Ministry of Education and the local town or city councils, primary schools do not have principals and school background data are not available.

\section{How Well Prepared Are Students to Learn to Read?}

Preprimary education, in the form of preschool, kindergarten, or an early childhood education center, plays an important role in preparing children for primary school. According to the PIRLS 2006 Encyclopedia, many countries have compulsory preprimary education, and enrollment rates are increasing in countries where preprimary education is voluntary. In many countries, Ministries of Education have published curriculum guidelines for preprimary education, much as they do for primary education.

Exhibit 5.1 contains parents' reports of the number of years their children participated in preprimary education. Although attendance differed dramatically from country to country, on average internationally, 45 percent of fourth-grade students had at least 3 years of preprimary education. In Belgium (Flemish) and Hungary, 85 percent of students had attended at least 3 years of primary education, and $75-78$ percent had in Belgium (French), Denmark, and France. Interestingly, on average internationally, reading achievement increased with the amount of time spent in preprimary education, with students who had not attended preprimary school having an international average reading score of 455 , compared to 510 for those students receiving 3 years or more of preprimary education.

Exhibit 5.2 presents parents' responses to the question about the age at which their children started formal primary school. Parents in New Zealand, Scotland, and Trinidad and Tobago reported that 90 percent or more of their children began school at age 5 or younger (parents' reports were not available for England, the other PIRLS 2006 country where students typically start school at age 5). According to parents, age 5 also was the predominant school entry age (63 to $91 \%$ of the students) in the five Canadian provinces. In 18 countries, parents reported age 6 as the predominant entry age (65 to $89 \%$ of the students). The 12 countries where parents reported children started 
school predominantly at age 7 or older (6o to $89 \%$ of students) included eight Eastern European countries (Bulgaria, Hungary, Latvia, Lithuania, Macedonia, Moldova, Romania, and the Russian Federation), two Asian countries (Chinese Taipei and Singapore), Iran, and Sweden.

Regardless of the age at which they begin primary school, children enter with a range of literacy skills acquired at home or in preprimary education. To examine to what extent children are prepared for school and equipped with some basic skills as a foundation for formal reading instruction, PIRLS asked parents how well their child could do each of the following early literacy activities when he or she first entered primary school:

- Recognize most of the alphabet,

- Write letters of the alphabet,

- Read some words,

- Write some words, and

- Read sentences.

For each of the activities, parents were given four response options, including very well, moderately well, not very well, and not at all. PIRLS averaged parents' responses across the five activities to form a 4-point scale summarizing the children's early literacy skills. The results are presented in Exhibit 5.3. The first column in Exhibit 5.3 shows the percentage of fourthgrade students whose parents reported that their children entered primary school able to do the five activities very well, together with the average reading achievement for those students. For countries that collected this information in both PIRLS 2001 and PIRLS 2006, changes are shown, with an indication of whether or not that difference was statistically significant. For PIRLS 2006, the percentages of fourth-grade students who entered school able to perform the literacy skills very well are shown in the graph by the red bar and, when corresponding PIRLS 2001 results were available, the white bar shows the percentage from PIRLS 2001. The second page of the exhibit presents the data for the remaining three categories-moderately well, not very well, and not at all. 
In PIRLS 2006, on average internationally, parents reported that nearly one third of their children could perform the early literacy activities very well. On average internationally, students were distributed fairly evenly across categories, with about another one third in the "moderately well" category, one fourth in the "not very well" category, and 12 percent in the "not at all" category. More than half the students in Trinidad and Tobago, Israel, Singapore, Macedonia, Hong Kong SAR, and Spain had parents who reported that their children could perform the early literacy activities very well. In contrast, according to their parents, one fifth or more of the students in Morocco, Iran, Georgia, Romania, Norway, Hungary, Belgium (Flemish), and the Slovak Republic could not perform the early literacy activities at all beginning school.

Parents' assessments of their children's early literacy skills corresponded well with reading achievement at the fourth grade. Reading achievement, on average internationally, was 525 for those students whose parents reported their children could perform the activities very well, 499 for performing the activities moderately well, 484 for performing them not very well, and 474 for not being able to perform the activities at all.

Given the strong association between parents' assessments of early literacy skills and students' reading achievement in the fourth year of schooling, the changes between 2001 and 2006 are encouraging. In 17 of the 22 participants that also had data from 2001, there were increases in the "very well" category, with seven of these also having increases in the "moderately well" category, including Moldova, Lithuania, the Russian Federation, Iceland, Scotland, Germany, and Hungary. However, in the Canadian province of Ontario, parents reported increases in both the percentages of their children entering school unable to perform the literacy skills and not being able to perform them very well.

PIRLS asked school principals to estimate the percentages of students entering their schools with each of the same five early literacy skills. There were four response options-more than $75 \%, 51-75 \%, 25-50 \%$, and less than $25 \%$. The responses were averaged across the five activities to summarize the 
results. Exhibit 5.4 presents the principals' reports for PIRLS 2006 together with changes from PIRLS 2001. In Chinese Taipei, 94 percent of students were in schools where principals reported that most children (more than $75 \%$ ) entered school with early literacy skills, and 86 percent were in such schools in Hong Kong SAR. More than half were in such schools in England (56\%), Singapore (70\%), Spain (56\%), and the United States (65\%). Iceland, Israel, Latvia, Singapore, and the United States had a significant increase from 2001 in the percentage of students in this category.

On average internationally, however, 44 percent of the fourth-grade students were in schools where relatively few children (less than 25\%) entered school with these literacy skills. In 10 countries, two thirds or more of students were in schools where relatively few students beginning school had these literacy skills. These countries included Austria, Belgium (Flemish), Georgia, Germany, Hungary, Iran, New Zealand, Scotland, the Slovak Republic, and Slovenia. Three Canadian provinces were also in this situation (Alberta, Ontario, and Quebec). As might be anticipated, principals were not as positive about students' early literacy skills as were students' parents. Similarly, the principals agreed with parents about improvements in early literacy skills, but the picture was slightly less positive. According to school principals, the percentages of students in schools where relatively few students begin school with literacy skills decreased between 2001 and 2006 in Bulgaria, Germany, Iceland, Israel, Latvia, the Netherlands, Norway, Romania, and the Russian Federation. 
Exhibit 5.1 Number of Years Children Attended Preprimary Education (Preschool, Kindergarten, and Other Similar Programs)

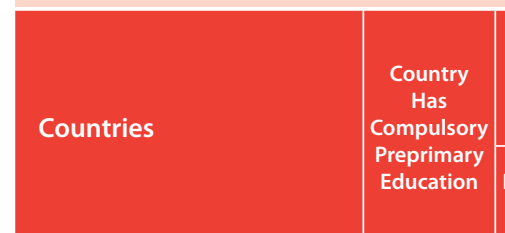

\section{Austria}

Belgium (Flemish)

Belgium (French)

1 Bulgaria

Canada, Alberta

Canada, British Columbia r

Canada, Nova Scotia

Canada, Ontario

Canada, Quebec

Chinese Taipei

Denmark

England

France

Georgia

Germany

Hong Kong SAR

Hungary

Iceland

Indonesia

Iran, Islamic Rep. of

Israel

Italy

Kuwait

Latvia

Lithuania

Luxembourg

${ }^{1}$ Macedonia, Rep. of

Moldova, Rep. of

Morocco

Netherlands

New Zealand

Norway

Poland

Qatar

Romania

Russian Federation

Scotland

Singapore

Slovak Republic

Slovenia

South Africa

Spain

Sweden

Trinidad and Tobago

United States
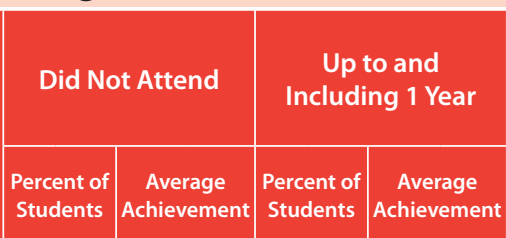

\begin{tabular}{|c|c|}
\hline \multicolumn{2}{|c|}{$\begin{array}{c}\text { More than } 1 \text { Year } \\
\text { Up to and } \\
\text { Including } 2 \text { Years }\end{array}$} \\
\hline Percent of Average
\end{tabular}

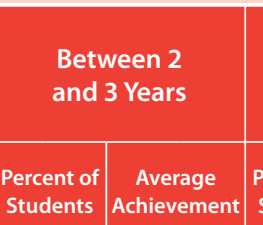

\begin{tabular}{ll}
$2(0.2)$ & $\sim \sim$ \\
$1(0.2)$ & $\sim \sim$ \\
\hline $1(0.2)$ & $\sim \sim$
\end{tabular}

$1(0.2)$

7 (0.6) $527(4.8)$

Students

$13(1.2) \quad 537(9.7)$

$0(0.1)$

$\sim \quad 2(0.3)$

Achievement

$4(0.4)$

$535(3.1)$

$5(0.4)$

$542(5.2)$

$7(0.8)$

$525(8.3)$

$12(0.8)$

$486(10.5) \quad 17(0.8)$

$539(4.5) \quad 85(0.8)$

$32(1.3) \quad 559(3.8) \quad 27(1.0) \quad 564(3.4) \quad 31(1.1)$

$543(7.5)$

$15(0.9)$

$542(6.9) \quad 53(1.8$

0

$25(1.4) \quad 554(4.3) \quad 20(0.9)$

$564(4.6) \quad 36(1.2)$

$570(3.3)$

$6(0.4)$

$574(5.8)$

$23(0.8) \quad 539(3.4) \quad 24(0.9) \quad 545(3.2) \quad 33(1.0)$

566 (3.3)

$12(0.9)$

$568(5.1)$

\begin{tabular}{|l|l|l|l|l|}
\hline $47(1.5)$ & $556(3.2)$ & $13(0.7)$ & $563(5.8)$ & $23(0.8)$ \\
\hline
\end{tabular}

$553(3.5)$

$8(0.7)$

$551(4.3)$

$35(1.5) \quad 530(3.9) \quad 27(1.3)$

$542(3.7) \quad 20(1.0)$

$1(0.2)$

$\sim \quad 6(0.4)$

$524(5.9) \quad 42(1.0)$

539 (3.4)

$6(0.7)$

$3(0.3)$

$556(7.5)$

$4(0.5)$

$534(2.3)$

$26(0.6)$

$538(2.7) \quad 25(0.8)$

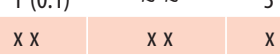

$\mathrm{xx}$

\begin{tabular}{|l|l|l}
$\mathrm{xx}$ & $\mathrm{xx}$ & $\mathrm{xx}$
\end{tabular}

\begin{tabular}{c|c|c|c|r|}
\hline $1(0.1)$ & $\sim \sim$ & $0(0.1)$ & $\sim \sim$ & $4(0.4)$ \\
\hline $35(2.0)$ & $471(5.0)$ & $7(0.7)$ & $465(8.1)$ & $20(1.1)$ \\
\hline
\end{tabular}

$518(6.6)$

$14(0.8)$

$543(4.5)$

470 (3.5)

$20(0.7)$

$1(0.2) \quad \sim \sim$

$11(0.8)$

547 (4.7)

$9(0.6)$

$477(5.8) \quad 29(1.4)$

$\begin{array}{lll}0(0.1) & \sim \sim & 2(0.3) \\ 1(0.2) & \sim \sim & 2(0.3)\end{array}$

$\sim \quad 3(0.4)$

$559(6.8)$

$8(0.7)$

$549(4.3)$

$\sim \quad 5(0.4)$

$531(9.9)$

$8(0.6)$

$\sim \quad 5(0.4)$

$433(3.9)$

$20(0.7)$

$38(2.8) \quad 381(5.0) \quad 13(1.3)$

$421(7.0) \quad 33(2.5)$

$433(3.9)$

$5(0.6)$

$419(8.9)$

\begin{tabular}{|l|l|l|l|l|}
\hline $49(2.1)$ & $392(3.8)$ & $30(1.3)$ & $440(3.9)$ & $14(0.8)$
\end{tabular}

$482(7.9)$

$2(0.3)$

444 (11.6) $15(1.1)$

\begin{tabular}{ccc}
$8(1.2)$ & $444(10.2)$ & $5(0.6)$ \\
\hline $2(0.3)$ & $\sim \sim$ & $2(0.3)$
\end{tabular}

$13(0.8) \quad 325(8.5) \quad 8(0.6)$ $\sim \quad 9(0.6)$

$11(0.8)$

$\sim \sim$

$341(10.9) \quad 69(1.1)$

$546(7.8) \quad 17(0.7)$

\begin{tabular}{|l|l|l|}
\hline $15(1.0)$ & $532(4.8)$ & $6(0.5)$ \\
\hline
\end{tabular}

$530(7.2) \quad 18(0.9)$

337 (4.4)

$5(0.5)$

$\begin{array}{lllll}15(1.0) & 532(4.8) & 6(0.5) & 530(7.2) & 18(0.9) \\ 30(1.4) & 524(3.0) & 6(0.4) & 531(3.9) & 10(0.6)\end{array}$

$532(4.5)$

$12(0.7)$

$\bigcirc$

$13(0.5) \quad 541(3.1) \quad 4(0.3)$

$10(0.6) \quad 537(3.6)$
$531(3.9)-10.7)$

$9(0.5)$

$547(3.4)$

$431(4.8) \quad 18(0.8)$

$565(1.3) \quad 7(0.4)$

$24(1.2) \quad 426(5.9) \quad 37(1.3)$

$491(6.3) \quad 16(1.0)$

$461(5.8)$

$6(0.5)$

$558(4.4) \quad 7(0.3)$

\begin{tabular}{|l|l|l|l}
\hline & $14(1.7)$ & $490(8.1)$ & $10(1.1)$
\end{tabular}

$28(2.2) \quad 296(12.8) \quad 9(0.9)$

$299(8.2) \quad 29(1.4)$

$503(4.9)$

$13(0.8)$

$550(7.5) \quad 56(1.4)$

$336(7.0)$

$13(0.9)$

$349(8.2)$

$4(0.4) \quad 532(7.9) \quad 5(0.5)$

$522(9.2) \quad 30(0.9)$

$555(2.0)$

34 (1.2)

$490(8.5) \quad 11(0.7)$

$484(3.5)$

$31(0.9)$

$9(0.9) \quad 490(6.9) \quad 3(0.3)$

$506(4.2) \quad 16(1.0)$

$519(4.5)$

$15(1.0)$

$491(5.5$

\begin{tabular}{|l|l|l|l|l|}
$34(1.9)$ & $513(3.2)$ & $17(0.9)$ & $506(4.2)$ & $16(1.0)$ \\
\hline $33(0.5)$ & $345(2.6)$ & $25(0.7)$ & $355(2.9)$ & $29(0.6)$
\end{tabular}

$371(3.3)$

$5(0.4)$

$452(12.4) \quad 19(1.2)$

$471(9.7)$

$6(0.3)$

$6(1.0) \quad 401(14.7) \quad 5(0.6)$

$561(7.2) \quad 8(0.7)$

$563(6.6)$

$18(1.4)$

$\begin{array}{rcr}2(1.2) & 545(6.3) & 4(0.3) \\ 2(0.3) & \sim \sim & 14(1.2)\end{array}$

$556(6.5) \quad 52(1.5)$

$534(4.6) \quad 19(1.0)$

$\begin{array}{lll}2(0.1) & \sim \sim & 1(0.2)\end{array}$

$\sim \quad 13(0.5)$

531 (4.2)

$26(0.6)$

$554(3.1)-57(0.8)$

\begin{tabular}{|r|r|r|r|r|r|r|r|r|r|}
\hline $5(0.6)$ & $473(11.9)$ & $11(0.8)$ & $515(6.7)$ & $15(0.7)$ & $523(4.3)$ & $11(0.7)$ & $529(4.8)$ & $59(1.3)$ & $545(2.3)$ \\
\hline $14(1.2)$ & $513(4.3)$ & $5(0.4)$ & $498(5.4)$ & $13(0.7)$ & $520(3.3)$ & $14(0.7)$ & $524(3.8)$ & $54(1.3)$ & $528(2.2)$ \\
\hline
\end{tabular}

\begin{tabular}{|r|l|r|r|r|r|r|r|r|r|}
\hline $5(0.6)$ & $473(11.9)$ & $11(0.8)$ & $515(6.7)$ & $15(0.7)$ & $523(4.3)$ & $11(0.7)$ & $529(4.8)$ & $59(1.3)$ & $545(2.3)$ \\
\hline $14(1.2)$ & $513(4.3)$ & $5(0.4)$ & $498(5.4)$ & $13(0.7)$ & $520(3.3)$ & $14(0.7)$ & $524(3.8)$ & $54(1.3)$ & $528(2.2)$ \\
\hline
\end{tabular}

$13(0.5) \quad 281(5.4) \quad 18(0.6)$

$307(6.6) \quad 23(0.7)$

$308(7.0)$

$14(0.4)$

$524(3.8) \quad 54(1.3)$

\begin{tabular}{l|l|l}
$4(0.6)$ & $480(9.0)$ & $3(0.4)$ \\
\hline
\end{tabular}

$500(10.2) \quad 17(1.0)$

$511(5.4)$

$16(0.9)$

$515(3.8) \quad 59(1.6)$

$4(0.4) \quad 516(7.0) \quad 5(0.5)$

$540(6.9) \quad 20(1.1)$

539 (3.6)

$12(0.6)$

$6(0.7) \quad 375(11.6) \quad 8(0.5)$

$456(9.2) \quad 43(1.2)$

446 (5.4)

$27(1.0)$

4th Grade

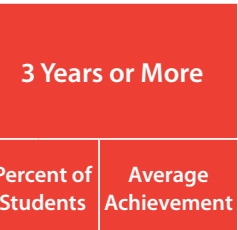

545 (2.4)

491 (3.8) $\quad 77(1.1)$

551 (1.9)

$4(0.7)$

$06(2.6)$

$8(0.6)$

$9(0.5)$

$571(7.5) \quad 9(0.7)$

$541(6.1) \quad 12(1.1)$

$78(1.2)$

$551(2.4)$

\begin{tabular}{ccccc}
$x \times$ & $x x$ & $x x$ & $x x$ & $x x$ \\
\hline
\end{tabular}

$514(3.4) \quad 75(0.8) \quad 528(2.2)$

$476(4.8)$

$67(1.1)$

$557(2.2)$

$561(2.6) \quad 66(0.8)$

567 (2.4)

$543(7.7) \quad 85(0.9)$

$556(2.8)$

$72(0.8)$

$520(1.8)$

$12(1.1)$

$5(0.6)$

$516(7.8) \quad 61(1.6)$

$474(9.5)$

\begin{tabular}{l|l|l}
$540(5.0)$ & $70(1.1)$ & $558(2.7)$ \\
\hline
\end{tabular}

$353(12.2) \quad 5(0.5) \quad 355(10.8)$

$545(4.6) \quad 49(1.2)$

\begin{tabular}{ll|l}
$545(4.6)$ & $49(1.2)$ & $551(2.5)$
\end{tabular}

$5(1.4)$

\begin{tabular}{l|l|l}
$502(4.5)$ & $47(1.8)$ & $504(3.4)$
\end{tabular}

$\begin{array}{ll}47(1.8) & 504(3.4) \\ 20(1.2) & 339(6.9)\end{array}$

$561(2.2) \quad 5(0.5)$

$536(5.9)$

$552(3.8) \quad 29(1.0) \quad 548(3.1)$

2 (1.9)

$529(5.6) \quad 28(1.4) \quad 543(3.6)$

$369(7.0) \quad 6(0.3)$

$6(0.3) \quad 352(6.7)$

$498(6.1) \quad 51(1.9)$

$511(3.8)$

\begin{tabular}{l|l|l|}
$574(5.4)$ & $59(1.3)$ & $571(3.0)$ \\
\hline
\end{tabular}

$\begin{array}{lll}549(4.9) & 13(1.0) & 552(8.1)\end{array}$

$554(3.1) \quad 57(0.8)$

$571(2.9)$

$31(0.7) \quad 318(8.1)$

\begin{tabular}{|l|l|l|}
$515(3.8)$ & $59(1.6)$ & $530(3.1)$ \\
\hline
\end{tabular}

$549(3.5) \quad 60(1.5) \quad 559(2.5)$

$450(5.7) \quad 16(0.9)$

$423(7.5)$

International Avg.

\begin{tabular}{ll|l}
$12(0.2)$ & $455(1.6)$ & $8(0.1)$
\end{tabular}

\begin{tabular}{l}
$479(1.5) \quad 21(0.2)$ \\
\hline
\end{tabular}

$496(1.0)$

$505(1.0$ 
Exhibit 5.2 Age Students Began Primary Schoo

PIRLS 2006

\begin{tabular}{|c|c|c|c|c|c|c|c|c|c|}
\hline \multirow{2}{*}{ Countries } & & \multicolumn{2}{|c|}{$\begin{array}{l}5 \text { Years Old } \\
\text { or Younger }\end{array}$} & \multicolumn{2}{|c|}{6 Years Old } & \multicolumn{2}{|c|}{7 Years Old } & \multicolumn{2}{|c|}{$\begin{array}{l}8 \text { Years Old } \\
\text { or Older }\end{array}$} \\
\hline & & $\begin{array}{l}\text { Percent of } \\
\text { Students }\end{array}$ & $\begin{array}{c}\text { Average } \\
\text { Achievement }\end{array}$ & $\begin{array}{l}\text { Percent of } \\
\text { Students }\end{array}$ & $\begin{array}{c}\text { Average } \\
\text { Achievement }\end{array}$ & $\begin{array}{l}\text { Percent of } \\
\text { Students }\end{array}$ & $\begin{array}{c}\text { Average } \\
\text { Achievement }\end{array}$ & $\begin{array}{l}\text { Percent of } \\
\text { Students }\end{array}$ & $\begin{array}{c}\text { Average } \\
\text { Achievement }\end{array}$ \\
\hline Austria & & $3(0.2)$ & $543(7.8)$ & $77(0.8)$ & $544(2.2)$ & $20(0.8)$ & $528(3.2)$ & $1(0.1)$ & $\sim \sim$ \\
\hline Belgium (Flemish) & & $17(0.6)$ & $548(2.8)$ & $78(0.7)$ & $550(2.0)$ & $4(0.3)$ & $525(4.1)$ & $0(0.1)$ & $\sim \sim$ \\
\hline Belgium (French) & & $19(0.7)$ & $504(3.9)$ & $77(0.8)$ & $504(2.9)$ & $4(0.4)$ & $453(8.9)$ & $0(0.0)$ & $\sim \sim$ \\
\hline Bulgaria & & $0(0.1)$ & $\sim \sim$ & $11(0.8)$ & $553(7.3)$ & $84(1.0)$ & $551(4.3)$ & $5(0.7)$ & $518(8.8)$ \\
\hline Canada, Alberta & $\mathrm{r}$ & $74(0.9)$ & $563(2.6)$ & $25(0.9)$ & $570(3.7)$ & $1(0.2)$ & $\sim \sim$ & $0(0.1)$ & $\sim \sim$ \\
\hline Canada, British Columbia & $r$ & $80(1.1)$ & $563(2.9)$ & $18(1.1)$ & $563(4.9)$ & $1(0.3)$ & $\sim \sim$ & $0(0.1)$ & $\sim \sim$ \\
\hline Canada, Nova Scotia & & $91(0.5)$ & $547(2.1)$ & $9(0.5)$ & $533(5.2)$ & $0(0.1)$ & $\sim \sim$ & $0(0.1)$ & $\sim \sim$ \\
\hline Canada, Ontario & & $88(1.0)$ & $558(2.7)$ & $11(1.0)$ & $556(5.5)$ & $1(0.2)$ & $\sim \sim$ & $0(0.1)$ & $\sim \sim$ \\
\hline Canada, Quebec & & $63(1.4)$ & $533(3.3)$ & $35(1.3)$ & $543(3.4)$ & $1(0.2)$ & $\sim \sim$ & $0(0.1)$ & $\sim \sim$ \\
\hline Chinese Taipei & & $5(0.4)$ & $531(4.5)$ & $16(0.8)$ & $546(3.9)$ & $57(0.8)$ & $535(2.1)$ & $22(0.7)$ & $535(2.9)$ \\
\hline Denmark & & $5(0.4)$ & $546(6.7)$ & $44(1.1)$ & $547(2.5)$ & $48(1.1)$ & $552(2.5)$ & $2(0.3)$ & $\sim \sim$ \\
\hline England & & $x x$ & $x x$ & $x x$ & $x x$ & $x x$ & $x x$ & $x x$ & $x x$ \\
\hline France & & $13(0.5)$ & $523(3.6)$ & $84(0.6)$ & $525(2.1)$ & $3(0.4)$ & $494(7.3)$ & $0(0.1)$ & $\sim \sim$ \\
\hline Georgia & & $6(0.4)$ & $483(6.8)$ & $73(1.1)$ & $474(3.5)$ & $20(1.2)$ & $457(4.7)$ & $1(0.3)$ & $\sim \sim$ \\
\hline Germany & & $4(0.3)$ & $563(5.5)$ & $73(0.8)$ & $555(2.3)$ & $22(0.7)$ & $544(2.9)$ & $1(0.2)$ & $\sim \sim$ \\
\hline Hong Kong SAR & & $10(0.5)$ & $558(3.9)$ & $80(0.7)$ & $567(2.3)$ & $9(0.6)$ & $559(5.0)$ & $1(0.2)$ & $\sim \sim$ \\
\hline Hungary & & $1(0.1)$ & $\sim \sim$ & $30(1.0)$ & $555(4.0)$ & $67(1.0)$ & $553(3.1)$ & $2(0.3)$ & $\sim \sim$ \\
\hline Iceland & $r$ & $13(0.6)$ & $514(3.7)$ & $86(0.6)$ & $517(1.6)$ & $0(0.1)$ & $\sim \sim$ & $0(0.0)$ & $\sim \sim$ \\
\hline Indonesia & & $6(0.5)$ & $414(6.6)$ & $58(1.7)$ & $410(4.0)$ & $34(1.7)$ & $398(4.8)$ & $2(0.3)$ & $\sim \sim$ \\
\hline Iran, Islamic Rep. of & & $1(0.1)$ & $\sim \sim$ & $39(1.6)$ & $411(4.9)$ & $58(1.6)$ & $426(3.7)$ & $3(0.3)$ & $416(11.6)$ \\
\hline Israel & s & $15(1.2)$ & $468(8.8)$ & $73(1.3)$ & $541(3.3)$ & $12(0.9)$ & $525(8.3)$ & $1(0.2)$ & $\sim \sim$ \\
\hline Italy & & $14(0.7)$ & $539(5.0)$ & $83(0.7)$ & $556(2.9)$ & $2(0.3)$ & $\sim \sim$ & $0(0.1)$ & $\sim \sim$ \\
\hline Kuwait & $r$ & $17(0.8)$ & $331(8.1)$ & $76(0.9)$ & $337(4.5)$ & $7(0.4)$ & $338(11.0)$ & $0(0.1)$ & $\sim \sim$ \\
\hline Latvia & & $1(0.3)$ & $\sim \sim$ & $22(0.9)$ & $548(2.7)$ & $74(0.9)$ & $542(2.5)$ & $3(0.4)$ & $503(8.9)$ \\
\hline Lithuania & & $3(0.3)$ & $521(6.4)$ & $31(1.3)$ & $534(2.2)$ & $65(1.3)$ & $541(1.9)$ & $1(0.2)$ & $\sim \sim$ \\
\hline Luxembourg & & $18(0.7)$ & $551(2.6)$ & $70(0.8)$ & $564(1.4)$ & $12(0.5)$ & $549(3.1)$ & $0(0.1)$ & $\sim \sim$ \\
\hline Macedonia, Rep. of & & $3(0.4)$ & $453(12.1)$ & $38(1.1)$ & $457(4.6)$ & $58(1.2)$ & $442(4.2)$ & $2(0.4)$ & $\sim \sim$ \\
\hline Moldova, Rep. of & & $1(0.2)$ & $\sim \sim$ & $22(1.5)$ & $504(4.8)$ & $72(1.5)$ & $501(3.1)$ & $4(0.4)$ & $469(7.1)$ \\
\hline Morocco & & $5(0.5)$ & $336(13.5)$ & $65(1.6)$ & $333(6.3)$ & $27(1.5)$ & $302(7.7)$ & $3(0.4)$ & $280(21.6)$ \\
\hline Netherlands & $s$ & $13(0.8)$ & $557(3.9)$ & $79(0.9)$ & $558(1.7)$ & $8(0.8)$ & $532(4.4)$ & $0(0.1)$ & $\sim \sim$ \\
\hline New Zealand & $s$ & $97(0.4)$ & $548(2.1)$ & $3(0.3)$ & $545(9.9)$ & $1(0.1)$ & $\sim \sim$ & $0(0.1)$ & $\sim \sim$ \\
\hline Norway & & $20(0.9)$ & $496(3.7)$ & $79(0.9)$ & $502(2.6)$ & $2(0.3)$ & $\sim \sim$ & $0(0.0)$ & $\sim \sim$ \\
\hline Poland & & $9(0.6)$ & $523(5.5)$ & $89(0.6)$ & $520(2.4)$ & $2(0.3)$ & $\sim \sim$ & $0(0.1)$ & $\sim \sim$ \\
\hline Qatar & $r$ & $14(0.5)$ & $354(4.1)$ & $71(0.6)$ & $359(1.7)$ & $14(0.4)$ & $355(3.7)$ & $1(0.1)$ & $\sim \sim$ \\
\hline Romania & & $1(0.2)$ & $\sim \sim$ & $19(1.3)$ & $489(5.8)$ & $77(1.3)$ & $492(5.8)$ & $4(0.4)$ & $461(11.3)$ \\
\hline Russian Federation & & $1(0.1)$ & $\sim \sim$ & $30(0.9)$ & $560(5.0)$ & $66(0.9)$ & $568(3.3)$ & $3(0.3)$ & $533(7.4)$ \\
\hline Scotland & s & $99(0.3)$ & $543(3.3)$ & $1(0.3)$ & $\sim \sim$ & $0(0.1)$ & $\sim \sim$ & $0(0.0)$ & $\sim \sim$ \\
\hline Singapore & & $4(0.3)$ & $536(5.5)$ & $31(0.5)$ & $571(3.2)$ & $64(0.6)$ & $556(3.0)$ & $1(0.1)$ & $\sim \sim$ \\
\hline Slovak Republic & & $2(0.3)$ & $\sim \sim$ & $71(0.8)$ & $534(3.1)$ & $26(0.7)$ & $530(2.8)$ & $0(0.2)$ & $\sim \sim$ \\
\hline Slovenia & & $7(0.4)$ & $522(5.0)$ & $56(0.8)$ & $525(3.0)$ & $36(0.7)$ & $521(2.4)$ & $0(0.1)$ & $\sim \sim$ \\
\hline South Africa & r & $12(0.5)$ & $270(7.0)$ & $35(0.9)$ & $331(8.9)$ & $42(0.8)$ & $310(5.2)$ & $12(0.6)$ & $245(4.9)$ \\
\hline Spain & $s$ & $50(1.3)$ & $515(2.6)$ & $48(1.3)$ & $529(3.3)$ & $1(0.3)$ & $\sim \sim$ & $1(0.3)$ & $\sim \sim$ \\
\hline Sweden & & $1(0.2)$ & $\sim \sim$ & $21(1.2)$ & $543(3.8)$ & $78(1.2)$ & $554(2.5)$ & $1(0.1)$ & $\sim \sim$ \\
\hline Trinidad and Tobago & & $91(0.6)$ & $442(4.9)$ & $7(0.6)$ & $420(9.5)$ & $1(0.2)$ & $\sim \sim$ & $1(0.2)$ & $\sim \sim$ \\
\hline United States & & -- & -- & -- & -- & -- & -- & -- & -- \\
\hline International Avg. & & $16(0.1)$ & $491(1.1)$ & $51(0.2)$ & $503(0.7)$ & $31(0.1)$ & $491(0.9)$ & $2(0.0)$ & $\sim \sim$ \\
\hline
\end{tabular}

Background data provided by parents.

() Standard errors appear in parentheses. Because results are rounded to the nearest whole number, some totals may appear inconsistent.

$A n$ " $r$ " indicates data are available for $70-84 \%$ of the students. An " $s$ " indicates data are available for $50-69 \%$ of the students. An " $\mathrm{x}$ " indicates data are available for less than $50 \%$ of the students.
A dash (-) indicates comparable data are not available. A tilde $(\sim)$ indicates insufficient data to report achievement.

NOTE: The International Average does not include the results from the Canadian provinces. 
Exhibit 5.3 How Well Students Could Perform Beginning Literacy Activities

PIRLS 2006 When They Entered School with Trends

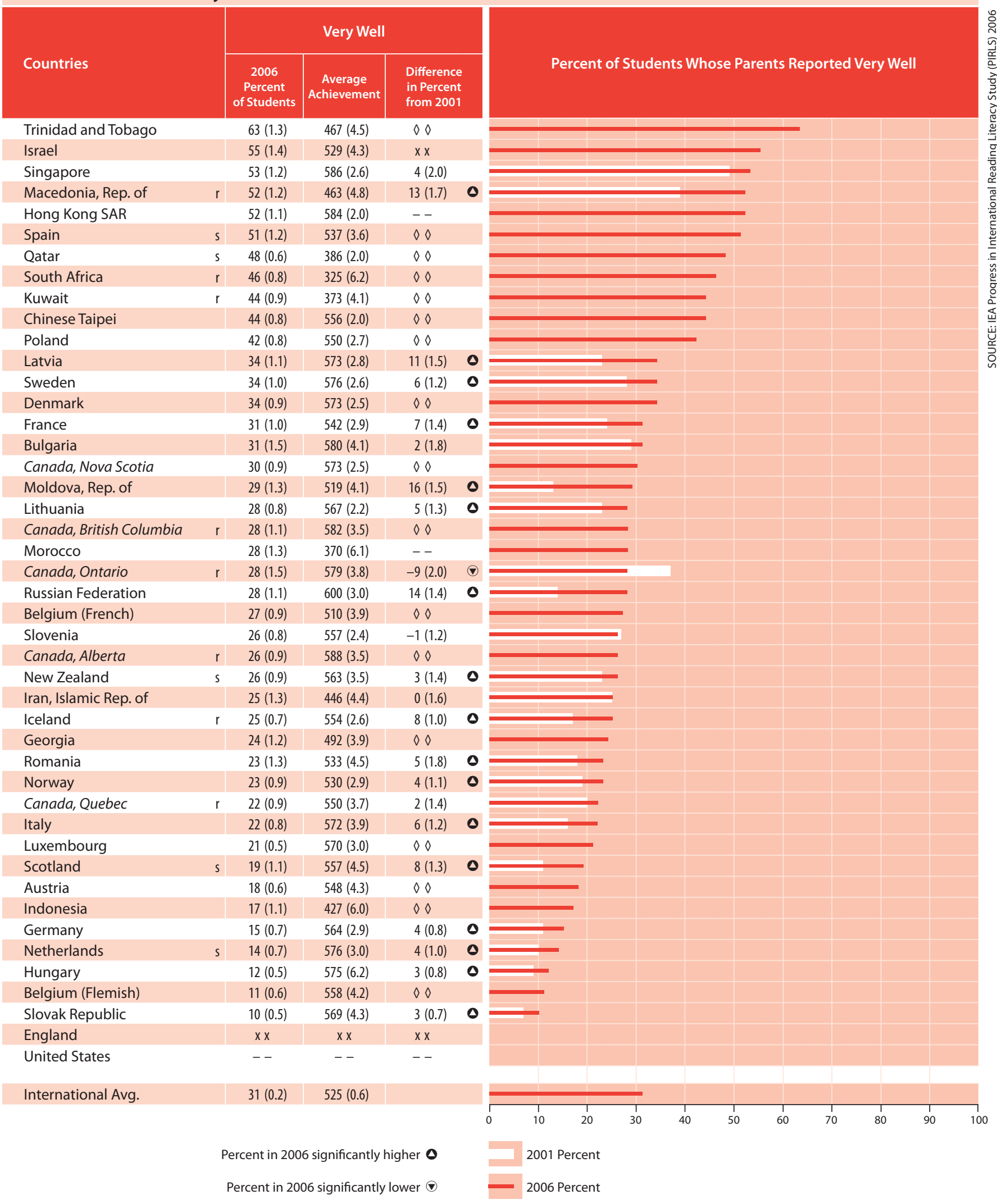

Based on parents' assessments of how well their child could do the following when he/she began primary school: recognize most of the letters of the alphabet, write letters of the alphabet, read some words, write some words, and read sentences. Average is computed across the 5 items based on a 4-point scale: Not at all $=1$, Not very well $=2$, Moderately well $=3$, and Very well $=4$. Very well indicates an average response score of greater than 3.25 through 4 . Moderately well indicates an average response score of greater than 2.5 through 3.25. Not very well indicates an average of 1.75 through 2.5. Not at all indicates an average of 1 to less than 1.75 .

() Standard errors appear in parentheses. Because results are rounded to the nearest whole number, some totals may appear inconsistent. 


\begin{tabular}{|c|c|c|c|c|c|c|c|c|c|c|c|c|c|}
\hline \multirow[t]{3}{*}{ Exhibit 5.3} & & $\begin{array}{l}\text { tudents } \\
\text { Entered }\end{array}$ & $\begin{array}{l}\text { Could Perf } \\
\text { School wi }\end{array}$ & $\begin{array}{l}\text { m Beg } \\
\text { Trend }\end{array}$ & jinr & $\begin{array}{l}\text { ning Lite } \\
\text { Continue }\end{array}$ & $\begin{array}{l}\text { racy Activ } \\
\text { d) }\end{array}$ & & & & & \multicolumn{2}{|c|}{$\begin{array}{l}\text { PIRLS } 2006 \\
\text { 4th Grade }\end{array}$} \\
\hline & \multicolumn{5}{|c|}{ Moderately Well } & \multicolumn{4}{|c|}{ Not Very Well } & \multicolumn{4}{|c|}{ Not at All } \\
\hline & & $\begin{array}{c}2006 \\
\text { Percent } \\
\text { of Students }\end{array}$ & $\begin{array}{c}\text { Average } \\
\text { Achievement }\end{array}$ & $\begin{array}{l}\text { Difference } \\
\text { in Percent } \\
\text { from } 2001\end{array}$ & & $\begin{array}{c}2006 \\
\text { Percent } \\
\text { of Students }\end{array}$ & $\begin{array}{c}\text { Average } \\
\text { Achievement }\end{array}$ & $\begin{array}{l}\text { Difference } \\
\text { in Percent } \\
\text { from } 2001\end{array}$ & & $\begin{array}{c}2006 \\
\text { Percent } \\
\text { of Students }\end{array}$ & $\begin{array}{c}\text { Average } \\
\text { Achievement }\end{array}$ & $\begin{array}{l}\text { Difference } \\
\text { in Percent } \\
\text { from } 2001\end{array}$ & \\
\hline Trinidad and Tobago & & $29(1.0)$ & $406(5.6)$ & $\Delta \diamond$ & & $7(0.6)$ & $363(12.2)$ & $\Delta \diamond$ & & $1(0.4)$ & $\sim \sim$ & $\Delta \diamond$ & \\
\hline Israel & & $26(1.0)$ & $520(5.8)$ & $x x$ & & $13(0.7)$ & $538(6.2)$ & $x x$ & & $5(0.5)$ & $541(10.4)$ & $x x$ & \\
\hline Singapore & & $37(0.9)$ & $541(3.0)$ & $-1(1.4)$ & & $9(0.5)$ & $494(5.5)$ & $-2(1.0)$ & $(-)$ & $1(0.1)$ & $\sim \sim$ & $-1(0.2)$ & \\
\hline Macedonia, Rep. of & $r$ & $31(0.9)$ & $442(4.2)$ & $-3(1.5)$ & $(\uparrow$ & $13(0.7)$ & $433(6.7)$ & $-6(1.1)$ & $(\nabla$ & $4(0.5)$ & $413(12.7)$ & $-3(1.1)$ & ( \\
\hline Hong Kong SAR & & $42(0.8)$ & $552(2.4)$ & -- & & $6(0.5)$ & $515(4.8)$ & -- & & $1(0.1)$ & $\sim \sim$ & -- & \\
\hline Spain & $s$ & $35(1.0)$ & $510(3.2)$ & $\Delta 0$ & & $11(0.8)$ & $489(4.9)$ & $\Delta \Delta$ & & $3(0.4)$ & $487(12.4)$ & $\Delta \Delta$ & \\
\hline Qatar & s & $29(0.7)$ & $345(3.0)$ & $\Delta \Delta$ & & $18(0.5)$ & $318(3.0)$ & $\Delta \Delta$ & & $5(0.4)$ & $317(7.6)$ & $\Delta \Delta$ & \\
\hline South Africa & $r$ & $35(0.6)$ & $293(6.3)$ & $\Delta \Delta$ & & $15(0.4)$ & $292(8.0)$ & $\Delta \Delta$ & & $4(0.2)$ & $258(9.1)$ & $\Delta \Delta$ & \\
\hline Kuwait & $r$ & $28(0.9)$ & $319(6.0)$ & $\Delta \diamond$ & & $20(0.9)$ & $302(8.3)$ & $\Delta \diamond$ & & $8(0.5)$ & $299(10.6)$ & $\Delta \diamond$ & \\
\hline Chinese Taipei & & $45(0.8)$ & $526(2.5)$ & $\Delta \Delta$ & & $10(0.5)$ & $507(4.4)$ & $\Delta \Delta$ & & $1(0.2)$ & $\sim \sim$ & $\Delta \Delta$ & \\
\hline Poland & & $40(0.8)$ & $505(3.4)$ & $\Delta \diamond$ & & $14(0.6)$ & $492(3.9)$ & $\Delta \diamond$ & & $4(0.3)$ & $494(9.3)$ & $\Delta \diamond$ & \\
\hline Latvia & & $41(0.8)$ & $538(2.6)$ & $-2(1.2)$ & & $20(0.9)$ & $511(3.5)$ & $-8(1.4)$ & ( & $5(0.5)$ & $491(7.5)$ & $-2(0.8)$ & ( \\
\hline Sweden & & $36(0.9)$ & $549(3.1)$ & $1(1.1)$ & & $24(1.0)$ & $534(3.3)$ & $-4(1.3)$ & $(\nabla$ & $6(0.5)$ & $508(5.8)$ & $-3(0.7)$ & ( \\
\hline Denmark & & $43(1.1)$ & $546(2.5)$ & $\Delta 0$ & & $20(0.9)$ & $519(3.6)$ & $\Delta \Delta$ & & $4(0.4)$ & $508(8.4)$ & $\Delta \Delta$ & \\
\hline France & & $41(0.9)$ & $522(2.7)$ & $0(1.4)$ & & $23(0.8)$ & $510(2.7)$ & $-5(1.3)$ & $(\nabla$ & $5(0.4)$ & $502(8.6)$ & $-1(0.6)$ & \\
\hline Bulgaria & & $31(1.1)$ & $557(4.2)$ & $-2(1.5)$ & & $21(1.0)$ & $532(5.7)$ & $-2(1.4)$ & & $18(1.9)$ & $514(10.1)$ & $2(2.6)$ & \\
\hline Canada, Nova Scotia & & $42(0.8)$ & $545(2.5)$ & $\Delta \Delta$ & & $24(0.7)$ & $521(3.5)$ & $\Delta \diamond$ & & $5(0.3)$ & $505(5.6)$ & $\Delta \diamond$ & \\
\hline Moldova, Rep. of & & $35(0.9)$ & $502(3.4)$ & $7(1.4)$ & $\theta$ & $26(1.0)$ & $486(3.8)$ & $-11(1.7)$ & ( & $11(1.1)$ & $479(8.4)$ & $-13(1.9)$ & ( \\
\hline Lithuania & & $46(0.8)$ & $537(1.7)$ & $7(1.5)$ & 0 & $23(0.7)$ & $509(2.4)$ & $-3(1.2)$ & $(-$ & $3(0.4)$ & $485(7.1)$ & $-9(0.7)$ & ( \\
\hline Canada, British Columbia & $r$ & $40(1.0)$ & $563(3.5)$ & $\Delta \Delta$ & & $25(0.9)$ & $548(3.9)$ & $\Delta \Delta$ & & $7(0.5)$ & $543(6.5)$ & $\Delta \Delta$ & \\
\hline Morocco & & $35(1.6)$ & $318(7.3)$ & -- & & $15(1.0)$ & $303(11.1)$ & -- & & $21(2.3)$ & $286(13.6)$ & -- & \\
\hline Canada, Ontario & $r$ & $38(1.2)$ & $556(3.1)$ & $-3(1.6)$ & & $25(1.3)$ & $544(3.8)$ & $7(1.5)$ & 0 & $8(0.7)$ & $524(7.3)$ & $5(0.8)$ & 0 \\
\hline Russian Federation & & $37(1.0)$ & $570(3.3)$ & $7(1.7)$ & 0 & $25(0.9)$ & $541(4.4)$ & $-8(1.5)$ & 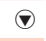 & $10(0.9)$ & $517(4.6)$ & $-14(1.9)$ & ( \\
\hline Belgium (French) & & $37(0.8)$ & $500(3.1)$ & $\Delta \Delta$ & & $26(0.8)$ & $497(3.9)$ & $\Delta \Delta$ & & $10(0.7)$ & $498(5.4)$ & $\Delta \diamond$ & \\
\hline Slovenia & & $34(0.7)$ & $525(2.3)$ & $-2(1.3)$ & & $26(0.8)$ & $503(2.9)$ & $0(1.2)$ & & $13(0.5)$ & $497(3.6)$ & $3(0.9)$ & 0 \\
\hline Canada, Alberta & $r$ & $39(1.0)$ & $565(3.2)$ & $\Delta \Delta$ & & $27(0.8)$ & $551(3.2)$ & $\Delta \Delta$ & & $7(0.5)$ & $536(5.5)$ & $\Delta \Delta$ & \\
\hline New Zealand & s & $40(0.9)$ & $551(2.7)$ & $-1(1.5)$ & & $26(0.8)$ & $535(3.8)$ & $-2(1.4)$ & & $8(0.6)$ & $528(5.9)$ & $-1(0.9)$ & \\
\hline Iran, Islamic Rep. of & & $29(1.1)$ & $427(4.0)$ & $-1(1.6)$ & & $21(0.9)$ & $421(4.4)$ & $1(1.3)$ & & $25(1.7)$ & $392(6.3)$ & $0(2.4)$ & \\
\hline Iceland & $\mathrm{r}$ & $31(0.8)$ & $516(2.1)$ & $3(1.2)$ & 0 & $32(0.9)$ & $502(2.1)$ & $-5(1.2)$ & $(\nabla$ & $12(0.6)$ & $480(4.4)$ & $-6(0.9)$ & 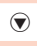 \\
\hline Georgia & & $28(1.0)$ & $479(4.2)$ & $\Delta \Delta$ & & $25(1.0)$ & $468(3.7)$ & $\Delta \Delta$ & & $22(1.3)$ & $458(7.3)$ & $\Delta \diamond$ & \\
\hline Romania & & $30(1.3)$ & $505(4.4)$ & $1(1.8)$ & & $25(1.2)$ & $484(5.4)$ & $-7(1.8)$ & $(\nabla$ & $22(2.0)$ & $433(10.3)$ & $0(2.4)$ & \\
\hline Norway & & $28(0.7)$ & $504(3.7)$ & $-9(1.4)$ & ( & $29(0.7)$ & $491(3.6)$ & $-1(1.3)$ & & $20(1.0)$ & $476(4.7)$ & $7(1.2)$ & 0 \\
\hline Canada, Quebec & $\mathrm{r}$ & $36(1.1)$ & $540(3.3)$ & $3(1.6)$ & 0 & $30(1.0)$ & $529(3.8)$ & $-6(1.5)$ & $(\nabla$ & $11(0.6)$ & $515(6.1)$ & $1(1.1)$ & \\
\hline Italy & & $38(0.8)$ & $555(3.4)$ & $2(1.2)$ & & $27(0.7)$ & $546(3.4)$ & $-5(1.2)$ & ( & $13(0.5)$ & $540(4.0)$ & $-3(0.8)$ & ( \\
\hline Luxembourg & & $37(0.7)$ & $555(2.1)$ & $\Delta \Delta$ & & $30(0.7)$ & $560(1.8)$ & $\Delta \diamond$ & & $12(0.4)$ & $559(3.0)$ & $\Delta \Delta$ & \\
\hline Scotland & $s$ & $45(1.2)$ & $546(4.4)$ & $4(1.7)$ & 0 & $25(1.2)$ & $531(5.2)$ & $-10(1.7)$ & $(\nabla$ & $11(0.7)$ & $528(9.0)$ & $-2(1.3)$ & \\
\hline Austria & & $31(0.8)$ & $540(2.6)$ & $\Delta \Delta$ & & $33(0.8)$ & $538(2.3)$ & $\Delta \diamond$ & & $17(0.7)$ & $536(3.4)$ & $\Delta \Delta$ & \\
\hline Indonesia & & $51(1.7)$ & $416(3.8)$ & $\Delta 0$ & & $22(1.6)$ & $386(5.7)$ & $\Delta \Delta$ & & $10(1.7)$ & $369(6.5)$ & $\Delta \diamond$ & \\
\hline Germany & & $32(0.8)$ & $552(2.7)$ & $3(1.1)$ & 0 & $34(0.6)$ & $551(2.8)$ & $-1(1.0)$ & & $18(0.9)$ & $551(3.6)$ & $-6(1.1)$ & ( \\
\hline Netherlands & s & $35(1.1)$ & $555(2.4)$ & $6(1.5)$ & 0 & $35(0.9)$ & $551(2.3)$ & $-3(1.4)$ & $(\uparrow$ & $16(0.9)$ & $550(3.5)$ & $-6(1.4)$ & ( \\
\hline Hungary & & $20(0.7)$ & $556(4.6)$ & $3(1.0)$ & 0 & $31(0.8)$ & $549(3.5)$ & $-2(1.2)$ & & $37(0.9)$ & $549(2.8)$ & $-4(1.3)$ & ( \\
\hline Belgium (Flemish) & & $29(0.8)$ & $545(2.5)$ & $\Delta \Delta$ & & $34(0.8)$ & $548(2.1)$ & $\Delta \Delta$ & & $25(0.9)$ & $549(2.7)$ & $\Delta \Delta$ & \\
\hline Slovak Republic & & $21(0.7)$ & $547(3.1)$ & $2(1.1)$ & & $39(0.9)$ & $534(2.6)$ & $1(1.2)$ & & $30(1.0)$ & $512(4.1)$ & $-6(1.4)$ & ( \\
\hline England & & $x x$ & $x x$ & $x x$ & & $x x$ & $x x$ & $x x$ & & $x x$ & $x x$ & $x x$ & \\
\hline United States & & -- & -- & -- & & -- & -- & -- & & -- & -- & -- & \\
\hline International Avg. & & $35(0.2)$ & $499(0.6)$ & & & $23(0.1)$ & $484(0.8)$ & & & $12(0.2)$ & $474(1.3)$ & & \\
\hline
\end{tabular}

$A n$ " $r$ " indicates data are available for $70-84 \%$ of the students. An "s" indicates data are available for $50-69 \%$ of the students. An " $x$ " indicates data are available for less than $50 \%$ of the students.

A dash (-) indicates comparable data are not available. A tilde $(\sim)$ indicates insufficient data to report achievement.
A diamond $(\diamond)$ indicates the country did not participate in the 2001 assessment NOTE: The International Average does not include the results from the Canadian provinces. Trend Note: The primary education systems of the Russian Federation and Slovenia underwent structural changes. Data for Canada, Ontario include only public schools. 
Exhibit 5.4 Principals' Estimates of the Percentage of Students Entering School Able to Perform Beginning Literacy Skills with Trends

\begin{tabular}{|c|c|c|c|c|c|c|c|c|c|c|c|c|c|}
\hline \multirow{2}{*}{ Countries } & & \multicolumn{3}{|c|}{$\begin{array}{l}\text { More than } 75 \% \text { Begin } \\
\text { School with Skills }\end{array}$} & \multicolumn{3}{|c|}{$\begin{array}{l}51-75 \% \text { Begin } \\
\text { School with Skills }\end{array}$} & \multicolumn{3}{|c|}{$\begin{array}{l}25-50 \% \text { Begin } \\
\text { School with Skills }\end{array}$} & \multicolumn{3}{|c|}{$\begin{array}{l}\text { Less than } 25 \% \text { Begin } \\
\text { School with Skills }\end{array}$} \\
\hline & & $\begin{array}{l}\text { Percent of } \\
\text { Students }\end{array}$ & $\begin{array}{l}\text { Difference } \\
\text { in Percent } \\
\text { From } 2001\end{array}$ & & $\begin{array}{l}\text { Percent of } \\
\text { Students }\end{array}$ & $\begin{array}{l}\text { Difference } \\
\text { in Percent } \\
\text { From } 2001\end{array}$ & & $\begin{array}{l}\text { Percent of } \\
\text { Students }\end{array}$ & $\begin{array}{l}\text { Difference } \\
\text { in Percent } \\
\text { From } 2001\end{array}$ & & $\begin{array}{l}\text { Percent of } \\
\text { Students }\end{array}$ & $\begin{array}{l}\text { Difference } \\
\text { in Percent } \\
\text { From } 2001\end{array}$ & \\
\hline Austria & & $0(0.0)$ & $\Delta \diamond$ & & $2(1.2)$ & $\Delta \diamond$ & & $14(3.4)$ & $\Delta \diamond$ & & $84(3.6)$ & $\Delta \diamond$ & \\
\hline Belgium (Flemish) & & $2(1.2)$ & $\Delta \Delta$ & & $5(2.2)$ & $\Delta \diamond$ & & $19(3.7)$ & $\Delta \Delta$ & & $73(4.1)$ & $\Delta \Delta$ & \\
\hline Belgium (French) & $r$ & $6(2.3)$ & $\Delta \diamond$ & & $20(3.6)$ & $\Delta \Delta$ & & $35(4.7)$ & $\Delta \diamond$ & & $39(4.6)$ & $\Delta \diamond$ & \\
\hline Bulgaria & & $7(2.2)$ & $2(2.8)$ & & $19(3.6)$ & $8(4.5)$ & & $32(3.7)$ & $6(5.0)$ & & $42(4.0)$ & $-16(5.2)$ & ( \\
\hline Canada, Alberta & & $2(1.3)$ & $\Delta 0^{\circ}$ & & $5(1.4)$ & $\Delta \Delta$ & & $23(3.3)$ & $\Delta \diamond$ & & $70(3.7)$ & $\Delta 0$ & \\
\hline Canada, British Columbia & & $3(1.5)$ & $\Delta \Delta$ & & $7(2.1)$ & $\Delta \diamond$ & & $29(3.4)$ & $\Delta \Delta$ & & $62(3.9)$ & $\Delta \Delta$ & \\
\hline Canada, Nova Scotia & & $3(1.7)$ & $\Delta \diamond$ & & $7(2.3)$ & $\diamond \diamond$ & & $30(4.0)$ & $\Delta \diamond$ & & $60(4.3)$ & $\Delta \diamond$ & \\
\hline Canada, Ontario & & $2(1.7)$ & $-32(5.1)$ & ( & $4(1.9)$ & $-28(5.4)$ & ( & $14(3.7)$ & $-10(5.8)$ & & $79(4.2)$ & $70(4.8)$ & 0 \\
\hline Canada, Quebec & & $2(1.5)$ & $-15(4.1)$ & 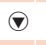 & $2(1.3)$ & $-11(3.6)$ & 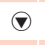 & $9(2.8)$ & $-17(5.6)$ & 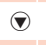 & $87(3.5)$ & $43(6.4)$ & 0 \\
\hline Chinese Taipei & & $94(2.0)$ & $\Delta \Delta$ & & $5(2.0)$ & $\Delta \diamond$ & & $0(0.0)$ & $\Delta \diamond$ & & $1(0.5)$ & $\Delta \Delta$ & \\
\hline Denmark & & $35(4.4)$ & $\Delta \diamond$ & & $37(4.6)$ & $\Delta \Delta$ & & $21(3.1)$ & $\Delta \diamond$ & & $7(2.3)$ & $\Delta \Delta$ & \\
\hline England & $s$ & $56(5.4)$ & $-5(7.6)$ & & $22(4.3)$ & $4(6.2)$ & & $14(3.3)$ & $3(4.6)$ & & $8(2.8)$ & $-1(4.1)$ & \\
\hline France & & $19(3.2)$ & $-2(4.8)$ & & $32(4.0)$ & $4(5.6)$ & & $25(4.1)$ & $-9(6.5)$ & & $23(4.2)$ & $6(5.3)$ & \\
\hline Georgia & & $3(1.1)$ & $\Delta \Delta$ & & $3(1.4)$ & $\Delta \diamond$ & & $20(3.9)$ & $\diamond \diamond$ & & $74(4.1)$ & $\Delta \diamond$ & \\
\hline Germany & & $2(1.2)$ & $1(1.4)$ & & $2(0.8)$ & $1(0.8)$ & & $24(3.7)$ & $16(4.1)$ & 0 & $73(3.7)$ & $-19(4.1)$ & ( \\
\hline Hong Kong SAR & & $86(3.0)$ & -- & & $14(3.0)$ & -- & & $0(0.0)$ & -- & & $0(0.0)$ & -- & \\
\hline Hungary & & $0(0.0)$ & $0(0.0)$ & & $1(0.8)$ & $0(1.3)$ & & $6(2.1)$ & $1(2.7)$ & & $93(2.2)$ & $-1(3.0)$ & \\
\hline Iceland & $r$ & $7(0.2)$ & $2(0.2)$ & $\Delta$ & $36(0.3)$ & $14(0.5)$ & 0 & $43(0.3)$ & $4(0.5)$ & 0 & $14(0.3)$ & $-21(0.4)$ & ( ) \\
\hline Indonesia & & $16(2.8)$ & $\Delta \diamond$ & & $16(3.4)$ & $\Delta \Delta$ & & $18(3.7)$ & $\Delta \diamond$ & & $50(4.5)$ & $\Delta \diamond$ & \\
\hline Iran, Islamic Rep. of & & $4(1.4)$ & $0(2.1)$ & & $4(1.2)$ & $3(1.3)$ & 0 & $4(1.3)$ & $1(2.1)$ & & $88(2.2)$ & $-4(3.1)$ & \\
\hline Israel & & $16(3.4)$ & $9(4.0)$ & 0 & $17(3.4)$ & $9(4.1)$ & 0 & $30(4.0)$ & $16(4.9)$ & 0 & $37(4.1)$ & $-33(5.7)$ & 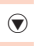 \\
\hline Italy & & $2(1.2)$ & $0(1.6)$ & & $15(3.2)$ & $1(3.9)$ & & $29(3.6)$ & $1(5.2)$ & & $54(4.4)$ & $-2(6.0)$ & \\
\hline Kuwait & & $20(3.4)$ & $\Delta \diamond$ & & $16(3.1)$ & $\Delta \diamond$ & & $12(2.8)$ & $\Delta \diamond$ & & $52(4.2)$ & $\Delta \diamond$ & \\
\hline Latvia & & $40(4.9)$ & $31(5.3)$ & 0 & $33(4.1)$ & $2(5.5)$ & & $20(3.5)$ & $-13(5.5)$ & ( & $7(1.9)$ & $-20(4.4)$ & 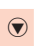 \\
\hline Lithuania & & $7(2.2)$ & $-1(2.8)$ & & $15(3.4)$ & $4(4.4)$ & & $32(3.9)$ & $5(5.5)$ & & $46(4.0)$ & $-9(6.0)$ & \\
\hline${ }^{1}$ Luxembourg & & -- & -- & & -- & -- & & -- & -- & & -- & -- & \\
\hline Macedonia, Rep. of & $r$ & $4(2.1)$ & $-1(2.9)$ & & $15(3.6)$ & $0(4.9)$ & & $30(3.7)$ & $-6(5.8)$ & & $51(4.2)$ & $6(6.3)$ & \\
\hline Moldova, Rep. of & & $11(2.9)$ & $-8(4.7)$ & & $20(3.7)$ & $6(4.7)$ & & $28(3.9)$ & $-8(5.8)$ & & $42(4.5)$ & $10(6.1)$ & \\
\hline Morocco & $r$ & $31(5.1)$ & $9(6.7)$ & & $15(3.2)$ & $-2(5.0)$ & & $19(3.7)$ & $1(4.5)$ & & $36(4.9)$ & $-8(7.0)$ & \\
\hline Netherlands & $r$ & $2(1.2)$ & $0(1.7)$ & & $10(3.1)$ & $5(3.6)$ & & $27(4.3)$ & $10(5.5)$ & & $61(5.1)$ & $-14(6.5)$ & ( \\
\hline New Zealand & & $5(1.6)$ & $-1(2.4)$ & & $9(2.3)$ & $1(3.1)$ & & $14(2.6)$ & $-7(4.2)$ & & $72(3.3)$ & $7(4.9)$ & \\
\hline Norway & & $3(1.6)$ & $3(1.6)$ & & $10(3.1)$ & $3(4.0)$ & & $49(4.9)$ & $11(6.6)$ & & $38(4.2)$ & $-17(6.4)$ & ( \\
\hline Poland & $r$ & $16(3.7)$ & $\diamond \diamond$ & & $18(3.6)$ & $\diamond \diamond$ & & $13(3.4)$ & $\Delta \diamond$ & & $53(5.3)$ & $\Delta \diamond$ & \\
\hline Qatar & & $26(0.2)$ & $\Delta \Delta$ & & $17(0.2)$ & $\Delta \Delta$ & & $10(0.2)$ & $\Delta \Delta$ & & $46(0.2)$ & $\Delta \Delta$ & \\
\hline Romania & & $2(1.1)$ & $-4(2.3)$ & & $15(3.3)$ & $6(4.1)$ & & $30(4.3)$ & $10(5.3)$ & & $53(4.5)$ & $-12(5.9)$ & $(-$ \\
\hline Russian Federation & & $11(2.4)$ & $4(3.0)$ & & $27(3.0)$ & $8(4.1)$ & & $31(3.2)$ & $-2(4.8)$ & & $31(3.3)$ & $-10(5.0)$ & ( \\
\hline Scotland & $r$ & $4(2.3)$ & $3(2.6)$ & & $7(2.4)$ & $1(3.5)$ & & $16(4.0)$ & $6(5.1)$ & & $72(4.9)$ & $-10(6.4)$ & \\
\hline Singapore & & $70(0.0)$ & $7(3.5)$ & $\boldsymbol{\Delta}$ & $22(0.0)$ & $-5(3.5)$ & & $6(0.0)$ & $-2(2.0)$ & & $1(0.0)$ & $0(0.8)$ & \\
\hline Slovak Republic & & $0(0.0)$ & $-1(1.0)$ & & $0(0.0)$ & $0(0.0)$ & & $11(2.6)$ & $7(3.2)$ & 0 & $89(2.6)$ & $-5(3.3)$ & \\
\hline Slovenia & & $0(0.0)$ & $-61(4.3)$ & ( & $7(2.2)$ & $-8(3.9)$ & ( & $27(3.7)$ & $13(4.7)$ & 0 & $67(4.1)$ & $55(4.8)$ & 0 \\
\hline South Africa & & $7(1.9)$ & $\diamond \diamond$ & & $9(2.1)$ & $\Delta \Delta$ & & $19(2.3)$ & $\Delta \diamond$ & & $65(3.0)$ & $\Delta \diamond$ & \\
\hline Spain & & $56(3.6)$ & $\Delta \Delta$ & & $20(3.3)$ & $\Delta \diamond$ & & $14(2.8)$ & $\Delta \Delta$ & & $10(2.3)$ & $\Delta \Delta$ & \\
\hline Sweden & & $15(3.3)$ & $2(4.5)$ & & $28(4.1)$ & $-6(6.3)$ & & $42(4.2)$ & $6(6.5)$ & & $15(3.3)$ & $-2(5.1)$ & \\
\hline Trinidad and Tobago & & $12(2.7)$ & $\Delta 0$ & & $28(4.0)$ & $\Delta \Delta$ & & $26(4.0)$ & $\Delta \Delta$ & & $34(4.3)$ & $\Delta \Delta$ & \\
\hline United States & & $65(3.7)$ & $17(5.8)$ & $\boldsymbol{\theta}$ & $12(2.6)$ & $-10(4.4)$ & $\nabla$ & $10(2.5)$ & $-4(3.9)$ & & $13(2.6)$ & $-3(4.1)$ & \\
\hline International Avg. & & $20(0.4)$ & & & $15(0.5)$ & & & $21(0.5)$ & & & $44(0.6)$ & & \\
\hline
\end{tabular}

Based on principals' responses to questions about how many of the students in their schools can do the following when they begin the first year of formal schooling: recognize most of the letters of the alphabet, write letters of the alphabet, read some words, write some words, and read sentences. Average is computed across the five items based on a 4-point scale: Less than $25 \%=1,25-50 \%=2,51-75 \%=3$, and More than $75 \%=4$. More than $75 \%$ indicates an average response score of greater than 3.25 through $4.51-75 \%$ indicates an average of greater than 2.5 through $3.25 .25-50 \%$ indicates an average of 1.75 through 2.5. Less than $25 \%$ indicates an average of 1 to less than 1.75 .

() Standard errors appear in parentheses. Because results are rounded to the nearest whole number, some totals may appear inconsistent.
An " $r$ " indicates data are available for $70-84 \%$ of the students. An " $s$ " indicates data are available for $50-69 \%$ of the students. An " $x$ " indicates data are available for less than $50 \%$ of the students.

A dash (-) indicates comparable data are not available.

A diamond $(\diamond)$ indicates the country did not participate in the 2001 assessment.

NOTE: The International Average does not include the results from the Canadian provinces. Trend Note: The primary education systems of the Russian Federation and Slovenia underwent structural changes. Data for Canada, Ontario include only public schools. 1 Primary schools in Luxembourg do not have principals. 


\section{How Much Is Reading Emphasized in the School Curriculum?}

In most countries, the Ministry of Education (or the government department responsible for education) is responsible for developing a national curriculum, and monitoring its implementation. Typically, the curriculum is prescribed for a range of grades, or grade by grade, and covers reading either as a separate subject or as part of a language arts curriculum. A substantial portion of each country's chapter of the PIRLS 2006 Encyclopedia is devoted to describing the reading curriculum that covers reading instruction in the fourth grade. In addition, PIRLS asked each country to indicate to what extent curricular emphasis is placed on various reading purposes and processes. School principals reported the emphasis placed on the prescribed reading curriculum within the school, and the grade-by-grade emphases on specific reading skills and strategies in the school's primary grades.

Exhibit 5.5 presents basic information about the reading curriculum within each country or province. Nearly all countries have a national curriculum that covers reading instruction at the fourth grade. Canada, Germany, and the United States define curricula at a regional levelaccording to province (Canada), Laender (Germany), or state (United States). In Belgium, the Flemish and French linguistic and cultural communities have their own governments that are responsible for education, each with its own document that defines minimum attainment targets for reading. However, within each community, different networks of schools have the authority to develop their own curricula. In Luxembourg, while the reading curriculum is developed by the Ministry of Education for all schools, the local town and city councils are responsible for the administration of the schools. In Qatar, the Ministry of Education developed a reading curriculum in 1995 that is followed by public and private schools under its jurisdiction. Separate Qatar curriculum standards were developed in 2005 specifically for the small number of independent schools, but individual schools may choose to what extent they adhere to these standards.

Between PIRLS 2001 and PIRLS 2006, the reading curriculum around the world seems to have been in considerable flux. During that time period, 
25 countries and 3 provinces either introduced a new curriculum or were in the curriculum revision process (or both). Most of the PIRLS 2006 participants considered the reading curriculum to be part of the language arts curriculum. Those with reading as a separate curriculum area included the Canadian province of Ontario, the Netherlands, the Russian Federation, and Sweden. In the United States, the structure of the reading curriculum varied by state. There was substantial variation across participants in the grade-tograde structure of the reading curriculum, depending on the structure of the primary school system.

Exhibit 5.6 shows principals' reports about the emphasis schools place on the reading curriculum. Four fifths of students, on average internationally, were taught in schools that had informal initiatives to encourage students to read. Nearly half of the fourth-grade students were in schools with school-based programs for teachers to improve reading instruction, and the same percentage were in schools with guidelines that coordinated reading instruction among teachers within a grade, or across grades.

Reading, writing, and oral language are the most prominent literacy skills included in language arts curricula. The greatest percentages of students were in schools that placed comparatively more emphasis on reading than on other school subject areas. Almost three fourths of the fourth-grade students, on average internationally, were in schools that placed more emphasis on reading compared to other areas. More than 90 percent of students were in such schools in all five Canadian provinces, Iceland, Latvia, New Zealand, Norway, and the United States.

To obtain some information about the alignment of the PIRLS 2006 assessment with their reading curricula, PIRLs asked the participants about the curricular emphases placed on the reading purposes and processes that provided the foundation for the assessment. As shown in Exhibit 5.7, the purposes specified in the PIRLS 2006 Framework and Assessment Specifications - reading for literary experience and to acquire informationreceived major emphasis in 25 of the participants' reading curricula. Countries with a major emphasis on reading for literary experience but less emphasis 
Exhibit 5.5 Structural Characteristics of Reading Curricula for Primary/Elementary Grades

PIRLS 2006

4th Grade

\begin{tabular}{|c|c|c|c|c|c|}
\hline Countries & $\begin{array}{c}\text { National } \\
\text { Curriculum }\end{array}$ & $\begin{array}{l}\text { Grade to Grade } \\
\text { Structure }\end{array}$ & $\begin{array}{c}\text { Year } \\
\text { Introduced }\end{array}$ & $\begin{array}{l}\text { Under } \\
\text { Revision }\end{array}$ & $\begin{array}{c}\text { Reading as } \\
\text { a Separate } \\
\text { Curriculum } \\
\text { Area }\end{array}$ \\
\hline Austria & - & $1-2,3,4$ & 2003 & - & 0 \\
\hline Belgium (Flemish) & 0 & $1,2,3,4,5,6$ & 1989 & 0 & 0 \\
\hline Belgium (French) & 0 & $1-2,3-6$ & 1999 & 0 & 0 \\
\hline Bulgaria & - & $1-4$ & 2002 & 0 & 0 \\
\hline Canada, Alberta & 0 & $1-9$ & 2000 & 0 & 0 \\
\hline Canada, British Columbia & 0 & $1-7$ & 1996 & - & 0 \\
\hline Canada, Nova Scotia & 0 & $1-3,4-6,7-9,10-12$ & 1997 & 0 & 0 \\
\hline Canada, Ontario & 0 & $1-8$ & 2006 & 0 & - \\
\hline Canada, Quebec & 0 & $1-2,3-4,5-6$ & 2001 & - & 0 \\
\hline Chinese Taipei & - & $1-3,4-6,7-9$ & 2003 & 0 & 0 \\
\hline Denmark & - & $1-2,3-4,5-6$ & 2003 & - & 0 \\
\hline England & - & $1-2,3-6$ & 1988 & 0 & 0 \\
\hline France & - & $1-2,3-5$ & 2002 & - & 0 \\
\hline Georgia & 0 & $1,2,3,4,5,6$ & 1997 & - & 0 \\
\hline Germany & 0 & $1-6$ & 2006 & 0 & 0 \\
\hline Hong Kong SAR & - & $1-6$ & 2000 & 0 & 0 \\
\hline Hungary & $\bullet$ & $1-4,5-6,7-8$ & 2003 & O & 0 \\
\hline Iceland & $\bullet$ & $\begin{array}{l}\text { 1-4,5-7,8-10; Enabling } \\
\text { objectives for each grade }\end{array}$ & 1999 & $\bullet$ & 0 \\
\hline Indonesia & - & $1-3,4-6$ & 2004 & - & 0 \\
\hline Iran, Islamic Rep. of & - & $1-5$ & 2000 & 0 & 0 \\
\hline Israel & - & $1,2,3,4,5,6$ & 2003 & - & 0 \\
\hline Italy & - & $1,2-3,4-5$ & 2004 & - & 0 \\
\hline Kuwait & - & $1-5$ & 1992 & 0 & 0 \\
\hline Latvia & - & $1-3,4-6,7-9$ & 2004 & 0 & 0 \\
\hline Lithuania & - & $1-2,3-4$ & 2003 & - & 0 \\
\hline Luxembourg & - & $1-2,3-6$ & 1989 & - & 0 \\
\hline Macedonia, Rep. of & - & $1-4,5-8$ & 1996 & 0 & 0 \\
\hline Moldova, Rep. of & - & $1-4$ & 1996 & - & 0 \\
\hline Morocco & - & - & - & - & - \\
\hline Netherlands & - & $1-8$ & 2006 & 0 & - \\
\hline New Zealand & - & $1-13$ & 1996 & - & 0 \\
\hline Norway & - & $1-4,5-7$ & 2006 & 0 & 0 \\
\hline Poland & 0 & $0,1,2,3$ & 1999 & 0 & 0 \\
\hline Qatar & 0 & $1-6$ & 1995 & 0 & 0 \\
\hline Romania & - & $1-4$ & 1998 & - & 0 \\
\hline Russian Federation & - & $1-4$ & 2004 & - & - \\
\hline Scotland & 0 & $1-3,4-7$ & 1991 & - & 0 \\
\hline Singapore & - & $1-6$ & 2001 & - & 0 \\
\hline Slovak Republic & - & $1-4,5-9$ & 1997 & - & 0 \\
\hline Slovenia & - & $1-3,4-6,7-9$ & 1998 & 0 & 0 \\
\hline South Africa & - & $1-3,4-6,7-9$ & 2002 & 0 & 0 \\
\hline Spain & - & $1-2,3-4,5-6$ & 1993 & - & 0 \\
\hline Sweden & - & $1-5$ & 1994 & 0 & - \\
\hline Trinidad and Tobago & - & $1-4,5-6$ & 1999 & 0 & 0 \\
\hline United States & 0 & Varies by state & - & 0 & $\bullet$ \\
\hline
\end{tabular}

Yes

$\bigcirc$ No

Background data provided by National Research Coordinators.

The Netherlands does not have a national reading curriculum that covers reading in the fourth grade. The Ministry of Education imposes a number of attainment targets students should reach before they enter secondary school at age 12 . Freedom of education, guaranteed under article 23 of the Constitution, allows schools to determine their own educational content and how to attain these targets.

A dash (-) indicates comparable data are not available. 
Exhibit 5.6 Emphasis on Reading Curriculum in the Schools

PIRLS 2006

\begin{tabular}{|c|c|c|c|c|c|c|c|}
\hline \multirow{2}{*}{ Countries } & \multicolumn{4}{|c|}{$\begin{array}{l}\text { Percentage of Students Whose Schools Have } \\
\text { Various Policies and Procedures }\end{array}$} & \multicolumn{3}{|c|}{$\begin{array}{c}\text { Percentage of Students Whose Schools Place } \\
\text { More Emphasis on Various Literacy Skills } \\
\text { Compared with Other Areas }\end{array}$} \\
\hline & $\begin{array}{c}\text { Written Statement } \\
\text { of the Reading } \\
\text { Curriculum to Be } \\
\text { Taught in the } \\
\text { School }\end{array}$ & $\begin{array}{l}\text { Informal Initiatives } \\
\text { to Encourage } \\
\text { Students to Read }\end{array}$ & $\begin{array}{l}\text { School-Based } \\
\text { Programs for } \\
\text { Teachers to } \\
\text { Improve Reading } \\
\text { Instruction }\end{array}$ & $\begin{array}{c}\text { Guidelines on How } \\
\text { to Coordinate } \\
\text { Reading } \\
\text { Instruction } \\
\text { Across Teachers }\end{array}$ & Reading & $\begin{array}{c}\text { Writing } \\
\text { (Not Handwriting) }\end{array}$ & $\begin{array}{c}\text { Speaking / } \\
\text { Listening } \\
\text { (Oral Language) }\end{array}$ \\
\hline Austria & $7(2.1)$ & $88(2.7)$ & $50(4.2)$ & $29(3.5)$ & $65(4.0)$ & $20(3.3)$ & $35(4.1)$ \\
\hline Belgium (Flemish) & $32(4.4)$ & $88(2.7)$ & $31(3.9)$ & $64(4.0)$ & $50(4.2)$ & $13(3.3)$ & $13(3.0)$ \\
\hline Belgium (French) & $21(3.8)$ & $83(3.2)$ & $35(4.3)$ & $48(4.7)$ & $78(3.9)$ & $22(3.9)$ & $24(3.8)$ \\
\hline Bulgaria & $10(2.6)$ & $52(4.9)$ & $31(4.5)$ & $47(4.5)$ & $75(3.8)$ & $65(4.6)$ & $54(4.6)$ \\
\hline Canada, Alberta & $23(3.5)$ & $97(1.5)$ & $80(3.6)$ & $53(4.4)$ & $91(2.7)$ & $81(3.5)$ & $50(4.4)$ \\
\hline Canada, British Columbia & $34(4.2)$ & $99(1.0)$ & $82(3.5)$ & $50(4.7)$ & $92(2.4)$ & $68(3.9)$ & $45(4.5)$ \\
\hline Canada, Nova Scotia & $29(3.8)$ & $87(2.0)$ & $86(2.3)$ & $56(3.8)$ & $95(1.8)$ & $76(3.2)$ & $68(3.3)$ \\
\hline Canada, Ontario & $29(4.5)$ & $94(2.5)$ & $94(2.4)$ & $59(4.5)$ & $92(2.9)$ & $74(4.2)$ & $60(4.7)$ \\
\hline Canada, Quebec & $18(2.6)$ & $91(2.5)$ & $58(4.0)$ & $46(4.5)$ & $90(2.4)$ & $56(4.9)$ & $25(4.2)$ \\
\hline Chinese Taipei & $66(4.1)$ & $95(2.0)$ & $66(3.7)$ & $42(4.0)$ & $46(4.3)$ & $14(2.8)$ & $19(3.2)$ \\
\hline Denmark & $26(4.2)$ & $57(4.2)$ & $78(3.4)$ & $44(4.7)$ & $84(3.2)$ & $34(4.3)$ & $46(4.7)$ \\
\hline England & $62(4.6)$ & $96(1.8)$ & $73(4.3)$ & $63(4.0)$ & $73(4.3)$ & $63(4.8)$ & $46(4.8)$ \\
\hline France & $54(4.1)$ & $81(3.7)$ & $30(3.9)$ & $49(4.4)$ & $69(4.1)$ & $36(4.2)$ & $37(4.2)$ \\
\hline Georgia & $37(4.1)$ & $75(3.9)$ & $49(4.2)$ & $44(4.9)$ & $59(4.7)$ & $41(5.0)$ & $48(5.1)$ \\
\hline Germany & $31(2.6)$ & $87(2.4)$ & $32(2.9)$ & $20(2.2)$ & $53(3.8)$ & $27(3.7)$ & $38(3.7)$ \\
\hline Hong Kong SAR & $66(3.8)$ & $100(0.0)$ & $62(4.3)$ & $52(4.2)$ & $87(3.1)$ & $68(4.3)$ & $56(4.7)$ \\
\hline Hungary & $51(4.7)$ & $82(3.1)$ & $62(4.2)$ & $60(4.0)$ & $71(3.8)$ & $37(4.6)$ & $64(4.1)$ \\
\hline Iceland & $65(0.3)$ & $94(0.1)$ & $62(0.3)$ & $63(0.3)$ & $93(0.3)$ & $37(0.3)$ & $42(0.3)$ \\
\hline Indonesia & $34(4.3)$ & $72(3.4)$ & $83(3.0)$ & $70(3.4)$ & $83(3.2)$ & $68(4.0)$ & $56(4.2)$ \\
\hline Iran, Islamic Rep. of & $37(3.7)$ & $58(3.5)$ & $43(4.0)$ & $36(3.6)$ & $51(3.9)$ & $42(3.3)$ & $63(3.6)$ \\
\hline Israel & $69(4.5)$ & $84(2.8)$ & $69(3.9)$ & $81(3.6)$ & $83(3.2)$ & $66(4.1)$ & $53(4.4)$ \\
\hline Italy & $51(3.8)$ & $77(3.4)$ & $19(3.3)$ & $30(3.9)$ & $57(4.4)$ & $27(4.1)$ & $43(4.5)$ \\
\hline Kuwait & $53(4.6)$ & $72(4.0)$ & $51(4.5)$ & $57(4.3)$ & $73(3.6)$ & $65(3.9)$ & $54(4.2)$ \\
\hline Latvia & $10(2.3)$ & $80(3.4)$ & $21(3.7)$ & $43(4.6)$ & $94(2.0)$ & $75(3.7)$ & $80(3.5)$ \\
\hline Lithuania & $4(1.6)$ & $64(3.5)$ & $4(1.5)$ & $27(3.8)$ & $51(4.1)$ & $44(4.3)$ & $32(4.0)$ \\
\hline${ }^{1}$ Luxembourg & -- & -- & -- & -- & -- & -- & -- \\
\hline Macedonia, Rep. of & $23(4.2)$ & $72(4.2)$ & $34(4.3)$ & $61(4.2)$ & $65(3.7)$ & $41(4.4)$ & $45(4.4)$ \\
\hline Moldova, Rep. of & $18(3.1)$ & $67(4.1)$ & $33(4.2)$ & $30(4.3)$ & $87(2.8)$ & $30(4.3)$ & $75(3.9)$ \\
\hline Morocco & $17(3.6)$ & $34(5.4)$ & $14(3.5)$ & $23(4.3)$ & $88(3.4)$ & $56(4.8)$ & $58(5.0)$ \\
\hline Netherlands & $32(4.8)$ & $68(4.7)$ & $46(5.3)$ & $46(4.6)$ & $84(3.7)$ & $16(3.6)$ & $28(4.7)$ \\
\hline New Zealand & $89(2.1)$ & $84(2.8)$ & $86(2.4)$ & $72(3.3)$ & $90(2.2)$ & $78(2.8)$ & $62(3.5)$ \\
\hline Norway & $46(5.0)$ & $82(4.0)$ & $62(5.3)$ & $62(5.2)$ & $92(2.6)$ & $64(5.1)$ & $59(4.7)$ \\
\hline Poland & $7(2.1)$ & $90(2.5)$ & $14(2.7)$ & $32(4.0)$ & $59(4.4)$ & $29(4.0)$ & $23(3.5)$ \\
\hline Qatar & $55(0.2)$ & $85(0.2)$ & $67(0.2)$ & $46(0.2)$ & $73(0.2)$ & $73(0.2)$ & $66(0.2)$ \\
\hline Romania & $23(4.3)$ & $57(4.4)$ & $30(3.7)$ & $25(4.1)$ & $65(4.4)$ & $55(4.6)$ & $53(4.6)$ \\
\hline Russian Federation & $4(1.3)$ & $76(3.0)$ & $32(2.8)$ & $90(1.7)$ & $54(3.3)$ & $31(3.2)$ & $41(4.1)$ \\
\hline Scotland & $90(2.7)$ & $96(1.8)$ & $69(5.0)$ & $64(5.1)$ & $84(3.4)$ & $72(4.5)$ & $49(4.9)$ \\
\hline Singapore & $60(0.0)$ & $97(0.0)$ & $77(0.0)$ & $60(0.0)$ & $80(0.0)$ & $48(0.0)$ & $69(0.0)$ \\
\hline Slovak Republic & $15(2.9)$ & $89(2.4)$ & $51(4.1)$ & $15(3.1)$ & $53(4.0)$ & $19(3.5)$ & $40(4.0)$ \\
\hline Slovenia & $19(3.3)$ & $100(0.0)$ & $26(3.8)$ & $39(3.9)$ & $58(4.4)$ & $36(3.8)$ & $47(4.5)$ \\
\hline South Africa & $39(2.8)$ & $69(3.1)$ & $57(2.8)$ & $51(2.8)$ & $64(2.5)$ & $55(3.0)$ & $62(2.7)$ \\
\hline Spain & $62(4.2)$ & $85(2.9)$ & $39(4.0)$ & $55(4.3)$ & $80(3.7)$ & $61(4.7)$ & $38(4.0)$ \\
\hline Sweden & $67(4.6)$ & $91(2.9)$ & $79(3.4)$ & $46(4.5)$ & $86(3.1)$ & $42(5.4)$ & $48(4.9)$ \\
\hline Trinidad and Tobago & $49(4.1)$ & $83(3.2)$ & $66(4.4)$ & $50(4.4)$ & $81(3.7)$ & $30(3.9)$ & $47(4.5)$ \\
\hline United States & $73(3.9)$ & $95(1.5)$ & $87(2.6)$ & $76(4.1)$ & $91(2.0)$ & $57(3.5)$ & $31(3.4)$ \\
\hline International Avg. & $40(0.6)$ & $80(0.5)$ & $49(0.6)$ & $49(0.6)$ & $73(0.6)$ & $45(0.6)$ & $47(0.6)$ \\
\hline
\end{tabular}

Background data provided by schools

() Standard errors appear in parentheses. Because results are rounded to the nearest whole number, some totals may appear inconsistent.

$A n$ " $r$ " indicates data are available for $70-84 \%$ of the students. An " $s$ " indicates data are available for $50-69 \%$ of the students. An " $x$ " indicates data are available for less than $50 \%$ of the students.
A dash (-) indicates comparable data are not available.

NOTE: The International Average does not include the results from the Canadian provinces. 1 Primary schools in Luxembourg do not have principals. 
Exhibit 5.7 Emphasis on Purposes for Reading in the Reading Curriculum

PIRLS 2006

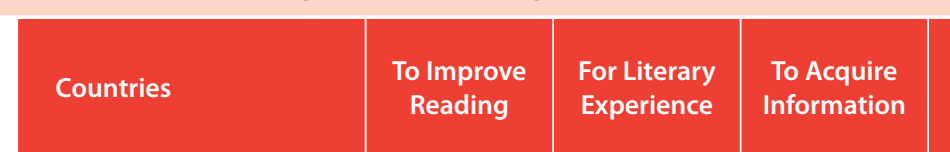

For Social

Awareness / For Enjoyment

Civic Duty

\begin{tabular}{|c|c|c|c|c|c|}
\hline Austria & 0 & ○ & O & 0 & O \\
\hline Belgium (Flemish) & 0 & O & 0 & $\bullet$ & $\odot$ \\
\hline Belgium (French) & $\bigcirc$ & 0 & ○ & $\odot$ & ○ \\
\hline Bulgaria & 0 & ○ & ○ & 0 & 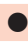 \\
\hline Canada, Alberta & $\bullet$ & ○ & ○ & $\odot$ & ○ \\
\hline Canada, British Columbia & 0 & - & 0 & $\odot$ & - \\
\hline Canada, Nova Scotia & 0 & - & 0 & 0 & ○ \\
\hline Canada, Ontario & $\bullet$ & $\odot$ & $\odot$ & $\odot$ & $\odot$ \\
\hline Canada, Quebec & 0 & ○ & 0 & $\bigcirc$ & ○ \\
\hline Chinese Taipei & 0 & - & 0 & $\odot$ & $\odot$ \\
\hline Denmark & 0 & $\odot$ & $\odot$ & $\bigcirc$ & 0 \\
\hline England & 0 & ○ & ○ & $\bigcirc$ & 0 \\
\hline France & $\bullet$ & $\bullet$ & $\bullet$ & $\odot$ & O \\
\hline Georgia & 0 & $\odot$ & $\odot$ & $\bigcirc$ & O \\
\hline Germany & 0 & ○ & ○ & $\odot$ & 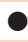 \\
\hline Hong Kong SAR & 0 & - & 0 & $\bullet$ & - \\
\hline Hungary & 0 & $\odot$ & O & $\bigcirc$ & $\odot$ \\
\hline Iceland & 0 & - & 0 & 0 & O \\
\hline Indonesia & 0 & ○ & O & $\bullet$ & ○ \\
\hline Iran, Islamic Rep. of & 0 & $\odot$ & $\odot$ & 0 & $\odot$ \\
\hline Israel & $\bullet$ & ○ & ○ & $\odot$ & $\odot$ \\
\hline Italy & $\bullet$ & ○ & 0 & $\odot$ & $\odot$ \\
\hline Kuwait & 0 & - & $\bigcirc$ & $\bullet$ & $\odot$ \\
\hline Latvia & 0 & ○ & ○ & $\odot$ & 0 \\
\hline Lithuania & 0 & ○ & $\odot$ & $\odot$ & 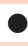 \\
\hline Luxembourg & 0 & - & O & 0 & - \\
\hline Macedonia, Rep. of & 0 & ○ & $\odot$ & $\bullet$ & - \\
\hline Moldova, Rep. of & 0 & $\odot$ & $\bullet$ & $\odot$ & $\odot$ \\
\hline Morocco & - & - & - & - & - \\
\hline Netherlands & 0 & $\odot$ & O & $\bigcirc$ & $\odot$ \\
\hline New Zealand & $\bullet$ & $\odot$ & $\odot$ & $\bigcirc$ & $\odot$ \\
\hline Norway & 0 & - & 0 & $\bullet$ & 0 \\
\hline Poland & $\odot$ & 0 & $\bullet$ & $\odot$ & $\odot$ \\
\hline Qatar & $\bullet$ & 0 & $\odot$ & $\bigcirc$ & $\odot$ \\
\hline Romania & 0 & $\odot$ & $\odot$ & $\odot$ & ○ \\
\hline Russian Federation & 0 & - & $\odot$ & $\bullet$ & $\odot$ \\
\hline Scotland & 0 & ○ & ○ & $\bigcirc$ & ○ \\
\hline Singapore & 0 & - & 0 & 0 & - \\
\hline Slovak Republic & 0 & ○ & $\odot$ & $\bigcirc$ & 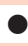 \\
\hline Slovenia & $\odot$ & $\odot$ & $\odot$ & $\odot$ & O \\
\hline South Africa & 0 & ○ & 0 & 0 & ○ \\
\hline Spain & 0 & $\odot$ & 0 & 0 & ○ \\
\hline Sweden & $\bigcirc$ & ○ & ○ & 0 & 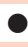 \\
\hline Trinidad and Tobago & 0 & ○ & ○ & 0 & ○ \\
\hline United States & 0 & ○ & ○ & $\bigcirc$ & ○ \\
\hline
\end{tabular}

Major Emphasis

- Some Emphasis

Little or No Emphasis 
on reading to acquire information included Kuwait, Lithuania, Macedonia, the Russian Federation, and the Slovak Republic. Countries with a major emphasis on reading to acquire information but less emphasis on reading for literary experience included Belgium (French), the Netherlands, Poland, and Spain. Not surprisingly, many of the participants emphasized reading in order to improve reading. Twenty-six participants reported a major emphasis on reading for enjoyment in the reading curriculum, while only 11 reported a major emphasis on reading for social awareness or civic duty.

The corresponding information about the reading processes assessed by PIRLS is found in Exhibit 5.8. Participants were asked to provide relatively detailed information about the extent to which their curriculum emphasized various aspects of the four broad reading processes assessed by PIRLS 2006: 1) focus on and retrieve explicitly stated information, 2) make straightforward inferences, 3 ) interpret and integrate ideas and information, and 4) examine and evaluate content, language, and textual elements.

More than two thirds of participants reported placing major emphasis in the reading curriculum on the processes related to focusing on and retrieving information, including identifying specific ideas, searching for definitions of words or phrases, and finding the topic sentence or main idea in a text. Of the straightforward inferencing processes, summarizing the main point was most widely reported as a major emphasis (27 participants), followed by evaluating cause and effect (18 participants), determining the referent of a pronoun (12 participants), and identifying generalizations (11 participants).

The most highly emphasized process within the category of interpreting and integrating ideas and information was discerning the overall message or theme, which received a major emphasis in more than two thirds of curricula. Interpreting a real-world application of information from the text and describing the relationship between two characters received a major emphasis in 17 and 16 of the reading curricula, respectively. Inferring mood or tone in a story, or comparing and contrasting information from a text received major emphasis in less than one third of the curricula. 
Considering that these curricula are for fourth-grade students in transition from learning to read to reading to learn, processes requiring students to examine and evaluate content, language, and textual elements received the least emphasis across the four categories. Less than one third of the participants reported placing a major emphasis on evaluating the likelihood that story events could really happen, judging completeness or clarity of information in a text, or determining an author's perspective. Countries reporting little or no emphasis on any of the examining and evaluating processes included Denmark, Georgia, Hungary, Iceland, Indonesia, Kuwait, Lithuania, Luxembourg, Macedonia, Qatar, the Slovak Republic, and South Africa. This also was the case for the Canadian province of Quebec.

Exhibit 5.9 shows principals' reports of the grade by which certain reading skills or strategies were emphasized for at least half of the students in the school. In nearly all countries and provinces, knowing letters of the alphabet and letter-sound relationships, and reading words, isolated sentences, or connected text were emphasized for at least half the students by first grade. By second grade, a number of participants emphasized identifying the main idea of the text, explaining or supporting understanding of a text, and comparing text with personal experience. By third grade, most participants emphasized making predictions about a story's plot sequence or story resolution, comparing different texts, and making generalizations and inferences based on text. Often, describing the style and structure of a text was emphasized by fourth grade. All reading comprehension skills or strategies queried were emphasized for at least half the students by third grade in England, Israel, Latvia, New Zealand, the Russian Federation, Scotland, and the United States, as well as three of the Canadian provinces, Alberta, Nova Scotia, and Ontario. 
Exhibit 5.8 Emphasis on Processes of Comprehension in the Reading Curriculum

PIRLS 2006

4th Grade
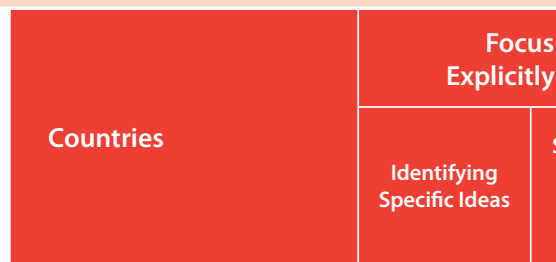

Focus on and Retrieve

Explicitly Stated Information

\section{Austria}

Belgium (Flemish)

Belgium (French)

$$
\text { Bulgaria }
$$

Canada, Alberta

Canada, British Columbia

Canada, Nova Scotia

Canada, Ontario

Canada, Quebec

Chinese Taipei

Denmark

England

France

Georgia

Germany

Hong Kong SAR

Hungary

Iceland

$$
\text { Indonesia }
$$

Iran, Islamic Rep. of

Israel

$$
\text { Italy }
$$

$$
\text { Kuwait }
$$

Latvia

\section{Lithuania}

Luxembourg

Macedonia, Rep. of

Moldova, Rep. of

Morocco

Netherlands

New Zealand

Norway

Poland

Qatar

Romania

Russian Federation

Scotland

Singapore

Slovak Republic

Slovenia

South Africa

Spain

Sweden

Trinidad and Tobago

United States

Make Straightforward Inferences

Evaluating
Cause/
Effect

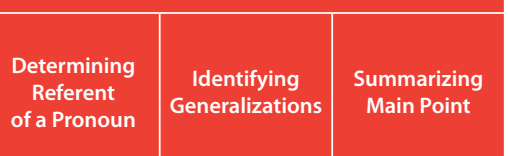

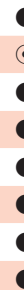

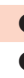

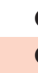

0

○

$\odot$

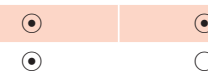

9

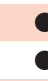

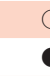

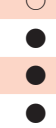

$\odot$

$\odot$
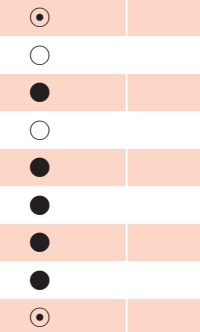

$\odot$
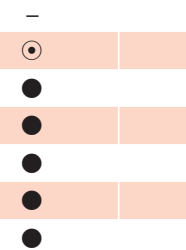

○
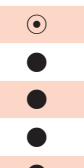

$\bullet$

$\odot$

$\odot$

-

\section{- Major Emphasis \\ • Some Emphasis \\ $\bigcirc$ Little or No Emphasis}

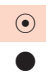

$\bullet$

sis

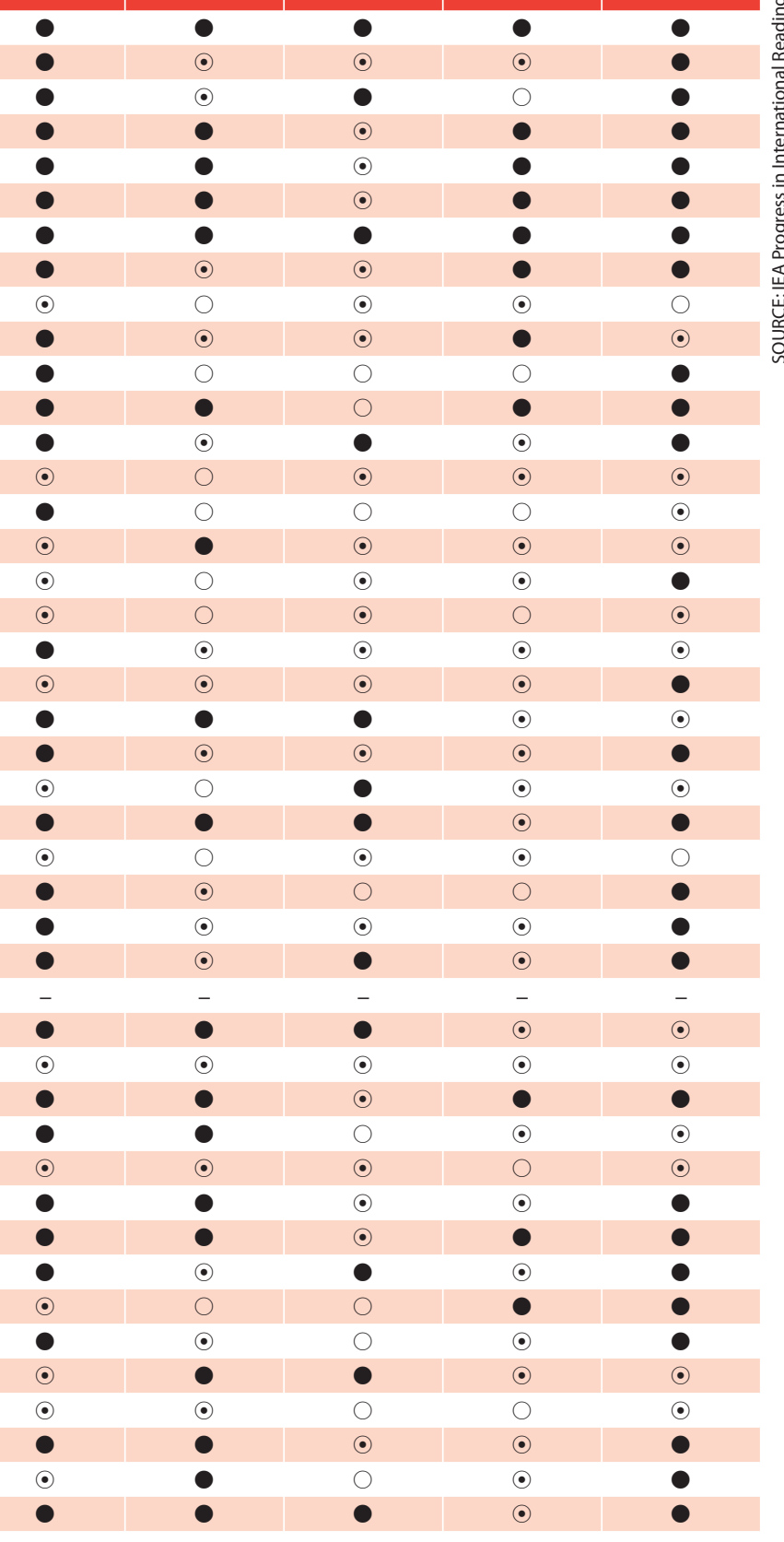

\begin{tabular}{c|c|}
$\begin{array}{c}\text { Searching for } \\
\text { Definitions } \\
\text { of Words } \\
\text { or Phrases }\end{array}$ & $\begin{array}{c}\text { Finding Topic } \\
\text { Sentence or } \\
\text { Main Idea }\end{array}$ \\
\end{tabular}
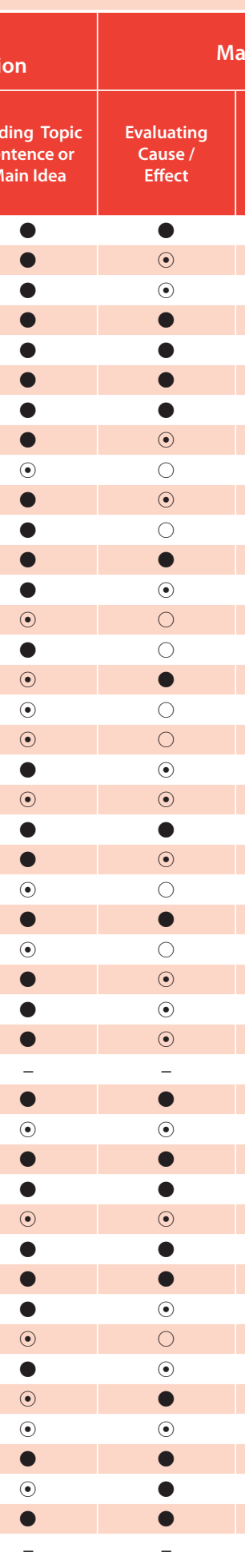

$\bullet$
$\bullet$
$\bullet$
$\odot$
$\bullet$
$\bullet$
$\bullet$
$\bullet$
$\odot$

○
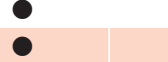

$\odot$

0

$\odot$
$\bullet$
$\bullet$
$\bullet$
$\bullet$
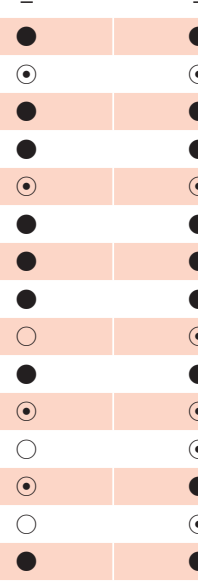
Exhibit 5.8 Emphasis on Processes of Comprehension in the Reading Curriculum (Continued)

PIRLS 2006

Countries

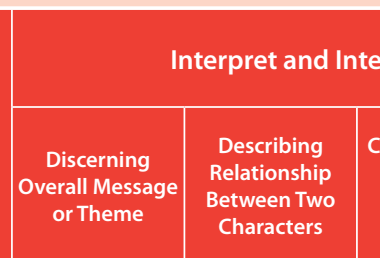

Interpret and Integrate Ideas and Information

Examine and Evaluate Content,

Language, and Textual Elements

Austria

Belgium (Flemish)

Belgium (French)

Bulgaria

Canada, Alberta

Canada, British Columbia

Canada, Nova Scotia

Canada, Ontario

Canada, Quebec

Chinese Taipei

Denmark

England

France

Georgia

Germany

Hong Kong SAR

Hungary

Iceland

Indonesia

Iran, Islamic Rep. of

Israel

Italy

Kuwait

Latvia

Lithuania

Luxembourg

Macedonia, Rep. of

Moldova, Rep. of

Morocco

Netherlands

New Zealand

Norway

Poland

Qatar

Romania

Russian Federation

Scotland

\begin{tabular}{|c|c|}
$\begin{array}{c}\text { Comparing and } \\
\text { Contrasting } \\
\text { Text } \\
\text { Information }\end{array}$ & $\begin{array}{c}\text { Inferring } \\
\text { Story's Mood } \\
\text { or Tone }\end{array}$ \\
\hline
\end{tabular}

\begin{tabular}{|c|} 
Interpreting \\
a Real-World \\
Application \\
of Text \\
Information
\end{tabular}

\begin{tabular}{|c|}
\hline Evaluating \\
Likelihood \\
that Events \\
Described Could \\
Really Happen
\end{tabular}

Judging

Completeness Determining

or Clarity of an Author's in Text
Singapore

Slovak Republic

Slovenia

South Africa

Spain

Sweden

Trinidad and Tobago

United States
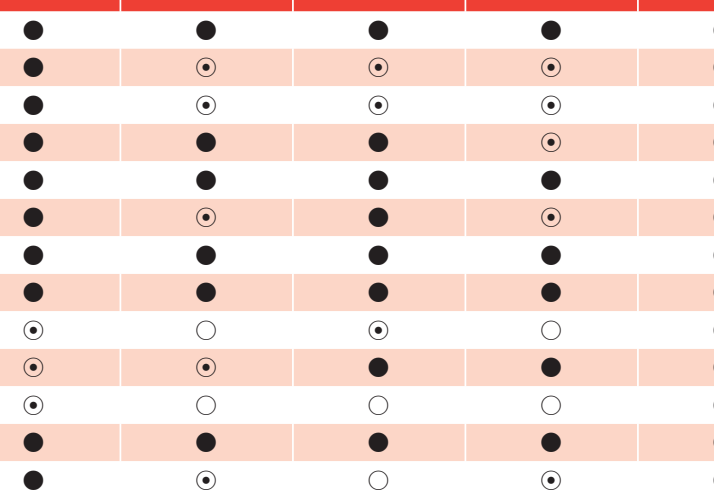

$\odot$

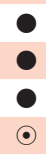

(7)
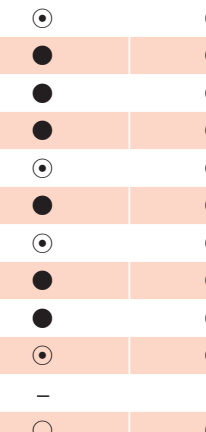

$\odot$

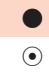

$\odot$
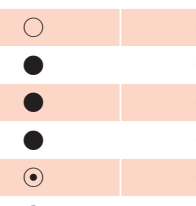

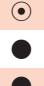

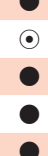

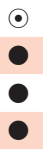
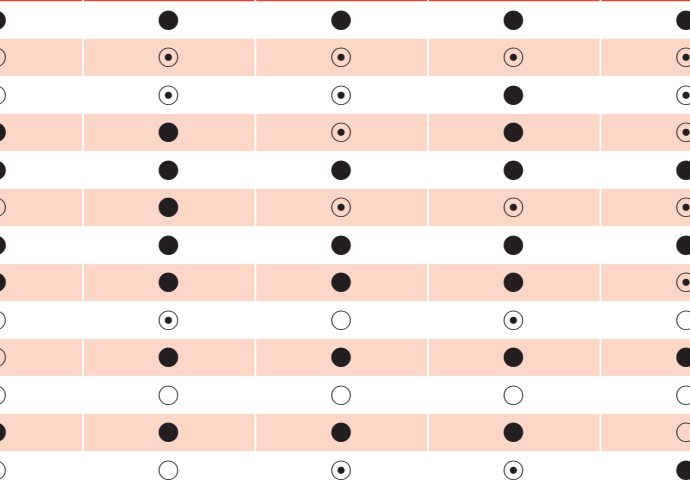

$$
\text { : }
$$
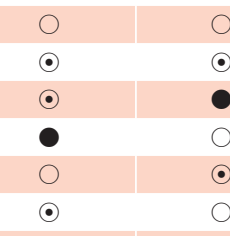
Exhibit 5.9 Schools' Grade-by-Grade Emphases on Reading Comprehension Skills or Strategies

\begin{tabular}{|c|c|c|c|c|c|c|}
\hline \multirow[b]{2}{*}{ Countries } & \multicolumn{6}{|c|}{ Grade by Which Skill or Strategy Is Emphasized for at Least $50 \%$ of the Students } \\
\hline & $\begin{array}{c}\text { Knowing } \\
\text { Letters } \\
\text { of the Alphabet }\end{array}$ & $\begin{array}{l}\text { Knowing } \\
\text { Letter-Sound } \\
\text { Relationships }\end{array}$ & Reading Words & $\begin{array}{l}\text { Reading } \\
\text { Isolated } \\
\text { Sentences }\end{array}$ & $\begin{array}{l}\text { Reading } \\
\text { Connected } \\
\text { Text }\end{array}$ & $\begin{array}{l}\text { Identifying } \\
\text { the Main } \\
\text { Idea of Text }\end{array}$ \\
\hline Austria & 1 & 1 & 1 & 1 & 1 & 2 \\
\hline Belgium (Flemish) & 1 & 1 & 1 & 1 & 1 & 2 \\
\hline Belgium (French) & 1 & 1 & 1 & 1 & 1 & 2 \\
\hline Bulgaria & 1 & 1 & 1 & 1 & 1 & 2 \\
\hline Canada, Alberta & 1 & 1 & 1 & 1 & 1 & 2 \\
\hline Canada, British Columbia & 1 & 1 & 1 & 1 & 1 & 2 \\
\hline Canada, Nova Scotia & 1 & 1 & 1 & 1 & 1 & 2 \\
\hline Canada, Ontario & 1 & 1 & 1 & 1 & 1 & 2 \\
\hline Canada, Quebec & 1 & 1 & 1 & 1 & 1 & 2 \\
\hline Chinese Taipei & 1 & 1 & 1 & 1 & 2 & 3 \\
\hline Denmark & 1 & 1 & 1 & 1 & 1 & 2 \\
\hline England & 1 & 1 & 1 & 1 & 1 & 1 \\
\hline France & 1 & 1 & 1 & 1 & 1 & 2 \\
\hline Georgia & 1 & 1 & 1 & 1 & 1 & 2 \\
\hline Germany & 1 & 1 & 1 & 1 & 1 & 2 \\
\hline Hong Kong SAR & - & - & - & - & - & - \\
\hline Hungary & 1 & 1 & 1 & 1 & 1 & 2 \\
\hline Iceland & 1 & 1 & 1 & 1 & 1 & 2 \\
\hline Indonesia & 1 & 1 & 1 & 1 & 2 & 3 \\
\hline Iran, Islamic Rep. of & 1 & 1 & 1 & 1 & 2 & 3 \\
\hline Israel & 1 & 1 & 1 & 1 & 1 & 2 \\
\hline Italy & 1 & 1 & 1 & 1 & 1 & 2 \\
\hline Kuwait & - & - & - & - & - & - \\
\hline Latvia & 1 & 1 & 1 & 1 & 1 & 2 \\
\hline Lithuania & 1 & 1 & 1 & 1 & 1 & 2 \\
\hline${ }^{1}$ Luxembourg & - & - & - & - & - & - \\
\hline Macedonia, Rep. of & 1 & 1 & 1 & 1 & 1 & 2 \\
\hline Moldova, Rep. of & 1 & 1 & 1 & 1 & 1 & 2 \\
\hline Morocco & 1 & 1 & 1 & 1 & 2 & 3 \\
\hline Netherlands & 1 & 1 & 1 & 1 & 1 & 2 \\
\hline New Zealand & 1 & 1 & 1 & 1 & 1 & 1 \\
\hline Norway & 1 & 1 & 1 & 2 & 2 & 3 \\
\hline Poland & 1 & 1 & 1 & 2 & 2 & 2 \\
\hline Qatar & 1 & 1 & 1 & 1 & 2 & 3 \\
\hline Romania & 1 & 1 & 1 & 1 & 1 & 2 \\
\hline Russian Federation & 1 & 1 & 1 & 1 & 1 & 2 \\
\hline Scotland & 1 & 1 & 1 & 1 & 1 & 1 \\
\hline Singapore & 1 & 1 & 1 & 1 & 1 & 2 \\
\hline Slovak Republic & 1 & 1 & 1 & 1 & 1 & 2 \\
\hline Slovenia & 1 & 1 & 2 & 2 & 2 & 2 \\
\hline South Africa & 1 & 1 & 1 & 2 & 2 & 3 \\
\hline Spain & 1 & 1 & 1 & 1 & 1 & 2 \\
\hline Sweden & 1 & 1 & 1 & 1 & 1 & 2 \\
\hline Trinidad and Tobago & 1 & 1 & 1 & 1 & 2 & 2 \\
\hline United States & 1 & 1 & 1 & 1 & 1 & 2 \\
\hline International Avg. & 1 & 1 & 1 & 1 & 1 & 2 \\
\hline
\end{tabular}

$\mathrm{N}=$ Not by Grade 4

Background data provided by schools.

An " $r$ " indicates data are available for $70-84 \%$ of the students. An " $\mathrm{s}$ " indicates data are available for $50-69 \%$ of the students. An " $\mathrm{x}$ " indicates data are available for less than $50 \%$ of the students.

A dash (-) indicates comparable data are not available.

1 Primary schools in Luxembourg do not have principals. 


\begin{tabular}{|c|c|c|c|c|c|c|}
\hline $\begin{array}{l}\text { Schools } \\
\text { or Strat }\end{array}$ & $\begin{array}{l}\text { Grade-by- } \\
\text { gies (Conti }\end{array}$ & $\begin{array}{l}\text { ide Emp } \\
\text { ed) }\end{array}$ & es on $\mathrm{Re}$ & g Comp & ension Skill & \\
\hline \multirow[b]{2}{*}{ Countries } & \multicolumn{6}{|c|}{ Grade by Which Skill or Strategy Is Emphasized for at Least $50 \%$ of the Students } \\
\hline & $\begin{array}{l}\text { Explaining or } \\
\text { Supporting } \\
\text { Understanding } \\
\text { of Text }\end{array}$ & $\begin{array}{l}\text { Comparing } \\
\text { Text with } \\
\text { Personal } \\
\text { Experience }\end{array}$ & $\begin{array}{l}\text { Making } \\
\text { Predictions } \\
\text { About What } \\
\text { Will Happen } \\
\text { Next in Text }\end{array}$ & $\begin{array}{l}\text { Comparing } \\
\text { Different } \\
\text { Texts }\end{array}$ & $\begin{array}{c}\text { Making } \\
\text { Generalizations } \\
\text { and Inferences } \\
\text { Based on Text }\end{array}$ & $\begin{array}{l}\text { Describing Style } \\
\text { and Structure } \\
\text { of Text }\end{array}$ \\
\hline Austria & 2 & 2 & 3 & 3 & 3 & 4 \\
\hline Belgium (Flemish) & 2 & 3 & 2 & 3 & 4 & 4 \\
\hline Belgium (French) & 2 & 2 & 2 & 3 & 3 & 4 \\
\hline Bulgaria & 2 & 3 & 3 & 3 & 3 & 4 \\
\hline Canada, Alberta & 2 & 1 & 1 & 2 & 2 & 3 \\
\hline Canada, British Columbia & 2 & 2 & 1 & 3 & 2 & 4 \\
\hline Canada, Nova Scotia & 2 & 2 & 1 & 2 & 2 & 3 \\
\hline Canada, Ontario & 2 & 1 & 1 & 2 & 2 & 3 \\
\hline Canada, Quebec & 2 & 2 & 2 & 3 & 3 & 4 \\
\hline Chinese Taipei & 3 & 3 & 4 & 4 & 4 & $\mathrm{~N}$ \\
\hline Denmark & 2 & 2 & 3 & 3 & 4 & 4 \\
\hline England & 1 & 1 & 1 & 2 & 2 & 2 \\
\hline France & 3 & 3 & 3 & 3 & 4 & 4 \\
\hline Georgia & 2 & 3 & 3 & 3 & 3 & 4 \\
\hline Germany & 2 & 2 & 2 & 3 & 3 & 4 \\
\hline Hong Kong SAR & - & - & - & - & - & - \\
\hline Hungary & 2 & 2 & 3 & 3 & 3 & 4 \\
\hline Iceland & 2 & 3 & 3 & 4 & 4 & N \\
\hline Indonesia & 3 & 4 & 4 & 4 & 4 & 4 \\
\hline Iran, Islamic Rep. of & 3 & 4 & 4 & 4 & 4 & 4 \\
\hline Israel & 2 & 2 & 2 & 3 & 3 & 3 \\
\hline Italy & 2 & 2 & 3 & 3 & 3 & 4 \\
\hline Kuwait & - & - & - & - & - & - \\
\hline Latvia & 1 & 1 & 2 & 2 & 2 & 3 \\
\hline Lithuania & 2 & 2 & 3 & 3 & 3 & 4 \\
\hline${ }^{1}$ Luxembourg & - & - & - & - & - & - \\
\hline Macedonia, Rep. of & 2 & 3 & 3 & 3 & 3 & 4 \\
\hline Moldova, Rep. of & 2 & 2 & 3 & 3 & 3 & 4 \\
\hline Morocco & 4 & 4 & $\mathrm{~N}$ & $\mathrm{~N}$ & $\mathrm{~N}$ & $\mathrm{~N}$ \\
\hline Netherlands & 2 & 2 & 2 & 3 & 4 & 4 \\
\hline New Zealand & 1 & 1 & 1 & 2 & 2 & 3 \\
\hline Norway & 3 & 3 & 3 & 4 & 4 & $\mathrm{~N}$ \\
\hline Poland & 2 & 2 & 3 & 3 & 3 & 4 \\
\hline Qatar & 3 & 3 & 4 & 4 & 4 & 4 \\
\hline Romania & 2 & 2 & 3 & 3 & 3 & 4 \\
\hline Russian Federation & 2 & 2 & 2 & 2 & 2 & 3 \\
\hline Scotland & 2 & 2 & 2 & 2 & 3 & 3 \\
\hline Singapore & 2 & 2 & 1 & 3 & 3 & 4 \\
\hline Slovak Republic & 2 & 2 & 3 & 3 & 3 & 4 \\
\hline Slovenia & 3 & 2 & 3 & 3 & 3 & 4 \\
\hline South Africa & 4 & 4 & 4 & 4 & 4 & $\mathrm{~N}$ \\
\hline Spain & 2 & 2 & 2 & 3 & 3 & 4 \\
\hline Sweden & 2 & 2 & 2 & 3 & 3 & 4 \\
\hline Trinidad and Tobago & 2 & 2 & 2 & 4 & 3 & 4 \\
\hline United States & 2 & 2 & 1 & 2 & 2 & 3 \\
\hline International Avg. & 2 & 2 & 3 & 3 & 3 & 4 \\
\hline
\end{tabular}




\section{How Much Instructional Time Is Devoted to Reading?}

Exhibit 5.10 shows the participants' reports of the number of hours specified for instruction per week overall, and the percent of the total that is designated for language and reading instruction. This is considered the intended time for instruction, which is typically established at the national level by the country's Ministry of Education, and, in some cases, represents minimum requirements. In addition, the exhibit shows principals' reports of the actual or implemented instructional time spent in schools overall, as well as teachers' reports of the percent of classroom instructional time spent separately for language and reading.

Four fifths of the participants specified a weekly number of hours for instruction across subject areas. On average across these participants, the total intended instructional time was 22 hours per week. Typically, the total instructional time implemented in the classroom matched closely, or exceeded the intended instructional time. Countries where principals reported at least 30 hours of instructional time per week included Italy, Indonesia, and the United States.

Not all participants had official policies about the percentage of the total amount of time to be devoted to language or reading instruction. The percentage of total instructional time devoted to language ranged from 16 to 50 percent. For the few countries specifying instructional time for reading, it ranged from 10 percent in Trinidad and Tobago to 60 percent in Indonesia. There is some overlap across reporting categories, because in several countries reading was included as part of language instruction or was taught across the curriculum.

Teachers' reports of the percentage of total instructional time spent on language and reading indicate that more time was spent on language instruction than reading. On average internationally, 30 percent of total instructional time was spent on language and 20 percent on reading. Interestingly, the percent of time spent on reading instruction in the classroom often was less than the intended percent of total instructional time specified at the national level. 
Exhibit 5.11 presents teachers' reports of the number of hours weekly spent teaching reading, either formally or integrated as part of reading for other subjects. For each category of hours-more than 6, more than 3 up to and including 6 , and up to and including 3 -the exhibit presents the percentage of students receiving that amount of weekly reading instruction in 2006, together with average reading achievement for those students. In addition, for countries that participated in PIRLS 2001, the exhibit presents the difference in the percent of students from 2001 and indicates if the difference was statistically significant. Countries are ordered according to the highest percentage of students receiving more than 6 hours of reading instruction each week.

Internationally on average, about one fourth of the students were taught reading for more than 6 hours per week. In the United States, approximately two thirds of students were taught reading for more than 6 hours weekly. The percentage of students taught reading for more than 6 hours a week increased from 2001 in Hungary, Moldova, Bulgaria, France, Macedonia, and the Netherlands. Countries where two thirds or more of students were taught reading for 3 hours or less per week included Slovenia, South Africa, Indonesia, England, Germany, Hong Kong SAR, Austria, and Chinese Taipei. In Iran and Latvia, the percentage of students taught reading for 3 hours or less increased since 2001 by 38 and 30 percentage points, respectively. The PIRLS 2006 results show little, if any, relationship between amount of instructional time and reading achievement. This is a frequent research finding, because there are many complex factors involved. For example, additional instruction often is provided to low-achieving students for remediation, and also, instructional time is not always spent in effective, productive ways.

As shown in Exhibit 5.12, on average internationally 77 percent of students were in classrooms where teachers reported explicitly spending time on formal reading instruction. More than 90 percent of students in Belgium (Flemish), Hungary, Luxembourg, Moldova, the Netherlands, New Zealand, the Russian Federation, Slovak Republic, Trinidad and Tobago, 
and the United States received formal reading instruction each week. In comparison, teachers reported that more than half the students in Germany, Poland, and Slovenia did not receive any formal reading instruction. On average internationally, teachers spent 2.5 hours weekly on formal reading instruction. Teachers in Kuwait, Moldova, Qatar, and United States averaged more than 4 hours per week, whereas those in Chinese Taipei averaged only 1 hour per week. However, principals in Chinese Taipei did report that most students entered school with beginning literacy skills (see Exhibit 5.4).

As shown in Exhibit 5.13, teachers reported that more than half the fourth-grade students received daily instruction in reading. On average internationally, another one third were taught reading 3 to 4 days each week. Countries where one fifth or more of the students were taught reading fewer than 3 days a week included Belgium (Flemish), Chinese Taipei, Georgia, Hong Kong SAR, Singapore, Slovenia, and South Africa.

Teachers' time spent on instruction is only a portion of the time spent with students in the classroom. Exhibit 5.14 contains teachers' reports of the percentage of time spent on instructional activities as well as other classroom duties. About four fifths of the time spent in the classroom was dedicated to teaching the class as a whole or working with individuals or small groups of students. On average, the remaining time was shared equally among administrative or other duties and disciplinary responsibilities. Participating entities where teachers spent 10 percent or more of their in-class time maintaining discipline included the Canadian province of Quebec, Iceland, Indonesia, Iran, Kuwait, South Africa, and Trinidad and Tobago. 
Exhibit 5.10 Intended and Implemented Weekly Instructional Time for Language and Reading (Formal and Integrated)

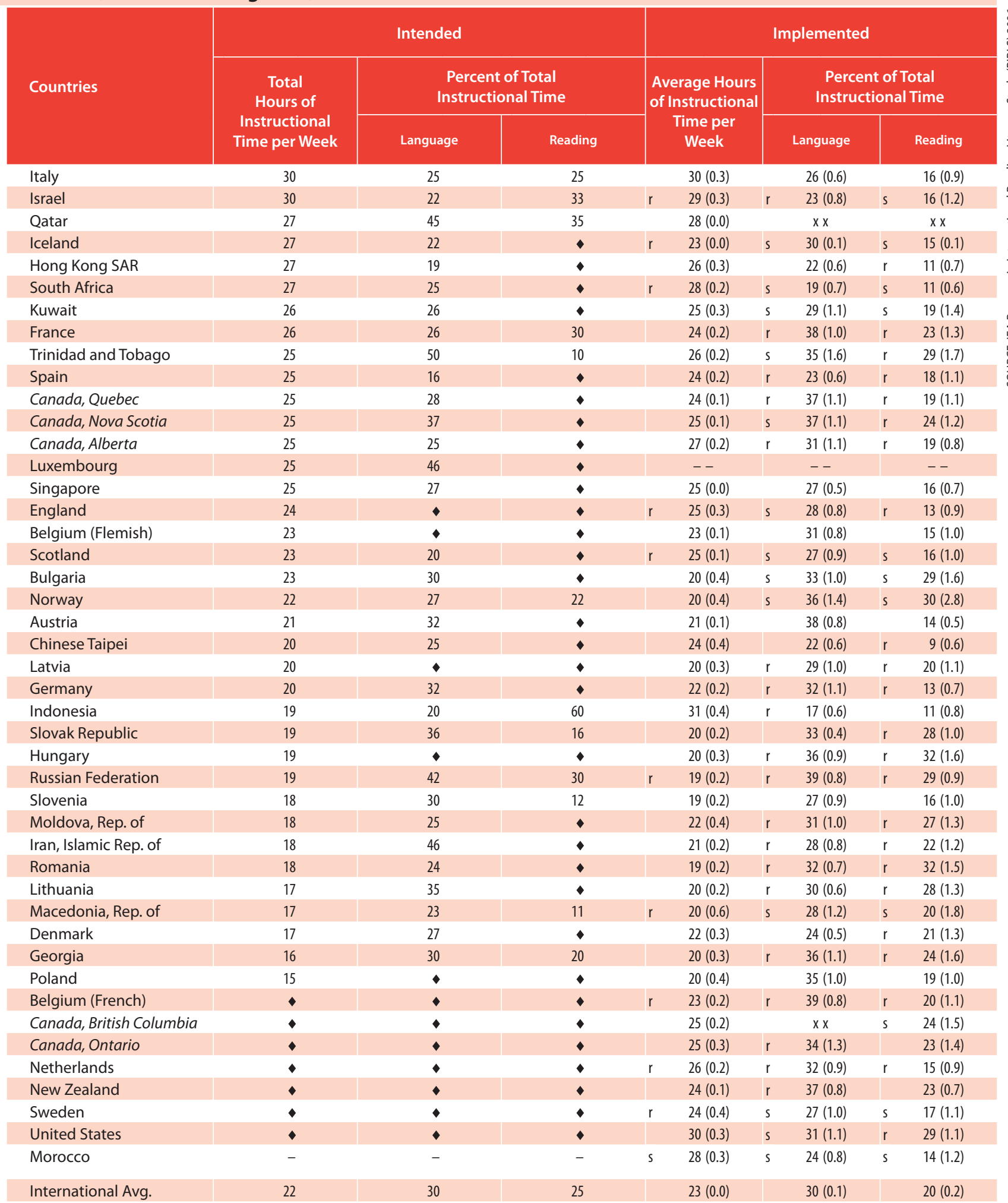

National Research Coordinators provided the intended instructional time per week and the proportion of time intended for language and reading.

Total hours of implemented instruction per week is based on principals' reports of the number of hours spent on instruction per day multiplied by the number of days per week the school is open for instruction. The percents of total instructional time for language and reading are based on teachers' reports of time spent weekly on language and reading instruction, respectively, divided by the principals' reports of total instructional time per week.

() Standard errors appear in parentheses. Because results are rounded to the nearest whole number, some totals may appear inconsistent.
An " $r$ " indicates data are available for $70-84 \%$ of the students. An "s" indicates data are available for $50-69 \%$ of the students. An " $x$ " indicates data are available for less than $50 \%$ of the students.

A dash (-) indicates comparable data are not available.

A black diamond $(\bullet)$ indicates instructional time is not specified.

NOTE: The International Average does not include the results from the Canadian provinces. 
Exhibit 5.11 Number of Hours Reading Is Taught Weekly (Formal and Integrated) with Trends

PIRLS 2006

Countries

\begin{tabular}{|c|c|c|}
\hline \multicolumn{3}{|c|}{ More than 6} \\
\hline $\begin{array}{c}2006 \\
\text { Percent } \\
\text { of Students }\end{array}$ & $\begin{array}{c}\text { Average } \\
\text { Achievement }\end{array}$ & $\begin{array}{c}\text { Difference } \\
\text { in Percent } \\
\text { from 2001 }\end{array}$ \\
\hline
\end{tabular}

United States

$68(3.4)$

$538(4.1)$

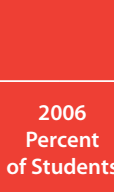

More than 3 Up to

and Including 6

Hungary

$56(4.5)$

Romania

$54(4.1)$

$551(4.1)$

$3(6.0)$

$22(3.0)$

\begin{tabular}{c|c} 
Average \\
Achievement
\end{tabular}

Difference
in Percent
from 2001

Up to and Including 3

Moldova, Rep. of

$50(3.8)$

Bulgaria

$46(4.4)$

$546(5.4)$

2006

Percent from 2001

of Students

Average

Difference

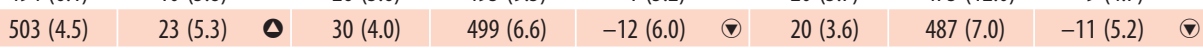

\begin{tabular}{l|l} 
Trinidad and Tobago & $46(4.6)$ \\
\hline
\end{tabular}

Norway

$44(4.5)$

$551(6.4) \quad 15(5.6) \quad$ ○ $33(4.2)$

\begin{tabular}{l|l|l}
$428(8.7)$ & $\Delta \diamond$ & $32(4.3)$
\end{tabular}

Canada, Nova Scotia

$498(3.9)$

$32(4.3)$

\begin{tabular}{l|l|l}
$545(3.1)$ & $\diamond \diamond$ & $34(3.7)$
\end{tabular}

$34(3.7)$

Columbia $r \quad 40(4.0)$

Canada, Ontario $39(5.1)$

$551(5.4) \quad \Delta \diamond$

$557(4.4) \quad 4(7.1)$

Lithuania

$39(3.3)$

$537(3.0)$

$4(7.1)$
$-7(5.5)$

$34(5.0)$

Qata

$345(2.4)$

$\Delta 0$

Slovak Republic 37 (3.1)

Georgia

$33(3.9)$

Canada, Alberta

$30(3.4)$

$534(4.2)$

$\Delta 0 \quad-31(0.2)$

$31(0.2)$

$546(5.4)-6(5.2)$

$10(2.7)$

$540(8.2)$

from 2001

$29(2.7)$

$467(6.0)$

$1(4.8)$

$43(3.6)$

$553(3.1) \quad \Delta \diamond \quad 40(3.4)$

$40(3.4)$

$543(9.6)$

$449(8.8)$

$\diamond 0$

$-4(5.9)$

$538(4.3)$

$561(4.9)$

$552(3.9)--12(6.8)$

$538(3.3)--12(6.8)$

$538(3.3)$

$-2(5.2)$

$346(2.9)$

0
$-9(5.5)$

$475(6.3)$

$-9(5.5)$

$562(4.8)$

$\diamond \diamond$

$\Delta \diamond$

$1(5.4)$

\begin{tabular}{rrr|r|}
$526(4.3)$ & $2(4.9)$ & & $56(3.0)$ \\
$523(4.6)$ & $18(4.7)$ & 0 & $48(4.1)$
\end{tabular}

$537(3.1)$

$8(6.2)$

\begin{tabular}{l|l}
\hline Russian Federation & $28(3.2)$ \\
\hline
\end{tabular}

\begin{tabular}{l|l|l|}
$563(6.9)$ & $-1(4.7)$ & $60(2.9)$
\end{tabular}

$519(3.1)$

$32(4.5) \quad 331(9.1)$

$567(4.6)$

$1(4.5)$

$329(9.2) \quad \diamond \diamond$

$\diamond \diamond$

$423(7.5) \quad-43(5.4) \quad \odot \quad 35(3.8)$

$425(8.0)$

$5(5.8)$

$\begin{array}{ll}\text { Iran, Islamic Rep. of } & 27(3.2) \\ \text { Denmark } & 25(3.8)\end{array}$

$545(5.2) \quad \Delta \diamond \quad 31(3.7)$

548 (4.1)

$\diamond \diamond$

\begin{tabular}{ll|l}
$504(6.9)$ & $\diamond \diamond$ & $36(3.2)$ \\
$545(8.2)$ & $-4(6.1)$ & $24(4.7)$
\end{tabular}

$501(4.2)$

$\diamond \diamond$

Israel

$24(4.5)$

$545(8.2)$

$-4(6.1)$

$24(3.6)$

$532(13.9)$

$-11(6.4)$

$546(4.3) \quad-7(5.5) \quad 27(4.3)$

$507(5.8) \quad \Delta \diamond$

$\Delta \diamond$

535 (4.2)

$-23(6.6)$

Latvia

$22(3.7)$

$533(5.7)$

$-14(5.9)$

Canada, Quebec

$20(3.8)$

$43(4.9)$

$529(3.9)$

$2(6.8)$
$-6(5.2)$

$30(3.7)$

$550(4.9)$

Sweden

$18(3.1)$

$551(6.7) \quad 0(4.3)$

$27(4.0)$

Singapore

$17(2.1)$

$\begin{array}{llll}558(8.1) & -8(3.9) & \bigcirc & 19(2.1)\end{array}$

$550(4.0) \quad-5(5.0)$

$429(13.4) \quad 17(3.0) \quad 0$

Macedonia, Rep. of

$17(3.0)$

$33(4.0)$

$561(7.0)$

Luxembourg

$16(0.1)$

Slovenia

$14(2.4)$

$556(2.1) \quad \Delta 0$

$39(0.2)$

$432(11.0) \quad 33(4.0)$

$33(4.0)$

\begin{tabular}{|l|l|l|}
\hline $512(5.4)$ & $1(3.9)$ & $16(2.2)$ \\
\hline
\end{tabular}

$556(1.5) \quad \Delta \diamond$

$323(20.7) \quad-13(5.7) \quad$ ๑ $31(4.3)$

$520(4.4) \quad-10(4.4)$

Morocco $\quad$ r $\quad 14(3.0)$

Poland

Netherlands

$13(2.7)$

$509(8.1) \quad \diamond \diamond$

Belgium (Flemish)

$13(3.3)$

$00 \quad 40(3.9)$

$318(15.1)$

$9(6.0)$

$547(2.4) \quad-4(6.4)$

$26(4.0) \quad 546(4.6) \quad \nabla 0$

\begin{tabular}{l|l|l|}
$542(5.9)$ & $\Delta \diamond$ & $26(4.0)$
\end{tabular}

$546(4.6)$

$\diamond \diamond$

$30(0.4)$

$530(4.2) \quad 3(6.8)$

$(6.8)$

$533(10.1) \quad-2(4.6)$

$30(0.4)$

$509(2.2)$

$-12(0.5)-45(4.9)$

$\begin{array}{lll}\text { Iceland } & r & 10(0.2) \\ \text { South Africa } & r & 10(1.9)\end{array}$

$511(3.4)$

$1(0.3)$

$288(16.1)$

$-12(0.5)$

$23(3.0) \quad 404(9.1) \quad \Delta \diamond$

\begin{tabular}{l|l|l}
$397(11.0)$ & $\Delta \diamond$ & $23(3.0)$
\end{tabular}

England

$8(2.3)$

$522(12.2) \quad-5(3.9)$

$25(4.0)$

$544(6.7)$

$-9(5.9)$

$547(6.4) \quad-5(3.0)$

$23(2.8)$

$544(6.7)$

$-9(5.9)$

$72(2.7)$

$69(2.7)$

Germany

$5(2.0)$

$567(6.7) \quad 2(2.6)$

$22(3.6)$

$537(5.6)$

$-5(4.3)$
$1(5.4)$

$67(4.3)$

Austria $4(1.3)$

Chinese Taipei

$531(8.5) \quad \diamond$

$541(12.9) \quad \diamond 0$

$28(3.4)$

$572(6.4)$

00

$537(6.2) \quad \diamond \diamond$

$\diamond \diamond$

$31(0.6) \quad 501(1.1)$

\begin{tabular}{|l|l|l|}
\hline $44(0.6)$ & $500(0.9)$ \\
\hline
\end{tabular}

International Avg.

$25(0.5)$

$500(1.3)$

rcent in 2006 significantly higher

- Percent in 2006 significantly lower

Background data provided by teachers.

() Standard errors appear in parentheses. Because results are rounded to the nearest whole number, some totals may appear inconsistent.

An " $r$ " indicates data are available for $70-84 \%$ of the students. An " $s$ " indicates data are available for $50-69 \%$ of the students. An " $x$ " indicates data are available for less than $50 \%$ of the students.
A diamond $(\diamond)$ indicates the country did not participate in the 2001 assessment.

NOTE: The International Average does not include the results from the Canadian provinces. Trend Note: The primary education systems of the Russian Federation and Slovenia underwent structural changes. Data for Canada, Ontario include only public schools. 
Exhibit 5.12 Teachers Spend Time for Formal Reading Instruction

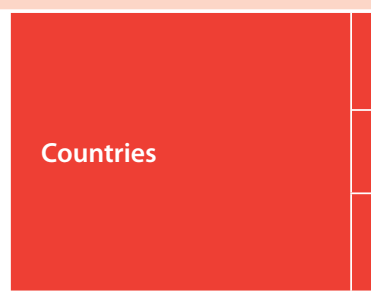

Austria

Belgium (Flemish)

Belgium (French)

Bulgaria

Canada, Alberta

Canada, British Columbia

Canada, Nova Scotia

Canada, Ontario

Canada, Quebec

Chinese Taipei

Denmark

England

France

Georgia

Germany

Hong Kong SAR

Hungary

Iceland

Indonesia

Iran, Islamic Rep. of

Israel

Italy

Kuwait

Latvia

Lithuania

Luxembourg

Macedonia, Rep. of

Moldova, Rep. of

Morocco

Netherlands

New Zealand

Norway

Poland

Qatar

Romania

Russian Federation

Scotland

Singapore

Slovak Republic

Slovenia

South Africa

Spain

Sweden

Trinidad and Tobago

United States

International Avg.

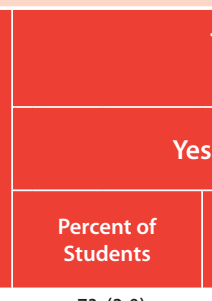

$73(2.9)$

$91(2.0)$

65 (4.1)

$89(2.9)$

$69(3.9)$

$83(3.0)$

$87(2.7)$

$82(4.1)$

$73(4.0)$

$75(3.6)$

$61(3.7)$

80 (3.6)

$88(2.5)$

$84(3.2)$

$43(3.5)$

$73(4.0)$

$99(0.8)$

r $\quad 75(0.3)$

$73(3.7)$

$84(2.7)$

$69(3.9)$

$79(3.2)$

$\quad 79(3.2)$
$\mathrm{r} \quad 71(3.6)$

$61(3.4)$

$68(3.4)$

$100(0.0)$

$64(3.6)$

$93(1.6)$

$88(2.7)$

$96(1.6)$

$96(0.8)$

68 (3.7)

$49(4.2)$

s

$79(0.3)$

$77(3.3)$

$94(1.6)$

$87(3.2)$

$62(3.1)$

$94(1.5)$

42 (3.2)

$70(2.8)$

$68(3.6)$

$79(3.5)$

$92(2.1)$

$94(2.0)$

$77(0.5)$
Time Explicitly for Formal

Reading Instruction

Yes

|

Averag
Achiever

$539(2.7)$

$548(2.1)$

$498(3.7)$

$547(4.4)$

$561(2.7)$

557 (3.3)

543 (2.4)

$555(3.0)$

$531(3.2)$

$536(2.4)$

$550(3.3)$

$542(3.7)$

$524(2.4)$

$472(3.7)$

$548(3.8)$

$567(2.7)$

551 (3.1)

$512(1.7)$

$400(5.4)$

$421(3.9)$

$506(6.2)$

$553(3.3)$

$333(5.8)$

$540(3.5)$

$537(2.0)$

$557(1.1)$

$432(6.5)$

498 (3.3)

$325(6.8)$

$546(1.7)$

$535(2.0)$

497 (3.4)

$515(3.2)$

$350(1.5)$

$487(5.8)$

566 (3.3)

$527(3.1)$

$554(3.4)$

$531(2.9)$

$521(3.6)$

$305(8.8)$

$516(3.4)$

$548(2.8)$

$436(5.5)$

539 (3.9)

$500(0.7)$
No

Percent of
Students

$27(2.9)$

$9(2.0)$

35 (4.1)

$11(2.9)$

$31(3.9)$

$17(3.0)$

13 (2.7)

$18(4.1)$

$27(4.0)$

25 (3.6)

$39(3.7)$

$20(3.6)$

$12(2.5)$

$16(3.2)$

57 (3.5)

$27(4.0)$

$1(0.8)$

$25(0.3)$

$25(0.3)$
$27(3.7)$

$16(2.7)$

$31(3.9)$

$21(3.2)$

$29(3.6)$

39 (3.4)

$32(3.4)$

$0(0.0)$

$36(3.6)$

$7(1.6)$

$12(2.7)$

4 (1.6)

$4(0.8)$

32 (3.7)

$51(4.2)$

$21(0.3)$

$23(3.3)$

$6(1.6)$

$13(3.2)$

$38(3.1)$

$6(1.5)$

58 (3.2)

$30(2.8)$

$32(3.6)$

21 (3.5)

$8(2.1)$

$6(2.0)$

$23(0.5)$
Average Achievement

$535(4.0)$

$1.6(0.06)$

\begin{tabular}{l|l}
$536(5.5)$ & $1.4(0.08)$
\end{tabular}

$507(4.7) \quad r \quad 1.8(0.11)$

\begin{tabular}{l|l}
$543(19.6)$ & $2.7(0.11)$ \\
\hline
\end{tabular}

$558(5.4) \quad 2.9(0.21)$

$562(7.7)$ s $3.1(0.19)$

$536(6.7) \quad r \quad 3.0(0.19)$

\begin{tabular}{l|l}
$557(7.0)$ & $3.1(0.27)$
\end{tabular}

$539(5.0) \quad r \quad 2.2(0.25)$

$535(3.4) \quad r \quad 1.0(0.06)$

$544(4.0) \quad s \quad 1.7(0.15)$

$540(11.2) \quad r \quad 1.8(0.08)$

$506(6.9) \quad r \quad 2.1(0.11)$

\begin{tabular}{ll|l}
$468(8.5)$ & $3.1(0.17)$
\end{tabular}

$549(2.3) \quad s \quad 1.4(0.07)$

\begin{tabular}{|l|l|}
\hline $555(5.1)$ & $1.7(0.14)$ \\
\hline
\end{tabular}

$\sim \quad 2.9(0.20)$

$502(2.7) \quad$ r $\quad 1.8(0.01)$

$419(7.2) \quad 2.6(0.23)$

$418(10.2) \quad 3.4(0.12)$

$519(11.0) \quad s \quad 1.8(0.12)$

\begin{tabular}{l|l}
$547(5.7)$ & $2.4(0.13)$
\end{tabular}

$324(9.0) \quad s \quad 4.4(0.32)$

$\begin{array}{llll}543(3.6) & r & 2.2(0.12)\end{array}$

$539(3.4) \quad r \quad 3.0(0.20)$

$\sim \quad 1.4(0.00)$

$465(8.5) \quad r \quad 2.5(0.19)$

$506(11.1) \quad 5.0(0.28)$

$295(14.1) \quad 2.2(0.06)$

$551(12.5) \quad$ r $\quad 1.5(0.05)$

$513(11.4) \quad 3.2(0.09)$

$\begin{array}{lll}501(3.6) & \text { r } & 2.7(0.20)\end{array}$

$523(3.6) \quad r \quad 2.6(0.20)$

$360(3.4)$ s $4.6(0.02)$

$489(9.2) \quad 3.2(0.21)$

\begin{tabular}{|l|l|}
\hline $540(17.8)$ & $3.1(0.05)$ \\
\hline
\end{tabular}

$525(8.9) \quad r \quad 2.5(0.17)$

\begin{tabular}{l|l}
$564(4.5)$ & $2.0(0.17)$
\end{tabular}

$532(9.7) \quad 3.0(0.03)$

$522(2.5) \quad$ r $\quad 1.9(0.19)$

$304(11.0) \quad r \quad 1.9(0.13)$

$504(4.7) \quad r \quad 2.0(0.15)$

$548(3.9) \quad r \quad 1.6(0.09)$

\begin{tabular}{l|r}
$427(16.8)$ & $3.7(0.21)$ \\
\hline &
\end{tabular}

$551(8.5) \quad$ r $4.8(0.19)$

$496(1.5)$

$496(1.5)$

$2.5(0.02)$
Background data provided by teachers.

() Standard errors appear in parentheses. Because results are rounded to the nearest whole number, some totals may appear inconsistent.

$\mathrm{An}$ " $\mathrm{r}$ " indicates data are available for $70-84 \%$ of the students. An " $s$ " indicates data are available for $50-69 \%$ of the students. An " $\mathrm{x}$ " indicates data are available for less than $50 \%$ of the students.
A tilde $(\sim)$ indicates insufficient data to report achievement.

NOTE: The International Average does not include the results from the Canadian provinces. 


\begin{tabular}{|c|c|c|c|c|c|c|c|}
\hline \multirow{2}{*}{ Countries } & & \multicolumn{2}{|c|}{ Every Day } & \multicolumn{2}{|c|}{ 3-4 Days a Week } & \multicolumn{2}{|c|}{$\begin{array}{c}\text { Fewer than } 3 \text { Days } \\
\text { a Week }\end{array}$} \\
\hline & & $\begin{array}{l}\text { Percent of } \\
\text { Students }\end{array}$ & $\begin{array}{c}\text { Average } \\
\text { Achievement }\end{array}$ & $\begin{array}{l}\text { Percent of } \\
\text { Students }\end{array}$ & $\begin{array}{c}\text { Average } \\
\text { Achievement }\end{array}$ & $\begin{array}{l}\text { Percent of } \\
\text { Students }\end{array}$ & $\begin{array}{c}\text { Average } \\
\text { Achievement }\end{array}$ \\
\hline Austria & & $58(3.6)$ & $538(2.7)$ & $35(3.5)$ & $539(3.5)$ & $7(1.8)$ & $532(7.4)$ \\
\hline Belgium (Flemish) & & $46(4.3)$ & $548(3.1)$ & $28(3.8)$ & $546(2.9)$ & $26(3.5)$ & $545(3.2)$ \\
\hline Belgium (French) & & $58(3.7)$ & $502(4.0)$ & $28(3.4)$ & $500(4.7)$ & $15(2.6)$ & $492(5.4)$ \\
\hline Bulgaria & & $66(3.6)$ & $555(5.5)$ & $32(3.5)$ & $532(7.1)$ & $2(1.0)$ & $\sim \sim$ \\
\hline Canada, Alberta & & $77(3.7)$ & $561(2.3)$ & $18(3.3)$ & $558(6.9)$ & $5(1.9)$ & $554(16.5)$ \\
\hline Canada, British Columbia & $r$ & $74(3.6)$ & $557(3.5)$ & $20(3.1)$ & $573(4.3)$ & $6(2.1)$ & $550(15.8)$ \\
\hline Canada, Nova Scotia & & $92(1.9)$ & $543(2.5)$ & $6(1.7)$ & $542(15.1)$ & $2(0.9)$ & $\sim \sim$ \\
\hline Canada, Ontario & & $80(4.0)$ & $554(2.8)$ & $16(3.4)$ & $561(9.1)$ & $5(2.3)$ & $544(6.7)$ \\
\hline Canada, Quebec & & $57(4.9)$ & $536(3.8)$ & $30(4.5)$ & $525(5.1)$ & $14(3.5)$ & $540(3.9)$ \\
\hline Chinese Taipei & & $19(3.2)$ & $536(4.8)$ & $27(3.8)$ & $539(4.3)$ & $54(4.2)$ & $535(2.6)$ \\
\hline Denmark & & $30(3.9)$ & $551(4.3)$ & $59(4.3)$ & $545(3.3)$ & $11(2.3)$ & $544(6.4)$ \\
\hline England & & $69(4.3)$ & $539(3.7)$ & $23(3.8)$ & $555(8.6)$ & $9(2.6)$ & $517(6.6)$ \\
\hline France & & $80(2.8)$ & $521(2.4)$ & $15(2.5)$ & $523(5.7)$ & $5(1.6)$ & $526(8.3)$ \\
\hline Georgia & & $43(4.7)$ & $470(4.9)$ & $35(4.0)$ & $472(5.4)$ & $22(3.2)$ & $469(7.7)$ \\
\hline Germany & & $57(3.4)$ & $547(2.7)$ & $25(2.9)$ & $545(4.0)$ & $17(2.9)$ & $554(3.9)$ \\
\hline Hong Kong SAR & & $24(3.7)$ & $568(6.0)$ & $39(3.9)$ & $565(4.1)$ & $36(4.1)$ & $561(4.1)$ \\
\hline Hungary & & $65(3.8)$ & $555(4.2)$ & $35(3.8)$ & $543(5.0)$ & $0(0.0)$ & $\sim \sim$ \\
\hline Iceland & & $40(0.4)$ & $510(2.1)$ & $41(0.4)$ & $510(1.8)$ & $19(0.3)$ & $510(3.1)$ \\
\hline Indonesia & & $48(4.4)$ & $406(5.8)$ & $38(4.6)$ & $403(7.0)$ & $14(2.7)$ & $405(10.4)$ \\
\hline Iran, Islamic Rep. of & & $41(3.8)$ & $420(6.5)$ & $47(3.8)$ & $423(5.0)$ & $12(2.5)$ & $418(9.6)$ \\
\hline Israel & & $49(4.3)$ & $511(7.3)$ & $38(4.7)$ & $508(10.5)$ & $14(2.8)$ & $527(11.4)$ \\
\hline Italy & & $48(3.7)$ & $548(5.0)$ & $38(3.6)$ & $556(3.7)$ & $14(2.6)$ & $553(6.6)$ \\
\hline Kuwait & $r$ & $92(2.3)$ & $331(5.0)$ & $4(1.6)$ & $318(26.5)$ & $4(1.9)$ & $344(22.9)$ \\
\hline Latvia & & $54(4.1)$ & $544(3.1)$ & $42(3.9)$ & $536(3.8)$ & $3(1.3)$ & $553(11.1)$ \\
\hline Lithuania & & $65(3.2)$ & $535(2.1)$ & $29(3.3)$ & $544(3.5)$ & $5(1.6)$ & $539(5.6)$ \\
\hline Luxembourg & & $29(0.2)$ & $557(1.9)$ & $53(0.2)$ & $558(1.3)$ & $18(0.1)$ & $555(2.3)$ \\
\hline Macedonia, Rep. of & & $64(3.9)$ & $455(6.2)$ & $24(3.4)$ & $433(10.5)$ & $12(2.7)$ & $396(19.5)$ \\
\hline Moldova, Rep. of & & $58(4.2)$ & $502(4.1)$ & $39(4.2)$ & $493(5.3)$ & $3(1.3)$ & $512(5.7)$ \\
\hline Morocco & & $39(4.1)$ & $336(10.8)$ & $60(4.1)$ & $313(7.2)$ & $1(0.0)$ & $\sim \sim$ \\
\hline Netherlands & & $62(4.6)$ & $544(2.3)$ & $36(4.6)$ & $550(2.5)$ & $2(1.0)$ & $\sim \sim$ \\
\hline New Zealand & & $58(3.4)$ & $532(2.8)$ & $40(3.4)$ & $536(3.6)$ & $2(0.8)$ & $\sim \sim$ \\
\hline Norway & & $73(4.3)$ & $497(2.5)$ & $24(4.1)$ & $503(5.6)$ & $3(1.7)$ & $489(12.9)$ \\
\hline Poland & & $64(3.8)$ & $519(3.0)$ & $28(3.5)$ & $522(4.3)$ & $8(2.5)$ & $507(5.7)$ \\
\hline Qatar & $s$ & $56(0.3)$ & $350(2.0)$ & $30(0.3)$ & $351(2.7)$ & $14(0.2)$ & $364(4.1)$ \\
\hline Romania & & $80(3.3)$ & $492(5.2)$ & $17(3.2)$ & $475(10.4)$ & $3(1.5)$ & $506(22.7)$ \\
\hline Russian Federation & & $87(2.6)$ & $565(3.3)$ & $12(2.6)$ & $560(12.5)$ & $0(0.3)$ & $\sim \sim$ \\
\hline Scotland & & $44(3.7)$ & $528(4.4)$ & $50(3.7)$ & $527(4.2)$ & $5(2.2)$ & $519(12.9)$ \\
\hline Singapore & & $36(3.0)$ & $550(5.6)$ & $41(3.2)$ & $562(4.2)$ & $22(2.4)$ & $566(5.9)$ \\
\hline Slovak Republic & & $46(3.5)$ & $537(3.6)$ & $51(3.6)$ & $528(3.9)$ & $3(1.3)$ & $493(28.4)$ \\
\hline Slovenia & & $51(3.4)$ & $523(2.9)$ & $29(3.1)$ & $520(3.8)$ & $20(2.5)$ & $519(3.8)$ \\
\hline South Africa & & $30(2.6)$ & $328(13.3)$ & $39(3.4)$ & $288(12.1)$ & $31(2.8)$ & $294(9.5)$ \\
\hline Spain & & $57(4.0)$ & $510(4.6)$ & $26(3.5)$ & $512(5.0)$ & $17(3.2)$ & $520(6.3)$ \\
\hline Sweden & & $65(3.4)$ & $549(2.6)$ & $26(3.5)$ & $546(4.4)$ & $9(2.2)$ & $553(7.3)$ \\
\hline Trinidad and Tobago & & $82(3.6)$ & $435(6.3)$ & $18(3.6)$ & $452(12.0)$ & $0(0.0)$ & $\sim \sim$ \\
\hline United States & & $93(1.6)$ & $538(3.6)$ & $7(1.5)$ & $540(11.5)$ & $0(0.0)$ & $\sim \sim$ \\
\hline International Avg. & & $56(0.6)$ & $502(0.8)$ & $33(0.5)$ & $499(1.2)$ & $12(0.3)$ & $497(1.9)$ \\
\hline
\end{tabular}

Background data provided by teachers.

() Standard errors appear in parentheses. Because results are rounded to the nearest whole number, some totals may appear inconsistent.

An " $r$ " indicates data are available for $70-84 \%$ of the students. An " $s$ " indicates data are available for $50-69 \%$ of the students. An " $\mathrm{x}$ " indicates data are available for less than $50 \%$ of the students.
A tilde $(\sim)$ indicates insufficient data to report achievement.

NOTE: The International Average does not include the results from the Canadian provinces. 


\begin{tabular}{|c|c|c|c|c|c|c|c|}
\hline \multirow[b]{2}{*}{ Countries } & \multicolumn{7}{|c|}{ Percent of Time Spent in Class with Students } \\
\hline & & $\begin{array}{l}\text { eaching the Class } \\
\text { as a Whole }\end{array}$ & & $\begin{array}{l}\text { Working with } \\
\text { dividual Students } \\
\text { or Small Groups }\end{array}$ & $\begin{array}{l}\text { Administrative } \\
\text { Duties }\end{array}$ & $\begin{array}{l}\text { Maintaining } \\
\text { Discipline }\end{array}$ & Other Duties \\
\hline Austria & & $58(1.2)$ & & $25(1.1)$ & $5(0.2)$ & $7(0.4)$ & $5(0.3)$ \\
\hline Belgium (Flemish) & & $55(1.6)$ & & $26(1.0)$ & $5(0.3)$ & $7(0.4)$ & $7(0.7)$ \\
\hline Belgium (French) & & $61(1.2)$ & & $22(1.1)$ & $5(0.3)$ & $7(0.4)$ & $5(0.4)$ \\
\hline Bulgaria & & $59(1.2)$ & & $24(0.9)$ & $5(0.3)$ & $7(0.5)$ & $6(0.5)$ \\
\hline Canada, Alberta & & $55(1.6)$ & & $26(1.2)$ & $6(0.3)$ & $8(0.6)$ & $5(0.4)$ \\
\hline Canada, British Columbia & r & $56(1.8)$ & r & $25(1.5)$ & $5(0.3)$ & $8(0.6)$ & $5(0.5)$ \\
\hline Canada, Nova Scotia & & $55(1.4)$ & & $27(1.1)$ & $5(0.3)$ & $8(0.6)$ & $5(0.4)$ \\
\hline Canada, Ontario & & $49(1.3)$ & & $28(1.3)$ & $7(0.4)$ & $9(0.7)$ & $7(0.4)$ \\
\hline Canada, Quebec & & $50(1.8)$ & & $24(1.5)$ & $6(0.4)$ & $12(0.9)$ & $8(0.7)$ \\
\hline Chinese Taipei & & $72(1.0)$ & & $13(0.6)$ & $4(0.3)$ & $6(0.3)$ & $5(0.4)$ \\
\hline Denmark & & $46(1.4)$ & & $33(1.5)$ & $6(0.3)$ & $9(0.7)$ & $5(0.5)$ \\
\hline England & r & $53(1.2)$ & r & $30(1.3)$ & $5(0.3)$ & $6(0.5)$ & $6(0.5)$ \\
\hline France & & $65(1.2)$ & & $20(0.9)$ & $3(0.2)$ & $7(0.6)$ & $4(0.5)$ \\
\hline Georgia & & $60(1.6)$ & & $18(0.9)$ & $8(0.4)$ & $7(0.4)$ & $7(0.4)$ \\
\hline Germany & & $57(1.5)$ & & $24(1.3)$ & $6(0.3)$ & $6(0.4)$ & $6(0.4)$ \\
\hline Hong Kong SAR & & $64(1.8)$ & & $7(0.5)$ & $12(1.3)$ & $6(0.5)$ & $11(0.9)$ \\
\hline Hungary & & $56(1.3)$ & & $32(1.3)$ & $3(0.2)$ & $5(0.4)$ & $4(0.3)$ \\
\hline Iceland & $r$ & $42(0.2)$ & r & $28(0.2)$ & $7(0.0)$ & $13(0.1)$ & $11(0.1)$ \\
\hline Indonesia & & $55(1.4)$ & & $18(0.8)$ & $8(0.5)$ & $10(0.5)$ & $8(0.4)$ \\
\hline Iran, Islamic Rep. of & & $52(1.4)$ & & $20(0.9)$ & $7(0.3)$ & $11(0.5)$ & $10(0.5)$ \\
\hline Israel & $r$ & $57(1.6)$ & r & $24(1.4)$ & $6(0.3)$ & $8(0.6)$ & $5(0.4)$ \\
\hline Italy & & $68(1.1)$ & & $17(0.8)$ & $3(0.2)$ & $7(0.5)$ & $5(0.5)$ \\
\hline Kuwait & $s$ & $55(2.5)$ & s & $18(1.4)$ & $6(0.8)$ & $11(1.1)$ & $10(1.0)$ \\
\hline Latvia & & $60(1.4)$ & & $22(0.9)$ & $6(0.3)$ & $7(0.5)$ & $5(0.4)$ \\
\hline Lithuania & & $63(1.3)$ & & $26(1.1)$ & $3(0.2)$ & $4(0.3)$ & $4(0.4)$ \\
\hline Luxembourg & & $62(0.1)$ & & $21(0.1)$ & $4(0.0)$ & $5(0.0)$ & $8(0.0)$ \\
\hline Macedonia, Rep. of & r & $55(1.5)$ & r & $25(1.3)$ & $6(0.3)$ & $8(0.5)$ & $6(0.4)$ \\
\hline Moldova, Rep. of & & $49(1.6)$ & & $27(1.2)$ & $8(0.7)$ & $7(0.6)$ & $9(0.8)$ \\
\hline Morocco & $s$ & $60(1.8)$ & s & $22(1.5)$ & $5(0.6)$ & $7(0.7)$ & $6(0.8)$ \\
\hline Netherlands & r & $49(1.6)$ & r & $29(1.5)$ & $6(0.4)$ & $6(0.5)$ & $9(0.6)$ \\
\hline New Zealand & & $39(0.9)$ & & $41(1.0)$ & $7(0.4)$ & $8(0.3)$ & $6(0.3)$ \\
\hline Norway & & $54(1.6)$ & & $30(1.6)$ & $4(0.4)$ & $7(0.6)$ & $5(0.4)$ \\
\hline Poland & & $64(1.0)$ & & $21(0.8)$ & $4(0.2)$ & $5(0.4)$ & $5(0.3)$ \\
\hline Qatar & & $x x$ & & $x x$ & $x x$ & $\mathrm{xx}$ & $x x$ \\
\hline Romania & & $55(1.3)$ & & $30(1.1)$ & $4(0.3)$ & $5(0.3)$ & $5(0.4)$ \\
\hline Russian Federation & & $64(1.1)$ & & $24(1.0)$ & $4(0.2)$ & $4(0.2)$ & $4(0.4)$ \\
\hline Scotland & r & $44(1.4)$ & r & $40(1.2)$ & $5(0.3)$ & $7(0.6)$ & $5(0.5)$ \\
\hline Singapore & & $60(0.8)$ & & $16(0.5)$ & $9(0.4)$ & $9(0.3)$ & $6(0.3)$ \\
\hline Slovak Republic & & $64(1.0)$ & & $21(0.8)$ & $5(0.2)$ & $6(0.4)$ & $4(0.3)$ \\
\hline Slovenia & & $67(1.1)$ & & $20(0.9)$ & $5(0.4)$ & $6(0.3)$ & $2(0.3)$ \\
\hline South Africa & & $48(1.1)$ & & $23(0.9)$ & $10(0.5)$ & $11(0.5)$ & $8(0.4)$ \\
\hline Spain & & $61(1.4)$ & & $20(1.2)$ & $6(0.7)$ & $8(0.5)$ & $5(0.5)$ \\
\hline Sweden & & $59(1.9)$ & & $24(1.4)$ & $4(0.3)$ & $8(0.6)$ & $5(0.5)$ \\
\hline Trinidad and Tobago & & $63(1.1)$ & & $16(0.8)$ & $5(0.2)$ & $10(0.6)$ & $6(0.3)$ \\
\hline United States & & $55(1.6)$ & & $25(1.4)$ & $7(0.4)$ & $9(0.5)$ & $5(0.3)$ \\
\hline International Avg. & & $57(0.2)$ & & $24(0.2)$ & $6(0.1)$ & $7(0.1)$ & $6(0.1)$ \\
\hline
\end{tabular}

Background data provided by teachers.

( ) Standard errors appear in parentheses. Because results are rounded to the nearest whole number, some totals may appear inconsistent.
An " $r$ " indicates data are available for $70-84 \%$ of the students. An "s" indicates data are available for $50-69 \%$ of the students. An " $x$ " indicates data are available for less than $50 \%$ of the students.

NOTE: The International Average does not include the results from the Canadian provinces. 


\section{How Are Classes Organized for Reading Instruction?}

Teachers employ a variety of approaches in organizing their classrooms for reading instruction. Often, the decision of how to organize students is based on the students' level of ability and class size. Exhibit 5.15 presents teachers' reports of how they grouped their fourth-grade students for reading instruction or reading activities. The most popular approach, used for 78 percent of the students, on average internationally, was to employ a variety of strategies for organizing students. The next most common approach was to teach reading as a whole-class activity. On average internationally, about one third of students were taught reading in classes that were always or almost always organized as an entire class. More than two thirds of students in Bulgaria, Kuwait, and Romania were taught reading as a whole-class activity.

In general, teachers reported that creating either same-ability or mixedability groups of students to teach reading was relatively rare. However, in New Zealand and Scotland, more than half the students were in classes where same-ability groups were always or almost always created for reading instruction. In Iran and Qatar, more than one third of students were always or almost always taught reading instruction in mixed-ability groups. Similarly, in most countries, teachers reported always or almost always using individualized instruction for only small percentages of students. However, individualized instruction for reading was used often for more than one third of the students in Kuwait and Morocco, and almost half the students in Qatar.

Exhibit 5.16 presents trends in class size for reading and language instruction. Countries are ordered by average class size in PIRLS 2006 from smallest to largest. The difference is shown from PIRLS 2001 for trend participants, with an indication as to whether or not the difference was statistically significant. Average class sizes in 2006 and 2001 are displayed graphically. The exhibit also shows the percentages of fourth-grade students in classes from 1-20 students, 21-30 students, and 31 or more students. For each category of class size, the percentage of students in 2006 is shown together 
Exhibit 5.15 Organization of Students for Reading Instruction
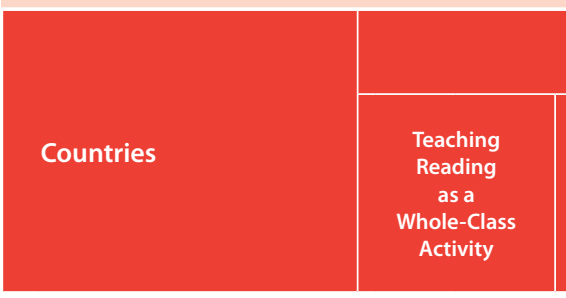

Percentage of Students Whose Teachers Reported Always or Almost Always

$$
\text { Austria }
$$

$16(2.3)$

Belgium (Flemish)

Belgium (French)

Bulgaria

Canada, Alberta

$7(2.0)$
$37(3.4)$

$75(3.6)$

Canada, Nova Scotia

Canada, Ontario

Canada, Quebec

Chinese Taipei

Denmark

England

France

Georgia

Germany

Hong Kong SAR

$24(3.8)$

Hungary

Iceland

Indonesia

Iran, Islamic Rep. of

Israel

Italy

$$
\text { Kuwait }
$$

Latvia

Lithuania

Luxembourg

Macedonia, Rep. of

Moldova, Rep. of

\section{$10(2.4)$}

$18(3.6)$

$35(4.5)$
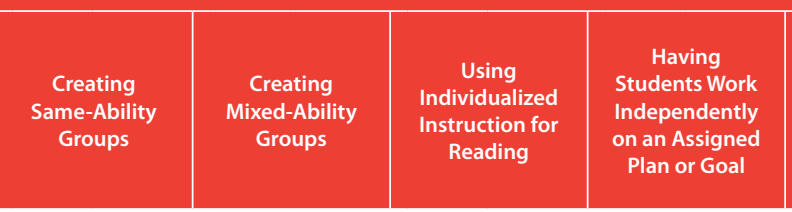

Having Having
Students Work Independently Using a Variety

on a Goal
They Choose

They Choose Themselves

4th Grade

Morocco

$50(4.1)$

$1(0.6)$

$1(0.6)$

$2(0.9)$

$1(0.7)$

$7(1.8)$

$3(1.1)$

$10(2.1)$

$1(0.6)$

$2(0.6)$

$4(1.7)$

$6(1.8)$

$6(1.9)$

$6(1.7)$

$3(1.2)$

$5(1.2)$

$5(1.5)$
$1(0.5)$

$5(1.2)$
$7(2.1)$

$16(2.7)$

$4(1.3)$

$4(1.7)$

$6(1.9)$

5 (1.4)

$3(1.5)$

$1(0.7)$

$11(2.3)$

$6(2.0)$

$4(1.2)$

$4(1.2)$
$27(4.5)$

$25(3.5)$

4 (1.1)

63 (3.9)

5 (1.9)

$3(1.0)$

$5(1.1)$

$2(0.7)$

$16(2.9)$

$0(0.3)$

$0(0.4)$

$4(1.5)$

$5(2.1)$

$2(0.7)$

$22(3.0)$

$0(0.0)$

5 (1.7)

$7(2.2)$

$17(0.3)$
$43(3.8)$

$6(0.3)$

$6(0.3)$
$6(2.0)$

$59(3.4)$

$1(0.7)$

$21(3.0)$

$4(1.7)$

63 (3.4)

$0(0.3)$
$11(2.8)$

$69(3.7)$
$48(4.2)$

Netherlands

New Zealand

Norway

Poland

$35(3.0)$

$2(0.9)$

$2(0.9)$

$2(0.9)$
$1(0.0)$

$31(0.2)$

$49(4.4)$

$9(2.7)$
$12(2.9)$

$62(3.9)$
$61(3.9)$

$8(2.0)$

8 (2.4)

$8(2.4)$
$6(1.3)$

$2(0.6)$

$61(2.7)$

$12(2.2)$

Qatar

$38(4.3)$

$6(1.7)$

$1(0.7)$
$6(2.0)$

$5(1.6)$

$4(0.1)$
$8(2.1)$

$38(3.6)$

$9(2.6)$

$10(2.2)$

$10(2.2)$

$8(2.3)$

$5(1.5)$

$4(0.1)$

$7(2.3)$

$15(3.2)$

$15(3.2)$

$1(0.7)$

$1(0.7)$
$4(1.7)$

4 (1.6)

$5(2.2)$
$0(0.2)$

$5(2.1)$

$5(2.1)$
$3(1.2)$

$5(2.0)$

$2(1.2)$
$14(2.2)$

$4(1.7)$

$2(1.3)$

$14(2.6)$
$6(2.2)$

$3(0.7)$

$2(1.0) \quad 5(1.4)$

$32(4.2)$

$2(0.8)$

$2(1.1)$

$16(2.9)$

$10(2.3)$

$16(3.2)$

$32(0.3)$
$27(4.0)$

$5(1.9)$

$3(1.4)$

$3(1.4)$
$22(0.3)$

$10(2.3)$

$1(1.0)$

$36(4.3)$

$21(3.3)$

$14(3.2)$

$3(1.3)$

$30(3.0)$

$35(3.8)$

$30(3.0)$
$18(3.3)$

$2(0.9)$

$1(0.5)$
$3(1.3)$

$1(0.9)$
$1(0.7)$

$1(0.7)$
$0(0.0)$
$0(0.2)$

4 (1.6)

4 (1.4)

3 (1.2)

$3(1.2)$
$9(2.2)$

$0(0.2)$

$10(2.5)$

$1(0.5)$

$0(0.0)$

$0(0.2)$
$20(3.5)$

$5(1.5)$
$3(1.6)$

$1(0.9)$

$7(0.3)$

$18(2.8)$
$4(1.2)$

$3(1.5)$

$5(1.7)$

$8(2.5)$ of Organizational

Approache

\section{Romania}

s $51(0.3)$

$3(1.3)$

$72(3.4)$

s $\quad 34(0.3)$

$6(1.9)$

$4(1.5)$

Scotland

Singapore

Slovak Republic

$63(4.1)$

$4(1.5)$

Slovenia

South Africa

Spain

Sweden

Trinidad and Tobago

United States

$6(2.7)$
$29(2.6)$

$54(4.5)$

$0(0.0)$

$7(0.1)$

$16(3.1)$

$29(4.4)$

$33(3.8)$

$3(1.3)$

$35(4.2)$

$14(3.1)$

$5(1.4)$
$8(1.5)$

$6(1.4)$

$2(1.3)$

$4(2.2)$

$5(2.0)$
$12(2.3)$

$5(1.4) \quad 12(2.4)$

$1(1.0)$

$3(1.2)$

$3(1.2)$

$14(3.1)$

$19(3.6)$

$74(3.1)$

$80(2.6)$

$55(3.4)$

$94(1.9)$

$70(3.4)$

$65(4.1)$

$67(3.7)$

$72(3.9)$

$68(3.8)$

$82(3.0)$

$62(4.1)$

66 (4.1)

$66(3.2)$

$66(3.2)$
$92(2.0)$

$68(3.5)$

$55(4.3)$

International Avg.

$29(2.6)$
$40(3.6)$

$1(0.7)$

$43(0.3)$

$4(1.5)$

$49(0.3)$

$23(0.2)$

\begin{tabular}{l|l}
$3(0.9)$ & $11(2.5)$
\end{tabular}

$\begin{array}{lll}3(0.9) & & 11(2.5) \\ 7(0.6) & r & 5(2.3)\end{array}$

$27(3.8)$

$6(1.8)$

$7(1.4) \quad 1(0.7)$

$3(1.1) \quad 14(2.3)$

$9(3.1)$
$5(1.2)$

$15(2.7)$

$3(1.4)$

$90(2.2)$
$79(0.3)$

$11(1.9) \quad 1(0.5)$

\begin{tabular}{l|l|l|}
$1(0.5)$ & $8(1.6)$ & $3(0.9)$ \\
\hline
\end{tabular}


Exhibit 5.16 Class Size for Reading and Language Instruction with Trends*

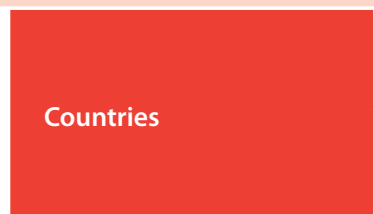

Luxembourg

Romania

Bulgaria

Slovenia

Italy

Norway

Lithuania

Denmark

Latvia

Iceland

Belgium (Flemish)

Austria

Belgium (French)

Georgia

Poland

Trinidad and Tobago

Germany

Hungary

Russian Federation

Moldova, Rep. of

Slovak Republic

Spain

United States

Sweden

Canada, Alberta

Canada, Nova Scotia

France

Netherlands

Macedonia, Rep. of

Kuwait

Iran, Islamic Rep. of

Canada, Quebec

Canada, British Columbia

Canada, Ontario

Scotland

Qatar

New Zealand

England

Israel

Morocco

Indonesia

Chinese Taipei

Hong Kong SAR

Singapore

South Africa

International Avg.

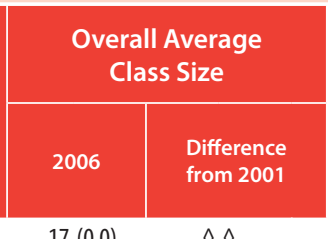

$17(0.0)$

$\diamond \diamond$

$19(0.4)$

$20(0.3)$

$20(0.3)$

$20(0.3)$

$20(0.5)$

$20(0.3)$

$20(0.3)$

$20(0.4)$

$21(0.0)$

$21(0.3)$

$21(0.3)$

$21(0.4)$

$21(0.5)$

$22(0.4)$

$22(0.4)$

$22(0.3)$

$22(0.4)$

$22(0.3)$

$22(0.4)$

$22(0.3)$

$22(0.3)$

$23(0.4)$

$23(0.4)$

$23(0.3)$

$24(0.3)$

$24(0.2)$

$24(0.4)$

$25(0.4)$

$25(0.3)$

$25(0.5)$

$25(0.3)$

$26(0.4)$

$26(0.5)$

$26(0.4)$

$26(0.0)$

$27(0.3)$

$27(0.3)$

$30(0.4)$

$30(0.6)$

$31(0.8)$

$32(0.3)$

$35(0.5)$

$38(0.2)$

$42(0.8)$

$24(0.1)$

Average in 2006 significantly higher 0

Average in 2006 significantly lower $\mathbb{}$

Background data provided by teachers.

* Results are for entire classes, which included some multi-grade classrooms. To take the possibility of multi-grade classrooms into consideration, PIRLS also asked teachers to report the number of 4 th-grade students. There was little difference in most countries

$0.6)$

(0.7)

$-1(0.6)$

00

$-1(0.6)$

00

$0(0.3)$

00

$-3(0.7)$

$0(0.4)$

$\Delta 0$

0

$-1(0.4)$

$-2(0.5)$

$-1(0.6)$

$-1(1.0)$

00

$0(0.6)$

$1(0.4)$

$\Delta \diamond$
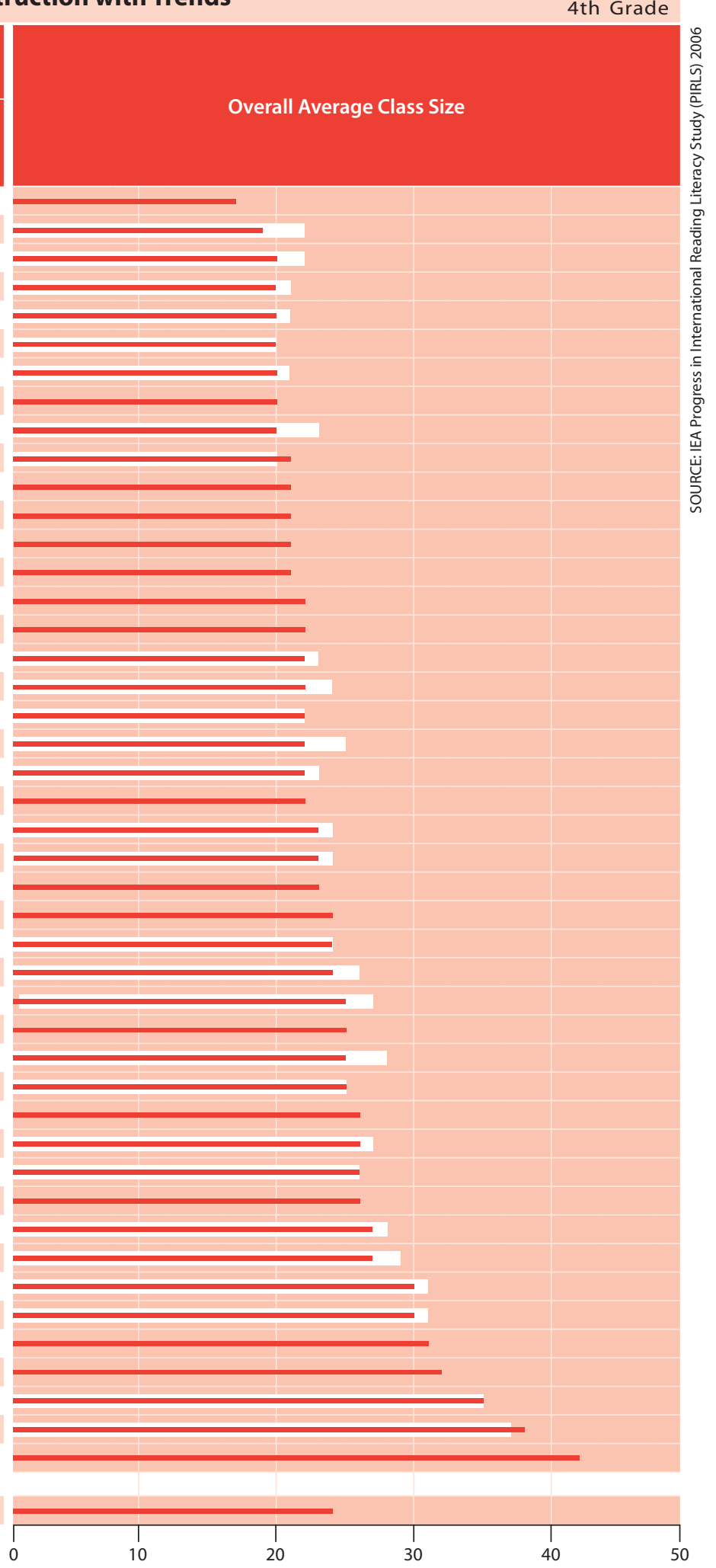

2001 Average

2006 Average

between the size of the entire classes and just the 4 th graders. Across countries, the average number of fourth graders in a class and the average class size were both 24

( ) Standard errors appear in parentheses. Because results are rounded to the nearest whole number, some totals may appear inconsistent. 
Exhibit 5.16 Class Size for Reading and Language Instruction with Trends (Continued)

\begin{tabular}{|c|c|c|c|c|c|c|c|c|c|c|c|c|}
\hline \multirow{2}{*}{ Countries } & \multicolumn{4}{|c|}{ 1-20 Students } & \multicolumn{4}{|c|}{ 21-30 Students } & \multicolumn{4}{|c|}{31 or More Students } \\
\hline & $\begin{array}{c}2006 \\
\text { Percent } \\
\text { of Students }\end{array}$ & $\begin{array}{c}\text { Average } \\
\text { Achievement }\end{array}$ & $\begin{array}{l}\text { Difference } \\
\text { in Percent } \\
\text { from } 2001\end{array}$ & & $\begin{array}{l}2006 \\
\text { Percent } \\
\text { of Students }\end{array}$ & $\begin{array}{c}\text { Average } \\
\text { Achievement }\end{array}$ & $\begin{array}{l}\text { Difference } \\
\text { in Percent } \\
\text { from } 2001\end{array}$ & & $\begin{array}{c}2006 \\
\text { Percent } \\
\text { of Students }\end{array}$ & $\begin{array}{c}\text { Average } \\
\text { Achievement }\end{array}$ & $\begin{array}{l}\text { Difference } \\
\text { in Percent } \\
\text { from } 2001\end{array}$ & \\
\hline Luxembourg & $92(0.1)$ & $557(1.2)$ & $\Delta \diamond$ & & $6(0.1)$ & $554(2.8)$ & $\Delta \Delta$ & & $1(0.0)$ & $\sim \sim$ & $\Delta \Delta$ & \\
\hline Romania & $57(3.3)$ & $474(6.7)$ & $23(4.3)$ & 0 & $41(3.4)$ & $508(7.0)$ & $-13(5.0)$ & ( & $2(1.1)$ & $\sim \sim$ & $-9(3.0)$ & $(\nabla$ \\
\hline Bulgaria & $59(4.0)$ & $541(5.5)$ & $22(5.1)$ & 0 & $41(4.0)$ & $556(6.7)$ & $-21(5.2)$ & ( & $0(0.0)$ & $\sim \sim$ & $-1(1.2)$ & \\
\hline Slovenia & $57(3.4)$ & $519(2.5)$ & $14(5.1)$ & 0 & $42(3.4)$ & $525(3.1)$ & $-16(5.1)$ & ( & $1(0.9)$ & $\sim \sim$ & $1(0.9)$ & \\
\hline Italy & $53(3.8)$ & $548(4.0)$ & $3(5.2)$ & & $47(3.8)$ & $555(3.7)$ & $-3(5.2)$ & & $0(0.0)$ & $\sim \sim$ & $0(0.4)$ & \\
\hline Norway & $56(4.4)$ & $496(4.1)$ & $6(6.1)$ & & $42(4.1)$ & $500(3.3)$ & $-7(6.0)$ & & $2(1.5)$ & $\sim \sim$ & $2(1.7)$ & \\
\hline Lithuania & $40(3.0)$ & $526(2.7)$ & $6(4.3)$ & & $60(3.0)$ & $545(2.0)$ & $-4(4.4)$ & & $0(0.0)$ & $\sim \sim$ & $-1(1.0)$ & \\
\hline Denmark & $51(3.9)$ & $541(3.4)$ & $\Delta \Delta$ & & $49(4.0)$ & $553(3.5)$ & $\Delta \Delta$ & & $0(0.5)$ & $\sim \sim$ & $\Delta \Delta$ & \\
\hline Latvia & $51(3.0)$ & $531(3.6)$ & $19(4.4)$ & 0 & $45(3.0)$ & $550(2.8)$ & $-13(4.9)$ & $(\nabla$ & $4(1.5)$ & $564(11.8)$ & $-7(3.0)$ & $(\nabla$ \\
\hline Iceland & $48(0.4)$ & $509(1.9)$ & $-2(0.5)$ & ( & $49(0.4)$ & $511(1.8)$ & $-1(0.5)$ & & $3(0.1)$ & $504(10.6)$ & $3(0.1)$ & 0 \\
\hline Belgium (Flemish) & $47(3.9)$ & $546(2.7)$ & $\Delta \diamond$ & & $52(3.9)$ & $548(2.6)$ & $\Delta \diamond$ & & $0(0.3)$ & $\sim \sim$ & $\Delta \diamond$ & \\
\hline Austria & $42(3.5)$ & $542(3.2)$ & $\Delta \Delta$ & & $58(3.5)$ & $536(2.8)$ & $\Delta 0$ & & $0(0.0)$ & $\sim \sim$ & $\Delta \Delta$ & \\
\hline Belgium (French) & $39(3.8)$ & $496(5.5)$ & $\Delta \diamond$ & & $59(3.7)$ & $502(3.3)$ & $\Delta \Delta$ & & $2(1.3)$ & $\sim \sim$ & $\diamond \diamond$ & \\
\hline Georgia & $51(3.1)$ & $467(4.9)$ & $\Delta 0$ & & $29(3.3)$ & $464(4.1)$ & $\Delta \Delta$ & & $20(2.9)$ & $489(6.2)$ & $\Delta \Delta$ & \\
\hline Poland & $38(3.6)$ & $512(3.9)$ & $\Delta \diamond$ & & $60(3.7)$ & $524(3.2)$ & $\Delta \Delta$ & & $2(0.8)$ & $\sim \sim$ & $\Delta \Delta$ & \\
\hline Trinidad and Tobago & $41(3.1)$ & $407(9.8)$ & $\Delta \Delta$ & & $53(3.4)$ & $456(6.0)$ & $\Delta \Delta$ & & $7(2.0)$ & $485(14.4)$ & $\Delta \Delta$ & \\
\hline Germany & $33(2.8)$ & $541(4.1)$ & $2(4.1)$ & & $67(2.8)$ & $553(2.5)$ & $1(4.1)$ & & $0(0.1)$ & $\sim \sim$ & $-2(0.9)$ & $\uparrow$ \\
\hline Hungary & $38(3.6)$ & $529(5.6)$ & $12(4.8)$ & 0 & $58(3.8)$ & $563(3.5)$ & $-6(5.6)$ & & $4(1.7)$ & $568(5.7)$ & $-6(3.3)$ & \\
\hline Russian Federation & $35(2.3)$ & $542(4.9)$ & $-2(4.2)$ & & $62(2.5)$ & $577(4.0)$ & $7(4.5)$ & & $2(1.1)$ & $\sim \sim$ & $-5(2.2)$ & $(\nabla$ \\
\hline Moldova, Rep. of & $35(3.5)$ & $495(5.9)$ & $13(5.3)$ & 0 & $55(3.8)$ & $499(4.2)$ & $-5(6.2)$ & & $10(2.4)$ & $513(8.3)$ & $-8(4.1)$ & ( \\
\hline Slovak Republic & $34(2.9)$ & $520(5.4)$ & $3(4.3)$ & & $59(3.3)$ & $534(3.6)$ & $2(5.3)$ & & $7(1.8)$ & $553(3.9)$ & $-5(3.4)$ & \\
\hline Spain & $32(3.6)$ & $498(6.2)$ & $\Delta \Delta$ & & $68(3.6)$ & $519(2.8)$ & $\Delta \Delta$ & & $0(0.0)$ & $\sim \sim$ & $\Delta \Delta$ & \\
\hline United States & $26(3.4)$ & $535(6.4)$ & $3(5.4)$ & & $68(3.7)$ & $541(3.9)$ & $1(5.4)$ & & $5(1.6)$ & $540(10.7)$ & $-3(2.9)$ & \\
\hline Sweden & $33(3.5)$ & $542(3.8)$ & $8(4.5)$ & & $61(4.1)$ & $553(2.9)$ & $-5(5.2)$ & & $6(2.3)$ & $545(8.5)$ & $-2(3.3)$ & \\
\hline Canada, Alberta & $24(2.5)$ & $558(4.2)$ & $\Delta \diamond$ & & $74(2.7)$ & $561(3.0)$ & $\Delta \Delta$ & & $3(1.2)$ & $542(22.9)$ & $\Delta \Delta$ & \\
\hline Canada, Nova Scotia & $19(2.3)$ & $536(6.1)$ & $\Delta \diamond$ & & $79(2.5)$ & $544(2.5)$ & $\Delta 0$ & & $2(0.9)$ & $\sim \sim$ & $\Delta \Delta$ & \\
\hline France & $14(1.9)$ & $505(4.8)$ & $-3(3.1)$ & & $85(2.2)$ & $524(2.6)$ & $3(3.4)$ & & $1(1.0)$ & $\sim \sim$ & $0(1.4)$ & \\
\hline Netherlands & $18(3.3)$ & $527(4.1)$ & $4(4.2)$ & & $71(3.8)$ & $551(2.0)$ & $5(5.5)$ & & $11(2.6)$ & $551(5.1)$ & $-10(4.9)$ & ( \\
\hline Macedonia, Rep. of & $24(3.3)$ & $430(10.6)$ & $9(4.3)$ & 0 & $58(4.0)$ & $454(7.4)$ & $0(5.7)$ & & $17(3.0)$ & $427(14.8)$ & $-9(4.8)$ & \\
\hline Kuwait & $5(1.8)$ & $315(23.7)$ & $\Delta \Delta$ & & $91(2.5)$ & $331(4.4)$ & $\Delta \Delta$ & & $4(1.8)$ & $345(15.5)$ & $\Delta \Delta$ & \\
\hline Iran, Islamic Rep. of & $28(2.8)$ & $401(6.8)$ & $5(4.4)$ & & $44(3.7)$ & $422(5.3)$ & $5(6.0)$ & & $28(3.4)$ & $439(7.9)$ & $-10(5.0)$ & $\odot$ \\
\hline Canada, Quebec & $7(1.7)$ & $528(9.1)$ & $3(2.4)$ & & $91(2.1)$ & $535(3.1)$ & $-3(3.0)$ & & $2(1.3)$ & $\sim \sim$ & $0(1.8)$ & \\
\hline Canada, British Columbia $\quad r$ & $10(2.4)$ & $570(6.9)$ & $\Delta \diamond$ & & $83(3.2)$ & $558(3.3)$ & $\Delta \diamond$ & & $7(2.3)$ & $556(11.8)$ & $\Delta \diamond$ & \\
\hline Canada, Ontario & $6(1.9)$ & $539(6.1)$ & $2(2.3)$ & & $85(3.4)$ & $554(3.2)$ & $-3(4.5)$ & & $9(3.1)$ & $563(7.2)$ & $1(4.1)$ & \\
\hline Scotland & $11(2.6)$ & $525(10.7)$ & $0(3.9)$ & & $71(4.3)$ & $528(3.3)$ & $0(6.0)$ & & $18(3.8)$ & $524(7.2)$ & $0(5.2)$ & \\
\hline Qatar & $12(0.2)$ & $352(5.3)$ & $\Delta \Delta$ & & $68(0.3)$ & $354(1.5)$ & $\Delta \Delta$ & & $20(0.2)$ & $344(3.1)$ & $\Delta \Delta$ & \\
\hline New Zealand & $10(1.2)$ & $508(8.4)$ & $1(2.6)$ & & $71(2.5)$ & $535(2.6)$ & $2(4.7)$ & & $19(2.4)$ & $539(4.4)$ & $-3(4.2)$ & \\
\hline England & $8(1.6)$ & $566(17.1)$ & $-1(2.8)$ & & $71(3.8)$ & $536(3.7)$ & $23(5.8)$ & 0 & $21(3.5)$ & $548(6.3)$ & $-22(5.8)$ & ( ) \\
\hline Israel & $6(1.9)$ & $497(23.2)$ & $2(2.4)$ & & $49(4.1)$ & $527(6.4)$ & $7(6.1)$ & & $45(4.0)$ & $499(8.3)$ & $-9(5.9)$ & \\
\hline Morocco & $15(2.8)$ & $318(15.8)$ & $-1(4.2)$ & & $35(3.9)$ & $338(10.5)$ & $4(6.5)$ & & $50(3.8)$ & $318(7.7)$ & $-3(6.7)$ & \\
\hline Indonesia & $19(2.3)$ & $391(8.1)$ & $\Delta \diamond$ & & $32(3.8)$ & $408(7.2)$ & $\Delta \diamond$ & & $49(3.7)$ & $408(6.3)$ & $\Delta \Delta$ & \\
\hline Chinese Taipei & $3(0.7)$ & $504(6.8)$ & $\Delta \Delta$ & & $22(2.9)$ & $530(4.6)$ & $\Delta \Delta$ & & $75(2.8)$ & $539(2.4)$ & $\Delta \Delta$ & \\
\hline Hong Kong SAR & $1(0.6)$ & $\sim \sim$ & $1(0.6)$ & & $18(3.3)$ & $544(6.1)$ & $5(5.2)$ & & $81(3.3)$ & $568(2.6)$ & $-6(5.2)$ & \\
\hline Singapore & $3(0.9)$ & $583(11.0)$ & $2(1.1)$ & 0 & $1(0.5)$ & $\sim \sim$ & $-5(1.6)$ & ( & $96(1.1)$ & $558(3.0)$ & $2(2.0)$ & \\
\hline South Africa & $3(0.8)$ & $244(31.8)$ & $\Delta \Delta$ & & $16(2.4)$ & $355(21.7)$ & $\Delta \Delta$ & & $81(2.4)$ & $292(6.3)$ & $\Delta \Delta$ & \\
\hline International Avg. & $32(0.5)$ & $489(1.6)$ & & & $51(0.5)$ & $504(0.9)$ & & & $17(0.3)$ & $486(1.7)$ & & \\
\hline
\end{tabular}

An " $\mathrm{r}$ " indicates data are available for $70-84 \%$ of the students. An " $\mathrm{s}$ " indicates data are available for $50-69 \%$ of the students. An " $x$ " indicates data are available for less than $50 \%$ of the students.

A tilde $(\sim)$ indicates insufficient data to report achievement.
A diamond $(0)$ indicates the country did not participate in the 2001 assessment. NOTE: The International Average does not include the results from the Canadian provinces. Trend Note: The primary education systems of the Russian Federation and Slovenia underwent structural changes. Data for Canada, Ontario include only public schools. 
with the average reading achievement for those students. In addition, for countries and provinces that participated in PIRLS 2001, the exhibit includes the change in the percent of students, and indicates whether the change was statistically significant.

Across the PIRLS 2006 participants, the average class size for fourth-grade reading instruction was 24 students. The range in average class size varied from 17 students in Luxembourg to 42 in South Africa. Among the trend participants, more than half had a reduction in average class size between one and three students since 2001. Only Iceland and Singapore had small, but statistically significant, class size increases from 2001 to 2006, both with an average increase of one student. About half the students, internationally on average, were in classes with between 21 and 30 students, and about one third were in classes of 20 students or fewer. Countries where more than half the students were in classes with 20 students or fewer included Luxembourg, Romania, Bulgaria, Slovenia, Italy, Norway, Denmark, Latvia, and Georgia. On average internationally, 17 percent of students were in classes of 31 or more students. However, Israel, Morocco, and Indonesia had approximately half of their students in classes this large. The following countries had most of their students in classes of 31 or more: Chinese Taipei (75\%), Hong Kong SAR (81\%), Singapore (96\%), and South Africa (81\%).

The reduction in average class size between PIRLS 2001 and PIRLS 2006 was reflected in changes for the three ranges of class size, with students moving from larger to smaller classes. There was an increase in the percentage of Singaporean fourth graders in classes with 1-20 students, as well as increases in several Eastern European countries, including Romania, Bulgaria, Slovenia, Latvia, Hungary, Moldova, and Macedonia. Iceland was the only country with a decrease, but it was small and nearly half of the students were still in small classes in 2006. Most of the increases in percentages of students in classes of 1 to 20 were accompanied by commensurate decreases in the percentages of students in larger classes (with 21-30 students, 31 or more students, or both). In England, the increase in the percentage of students in classes with 21 to 30 students was accompanied by a decrease in the percentage of students in classes with 31 or more students. 


\section{How Do Schools Help Students with Reading Difficulties?}

Countries differ in their policies and approaches to diagnostic screening and provisions for students with reading difficulties, as explained in more detail in the PIRLS 2006 Encyclopedia. Exhibit 5.17 presents teachers' reports of the percent of students needing and receiving remedial reading instruction. On average internationally, teachers estimated that about one tenth of the students were likely to have experienced difficulty understanding the spoken language of the test. Teachers' estimates were 20 percent or higher in Indonesia, Iran, Kuwait, Morocco, and South Africa. Internationally on average, teachers reported that 17 percent of their students were in need of remedial reading instruction. However, the percentage of students needing remedial reading instruction exceeded the percentage who received remedial instruction in nearly every country.

Exhibit 5.18 shows teachers' reports of the availability of specialists either in the regular classroom or in a separate remedial reading classroom. On average internationally, 41 percent of students did not have access to any type of specialist. However, internationally on average, about 40 percent of students were in classrooms where a remedial reading specialist was either sometimes or always available. Also, an equivalent percent had access, either sometimes or always, to another type of specialist, such as a learning specialist or speech therapist. 
Exhibit 5.17 Students In Class Needing Special Instruction

PIRLS 2006

4th Grade
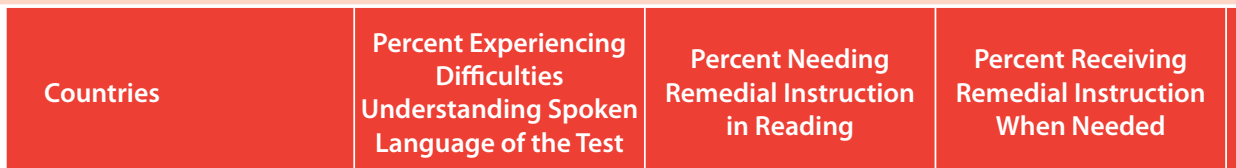

Austria

Belgium (Flemish)

Belgium (French)

Bulgaria

Canada, Alberta

Canada, British Columbia

Canada, Nova Scotia

Canada, Ontario

Canada, Quebec

Chinese Taipei

Denmark

England

France

Georgia

Germany

Hong Kong SAR

Hungary

Iceland

Indonesia

Iran, Islamic Rep. of

Israel

Italy

Kuwait

Latvia

Lithuania

Luxembourg

Macedonia, Rep. of

Moldova, Rep. of

Morocco

Netherlands

New Zealand

Norway

Poland

Qatar

Romania

Russian Federation

Scotland

Singapore

Slovak Republic

Slovenia

South Africa

Spain

Sweden

Trinidad and Tobago

United States

International Avg.

$7(0.7)$
$6(0.8)$
$5(0.6)$

$5(0.6)$

$10(1.5)$

$5(1.0)$

$5(0.7)$

$1(0.3)$

$4(0.7)$

$3(1.0)$

$7(0.6)$

s $\quad 7(1.0)$

$\begin{array}{ll}5 & 7(1.0) \\ & 3(0.6)\end{array}$

$3(0.6)$

$2(0.2)$

$5(1.4)$

$5(0.6)$

$4(0.8)$

r $\quad 11(1.4)$

$3(0.1)$

$21(1.7)$

$20(0.9)$

$12(1.2)$

$10(1.0)$

$30(4.9)$

$9(1.2)$

$9(0.8)$

$14(0.1)$

r $\quad 8(0.7)$

$13(1.3)$

$27(1.6)$

$5(0.5)$

$3(0.5)$

$4(0.7)$

$14(1.3)$

$13(0.1)$

$10(1.0)$

$6(1.0)$

$2(0.4)$

$6(0.7)$

$4(0.6)$

$10(0.7)$

$20(1.8)$

$4(0.8)$

$3(0.6)$

$10(1.5)$

$4(0.6)$

$9(0.2)$

$17(0.2)$

\begin{tabular}{l|}
$15(0.9)$ \\
$18(0.9)$ \\
$17(1.0)$ \\
$17(1.0)$ \\
$19(1.0)$
\end{tabular}

$12(0.7) \quad 6(1.0)$

\begin{tabular}{l|r}
$14(0.8)$ & $16(2.0)$ \\
\hline $8(0.9)$
\end{tabular}

$8(0.9) \quad 3(0.7)$

$15(1.0) \quad 22(2.3)$

$14(0.9) \quad 4(0.8)$

\begin{tabular}{l|l|l|}
\hline $20(1.1)$ & $15(0.9)$ & $3(0.9)$ \\
\hline $20(0.8)$ & $17(0.8)$ & $2(0.5)$
\end{tabular}

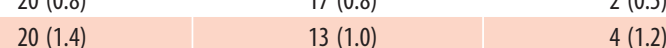

$22(1.4) \quad 14(1.1) \quad 1(0.3)$

\begin{tabular}{l|l|l}
$12(0.8)$ & $3(0.6)$ & $2(0.7)$
\end{tabular}

$\begin{array}{lll}16(0.6) & 12(0.7) & 0(0.0)\end{array}$

\begin{tabular}{l|l|l}
\hline $18(1.0)$ & $15(1.0)$ & $2(0.6)$ \\
\hline $13(0.8)$ & $3(0.4)$
\end{tabular}

$13(0.8) \quad 3(0.4) \quad 1(0.3)$

\begin{tabular}{r|rr|rr}
$17(1.6)$ & $r$ & $12(1.1)$ & $r$ & $22(2.6)$ \\
\hline $21(1.4)$ & & $13(1.6)$ & &
\end{tabular}

$21(1.4) \quad 13(1.6)-4(0.9)$

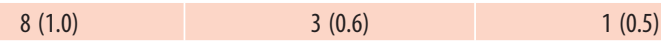

$20(1.0)$

$17(0.1)$

21 (1.5)

$18(0.9)$

$18(0.9)$

$14(0.7)$
$26(1.4)$

$17(1.2)$

$9(0.6)$

$15(1.0)$

$17(1.2)$

$31(1.3)$

$17(0.7)$

$10(0.4)$

$15(1.0)$

$23(1.3)$

$20(0.1)$

$20(1.1)$

$15(1.0)$

$13(1.0)$
$9(0.7)$

17 (1.1)

$16(0.6)$

$26(1.5)$

$17(1.0)$

$13(0.8)$

$26(1.6)$

$24(1.0)$

$3(0.6)$
$16(1.0)$

$15(0.1)$

$18(1.4)$

$12(0.8)$

$10(0.6)$

$10(0.6)$
$17(1.4)$

$7(0.8)$

$7(0.6)$

$6(0.0)$

$15(1.0)$

$12(1.0)$

$15(1.3)$

$16(0.7)$

$7(0.3)$

$11(1.0)$

19 (1.2)

$12(0.1)$

$15(1.1)$

$8(0.9)$

$10(0.9)$

$5(0.7)$

$8(0.9)$

$14(0.7)$

$15(1.3)$

$14(1.0)$

$9(0.6)$

$14(1.2)$

$18(1.2)$

$12(0.1)$
Background data provided by teachers.

( ) Standard errors appear in parentheses. Because results are rounded to the nearest whole number, some totals may appear inconsistent.
An " $r$ " indicates data are available for $70-84 \%$ of the students. An " $s$ " indicates data are available for $50-69 \%$ of the students. An " $x$ " indicates data are available for less than $50 \%$ of the students.

NOTE: The International Average does not include the results from the Canadian provinces. 


\begin{tabular}{|c|c|c|c|c|c|c|c|}
\hline \multirow{3}{*}{ Countries } & \multicolumn{7}{|c|}{ Percentage of Students in Classrooms with } \\
\hline & \multicolumn{3}{|c|}{ Remedial Reading Specialist Available* } & \multicolumn{3}{|c|}{ Other Specialist(s) Available } & \multirow{2}{*}{$\begin{array}{c}\text { No Access to } \\
\text { Any } \\
\text { Specialist }\end{array}$} \\
\hline & Always & Sometimes & Never & Always & Sometimes & Never & \\
\hline Austria & $2(1.1)$ & $21(2.6)$ & $77(2.7)$ & $2(0.8)$ & $44(3.3)$ & $53(3.3)$ & $45(3.3)$ \\
\hline Belgium (Flemish) & $18(3.1)$ & $61(4.4)$ & $20(3.4)$ & $7(2.0)$ & $37(3.9)$ & $56(4.1)$ & $15(3.0)$ \\
\hline Belgium (French) & $3(1.3)$ & $14(2.4)$ & $82(2.8)$ & $17(2.4)$ & $53(4.2)$ & $30(4.0)$ & $24(3.8)$ \\
\hline Bulgaria & $4(1.4)$ & $7(2.3)$ & $89(2.7)$ & $7(2.1)$ & $24(3.4)$ & $69(3.6)$ & $62(3.7)$ \\
\hline Canada, Alberta & $13(2.7)$ & $44(4.0)$ & $42(3.7)$ & $5(1.7)$ & $70(3.9)$ & $26(3.6)$ & $15(2.7)$ \\
\hline Canada, British Columbia & $23(3.3)$ & $58(4.0)$ & $19(3.3)$ & $9(2.7)$ & $77(3.9)$ & $14(3.3)$ & $3(1.6)$ \\
\hline Canada, Nova Scotia & $25(3.1)$ & $61(3.4)$ & $14(2.6)$ & $9(2.2)$ & $80(3.3)$ & $11(2.6)$ & $3(1.1)$ \\
\hline Canada, Ontario & $17(3.9)$ & $41(4.9)$ & $42(5.1)$ & $7(2.9)$ & $63(5.3)$ & $30(4.6)$ & $17(3.4)$ \\
\hline Canada, Quebec & $5(1.6)$ & $40(4.5)$ & $56(4.8)$ & $15(3.0)$ & $69(4.3)$ & $16(3.3)$ & $7(2.0)$ \\
\hline Chinese Taipei & $7(2.1)$ & $21(3.6)$ & $73(3.8)$ & $2(1.2)$ & $12(2.6)$ & $86(2.7)$ & $69(3.9)$ \\
\hline Denmark & $30(3.2)$ & $65(3.1)$ & $5(1.7)$ & $5(2.1)$ & $53(3.9)$ & $42(3.6)$ & $4(1.5)$ \\
\hline England & $24(3.7)$ & $60(4.5)$ & $16(3.0)$ & $2(1.1)$ & $62(3.8)$ & $36(3.9)$ & $8(1.9)$ \\
\hline France & $6(1.4)$ & $33(3.2)$ & $61(3.5)$ & $1(0.6)$ & $12(2.7)$ & $87(2.8)$ & $50(3.7)$ \\
\hline Georgia & $6(1.9)$ & $23(3.7)$ & $70(4.1)$ & $3(1.2)$ & $40(4.4)$ & $58(4.5)$ & $50(4.9)$ \\
\hline Germany & $5(1.6)$ & $35(3.4)$ & $59(3.6)$ & $3(1.1)$ & $11(2.2)$ & $86(2.4)$ & $58(3.7)$ \\
\hline Hong Kong SAR & $2(1.1)$ & $4(1.3)$ & $94(1.7)$ & $1(0.9)$ & $6(2.1)$ & $93(2.3)$ & $89(2.7)$ \\
\hline Hungary & $8(2.2)$ & $15(3.0)$ & $77(3.4)$ & $22(3.6)$ & $43(3.6)$ & $35(3.8)$ & $29(3.8)$ \\
\hline Iceland & $35(0.4)$ & $59(0.4)$ & $6(0.1)$ & $7(0.2)$ & $40(0.4)$ & $53(0.4)$ & $5(0.1)$ \\
\hline Indonesia & $15(2.6)$ & $23(3.5)$ & $62(3.7)$ & $2(0.9)$ & $10(2.8)$ & $89(3.0)$ & $63(3.9)$ \\
\hline Iran, Islamic Rep. of & $4(1.5)$ & $13(2.6)$ & $83(3.2)$ & $0(0.0)$ & $6(1.9)$ & $94(1.9)$ & $82(3.5)$ \\
\hline Israel & $36(4.0)$ & $41(4.0)$ & $24(3.4)$ & $3(1.6)$ & $29(3.9)$ & $68(3.9)$ & $22(3.3)$ \\
\hline Italy & $0(0.0)$ & $5(1.7)$ & $95(1.7)$ & $2(1.0)$ & $6(1.9)$ & $92(2.2)$ & $89(2.7)$ \\
\hline Kuwait & $3(1.4)$ & $7(2.3)$ & $90(2.7)$ & $5(1.9)$ & $18(2.9)$ & $77(3.3)$ & $73(3.7)$ \\
\hline Latvia & $13(2.7)$ & $16(2.8)$ & $71(3.3)$ & $39(4.0)$ & $41(4.4)$ & $20(3.4)$ & $16(3.1)$ \\
\hline Lithuania & $7(1.7)$ & $16(2.1)$ & 77 (2.7) & $44(3.1)$ & $45(3.3)$ & $11(2.0)$ & $10(1.8)$ \\
\hline Luxembourg & $11(0.1)$ & $21(0.1)$ & $68(0.2)$ & $3(0.1)$ & $10(0.1)$ & $87(0.1)$ & $62(0.2)$ \\
\hline Macedonia, Rep. of & $5(1.7)$ & $9(2.5)$ & $86(3.0)$ & $12(2.8)$ & $48(4.0)$ & $41(3.8)$ & $37(4.0)$ \\
\hline Moldova, Rep. of & $5(1.5)$ & $8(2.3)$ & $87(2.6)$ & $3(1.1)$ & $13(2.8)$ & $84(3.0)$ & $74(3.6)$ \\
\hline Morocco & $1(0.1)$ & $6(1.7)$ & $93(1.7)$ & $1(0.9)$ & $1(0.8)$ & 98 (1.2) & $91(2.1)$ \\
\hline Netherlands & $29(4.0)$ & $57(4.5)$ & $14(3.0)$ & $1(0.0)$ & $35(4.3)$ & $64(4.4)$ & $12(2.9)$ \\
\hline New Zealand & $27(2.4)$ & $40(3.1)$ & $33(2.7)$ & $11(1.8)$ & $60(3.2)$ & $28(2.9)$ & $16(2.2)$ \\
\hline Norway & $7(2.5)$ & $42(4.4)$ & $51(4.1)$ & $3(1.4)$ & $51(5.1)$ & $46(4.8)$ & $30(4.3)$ \\
\hline Poland & $26(3.6)$ & $26(3.1)$ & $48(3.6)$ & $23(3.4)$ & $61(4.1)$ & $15(2.8)$ & $8(1.8)$ \\
\hline Qatar & $6(0.1)$ & $7(0.2)$ & $86(0.2)$ & $12(0.2)$ & $14(0.2)$ & $74(0.3)$ & $71(0.3)$ \\
\hline Romania & $3(1.1)$ & $13(2.4)$ & $84(2.6)$ & $4(1.4)$ & $22(3.2)$ & $74(3.4)$ & $66(3.8)$ \\
\hline Russian Federation & $15(2.3)$ & $22(2.5)$ & $63(3.3)$ & $27(3.1)$ & $49(3.4)$ & $24(2.9)$ & $24(3.2)$ \\
\hline Scotland & $18(3.2)$ & $36(4.5)$ & $46(4.4)$ & $10(3.2)$ & $72(4.1)$ & $18(3.2)$ & $8(2.5)$ \\
\hline Singapore & $7(1.5)$ & $14(1.7)$ & $79(2.0)$ & $5(1.3)$ & $18(2.5)$ & $77(2.5)$ & $69(2.5)$ \\
\hline Slovak Republic & $5(1.4)$ & $19(2.8)$ & $76(2.9)$ & $12(2.4)$ & $50(3.9)$ & $38(3.5)$ & $37(3.5)$ \\
\hline Slovenia & $18(2.5)$ & $56(3.2)$ & $27(2.9)$ & $15(2.5)$ & $52(3.2)$ & $32(3.3)$ & $10(1.8)$ \\
\hline South Africa & $10(1.7)$ & $13(1.9)$ & $77(2.5)$ & $4(1.3)$ & $10(1.8)$ & $86(2.1)$ & $74(2.6)$ \\
\hline Spain & $27(3.5)$ & $59(4.1)$ & $14(3.0)$ & $19(2.7)$ & $51(3.9)$ & 31 (3.6) & $6(2.0)$ \\
\hline Sweden & $8(2.0)$ & $80(3.0)$ & $13(2.6)$ & $4(1.5)$ & $53(4.0)$ & $43(4.0)$ & $9(2.0)$ \\
\hline Trinidad and Tobago & $5(1.9)$ & $10(2.4)$ & $85(2.9)$ & $1(0.9)$ & $6(1.8)$ & $93(2.0)$ & $82(2.9)$ \\
\hline United States & $34(4.2)$ & $37(4.2)$ & $29(2.9)$ & $27(2.9)$ & $60(3.4)$ & $13(2.3)$ & $8(1.9)$ \\
\hline International Avg. & $12(0.4)$ & $28(0.5)$ & $60(0.5)$ & $9(0.3)$ & $33(0.5)$ & $58(0.5)$ & $41(0.5)$ \\
\hline
\end{tabular}

Background data provided by teachers.

* Remedial Reading Specialist Available indicates that a specialist was available either in the classroom or in a remedial reading classroom.

() Standard errors appear in parentheses. Because results are rounded to the nearest whole number, some totals may appear inconsistent.
An " $r$ " indicates data are available for $70-84 \%$ of the students. An "s" indicates data are available for $50-69 \%$ of the students. An " $x$ " indicates data are available for less than $50 \%$ of the students.

NOTE: The International Average does not include the results from the Canadian provinces. 



\section{Chapter 6}

\section{Teachers and Reading Instruction}

Although children are presented with an array of literacy-related experiences in the home and community before they begin school, the teacher in the classroom plays a central role in children's reading literacy development. It is primarily the teacher's responsibility to structure the classroom environment to support reading development and to provide opportunities for learning to read. There is a wide variety of instructional approaches and materials for helping students develop their reading skills and strategies. Depending on their classroom situations, teachers may draw on an extensive repertoire of reading activities or primarily rely on several approaches to engage students in reading and monitor their performance and progress in reading. The classroom environment in which reading instruction takes place also can be influenced by school policy, curriculum, and the teacher's preparation and training.

To collect information about instruction, the teachers responsible for teaching reading to the class of students participating in PIRLS 2006 completed a Teacher Questionnaire that asked them about their preparation for teaching reading, as well as their use of instructional materials and strategies and activities for teaching reading, specifically with regard to the PIRLS class. Chapter 6 presents the results for a subset of these questions.

\section{What Education and Training Do Teachers Have for Teaching Reading?}

This section presents information about teachers' preparation to teach reading, including their highest level of formal education, certification as a teacher, and the emphasis on various content areas in their studies. 
As described in the PIRLS 2006 Encyclopedia, there is variation from country to country in the path(s) for teachers to receive their education, training, and certification. Exhibit 6.1 presents teachers' reports about their highest level of education-a 3- or 4-year university or university college degree program or a program of 2 or 3 years in a teacher training college, university, or vocational institution. Some countries included both types of settings, whereas others included only one.

On average internationally, 6o percent of students had teachers with a university degree and 30 percent had teachers who graduated from a 2- or 3-year college or university program. Many countries fit this pattern, with most students having teachers with a university degree and nearly all the rest having teachers educated in a 2- or 3-year college or university program. However, nearly all students ( $98 \%$ or more) were taught by teachers with a university degree in Georgia, Hungary, Poland, the United States, and the Canadian provinces except Nova Scotia. In Indonesia, Luxembourg, South Africa, and Trinidad and Tobago, some students had teachers with a 4-year degree, but the majority had teachers educated in a 2- or 3-year program. Nearly all the students (94-100\%) were taught by teachers educated in a 2- to 3-year program in Austria and Belgium (Flemish and French). In several countries, as many as one fifth of the students (or more) were taught by teachers without a tertiary degree. Sometimes, as was the case for teachers in the former German Democratic Republic, existing policies allowed previous generations of teachers to enter primary education teaching service without a university or college degree. Regardless of their educational path, however, nearly all students (97\%), on average internationally, were taught by teachers who were certified.

Exhibit 6.2 shows the percentages of students whose teachers had various areas of specialization in their studies. As might be anticipated, the majority of students were taught reading by teachers whose studies emphasized a combination of pedagogy, language, and literature. Across countries, the majority of students $(57 \%)$ had teachers whose studies emphasized pedagogy in combination with language, literature, or both, and another 19 percent had 
teachers whose studies emphasized language and/or literature. Few students $(7 \%)$, on average internationally, had teachers whose studies focused on pedagogy without an emphasis on either language or literature.

Interestingly, substantial percentages of fourth-grade students had teachers whose studies emphasized a variety of content areas other than pedagogy, language, or literature. More specifically, teachers were asked about psychology, remedial reading, reading theory, children's language development, special education, and second language learning. The last three columns of Exhibit 6.2 provide the percentages of students taught by teachers whose education program emphasized one (24\%), two (18\%), or three or more (29\%) of these other content areas. 
Exhibit 6.1 Reading Teachers' Formal Education

PIRLS 2006

4th Grade
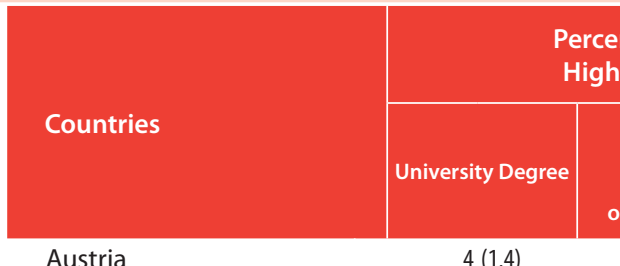

Percentage of Students by Teachers'

Highest Level of Formal Education

Belgium (Flemish)

Bulgaria

Canada, Alberta

Canada, British Columbia

Canada, Nova Scotia

Canada, Ontario

Canada, Quebec

Chinese Taipei

Denmark

England

France

Georgia

Germany

Hong Kong SAR

Hungary

Iceland

Indonesia

4 (1.4)

College or

University

Program* of 2 or 3 Years

\begin{tabular}{|c|c|}
\hline Completed & Did Not Complete \\
Upper-secondary & Upper-secondary \\
School & School
\end{tabular}

Percentage of

Students

Taught by

Certified

Teachers

Iran, Islamic Rep. of

$$
\text { Israel }
$$

Italy

Kuwait

$5(1.3)$

$94(1.8)$

$2(1.2)$

$0(0.0)$

$100(0.0)$

$0(0.0)$

$95(1.3)$

$81(3.5)$

$100(0.0)$

$100(0.0)$

$19(3.5)$

$98(1.2)$

$0(0.0)$

$0(0.0)$

$0(0.0)$
$0(0.0)$

$0(0.0)$

$0(0.0)$

$0(0.0)$

$100(0.0)$

$98(0.7)$

Latvia

$98(1.2)$

$2(1.2)$

$0(0.0)$

$0(0.0)$

$0(0.0)$

$0(0.0)$

$100(0.0)$

$10(2.2)$

$100(0.2)$

$2(1.2)$

$96(1.7)$

$93(2.5)$

$0(0.2)$

$0(0.0)$

$0(0.0)$

$0(0.0)-r-100(0.0)$

$0(0.0)$

$0(0.0)$
$0(0.0)$

$4(1.7)$

$\begin{array}{ll}0(0.0) & 0(0.0) \\ 0(0.0) & 0(0.0)\end{array}$

$r \quad 100(0.0)$
$\quad 100(0.2)$

$100(0.0)$

86 (3.1)

$5(1.9)$

$0(0.0)$

$0(0.0)$

$\begin{array}{ll}2(1.7) & 0(0.0) \\ 1(0.0) & 0(0.0)\end{array}$

$96(1.5)$

$78(2.8)$

$13(3.1)$

$99(0.4)$

$4(1.3)$

$0(0.0)$

$18(2.6)$

$0(0.0)$

\begin{tabular}{r|r}
$1(0.4)$ & $0(0.0)$ \\
$24(2.9)$ & $2(1.1)$
\end{tabular}

\begin{tabular}{r|r|r|}
\hline $75(3.0)$ & $24(2.9)$ & $2(1.1)$ \\
\hline $31(3.5)$ & $3(1.3)$ & $0(0.0)$ \\
\hline
\end{tabular}

$67(3.6)$

$67(3.6)$
$100(0.0)$

$88(0.3)$

$16(2.8)$

$0(0.0)$

$7(0.2)$

$7(0.2)$
$59(3.7)$

$0(0.0)$

$0(0.0)$

$0(0.0) \quad 0(0.0)$

\begin{tabular}{r|r}
\hline $4(0.2)$ & $1(0.0)$ \\
\hline $23(3.4)$ & $1(0.7)$ \\
\hline $34(3.9)$ & $0(0.0)$
\end{tabular}

$40(4.1)$

$26(3.5)$

$26(3.5)$
$81(3.5)$

$40(4.1)$

$34(3.9)$

$9(2.0)$

$24(3.5)$
$89(2.8)$

$9(2.0)$

\begin{tabular}{r|r}
$34(3.9)$ & $0(0.0)$ \\
$0(0.0)$ & $0(0.0)$ \\
$66(3.7)$ & $1(0.0)$
\end{tabular}

$1(0.7)$

$98(1.4)$

Lithuania
Luxembourg
Macedonia, Rep. of

Moldova, Rep. of

Morocco

Netherlands

$83(3.0)$

$80(2.8)$

$17(3.0)$

$42(3.8)$

$20(2.8)$
$85(0.2)$

$66(3.7)$
$1(0.0)$

$1(0.0)$
$2(1.0)$

$92(2.3)$

$100(0.0)$

New Zealand

\begin{tabular}{l|l} 
Norway & 9311 \\
Poland & 9810
\end{tabular}

\begin{tabular}{ll} 
Qatar & r \\
Romania & 9110 \\
\hline
\end{tabular}

Russian Federation
Scotland
Singapore

Singapore

Slovak Republic

Slovenia

South Africa

$59(4.2)$

$50(3.6)$

$0(0.3)$

$40(4.1)$

$0(0.0)$

$0(0.0)$

$\begin{array}{ll}0(0.1) & 0(0.0) \\ 70 & 0(0.0)\end{array}$

$92(1.9)$

$93(1.9)$

$99(0.6)$

Spain $r$

Sweden

Trinidad and Tobago

United States

$21(3.4)$

$95(1.9)$

$71(2.6)$

$9(2.0)$

$5(1.9)$

$29(2.6)$

$93(1.6) \quad 6(1.5)$

$98(0.8)$

$91(0.2)$

$6(1.3)$

$70(3.3)$

$86(2.8)$

$59(2.7)$

$94(1.6)$

54 (3.2)

$33(3.2)$

$61(4.2)$

$68(3.8)$

$14(3.0)$

$99(0.6)$

$2(0.7)$

$8(0.2)$

$10(0.1)$
$7(2.4)$

$0(0.0)$

\begin{tabular}{r|r|}
$7(2.4)$ & $1(0.8)$ \\
\hline $1(0.8)$ & $0(0.0)$ \\
\hline $58(4.1)$ & $11(2.7)$ \\
\hline $0(0.0)$ & $0(0.0)$
\end{tabular}

$98(1.1)$

International Avg.

$60(0.4)$

$40(3.7)$

\begin{tabular}{|l|l|l|}
\hline $0(0.0)$ & $0(0.0)$ & $100(0.4)$ \\
\hline $0(0.0)$ & $0(0.0)$ & $100(0.0)$ \\
\hline $1(0.6)$ & $0(0.4)$ & $100(0.4)$ \\
\hline $0(0.4)$ & $0(0.0)$ & $100(0.0)$ \\
\hline
\end{tabular}

\begin{tabular}{|l|l|l|}
\hline $0(0.0)$ & $0(0.0)$ & $100(0.4)$ \\
\hline $0(0.0)$ & $0(0.0)$ & $100(0.0)$ \\
\hline $1(0.6)$ & $0(0.4)$ & $100(0.4)$ \\
\hline $0(0.4)$ & $0(0.0)$ & $100(0.0)$ \\
\hline
\end{tabular}

International Avg.

$30(3.2)$
$6(2.0)$

$40(2.7)$

$0(0.4)$
$1(0.0)$

$0(0.0)$

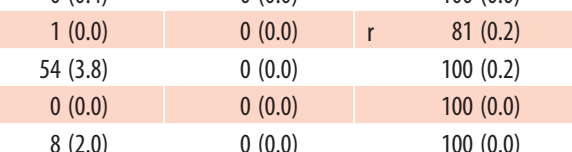


Exhibit 6.2 Areas of Emphasis in Teachers' Formal Education and Training

\begin{tabular}{|c|c|c|c|c|c|c|c|c|}
\hline \multirow[b]{2}{*}{ Countries } & \multicolumn{8}{|c|}{ Percentage of Students Whose Teachers' Studies Emphasized } \\
\hline & & $\begin{array}{l}\text { Reading } \\
\text { Pedagogy with } \\
\text { anguage and/or } \\
\text { Literature }\end{array}$ & $\begin{array}{l}\text { Reading } \\
\text { Pedagogy } \\
\text { Without } \\
\text { Language or } \\
\text { Literature }\end{array}$ & $\begin{array}{c}\text { Language } \\
\text { and/or Literature } \\
\text { Without Reading } \\
\text { Pedagogy }\end{array}$ & & $\begin{array}{l}1 \text { Other } \\
\text { Related } \\
\text { Area* }\end{array}$ & $\begin{array}{l}2 \text { Other } \\
\text { Related } \\
\text { Areas }\end{array}$ & $\begin{array}{c}3 \text { or More } \\
\text { Other Related } \\
\text { Areas }\end{array}$ \\
\hline Austria & & $49(3.7)$ & $13(2.3)$ & $13(2.5)$ & & $24(2.4)$ & $21(2.7)$ & $22(3.1)$ \\
\hline Belgium (Flemish) & & $58(4.0)$ & $6(1.8)$ & $18(2.9)$ & & $21(3.2)$ & $17(3.1)$ & $31(4.1)$ \\
\hline Belgium (French) & & $64(3.1)$ & $1(0.4)$ & $25(2.9)$ & & $23(2.7)$ & $19(2.4)$ & $26(3.1)$ \\
\hline Bulgaria & & $90(2.6)$ & $4(1.8)$ & $5(1.8)$ & & $24(3.3)$ & $22(3.0)$ & $49(4.2)$ \\
\hline Canada, Alberta & & $41(4.4)$ & $8(2.3)$ & $18(3.3)$ & & $30(3.3)$ & $11(2.1)$ & $16(2.7)$ \\
\hline Canada, British Columbia & r & $44(4.3)$ & $10(2.7)$ & $21(3.5)$ & r & $26(3.7)$ & $14(2.8)$ & $26(3.5)$ \\
\hline Canada, Nova Scotia & & $62(3.9)$ & $8(2.1)$ & $18(2.8)$ & & $25(3.0)$ & $20(3.3)$ & $25(3.4)$ \\
\hline Canada, Ontario & & $48(4.9)$ & $8(2.4)$ & $23(4.1)$ & & $19(3.8)$ & $21(4.2)$ & $29(4.9)$ \\
\hline Canada, Quebec & & $65(4.2)$ & $3(1.5)$ & $25(3.5)$ & & $34(4.6)$ & $20(3.9)$ & $20(3.7)$ \\
\hline Chinese Taipei & & $23(3.5)$ & $17(3.2)$ & $11(2.8)$ & & $32(3.4)$ & $19(3.5)$ & $11(2.7)$ \\
\hline Denmark & & $35(4.0)$ & $3(1.2)$ & $48(4.1)$ & & $34(3.9)$ & $22(3.0)$ & $24(3.4)$ \\
\hline England & & $43(4.3)$ & $8(2.3)$ & $31(3.5)$ & & $24(3.7)$ & $16(3.1)$ & $15(2.5)$ \\
\hline France & & $33(3.4)$ & $3(1.1)$ & $35(3.5)$ & & $26(3.0)$ & $12(2.4)$ & $12(2.5)$ \\
\hline Georgia & & $85(3.0)$ & $2(1.0)$ & $6(2.1)$ & & $13(2.6)$ & $13(3.2)$ & $66(4.1)$ \\
\hline Germany & & $34(3.4)$ & $8(2.0)$ & $19(3.1)$ & & $33(3.6)$ & $12(2.1)$ & $13(2.4)$ \\
\hline Hong Kong SAR & & $59(4.5)$ & $7(2.2)$ & $16(3.6)$ & & $20(3.6)$ & $16(3.4)$ & $13(2.8)$ \\
\hline Hungary & & $74(3.5)$ & $7(2.3)$ & $15(3.1)$ & & $40(4.0)$ & $21(2.9)$ & $18(2.8)$ \\
\hline Iceland & $r$ & $39(0.4)$ & $16(0.3)$ & $15(0.3)$ & r & $23(0.2)$ & $17(0.2)$ & $15(0.3)$ \\
\hline Indonesia & & $59(4.0)$ & $11(2.4)$ & $13(2.6)$ & & $18(3.2)$ & $17(3.2)$ & $47(4.3)$ \\
\hline Iran, Islamic Rep. of & & $35(3.7)$ & $20(3.3)$ & $11(2.7)$ & & $23(3.0)$ & $13(2.8)$ & $18(2.8)$ \\
\hline Israel & & $26(3.7)$ & $8(2.7)$ & $26(4.1)$ & & $13(2.9)$ & $10(2.3)$ & $13(3.0)$ \\
\hline Italy & & $35(3.6)$ & $2(1.1)$ & $51(3.4)$ & & $19(3.0)$ & $17(2.7)$ & $24(3.4)$ \\
\hline Kuwait & $r$ & $74(4.1)$ & $2(1.1)$ & $23(3.8)$ & r & $24(3.4)$ & $21(3.7)$ & $30(4.2)$ \\
\hline Latvia & & $65(3.9)$ & $12(2.4)$ & $5(1.7)$ & & $32(3.7)$ & $18(3.1)$ & $26(3.5)$ \\
\hline Lithuania & & $84(2.9)$ & $3(1.1)$ & $10(2.3)$ & & $25(3.3)$ & $25(3.0)$ & $43(3.7)$ \\
\hline Luxembourg & & $40(0.2)$ & $20(0.1)$ & $18(0.2)$ & & $17(0.1)$ & $19(0.1)$ & $36(0.1)$ \\
\hline Macedonia, Rep. of & $r$ & $79(3.7)$ & $6(2.4)$ & $7(2.5)$ & $r$ & $19(3.3)$ & $12(2.7)$ & $58(4.6)$ \\
\hline Moldova, Rep. of & & $76(3.9)$ & $7(2.2)$ & $6(2.0)$ & & $20(2.7)$ & $16(2.9)$ & $45(4.1)$ \\
\hline Morocco & & $36(4.0)$ & $5(2.2)$ & $34(4.3)$ & & $18(3.2)$ & $8(2.4)$ & $21(3.6)$ \\
\hline Netherlands & $r$ & $40(3.6)$ & $6(1.5)$ & $11(2.7)$ & & $17(3.3)$ & $13(2.8)$ & $18(3.7)$ \\
\hline New Zealand & & $45(2.6)$ & $15(2.3)$ & $17(2.1)$ & & $27(2.4)$ & $19(2.0)$ & $20(2.1)$ \\
\hline Norway & & $67(5.0)$ & $8(2.3)$ & $16(3.7)$ & & $22(4.0)$ & $23(3.5)$ & $39(4.3)$ \\
\hline Poland & & $55(4.0)$ & $25(3.3)$ & $3(1.3)$ & & $29(3.4)$ & $18(2.7)$ & $34(3.6)$ \\
\hline Qatar & s & $55(0.3)$ & $4(0.1)$ & $23(0.2)$ & s & $22(0.2)$ & $14(0.1)$ & $23(0.3)$ \\
\hline Romania & & $92(2.1)$ & $4(1.6)$ & $2(0.9)$ & & $22(3.1)$ & $19(3.6)$ & $55(3.7)$ \\
\hline Russian Federation & & $87(2.4)$ & $5(1.4)$ & $4(1.3)$ & & $21(2.3)$ & $23(2.9)$ & $45(2.9)$ \\
\hline Scotland & & $59(4.4)$ & $6(2.1)$ & $22(3.8)$ & & $17(3.3)$ & $28(3.7)$ & $24(3.6)$ \\
\hline Singapore & r & $66(2.8)$ & $6(1.5)$ & $17(2.3)$ & $r$ & $22(2.6)$ & $16(2.1)$ & $20(2.5)$ \\
\hline Slovak Republic & & $90(2.1)$ & $0(0.0)$ & $8(1.9)$ & & $38(3.7)$ & $21(3.1)$ & $32(3.6)$ \\
\hline Slovenia & & $37(3.2)$ & $2(0.8)$ & $40(3.3)$ & & $33(3.3)$ & $14(2.0)$ & $11(2.0)$ \\
\hline South Africa & r & $60(2.9)$ & $4(1.7)$ & $25(2.7)$ & $r$ & $23(2.9)$ & $19(2.5)$ & $38(3.9)$ \\
\hline Spain & & $40(4.2)$ & $1(0.9)$ & $39(3.9)$ & & $33(3.8)$ & $19(3.3)$ & $27(3.7)$ \\
\hline Sweden & & $46(4.1)$ & $2(1.0)$ & $33(3.7)$ & & $33(3.5)$ & $19(2.2)$ & $19(2.7)$ \\
\hline Trinidad and Tobago & & $73(3.5)$ & $2(1.3)$ & $15(2.8)$ & & $19(3.3)$ & $13(2.8)$ & $48(3.6)$ \\
\hline United States & & $54(3.7)$ & $13(2.5)$ & $16(2.4)$ & & $24(2.9)$ & $20(2.8)$ & $25(3.1)$ \\
\hline International Avg. & & $57(0.5)$ & $7(0.3)$ & $19(0.4)$ & & $24(0.5)$ & $18(0.4)$ & $29(0.5)$ \\
\hline
\end{tabular}

Background data provided by teachers.

* Other related areas include psychology, remedial reading, reading theory, children's language development, special education, and second language learning.

() Standard errors appear in parentheses. Because results are rounded to the nearest whole number, some totals may appear inconsistent.
$A n$ " $r$ " indicates data are available for $70-84 \%$ of the students. An "s" indicates data are available for $50-69 \%$ of the students. An " $x$ " indicates data are available for less than $50 \%$ of the students.

NOTE: The International Average does not include the results from the Canadian provinces. 


\section{What Are the Background Characteristics and Responsibilities of Reading Teachers?}

This section provides information about the gender, age, experience, and teaching assignment of the fourth-grade teachers. As shown in Exhibit 6.3, internationally on average, 83 percent of students were taught reading by women, and 17 percent were taught by men. Countries where students were taught reading exclusively or almost exclusively ( $98 \%$ or more) by female teachers included Georgia, Italy, Latvia, Lithuania, Poland, the Russian Federation, and Slovenia.

Internationally on average, slightly less than half the students (45\%) were taught by teachers under the age of 40 , and the remainder were taught by teachers 40 or older. Fifteen percent of students were taught by teachers under the age of 30 . Considering that a sustainable teaching force should have about one fourth of students taught by teachers in their 20s, 30s, 40s, and 50s, the international distribution suggests that there may be a problem in coming years without recruitment of younger teachers. This may be particularly true for several participants where less than 10 percent of students were taught by teachers under age 30, including Austria, Bulgaria, Georgia, Germany, Hungary, Iran, Israel, Italy, Latvia, Lithuania, Macedonia, Moldova, Norway, Poland, the Russian Federation, South Africa, and the Canadian province of British Columbia.

Internationally, teachers had been teaching for an average of 17 years. Countries where teachers averaged more than 20 years of experience teaching included Austria, Bulgaria, Hungary, Italy, Latvia, Lithuania, Moldova, Romania, and the Russian Federation. Teachers in two countries, Kuwait and Singapore, averaged less than 10 years of teaching experience. The average number of years teaching increased from 2001 in Bulgaria, Hungary, Iran, Moldova, and the Russian Federation. Average decreases from 2001 were evident in France, Germany, Iceland, Singapore, the United States, and the Canadian province of Ontario.

Exhibit 6.4 reveals that teachers of fourth-grade reading typically are full-time teachers. Internationally on average, 91 percent of students were 
Exhibit 6.3 Teachers' Gender, Age, and Number of Years Teaching

PIRLS 2006

4th Grade

\begin{tabular}{|c|c|c|c|c|c|c|c|c|c|}
\hline \multirow{3}{*}{ Countries } & \multicolumn{6}{|c|}{ Percentage of Students by Teacher Characteristics } & \multirow{2}{*}{\multicolumn{3}{|c|}{$\begin{array}{l}\text { Trends in Number } \\
\text { of Years Teaching } \\
\text { All Grades }\end{array}$}} \\
\hline & \multicolumn{2}{|c|}{ Gender } & \multicolumn{4}{|c|}{ Age } & & & \\
\hline & Female & Male & $\begin{array}{l}29 \text { Years } \\
\text { or Under }\end{array}$ & $\begin{array}{l}30-39 \\
\text { Years }\end{array}$ & $\begin{array}{l}40-49 \\
\text { Years }\end{array}$ & $\begin{array}{l}50 \text { Years } \\
\text { or Older }\end{array}$ & 2006 & $\begin{array}{l}\text { Difference } \\
\text { from } 2001\end{array}$ & \\
\hline Austria & $88(2.1)$ & $12(2.1)$ & $6(1.7)$ & $17(2.5)$ & $39(3.0)$ & $38(3.2)$ & $22(0.7)$ & $\Delta \diamond$ & \\
\hline Belgium (Flemish) & $75(3.0)$ & $25(3.0)$ & $28(3.2)$ & $29(3.3)$ & $29(2.9)$ & $14(2.2)$ & $16(0.6)$ & $\Delta \diamond$ & \\
\hline Belgium (French) & $80(3.0)$ & $20(3.0)$ & $16(2.2)$ & $33(3.2)$ & $37(3.5)$ & $14(2.6)$ & $17(0.6)$ & $\Delta \Delta$ & \\
\hline Bulgaria & $94(1.8)$ & $6(1.8)$ & $3(1.4)$ & $26(2.8)$ & $40(4.0)$ & $31(3.9)$ & $21(0.6)$ & $4(0.9)$ & 0 \\
\hline Canada, Alberta & $80(3.2)$ & $20(3.2)$ & $22(3.7)$ & $20(3.3)$ & $26(3.6)$ & $32(3.3)$ & $15(0.8)$ & $\Delta \diamond$ & \\
\hline Canada, British Columbia & $72(3.6)$ & $28(3.6)$ & $9(2.2)$ & $21(3.0)$ & $32(4.1)$ & $38(4.2)$ & $17(0.8)$ & $\Delta \Delta$ & \\
\hline Canada, Nova Scotia & $84(2.9)$ & $16(2.9)$ & $12(2.4)$ & $22(3.4)$ & $25(3.0)$ & $40(3.4)$ & $18(0.7)$ & $\Delta \Delta$ & \\
\hline Canada, Ontario & $75(4.8)$ & $25(4.8)$ & $18(3.9)$ & $42(5.2)$ & $21(4.3)$ & $20(4.3)$ & $12(0.9)$ & $-4(1.3)$ & ( \\
\hline Canada, Quebec & $86(3.2)$ & $14(3.2)$ & $12(2.3)$ & $37(4.0)$ & $19(3.5)$ & $32(3.7)$ & $17(0.8)$ & $-1(1.2)$ & \\
\hline Chinese Taipei & $83(3.2)$ & $17(3.2)$ & $24(3.7)$ & $44(4.1)$ & $27(3.5)$ & $5(1.6)$ & $12(0.6)$ & $\Delta \Delta$ & \\
\hline Denmark & $90(2.1)$ & $10(2.1)$ & $16(2.9)$ & $24(3.4)$ & $19(2.9)$ & $41(4.3)$ & $16(1.1)$ & $\Delta \diamond$ & \\
\hline England & $75(3.5)$ & $25(3.5)$ & $30(3.8)$ & $33(4.1)$ & $14(2.9)$ & $23(3.7)$ & $12(0.9)$ & $-2(1.3)$ & \\
\hline France & $71(3.3)$ & $29(3.3)$ & $17(2.9)$ & $31(3.0)$ & $34(3.4)$ & $18(2.6)$ & $15(0.7)$ & $-3(1.1)$ & $(\nabla$ \\
\hline Georgia & $100(0.3)$ & $0(0.0)$ & $9(1.6)$ & $28(3.4)$ & $24(3.4)$ & $39(3.6)$ & $20(0.9)$ & $\Delta \Delta$ & \\
\hline Germany & $89(2.4)$ & $11(2.4)$ & $5(1.7)$ & $23(2.9)$ & $22(3.0)$ & $49(3.8)$ & $20(0.9)$ & $-3(1.1)$ & ( \\
\hline Hong Kong SAR & $78(3.7)$ & $22(3.7)$ & $29(4.2)$ & $33(4.1)$ & $19(3.5)$ & $19(2.6)$ & $13(0.7)$ & $0(1.2)$ & \\
\hline Hungary & $97(1.6)$ & $3(1.6)$ & $6(2.1)$ & $21(3.1)$ & $48(3.7)$ & $24(3.5)$ & $21(0.7)$ & $3(1.0)$ & 0 \\
\hline Iceland & $93(0.2)$ & $7(0.2)$ & $13(0.3)$ & $31(0.4)$ & $35(0.4)$ & $22(0.3)$ & $12(0.1)$ & $-1(0.1)$ & ( \\
\hline Indonesia & $56(3.9)$ & $44(3.9)$ & $13(2.2)$ & $31(4.1)$ & $39(3.9)$ & $17(3.0)$ & $16(0.8)$ & $\Delta \Delta$ & \\
\hline Iran, Islamic Rep. of & $50(2.3)$ & $50(2.3)$ & $8(1.6)$ & $44(3.6)$ & $41(3.5)$ & $7(1.9)$ & $17(0.5)$ & $3(0.8)$ & 0 \\
\hline Israel & $92(1.9)$ & $8(1.9)$ & $8(1.8)$ & $41(4.0)$ & $33(4.1)$ & $18(3.4)$ & $16(0.8)$ & $2(1.1)$ & \\
\hline Italy & $98(1.1)$ & $2(1.1)$ & $2(1.3)$ & $14(2.5)$ & $37(3.5)$ & $47(3.6)$ & $22(0.7)$ & $1(1.0)$ & \\
\hline Kuwait & $86(2.3)$ & $14(2.3)$ & $36(3.8)$ & $48(3.8)$ & $15(3.1)$ & $1(0.0)$ & $8(0.6)$ & $\Delta \Delta$ & \\
\hline Latvia & $99(0.6)$ & $1(0.0)$ & $7(2.0)$ & $30(3.6)$ & $34(3.6)$ & $29(3.4)$ & $21(0.8)$ & $2(1.3)$ & \\
\hline Lithuania & $99(0.5)$ & $1(0.5)$ & $1(0.7)$ & $35(3.4)$ & $40(3.2)$ & $23(3.2)$ & $21(0.6)$ & $1(1.1)$ & \\
\hline Luxembourg & $55(0.2)$ & $45(0.2)$ & $32(0.2)$ & $24(0.1)$ & $16(0.1)$ & $27(0.1)$ & $15(0.0)$ & $\Delta \Delta$ & \\
\hline Macedonia, Rep. of & $70(3.5)$ & $30(3.5)$ & $5(1.7)$ & $24(3.4)$ & $40(4.1)$ & $31(4.2)$ & $20(1.0)$ & $0(1.5)$ & \\
\hline Moldova, Rep. of & $90(2.8)$ & $10(2.8)$ & $8(2.3)$ & $23(3.7)$ & $36(4.3)$ & $33(4.2)$ & $24(0.9)$ & $5(1.2)$ & 0 \\
\hline Morocco & $56(4.0)$ & $44(4.0)$ & $18(3.0)$ & $25(3.5)$ & $40(3.4)$ & $17(2.9)$ & $17(0.6)$ & $1(1.0)$ & \\
\hline Netherlands & $68(3.4)$ & $32(3.4)$ & $26(3.5)$ & $19(3.0)$ & $19(3.5)$ & $36(4.0)$ & $17(1.0)$ & $0(1.4)$ & \\
\hline New Zealand & $77(2.7)$ & $23(2.7)$ & $22(2.1)$ & $28(2.6)$ & $26(2.4)$ & $25(2.4)$ & $12(0.6)$ & $-1(1.1)$ & \\
\hline Norway & $91(1.9)$ & $9(1.9)$ & $9(2.7)$ & $28(3.3)$ & $29(3.9)$ & $35(3.9)$ & $16(1.0)$ & $-1(1.3)$ & \\
\hline Poland & $100(0.3)$ & $0(0.3)$ & $2(1.1)$ & $31(3.4)$ & $58(4.0)$ & $8(2.3)$ & $20(0.5)$ & $\Delta \diamond$ & \\
\hline Qatar & $90(0.1)$ & $10(0.1)$ & $27(0.2)$ & $54(0.3)$ & $16(0.2)$ & $3(0.1)$ & $11(0.0)$ & $\Delta \Delta$ & \\
\hline Romania & $89(2.4)$ & $11(2.4)$ & $18(3.1)$ & $25(3.3)$ & $27(3.5)$ & $30(3.2)$ & $22(0.9)$ & $2(1.2)$ & \\
\hline Russian Federation & $98(1.1)$ & $2(1.1)$ & $6(1.6)$ & $38(3.7)$ & $35(3.5)$ & $21(2.8)$ & $22(0.6)$ & $2(1.0)$ & 0 \\
\hline Scotland & $96(1.6)$ & $4(1.6)$ & $26(3.8)$ & $19(2.8)$ & $18(3.5)$ & $37(4.2)$ & $16(1.1)$ & $-2(1.5)$ & \\
\hline Singapore & $75(2.2)$ & $25(2.2)$ & $37(2.6)$ & $42(3.0)$ & $16(2.4)$ & $6(1.0)$ & $9(0.4)$ & $-2(1.0)$ & (1) \\
\hline Slovak Republic & $93(1.7)$ & $7(1.7)$ & $13(2.4)$ & $37(3.5)$ & $25(2.7)$ & $25(3.2)$ & $17(0.8)$ & $0(1.2)$ & \\
\hline Slovenia & $98(1.1)$ & $2(1.1)$ & $12(2.2)$ & $24(2.9)$ & $44(3.0)$ & $19(2.4)$ & $19(0.7)$ & $0(1.0)$ & \\
\hline South Africa & $71(2.5)$ & $29(2.5)$ & $4(1.2)$ & $44(2.8)$ & $32(2.9)$ & $20(2.3)$ & $15(0.4)$ & $\Delta \diamond$ & \\
\hline Spain & $78(3.6)$ & $22(3.6)$ & $11(2.4)$ & $16(2.6)$ & $25(3.4)$ & $49(3.8)$ & $22(0.9)$ & $\Delta \Delta$ & \\
\hline Sweden & $84(2.8)$ & $16(2.8)$ & $9(1.7)$ & $29(3.3)$ & $24(2.9)$ & $38(3.6)$ & $17(1.0)$ & $1(1.2)$ & \\
\hline Trinidad and Tobago & $82(3.0)$ & $18(3.0)$ & $11(2.5)$ & $37(3.6)$ & $28(3.3)$ & $24(3.0)$ & $19(0.7)$ & $\Delta \Delta$ & \\
\hline United States & $85(2.7)$ & $15(2.7)$ & $21(2.8)$ & $27(2.8)$ & $28(3.7)$ & $25(3.4)$ & $12(0.7)$ & $-3(1.1)$ & ( \\
\hline International Avg. & $83(0.4)$ & $17(0.4)$ & $15(0.4)$ & $30(0.5)$ & $30(0.5)$ & $25(0.5)$ & $17(0.1)$ & $0(0.0)$ & \\
\hline
\end{tabular}

Background data provided by teachers.

() Standard errors appear in parentheses. Because results are rounded to the nearest whole number, some totals may appear inconsistent.

$\mathrm{An}$ " $\mathrm{r}$ " indicates data are available for $70-84 \%$ of the students. An " $s$ " indicates data are available for $50-69 \%$ of the students. An " $x$ " indicates data are available for less than $50 \%$ of the students.
A diamond $(\diamond)$ indicates the country did not participate in the 2001 assessment. NOTE: The International Average does not include the results from the Canadian provinces. Trend Note: The primary education systems of the Russian Federation and Slovenia underwent structural changes. Data for Canada, Ontario include only public schools. 
Exhibit 6.4 Teachers' Teaching Responsibilities

PIRLS 2006

4th Grade

\begin{tabular}{|c|c|c|c|c|c|c|c|}
\hline \multirow[b]{2}{*}{ Countries } & & \multicolumn{2}{|c|}{$\begin{array}{l}\text { Percentage of Students } \\
\text { Whose Teachers Work }\end{array}$} & \multicolumn{4}{|c|}{$\begin{array}{l}\text { Percentage of Students } \\
\text { Whose Teachers Reported }\end{array}$} \\
\hline & & Full Time & Part Time & $\begin{array}{l}\text { Being the Only } \\
\text { Teacher for All or } \\
\text { Most of the Time }\end{array}$ & $\begin{array}{l}\text { That Students } \\
\text { Have Specialist } \\
\text { Teachers for } \\
\text { Core Subjects }\end{array}$ & $\begin{array}{l}\text { Sharing Teaching } \\
\text { Responsibilities } \\
\text { with Another } \\
\text { Teacher }\end{array}$ & $\begin{array}{l}\text { Other } \\
\text { Situations }\end{array}$ \\
\hline Austria & & $90(2.2)$ & $10(2.2)$ & $67(3.6)$ & $10(2.0)$ & $9(1.5)$ & $15(2.8)$ \\
\hline Belgium (Flemish) & & $91(1.7)$ & $9(1.7)$ & $50(3.5)$ & $20(3.5)$ & $22(3.2)$ & $8(1.7)$ \\
\hline Belgium (French) & & $91(1.9)$ & $9(1.9)$ & $27(3.1)$ & $42(3.1)$ & $17(2.6)$ & $14(2.3)$ \\
\hline Bulgaria & & $99(0.9)$ & $1(0.9)$ & $26(3.7)$ & $55(4.5)$ & $18(3.1)$ & $2(1.0)$ \\
\hline Canada, Alberta & & $92(2.4)$ & $8(2.4)$ & $61(3.7)$ & $13(2.5)$ & $15(2.7)$ & $10(2.2)$ \\
\hline Canada, British Columbia & $r$ & $89(2.3)$ & $11(2.3)$ & $63(4.0)$ & $10(2.6)$ & $17(3.0)$ & $10(2.4)$ \\
\hline Canada, Nova Scotia & & $94(1.7)$ & $6(1.7)$ & $68(3.6)$ & $17(3.0)$ & $8(2.0)$ & $6(1.2)$ \\
\hline Canada, Ontario & & $98(1.1)$ & $2(1.1)$ & $62(4.8)$ & $19(4.0)$ & $11(3.2)$ & $8(2.5)$ \\
\hline Canada, Quebec & & $89(2.9)$ & $11(2.9)$ & $44(4.4)$ & $32(4.3)$ & $15(3.2)$ & $9(2.6)$ \\
\hline Chinese Taipei & & $98(1.1)$ & $2(1.1)$ & $53(3.9)$ & $41(4.0)$ & $3(1.6)$ & $3(1.4)$ \\
\hline Denmark & & $95(1.6)$ & $5(1.6)$ & $2(1.0)$ & $97(1.3)$ & $0(0.0)$ & $1(0.7)$ \\
\hline England & & $91(2.6)$ & $9(2.6)$ & $51(4.5)$ & $20(2.9)$ & $14(3.4)$ & $15(3.0)$ \\
\hline France & & $91(2.0)$ & $9(2.0)$ & $30(3.7)$ & $44(3.8)$ & $21(3.0)$ & $6(1.3)$ \\
\hline Georgia & & $69(3.5)$ & $31(3.5)$ & $63(3.7)$ & $29(3.9)$ & $4(1.7)$ & $4(1.8)$ \\
\hline Germany & & $57(4.0)$ & $43(4.0)$ & $30(3.3)$ & $60(3.5)$ & $3(0.9)$ & $7(1.9)$ \\
\hline Hong Kong SAR & & $99(0.6)$ & $1(0.0)$ & $6(2.1)$ & $91(2.8)$ & $3(1.9)$ & $0(0.0)$ \\
\hline Hungary & & $98(1.3)$ & $2(1.3)$ & $32(3.8)$ & $48(4.0)$ & $7(1.6)$ & $12(2.4)$ \\
\hline Iceland & & $85(0.3)$ & $15(0.3)$ & $69(0.4)$ & $8(0.2)$ & $18(0.3)$ & $5(0.2)$ \\
\hline Indonesia & & $70(3.9)$ & $30(3.9)$ & $42(4.1)$ & $39(3.8)$ & $16(2.8)$ & $3(1.2)$ \\
\hline Iran, Islamic Rep. of & & $84(2.9)$ & $16(2.9)$ & $90(2.0)$ & $3(1.1)$ & $3(1.2)$ & $4(1.4)$ \\
\hline Israel & & $90(2.8)$ & $10(2.8)$ & $41(4.2)$ & $53(4.2)$ & $3(1.4)$ & $3(1.3)$ \\
\hline Italy & & $90(2.1)$ & $10(2.1)$ & $14(2.4)$ & $63(3.3)$ & $20(2.9)$ & $2(1.2)$ \\
\hline Kuwait & & $83(3.2)$ & $17(3.2)$ & $48(3.8)$ & $48(3.8)$ & $3(1.4)$ & $1(0.0)$ \\
\hline Latvia & & $97(1.4)$ & $3(1.4)$ & $57(3.7)$ & $32(3.7)$ & $0(0.0)$ & $11(2.4)$ \\
\hline Lithuania & & $98(1.1)$ & $2(1.1)$ & $75(3.0)$ & $1(0.6)$ & $9(2.3)$ & $14(2.7)$ \\
\hline Luxembourg & & $95(0.1)$ & $5(0.1)$ & $59(0.2)$ & $10(0.1)$ & $10(0.1)$ & $21(0.2)$ \\
\hline Macedonia, Rep. of & & $100(0.0)$ & $0(0.0)$ & $61(3.4)$ & $30(3.3)$ & $1(0.9)$ & $8(2.1)$ \\
\hline Moldova, Rep. of & & $93(2.2)$ & $7(2.2)$ & $69(3.7)$ & $28(3.5)$ & $1(0.7)$ & $2(1.2)$ \\
\hline Morocco & & $91(2.7)$ & $9(2.7)$ & $30(4.0)$ & $59(4.3)$ & $7(2.0)$ & $4(1.7)$ \\
\hline Netherlands & & $64(3.9)$ & $36(3.9)$ & $49(4.5)$ & $3(1.1)$ & $42(4.2)$ & $6(2.1)$ \\
\hline New Zealand & & $97(0.8)$ & $3(0.8)$ & $75(2.5)$ & $10(1.9)$ & $8(1.4)$ & $7(1.4)$ \\
\hline Norway & & $77(3.4)$ & $23(3.4)$ & $43(4.5)$ & $21(3.4)$ & $33(4.4)$ & $4(1.5)$ \\
\hline Poland & & $98(0.8)$ & $2(0.8)$ & $17(3.0)$ & $70(3.8)$ & $6(1.8)$ & $6(2.0)$ \\
\hline Qatar & $r$ & $95(0.1)$ & $5(0.1)$ & $27(0.3)$ & $71(0.3)$ & $1(0.0)$ & $1(0.0)$ \\
\hline Romania & & $100(0.0)$ & $0(0.0)$ & $41(4.3)$ & $52(4.3)$ & $1(0.6)$ & $6(1.7)$ \\
\hline Russian Federation & & $94(1.4)$ & $6(1.4)$ & $97(1.2)$ & $2(1.0)$ & $1(0.0)$ & $0(0.0)$ \\
\hline Scotland & & $93(2.1)$ & $7(2.1)$ & $59(4.6)$ & $7(2.3)$ & $23(3.7)$ & $11(2.9)$ \\
\hline Singapore & & $99(0.7)$ & $1(0.7)$ & $54(2.8)$ & $38(2.7)$ & $3(0.8)$ & $5(1.4)$ \\
\hline Slovak Republic & & $99(0.5)$ & $1(0.5)$ & -- & -- & -- & -- \\
\hline Slovenia & & $99(0.6)$ & $1(0.6)$ & $53(3.7)$ & $35(3.3)$ & $3(1.1)$ & $10(2.3)$ \\
\hline South Africa & & $96(1.3)$ & $4(1.3)$ & $15(2.2)$ & $69(2.7)$ & $14(1.9)$ & $2(0.7)$ \\
\hline Spain & & $91(2.3)$ & $9(2.3)$ & $48(4.1)$ & $23(3.6)$ & $12(2.6)$ & $17(2.9)$ \\
\hline Sweden & & $84(2.8)$ & $16(2.8)$ & $38(4.2)$ & $33(3.4)$ & $26(3.3)$ & $4(1.3)$ \\
\hline Trinidad and Tobago & & $100(0.4)$ & $0(0.0)$ & $83(3.0)$ & $9(2.5)$ & $5(1.5)$ & $4(1.6)$ \\
\hline United States & & $98(0.9)$ & $2(0.9)$ & $49(3.6)$ & $24(3.8)$ & $15(2.6)$ & $12(3.2)$ \\
\hline International Avg. & & $91(0.3)$ & $9(0.3)$ & $47(0.5)$ & $36(0.5)$ & $10(0.3)$ & $7(0.3)$ \\
\hline
\end{tabular}

Background data provided by teachers.

() Standard errors appear in parentheses. Because results are rounded to the nearest whole number, some totals may appear inconsistent.

$A n$ " $r$ " indicates data are available for $70-84 \%$ of the students. An " $s$ " indicates data are available for $50-69 \%$ of the students. An " $x$ " indicates data are available for less than $50 \%$ of the students.
A dash (-) indicates comparable data are not available.

NOTE: The International Average does not include the results from the Canadian provinces. 
taught by full-time teachers. Only in Germany and the Netherlands were more than one third of the students taught by part-time teachers. In terms of teaching assignments, 47 percent of students, internationally on average, were taught by the same teacher for all or most subjects, while 36 percent were taught by specialist teachers for core subjects. There were few countries where, almost exclusively, students were taught by the same teacher for all or most subjects (the Russian Federation with $97 \%$ of students and Iran with 90\%) or almost exclusively taught by a specialist teacher for core subjects (Denmark with $97 \%$ of students and Hong Kong SAR with 91\%). One fourth or more of the students in the Netherlands, Norway, and Sweden had teachers who shared teaching responsibilities with another teacher, but this was relatively rare ( $10 \%$ of students), internationally on average. 


\section{What Instructional Resources Do Teachers Use?}

Primary schools use a variety of instructional materials for their reading instructional programs. Exhibit 6.5 presents principals' reports of the percentage of students in schools using a reading series, textbooks, children's books, or other materials for reading instruction, either as a basis or a supplement. Textbooks and reading series are the materials most widely used for reading instruction across countries, with 77 percent and 42 percent of students, on average respectively, attending schools that use them as a basis for instruction. Many countries use not one but several types of reading materials as supplement(s) for instruction, including a variety of children's books, children's newspapers or magazines, and materials from different curricular areas. Countries where more than 80 percent of students were in schools using a variety of children's books as a supplement for instruction included Belgium (Flemish), Bulgaria, Georgia, Hungary, Iceland, Latvia, Lithuania, Norway, Poland, the Russian Federation, the Slovak Republic, and Slovenia. On average internationally, more than half of the students attended schools where children's newspapers or magazines and materials from other subject areas were supplemental materials for reading instruction. Also, more than one third of students across countries attended schools that used computer programs as a supplemental resource for teaching reading.

As shown in Exhibit 6.6, teachers' reports about the materials used for reading instruction corresponded to their principals' reports, in that textbooks were the foundation of reading instruction supplemented with other materials. On average internationally, 90 percent of students had reading teachers who used textbooks at least once or twice a week for reading instruction, while 82 percent had teachers who used workbooks and worksheets, and 60 percent had teachers who used a reading series at least weekly. More than half the students, on average across countries, had teachers who used a reading series (6o\%), a variety of children's books (55\%), or materials from other subjects (53\%) at least weekly. Two countries (Austria and the Slovak Republic) had at least half the students with teachers who used children's newspapers or magazines at least once a week, and another eight 
countries (Georgia, Kuwait, Macedonia, Moldova, Qatar, Singapore, South Africa, and the United States) had between one third and one half using this resource. Only 11 percent of students, on average internationally, had teachers who used computer software at least once a week, whereas approximately one fourth to one third did in Austria, Chinese Taipei, England, Hong Kong SAR, the Netherlands, Singapore, and the United States. 
Exhibit 6.5 Materials Schools Used for Their Reading Instructional Programs

PIRLS 2006

4th Grade

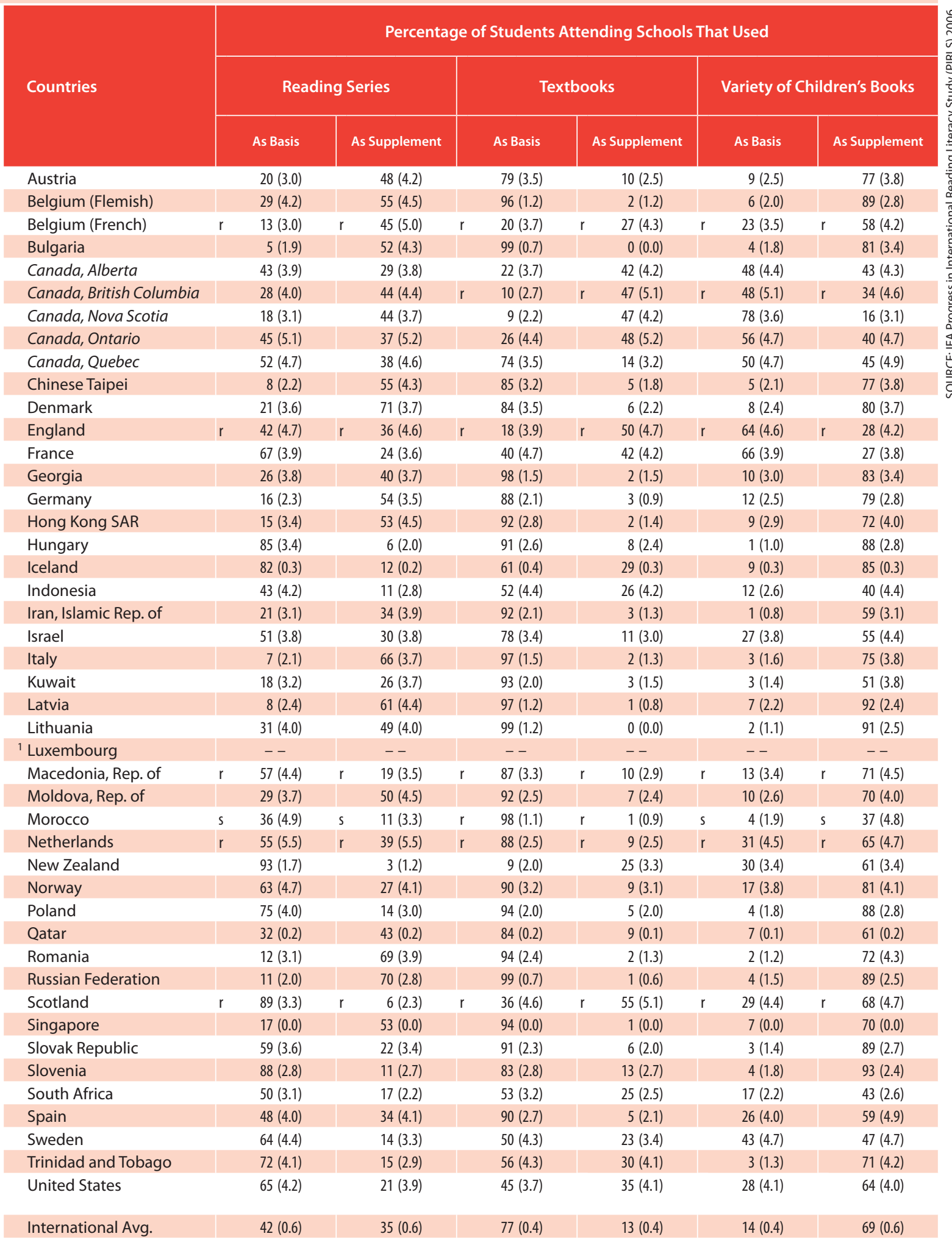

Background data provided by schools.

() Standard errors appear in parentheses. Because results are rounded to the nearest whole number, some totals may appear inconsistent.

$\mathrm{An}$ " $\mathrm{r}$ " indicates data are available for $70-84 \%$ of the students. An "s" indicates data are available for $50-69 \%$ of the students. An " $x$ " indicates data are available for less than $50 \%$ of the students.
A dash (-) indicates comparable data are not available.

NOTE: The International Average does not include the results from the Canadian provinces. 1 Primary schools in Luxembourg do not have principals. 
Exhibit 6.5 Materials Schools Used for Their Reading Instructional Programs (Continued)
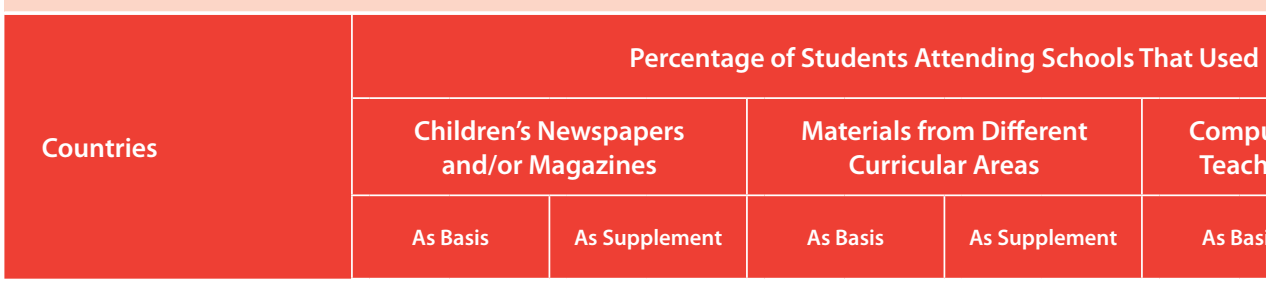

\begin{tabular}{|l|r|}
\hline Austria & \\
\hline Belgium (Flemish) & \\
\hline Belgium (French) & $r$ \\
\hline
\end{tabular}

Bulgaria

Canada, Alberta

$25(3.5) \quad 50(4.3)$

Canada, British Columbia

Canada, Nova Scotia

Canada, Ontario

Canada, Quebec

$4(1.8)$

$75(3.6)$

\begin{tabular}{|l|l|l|}
\hline \multicolumn{2}{|c|}{$\begin{array}{c}\text { Materials from Different } \\
\text { Curricular Areas }\end{array}$} & \\
\hline As Basis & As Supplement & \\
\hline
\end{tabular}

Computer Programs That Teach Students to Read

Chinese Taipei

$0(0.0)$

Denmark

England

France

$4(1.8)$

r $\quad 51(4.5)$

$21(4.0)$

$9(2.5)$

$57(4.5)$

$52(4.5)$

$r$

\begin{tabular}{l}
$65(4.4)$ \\
\hline
\end{tabular}

$52(4.5)$

$2(1.3)$

$12(3.1)$

$63(4.0)$

$6(2.3)$

$66(4.5)$

$7(2.4)$

$56(5.0)$

$3(1.4)$

55 (4.4)

$0(0.0)$

$55(4.4)$

$40(4.2)$

$9(2.3)$
$20(3.7)$

$18(3.5)$

$39(4.4)$
$28(4.1)$

$28(4.1)$
$17(2.9)$

$6(1.8)$

$49(4.5)$

$7(2.4)$
$22(3.7)$

Georgia

7 (2.1)

$54(4.8)$

$79(3.4)$

$2(1.2)$

Germany

$1(0.9)$

Hong Kong SAR

$2(1.2)$

Iceland

$0(0.0)$

Indonesia

$2(1.1)$

Iran, Islamic Rep. of

$5(1.3)$

Israel

$6(2.0)$

$60(3.8)$

$61(4.0)$

$73(4.0)$

$51(0.3)$

$9(2.4)$

$43(3.7)$

29

$29(4.0)$
$42(4.0)$

12 (2.6)

$55(3.5)$

Italy

Kuwait

$2(1.1)$

$49(3.8)$

$27(3.3)$

$32(3.7)$

Latvia

$4(1.7)$

Lithuania

$0(0.0)$

$37(4.2)$

$86(2.7)$

$69(3.7)$

${ }^{1}$ Luxembourg

Macedonia, Rep. of

$--$

Moldova, Rep. of

Morocco

$13(3.0)$

Netherlands

New Zealand

Norway

Poland

Qatar

Romania

$--$

Russian Federation

Scotland

Singapore

Slovak Republic

Slovenia

South Africa

$72(4.2)$

$\begin{array}{rr}r & 13(3.0) \\ & 2(1.3) \\ s & 0(0.0)\end{array}$

$69(4.0)$

$4(1.6)$

$4(1.6)$

\begin{tabular}{l}
$9(2.4) \quad 67(4.2)$ \\
\hline
\end{tabular}

$\begin{array}{lllll}39(4.9) & r & 1(0.0) & r & 24(4.2)\end{array}$

66

$\begin{array}{lll}66(4.2) & 0(0.0) & 18(3.2)\end{array}$

$\begin{array}{lll}59(4.4) & 3(1.4) & 40(4.3)\end{array}$

$55(4.3)$

$49(4.3)$

$61(4.5)$

$66(4.2)$

$58(4.6)$

$56(4.5)$

$1(1.1)$

$43(4.1)$

$62(4.3)$

$53(4.0)$

$56(4.4)$

$6(0.2)$

$61(4.3)$

$17(3.1)$

38

$58(0.4)$

$31(4.0)$

$31(4.0)$
$52(4.0)$
$45(4.8)$

$30(4.1)$

$45(4.8)$

$10(2.4)$

$65(4.1)$

$65(4.1)$
$28(3.4)$

$8(2.6)$

$69(4.0)$

$1(1.0)$

$86(3.0)$

$1(0.8)$

$71(4$.

$21(3.6)$

\begin{tabular}{l|l}
1 & \\
- & -
\end{tabular}

\begin{tabular}{lll}
$8(3.0)$ & $0(0.3)$ & $13(3.0)$ \\
\hline-14.1$)$ & $0(0.0)$ & $23(3.6)$
\end{tabular}

\begin{tabular}{|c|c|c|c|c|}
\hline-- & & -- & & -- \\
\hline $52(4.5)$ & $r$ & $5(2.0)$ & $r$ & $8(2.7)$ \\
\hline $46(4.7)$ & & $1(0.8)$ & & $3(1.5)$ \\
\hline
\end{tabular}

$16(3.4)$

\begin{tabular}{|c|c|c|c|c|c|c|c|c|c|c|}
\hline $2(1.3)$ & & $69(4.0)$ & & $16(3.4)$ & & $46(4.7)$ & & $1(0.8)$ & & $3(1.5)$ \\
\hline $0(0.0)$ & $S$ & $30(5.0)$ & s & $14(3.4)$ & $S$ & $28(5.0)$ & $s$ & $1(0.9)$ & $S$ & $10(3.1)$ \\
\hline$(0.0)$ & $r$ & $54(4.1)$ & $r$ & $17(3.5)$ & $r$ & $67(4.9)$ & $r$ & $33(4.8)$ & $r$ & $50(4.7)$ \\
\hline
\end{tabular}

$60(3.9)$

\begin{tabular}{l|l}
$5(1.3)$ & 48 \\
$2(1.2)$ & 4
\end{tabular}

\begin{tabular}{|l|l|}
\hline $60(3.9)$ & $15(2.6)$ \\
\hline $48(4.8)$ & $14(3.3)$ \\
\hline
\end{tabular}

\begin{tabular}{r|r|r|}
\hline $63(3.5)$ & $4(1.3)$ & $52(3.1)$ \\
\hline $58(5.4)$ & $13(3.6)$ & $63(5.2)$ \\
\hline
\end{tabular}

$1(1.0)$

$75(4.2)$

\begin{tabular}{r|r|}
\hline $50(0.3)$ & $8(2.4)$ \\
\hline & $28(0.2)$ \\
\hline
\end{tabular}

\begin{tabular}{|c|c|c|}
\hline & & \\
\hline $52(4.4)$ & $1(0.9)$ & $38(4.1)$ \\
\hline $27(0.2)$ & $13(0.1)$ & $40(0.2)$ \\
\hline
\end{tabular}

$10(0.1) \quad 50$

\begin{tabular}{|c|c|}
\hline & $\angle 010$. \\
\hline $8(4.8)$ & $6(2.2)$ \\
\hline 1 (2.8) & $4(1.3)$ \\
\hline
\end{tabular}

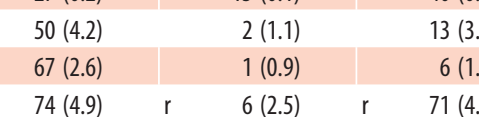

\begin{tabular}{|c|c|c|c|c|c|c|c|c|}
\hline $71(2.8)$ & & $4(1.3)$ & & $67(2.6)$ & & $1(0.9)$ & & $6(1.8)$ \\
\hline $50(5.0)$ & $r$ & $13(3.3)$ & $r$ & $74(4.9)$ & $r$ & $6(2.5)$ & $r$ & $71(4.3)$ \\
\hline $66(0.0)$ & & $13(0.0)$ & & $50(0.0)$ & & $8(0.0)$ & & $46(0.0)$ \\
\hline $75(3.5)$ & & $2(1.0)$ & & $70(3.5)$ & & $1(1.0)$ & & $32(3.9)$ \\
\hline
\end{tabular}

75

\begin{tabular}{|c|c|}
\hline $4(31)$ & $5(1.8)$ \\
\hline
\end{tabular}

Spain

Sweden

$3(0.8)$

\begin{tabular}{l|l} 
Trinidad and Tobago & $0(0.0)$
\end{tabular}

United States

$3(1.2)$

$47(2.8)$

$47(2.8)$

$21(2.5)$

$68(4.3)$

$39(2.9)$

\begin{tabular}{ll}
$5(1.5)$ & $65(4.2)$ \\
\hline $4(1.6)$ & $13(1.7)$ \\
\hline $4(1.9)$ & $49(3.8)$ \\
\hline
\end{tabular}

$32(4.5)$

$22(3.9)$
$16(3.2)$

$63(4.4)$

\begin{tabular}{r|r}
\hline $70(2.6)$ & $7(2.2)$ \\
\hline
\end{tabular}

$16(3.0)$

$68(3.8)$

$57(0.6) \quad 13(0.4)$

$55(0.7)$

$6(0.3)$

$4(0.3)$

$55(0.7)$


Exhibit 6.6 Materials Used by Teachers for Reading Instruction

PIRLS 2006
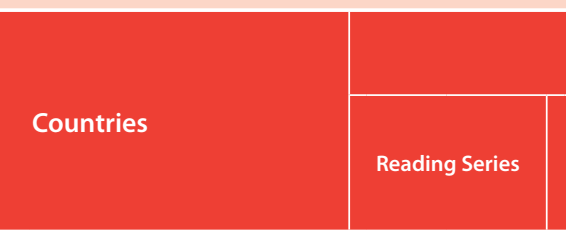

Percentage of Students Whose Teacher Used at Least Weekly

Austria
Belgium (Flemish)
Belgium (French)
Bulgaria
Canada, Alberta
Canada, British Columbia
Canada, Nova Scotia

Canada, Ontario

Canada, Quebec

$30(3.2)$
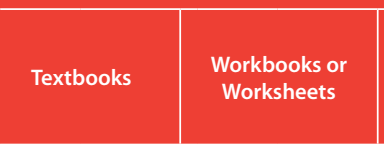

\begin{tabular}{|c|}
$\begin{array}{c}\text { Variety of } \\
\text { Children's } \\
\text { Books }\end{array}$ \\
\hline
\end{tabular}

\begin{tabular}{c|} 
Children's \\
Newspapers \\
and/or \\
Magazines
\end{tabular}

\begin{tabular}{c|c} 
Materials \\
from Other \\
Subjects
\end{tabular}

4th Grade

\section{Chinese Taipei}

$35(4.6) \quad 92(1.6)$

$30(3.4)$

$92(1.6)$

$95(1.8)$

$50(3.7)$

$89(2.4)$

$52(3.4)$

$61(3.4)$

$43(3.7)$

$100(0.0)$

$91(2.1)$

$47(4.8) \quad r \quad 66(3.6)$

Denmark

England

$35(3.5)$

$55(4.9)$

4714

$66(3.6) \quad r$

France

Georgia

$45(4.8)$

$68(4.5)$

$68(4.5)$
$89(2.1)$

$37(4.1)$

95 (1.9)

$88(2.4)$

69 (4.1)

$49(4.1)$

$74(3.3)$

$66(4.2)$

Germany

$63(4.1)$

Hong Kong SAR

Hungary

$20(3.6)$

$62(3.2)$

$99(0.7)$

$99(0.7)$
$83(2.7)$

$36(3.8) \quad 97(1.5)$

$99(0.8)$

$100(0.0)$

Iceland

Indonesia

$80(0.3)$

Iran, Islamic Rep. of

$57(3.8)$

$96(0.2)$

Israel

$51(3.7)$

$67(4.0)$

96 (1.6)

Italy

Kuwait

$47(3.6)$

Latvia

Lithuania

69 (3.4)

$98(1.0)$

$96(1.8)$

$64(4.2)$

52 (3.8)

$99(0.5)$

$68(3.6)$

$65(4.4) \quad r \quad 91(2.7)$

68 (3.8)

$77(4.4)$

$82(3.3)$

$61(4.2)$

$74(3.4)$

$65(4.0)$

$63(3.3)$

$68(3.8)$
$92(1.4)$

$71(3.7)$

$99(0.8)$

$86(0.2)$
$73(4.0)$

$64(3.8)$
$93(2.3)$

$99(1.0)$

$92(2.1)$
$89(2.3)$

$99(1.0)$

\begin{tabular}{l|l}
$31(3.1)$ & $100(0.0)$
\end{tabular}

$74(2.7)$

$41(3.6) \quad 100(0.0)$

Luxembourg

Macedonia, Rep. of

\begin{tabular}{l|l}
$8(0.1)$ & $95(0.1)$
\end{tabular}

Moldova, Rep. of

$36(4.0)$

$100(0.0)$

Morocco

$81(3.3) \quad 100(0.3)$

Netherlands

$74(4.0)$

$86(3.1)$

$74(2.7)$

$95(1.8)$

$85(0.1)$
$80(3.4)$

$86(2.7)$

$91(2.6)$

$94(1.8)$

New Zealand

$51(4.3)$

$20(2.3)$

Norway

Poland

$76(3.5) \quad 98(1.3)$

$95(1.6) \quad 100(0.0)$

Qatar

Romania

s $\quad 62(0.2)$

$100(0.0)$

Russian Federation

$92(1.9) \quad 100(0.1)$

\begin{tabular}{l|l}
$56(3.7)$ & $100(0.0)$
\end{tabular}

Scotland

Singapore

Slovak Republic

$95(1.8)$

$100(0.0)$

\begin{tabular}{l|l}
$34(2.5)$ & $97(1.0)$
\end{tabular}

$16(2.4) \quad 100(0.0)$

$95(1.5) \quad 94(1.6)$

Slovenia

South Africa

63 (3.2)

$94(1.6)$

Spain

Sweden

$75(3.9)$

$94(1.4)$

$52(3.7)$

$82(2.7)$

\begin{tabular}{l|l}
$88(2.6)$ & $99(0.5)$
\end{tabular}

Trinidad and Tobago

United States

$69(4.1)$

$82(1.8)$

$60(0.5)$

$90(0.3)$

$76(3.5)$

$65(2.8)$

$90(2.9)$

\section{$97(1.2)$}

s $\quad 96(0.1)$

$91(2.4)$

$53(3.6)$

$53(3.6)$
$82(3.6)$

$97(1.0)$

$77(3.3)$

$97(1.4)$
$80(2.3)$

$84(3.2)$

$96(1.4)$
$91(2.8)$

$70(4.3)$

$49(4.3)$

$60(3.7)$

$60(3.7)$
$93(1.7)$

$72(3.0)$

\section{$67(3.8)$}

$42(3.7)$

$25(3.6)$

$63(3.7)$

$83(0.3)$

$19(3.2)$

$19(3.2)$
$31(2.9)$

$18(2.8)$

$+25$

$58(3.3) \quad 26(2.8)$

\begin{tabular}{l|l}
$50(3.9)$ & $8(2.1)$
\end{tabular}

$\begin{array}{ll}50(3.9) & 8(2.1) \\ 55(3.5) & 4(1.6)\end{array}$

\begin{tabular}{l|l}
$57(3.7)$ & $4(1.8)$
\end{tabular}

$13(3.0)$

$13(3.0) \quad r$

$20(3.2)$

$20(4.5)$

\section{$14(2.9)$}

$31(3.9)$

$2(1.1)$
$9(2.6)$

$20(3.3)$

$40(4.4)$

$24(3.5)$

$8(2.0)$

$26(3.5)$

$44(3.5)$

$5(0.2)$

$5(0.2)$
$9(2.4)$

$51(4.4)$

$25(3.2)$

$54(4.0)$

$22(3.6)$

$41(3.9)$

\section{$45(3.7)$}

$45(3.5)$

$16(2.9)$

$16(2.9)$

11 (2.1)

$34(0.2)$

$14(2.3)$

$29(3.8)$

$15(0.1)$

$53(4.2)$

$10(2.6)$

$37(4.1)$

$46(4.3)$

$79(3.0)$

5 (1.7)

82 (3.2)

$78(2.5)$

$9(2.4)$

$84(3.5)$

$9(2.4)$
$18(2.2)$

\section{$36(3.9)$}

7 (2.4)

$79(3.9) \quad r \quad 15(3.2)$

International Avg.

$60(0.5)$

$90(0.3)$

$71(3.6)$

$39(0.3)$
$66(4.1)$

$6(1.7)$

\begin{tabular}{ll}
$87(2.4)$ & $14(2.4)$ \\
\hline $83(4.1)$ & $17(3.6)$ \\
\hline
\end{tabular}

\begin{tabular}{l|l}
$83(4.1)$ & $17(3.6)$
\end{tabular}

$\begin{array}{ll}83(4.1) & 17(3.6) \\ 69(4.0) & 4(1.4)\end{array}$

\begin{tabular}{r|r}
$23(3.6)$ & $24(3.5)$ \\
$21(3.5)$ & $4(1.3)$
\end{tabular}

$\begin{array}{rr}21(3.5) & 4(1.3) \\ 75(4.1) & 32(4.0)\end{array}$

\begin{tabular}{l|l}
$82(2.5)$ & $3(1.2)$ \\
$52(3.8)$ & $0(0.0)$
\end{tabular}

\begin{tabular}{l|l}
$52(3.8)$ & $0(0.0)$ \\
\hline $68(3.5)$
\end{tabular}

\begin{tabular}{|l|l|}
\hline $68(3.5)$ & $15(2.2)$ \\
\hline $12(2.7)$ & $36(4.2)$ \\
\hline
\end{tabular}

\begin{tabular}{|r|r|}
\hline $12(2.7)$ & $36(4.2)$ \\
\hline $67(3.6)$ & $2(0.7)$ \\
\hline
\end{tabular}

\begin{tabular}{r|r|}
\hline $82(0.3)$ & $12(0.2)$ \\
\hline $29(3.8)$ & $3(1.6)$ \\
\hline $27(3.5)$ & $4(1.5)$ \\
\hline
\end{tabular}

\begin{tabular}{|l|l|}
\hline $27(3.5)$ & $4(1.5)$ \\
\hline $45(4.1)$ & $6(2.1)$ \\
\hline $52(4.1)$ & $5(1.8)$ \\
\hline
\end{tabular}

\begin{tabular}{|l|l|}
\hline $52(4.1)$ & $5(1.8)$ \\
\hline $27(3.8)$ & $7(2.1)$ \\
\hline
\end{tabular}

\begin{tabular}{|l|l|}
\hline $27(3.8)$ & $7(2.1)$ \\
\hline $49(4.1)$ & $2(1.2)$ \\
\hline $47(3.7)$ & $2(0.9)$ \\
\hline
\end{tabular}

\begin{tabular}{|l|l|}
\hline $47(3.7)$ & $2(0.9)$ \\
\hline $33(0.2)$ & $3(0.1)$ \\
\hline
\end{tabular}

\begin{tabular}{|l|l|}
\hline $33(0.2)$ & $3(0.1)$ \\
\hline $46(4.0)$ & $6(2.4)$ \\
\hline $63(3.9)$ & $2(0.4)$ \\
\hline $35(4.4)$ & $2(1.1)$ \\
\hline
\end{tabular}

\begin{tabular}{|r|r|}
\hline $63(3.9)$ & $2(0.4)$ \\
\hline $35(4.4)$ & $2(1.1)$ \\
\hline $64(4.1)$ & $26(4.2)$ \\
\hline $61(2.8)$ & $18(1.9)$ \\
\hline
\end{tabular}

\begin{tabular}{ll}
$61(2.8)$ & $26(4.2)$ \\
$82(3.1)$ & $18(1.9)$ \\
\hline $43(4.0)$ & $20(4.0)$
\end{tabular}


Exhibits 6.7 through 6.9 present teachers' reports of how often they asked students to read literary and informational texts, as well as trends in the percentages of students whose teachers used literary and informational texts at least once a week. To gather information about the use of different types of texts for the two major reading purposes in primary schools, PIRLS asked teachers about the frequency with which they used different types of texts with the following response options: every day or almost every day, once or twice a week, once or twice a month, and never or almost never. For reporting purposes, the first two response categories were combined into the reporting category at least weekly, and the last two were combined into less than weekly.

Exhibit 6.7 shows the percentage of students whose teachers asked them to read the following types of literary texts at least once a week:

- Short stories

- Longer books with chapters

- Poems

- Plays

On average internationally, 70 percent of the students were asked to read short stories at least weekly. About one third (36\%) were asked to read chapter books, and about one third (36\%) to read poems. Just 8 percent of students, on average across countries, were asked to read plays at least once a week, although in Macedonia, Moldova, New Zealand, and South Africa, the percentage was greater than one fifth (21-36\%). On average across countries, those students asked to read fiction at least weekly had somewhat higher achievement (difference of eight points) than those asked to read it less than weekly.

Exhibit 6.8 shows the percentage of students whose teachers asked them to read the following types of informational texts at least once a week:

- Descriptions and explanations about things, people, or events

- Instructions of manuals about how things work

- Charts, diagrams, or graphs 
On average internationally, about half the students (49\%) were asked to read descriptions and explanations at least weekly, while fewer were asked to read instructions or manuals (19\%) or charts, diagrams, or graphs $(26 \%)$. Most of the students (82 to $85 \%$ ) in Austria, Germany, Hungary, and Italy were asked to read descriptions and explanations about things, people, or events at least weekly.

The percentage of students whose teachers asked them to read informational texts at least once a week was less than those asked to read literary texts ( $58 \%$ vs. $84 \%$ ). Interestingly, the six countries and two provinces with the highest percentage of students (between 80 and 90\%) who were asked to read nonfiction at least once a week were among those with the smallest relative differences between informational and literary achievement scores (less than 5 points, though nonsignificant only in Austria in Italy). The average achievement across countries of those students asked to read nonfiction at least weekly differed only slightly from the achievement of those asked to read nonfiction less than weekly.

Exhibit 6.9 shows changes from 2001 in the percentage of students whose teachers asked them to read literary and informational texts at least once a week. Although, on average internationally, there was no change over the 5-year period in the percentage of students asked to read fiction, there were changes for a number of participants. Increases occurred in France, the Netherlands, Singapore, Sweden, and the Canadian province of Quebec, while decreases occurred in Latvia, Morocco, New Zealand, and Romania. There were more increases across participants in weekly nonfiction reading assignments, including Bulgaria, Germany, Hungary, Israel, Italy, Moldova, the Russian Federation, Slovenia, Sweden, and the Canadian province of Quebec. Only in Iceland was there a significant decrease in the percentage of students asked to read nonfiction at least weekly. An increase in the percentage of students asked to read both fiction and nonfiction took place in Sweden and the Canadian province of Quebec. 
Exhibit 6.7 Teachers' Use of Literary Texts for Reading Instruction

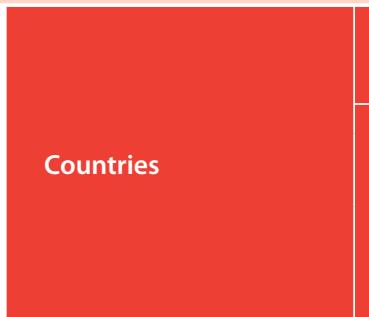

Percentage of Students Whose Teachers Asked Them to Read at Least Weekly

Austria
Belgium (Flemish)
Belgium (French)

Belgium (French)

Bulgaria

Canada, Alberto

Canada, British Colur

Canada, Nova Scotia

Canada, Ontario

Canada, Quebec

Chinese Taipei

Denmark

England

France

Georgia

Germany

Hong Kong SAR

Hungary

Iceland

Indonesia

Iran, Islamic Rep. of

Israel

Italy

Kuwait

Latvia

Lithuania

Luxembourg

Macedonia, Rep. of

Moldova, Rep. of

Morocco

Netherlands

New Zealand

Norway

Poland

Qatar

Romania

Russian Federation

Scotland

Singapore

Slovak Republic

Slovenia

South Africa

Spain

Sweden

Trinidad and Tobago

United States

International Avg.
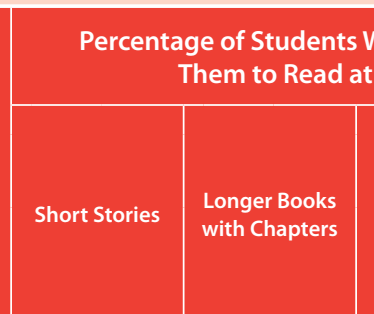

$83(2.9)$

$57(4.3)$

$59(3.3)$

$91(2.3)$

$71(3.9)$

$74(3.7)$
$67(3.9)$

$66(4.5)$

$51(4.5)$

$61(4.6)$

$76(3.3)$

$55(4.0)$

$66(3.6)$

$92(1.6)$

$60(3.4)$

$42(4.0)$

$92(1.8)$

$63(0.4)$

$65(3.4)$

$60(3.5)$

78 (3.3)

$95(1.4)$

$20(3.6)$

$83(2.8)$

$83(2.7)$

$68(0.2)$

$76(3.5)$

$92(2.4)$

25 (3.8)

$60(4.4)$

$55(3.0)$

$73(3.5)$

84 (3.5)

5 $\quad 39(0.3)$

$88(2.4)$

$80(2.1)$

63 (4.4)

76 (2.4)

$83(2.7)$

$83(2.5)$

$72(2.8)$

86 (2.8)

51 (4.3)

$86(2.9)$

$70(4.0)$

$70(4.0)$

$27(3.0)$

$29(3.7)$

$16(2.8)$

$14(2.8)$

$81(3.0)$

84 (3.4)

$87(2.2)$

$75(4.5)$

31 (3.8)

$15(3.1)$

$63(3.6)$

$60(4.3)$

69 (3.2)

$13(2.5)$

$18(3.0)$

$5(1.9)$

$29(3.6)$

$75(0.3)$

$17(3.0)$

$23(3.3)$

$34(3.8)$

$32(3.8)$

$5(1.9)$

$26(3.1)$

$33(3.0)$

$21(0.2)$

$11(3.0)$

$16(2.9)$

$26(4.0)$

$85(2.6)$

$66(3.0)$

83 (3.4)

$24(3.3)$

$4(0.1)$

$21(3.3)$

68 (3.7)

$77(4.2)$

$27(2.4)$

$28(2.7)$

7 (1.7)

$32(3.1)$

$51(4.4)$

$97(1.1)$

$35(3.8)$

74 (3.8)

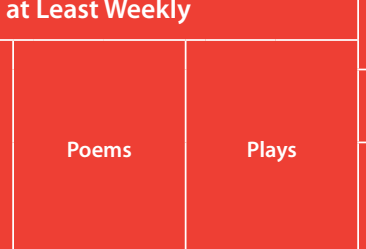

22 (2.9)

$20(3.1)$

8 (2.1)

$75(3.2)$

$26(3.5)$

$24(3.8)$

$31(3.5)$

$13(2.7)$

$7(2.0)$

15 (3.1)

19 (3.3)

$20(3.4)$

35 (3.6)

$72(4.1)$

$13(2.8)$

$15(3.3)$

$55(4.1)$

$34(0.4)$

44 (4.4)

$44(3.6)$

31 (3.7)

39 (3.9)

$41(4.3)$

$29(3.2)$

$57(3.5)$

$4(0.1)$

$60(4.1)$

$88(2.8)$

$26(3.9)$

$4(1.5)$

$43(3.0)$

$25(3.9)$

$85(2.8)$

$26(0.2)$

$$
68(3.7)
$$

$68(3.8)$

$14(2.5)$

$27(2.2)$

$67(3.3)$

17 (2.7)

$32(3.2)$

$26(3.4)$

$3(1.1)$

$64(3.7)$

$20(3.2)$

$1(0.5)$
$0(0.0)$
$2(1.1)$

$0(0.0)$
$2(1.1)$

$4(1.5)$

$6(1.6)$

$9(2.7)$
$4(1.5)$

$10(2.7)$

$3(1.3)$

$1(0.6)$
$1(0.6)$

$6(2.1)$

$11(2.4)$

$11(2.8)$

$1(0.4)$

2 (1.1)

$2(1.1)$

$2(0.1)$

$13(2.6)$

$17(2.9)$

$6(2.2)$

$6(1.7)$

$13(2.7)$

$3(1.3)$

$13(2.3)$

$3(0.0)$

$36(4.0)$

$21(3.8)$

$3(1.3)$

$1(0.8)$

$24(3.0)$

$2(1.3)$

$3(1.1)$

$10(0.2)$

$36(0.5)$

$36(0.5)$

$8(0.3)$

PIRLS 2006

4th Grade
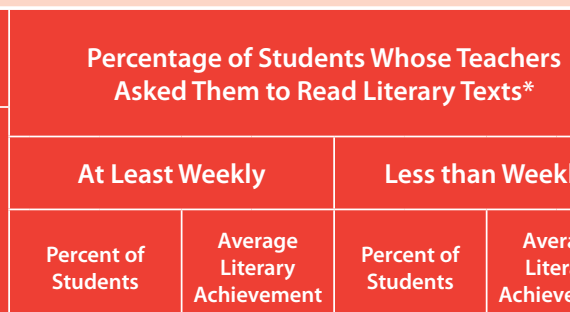

$90(1.9) \quad 537(2.2)$

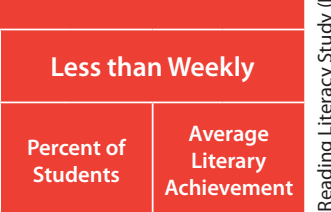

$10(1.9) \quad 535(9.1)$

$543(2.0)$

$30(3.8)$

$545(3.8)$

$67(3.2) \quad 499(2.9)$

$33(3.2)$

$500(5.0)$

\begin{tabular}{|l|l|l|l|}
$94(1.9)$ & $543(4.9)$ & $6(1.9)$ & $513(14.4)$ \\
\hline
\end{tabular}

$92(2.4)$

$563(2.6)$

$96(1.5) \quad 560(3.2)$

$96(1.3)$

544 (2.7)

8 (2.4)

$539(12.6)$

$87(3.9)$

$67(3.9)$

$557(2.9)$

$4(1.5)$

$561(11.3)$

$4(1.3) \quad 520(12.3)$

$67(4.2)$

$530(3.3)$

$13(3.9)$

$33(3.9)$

540 (12.7)

$90(2.4) \quad 548(2.8)$

$33(4.2)$

$533(4.9)$

$75(3.8)$

$91(2.2)$

$542(3.5)$

$33(4.2)$

$532(2.8)$

$97(1.2)$

$516(2.6)$

$10(2.4) \quad 546(6.6)$

$69(3.4)$

478 (3.4)

$25(3.8)$

537 (6.6)

50 (4.1)

$50(4.1)$
$97(1.0)$

$549(2.5)$

$9(2.2)$

$518(4.7)$

$$
91(0.2)
$$

561 (3.6)

$3(1.2) \quad 454(14.3)$

$74(3.4)$

$555(3.1)$

31 (3.4)

$550(3.2)$ 
Exhibit 6.8 Teachers' Use of Informational Texts for Reading Instruction

\begin{tabular}{|c|c|c|c|c|c|c|c|}
\hline \multirow{3}{*}{ Countries } & \multicolumn{3}{|c|}{$\begin{array}{l}\text { Percentage of Students Whose Teachers } \\
\text { Asked Them to Read at Least Weekly }\end{array}$} & \multicolumn{4}{|c|}{$\begin{array}{l}\text { Percentage of Students Whose Teachers } \\
\text { Asked Them to Read Informational Texts* }\end{array}$} \\
\hline & \multirow{2}{*}{$\begin{array}{l}\text { Descriptions } \\
\text { and } \\
\text { Explanations } \\
\text { About Things, } \\
\text { People, or } \\
\text { Events }\end{array}$} & \multirow{2}{*}{$\begin{array}{l}\text { Instructions or } \\
\text { Manuals About } \\
\text { How Things } \\
\text { Work }\end{array}$} & \multirow{2}{*}{$\begin{array}{l}\text { Charts, } \\
\text { Diagrams, } \\
\text { Graphs }\end{array}$} & \multicolumn{2}{|c|}{ At Least Weekly } & \multicolumn{2}{|c|}{ Less than Weekly } \\
\hline & & & & $\begin{array}{l}\text { Percent of } \\
\text { Students }\end{array}$ & \begin{tabular}{c|} 
Average \\
Informational \\
Achievement
\end{tabular} & $\begin{array}{l}\text { Percent of } \\
\text { Students }\end{array}$ & $\begin{array}{c}\text { Average } \\
\text { Informationa } \\
\text { Achievement }\end{array}$ \\
\hline Austria & $83(2.4)$ & $14(2.1)$ & $7(1.7)$ & $83(2.4)$ & $537(2.6)$ & $17(2.4)$ & $536(4.5)$ \\
\hline Belgium (Flemish) & $50(4.2)$ & $12(2.5)$ & $18(3.0)$ & $55(4.0)$ & $547(2.9)$ & $45(4.0)$ & $547(2.6)$ \\
\hline Belgium (French) & $27(3.6)$ & $15(2.5)$ & $16(2.5)$ & $40(3.9)$ & $503(4.5)$ & $60(3.9)$ & $495(3.9)$ \\
\hline Bulgaria & $47(3.8)$ & $13(2.6)$ & $17(3.0)$ & $52(3.6)$ & $550(6.7)$ & $48(3.6)$ & $549(5.4)$ \\
\hline Canada, Alberta & $71(3.3)$ & $29(3.4)$ & $66(3.8)$ & $84(2.7)$ & $555(2.8)$ & $16(2.7)$ & $563(6.1)$ \\
\hline Canada, British Columbia & $66(4.4)$ & $24(3.9)$ & $57(4.0)$ & $81(3.2)$ & $553(3.3)$ & $19(3.2)$ & $559(7.5)$ \\
\hline Canada, Nova Scotia & $70(3.2)$ & $27(3.4)$ & $56(3.1)$ & $79(2.9)$ & $539(2.7)$ & $21(2.9)$ & $540(5.5)$ \\
\hline Canada, Ontario & $62(4.2)$ & $20(3.8)$ & $56(5.0)$ & $77(4.1)$ & $552(3.3)$ & $23(4.1)$ & $555(6.7)$ \\
\hline Canada, Quebec & $30(4.1)$ & $36(4.7)$ & $27(3.9)$ & $57(4.5)$ & $535(3.2)$ & $43(4.5)$ & $534(4.1)$ \\
\hline Chinese Taipei & $24(3.1)$ & $11(2.7)$ & $8(2.2)$ & $30(3.3)$ & $538(3.8)$ & $70(3.3)$ & $539(2.1)$ \\
\hline Denmark & $34(3.6)$ & $6(1.4)$ & $4(1.3)$ & $37(3.6)$ & $539(3.6)$ & $63(3.6)$ & $544(3.0)$ \\
\hline England & $45(4.4)$ & $15(3.0)$ & $37(4.4)$ & $55(4.4)$ & $536(4.3)$ & $45(4.4)$ & $543(5.1)$ \\
\hline France & $26(3.3)$ & $5(1.5)$ & $19(3.2)$ & $37(3.9)$ & $527(3.7)$ & $63(3.9)$ & $526(2.7)$ \\
\hline Georgia & $10(2.7)$ & $26(3.9)$ & $36(4.3)$ & $46(4.2)$ & $471(5.2)$ & $54(4.2)$ & $463(5.7)$ \\
\hline Germany & $82(2.5)$ & $19(2.6)$ & $31(3.5)$ & $84(2.5)$ & $546(2.4)$ & $16(2.5)$ & $543(3.8)$ \\
\hline Hong Kong SAR & $21(3.7)$ & $9(2.5)$ & $1(0.0)$ & $22(3.8)$ & $572(4.2)$ & $78(3.8)$ & $568(2.7)$ \\
\hline Hungary & $71(3.7)$ & $24(3.4)$ & $11(3.0)$ & $74(3.6)$ & $543(3.8)$ & $26(3.6)$ & $536(5.3)$ \\
\hline Iceland & $20(0.3)$ & $8(0.2)$ & $15(0.3)$ & $28(0.4)$ & $511(2.9)$ & $72(0.4)$ & $502(1.5)$ \\
\hline Indonesia & $38(4.3)$ & $45(4.3)$ & $17(3.4)$ & $60(4.4)$ & $421(4.9)$ & $40(4.4)$ & $412(6.9)$ \\
\hline Iran, Islamic Rep. of & $35(3.8)$ & $22(3.6)$ & $28(3.7)$ & $51(4.4)$ & $426(5.5)$ & $49(4.4)$ & $416(5.0)$ \\
\hline Israel & $55(3.9)$ & $21(3.7)$ & $24(3.9)$ & $63(4.1)$ & $523(5.9)$ & $37(4.1)$ & $480(9.3)$ \\
\hline Italy & $85(2.7)$ & $15(2.6)$ & $39(3.5)$ & $88(2.4)$ & $548(3.2)$ & $12(2.4)$ & $555(9.3)$ \\
\hline Kuwait & $46(4.2)$ & $20(3.5)$ & $8(2.3)$ & $51(4.1)$ & $327(6.8)$ & $49(4.1)$ & $328(7.0)$ \\
\hline Latvia & $30(3.1)$ & $9(2.2)$ & $33(3.8)$ & $45(3.6)$ & $548(3.8)$ & $55(3.6)$ & $534(3.3)$ \\
\hline Lithuania & $38(3.6)$ & $11(2.5)$ & $28(2.8)$ & $54(3.4)$ & $531(2.1)$ & $46(3.4)$ & $529(2.6)$ \\
\hline Luxembourg & $46(0.2)$ & $7(0.1)$ & $15(0.1)$ & $49(0.2)$ & $556(1.3)$ & $51(0.2)$ & $558(1.3)$ \\
\hline Macedonia, Rep. of & $46(4.0)$ & $22(3.5)$ & $23(3.8)$ & $54(4.1)$ & $453(6.5)$ & $46(4.1)$ & $456(8.6)$ \\
\hline Moldova, Rep. of & $58(4.4)$ & $37(4.4)$ & $56(4.3)$ & $79(3.2)$ & $511(3.2)$ & $21(3.2)$ & $494(6.4)$ \\
\hline Morocco & $41(4.4)$ & $8(2.6)$ & $8(2.3)$ & $44(4.4)$ & $331(8.0)$ & $56(4.4)$ & $336(10.2)$ \\
\hline Netherlands & $47(4.1)$ & $19(3.7)$ & $36(4.1)$ & $59(4.1)$ & $544(2.0)$ & $41(4.1)$ & $552(2.5)$ \\
\hline New Zealand & $65(2.8)$ & $23(2.5)$ & $26(3.0)$ & $68(2.9)$ & $534(3.0)$ & $32(2.9)$ & $538(3.8)$ \\
\hline Norway & $56(4.7)$ & $5(1.5)$ & $10(2.6)$ & $59(5.0)$ & $495(3.5)$ & $41(5.0)$ & $492(3.8)$ \\
\hline Poland & $50(3.9)$ & $10(2.2)$ & $27(3.3)$ & $62(3.8)$ & $516(3.2)$ & $38(3.8)$ & $514(2.9)$ \\
\hline Qatar & $31(0.2)$ & $36(0.3)$ & $21(0.2)$ & $51(0.3)$ & $358(2.4)$ & $49(0.3)$ & $352(2.2)$ \\
\hline Romania & $44(4.0)$ & $13(2.9)$ & $43(3.5)$ & $61(3.7)$ & $485(5.5)$ & $39(3.7)$ & $492(8.5)$ \\
\hline Russian Federation & $64(3.6)$ & $33(3.0)$ & $39(3.2)$ & $77(2.6)$ & $568(3.4)$ & $23(2.6)$ & $550(7.0)$ \\
\hline Scotland & $58(4.8)$ & $14(3.1)$ & $29(3.9)$ & $64(4.5)$ & $527(3.2)$ & $36(4.5)$ & $524(4.8)$ \\
\hline Singapore & $60(2.7)$ & $34(2.8)$ & $37(3.0)$ & $66(2.6)$ & $562(3.3)$ & $34(2.6)$ & $566(5.2)$ \\
\hline Slovak Republic & $36(3.2)$ & $10(1.9)$ & $5(1.4)$ & $41(3.1)$ & $537(3.6)$ & $59(3.1)$ & $520(4.0)$ \\
\hline Slovenia & $57(3.4)$ & $28(3.1)$ & $33(3.3)$ & $69(3.1)$ & $523(2.9)$ & $31(3.1)$ & $523(3.5)$ \\
\hline South Africa & $53(2.9)$ & $44(2.8)$ & $51(3.1)$ & $72(2.5)$ & $308(6.6)$ & $28(2.5)$ & $340(11.5)$ \\
\hline Spain & $53(3.5)$ & $16(3.3)$ & $30(3.8)$ & $60(3.8)$ & $511(3.4)$ & $40(3.8)$ & $503(5.7)$ \\
\hline Sweden & $75(3.4)$ & $36(3.5)$ & $17(2.7)$ & $78(3.3)$ & $549(2.9)$ & $22(3.3)$ & $546(4.1)$ \\
\hline Trinidad and Tobago & $61(3.8)$ & $27(3.4)$ & $62(4.1)$ & $79(3.1)$ & $444(4.9)$ & $21(3.1)$ & $419(9.6)$ \\
\hline United States & $72(3.7)$ & $16(2.8)$ & $76(2.8)$ & $85(2.6)$ & $537(3.6)$ & $15(2.6)$ & $530(5.9)$ \\
\hline International Avg. & $49(0.6)$ & $19(0.4)$ & $26(0.5)$ & $58(0.6)$ & $502(0.7)$ & $42(0.6)$ & $499(0.9)$ \\
\hline
\end{tabular}

* Based on teachers' responses to having students read the following when having reading instruction and/or doing reading activities: descriptions and explanations about things, people, or events; instructions or manuals about how things work; and charts, diagrams, graphs.

Response options Every day or almost every day and Once or twice a week were combined as At least weekly. Response options Once or twice a month and Never or almost never were combined as Less than weekly.
() Standard errors appear in parentheses. Because results are rounded to the nearest whole number, some totals may appear inconsistent.

$A n$ " $r$ " indicates data are available for $70-84 \%$ of the students. An " $s$ " indicates data are available for $50-69 \%$ of the students. An " $x$ " indicates data are available for less than $50 \%$ of the students.

NOTE: The International Average does not include the results from the Canadian provinces. 
Exhibit 6.9 Trends in Teachers' Use of Literary and Informational Texts for Reading Instruction*

\begin{tabular}{|c|c|c|c|c|c|c|c|c|c|c|c|c|}
\hline \multirow{4}{*}{ Countries } & \multicolumn{12}{|c|}{ Percentage of Students Whose Teachers Asked Them to Read } \\
\hline & \multicolumn{6}{|c|}{ Literary Texts } & \multicolumn{6}{|c|}{ Informational Texts } \\
\hline & \multicolumn{3}{|c|}{ At Least Weekly } & \multicolumn{3}{|c|}{ Less than Weekly } & \multicolumn{3}{|c|}{ At Least Weekly } & \multicolumn{3}{|c|}{ Less than Weekly } \\
\hline & $\begin{array}{l}\text { Percent } \\
\text { in } 2006\end{array}$ & $\begin{array}{l}\text { Difference } \\
\text { in Percent } \\
\text { From } 2001\end{array}$ & & $\begin{array}{l}\text { Percent } \\
\text { in } 2006\end{array}$ & $\begin{array}{l}\text { Difference } \\
\text { in Percent } \\
\text { From } 2001\end{array}$ & & $\begin{array}{l}\text { Percent of } \\
\text { Students }\end{array}$ & $\begin{array}{l}\text { Difference } \\
\text { in Percent } \\
\text { From } 2001\end{array}$ & & $\begin{array}{l}\text { Percent of } \\
\text { Students }\end{array}$ & $\begin{array}{l}\text { Difference } \\
\text { in Percent } \\
\text { From } 2001\end{array}$ & \\
\hline Bulgaria & $94(1.9)$ & $3(3.2)$ & & $6(1.9)$ & $-3(3.2)$ & & $52(3.6)$ & $15(5.4)$ & $\boldsymbol{\theta}$ & $48(3.6)$ & $-15(5.4)$ & $(\bullet$ \\
\hline Canada, Ontario & $87(4.0)$ & $-2(4.9)$ & & $13(4.0)$ & $2(4.9)$ & & $77(4.2)$ & $1(5.5)$ & & $23(4.2)$ & $-1(5.5)$ & \\
\hline Canada, Quebec & $67(3.9)$ & $16(6.1)$ & $\mathbf{0}$ & $33(3.9)$ & $-16(6.1)$ & 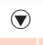 & $57(4.5)$ & $25(6.3)$ & 0 & $43(4.5)$ & $-25(6.3)$ & $(\nabla$ \\
\hline England & $75(3.8)$ & $-5(5.4)$ & & $25(3.8)$ & $5(5.4)$ & & $55(4.4)$ & $-1(6.7)$ & & $45(4.4)$ & $1(6.7)$ & \\
\hline France & $91(2.2)$ & $13(4.4)$ & 0 & $9(2.2)$ & $-13(4.4)$ & 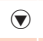 & $37(3.9)$ & $3(5.6)$ & & $63(3.9)$ & $-3(5.6)$ & \\
\hline Germany & $69(3.4)$ & $1(4.6)$ & & $31(3.4)$ & $-1(4.6)$ & & $84(2.5)$ & $9(3.7)$ & 0 & $16(2.5)$ & $-9(3.7)$ & $(\bullet$ \\
\hline Hong Kong SAR & $50(4.1)$ & $6(5.9)$ & & $50(4.1)$ & $-6(5.9)$ & & $22(3.8)$ & $-5(5.4)$ & & $78(3.8)$ & $5(5.4)$ & \\
\hline Hungary & $97(1.0)$ & $-1(1.4)$ & & $3(1.0)$ & $1(1.4)$ & & $74(3.6)$ & $15(5.5)$ & 0 & $26(3.6)$ & $-15(5.5)$ & ( \\
\hline Iceland & $91(0.2)$ & $-1(0.3)$ & & $9(0.2)$ & $1(0.3)$ & & $28(0.4)$ & $-2(0.5)$ & $(\nabla$ & $72(0.4)$ & $2(0.5)$ & 0 \\
\hline Iran, Islamic Rep. of & $74(2.9)$ & $5(4.4)$ & & $26(2.9)$ & $-5(4.4)$ & & $51(4.4)$ & $3(5.8)$ & & $49(4.4)$ & $-3(5.8)$ & \\
\hline Israel & $85(3.0)$ & $-4(4.3)$ & & $15(3.0)$ & $4(4.3)$ & & $63(4.1)$ & $13(5.6)$ & 0 & $37(4.1)$ & $-13(5.6)$ & ( \\
\hline Italy & $98(1.0)$ & $1(1.8)$ & & $2(1.0)$ & $-1(1.8)$ & & $88(2.4)$ & $11(3.9)$ & 0 & $12(2.4)$ & $-11(3.9)$ & ( \\
\hline Latvia & $88(2.4)$ & $-8(3.0)$ & $(-$ & $12(2.4)$ & $8(3.0)$ & 0 & $45(3.6)$ & $9(6.0)$ & & $55(3.6)$ & $-9(6.0)$ & \\
\hline Lithuania & $92(2.0)$ & $-4(2.7)$ & & $8(2.0)$ & $4(2.7)$ & & $54(3.4)$ & $2(5.4)$ & & $46(3.4)$ & $-2(5.4)$ & \\
\hline Macedonia, Rep. of & $85(3.1)$ & $5(4.5)$ & & $15(3.1)$ & $-5(4.5)$ & & $54(4.1)$ & $-10(6.2)$ & & $46(4.1)$ & $10(6.2)$ & \\
\hline Moldova, Rep. of & $97(1.5)$ & $-2(1.7)$ & & $3(1.5)$ & $2(1.7)$ & & $79(3.2)$ & $15(5.1)$ & 0 & $21(3.2)$ & $-15(5.1)$ & 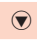 \\
\hline Morocco & $48(4.7)$ & $-18(6.8)$ & $(\nabla$ & $52(4.7)$ & $18(6.8)$ & 0 & $44(4.4)$ & $4(6.9)$ & & $56(4.4)$ & $-4(6.9)$ & \\
\hline Netherlands & $91(2.1)$ & $10(4.2)$ & 0 & $9(2.1)$ & $-10(4.2)$ & ( & $59(4.1)$ & $7(6.2)$ & & $41(4.1)$ & $-7(6.2)$ & \\
\hline New Zealand & $85(2.1)$ & $-8(3.0)$ & ( & $15(2.1)$ & $8(3.0)$ & 0 & $68(2.9)$ & $6(5.1)$ & & $32(2.9)$ & $-6(5.1)$ & \\
\hline Norway & $95(1.6)$ & $4(2.7)$ & & $5(1.6)$ & $-4(2.7)$ & & $59(5.0)$ & $-1(6.7)$ & & $41(5.0)$ & $1(6.7)$ & \\
\hline Romania & $93(1.8)$ & $-4(2.1)$ & $(\nabla$ & $7(1.8)$ & $4(2.1)$ & 0 & $61(3.7)$ & $3(5.7)$ & & $39(3.7)$ & $-3(5.7)$ & \\
\hline Russian Federation & $95(1.7)$ & $-3(1.9)$ & & $5(1.7)$ & $3(1.9)$ & & $77(2.6)$ & $27(4.1)$ & 0 & $23(2.6)$ & $-27(4.1)$ & 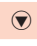 \\
\hline Scotland & $89(2.8)$ & $-1(4.1)$ & & $11(2.8)$ & $1(4.1)$ & & $64(4.5)$ & $-5(6.2)$ & & $36(4.5)$ & $5(6.2)$ & \\
\hline Singapore & $82(1.8)$ & $10(3.9)$ & $\theta$ & $18(1.8)$ & $-10(3.9)$ & ( & $66(2.6)$ & $7(4.8)$ & & $34(2.6)$ & $-7(4.8)$ & \\
\hline Slovak Republic & $93(1.5)$ & $4(2.9)$ & & $7(1.5)$ & $-4(2.9)$ & & $41(3.1)$ & $9(4.9)$ & & $59(3.1)$ & $-9(4.9)$ & \\
\hline Slovenia & $85(2.4)$ & $-6(3.4)$ & & $15(2.4)$ & $6(3.4)$ & & $69(3.1)$ & $11(5.1)$ & 0 & $31(3.1)$ & $-11(5.1)$ & ( \\
\hline Sweden & $99(0.3)$ & $3(1.4)$ & 0 & $1(0.3)$ & $-3(1.4)$ & ( & $78(3.3)$ & $11(4.6)$ & 0 & $22(3.3)$ & $-11(4.6)$ & ( \\
\hline United States & $92(2.4)$ & $-2(3.8)$ & & $8(2.4)$ & $2(3.8)$ & & $85(2.6)$ & $-3(4.0)$ & & $15(2.6)$ & $3(4.0)$ & \\
\hline International Avg. & $86(0.5)$ & $0(0.7)$ & & $14(0.5)$ & $0(0.7)$ & & $60(0.7)$ & $6(1.0)$ & 0 & $40(0.7)$ & $-6(1.0)$ & ( \\
\hline
\end{tabular}

( Percent in 2006 significantly higher

( ) Percent in 2006 significantly lower

Background data provided by teachers

* For description of types of literary and informational texts, see Exhibits 6.7 and 6.8 . Please note that in 2001, "short stories" was not included as part of literary texts, but "fables and fairy tales" and "other stories" were.

() Standard errors appear in parentheses. Because results are rounded to the nearest whole number, some totals may appear inconsistent.
An " $r$ " indicates data are available for $70-84 \%$ of the students. An "s" indicates data are available for $50-69 \%$ of the students. An " $x$ " indicates data are available for less than $50 \%$ of the students.

NOTE: The International Average does not include the results from the Canadian provinces. Trend Note: The primary education systems of the Russian Federation and Slovenia underwent structural changes. Data for Canada, Ontario include only public schools. 


\section{How Often Do Students Read Aloud and Independently in Class?}

This section describes how often either teachers or students read aloud in the classroom to the entire class or a small group, or students read silently on their own, as part of reading instruction. Exhibits 6.10 and 6.11 present teachers' reports of their emphasis on teaching strategies for decoding, understanding new vocabulary, and using different skills and strategies to develop or improve reading comprehension during reading instruction. As shown in Exhibit 6.10, only one fourth of the fourth-grade students, on average internationally, were taught strategies for decoding sounds and words every day, although more than half the students in Kuwait, Morocco, Poland, and Trinidad and Tobago were taught these strategies on a daily basis. Across countries, 69 percent of the students were taught strategies daily to understand new vocabulary, with content vocabulary strategies receiving a daily emphasis for the fewest students ( 38 to $45 \%$ ) in Chinese Taipei, Denmark, Iceland, Indonesia, Luxembourg, and Norway.

Exhibit 6.11 presents the percentage of students whose teachers asked them to use a number of skills and strategies at least once or twice a week. On average internationally, the most popular skills and strategies, used with 90 and 91 percent of the students, respectively, were asking students to identify main ideas or to explain or support their understanding of what they read with support from the text or personal experience. The next most common strategies were comparing reading with their own experiences and making generalizations and drawing inferences ( $72 \%$ and $71 \%$, respectively). Fewer students, but still the majority, on average across countries, were asked to make predictions about outcomes (61\%), to compare reading with other things they had read (58\%), or to describe the style or structure of a text $(53 \%)$.

Reading by teachers and students in the classroom can take a variety of forms. For example, teachers can read aloud to the class, ask students to read aloud to the whole class or in small groups or pairs, or ask them to read silently on their own. As shown in Exhibit 6.12, according to teachers, on average internationally, both listening to their teachers read aloud (58\%) and 
reading aloud themselves to the whole class (56\%) were daily activities for the majority of students. Also, silent independent reading was assigned daily to the majority of the students (59\%). Across countries, teachers reported asking students to read aloud in small groups or pairs less frequently, with nearly half the students (47\%), on average, reading aloud in small groups less than weekly.

To report how often students were asked to read aloud in class (see Exhibit 6.13), students' responses to separate questions about reading aloud to the whole class or to a small group of students were combined and averaged. Students themselves reported reading aloud in class much less frequently than did their teachers. However, on average internationally, the majority (54\%) reported reading aloud in class at least weekly. In general, the frequency of reading aloud was inversely related to achievement, probably because teachers do not need to monitor the more fluent readers as often. More than half the fourth-grade students reported never (or almost never) reading aloud in class in Austria, Chinese Taipei, Germany, Hong Kong SAR, Iceland, the Netherlands, New Zealand, Singapore, and the Canadian provinces of British Columbia and Nova Scotia.

Exhibit 6.14 shows students' reports about their independent reading as a combination of responses to how often they were asked to read silently on their own and how often they read books they chose themselves. Here students' reports agreed with their teachers' reports. On average internationally, 65 percent of students reported that independent reading was a daily activity, and another 27 percent reported reading independently in class at least weekly. Again, because by fourth grade students should be making the transition from learning to read to reading to learn, it would follow that independent reading would be more frequent than reading aloud. 
Exhibit 6.10 Emphasis on Decoding and Vocabulary

PIRLS 2006

4th Grade

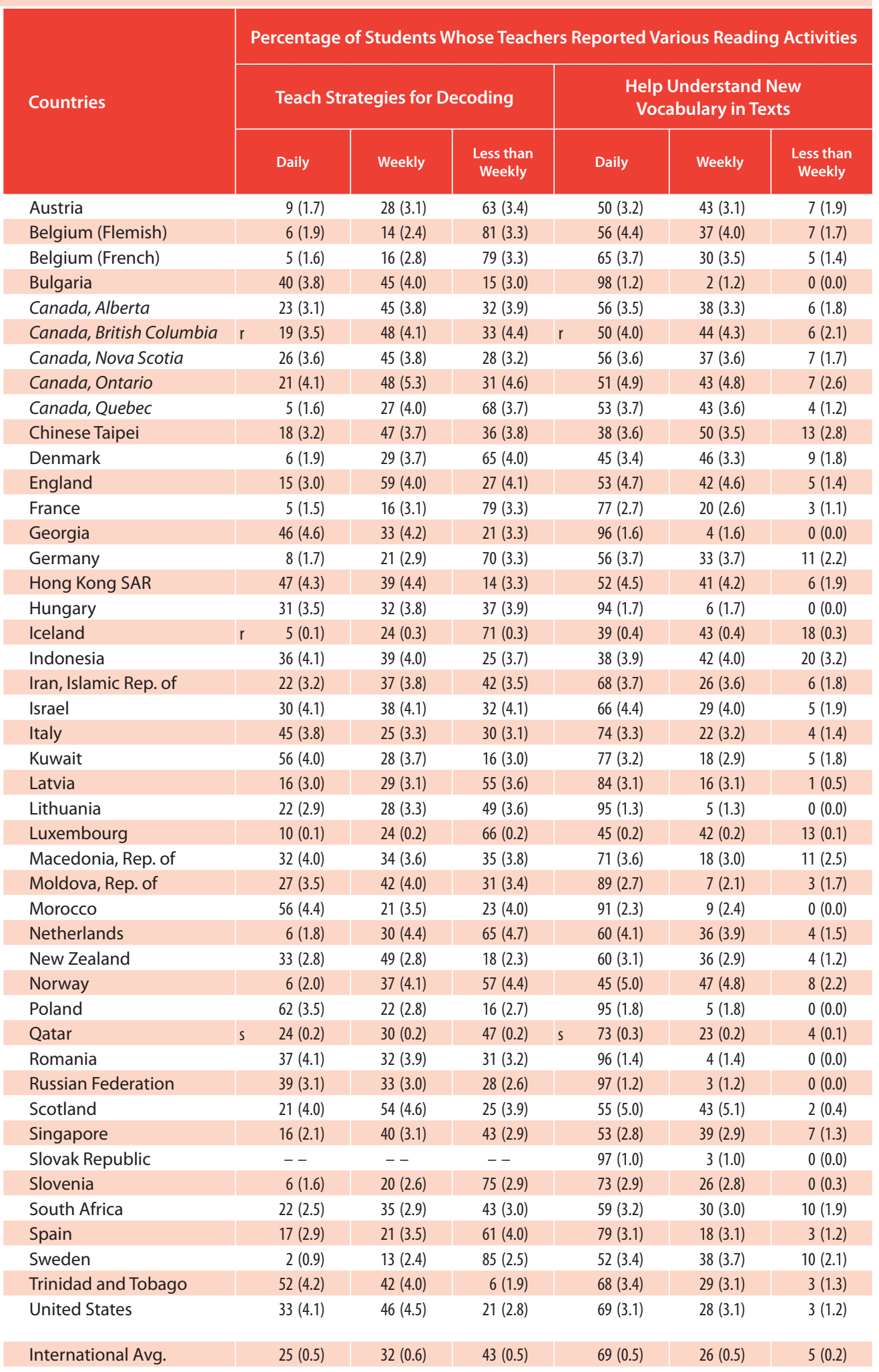

Background data provided by teachers.

() Standard errors appear in parentheses. Because results are rounded to the nearest whole number, some totals may appear inconsistent.

An " $r$ " indicates data are available for $70-84 \%$ of the students. An "s" indicates data are available for $50-69 \%$ of the students. An " $x$ " indicates data are available for less than $50 \%$ of the students.
A dash (-) indicates comparable data are not available.

NOTE: The International Average does not include the results from the Canadian provinces. 


\begin{tabular}{|c|c|c|c|c|c|c|c|}
\hline \multirow[t]{3}{*}{ Exhibit 6.11} & $\begin{array}{l}\text { Ask Stud } \\
\text { hension Sk }\end{array}$ & $\begin{array}{l}\text { ts to Do Clas } \\
\text { s or Strateg }\end{array}$ & $\begin{array}{l}\text { room Activiti } \\
\text { S }\end{array}$ & les at Least & eekly to Deve & elop Reading & $\begin{array}{l}\text { PIRLS } 2006 \\
\text { 4th Grade }\end{array}$ \\
\hline & \multicolumn{7}{|c|}{ Percentage of Students Whose Teachers Ask Them to Do the Activities } \\
\hline & $\begin{array}{l}\text { Identify } \\
\text { Main Ideas }\end{array}$ & $\begin{array}{l}\text { Explain or } \\
\text { Support Their } \\
\text { Understanding }\end{array}$ & $\begin{array}{l}\text { Compare Reading } \\
\text { with Own } \\
\text { Experiences }\end{array}$ & $\begin{array}{l}\text { Compare with } \\
\text { Other Things } \\
\text { Read }\end{array}$ & $\begin{array}{l}\text { Make Predictions } \\
\text { About What } \\
\text { Will Happen Next }\end{array}$ & $\begin{array}{c}\text { Make } \\
\text { Generalizations } \\
\text { and Draw } \\
\text { Inferences }\end{array}$ & $\begin{array}{l}\text { Describe Text } \\
\text { Style or } \\
\text { Structure }\end{array}$ \\
\hline Austria & $80(2.6)$ & $88(2.3)$ & $57(3.8)$ & $32(3.2)$ & $31(2.9)$ & $49(3.3)$ & $17(2.6)$ \\
\hline Belgium (Flemish) & $77(3.8)$ & $81(3.0)$ & $60(4.6)$ & $36(4.3)$ & $50(3.7)$ & $51(3.9)$ & $34(3.9)$ \\
\hline Belgium (French) & $77(2.6)$ & $83(2.7)$ & $34(3.9)$ & $27(3.4)$ & $31(3.7)$ & $27(3.4)$ & $30(3.5)$ \\
\hline Bulgaria & $100(0.0)$ & $100(0.0)$ & $92(2.3)$ & $87(2.6)$ & $74(3.3)$ & $100(0.0)$ & $77(3.6)$ \\
\hline Canada, Alberta & $90(2.3)$ & $93(2.0)$ & $83(2.8)$ & $67(3.9)$ & $89(2.2)$ & $86(2.2)$ & $45(3.8)$ \\
\hline Canada, British Columbia & $90(2.3)$ & $92(2.1)$ & $84(3.2)$ & $68(3.7)$ & $90(2.3)$ & $82(3.2)$ & $44(4.4)$ \\
\hline Canada, Nova Scotia & $89(2.4)$ & $94(1.7)$ & $86(2.7)$ & $79(3.1)$ & $90(2.4)$ & $86(2.5)$ & $48(4.0)$ \\
\hline Canada, Ontario & $84(4.0)$ & $89(3.6)$ & $76(4.9)$ & $70(4.9)$ & $88(3.5)$ & $82(4.2)$ & $53(5.2)$ \\
\hline Canada, Quebec & $74(3.8)$ & $82(3.3)$ & $54(4.7)$ & $41(4.4)$ & $58(4.3)$ & $55(4.5)$ & $39(3.4)$ \\
\hline Chinese Taipei & $87(2.6)$ & $61(4.2)$ & $67(3.7)$ & $53(4.1)$ & $42(4.3)$ & $66(4.0)$ & $55(4.5)$ \\
\hline Denmark & $79(3.3)$ & $80(3.2)$ & $68(3.9)$ & $51(3.9)$ & $43(3.8)$ & $52(3.8)$ & $39(3.7)$ \\
\hline England & $92(1.8)$ & $97(1.4)$ & $71(4.1)$ & $63(4.2)$ & $87(2.7)$ & $89(2.7)$ & $74(3.7)$ \\
\hline France & $87(2.0)$ & $91(1.9)$ & $35(3.6)$ & $38(2.8)$ & $53(3.9)$ & $35(3.4)$ & $34(3.4)$ \\
\hline Georgia & $98(0.9)$ & $96(1.9)$ & $92(2.4)$ & $85(3.1)$ & $72(3.9)$ & $89(2.6)$ & $70(3.9)$ \\
\hline Germany & $95(1.7)$ & $94(1.7)$ & $75(3.2)$ & $49(3.7)$ & $55(3.8)$ & $69(3.2)$ & $26(3.0)$ \\
\hline Hong Kong SAR & $89(2.5)$ & $80(3.3)$ & $65(4.0)$ & $50(4.2)$ & $51(4.5)$ & $64(4.6)$ & $51(4.4)$ \\
\hline Hungary & $97(1.9)$ & $100(0.0)$ & $94(2.2)$ & $92(2.2)$ & $75(3.6)$ & $96(1.7)$ & $88(2.7)$ \\
\hline Iceland & $40(0.3)$ & $60(0.4)$ & $34(0.4)$ & $8(0.3)$ & $29(0.3)$ & $26(0.3)$ & $1(0.0)$ \\
\hline Indonesia & $86(3.1)$ & $87(2.9)$ & $56(4.1)$ & $49(4.6)$ & $36(3.7)$ & $57(4.1)$ & $44(4.2)$ \\
\hline Iran, Islamic Rep. of & $93(1.8)$ & $88(2.2)$ & $69(3.8)$ & $60(4.0)$ & $47(3.7)$ & $80(3.3)$ & $67(3.3)$ \\
\hline Israel & $99(0.7)$ & $99(1.0)$ & $82(3.5)$ & $70(3.7)$ & $84(3.3)$ & $93(2.4)$ & $88(2.8)$ \\
\hline Italy & $98(1.0)$ & $96(1.4)$ & $79(3.1)$ & $66(3.4)$ & $71(3.5)$ & $59(3.7)$ & $70(3.5)$ \\
\hline Kuwait & $85(3.0)$ & $90(2.6)$ & $60(4.1)$ & $55(3.6)$ & $47(4.0)$ & $67(4.0)$ & $51(4.5)$ \\
\hline Latvia & $97(1.3)$ & $98(1.2)$ & $92(2.1)$ & $65(3.5)$ & $70(3.1)$ & $89(2.7)$ & $35(3.6)$ \\
\hline Lithuania & $100(0.0)$ & $100(0.4)$ & $85(2.5)$ & $77(3.2)$ & $75(3.0)$ & $97(1.3)$ & $73(3.3)$ \\
\hline Luxembourg & $89(0.1)$ & $90(0.1)$ & $64(0.2)$ & $37(0.2)$ & $70(0.2)$ & $66(0.2)$ & $26(0.1)$ \\
\hline Macedonia, Rep. of & $99(0.5)$ & $100(0.0)$ & $90(2.4)$ & $82(3.2)$ & $74(3.9)$ & $94(2.1)$ & $76(3.6)$ \\
\hline Moldova, Rep. of & $100(0.0)$ & $100(0.0)$ & $95(1.7)$ & $87(2.8)$ & $83(3.2)$ & $99(0.9)$ & $89(2.9)$ \\
\hline Morocco & $95(1.8)$ & $93(2.3)$ & $55(5.0)$ & $47(5.0)$ & $58(4.4)$ & $63(4.4)$ & $59(4.4)$ \\
\hline Netherlands & $84(3.4)$ & $87(2.9)$ & $65(4.7)$ & $34(4.1)$ & $50(4.5)$ & $66(3.6)$ & $34(4.0)$ \\
\hline New Zealand & $95(1.3)$ & $94(1.4)$ & $82(2.4)$ & $60(2.8)$ & $94(1.2)$ & $87(2.0)$ & $65(2.9)$ \\
\hline Norway & $67(4.4)$ & $66(4.6)$ & $38(4.3)$ & $19(3.8)$ & $28(3.7)$ & $28(4.4)$ & $11(2.3)$ \\
\hline Poland & $100(0.2)$ & $99(0.6)$ & $95(1.9)$ & $66(3.7)$ & $62(4.1)$ & $92(2.1)$ & $56(4.2)$ \\
\hline Qatar & $100(0.1)$ & $97(0.1)$ & $86(0.2)$ & $74(0.2)$ & $69(0.2)$ & $67(0.2)$ & $72(0.2)$ \\
\hline Romania & $99(0.6)$ & $98(1.2)$ & $84(2.5)$ & $81(3.0)$ & $73(3.4)$ & $88(2.7)$ & $80(3.3)$ \\
\hline Russian Federation & $100(0.5)$ & $100(0.3)$ & $92(2.0)$ & $87(2.0)$ & $77(3.2)$ & $99(0.9)$ & $82(2.7)$ \\
\hline Scotland & $93(2.3)$ & $95(2.0)$ & $71(4.2)$ & $55(4.4)$ & $85(2.8)$ & $79(3.8)$ & $68(4.2)$ \\
\hline Singapore & $95(1.1)$ & $95(1.2)$ & $80(2.4)$ & $70(2.8)$ & $84(2.0)$ & $83(2.0)$ & $64(2.7)$ \\
\hline Slovak Republic & $97(0.9)$ & $99(0.6)$ & $84(2.5)$ & $62(3.3)$ & $53(3.8)$ & $79(2.8)$ & $56(3.6)$ \\
\hline Slovenia & $85(2.0)$ & $95(1.4)$ & $84(2.3)$ & $52(3.3)$ & $46(3.3)$ & $74(2.5)$ & $55(2.9)$ \\
\hline South Africa & $79(2.3)$ & $79(2.3)$ & $66(2.9)$ & $65(3.2)$ & $64(2.7)$ & $54(3.1)$ & $46(2.9)$ \\
\hline Spain & $95(1.7)$ & $97(1.4)$ & $65(4.1)$ & $47(4.4)$ & $48(4.2)$ & $46(4.0)$ & $39(4.1)$ \\
\hline Sweden & $59(4.0)$ & $81(2.9)$ & $39(3.8)$ & $25(3.5)$ & $19(3.4)$ & $29(3.8)$ & $5(1.6)$ \\
\hline Trinidad and Tobago & $96(1.9)$ & $97(1.5)$ & $85(2.6)$ & $73(3.7)$ & $86(3.0)$ & $86(2.9)$ & $38(3.6)$ \\
\hline United States & $98(1.0)$ & $97(1.0)$ & $90(2.1)$ & $81(3.2)$ & $96(1.4)$ & $93(1.6)$ & $71(3.2)$ \\
\hline International Avg. & $90(0.3)$ & $91(0.3)$ & $72(0.5)$ & $58(0.5)$ & $61(0.5)$ & $71(0.5)$ & $53(0.5)$ \\
\hline
\end{tabular}

Background data provided by teachers.

() Standard errors appear in parentheses. Because results are rounded to the nearest whole number, some totals may appear inconsistent.
An " $r$ " indicates data are available for $70-84 \%$ of the students. An " $s$ " indicates data are available for $50-69 \%$ of the students. An " $x$ " indicates data are available for less than $50 \%$ of the students.

NOTE: The International Average does not include the results from the Canadian provinces. 
Exhibit 6.12 Teachers' Reports About Reading Aloud or Silently in Class

PIRLS 2006

4th Grade

\begin{tabular}{|c|c|c|c|c|c|c|}
\hline \multirow{3}{*}{ Countries } & \multicolumn{6}{|c|}{ Percentage of Students Whose Teachers Reported Various Reading Activities } \\
\hline & \multicolumn{3}{|c|}{ Teacher Reads Aloud to Whole Class } & \multicolumn{3}{|c|}{ Students Read Aloud to Whole Class } \\
\hline & Daily & Weekly & $\begin{array}{l}\text { Less than } \\
\text { Weekly }\end{array}$ & Daily & Weekly & $\begin{array}{l}\text { Less than } \\
\text { Weekly }\end{array}$ \\
\hline Austria & $14(2.5)$ & $35(3.4)$ & $51(3.8)$ & $31(3.5)$ & $50(3.6)$ & $19(2.8)$ \\
\hline Belgium (Flemish) & $42(3.8)$ & $39(3.5)$ & $20(3.2)$ & $61(4.2)$ & $25(3.8)$ & $14(2.8)$ \\
\hline Belgium (French) & $53(3.4)$ & $29(2.8)$ & $18(2.8)$ & $62(3.2)$ & $28(3.3)$ & $10(2.1)$ \\
\hline Bulgaria & $89(2.6)$ & $8(2.3)$ & $3(1.4)$ & $90(1.9)$ & $10(1.9)$ & $0(0.0)$ \\
\hline Canada, Alberta & $75(3.1)$ & $24(3.1)$ & $1(0.5)$ & $32(3.9)$ & $50(4.2)$ & $18(2.8)$ \\
\hline Canada, British Columbia & $72(3.9)$ & $24(3.8)$ & $4(1.8)$ & $32(3.5)$ & $49(4.2)$ & $19(3.6)$ \\
\hline Canada, Nova Scotia & $81(2.7)$ & $18(2.6)$ & $1(0.6)$ & $29(3.4)$ & $43(3.4)$ & $27(3.5)$ \\
\hline Canada, Ontario & $78(3.9)$ & $17(3.5)$ & $5(2.2)$ & $41(4.5)$ & $35(4.5)$ & $25(4.2)$ \\
\hline Canada, Quebec & $50(4.8)$ & $40(4.6)$ & $9(2.6)$ & $40(4.0)$ & $45(4.4)$ & $16(3.3)$ \\
\hline Chinese Taipei & $25(3.4)$ & $40(3.7)$ & $35(3.8)$ & $33(4.4)$ & $44(4.2)$ & $23(3.5)$ \\
\hline Denmark & $22(3.3)$ & $53(4.2)$ & $25(3.9)$ & $18(3.3)$ & $48(4.2)$ & $35(4.1)$ \\
\hline England & $70(3.9)$ & $29(3.9)$ & $1(0.8)$ & $49(4.5)$ & $38(4.3)$ & $13(2.9)$ \\
\hline France & $54(3.4)$ & $37(3.4)$ & $9(1.6)$ & $76(2.7)$ & $22(2.7)$ & $2(0.8)$ \\
\hline Georgia & $83(3.4)$ & $16(3.3)$ & $1(0.6)$ & $87(2.8)$ & $11(2.6)$ & $2(1.2)$ \\
\hline Germany & $14(2.1)$ & $41(3.8)$ & $45(3.9)$ & $49(3.9)$ & $38(3.7)$ & $13(2.4)$ \\
\hline Hong Kong SAR & $44(4.4)$ & $46(4.5)$ & $10(2.8)$ & $49(4.7)$ & $45(4.6)$ & $6(2.1)$ \\
\hline Hungary & $32(3.5)$ & $56(3.9)$ & $13(2.9)$ & $62(3.7)$ & $33(4.0)$ & $5(1.7)$ \\
\hline Iceland & $75(0.3)$ & $20(0.3)$ & $5(0.2)$ & $19(0.3)$ & $53(0.4)$ & $28(0.4)$ \\
\hline Indonesia & $45(3.8)$ & $45(4.1)$ & $10(2.1)$ & $42(3.7)$ & $39(4.4)$ & $19(3.3)$ \\
\hline Iran, Islamic Rep. of & $54(3.9)$ & $41(3.9)$ & $5(1.5)$ & $58(3.8)$ & $38(3.8)$ & $4(1.3)$ \\
\hline Israel & $72(4.0)$ & $25(3.7)$ & $4(1.5)$ & $71(3.7)$ & $28(3.8)$ & $1(0.0)$ \\
\hline Italy & $72(3.3)$ & $24(3.0)$ & $4(1.6)$ & $75(2.8)$ & $20(2.6)$ & $4(1.7)$ \\
\hline Kuwait & $94(2.0)$ & $3(1.3)$ & $4(1.5)$ & $75(3.5)$ & $14(3.0)$ & $11(2.7)$ \\
\hline Latvia & $25(3.2)$ & $50(3.8)$ & $25(3.2)$ & $76(3.5)$ & $22(3.4)$ & $2(0.9)$ \\
\hline Lithuania & $36(3.4)$ & $43(3.4)$ & $21(2.8)$ & $82(2.6)$ & $17(2.6)$ & $1(0.5)$ \\
\hline Luxembourg & $13(0.1)$ & $46(0.2)$ & $40(0.2)$ & $34(0.2)$ & $48(0.2)$ & $18(0.1)$ \\
\hline Macedonia, Rep. of & $73(3.7)$ & $26(3.6)$ & $2(1.1)$ & $72(3.6)$ & $24(3.4)$ & $5(1.9)$ \\
\hline Moldova, Rep. of & $76(3.1)$ & $18(3.0)$ & $6(2.2)$ & $82(3.0)$ & $13(2.5)$ & $4(1.9)$ \\
\hline Morocco & $96(1.7)$ & $4(1.7)$ & $0(0.0)$ & $96(1.6)$ & $2(1.2)$ & $1(1.0)$ \\
\hline Netherlands & $62(3.9)$ & $33(3.9)$ & $5(1.7)$ & $33(3.6)$ & $53(3.9)$ & $13(2.6)$ \\
\hline New Zealand & $77(2.4)$ & $19(2.2)$ & $3(1.0)$ & $9(1.5)$ & $44(2.5)$ & $47(2.7)$ \\
\hline Norway & $76(4.0)$ & $22(3.9)$ & $1(0.8)$ & $24(3.7)$ & $52(4.0)$ & $24(3.8)$ \\
\hline Poland & $70(3.5)$ & $24(3.0)$ & $6(2.0)$ & $77(3.5)$ & $21(3.4)$ & $2(0.8)$ \\
\hline Qatar & $82(0.2)$ & $17(0.2)$ & $0(0.1)$ & $54(0.3)$ & $36(0.3)$ & $11(0.1)$ \\
\hline Romania & $72(3.7)$ & $24(3.6)$ & $4(1.2)$ & $93(2.0)$ & $4(1.5)$ & $3(1.5)$ \\
\hline Russian Federation & $61(3.7)$ & $33(3.5)$ & $7(2.0)$ & $96(1.5)$ & $3(1.2)$ & $1(0.7)$ \\
\hline Scotland & $49(4.8)$ & $48(4.8)$ & $3(1.6)$ & $21(4.1)$ & $46(4.8)$ & $33(4.3)$ \\
\hline Singapore & $65(2.7)$ & $31(2.8)$ & $4(1.1)$ & $39(2.5)$ & $52(2.6)$ & $9(1.8)$ \\
\hline Slovak Republic & $41(3.6)$ & $42(3.3)$ & $17(2.7)$ & $78(2.9)$ & $16(2.2)$ & $5(2.0)$ \\
\hline Slovenia & $49(3.2)$ & $45(3.3)$ & $6(1.4)$ & $57(3.2)$ & $36(3.2)$ & $8(1.6)$ \\
\hline South Africa & $54(3.4)$ & $40(3.2)$ & $7(1.6)$ & $41(3.5)$ & $48(3.6)$ & $12(1.9)$ \\
\hline Spain & $76(3.0)$ & $22(2.8)$ & $2(1.4)$ & $82(2.9)$ & $16(2.8)$ & $2(1.2)$ \\
\hline Sweden & $55(3.5)$ & 40 (3.2) & $5(1.6)$ & $4(1.6)$ & $59(3.7)$ & $37(3.8)$ \\
\hline Trinidad and Tobago & $84(3.1)$ & $15(3.0)$ & $1(0.0)$ & $68(3.6)$ & $31(3.5)$ & $1(1.1)$ \\
\hline United States & $76(2.9)$ & $21(3.0)$ & $3(1.2)$ & $38(4.1)$ & $46(3.9)$ & $16(2.7)$ \\
\hline International Avg. & $58(0.5)$ & $31(0.5)$ & $11(0.3)$ & $56(0.5)$ & $32(0.5)$ & $12(0.3)$ \\
\hline
\end{tabular}

Background data provided by teachers.

() Standard errors appear in parentheses. Because results are rounded to the nearest whole number, some totals may appear inconsistent.
An " $r$ " indicates data are available for $70-84 \%$ of the students. An " $s$ " indicates data are available for $50-69 \%$ of the students. An " $x$ " indicates data are available for less than $50 \%$ of the students.

NOTE: The International Average does not include the results from the Canadian provinces. 
Exhibit 6.12 Teachers' Reports About Reading Aloud or Silently in Class (Continued)

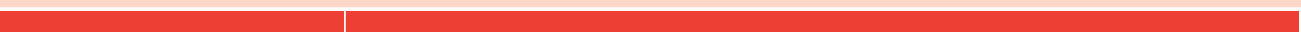

\begin{tabular}{|c|c|c|c|c|c|c|c|}
\hline \multirow{3}{*}{ Countries } & \multicolumn{7}{|c|}{ Percentage of Students Whose Teachers Reported Various Reading Activities } \\
\hline & \multicolumn{3}{|c|}{$\begin{array}{l}\text { Students Read Aloud } \\
\text { in Small Groups or Pairs }\end{array}$} & \multicolumn{4}{|c|}{$\begin{array}{l}\text { Students Read Silently } \\
\text { on Their Own }\end{array}$} \\
\hline & Daily & Weekly & $\begin{array}{l}\text { Less than } \\
\text { Weekly }\end{array}$ & & Daily & Weekly & $\begin{array}{l}\text { Less than } \\
\text { Weekly }\end{array}$ \\
\hline Austria & $5(1.4)$ & $38(3.4)$ & $58(3.4)$ & & $58(3.4)$ & $38(3.4)$ & $4(1.5)$ \\
\hline Belgium (Flemish) & $6(1.8)$ & $45(3.8)$ & $50(3.7)$ & & $60(4.0)$ & $37(4.0)$ & $3(0.9)$ \\
\hline Belgium (French) & $5(1.4)$ & $17(2.8)$ & $78(3.0)$ & & $63(3.0)$ & $32(2.9)$ & $4(1.3)$ \\
\hline Bulgaria & $17(3.0)$ & $45(3.9)$ & $38(3.9)$ & & $79(3.3)$ & $16(2.8)$ & $5(1.8)$ \\
\hline Canada, Alberta & $23(3.4)$ & $47(3.9)$ & $30(3.6)$ & & $85(3.2)$ & $14(3.1)$ & $1(0.7)$ \\
\hline Canada, British Columbia & $21(3.2)$ & $46(3.7)$ & $33(3.6)$ & $r$ & $87(2.5)$ & $13(2.5)$ & $0(0.0)$ \\
\hline Canada, Nova Scotia & $13(2.5)$ & $56(3.8)$ & $30(3.5)$ & & $89(2.1)$ & $10(2.1)$ & $1(0.5)$ \\
\hline Canada, Ontario & $14(3.5)$ & $56(4.2)$ & $29(4.5)$ & & $76(4.5)$ & $24(4.5)$ & $0(0.0)$ \\
\hline Canada, Quebec & $4(1.8)$ & $34(4.4)$ & $61(4.7)$ & & $75(4.1)$ & $23(3.9)$ & $2(1.2)$ \\
\hline Chinese Taipei & $12(2.7)$ & $26(3.4)$ & $63(4.1)$ & & $23(3.5)$ & $52(4.3)$ & $25(3.8)$ \\
\hline Denmark & $14(3.0)$ & $47(4.3)$ & $39(4.1)$ & & $38(4.2)$ & $49(4.2)$ & $13(2.5)$ \\
\hline England & $24(3.7)$ & $54(4.0)$ & $21(3.6)$ & & $64(4.2)$ & $31(4.2)$ & $4(1.8)$ \\
\hline France & $3(1.2)$ & $19(2.7)$ & $77(2.8)$ & & $75(2.4)$ & $22(2.5)$ & $2(1.0)$ \\
\hline Georgia & $32(4.2)$ & $35(3.6)$ & $33(4.1)$ & & $44(4.1)$ & $37(3.9)$ & $20(3.4)$ \\
\hline Germany & $8(1.9)$ & $43(3.4)$ & $49(3.5)$ & & $61(3.3)$ & $34(3.1)$ & $5(1.7)$ \\
\hline Hong Kong SAR & $10(2.8)$ & $48(4.1)$ & $42(4.0)$ & & $44(4.6)$ & $44(4.3)$ & $12(2.9)$ \\
\hline Hungary & $8(2.1)$ & $36(3.4)$ & $56(3.7)$ & & $64(4.2)$ & $32(4.1)$ & $4(1.7)$ \\
\hline Iceland & $4(0.1)$ & $16(0.3)$ & $80(0.3)$ & & $63(0.3)$ & $28(0.3)$ & $8(0.2)$ \\
\hline Indonesia & $7(2.0)$ & $45(3.9)$ & $49(3.5)$ & & $47(4.2)$ & $40(4.6)$ & $13(2.7)$ \\
\hline Iran, Islamic Rep. of & $12(2.7)$ & $37(3.6)$ & $51(3.5)$ & & $30(3.7)$ & $50(3.7)$ & $20(2.9)$ \\
\hline Israel & $18(3.0)$ & $44(4.4)$ & $38(4.5)$ & & $56(4.0)$ & $40(4.1)$ & $4(1.7)$ \\
\hline Italy & $5(1.6)$ & $20(3.1)$ & $74(3.4)$ & & $50(4.0)$ & $39(4.0)$ & $11(2.4)$ \\
\hline Kuwait & $18(3.2)$ & $32(4.2)$ & $50(4.4)$ & & $24(3.5)$ & $34(4.0)$ & $42(3.9)$ \\
\hline Latvia & $10(2.6)$ & $38(3.6)$ & $52(3.7)$ & & $66(3.4)$ & $25(3.2)$ & $9(2.2)$ \\
\hline Lithuania & $9(1.9)$ & $48(3.7)$ & $43(3.8)$ & & $52(3.4)$ & $36(3.0)$ & $12(2.5)$ \\
\hline Luxembourg & $2(0.0)$ & $14(0.1)$ & $84(0.1)$ & & $41(0.2)$ & $48(0.2)$ & $11(0.1)$ \\
\hline Macedonia, Rep. of & $13(2.9)$ & $42(4.2)$ & $46(4.0)$ & & $41(4.0)$ & $37(4.4)$ & $23(3.7)$ \\
\hline Moldova, Rep. of & $39(4.3)$ & $51(3.9)$ & $10(2.4)$ & & $83(3.3)$ & $10(2.5)$ & $7(2.2)$ \\
\hline Morocco & $5(1.7)$ & $12(3.2)$ & $83(3.6)$ & & $75(3.6)$ & $21(3.3)$ & $4(1.9)$ \\
\hline Netherlands & $10(2.8)$ & $43(4.4)$ & $46(4.3)$ & & $64(4.6)$ & $35(4.6)$ & $1(0.6)$ \\
\hline New Zealand & $21(2.1)$ & $55(3.1)$ & $24(2.6)$ & & $89(1.8)$ & $10(1.8)$ & $0(0.1)$ \\
\hline Norway & $15(2.9)$ & $45(3.7)$ & $40(4.2)$ & & $67(3.3)$ & $29(3.4)$ & $3(1.6)$ \\
\hline Poland & $3(1.1)$ & $44(3.8)$ & $54(3.8)$ & & $68(3.6)$ & $30(3.5)$ & $2(1.1)$ \\
\hline Qatar & $34(0.3)$ & $51(0.3)$ & $15(0.2)$ & s & $48(0.3)$ & $50(0.3)$ & $2(0.0)$ \\
\hline Romania & $17(3.2)$ & $42(4.2)$ & $41(4.3)$ & & $75(3.2)$ & $19(2.8)$ & $6(1.8)$ \\
\hline Russian Federation & $31(2.9)$ & $46(3.5)$ & $23(3.2)$ & & $84(2.9)$ & $13(2.5)$ & $4(1.3)$ \\
\hline Scotland & $26(4.0)$ & $55(4.6)$ & $19(3.7)$ & & $65(3.6)$ & $32(3.8)$ & $3(1.7)$ \\
\hline Singapore & $11(1.7)$ & $53(2.9)$ & $36(2.7)$ & & $59(2.7)$ & $34(2.7)$ & $7(1.6)$ \\
\hline Slovak Republic & $1(0.7)$ & $27(3.5)$ & $72(3.5)$ & & $42(3.3)$ & $42(3.2)$ & $16(2.4)$ \\
\hline Slovenia & $9(1.7)$ & $40(3.3)$ & $51(3.5)$ & & $66(3.5)$ & $30(3.3)$ & $4(1.2)$ \\
\hline South Africa & $22(2.6)$ & $52(3.4)$ & $26(2.8)$ & & $21(2.5)$ & $43(3.2)$ & $36(2.8)$ \\
\hline Spain & $4(1.3)$ & $21(3.6)$ & $75(3.7)$ & & $57(4.1)$ & $31(3.7)$ & $12(2.7)$ \\
\hline Sweden & $3(1.3)$ & $46(3.6)$ & $50(3.9)$ & & $83(2.6)$ & $16(2.6)$ & $2(0.9)$ \\
\hline Trinidad and Tobago & $25(3.5)$ & $64(3.8)$ & $11(2.4)$ & & $79(3.0)$ & $19(3.0)$ & $2(0.9)$ \\
\hline United States & $22(2.5)$ & $56(3.7)$ & $22(3.1)$ & & $79(3.3)$ & $18(3.3)$ & $3(1.2)$ \\
\hline International Avg. & $14(0.4)$ & $40(0.6)$ & $47(0.6)$ & & $59(0.5)$ & $32(0.5)$ & $9(0.3)$ \\
\hline
\end{tabular}


Exhibit 6.13 Students' Reports About Students Reading Aloud in Class

PIRLS 2006

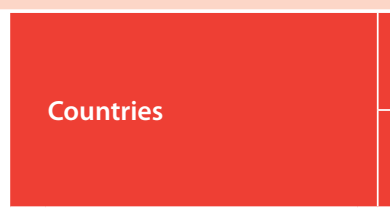

\begin{tabular}{|c|c|}
\multicolumn{2}{|c|}{$\begin{array}{c}\text { Every Day or Almost } \\
\text { Every Day }\end{array}$} \\
\hline $\begin{array}{c}\text { Percent of } \\
\text { Students }\end{array}$ & $\begin{array}{c}\text { Average } \\
\text { Achievement }\end{array}$ \\
\hline
\end{tabular}

\begin{tabular}{c|c|}
\multicolumn{2}{|c|}{$\begin{array}{c}\text { Once or Twice } \\
\text { a Week }\end{array}$} \\
\hline $\begin{array}{c}\text { Percent of } \\
\text { Students }\end{array}$ & $\begin{array}{c}\text { Average } \\
\text { Achievement }\end{array}$ \\
\hline
\end{tabular}

\begin{tabular}{|c|c|}
\hline \multicolumn{2}{|c|}{$\begin{array}{c}\text { Once or } \\
\text { Twice a Month }\end{array}$} \\
\hline $\begin{array}{c}\text { Percent of } \\
\text { Students }\end{array}$ & $\begin{array}{c}\text { Average } \\
\text { Achievement }\end{array}$ \\
\hline $21(0.9)$ & $547(2.9)$ \\
\hline
\end{tabular}

Austria
Belgium (Flemish)
Belgium (French)

$\begin{array}{llll}4(0.3) & 507(6.8) & 21(0.8) & 534(3.5)\end{array}$

\begin{tabular}{ll|l}
$4(0.3)$ & $507(6.8)$ \\
$9(0.9)$ & $529(4.9)$
\end{tabular}

Bulgaria $19(1.1)$

Canada, Alberta

$37(1.9)$

$473(4.0)$

$33(1.6)$

$544(2.9)$

$22(1.0)$

$548(2.7)$

$39(1.2) \quad 500(3.0)$

$19(0.8)$

Canada, British Columbia

$5(0.6)$

$541(5.9)$

$42(1.6)$

$552(5.0)$

$15(0.9)$

$511(3.4)$

$511(3.4)$

$20(0.8)$

$563(3.4)$

Canada, Nova Scotia

$3(0.5)$

$554(8.9)$

$30(1.2)$

$557(3.2)$

Canada, Ontario

$4(0.4) \quad 505(7.3)$

$\begin{array}{ll}4(0.4) & 505(7.3) \\ 5(0.5) & 522(6.8)\end{array}$

$23(1.2)$

Canada, Quebec

$13(0.9) \quad 511(5.2)$

$28(1.6)$

$559(3.6)$

$21(1.0)$

$565(3.3)$

$541(3.2)$

$21(0.8)$

Chinese Taipei

$3(0.3)$

Denmark

England

$5(0.7)$

511 (8.6)

$33(1.4)$

557 (4.4)

$23(0.8)$

$551(3.1)$

$559(4.3)$

$22(0.9)$

$543(3.3)$

$11(0.5) \quad 526(3.4)$

$15(0.9)$

$543(3.4)$

$24(0.8)$

$547(3.8)$

France

$3(0.4)$

$532(6.6) \quad 33(1.3)$

$546(2.8)$

$26(1.1)$

$546(4.0)$

Georgia

$15(0.8)$

495 (3.9)

$39(0.9)$

$533(3.9)$

$25(0.9)$

$528(3.0)$

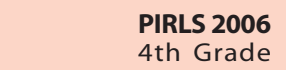

Germany

$5(0.4)$

464 (3.3)

$38(2.0)$

$475(5.3)$

$12(1.4)$

$489(7.7)$

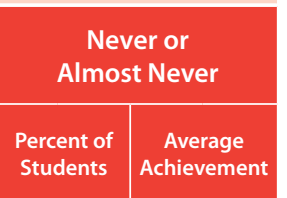

Hong Kong SAR

$23(1.1)$

$475(5.3)$

$20(0.6)$

556 (3.1)

Hungary

$16(1.1)$

$552(6.2)$

$18(0.8) \quad 565(3.5)$

$17(0.7)$

570 (3.2)

$551(3.4)$

$21(0.8)$

$562(3.5)$

$24(0.7)$

$517(2.3)$
$415(6.5)$

Indonesia

$57(0.3)$

485 (5.9)

$19(0.6)$

Iran, Islamic Rep. of

$400(4.6) \quad 41(1.2)$

$408(5.0)$

$10(0.6)$

$420(8.8)$

$55(1.3) \quad 540(2.2)$

Israe

Italy

$$
\text { Kautrit }
$$

Lithuania

Luxembourg

Macedonia, Rep. of

$30(1.6)$

$418(4.4)$

$47(1.3) \quad 428(4.4)$

$13(1.0)$

$37(1.7) \quad 554(2.1)$

$23(1.2)$

$6(0.5) \quad 541(8.8)$

$45(1.5) \quad 564(2.7)$

$51(1.7) \quad 557(3.2)$

$52(1.7) \quad 545(2.5)$

$43(1.8) \quad 558(2.7)$

$32(1.6) \quad 541(4.0)$

$71(1.3) \quad 537(2.1)$

$38(1.7) \quad 549(2.9)$

$42(2.0) \quad 548(3.6)$

$21(1.0) \quad 532(2.8)$

$12(0.7) \quad 477(5.3)$

$52(1.3) \quad 556(2.3)$

Moldova, Rep. of

Morocco

Netherlands

$30(1.7)$

$543(4.8)$

37 (1.1)

$518(3.9)$

$18(0.8)$

$539(4.0)$

$62(1.0) \quad 563(2.4)$

$26(1.3) \quad 560(3.3)$

$52(0.8) \quad 513(1.9)$

$\begin{array}{ll}52(0.8) & 513(1.9) \\ 11(0.8) & 418(6.1)\end{array}$

\begin{tabular}{l|l}
$10(0.7)$ & $417(5.6)$ \\
$21(0.9)$ & $543(4.2)$
\end{tabular}

Netherlands

New Zealand

$\begin{array}{ll}33(1.4) & 332(6.2) \\ 19(1.2) & 528(4.9)\end{array}$

$43(1.1)$

$558(3.3)$

$16(1.0)$

$558(4.3)$

$11(0.8) \quad 545(5.2)$

Poland

$35(1.7) \quad 524(2.3)$

$40(1.2)$

$547(3.2)$

$11(0.7)$

$343(6.6)$

$12(0.7)$

$355(6.4)$

$43(1.3)$

$22(1.2)$

$547(3.1)$

$19(0.9)$

$536(3.0)$

Qatar

Romania

$3(0.2) \quad 521(5.4)$

$24(0.6) \quad 554(2.2)$

$15(0.8)$

$547(3.3)$

$7(0.6)$

$545(4.9)$

$53(1.9) \quad 423(4.5)$

$35(1.5)$

$554(2.2)$
$467(4.7)$

$28(0.6)$

$563(2.0)$

$45(0.7)$

$559(1.5)$

\begin{tabular}{l|l|}
$55(1.8)$ & $501(3.8)$
\end{tabular}

$40(1.4)$

$500(3.6)$

$7(0.6)$

$503(7.3)$

$45(0.7)$

$475(10.2)$

$42(2.0) \quad 318(5.8) \quad 43(1.8)$

$331(8.6) \quad 9(0.7)$

$9(0.8)$

$504(4.3)$

$6(0.4)$

$490(7.6)$

$3(0.4) \quad 518(7.3)$

$43(1.8)$

$341(10.3)$

$6(0.5)$

$309(11.2)$

\begin{tabular}{|l|l|l|l|}
$3(0.4)$ & $518(7.3)$ & $12(0.9)$ & $542(3.4)$
\end{tabular}

$16(0.9)$

$552(2.9)$

$69(1.8)$

$549(1.6)$

$5(0.4) \quad 460(6.8) \quad 22(0.9) \quad 519(3.6)$

$23(0.6)$

$540(3.0)$

$51(1.1)$

$543(2.5)$

\begin{tabular}{|l|l|l|l|}
\hline $8(1.2)$ & $474(6.0)$ & $34(2.0)$ & $500(2.9)$ \\
\hline
\end{tabular}

$21(1.0)$

$503(3.6)$

$38(2.5)$

$501(3.2)$

$20(1.2)$

$498(4.2)$

$41(0.9)$

$522(2.6)$

$21(0.8)$

535 (3.6)

$19(1.1)$

527 (3.9)

The

$39(2.2)$

$350(2.0)$

$40(0.6) \quad 357(1.6)$

$11(0.4)$

$366(3.5)$

$12(0.4)$

$368(3.8)$

Russian Federation

Scotland

$30(1.6)$

$552(5.1)$

$39(1.7) \quad 499(5.0)$

$12(0.9)$

$511(6.3)$

$9(0.7)$

$492(10.1)$

Scotland

$6(0.8)$

$492(8.7) \quad 41(1.8)$

$41(1.8) \quad 530(3.1)$

$21(0.9)$

$576(3.4)$

$8(0.6) \quad 570(4.0)$

Slovak Repub

$6(0.3)$

$530(3.1) \quad 28(1.4)$

$537(4.1)$

$25(1.7)$

$524(4.3)$

Slovenia

South Africa

$32(1.4) \quad 509(4.2)$

$24(0.6) \quad 551(3.6)$

$20(0.6)$

$562(3.8)$

$25(1.7)$

$15(0.8)$

506 (3.6)

$43(1.1) \quad 537(2.7)$

$16(0.7)$

$547(5.3)$

$\begin{array}{ll}9(0.6) & 558(4.7)\end{array}$

Spain

$43(1.1) \quad 280(4.0)$

$35(0.6)$

$522(2.7)$

$24(0.8)$

$529(2.8)$

$20(1.0) \quad 525(3.1)$

Spain - 23

Sweden $23(1.1) \quad 490(4.1)$

$41(1.2) \quad 512(2.9)$

$12(0.6)$

$359(13.0)$ 
Exhibit 6.14 Students' Reports About Independent Reading

PIRLS 2006

4th Grade

\begin{tabular}{|c|c|c|c|c|c|c|c|c|}
\hline \multirow{2}{*}{ Countries } & \multicolumn{2}{|c|}{$\begin{array}{c}\text { Every Day or } \\
\text { Almost Every Day }\end{array}$} & \multicolumn{2}{|c|}{$\begin{array}{c}\text { Once or Twice } \\
\text { a Week }\end{array}$} & \multicolumn{2}{|c|}{$\begin{array}{c}\text { Once or } \\
\text { Twice a Month }\end{array}$} & \multicolumn{2}{|c|}{$\begin{array}{c}\text { Never or } \\
\text { Almost Never }\end{array}$} \\
\hline & $\begin{array}{l}\text { Percent of } \\
\text { Students }\end{array}$ & $\begin{array}{c}\text { Average } \\
\text { Achievement }\end{array}$ & $\begin{array}{l}\text { Percent of } \\
\text { Students }\end{array}$ & \begin{tabular}{c|} 
Average \\
Achievement
\end{tabular} & $\begin{array}{l}\text { Percent of } \\
\text { Students }\end{array}$ & \begin{tabular}{c|} 
Average \\
Achievement
\end{tabular} & $\begin{array}{l}\text { Percent of } \\
\text { Students }\end{array}$ & $\begin{array}{c}\text { Average } \\
\text { Achievement }\end{array}$ \\
\hline Austria & $63(0.9)$ & $538(2.5)$ & $28(0.7)$ & $541(2.7)$ & $5(0.4)$ & $542(5.8)$ & $4(0.3)$ & $528(5.1)$ \\
\hline Belgium (Flemish) & $62(1.4)$ & $548(2.4)$ & $30(1.0)$ & $547(2.5)$ & $5(0.5)$ & $548(4.4)$ & $3(0.4)$ & $534(5.3)$ \\
\hline Belgium (French) & $65(1.2)$ & $505(2.9)$ & $28(1.2)$ & $494(3.1)$ & $4(0.3)$ & $488(5.5)$ & $3(0.3)$ & $472(7.1)$ \\
\hline Bulgaria & $66(1.5)$ & $553(4.3)$ & $28(1.4)$ & $542(5.7)$ & $4(0.4)$ & $529(11.0)$ & $2(0.4)$ & $\sim \sim$ \\
\hline Canada, Alberta & $88(0.8)$ & $565(2.4)$ & $10(0.7)$ & $537(4.9)$ & $1(0.3)$ & $\sim \sim$ & $1(0.2)$ & $\sim \sim$ \\
\hline Canada, British Columbia & $90(0.8)$ & $563(2.6)$ & $9(0.7)$ & $533(4.9)$ & $1(0.2)$ & $\sim \sim$ & $1(0.1)$ & $\sim \sim$ \\
\hline Canada, Nova Scotia & $87(0.8)$ & $549(2.0)$ & $11(0.7)$ & $512(4.2)$ & $1(0.1)$ & $\sim \sim$ & $1(0.2)$ & $\sim \sim$ \\
\hline Canada, Ontario & $83(1.0)$ & $560(2.8)$ & $14(0.9)$ & $542(3.9)$ & $2(0.3)$ & $\sim \sim$ & $1(0.2)$ & $\sim \sim$ \\
\hline Canada, Quebec & $85(1.1)$ & $537(2.7)$ & $13(1.0)$ & $519(4.6)$ & $2(0.2)$ & $\sim \sim$ & $1(0.2)$ & $\sim \sim$ \\
\hline Chinese Taipei & $50(1.2)$ & $550(2.2)$ & $33(0.8)$ & $532(2.4)$ & $9(0.6)$ & $513(4.4)$ & $8(0.5)$ & $493(4.2)$ \\
\hline Denmark & $67(1.6)$ & $546(2.5)$ & $28(1.3)$ & $550(2.9)$ & $4(0.4)$ & $546(7.2)$ & $2(0.3)$ & $\sim \sim$ \\
\hline England & $79(1.4)$ & $547(2.8)$ & $17(1.2)$ & $522(5.0)$ & $2(0.4)$ & $\sim \sim$ & $2(0.2)$ & $\sim \sim$ \\
\hline France & $66(1.1)$ & $524(2.3)$ & $26(0.9)$ & $519(2.8)$ & $5(0.4)$ & $515(6.4)$ & $3(0.2)$ & $511(6.6)$ \\
\hline Georgia & $65(1.6)$ & $476(3.2)$ & $27(1.1)$ & $470(4.4)$ & $5(1.1)$ & $473(7.4)$ & $3(0.4)$ & 449 (11.2) \\
\hline Germany & $60(1.0)$ & $548(2.5)$ & $29(0.9)$ & $554(2.9)$ & $7(0.5)$ & $551(4.6)$ & $4(0.3)$ & $548(5.6)$ \\
\hline Hong Kong SAR & $55(1.2)$ & $573(2.2)$ & $32(0.9)$ & $560(3.2)$ & $7(0.5)$ & $546(4.4)$ & $6(0.5)$ & $522(5.1)$ \\
\hline Hungary & $59(1.1)$ & $552(3.5)$ & $33(1.0)$ & $550(3.3)$ & $5(0.4)$ & $551(6.2)$ & $3(0.3)$ & $544(9.2)$ \\
\hline Iceland & $74(0.7)$ & $514(1.3)$ & $21(0.6)$ & $505(2.9)$ & $3(0.3)$ & $507(6.3)$ & $3(0.2)$ & $487(9.3)$ \\
\hline Indonesia & $62(1.3)$ & $418(4.5)$ & $30(1.1)$ & $394(4.5)$ & $5(0.4)$ & $374(9.0)$ & $3(0.3)$ & $352(9.3)$ \\
\hline Iran, Islamic Rep. of & $52(1.4)$ & $434(4.2)$ & $38(1.2)$ & $415(3.8)$ & $7(0.5)$ & $395(7.5)$ & $3(0.3)$ & $386(10.5)$ \\
\hline Israel & $67(1.1)$ & $531(3.2)$ & $25(0.9)$ & $500(4.5)$ & $4(0.3)$ & $475(9.0)$ & $4(0.3)$ & $465(9.9)$ \\
\hline Italy & $58(1.4)$ & $554(2.7)$ & $32(1.1)$ & $551(3.7)$ & $5(0.5)$ & $559(9.0)$ & $4(0.4)$ & $523(9.1)$ \\
\hline Kuwait & $47(1.2)$ & $361(4.8)$ & $39(1.0)$ & $329(5.0)$ & $8(0.5)$ & $318(8.8)$ & $6(0.7)$ & $281(9.8)$ \\
\hline Latvia & $63(1.4)$ & $543(2.6)$ & $30(1.1)$ & $537(2.7)$ & $4(0.5)$ & $548(9.3)$ & $3(0.3)$ & $524(8.3)$ \\
\hline Lithuania & $76(0.9)$ & $538(1.8)$ & $21(0.7)$ & $535(2.8)$ & $2(0.2)$ & $\sim \sim$ & $1(0.2)$ & $\sim \sim$ \\
\hline Luxembourg & $39(0.6)$ & $554(1.7)$ & $41(0.6)$ & $560(1.5)$ & $12(0.5)$ & $561(3.0)$ & $8(0.4)$ & $552(3.6)$ \\
\hline Macedonia, Rep. of & $72(1.4)$ & $448(4.4)$ & $24(1.3)$ & $448(5.6)$ & $2(0.3)$ & $\sim \sim$ & $1(0.2)$ & $\sim \sim$ \\
\hline Moldova, Rep. of & $77(1.3)$ & $503(3.1)$ & $20(1.2)$ & $493(4.5)$ & $2(0.3)$ & $\sim \sim$ & $1(0.2)$ & $\sim \sim$ \\
\hline Morocco & $34(1.6)$ & $338(5.9)$ & $47(1.6)$ & $318(6.5)$ & $12(1.1)$ & $336(12.0)$ & $7(1.0)$ & $294(18.0)$ \\
\hline Netherlands & $73(1.5)$ & $549(1.7)$ & $23(1.2)$ & $545(2.3)$ & $3(0.4)$ & $536(9.7)$ & $2(0.3)$ & $\sim \sim$ \\
\hline New Zealand & $83(0.8)$ & $541(1.9)$ & $14(0.7)$ & $502(4.6)$ & $2(0.2)$ & $\sim \sim$ & $1(0.2)$ & $\sim \sim$ \\
\hline Norway & $77(1.5)$ & $499(2.4)$ & $19(1.3)$ & $502(4.8)$ & $2(0.4)$ & $\sim \sim$ & $1(0.4)$ & $\sim \sim$ \\
\hline Poland & $64(1.0)$ & $527(2.3)$ & $29(0.9)$ & $511(3.5)$ & $4(0.3)$ & $500(8.9)$ & $3(0.3)$ & $497(6.8)$ \\
\hline Qatar & $57(0.6)$ & $372(1.5)$ & $32(0.6)$ & $347(2.3)$ & $6(0.3)$ & $319(5.3)$ & $6(0.3)$ & $306(5.2)$ \\
\hline Romania & $71(1.5)$ & $496(4.9)$ & $24(1.4)$ & $489(6.3)$ & $3(0.3)$ & $459(18.9)$ & $2(0.4)$ & $\sim \sim$ \\
\hline Russian Federation & $67(1.0)$ & $565(3.9)$ & $26(0.8)$ & $563(3.3)$ & $4(0.3)$ & $568(6.8)$ & $3(0.3)$ & $580(6.8)$ \\
\hline Scotland & $71(1.8)$ & $534(3.0)$ & $24(1.4)$ & $523(4.4)$ & $3(0.5)$ & $504(10.2)$ & $2(0.4)$ & $\sim \sim$ \\
\hline Singapore & $77(0.8)$ & $570(2.8)$ & $19(0.6)$ & $531(3.6)$ & $3(0.2)$ & $504(6.8)$ & $2(0.2)$ & $\sim \sim$ \\
\hline Slovak Republic & $58(1.3)$ & $535(2.8)$ & $34(1.1)$ & $525(4.2)$ & $5(0.5)$ & $538(6.9)$ & $2(0.3)$ & $\sim \sim$ \\
\hline Slovenia & $64(1.1)$ & $526(2.1)$ & $29(0.9)$ & $516(3.1)$ & $4(0.3)$ & $519(5.1)$ & $3(0.3)$ & $494(10.1)$ \\
\hline South Africa & $58(1.2)$ & $335(6.8)$ & $30(0.8)$ & $275(4.9)$ & $7(0.4)$ & $257(7.4)$ & $5(0.4)$ & $247(6.3)$ \\
\hline Spain & $68(1.1)$ & $516(2.6)$ & $25(0.9)$ & $511(3.5)$ & $4(0.3)$ & $494(8.7)$ & $3(0.3)$ & $492(8.9)$ \\
\hline Sweden & $73(1.7)$ & $548(2.6)$ & $24(1.5)$ & $555(3.8)$ & $2(0.3)$ & $\sim \sim$ & $1(0.2)$ & $\sim \sim$ \\
\hline Trinidad and Tobago & $76(1.4)$ & $448(4.7)$ & $19(1.0)$ & $408(6.8)$ & $3(0.4)$ & $393(15.1)$ & $2(0.4)$ & $\sim \sim$ \\
\hline United States & $85(0.9)$ & $547(3.5)$ & $12(0.8)$ & $514(4.3)$ & $2(0.2)$ & $\sim \sim$ & $1(0.2)$ & $\sim \sim$ \\
\hline International Avg. & $65(0.2)$ & $508(0.6)$ & $27(0.2)$ & $495(0.7)$ & $5(0.1)$ & $483(1.5)$ & $3(0.1)$ & $462(1.6)$ \\
\hline
\end{tabular}

Based on students' responses to how often they do the following: read silently on their own; and read books of their own choosing. Average is computed based on a 4-point scale: Never or almost never $=1$, Once or twice a month $=2$, Once or twice a week $=3$, and Every day or almost every day $=4$. Never or almost never indicates an average of 1 to less than 1.75. Once or twice a month indicates an average of 1.75 through 2.5. Once or twice a week indicates an average of greater than 2.5 through 3.25. Every day or almost every day indicates an average of greater than 3.25 through 4 .
() Standard errors appear in parentheses. Because results are rounded to the nearest whole number, some totals may appear inconsistent.

A tilde $(\sim)$ indicates insufficient data to report achievement.

NOTE: The International Average does not include the results from the Canadian provinces. 


\section{What Activities Do Students Do in Response to Class Reading?}

As part of reading instruction, teachers usually provide students with a number of ways to demonstrate their understanding of what they have read. Exhibits 6.15 through 6.19 present teachers' and students' reports of how often students were asked to do various activities after reading, both informal and formal, with changes from 2001.

Exhibit 6.15 shows teachers' reports about the types of activities used at least weekly to monitor students' reading comprehension, including changes from 2001. Most students (89\%), on average internationally, were asked to answer questions aloud or give an oral summary, or to write something (either in short answers or paragraphs or in a workbook or worksheet) at least once or twice a week. Approximately two thirds of the students internationally were asked to talk with other students, which included an increase from 2001 in 10 countries.

Exhibit 6.16 presents students' reports about how often they answer questions aloud about their reading. Students' reports support those of their teachers, with more than half the students $(62 \%)$, on average internationally, reporting having answered questions aloud at least weekly. Internationally, average achievement for those students answering questions aloud every day or almost every day does not differ greatly from those answering questions less frequently (a difference of 11 points), but differences do occur within many countries, with those students answering aloud daily having lower achievement than students answering aloud less often. It appears that in these countries, teachers are monitoring the less proficient readers more frequently than they are the more proficient readers.

As shown in Exhibit 6.17, students' reports of how often they were asked to answer questions about class reading in workbooks or worksheets confirms teachers' reports of the use of workbooks or worksheets as a basis for instruction (see Exhibit 6.6). Three fourths of students, on average internationally, reported writing answers to questions in workbooks or worksheets at least weekly. 
Exhibit 6.18 shows the percentage of students who reported writing responses to class reading (not in workbooks or worksheets) at least weekly. More than half the students (57\%), internationally on average, wrote responses to what they read at least once or twice a week. Countries where the greatest percentages of students (41 to $46 \%$ ) reported daily writing activities included Bulgaria, Indonesia, Macedonia, and South Africa. On average internationally, those students who reported doing writing activities once or twice a month had higher reading achievement than students who reported writing more or less often.

Exhibit 6.19 shows teachers' reports of how often they gave a written quiz or test after students read something in class. Internationally on average, about one fourth of students were given a weekly written quiz or test about what they read. Interestingly, more than half the students in Denmark (79\%), England (55\%), Iceland (71\%), New Zealand (79\%), Norway (59\%), Scotland $(54 \%)$, Sweden (54\%), and the Canadian province of Nova Scotia $(56 \%)$ were never given a written quiz or test. 
Exhibit 6.15 Teachers' Reports About Reading Comprehension Activities After Instruction with Trends PIRLS 2006

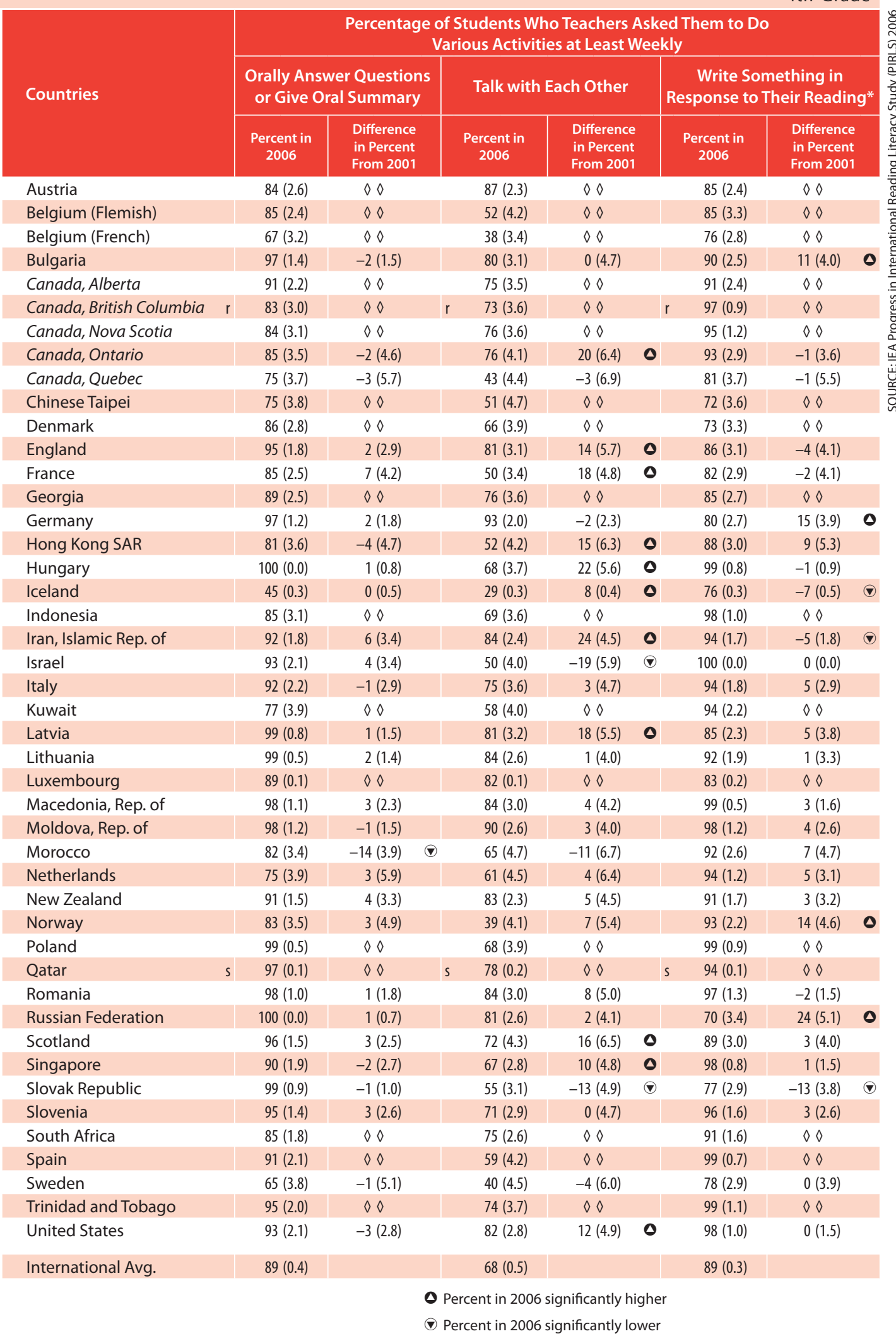

Background data provided by teachers.

* "Write something in response to their reading" is a combination of the teacher's responses to the following about activities after instruction: answer reading comprehension questions in a workbook or on a worksheet about what they have read; and write something about or in response to what they have read.

() Standard errors appear in parentheses. Because results are rounded to the nearest whole number, some totals may appear inconsistent.
An " $r$ " indicates data are available for $70-84 \%$ of the students. An "s" indicates data are available for $50-69 \%$ of the students. An " $x$ " indicates data are available for less than $50 \%$ of the students.

A diamond $(\nabla)$ indicates the country did not participate in the 2001 assessment. NOTE: The International Average does not include the results from the Canadian provinces. Trend Note: The primary education systems of the Russian Federation and Slovenia underwent structural changes. Data for Canada, Ontario include only public schools. 
Exhibit 6.16 Students Answer Questions Aloud About Class Reading

PIRLS 2006

4th Grade

\begin{tabular}{|c|c|c|c|c|c|c|c|c|}
\hline \multirow{2}{*}{ Countries } & \multicolumn{2}{|c|}{$\begin{array}{c}\text { Every Day or } \\
\text { Almost Every Day }\end{array}$} & \multicolumn{2}{|c|}{$\begin{array}{c}\text { Once or Twice } \\
\text { a Week }\end{array}$} & \multicolumn{2}{|c|}{$\begin{array}{c}\text { Once or } \\
\text { Twice a Month }\end{array}$} & \multicolumn{2}{|c|}{$\begin{array}{c}\text { Never or } \\
\text { Almost Never }\end{array}$} \\
\hline & $\begin{array}{l}\text { Percent of } \\
\text { Students }\end{array}$ & $\begin{array}{c}\text { Average } \\
\text { Achievement }\end{array}$ & $\begin{array}{l}\text { Percent of } \\
\text { Students }\end{array}$ & \begin{tabular}{c|} 
Average \\
Achievement
\end{tabular} & $\begin{array}{l}\text { Percent of } \\
\text { Students }\end{array}$ & \begin{tabular}{c|} 
Average \\
Achievement
\end{tabular} & $\begin{array}{l}\text { Percent of } \\
\text { Students }\end{array}$ & $\begin{array}{c}\text { Average } \\
\text { Achievement }\end{array}$ \\
\hline Austria & $16(0.7)$ & $517(3.1)$ & $30(0.9)$ & $533(2.6)$ & $26(0.8)$ & $545(3.1)$ & $28(1.0)$ & $551(2.4)$ \\
\hline Belgium (Flemish) & $18(1.5)$ & $537(3.1)$ & $27(1.1)$ & $547(3.1)$ & $17(0.9)$ & $551(3.1)$ & $38(1.7)$ & $551(2.2)$ \\
\hline Belgium (French) & $21(1.4)$ & $480(3.4)$ & $28(0.9)$ & $499(3.1)$ & $20(1.0)$ & $507(3.5)$ & $31(1.4)$ & $510(3.3)$ \\
\hline Bulgaria & $65(1.6)$ & $553(4.6)$ & $24(1.2)$ & $546(5.1)$ & $7(0.6)$ & $530(7.3)$ & $4(0.4)$ & $519(9.8)$ \\
\hline Canada, Alberta & $11(0.7)$ & $541(4.5)$ & $23(0.7)$ & $554(3.5)$ & $23(0.8)$ & $564(3.5)$ & $43(1.3)$ & $568(2.8)$ \\
\hline Canada, British Columbia & $10(0.9)$ & $534(5.7)$ & $25(1.1)$ & $554(3.3)$ & $26(1.2)$ & $564(4.6)$ & $39(1.3)$ & $566(3.1)$ \\
\hline Canada, Nova Scotia & $12(0.6)$ & $507(5.0)$ & $25(0.8)$ & $536(3.0)$ & $23(0.8)$ & $552(2.9)$ & $41(1.1)$ & $554(2.2)$ \\
\hline Canada, Ontario & $13(0.9)$ & $529(6.0)$ & $27(1.1)$ & $554(4.0)$ & $24(0.9)$ & $562(3.5)$ & $36(1.3)$ & $563(2.7)$ \\
\hline Canada, Quebec & $12(0.9)$ & $506(4.8)$ & $25(1.3)$ & $530(3.3)$ & $23(1.1)$ & $538(3.6)$ & $40(1.6)$ & $543(3.5)$ \\
\hline Chinese Taipei & $13(0.7)$ & $526(3.3)$ & $29(1.2)$ & $539(2.6)$ & $25(0.8)$ & $539(3.1)$ & $32(1.1)$ & $534(2.5)$ \\
\hline Denmark & $15(1.2)$ & $539(4.3)$ & $35(1.2)$ & $539(3.0)$ & $28(1.3)$ & $556(3.4)$ & $22(1.0)$ & $554(3.3)$ \\
\hline England & $11(0.9)$ & $501(7.9)$ & $28(1.0)$ & $533(3.2)$ & $25(0.9)$ & $549(3.5)$ & $35(1.4)$ & $553(3.5)$ \\
\hline France & $20(1.2)$ & $507(3.3)$ & $32(1.1)$ & $524(3.0)$ & $20(0.9)$ & $526(3.3)$ & $28(1.0)$ & $529(2.6)$ \\
\hline Georgia & $58(1.7)$ & $470(3.7)$ & $24(1.1)$ & $476(4.6)$ & $8(0.6)$ & $463(5.6)$ & $10(0.7)$ & $483(6.4)$ \\
\hline Germany & $21(0.8)$ & $540(3.4)$ & $35(0.9)$ & $551(2.9)$ & $25(0.6)$ & $557(3.2)$ & $19(0.8)$ & $551(3.1)$ \\
\hline Hong Kong SAR & $15(0.8)$ & $567(3.6)$ & $25(0.7)$ & $566(3.1)$ & $27(0.9)$ & $565(3.1)$ & $34(1.0)$ & $561(2.5)$ \\
\hline Hungary & $36(1.5)$ & $543(4.3)$ & $39(1.1)$ & $555(3.4)$ & $16(0.9)$ & $555(4.0)$ & $8(0.7)$ & $560(4.7)$ \\
\hline Iceland & $6(0.4)$ & $478(5.6)$ & $17(0.7)$ & $505(3.3)$ & $18(0.6)$ & $513(2.9)$ & $59(0.8)$ & $516(1.6)$ \\
\hline Indonesia & $41(1.4)$ & $409(4.7)$ & $35(1.3)$ & $407(4.6)$ & $13(0.7)$ & $396(5.9)$ & $11(0.7)$ & $409(6.5)$ \\
\hline Iran, Islamic Rep. of & $35(1.6)$ & $417(5.2)$ & $37(1.4)$ & $424(3.9)$ & $13(0.7)$ & $430(5.3)$ & $15(1.1)$ & $422(5.8)$ \\
\hline Israel & $38(1.3)$ & $507(4.4)$ & $31(0.9)$ & $520(3.9)$ & $18(0.7)$ & $523(5.4)$ & $13(0.7)$ & $535(5.5)$ \\
\hline Italy & $45(1.8)$ & $549(4.1)$ & $35(1.4)$ & $558(3.3)$ & $12(0.8)$ & $553(4.7)$ & $8(0.7)$ & $538(4.8)$ \\
\hline Kuwait & $33(1.4)$ & $330(6.4)$ & $29(1.2)$ & $339(5.1)$ & $18(0.8)$ & $318(6.3)$ & $21(1.1)$ & $364(6.0)$ \\
\hline Latvia & $31(1.3)$ & $541(3.5)$ & $41(1.0)$ & $545(2.8)$ & $19(0.8)$ & $539(3.1)$ & $10(0.7)$ & $529(4.5)$ \\
\hline Lithuania & $42(1.4)$ & $534(2.1)$ & $36(1.0)$ & $539(2.2)$ & $13(0.6)$ & $545(3.1)$ & $9(0.6)$ & $534(3.9)$ \\
\hline Luxembourg & $13(0.4)$ & $540(2.7)$ & $37(0.7)$ & $558(1.8)$ & $30(0.7)$ & $563(1.8)$ & $21(0.5)$ & $559(1.7)$ \\
\hline Macedonia, Rep. of & $63(1.4)$ & $444(4.3)$ & $28(1.1)$ & $458(4.8)$ & $7(0.6)$ & $434(9.0)$ & $2(0.3)$ & $\sim \sim$ \\
\hline Moldova, Rep. of & $59(1.5)$ & $506(3.6)$ & $31(1.3)$ & $496(3.0)$ & $6(0.5)$ & $480(7.3)$ & $3(0.4)$ & $475(10.4)$ \\
\hline Morocco & $57(2.0)$ & $338(4.9)$ & $28(1.4)$ & $310(10.2)$ & $10(0.8)$ & $295(13.5)$ & $6(0.5)$ & $314(10.9)$ \\
\hline Netherlands & $7(0.7)$ & $527(4.7)$ & $16(0.9)$ & $546(3.0)$ & $13(0.6)$ & $547(3.3)$ & $64(1.3)$ & $550(1.6)$ \\
\hline New Zealand & $15(0.8)$ & $482(3.8)$ & $29(0.8)$ & $530(2.7)$ & $24(0.8)$ & $548(3.0)$ & $32(0.9)$ & $547(2.8)$ \\
\hline Norway & $15(1.3)$ & $486(4.3)$ & $31(1.2)$ & $498(4.2)$ & $22(1.0)$ & $505(3.0)$ & $33(1.7)$ & $501(3.5)$ \\
\hline Poland & $40(1.3)$ & $523(2.7)$ & $40(1.1)$ & $521(3.0)$ & $13(0.7)$ & $510(4.2)$ & $6(0.5)$ & $517(6.5)$ \\
\hline Qatar & $35(0.5)$ & $357(2.0)$ & $28(0.6)$ & $358(2.9)$ & $17(0.5)$ & $339(3.5)$ & $20(0.6)$ & $367(3.0)$ \\
\hline Romania & $60(1.9)$ & $496(4.8)$ & $27(1.5)$ & $487(6.4)$ & $8(0.6)$ & $487(8.9)$ & $5(0.4)$ & $456(17.0)$ \\
\hline Russian Federation & $57(1.4)$ & $569(3.7)$ & $31(1.1)$ & $559(4.0)$ & $8(0.5)$ & $562(5.4)$ & $5(0.5)$ & $556(7.4)$ \\
\hline Scotland & $19(1.6)$ & $498(4.8)$ & $36(1.6)$ & $537(3.8)$ & $22(1.2)$ & $538(3.7)$ & $23(1.5)$ & $529(4.6)$ \\
\hline Singapore & $11(0.6)$ & $534(4.4)$ & $23(0.6)$ & $556(3.5)$ & $24(0.6)$ & $559(3.7)$ & $41(0.7)$ & $567(3.0)$ \\
\hline Slovak Republic & $28(1.6)$ & $513(5.5)$ & $39(1.3)$ & $535(3.0)$ & $23(1.1)$ & $546(3.7)$ & $10(0.6)$ & $536(3.8)$ \\
\hline Slovenia & $19(1.0)$ & $507(3.2)$ & $42(1.0)$ & $526(2.6)$ & $25(0.9)$ & $528(2.9)$ & $14(0.9)$ & $520(4.0)$ \\
\hline South Africa & $48(1.1)$ & $288(4.3)$ & $26(0.6)$ & $305(6.4)$ & $14(0.6)$ & $315(11.6)$ & $12(0.7)$ & $363(12.7)$ \\
\hline Spain & $35(1.3)$ & $501(3.4)$ & $29(1.0)$ & $520(3.0)$ & $13(0.7)$ & $522(4.2)$ & $22(0.9)$ & $517(4.3)$ \\
\hline Sweden & $20(1.2)$ & $537(3.6)$ & $34(1.3)$ & $545(2.9)$ & $27(1.1)$ & $553(2.9)$ & $19(1.1)$ & $569(3.8)$ \\
\hline Trinidad and Tobago & $41(1.7)$ & $424(6.0)$ & $32(1.0)$ & $441(4.9)$ & $13(0.7)$ & $444(9.1)$ & $15(1.1)$ & $458(7.7)$ \\
\hline United States & $19(1.2)$ & $513(3.9)$ & $25(0.7)$ & $545(3.8)$ & $17(0.8)$ & $547(4.3)$ & $38(1.1)$ & $549(3.6)$ \\
\hline International Avg. & $31(0.2)$ & $491(0.8)$ & $31(0.2)$ & $502(0.6)$ & $18(0.1)$ & $502(0.9)$ & $21(0.1)$ & $506(1.0)$ \\
\hline
\end{tabular}

Background data provided by students.

() Standard errors appear in parentheses. Because results are rounded to the nearest whole number, some totals may appear inconsistent.

A tilde $(\sim)$ indicates insufficient data to report achievement.

NOTE: The International Average does not include the results from the Canadian provinces. 
Exhibit 6.17 Students Answer Questions in Workbooks or Worksheets About Class Reading

PIRLS 2006

\begin{tabular}{|c|c|c|c|c|c|c|c|c|}
\hline \multirow{2}{*}{ Countries } & \multicolumn{2}{|c|}{$\begin{array}{c}\text { Every Day or } \\
\text { Almost Every Day }\end{array}$} & \multicolumn{2}{|c|}{$\begin{array}{l}\text { Once or Twice } \\
\text { a Week }\end{array}$} & \multicolumn{2}{|c|}{$\begin{array}{c}\text { Once or } \\
\text { Twice a Month }\end{array}$} & \multicolumn{2}{|c|}{$\begin{array}{c}\text { Never or } \\
\text { Almost Never }\end{array}$} \\
\hline & $\begin{array}{l}\text { Percent of } \\
\text { Students }\end{array}$ & $\begin{array}{c}\text { Average } \\
\text { Achievement }\end{array}$ & $\begin{array}{l}\text { Percent of } \\
\text { Students }\end{array}$ & $\begin{array}{c}\text { Average } \\
\text { Achievement }\end{array}$ & $\begin{array}{l}\text { Percent of } \\
\text { Students }\end{array}$ & \begin{tabular}{c|} 
Average \\
Achievement
\end{tabular} & $\begin{array}{l}\text { Percent of } \\
\text { Students }\end{array}$ & $\begin{array}{c}\text { Average } \\
\text { Achievement }\end{array}$ \\
\hline Austria & $17(0.9)$ & $516(2.9)$ & $43(1.1)$ & $539(2.7)$ & $24(0.9)$ & $550(2.9)$ & $16(0.8)$ & $544(3.2)$ \\
\hline Belgium (Flemish) & $34(1.8)$ & $543(2.8)$ & $41(1.3)$ & $550(2.3)$ & $14(1.0)$ & $557(3.3)$ & $10(0.8)$ & $538(4.7)$ \\
\hline Belgium (French) & $35(1.4)$ & $488(3.3)$ & $41(1.1)$ & $504(2.9)$ & $14(0.8)$ & $515(4.7)$ & $10(0.7)$ & $503(5.0)$ \\
\hline Bulgaria & $52(1.8)$ & $548(5.3)$ & $33(1.7)$ & $555(4.0)$ & $9(0.9)$ & $559(8.4)$ & $7(0.9)$ & $505(9.3)$ \\
\hline Canada, Alberta & $26(1.1)$ & $547(3.4)$ & $37(1.2)$ & $564(3.3)$ & $21(1.0)$ & $573(2.9)$ & $16(0.9)$ & $559(4.3)$ \\
\hline Canada, British Columbia & $26(1.4)$ & $545(4.1)$ & $41(1.3)$ & $563(3.2)$ & $19(1.0)$ & $570(4.4)$ & $14(0.9)$ & $559(4.0)$ \\
\hline Canada, Nova Scotia & $29(1.3)$ & $524(3.4)$ & $36(1.0)$ & $549(2.6)$ & $21(0.9)$ & $557(3.2)$ & $15(0.8)$ & $546(3.7)$ \\
\hline Canada, Ontario & $29(1.7)$ & $545(4.9)$ & $38(1.4)$ & $557(3.0)$ & $19(1.0)$ & $570(4.6)$ & $14(1.1)$ & $553(4.9)$ \\
\hline Canada, Quebec & $26(1.2)$ & $514(4.0)$ & $41(1.5)$ & $541(2.8)$ & $21(1.6)$ & $547(4.2)$ & $12(0.8)$ & $529(5.1)$ \\
\hline Chinese Taipei & $37(1.0)$ & $534(2.3)$ & $29(1.0)$ & $541(2.8)$ & $15(0.6)$ & $539(3.7)$ & $19(0.8)$ & $530(3.0)$ \\
\hline Denmark & $18(1.4)$ & $545(3.9)$ & $42(1.0)$ & $546(2.9)$ & $25(1.1)$ & $550(3.6)$ & $16(1.1)$ & $543(3.7)$ \\
\hline England & $17(1.2)$ & $510(6.3)$ & $41(1.4)$ & $535(3.1)$ & $26(1.0)$ & $559(3.6)$ & $16(1.0)$ & $553(4.9)$ \\
\hline France & $31(1.5)$ & $512(2.7)$ & $45(1.3)$ & $528(2.6)$ & $15(0.9)$ & $532(3.6)$ & $9(0.6)$ & $510(5.4)$ \\
\hline Georgia & $44(1.7)$ & $464(3.2)$ & $36(1.7)$ & $482(4.5)$ & $9(0.6)$ & $488(6.5)$ & $10(0.7)$ & $459(7.0)$ \\
\hline Germany & $25(0.8)$ & $531(3.3)$ & $40(1.0)$ & $554(2.8)$ & $23(0.7)$ & $563(2.9)$ & $13(0.7)$ & $549(3.4)$ \\
\hline Hong Kong SAR & $27(1.1)$ & $565(2.7)$ & $37(0.8)$ & $570(2.6)$ & $22(0.9)$ & $561(2.9)$ & $14(0.7)$ & $551(4.0)$ \\
\hline Hungary & $62(1.6)$ & $554(3.5)$ & $29(1.3)$ & $551(3.5)$ & $5(0.5)$ & $537(6.3)$ & $4(0.4)$ & $534(7.1)$ \\
\hline Iceland & $28(0.7)$ & $499(2.6)$ & $37(0.8)$ & $513(1.7)$ & $16(0.6)$ & $523(3.2)$ & $19(0.7)$ & $514(2.8)$ \\
\hline Indonesia & $53(1.3)$ & $413(4.4)$ & $34(1.2)$ & $404(4.5)$ & $7(0.5)$ & $392(6.3)$ & $6(0.5)$ & $374(6.8)$ \\
\hline Iran, Islamic Rep. of & $37(1.5)$ & $423(4.8)$ & $46(1.3)$ & $424(3.3)$ & $12(0.9)$ & $419(6.6)$ & $6(0.8)$ & $409(10.5)$ \\
\hline Israel & $67(1.1)$ & $521(3.3)$ & $24(0.9)$ & $510(4.6)$ & $5(0.4)$ & $501(13.2)$ & $4(0.3)$ & $511(10.8)$ \\
\hline Italy & $41(1.9)$ & $548(4.3)$ & $40(1.8)$ & $556(3.3)$ & $11(1.0)$ & $555(4.2)$ & $8(0.7)$ & $542(6.4)$ \\
\hline Kuwait & $50(1.5)$ & $338(5.3)$ & $38(1.2)$ & $339(4.6)$ & $7(0.5)$ & $331(9.9)$ & $5(0.6)$ & $303(15.9)$ \\
\hline Latvia & $32(1.5)$ & $539(3.9)$ & $45(1.1)$ & $539(2.5)$ & $17(0.8)$ & $555(3.8)$ & $7(0.6)$ & $531(5.5)$ \\
\hline Lithuania & $36(1.2)$ & $526(2.2)$ & $37(1.0)$ & $540(2.1)$ & $14(0.7)$ & $551(3.9)$ & $14(0.8)$ & $543(3.3)$ \\
\hline Luxembourg & $13(0.4)$ & $548(3.4)$ & $50(0.6)$ & $557(1.5)$ & $27(0.6)$ & $567(2.0)$ & $10(0.4)$ & $542(3.1)$ \\
\hline Macedonia, Rep. of & $67(1.7)$ & $441(4.1)$ & $28(1.4)$ & $457(5.4)$ & $4(0.6)$ & $446(16.5)$ & $1(0.2)$ & $\sim \sim$ \\
\hline Moldova, Rep. of & $49(1.9)$ & $497(4.0)$ & $37(1.6)$ & $503(3.3)$ & $9(1.2)$ & $506(5.1)$ & $5(0.5)$ & $495(8.9)$ \\
\hline Morocco & $51(2.3)$ & $329(6.8)$ & $39(2.2)$ & $324(7.5)$ & $8(1.0)$ & $300(11.8)$ & $3(0.4)$ & $305(17.1)$ \\
\hline Netherlands & $18(1.0)$ & $537(4.1)$ & $31(1.0)$ & $550(1.9)$ & $19(1.1)$ & $552(2.9)$ & $33(1.4)$ & $547(2.0)$ \\
\hline New Zealand & $32(1.3)$ & $520(3.2)$ & $42(0.8)$ & $541(2.5)$ & $16(0.9)$ & $540(3.8)$ & $10(0.6)$ & $524(5.3)$ \\
\hline Norway & $27(1.4)$ & $486(3.3)$ & $43(1.1)$ & $504(3.2)$ & $16(1.0)$ & $505(4.0)$ & $13(0.8)$ & $502(3.8)$ \\
\hline Poland & $42(1.3)$ & $528(2.8)$ & $41(1.2)$ & $516(3.2)$ & $12(0.6)$ & $516(4.4)$ & $6(0.5)$ & $500(7.3)$ \\
\hline Qatar & $54(0.6)$ & $353(1.8)$ & $31(0.6)$ & $361(2.0)$ & $8(0.4)$ & $355(5.0)$ & $7(0.3)$ & $347(5.3)$ \\
\hline Romania & $47(2.2)$ & $485(5.3)$ & $35(1.7)$ & $503(5.0)$ & $8(0.8)$ & $496(8.9)$ & $10(0.7)$ & $476(12.0)$ \\
\hline Russian Federation & $23(1.5)$ & $548(4.6)$ & $44(1.4)$ & $564(4.6)$ & $17(0.9)$ & $584(3.4)$ & $16(1.0)$ & $572(3.7)$ \\
\hline Scotland & $23(1.5)$ & $500(4.2)$ & $46(1.7)$ & $535(3.4)$ & $21(1.5)$ & $545(4.4)$ & $10(0.9)$ & $520(6.3)$ \\
\hline Singapore & $39(0.8)$ & $555(2.7)$ & $37(0.7)$ & $563(3.6)$ & $12(0.4)$ & $564(4.2)$ & $11(0.4)$ & $551(4.5)$ \\
\hline Slovak Republic & $22(1.5)$ & $522(5.7)$ & $44(1.4)$ & $531(3.2)$ & $23(1.3)$ & $539(5.6)$ & $11(0.9)$ & $534(4.3)$ \\
\hline Slovenia & $38(1.3)$ & $517(2.9)$ & $47(1.2)$ & $527(2.2)$ & $13(0.8)$ & $521(3.5)$ & $3(0.3)$ & $499(9.1)$ \\
\hline South Africa & $60(1.0)$ & $302(5.3)$ & $26(0.6)$ & $312(7.1)$ & $8(0.4)$ & $312(11.8)$ & $6(0.4)$ & $279(9.9)$ \\
\hline Spain & $52(1.5)$ & $506(3.1)$ & $29(1.2)$ & $523(3.2)$ & $9(0.6)$ & $525(6.0)$ & $10(0.9)$ & $509(6.3)$ \\
\hline Sweden & $17(1.2)$ & $532(5.1)$ & $42(1.3)$ & $549(2.6)$ & $23(1.1)$ & $558(3.3)$ & $19(1.4)$ & $558(3.9)$ \\
\hline Trinidad and Tobago & $44(1.6)$ & $429(6.7)$ & $41(1.2)$ & $442(4.9)$ & $9(0.8)$ & $453(10.4)$ & $5(0.6)$ & $429(11.4)$ \\
\hline United States & $38(1.8)$ & $522(4.4)$ & $35(1.0)$ & $552(3.6)$ & $13(0.8)$ & $556(4.4)$ & $13(0.8)$ & $546(4.4)$ \\
\hline International Avg. & $37(0.2)$ & $494(0.7)$ & $38(0.2)$ & $505(0.6)$ & $14(0.1)$ & $507(1.0)$ & $10(0.1)$ & $495(1.2)$ \\
\hline
\end{tabular}

Background data provided by students.

() Standard errors appear in parentheses. Because results are rounded to the nearest whole number, some totals may appear inconsistent.

A tilde $(\sim)$ indicates insufficient data to report achievement.

NOTE: The International Average does not include the results from the Canadian provinces. 
Exhibit 6.18 Students Write Something About Class Reading

PIRLS 2006

4th Grade

\begin{tabular}{|c|c|c|c|c|c|c|c|c|}
\hline \multirow{2}{*}{ Countries } & \multicolumn{2}{|c|}{$\begin{array}{c}\text { Every Day or } \\
\text { Almost Every Day }\end{array}$} & \multicolumn{2}{|c|}{$\begin{array}{c}\text { Once or Twice } \\
\text { a Week }\end{array}$} & \multicolumn{2}{|c|}{$\begin{array}{c}\text { Once or } \\
\text { Twice a Month }\end{array}$} & \multicolumn{2}{|c|}{$\begin{array}{c}\text { Never or } \\
\text { Almost Never }\end{array}$} \\
\hline & $\begin{array}{l}\text { Percent of } \\
\text { Students }\end{array}$ & $\begin{array}{c}\text { Average } \\
\text { Achievement }\end{array}$ & $\begin{array}{l}\text { Percent of } \\
\text { Students }\end{array}$ & $\begin{array}{c}\text { Average } \\
\text { Achievement }\end{array}$ & $\begin{array}{l}\text { Percent of } \\
\text { Students }\end{array}$ & $\begin{array}{c}\text { Average } \\
\text { Achievement }\end{array}$ & $\begin{array}{l}\text { Percent of } \\
\text { Students }\end{array}$ & $\begin{array}{c}\text { Average } \\
\text { Achievement }\end{array}$ \\
\hline Austria & $9(0.4)$ & $509(3.9)$ & $30(1.1)$ & $530(3.2)$ & $29(0.9)$ & $547(2.3)$ & $32(1.2)$ & $549(2.6)$ \\
\hline Belgium (Flemish) & $8(0.9)$ & $526(5.2)$ & $26(1.1)$ & $539(2.7)$ & $31(1.1)$ & $555(2.2)$ & $35(1.2)$ & $552(2.5)$ \\
\hline Belgium (French) & $15(0.7)$ & $471(4.1)$ & $34(1.3)$ & $491(3.0)$ & $25(0.9)$ & $514(3.1)$ & $26(1.0)$ & $515(3.1)$ \\
\hline Bulgaria & $41(2.1)$ & $547(5.8)$ & $43(2.0)$ & $555(4.7)$ & $9(0.8)$ & $544(10.7)$ & $6(0.7)$ & $522(9.4)$ \\
\hline Canada, Alberta & $14(0.8)$ & $544(3.6)$ & $31(1.0)$ & $554(3.4)$ & $29(0.9)$ & $573(3.0)$ & $27(1.2)$ & $564(3.1)$ \\
\hline Canada, British Columbia & $14(1.2)$ & $541(5.1)$ & $37(1.4)$ & $559(3.3)$ & $30(1.3)$ & $565(3.5)$ & $19(1.1)$ & $564(3.2)$ \\
\hline Canada, Nova Scotia & $19(1.1)$ & $525(3.6)$ & $36(1.2)$ & $547(2.8)$ & $26(1.0)$ & $554(3.3)$ & $19(0.9)$ & $539(3.5)$ \\
\hline Canada, Ontario & $16(1.1)$ & $541(4.5)$ & $38(1.3)$ & $553(3.3)$ & $27(1.1)$ & $569(3.7)$ & $18(1.1)$ & $554(4.2)$ \\
\hline Canada, Quebec & $12(0.8)$ & $508(4.1)$ & $28(1.2)$ & $525(3.3)$ & $30(1.3)$ & $544(3.0)$ & $30(1.4)$ & $543(4.2)$ \\
\hline Chinese Taipei & $13(0.5)$ & $518(3.6)$ & $41(1.3)$ & $542(2.3)$ & $26(1.0)$ & $542(2.7)$ & $21(0.9)$ & $526(2.8)$ \\
\hline Denmark & $8(0.7)$ & $523(5.1)$ & $34(1.4)$ & $543(3.2)$ & $36(1.2)$ & $555(3.0)$ & $22(1.0)$ & $545(2.8)$ \\
\hline England & $13(0.9)$ & $508(5.1)$ & $32(1.1)$ & $530(3.4)$ & $33(1.1)$ & $560(4.0)$ & $23(1.2)$ & $546(3.7)$ \\
\hline France & $14(0.9)$ & $491(4.0)$ & $36(1.1)$ & $519(2.4)$ & $29(1.1)$ & $537(2.8)$ & $21(0.9)$ & $529(3.1)$ \\
\hline Georgia & $27(1.6)$ & $464(3.4)$ & $41(1.4)$ & $481(4.3)$ & $16(1.1)$ & $476(5.0)$ & $16(1.2)$ & $465(6.0)$ \\
\hline Germany & $11(0.6)$ & $522(4.3)$ & $27(0.8)$ & $544(3.2)$ & $30(0.8)$ & $560(2.8)$ & $32(1.0)$ & $556(2.5)$ \\
\hline Hong Kong SAR & $11(0.6)$ & $556(3.7)$ & $30(1.1)$ & $564(3.2)$ & $31(0.8)$ & $565(2.6)$ & $28(1.0)$ & $565(2.9)$ \\
\hline Hungary & $16(1.0)$ & $516(4.5)$ & $42(1.2)$ & $546(3.8)$ & $24(0.9)$ & $567(3.3)$ & $18(1.0)$ & $573(4.9)$ \\
\hline Iceland & $10(0.4)$ & $475(3.8)$ & $28(0.8)$ & $507(2.8)$ & $26(0.7)$ & $520(2.0)$ & $36(0.8)$ & $517(2.0)$ \\
\hline Indonesia & $42(1.2)$ & $409(4.6)$ & $38(1.0)$ & $409(4.4)$ & $12(0.8)$ & $395(5.7)$ & $8(0.7)$ & $398(8.5)$ \\
\hline Iran, Islamic Rep. of & $15(1.0)$ & $419(5.0)$ & $46(1.6)$ & $429(4.1)$ & $26(1.3)$ & $426(4.3)$ & $13(1.1)$ & $397(8.6)$ \\
\hline Israel & $25(0.9)$ & $505(4.2)$ & $40(0.9)$ & $511(4.4)$ & $20(0.8)$ & $531(5.2)$ & $15(0.7)$ & $539(5.1)$ \\
\hline Italy & $27(1.6)$ & $543(4.9)$ & $44(1.9)$ & $559(3.7)$ & $16(0.9)$ & $553(3.8)$ & $12(0.9)$ & $544(4.7)$ \\
\hline Kuwait & $24(1.1)$ & $339(5.4)$ & $41(1.0)$ & $337(5.1)$ & $23(1.0)$ & $335(6.3)$ & $12(1.0)$ & $339(6.9)$ \\
\hline Latvia & $16(0.9)$ & $522(4.6)$ & $42(1.3)$ & $544(2.7)$ & $28(1.0)$ & $549(3.7)$ & $14(0.8)$ & $540(4.5)$ \\
\hline Lithuania & $19(1.1)$ & $510(3.0)$ & $42(1.1)$ & $535(2.1)$ & $27(1.0)$ & $554(2.1)$ & $12(0.7)$ & $550(3.4)$ \\
\hline Luxembourg & $4(0.3)$ & $520(4.7)$ & $22(0.5)$ & $540(2.1)$ & $40(0.7)$ & $567(1.5)$ & $33(0.7)$ & $562(1.7)$ \\
\hline Macedonia, Rep. of & $46(1.6)$ & $445(5.3)$ & $39(1.4)$ & $447(4.6)$ & $12(1.4)$ & $437(8.9)$ & $3(0.3)$ & $483(13.2)$ \\
\hline Moldova, Rep. of & $31(1.9)$ & $501(4.9)$ & $46(1.8)$ & $500(3.5)$ & $18(1.7)$ & $505(4.2)$ & $5(0.5)$ & $476(9.2)$ \\
\hline Morocco & $27(1.5)$ & $330(6.8)$ & $42(1.7)$ & $331(6.9)$ & $19(1.4)$ & $320(8.9)$ & $13(1.6)$ & $302(15.3)$ \\
\hline Netherlands & $8(0.4)$ & $525(4.8)$ & $23(0.9)$ & $539(2.4)$ & $26(1.2)$ & $555(2.3)$ & $43(1.5)$ & $551(1.9)$ \\
\hline New Zealand & $17(1.0)$ & $496(4.3)$ & $38(1.0)$ & $535(2.5)$ & $26(0.9)$ & $550(2.6)$ & $19(0.7)$ & $537(4.0)$ \\
\hline Norway & $13(1.2)$ & $475(4.1)$ & $36(1.4)$ & $496(2.9)$ & $27(1.0)$ & $512(3.3)$ & $24(1.2)$ & $502(5.1)$ \\
\hline Poland & $20(1.0)$ & $506(3.4)$ & $41(1.0)$ & $526(2.8)$ & $25(0.9)$ & $523(3.6)$ & $13(0.8)$ & $517(5.3)$ \\
\hline Qatar & $34(0.6)$ & $350(2.5)$ & $40(0.5)$ & $354(1.8)$ & $15(0.5)$ & $364(3.2)$ & $11(0.4)$ & $368(3.6)$ \\
\hline Romania & $36(2.0)$ & $487(5.3)$ & $42(1.5)$ & $500(5.4)$ & $13(1.0)$ & $486(8.6)$ & $9(0.7)$ & $470(9.9)$ \\
\hline Russian Federation & $15(1.1)$ & $538(5.4)$ & $41(1.3)$ & $561(4.0)$ & $29(1.8)$ & $581(4.1)$ & $15(0.7)$ & $573(4.0)$ \\
\hline Scotland & $8(0.7)$ & $469(6.2)$ & $38(2.0)$ & $527(3.4)$ & $35(1.7)$ & $542(3.3)$ & $20(1.3)$ & $527(4.6)$ \\
\hline Singapore & $11(0.4)$ & $526(3.9)$ & $26(0.6)$ & $546(3.6)$ & $27(0.7)$ & $572(3.5)$ & $36(0.8)$ & $567(2.7)$ \\
\hline Slovak Republic & $16(0.9)$ & $509(6.3)$ & $39(1.3)$ & $532(2.8)$ & $31(1.3)$ & $542(3.8)$ & $15(0.9)$ & $532(5.4)$ \\
\hline Slovenia & $12(0.6)$ & $496(3.6)$ & $47(1.0)$ & $522(2.7)$ & $33(1.0)$ & $533(2.5)$ & $8(0.5)$ & $518(5.5)$ \\
\hline South Africa & $41(1.0)$ & $288(4.6)$ & $35(0.7)$ & $308(6.0)$ & $14(0.5)$ & $325(10.0)$ & $10(0.5)$ & $340(13.1)$ \\
\hline Spain & $29(1.3)$ & $493(3.5)$ & $37(1.1)$ & $517(3.2)$ & $20(0.9)$ & $533(3.5)$ & $14(0.9)$ & $516(4.6)$ \\
\hline Sweden & $7(0.5)$ & $523(6.7)$ & $30(1.4)$ & $542(3.0)$ & $39(1.0)$ & $556(2.8)$ & $24(1.3)$ & $557(3.1)$ \\
\hline Trinidad and Tobago & $27(1.2)$ & $426(7.5)$ & $42(1.4)$ & $436(5.0)$ & $18(1.0)$ & $454(7.4)$ & $13(0.9)$ & $438(8.5)$ \\
\hline United States & $23(1.1)$ & $513(3.8)$ & $30(1.0)$ & $542(4.3)$ & $25(1.1)$ & 558 (3.6) & $22(1.0)$ & $549(4.3)$ \\
\hline International Avg. & $20(0.2)$ & $482(0.8)$ & $37(0.2)$ & $500(0.7)$ & $25(0.2)$ & $510(0.8)$ & $19(0.1)$ & $504(1.0)$ \\
\hline
\end{tabular}

Background data provided by students.

() Standard errors appear in parentheses. Because results are rounded to the nearest whole number, some totals may appear inconsistent. 
Exhibit 6.19 Teachers' Reports on Giving a Written Quiz or Test After Students Read

PIRLS 2006

4th Grade

\begin{tabular}{|c|c|c|c|c|c|c|c|}
\hline \multirow{2}{*}{ Countries } & & \multicolumn{2}{|c|}{$\begin{array}{l}\text { At Least } \\
\text { Weekly }\end{array}$} & \multicolumn{2}{|c|}{$\begin{array}{c}\text { Once or Twice } \\
\text { a Month }\end{array}$} & \multicolumn{2}{|c|}{$\begin{array}{c}\text { Never or } \\
\text { Almost Never }\end{array}$} \\
\hline & & $\begin{array}{l}\text { Percent of } \\
\text { Students }\end{array}$ & $\begin{array}{c}\text { Average } \\
\text { Achievement }\end{array}$ & $\begin{array}{l}\text { Percent of } \\
\text { Students }\end{array}$ & $\begin{array}{c}\text { Average } \\
\text { Achievement }\end{array}$ & $\begin{array}{l}\text { Percent of } \\
\text { Students }\end{array}$ & $\begin{array}{c}\text { Average } \\
\text { Achievement }\end{array}$ \\
\hline Austria & & $13(2.7)$ & $538(5.1)$ & $44(3.4)$ & $535(3.2)$ & $43(3.4)$ & $542(3.2)$ \\
\hline Belgium (Flemish) & & $44(4.0)$ & $549(2.8)$ & $52(3.9)$ & $545(2.8)$ & $4(1.3)$ & $547(5.6)$ \\
\hline Belgium (French) & & $26(3.2)$ & $496(4.6)$ & $61(3.6)$ & $500(3.8)$ & $14(2.6)$ & $501(8.9)$ \\
\hline Bulgaria & & $14(2.8)$ & $555(14.1)$ & $79(3.4)$ & $547(4.8)$ & $7(2.0)$ & $533(10.5)$ \\
\hline Canada, Alberta & & $15(2.8)$ & $555(6.2)$ & $60(3.5)$ & $558(3.1)$ & $25(3.5)$ & $568(5.2)$ \\
\hline Canada, British Columbia & $r$ & $13(2.6)$ & $564(10.0)$ & $61(3.6)$ & $557(3.7)$ & $27(3.7)$ & $561(5.9)$ \\
\hline Canada, Nova Scotia & & $5(1.6)$ & $515(10.1)$ & $39(3.8)$ & $542(3.7)$ & $56(3.8)$ & $544(3.2)$ \\
\hline Canada, Ontario & & $10(3.0)$ & $550(7.8)$ & $55(4.9)$ & $556(4.0)$ & $35(4.8)$ & $556(4.0)$ \\
\hline Canada, Quebec & & $35(4.7)$ & $536(4.6)$ & $52(4.6)$ & $533(3.5)$ & $14(2.9)$ & $534(6.5)$ \\
\hline Chinese Taipei & & $35(4.0)$ & $533(3.6)$ & $31(3.9)$ & $536(3.3)$ & $34(3.9)$ & $538(3.2)$ \\
\hline Denmark & & $1(0.7)$ & $\sim \sim$ & $20(3.0)$ & $550(5.2)$ & $79(3.1)$ & $546(2.7)$ \\
\hline England & & $7(2.2)$ & $520(13.8)$ & $38(4.6)$ & $535(6.0)$ & $55(4.5)$ & $548(4.0)$ \\
\hline France & & $19(2.3)$ & $518(5.4)$ & $67(3.4)$ & $524(2.3)$ & $14(2.5)$ & $517(4.7)$ \\
\hline Georgia & & $57(4.1)$ & $475(4.3)$ & $33(3.7)$ & $466(5.3)$ & $10(2.5)$ & $467(11.9)$ \\
\hline Germany & & $3(0.8)$ & $523(12.6)$ & $70(3.2)$ & $552(2.4)$ & $28(3.3)$ & $540(4.9)$ \\
\hline Hong Kong SAR & & $6(2.2)$ & $578(8.1)$ & $58(4.2)$ & $564(2.9)$ & $36(3.9)$ & $563(3.6)$ \\
\hline Hungary & & $14(2.6)$ & $550(8.0)$ & $76(3.5)$ & $550(3.8)$ & $10(2.5)$ & $554(10.1)$ \\
\hline Iceland & & $3(0.1)$ & $500(8.2)$ & $26(0.3)$ & $510(2.1)$ & $71(0.3)$ & $511(1.7)$ \\
\hline Indonesia & & $40(4.1)$ & $413(6.1)$ & $46(4.2)$ & $397(6.5)$ & $14(3.1)$ & $408(10.7)$ \\
\hline Iran, Islamic Rep. of & & $39(4.0)$ & $414(6.3)$ & $55(4.1)$ & $428(4.5)$ & $6(1.6)$ & $406(15.5)$ \\
\hline Israel & & $21(3.1)$ & $490(15.2)$ & $74(3.4)$ & $520(4.7)$ & $5(1.9)$ & $527(22.0)$ \\
\hline Italy & & $54(3.4)$ & $548(4.6)$ & $39(3.4)$ & $559(3.7)$ & $7(1.5)$ & $541(11.5)$ \\
\hline Kuwait & & $58(3.9)$ & $338(5.4)$ & $28(3.8)$ & $322(8.9)$ & $14(3.1)$ & $318(15.2)$ \\
\hline Latvia & & $21(3.0)$ & $544(4.9)$ & $76(3.2)$ & $540(3.0)$ & $3(1.1)$ & $539(7.9)$ \\
\hline Lithuania & & $11(2.2)$ & $525(4.8)$ & $72(3.2)$ & $539(1.9)$ & $18(2.6)$ & $539(4.1)$ \\
\hline Luxembourg & & $17(0.2)$ & $556(2.0)$ & $77(0.2)$ & $557(1.3)$ & $6(0.1)$ & $557(3.2)$ \\
\hline Macedonia, Rep. of & & $18(3.1)$ & $407(15.1)$ & $70(3.6)$ & $455(5.2)$ & $12(2.8)$ & 447 (15.3) \\
\hline Moldova, Rep. of & & $45(4.5)$ & $496(4.3)$ & $49(4.3)$ & $502(4.8)$ & $6(2.1)$ & $495(15.1)$ \\
\hline Morocco & & $30(4.1)$ & $313(9.5)$ & $50(4.5)$ & $322(10.3)$ & $20(3.3)$ & $331(15.1)$ \\
\hline Netherlands & & $24(3.6)$ & $549(4.5)$ & $66(4.0)$ & $547(2.0)$ & $10(2.5)$ & $539(4.0)$ \\
\hline New Zealand & & $3(0.9)$ & $489(44.1)$ & $19(2.3)$ & $532(6.0)$ & $79(2.3)$ & $536(2.6)$ \\
\hline Norway & & $8(2.2)$ & $481(5.3)$ & $34(4.0)$ & $498(3.9)$ & $59(4.3)$ & $500(3.4)$ \\
\hline Poland & & $46(3.7)$ & $521(3.3)$ & $48(4.0)$ & $519(3.5)$ & $5(1.7)$ & $515(8.4)$ \\
\hline Qatar & $s$ & $46(0.3)$ & $356(2.3)$ & $44(0.3)$ & $349(1.9)$ & $11(0.2)$ & $352(4.8)$ \\
\hline Romania & & $43(3.9)$ & $480(8.9)$ & $53(4.0)$ & $498(5.7)$ & $4(1.5)$ & $483(19.3)$ \\
\hline Russian Federation & & $16(2.7)$ & $554(8.5)$ & $73(3.2)$ & $567(3.3)$ & $11(1.9)$ & $566(9.9)$ \\
\hline Scotland & & $6(2.2)$ & $542(9.4)$ & $40(4.8)$ & $522(4.2)$ & $54(4.6)$ & $528(4.4)$ \\
\hline Singapore & & $18(2.2)$ & $548(8.9)$ & $49(3.2)$ & $559(4.4)$ & $33(2.6)$ & $562(4.4)$ \\
\hline Slovak Republic & & $14(2.6)$ & $539(6.2)$ & $60(3.4)$ & $532(3.5)$ & $26(2.9)$ & $523(5.8)$ \\
\hline Slovenia & & $26(3.1)$ & $523(4.0)$ & $61(3.5)$ & $521(2.5)$ & $13(2.1)$ & $520(4.7)$ \\
\hline South Africa & & $37(3.5)$ & $294(10.4)$ & $57(3.4)$ & $299(9.6)$ & $6(1.1)$ & $382(27.4)$ \\
\hline Spain & & $25(3.2)$ & $516(6.1)$ & $63(4.0)$ & $512(3.5)$ & $12(2.7)$ & $512(7.7)$ \\
\hline Sweden & & $8(2.2)$ & $551(7.8)$ & $38(3.5)$ & $550(3.0)$ & $54(3.6)$ & $548(2.7)$ \\
\hline Trinidad and Tobago & & $43(4.0)$ & $441(9.1)$ & $38(4.2)$ & $433(8.4)$ & $19(3.2)$ & $431(14.2)$ \\
\hline United States & & $67(3.9)$ & $534(4.2)$ & $24(3.4)$ & $551(5.2)$ & $9(2.4)$ & $541(10.2)$ \\
\hline International Avg. & & $26(0.5)$ & $495(1.7)$ & $51(0.6)$ & $501(0.8)$ & $23(0.4)$ & $501(1.7)$ \\
\hline
\end{tabular}

Background data provided by teachers.

() Standard errors appear in parentheses. Because results are rounded to the nearest whole number, some totals may appear inconsistent.

An " $r$ " indicates data are available for $70-84 \%$ of the students. An "s" indicates data are available for $50-69 \%$ of the students. An " $x$ " indicates data are available for less than $50 \%$ of the students.
A tilde ( ) indicates insufficient data to report achievement.

NOTE: The International Average does not include the results from the Canadian provinces. 


\section{What Library Resources Are Available and How Are They Used?}

Libraries, both within the school and in the local community, provide a range of reading materials and other resources from which students can choose books for their own learning and enjoyment. Because libraries expand students' opportunities to access a variety of materials and can promote positive reading habits and attitudes, PIRLS 2006 asked a series of questions about students' access to and use of libraries.

Exhibit 6.20 contains reports about school and classroom libraries. In a number of countries, principals reported that more than 95 percent of the schools attended by fourth-grade students had libraries. Principals reported 100 percent of students attending schools with libraries in Latvia, Moldova, the Russian Federation, Singapore, Slovenia, and the Canadian province of Alberta. On average internationally, school principals reported that most fourth-grade students (89\%) attended a school with a library, and for many students (71\%), their school library had more than 500 books. Teachers reported that approximately two thirds of the students (69\%) had libraries in their classrooms (averaging 52 books and 3 magazines). About half the students (55\%), internationally on average, could borrow books from their classroom library to take home.

Exhibit 6.21 shows the percentage of students whose teachers took or sent them to a library other than the classroom library according to four response categories: every day or almost every day, once or twice a week, once or twice a month, or never or almost never. Internationally on average, teachers reported that 50 percent of the students were given opportunities for library visits at least weekly ( $8 \%$ daily and $42 \%$ once or twice a week), and another 32 percent were given opportunities for visits at least monthly. Eighty percent or more of students in Denmark, Iceland, Moldova, New Zealand, the United States, and the five Canadian provinces visited a library other than the classroom library at least once a week. On average internationally, 40 percent of the fourth-grade students reported borrowing library books on a weekly basis, and another 28 percent reported borrowing library books once or twice a month. 
Exhibit 6.20 Access to School and Classroom Libraries

PIRLS 2006
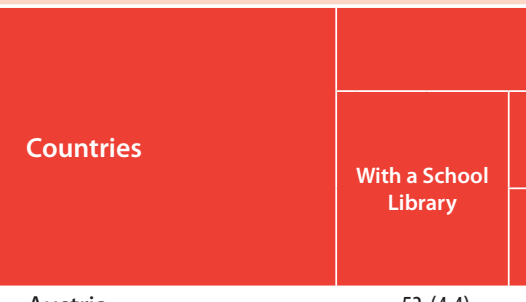

Percentage of Students

Austria

$53(4.4)$

With a School Library

Having More than

Belgium (Flemish)

$43(4.4)$

500 Books
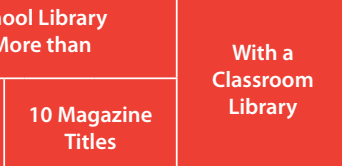

Size of Classroom Library

Percentage of

Students Who

Can Borrow

\begin{tabular}{|c|c} 
Average & $\begin{array}{c}\text { Average } \\
\text { Number } \\
\text { of Books }\end{array}$ \\
of Magarine \\
Titles
\end{tabular}

Books

from Their

Classroom

Home

Belgium (French)

$41(3.9)$

$2(1.1)$

$73(3.2)$

$62(5.9)$

$2(0.3)$

$67(3.3)$

Bulgaria

Canada, Alberta

$72(4.1)$

$16(3.3)$

$3(1.6)$

96 (1.8)

$114(6.9)$

$2(0.2)$

$60(4.2)$

Canada, British Columbia

$100(0.4)$

$34(4.8)$

$5(2.1)$

$91(1.6)$

$90(6.5)$

$9(0.6)$

$64(3.3)$

Canada, Nova Scotia

$98(1.0) \quad 37(4.5)$

$95(1.7)$

$179(10.7)$

Canada, Ontario

$99(0.6)$

Canada, Quebec

$99(0.5)$

$99(0.7)$

$37(4.5)$

$3(0.5)$

$33(3.7)$

Chinese Taipei

$91(2.8)$

$94(1.9)$

$26(3.0)$

$98(0.9)$

$174(10.8)-5$

$77(3.1)$

Denmark

$99(0.8)$

$96(2.3)$

$27(4.0)$

$98(1.3)$

27

$72(11.4)$ s $\quad 8(0.6)$

$82(3.9)$

England

France

$97(1.5)$

$87(3.5) \quad r \quad 12(2.8)$

$98(1.3)$

$164(13.2)$ r $6(0.7)$

$94(1.7)$

Georg

Germany
Hong Kong SAR
Hungary

Iceland

\section{Indonesia}

Iran, Islamic Rep. of

$96(1.7) \quad r \quad 80(3.6) \quad r \quad 3(1.3)$

$88(3.0)$

$95(7.4) \quad r \quad 4(0.6)$

$84(3.3)$

Israel

Italy

Italy

Latvia

Lithuania
${ }^{1}$ Luxembourg

Macedonia, Rep. of r $93(2.5)$

Moldova, Rep. of 100

\begin{tabular}{|l|l|}
\hline Morocco & $\mathrm{r}$ \\
\hline Netherlands & $\mathrm{r}$ \\
\hline New Zealand &
\end{tabular}

$90(2.4)$

$93(2.6)$

$79(2.8)$

$98(1.3)$

$98(1.1)$

$99(0.0)$

$77(3.5)$

$89(2.0)$

$89(2.8)$

$94(2.0)$

$99(0.7)$

$100(0.3)$

$80(3.6)$

$63(3.9)$

$76(3.4)$

$76(3.4)$
$34(3.6)$

$98(1.3)$

$10(2.5)$

$35(3.8)$

$124(7.6)$

$16(2.3)$

$4(0.4)$

$72(4.3)$
$75(3.6)$

$85(2.4)$

$118(9.6)$

$1(0.3)$

$30(3.6)$

Norway

Poland

Romania

Russian Federation

Scotland

Singapore

Slovak Republic

Slovenia

South Africa

Spain

Sweden

Trinidad and Tobago

United States

$94(2.0)$

$98(0.1)$

$21(3.4)$

$13(3.2)$

$55(4.3)$

$78(7.0)$

$55(4.3)$

$22(4.6)$

$3(0.5)$

$70(4.2)$

$3(1.0)$
$25(4.0)$

$92(2.2)$

$52(3.7)$

$5(0.4)$

$37(4.1)$

$70(3.7)$

$\quad 15(3.3)$

$51(0.3)$

$\begin{array}{lll}133(9.6) & r & 2(0.5)\end{array}$

$3(0.4)$

$71(3.2)$

\begin{tabular}{l|l}
$39(3.2)$ & $12(2.2)$ \\
\hline
\end{tabular}

$51(0.3)$

$51(4.5)$

$63(3.7) \quad 9(2.2)$

$46(3.8)$

$\begin{array}{llll}26(1.9) & r & 3(0.3) & 68(4.4)\end{array}$

\begin{tabular}{r|r|}
\hline $63(3.7)$ & $9(2.2)$ \\
\hline $52(4.0)$ & $7(2.3)$ \\
\hline $64(4.1)$ & $73(3.6)$ \\
\hline
\end{tabular}

$66(3.9)$

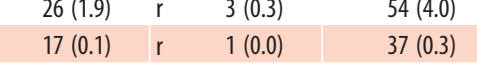

$\begin{array}{llll}17(0.1) & r & 1(0.0) & 37(0.3) \\ 51(8.4) & r & 2(0.4) & 42(3.7)\end{array}$

\begin{tabular}{l|l|l|l|}
$30(3.7)$ & $r$ & $1(0.3)$ & $44(3.6)$
\end{tabular}

\begin{tabular}{|c|c|c|c|c|}
\hline $66(3.9)$ & $30(4.1)$ & $r$ & $2(0.4)$ & $49(4.0)$ \\
\hline $69(3.2$ & $28(1.4)$ & $s$ & $2(0.4)$ & $67(3.3)$ \\
\hline
\end{tabular}

$\begin{array}{rrrrrr}20(3.4) & \mathrm{r} & 4(0.9) & \mathrm{r} & 2(0.4) & 16(3.2)\end{array}$

\begin{tabular}{r|r|r|r|rrr|r|}
$64(4.1)$ & $73(3.6)$ & $20(3.4)$ & $\mathrm{r}$ & $4(0.9)$ & $\mathrm{r}$ & $2(0.4)$ & $16(3.2)$ \\
\hline $96(1.5)$ & $45(4.2)$ & $60(3.5)$ & & $45(4.7)$ & $\mathrm{r}$ & $2(0.3)$ & $54(3.7)$
\end{tabular}

International Avg.

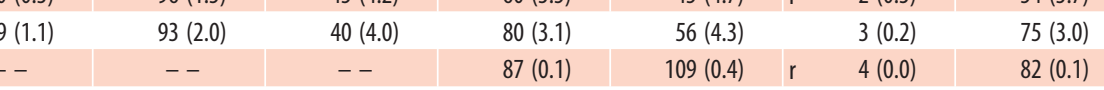

$80(3.1)$

$54(3.7)$

\begin{tabular}{|c|c|c|r|r|rr|r|}
\hline-- & & -- & $87(0.1)$ & $109(0.4)$ & $\mathrm{r}$ & $4(0.0)$ & $82(0.1)$ \\
\hline $80(3.2)$ & $\mathrm{r}$ & $6(2.3)$ & $32(4.1)$ & $18(5.8)$ & $\mathrm{r}$ & $1(0.3)$ & $28(4.2)$ \\
\hline
\end{tabular}

\begin{tabular}{|c|c|c|c|c|c|c|c|c|c|c|}
\hline $93(2.5)$ & $r$ & $80(3.2)$ & $r$ & $6(2.3)$ & $32(4.1)$ & $18(5.8)$ & $r$ & $1(0.3)$ & & $28(4.2)$ \\
\hline $100(0.0)$ & & $91(2.6)$ & & $40(4.6)$ & $93(2.0)$ & $36(3.8)$ & & $6(0.4)$ & $r$ & $87(3.3)$ \\
\hline
\end{tabular}

\begin{tabular}{r|rrrrr|r|r|r|r|r|}
$39(4.3)$ & $\mathrm{r}$ & $9(2.9)$ & $\mathrm{r}$ & $14(3.9)$ & $35(3.8)$ & $8(1.3)$ & $2(0.4)$ & $33(3.9)$ \\
\hline $76(4.5)$ & $\mathrm{r}$ & $39(4.6)$ & $\mathrm{r}$ & $1(1.0)$ & $88(3.1)$ & $\mathrm{r}$ & $78(7.0)$ & $\mathrm{r}$ & $3(0.5)$ & $18(3.3)$ \\
\hline
\end{tabular}

\begin{tabular}{|c|c|c|c|c|c|c|c|c|c|}
\hline & $91(2.6)$ & & $40(4.6)$ & $93(2.0)$ & & $36(3.8)$ & & $6(0.4)$ & $87(3.3)$ \\
\hline .3) & $9(2.9)$ & r & $14(3.9)$ & $35(3.8)$ & & $8(1.3)$ & & $2(0.4)$ & $33(3.9)$ \\
\hline (4.5) & $39(4.6)$ & $r$ & $1(1.0)$ & 88 (3.1) & $r$ & $78(7.0)$ & $r$ & $3(0.5)$ & $18(3.3)$ \\
\hline
\end{tabular}

\begin{tabular}{|c|c|c|c|c|c|c|c|c|c|}
\hline & $91(2.6)$ & & $40(4.6)$ & $93(2.0)$ & & $36(3.8)$ & & $6(0.4)$ & $87(3.3)$ \\
\hline .3) & $9(2.9)$ & r & $14(3.9)$ & $35(3.8)$ & & $8(1.3)$ & & $2(0.4)$ & $33(3.9)$ \\
\hline (4.5) & $39(4.6)$ & $r$ & $1(1.0)$ & 88 (3.1) & $r$ & $78(7.0)$ & $r$ & $3(0.5)$ & $18(3.3)$ \\
\hline
\end{tabular}

\begin{tabular}{|c|c|c|c|c|c|c|c|c|c|}
\hline $76(4.5)$ & $r$ & $39(4.6)$ & $1(1.0)$ & $88(3.1)$ & $r$ & $78(7.0)$ & $r$ & $3(0.5)$ & $18(3.3)$ \\
\hline $99(0.5)$ & & $99(0.7)$ & $22(3.1)$ & $99(0.6)$ & & $57(2.5)$ & $r$ & $5(0.4)$ & $64(2.8)$ \\
\hline $98(1.0)$ & & $92(2.9)$ & $10(3.1)$ & $58(4.9)$ & & $27(3.6)$ & $r$ & $2(0.4)$ & $44(4.9)$ \\
\hline
\end{tabular}

Background data provided by teachers and schools.

1 Primary schools in Luxembourg do not have principals.

() Standard errors appear in parentheses. Because results are rounded to the nearest whole number, some totals may appear inconsistent.

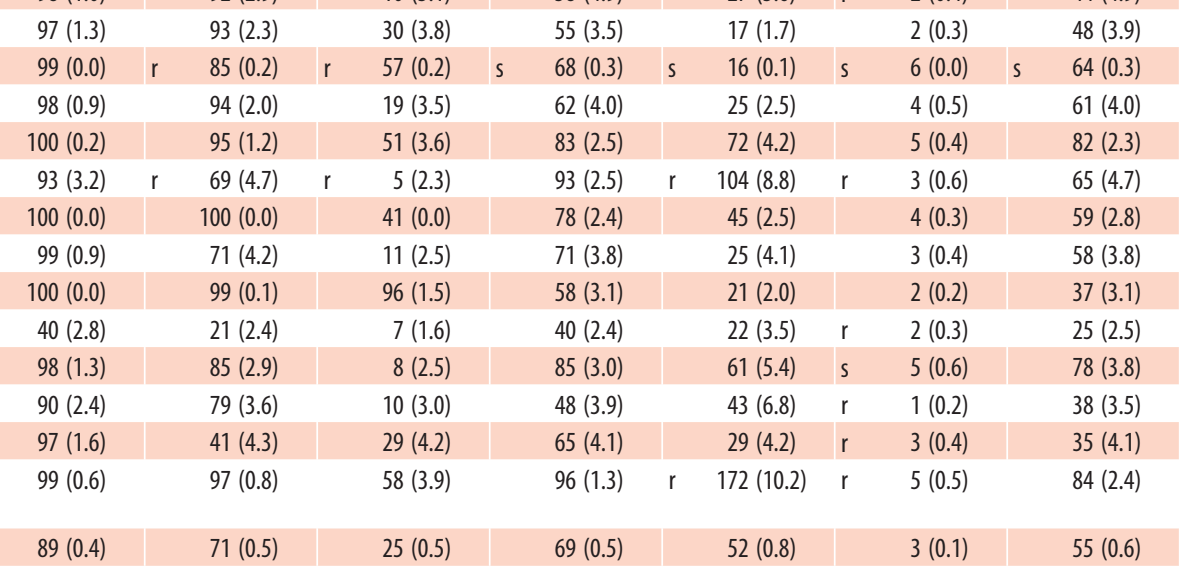


Exhibit 6.21 Students Visit and Borrow Books from School or Local Library

PIRLS 2006

4th Grade

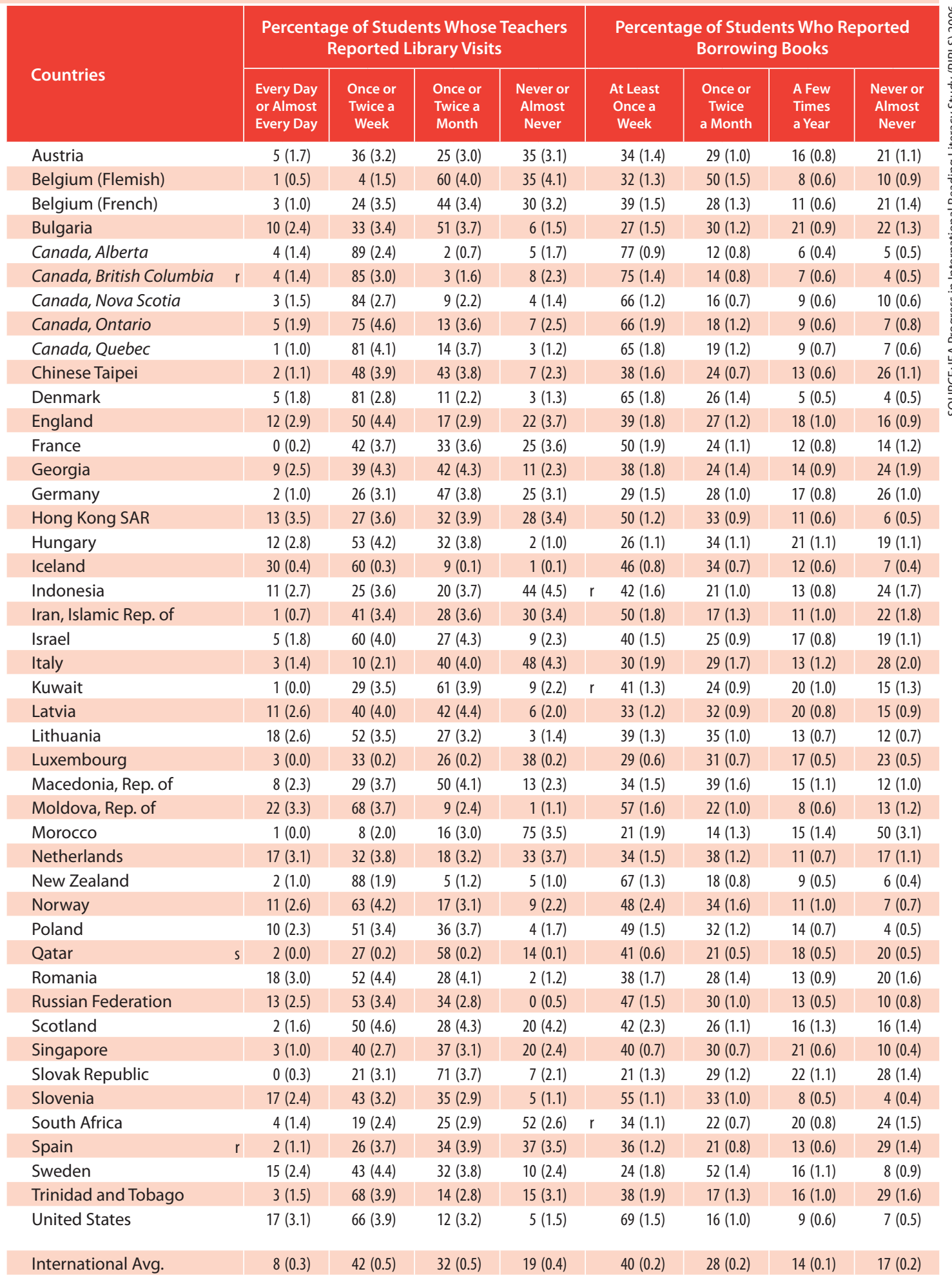

Background data provided by teachers and students.

() Standard errors appear in parentheses. Because results are rounded to the nearest whole number, some totals may appear inconsistent.
An " $r$ " indicates data are available for $70-84 \%$ of the students. An "s" indicates data are available for $50-69 \%$ of the students. An " $x$ " indicates data are available for less than $50 \%$ of the students.

NOTE: The International Average does not include the results from the Canadian provinces. 


\section{How Are Computers Used for Reading Instruction?}

Computers and other information technologies have expanded in format and function since the last PIRLS assessment in 2001, leading to the emergence of new modes of literacy in addition to new considerations of computer access and technological equity. Exhibit 6.22 presents teachers' reports of the percentages of students in schools with computers available for students' use, as well as the percentages in schools having access to the Internet, with trends from 2001. In PIRLS 2006, the percentage of fourth-grade students with computer access in schools differed greatly across participants, ranging between 5 percent in Iran to 100 percent in the Canadian province of Nova Scotia. Nearly all students (96 to 99\%) had computer access in Belgium (Flemish), England, the Netherlands, New Zealand, Scotland, Sweden, the United States, and the Canadian provinces of Alberta and Ontario. In contrast, less than 20 percent of students had computer access at school in Georgia, Indonesia, Iran, Kuwait, Macedonia, Moldova, and Morocco, and from 23 to 29 percent had access in Israel, the Russian Federation, and South Africa.

More than half the students (57\%) on average internationally attended schools with computers having Internet access. But, this also varied dramatically from country to country. The vast majority (at least 95\%) of students in England, the Netherlands, New Zealand, Scotland, Sweden, and the United States had Internet access on computers in their schools, compared to less than 10 percent of students in Georgia, Indonesia, Iran, Kuwait, and Morocco.

There has been a substantial increase, primarily in the Eastern European countries, between PIRLS 2001 and PIRLS 2006 in the percentages of students having access to computers and the Internet. In particular, the percentage of students in the Slovak Republic with access to computers increased by 70 percentage points, and with access to the Internet by 80 points, and in Bulgaria, by 53 percentage points for computers and 45 points for the Internet. Lithuania had increases of 33 percentage points for computer access and 36 points for Internet access; Germany had a 24-point increase for 
Exhibit 6.22 Computer Availability and Instructional Use with Trends

PIRLS 2006

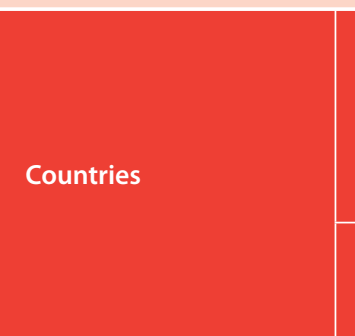

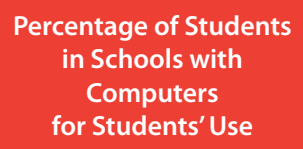

Percentage of Students

in Schools with

Computers

for Students' Use

Percentage of Students in Schools with

Computers Having

Internet Access

\section{Austria}

Belgium (Flemish)

Belgium (French)

Bulgaria

Canada, Alberto

Canada, British Columbia

Canada, Nova Scotia

Canada, Ontario

Canada, Quebec

Chinese Taipei

Denmark

England

France

Georgia

Germany

Hong Kong SAR

Hungary

Iceland

Indonesia

Iran, Islamic Rep. of

Israel

Italy

Kuwait

Latvia

Lithuania

Luxembourg

Macedonia, Rep. of

Moldova, Rep. of

Morocco

Netherlands

New Zealand

Norway

Poland

Qatar

Romania

Russian Federation

Scotland

Singapore

Slovak Republic

Slovenia

South Africa

Spain

Sweden

Trinidad and Tobago

United States

International Avg

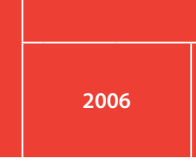

$90(2.3)$

$99(0.5)$

$58(3.4)$

$72(3.6)$

$99(0.8)$

$94(2.3)$

$100(0.3)$

$98(1.0)$

$92(2.6)$

$86(3.0)$

$91(2.0)$

$98(1.1)$

$93(2.0)$

$10(2.4)$

$85(2.8)$

$93(1.8)$

$52(4.0)$

$93(0.2)$

$14(2.9)$

$5(1.7)$

$26(3.8)$
$80(3.0)$

$15(2.5)$

$54(3.8)$

$57(3.8)$

$89(0.1)$

$19(3.2)$

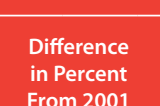

From 2001

$\diamond \diamond$

$\Delta 0$

$\Delta \diamond$

$53(4.7)$

$\Delta 0$

$\Delta 0$

$\Delta \diamond$

0 (1.5)

$-3(3.4)$

$\Delta 0$

00

$-1(1.6)$

$10(4.1)$

00

$24(4.1)$

$13(4.6)$

$13(6.2)$

$1(0.3)$

$\Delta \diamond$

$4(1.9)$

$-23(5.1)$
$17(4.4)$

00

$r \quad 6(1.9)$

$16(5.4)$

$33(5.3)$

$\triangle 0$

$4(4.5)$

$4(3.9)$

$11(2.1)$

$97(1.5)$

$-13(5.1)$

0 (2.5)

$0(2.5)$
$-2(1.1)$

$97(1.0)$

$91(2.8) \quad 5(4.3)$

$68(4.0)$

s $59(0.3)$

$46(3.3)$

$29(3.0)$

$98(1.3)$

$93(1.4) \quad 2(27)$

$86(2.6) \quad 70(4.0)$

$90(1.7) \quad 23(4.2) \quad 0$

$23(2.4) \quad \Delta 0$

$78(3.0) \quad 00$

$96(1.7) \quad-3(1.8)$

$55(4.0) \quad 00$

$98(0.8) \quad 0(1.4)$

$65(0.4)$

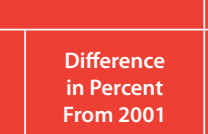

2006

$62(3.1)$

$91(2.5)$

$46(3.8)$

$52(3.7)$

$99(0.8)$

$93(2.2)$

$99(0.6)$

$98(1.0)$

$92(2.6)$

$82(3.3)$

$91(2.0)$

$98(1.1)$

$84(3.0)$

$$
3(1.4)
$$

$65(3.7)$

$90(2.1)$

$49(4.0)$

$93(0.2)$

$2(1.0)$

$1(0.8)$

$23(3.6)$

$60(3.4)$

$6(1.9) \quad \Delta \diamond$

\begin{tabular}{l|l|}
$49(3.8)$ & $22(5.2)$ \\
\hline
\end{tabular}

$51(4.0)$

$79(0.1)$

$14(3.0)$

$11(2.4)$

$4(1.3)$

$95(2.4)$

$95(1.4)$

88 (3.1)

$63(4.1)$

$25(0.2)$

$30(4.1)$

$19(2.6)$

$97(1.5)$

$93(1.5)$

$85(2.8)$

$85(2.1)$

$11(1.7)$

$69(3.2)$

$95(1.9)$

24 (3.5)

$97(1.1)$

$57(0.4)$

- Percent in 2006 significantly higher

$\Delta \Delta$

00

$2(2.5)$

00

$5(2.1)$

0

Percentage of Students Whose Teachers Reported Instructional Uses at Least Monthly

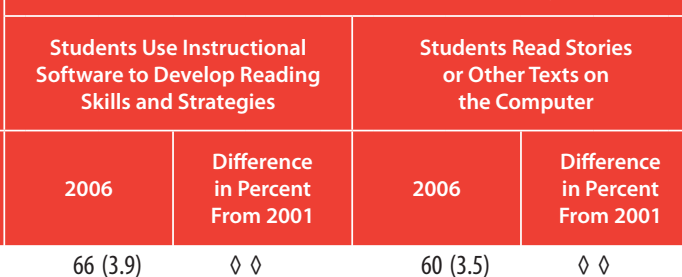

$\Delta \diamond \quad 66(3.9)$

$38(3.7)$

$17(2.5)$

$28(3.5)$

$44(4.1)$

$35(4.2)$

$42(3.9)$

$46(5.3)$

$22(3.6)$

$41(4.3)$

$29(3.6)$

$53(4.2)$

$28(3.2)$

$1(0.7)$

$48(3.5)$

$68(3.8)$

$12(2.7)$

$51(0.4)$

$6(2.1)$

$2(0.9)$

$11(2.6)$

$28(3.7)$

$8(2.2)$

$14(2.7)$

$21(3.1)$

$\begin{array}{lll}00 & & 19(0.1) \\ 12(3.2) & 0 & 5(1.7)\end{array}$

$11(2.4) \quad \mathrm{r} \quad 4(1.8)$

$3(1.3) \quad 0 \quad 3(1.4)$

$48(5.2) \quad 0 \quad 52(4.5)$

$44(2.9)$

$16(5.3) \quad 0 \quad 61(4.4)$

$\Delta \diamond \quad 19(2.9)$

$\Delta \Delta \quad$ s $38(0.3)$

$25(4.5) \quad 0 \quad 17(3.4)$

$18(2.6) \quad 0 \quad 14(2.6)$

$37(4.2) \quad 0 \quad 47(4.6)$

$15(3.3) \quad 0 \quad 60(3.2)$

$80(3.3) \quad 0 \quad 30(3.9)$

$21(4.5) \quad 0 \quad 38(3.1)$

$12(1.7)$

$50(4.2)$

$35(3.7)$

$29(3.4)$

$56(3.6)$

$30(0.5)$

A diamond $(\diamond)$ indicates the country did not participate in the 2001 assessment.

NOTE: The International Average does not include the results from the Canadian provinces.

Trend Note: The primary education systems of the Russian Federation and Slovenia

underwent structural changes. Data for Canada, Ontario include only public schools.
() Standard errors appear in parentheses. Because results are rounded to the nearest whole number, some totals may appear inconsistent.

$\mathrm{An}$ " $\mathrm{r}$ " indicates data are available for $70-84 \%$ of the students. An "s" indicates data are available for $50-69 \%$ of the students. An " $x$ " indicates data are available for less than $50 \%$ of the students. 
computer access and 38-point increase for Internet access; Latvia, Romania, the Russian Federation, and Slovenia had increases of about 20 percentage points (ranging from 16 to 25 points) for both computer and Internet access; and Hungary had increases of 13 and 23 percentage points, respectively.

Teachers reported how often they used computers for reading instruction: every day or almost every day, once or twice a week, once or twice a month, and never or almost never. Changes in the percentages of students whose teachers reported using computers for reading instruction at least monthly are shown in the second half of Exhibit 6.22. In PIRLS 2006, teachers reported that 30 percent of the students, on average internationally, had used instructional software to develop their reading skills, and slightly more $(39 \%)$ had read stories or other texts on the computer.

Use of computer technology in reading instruction increased between PIRLS 2001 and PIRLS 2006. Fourteen countries had increases from 2001 in the percentages of students using instructional software to develop reading skills and strategies, while only three (Iceland, Israel, and Sweden) showed a decrease. Similarly, 17 countries had increases in the percentages of students reading texts on the computer.

\section{What Is the Role of Reading Homework?}

PIRLS 2001 established an Index of Reading for Homework by combining teachers' responses to individual questions about reading homework. Teachers were asked how often they assigned reading as part of homework (for any subject), and how much time they expected students to spend on the homework each time it was assigned. Students were placed in one of three categories of the index: high, medium, or low, according to their teachers' responses. Cutoff points were established such that the "high" level of the index corresponds to relatively high amounts of reading for homework. Students assigned to the high level were expected to spend more than 30 minutes on reading for homework at least once a week. Students assigned to the low level were expected to spend no more than 30 minutes less than once a week. The remaining students were assigned to the medium level. 
Exhibit 6.23 shows the percentages of students in 2006 at each level of the index, together with the average reading achievement associated with doing that much homework, and differences in the percentages of students at each level compared to 2001. On average across countries, 24 percent of students were at the high level, 62 percent at the medium level, and 14 percent at the low level. Percentages of students at the high level ranged from 1 percent in Austria and Belgium (Flemish and French) to at least 75 percent in the Russian Federation, Romania, and Macedonia. Such a range is evidence of the diversity of policies and practices of assigning homework in the primary grades. There were only slight differences in achievement among the categories, but lower achievement was associated with high amounts of homework, presumably for remediation, and also with low amounts of homework.

Trends from 2001 reveal a pervasive decline across countries (20 countries and 1 province) in the percentages of students at the high level on the reading for homework index, with the greatest decreases in Hungary (6o percentage points), Sweden ( 42 points), and Italy ( 36 points). These decreases were accompanied by pervasive increases in the percentages of students in the medium level ( 15 countries) and, in some cases, the low level (4 countries).

\section{How Do Teachers Assess Reading Progress?}

Teachers use a combination of informal and formal methods to monitor students' progress in reading. Typically, the choice of a particular method is guided by the manner in which the assessment information will be used. This section reports trends in the emphasis on various sources used by teachers to monitor students' progress in reading, how often different types of assessment are used in the classroom, and for what purpose teachers use results of classroom assessment.

PIRLS 2006 asked teachers whether they placed a major emphasis, some emphasis, or little or no emphasis on a variety of sources to monitor students' progress in reading. Exhibit 6.24 presents the percentage of 
Exhibit 6.23 Index of Reading for Homework (RFH) with Trends

PIRLS 2006

4th Grade

\begin{tabular}{|c|c|c|c|c|c|c|c|c|c|c|c|c|c|}
\hline \multirow{2}{*}{ Countries } & \multicolumn{5}{|c|}{ High RFH } & \multicolumn{4}{|c|}{ Medium RFH } & \multicolumn{4}{|c|}{ Low RFH } \\
\hline & & $\begin{array}{c}2006 \\
\text { Percent } \\
\text { of Students }\end{array}$ & $\begin{array}{c}\text { Average } \\
\text { Achievement }\end{array}$ & $\begin{array}{l}\text { Difference } \\
\text { in Percent } \\
\text { from } 2001\end{array}$ & & $\begin{array}{c}2006 \\
\text { Percent } \\
\text { of Students }\end{array}$ & $\begin{array}{c}\text { Average } \\
\text { Achievement }\end{array}$ & $\begin{array}{l}\text { Differenc } \\
\text { in Percen } \\
\text { from } 200\end{array}$ & & $\begin{array}{c}2006 \\
\text { Percent } \\
\text { of Students }\end{array}$ & $\begin{array}{c}\text { Average } \\
\text { Achievement }\end{array}$ & $\begin{array}{l}\text { Difference } \\
\text { in Percent } \\
\text { from } 2001\end{array}$ & \\
\hline Russian Federation & & $78(2.7)$ & $565(4.1)$ & $6(4.5)$ & & $21(2.7)$ & $563(5.6)$ & $-5(4.4)$ & & $1(0.6)$ & $\sim \sim$ & $-1(0.9)$ & \\
\hline Romania & & $76(3.4)$ & $491(5.7)$ & $4(4.8)$ & & $19(3.2)$ & $488(10.7)$ & $-6(4.7)$ & & $5(1.9)$ & $488(12.5)$ & $2(2.4)$ & \\
\hline Macedonia, Rep. of & & $75(3.3)$ & $449(5.5)$ & $-8(4.7)$ & & $21(2.9)$ & $434(14.0)$ & $4(4.3)$ & & $5(1.6)$ & $406(21.6)$ & $3(1.8)$ & \\
\hline Moldova, Rep. of & & $72(3.6)$ & $502(4.1)$ & $-18(4.5)$ & ( & $28(3.6)$ & $493(5.5)$ & $18(4.6)$ & $\theta$ & $0(0.2)$ & $\sim \sim$ & $0(0.2)$ & \\
\hline Georgia & & $55(4.4)$ & $467(4.4)$ & $\Delta \Delta$ & & $40(4.2)$ & $473(6.1)$ & $\Delta \diamond$ & & $5(1.7)$ & $492(11.1)$ & $\Delta \Delta$ & \\
\hline Bulgaria & & $51(4.0)$ & $553(5.7)$ & $-24(5.5)$ & $\nabla$ & $46(3.8)$ & $541(7.6)$ & $24(5.2)$ & $\theta$ & $4(1.8)$ & $525(13.9)$ & $1(2.4)$ & \\
\hline Indonesia & & $37(4.2)$ & $400(6.5)$ & $\Delta \Delta$ & & $60(4.3)$ & $405(5.5)$ & $\Delta \Delta$ & & $3(1.3)$ & $403(18.6)$ & $\Delta \Delta$ & \\
\hline Latvia & & $36(3.5)$ & $538(3.4)$ & $-13(5.1)$ & $\nabla$ & $63(3.5)$ & $542(3.5)$ & $14(5.3)$ & $\theta$ & $1(0.7)$ & $\sim \sim$ & $0(1.1)$ & \\
\hline Trinidad and Tobago & & $36(3.3)$ & $444(8.7)$ & $\Delta \Delta$ & & $60(3.4)$ & $437(6.2)$ & $\Delta \Delta$ & & $4(1.7)$ & $399(40.5)$ & $\Delta \Delta$ & \\
\hline Morocco & $r$ & $35(4.6)$ & $308(13.7)$ & $-15(6.9)$ & 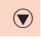 & $53(4.8)$ & $328(9.2)$ & $13(6.9)$ & & $12(2.7)$ & $341(18.4)$ & $3(4.1)$ & \\
\hline Lithuania & & $32(3.2)$ & $534(3.8)$ & $-18(5.1)$ & 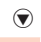 & $65(3.2)$ & $538(1.9)$ & $18(5.2)$ & $\theta$ & $3(1.4)$ & $541(5.3)$ & $1(2.0)$ & \\
\hline Hungary & & $30(4.1)$ & $548(6.3)$ & $-60(4.9)$ & ( & $69(4.2)$ & $552(3.7)$ & $60(4.8)$ & $\theta$ & $1(0.6)$ & $\sim \sim$ & $0(0.6)$ & \\
\hline Israel & & $29(3.7)$ & $521(9.5)$ & $-22(5.9)$ & $(\nabla$ & $64(3.9)$ & $513(6.2)$ & $23(6.1)$ & $\theta$ & $6(2.2)$ & $501(29.1)$ & $-1(3.3)$ & \\
\hline Iran, Islamic Rep. of & & $29(3.2)$ & $422(8.3)$ & $-29(5.3)$ & ( & $57(3.6)$ & $422(4.3)$ & $24(5.0)$ & $\theta$ & $14(2.2)$ & $417(8.0)$ & $5(3.5)$ & \\
\hline Spain & & $28(3.9)$ & $511(5.1)$ & $\Delta \Delta$ & & $67(3.8)$ & $514(3.1)$ & $\Delta \diamond$ & & $5(1.8)$ & $513(13.0)$ & $\Delta \Delta$ & \\
\hline South Africa & & $27(2.7)$ & $267(8.9)$ & $\Delta \Delta$ & & $56(3.0)$ & $315(10.9)$ & $\Delta \Delta$ & & $17(1.9)$ & $320(13.3)$ & $\Delta \Delta$ & \\
\hline Poland & & $26(3.3)$ & $517(5.1)$ & $\Delta \Delta$ & & $70(3.1)$ & $520(2.8)$ & $\Delta \diamond$ & & $5(1.6)$ & $518(7.3)$ & $\Delta \diamond$ & \\
\hline Chinese Taipei & & $23(3.6)$ & $542(3.1)$ & $\Delta \Delta$ & & $48(4.4)$ & $536(3.3)$ & $\Delta \Delta$ & & $29(4.2)$ & $530(4.5)$ & $\Delta \Delta$ & \\
\hline United States & & $22(3.1)$ & $532(7.0)$ & $-12(4.8)$ & ( & $73(3.5)$ & $541(3.3)$ & $20(5.2)$ & $\theta$ & $5(1.4)$ & $557(9.3)$ & $-9(3.3)$ & $\nabla$ \\
\hline Slovak Republic & & $14(2.9)$ & $528(6.7)$ & $-17(5.0)$ & $(\nabla$ & $83(3.1)$ & $532(3.2)$ & $15(5.2)$ & $\theta$ & $3(1.0)$ & $502(45.1)$ & $2(1.4)$ & \\
\hline Qatar & $S$ & $13(0.1)$ & $334(4.9)$ & $\Delta \Delta$ & & $71(0.3)$ & $353(1.6)$ & $\Delta \diamond$ & & $16(0.2)$ & $359(3.7)$ & $\Delta \Delta$ & \\
\hline Canada, British Columbia & $\mathrm{r}$ & $12(2.7)$ & $565(6.0)$ & $\Delta \Delta$ & & $71(4.1)$ & $556(3.6)$ & $\Delta \Delta$ & & $16(3.4)$ & $569(7.4)$ & $\Delta \Delta$ & \\
\hline Canada, Nova Scotia & & $12(2.3)$ & $557(5.9)$ & $\Delta \Delta$ & & $83(2.7)$ & $541(2.5)$ & $\Delta \diamond$ & & $5(1.5)$ & $522(9.7)$ & $\Delta \Delta$ & \\
\hline Italy & & $11(2.5)$ & $550(11.1)$ & $-36(4.6)$ & ( ) & $87(2.7)$ & $552(3.0)$ & $39(4.7)$ & $\theta$ & $1(0.9)$ & $\sim \sim$ & $-3(1.7)$ & \\
\hline Denmark & & $11(2.7)$ & $537(9.3)$ & $\Delta \Delta$ & & $87(3.0)$ & $548(2.3)$ & $\Delta \diamond$ & & $2(1.2)$ & $\sim \sim$ & $\Delta \Delta$ & \\
\hline Canada, Quebec & & $11(2.9)$ & $536(11.5)$ & $-3(4.7)$ & & $76(4.3)$ & $535(2.8)$ & $6(6.5)$ & & $14(3.4)$ & $532(6.4)$ & $-3(5.2)$ & \\
\hline Canada, Alberta & & $10(2.6)$ & $550(5.5)$ & $\Delta \Delta$ & & $76(3.5)$ & $562(3.0)$ & $\Delta \Delta$ & & $14(2.4)$ & $558(6.9)$ & $\Delta \diamond$ & \\
\hline New Zealand & & $10(1.6)$ & $544(7.2)$ & $-5(3.6)$ & & $77(2.5)$ & $534(2.2)$ & $8(5.0)$ & & $13(2.0)$ & $521(7.8)$ & $-4(4.1)$ & \\
\hline Sweden & & $10(2.1)$ & $548(6.8)$ & $-42(3.6)$ & ( & $71(3.4)$ & $551(2.6)$ & $34(4.5)$ & $\theta$ & $19(3.1)$ & $544(4.9)$ & $8(3.8)$ & $\theta$ \\
\hline England & & $9(2.5)$ & $533(11.2)$ & $-16(4.5)$ & 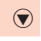 & $65(4.3)$ & $543(3.8)$ & $4(6.1)$ & & $25(4.1)$ & $541(8.1)$ & $12(5.2)$ & 0 \\
\hline Singapore & & $8(1.7)$ & $552(12.7)$ & $-31(4.1)$ & ( & $44(3.1)$ & $554(4.8)$ & $14(4.7)$ & $\theta$ & $48(3.2)$ & $563(4.3)$ & $17(4.6)$ & $\theta$ \\
\hline Norway & & $8(2.1)$ & $483(5.2)$ & $-14(4.0)$ & ( & $91(2.3)$ & $499(2.7)$ & $13(4.0)$ & $\theta$ & $1(0.9)$ & $\sim \sim$ & $0(1.1)$ & \\
\hline Hong Kong SAR & & $8(2.9)$ & $580(9.8)$ & $-22(5.4)$ & $(\nabla$ & $56(4.4)$ & $568(3.5)$ & $12(6.2)$ & & $36(3.9)$ & $553(3.5)$ & $10(5.2)$ & \\
\hline Canada, Ontario & & $7(2.3)$ & $545(6.7)$ & $-15(4.9)$ & ( & $75(4.8)$ & $553(3.3)$ & $12(6.9)$ & & $18(4.2)$ & $564(4.8)$ & $3(5.2)$ & \\
\hline Scotland & & $6(2.5)$ & $514(11.4)$ & $-8(4.2)$ & & $84(3.3)$ & $527(3.2)$ & $9(5.3)$ & & $9(2.5)$ & $527(9.0)$ & $-1(4.0)$ & \\
\hline Iceland & & $5(0.1)$ & $520(6.2)$ & $-24(0.3)$ & ( & $89(0.2)$ & $510(1.4)$ & $21(0.4)$ & $\theta$ & $6(0.2)$ & $504(6.4)$ & $3(0.2)$ & $\theta$ \\
\hline France & & $5(1.4)$ & $530(14.6)$ & $-8(3.1)$ & $\nabla$ & $72(3.4)$ & $521(2.8)$ & $8(5.3)$ & & $23(3.3)$ & $523(4.2)$ & $0(4.7)$ & \\
\hline Luxembourg & & $4(0.1)$ & $571(6.8)$ & $\Delta \Delta$ & & $64(0.2)$ & $557(1.2)$ & $\Delta \Delta$ & & $33(0.2)$ & $556(1.8)$ & $\Delta \Delta$ & \\
\hline Slovenia & & $3(1.3)$ & $509(6.9)$ & $-29(4.0)$ & $\nabla$ & $88(2.4)$ & $522(2.3)$ & $25(4.6)$ & $\theta$ & $9(1.9)$ & $522(6.1)$ & $3(2.7)$ & \\
\hline Germany & & $2(0.9)$ & $\sim \sim$ & $-4(2.0)$ & ( ) & $88(2.1)$ & $548(2.5)$ & $6(3.3)$ & & $10(2.2)$ & $550(3.4)$ & $-2(2.9)$ & \\
\hline Netherlands & $r$ & $2(1.2)$ & $\sim \sim$ & $-3(2.2)$ & & $30(3.8)$ & $546(4.1)$ & $7(5.2)$ & & $67(3.7)$ & $547(2.5)$ & $-3(5.1)$ & \\
\hline Belgium (French) & $r$ & $1(0.8)$ & $\sim \sim$ & $\Delta \Delta$ & & $45(4.3)$ & $497(4.6)$ & $\Delta \Delta$ & & $53(4.3)$ & $503(3.6)$ & $\Delta \Delta$ & \\
\hline Belgium (Flemish) & & $1(0.0)$ & $\sim \sim$ & $\Delta \Delta$ & & $45(4.1)$ & $546(3.0)$ & $\Delta \Delta$ & & $54(4.1)$ & $547(2.8)$ & $\Delta \Delta$ & \\
\hline Austria & & $1(0.5)$ & $\sim \sim$ & $\Delta \Delta$ & & $93(1.5)$ & $538(2.2)$ & $\Delta \Delta$ & & $7(1.4)$ & $546(8.5)$ & $\Delta \Delta$ & \\
\hline Kuwait & & $x x$ & $x x$ & $\Delta \Delta$ & & $x x$ & $x x$ & $\Delta \Delta$ & & $x x$ & $x x$ & $\Delta \Delta$ & \\
\hline International Avg. & & $24(0.5)$ & $498(1.4)$ & & & $62(0.5)$ & $505(0.8)$ & & & $14(0.4)$ & $496(2.7)$ & & \\
\hline
\end{tabular}

Based on teachers' responses to two questions: How often do you assign reading as part of homework (for any subject)? In general, how much time do you expect students to spend on homework involving reading (for any subject) each time you assign it? High level indicates students are expected to spend more than 30 minutes at least 1-2 times a week. Low level indicates students are never assigned homework or are expected to spend no more than 30 minutes less than once a week. Medium level indicates all other combinations of the frequencies.

() Standard errors appear in parentheses. Because results are rounded to the nearest whole number, some totals may appear inconsistent.
An " $r$ " indicates data are available for $70-84 \%$ of the students. An " $s$ " indicates data are available for $50-69 \%$ of the students. An " $x$ " indicates data are available for less than $50 \%$ of the students.

A tilde $(\sim)$ indicates insufficient data to report achievement.

A diamond $(\diamond)$ indicates the country did not participate in the 2001 assessment.

NOTE: The International Average does not include the results from the Canadian provinces. Trend Note: The primary education systems of the Russian Federation and Slovenia underwent structural changes. Data for Canada, Ontario include only public schools. 
students whose teachers reported placing major emphasis on their own professional judgment, classroom tests, national or regional achievement tests, and diagnostic tests (e.g., miscue analysis) with trends. In 2006, about two thirds of students (68\%) had teachers who placed major emphasis on their own professional judgment in monitoring students' progress in reading. In comparison, fewer had teachers placing emphasis on testing. Slightly more than half $(53 \%)$ had teachers who emphasized classroom tests, slightly less than half ( $46 \%)$ had teachers who emphasized diagnostic tests, and approximately one fourth $(27 \%)$ had teachers who emphasized national or regional achievement tests.

The percentages of students whose teachers placed major emphasis on their own judgment increased in Hong Kong SAR, Iceland, Morocco, the Russian Federation, and Singapore, but decreased in Latvia and Lithuania. Reliance on classroom tests decreased in Bulgaria, Scotland and the Canadian province of Quebec and increased in Iceland, New Zealand, and Norway. For national or regional achievement tests, between 2001 and 2006 there were increases in six countries and decreases in three countries. There were a considerable number of increases (14 countries and 1 province) in the percentages of students whose teachers emphasized the use of diagnostic tests, including increases of 20 percentage points or more in Bulgaria, Italy, Moldova, Norway, Romania, and Slovenia.

Teachers reported how often they used different approaches to assess students' reading performance based on the response options: at least once a week, once or twice a month, once or twice a year, and never or almost never. The different approaches included:

- Listening to students read aloud

- Oral questioning of students

- Students give an oral summary or report of what they have read

- Multiple-choice questions on material read

- Short-answer written questions on material read

- Paragraph-length written responses about what students have read. 
Exhibit 6.24 Emphasis on Sources to Monitor Students' Progress in Reading with Trends
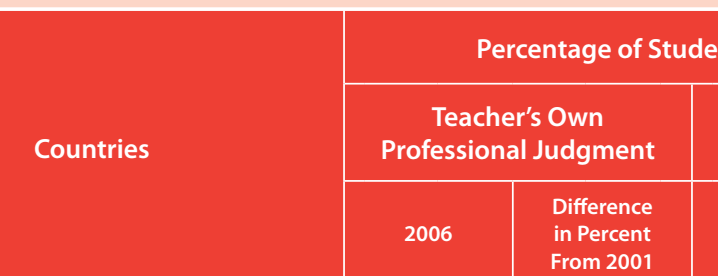

Countries

Percentage of Students Whose Teachers Reported Placing Major Emphasis on Various Sources*

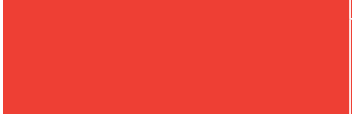

Austria

$(4,2)$ From 2001

Belgium (Flemish)

Belgium (French)

$44(3.2)$

$\Delta \Delta$

\begin{tabular}{l|l|}
\multicolumn{2}{|c}{ Classroom Tests } \\
\hline 2006 & $\begin{array}{l}\text { Difference } \\
\text { in Percent } \\
\text { From } 2001\end{array}$ \\
\hline
\end{tabular}

\begin{tabular}{|l|l|}
\hline \multicolumn{2}{|c|}{$\begin{array}{c}\text { National or Regional } \\
\text { Achievement Tests }\end{array}$} \\
\hline 2006 & $\begin{array}{r}\text { Difference } \\
\text { in Percent } \\
\text { From 2001 }\end{array}$ \\
\hline
\end{tabular}

Diagnostic Tests

Bulgaria

Canada, Alberta

$74(3.8)$

$\Delta 0$

$17(2.7)$

$\diamond \diamond$

$6(1.4)$

$\diamond \diamond$

$43(3.8)$

$\Delta \Delta$

$58(2.7)$

$\Delta \Delta$

$80(3.0)$

$\Delta \Delta$

$58(4.2)$

$45(3.5)$

00

$17(3.2) \quad-11(5.0)$

Canada, British Columbia

$70(3.9)$

$\Delta \diamond \quad 33(3.5)$

$-13(5.7)$

$8(2.1)$

$(5.0) \quad(1)$

Canada, Nova Scotia

$83(3.5)$

$\Delta \diamond \quad$ r $33(4.1)$

Canada, Ontario

Canada, Quebec

$74(3.9)$

$\Delta \nabla$

$17(2.9)$

$\Delta 0 \quad$ r $\quad 4(1.6)$

$\Delta \diamond \quad 1(0.8)$

$\diamond \diamond$

$\Delta \Delta \quad r$

$\Delta 0$

$5(2.3) \quad 3(2.6)$

$86(3.2) \quad-2(4.5)$

$44(4.0)$

$-10(6.8)$

Chinese Taipei

$40(4.2)$

$\Delta \Delta$

$49(4.0)$

$-32(5.4) \quad \uparrow \quad 11(3.2)$

$-14(5.8)$

$23(3.6)$

00

$\begin{array}{ll}\Delta \diamond & 23(3.6) \\ \Delta \diamond & 14(2.6)\end{array}$

$\Delta \diamond$

$15(3.3) \quad-10(5.6)$

$33(4.2)$

$79(2.7) \quad 5(4.4)$

France

$82(2.4)$

$1(5.3)$

\section{5 (3.2)}

Georgia

$51(4.1)$

Hong Kong SAR

Hungary

$2(5.2)$

$67(4.1)$

$5(4.4)$

\section{$36(3.6)$}

$-7(6.7)$

$10(5.0)$

\section{$38(3.6)$}

$\checkmark 0 \quad 42(4.6)$

$5(4.8)$

$11(2.3)$

00

$12(6.3)$

$16(3.1)$

\section{$79(3.1)$}

$0(4.5)$

$80(3.5)$

$3(5.2)$

Iceland

$32(3.9)$

$5(0.5)$

0

$18(0.3)$

$4(0.4)$

$36(4.2)$

$7(2.7)$

$5(4.0)$
$8(5.7)$

$9(0.2)$

$-1(0.3)$

$31(3.5)$

$\diamond \diamond$

$22(3.3) \quad 0(4.4)$

$-5(4.8)$

$60(4.2) \quad-4(5.9)$

Israel

\section{$72(3.8)$}

$0(5.8)$

Kuwait

$82(3.2)$
$68(4.3)$

$5(4.7)$

$-4(5.9)$

\section{$27(3.7)$}

$8(5.2)$

$\Delta \diamond$

$78(3.3)$

$\Delta 0$

$15(2.9)$

11 (3.4)

$21(3.5) \quad \Delta \Delta$

$-2(5.1)$
$-5(5.4)$

$21(3.1) \quad-3(4.3)$

$-20(5.5)$

$42(3.5)$

$61(3.4)$

\section{$63(3.4)$}

$\begin{array}{cc}-17(5.2) & 61(3.4) \\ \Delta \diamond & 39(0.2)\end{array}$

$-5(5.4)$

$66(4.0) \quad-5(5.6)$

$22(3.0)$

$10(0.1)$

Macedonia, Rep. of

$79(3.2) \quad 0(5.0)$

$0(5.0)$

$89(2.6) \quad-3(3.5)$

$24(3.8)$

$--$

$\Delta \diamond$

$-2(5.5)$

$64(4.1) \quad-1(6.1)$

\begin{tabular}{r|r}
$61(3.7)$ & $-11(5.8)$ \\
\hline $64(3.9)$ & $6(5.9)$
\end{tabular}

Metherlands

$95(1.8)$

$18(4.8)$

New Zealand

$80(3.3) \quad 2(4.9)$

$62(3.1) \quad-6(5.3)$

$75(4.0) \quad 5(5.6)$

$6(5.9)$
$16(4.5)$

Norway

Poland

$59(4.0)$

$5(5.6) \quad 26(3.5)$

$13(4.6)$

$25(4.3)$

$-1(6.1)$
$9(5.8)$

$\begin{array}{ll}64(4.3) & -8(5.8)\end{array}$

$43(4.2)$
$31(4.5)$

\begin{tabular}{l|l}
2006 & $\begin{array}{l}\text { Difference } \\
\text { in Percent } \\
\text { From } 2001\end{array}$
\end{tabular}

Qatar

$\Delta \diamond \quad 45(3.9)$

$--$

$32(3.9)$

$26(2.9)$

$\diamond \diamond$

Romania

\section{$63(4.0)$}

$\Delta \diamond \quad s \quad 81(0.2)$

$\Delta 0 \quad 22(3.6)$

$-9(5.9) \quad 89(2.5)$

$--$

$--$

$55(4.1)$

$60(3.7)$

$\Delta \Delta$

Russian Federation

Scotland

$84(2.4) \quad 13(4.3)$

$89(3.0) \quad-4(3.9)$

Singapore

Slovak Republic

$59(2.5) \quad 13(4.3)$

Slovenia

South Africa

$55(3.5) \quad 8(5.6)$

- $71(3.1)$

4 (4.2)

$31(0.3)$

$\Delta \diamond$

$\Delta \diamond \quad 35(3.7) \quad \Delta 0$

$31(0.3) \quad \Delta \Delta \quad s$

\begin{tabular}{|c|c|c|}
\hline $4(4.2)$ & $26(3.7)$ & $6(5.3)$ \\
\hline $1(4.6)$ & $49(3.6)$ & $22(4.8)$ \\
\hline (1) & $24(3.6)$ & $-27(6.4)$ \\
\hline
\end{tabular}

49 (3.6)

$22(4.8)$
$-27(6.4)$

$64(4.0) \quad 24(6.1)$

40

Africa

Spain

Sweden

Trinidad and Tobago

$\begin{array}{rr}62(3.3) & -5 \\ 61(3.4) & 0\end{array}$

$-5(5.2)$

$40(2.6) \quad-7(4.6)$

$\begin{array}{cc}24(3.6) & -27(6.4) \\ -- & --\end{array}$

$-7(5.1)$

$28(3.4)$

$-7(5.1)$

$14(2.7)$

$\Delta 0$

50 (3.4)

$1(5.3)$

$11(2.1)$

$95(1.7) \quad 0$

$\begin{array}{rr}84(3.0) & -5 \\ 63(3.8) & 0 \\ 79(3.2) & 10(5.5)\end{array}$

$\diamond \diamond \quad 86(2.8)$

$\Delta \diamond$

$12(2.1)$

\begin{tabular}{|c|c|c|c|c|}
\hline-- & & $13(1.7)$ & $-1(3.3)$ & \\
\hline 11 (3.1) & 0 & $29(3.0)$ & $10(4.3)$ & 0 \\
\hline $8(2.6)$ & 0 & $34(2.9)$ & $20(4.2)$ & 0 \\
\hline$\Delta \Delta$ & & $32(3.4)$ & $\Delta 0$ & \\
\hline$\Delta \Delta$ & & 76 (3.5) & $\Delta 0$ & \\
\hline $3(5.0)$ & & $36(3.9)$ & $13(4.7)$ & 0 \\
\hline$\Delta \Delta$ & & $26(3.7)$ & 00 & \\
\hline $9(4.6)$ & & 37 (3.7) & $13(5.6)$ & 0 \\
\hline
\end{tabular}

\begin{tabular}{rr}
$64(3.1)$ & $24(6.1)$ \\
\hline 66 & $2(4.9)$ \\
\hline
\end{tabular}

$10(2.6) \quad-4(3.6)$

$-5(3.5)$

$11(2.2)$

$-1(2.9)$

$30(3.6)$

$34(3.9)$
$28(3.6)$
$24(3.5)$

International Avg.

$68(0.5)$

$10(5.5)$

$57(3.1) \quad 5(4.5)$

$53(0.5)$

\begin{tabular}{|c|r|r|c|c|}
\hline & $53(0.5)$ & $27(0.5)$ & $46(0.5)$ \\
\hline Percent in 2006 significantly higher & $\odot$ & Percent in 2006 significantly lower
\end{tabular}

\begin{tabular}{|c|c|c|c|c|}
\hline $53(0.5)$ & $27(0.5)$ & $46(0.5)$ \\
\hline - Percent in 2006 significantly higher & $\nabla$ & Percent in 2006 significantly lower
\end{tabular}

Background data provided by teachers. * In 2001, "professional judgment" was worded as "professional opinion" and "national or
regional achievement tests" was worded as "national or regional examinations".

() Standard errors appear in parentheses. Because results are rounded to the nearest whole number, some totals may appear inconsistent.

An " $r$ " indicates data are available for $70-84 \%$ of the students. An "s" indicates data are available for $50-69 \%$ of the students. An " $x$ " indicates data are available for less than

$50 \%$ of the students.

A dash (-) indicates comparable data are not available.
A diamond $(\diamond)$ indicates the country did not participate in the 2001 assessment.

NOTE: The International Average does not include the results from the Canadian provinces. NOTE: The International Average does not include the results from the Canadian province
Trend Note: The primary education systems of the Russian Federation and Slovenia
underwent structural changes. Data for Canada, Ontario include only public schools. 
For oral questioning and oral summary, teachers' responses were averaged and combined as one reporting category. Similarly, teachers' responses for short-answer, and paragraph-length written responses were averaged to create a constructed-response category. Then, the response options of at least once a week and once or twice a month were combined.

Exhibit 6.25 presents the summary of teachers' responses regarding their approaches to assessing students' reading performance. More than three fourths of students (78\%), on average internationally, had teachers who listened to them read aloud at least weekly. Eighty-five percent had teachers who used oral assessment techniques (oral questioning or summary reports) at least weekly, and 63 percent had teachers who used written assessment (short or paragraph length). On average, multiple-choice questions were used with less than half the students ( $42 \%)$, but this varied considerably across countries.

Two countries (Bulgaria and the Netherlands) had increases from 2001 in the percentage of students whose teachers listened to students read aloud, while only Iceland had a decrease (16 percentage points). The percentage of students whose teachers used oral questioning or summary reports to monitor students' progress increased from 2001 in several countries, including Hungary, Iceland, Macedonia, New Zealand, Norway, and the Slovak Republic. Only in Latvia did the percentage decrease. The percentages of students assessed with multiple-choice questions increased from 2001 in Bulgaria, Germany, Morocco and the United States, whereas decreases occurred in France, the Slovak Republic, and Slovenia. Since 2001 the percentage of students whose teachers asked them to write responses to what they read increased in Germany, Italy, and Latvia.

Exhibit 6.26 presents teachers' reports about how they use information from classroom assessment to make decisions about instruction. On average internationally, most students had teachers who used classroom assessment in reading to adapt instruction (91\%), inform parents of students' progress $(92 \%)$, and identify students in need of remediation (91\%). On average internationally, teachers used assessments for 72 percent of students to 
assign marks or grades, and for about two thirds (66\%) they used reading assessment information to group students for reading instruction. A smaller percentage of students across countries $(35 \%)$ had teachers who provided data from classroom assessments for national or local monitoring programs. Countries where more than half the students' teachers were required to provide such data included Hungary, Indonesia, Macedonia, Moldova, the Netherlands, Poland, Qatar, Scotland, and Trinidad and Tobago. 
Exhibit 6.25 Approaches to Assessing Students' Performance in Reading with Trends

PIRLS 2006
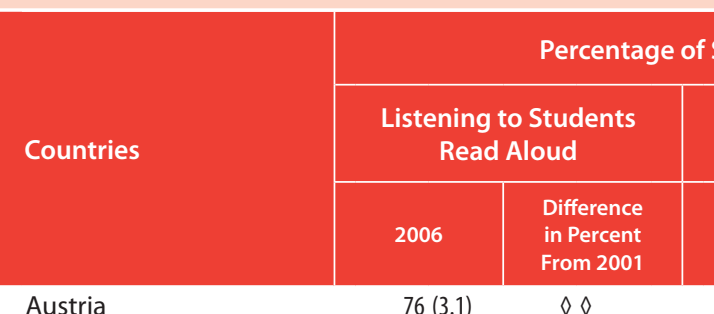

Austria

$76(3.1) \quad \Delta \diamond$

$68(3.0)$

$68(3.0)$

Belgium (Flemish)

$68(3.5)$

$\Delta 0$

$66(3.7)$

$62(3.6)$

$\diamond \diamond$

Bulgaria

Canada, Alberta

$99(0.0) \quad 3(1.6)$

Canada, British Columbia

$56(4.0)$

$\diamond \diamond$

○

$54(3.7)$

$97(1.1)$

$74(3.4)$

Whose Teachers Reported Using Approach at Least Weekly

Canada, Nova Scotia

$63(4.1)$

$\Delta \Delta$

r $\quad 72(3.3)$

$81(3.4)$

$58(4.9) \quad 2(6.8)$

Canada, Quebec

$47(4.4) \quad-5(6.9)$

Chinese Taipei

78 (3.6)

$\Delta \diamond$

$73(4.3)$

Denmark

$53(3.5)$

$\diamond \Delta$

England

$67(3.8) \quad 1(5.8)$

France

74 (3.4)

Georgia

$90(2.6)$

$9(5.1)$

Germany

$75(3.3)$

$-5(4.4)$

$57(4.6)$

85 (2.7)

stioning

Hong Kong SAR

Hungary

Iceland

Indonesia

$68(3.5) \quad 10(5.5)$

Iran, Islamic Rep. of

$41(3.9)$

2 (5.6)

$56(0.4)$

$-17(0.5)$

$74(3.3) \quad \Delta \diamond$

Israel

$89(2.3) \quad-1(3.4)$

Italy

Kuwait

$79(3.5)$

$10(5.5)$

$90(2.5) \quad 2(3.4)$

$88(2.6)$

ary Reports*

\begin{tabular}{|l|l}
\hline & Mul \\
\hline & \\
\hline
\end{tabular}

\begin{tabular}{|r|r}
\hline Multipl \\
\hline 200 \\
\hline
\end{tabular}

Difference
in Percent
From 2001
$\diamond \diamond$

$84(3.0)$

$80(3.0)$

$\Delta 0$

$\Delta \diamond$

0 (1.7)

$34(3.6)$
$-\quad 30(3.3)$

$30(3.3)$

$25(2.7)$

$\diamond \diamond$

$\Delta \diamond \quad 16(2.6)$

$\diamond \diamond \quad$ r $14(2.4)$

$\Delta 0$

$-3(5.8)$

$14(2.4)$

$-3(6.6)$

00

$\Delta \Delta$
$8(5.2)$

$8(5.2)$

$$
96(1.5)
$$

$3(4.3)$

$3(4.3)$

$$
69(3.3)
$$

$\begin{array}{lll}-5(4.2) & \text { r } & 17(2.9)\end{array}$

$84(2.8)$

$66(3.5)$

$4(5.0)$

$41(0.4)$

$10(4.9)$

$87(2.9)$

$10(0.5)$

0

$9(3.2)$

$96(1.3)$

$58(3.9)$

$9(2.7)$
$51(4.5)$

$92(2.4)$

$1(2.0)$

$-1(3.5)$

$93(1.9)$

$-1(3.5)$
$4(3.1)$

$94(2.0)$

00

10

$62(4.1)$
$55(3.2)$

$\begin{array}{llll}94(2.0) & \diamond \diamond & 57(4.0) \\ 86(2.6) & -8(3.3) \quad & 33(3.4)\end{array}$

$77(3.4) \quad-9(4.7)$

Lithuania

$82(2.8)$

$-9(4.7)$

$\begin{array}{ll}0(2.0) & 33(3.4) \\ 0 & 50(3.4)\end{array}$

$96(1.3)$

$\begin{array}{ll}0(2.0) & 50(3.4) \\ 00 & 23(0.2)\end{array}$

$99(1.0) \quad 6(2.6) \quad 0 \quad 65(3.9)$

$94(1.9) \quad 5(3.2)$

$98(1.1)$

$1(1.8)$

$91(2.3) \quad-1(3.2)$

Moldova, Rep. of

$96(1.8)$

$-1(3.2)$

$98(1.1)$

$1(1.8)$
$-2(3.3)$

\begin{tabular}{|c|c|c|}
\hline 65 (3.9) & $6(5.6)$ & \\
\hline 68 (3.7) & $8(5.7)$ & \\
\hline $85(2.8)$ & $24(5.6)$ & 0 \\
\hline $21(3.2)$ & $-1(4.9)$ & \\
\hline $6(1.1)$ & $1(2.0)$ & \\
\hline
\end{tabular}

$\begin{array}{ll}93(2.5) & -2(3.3) \\ 70(4.1) & -5(5.6)\end{array}$

$68(3.7)$
$85(2.8)$

\begin{tabular}{r|rr}
$96(1.8)$ & $2(2.9)$ & \\
$56(4.4)$ & $17(6.5) \quad 0$
\end{tabular}

$81(2.6)$

$-5(5.6)$

$56(2.7) \quad-3(4.9)$

New Zealand

$88(2.7) \quad 3(4.4)$

$93(1.8)$

\begin{tabular}{|c|c|c|}
\hline $65(3.9)$ & $6(5.6)$ & \\
\hline $68(3.7)$ & $8(5.7)$ & \\
\hline $85(2.8)$ & $24(5.6)$ & 0 \\
\hline $21(3.2)$ & $-1(4.9)$ & \\
\hline $6(1.1)$ & $1(2.0)$ & \\
\hline
\end{tabular}

$13(4.7) \quad \mathbf{0}$

\begin{tabular}{c|c}
\hline Difference & \\
in Percent \\
From 2001 \\
\hline$\diamond$
\end{tabular}

Constructed-response Questions**

Norway

Poland

$97(1.2) \quad \diamond \diamond$

$93(1.8)$

$\begin{array}{lll}23(4.3) & \bullet & 24(3.9) \\ \Delta \diamond & & 36(3.9) \\ \Delta \diamond & & 72(0.2)\end{array}$

$24(164$

\begin{tabular}{l|l}
2006 & $\begin{array}{l}\text { Difference } \\
\text { in Percent } \\
\text { From } 2001\end{array}$
\end{tabular}

Qatar s

$96(1.5)$

$93(0.1) \quad \diamond$

$93(0.2)$

00

39 (3.1)

$\Delta \diamond$

$58(3.8) \quad 00$

$54(3.6) \quad \diamond \diamond$

$12(5.1) \quad 0 \quad 61(3.6) \quad 7(5.3)$

$\Delta \Delta \quad 52(4.0) \quad \Delta \Delta$

$\begin{array}{llll}\Delta \diamond & r & 61(4.0) & \Delta \Delta \\ \Delta \diamond & & 67(3.7) & \Delta \Delta\end{array}$

$\Delta \diamond$

$67(3.7)$

$-1(4.3)$

$55(5.5) \quad-4(7.1)$

$95(1.5) \quad-1(1.9)$

\begin{tabular}{|c|c|c|}
\hline$\Delta \diamond$ & & $36(3.9)$ \\
\hline$\Delta \Delta$ & $s$ & $72(0.2)$ \\
\hline $0(1.5)$ & & $67(3.6)$ \\
\hline $1(0.6)$ & & $70(2.8)$ \\
\hline
\end{tabular}

$98(1.0)$

$100(0.3)$

$\Delta \diamond$

$\begin{array}{cc}48(4.6) & -11(6.8) \\ 60(4.3) & \diamond \diamond\end{array}$

$60(4.3) \quad \Delta \diamond$

$29(3.6) \quad \Delta \Delta$

Scotland

Singapore

\begin{tabular}{lr}
$86(3.2)$ & $-6(4.4)$ \\
$71(2.6)$ & $2(4.7)$ \\
\hline
\end{tabular}

$94(2.0)$
$83(2.0)$

\begin{tabular}{l|l}
$1(0.6)$ & $70(2.8)$ \\
$0(2.9)$ & $12(2.8)$
\end{tabular}

$4(3.5)$

$32(3.9) \quad-8(5.9)$

Slovak Republic

Slovenia

Slovenia
South Africa

$97(1.1) \quad 5(2.7)$

\begin{tabular}{r|r|}
$0(2.9)$ & $12(2.8)$ \\
$-1(3.4)$ & $53(3.4)$ \\
\hline $6(2.0)$ & 0
\end{tabular}

\begin{tabular}{r|r|}
\hline $83(2.0)$ & $-1(3.4)$ \\
\hline $98(0.8)$ & $6(2.0) \quad 0$ \\
\hline $82(2.6)$ & $2(4.5)$
\end{tabular}

$40(3.8)$

$-10(4.7)$

$\begin{array}{ll}32(3.9) & -8(5.9) \\ 64(3.7) & 5(5.6)\end{array}$

\begin{tabular}{l|ll}
$\Delta \diamond$ & $75(4.2)$ & $\Delta(5.6)$ \\
$9(3.4) \quad \diamond$ & $33(3.7)$ & $19(4.4)$ \\
\hline
\end{tabular}

Sweden

Trinidad and Tobago

\begin{tabular}{|c|c|c|}
\hline $74(3.0)$ & $-5(4.5)$ & $82(2.6)$ \\
\hline $70(2.9)$ & $\diamond \diamond$ & $77(2.3)$ \\
\hline $91(2.1)$ & $\diamond \diamond$ & $90(2.3)$ \\
\hline
\end{tabular}

$82(2.6)$
$77(2.3)$

\begin{tabular}{|l|l|l|}
\hline $91(2.1)$ & $\diamond \diamond$ & $90(2.3)$ \\
\hline $44(4.0)$ & $-7(5.1)$ & $70(3.3)$ \\
\hline $93(1.9)$ & 00 & $98(1.2)$ \\
\hline
\end{tabular}

United States

$93(1.9)$
$76(3.1)$

International Avg.

• Percent in 2006 significantly lower An " $r$ " indicates data are available for $70-84 \%$ of the students. An "s" indicates data are
available for $50-69 \%$ of the students. An " $x$ " indicates data are available for less than
$50 \%$ of the students.
A diamond $(0)$ indicates the country did not participate in the 2001 assessment.

available for $50-69 \%$ of the students. An " $x$ " indicates data are available for less than
$50 \%$ of the students.
A diamond $(0)$ indicates the country did not participate in the 2001 assessment.
NOTE: The International Average does not include the results from the Canadian provinces.
Trend Note: The primary education systems of the Russian Federation and Slovenia

Trend Note: The primary education systems of the Russian Federation and Slovenia
underwent structural changes. Data for Canada, Ontario include only public schools.

* Includes oral questioning of students and students giving an oral summary/report.

** Includes short-answer written questions on materials read and paragraph-length

Standard errors appear in parentheses. Because results are rounded to the nearest whole number, some totals may appear inconsistent. 
Exhibit 6.26 Teachers' Reports on Uses to Which Classroom Assessment of Reading Is Put

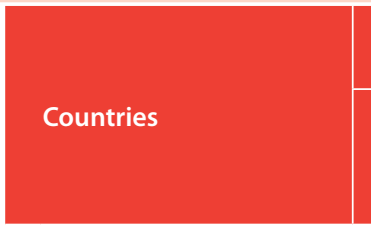

Percentage of Students Whose Teachers Reported Using Assessment Information

\begin{tabular}{|c|c|c|c|c|c|c|}
\hline Austria & $89(2.2)$ & $90(1.9)$ & $94(1.8)$ & $87(2.2)$ & $44(3.5)$ & $11(2.0)$ \\
\hline Belgium (Flemish) & $76(3.2)$ & $86(2.3)$ & $88(2.5)$ & $90(2.2)$ & $68(3.7)$ & $14(2.5)$ \\
\hline Belgium (French) & $79(2.6)$ & $95(1.4)$ & $85(2.7)$ & $92(1.8)$ & $40(3.9)$ & $8(2.2)$ \\
\hline Bulgaria & $86(2.7)$ & $89(2.2)$ & $93(2.3)$ & $100(0.3)$ & $78(2.9)$ & $35(3.7)$ \\
\hline Canada, Alberta & $95(1.7)$ & $97(1.3)$ & $99(0.4)$ & $98(1.0)$ & $78(3.5)$ & $27(3.6)$ \\
\hline Canada, British Columbia & $96(1.3)$ & $100(0.0)$ & $100(0.3)$ & $99(1.0)$ & $74(3.9)$ & $28(3.7)$ \\
\hline Canada, Nova Scotia & $86(2.5)$ & $100(0.2)$ & $100(0.2)$ & $98(1.1)$ & $87(2.6)$ & $22(3.2)$ \\
\hline Canada, Ontario & $95(2.4)$ & $100(0.0)$ & $95(2.3)$ & $97(1.6)$ & $86(3.6)$ & $29(4.6)$ \\
\hline Canada, Quebec & $92(2.6)$ & $99(0.7)$ & $99(0.9)$ & $97(1.5)$ & $61(4.1)$ & $14(2.8)$ \\
\hline Chinese Taipei & $83(3.1)$ & $92(2.5)$ & $64(3.8)$ & $89(2.6)$ & $61(4.2)$ & $9(2.3)$ \\
\hline Denmark & $4(1.5)$ & $97(1.7)$ & $99(0.8)$ & $97(1.2)$ & $73(3.5)$ & $21(2.9)$ \\
\hline England & $54(4.2)$ & $97(1.4)$ & $96(2.0)$ & $96(1.8)$ & $92(2.0)$ & $46(4.2)$ \\
\hline France & $62(3.8)$ & $98(1.0)$ & $88(2.3)$ & $97(1.1)$ & $58(3.7)$ & $11(2.4)$ \\
\hline Georgia & $90(2.6)$ & $80(3.4)$ & $96(1.5)$ & $96(1.7)$ & $65(3.9)$ & $29(3.6)$ \\
\hline Germany & $94(1.3)$ & $95(1.5)$ & $90(2.3)$ & $86(2.4)$ & $63(4.1)$ & $13(2.4)$ \\
\hline Hong Kong SAR & $81(3.3)$ & $95(1.7)$ & $62(4.1)$ & $76(3.3)$ & $40(4.5)$ & $4(1.7)$ \\
\hline Hungary & $81(3.2)$ & $99(0.9)$ & $98(1.0)$ & $91(2.6)$ & $76(3.7)$ & $58(3.5)$ \\
\hline Iceland & $63(0.4)$ & $83(0.2)$ & $96(0.1)$ & $97(0.1)$ & $53(0.4)$ & $18(0.3)$ \\
\hline Indonesia & $90(2.6)$ & $91(2.5)$ & $94(2.0)$ & $92(2.3)$ & $76(3.3)$ & $69(3.7)$ \\
\hline Iran, Islamic Rep. of & $82(3.0)$ & $94(1.5)$ & $94(2.1)$ & $92(1.8)$ & $92(1.8)$ & $43(3.7)$ \\
\hline Israel & $90(2.6)$ & $93(2.3)$ & $97(1.4)$ & $47(5.1)$ & $79(3.6)$ & $41(4.6)$ \\
\hline Italy & $73(3.4)$ & $100(0.0)$ & $87(2.6)$ & $95(1.8)$ & $37(4.1)$ & $24(3.1)$ \\
\hline Kuwait & $99(1.0)$ & $61(4.4)$ & $97(1.2)$ & $96(1.4)$ & $48(4.3)$ & $39(4.1)$ \\
\hline Latvia & $80(3.3)$ & $85(2.4)$ & $96(1.4)$ & $58(3.5)$ & $32(3.5)$ & $15(2.7)$ \\
\hline Lithuania & $31(3.4)$ & $98(0.9)$ & $100(0.0)$ & $98(0.9)$ & $66(3.3)$ & $25(2.9)$ \\
\hline Luxembourg & $80(0.1)$ & $95(0.1)$ & $85(0.1)$ & $60(0.2)$ & $44(0.2)$ & $7(0.1)$ \\
\hline Macedonia, Rep. of & $96(1.7)$ & $97(1.4)$ & $99(0.9)$ & $97(1.4)$ & $90(2.4)$ & $58(4.4)$ \\
\hline Moldova, Rep. of & $88(2.8)$ & $85(3.0)$ & 95 (1.6) & $86(2.9)$ & $86(2.9)$ & $55(4.0)$ \\
\hline Morocco & $68(4.3)$ & $82(3.0)$ & $69(4.2)$ & $96(1.6)$ & $57(4.8)$ & $44(3.9)$ \\
\hline Netherlands & $68(4.2)$ & $89(3.4)$ & $97(1.8)$ & $92(2.6)$ & $64(4.2)$ & $89(2.7)$ \\
\hline New Zealand & $41(3.2)$ & $99(0.4)$ & $95(1.2)$ & $97(0.9)$ & $95(1.2)$ & $49(3.0)$ \\
\hline Norway & $0(0.0)$ & $100(0.5)$ & $100(0.0)$ & $100(0.5)$ & $63(4.4)$ & $48(4.1)$ \\
\hline Poland & $91(1.7)$ & $97(1.0)$ & $98(0.8)$ & $98(0.9)$ & 77 (3.2) & $58(4.2)$ \\
\hline Qatar & $89(0.2)$ & $86(0.2)$ & $93(0.1)$ & $89(0.1)$ & $75(0.2)$ & $53(0.3)$ \\
\hline Romania & $84(3.1)$ & $91(2.6)$ & $97(1.1)$ & $98(1.3)$ & $82(3.0)$ & $44(3.9)$ \\
\hline Russian Federation & $99(0.7)$ & $65(3.3)$ & $99(0.9)$ & $93(1.4)$ & $66(3.6)$ & $37(3.6)$ \\
\hline Scotland & $34(4.1)$ & $98(1.3)$ & $97(1.6)$ & $100(0.2)$ & $95(2.1)$ & $56(3.8)$ \\
\hline Singapore & $66(2.5)$ & $93(1.5)$ & $85(2.4)$ & $90(1.7)$ & $74(3.0)$ & $36(2.8)$ \\
\hline Slovak Republic & $83(2.7)$ & $90(2.1)$ & $93(1.8)$ & $81(3.1)$ & $56(3.8)$ & $31(3.4)$ \\
\hline Slovenia & $30(3.3)$ & $90(2.0)$ & $98(0.8)$ & $97(1.0)$ & $68(3.2)$ & $6(1.4)$ \\
\hline South Africa & $94(1.5)$ & $85(2.0)$ & $97(1.1)$ & $96(1.1)$ & $85(1.7)$ & $50(3.4)$ \\
\hline Spain & $84(2.9)$ & $96(1.5)$ & $97(1.2)$ & $98(1.2)$ & $33(4.0)$ & $29(4.1)$ \\
\hline Sweden & $34(3.8)$ & $97(1.3)$ & $98(0.9)$ & $97(1.4)$ & $37(4.1)$ & $41(4.2)$ \\
\hline Trinidad and Tobago & $78(3.3)$ & $95(1.6)$ & $94(2.0)$ & 97 (1.6) & $86(2.7)$ & $56(4.1)$ \\
\hline United States & $96(0.8)$ & $97(1.3)$ & $98(1.1)$ & $97(1.0)$ & $83(3.1)$ & $39(4.0)$ \\
\hline International Avg. & $72(0.4)$ & $91(0.3)$ & $92(0.3)$ & $91(0.3)$ & $66(0.5)$ & $35(0.5)$ \\
\hline
\end{tabular}

Background data provided by teachers.

() Standard errors appear in parentheses. Because results are rounded to the nearest whole number, some totals may appear inconsistent.

An " $r$ " indicates data are available for $70-84 \%$ of the students. An "s" indicates data are available for $50-69 \%$ of the students. An " $x$ " indicates data are available for less than $50 \%$ of the students.

NOTE: The International Average does not include the results from the Canadian provinces. 




\section{Chapter 7}

\section{School Contexts}

While the foundation for literacy is laid in the home and the home is a continuing source of support for literacy activities, formal instruction in reading takes place in the school. Thus, the learning environment that the school provides is a crucial factor in supporting reading achievement and establishing a positive orientation toward reading. This chapter provides reports from school principals, teachers, students, and parents on aspects of the school context, particularly school demographics, the role of the principal, school resources, home-school involvement, school attendance, school climate for learning, and school safety.

\section{What Are the Schools' Demographic Characteristics?}

To provide information on the demographic context for the schools attended by fourth-grade students, PIRLS 2006 collected data on school location (urban, suburban, and rural) and the composition of the student body in terms of the percentage of students from economically disadvantaged homes and the percentage of students who do not speak the language of the PIRLS test as their first language.

Exhibit 7.1 presents, for the PIRLS 2006 countries, the percentage of fourth-grade students in schools characterized by their principals as urban, suburban, and rural, together with their average reading achievement, as well as the change in the percentage of students in such schools since 2001. On average internationally, more than two fifths of students (43\%) attended schools in urban areas, almost one quarter (24\%) in suburban 
areas, and about one third (33\%) in rural areas. The majority of students in Bulgaria, Hong Kong SAR, Italy, Latvia, Lithuania, Macedonia, Poland, Qatar, the Russian Federation, Singapore, the Slovak Republic, Spain, and the Canadian provinces of Ontario and Quebec attended schools in an urban setting, whereas in Indonesia, Moldova, and South Africa, the majority were attending rural schools. Countries with increased percentages of students in 2006 attending urban schools included Latvia, the Russian Federation, Sweden, and the Canadian province of Quebec. Iran and the United States had increased percentages of students in suburban schools, and only Iceland had proportionately more students in rural schools in 2006 than in 2001.

As in 2001, average reading achievement across countries was highest among students attending urban schools, next highest among those attending suburban schools, and lowest for those in rural schools. Although this pattern of achievement was apparent in almost half of the PIRLS 2006 countries, it was not universal. Particularly in some of the industrialized countries, where urban decay in large cities may have offset any advantage of an urban setting, average student achievement in urban schools was lower than in suburban or rural schools. For example, in Germany, the Netherlands, Scotland, the United States, and the Canadian province of Ontario, average reading achievement was highest among students in suburban schools, next highest in rural schools, and lowest in urban schools, and in Austria, Belgium (French and Flemish), England, and France, average achievement was highest among students in rural schools.

In order to provide information on the socioeconomic composition of the student body, school principals were asked to estimate the percentage of students in their schools that came from economically disadvantaged homes. Because of the range of economic development across the PIRLS countries, and because ideas of economic disadvantage vary from country to country, it was not possible to have a definition of economic disadvantage that would have the same meaning in all countries. PIRLs relied on principals' perceptions of disadvantage, therefore, in gathering this information. Exhibit 7.2 summarizes the results in terms of four categories of schools: 
- Schools where, according to principals' reports, no more than 10 percent of the students came from economically disadvantaged homes,

- Schools where 11 to 25 percent of the students came from disadvantaged homes,

- Schools where 26 to 50 percent of the students came from disadvantaged homes, and

- Schools with more than 50 percent disadvantaged students.

According to school principals, almost 40 percent of students, on average, across countries, were in schools with few (no more than 10\%) students from disadvantaged homes. In Iceland and Norway, the countries with the highest percentages, 84 percent of students were in such schools. Also, at least 55 percent of students attended such schools in Austria, the Flemish part of Belgium, Chinese Taipei, Denmark, Kuwait, the Netherlands, Singapore, Spain, and the Canadian province of Alberta. Since PIRLS 2001, there was an increase in the percentage of students in schools with few disadvantaged students in a number of countries, including Bulgaria, England, Hong Kong SAR, Latvia, Lithuania, the Russian Federation, and the Slovak Republic. On average, 18 percent of students were in schools where more than 50 percent of the students were from economically disadvantaged homes, with the greatest percentages (more than 60\%) in Indonesia and South Africa.

On average internationally, the reading achievement of students attending schools with a high proportion of disadvantaged students was lower than for students with fewer disadvantaged schoolmates. There was a difference of more than 50 points between the average achievement of students attending schools with few disadvantaged schoolmates (521 points) and those in schools where the majority of the students were from disadvantaged homes (465 points).

In addition to estimating the percentage of students in their schools from economically disadvantaged homes, school principals also provided the approximate percentage of their students who did not speak the language 
of the PIRLS assessment as their first language. Using the same percentage categories as the previous exhibit $(0-10 \%, 11-25 \%, 26-50 \%$, and more than $50 \%$ ), Exhibit 7.3 presents for each country the percentage of students in each category, their average reading achievement, and the change in percentage since 2001, if applicable.

According to school principals, almost three quarters of the fourth-grade students ( $73 \%$ on average internationally) were in schools with just a few students (no more than 10\%) whose first language was not the language of the PIRLS assessment. Almost all students (more than 90\%) in Hong Kong SAR, Hungary, Iceland, Kuwait, Lithuania, Poland, Scotland and the Canadian province of Nova Scotia were in such schools, as well as between 80 and 90 percent of the students in Chinese Taipei, France, Georgia, Italy, Moldova, Norway, Romania, the Slovak Republic, and Trinidad and Tobago. In contrast, there were some countries with substantial percentages of students in schools where the PIRLS assessment language was not the first language for the majority of students-notably Indonesia (54\%), Iran (36\%), Morocco (31\%), and Singapore (45\%). However, many countries had just a small percentage of students in such schools. Compared to 2001, Israel and Latvia had increased percentages of students in 2006 in schools where the PIRLs language was not the first language for the majority of students, and Hong Kong SAR, Macedonia, and the Slovak Republic had decreased percentages.

Perhaps not surprisingly, on average internationally, reading achievement was highest for students in schools where only a few schoolmates did not speak the PIRLs language as a first language (504 points), and was progressively lower across categories of schools with greater percentages of such students. This pattern was relatively consistent across countries, although there were several exceptions. For students in schools where the majority of students did not speak the PIRLs language as a first language, average reading achievement was 471 points, a difference of 33 points lower than the highest achieving group. 
Exhibit 7.1 Principals' Reports on Their Schools' Locations with Trends

PIRLS 2006
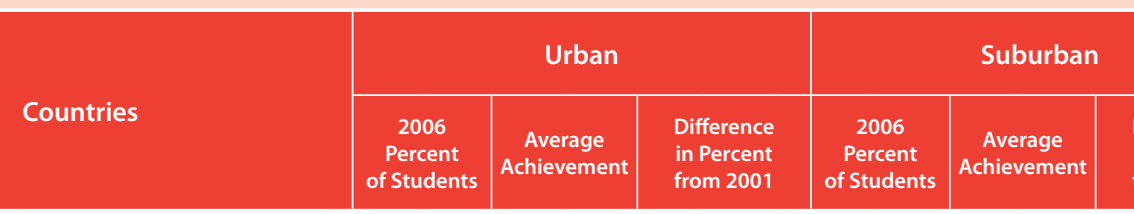

4th Grade

\section{Austria}

$31(3.4)$

$529(4.4)$

from 2001

of Students

\begin{tabular}{|l|l|l|l|}
\hline Belgium (Flemish) & $21(3.6)$ & $541(5.4)$ & $\Delta \diamond$ \\
\hline
\end{tabular}

Belgium (French)

Bulgaria

Canada, Alberta

$47(4.0)$

$494(5.5)$

$37(4.7)$

$21(3.9)$

$70(3.0)$

Canada, British Columbia

$46(4.5)$

$557(5.2)$

00

$21(3.9)$

$542(5.5)$

$559(3.8)$

$6(4.1)$

$5(1.7)$

$546(3.7)$

$496(5.7)$

$550(10.8)$

$26(3.5)$
$46(4.7)$

Canada, Nova Scotia

Canada, Ontario

$38(4.3)$

$555(4.3)$

$\Delta 0$

Canada, Quebec

$51(4.9)$

$542(6.5)$

$\Delta \diamond \quad 26(3.2)$

572 (4.4)

$36(5.0)-551(3.9)$

Difference
in Percent
from 2001

Chinese Taipei

Denmark

England

51 (4.8)

$549(4.1) \quad 8(6.9)$

$36(5.0)$

$563(4.3)$

$\Delta \Delta$

$15(6.7)$

$33(4.1)$

France

r $\quad 45(4.2)$

- - - -

Georgia

Germany

Hong Kong SAR

$545(3.4)$

$\Delta \diamond$

$-130$

Hungary

Iceland

$34(4.0)$

$523(5.3) \quad-2(6.5)$

$30(3.4)$

$538(6.5)$

$522(4.3)$

$-2(6.5)$
$-2(5.7)$

$35(3.9)$

555 (3.9)

$486(4.5)$

$\diamond \diamond$

$25(3.9)$

$553(5.1)$

$518(6.3)$

$15(2.7)$

465 (8.1)

$37(3.3)$

535 (4.6)

4 (4.4)

19 (3.1)

557 (3.9)

$6(5.3)$

$37(4.1)$

$555(4.5)$

$40(2.5)$

$557(5.0)$

$28(2.2)$

$565(6.6)$

$0(3.3)$

$-3(0.5) \odot \quad 37(0.3)$

Indonesia

$12(2.2)$

$518(2.0)$

$509(2.3)$

$509(2.3)$

$14(2.7)$

$425(9.6) \quad \diamond \Delta$

$\begin{array}{ll}451(9.7) & \diamond \diamond \\ 454(4.1) & 1(4.7)\end{array}$

$18(2.8)$

$415(10.3)$
$529(13.1)$

$15(2.8)$

$-4(4.3)$

$61(4.0)$

$555(9.2)$

\begin{tabular}{l}
$554(2.9) \quad-6(4.8)$ \\
\hline
\end{tabular}

Kuwait

$26(3.6)$

$355(7.6)$

Latvia

$70(0.7)$

\begin{tabular}{l|l|}
$548(2.4)$ & $26(3.9)$ \\
\hline
\end{tabular}

${ }^{1}$ Luxembourg

72 (2.3)

$544(1.9)$

$1(3.6)$

$3(1.5)$

$321(5.8)$

1 (3.8)

$1(3.8)$

$3(1.4)$

$528(6.6) \quad-15(4.2) \quad$ ○

\begin{tabular}{c|c|c|}
\multicolumn{3}{c}{ Rural } \\
$\begin{array}{c}\text { Percent } \\
\text { Peo6 } \\
\text { of Students }\end{array}$ & $\begin{array}{c}\text { Average } \\
\text { Achievement }\end{array}$ & \\
\hline
\end{tabular}

\begin{tabular}{|l|l|l}
\hline Moldova, Rep. of & & \\
\hline
\end{tabular}

Morocco r $37(3.3)$

Netherlands

Norway

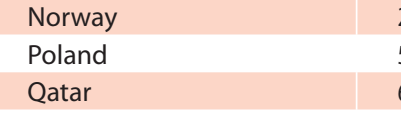

\section{Rom}

Russian Federation

$\begin{array}{cc}-- & -- \\ 477(6.9) & -6(4.9)\end{array}$

$--$

$549(10.7) \quad-3(2.5)$

$48(3.7)$

$42(4.8)$

$33(3.8)$

$543(2.5)$

$551(2.4)$

$512(4.2)$

$24(2.5)$

$516(10.8)$

$28(3.5) \quad 550(4.1) \quad \Delta \diamond$

\begin{tabular}{l|l|l}
\hline $17(3.2)$ & $545(6.2) \quad \diamond \diamond$ \\
\hline $50(3.5)$ & $537(2.6)$ & $\diamond \diamond$
\end{tabular}

$\begin{array}{lll}50(3.5) & 537(2.6) & \diamond \diamond \\ 14(3.2) & 552(4.9) & -9(5.1)\end{array}$

\begin{tabular}{lll|r|r}
\hline$(7.0)$ & & $14(3.2)$ & $552(4.9)$ & $-9(5.1)$ \\
\hline$(6.3)$ & $\quad$ & $20(3.6)$ & $528(5.6)$ & $3(5.0)$
\end{tabular}

$\begin{array}{ccccc}-- & -- & -- & -- \\ \diamond \diamond & 37(4.0) & 542(4.4) & \Delta \diamond\end{array}$

\begin{tabular}{|l|l|l|l|}
\hline $4(6.0)$ & $19(3.7)$ & $564(5.2)$ & $-1(5.2)$ \\
\hline
\end{tabular}

$\Delta \diamond$

$-5(5.7) \quad 41(3.9) \quad 524(2.5) \quad 6(5.3)$

\begin{tabular}{ll|l}
$43(2.6)$ & $459(5.1)$ & 0
\end{tabular}

$-3(4.3) \quad 44(4.0) \quad 555(2.3) \quad-1(5.5)$

\begin{tabular}{l|l|l|r|}
$-9(4.9)$ & $5(1.9)$ & $540(11.0)$ & $3(2.2)$
\end{tabular}

$31(1.8)$

528 (4.7)

$3(2.2)$
$-5(2.5)$

1 (3.6)

$\begin{array}{lll}477(6.9) & -6(4.9) & 18(3.5)\end{array}$

$--$

$\begin{array}{cc}-- & -- \\ 443(13.8) & 3(4.5)\end{array}$

\begin{tabular}{l|l|r|}
\hline $6(2.4)$ & $498(17.6)$ & $-8(4.5)$ \\
\hline
\end{tabular}

$18(3.6) \quad 334(15.8) \quad-3(5.7)$

$30(0.4)$

$74(2.9)$

$506(2.3)$

$9(0.5)$

$9(0.5)$

\begin{tabular}{|l|l|l|}
\hline $35(2.8)$ & $376(5.7)$ & $-8(4.2)$ \\
\hline
\end{tabular}

$33(3.8) \quad 472(10.2) \quad 7(5.0)$

\begin{tabular}{l|l|l|}
$15(3.1)$ & $533(9.9)$ & $5(3.8)$ \\
\hline
\end{tabular}

$14(3.0) \quad 311(12.1) \quad \Delta 0$

\begin{tabular}{l|l|l|}
\hline $27(1.7)$ & $525(5.9)$ & $-10(3.4) \quad \bigcirc$
\end{tabular}

$37(3.3)$

$363(7.2) \quad 2(4.1)$

$363(7.2) \quad-3(5.5)$

$18(3.6)$

$26(2.1)$

26

$\begin{array}{ccc}26(2.1) & 516(3.4) & 3(3.2) \\ -- & -- & -\end{array}$

\begin{tabular}{|c|c|c|}
\hline Russian Federation & & $63(2.0)$ \\
\hline Scotland & $r$ & $32(3.5)$ \\
\hline Singapore & & $100(0.0)$ \\
\hline
\end{tabular}

Slovak Republic $\quad 52(3.0)$

Slovenia $36(4.2)$

South Africa

Spain

Sweden

$17(1.8)$

\begin{tabular}{l}
$538(4.1) \quad-5(5.6)$ \\
\hline
\end{tabular}

$33(4.7)$

$553(3.2) \quad-10(6.0)$

$\begin{array}{lll}31(2.6) & 401(9.5) \quad 3(4.1)\end{array}$

\begin{tabular}{l|l|l|}
\hline $65(2.5)$ & $492(3.9)$ & $6(4.5)$ \\
\hline
\end{tabular}

$26(4.0)$

$536(3.3) \quad 3(5.1)$

$39(3.0)$

$553(3.2)$

$10(6.0)$
$-1(4.7)$

$45(3.7)$

$6(13.5)-6(4.5)$

20 (3.6)

\begin{tabular}{|l|l|l|}
\hline $502(3.8)$ & $1(5.0)$ & $30(3.9)$ \\
\hline
\end{tabular}

\begin{tabular}{rr|}
$527(3.6)$ & $-1(4.7)$ \\
\hline $504(3.9)$ & $4(5.5)$ \\
\hline
\end{tabular}

$41(3.5)$

$\begin{array}{r}-5(5.5) \\ \hline 5(2.5)\end{array}-5(5.2)$

\begin{tabular}{l|l|l|}
$41(3.5)$ & $547(2.5)$ & $-5(5.2)$ \\
\hline $21(2.3)$ & $535(5.3)$ & $-2(3.7)$
\end{tabular}

Trinidad and Tobago

United States

\begin{tabular}{|c|c|c|c|c|c|c|c|c|c|c|}
\hline $17(1.8)$ & 350 (19.5) & $\Delta \Delta$ & & $21(2.2)$ & $381(14.9)$ & $\Delta \Delta$ & & $62(2.0)$ & $261(3.8)$ & 00 \\
\hline $58(4.3)$ & 524 (3.4) & $\Delta 0$ & & $20(3.3)$ & $497(6.8)$ & $\Delta \Delta$ & & $21(3.4)$ & $498(7.1)$ & $\Delta 0$ \\
\hline $27(4.1)$ & $549(3.8)$ & $12(5.1)$ & 0 & $55(4.1)$ & $549(3.4)$ & $-12(5.6)$ & ( ) & $18(2.8)$ & $550(4.7)$ & $0(4.5)$ \\
\hline $19(2.5)$ & 470 (13.0) & $\Delta \Delta$ & & $50(3.7)$ & $441(7.0)$ & $\Delta \Delta$ & & $32(3.0)$ & $408(8.9)$ & $\Delta \theta$ \\
\hline
\end{tabular}

\begin{tabular}{|c|c|c|c|c|c|c|c|}
$502(3.8)$ & $1(5.0)$ & $30(3.9)$ & $504(3.9)$ & $4(5.5)$ & $50(4.2)$ & $492(4.2)$ & $-6(5.5)$ \\
\hline $528(2.9)$ & $\diamond \diamond$ & $5(1.7)$ & $529(11.0)$ & $\diamond \diamond$ & $43(1.9)$ & $508(3.8)$ & $\diamond \diamond$ \\
\hline
\end{tabular}

\begin{tabular}{|c|c|c|c|c|c|c|c|}
$502(3.8)$ & $1(5.0)$ & $30(3.9)$ & $504(3.9)$ & $4(5.5)$ & $50(4.2)$ & $492(4.2)$ & $-6(5.5)$ \\
\hline $528(2.9)$ & $\diamond \diamond$ & $5(1.7)$ & $529(11.0)$ & $\diamond \diamond$ & $43(1.9)$ & $508(3.8)$ & $\diamond \diamond$ \\
\hline
\end{tabular}

$535(5.3)$

$32(0.2)$

$336(2.0) \quad \Delta \diamond \quad-3(0.0)$

\begin{tabular}{rrrr}
$581(3.4)$ & $8(3.2)$ & 0 & $6(1.3)$ \\
\hline
\end{tabular}

\begin{tabular}{|c|c|c|c|c|c|c|c|c|}
\hline $2(1.5)$ & $\Delta \Delta$ & & $32(0.2)$ & $336(2.0)$ & $\Delta \Delta$ & $3(0.0)$ & $318(8.4)$ & 00 \\
\hline ( & $-3(3.4)$ & & $5(1.9)$ & $498(14.8)$ & $1(2.8)$ & $48(2.4)$ & $462(8.0)$ & $2(3.6)$ \\
\hline (3.4) & $8(3.2)$ & 0 & $6(1.3)$ & $563(8.8)$ & $4(2.3)$ & $31(2.2)$ & $532(6.1)$ & $-12(3.3)$ \\
\hline 4.8) & $-2(5.6)$ & & $36(4.3)$ & $539(5.3)$ & $-3(6.9)$ & $32(3.9)$ & $528(6.8)$ & $6(5.9)$ \\
\hline
\end{tabular}

\begin{tabular}{|c|c|c|c|c|c|c|c|c|}
\hline $362(1.5)$ & $\Delta 0$ & & $32(0.2)$ & $336(2.0)$ & $\Delta \Delta$ & $3(0.0)$ & $318(8.4)$ & $\Delta 0$ \\
\hline $515(6.5)$ & $-3(3.4)$ & & $5(1.9)$ & 498 (14.8) & $1(2.8)$ & $48(2.4)$ & $462(8.0)$ & $2(3.6)$ \\
\hline $581(3.4)$ & $8(3.2)$ & 0 & $6(1.3)$ & $563(8.8)$ & $4(2.3)$ & $31(2.2)$ & $532(6.1)$ & $-12(3.3)$ \\
\hline $517(4.8)$ & $-2(5.6)$ & & $36(4.3)$ & $539(5.3)$ & $-3(6.9)$ & $32(3.9)$ & $528(6.8)$ & $6(5.9)$ \\
\hline
\end{tabular}

\begin{tabular}{|c|c|c|c|c|c|c|c|c|}
\hline $362(1.5)$ & $\Delta 0$ & & $32(0.2)$ & $336(2.0)$ & $\Delta \Delta$ & $3(0.0)$ & $318(8.4)$ & $\Delta 0$ \\
\hline $515(6.5)$ & $-3(3.4)$ & & $5(1.9)$ & 498 (14.8) & $1(2.8)$ & $48(2.4)$ & $462(8.0)$ & $2(3.6)$ \\
\hline $581(3.4)$ & $8(3.2)$ & 0 & $6(1.3)$ & $563(8.8)$ & $4(2.3)$ & $31(2.2)$ & $532(6.1)$ & $-12(3.3)$ \\
\hline $517(4.8)$ & $-2(5.6)$ & & $36(4.3)$ & $539(5.3)$ & $-3(6.9)$ & $32(3.9)$ & $528(6.8)$ & $6(5.9)$ \\
\hline
\end{tabular}

\begin{tabular}{|c|c|c|c|c|c|c|c|c|}
\hline (2.9) & $\Delta \diamond$ & & $5(1.7)$ & $529(11.0)$ & 0 & $43(1.9)$ & $508(3.8)$ & $\diamond \diamond$ \\
\hline $52(1.5)$ & 00 & & $32(0.2)$ & $336(2.0)$ & $\Delta 0$ & $3(0.0)$ & $318(8.4)$ & $\Delta \Delta$ \\
\hline (6.5) & $-3(3.4)$ & & $5(1.9)$ & $498(14.8)$ & $1(2.8)$ & $48(2.4)$ & $462(8.0)$ & $2(3.6)$ \\
\hline (3.4) & $8(3.2)$ & 0 & $6(1.3)$ & $563(8.8)$ & $4(2.3)$ & $31(2.2)$ & $532(6.1)$ & $-12(3.3)$ \\
\hline (4.8) & $-2(5.6)$ & & $36(4.3)$ & $539(5.3)$ & $-3(6.9)$ & $32(3.9)$ & $528(6.8)$ & $6(5.9)$ \\
\hline
\end{tabular}

\begin{tabular}{|c|c|c|c|c|c|c|c|c|}
\hline $581(3.4)$ & $8(3.2)$ & 0 & $6(1.3)$ & $563(8.8)$ & $4(2.3)$ & $31(2.2)$ & $532(6.1)$ & $-12(3.3)$ \\
\hline $517(4.8)$ & $-2(5.6)$ & & $36(4.3)$ & $539(5.3)$ & $-3(6.9)$ & $32(3.9)$ & $528(6.8)$ & $6(5.9)$ \\
\hline $558(2.9)$ & $0(0.0)$ & & $0(0.0)$ & $\sim \sim$ & $0(0.0)$ & $0(0.0)$ & $\sim \sim$ & $0(0.0)$ \\
\hline $544(2.9)$ & $2(4.8)$ & & $8(2.5)$ & $537(8.0)$ & $-2(3.5)$ & $40(3.3)$ & $512(5.9)$ & $0(4.5)$ \\
\hline $529(3.6)$ & $-4(5.3)$ & & $37(4.0)$ & $520(3.1)$ & $10(5.5)$ & $27(3.7)$ & $512(3.6)$ & $-6(4.7)$ \\
\hline 350 (19.5) & $\Delta 0$ & & $21(2.2)$ & $381(14.9)$ & $\Delta 0$ & $62(2.0)$ & $261(3.8)$ & $\Delta 0$ \\
\hline
\end{tabular}

\begin{tabular}{|l|l|l|l|l|}
\hline $529(3.6)$ & $-4(5.3)$ & $37(4.0)$ & $520(3.1)$ & $10(5.5)$ \\
\hline $350(19.5)$ & $\diamond \diamond$ & $21(2.2)$ & $381(14.9)$ & $\diamond \diamond$ \\
\hline
\end{tabular}

\begin{tabular}{|c|c|c|c|c|c|c|c|}
\hline 9 (3.6) & $-4(5.3)$ & $37(4.0)$ & $520(3.1)$ & $10(5.5)$ & $27(3.7)$ & $512(3.6)$ & $-6(4.7)$ \\
\hline 19.5) & $\Delta \Delta$ & $21(2.2)$ & $381(14.9)$ & $\Delta \Delta$ & $62(2.0)$ & $261(3.8)$ & $\Delta \Delta$ \\
\hline 3.4) & $\Delta \Delta$ & $20(3.3)$ & $497(6.8)$ & $\Delta \Delta$ & $21(3.4)$ & $498(7.1)$ & 00 \\
\hline (3.8) & $12(5.1)$ & $55(4.1)$ & 549 (3.4) & $-12(5.6)$ & $18(2.8)$ & $550(4.7)$ & 0 \\
\hline
\end{tabular}

\begin{tabular}{|c|c|c|c|c|c|c|c|c|}
\hline (4.2) & $529(3.6)$ & $-4(5.3)$ & $37(4.0)$ & $520(3.1)$ & $10(5.5)$ & 27 (3.7) & $512(3.6)$ & $-6(4.7)$ \\
\hline (1.8) & $350(19.5)$ & $\Delta \Delta$ & $21(2.2)$ & $381(14.9)$ & $\Delta \Delta$ & $62(2.0)$ & $261(3.8)$ & $\Delta \diamond$ \\
\hline (4.3) & $524(3.4)$ & $\Delta \Delta$ & $20(3.3)$ & $497(6.8)$ & $\Delta 0$ & $21(3.4)$ & $498(7.1)$ & $\Delta 0$ \\
\hline 4.1) & $549(3.8)$ & $12(5.1)$ & $55(4.1)$ & $549(3.4)$ & $-12(5.6)$ & $18(2.8)$ & $550(4.7)$ & $0(4.5)$ \\
\hline 2.5) & $470(13.0)$ & $\Delta \Delta$ & $50(3.7)$ & $441(7.0)$ & $\Delta \Delta$ & $32(3.0)$ & $408(8.9)$ & $\Delta \Delta$ \\
\hline
\end{tabular}

\begin{tabular}{|c|c|c|c|}
\hline $27(4.1)$ & $549(3.8)$ & $12(5.1)$ & 55 (4.1) \\
\hline $19(2.5)$ & $470(13.0)$ & $\Delta 0$ & $50(3.7)$ \\
\hline 28 (3.5) & $524(4.4)$ & $-5(4.9)$ & $47(3.9)$ \\
\hline
\end{tabular}

$\begin{array}{ll}441(7.0) & 00 \\ 550(3.2) & 13(6.1)\end{array}$

\begin{tabular}{|l|l|l|}
\hline $13(6.1) \quad \bullet \quad 25(2.7)$ & $539(9.1)$ \\
\hline & $33(0.5)$ & $483(1.1)$ \\
\hline
\end{tabular}

International Avg.

$43(0.5) \quad 508(1.0)$

$24(0.5) \quad 501(1.4)$
Percent in 2006 significantly higher
Percent in 2006 significantly lower

Background data provided by schools.

() Standard errors appear in parentheses. Because results are rounded to the nearest whole number, some totals may appear inconsistent.

An " $r$ " indicates data are available for $70-84 \%$ of the students. An "s" indicates data are available for $50-69 \%$ of the students. An " $x$ " indicates data are available for less than

A dash (-) indicates comparable data are not available. A tilde $(\sim)$ indicates insufficient

data to report achievement.

A diamond $(\diamond)$ indicates the country did not participate in the 2001 assessment.
NOTE: The International Average does not include the results from the Canadian provinces. Trend Note: The primary education systems of the Russian Federation and Slovenia Trend Note: The primary education systems of the Russian Federation and Slovenia
underwent structural changes. Data for Canada, Ontario include only public schools.
1 Primary schools in Luxembourg do not have principals. 
Exhibit 7.2 Principals' Reports on Their Primary Grade Students Coming from Economically

PIRLS 2006 Disadvantaged Homes with Trends

4th Grade

\begin{tabular}{|c|c|c|c|c|c|c|c|c|c|}
\hline \multirow{2}{*}{ Countries } & \multicolumn{5}{|c|}{$\begin{array}{l}\text { 0-10\% Economically } \\
\text { Disadvantaged }\end{array}$} & \multicolumn{4}{|c|}{$\begin{array}{c}11-25 \% \text { Economically } \\
\text { Disadvantaged }\end{array}$} \\
\hline & & $\begin{array}{c}2006 \\
\text { Percent } \\
\text { of Students }\end{array}$ & $\begin{array}{c}\text { Average } \\
\text { Achievement }\end{array}$ & $\begin{array}{l}\text { Differenc } \\
\text { in Percen } \\
\text { from } 200\end{array}$ & & $\begin{array}{c}2006 \\
\text { Percent } \\
\text { of Students }\end{array}$ & $\begin{array}{c}\text { Average } \\
\text { Achievement }\end{array}$ & $\begin{array}{l}\text { Difference } \\
\text { in Percent } \\
\text { from } 2001\end{array}$ & \\
\hline Austria & & $56(4.1)$ & $543(3.0)$ & $\Delta \Delta$ & & $28(4.1)$ & $536(4.2)$ & $\Delta \diamond$ & \\
\hline Belgium (Flemish) & & $64(4.8)$ & $552(2.0)$ & $\Delta \Delta$ & & $23(4.4)$ & $544(4.1)$ & $\Delta \Delta$ & \\
\hline Belgium (French) & $r$ & $46(4.7)$ & $517(4.0)$ & $\Delta \Delta$ & & $23(3.7)$ & $506(5.1)$ & $\Delta \Delta$ & \\
\hline Bulgaria & & $28(4.1)$ & $576(5.4)$ & $10(4.7)$ & $\theta$ & $29(3.8)$ & $564(6.9)$ & $6(4.8)$ & \\
\hline Canada, Alberta & & $57(3.8)$ & $568(3.3)$ & $\Delta \Delta$ & & $28(3.7)$ & $557(3.4)$ & $\Delta \Delta$ & \\
\hline Canada, British Columbia & & $50(4.2)$ & $572(3.0)$ & $\Delta \Delta$ & & $25(3.7)$ & $557(4.5)$ & $\Delta \Delta$ & \\
\hline Canada, Nova Scotia & & $38(3.6)$ & $551(3.1)$ & $\Delta \diamond$ & & $32(3.6)$ & $542(3.7)$ & $\Delta \Delta$ & \\
\hline Canada, Ontario & & $47(5.0)$ & $564(4.2)$ & $-9(7.1)$ & & $22(4.5)$ & $551(5.7)$ & $-2(6.2)$ & \\
\hline Canada, Quebec & & $40(4.0)$ & $548(4.6)$ & $7(5.7)$ & & $28(4.1)$ & $532(6.2)$ & $-8(6.1)$ & \\
\hline Chinese Taipei & & $61(4.2)$ & $539(2.6)$ & $\Delta \Delta$ & & $29(3.9)$ & $532(3.5)$ & $\Delta 0$ & \\
\hline Denmark & & $59(4.6)$ & $551(3.1)$ & $\Delta \Delta$ & & $31(4.2)$ & $546(4.4)$ & $\Delta \Delta$ & \\
\hline England & $r$ & $47(4.0)$ & $573(3.5)$ & $13(5.7)$ & 0 & $14(3.2)$ & $534(5.0)$ & $-21(5.5)$ & ( \\
\hline France & & $45(4.3)$ & $536(2.5)$ & $-9(5.8)$ & & $30(3.8)$ & $527(2.8)$ & $7(5.4)$ & \\
\hline Georgia & & $21(3.1)$ & $484(7.3)$ & $\Delta \Delta$ & & $26(3.7)$ & $474(9.6)$ & $\Delta \Delta$ & \\
\hline Germany & $r$ & $43(3.5)$ & $558(2.6)$ & $-3(5.3)$ & & $38(3.6)$ & $550(4.2)$ & $1(5.4)$ & \\
\hline Hong Kong SAR & & $40(4.2)$ & $574(2.4)$ & $13(5.5)$ & $\theta$ & $26(3.4)$ & $559(5.7)$ & $7(4.9)$ & \\
\hline Hungary & & $18(3.0)$ & $574(4.8)$ & $-5(4.6)$ & & $35(4.3)$ & $563(4.8)$ & $-6(5.8)$ & \\
\hline Iceland & $r$ & $84(0.3)$ & $513(1.5)$ & $-5(0.4)$ & 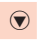 & $13(0.3)$ & $499(3.1)$ & $4(0.3)$ & $\theta$ \\
\hline Indonesia & & $7(1.9)$ & $425(13.3)$ & $\Delta \diamond$ & & $13(3.2)$ & $437(9.7)$ & $\Delta \diamond$ & \\
\hline Iran, Islamic Rep. of & & $24(3.0)$ & $481(5.1)$ & $-1(5.0)$ & & $20(3.3)$ & $422(8.2)$ & $10(4.3)$ & $\theta$ \\
\hline Israel & & $20(3.1)$ & $546(10.9)$ & $-3(4.7)$ & & $31(3.8)$ & $537(7.4)$ & $2(5.5)$ & \\
\hline Italy & & $50(3.8)$ & $556(3.3)$ & $-6(5.2)$ & & $28(3.5)$ & $551(5.7)$ & $-2(4.7)$ & \\
\hline Kuwait & & $68(4.2)$ & $333(4.9)$ & $\Delta \diamond$ & & $17(3.2)$ & $314(11.4)$ & $\Delta \Delta$ & \\
\hline Latvia & & $34(3.6)$ & $555(4.5)$ & $22(4.3)$ & $\theta$ & $42(4.0)$ & $535(3.3)$ & $-7(5.4)$ & \\
\hline Lithuania & & $31(3.4)$ & $554(3.0)$ & $15(4.6)$ & $\theta$ & $33(3.9)$ & $532(3.1)$ & $-3(5.7)$ & \\
\hline${ }^{1}$ Luxembourg & & -- & -- & -- & & -- & -- & -- & \\
\hline Macedonia, Rep. of & $r$ & $13(3.0)$ & $489(13.7)$ & $0(4.0)$ & & $28(4.3)$ & $470(11.2)$ & $10(5.4)$ & \\
\hline Moldova, Rep. of & & $25(3.9)$ & $506(7.5)$ & $9(4.8)$ & & $37(4.1)$ & $502(5.1)$ & $11(5.8)$ & $\theta$ \\
\hline Morocco & $r$ & $14(3.4)$ & $348(21.4)$ & $5(4.5)$ & & $15(3.9)$ & $351(16.9)$ & $8(4.4)$ & \\
\hline Netherlands & $r$ & $55(4.3)$ & $558(1.8)$ & $-4(6.3)$ & & $24(4.0)$ & $541(3.5)$ & $-1(5.7)$ & \\
\hline New Zealand & & $51(2.9)$ & $557(3.0)$ & $5(4.5)$ & & $19(2.6)$ & $526(5.6)$ & $-5(4.6)$ & \\
\hline Norway & $r$ & $84(3.7)$ & $498(3.0)$ & $-1(5.4)$ & & $15(3.8)$ & $490(6.7)$ & $3(5.2)$ & \\
\hline Poland & & $16(3.2)$ & $528(6.4)$ & $\Delta \Delta$ & & $41(4.4)$ & $524(3.8)$ & $\Delta \Delta$ & \\
\hline Qatar & & $19(0.2)$ & $367(3.1)$ & $\Delta \Delta$ & & $27(0.2)$ & $348(2.6)$ & $\Delta \Delta$ & \\
\hline Romania & & $12(3.0)$ & $521(11.4)$ & $6(3.5)$ & & $24(3.7)$ & $510(6.9)$ & $7(5.0)$ & \\
\hline Russian Federation & & $33(3.5)$ & $585(5.1)$ & $10(4.5)$ & $\theta$ & $30(3.2)$ & $566(5.8)$ & $2(4.9)$ & \\
\hline Scotland & $r$ & $46(4.4)$ & $547(5.0)$ & $8(6.0)$ & & $21(4.3)$ & $533(3.6)$ & $-4(6.3)$ & \\
\hline Singapore & & $63(0.0)$ & $568(3.8)$ & $-3(3.7)$ & & $29(0.0)$ & $547(5.9)$ & $8(3.0)$ & $\theta$ \\
\hline Slovak Republic & & $38(4.0)$ & $548(3.5)$ & $20(5.2)$ & 0 & $29(3.8)$ & $533(4.6)$ & $0(5.3)$ & \\
\hline Slovenia & & $30(3.8)$ & $524(4.4)$ & $-5(5.7)$ & & $44(3.7)$ & $522(2.7)$ & $-6(5.9)$ & \\
\hline South Africa & & $9(1.6)$ & $498(23.4)$ & $\Delta \Delta$ & & $5(1.4)$ & $377(47.0)$ & $\Delta \Delta$ & \\
\hline Spain & & $72(3.8)$ & $520(3.2)$ & $\Delta \Delta$ & & $16(3.3)$ & $505(5.0)$ & $\Delta \Delta$ & \\
\hline Sweden & & $51(4.8)$ & $555(2.9)$ & $5(6.5)$ & & $33(4.6)$ & $545(3.0)$ & $0(6.2)$ & \\
\hline Trinidad and Tobago & & $18(2.9)$ & $491(10.9)$ & $\Delta \Delta$ & & $21(3.6)$ & $444(13.3)$ & $\Delta \Delta$ & \\
\hline United States & & $26(3.9)$ & $566(4.2)$ & $1(5.4)$ & & $12(2.5)$ & $559(5.8)$ & $-8(5.3)$ & \\
\hline International Avg. & & $39(0.6)$ & $521(1.2)$ & & & $26(0.6)$ & $504(1.6)$ & & \\
\hline
\end{tabular}

Background data provided by schools.

() Standard errors appear in parentheses. Because results are rounded to the nearest whole number, some totals may appear inconsistent.

$A n$ " $r$ " indicates data are available for $70-84 \%$ of the students. An " $s$ " indicates data are available for $50-69 \%$ of the students. An " $x$ " indicates data are available for less than $50 \%$ of the students.

A dash (-) indicates comparable data are not available. A tilde ( ) indicates insufficient data to report achievement.
A diamond $(\diamond)$ indicates the country did not participate in the 2001 assessment.

NOTE: The International Average does not include the results from the Canadian provinces. Trend Note: The primary education systems of the Russian Federation and Slovenia underwent structural changes. Data for Canada, Ontario include only public schools. 1 Primary schools in Luxembourg do not have principals. 


\begin{tabular}{|c|c|c|c|c|c|c|c|c|c|}
\hline $\begin{array}{l}\text { Princip } \\
\text { Disadv }\end{array}$ & & $\begin{array}{l}\text { Repor } \\
\text { aged H }\end{array}$ & $\begin{array}{l}\text { s on Thei } \\
\text { omes wit }\end{array}$ & $\begin{array}{l}\text { Primary } \\
\text { Trends }\end{array}$ & & $\begin{array}{l}\text { rade Stud } \\
\text { ontinued }\end{array}$ & dents Co & ing fr & \\
\hline \multirow{2}{*}{ Countries } & & \multicolumn{4}{|c|}{$\begin{array}{l}26-50 \% \text { Economically } \\
\text { Disadvantaged }\end{array}$} & \multicolumn{4}{|c|}{$\begin{array}{c}\text { More than } 50 \% \text { Economically } \\
\text { Disadvantaged }\end{array}$} \\
\hline & \multirow{2}{*}{\multicolumn{2}{|c|}{$\begin{array}{c}2006 \\
\begin{array}{c}\text { Percent } \\
\text { of Students }\end{array} \\
12(2.8)\end{array}$}} & \multirow{2}{*}{$\begin{array}{c}\begin{array}{c}\text { Average } \\
\text { Achievement }\end{array} \\
537(7.2)\end{array}$} & \multicolumn{2}{|c|}{$\begin{array}{l}\text { Difference } \\
\text { in Percent } \\
\text { from } 2001\end{array}$} & \multirow{2}{*}{\begin{tabular}{|c|}
2006 \\
$\begin{array}{c}\text { Percent } \\
\text { of Students }\end{array}$ \\
$4(1.3)$
\end{tabular}} & \multirow{2}{*}{$\begin{array}{c}\text { Average } \\
\text { Achievement }\end{array}$} & \multicolumn{2}{|c|}{$\begin{array}{l}\text { Difference } \\
\text { in Percent } \\
\text { from } 2001\end{array}$} \\
\hline Austria & & & & $\Delta \Delta$ & & & & $\Delta \diamond$ & \\
\hline Belgium (Flemish) & & $11(2.7)$ & $533(7.5)$ & $\Delta \Delta$ & & $2(1.3)$ & $\sim \sim$ & $\Delta \Delta$ & \\
\hline Belgium (French) & $r$ & $20(3.5)$ & $480(6.5)$ & $\Delta \Delta$ & & $11(2.8)$ & $453(7.3)$ & $\Delta \diamond$ & \\
\hline Bulgaria & & $15(3.0)$ & $509(11.1)$ & $-14(4.6)$ & $(-)$ & $29(3.4)$ & $522(10.1)$ & $-2(4.9)$ & \\
\hline Canada, Alberta & & $11(2.6)$ & $545(6.6)$ & $\Delta \diamond$ & & $4(1.3)$ & $516(14.9)$ & $\Delta \Delta$ & \\
\hline Canada, British Columbia & & $19(3.3)$ & $533(5.2)$ & $\Delta \Delta$ & & $6(1.9)$ & $530(8.2)$ & $\Delta \Delta$ & \\
\hline Canada, Nova Scotia & & $22(3.0)$ & $531(4.0)$ & $\Delta \diamond$ & & $7(2.1)$ & $527(7.8)$ & $\Delta \Delta$ & \\
\hline Canada, Ontario & & $16(3.7)$ & $542(4.9)$ & $7(4.5)$ & & $15(3.9)$ & $538(6.5)$ & $3(5.0)$ & \\
\hline Canada, Quebec & & $20(4.2)$ & $522(4.8)$ & $0(5.5)$ & & $12(3.1)$ & $512(6.0)$ & $2(4.6)$ & \\
\hline Chinese Taipei & & $7(2.2)$ & $515(3.7)$ & $\Delta \Delta$ & & $2(1.0)$ & $\sim \sim$ & $\Delta \Delta$ & \\
\hline Denmark & & $8(2.1)$ & $526(9.6)$ & $\Delta \Delta$ & & $2(1.2)$ & $\sim \sim$ & $\Delta \diamond$ & \\
\hline England & $r$ & $21(3.7)$ & $511(6.5)$ & $3(5.1)$ & & $18(3.5)$ & $501(7.4)$ & $5(4.6)$ & \\
\hline France & & $9(3.0)$ & $496(5.0)$ & $-4(4.5)$ & & $16(2.9)$ & $485(5.6)$ & $7(3.7)$ & \\
\hline Georgia & & $24(3.8)$ & $467(7.7)$ & $\Delta \diamond$ & & $28(3.9)$ & $462(4.9)$ & $\Delta \diamond$ & \\
\hline Germany & $r$ & $12(2.3)$ & $540(3.5)$ & $-1(3.7)$ & & $7(1.3)$ & $465(10.2)$ & $3(2.2)$ & \\
\hline Hong Kong SAR & & $18(3.5)$ & $559(7.5)$ & $-9(5.8)$ & & $15(3.6)$ & $550(5.0)$ & $-10(5.3)$ & \\
\hline Hungary & & $30(4.0)$ & $544(4.9)$ & $1(5.3)$ & & $17(3.5)$ & $518(9.9)$ & $10(3.9)$ & $\theta$ \\
\hline Iceland & $r$ & $2(0.1)$ & $\sim \sim$ & $0(0.1)$ & & $1(0.1)$ & $\sim \sim$ & $1(0.1)$ & \\
\hline Indonesia & & $18(3.3)$ & $413(8.9)$ & $\Delta \Delta$ & & $62(3.9)$ & $393(4.9)$ & $\Delta \Delta$ & \\
\hline Iran, Islamic Rep. of & & $15(2.6)$ & $412(6.6)$ & $-7(4.4)$ & & $41(4.0)$ & $390(6.0)$ & $-2(5.9)$ & \\
\hline Israel & & $26(3.9)$ & $513(9.7)$ & $-5(5.3)$ & & $23(3.1)$ & $449(10.5)$ & $6(4.5)$ & \\
\hline Italy & & $14(2.9)$ & $537(9.3)$ & $2(3.7)$ & & $8(1.7)$ & $546(14.0)$ & $5(2.0)$ & $\theta$ \\
\hline Kuwait & & $11(2.7)$ & $304(20.5)$ & $\Delta \Delta$ & & $4(1.7)$ & $318(19.5)$ & $\Delta \Delta$ & \\
\hline Latvia & & $17(3.3)$ & $535(4.9)$ & $-6(4.8)$ & & $7(2.1)$ & $521(8.5)$ & $-9(3.4)$ & ( \\
\hline Lithuania & & $25(3.3)$ & $529(3.7)$ & $-7(5.0)$ & & $11(2.6)$ & $525(5.9)$ & $-5(3.4)$ & \\
\hline${ }^{1}$ Luxembourg & & -- & -- & -- & & -- & -- & -- & \\
\hline Macedonia, Rep. of & $r$ & $32(4.5)$ & $420(10.3)$ & $1(6.2)$ & & $27(4.2)$ & $422(9.5)$ & $-11(6.2)$ & \\
\hline Moldova, Rep. of & & $21(3.7)$ & $487(5.5)$ & $-5(5.2)$ & & $17(3.2)$ & $502(9.5)$ & $-15(5.1)$ & $\nabla$ \\
\hline Morocco & $r$ & $23(5.1)$ & $298(17.9)$ & $0(6.6)$ & & $47(5.3)$ & $318(9.8)$ & $-14(7.1)$ & \\
\hline Netherlands & $r$ & $11(3.3)$ & $534(6.7)$ & $3(4.3)$ & & $10(3.3)$ & $510(6.9)$ & $2(4.2)$ & \\
\hline New Zealand & & $14(2.4)$ & $516(5.1)$ & $2(3.4)$ & & $16(2.0)$ & $475(7.0)$ & $-2(3.2)$ & \\
\hline Norway & $r$ & $1(1.0)$ & $\sim \sim$ & $-1(2.0)$ & & $0(0.0)$ & $\sim \sim$ & $0(0.0)$ & \\
\hline Poland & & $29(4.2)$ & $511(4.6)$ & $\Delta \Delta$ & & $14(3.1)$ & $510(6.2)$ & $\Delta \Delta$ & \\
\hline Qatar & & $36(0.2)$ & $351(2.0)$ & $\Delta \diamond$ & & $18(0.2)$ & $352(2.6)$ & $\Delta \Delta$ & \\
\hline Romania & & $21(3.9)$ & $503(6.8)$ & $-14(5.6)$ & ( & $43(4.5)$ & $462(8.1)$ & $1(6.6)$ & \\
\hline Russian Federation & & $23(2.6)$ & $553(6.4)$ & $-7(4.6)$ & & $14(2.1)$ & $534(8.5)$ & $-5(4.0)$ & \\
\hline Scotland & $r$ & $25(4.7)$ & $505(6.2)$ & $9(5.6)$ & & $8(3.1)$ & $497(8.9)$ & $-13(5.3)$ & $\nabla$ \\
\hline Singapore & & $7(0.0)$ & $531(14.4)$ & $-4(2.1)$ & & $1(0.0)$ & $\sim \sim$ & $-2(1.1)$ & \\
\hline Slovak Republic & & $23(3.5)$ & $525(5.8)$ & $-9(5.4)$ & & $10(2.6)$ & $470(14.8)$ & $-11(4.5)$ & 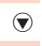 \\
\hline Slovenia & & $20(3.5)$ & $517(5.4)$ & $5(4.8)$ & & $6(2.1)$ & $520(10.2)$ & $5(2.2)$ & $\theta$ \\
\hline South Africa & & $12(2.0)$ & $305(16.8)$ & $\Delta \Delta$ & & $74(2.7)$ & $272(4.4)$ & $\Delta \Delta$ & \\
\hline Spain & & $6(1.7)$ & $490(5.7)$ & $\Delta \Delta$ & & $6(2.1)$ & $458(8.5)$ & $\Delta \Delta$ & \\
\hline Sweden & & $8(2.9)$ & $558(9.3)$ & $-4(4.1)$ & & $8(2.6)$ & $522(11.2)$ & $0(3.7)$ & \\
\hline Trinidad and Tobago & & $22(3.5)$ & $440(9.9)$ & $\Delta \diamond$ & & $39(3.6)$ & $405(7.9)$ & $\Delta 0_{0}$ & \\
\hline United States & & $25(3.4)$ & $546(4.9)$ & $6(5.3)$ & & $37(4.2)$ & $511(3.7)$ & $1(5.7)$ & \\
\hline International Avg. & & $17(0.5)$ & $488(1.4)$ & & & $18(0.5)$ & 465 (1.6) & & \\
\hline
\end{tabular}


Exhibit 7.3 Principals' Reports on Primary Grade Students Not Speaking the Language of the Test as Their First Language with Trends

PIRLS 2006

4th Grade

\begin{tabular}{|c|c|c|c|c|c|c|c|c|c|}
\hline \multirow{2}{*}{ Countries } & & \multicolumn{4}{|c|}{$\begin{array}{l}\text { 0-10\% First Language } \\
\text { Not Language of Test }\end{array}$} & \multicolumn{4}{|c|}{$\begin{array}{c}11-25 \% \text { First Language } \\
\text { Not Language of Test }\end{array}$} \\
\hline & & $\begin{array}{c}2006 \\
\text { Percent } \\
\text { of Students }\end{array}$ & $\begin{array}{c}\text { Average } \\
\text { Achievement }\end{array}$ & $\begin{array}{l}\text { Difference } \\
\text { in Percent } \\
\text { from } 2001\end{array}$ & & $\begin{array}{c}2006 \\
\text { Percent } \\
\text { of Students }\end{array}$ & $\begin{array}{c}\text { Average } \\
\text { Achievement }\end{array}$ & $\begin{array}{l}\text { Difference } \\
\text { in Percent } \\
\text { from } 2001\end{array}$ & \\
\hline Austria & & $45(3.7)$ & $549(2.8)$ & $\Delta \Delta$ & & $27(3.8)$ & $541(3.7)$ & $\Delta \Delta$ & \\
\hline Belgium (Flemish) & & $72(4.3)$ & $553(1.8)$ & $\Delta \Delta$ & & $15(3.5)$ & $544(4.7)$ & $\Delta \Delta$ & \\
\hline Belgium (French) & $r$ & $75(3.6)$ & $509(3.1)$ & $\Delta \diamond$ & & $9(2.7)$ & $494(8.1)$ & $\Delta \diamond$ & \\
\hline Bulgaria & & $62(3.9)$ & $566(5.0)$ & $1(5.5)$ & & $7(2.0)$ & $514(10.4)$ & $-2(3.1)$ & \\
\hline Canada, Alberta & & $74(3.9)$ & $564(2.7)$ & $\Delta \Delta$ & & $16(3.2)$ & $560(5.9)$ & $\Delta \Delta$ & \\
\hline Canada, British Columbia & & $59(4.9)$ & $558(3.5)$ & $\Delta \Delta$ & & $19(3.5)$ & $567(6.3)$ & $\Delta \Delta$ & \\
\hline Canada, Nova Scotia & & $93(1.8)$ & $545(2.4)$ & $\Delta \diamond$ & & $2(1.2)$ & $\sim \sim$ & $\Delta \diamond$ & \\
\hline Canada, Ontario & & $67(5.2)$ & $559(3.0)$ & $-11(6.4)$ & & $13(4.0)$ & $560(9.2)$ & $5(4.8)$ & \\
\hline Canada, Quebec & & $79(3.5)$ & $536(3.6)$ & $-3(4.7)$ & & $7(2.2)$ & $541(6.3)$ & $-3(3.2)$ & \\
\hline Chinese Taipei & & $80(3.5)$ & $537(2.3)$ & $\Delta \Delta$ & & $11(2.7)$ & $536(6.5)$ & $\Delta \Delta$ & \\
\hline Denmark & & $75(3.6)$ & $549(2.9)$ & $\Delta \diamond$ & & $18(3.1)$ & $545(4.4)$ & $\Delta \diamond$ & \\
\hline England & $r$ & $77(3.3)$ & $551(3.2)$ & $-11(4.3)$ & ( & $12(2.6)$ & $535(9.1)$ & $9(3.0)$ & 0 \\
\hline France & & $87(2.9)$ & $524(2.4)$ & $-2(4.0)$ & & $7(2.3)$ & $502(12.0)$ & $-2(3.6)$ & \\
\hline Georgia & $s$ & $89(3.3)$ & $476(4.5)$ & $\Delta \diamond$ & & $6(2.5)$ & $450(12.0)$ & $\Delta \Delta$ & \\
\hline Germany & & $52(3.2)$ & $559(2.0)$ & $-15(4.8)$ & ( & $24(3.1)$ & $546(5.3)$ & $5(4.4)$ & \\
\hline Hong Kong SAR & & $96(1.3)$ & $564(2.3)$ & $85(3.2)$ & 0 & $1(0.5)$ & $\sim \sim$ & $1(0.5)$ & \\
\hline Hungary & & $94(2.0)$ & $554(3.1)$ & $0(2.8)$ & & $2(1.2)$ & $\sim \sim$ & $1(1.3)$ & \\
\hline Iceland & $r$ & $95(0.2)$ & $512(1.4)$ & $-3(0.2)$ & ( & $5(0.2)$ & $501(5.3)$ & $5(0.2)$ & 0 \\
\hline Indonesia & & $28(4.2)$ & 409 (8.1) & $\Delta \Delta$ & & $8(2.3)$ & $399(15.6)$ & $\Delta \Delta$ & \\
\hline Iran, Islamic Rep. of & & $52(3.4)$ & $446(4.0)$ & $-2(6.1)$ & & $7(1.7)$ & $422(9.5)$ & $-2(3.7)$ & \\
\hline Israel & & $48(4.2)$ & $538(7.5)$ & $-11(5.9)$ & & $15(3.0)$ & $543(4.8)$ & $-2(4.4)$ & \\
\hline Italy & & $81(3.3)$ & $550(3.5)$ & $-13(3.8)$ & ( & $16(3.2)$ & $547(7.8)$ & $13(3.5)$ & 0 \\
\hline Kuwait & $r$ & $94(2.2)$ & $326(5.0)$ & $\Delta \Delta$ & & $1(0.9)$ & $\sim \sim$ & $\Delta \Delta$ & \\
\hline Latvia & & $53(3.4)$ & $541(3.6)$ & $-34(4.2)$ & ( & $17(2.7)$ & $536(4.1)$ & $9(3.5)$ & $\theta$ \\
\hline Lithuania & $r$ & $93(2.2)$ & $538(2.1)$ & $1(3.3)$ & & $4(1.8)$ & $559(8.5)$ & $-1(2.6)$ & \\
\hline${ }^{1}$ Luxembourg & & -- & -- & -- & & -- & -- & -- & \\
\hline Macedonia, Rep. of & $s$ & $76(4.5)$ & $461(7.7)$ & $22(5.8)$ & $\theta$ & $8(2.5)$ & 409 (11.9) & $-2(3.8)$ & \\
\hline Moldova, Rep. of & $r$ & $87(3.2)$ & $499(3.8)$ & $4(4.7)$ & & $1(0.8)$ & $\sim \sim$ & $-7(2.5)$ & ( \\
\hline Morocco & $s$ & $55(5.8)$ & $324(9.2)$ & $16(7.6)$ & $\theta$ & $7(3.4)$ & $291(19.6)$ & $-1(4.4)$ & \\
\hline Netherlands & $r$ & $79(4.1)$ & $554(1.8)$ & $-5(5.2)$ & & $4(1.9)$ & $537(14.2)$ & $-2(2.8)$ & \\
\hline New Zealand & & $74(3.3)$ & $537(2.7)$ & $-5(4.6)$ & & $15(2.8)$ & $541(7.5)$ & $1(3.9)$ & \\
\hline Norway & & $87(3.2)$ & $499(3.2)$ & $-2(4.3)$ & & $10(2.8)$ & $488(7.5)$ & $2(4.0)$ & \\
\hline Poland & $r$ & 97 (1.4) & $520(2.7)$ & $\Delta \Delta$ & & $0(0.0)$ & $\sim \sim$ & $\Delta \Delta$ & \\
\hline Qatar & & $67(0.2)$ & $354(1.6)$ & $\Delta \diamond$ & & $14(0.2)$ & $352(2.9)$ & $\Delta \diamond$ & \\
\hline Romania & & $80(4.1)$ & $496(5.9)$ & $-3(5.5)$ & & $6(2.1)$ & $492(15.9)$ & $-4(3.8)$ & \\
\hline Russian Federation & & $73(3.4)$ & $571(4.2)$ & $-4(5.4)$ & & $8(2.3)$ & $561(12.2)$ & $2(2.8)$ & \\
\hline Scotland & $r$ & $95(1.9)$ & $526(3.5)$ & $-1(2.8)$ & & $4(1.6)$ & $545(8.0)$ & $1(2.5)$ & \\
\hline Singapore & & $11(0.0)$ & $573(8.0)$ & $-10(3.0)$ & ( & $18(0.0)$ & $573(8.6)$ & $5(2.8)$ & \\
\hline Slovak Republic & & $85(2.9)$ & $539(2.6)$ & $3(3.9)$ & & $6(1.8)$ & $500(7.6)$ & $1(2.6)$ & \\
\hline Slovenia & & $76(3.6)$ & $521(2.5)$ & $-8(4.9)$ & & $11(2.6)$ & $527(6.2)$ & $3(3.5)$ & \\
\hline South Africa & & $63(2.3)$ & $284(7.4)$ & $\Delta \diamond$ & & $10(1.9)$ & $342(19.9)$ & $\Delta \Delta$ & \\
\hline Spain & & $66(3.0)$ & $518(3.2)$ & $\Delta \Delta$ & & $12(2.5)$ & $499(9.2)$ & $\Delta \Delta$ & \\
\hline Sweden & & $63(4.2)$ & $555(2.6)$ & $-13(5.7)$ & $(\nabla$ & $18(3.6)$ & $542(4.2)$ & $7(4.4)$ & \\
\hline Trinidad and Tobago & & $86(3.1)$ & $439(6.0)$ & $\Delta \diamond$ & & $1(0.0)$ & $\sim \sim$ & $\Delta \Delta$ & \\
\hline United States & & $71(3.1)$ & $548(4.5)$ & $-7(4.4)$ & & $11(2.4)$ & $540(6.3)$ & $-1(3.4)$ & \\
\hline International Avg. & & $73(0.5)$ & $504(0.7)$ & & & $10(0.4)$ & $499(1.7)$ & & \\
\hline
\end{tabular}

Background data provided by schools.

() Standard errors appear in parentheses. Because results are rounded to the nearest whole number, some totals may appear inconsistent.

An " $r$ " indicates data are available for $70-84 \%$ of the students. An " $s$ " indicates data are available for $50-69 \%$ of the students. An " $\mathrm{x}$ " indicates data are available for less than $50 \%$ of the students.

A dash (-) indicates comparable data are not available. A tilde ( ) indicates insufficient data to report achievement.
A diamond $(\diamond)$ indicates the country did not participate in the 2001 assessment.

NOTE: The International Average does not include the results from the Canadian provinces. Trend Note: The primary education systems of the Russian Federation and Slovenia underwent structural changes. Data for Canada, Ontario include only public schools. 1 Primary schools in Luxembourg do not have principals. 


\begin{tabular}{|c|c|c|c|c|c|c|c|c|c|}
\hline \multirow[t]{3}{*}{ Exhibit 7.3} & \multicolumn{9}{|c|}{$\begin{array}{l}\text { als' Reports on Primary Grade Students Not Speaking the La } \\
\text { est as Their First Language with Trends (Continued) }\end{array}$} \\
\hline & & \multicolumn{4}{|c|}{$\begin{array}{c}26-50 \% \text { First Language } \\
\text { Not Language of Test }\end{array}$} & \multicolumn{4}{|c|}{$\begin{array}{c}\text { More than } 50 \% \text { First Language } \\
\text { Not Language of Test }\end{array}$} \\
\hline & & $\begin{array}{c}2006 \\
\text { Percent } \\
\text { of Students }\end{array}$ & \begin{tabular}{c|} 
Average \\
Achievement
\end{tabular} & $\begin{array}{l}\text { Difference } \\
\text { in Percent } \\
\text { from } 2001\end{array}$ & & $\begin{array}{c}2006 \\
\text { Percent } \\
\text { of Students }\end{array}$ & $\begin{array}{c}\text { Average } \\
\text { Achievement }\end{array}$ & $\begin{array}{l}\text { Difference } \\
\text { in Percent } \\
\text { from } 2001\end{array}$ & \\
\hline Austria & & $15(3.0)$ & $530(4.2)$ & $\Delta \Delta$ & & $13(2.4)$ & $505(5.5)$ & $\Delta \theta_{0}$ & \\
\hline Belgium (Flemish) & & $9(2.7)$ & $525(8.6)$ & $\Delta \Delta$ & & $4(1.4)$ & $501(9.0)$ & $\Delta \Delta$ & \\
\hline Belgium (French) & $r$ & $6(1.8)$ & $473(7.4)$ & $\Delta \Delta$ & & $11(2.6)$ & $458(14.0)$ & $\Delta \Delta$ & \\
\hline Bulgaria & & $9(2.7)$ & $502(7.6)$ & $2(3.5)$ & & $22(3.1)$ & $521(9.9)$ & $-1(4.5)$ & \\
\hline Canada, Alberta & & $7(2.2)$ & $547(10.0)$ & $\Delta \diamond$ & & $4(1.8)$ & $519(10.6)$ & $\diamond \diamond$ & \\
\hline Canada, British Columbia & & $12(3.1)$ & $548(7.5)$ & $\Delta \Delta$ & & $10(2.6)$ & $556(9.3)$ & $\Delta \Delta$ & \\
\hline Canada, Nova Scotia & & $3(1.3)$ & $531(13.7)$ & $\Delta \diamond$ & & $2(0.8)$ & $\sim \sim$ & $\Delta \diamond$ & \\
\hline Canada, Ontario & & $9(2.9)$ & $537(7.8)$ & $1(3.9)$ & & $10(3.2)$ & $531(9.0)$ & $6(3.6)$ & \\
\hline Canada, Quebec & & $5(1.9)$ & $517(9.4)$ & $3(2.3)$ & & $9(2.6)$ & $522(7.4)$ & $4(3.2)$ & \\
\hline Chinese Taipei & & $1(0.0)$ & $\sim \sim$ & $\Delta \Delta$ & & $8(2.2)$ & $512(9.3)$ & $\Delta \diamond$ & \\
\hline Denmark & & $4(1.4)$ & $536(10.7)$ & $\Delta \Delta$ & & $3(1.2)$ & $527(13.9)$ & $\Delta \Delta$ & \\
\hline England & $r$ & $3(1.6)$ & $514(18.7)$ & $-1(2.4)$ & & $9(2.7)$ & $483(13.5)$ & $3(3.2)$ & \\
\hline France & & $5(2.2)$ & $480(10.0)$ & $5(2.2)$ & 0 & $1(0.0)$ & $\sim \sim$ & $-1(1.2)$ & \\
\hline Georgia & $s$ & $3(1.6)$ & $467(10.4)$ & $\Delta \diamond$ & & $2(1.4)$ & $\sim \sim$ & $\Delta \Delta$ & \\
\hline Germany & & $17(2.9)$ & $534(4.6)$ & $7(3.9)$ & & $7(2.0)$ & $503(7.6)$ & $4(2.6)$ & \\
\hline Hong Kong SAR & & $0(0.0)$ & $\sim \sim$ & $0(0.0)$ & & $3(1.2)$ & $549(12.8)$ & $-85(3.2)$ & $\nabla$ \\
\hline Hungary & & $0(0.0)$ & $\sim \sim$ & $0(0.0)$ & & $4(1.6)$ & $543(16.3)$ & $-1(2.5)$ & \\
\hline Iceland & $r$ & $0(0.0)$ & $\sim \sim$ & $0(0.0)$ & & $0(0.0)$ & $\sim \sim$ & $-1(0.1)$ & \\
\hline Indonesia & & $10(2.5)$ & $406(15.2)$ & $\Delta \Delta$ & & $54(4.1)$ & $404(5.5)$ & $\Delta \Delta$ & \\
\hline Iran, Islamic Rep. of & & $5(1.8)$ & $436(10.8)$ & $-2(2.9)$ & & $36(3.0)$ & $383(5.9)$ & $7(5.3)$ & \\
\hline Israel & & $11(2.9)$ & $521(9.5)$ & $2(3.8)$ & & $26(3.3)$ & $448(10.9)$ & $11(4.1)$ & $\theta$ \\
\hline Italy & & $2(1.2)$ & $\sim \sim$ & $1(1.4)$ & & $1(0.8)$ & $\sim \sim$ & $0(1.2)$ & \\
\hline Kuwait & $r$ & $2(1.2)$ & $\sim \sim$ & $\Delta \diamond$ & & $3(1.6)$ & $351(34.8)$ & $\Delta \Delta$ & \\
\hline Latvia & & $5(1.8)$ & $536(10.3)$ & $1(2.0)$ & & $24(1.7)$ & $547(5.4)$ & $23(2.2)$ & 0 \\
\hline Lithuania & $r$ & $1(1.0)$ & $\sim \sim$ & $1(1.0)$ & & $2(1.2)$ & $\sim \sim$ & $-2(2.0)$ & \\
\hline${ }^{1}$ Luxembourg & & -- & -- & -- & & -- & -- & -- & \\
\hline Macedonia, Rep. of & $s$ & $6(2.7)$ & $413(25.3)$ & $-2(4.0)$ & & $10(3.2)$ & $392(19.2)$ & $-18(4.8)$ & 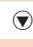 \\
\hline Moldova, Rep. of & $r$ & $6(2.3)$ & $518(27.3)$ & $3(2.7)$ & & $7(2.5)$ & $507(14.6)$ & $-1(3.5)$ & \\
\hline Morocco & $s$ & $7(2.7)$ & $336(28.4)$ & $-3(4.2)$ & & $31(5.4)$ & $315(19.1)$ & $-13(7.3)$ & \\
\hline Netherlands & $r$ & $9(2.9)$ & $517(7.1)$ & $6(3.3)$ & & $9(3.2)$ & $515(8.0)$ & $2(4.0)$ & \\
\hline New Zealand & & $6(1.9)$ & $527(9.3)$ & $1(2.7)$ & & $5(1.2)$ & $451(13.8)$ & $2(1.7)$ & \\
\hline Norway & & $2(0.9)$ & $\sim \sim$ & $-1(1.9)$ & & $2(1.1)$ & $\sim \sim$ & $2(1.1)$ & \\
\hline Poland & $r$ & $0(0.0)$ & $\sim \sim$ & $\Delta \Delta$ & & $3(1.4)$ & $525(18.0)$ & $\Delta \Delta$ & \\
\hline Qatar & & $4(0.1)$ & $385(8.1)$ & $\Delta \Delta$ & & $15(0.2)$ & $346(3.0)$ & $\Delta \diamond$ & \\
\hline Romania & & $4(2.3)$ & $463(18.2)$ & $3(2.6)$ & & $9(3.1)$ & $448(14.0)$ & $4(3.8)$ & \\
\hline Russian Federation & & $8(2.5)$ & $562(10.4)$ & $4(2.9)$ & & $11(1.6)$ & $537(11.0)$ & $-2(2.9)$ & \\
\hline Scotland & $r$ & $1(1.0)$ & $\sim \sim$ & $1(1.4)$ & & $0(0.0)$ & $\sim \sim$ & $0(0.0)$ & \\
\hline Singapore & & $26(0.0)$ & $566(5.4)$ & $7(3.6)$ & & $45(0.0)$ & $544(5.0)$ & $-2(4.1)$ & \\
\hline Slovak Republic & & $6(2.0)$ & $500(14.6)$ & $2(2.7)$ & & $3(1.6)$ & $450(41.0)$ & $-6(2.2)$ & $\nabla$ \\
\hline Slovenia & & $8(2.2)$ & $520(8.2)$ & $2(3.1)$ & & $5(2.0)$ & $521(7.4)$ & $3(2.3)$ & \\
\hline South Africa & & $9(1.8)$ & $348(24.2)$ & $\Delta \Delta$ & & $18(2.1)$ & $323(15.0)$ & $\Delta \diamond$ & \\
\hline Spain & & $6(2.1)$ & $521(10.8)$ & $\Delta \Delta$ & & $16(2.8)$ & $498(6.5)$ & $\Delta \Delta$ & \\
\hline Sweden & & $11(2.5)$ & $545(8.4)$ & $7(3.0)$ & $\theta$ & $7(2.4)$ & $526(12.5)$ & $-1(3.6)$ & \\
\hline Trinidad and Tobago & & $3(1.6)$ & $437(23.3)$ & $\Delta \Delta$ & & $10(2.7)$ & $434(13.2)$ & $\Delta \Delta$ & \\
\hline United States & & $8(2.0)$ & $510(6.4)$ & $3(2.6)$ & & $10(2.5)$ & $509(6.7)$ & $5(2.9)$ & \\
\hline International Avg. & & $6(0.3)$ & $487(2.7)$ & & & $12(0.4)$ & $471(2.6)$ & & \\
\hline
\end{tabular}




\section{What Is the Role of the School Principal?}

Exhibit 7.4 summarizes principals' reports of how much time each week they devoted to a range of professional activities, including curriculum and staff development, administrative duties, community relations, teaching, and interacting with students. Principals reported spending a considerable amount of time, 39 hours per week, on average internationally, on such activities. For several participants, including Belgium (Flemish), England, New Zealand, the United States, as well as the Canadian provinces of Alberta, British Columbia, Nova Scotia, and Ontario, principals reported spending 50 hours per week or more, on average, on these activities. By contrast, principals in Indonesia, Kuwait, Qatar, and South Africa reported spending less than 25 hours per week on school-related activities.

In most countries (and in the five Canadian provinces), administrative duties (such as hiring and budgeting) were the most time-consuming activities (22\% of principals' time, on average). Across countries this was followed by managing staff or staff development (18\%, on average). Developing curriculum and pedagogy for the school (16\% of time, on average) was the most time-consuming activity in four countries-Bulgaria, Georgia, Indonesia and Israel. Principals reported devoting 13 percent of their time, on average, to parent and community relations, 10 percent to interacting with individual students, and 13 percent to teaching. Countries where principals reported spending more time on teaching than on any of the other activities included France, Germany, Kuwait, Macedonia, Romania, and Spain.

\section{What Shortages of School Resources Affect Reading Instruction?}

To provide information on the extent to which school resources are available to support reading instruction, PIRLS constructed an Index of Availability of School Resources based on principals' responses about shortages of or inadequacies in accommodation, staff, equipment, and instructional materials. More specifically, principals were asked to indicate if their school's capacity to provide instruction was affected by shortages or inadequacies 
in qualified teaching staff, teachers with a specialization in reading, second language teachers, instructional materials (e.g., textbooks), supplies (e.g., papers, pencils), school buildings and grounds, heating/cooling and lighting systems, instructional space (e.g., classrooms), special equipment for physically disabled students, computers for instructional purposes, computer software for instructional purposes, computer support staff, library books, and audio-visual resources. Principals' average responses were computed on a 4-point scale: not at all $=1$, a little $=2$, some $=3$, and a lot $=4$. Students were assigned to the high level of the index if their school's principal had an average response of less than 2. To achieve such a response average, a principal would have to reply not at all or some to most of the questions, implying that resource shortages were not a problem for the school. Students were assigned to the medium level if their principal's average was 2 or more but not less than 3 , and to the low level if the average was 3 or more.

Exhibit 7.5 presents, for every participant, the percentage of PIRLS 2006 students at each level of the index, together with their average reading achievement. For those that also participated in PIRLS 2001, the exhibit shows the change in percentage of students at each level, and an indication of whether the change was statistically significant. On average across countries, the majority (52\%) of students were at the high level of the index (i.e., were attending schools that were not hampered by resource shortages). Eighty percent or more of the students were at the high level of the index in the Netherlands, Scotland, Denmark, Belgium (Flemish), New Zealand, Iceland, Sweden, Slovenia, the United States, England, Austria, and the Canadian province of British Columbia. Although, on average, there were just 15 percent of students at the low level of the index (i.e., attending schools where principals reported that resource shortages greatly affected the provision of instruction), there were some countries with a substantial percentage of students at this level. Countries with more than 20 percent of students at the low level included Israel, Chinese Taipei, South Africa, Qatar, the Russian Federation, Moldova, Iran, Kuwait, Morocco, and Hong Kong SAR. 
Exhibit 7.4 Principals'Time Spent on Various School-related Activities

PIRLS 2006

Countries

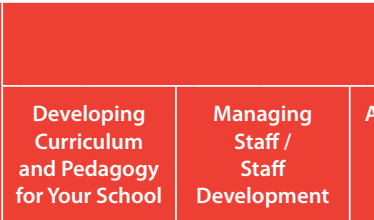

Percent of Time

Average

Austria

$11(0.6)$

$13(0.7)$

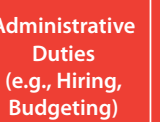

Parent and
Community
Relations

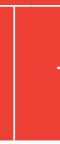

Teaching

Hours per

Belgium (Flemish)

$16(0.8) \quad 13(0.6)$

$29(1.4)$

$12(0.5)$

20 (1.6)

Belgium (French)

$16(0.8)$

32 (1.6)

$13(0.6)$

$20(1.6)$
$2(0.5)$

Interacting
with
Individual
Students

Week

Bulgaria

Canada, Alberta

$29(1.1)$

$41(1.8)$

$13(0.6)$

$9(0.4)$

Canada, British

$29(1.1)$

$9(0.4)$

20 (1.1)

$14(0.6)$

$r \quad 7(1.1)$

$8(0.4)$

Nova Scotia

\begin{tabular}{l|l}
$16(0.7)$ & $28(1.3)$
\end{tabular}

$14(0.6)$

$11(0.5)$

$8(0.6)$

$9(0.4)$

$13(1.0)$

$11(0.5) \quad 15(0.6)$

27 (1.3)

$15(0.7)$

$13(1.1)$

$16(0.7)$

Canada, Ontario

$12(0.8)$

$18(0.7)$

28 (1.7)

$15(0.6)$

$18(0.8)$

Canada, Quebec

$11(0.5)$

$20(1.3)$

$15(0.8)$

$6(0.6)$

21 (1.1)

$2(0.4)$

$21(1.2)$

Chinese Taipei

$17(0.6) \quad 17(0.5)$

Denmark

$15(0.9)$

$17(0.5)$

$21(1.0)$

$16(0.7)$

$10(0.5)$

England

r $\quad 16(0.8)$

France

$6(0.5)$

$16(0.9)$

30 (1.4)

$16(0.6)$

$10(0.5)$

$12(0.6)$

$4(0.6)$

$17(0.9)$

$11(0.6)$

Georgia

$22(0.9)$

$5(0.5)$

$27(1.4)$

$r \quad 13(0.7)$
$r \quad 11(0.7)$

r $8(0.8)$

$47(1.9)$

$9(0.5)$

$11(0.6)$

$14(0.5)$

$15(0.7)$

$6(0.5)$

$15(0.8)$

$15(0.7)$

$11(0.6)$

\begin{tabular}{l|l}
$11(0.4)$ & $38(1.4)$ \\
$13(0.5)$ & $5(0.5)$
\end{tabular}

$7(0.3)$

$21(0.8) \quad 20(0.6)$

$20(0.8)$

$5(0.5)$

$9(0.5)$

\begin{tabular}{ll} 
Hong Kong SAR & $21(0.8)$ \\
\hline Hungary & $11(0.7)$
\end{tabular}

$13(0.5) \quad 22(1.0)$

$14(0.6)$

$13(0.6)$

Other

Spent on

These

Activities

\begin{tabular}{l|lll} 
Iceland & r & $12(0.1)$ & r
\end{tabular}

28 (1.4)

$0(0.1)$

$18(0.1)$

$13(0.0)$

$4(0.0)$

$13(0.1)$

$6(0.6)$

$6(0.6) \quad 39(1.0)$

$15(1.2) \quad 50(1.0)$

$15(0.7)$

$15(1.0)$

$12(0.5)$

$4(0.0)$

$5(0.6)$

\section{$8(0.6)$}

$4(0.6)$

$5(0.7)$

$45(0.8)$

$38(1.0)$

Iran, Islamic Rep. of

Italy

$23(0.9)$

$20(0.7) \quad 10(0.6)$

$14(0.9)$

$10(0.7)$

r $\quad 54(1.1)$

$4(0.6) \quad 53(0.8)$

$5(0.7) \quad r \quad 54(1.1)$

$5(0.6) \quad r \quad 48(0.7)$

$8(0.6)$

40 (1.3)

$8(0.6)$

$47(0.5)$

r $\quad 9(1.0) \quad$ r $\quad 54(1.1)$

$2(0.4) \quad 40(0.8)$

Kuwait

$15(0.7)$

$12(0.7)$

$17(0.7)$

$14(0.6)$

$12(0.5)$

$5(0.4)$

$41(0.9)$

Latvia

$15(1.6) \quad s \quad 18(1.1)$

25 (1.1)

$19(0.6) \quad 2(0.3)$

$10(0.6)$

$5(0.5)$

$47(0.7)$

Lithuania

$19(0.6)$

\begin{tabular}{l|l}
$17(0.7)$ & $20(0.9)$
\end{tabular}

$16(0.9)$

$2(0.3)$

$7(0.5)$

r $29(2.2)$

(1.3) $\quad 43(0.8)$

${ }^{1}$ Luxembourg

$18(0.8)$

$19(0.9)$

$13(0.5)$

$15(0.7)$

$13(0.7)$
$10(0.5)$

$12(0.7)$

$10(0.4)$

$10(0.1)$

$42(0.1)$

$22(1.3) \quad r \quad 17(0.8)$

$--$

$--$

$--$

$--$

$6(0.4)$

22 (1.3)

$13(0.7) \quad 27(1.0)$

$14(0.7)$

$12(0.6) \quad r \quad 22(1.1)$

$10(0.8)$

$28(1.1)$

Moldova, Rep. of

Morocco

10

Netherlands

$x x-27$

$10(0.4) \quad 13(0.5)$

$17(0.9)$

$8(0.4)$

$5(0.6) \quad r \quad 45(1.4)$

$4(0.5) \quad 38(1.0)$

Nor

Norway

Poland

$\begin{array}{llllll}r & 13(0.6) & r & 17(1.1) & r & 29\end{array}$

$x x$

$\begin{array}{ll}x x & 17 \\ \end{array}$

Qat

Ro

Russian Federation

Scotland

Singapore

Slovak Republic

South Africa

Spain

Sweden

$15(0.7) \quad 17(0.6)$

29 (1.6)

r $12(0.7)$

$\mathrm{x} \mathrm{x}$

\begin{tabular}{|l|l|l}
\hline $8(0.4)$ & $7(0.5)$ & $40(1.4)$ \\
\hline $13(0.6)$ & $x x$ & 5
\end{tabular}

Trinidad and Tobago

United States

$15(0.9)$

\begin{tabular}{|l|l|l|}
\hline $21(0.9)$ & $34(1.3)$ & $9(0.4)$ \\
\hline
\end{tabular}

$32(1.2)$

$12(0.7)$

r $\quad 7(1.2)$

$7(1.2)$
$8(0.8)$

$\mathrm{xx}$

$5(0.5)$

$14(1.2)$

$15(0.7) \quad 20(0.6) \quad 23(1.1)$

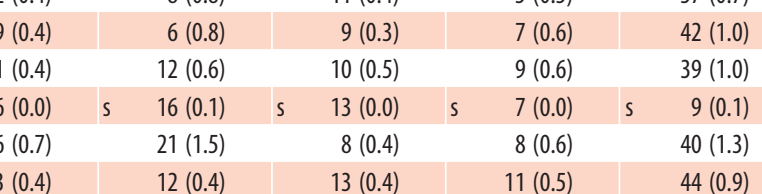

$7(0.5)$
$11(0.4)$

\begin{tabular}{|c|c|c|c|c|c|c|c|c|}
\hline (0.4) & & $8(0.8)$ & & $11(0.4)$ & & $5(0.5)$ & & $57(0.7)$ \\
\hline (0.4) & & $6(0.8)$ & & $9(0.3)$ & & $7(0.6)$ & & $42(1.0)$ \\
\hline$(0.4)$ & & $12(0.6)$ & & $10(0.5)$ & & $9(0.6)$ & & $39(1.0)$ \\
\hline$(0,0)$ & $S$ & $16(0.1)$ & $S$ & $13(0.0)$ & $S$ & $7(0.0)$ & $S$ & $9(0.1)$ \\
\hline (07) & & $21(1.5)$ & & $8(0.4)$ & & $8(0.6)$ & & $40(1.3)$ \\
\hline & & $12(0.4)$ & & $13(0.4)$ & & $11(0.5)$ & & $44(0.9)$ \\
\hline
\end{tabular}

$8(0.6)$

$36(1.5)$

$41(1.0)$

International Avg.

s $16(0.1)$

$22(0.0)$

$\begin{array}{lllll}23(1.1) & & 11(0.4) & & 12(0.6) \\ 11(0.0) & s & 16(0.0) & s & 16(0.1) \\ 17(1.0) & & 16(0.7) & & 21(1.5)\end{array}$

$6(0.8)$

$10(0.5)$

$10(0.5)$

$--$

$\begin{array}{ll}-- & -- \\ 6(0.4) & \quad\end{array}$

$13(0.6)$

\begin{tabular}{lll}
\hline $8(0.9)$ & $17(1.0)$ & $16(()$ \\
\hline $8(0.6)$ & $17(0.7)$
\end{tabular}

\begin{tabular}{|r|r|r|r|r|r|r|r|r|r|r|r|r|r|r|}
\hline $17(0.6)$ & & $18(0.6)$ & & $17(0.7)$ & & $13(0.4)$ & & $12(0.4)$ & & $13(0.4)$ & & $11(0.5)$ & $44(0.9)$ \\
\hline $16(0.9)$ & $\mathrm{r}$ & $14(0.7)$ & $\mathrm{r}$ & $28(1.4)$ & $\mathrm{r}$ & $13(0.8)$ & $\mathrm{r}$ & $10(1.1)$ & $\mathrm{r}$ & $12(0.9)$ & $\mathrm{r}$ & $7(0.9)$ & $\mathrm{r}$ & $49(0.8)$ \\
\hline $1(0.0)$ & & $24(0.0)$ & & $25(0.0)$ & & $14(0.0)$ & & $3(0.0)$ & & $10(0.0)$ & $9(0.0)$ & $49(0.0)$ \\
\hline $11(0.5)$ & & $21(0.7)$ & & $25(1.0)$ & & $12(0.4)$ & & $15(0.7)$ & & $9(0.4)$ & & $7(0.5)$ &
\end{tabular}

\begin{tabular}{|r|r|r|r|r|r|r|r|r|}
\hline $14(0.0)$ & $24(0.0)$ & $25(0.0)$ & $14(0.0)$ & $3(0.0)$ & $10(0.0)$ & $9(0.0)$ & $49(0.0)$ \\
\hline $11(0.5)$ & $21(0.7)$ & $25(1.0)$ & $12(0.4)$ & $15(0.7)$ & $9(0.4)$ & $7(0.5)$ & $41(1.1)$ \\
\hline $22(0.9)$ & $19(0.7)$ & $29(1.2)$ & $11(0.4)$ & $4(0.4)$ & $8(0.3)$ & $7(0.7)$ & $42(1.2)$ \\
\hline
\end{tabular}

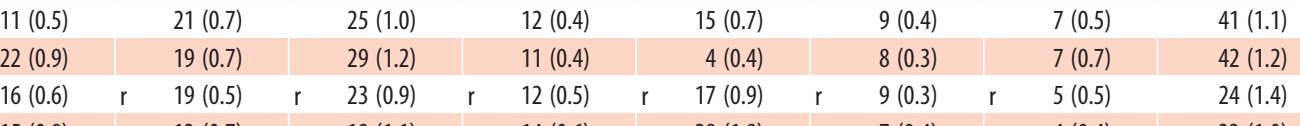

\begin{tabular}{|c|c|c|c|c|c|c|c|c|c|c|c|c|c|c|}
\hline $2(0.9)$ & & $19(0.7)$ & & $29(1.2)$ & & $11(0.4)$ & & $4(0.4)$ & & $8(0.3)$ & & $7(0.7)$ & & $42(1.2)$ \\
\hline $6(0.6)$ & $r$ & $19(0.5)$ & $r$ & $23(0.9)$ & $r$ & $12(0.5)$ & $r$ & $17(0.9)$ & $r$ & $9(0.3)$ & $r$ & $5(0.5)$ & & 24 (1.4) \\
\hline $5(0.8)$ & & $13(0.7)$ & & 19 (1.1) & & $14(0.6)$ & & $28(1.8)$ & & $7(0.4)$ & & $4(0.4)$ & $r$ & $33(1.0)$ \\
\hline $15(0.7)$ & & $22(0.9)$ & & $28(1.2)$ & & $12(0.6)$ & & $2(0.5)$ & & $11(0.5)$ & & $9(1.0)$ & & $43(0.6)$ \\
\hline
\end{tabular}

\begin{tabular}{|c|c|c|c|c|c|c|c|}
\hline & & & & & & & \\
\hline $15(0.7)$ & $22(0.9)$ & $28(1.2)$ & $12(0.6)$ & $2(0.5)$ & $11(0.5)$ & $9(1.0)$ & $43(0.6)$ \\
\hline $17(0.9)$ & $16(0.6)$ & $23(1.1)$ & $12(0.4)$ & $11(0.7)$ & $15(0.7)$ & $7(0.5)$ & $33(1.7)$ \\
\hline
\end{tabular}

$15(0.7)$

$5(0.6)$

$18(1.0)$

$6(0.8)$

$51(1.3)$

\begin{tabular}{|l|l|l|}
\hline $16(0.1)$ & $18(0.1)$ & $22(0.2)$ \\
\hline
\end{tabular}

$13(0.1)$

$13(0.2)$

$10(0.1)$

$7(0.1)$

$39(0.2)$ 
Several participants showed improvement from 2001 in terms of an increase in the percentage of students attending schools with few resources problems (i.e., at the high level of the index). These included the Netherlands, Scotland, Iceland, Slovenia, Norway, the Slovak Republic, Italy, Lithuania, Romania, Israel, and the Canadian province of Ontario. Participants with decreased percentages of students at the high level since 2001 included Macedonia, the Russian Federation, Morocco, and the Canadian province of Quebec. Although the situation varied from country to country, on average, there was a positive relationship between absence of school resource shortages and average reading achievement. At 505 points, the achievement average for students at the high level of the index was 29 points above the average for students at the low level of the index.

Related to the issue of school resources and facilities, Exhibit 7.6 presents schools' reports on the provision of workspace to teachers, and teachers' reports of where they usually prepared materials for class. In general, schools around the world provided teachers with a workspace in the classroom $(81 \%$ of students attended such schools), and many also provided a workspace shared by several teachers ( $71 \%$ of students in such schools). Having a separate workspace for each teacher was far less common, although in a number of countries (Hong Kong SAR, Hungary, Lithuania, Norway, Qatar, the Russian Federation, and Singapore), the majority of students were in schools where such a facility was provided.

Whatever the provision of workspace in schools, many teachers still did much of their class preparation at home. On average internationally, 13 percent of students were taught by teachers who prepared instructional materials only at home, 30 percent by teachers who did so mostly at home, and 40 percent by teachers who prepared materials at home and at school about equally. Although preparing instructional materials mostly or only at school was less common (only $16 \%$ of students were taught by teachers who prepared mostly at school and 1 percent by those preparing only at school), in Iceland, the Netherlands, and Norway, the majority of students were taught by teachers who mostly prepared at school. 
Given the increasingly widespread availability of literacy materials on the Internet, access to computers that may be used for instructional purposes can be a crucial school resource. Exhibit 7.7 provides principals' reports on the availability of computers for fourth-grade students in 2006, as well as changes since 2001. Results are presented in terms of number of students per computer-fewer than 5 students, 5-10 students, 11-20 students, more than 20 students, and students in schools without any computers. Although on average internationally, more than half the students $(53 \%)$ were in relatively well-resourced schools (fewer than five students per computer), the situation varied enormously across countries. More than 90 percent of students in Denmark, England, Iceland, and the Canadian provinces of Alberta, British Columbia, and Ontario were in such schools, as well as 80 percent or more in Scotland, the United States, Hong Kong SAR, Singapore, and the Canadian province of Quebec. In contrast, however, less than 10 percent of students were in such schools in Moldova, Macedonia, Indonesia, Georgia, and Iran. In these countries, the majority of students attended schools with no computers at all. 
Exhibit 7.5 Index of Availability of School Resources (ASR) with Trends

PIRLS 2006

Countries

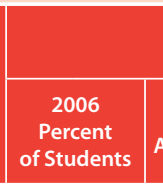

High ASR

Medium ASR

4th Grade

Netherlands

$93(2.5)$

\begin{tabular}{c|c} 
Average \\
Achievement
\end{tabular}

Scotland

$r \quad 88(3.3)$

$546(1.8)$

Denmark

$86(2.9)$

$528(3.8)$

Belgium (Flemish)

$86(2.9)$

$546(2.6)$

\begin{tabular}{|l|c|}
\hline $\begin{array}{c}\text { Difference } \\
\text { in Percent } \\
\text { from } 2001\end{array}$ & $\begin{array}{c}2006 \\
\text { Percent } \\
\text { of Students }\end{array}$ \\
\hline $11(4.6) \quad 0$ & $7(2.5)$ \\
\hline
\end{tabular}

Medium ASR

New Zealand

$86(2.5)$

$546(2.2)$

$11(5.3)$

$7(2.5)$

\begin{tabular}{|c|c|}
\hline Average & $\begin{array}{c}\text { Difference } \\
\text { in Percent }\end{array}$ \\
\hline
\end{tabular}

Iceland

$85(0.3)$

$533(2.2)$

$\diamond \diamond$

$14(2.9)$

$552(9.8)$

from 2001

$552(9.8) \quad-10(4.5) \quad$ ๑ $\quad 0(0.0)$

$\diamond \diamond \quad 13(2.9)$

$525(8.4)$

$-12(5.2)$

$551(7.3)$

00

$13(2.3)$

$554(4.9)$

$1(4.1)$

$533(7.8)$

Sweden

$82(3.7)$

$549(2.8)$

$4(0.4)$

$502(3.3)$

00

$5(5.2) \quad 15(3.5)$

$552(3.8)$

$-3(0.4)$

Canada, British Columbia

$82(3.2) \quad 556(3.1)$

$\Delta \diamond \quad 17(3.1)$

Slovenia

$81(3.2)$

$520(2.3)$

$18(5.7) \quad 0 \quad 16(2.9)$

United States

$81(2.8)$

$543(4.1)$

$4(4.5)$

81 (3.8)

$543(3.4)$

Austria

80 (3.4)

$541(2.3)$

$--$

$18(3.1)$

Norway

$79(3.5)$

$500(3.2)$

19 (3.8)

$563(4.9)$

$-3(4.8)$

$529(5.8)$

00

$520(6.1)$

$-19(5.4)$

537 (7.9)

$-5(4.7)$

$20(3.4)$

$531(6.1)$

$--$

Canada, Alberta

$78(3.6)$

$562(2.6)$

$\Delta 0$

$20(3.4)$

Canada, Ontario

$76(5.0)$

$556(3.0)$

$14(6.7) \quad 0 \quad 20(4.5)$

491 (4.4)

$-8(5.9)$

$554(6.4)$

$548(6.7)$

$\Delta 0$

$541(4.5)$

Canada, Nova Scotia

$73(0.0)$

$560(3.2)$

$00 \quad 23(3.5)$

$553(11.1)$

00

\begin{tabular}{|c|c|c|}
\hline \multicolumn{2}{|c}{ Low ASR } \\
\hline $\begin{array}{c}2006 \\
\text { Percent } \\
\text { of Students }\end{array}$ & $\begin{array}{c}\text { Average } \\
\text { Achievement }\end{array}$ & Diff \\
\hline
\end{tabular}

Canada, Quebec

$73(3.9)$

$537(3.0)$

1 (3.4)

$12(0.0)$

Poland

$72(4.0)$

$520(2.9)$

$-11(5.4) \odot \quad 24(3.6)$

Germany

$71(3.2)$

$553(2.4)$

$\Delta \diamond \quad 27(4.0)$

Hungary

$71(4.4)$

$553(4.0)$

$3(4.6)$

$27(3.2)$

$526(7.2)$

00

$520(4.2)$

$-6(2.9)$

$1(0.0)$

$\sim \sim$
$\sim \sim$

$0(0.0)$

$\sim \sim$

$2(1.1)$

$\sim \sim$

Slovak Republic

$65(3.8)$

$532(3.8)$

$8(5.7)$

$15(3.2)$

$535(4.5)$

$\Delta \diamond$

$539(6.3)$

$-4(4.6)$

$34(5.3) \quad 0 \quad 33(3.8)$

$64(4.3)$

$518(2.9)$

$\Delta \diamond$

France

$60(4.1)$

$526(2.8)$

$-12(6.3)$

25 (3.7)

$530(5.0)$

$-13(4.5)$

$0(0.0)$

$\begin{array}{ll}\sim & \sim \\ \sim & \sim \diamond \\ \sim & \sim\end{array}$

Italy

$56(4.2)$

$552(4.1)$

$39(4.1)$

$501(7.3)$

$-32(5.3)$

Lithuania

Latvia

$49(4.5)$

538 (2.6)

$20(5.5) \quad 0 \quad 42(4.2)$

$516(4.0)$

00

$551(4.2)$

$12(6.3)$

$28(5.6) \quad 0 \quad 40(4.1)$

$537(3.0)$

Romania

$48(4.1)$

$544(2.7)$

$-3(5.7)$

$34(3.9)$

$539(5.0)$

$-16(5.7)$

$-25(5.6)$

$-25(5.6)$
$-7(5.9)$

$469(8.6)$

$-16(6.4)$

$3(1.5)$

$\sim \sim$

$-2(0.0)$

Georgia

$46(4.3)$

$479(5.5)$

$(6.1) \quad 0 \quad 40(4.7)$

Belgium (French)

$42(4.4) \quad 504(5.6)$

$51(4.2)$

$462(4.4)$

$\Delta \diamond$

$500(3.9)$

$\Delta 0 \quad 52(4.2)$

$420(5.5)$

$\Delta \Delta$

Indonesia

$38(4.6)$

$386(6.1)$

$\Delta \diamond \quad 51(4.0)$

$546(6.1)$

$-4(6.3)$

$507(9.1) \quad-9(6.2)$

$1(0.0)$

$540(11.5)$

Bulgaria

$37(4.0)$

$532(7.2)$

11(5.9) $\quad 44(4.9)$

Chinese Taipei

$30(3.7)$

$537(3.4)$

$0(5.1) 0 \quad 40(4.1)$

$535(3.6)$

$51(2.7)$

$299(6.8)$

$\Delta \diamond$

$2(1.3)$

$\sim \sim \Delta \Delta$

$\begin{array}{lrr}\text { South Africa } & 26(2.9) \\ \text { Macedonia, Rep. of } & \mathrm{r} & 17(3.2)\end{array}$

Qatar $\quad$ r $16(0.2)$

\begin{tabular}{l|l}
\hline Trinidad and Tobago & $15(3.3)$
\end{tabular}

$424(13.1) \quad-24(5.4) \quad \odot \quad 67(4.1)$

$447(6.5)$

$\Delta \Delta$

$345(3.3) \quad \Delta \diamond \quad 39(0.2)$

\section{$346(1.8)$}

$18(6.0)$

$67(4.0)$

$431(6.1)$

- $16(3.3)$

$\sim \sim$

$2(1.3)$

Russian Federation $\quad 14(2.7)$

\begin{tabular}{|l|l|} 
Moldova, Rep. of & $12(2.8)$ \\
\hline
\end{tabular}

$\begin{array}{llll}454(9.0) & \Delta \Delta & & \\ 582(6.0) & -9(3.4) \quad \odot \quad 22(2.2)\end{array}$

$560(7.0)$

00

$\Delta \diamond$

$+18$

$46(0.2)$

$\sim \sim$

Moldova, Rep. of

$517(8.0)$

$6(4.0) \quad 65(3.9)$

Iran, Islamic Rep. of

$9(1.8)$

474 (9.7)

$65(3.9)$

Kuwait

$7(1.9)$

$343(12.3)$

$3(2.3)$

496 (3.8)

$-19(4.4)$

Morocco

\begin{tabular}{|c|c|c|c|c|c|c|}
\hline Morocco & $r$ & $5(2.0)$ & 354 (14.4) & $-56(5.5)$ & $(\nabla$ & $20(4.6)$ \\
\hline Hong Kong SAR & & $4(1.6)$ & $564(11.9)$ & $1(2.1)$ & & $56(4.1)$ \\
\hline Luxembourg & & -- & -- & -- & & - - \\
\hline
\end{tabular}

354 (14.4)

$\Delta \diamond$

$46(3.4)$

$422(4.6)$

$15(6.2)$

$64(3.5)$

$84(3.3)$

$\sim \sim$

International Avg.

$52(0.5) \quad 505(1.0)$

$32(0.6) \quad 496(1.1)$

$3(5.9)$

$24(3.5)$
$45(3.5)$

\begin{tabular}{|l|c|c|}
\hline $422(4.6)$ & $3(5.9)$ & $45(3.5)$ \\
\hline $327(6.8)$ & 00 & $51(4.7)$ \\
\hline $318(22.9)$ & $-5(7.1)$ & $75(4.9)$ \\
\hline
\end{tabular}

$\sim \sim$

$\sim \sim$

Difference

in Percent
from 2001 
Exhibit 7.6 Workspace for Teachers Provided by Schools

PIRLS 2006

4th Grade

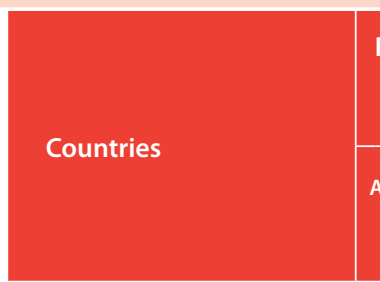

Percent of Students Whose Schools

Provide Teachers with the

Following Facilities

\begin{tabular}{|l|l|}
\hline Austria \\
\hline Belgium (Flemish) \\
\hline Belgium (French) \\
\hline Bulgaria
\end{tabular}

\begin{tabular}{|c|c|c|}
\hline at & $\begin{array}{l}\text { A Workspace } \\
\text { Shared by } \\
\text { Several }\end{array}$ & $\begin{array}{r}\text { A } \\
\text { Wor } \\
\text { Eac }\end{array}$ \\
\hline
\end{tabular}

Percent of Students Whose Teachers

Prepare Materials for Instruction

\section{Canada, Alberta}

$93(2.3)$

$63(3.5)$

$11(2.7)$

\begin{tabular}{l|l|r|}
$84(3.4)$ & $78(4.0)$ & $2(1.4)$
\end{tabular}

$41(4.3)$

$89(3.0)$

$79(3.8)$

$43(4.4)$

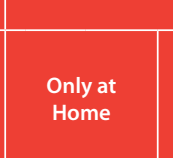

Canada, British Columbia

$92(2.3)$

$82(3.2)$

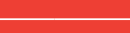

Canada, Nova Scotia

Canada, Ontario

$89(2.7) \quad 71(4.3)$

$6(2.0)$

$19(2.6)$
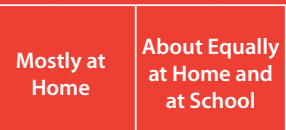

Mostly
at School

Canada, Quebec

$81(2.9)$

$71(4.3)$

$6(2.2)$ ir $0(0.0)$

$37(3.4)$

36 (3.4)

7 (1.7)

$2(1.0)$

Chinese Taipei

$80(4.4) \quad 71(5.0)$

$9(2.3)$

$29(3.2)$

$45(3.3)$

$19(2.9)$

\begin{tabular}{l|l}
$7(1.9)$ & $1(0.4)$
\end{tabular}

Denmark

$97(1.5)-74(4.4)$

$14(3.7)$

$x^{2}$

\section{England}

France

$78(3.2)$

$74(4.4)$

$27(4.1)$

$78(3.2)$

$73(3.4)$

$47(4.3)$

$25(2.8)$

$44(3.2)$

$18(2.7)$

$10(2.4)$

$3(1.1)$

Georg

Germany

79

Hong Kong SAR

Hungary

Iceland

Indonesia

$73(3.4)$
$-82(3.7) \quad 6(2.2)$

$83(3.5) \quad r \quad 4(1.8)$

$79(3.8)$

$83(3.5)$

$4(1.8)$
$36(4.7)$

$29(3.9)$

$49(4.1)$

$21(3.3)$

$12(2.2) \quad 43(3.2)$

$1(0.9)$

$10(2.1) \quad 45(3.8)$

$39(3.3)$

$0(0.0)$

$12(2.3)$

$52(3.8)$

$40(3.9)-\quad 5(1.7)$

Indonia

Iran, Islamic Rep of

$97(1.6)$

$74(3.8)$

$14(3.1)$

$1(0.5)$

$13(3.1)$

$0(0.0)$

$59(4.7)$

$40(3.9)$

$15(2.7)$

$47(4.1)$

$35(3.5)$

$2(1.1)$

81 (3.3)

$26(3.9)$

$2(1.0)$

Israe

Italy

Kuwait

$76(3.5)$

$74(3.8)$

$14(3.1)$

$1(0.8)$
$4(1.7)$

$54(3.7)$

$38(4.0)$

$30(3.9)$

$(3.9) \quad-\quad 7(2.7)$

$1(0.9)$

$22(3.8)$

$51(4.1)$

$17(3.0) \quad 0(0.0)$

$10(2.1)$

48 (3.6)

$4(1.6)$

$10(2.1)$

$23(3.0)$

$30(3.4)$
$56(3.9)$

$12(2.4)$

$12(2.4) \quad 1(0.7)$

$\begin{array}{lll}1(0.2) & 35(3.4)\end{array}$

$45(3.6)$

$17(2.4)$

$6(2.1) \quad 1(0.8)$

$98(1.1)$

$73(3.8)$

$71(4.2)$

$3(1.5)$

$11(2.9)$

$53(4.4)$

$3(1.0) \quad 1(0.4)$

$97(0.1)$

$97(1.5)$

$54(4.4) \quad 21(3.6)$

$53(4.5)$

$22(3.5)$

$32(3.7)$

\begin{tabular}{l}
$90(0.2)-15(0.3)$ \\
\hline
\end{tabular}

$1(0.1) \quad 5(0.2)$

$26(0.3)$

$3(1.6) \quad 1(0.0)$

$\begin{array}{lll}81(3.0) & 78(3.1) & 38(3.8)\end{array}$

$8(2.0) \quad 33(3.8)$

$41(3.9)$

$\begin{array}{ll}61(0.4) & 7(0.2)\end{array}$

\begin{tabular}{l|l}
$70(3.5)$ & 35 \\
$31(3.9)$ & $67(4)$
\end{tabular}

\begin{tabular}{|r|r|r|}
\hline $35(3.6)$ & $42(4.5)$ & $5(1.9)$ \\
\hline
\end{tabular}

$10(2.3)$

$69(3.6)$

$18(3.4) \quad 0(0.0)$

$35(3.6) \quad 42(4.5)$

$42(4.0)$

$69(3.6)$

$12(2.4) \quad 2(1.0)$

$75(3.8)$

$88(2.5) \quad 4(1.6)$

$13(2.4) \quad 50(3.6)$

$34(2.7)$

$1(1.1)$

$\begin{array}{ll}1(1.1) & 0(0.0) \\ 3(1.2) & 0(0.0)\end{array}$

Latvi

Lithuania

$72(4.1)$

$95(1.8) \quad 9(2.3)$

$6(1.9)$

$14(2.8)$

$62(4.0)$

$18(3.2) \quad 1(0.6)$

${ }^{1}$ Luxembourg

Macedonia, Rep. of

$77(3.8)$

\begin{tabular}{l|l|}
$80(3.5)$ & $24(3.8)$
\end{tabular}

$7(2.0)$

$31(3.7)$

$49(3.6)$

$11(2.6)$

$7(2.0)$

$32(3.0)$

$49(3.6)$

\begin{tabular}{l|l}
$11(2.6)$ & $1(1.0)$
\end{tabular}

\begin{tabular}{c|c|r|}
$77(3.9)$ & $56(4.3)$ & $9(1.5)$ \\
\hline-- & -- & $35(0.2)$
\end{tabular}

$40(0.2)$

$22(0.2)$

$5(1.4)$

\begin{tabular}{lll|l}
\hline $0(0.2)$ & $22(0.2)$ & $2(0.0)$ & $0(0.0)$ \\
\hline $5(4.4)$ & $24(3.4)$ & $1(0.7)$ & $0(0.0)$
\end{tabular}

Moldova, Rep. of

$85(3.6) \quad r \quad 60(4.5) \quad r \quad 11(2.6)$

$30(3.7)$

$45(4.4) \quad 24(3.4)$

$1(0.7) \quad 0(0.0)$

\begin{tabular}{l|l|l|}
$98(1.2)$ & $42(4.6)$ & $26(4.1)$ \\
\hline
\end{tabular}

$15(3.0)$

$14(2.7) \quad 66(3.8)$

$27(4.0)$

$66(3.8)$
$3(1.4)$

$6(1.7) \quad 0(0.0)$

Netherlands

New Zealand

$84(3.2)$
$70(4.4)$

$35(4.9) \quad r \quad 50(4.0) \quad 70(4.2)$

\begin{tabular}{|c|c|}
\hline $67(4.2)$ & $r \quad 0(0.0)$ \\
\hline
\end{tabular}

$13(2.9)$

$31(4.1)$

$0(0.0) \quad 0(0.0)$

Norway

Poland

$79(2.8)$

$64(2.8) \quad 6(1.5)$

$1(0.4)$

$15(2.1)$

$47(2.9)$

$51(4.4) \quad 3(1.4)$

$$
\text { Qata }
$$

Romania

Russian Federation

$37(4.2)$

\begin{tabular}{|r|r|r|}
\hline $37(4.5)$ & $62(4.9)$ & $0(0.0)$ \\
\hline $63(3.3)$ & $13(2.9)$ & $31(3.1)$ \\
\hline
\end{tabular}

\begin{tabular}{r|r|r|}
\hline $37(4.5)$ & $62(4.9)$ & $0(0.0)$ \\
\hline $63(3.3)$ & $13(2.9)$ & $31(3.1)$
\end{tabular}

$7(2.0)$

$36(4.1)$

$35(2.7) \quad 3(0.9)$

$7(2.0)$
$45(3.9)$

$36(4.1)$

\begin{tabular}{ll}
$57(4.3) \quad 0(0.0)$ \\
\hline
\end{tabular}

\begin{tabular}{|c|c|c|c|}
\hline & & & קוינן ורנ \\
\hline $79(0.2)$ & $80(0.2)$ & $78(0.2)$ & \\
\hline
\end{tabular}

Scotland

$78(3.2)$

$23(3.2)$

$14(0.2)$

$60(0.2)$

$0(0.0) \quad 0(0.1)$

\begin{tabular}{|c|}
\hline Scotland \\
\hline Singapore \\
\hline
\end{tabular}

Slovak Republic

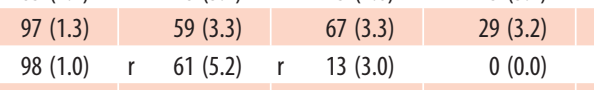

$3(1.6)$

$23(3.2)$

$45(3.6)$

$30(3.5)$

$15(0.2)$

$15(0.2)$

$2(0.1)$

\begin{tabular}{r|r|r|r}
$45(3.6)$ & $30(3.5)$ & $2(1.2)$ & $0(0.1)$ \\
\hline $39(3.7)$ & $28(3.1)$ & $4(1.4)$ & $0(0.0)$ \\
$20(3.5)$ & $55(4.4)$ & $23(3.9)$ & $2(1.0)$
\end{tabular}

\begin{tabular}{|c|c|c|c|}
\hline (3.6) & $30(3.5)$ & $2(1.2)$ & $0(0.1)$ \\
\hline & $28(3.1)$ & $4(1.4)$ & $0(0.0)$ \\
\hline & $55(4.4)$ & $23(3.9)$ & $2(1.0)$ \\
\hline
\end{tabular}

Slovak Republic

Slovenia

\begin{tabular}{|r|r|r|r|r|}
\hline $88(0.0)$ & $76(0.0)$ & $93(0.0)$ & $2(0.7)$ & $24(2.2)$ \\
\hline $73(3.0)$ & $87(2.8)$ & $3(1.4)$ & $7(2.0)$ & $36(3.4)$ \\
\hline $87(2.7)$ & $92(2.1)$ & $24(2.8)$ & $8(2.0)$ & $60(2.9)$ \\
\hline
\end{tabular}

\begin{tabular}{|r|r|r|r|r|}
\hline $88(0.0)$ & $76(0.0)$ & $93(0.0)$ & $2(0.7)$ & $24(2.2)$ \\
\hline $73(3.0)$ & $87(2.8)$ & $3(1.4)$ & $7(2.0)$ & $36(3.4)$ \\
\hline $87(2.7)$ & $92(2.1)$ & $24(2.8)$ & $8(2.0)$ & $60(2.9)$ \\
\hline
\end{tabular}

\begin{tabular}{l|l|l|}
$55(4.4)$ & $23(3.9)$ & $2(1.0)$ \\
\hline $52(2.8)$ & $21(2.5)$ & $1(0.5)$
\end{tabular}

South Africa

\begin{tabular}{l|l} 
Spain & 8013 \\
\hline
\end{tabular}

Spain

\begin{tabular}{|c|c|c|c|c|c|c|c|}
\hline $88(0.0)$ & $76(0.0)$ & $93(0.0)$ & $2(0.7)$ & $24(2.2)$ & $52(2.8)$ & $21(2.5)$ & $1(0.5)$ \\
\hline $73(3.0)$ & $87(2.8)$ & $3(1.4)$ & $7(2.0)$ & $36(3.4)$ & $50(3.8)$ & 7 (1.5) & $0(0.0)$ \\
\hline $87(2.7)$ & $92(2.1)$ & $24(2.8)$ & $8(2.0)$ & $60(2.9)$ & $26(3.0)$ & $6(1.5)$ & $0(0.0)$ \\
\hline $84(2.0)$ & $54(3.3)$ & $16(2.1)$ & $5(1.7)$ & $22(2.4)$ & $60(3.1)$ & $13(2.0)$ & $0(0.1)$ \\
\hline $80(3.5)$ & 95 (1.4) & $5(1.6)$ & $2(1.3)$ & $21(3.3)$ & $48(4.2)$ & $28(3.7)$ & $1(0.7)$ \\
\hline $74(3.9)$ & $79(3.4)$ & $27(4.3)$ & $0(0.0)$ & $15(2.7)$ & $40(4.0)$ & $42(3.6)$ & $2(1.0)$ \\
\hline $59(3.8)$ & $38(3.8)$ & $9(2.6)$ & $2(1.3)$ & $29(3.8)$ & $49(3.7)$ & $19(3.0)$ & $1(0.5)$ \\
\hline $91(2.0)$ & $74(3.4)$ & $14(3.1)$ & $0(0.0)$ & $13(2.5)$ & $47(3.4)$ & $36(3.4)$ & $3(0.9)$ \\
\hline
\end{tabular}

International Avg.

$81(0.5)$

$14(3.1)$

\begin{tabular}{|l|l|l|}
\hline $13(0.4)$ & $30(0.5)$ & $40(0.5)$ \\
\hline
\end{tabular}

$1(0.1)$

Background data provided by teachers and schools.

() Standard errors appear in parentheses. Because results are rounded to the nearest whole number, some totals may appear inconsistent.

An " $r$ " indicates data are available for $70-84 \%$ of the students. An " $s$ " indicates data are An " $r$ " indicates data are available for $70-84 \%$ of the students. An "s" indicates data ar
available for $50-69 \%$ of the students. An " $x$ " indicates data are available for less than
$50 \%$ of the students. 
Exhibit 7.7 Schools' Reports of Availability of Computers for Instructional Purposes with Trends

PIRLS 2006

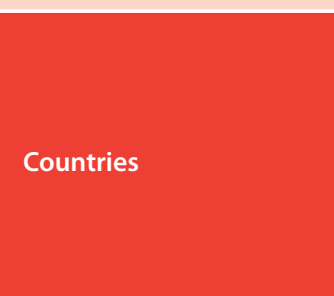

Percentage of Students by Number of Fourth-Grade* Students per Computer

Canada, Alberta
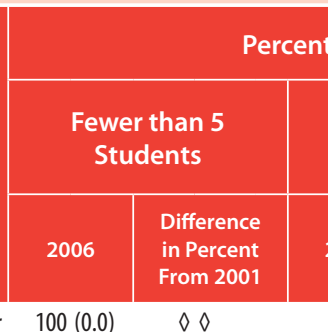

Denmark

$97(1.5)$

$\Delta \Delta$

Canada, British Columbia r

$95(2.0)$

00

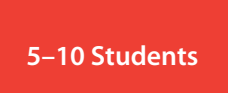

11

Canada, Ontario

$95(2.4) \quad 13(5.0) \quad 0$

England

Iceland

Scotland

$94(1.8) \quad 35(5.0) \quad 0$

$4(2.2) \quad-7(4.2)$

United States

$92(0.3)$

$15(0.4) \quad 0$

$5(1.6)$

$0(0.0)$

$2(1.2)$

$\Delta \Delta$

$46(6.2) \quad 0$

$5(0.2)$

$-19(4.1)$

20 (4.9) 0

$6(2.7)$

$-4(0.3)$

\begin{tabular}{|c|c|}
\hline $11-20$ & Students \\
2006 & $\begin{array}{l}\text { Difference } \\
\text { in Percent } \\
\text { From } 2001\end{array}$ \\
\hline $0(0.0)$ & $\diamond \diamond$ \\
\hline
\end{tabular}

Hong Kong SAR

$84(3.2)$

$27(5.9) \quad 14(3.1)$

Singapore

Canada, Quebec

$82(3.6)$

$81(0.0)$

$14(4.6)$

$-10(4.3) \odot$

$0(0.0)$

$1(0.0)$

$1(0.1)$

00

$\Delta \Delta$

$2(0.9)--11(3.7)$

More than

20 Students

Students in Schools

$79(3.9)$

$-17(5.0)$

$1(0.2)$

$-11(3.7)$

2006

Difference

in Percent From 2001

Spain

$79(2.9)$

$15(6.4) \quad 0 \quad 17(4.2)$

$-9(4.1)$

5 (2.4)

$-3(0.2)$

$0(0.0)$

$\Delta \Delta$

$1(0.0)$

$\Delta 0$

$\Delta \Delta$

$0(0.0) \quad-2(0.1)$

$0(0.0) \quad-5(2.0) \quad-\quad$

$2(0.0)$

$-2(0.1)$

$\begin{array}{llllll}4(1.6) & -7(3.0) & 0 & 0(0.0) & -3(1.5) & \odot\end{array}$

$1(1.0) \quad-1(1.6)$

$3(0.0)$

$-2(2.1)$

$3(1.6)$

$-3(1.5)$

$0(0.0)$

$-2(1.2)$

$2(1.0)$

$-10(3.6) \odot \quad 1(0.1)$

0 (1.1)

$3(1.4)$

$15(2.7)$

$-3(5.5)$

$\diamond \diamond$

$-14(3.6)$

$2(1.4)$

$-2(2.5)$

$1(0.7)$

$\Delta \diamond$

$1(0.7)$

$-15(4.1) \odot \quad 1(0.6)$

$-2(1.9)$

$5(2.0) \quad \Delta 0$

Canada, Nova Scotia

$74(3.7)$

$72(3.8) \bullet \quad 23(3.6)$

$6(5.2)$

$21(3.2) \quad \Delta 0$

$1(0.0) \quad \Delta \diamond$

$\Delta \Delta$

$27(4.2) \quad \Delta 0$

$1(0.5)$

$\Delta \Delta$

$0(0.0)$

$\Delta 0$

$0(0.0) \quad \Delta 0$

$\Delta \Delta$

$0(0.0)$

$3(2.7)$

$2(1.3) \quad 2(1.3)$

$2(1.3)$
$-3(2.0)$

$10(3.1)$

$-4(3.2)$

$1(0.0)$

$-3(2.0)$

$24(6.7) \bigcirc \quad 26(4.5) \quad-12(7.0)$

Bulgaria

Belgium (Flemish)

$61(3.7)$

$56(4.2) \otimes$

France

Qatar

$61(4.8)$

$\Delta \Delta$

$26(4.4)$

$18(4.9)$

$3(1.5)$

$-10(5.2)$

$3(1.0)$

$\Delta \diamond$

$6(2.3) \quad-7(4.4)$

$2(1.1)$

$4(1.9) \quad-10(4.1) \quad \odot$

\begin{tabular}{lllll|l|l}
\hline $9(6.6)$ & $\bullet$ & $26(4.7)$ & $-3(6.5)$ & $6(2.3)$ & $-7(4.4)$ \\
\hline$\Delta$ & $30(0.3)$ & $\Delta \diamond$ & $3(0.1)$ & $\Delta \diamond$
\end{tabular}

$57(0.3) \quad \Delta 0$

$3(0.1)$

$2(1.7)$

$1(0.1)$

00

$1(2.2)$

$5(2.1) \quad \diamond \diamond$

$6(1.6)$

$\diamond \diamond$

\begin{tabular}{l|lll|l}
$11(3.0)$ & $-18(5.5)$ & $\odot$ & $2(1.0)$ & $-3(2.4)$
\end{tabular}

$2(3.4)$

$4(7.0)$

$\begin{array}{rr}11(3.0) & -18(5.5) \\ 14(2.9) & 0(4.4)\end{array}$

$8(2.5)$

$2(3.4)$

$19(5.9) \quad 0 \quad 27(4.0)$

$-2(5.8)$

Italy

$42(4.2)$

$28(4.7) \quad 0 \quad 44(4.3)$

$\begin{array}{llll}-3(5.1) & & 3(1.5) & -3(3.0) \\ 15(5.5) & 0 & 8(3.3) & -8(4.8)\end{array}$

(2.6) $-18(4.2)$

$4(1.8)$

$\begin{array}{rrrrrr}4(1.8) & -17(3.3) & \uparrow & 1(0.8) & -7(1.7) & \uparrow \\ 4(1.8) & 1(2.4) & & 34(4.3) & -18(6.0) & \bullet\end{array}$

$22(4.9) \quad 0 \quad 17(3.1)$

Romania

$41(4.8)$

$36(5.3) \circlearrowright \quad 28(3.9)$

$\begin{array}{rrrr}15(5.5) & 0 & 8(3.3) & -8(4.8)\end{array}$

$2(1.4)$

$-13(3.8) \odot \quad 20(3.3) \quad-30(5.5)$

Russian Federation

$40(3.5) \quad 36(4.2) \quad 0 \quad 28(2.8)$

Germany

Chinese Taipei

$39(3.1) \quad 26(4.2) \quad 0$

$17(5.0) \quad 14(2.8) \quad-3(4.5)$

$2(1.0)$

$2(1.0) \quad 0$

$20(3.3)-30(5.5) \quad-$

$26(4.2) \quad 40(3.5)$

$\Delta 0$

$9(2.1) \quad \Delta \Delta$

Poland

Austria

$35(4.4) \quad 0$

00

$48(4.0)$

$\diamond \diamond$

$12(2.5)$

$\diamond \diamond$

$23(3.9)$

$\Delta \diamond$

$8(1.8)-21(3.9) \quad-$

$1(0.4) \quad-19(3.3)$

Trinidad and Tobago

$30(4.2) \quad \Delta \Delta$

$33(4.0)$

$\Delta \Delta$

$15(3.6)$

$\Delta 0$

$14(3.4) \quad x x$

Morocco

$11(2.7)$

$x x \quad 20(3.6)$

$x \times$

$2(0.8)$

$x x$
$-4(2.6)$

$9(3.0)$

Macedonia, Rep. of $\quad s \quad 6(2.4)$

Indonesia

Georgia

$4(1.6)$

$6(2.4) \otimes 24(4.5)$

$-8(5.0)$

$9(3.0)$

$\Delta 0$

$4(1.7) \quad \diamond \diamond$

$4(1.3) \quad \Delta 0$

$\Delta \Delta$

$1(0.3)$

$1(0.3)$

$4(1.2)$

4 (1.2)

$2(1.0) \quad 2(1.0)$

$5(1.9) \quad \diamond \diamond$

$7(2.3)$

$7(2.3)$

$\Delta 0$

$0(0.0) \quad \diamond 0$

1 Luxembourg

International Avg.

$53(0.6)$

$21(0.5)$

$6(0.3)$

$4(2.0)$

$28(5.2)$

$4(1.8) \quad 3(18)$

$\Delta \diamond$
$\times x$
$3(1.8)$

$3(1.0) \quad-3(2.5)$

$5(1.8) \quad \diamond 0$

$9(2.1) \quad \diamond \diamond$

$12(2.7) \quad 10(2.9) \quad 0$

$22(4.0) \quad \Delta \Delta$

$1(0.6) \quad \diamond \diamond$

$19(3.9) \quad \diamond \diamond$

\begin{tabular}{ll}
$27(4.2)$ & $x x$ \\
\hline $77(3.8)$ & $2(6.5)$
\end{tabular}

$58(5.1) \quad-34(5.8)$

$--$

$--\quad--$

$2(1.0)$

$--$

$84(2.8) \quad \Delta 0$

$80(3.2) \quad \diamond \diamond$

$81(3.1) \quad-16(3.3) \quad \odot$

Dercent in 2006 significantly higher

( Percent in 2006 significantly lower

Background data provided by schools.

* Fourth grade in most countries.

() Standard errors appear in parentheses. Because results are rounded to the nearest whole number, some totals may appear inconsistent.

An " $r$ " indicates data are available for $70-84 \%$ of the students. An "s" indicates data are available for $50-69 \%$ of the students. An " $x$ " indicates data are available for less than $50 \%$ of the students.
A dash (-) indicates comparable data are not available.

A diamond $(\diamond)$ indicates the country did not participate in the 2001 assessment.

NOTE: The International Average does not include the results from the Canadian provinces. Trend Note: The primary education systems of the Russian Federation and Slovenia underwent structural changes. Data for Canada, Ontario include only public schools.

1 Primary schools in Luxembourg do not have principals. 
It is clear from Exhibit 7.7 that the student-to-computer ratio has improved since 2001. Every country that participated in PIRLS 2001 had a greater percentage of students in 2006 in schools with fewer than five students per computer-most notably the Slovak Republic (an increase of 72 percentage points) and Bulgaria (a 56-point increase). A number of countries also made good progress in reducing the percentage of students in schools without any computers, especially the Slovak Republic (a 66-point decrease), the Russian Federation (a 65-point decrease), and Bulgaria (a 58-point decrease), but also Macedonia (34-point decrease) and Romania (3o-point decrease).

\section{What Is the Level of Home-School Involvement?}

To provide information on parents' involvement with their children's school and on the degree of communication between the home and the school, PIRLS 2006 created an Index of Home-School Involvement. As described in Exhibit 7.8, the index is based on principals' responses to seven questions, including four questions about frequency of communication from the school to the home (teacher-parent conferences; letters, newsletters, etc., sent home; written reports of child's performance; events at school to which parents are invited) and three questions about the percentage of students with parents who participate in the life of the school (volunteer regularly to help in the classroom or school; attend teacher-parent conferences; attend cultural, sporting, or social events at the school).

Students were assigned to the high level of the index if they attended a school that, at least four times a year, held teacher-parent conferences and school events that were attended by more than half the parents; sent home written report cards of the child's performance at least four times a year; and sent home letters, calendars, newsletters, etc., with news about the school at least seven times a year. Students were assigned to the low level of the index if their school never held a teacher-parent conference, or if it did, no more than 25 percent of parents attended; held school events no more than once a year that were attended by no more than 25 percent of parents; sent home 
letters or newsletters no more than three times a year; and written reports of the child's performance no more than once a year. Students in schools with other combinations were assigned to the medium level.

As shown in Exhibit 7.8, almost half the students (48\%), on average internationally, were at the high level of the Home-School Involvement Index, one quarter $(25 \%)$ at the medium level, and about the same $(27 \%)$ at the low level. Within the levels, however, there was great variation across countries. Home-school involvement appeared to be strongest in the Netherlands, the United States, and four of the Canadian provinces (British Columbia, Nova Scotia, Alberta, and Ontario), where more than 90 percent of students were at the high level of the index. In contrast, more than 60 percent of students in Poland, Bulgaria, Moldova, Macedonia, Morocco, Georgia, and Indonesia were at the low level of the index. Among countries that participated also in PIRLS 2001, increased percentages of students at the high level of the index were found in Iceland, Singapore, Slovenia, Sweden, Germany, Italy, Hungary, Latvia, Norway, England, and Bulgaria. On average across countries, there was a modest positive relationship between the level of home-school involvement and average reading achievement-an average of 504 points for the high level, 499 for the medium level, and 490 for the low level.

Further details on communications with parents are provided in Exhibit 7.9, which presents teachers' reports on meeting or talking individually with the child's parents to discuss progress in reading, and on sending home to the child's parents progress reports on reading. For most teachers in most countries, meeting individually with parents was at most a bimonthly affair. On average across countries, 71 percent of students had teachers who reported meeting or talking individually with parents no more often than six times a year. More frequent encounters were reported by teachers in several of the Eastern European countries (Bulgaria, Georgia, Hungary, Macedonia, Moldova, Poland, Romania, the Russian Federation, and Slovenia) and in Iran and Kuwait, where approximately half the students had teachers reporting monthly meetings. On average, 77 percent of students had teachers reporting sending home progress reports on reading six times a 
Exhibit 7.8 Index of Home-School Involvement (HSI) with Trends

PIRLS 2006

Countries

\begin{tabular}{|c|c|c|}
\multicolumn{2}{|c}{ High HSI } \\
\hline $\begin{array}{c}2006 \\
\text { Percent } \\
\text { of Students }\end{array}$ & $\begin{array}{c}\text { Average } \\
\text { Achievement }\end{array}$ & \\
\hline
\end{tabular}

\begin{tabular}{l|l} 
& \\
\hline Difference & \\
in Percent & \\
from 2001 &
\end{tabular}

\begin{tabular}{|c|c|}
\hline $\begin{array}{c}2006 \\
\text { Percent } \\
\text { of Students }\end{array}$ & Ach \\
\hline $1(0.0)$ \\
\hline
\end{tabular}

Canada, British Columbia

$99(0.7)$

Canada, Nova Scotia

$98(1.0)$

558 (2.6)

$\Delta \diamond$

Canada, Alberta

97 (1.1)

Canada, Ontario

$543(2.2)$

$\Delta \nabla$

96 (1.9)

561 (2.4)

$\Delta \Delta$

$1(0.7)$

Netherlands

555 (2.9)

$-2(2.2)$

2 (0.6)

United States

94 (2.0)

546 (1.7)

2 (3.2)

4 (1.9)

Belgium (Flemish)

$93(2.0)$

542 (3.7)

-4 (2.4)

5 (1.9)

Canada, Quebec

$81(4.0)$

$546(2.2)$

$\diamond \diamond$

$5(1.4)$

Iceland

$535(3.0)-11(5.0)$

Medium HSI

$\begin{array}{rrrr}535(3.0) & -11(5.0) & \nabla & 16(3.9) \\ 511(1.5) & 29(0.5) & \bigotimes & 11(0.2)\end{array}$

Kuwait

$80(0.3)$

Singapore

$75(0.0)$

$323(5.3)$

$19(3.5)$

Israel

Spain

$74(3.3)$

$559(3.5)$

$12(4.4) \quad 0 \quad 20(0.0)$

\begin{tabular}{l|l|l|}
$529(4.5)$ & $8(5.1)$ & $13(2.8)$
\end{tabular}

$66(4.5)$

$514(2.9)$

$\diamond \diamond$

19 (3.6)

New Zealand

$66(3.4)$

Slovenia

64 (4.2)

$534(2.9) \quad 0(5.3)$

$32(3.2)$

Average

$523(2.6) \quad 33(5.6) \quad$ ○ $\quad 19(3.4)$

Achievement

$503(3.5) \quad \Delta \nabla$

$62(4.3)$

551 (2.9)

$26(4.4)$

Sweden

$59(4.3)$

565 (3.1)

$9(5.8) \quad 0 \quad 25(3.7)$

France

56 (3.8)

$519(3.1)$

$6(6.4)$

19 (3.3)

$\sim \sim$

$\sim \sim \Delta$

$\sim \sim \Delta$

545 (14.8) $\quad 2(2.2)$

$556(6.5)$

$514(9.6) \quad 2(2.0)$

$551(5.2) \quad \Delta \diamond$

$528(8.1)$

510 (3.7)

$339(10.1)$

$8(5.0)$

$561(6.2)$

$\diamond \diamond$

$479(12.8)-12(4.8)-5(0.0)$

$507(9.1)$

$-12(4.8)$

$527(5.6)$

$\diamond \diamond$

$520(5.8)$

$1(5.1)$

$496(7.3)$

$-10(5.1)$

$546(3.8)$

$\diamond 0$

० $11(3.0)$

$11(3.0)$

$562(6.3) \quad-4(5.1)$

$20(3.6)$

$523(5.5) \quad-4(5.9)$

$0(6.2)$

$\begin{array}{llll}553(3.2) & 16(5.1) & \mathbf{0} & 30(2.9)\end{array}$

545 (3.4)

1 (4.6)

$\begin{array}{lllll}54(3.7) & 552(4.3) & 12(5.0) & \text { ○ } & 27(3.6)\end{array}$

Italy

\begin{tabular}{lll|l}
$553(4.9)$ & $20(5.4)$ & 0 & $23(3.6)$
\end{tabular}

South Africa

$51(2.7)$

$349(10.9)$

$551(5.0)$

$-3(4.8)$

$28(2.5)$

$541(7.3)$

$-17(5.2)$

$260(8.3)$

$\diamond \diamond$

\begin{tabular}{l|ll|l}
$541(4.0)$ & $21(5.9)$ & Q & $33(3.9)$
\end{tabular}

541 (3.4)

$2(6.0)$

21 (3.2)

$419(9.3)$

$2(6.0)$
$-4(5.0)$

551 (3.5)

$\Delta 0$

44 (5.2)

532 (4.1)

$-9(6.5)$

$522(5.4) \quad 11(6.8)$

Scotland

46 (5.3)

499 (3.5)

$21(6.8) \quad$ Q $30(4.4)$

496 (4.7)

20 (3.5)

$496(4.7) \quad-5(6.7)$

45 (4.1)

$433(7.4) \quad \Delta \diamond$

$34(0.2)$

$343(1.9)$

$\diamond \diamond$

42 (3.4)

343 (1.9)

$\diamond \diamond$

0 (5.5)

564 (4.3)

$-1(5.8)$

\begin{tabular}{ll|l}
$568(5.1)$ & $0(5.5)$ & $32(3.4)$ \\
$540(2.9)$ & $\Delta \diamond$ & $33(3.9)$
\end{tabular}

$532(3.3)$

$\diamond \diamond$

$33(3.9)$

$536(3.6)$

$\Delta \Delta$

$52(4.6)$

$538(2.6)$

$-2(6.1)$
$-9(5.4)$

$26(3.4)$

$536(5.4)$

$-9(5.4)$

$537(3.8) \quad-13(5.9)$

$34(4.4)$

$483(7.9) \quad-7(6.5)$

Romania 26 (4.2)

$\begin{array}{llll}555(7.3) & 12(5.2) & 0 & 58(4.2) \\ 508(7.6) & -1(5.8) & & 34(4.4)\end{array}$

$\begin{array}{llll}519(4.0) & \Delta \diamond & & 16(3.4) \\ 558(10.0) & 9(3.8) & \bullet & 19(3.3)\end{array}$

$511(7.8)$

$\diamond \diamond$

Moldova, Rep. of $17(3.2)$

\begin{tabular}{|l|l|l|}
\hline $493(6.8)$ & $1(4.4)$ & $24(4.0)$ \\
\hline
\end{tabular}

$549(13.3)$

$9(4.0)$

$17(3.5)$

$515(7.6) \quad 5(5.2)$

Macedonia, Rep. of $\quad r \quad 14$ (3.2)

Morocco

$437(17.2) \quad 4(4.3)$

$10(2.7)$

466 (15.5)

0 (4.9)

(2)

$22(3.9)$

25 (3.4)

$16(2.6)$

$19(3.4)$

$26(3.5)$

$21(2.2)$

\begin{tabular}{l} 
Low HSI \\
\hline $\begin{array}{c}\text { Average } \\
\text { Achievement }\end{array}$ \\
\hline
\end{tabular}

4th Grade

Georgia

$11(2.7)$

381 (14.4)

$5(4.0)$

$24(3.9)$

349 (13.3) $\quad 1(4.0)$

$32(3.5)$

$10(2.7)$

$10(3.3)$

25 (4.6)

$\sim \sim$

in Percent

from 2001

Indonesia

8 (2.2)

$420(19.3) \quad \Delta \diamond$

$16(3.0)$

1 Luxembourg

International Avg.

$48(0.6)$

$504(1.1)$

$25(0.5)$

$488(7.8)$

$\Delta \diamond$

$415(9.1) \quad \diamond \diamond$

$35(4.3)$

$23(0.2)$

$17(3.1)$

$\begin{array}{lll}27(3.8) & 533(3.9) & \diamond \diamond\end{array}$

$30(3.8) \quad 538(5.1) \quad \diamond \diamond$

\begin{tabular}{l|l|l|l|}
$16(2.8)$ & $547(4.1)$ & $-4(4.4)$
\end{tabular}

$43(3.9) \quad 524(5.7) \quad 2(5.5)$

\begin{tabular}{l|l|l|}
$15(3.1)$ & $537(10.7)$ & $1(4.4)$
\end{tabular}

$40(4.2) \quad 482(7.7) \quad 8(6.1)$

\begin{tabular}{c|c|c}
$64(4.6)$ & $522(3.1)$ & $\diamond \diamond$ \\
\hline $64(4.0)$ & $544(4.9)$ & $-18(4.9)$
\end{tabular}

\begin{tabular}{l|l|l|}
$60(4.7)$ & $496(4.1)$ & $-6(6.1)$
\end{tabular}

$69(4.4) \quad 444(6.7) \quad-5(6.2)$

\begin{tabular}{l|l|l|}
$77(4.0)$ & $314(8.6)$ & $-7(5.6)$
\end{tabular}

$\begin{array}{lll}77(4.0) & 314(8.6) & -7(5.6) \\ 65(4.2) & 465(3.7) & \diamond \diamond\end{array}$

\begin{tabular}{l|l|l}
$76(3.5)$ & $401(4.8) \quad 0 \diamond$
\end{tabular}

$\begin{array}{ccc}76(3.5) & 401(4.8) & 0 \diamond \\ -- & -- & --\end{array}$

$27(0.5) \quad 490(1.5)$

Based on principals' responses to the following questions: How often is each of the following provided by your school for fourth-grade students and/or their families?

Approximately what percentage of students in your school have parents or guardians who do each of the following? High level indicates that 4 or more times a year, schools hold teacher-parent conferences and events at school to which parents are invited that are attended by more than half of the parents, send home letters, calendars, newsletters, etc. with information about the school 7 or more times a year, and send written reports (report cards) of child's performance 4 or more times a year. Low level indicates schools never hold teacher-parent conferences, or if they do, only between $0-25 \%$ of parents attend; schools hold events to which parents are invited once a year or less, to which $0-25 \%$ of parents attend; letters, calendars, newsletters, etc., are sent home 3 times a year or less; and written reports of children's performance is sent home once a year or less. Medium level indicates all other combinations.

() Standard errors appear in parentheses. Because results are rounded to the nearest whole number, some totals may appear inconsistent.

An " $r$ " indicates data are available for $70-84 \%$ of the students. An " $s$ " indicates data are available for $50-69 \%$ of the students. An " $x$ " indicates data are available for less than $50 \%$ of the students.

A dash (-) indicates comparable data are not available. A tilde $(\sim)$ indicates insufficient data to report achievement.

A diamond $(\nabla)$ indicates the country did not participate in the 2001 assessment.

NOTE: The International Average does not include the results from the Canadian provinces.

Trend Note: The primary education systems of the Russian Federation and Slovenia underwent structural changes. Data for Canada, Ontario include only public schools.

1 Primary schools in Luxembourg do not have principals. 


\begin{tabular}{|c|c|c|c|c|c|c|}
\hline \multirow{2}{*}{ Countries } & \multicolumn{3}{|c|}{$\begin{array}{c}\text { Meeting or Talking Individually } \\
\text { with the Child's Parents to Discuss } \\
\text { His/Her Progress in Reading }\end{array}$} & \multicolumn{3}{|c|}{$\begin{array}{c}\text { Sending a Progress Report on } \\
\text { the Child's Reading } \\
\text { Home to His/Her Parents }\end{array}$} \\
\hline & Weekly & Monthly & $\begin{array}{l}6 \text { Times } \\
\text { a Year } \\
\text { or Fewer }\end{array}$ & Weekly & Monthly & $\begin{array}{l}6 \text { Times } \\
\text { a Year } \\
\text { or Fewer }\end{array}$ \\
\hline Austria & $0(0.0)$ & $7(1.7)$ & $93(1.7)$ & $1(0.7)$ & $4(1.6)$ & $94(1.7)$ \\
\hline Belgium (Flemish) & $0(0.0)$ & $2(1.4)$ & $98(1.4)$ & $1(1.0)$ & $9(2.5)$ & $90(2.6)$ \\
\hline Belgium (French) & $1(0.7)$ & $6(1.6)$ & $93(1.6)$ & $0(0.0)$ & $2(0.8)$ & $98(0.8)$ \\
\hline Bulgaria & $11(2.3)$ & $48(3.8)$ & $41(3.5)$ & $13(2.7)$ & $37(3.9)$ & $51(4.1)$ \\
\hline Canada, Alberta & $1(0.7)$ & $6(1.8)$ & $93(1.8)$ & $1(0.6)$ & $3(1.2)$ & $96(1.3)$ \\
\hline Canada, British Columbia & $0(0.0)$ & $6(2.2)$ & $94(2.3)$ & $1(0.1)$ & $4(1.8)$ & $95(2.2)$ \\
\hline Canada, Nova Scotia & $0(0.0)$ & $3(1.3)$ & $97(1.3)$ & $0(0.0)$ & $3(1.5)$ & $96(1.5)$ \\
\hline Canada, Ontario & $0(0.0)$ & $4(1.7)$ & $96(1.7)$ & $1(0.2)$ & $6(1.7)$ & $94(1.6)$ \\
\hline Canada, Quebec & $1(0.0)$ & $6(2.2)$ & $93(2.5)$ & $18(3.8)$ & $30(4.5)$ & $52(4.4)$ \\
\hline Chinese Taipei & $0(0.0)$ & $16(3.2)$ & $84(3.2)$ & $5(1.9)$ & $21(3.6)$ & $74(3.6)$ \\
\hline Denmark & $0(0.0)$ & $2(1.0)$ & $98(1.0)$ & $0(0.0)$ & $3(1.4)$ & $97(1.4)$ \\
\hline England & $0(0.0)$ & $2(1.0)$ & $98(1.0)$ & $1(0.8)$ & $2(1.2)$ & $97(1.4)$ \\
\hline France & $0(0.0)$ & $7(1.8)$ & $93(1.8)$ & $1(0.0)$ & $3(1.2)$ & $96(1.2)$ \\
\hline Georgia & $45(4.0)$ & $49(3.9)$ & $7(2.0)$ & $25(3.5)$ & $44(3.9)$ & $31(3.7)$ \\
\hline Germany & $1(0.0)$ & $6(1.9)$ & $93(2.0)$ & $0(0.1)$ & $0(0.1)$ & $100(0.2)$ \\
\hline Hong Kong SAR & $1(0.0)$ & $7(1.9)$ & $92(2.1)$ & $0(0.0)$ & $3(1.5)$ & $97(1.5)$ \\
\hline Hungary & $5(1.5)$ & $43(4.0)$ & $52(4.2)$ & $2(0.9)$ & $21(3.4)$ & $77(3.4)$ \\
\hline Iceland & $0(0.0)$ & $3(0.1)$ & $97(0.1)$ & $0(0.1)$ & $4(0.1)$ & $96(0.2)$ \\
\hline Indonesia & $5(2.0)$ & $27(3.4)$ & $67(3.6)$ & $23(3.3)$ & $29(4.2)$ & $48(4.4)$ \\
\hline Iran, Islamic Rep. of & $10(1.7)$ & $62(3.7)$ & $28(3.5)$ & $17(2.6)$ & $51(3.3)$ & $32(2.9)$ \\
\hline Israel & $4(1.7)$ & $24(3.9)$ & $73(4.3)$ & $1(0.8)$ & $20(3.3)$ & $79(3.3)$ \\
\hline Italy & $2(1.1)$ & $21(2.7)$ & $77(2.7)$ & $10(2.3)$ & $24(3.1)$ & $66(3.3)$ \\
\hline Kuwait & $13(2.8)$ & $49(4.3)$ & $39(4.1)$ & $8(2.2)$ & $25(3.8)$ & $67(3.8)$ \\
\hline Latvia & $3(0.8)$ & $21(2.9)$ & $76(3.0)$ & $2(1.2)$ & $12(2.3)$ & $85(2.4)$ \\
\hline Lithuania & $4(1.5)$ & $35(3.4)$ & $61(3.4)$ & $29(3.4)$ & $32(3.4)$ & $40(3.1)$ \\
\hline Luxembourg & $1(0.1)$ & $7(0.1)$ & $92(0.1)$ & $1(0.0)$ & $0(0.0)$ & $99(0.0)$ \\
\hline Macedonia, Rep. of & $19(3.6)$ & $56(4.3)$ & $25(3.8)$ & $8(2.2)$ & $38(4.0)$ & $55(4.1)$ \\
\hline Moldova, Rep. of & $21(3.5)$ & $50(4.0)$ & $30(4.1)$ & $22(3.5)$ & $36(3.7)$ & $42(3.9)$ \\
\hline Morocco & $7(2.8)$ & $20(3.4)$ & $73(3.3)$ & $7(2.3)$ & $33(4.4)$ & $60(4.5)$ \\
\hline Netherlands & $0(0.0)$ & $1(0.9)$ & $99(0.9)$ & $1(0.9)$ & $7(2.1)$ & $92(2.3)$ \\
\hline New Zealand & $1(0.4)$ & $5(1.3)$ & $95(1.3)$ & $0(0.0)$ & $3(1.0)$ & $97(0.9)$ \\
\hline Norway & $0(0.0)$ & $0(0.5)$ & $100(0.5)$ & $0(0.3)$ & $2(1.1)$ & $98(1.1)$ \\
\hline Poland & $3(1.2)$ & $47(3.6)$ & $50(3.6)$ & $4(1.3)$ & $15(2.9)$ & $81(2.9)$ \\
\hline Qatar & $9(0.2)$ & $36(0.2)$ & $55(0.3)$ & $10(0.1)$ & $45(0.2)$ & $45(0.2)$ \\
\hline Romania & $22(3.4)$ & $66(4.1)$ & $12(2.3)$ & $14(3.2)$ & $33(4.1)$ & $53(4.1)$ \\
\hline Russian Federation & $13(2.3)$ & $55(3.2)$ & $32(3.2)$ & $12(2.5)$ & $36(3.2)$ & $53(3.5)$ \\
\hline Scotland & $0(0.0)$ & $0(0.0)$ & $100(0.4)$ & $4(2.0)$ & $1(0.8)$ & $95(2.1)$ \\
\hline Singapore & $3(0.8)$ & $14(1.9)$ & $83(1.9)$ & $0(0.0)$ & $6(1.4)$ & $94(1.4)$ \\
\hline Slovak Republic & $1(0.8)$ & $29(3.2)$ & $70(3.2)$ & $9(1.8)$ & $18(2.8)$ & $73(3.2)$ \\
\hline Slovenia & $0(0.0)$ & $53(3.1)$ & $47(3.1)$ & $0(0.0)$ & $7(1.4)$ & $93(1.4)$ \\
\hline South Africa & $3(0.9)$ & $29(2.7)$ & $69(2.6)$ & -- & -- & -- \\
\hline Spain & $5(1.8)$ & $15(2.7)$ & $80(3.3)$ & $4(1.5)$ & $10(2.6)$ & $86(3.0)$ \\
\hline Sweden & $0(0.1)$ & $1(0.5)$ & $99(0.5)$ & $1(0.7)$ & $0(0.2)$ & $99(0.7)$ \\
\hline Trinidad and Tobago & $0(0.4)$ & $27(3.6)$ & $72(3.6)$ & $4(1.3)$ & $15(2.8)$ & $81(2.9)$ \\
\hline United States & $3(1.2)$ & $12(2.4)$ & $85(2.7)$ & $12(2.4)$ & $14(2.3)$ & $74(3.2)$ \\
\hline International Avg. & $5(0.3)$ & $24(0.4)$ & $71(0.4)$ & $6(0.3)$ & $17(0.4)$ & $77(0.4)$ \\
\hline
\end{tabular}

Background data provided by teachers.

() Standard errors appear in parentheses. Because results are rounded to the nearest whole number, some totals may appear inconsistent.

An " $r$ " indicates data are available for $70-84 \%$ of the students. An " $s$ " indicates data are available for $50-69 \%$ of the students. An " $x$ " indicates data are available for less than $50 \%$ of the students.
A dash (-) indicates comparable data are not available.

NOTE: The International Average does not include the results from the Canadian provinces. 
year or fewer. Generally, teachers reported sending home reports somewhat less frequently than meeting or talking with parents about the child's progress in reading.

As shown in Exhibit 7.10, parents reported discussing their child's classroom reading work with him or her quite frequently-every day or almost every day for 44 percent of students, on average, and once or twice a week for an additional 35 percent. Only 7 percent of students had parents reporting never or almost never discussing classroom reading with their child.

\section{What Are the School Attendance Levels and Climate Characteristics?}

Because persistent student absenteeism can disrupt learning and retard learning progress, PIRLS asked school principals to what degree absenteeism was a problem in their schools. Exhibit 7.11 presents trends in the percentage of students in schools where the principal reported that student absenteeism was not a problem, a minor problem, a moderate problem, or a serious problem. The exhibit shows the percentage of students in 2006 in each of these categories, together with their average reading achievement, and for countries that also participated in PIRLS 2001, the change in student percentages over the intervening 5 years. Exhibit 7.11 also displays graphically the 2001 and 2006 percentages of students in schools where the principal reported that absenteeism was not a problem.

On average across countries, most students were in schools where principals reported that absenteeism either was not a problem $(37 \%$ of students) or was a minor problem (40\%). Countries with the least attendance problems included Hong Kong sar, Chinese Taipei, Scotland, Austria, Norway, the Netherlands, Sweden, and Belgium (Flemish), where 6o percent or more of students were in schools where absenteeism among fourth-grade students was not a problem. The percentage of students in this category increased since 2001 in the Russian Federation, Iceland, and Macedonia, and decreased in France, the Canadian province of Ontario, Romania, and Morocco. Although on average across countries, only 9 percent of students were in schools with serious absentee problems, more than 20 percent of 
Exhibit 7.10 Parents Discuss Their Child's Classroom Reading Work with Him or Her

PIRLS 2006

4th Grade

\begin{tabular}{|c|c|c|c|c|c|c|c|c|c|}
\hline \multirow{2}{*}{ Countries } & & \multicolumn{2}{|c|}{$\begin{array}{c}\text { Every Day or } \\
\text { Almost Every Day }\end{array}$} & \multicolumn{2}{|c|}{$\begin{array}{c}\text { Once or Twice } \\
\text { a Week }\end{array}$} & \multicolumn{2}{|c|}{$\begin{array}{l}\text { Once or Twice } \\
\text { a Month }\end{array}$} & \multicolumn{2}{|c|}{$\begin{array}{c}\text { Never or } \\
\text { Almost Never }\end{array}$} \\
\hline & & $\begin{array}{l}\text { Percent of } \\
\text { Students }\end{array}$ & $\begin{array}{c}\text { Average } \\
\text { Achievement }\end{array}$ & $\begin{array}{l}\text { Percent of } \\
\text { Students }\end{array}$ & $\begin{array}{c}\text { Average } \\
\text { Achievement }\end{array}$ & $\begin{array}{l}\text { Percent of } \\
\text { Students }\end{array}$ & $\begin{array}{c}\text { Average } \\
\text { Achievement }\end{array}$ & $\begin{array}{l}\text { Percent of } \\
\text { Students }\end{array}$ & $\begin{array}{c}\text { Average } \\
\text { Achievement }\end{array}$ \\
\hline Austria & & $27(0.9)$ & $530(2.7)$ & $39(0.8)$ & $541(2.6)$ & $20(0.6)$ & $549(2.7)$ & $14(0.6)$ & $543(3.8)$ \\
\hline Belgium (Flemish) & & $34(1.0)$ & $539(2.5)$ & $36(1.0)$ & $547(2.8)$ & $19(0.6)$ & $561(2.4)$ & $12(0.6)$ & $560(3.0)$ \\
\hline Belgium (French) & & $42(1.3)$ & $490(3.2)$ & $39(0.9)$ & $508(2.7)$ & $14(0.8)$ & $516(4.1)$ & $5(0.4)$ & $517(7.2)$ \\
\hline Bulgaria & & $59(1.5)$ & $550(4.1)$ & $25(1.0)$ & $557(6.0)$ & $9(1.0)$ & $551(10.3)$ & $7(1.0)$ & $525(9.5)$ \\
\hline Canada, Alberta & $r$ & $39(1.1)$ & $562(3.8)$ & $40(1.2)$ & $566(2.8)$ & $16(0.8)$ & $569(4.3)$ & $5(0.4)$ & $568(6.5)$ \\
\hline Canada, British Columbia & $r$ & $40(1.0)$ & $560(3.6)$ & $40(0.8)$ & $566(3.3)$ & $14(0.7)$ & $564(4.9)$ & $5(0.5)$ & $567(8.0)$ \\
\hline Canada, Nova Scotia & & $44(0.9)$ & $542(2.7)$ & $41(0.9)$ & $549(2.5)$ & $11(0.6)$ & $550(4.1)$ & $4(0.3)$ & $543(8.7)$ \\
\hline Canada, Ontario & & $44(1.3)$ & $557(3.0)$ & $39(1.2)$ & $558(3.5)$ & $13(0.9)$ & $560(4.9)$ & $4(0.5)$ & $552(8.8)$ \\
\hline Canada, Quebec & & $37(1.1)$ & $531(3.2)$ & $44(1.0)$ & $537(3.3)$ & $14(0.7)$ & $547(4.9)$ & $5(0.5)$ & $532(8.7)$ \\
\hline Chinese Taipei & & $30(0.8)$ & $547(2.7)$ & $41(0.7)$ & $537(2.8)$ & $20(0.6)$ & $530(3.0)$ & $9(0.5)$ & $518(4.5)$ \\
\hline Denmark & & $45(1.0)$ & $541(2.8)$ & $42(1.0)$ & $552(3.0)$ & $11(0.7)$ & $560(4.3)$ & $2(0.2)$ & $\sim \sim$ \\
\hline England & & $\mathrm{xx}$ & $x x$ & $x x$ & $\mathrm{xx}$ & $x x$ & $x x$ & $\mathrm{xx}$ & $x x$ \\
\hline France & & $35(1.0)$ & $515(2.5)$ & $43(0.9)$ & $529(2.2)$ & $15(0.6)$ & $531(3.7)$ & $7(0.5)$ & $526(4.7)$ \\
\hline Georgia & & $65(1.2)$ & $477(3.4)$ & $24(0.8)$ & $471(4.4)$ & $7(0.7)$ & $465(8.9)$ & $4(0.4)$ & $457(9.8)$ \\
\hline Germany & & $35(0.8)$ & $546(2.7)$ & $41(0.9)$ & $556(2.6)$ & $17(0.7)$ & $561(3.8)$ & $7(0.5)$ & $554(5.4)$ \\
\hline Hong Kong SAR & & $29(0.8)$ & $570(2.4)$ & $40(0.8)$ & $567(2.8)$ & $20(0.7)$ & $560(3.2)$ & $11(0.6)$ & $557(4.4)$ \\
\hline Hungary & & $40(1.0)$ & $541(3.2)$ & $40(0.8)$ & $555(3.5)$ & $16(0.7)$ & $571(4.7)$ & $4(0.4)$ & $581(7.1)$ \\
\hline Iceland & $r$ & $19(0.7)$ & $503(3.7)$ & $46(0.9)$ & $516(2.0)$ & $24(0.9)$ & $526(2.9)$ & $11(0.6)$ & $523(4.5)$ \\
\hline Indonesia & & $56(1.7)$ & $415(4.1)$ & $30(1.3)$ & $400(4.9)$ & $8(0.8)$ & $382(6.9)$ & $6(0.7)$ & $377(7.4)$ \\
\hline Iran, Islamic Rep. of & & $57(1.4)$ & $438(3.6)$ & $27(1.0)$ & $410(3.8)$ & $10(0.6)$ & $400(7.5)$ & $7(0.7)$ & $359(9.1)$ \\
\hline Israel & $s$ & $43(1.3)$ & $504(5.0)$ & $31(1.1)$ & $537(4.9)$ & $14(0.8)$ & $551(7.1)$ & $12(0.7)$ & $556(7.7)$ \\
\hline Italy & & $46(1.2)$ & $548(3.5)$ & $33(0.9)$ & $560(3.4)$ & $10(0.6)$ & $562(5.1)$ & $11(0.6)$ & $554(4.9)$ \\
\hline Kuwait & $r$ & $61(1.0)$ & $339(4.8)$ & $24(0.8)$ & $342(6.4)$ & $10(0.6)$ & $336(8.6)$ & $5(0.4)$ & 318 (14.3) \\
\hline Latvia & & $33(0.9)$ & $535(3.0)$ & $39(0.8)$ & $543(2.6)$ & $18(0.6)$ & $551(3.6)$ & $11(0.6)$ & $550(5.2)$ \\
\hline Lithuania & & $32(0.8)$ & $528(2.4)$ & $42(0.8)$ & $537(1.9)$ & $18(0.6)$ & $550(3.3)$ & $8(0.4)$ & $553(3.3)$ \\
\hline Luxembourg & & $42(0.7)$ & $552(1.6)$ & $38(0.7)$ & $566(1.8)$ & $14(0.5)$ & $569(2.7)$ & $6(0.4)$ & $558(3.8)$ \\
\hline Macedonia, Rep. of & & $80(0.9)$ & $454(4.0)$ & $16(0.6)$ & $434(6.2)$ & $3(0.4)$ & $439(13.4)$ & $2(0.3)$ & $\sim \sim$ \\
\hline Moldova, Rep. of & & $44(1.2)$ & $504(3.6)$ & $37(1.0)$ & $501(3.2)$ & $12(0.7)$ & $491(5.8)$ & $7(0.9)$ & $490(8.2)$ \\
\hline Morocco & & $46(1.4)$ & $341(5.3)$ & $25(1.1)$ & $312(7.2)$ & $11(0.9)$ & $301(15.3)$ & $18(1.3)$ & $307(11.4)$ \\
\hline Netherlands & $s$ & $28(1.0)$ & $547(2.6)$ & $41(0.9)$ & $558(2.1)$ & $22(0.9)$ & $561(2.5)$ & $9(0.6)$ & $558(5.5)$ \\
\hline New Zealand & $s$ & $30(0.9)$ & $544(2.9)$ & $49(0.9)$ & $548(2.7)$ & $17(0.7)$ & $553(4.0)$ & $4(0.4)$ & $554(9.7)$ \\
\hline Norway & & $23(1.0)$ & $488(4.0)$ & $53(0.9)$ & $502(2.8)$ & $20(1.0)$ & $509(3.6)$ & $5(0.4)$ & $501(7.7)$ \\
\hline Poland & & $86(0.6)$ & $517(2.6)$ & $12(0.5)$ & $535(4.5)$ & $2(0.2)$ & $\sim \sim$ & $1(0.2)$ & $\sim \sim$ \\
\hline Qatar & $s$ & $64(0.7)$ & $358(1.8)$ & $25(0.6)$ & $360(2.7)$ & $7(0.4)$ & $369(6.1)$ & $4(0.3)$ & $353(9.6)$ \\
\hline Romania & & $56(1.7)$ & $504(5.0)$ & $28(1.1)$ & $496(5.2)$ & $9(0.7)$ & $473(7.5)$ & $8(1.2)$ & $392(15.1)$ \\
\hline Russian Federation & & $49(1.0)$ & $563(3.2)$ & $34(0.8)$ & $567(4.8)$ & $10(0.6)$ & $574(4.8)$ & $7(0.5)$ & $563(4.9)$ \\
\hline Scotland & $s$ & $45(1.3)$ & $532(3.7)$ & $44(1.4)$ & $550(4.1)$ & $9(0.7)$ & $557(6.3)$ & $2(0.4)$ & $\sim \sim$ \\
\hline Singapore & & $31(0.7)$ & $566(3.1)$ & $36(0.6)$ & $560(3.2)$ & $21(0.5)$ & $553(3.7)$ & $13(0.5)$ & $557(4.1)$ \\
\hline Slovak Republic & & $34(0.9)$ & $524(2.9)$ & $40(0.9)$ & $535(2.9)$ & $16(0.8)$ & $545(5.1)$ & $9(0.5)$ & $537(9.0)$ \\
\hline Slovenia & & $41(0.9)$ & $512(2.7)$ & $41(0.7)$ & $526(2.2)$ & $14(0.6)$ & $545(3.4)$ & $4(0.3)$ & $546(4.9)$ \\
\hline South Africa & $r$ & $53(0.8)$ & $293(5.4)$ & $29(0.6)$ & $325(6.6)$ & $11(0.4)$ & $309(10.1)$ & $8(0.3)$ & $296(9.8)$ \\
\hline Spain & $s$ & $56(1.0)$ & $515(3.2)$ & $31(0.9)$ & $525(3.9)$ & $9(0.6)$ & $538(6.2)$ & $5(0.5)$ & $525(5.0)$ \\
\hline Sweden & & $25(1.1)$ & $537(3.3)$ & $43(1.0)$ & $553(3.1)$ & $25(0.9)$ & $558(2.9)$ & $8(0.6)$ & $569(5.1)$ \\
\hline Trinidad and Tobago & & $61(1.2)$ & $446(5.1)$ & $30(1.0)$ & $432(6.0)$ & $6(0.5)$ & $435(12.2)$ & $3(0.4)$ & $419(12.0)$ \\
\hline United States & & -- & -- & -- & -- & -- & -- & -- & -- \\
\hline International Avg. & & $44(0.2)$ & $499(0.6)$ & $35(0.1)$ & $504(0.7)$ & $14(0.1)$ & $505(1.1)$ & $7(0.1)$ & $496(1.5)$ \\
\hline
\end{tabular}

Background data provided by parents.

() Standard errors appear in parentheses. Because results are rounded to the nearest whole number, some totals may appear inconsistent.

An " $r$ " indicates data are available for $70-84 \%$ of the students. An "s" indicates data are available for $50-69 \%$ of the students. An " $x$ " indicates data are available for less than $50 \%$ of the students.
A dash (-) indicates comparable data are not available. A tilde ( ) indicates insufficient data to report achievement.

NOTE: The International Average does not include the results from the Canadian provinces. 
Exhibit 7.11 Seriousness of Absenteeism in Schools with Trends

PIRLS 2006
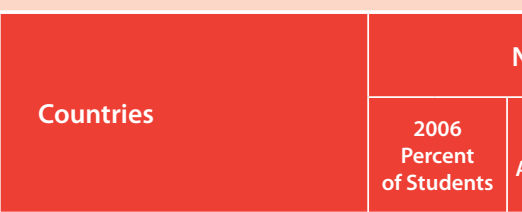

Not a Problem

Hong Kong SAR

Chinese Taipei

Scotland

Austria

Norway

Netherlands

Sweden

Belgium (Flemish)

Russian Federation

Iceland

Singapore

Germany

Spain

Canada, Quebec

Italy

Poland

Israel

Iran, Islamic Rep. of

New Zealand

England

Canada, Nova Scotia

France

Belgium (French)

Canada, British Columbia

Denmark

Lithuania

Slovenia

Canada, Alberta

Canada, Ontario

Macedonia, Rep. of

Slovak Republic

Bulgaria

United States

Latvia

Hungary

Indonesia

Qatar

Moldova, Rep. of

Georgia

Romania

South Africa

Trinidad and Tobago

Morocco

Kuwait

${ }^{1}$ Luxembourg

International Avg.

\section{2 (3.6)}

$73(4.1)$

$66(4.6)$

$65(4.3)$

$62(5.1)$

$62(4.5)$

$62(4.0)$

$60(4.7)$

$57(4.3)$

r $\quad 57(0.4)$

$56(0.0)$

$53(3.3)$

$52(4.5)$

$51(4.3)$

$50(3.8)$

$48(4.2)$

$45(4.7)$

$41(3.6)$

$40(3.7)$

$39(3.4)$

38 (3.6)

34 (3.9)

$33(4.5)$

$33(3.9)$

$$
30 \text { (4.1) }
$$

$27(3.9)$

$$
27(3.4)
$$

$27(4.3)$

$26(4.3)$

$25(3.4)$

$24(3.1)$

$23(3.8)$

$23(4.0)$

22 (3.4)

$16(3.0)$

$15(3.0)$

$15(0.2)$

$14(3.0)$

$13(2.9)$

$12(2.8)$

$$
12(1.9)
$$

$$
10(2.2)
$$

r $\quad 6(2.3)$

$5(1.9)$

$--$

$$
\begin{array}{l|l}
37(0.6) & 510(1.4)
\end{array}
$$

Percent in 2006 significantly higher $\boldsymbol{\theta}$

Percent in 2006 significantly lower

Background data provided by schools

() Standard errors appear in parentheses. Because results are rounded to the nearest whole number, some totals may appear inconsistent.

An " $r$ " indicates data are available for $70-84 \%$ of the students. An " $s$ " indicates data are available for $50-69 \%$ of the students. An " $x$ " indicates data are available for less than $50 \%$ of the students.

A dash (-) indicates comparable data are not available. A tilde $(\sim)$ indicates insufficient data to report achievement.

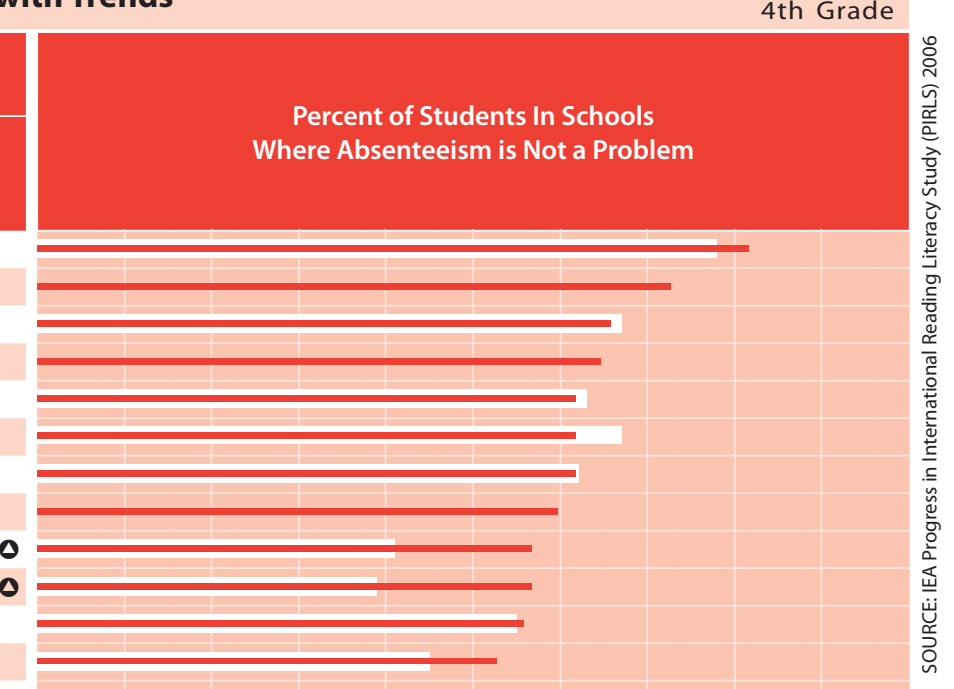

$(\nabla$

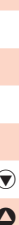

-
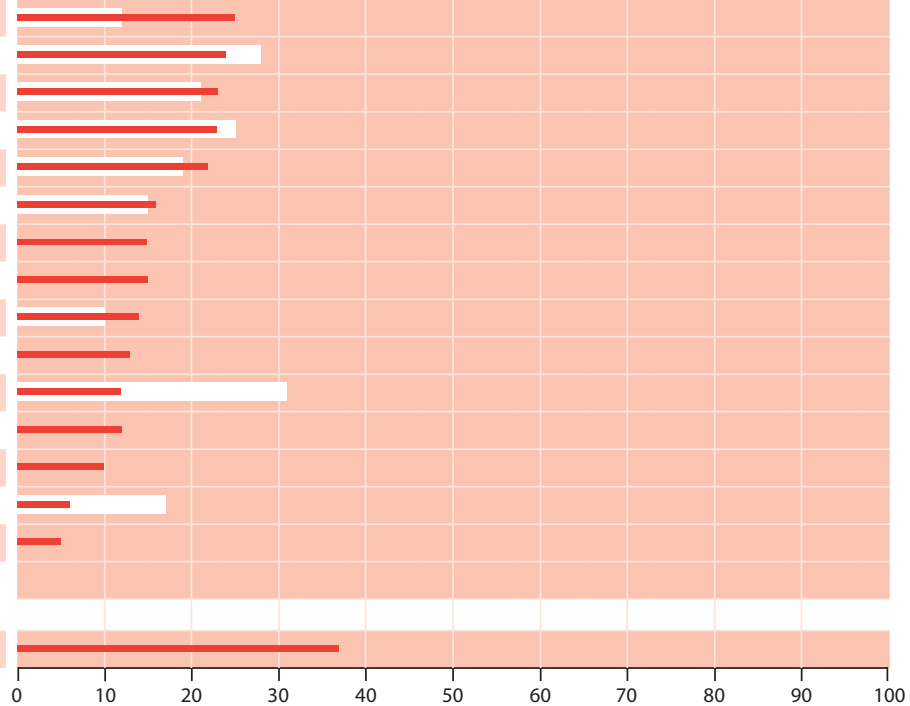

2001 Percent

2006 Percent

A diamond $(\diamond)$ indicates the country did not participate in the 2001 assessment. NOTE: The International Average does not include the results from the Canadian provinces. Trend Note: The primary education systems of the Russian Federation and Slovenia underwent structural changes. Data for Canada, Ontario include only public schools.

1 Primary schools in Luxembourg do not have principals. 
Exhibit 7.11 Seriousness of Absenteeism in Schools with Trends (Continued)

PIRLS 2006

\begin{tabular}{|c|c|c|c|c|c|c|}
\hline & \multicolumn{4}{|c|}{ Minor Problem } & \multicolumn{3}{c|}{ Moderate Problem } \\
\cline { 2 - 7 } Countries & $\begin{array}{c}2006 \\
\text { Percent } \\
\text { of Students }\end{array}$ & $\begin{array}{c}\text { Average } \\
\text { Achievement }\end{array}$ & $\begin{array}{c}\text { Difference } \\
\text { if Percent } \\
\text { from 2001 }\end{array}$ & $\begin{array}{c}2006 \\
\text { Percent } \\
\text { of Students }\end{array}$ & $\begin{array}{c}\text { Average } \\
\text { Achievement }\end{array}$ & $\begin{array}{c}\text { Difference } \\
\text { in Percent } \\
\text { from 2001 }\end{array}$ \\
\hline
\end{tabular}

4th Grade

\begin{tabular}{|c|c|c|c|c|c|c|c|c|c|c|c|c|c|}
\hline Hong Kong SAR & & $16(3.5)$ & $573(6.2)$ & $-4(5.3)$ & & $1(0.9)$ & $\sim \sim$ & $0(1.3)$ & & $0(0.0)$ & $\sim \sim$ & $0(0.0)$ & \\
\hline Chinese Taipei & & $27(4.1)$ & $531(3.5)$ & $\Delta 0$ & & $0(0.0)$ & $\sim \sim$ & $\Delta 0$ & & $1(0.0)$ & $\sim \sim$ & $\Delta \Delta$ & \\
\hline Scotland & $\mathrm{r}$ & $31(4.4)$ & $515(5.9)$ & $5(6.7)$ & & $2(1.6)$ & $\sim \sim$ & $-4(3.1)$ & & $0(0.0)$ & $\sim \sim$ & $0(0.0)$ & \\
\hline Austria & & $29(4.4)$ & $538(4.1)$ & $\Delta \Delta$ & & $5(1.7)$ & $509(7.5)$ & $\Delta \Delta$ & & $1(0.8)$ & $\sim \sim$ & $\Delta \diamond$ & \\
\hline Norway & & $37(5.1)$ & $498(5.0)$ & $4(7.0)$ & & $1(0.5)$ & $\sim \sim$ & $-3(1.8)$ & & $0(0.0)$ & $\sim \sim$ & $0(0.0)$ & \\
\hline Netherlands & & $32(3.8)$ & $549(3.2)$ & $3(5.7)$ & & $4(2.4)$ & $510(9.2)$ & $0(3.1)$ & & $2(2.0)$ & $\sim \sim$ & $2(2.0)$ & \\
\hline Sweden & & $30(3.9)$ & $546(4.2)$ & $0(6.0)$ & & $7(2.5)$ & $546(10.2)$ & $0(3.3)$ & & $1(0.8)$ & $\sim \sim$ & $0(1.3)$ & \\
\hline Belgium (Flemish) & & $37(4.8)$ & $545(3.6)$ & $\Delta \Delta$ & & $3(1.3)$ & $503(14.2)$ & $\Delta \Delta$ & & $0(0.0)$ & $\sim \sim$ & $\Delta \Delta$ & \\
\hline Russian Federation & & $41(4.1)$ & $554(5.8)$ & $-15(5.6)$ & (i) & $2(0.7)$ & $\sim \sim$ & $0(1.2)$ & & $0(0.0)$ & $\sim \sim$ & $-1(0.6)$ & \\
\hline Iceland & $r$ & $38(0.3)$ & $510(2.3)$ & $-14(0.6)$ & $(-)$ & $5(0.1)$ & $501(5.3)$ & $-3(0.3)$ & (1) & $0(0.0)$ & $\sim \sim$ & $0(0.0)$ & \\
\hline Singapore & & $42(0.0)$ & $553(5.0)$ & $-1(3.9)$ & & $2(0.0)$ & $\sim \sim$ & $-1(1.3)$ & & $0(0.0)$ & $\sim \sim$ & $0(0.0)$ & \\
\hline Germany & & $39(3.4)$ & $545(3.3)$ & $-6(5.9)$ & & $6(1.6)$ & $504(10.5)$ & $-3(3.0)$ & & $2(0.7)$ & $\sim \sim$ & $1(1.0)$ & \\
\hline Spain & & $39(4.2)$ & $513(4.2)$ & $\Delta \Delta$ & & $6(2.0)$ & $487(11.6)$ & $\Delta \diamond$ & & $3(1.5)$ & $477(28.6)$ & $\Delta \diamond$ & \\
\hline Canada, Quebec & & $41(4.1)$ & $527(4.5)$ & $-6(6.6)$ & & $8(2.5)$ & $527(7.0)$ & $2(3.6)$ & & $0(0.2)$ & $\sim \sim$ & $-1(0.8)$ & \\
\hline Italy & & $28(3.7)$ & $549(6.6)$ & $-4(5.2)$ & & $14(2.5)$ & $537(8.2)$ & $0(3.8)$ & & $8(2.0)$ & $560(14.4)$ & $-4(3.0)$ & \\
\hline Poland & & $48(4.4)$ & $519(3.3)$ & $\Delta 0$ & & $2(1.2)$ & $\sim \sim$ & $\Delta \Delta$ & & $1(0.0)$ & $\sim \sim$ & $\Delta \Delta$ & \\
\hline Israel & & $47(4.5)$ & $517(8.6)$ & $-1(6.4)$ & & $5(1.5)$ & $483(33.9)$ & $-6(3.0)$ & ( & $3(1.4)$ & $415(15.4)$ & $-2(2.0)$ & \\
\hline Iran, Islamic Rep. of & & $47(3.6)$ & $419(5.4)$ & $11(5.5)$ & & $9(2.2)$ & $393(11.4)$ & $-6(4.5)$ & & $3(1.0)$ & $374(24.7)$ & $-10(3.4)$ & (1) \\
\hline New Zealand & & $49(3.8)$ & $529(3.5)$ & $-1(5.7)$ & & $8(1.8)$ & $486(9.1)$ & $0(2.8)$ & & $2(0.9)$ & $\sim \sim$ & $1(1.3)$ & \\
\hline England & & $37(4.0)$ & $541(4.0)$ & -- & & $19(3.4)$ & $505(5.5)$ & -- & & $5(1.9)$ & $472(20.0)$ & -- & \\
\hline Canada, Nova Scotia & & $57(3.4)$ & $541(2.7)$ & $\Delta \diamond$ & & $4(1.4)$ & $508(10.1)$ & $\Delta \diamond$ & & $1(0.6)$ & $\sim \sim$ & $\Delta \diamond$ & \\
\hline France & & $54(4.5)$ & $518(3.0)$ & $15(6.9)$ & 0 & $5(1.9)$ & $514(9.9)$ & $-4(3.2)$ & & $6(2.1)$ & $496(11.8)$ & $5(2.3)$ & 0 \\
\hline Belgium (French) & & $47(4.5)$ & $497(4.4)$ & $\Delta \diamond$ & & $16(3.4)$ & $479(8.1)$ & $\diamond \diamond$ & & $4(1.9)$ & $472(10.7)$ & $\diamond \diamond$ & \\
\hline Canada, British Columbia & & $54(4.1)$ & $558(3.6)$ & $\Delta \diamond$ & & $13(2.4)$ & $546(9.1)$ & $\Delta \Delta$ & & $1(0.6)$ & $\sim \sim$ & $\Delta \diamond$ & \\
\hline Denmark & & $58(4.3)$ & $551(3.0)$ & $\diamond \diamond$ & & $10(2.8)$ & $531(6.9)$ & $\Delta \diamond$ & & $2(1.1)$ & $\sim \sim$ & $\Delta \diamond$ & \\
\hline Lithuania & & $30(4.2)$ & $532(3.9)$ & $-18(5.9)$ & $(\nabla$ & $33(4.2)$ & $538(3.7)$ & $11(5.6)$ & & $10(2.8)$ & $533(4.7)$ & $3(3.6)$ & \\
\hline Slovenia & & $62(4.0)$ & $521(2.9)$ & $-14(5.6)$ & $(\nabla$ & $11(2.7)$ & $534(6.7)$ & $6(3.2)$ & & $1(0.0)$ & $\sim \sim$ & $1(0.0)$ & \\
\hline Canada, Alberta & & $62(4.4)$ & $560(2.8)$ & $\Delta \Delta$ & & $8(2.2)$ & $553(6.5)$ & $\Delta \Delta$ & & $3(1.3)$ & $514(20.2)$ & $\Delta \Delta$ & \\
\hline Canada, Ontario & & $63(5.0)$ & $549(3.5)$ & $18(6.7)$ & 0 & $8(2.9)$ & $567(12.8)$ & $-1(4.0)$ & & $3(1.5)$ & $532(13.7)$ & $2(1.5)$ & \\
\hline Macedonia, Rep. of & $r$ & $36(4.2)$ & $436(9.6)$ & $-23(5.8)$ & $(\nabla)$ & $28(4.0)$ & $457(11.8)$ & $6(5.5)$ & & $12(3.0)$ & $424(13.6)$ & $5(3.7)$ & \\
\hline Slovak Republic & & $46(3.9)$ & $539(3.1)$ & $4(5.5)$ & & $22(3.4)$ & $506(8.5)$ & $0(4.7)$ & & $8(2.3)$ & $517(9.7)$ & $0(3.2)$ & \\
\hline Bulgaria & & $30(4.2)$ & $547(6.6)$ & $-5(5.7)$ & & $34(4.2)$ & $541(7.3)$ & $2(5.8)$ & & $13(3.1)$ & $532(11.5)$ & $1(4.1)$ & \\
\hline United States & & $59(4.1)$ & $537(3.9)$ & $-1(6.3)$ & & $15(2.7)$ & $525(7.6)$ & $1(4.0)$ & & $3(1.5)$ & $498(12.1)$ & $2(1.7)$ & \\
\hline Latvia & & $42(4.0)$ & $539(4.1)$ & $-22(5.6)$ & ( & $26(3.5)$ & $539(4.1)$ & $11(5.0)$ & 0 & $10(2.6)$ & $540(5.7)$ & $8(2.9)$ & 0 \\
\hline Hungary & & $71(4.0)$ & $555(3.3)$ & $-6(5.2)$ & & $10(2.7)$ & $538(11.0)$ & $3(3.4)$ & & $3(1.8)$ & $481(24.3)$ & $2(2.0)$ & \\
\hline Indonesia & & $26(3.8)$ & $408(8.7)$ & $\Delta 0$ & & $17(2.9)$ & $407(6.6)$ & $\Delta \Delta$ & & $42(4.3)$ & $399(7.0)$ & $\Delta \diamond$ & \\
\hline Qatar & & $35(0.2)$ & $354(2.5)$ & $\Delta \Delta$ & & $22(0.2)$ & $352(2.3)$ & $\Delta \Delta$ & & $28(0.2)$ & $344(2.7)$ & $\Delta \diamond$ & \\
\hline Moldova, Rep. of & & $45(4.5)$ & $505(4.0)$ & $7(6.2)$ & & $25(4.1)$ & $493(7.0)$ & $-18(6.0)$ & $(\nabla$ & $16(3.4)$ & $500(10.7)$ & $7(4.4)$ & \\
\hline Georgia & & $45(4.2)$ & $459(5.5)$ & $\Delta \diamond$ & & $29(4.3)$ & $476(7.8)$ & $\Delta \diamond$ & & $14(3.0)$ & $486(8.3)$ & $\Delta \diamond$ & \\
\hline Romania & & $35(4.3)$ & $496(7.7)$ & $-6(6.4)$ & & $31(4.0)$ & $492(7.7)$ & $8(5.4)$ & & $22(4.2)$ & $469(11.9)$ & $16(4.5)$ & 0 \\
\hline South Africa & & $43(3.0)$ & $320(9.4)$ & $\Delta \diamond$ & & $30(2.5)$ & $267(6.2)$ & $\Delta \diamond$ & & $16(2.6)$ & $252(10.4)$ & $\Delta \diamond$ & \\
\hline Trinidad and Tobago & & $48(3.5)$ & $448(6.4)$ & $\Delta \diamond$ & & $32(3.7)$ & $420(8.8)$ & $\Delta \diamond$ & & $10(2.7)$ & $370(14.6)$ & $\Delta \Delta$ & \\
\hline Morocco & $r$ & $12(3.0)$ & $308(10.8)$ & $-8(4.7)$ & & $17(3.4)$ & $356(15.1)$ & $-9(5.8)$ & & $66(4.3)$ & $320(9.9)$ & $28(6.4)$ & 0 \\
\hline Kuwait & & $28(3.3)$ & $330(8.6)$ & $\Delta \Delta$ & & $29(3.8)$ & $336(7.1)$ & $\Delta \Delta$ & & $38(3.9)$ & $320(7.6)$ & $\Delta \Delta$ & \\
\hline${ }^{1}$ Luxembourg & & -- & -- & -- & & -- & -- & -- & & -- & -- & -- & \\
\hline International Avg. & & $40(0.6)$ & $499(0.9)$ & & & $14(0.4)$ & $477(1.9)$ & & & $9(0.3)$ & $446(2.9)$ & & \\
\hline
\end{tabular}

- Percent in 2006 significantly higher

(7) Percent in 2006 significantly lower 
students attended such schools in Indonesia, Qatar, Romania, Morocco, and Kuwait.

Students in schools where absenteeism was problematic generally had lower average reading achievement than students in schools without problems with absenteeism. Average achievement in schools where absenteeism was not a problem was 510 points, compared to 499 points in schools where it was a minor problem, 477 where it was a moderate problem, and 446 where absenteeism was a serious problem.

The PIRLS 2006 Index of Principals' Perception of School Climate summarizes principals' characterization of their school in terms of teachers' job satisfaction, teachers' expectations for student achievement, parental support for student achievement, students' regard for school property, students' desire to do well in school, and students' regard for each other's welfare. An average was computed for each principal on a 5-point scale: very low $=1$, low $=2$, medium $=3$, high $=4$, and very high $=5$. Students whose school principal had an average response greater than 3.67 were assigned to the high level of the index, those where the average was below 2.33 to the low level, and the remainder to the medium level.

Exhibit 7.12 presents the percentage of students in each country in 2006 at each level of the index, together with their average achievement. For countries that participated in PIRLS 2001, the change in these percentages also is presented, with an indication of statistical significance where appropriate. In general, principals' perceptions of school climate were positive, with more than one third of students at the high level of the index, on average, across countries, and almost all of the rest at the medium level. Only 1 percent of students, on average, were at the low level of the index. There were, however, considerable differences across participants in principals' perception of school climate. In Iceland, Scotland, New Zealand, the United States, England, and the Canadian province of Alberta, 70 percent or more of students were at the high level, whereas no more than 20 percent were at this level in South Africa, Trinidad and Tobago, Poland, Bulgaria, Hungary, Lithuania, and less than 10 percent in Latvia, the Slovak Republic, and Moldova. Countries where the 
Exhibit 7.12 Index of Principal's Perception of School Climate (PPSC) with Trends

PIRLS 2006

Countries

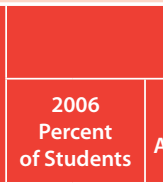

High PPSC

Medium PPSC

4th Grade

Iceland

$81(0.3)$

Achievement

Difference
in Percent
from 2001

\begin{tabular}{|c|c|}
\hline $\begin{array}{c}2006 \\
\text { Percent } \\
\text { of Students }\end{array}$ \\
\hline $19(0.3)$ \\
\hline
\end{tabular}

Scotland

r $74(4.0)$

$512(1.5)$

$5(0.4)$

$19(0.3)$

Ach

$71(3.1)$

$541(2.5)$

$11(6.3)$

$29(3.1)$

Canada, Alberta

$70(3.5)$

$569(2.6)$

$8(5.1)$

$30(3.5)$

United States

$70(3.9)$

549 (3.4)

$8(6.3)$

Chinese Taipei

$67(3.9)$

551 (4.1)

Canada, Nova Scotia

$67(3.8)$

$536(2.5)$

$--$

Singapore

Belgium (French)

$66(0.0)$

Canada, British Columbia

Sweden

$62(4.5)$

$547(2.5)$

$\Delta \diamond$

$30(3.9)$

Israel

$59(4.4)$

$506(3.6)$

$\Delta \Delta \quad 33(3.9)$

\begin{tabular}{c|c} 
Average & \\
Achievement &
\end{tabular}

Difference
in Percent
from 2001
$-5(0.4)$

$510(2.7)$

$566(3.1)$

$6(3.8)$

$\diamond \diamond$

$\Delta \Delta$

$53(4.1)$

$519(6.8)$

$3(6.8)$

$500(3.6)$

$--47(4.1)$

Norway

$51(5.1)$

$-21(6.2)$

$49(5.1)$

Canada, Ontario

$50(5.5)$

$558(3.8)$

$-6(7.4)$

$50(5.5)$

Indonesia

\begin{tabular}{l|l}
$48(4.2)$ & $409(7.1)$
\end{tabular}

$\Delta 0 \quad 52(4.2)$

Iran, Islamic Rep. of

$48(4.4)$

$555(3.3)$

$\Delta 0$

$52(4.4)$

Canada, Quebec

$47(4.0)$

$429(5.6)$

$1(6.1)$

$53(4.0)$

Austria

Hong Kong SAR

$46(4.8)$

$543(3.9)$

$-14(6.3) \odot 54(4.8)$

$45(4.3)$

$545(3.0)$

00

$36(4.4)$

$566(3.6)$

$-5(6.0)$

$54(4.4)$

$512(6.0)$

$-5(0.4)$
$-11(6.3)$

$512(5.6)$

$-8(5.1)$

$540(5.3)$

00

$521(5.7)$

$533(3.8)$

$--$

$531(4.5)$

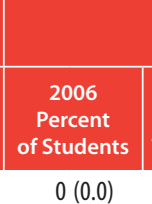

Low PPSC

Georgia

Spain

$32(4.2)$

$473(5.4)$

00

$57(3.7)$

Italy

$32(3.8)$

$532(3.6)$

$\Delta 0$

$63(4.4)$

Qatar

$31(0.2)$

$561(5.5)$

$12(4.7) \quad 0 \quad 68(3.8)$

$552(5.8)$

489 (5.1)

$547(4.7)$

$543(3.5)$

$495(3.6)$

$550(3.7)$

$401(4.8)$

$539(3.6)$

$414(5.3)$

$526(3.8)$

$534(3.2)$

$563(2.8)$

$\Delta \diamond \quad 0(0.0)$

$0(0.0)$

$-6(3.8)$

$-6(3.8)$

$-1(0.0)$

$\Delta \diamond \quad 1(0.0)$

$-3(6.8)$

$\begin{array}{cc}-3(6.8) & 0(0.0) \\ -- & 0(0.0)\end{array}$

$21(6.2)$

$6(7.3)$

$0(0.0)$

$0(0.0)$

00

$1(0.0)$

$1(0.4)$

$14(6.3) \quad 0$

$0(0.0)$

$1(0.0)$

$1(0.7)$

$471(4.5)$

$4(5.9)$

$1(0.8)$

$505(3.5)$

00

$547(3.4) \quad-12(4.7) \quad \nabla \quad 0(0.0)$

$67(0.2)$

$345(1.3)$

$-12(4.7)$
$\Delta \diamond$

$-11(6.3) \quad 69(4.2)$

$517(3.0)$

$11(6.4)$

$2(0.1)$

$318(5.6)$

Kuwait

$30(4.2)$

$349(7.9)$

$\diamond \diamond$

$70(4.2)$

$546(2.7)$

$\Delta 0$

Slovenia

$28(3.1)$

$57(3.2)$

$5(4.9)$

$71(3.1)$

$522(2.4)$

$-6(4.9)$

$-4(5.2)$

$312(8.7)$

$-14(5.7)$

Borocco

$26(3.8)$

356 (11.8)

$14(4.5) \quad 0 \quad 64(4.2)$

$74(4.0)$

Netherlands

Russian Federation

$6(4.0)$

$544(4.2)$

$\Delta \diamond$

$3(5.9)$

$76(4.0)$

$544(2.5)$

$-14(5.7)$

$548(2.4) \quad-3(5.9)$

$561(3.6)$

$-3(5.9)$

$437(6.2) \quad 7(5.3)$

$7(5.3)$

491 (4.6)

00

Romania

$21(3.9)$

\begin{tabular}{|l|l|l|}
\hline $479(12.1)$ & $-6(5.2)$ & $78(3.7)$ \\
\hline
\end{tabular}

$-6(5.2) \quad-12(5.8) \quad 74(3.9)$
$00 \quad 76(2.7)$

South Africa

$20(2.5)$

$510(9.6)$

$\Delta 0$

$76(2.7)$

Trinidad and Tobago

$17(3.1)$

$505(9.5)$

$\Delta \diamond \quad 80(3.4)$

$284(5.5)$

$423(5.8)$

$\Delta 0$

$\Delta 0$

$519(2.6)$

00

Poland

$16(3.2)$

$522(5.9)$

$00 \quad 84(3.2)$

$547(4.9)$

$6(5.0)$

$549(3.0)$

$8(4.1)$

$536(1.7)$

$2(4.0)$
$0(3.5)$

$91(2.5)$

$539(2.6)$

$4(3.7)$

499 (3.2)

$23(3.9)$

$--$

$1(1.1)$
$1(0.0)$
$1(0.2)$

Average

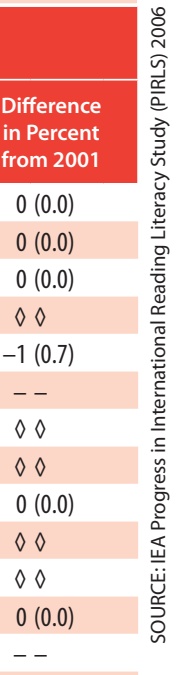

Slovak R

Moldova, Rep. of

\begin{tabular}{ll|l|l|l|}
\hline$(1.6)$ & $518(13.4)$ & $-23(3.9)$ &
\end{tabular}

1 Luxembourg

International Avg.

$37(0.6) \quad 513(1.1)$

$62(0.6) \quad 493(0.7)$

Percent in 2006 significantly higher

( Percent in 2006 significantly lower

Based on principals' characterization in their school: teachers' job satisfaction, teachers' expectations for student achievement, parental support for student achievement, students' regard for school property, students' desire to do well in school, and students' regard for each other's welfare. Average is computed on a 5-point scale: Very low $=1$, Low $=2$, Medium $=3$, High $=4$, and Very High $=5$. Responses for each activity were averaged across each principal. High level indicates an average of greater than 3.67 through 5 .

Medium level indicates an average of 2.33 through 3.67. Low level indicates an average of 1 to less than 2.33 .

"Students' regard for each other's welfare" was added to the index in PIRLS 2006 and is not included in the 2001 index calculations.

() Standard errors appear in parentheses. Because results are rounded to the nearest whole number, some totals may appear inconsistent.

An " $r$ " indicates data are available for $70-84 \%$ of the students. An " $s$ " indicates data are available for $50-69 \%$ of the students. An " $x$ " indicates data are available for less than $50 \%$ of the students.

A dash $(-)$ indicates comparable data are not available. A tilde $(\sim)$ indicates insufficient data to report achievement.

A diamond $(\diamond)$ indicates the country did not participate in the 2001 assessment.

NOTE: The International Average does not include the results from the Canadian provinces.

Trend Note: The primary education systems of the Russian Federation and Slovenia underwent structural changes. Data for Canada, Ontario include only public schools.

underwent structural changes. Data for Canada, Ontario include
1 Primary schools in Luxembourg do not have principals. 
percentage of students at the high level increased since 2001 included Iceland, Italy, and Morocco. Decreased percentages were found in Norway, Romania, Hungary, Moldova, and the Canadian province of Quebec. On average, students at the high level of the index had higher reading achievement than those at the medium level (513 vs. 493 points).

Exhibit 7.13 presents the results for the PIRLs Index of Teacher Career Satisfaction. Having teachers who are satisfied with their careers as teachers may be an important aspect of the school's climate for learning. The PIRLS 2006 Index of Teacher Career Satisfaction combined teachers' responses to five questions about being a teacher:

- I am content with my profession as a teacher.

- I am satisfied with being a teacher at this school.

- I would describe the teachers at this school as a satisfied group.

- I had more enthusiasm when I began teaching than I have now. (reverse coded)

- I do important work as a teacher.

Teachers' responses were averaged on a 4-point scale, as follows: disagree a lot $=1$, disagree a little $=2$, agree a little $=3$, and agree a lot $=4$. Students whose teacher's average was greater than 3 (i.e., they agreed either a little or a lot with all five statements) were assigned to the high level of the index, students with a teacher averaging 2 or more but less than 3 were at the medium level, and students with a teacher averaging less than 2 were assigned to the low level of the index. 
Exhibit 7.13 Index of Teacher Career Satisfaction (TCS)

PIRLS 2006

\begin{tabular}{|c|c|c|c|c|c|c|}
\hline \multirow{2}{*}{ Countries } & \multicolumn{2}{|c|}{ High TCS } & \multicolumn{2}{|c|}{ Medium TCS } & \multicolumn{2}{|c|}{ Low TCS } \\
\hline & $\begin{array}{l}\text { Percent of } \\
\text { Students }\end{array}$ & $\begin{array}{c}\text { Average } \\
\text { Achievement }\end{array}$ & $\begin{array}{l}\text { Percent of } \\
\text { Students }\end{array}$ & $\begin{array}{c}\text { Average } \\
\text { Achievement }\end{array}$ & $\begin{array}{l}\text { Percent of } \\
\text { Students }\end{array}$ & $\begin{array}{c}\text { Average } \\
\text { Achievement }\end{array}$ \\
\hline Norway & $84(2.7)$ & $497(3.0)$ & $15(2.8)$ & $504(5.5)$ & $0(0.4)$ & $\sim \sim$ \\
\hline Georgia & $83(3.4)$ & $469(4.0)$ & $17(3.4)$ & $476(8.1)$ & $0(0.0)$ & $\sim \sim$ \\
\hline Kuwait & $83(3.0)$ & $330(5.0)$ & $17(3.0)$ & $341(7.0)$ & $0(0.4)$ & $\sim \sim$ \\
\hline Macedonia, Rep. of & $82(3.3)$ & $453(5.6)$ & $15(3.0)$ & $411(11.9)$ & $2(1.7)$ & $\sim \sim$ \\
\hline Canada, Nova Scotia & $82(2.9)$ & $543(2.6)$ & $17(2.8)$ & $541(5.2)$ & $1(0.7)$ & $\sim \sim$ \\
\hline Israel & $81(3.2)$ & $520(5.4)$ & $19(3.1)$ & $487(13.6)$ & $1(0.7)$ & $\sim \sim$ \\
\hline Canada, Ontario & $80(4.0)$ & $556(3.0)$ & $17(3.8)$ & $548(5.7)$ & $2(1.4)$ & $\sim \sim$ \\
\hline Denmark & $78(3.1)$ & $547(2.8)$ & $20(3.1)$ & $543(4.2)$ & $2(0.9)$ & $\sim \sim$ \\
\hline Iceland & $77(0.2)$ & $507(1.4)$ & $23(0.2)$ & $520(2.5)$ & $1(0.1)$ & $\sim \sim$ \\
\hline Canada, British Columbia $\quad r$ & $76(3.5)$ & $562(3.1)$ & $21(3.2)$ & $552(7.6)$ & $3(1.7)$ & $563(8.1)$ \\
\hline Canada, Alberta & $74(3.2)$ & $563(2.7)$ & $24(3.1)$ & $553(4.4)$ & $2(1.0)$ & $\sim \sim$ \\
\hline Scotland & $73(3.4)$ & $522(3.8)$ & $23(3.0)$ & $531(4.7)$ & $4(1.6)$ & $532(6.9)$ \\
\hline United States & $73(3.3)$ & $542(4.1)$ & $24(3.6)$ & $532(4.3)$ & $3(1.3)$ & $544(16.3)$ \\
\hline Lithuania & $73(3.1)$ & $537(2.1)$ & $27(3.1)$ & $538(3.5)$ & $0(0.0)$ & $\sim \sim$ \\
\hline Luxembourg & $72(0.2)$ & $559(1.2)$ & $25(0.2)$ & $550(1.9)$ & $2(0.1)$ & $\sim \sim$ \\
\hline Netherlands & $71(3.4)$ & $548(1.9)$ & $27(3.6)$ & $542(3.5)$ & $2(1.0)$ & $\sim \sim$ \\
\hline Austria & $70(3.0)$ & $538(2.5)$ & $30(3.2)$ & $540(4.1)$ & $1(0.6)$ & $\sim \sim$ \\
\hline New Zealand & $69(2.4)$ & $533(2.8)$ & $29(2.5)$ & $536(4.0)$ & $2(0.7)$ & $\sim \sim$ \\
\hline Qatar & $68(0.2)$ & $360(1.4)$ & $29(0.2)$ & $346(2.4)$ & $3(0.1)$ & $325(9.5)$ \\
\hline South Africa & $68(2.8)$ & $291(7.4)$ & $28(2.9)$ & $321(13.5)$ & $4(1.0)$ & $367(34.6)$ \\
\hline Indonesia & $67(3.6)$ & $405(5.6)$ & $33(3.6)$ & $406(6.3)$ & $0(0.0)$ & $\sim \sim$ \\
\hline Russian Federation & $67(3.3)$ & $568(3.9)$ & $32(3.3)$ & $558(6.7)$ & $1(0.8)$ & $\sim \sim$ \\
\hline Germany & $67(3.3)$ & $546(2.9)$ & $31(3.0)$ & $549(3.4)$ & $2(1.4)$ & $\sim \sim$ \\
\hline England & $66(3.4)$ & $550(3.6)$ & $27(3.1)$ & $518(6.0)$ & $7(2.4)$ & $533(13.6)$ \\
\hline Canada, Quebec & $65(4.0)$ & $538(3.3)$ & $32(4.1)$ & $527(4.9)$ & $2(1.1)$ & $\sim \sim$ \\
\hline Iran, Islamic Rep. of & $64(3.3)$ & $421(4.3)$ & $34(3.5)$ & $420(7.6)$ & $2(0.9)$ & $\sim \sim$ \\
\hline Belgium (French) & $64(3.4)$ & $503(3.6)$ & $35(3.4)$ & $495(4.7)$ & $1(0.3)$ & $\sim \sim$ \\
\hline Belgium (Flemish) & $64(3.5)$ & $549(2.3)$ & $35(3.7)$ & $544(3.2)$ & $2(1.1)$ & $\sim \sim$ \\
\hline Romania & $63(4.0)$ & $495(5.6)$ & $36(3.9)$ & $480(8.2)$ & $1(0.4)$ & $\sim \sim$ \\
\hline Spain & $63(4.0)$ & $512(3.6)$ & $36(3.9)$ & $515(4.3)$ & $1(0.6)$ & $\sim \sim$ \\
\hline Slovenia & $62(3.0)$ & $521(2.8)$ & $36(2.8)$ & $523(2.8)$ & $2(0.7)$ & $\sim \sim$ \\
\hline Sweden & $60(4.5)$ & $549(3.0)$ & $38(4.5)$ & $546(3.6)$ & $2(0.4)$ & $\sim \sim$ \\
\hline Poland & $58(3.8)$ & $520(3.2)$ & $41(3.8)$ & $519(3.6)$ & $0(0.0)$ & $\sim \sim$ \\
\hline Latvia & $57(4.4)$ & $541(2.9)$ & $40(4.3)$ & $541(4.4)$ & $2(1.1)$ & $\sim \sim$ \\
\hline Slovak Republic & $57(3.6)$ & $534(3.7)$ & $41(3.5)$ & $529(4.5)$ & $2(0.7)$ & $\sim \sim$ \\
\hline Singapore & $55(2.9)$ & $555(4.3)$ & $40(3.0)$ & $564(4.1)$ & $5(1.0)$ & $549(12.0)$ \\
\hline Hungary & $55(4.3)$ & $554(4.5)$ & $42(4.2)$ & $547(4.3)$ & $3(1.3)$ & $542(19.7)$ \\
\hline Trinidad and Tobago & $55(3.9)$ & $437(8.5)$ & $39(3.9)$ & $435(7.0)$ & $7(2.0)$ & $428(11.8)$ \\
\hline France & $54(3.4)$ & $525(2.7)$ & $44(3.5)$ & $517(3.5)$ & $2(1.1)$ & $\sim \sim$ \\
\hline Italy & $52(3.9)$ & $554(4.5)$ & $44(3.9)$ & $550(3.7)$ & $3(1.4)$ & $531(14.9)$ \\
\hline Moldova, Rep. of & $50(4.1)$ & $500(4.3)$ & $49(3.9)$ & $500(4.5)$ & $2(0.8)$ & $\sim \sim$ \\
\hline Chinese Taipei & $44(4.1)$ & $539(3.2)$ & $54(4.1)$ & $533(2.5)$ & $2(1.2)$ & $\sim \sim$ \\
\hline Bulgaria & $42(4.1)$ & $557(6.7)$ & $55(4.1)$ & $542(6.0)$ & $3(1.2)$ & 515 (13.2) \\
\hline Morocco & $36(4.0)$ & $332(11.3)$ & $58(4.2)$ & $317(8.4)$ & $7(2.4)$ & $314(23.8)$ \\
\hline Hong Kong SAR & $32(4.4)$ & $560(4.4)$ & $66(4.4)$ & $566(2.9)$ & $2(1.1)$ & $\sim \sim$ \\
\hline International Avg. & $64(0.5)$ & $502(0.7)$ & $34(0.5)$ & $498(1.0)$ & $2(0.2)$ & $\sim \sim$ \\
\hline
\end{tabular}

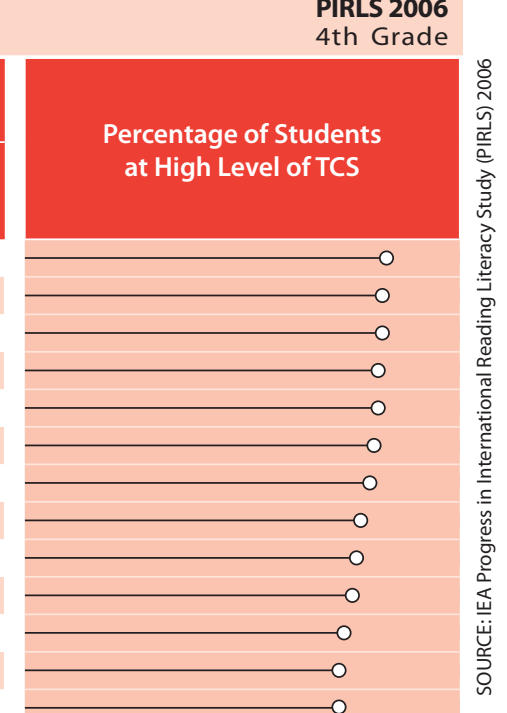


As shown in Exhibit 7.13, the majority of teachers had a positive view of the teaching profession and their career as a teacher. On average internationally, almost two thirds (64\%) of students were at the high level of the index (i.e., taught by teachers who agreed with the five statements) and one third at the medium level (34\%). Only 2 percent were at the low level. Highest levels of teacher career satisfaction were reported in Norway, Georgia, Kuwait, Macedonia, Israel, and the Canadian provinces of Nova Scotia and Ontario, where 80 percent or more of students were at the high level of the Teacher Career Satisfaction index. Less than half of the students in Chinese Taipei (44\%), Bulgaria (42\%), Morocco (36\%), and Hong Kong SAR $(32 \%)$ were at the high level. In a few countries, notably Macedonia, Israel, and England, average achievement of students taught by the most satisfied teachers was higher than that for other students. However, on average across all countries, the difference was negligible and in some countries, students at the medium level had higher achievement.

PIRLS 2006 also asked parents about their child's school. The Index of Parents' Perceptions of School Environment summarizes parents' agreement with four statements about their child's school:

- My child's school includes me in my child's education.

- My child's school should make a greater effort to include me in my child's education. (reverse coded)

- My child's school cares about my child's progress in school.

- My child's school does a good job in helping my child become better in reading.

Parents' responses were averaged on the following 4-point scale: disagree a lot $=1$, disagree a little $=2$, agree a little $=3$, and agree a lot $=4$. Students with parents averaging greater than 3 (i.e., they agreed either a little or a lot with all four statements) were assigned to the high level of the index, students whose parents averaged 2 or more but less than 3 were at the medium level, and students whose parents averaged less than 2 were assigned to the low level of the index. 
On average across countries, parents reported a good deal of satisfaction with their child's school and their involvement with it. As presented in Exhibit 7.14, 60 percent of students, on average internationally, were at the high level of the index and 38 percent at the medium level. Just 2 percent were at the low level. Parents' perceptions of the school environment were most positive in Romania, Macedonia, Trinidad and Tobago, Denmark, Georgia, Scotland, and the Canadian provinces of Alberta and Nova Scotia, where 70 percent or more of students were at the high level of the index. Fewer than 40 percent of students were at the high level in Luxembourg and Hong Kong SAR.

\section{How Safe Are Schools?}

A safe and secure school environment is a key aspect of a positive learning environment. As shown in Exhibit 7.15, the PIRLS 2006 Index of Student Safety in Schools is based on students' level of agreement with the statement "I feel safe when I am at school" and student reports of incidents of bullying, stealing, and injury to the student or someone in the student's class in the past month. Students at the high level of the index agreed (a little or a lot) that they felt safe at school, reported no incidents happening to them, and no more than one incident happening to a classmate. Students at the low level disagreed (a little or a lot) with feeling safe at school, and had two or more incidents happen to them and two or more incidents happen to a classmate during the past month. All other students were at the medium level.

Countries with the highest percentages of students at the high level of the school safety index (more than 60 percent) included the Scandinavian countries (Norway, Sweden, and Denmark) and several Eastern European countries (Georgia, Bulgaria, Poland, the Russian Federation, Macedonia, and Slovenia). However, fewer than 30 percent of students were at this level in Israel, Chinese Taipei, Trinidad and Tobago, and South Africa. On average across countries, there was a positive association between school safety and average reading achievement. Students at the high level of the school safety index had average achievement of 512 points, compared to 494 for students at the medium level, and 487 at the low level. 
Exhibit 7.14 Index of Parents' Perceptions of School Environment (PPSE)

PIRLS 2006

4th Grade

\begin{tabular}{|c|c|c|c|c|c|c|c|c|}
\hline \multirow{2}{*}{ Countries } & \multicolumn{3}{|c|}{ High PPSE } & \multicolumn{2}{|c|}{ Medium PPSE } & \multicolumn{2}{|c|}{ Low PPSE } & \multirow{2}{*}{$\begin{array}{l}\text { Percentage of Students at High } \\
\text { Level of PPSE }\end{array}$} \\
\hline & & $\begin{array}{l}\text { Percent of } \\
\text { Students }\end{array}$ & $\begin{array}{c}\text { Average } \\
\text { Achievement }\end{array}$ & $\begin{array}{l}\text { Percent of } \\
\text { Students }\end{array}$ & $\begin{array}{c}\text { Average } \\
\text { Achievement }\end{array}$ & $\begin{array}{l}\text { Percent of } \\
\text { Students }\end{array}$ & $\begin{array}{c}\text { Average } \\
\text { Achievement }\end{array}$ & \\
\hline Romania & & $81(1.5)$ & $494(5.2)$ & $19(1.5)$ & $475(8.0)$ & $0(0.1)$ & $\sim \sim$ & 0 \\
\hline Canada, Alberta & $r$ & $71(1.3)$ & $569(2.6)$ & $27(1.2)$ & $558(3.3)$ & $2(0.3)$ & $\sim \sim$ & $\longrightarrow$ \\
\hline Macedonia, Rep. of & & $71(1.1)$ & $451(4.1)$ & $28(1.0)$ & $446(5.6)$ & $1(0.2)$ & $\sim \sim$ & 0 \\
\hline Trinidad and Tobago & $r$ & $70(1.1)$ & $447(5.0)$ & $28(1.0)$ & $430(6.2)$ & $2(0.3)$ & $\sim \sim$ & $\longrightarrow$ \\
\hline Denmark & & $70(1.4)$ & $552(2.4)$ & $28(1.3)$ & $542(3.5)$ & $2(0.3)$ & $\sim \sim$ & 0 \\
\hline Canada, Nova Scotia & & $70(1.1)$ & $548(2.3)$ & $28(1.1)$ & $541(2.6)$ & $2(0.2)$ & $\sim \sim$ & $\longrightarrow$ \\
\hline Georgia & & $70(1.7)$ & $473(3.5)$ & $29(1.6)$ & $474(4.6)$ & $1(0.2)$ & $\sim \sim$ & 0 \\
\hline Scotland & s & $70(1.3)$ & $544(3.3)$ & $29(1.2)$ & $542(5.1)$ & $1(0.4)$ & $\sim \sim$ & $\longrightarrow$ \\
\hline Bulgaria & & $69(1.2)$ & $551(4.5)$ & $30(1.2)$ & $554(4.7)$ & $1(0.2)$ & $\sim \sim$ & 0 \\
\hline Norway & & $69(1.5)$ & $502(2.5)$ & $30(1.4)$ & $500(4.2)$ & $1(0.2)$ & $\sim \sim$ & 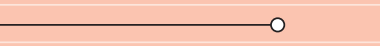 \\
\hline Sweden & & $69(1.5)$ & $552(2.7)$ & $30(1.3)$ & $552(2.7)$ & $1(0.3)$ & $\sim \sim$ & 0 \\
\hline New Zealand & $s$ & $67(1.1)$ & $551(2.3)$ & $30(1.0)$ & $544(3.3)$ & $2(0.3)$ & $\sim \sim$ & $\longrightarrow$ \\
\hline Indonesia & & $67(1.2)$ & $409(4.3)$ & $33(1.2)$ & $401(4.5)$ & $0(0.1)$ & $\sim \sim$ & $\longrightarrow$ \\
\hline Netherlands & s & $67(1.4)$ & $555(2.1)$ & $31(1.3)$ & $558(2.1)$ & $2(0.4)$ & $\sim \sim$ & $\longrightarrow$ \\
\hline Canada, Quebec & & $67(1.3)$ & $539(3.0)$ & $31(1.3)$ & $531(3.7)$ & $2(0.3)$ & $\sim \sim$ & $\longrightarrow$ \\
\hline Lithuania & & $66(1.3)$ & $538(1.9)$ & $32(1.2)$ & $538(2.1)$ & $1(0.2)$ & $\sim \sim$ & $\longrightarrow$ \\
\hline Moldova, Rep. of & & $66(1.2)$ & $504(3.3)$ & $33(1.1)$ & $494(3.8)$ & $1(0.3)$ & $\sim \sim$ & $\longrightarrow$ \\
\hline Iran, Islamic Rep. of & & $65(1.1)$ & $427(3.4)$ & $34(1.1)$ & $412(3.7)$ & $1(0.2)$ & $\sim \sim$ & $\longrightarrow$ \\
\hline Canada, British Columbia & r & $65(1.2)$ & $567(3.1)$ & $33(1.2)$ & $558(3.7)$ & $2(0.3)$ & $\sim \sim$ & $\longrightarrow$ \\
\hline South Africa & $r$ & $65(0.9)$ & $319(6.5)$ & $33(0.8)$ & $281(5.6)$ & $2(0.2)$ & $\sim \sim$ & 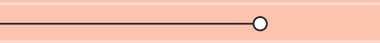 \\
\hline Canada, Ontario & & $64(1.2)$ & $561(2.9)$ & $34(1.1)$ & $555(3.7)$ & $2(0.3)$ & $\sim \sim$ & $\longrightarrow$ \\
\hline Russian Federation & & $64(1.2)$ & $567(3.6)$ & $35(1.2)$ & $565(3.5)$ & $1(0.2)$ & $\sim \sim$ & $\longrightarrow$ \\
\hline Slovak Republic & & $63(1.3)$ & $531(3.1)$ & $36(1.2)$ & $539(3.1)$ & $1(0.2)$ & $\sim \sim$ & $\longrightarrow$ \\
\hline Belgium (Flemish) & & $63(1.2)$ & $552(2.0)$ & $36(1.2)$ & $544(2.4)$ & $1(0.1)$ & $\sim \sim$ & $\longrightarrow$ \\
\hline Poland & & $61(1.1)$ & $522(2.8)$ & $38(1.0)$ & $521(2.8)$ & $1(0.3)$ & $\sim \sim$ & $\longrightarrow$ \\
\hline Spain & $s$ & $59(1.6)$ & $526(3.0)$ & $39(1.6)$ & $515(3.6)$ & $2(0.4)$ & $\sim \sim$ & 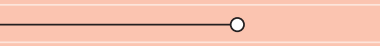 \\
\hline Austria & & $59(1.2)$ & $544(2.2)$ & $38(1.1)$ & $537(2.6)$ & $3(0.2)$ & $540(7.6)$ & $\longrightarrow$ \\
\hline Morocco & & $59(1.9)$ & $325(6.2)$ & $38(1.8)$ & $320(8.9)$ & $3(0.6)$ & $330(19.6)$ & 0 \\
\hline Italy & & $56(1.2)$ & $557(3.2)$ & $42(1.1)$ & $551(3.4)$ & $1(0.2)$ & $\sim \sim$ & $\longrightarrow$ \\
\hline Belgium (French) & $r$ & $56(1.3)$ & $505(3.2)$ & $43(1.3)$ & $500(2.7)$ & $1(0.2)$ & $\sim \sim$ & 0 \\
\hline Hungary & & $56(1.3)$ & $556(3.3)$ & $40(1.3)$ & $552(3.7)$ & $4(0.4)$ & $541(7.3)$ & $\longrightarrow$ \\
\hline Israel & s & $55(1.5)$ & $527(4.0)$ & $42(1.3)$ & $528(5.4)$ & $3(0.4)$ & $533(14.5)$ & 0 \\
\hline Slovenia & & $53(1.0)$ & $525(2.3)$ & $45(0.9)$ & $523(2.6)$ & $2(0.2)$ & $\sim \sim$ & $\longrightarrow$ \\
\hline Kuwait & s & $52(1.3)$ & $346(5.1)$ & $42(1.2)$ & $332(4.9)$ & $6(0.6)$ & $322(12.3)$ & 0 \\
\hline France & & $52(1.2)$ & $526(2.5)$ & $47(1.1)$ & $525(2.4)$ & $2(0.2)$ & $\sim \sim$ & 0 \\
\hline Chinese Taipei & & $52(1.0)$ & $539(2.4)$ & $48(1.0)$ & $536(2.4)$ & $1(0.1)$ & $\sim \sim$ & 0 \\
\hline Qatar & s & $52(0.7)$ & $366(2.2)$ & $44(0.8)$ & $353(2.2)$ & $4(0.3)$ & $329(8.8)$ & $\longrightarrow$ \\
\hline Germany & $r$ & $46(1.5)$ & $556(2.3)$ & $48(1.4)$ & $553(2.6)$ & $6(0.5)$ & $539(5.8)$ & 0 \\
\hline Iceland & $r$ & $45(0.8)$ & $515(2.2)$ & $52(0.8)$ & $519(2.3)$ & $2(0.3)$ & $\sim \sim$ & $\longrightarrow$ \\
\hline Latvia & & $44(1.2)$ & $547(2.9)$ & $54(1.2)$ & $540(2.9)$ & $3(0.3)$ & $528(8.4)$ & 0 \\
\hline Singapore & & $41(0.8)$ & $560(3.1)$ & $56(0.8)$ & $561(3.1)$ & $3(0.2)$ & $553(6.4)$ & 0 \\
\hline Luxembourg & & $39(0.8)$ & $553(2.0)$ & $55(0.8)$ & $565(1.5)$ & $6(0.3)$ & $571(3.7)$ & 0 \\
\hline Hong Kong SAR & & $39(1.1)$ & $569(2.7)$ & $59(1.0)$ & $564(2.4)$ & $2(0.3)$ & $\sim \sim$ & $\longrightarrow$ \\
\hline England & & $x x$ & $x x$ & $x x$ & $x x$ & $x x$ & $x x$ & \\
\hline United States & & -- & -- & -- & -- & -- & -- & \\
\hline \multirow[t]{2}{*}{ International Avg. } & & $60(0.2)$ & $505(0.6)$ & $38(0.2)$ & $500(0.8)$ & $2(0.0)$ & $\sim \sim$ & $\longrightarrow$ \\
\hline & & & & & & & & 50 \\
\hline
\end{tabular}

Based on parents' agreement with the following: my child's school includes me in my child's education, my child's school should make a greater effort to include me in my child's education, my child's school cares about my child's progress in school, and my child's school does a good job in helping my child become better in reading. Average is computed across the five items based on a 4-point scale: Disagree a lot $=1$, Disagree a little $=2$, Agree a little $=3$, Agree a lot $=4$. Responses for negative statements were reverse coded. High level indicates an average of greater than 3 through 4 . Medium level indicates an average of 2 through 3. Low level indicates an average of 1 to less than 2.

() Standard errors appear in parentheses. Because results are rounded to the nearest whole number, some totals may appear inconsistent.
An " $r$ " indicates data are available for $70-84 \%$ of the students. An " $\mathrm{s}$ " indicates data are available for $50-69 \%$ of the students. An " $x$ " indicates data are available for less than $50 \%$ of the students.

A dash $(-)$ indicates comparable data are not available. A tilde $(\sim)$ indicates insufficient data to report achievement.

NOTE: The International Average does not include the results from the Canadian provinces. 
Exhibit 7.15 Index of Student Safety in School (SSS)

PIRLS 2006

\begin{tabular}{|c|c|c|c|c|c|c|}
\hline \multirow{2}{*}{ Countries } & \multicolumn{2}{|c|}{ High SSS } & \multicolumn{2}{|c|}{ Medium SSS } & \multicolumn{2}{|c|}{ Low SSS } \\
\hline & $\begin{array}{l}\text { Percent of } \\
\text { Students }\end{array}$ & $\begin{array}{c}\text { Average } \\
\text { Achievement }\end{array}$ & $\begin{array}{l}\text { Percent of } \\
\text { Students }\end{array}$ & $\begin{array}{c}\text { Average } \\
\text { Achievement }\end{array}$ & $\begin{array}{l}\text { Percent of } \\
\text { Students }\end{array}$ & $\begin{array}{c}\text { Average } \\
\text { Achievemen }\end{array}$ \\
\hline Norway & $72(1.4)$ & $505(2.1)$ & $27(1.3)$ & $488(3.8)$ & $1(0.3)$ & $\sim \sim$ \\
\hline Sweden & $70(1.4)$ & $558(2.4)$ & $29(1.3)$ & $533(3.2)$ & $2(0.3)$ & $\sim \sim$ \\
\hline Denmark & $68(1.5)$ & $553(2.4)$ & $31(1.4)$ & $535(3.3)$ & $1(0.2)$ & $\sim \sim$ \\
\hline Georgia & $67(1.4)$ & $476(3.1)$ & $32(1.4)$ & $466(4.9)$ & $1(0.2)$ & $\sim \sim$ \\
\hline Bulgaria & $65(1.8)$ & $558(4.4)$ & $34(1.7)$ & $531(5.4)$ & $2(0.3)$ & $\sim \sim$ \\
\hline Poland & $65(1.3)$ & $527(2.8)$ & $33(1.2)$ & $508(3.1)$ & $2(0.3)$ & $\sim \sim$ \\
\hline Russian Federation & $63(1.5)$ & $569(4.0)$ & $36(1.5)$ & $558(3.5)$ & $1(0.2)$ & $\sim \sim$ \\
\hline Macedonia, Rep. of & $62(1.9)$ & $457(4.1)$ & $37(1.8)$ & $431(5.6)$ & $1(0.1)$ & $\sim \sim$ \\
\hline Slovenia & $61(1.5)$ & $528(2.3)$ & $37(1.4)$ & $511(2.5)$ & $2(0.2)$ & $\sim \sim$ \\
\hline Italy & $57(1.8)$ & $560(3.4)$ & $42(1.7)$ & $543(3.6)$ & $2(0.2)$ & $\sim \sim$ \\
\hline Moldova, Rep. of & $55(2.0)$ & $507(3.3)$ & $43(1.9)$ & $493(3.8)$ & $2(0.3)$ & $\sim \sim$ \\
\hline Romania & $54(2.1)$ & $493(6.2)$ & $44(2.0)$ & $490(5.1)$ & $2(0.2)$ & $\sim \sim$ \\
\hline Iceland & $54(0.8)$ & $518(1.7)$ & $42(0.7)$ & $504(1.9)$ & $4(0.3)$ & $504(7.1)$ \\
\hline Iran, Islamic Rep. of & $52(1.9)$ & $429(3.7)$ & $46(1.8)$ & $419(4.3)$ & $2(0.3)$ & $\sim \sim$ \\
\hline Germany & $51(1.2)$ & $566(2.3)$ & $45(1.1)$ & $540(2.5)$ & $4(0.3)$ & $514(6.5)$ \\
\hline Canada, British Columbia & $50(1.4)$ & $569(2.9)$ & $47(1.3)$ & $551(3.0)$ & $3(0.4)$ & $540(9.1)$ \\
\hline Lithuania & $49(1.3)$ & $545(2.1)$ & $48(1.3)$ & $529(2.0)$ & $4(0.3)$ & $532(4.5)$ \\
\hline Slovak Republic & $49(1.4)$ & $540(3.6)$ & $47(1.3)$ & $523(3.3)$ & $4(0.4)$ & $523(5.6)$ \\
\hline Canada, Quebec & $49(1.7)$ & $546(3.1)$ & $48(1.5)$ & $526(3.0)$ & $4(0.4)$ & $510(7.9)$ \\
\hline Austria & $48(1.3)$ & $547(2.7)$ & $47(1.0)$ & $531(2.5)$ & $5(0.4)$ & $528(4.5)$ \\
\hline United States & $48(1.6)$ & $557(3.0)$ & $49(1.4)$ & $528(3.8)$ & $3(0.4)$ & $505(8.6)$ \\
\hline Canada, Nova Scotia & $46(1.4)$ & $559(2.3)$ & $50(1.2)$ & $534(2.5)$ & $4(0.3)$ & $521(6.3)$ \\
\hline Netherlands & $46(1.5)$ & $555(1.9)$ & $50(1.2)$ & $542(2.2)$ & $5(0.5)$ & $532(6.0)$ \\
\hline Luxembourg & $46(0.7)$ & $567(1.4)$ & $50(0.7)$ & $550(1.5)$ & $4(0.2)$ & $536(6.0)$ \\
\hline Canada, Alberta & $45(1.4)$ & $576(2.6)$ & $52(1.3)$ & $551(2.7)$ & $3(0.3)$ & $535(6.7)$ \\
\hline Latvia & $45(1.6)$ & $550(3.0)$ & $51(1.5)$ & $536(2.7)$ & $5(0.5)$ & $522(5.3)$ \\
\hline Spain & $44(1.5)$ & $522(2.7)$ & $53(1.4)$ & $509(2.8)$ & $3(0.4)$ & $489(7.9)$ \\
\hline Scotland & $43(1.6)$ & $540(3.3)$ & $53(1.4)$ & $519(3.4)$ & $4(0.4)$ & $497(10.2)$ \\
\hline Belgium (Flemish) & $43(1.3)$ & $556(2.0)$ & $52(1.1)$ & $542(2.1)$ & $5(0.5)$ & $521(5.6)$ \\
\hline Hong Kong SAR & $42(1.3)$ & $573(2.6)$ & $53(1.2)$ & $558(2.5)$ & $5(0.5)$ & $544(5.7)$ \\
\hline Hungary & $41(1.3)$ & $567(3.5)$ & $54(1.2)$ & $541(3.1)$ & $5(0.4)$ & $537(6.3)$ \\
\hline France & $40(1.6)$ & $534(2.3)$ & $55(1.4)$ & $515(2.4)$ & $5(0.5)$ & $502(5.9)$ \\
\hline Canada, Ontario & $39(1.6)$ & $569(2.9)$ & $57(1.4)$ & $550(2.9)$ & $4(0.4)$ & $515(8.9)$ \\
\hline Morocco & $38(1.9)$ & $336(6.9)$ & $61(1.8)$ & $318(6.7)$ & $1(0.2)$ & $\sim \sim$ \\
\hline Singapore & $38(0.9)$ & $575(3.5)$ & $58(0.8)$ & $549(2.9)$ & $4(0.3)$ & $545(6.3)$ \\
\hline New Zealand & $37(1.1)$ & $551(2.8)$ & $58(0.9)$ & $523(2.2)$ & $4(0.4)$ & $516(6.9)$ \\
\hline England & $37(1.6)$ & $564(3.7)$ & $58(1.5)$ & $529(3.0)$ & $5(0.4)$ & $504(6.0)$ \\
\hline Belgium (French) & $34(1.2)$ & $512(3.3)$ & $59(1.1)$ & $495(2.7)$ & $6(0.5)$ & $490(5.4)$ \\
\hline Indonesia & $33(1.3)$ & $408(5.0)$ & $65(1.3)$ & $406(4.4)$ & $3(0.3)$ & $381(12.3)$ \\
\hline Kuwait & $31(1.3)$ & $346(6.2)$ & $66(1.3)$ & $339(4.9)$ & $3(0.4)$ & $318(14.9)$ \\
\hline Qatar & $31(0.5)$ & $367(2.4)$ & $63(0.5)$ & $353(1.5)$ & $6(0.3)$ & $354(5.3)$ \\
\hline Israel & $26(1.2)$ & $526(5.7)$ & $68(1.1)$ & $515(3.4)$ & $6(0.6)$ & $512(7.4)$ \\
\hline Chinese Taipei & $26(1.3)$ & $551(3.0)$ & $66(1.2)$ & $531(2.0)$ & $8(0.6)$ & $525(4.5)$ \\
\hline Trinidad and Tobago & $25(1.8)$ & $460(8.1)$ & $71(1.6)$ & $429(4.7)$ & $4(0.4)$ & $429(14.9)$ \\
\hline South Africa & $23(0.7)$ & $322(8.3)$ & $73(0.6)$ & $303(5.4)$ & $4(0.2)$ & $291(11.7)$ \\
\hline International Avg. & $47(0.2)$ & $512(0.7)$ & $50(0.2)$ & $494(0.6)$ & $3(0.1)$ & $487(1.5)$ \\
\hline
\end{tabular}
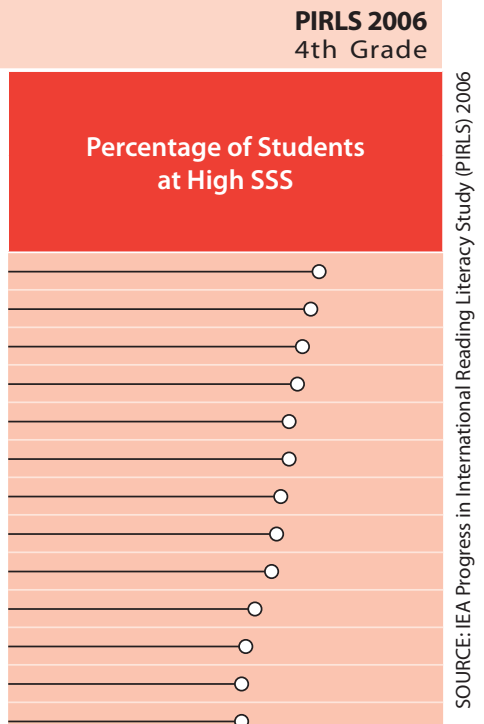

Iran, Islamic Rep. of

Canada, British Columbia

Lithuania

$48(1.5)$

$49(1.4)$

$531(2.5)$
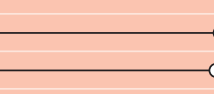

O
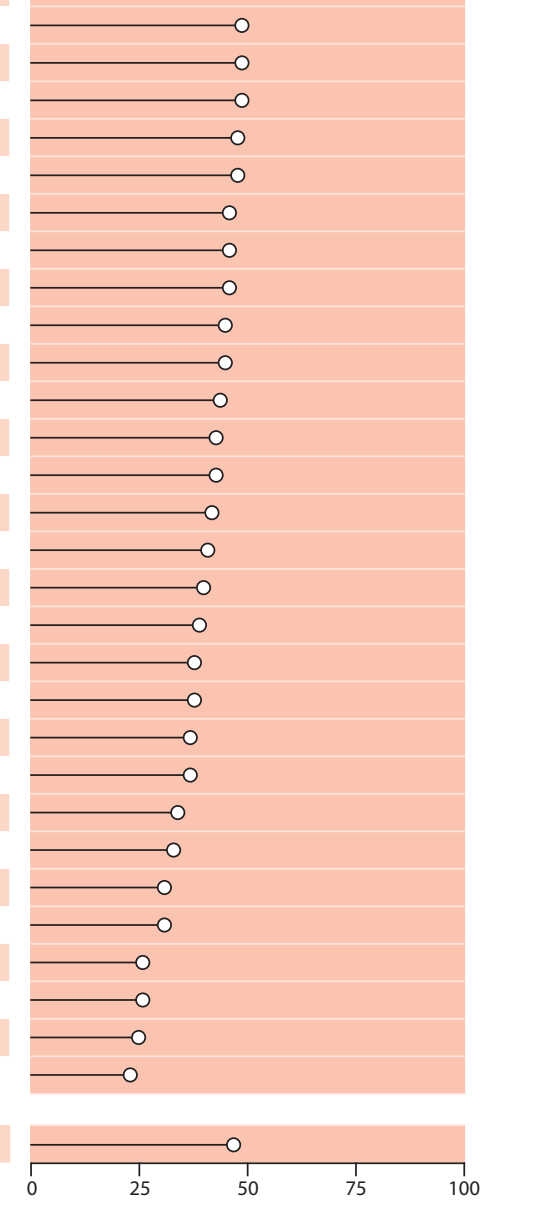

Based on students' agreement with the statement "I feel safe when I am at school" and incidents of stealing, bullying and injury happening to the student or someone in their class in the last month. High level indicates students agree a little or a lot with feeling safe at school, had one or fewer incidents happen to them, and had one or fewer incidents happen to someone in their class in the last month. Low level indicates that students disagree a little or a lot with feeling safe at school, had two or more incidents happen to them, and had two or more incidents happen to someone in their class in the last month. Medium level includes all other combinations of responses.

() Standard errors appear in parentheses. Because results are rounded to the nearest whole number, some totals may appear inconsistent.
An " $r$ " indicates data are available for $70-84 \%$ of the students. An " $s$ " indicates data are available for $50-69 \%$ of the students. An " $\mathrm{x}$ " indicates data are available for less than $50 \%$ of the students.

A tilde $(\sim)$ indicates insufficient data to report achievement.

NOTE: The International Average does not include the results from the Canadian provinces. 
Complementing students' perceptions, the PIRLS 2006 Index of Principals' Perception of School Safety (see Exhibit 7.16) is based on principals' characterizations of the extent to which a number of student behaviors, including classroom disturbance, cheating, profanity, vandalism, theft, intimidation or verbal abuse of other students, and physical conflict among students, are a problem in their school. Principals' average response was computed on a 4-point scale: serious problem $=1$, moderate problem $=$ 2 , minor problem $=3$, and not a problem $=4$. Students whose principal had an average greater than 3 were assigned to the high level of the index; those whose principal's average was between 2 and 3 were assigned to the medium category, and those with less than 2 to the low level.

On average across countries, principals reported a fairly high level of school safety, with 60 percent of students at the level of the school safety index and 32 percent at the medium level. Just 7 percent, on average, were at the low level. Despite the high percentage of students at the high level on average, there was a wide variation across countries, with the highest percentages (more than 80\%) in England, Hong Kong SAR, Iran, Chinese Taipei, Scotland, Belgium (Flemish), and the Russian Federation, and lower percentages (less than 40\%) in the Slovak Republic, South Africa, the Netherlands, Hungary, Kuwait, Indonesia, and Morocco. Unusually high percentages of students were at the low level of the index in Qatar $(32 \%)$, Kuwait (26\%), Indonesia (46\%), and Morocco (73\%). Participants showing improvement in school safety since 2001 (i.e., increased percentages at the high index level) included Iran, New Zealand, Italy, Iceland, Germany, and the Canadian province of Quebec, while those with decreased percentages were the Russian Federation, Singapore, Romania, Latvia, and Morocco. On average internationally, and, in many countries, there was a positive relationship between principals' perception of school safety and average reading achievement. 
Exhibit 7.16 Index of Principals' Perception of School Safety (PPSS) with Trends

PIRLS 2006

\begin{tabular}{|c|c|c|c|}
\hline \multirow{2}{*}{ Countries } & \multicolumn{3}{|c|}{ High PPSS } \\
\cline { 2 - 5 } & $\begin{array}{c}2006 \\
\text { Percent } \\
\text { of Students }\end{array}$ & $\begin{array}{c}\text { Average } \\
\text { Achievement }\end{array}$ & $\begin{array}{c}\text { Difference } \\
\text { in Percent } \\
\text { from 2001 }\end{array}$ \\
\hline
\end{tabular}

\begin{tabular}{|c|c|c|c|}
\hline \multicolumn{3}{|c|}{ Medium PPSS } & \\
\hline $\begin{array}{c}2006 \\
\text { Percent } \\
\text { of Students }\end{array}$ & $\begin{array}{c}\text { Average } \\
\text { Achievement }\end{array}$ & $\begin{array}{c}\text { Difference } \\
\text { in Percent } \\
\text { from 2001 }\end{array}$ & \\
\hline
\end{tabular}

4th Grade

England

Hong Kong SAR

Iran, Islamic Rep. of

Chinese Taipei

Scotland

Belgium (Flemish)

Russian Federation

Spain

Canada, British Columbia

Moldova, Rep. of

Singapore

New Zealand

United States

Italy

Canada, Nova Scotia

Georgia

Canada, Alberta

France

Denmark

Canada, Quebec

Canada, Ontario

Austria

Macedonia, Rep. of

Belgium (French)

Sweden

Iceland

Norway

Lithuania

Bulgaria

Romania

Germany

Slovenia

Latvia

Israel

Qatar

Trinidad and Tobago

Poland

Slovak Republic

South Africa

Netherlands

Hungary

Kuwait

Indonesia

Morocco

${ }^{1}$ Luxembourg

International Avg.

\begin{abstract}
$90(1.9)$
\end{abstract}
88 (2.9)

$88(2.2)$

$85(3.0)$

r $\quad 85(4.1)$

$84(3.4)$

$83(2.5)$

$79(3.2)$

$79(3.5)$

$77(3.8)$

$77(0.0)$

$77(2.8)$

$77(3.7)$

$76(3.6)$

75 (3.4)

$73(3.9)$

$72(4.0)$

$72(3.5)$

$71(3.8)$

$69(4.5)$

$68(5.3)$

$67(3.7)$

r $66(4.3)$

$65(4.7)$

$64(3.8)$

$r \quad 62(0.3)$

$61(4.8)$

57 (4.1)

55 (4.4)

$51(4.3)$

$50(3.0)$

$47(3.8)$

$47(3.8)$

$47(4.3)$

$43(0.2)$

$43(4.0)$

40 (4.4)

37 (3.9)

$36(2.8)$

r $33(4.3)$

$33(4.0)$

$32(3.7)$

$28(3.5)$

r $12(3.4)$

$--$
$547(3.0)$

564 (2.4)

422 (3.7)

535 (2.3)

531 (3.7)

547 (2.1)

$564(3.7)$

$517(2.6)$

$563(2.7)$

500 (3.3)

$558(3.0)$

541 (2.4)

$545(3.7)$

553 (3.1)

544 (2.6)

469 (3.9)

$563(2.7)$

529 (2.5)

$549(2.8)$

538 (3.4)

555 (3.6)

541 (2.8)

$448(6.8)$

507 (3.5)

$550(2.6)$

514 (1.6)

$498(3.2)$

$536(2.3)$

$553(5.9)$

$486(7.5)$

$557(2.7)$

$521(2.8)$

548 (3.6)

$519(7.2)$

$356(1.9)$

$452(7.5)$

$517(4.1)$

$531(6.0)$

326 (12.4)

$547(2.9)$

554 (5.9)

$341(8.6)$

$412(7.0)$

--
$-1(4.4)$

$13(4.5)$

$9(2.1)$

$11(2.8)$

$11(2.2)$

$14(2.9)$

$11(6.0)$

$\Delta 0 \quad 16(3.4)$

$-9(3.2) \quad 17 \quad 17(2.5)$

$\Delta 0 \quad 17(2.9)$

$\Delta \Delta$

1 (5.2)

$-7(3.3)$

$12(4.8)$

$6(5.4)$

$13(5.3)$

$\Delta 0$

$\Delta 0$

$-2(5.3)$

00

$14(7.0)$

$12(7.2)$

00

$0(5.7)$

$\Delta 0$

$7(5.9)$

$9(0.5)$

$9(0.5)$

$1(6.8)$
$-7(5.8)$
$-6(5.8)$

$-6(5.8)$

$-36(5.1)$

$11(4.8)$

$-1(5.3)$

$51(3.9)$

$3(6.4)-48(3.9)$

$00 \quad 24(0.2)$

$\Delta 0 \quad 56(4.0)$

$\Delta \diamond \quad 59(4.3)$

$10(5.3)$

$496(5.6)$

$572(7.4)$

417 (12.4)

$536(4.3)$

$517(10.7)$

$545(6.2)$

$570(8.0)$

$500(7.0)$

$542(6.0)$

$501(8.5)$

$559(7.4)$

$507(7.1)$

$525(5.7)$

$-6(5.2)$

$556(10.1) \quad-11(4.6)$

$536(4.4)$

481 (7.5)

$554(5.1)$

$505(5.2)$

$543(4.2)$

$521(4.6)$

$553(3.6)$

$533(4.4)$

$449(11.7) \quad-3(5.6)$

$-3(5.6)$

$547(3.9) \quad-7(6.0)$

$509(2.2)$
$498(4.2)$

$-9(0.5)$

$-9(0.5)$
$0(6.8)$

$7(5.7)$

$7(5.7)$

$537(6.9)$

$4(5.8)$

\begin{tabular}{|r|r|}
\hline $489(7.4)$ & $24(4.9)$ \\
\hline $542(3.2)$ & $-10(4.8)$ \\
\hline $522(3.5)$ & $-1(5.3)$
\end{tabular}

$522(3.5) \quad-1(5.3)$

$534(3.9)$

$514(4.8)$

$2(1.2)$

$19(5.8) \bigcirc \quad 5(1.9)$

$3(6.6)$

$346(2.3) \quad \diamond \diamond$

$427(7.7)$

$521(3.0)$

$\Delta 0$

$\Delta 0$

$\Delta 0$

$51(2.9)$

$530(3.3)$

$-9(5.6)$

$\Delta 0$

$547(2.3) \quad-9(6.0)$

$547(2.3)$

$-5(5.4)$

$66(4.1)$

$340(13,8)$

00

$42(4.4)$

$550(3.8)$

$332(6.7)$

$6(5.5)$

$\Delta \Delta$

$\Delta \diamond$

$326(17.4)$

$15(3.6)$

$--\quad--$

$326(17.4)$

$-13(6.3)$

$--$

$32(0.6) \quad 495(1.1)$

ه Percent in 2006 significantly higher

A dash (-) indicates comparable data are not available. A tilde $(\sim)$ indicates insufficient data to report achievement.

A diamond $(\diamond)$ indicates the country did not participate in the 2001 assessment.

NOTE: The International Average does not include the results from the Canadian provinces. Trend Note: The primary education systems of the Russian Federation and Slovenia underwent structural changes. Data for Canada, Ontario include only public schools.

1 Primary schools in Luxembourg do not have principals.
Based on principals' responses about the degree each was a school problem: classroom disturbances, cheating, profanity, vandalism, theft, intimidation or verbal abuse of other Serious problem $=1$, Moderate problem $=2$, Minor problem $=3$, Not a problem $=4$. High level indicates an average of greater than 3 through 4 . Medium level indicates an average of 2 through 3. Low level indicates an average of 1 to less than 2 .

() Standard errors appear in parentheses. Because results are rounded to the nearest whole number, some totals may appear inconsistent.

An " $r$ " indicates data are available for $70-84 \%$ of the students. An "s" indicates data are available for $50-69 \%$ of the students. An " $x$ " indicates data are available for less than $50 \%$ of the students. 

Appendix A

Supplementary Information About PIRLS 2006 Procedures 


\section{Participants in PIRLS 2006}

Building on two earlier IEA studies of reading achievement, the 1970 study of reading comprehension in 15 countries $^{1}$ and the 1991 Reading Literacy Study ${ }^{2}$ in 32 countries, the PIRLS assessment of student achievement in reading literacy at the fourth grade is an integral component of IEA's ongoing program of studies in the core subjects of mathematics, science, and reading. ${ }^{3}$ Beginning with PIRLS 2001, ${ }^{4}$ PIRLS was designed from the outset to monitor progress in reading achievement on a regular 5-year cycle. PIRLS 2006, the second study in this continuing cycle, was designed to measure children's reading literacy achievement, to provide information on changes in achievement since 2001, and to add to the store of knowledge about children's home and school experiences in learning to read.

Forty countries, including Belgium with 2 education systems and Canada with 5 provinces, participated in the 2006 PIRLS assessment for a total of 45 participants. Of these, 26 countries and 2 provinces had trend data from PIRLS 2001. ${ }^{5}$ Participating in PIRLS for the first time in 2006 were 13 countries (counting Belgium as one country) and 3 provinces (see Exhibit A.1).

\section{The PIRLS 2006 Test Instruments}

Across the PIRLS 2006 assessment, the questions on the reading passages enabled students to demonstrate a range of abilities and skills in constructing meaning from written texts. An important innovation in PIRLS 2006 was the ability to report achievement results according to reading comprehension processes, in addition to reading purposes. In PIRLS 2001, achievement results were reported for overall reading comprehension and separately by literary and informational purposes, but not by process of comprehension. Subsequently, research has indicated that it would be possible also to report comprehension processes separately by combining the retrieval and straightforward inferencing processes to make one scale and the interpreting and integrating and examining and evaluating processes to make another. ${ }^{6}$

Thorndike, R.L. (1973). Reading comprehension in fifteen countries: An empirical study. International studies in evaluation: Vol. 3. Stockholm: Almqvist \& Wiksell.

2 Elley, W.B. (Ed.). (1994). The IEA study of reading literacy: Achievement and instruction in thirty-two school systems. Oxford, England: Elsevier Science Ltd.

3 Mathematics and science are assessed at fourth and eighth grades by IEA's TIMSS (Trends in International Mathematics and Science Study).

4 Mullis, I.V.S., Martin, M.O., Gonzalez, E.J., \& Kennedy, A.M. (2003). PIRLS 2001 international report: IEA's study of reading literacy achievement in primary schools in 35 countries. Chestnut Hill: MA: Boston College.

5 Although Kuwait participated in PIRLS 2001, the data were not considered comparable for measuring trends, and so Kuwait does not appear in any trend exhibits.

6 Bos, W., Lankes, E. M., Prenzel, M., Schwippert, K., Walther, G., \& Valtin, R. (Hrsg.). (2003). Ergebnisse aus IGLU: Schülerleistungen am Ende der vierten Jahrgangsstufe im internationalen Vergleich. New York: Waxmann.

Mullis, I.V.S., Martin, M.O., \& Gonzalez, E.J. (2004). International achievement in the processes of reading comprehension: results from PIRLS 2001 in 35 countries. (2004). Chestnut Hill, MA: Boston College. 


\begin{tabular}{|c|c|c|}
\hline Countries & 2006 & 2001 \\
\hline \multicolumn{3}{|l|}{ Argentina } \\
\hline Austria & 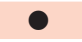 & \\
\hline Belgium (Flemish) & 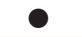 & \\
\hline Belgium (French) & 0 & \\
\hline Belize & & ○ \\
\hline Bulgaria & 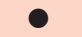 & ○ \\
\hline Canada, Alberta & ○ & \\
\hline Canada, British Columbia & ○ & \\
\hline Canada, Nova Scotia & ○ & \\
\hline Canada, Ontario & 0 & 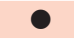 \\
\hline Canada, Quebec & ○ & ○ \\
\hline Chinese Taipei & ○ & \\
\hline Colombia & & ○ \\
\hline Cyprus & & ○ \\
\hline Czech Republic & & ○ \\
\hline Denmark & ○ & \\
\hline England & 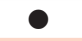 & $\bullet$ \\
\hline France & ○ & ○ \\
\hline Georgia & ○ & \\
\hline Germany & ○ & 0 \\
\hline Greece & & ○ \\
\hline Hong Kong SAR & ○ & ○ \\
\hline Hungary & $\bullet$ & 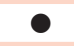 \\
\hline Iceland & ○ & ○ \\
\hline Indonesia & 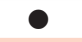 & \\
\hline Iran, Islamic Rep. of & ○ & ○ \\
\hline Israel & $\bullet$ & ○ \\
\hline Italy & ○ & ○ \\
\hline${ }^{1}$ Kuwait & ○ & \\
\hline Latvia & $\bullet$ & 0 \\
\hline Lithuania & ○ & ○ \\
\hline Luxembourg & ○ & \\
\hline Macedonia, Rep. of & 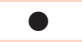 & ○ \\
\hline Moldova, Rep. of & ○ & ○ \\
\hline Morocco & ○ & ○ \\
\hline Netherlands & 0 & ○ \\
\hline New Zealand & ○ & ○ \\
\hline Norway & ○ & O \\
\hline Poland & ○ & \\
\hline Qatar & 0 & \\
\hline Romania & O & ○ \\
\hline Russian Federation & 0 & ○ \\
\hline Scotland & $\bullet$ & ○ \\
\hline Singapore & ○ & ○ \\
\hline Slovak Republic & ○ & ○ \\
\hline Slovenia & 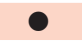 & ○ \\
\hline South Africa & ○ & \\
\hline Spain & ○ & \\
\hline Sweden & ○ & ○ \\
\hline Trinidad and Tobago & ○ & \\
\hline Turkey & & ○ \\
\hline United States & ○ & ○ \\
\hline
\end{tabular}


However, this necessitated increasing the number of passages and items in the assessment from 8 to 10 to ensure that there were sufficient items for the process scales.

Half of the passages and items from the 2001 assessment, two literary and two informational, have been published with the international report so that readers could appreciate the nature of the PIRLs reading tasks, and half were kept secure to serve as a basis for linking to the PIRLS 2006 assessment. The four secure passages and items (two literary and two informational) were available for use again in 2006. However, in addition, it was necessary to develop 6 new passages and items to replace the released passages and to expand the scope of the assessment from 8 to 10 passages.

The selection of the assessment passages and the development of the items and scoring guides were the result of an intensive process of collaboration, piloting, and review. ${ }^{7}$ Draft passages and items were subjected to full-scale field testing before the instruments for the main data collection were finalized. ${ }^{8}$ The final version of the assessment was endorsed by the NRCs of the participating countries.

Exhibit A.2 shows the distribution of the PIRLS 2006 test items by reading purpose and process category. There were 126 items in the assessment, approximately half of which were multiple-choice and half constructedresponse. The constructed-response items required students to generate and write their own answers. Some items required short answers while others demanded a more elaborate response. In scoring the test, correct answers to most questions (including all those in multiple-choice format) were worth one point. However, responses to questions seeking more elaborate responses were evaluated for partial credit, with a fully-correct answer being awarded two or three points. Thus, the total number of score points available for analyses (167) exceeds the number of items in the assessment. The student answer booklet provided an indication to the student of how many score points would be awarded for each answer, and how much writing was expected. About 60 percent of the score points came from constructedresponse items. Of the 126 items, 49 were trend items, that is, items from

7 For a full discussion of the PIRLS 2006 test development effort, see Kennedy, A.M. \& Sainsbury, M. (2007). Developing the PIRLS 2006 reading assessment and scoring guides. In M.O. Martin, I.V.S. Mullis, \& A.M. Kennedy (Eds.), PIRLS 2006 technical report. Chestnut Hill, MA: Boston College.

8 Approximately 50,000 students from almost 1,200 schools in 42 countries participated in the field test. 
Exhibit A.2 Distribution of Items by Reading Purpose and Process Category

\begin{tabular}{|c|c|c|c|c|}
\hline \multicolumn{5}{|c|}{ Items in the PIRLS 2006 Assessment } \\
\hline Reading Purpose & $\begin{array}{l}\text { Total Number } \\
\text { of Items }\end{array}$ & $\begin{array}{c}\text { Number of } \\
\text { Multiple-choice } \\
\text { Items }\end{array}$ & $\begin{array}{l}\text { Number of } \\
\text { Constructed- } \\
\text { response Items }\end{array}$ & $\begin{array}{l}\text { Total Number } \\
\text { Score Points }\end{array}$ \\
\hline Literary Experience & 64 & 34 & 30 & 85 \\
\hline Acquire and Use Information & 62 & 30 & 32 & 82 \\
\hline Total & 126 & 64 & 62 & 167 \\
\hline
\end{tabular}

\begin{tabular}{|l|c|c|c|c|c|}
\hline Reading Process & $\begin{array}{c}\text { Percentage } \\
\text { of Items }\end{array}$ & $\begin{array}{c}\text { Total Number } \\
\text { of Items }\end{array}$ & $\begin{array}{c}\text { Number of } \\
\text { Multiple-choice } \\
\text { Items }\end{array}$ & $\begin{array}{c}\text { Number of } \\
\text { Constructed- } \\
\text { response Items }\end{array}$ & $\begin{array}{c}\text { Number of } \\
\text { Score Points }\end{array}$ \\
\hline $\begin{array}{l}\text { Focus on and Retrieve Explicitly Stated } \\
\text { Information and Ideas }\end{array}$ & 22 & 31 & 19 & 12 \\
\hline $\begin{array}{l}\text { Make Straightforward Inferences } \\
\text { Interpret and Integrate Ideas } \\
\text { and Information }\end{array}$ & 28 & 43 & 34 & 29 & 14 \\
\hline $\begin{array}{l}\text { Examine and Evaluate Content, } \\
\text { Language, and Textual Elements }\end{array}$ & 14 & 18 & 6 & 28 \\
\hline Total & 100 & 126 & 64 & 87 \\
\hline
\end{tabular}

Trend Items in the PIRLS 2006 Assessment (Items also used in PIRLS 2001)

\begin{tabular}{|l|c|c|c|c} 
Reading Purpose & $\begin{array}{c}\text { Total Number } \\
\text { of Items }\end{array}$ & $\begin{array}{c}\text { Number of } \\
\text { Multiple-choice } \\
\text { Items }\end{array}$ & $\begin{array}{c}\text { Number of } \\
\text { Constructed- } \\
\text { response Items }\end{array}$ & $\begin{array}{c}\text { Total Number } \\
\text { Score Points }\end{array}$
\end{tabular}

\begin{tabular}{|l|l|l|l|l|}
\hline Literary Experience & 26 & 13 & 13 & 33 \\
\hline Acquire and Use Information & 23 & 10 & 13 & 33 \\
\hline Total & 49 & 23 & 26 & 66 \\
\hline
\end{tabular}

\begin{tabular}{|l|c|c|c|c|}
\hline Reading Process & $\begin{array}{c}\text { Total Number } \\
\text { of Items }\end{array}$ & $\begin{array}{c}\text { Number of } \\
\text { Multiple-choice } \\
\text { Items }\end{array}$ & $\begin{array}{c}\text { Number of } \\
\text { Constructed- } \\
\text { response Items }\end{array}$ & $\begin{array}{c}\text { Number of } \\
\text { Score Points }\end{array}$ \\
\hline $\begin{array}{l}\text { Focus on and Retrieve Explicitly Stated } \\
\text { Information and Ideas }\end{array}$ & 12 & 5 & 7 & 15 \\
\hline Make Straightforward Inferences & 18 & 10 & 8 & 21 \\
\hline $\begin{array}{l}\text { Interpret and Integrate Ideas } \\
\text { and Information }\end{array}$ & 12 & 3 & 10 & 22 \\
\hline $\begin{array}{l}\text { Examine and Evaluate Content, } \\
\text { language, and Textual Elements }\end{array}$ & 7 & 5 & 2 & 8 \\
\hline Total & 49 & 23 & 27 & 66 \\
\hline
\end{tabular}


the 4 passages that were retained from 2001 to serve as the basis of the link between the 2001 and 2006 assessments.

\section{PIRLS Testing Time and Booklet Design}

Given the broad coverage goals of the PIRLS 2006 framework and its emphasis on the use of authentic texts, the passages and accompanying items required extensive testing time. Students were given 40 minutes to complete a passage. With 10 passages, it would have taken 400 minutes to administer the entire assessment to a single child. However, so as not to overburden the relatively young children participating in PIRLS, and in line with the practice in 2001, the testing time was limited to 80 minutes (two passages) per student, with an additional 15-30 minutes allotted for a student questionnaire.

With 10 reading passages in total, but just 2 to be given to any one student, passages and their accompanying items were assigned to student test booklets according to a matrix sampling plan. The 10 passages were distributed across 13 booklets, 2 per booklet, so that passages were paired together in a booklet in as many different ways as possible. Each student booklet consisted of two 40-minute blocks of passages and items. So as to present at least some passages in a more natural, authentic setting, two blocks (one literary and one informational) were presented in colorized, magazinetype format, with the questions appearing in a separate booklet. This booklet, Booklet 13, is referred to as the PIRLs "Reader."

\section{Translation Verification}

The PIRLS 2006 instruments were prepared in English and translated into 45 languages (Exhibit A.3). Although most countries administered the assessment in just one language, nine countries and the five Canadian provinces administered it in two languages, and Spain administered the assessment in its five official languages: Spanish (Castilian), Catalonian, Galician, Basque, and Valencian. Most impressive of all, South Africa administered the PIRLS 2006 assessment in 11 languages: English, Afrikaans,

9 The PIRLS 2006 test booklet design is described in Mullis, I.V.S., Kennedy, A.M., Martin, M.O., \& Sainsbury, M. (2006). PIRLS 2006 assessment framework and specifications (2nd ed.). Chestnut Hill, MA: Boston College. 
isiZulu, isiXhosa, Sepedi, Sesotho, Setswana, isiNdebele, Siswati, Tshivenda, and Xitsonga.

The test was administered most often in English (seven countries, counting the Canadian provinces once), with Arabic and French second (three countries). In addition, it was sometimes necessary to modify the international versions for cultural reasons, even in the seven countries that tested in English.

The translation process represented an enormous effort for the national centers, with many checks along the way, including an exhaustive process of review and verification. ${ }^{10}$

The translation effort included: (1) developing explicit guidelines for translation and cultural adaptation, (2) translation of the instruments by the national centers in accordance with the guidelines-using two or more independent translations, (3) verification of translation quality by the IEA Secretariat using professional translators from an independent translation company, (4) corrections by national centers in accordance with the suggestions made, (5) verification by the TIMSS \& PIRLS International Study Center that the corrections were made and that the layout of the instruments corresponded to the international standard, and (6) a series of statistical checks after the testing to detect items that did not perform comparably across countries.

For the participating countries, the bulk of the translation effort took place prior to the field test. After the field test, countries needed only to make changes to the items or passages that resulted from analysis of the field test data.

The translations of the PIRLS 2006 data-collection instruments were verified twice-the field-test versions before the field test and the final versions before the main data collection. Countries, therefore, had the benefit of two careful reviews of their translations. They also had the benefit of diagnostic item statistics from the field test data analysis, which helped to identify mistranslations that could be corrected before the main data collection.

10 More details about the translation verification procedures can be found in Malak, B. \& Trong, K. L. (2007). Translating the PIRLS 2006 reading assessment and questionnaires. In M.O. Martin, I.V.S. Mullis, and A.M. Kennedy (Eds.), PIRLS 2006 technical report. Chestnut Hill, MA: Boston College. 
Exhibit A.3 Languages of Instruction and Testing

\begin{tabular}{|c|c|c|}
\hline Country & Language(s) of Instruction & Language(s) of Testing \\
\hline Austria & German & German \\
\hline Belgium (Flemish) & Dutch & Dutch \\
\hline Belgium (French) & French & French \\
\hline Bulgaria & Bulgarian & Bulgarian \\
\hline Canada, Alberta & English, French & English, French \\
\hline Canada, British Columbia & English, French & English, French \\
\hline Canada, Nova Scotia & English, French & English, French \\
\hline Canada, Ontario & English, French & English, French \\
\hline Canada, Quebec & English, French, Aboriginal languages & English, French \\
\hline Chinese Taipei & Mandarin & Chinese Mandarin \\
\hline Denmark & Danish & Danish \\
\hline England & English & English \\
\hline France & French & French \\
\hline Georgia & Georgian & Georgian \\
\hline Germany & German & German \\
\hline Hong Kong SAR & Chinese & Modern Standard Chinese \\
\hline Hungary & Hungarian & Hungarian \\
\hline Iceland & Icelandic & Icelandic \\
\hline Indonesia & Indonesian & Indonesian \\
\hline Iran, Islamic Rep. of & Farsi & Farsi \\
\hline Israel & Hebrew, Arabic & Hebrew, Arabic \\
\hline Italy & Italian, French, German, Ladin, and Slovenian & Italian \\
\hline Kuwait & Arabic and local dialects & Arabic \\
\hline Latvia & Latvian, Russian & Latvian, Russian \\
\hline Lithuania & Lithuanian, Russian, Polish & Lithuanian \\
\hline Luxembourg & Luxembourgish, French, German & German \\
\hline Macedonia, Rep. of & Macedonian, Albanian, Turkish and Serbian & Macedonian, Albanian \\
\hline Moldova, Rep. of & Romanian, Russian & Romanian, Russian \\
\hline Morocco & - & Arabic \\
\hline Netherlands & Dutch & Dutch \\
\hline New Zealand & English, Māori & English, Maori \\
\hline Norway & Bokmål, Nynorsk, Sámi & Bokmål, Nynorsk \\
\hline Qatar & Arabic, English & Arabic \\
\hline Poland & Polish & Polish \\
\hline Romania & Romanian, Hungarian & Romanian, Hungarian \\
\hline Russian Federation & Russian & Russian \\
\hline Scotland & English, Gaelic & English \\
\hline Singapore & English, Malay, Chinese (Mandarin), Tamil & English \\
\hline Slovak Republic & Slovak, Hungarian & Slovak, Hungarian \\
\hline Slovenia & Slovenian & Slovenian \\
\hline South Africa & $\begin{array}{l}\text { Afrikaans, English, isiZulu, isiXhosa, Sepedi, Sesotho, } \\
\text { Setswana, isiNdebele, SiSwati, Tshivenda, Xitsonga }\end{array}$ & $\begin{array}{l}\text { Afrikaans, English, isiZulu, isiXhosa, Sepedi, Sesotho, } \\
\text { Setswana, isiNdebele, SiSwati, Tshivenda, Xitsonga }\end{array}$ \\
\hline Spain & Spanish (Castilian), Catalonian, Galician, Basque, Valencian & Spanish (Castilian), Catalonian, Galician, Basque, Valencian \\
\hline Sweden & Swedish & Swedish \\
\hline Trinidad and Tobago & English & English \\
\hline United States & English & English \\
\hline
\end{tabular}




\section{Sample Implementation and Participation Rates}

PIRLS 2006 had as its target population students enrolled in the fourth grade of formal schooling, counting from the first year of primary school as defined by UNESCO's International Standard Classification for Education (ISCED). ${ }^{11}$ According to the ISCED classification, Level 1 corresponds to primary education or the first stage of basic education, and the first year of Level 1 should mark the beginning of formal instruction in reading, writing, and mathematics. Accordingly, the fourth year of Level 1 should be fourth grade in most countries. To avoid testing very young children, however, PIRLs has a policy that the average age of children in the grade tested should not be below 9.5 years old.

The PIRLS 2006 assessment was administered to carefully drawn random samples of students from the target population in each country. Because the accuracy of the PIRLS results depends on the quality of the national samples, the PIRLS team went to great lengths to work with participating countries to ensure efficient sampling design and implementation.

For PIRLS 2006, national research coordinators worked on all phases of sampling in conjunction with staff from Statistics Canada. National coordinators were trained in how to select the school and student samples, and in how to use the $\mathrm{Win}_{3} \mathrm{~S}$ within-school sampling software provided by the IEA Data Processing and Research Center. In consultation with the PIRLS 2006 sampling referee (Keith Rust, Westat, Inc.), staff from Statistics Canada reviewed the national sampling plans, sampling data, sampling frames, and sample selections. The sampling documentation was used by the TIMSS \& PIRLS International Study Center (in consultation with Statistics Canada and the sampling referee) to evaluate the quality of the samples.

In a few situations where it was not possible to test the entire internationally desired population (i.e., all students enrolled in the fourth grade), countries were permitted to define a national desired population that excluded part of the internationally desired population. Exhibit A.4 shows any differences in coverage between the international and national desired populations. Almost all participants achieved 100\% coverage, 
the exceptions being Georgia (tested only Georgian-speaking students), Lithuania (only Lithuanian-speaking students), and Moldova (did not include the Predniestrian republic).

Within the desired population, countries could define a population that excluded a small percentage (less than 5\%) of certain kinds of schools or students that would be very difficult or resource intensive to test (e.g., schools for students with special needs or schools that were very small or located in remote rural areas). Almost all countries kept their excluded students below the 5 percent limit, except for Bulgaria, Denmark, Georgia, the Russian Federation, and the United States, which just exceeded this figure, and Israel, which excluded more that 20 percent of its fourth-grade student population.

The basic design of the sample used in PIRLS 2006 was a two-stage stratified cluster design. ${ }^{12}$ The first stage was a sampling of schools, and the second stage a sampling of intact classrooms from the target grade in the sampled schools. Schools were selected with probability proportional to size, and classrooms with equal probabilities. Most countries sampled 150 schools and one or two intact classrooms from each school. ${ }^{13}$ This approach was designed to yield a representative sample of at least 4,000 students in each country.

Exhibits A.5 and A.6 present achieved sample sizes for schools and students, respectively. Exhibit A.7 shows the participation rates for schools, students, and overall, both with and without the use of replacement schools. Most countries achieved the minimum acceptable participation rates85 percent of both the schools and students, or a combined rate (the product of school and student participation) of 75 percent-although Belgium (Flemish), the Netherlands, Scotland, and the United States did so only after including replacement schools and have been annotated in the exhibits of this report. Norway had overall participation rates after including replacement schools of just below 75 percent $(71 \%)$ and has been annotated accordingly.

Because an important goal of the PIRLS 2006 assessment was to measure changes in fourth-grade students' reading achievement since 2001, it is important to track any changes in population composition and coverage since then that might be related to student achievement. Exhibit A.8 presents,

12 See Joncas, M. (2007). PIRLS 2006 sampling design. In M.O. Martin, I.V.S. Mullis, \& A.M. Kennedy (Eds.), PIRLS 2006 technical report. Chestnut Hill, MA: Boston College.

13 For further detail, see Joncas, M. (2007). PIRLS 2006 sampling weights and participation rates. In M.O. Martin, I.V.S. Mullis, \& A.M. Kennedy (Eds.), PIRLS 2006 technical report. Chestnut Hill, MA: Boston College. 
for each country, four attributes of the populations sampled in 2001 and 2006: number of years of formal schooling, average student age, the score on the United Nations Development Programme's (UNDP) human development index, and the percentage of students in the national desired population excluded from the assessment. Most countries and provinces were very similar with regard to these attributes across the two assessments, although it is noteworthy than the Russian Federation and Slovenia underwent structural changes in the age at which children enter schools that are reflected in their samples. In 2001, the Russian sample contained third-grade students from some regions and fourth-grade students from others, whereas all students were in fourth grade in 2006. Slovenia is in transition toward having all children begin school at an earlier age so that they all will have four years of primary schooling instead of three years, as was the case in 2001. However, the transition was not complete in 2006.

For analysis and reporting, students' questionnaire data, along with questionnaire data from their parents, teachers, and school principals, were linked to students' achievement data. Exhibit A.9 shows the percentage of students with available student, parent, teacher, and principal questionnaire data. Although the vast majority of students in PIRLS 2006 were taught by a single teacher, there were some students in some countries taught by more than one teacher. The percentage of students in each country taught by one, two, or three teachers is presented in Exhibit A.10. Only Scotland, Sweden, and the Canadian province of British Columbia had more than 10 percent of students with more than one teacher-21 percent, 13 percent, and 13 percent, respectively. If a student had more than one teacher, the student's data record was replicated so that there were as many student records as there were teacher records. Then each teacher record was merged with one of the student records. So as not to over count the student in analyses, the sampling weight for each student record was divided by the number of records that the student had. For example, if a student had two teachers, the student's record was replicated so that there were two records, and the first was merged with one teacher record and the second with the other teacher record. Each of the two student records was given half of the original student's weight. 
Exhibit A.4 Coverage of PIRLS Target Population

PIRLS 2006

4th Grade

\begin{tabular}{|c|c|c|c|c|c|}
\hline \multirow{2}{*}{ Countries } & \multicolumn{2}{|c|}{ International Desired Population } & \multicolumn{3}{|c|}{ National Desired Population } \\
\hline & Country Coverage & Notes on Coverage & $\begin{array}{l}\text { School-level } \\
\text { Exclusions }\end{array}$ & $\begin{array}{l}\text { Within-sample } \\
\text { Exclusions }\end{array}$ & Overall Exclusions \\
\hline Austria & $100 \%$ & & $1.4 \%$ & $3.8 \%$ & $5.1 \%$ \\
\hline Belgium (Flemish) & $100 \%$ & & $6.1 \%$ & $1.1 \%$ & $7.1 \%$ \\
\hline Belgium (French) & $100 \%$ & & $3.7 \%$ & $0.3 \%$ & $3.9 \%$ \\
\hline Bulgaria & $100 \%$ & & $2.2 \%$ & $4.3 \%$ & $6.4 \%$ \\
\hline Canada, Alberta & $100 \%$ & & $2.0 \%$ & $5.2 \%$ & $7.1 \%$ \\
\hline Canada, British Columbia & $100 \%$ & & $2.2 \%$ & $5.5 \%$ & $7.6 \%$ \\
\hline Canada, Nova Scotia & $100 \%$ & & $0.2 \%$ & $3.8 \%$ & $4.0 \%$ \\
\hline Canada, Ontario & $100 \%$ & & $1.6 \%$ & $6.8 \%$ & $8.3 \%$ \\
\hline Canada, Quebec & $100 \%$ & & $2.4 \%$ & $1.2 \%$ & $3.6 \%$ \\
\hline Chinese Taipei & $100 \%$ & & $1.8 \%$ & $1.1 \%$ & $2.9 \%$ \\
\hline England & $100 \%$ & & $1.6 \%$ & $0.9 \%$ & $2.4 \%$ \\
\hline France & $100 \%$ & & $3.4 \%$ & $0.4 \%$ & $3.8 \%$ \\
\hline Georgia & $80 \%$ & Students taught in Georgian & $2.4 \%$ & $5.0 \%$ & $7.3 \%$ \\
\hline Germany & $100 \%$ & & $0.4 \%$ & $0.3 \%$ & $0.7 \%$ \\
\hline Hong Kong SAR & $100 \%$ & & $3.0 \%$ & $0.9 \%$ & $3.9 \%$ \\
\hline Hungary & $100 \%$ & & $2.3 \%$ & $1.4 \%$ & $3.7 \%$ \\
\hline Iceland & $100 \%$ & & $1.3 \%$ & $2.5 \%$ & $3.8 \%$ \\
\hline Indonesia & $100 \%$ & & $3.2 \%$ & $0.0 \%$ & $3.2 \%$ \\
\hline Iran, Islamic Rep. of & $100 \%$ & & $2.9 \%$ & $0.9 \%$ & $3.8 \%$ \\
\hline Israel & $100 \%$ & & $17.5 \%$ & $6.1 \%$ & $22.5 \%$ \\
\hline Italy & $100 \%$ & & $0.1 \%$ & $5.2 \%$ & $5.3 \%$ \\
\hline Kuwait & $100 \%$ & & $0.3 \%$ & $0.0 \%$ & $0.3 \%$ \\
\hline Latvia & $100 \%$ & & $4.3 \%$ & $0.5 \%$ & $4.7 \%$ \\
\hline Lithuania & $93 \%$ & Students taught in Lithuanian & $0.9 \%$ & $4.2 \%$ & $5.1 \%$ \\
\hline Moldova, Rep. of & $91 \%$ & $\begin{array}{l}\text { Moldova less Predniestrian } \\
\text { - Moldovan Republic }\end{array}$ & $0.6 \%$ & $0.0 \%$ & $0.6 \%$ \\
\hline Morocco & $100 \%$ & & $1.1 \%$ & $0.0 \%$ & $1.1 \%$ \\
\hline Netherlands & $100 \%$ & & $3.5 \%$ & $0.1 \%$ & $3.6 \%$ \\
\hline New Zealand & $100 \%$ & & $1.4 \%$ & $3.9 \%$ & $5.3 \%$ \\
\hline Norway & $100 \%$ & & $1.0 \%$ & $2.8 \%$ & $3.8 \%$ \\
\hline Poland & $100 \%$ & & $0.9 \%$ & $4.2 \%$ & $5.1 \%$ \\
\hline Qatar & $100 \%$ & & $0.7 \%$ & $0.7 \%$ & $1.4 \%$ \\
\hline Romania & $100 \%$ & & $2.4 \%$ & $0.0 \%$ & $2.4 \%$ \\
\hline Russian Federation & $100 \%$ & & $6.8 \%$ & $1.0 \%$ & $7.7 \%$ \\
\hline Scotland & $100 \%$ & & $1.4 \%$ & $0.9 \%$ & $2.3 \%$ \\
\hline Singapore & $100 \%$ & & $0.9 \%$ & $0.0 \%$ & $0.9 \%$ \\
\hline Slovak Republic & $100 \%$ & & $1.8 \%$ & $1.9 \%$ & $3.6 \%$ \\
\hline Slovenia & $100 \%$ & & $0.2 \%$ & $0.5 \%$ & $0.8 \%$ \\
\hline South Africa & $100 \%$ & & $4.2 \%$ & $0.1 \%$ & $4.3 \%$ \\
\hline Spain & $100 \%$ & & $1.3 \%$ & $4.0 \%$ & $5.3 \%$ \\
\hline Sweden & $100 \%$ & & $2.4 \%$ & $1.5 \%$ & $3.9 \%$ \\
\hline Trinidad and Tobago & $100 \%$ & & $0.7 \%$ & $0.0 \%$ & $0.7 \%$ \\
\hline United States & $100 \%$ & & $3.2 \%$ & $2.8 \%$ & $5.9 \%$ \\
\hline
\end{tabular}


Exhibit A.5 School Sample Sizes

PIRLS 2006

\begin{tabular}{c|c|c|} 
Countries & $\begin{array}{c}\text { Number of } \\
\text { Schools in } \\
\text { Original Sample }\end{array}$ & $\begin{array}{c}\text { Number of } \\
\text { Eligible Schools } \\
\text { in Original } \\
\text { Sample }\end{array}$ \\
\hline
\end{tabular}

Number of

Original Sample

that Participated

4th Grade

$\begin{array}{lllll}\text { Austria } & 160 & 158 & 158 & 0\end{array}$

\section{Austria}

Belgium (Flemish)

Belgium (French)

Bulgaria

Canada, Alberta

Canada, British Columbia

Canada, Nova Scotia

Canada, Ontario

Canada, Quebec

Chinese Taipei

Denmark

England

France

Georgia

Germany

Hong Kong SAR

Hungary

Iceland

Indonesia

Iran, Islamic Rep. of

Israel

Italy

Kuwait

Latvia

Lithuania

Luxembourg

Macedonia, Rep. of

Moldova, Rep. of

Morocco

Netherlands

New Zealand

Norway

Poland

Qatar

Romania

Russian Federation

Scotland

Singapore

Slovak Republic

Slovenia

South Africa

Spain

Sweden

\begin{tabular}{l|l|}
160 & 149 \\
150 & 150 \\
150 & 147 \\
150 & 150 \\
\hline
\end{tabular}

150
150

150

150

201

200

200

150
150

150
150

175

175
152
410

410
150

150
150

150
136

136
170

170
240

240
150

150
150

150
150

150
150

150

183
150

150
150

160

160
150

150
250

250
178

150

150
123

123
150

150
232

150
178

178
174

150

150
441
152

Trinidad and Tobago

United States

152
150
222

\begin{tabular}{l|l}
149 & 102 \\
150 & 129 \\
\hline
\end{tabular}

147

150

150

201

201

194

150

146

150

150
175

149

149
407

407

407
144

149

131

131

168
236

236
149

150

150
150

158

Number of

Replacement

Schools that

Total Number of

Schools that

Participated

\begin{tabular}{l|l}
152 & 152 \\
150 & 147 \\
150 & 149 \\
222 & 214
\end{tabular}

\begin{tabular}{l}
146 \\
178 \\
\hline 150 \\
150 \\
\hline 160 \\
\hline 150 \\
\hline 250 \\
\hline 177 \\
\hline 148 \\
\hline 119 \\
\hline 147 \\
\hline 232 \\
\hline 150 \\
\hline 178 \\
\hline 171 \\
\hline 150 \\
\hline 410 \\
\hline 152 \\
\hline 147 \\
\hline 149 \\
\hline 214 \\
\hline
\end{tabular}

129

$0 \quad 158$

158
137

150

147

147

200

185

147

128

129

164

164

139

397

130

130
147

128

128
166

235

235

146

136

149
145

145
144

178

149
148

148
156
104

220
118

220
118

147

\begin{tabular}{|r|r|}
147 & 0 \\
119 & 0 \\
\hline 146 & 0 \\
\hline 101 & 29 \\
\hline
\end{tabular}

119
146

\begin{tabular}{|r|r|}
\hline 119 & 0 \\
146 & 0 \\
232 & 0 \\
101 & 29 \\
\hline
\end{tabular}

\begin{tabular}{r|r}
1147 & 0 \\
146 & 0 \\
\hline 232 & 0 \\
101 & 29 \\
\hline
\end{tabular}

178

178
155

155
140

140
391
149

149
147

147
120


Exhibit A.6 Student Sample Sizes

PIRLS 2006

\begin{tabular}{|c|c|c|}
\hline Countries & $\begin{array}{l}\text { Within-school } \\
\text { Student } \\
\text { Participation } \\
\text { (Weighted } \\
\text { Percentage) }\end{array}$ & $\begin{array}{l}\text { Number of } \\
\text { Sampled } \\
\text { Students in } \\
\text { Participating } \\
\text { Schools }\end{array}$ \\
\hline
\end{tabular}

\begin{tabular}{c|} 
Number of \\
Students \\
Withdrawn \\
from \\
Class/School
\end{tabular}

Austria
Belgium (Flemish)
Belgium (French)

Bulgaria

Canada, Alberta

\begin{tabular}{l|l|}
$98 \%$ \\
$99 \%$ \\
$95 \%$ \\
$97 \%$ & \\
$96 \%$ & \\
\hline $96 \%$ & \\
\hline
\end{tabular}

Canada, British Columbia

Canada, Nova Scotia

$95 \%$

Canada, Ontario

Canada, Quebec

5431

Chinese Taipei

Denmark

England

France

Georgia

Germany

Hong Kong SAR

\begin{tabular}{|l|l|}
\hline 4608 & 10 \\
\hline 4810 & 19 \\
\hline 4156 & 37 \\
\hline 4773 & 79 \\
\hline
\end{tabular}

$24-208$

Number of
Students
Excluded
208

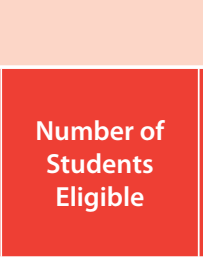

4th Grade

Hungary

Iceland

Indonesia

Iran, Islamic Rep. of

Israel

Italy

Kuwait

Latvia

Lithuania

Luxembourg

Macedonia, Rep. of

$96 \%$

4663

4884

4436
4639

$97 \%$

$99 \%$

$97 \%$

$93 \%$

$98 \%$

$98 \%$

$94 \%$

$97 \%$

$97 \%$

$91 \%$

$98 \%$

$99 \%$

$93 \%$

$97 \%$

$89 \%$

$94 \%$

$92 \%$

$99 \%$

$96 \%$

Moldova, Rep. of

$95 \%$

Morocco

Netherlands

New Zealand

$95 \%$

$97 \%$

Norway

Poland

Qatar

Romania

Russian Federation

Scotland

Singapore

Slovak Republic

Slovenia

South Africa

Spain

Sweden

Trinidad and Tobago

United States

$96 \%$

$87 \%$

$95 \%$

$94 \%$

$98 \%$

$97 \%$

$94 \%$

$95 \%$

$96 \%$

$96 \%$

$92 \%$

$97 \%$

$96 \%$

$95 \%$

$96 \%$

4746

62
-55

$4349 \quad 51 \quad 154$

\begin{tabular}{r|r|r}
\hline 4492 & 117 & 38 \\
4558 & 55 & 16
\end{tabular}

\begin{tabular}{|l|l|l}
\hline 4837 & 120 & 209 \\
\hline
\end{tabular}

$8395 \quad 49 \quad 44$

\begin{tabular}{|l|l|l|}
4917 & 25 & 34 \\
4265 & 17 & 46 \\
\hline
\end{tabular}

\begin{tabular}{l|l}
4200 & 47 \\
4981 & 99
\end{tabular}

\begin{tabular}{l|r}
5609 & 12 \\
4378
\end{tabular}

\begin{tabular}{|l|l|l|}
3882 & 31 & 153 \\
\hline
\end{tabular}

$\begin{array}{lrr}4467 & 0 & 0\end{array}$

\begin{tabular}{|l|l|r|}
\hline 4469 & 14 & 17 \\
5400 & 67 & 183 \\
\hline
\end{tabular}

5342

4209

4281

$3444 \quad 43$

4366

6872

$4570 \quad 130$

$5410 \quad 2$

\begin{tabular}{r|r}
7490 & 305 \\
4463 & 97
\end{tabular}

\begin{tabular}{|l|l|l|}
\hline 4463 & 20 & 35 \\
\hline 4911 & 66 & 4123 \\
\hline
\end{tabular}

\begin{tabular}{l|l}
6760 & 67 \\
5741 & 34 \\
\hline
\end{tabular}

\begin{tabular}{r|r}
5596 & 12 \\
16144 & 305 \\
\hline
\end{tabular}

4391

4653

\begin{tabular}{r|r}
\hline 4237 & 77 \\
\hline 5761 & 160 \\
\hline
\end{tabular}

$47 \quad 4551$

\begin{tabular}{|c|c|c|}
\hline 5199 & 132 & 5067 \\
\hline 4551 & 72 & 4479 \\
\hline 4777 & 225 & 4552 \\
\hline 3984 & 121 & 3863 \\
\hline 4444 & 201 & 4243 \\
\hline 4351 & 201 & 4150 \\
\hline 4616 & 180 & 4436 \\
\hline 4144 & 156 & 3988 \\
\hline 4490 & 742 & 3748 \\
\hline 4629 & 40 & 4589 \\
\hline 4144 & 143 & 4001 \\
\hline 4337 & 301 & 4036 \\
\hline
\end{tabular}

\begin{tabular}{r|r}
15 & 158 \\
33 & 11
\end{tabular}

0
0
5

196

\begin{tabular}{r|r}
5 & 4298 \\
196 & 6546 \\
\hline
\end{tabular}

\begin{tabular}{l|l}
\hline 134 & 440 \\
\hline 232 & 5157 \\
\hline
\end{tabular}

\begin{tabular}{r|r}
\hline 47 & 7138 \\
\hline 0 & 4366 \\
\hline
\end{tabular}

\begin{tabular}{r|r}
0 & 4366 \\
35 & 4856 \\
\hline
\end{tabular}

\begin{tabular}{r|r}
41 & 4016 \\
0 & 6693 \\
105 & -5602 \\
\hline
\end{tabular}

\begin{tabular}{|l|l|}
67 & 105 \\
\hline 34 & 27 \\
\hline 12 & \\
\hline
\end{tabular}

\begin{tabular}{|r|r|r|}
\hline 27 & 5602 & 2 \\
\hline 28 & 5557 & 220 \\
\hline 143 & 15811 & 1154 \\
\hline
\end{tabular}

Number of
Students
Absent

Number of

Students
Assessed

\begin{tabular}{r|r}
143 & 4236 \\
\hline 33 & 4587 \\
\hline 0 & 4160 \\
159 & 5442 \\
\hline
\end{tabular}

4587
4160
5442

$5442 \quad 209$
252

\begin{tabular}{|c|c|}
\hline 83 & 4404 \\
\hline 106 & 4402 \\
\hline 403 & 7899 \\
\hline 146 & 4712 \\
\hline 134 & 4068 \\
\hline 378 & 3673 \\
\hline 108 & 4774 \\
\hline 54 & 5411 \\
\hline 286 & 3908 \\
\hline 117 & 3581 \\
\hline 509 & 3958 \\
\hline 276 & 4162 \\
\hline 449 & 4701 \\
\hline 68 & 5101 \\
\hline 163 & 4002 \\
\hline 213 & 4036 \\
\hline 152 & 3249 \\
\hline 142 & 4156 \\
\hline 290 & 6256 \\
\hline 572 & 3837 \\
\hline 303 & 4854 \\
\hline 458 & 6680 \\
\hline 93 & 4273 \\
\hline 136 & 4720 \\
\hline 241 & 3775 \\
\hline 303 & 6390 \\
\hline 222 & 5380 \\
\hline 220 & 5337 \\
\hline 154 & 14657 \\
\hline 142 & 4094 \\
\hline 193 & 4394 \\
\hline 209 & 3951 \\
\hline 252 & 5190 \\
\hline
\end{tabular}


Exhibit A.7 Participation Rates (Weighted)

PIRLS 2006

4th Grade

\begin{tabular}{|c|c|c|c|c|c|c|}
\hline \multirow{2}{*}{ Countries } & \multicolumn{2}{|c|}{ School Participation } & \multirow{2}{*}{$\begin{array}{l}\text { Classroom } \\
\text { Participation }\end{array}$} & \multirow{2}{*}{$\begin{array}{c}\text { Student } \\
\text { Participation }\end{array}$} & \multicolumn{2}{|c|}{ Overall Participation } \\
\hline & Before Replacement & After Replacement & & & Before Replacement & After Replacement \\
\hline Austria & $100 \%$ & $100 \%$ & $99 \%$ & $98 \%$ & $97 \%$ & $97 \%$ \\
\hline Belgium (Flemish) & $69 \%$ & $92 \%$ & $100 \%$ & $99 \%$ & $68 \%$ & $91 \%$ \\
\hline Belgium (French) & $85 \%$ & $100 \%$ & $100 \%$ & $95 \%$ & $81 \%$ & $95 \%$ \\
\hline Bulgaria & $88 \%$ & $97 \%$ & $100 \%$ & $97 \%$ & $85 \%$ & $94 \%$ \\
\hline Canada, Alberta & $100 \%$ & $100 \%$ & $100 \%$ & $96 \%$ & $96 \%$ & $96 \%$ \\
\hline Canada, British Columbia & $98 \%$ & $99 \%$ & $100 \%$ & $95 \%$ & $93 \%$ & $94 \%$ \\
\hline Canada, Nova Scotia & $99 \%$ & $100 \%$ & $100 \%$ & $96 \%$ & $96 \%$ & $96 \%$ \\
\hline Canada, Ontario & $88 \%$ & $90 \%$ & $100 \%$ & $97 \%$ & $85 \%$ & $87 \%$ \\
\hline Canada, Quebec & $96 \%$ & $96 \%$ & $100 \%$ & $84 \%$ & $81 \%$ & $81 \%$ \\
\hline Chinese Taipei & $98 \%$ & $100 \%$ & $100 \%$ & $99 \%$ & $97 \%$ & $99 \%$ \\
\hline Denmark & $89 \%$ & $99 \%$ & $100 \%$ & $97 \%$ & $86 \%$ & $96 \%$ \\
\hline England & $86 \%$ & $99 \%$ & $100 \%$ & $93 \%$ & $80 \%$ & $92 \%$ \\
\hline France & $94 \%$ & $97 \%$ & $100 \%$ & $98 \%$ & $92 \%$ & $95 \%$ \\
\hline Georgia & $94 \%$ & $100 \%$ & $100 \%$ & $98 \%$ & $93 \%$ & $98 \%$ \\
\hline Germany & $97 \%$ & $99 \%$ & $100 \%$ & $94 \%$ & $90 \%$ & $92 \%$ \\
\hline Hong Kong SAR & $91 \%$ & $100 \%$ & $100 \%$ & $97 \%$ & $89 \%$ & $97 \%$ \\
\hline Hungary & $99 \%$ & $100 \%$ & $100 \%$ & $97 \%$ & $96 \%$ & $97 \%$ \\
\hline Iceland & $99 \%$ & $99 \%$ & $100 \%$ & $91 \%$ & $90 \%$ & $90 \%$ \\
\hline Indonesia & $99 \%$ & $100 \%$ & $100 \%$ & $98 \%$ & $97 \%$ & $98 \%$ \\
\hline Iran, Islamic Rep. of & $100 \%$ & $100 \%$ & $100 \%$ & $99 \%$ & $99 \%$ & $99 \%$ \\
\hline Israel & $98 \%$ & $100 \%$ & $100 \%$ & $93 \%$ & $91 \%$ & $93 \%$ \\
\hline Italy & $91 \%$ & $100 \%$ & $100 \%$ & $97 \%$ & $88 \%$ & $97 \%$ \\
\hline Kuwait & $99 \%$ & $99 \%$ & $99 \%$ & $89 \%$ & $88 \%$ & $88 \%$ \\
\hline Latvia & $97 \%$ & $98 \%$ & $100 \%$ & $94 \%$ & $91 \%$ & $92 \%$ \\
\hline Lithuania & $99 \%$ & $100 \%$ & $100 \%$ & $92 \%$ & $90 \%$ & $92 \%$ \\
\hline Luxembourg & $100 \%$ & $100 \%$ & $100 \%$ & $99 \%$ & $99 \%$ & $99 \%$ \\
\hline Macedonia, Rep. of & $100 \%$ & $100 \%$ & $100 \%$ & $96 \%$ & $96 \%$ & $96 \%$ \\
\hline Moldova, Rep. of & $98 \%$ & $100 \%$ & $100 \%$ & $95 \%$ & $93 \%$ & $95 \%$ \\
\hline Morocco & $98 \%$ & $99 \%$ & $100 \%$ & $95 \%$ & $93 \%$ & $94 \%$ \\
\hline Netherlands & $70 \%$ & $93 \%$ & $100 \%$ & $97 \%$ & $67 \%$ & $90 \%$ \\
\hline New Zealand & $92 \%$ & $99 \%$ & $100 \%$ & $96 \%$ & $88 \%$ & $95 \%$ \\
\hline Norway & $68 \%$ & $82 \%$ & $100 \%$ & $87 \%$ & $58 \%$ & $71 \%$ \\
\hline Poland & $99 \%$ & $100 \%$ & $100 \%$ & $95 \%$ & $94 \%$ & $95 \%$ \\
\hline Qatar & $100 \%$ & $100 \%$ & $100 \%$ & $94 \%$ & $94 \%$ & $94 \%$ \\
\hline Romania & $99 \%$ & $99 \%$ & $100 \%$ & $98 \%$ & $97 \%$ & $97 \%$ \\
\hline Russian Federation & $100 \%$ & $100 \%$ & $100 \%$ & $97 \%$ & $97 \%$ & $97 \%$ \\
\hline Scotland & $69 \%$ & $87 \%$ & $100 \%$ & $94 \%$ & $65 \%$ & $81 \%$ \\
\hline Singapore & $100 \%$ & $100 \%$ & $100 \%$ & $95 \%$ & $95 \%$ & $95 \%$ \\
\hline Slovak Republic & $93 \%$ & $98 \%$ & $100 \%$ & $96 \%$ & $89 \%$ & $94 \%$ \\
\hline Slovenia & $93 \%$ & $97 \%$ & $100 \%$ & $96 \%$ & $90 \%$ & $93 \%$ \\
\hline South Africa & $94 \%$ & $96 \%$ & $100 \%$ & $92 \%$ & $86 \%$ & $88 \%$ \\
\hline Spain & $99 \%$ & $100 \%$ & $100 \%$ & $97 \%$ & $95 \%$ & $97 \%$ \\
\hline Sweden & $100 \%$ & $100 \%$ & $100 \%$ & $96 \%$ & $96 \%$ & $96 \%$ \\
\hline Trinidad and Tobago & $99 \%$ & $99 \%$ & $100 \%$ & $95 \%$ & $94 \%$ & $94 \%$ \\
\hline United States & $57 \%$ & $86 \%$ & $100 \%$ & $96 \%$ & $54 \%$ & $82 \%$ \\
\hline
\end{tabular}


Exhibit A.8 Trends in Student Populations

PIRLS 2006

4th Grade

\begin{tabular}{|c|c|c|c|c|c|c|c|c|}
\hline \multirow{2}{*}{ Country } & \multicolumn{2}{|c|}{ Years of Formal Schooling } & \multicolumn{2}{|c|}{ Average Age } & \multicolumn{2}{|c|}{$\begin{array}{c}\text { Human Development } \\
\text { Index }\end{array}$} & \multicolumn{2}{|c|}{ Overall Exclusion Rate } \\
\hline & 2006 & 2001 & 2006 & 2001 & $2006^{1}$ & $2001^{2}$ & 2006 & 2001 \\
\hline Bulgaria & 4 & 4 & 10.9 & 10.9 & 0.816 & 0.772 & $6.4 \%$ & $2.7 \%$ \\
\hline Canada, Ontario & 4 & 4 & 9.8 & 9.9 & 0.950 & 0.936 & $8.3 \%$ & $6.6 \%$ \\
\hline Canada, Quebec & 4 & 4 & 10.1 & 10.2 & 0.950 & 0.936 & $3.6 \%$ & $3.3 \%$ \\
\hline England & 5 & 5 & 10.3 & 10.2 & 0.940 & 0.923 & $2.4 \%$ & $5.7 \%$ \\
\hline France & 4 & 4 & 10.0 & 10.1 & 0.942 & 0.924 & $3.8 \%$ & $5.3 \%$ \\
\hline Germany & 4 & 4 & 10.5 & 10.5 & 0.932 & 0.921 & $0.7 \%$ & $1.8 \%$ \\
\hline Hong Kong SAR & 4 & 4 & 10.0 & 10.2 & 0.927 & 0.880 & $3.9 \%$ & $2.8 \%$ \\
\hline Hungary & 4 & 4 & 10.7 & 10.7 & 0.869 & 0.829 & $3.7 \%$ & $2.1 \%$ \\
\hline Iceland & 4 & 4 & 9.8 & 9.7 & 0.960 & 0.932 & $3.8 \%$ & $3.1 \%$ \\
\hline Iran & 4 & 4 & 10.2 & 10.4 & 0.746 & 0.714 & $3.8 \%$ & $0.5 \%$ \\
\hline Israel & 4 & 4 & 10.1 & 10.0 & 0.927 & 0.893 & $22.5 \%$ & $22.4 \%$ \\
\hline Italy & 4 & 4 & 9.7 & 9.8 & 0.940 & 0.909 & $5.3 \%$ & $2.9 \%$ \\
\hline Kuwait & 4 & 4 & 9.8 & 9.9 & 0.871 & 0.818 & $0.3 \%$ & $0.0 \%$ \\
\hline Latvia & 4 & 4 & 11.0 & 11.0 & 0.845 & 0.791 & $4.7 \%$ & $4.6 \%$ \\
\hline Lithuania & 4 & 4 & 10.7 & 10.9 & 0.857 & 0.803 & $5.1 \%$ & $3.8 \%$ \\
\hline Macedonia & 4 & 4 & 10.6 & 10.7 & 0.796 & 0.766 & $4.9 \%$ & $4.2 \%$ \\
\hline Moldova & 4 & 4 & 10.9 & 10.8 & 0.694 & 0.699 & $0.6 \%$ & $0.5 \%$ \\
\hline Morocco & 4 & 4 & 10.8 & 11.2 & 0.640 & 0.596 & $1.1 \%$ & $1.0 \%$ \\
\hline Netherlands & 4 & 4 & 10.3 & 10.3 & 0.947 & 0.931 & $3.6 \%$ & $3.7 \%$ \\
\hline New Zealand & 5 & 5 & 10.0 & 10.1 & 0.936 & 0.913 & $5.3 \%$ & $3.2 \%$ \\
\hline Norway & 4 & 4 & 9.8 & 10.0 & 0.965 & 0.939 & $3.8 \%$ & $2.8 \%$ \\
\hline Romania & 4 & 4 & 10.9 & 11.1 & 0.805 & 0.772 & $2.4 \%$ & $4.5 \%$ \\
\hline Russian Federation & 4 & 3 or 4 & 10.8 & 10.3 & 0.797 & 0.775 & $7.7 \%$ & $6.6 \%$ \\
\hline Scotland & 5 & 5 & 9.9 & 9.8 & 0.940 & 0.923 & $2.3 \%$ & $4.7 \%$ \\
\hline Singapore & 4 & 4 & 10.4 & 10.1 & 0.916 & 0.876 & $0.9 \%$ & $1.4 \%$ \\
\hline Slovak Republic & 4 & 4 & 10.4 & 10.3 & 0.856 & 0.831 & $3.6 \%$ & $2.0 \%$ \\
\hline Slovenia & 3 or 4 & 3 & 9.9 & 9.8 & 0.910 & 0.874 & $0.8 \%$ & $0.3 \%$ \\
\hline Sweden & 4 & 4 & 10.9 & 10.8 & 0.951 & 0.936 & $3.9 \%$ & $5.0 \%$ \\
\hline United States & 4 & 4 & 10.1 & 10.2 & 0.948 & 0.934 & $5.9 \%$ & $5.3 \%$ \\
\hline
\end{tabular}

1 Taken from the United Nations Development Programme's Human Development Report 2006, p. 283-286

2 Taken from the United Nations Development Programme's Human Development Report 2001, p. 141-144 
Exhibit A.9 Percentage of Students with Any Available Student, Parent, Teacher, and Principal

PIRLS 2006 Questionnaire Data

\begin{tabular}{|c|c|c|c|c|}
\hline \multirow{2}{*}{ Countries } & \multicolumn{4}{|c|}{ Percent of Student with Any Available Data } \\
\hline & $\begin{array}{c}\text { Student } \\
\text { Questionnaire }\end{array}$ & $\begin{array}{c}\text { Parent } \\
\text { Questionnaire }\end{array}$ & $\begin{array}{c}\text { Teacher } \\
\text { Questionnaire }\end{array}$ & $\begin{array}{c}\text { Principal } \\
\text { Questionnaire }\end{array}$ \\
\hline Austria & 100 & 96 & 100 & 100 \\
\hline Belgium (Flemish) & 100 & 97 & 99 & 96 \\
\hline Belgium (French) & 100 & 90 & 96 & 87 \\
\hline Bulgaria & 99 & 96 & 97 & 96 \\
\hline Canada, Alberta & 99 & 80 & 99 & 99 \\
\hline Canada, British Columbia & 99 & 77 & 80 & 88 \\
\hline Canada, Nova Scotia & 99 & 91 & 91 & 96 \\
\hline Canada, Ontario & 100 & 90 & 99 & 95 \\
\hline Canada, Quebec & 99 & 90 & 95 & 92 \\
\hline Chinese Taipei & 100 & 97 & 99 & 100 \\
\hline Denmark & 100 & 94 & 95 & 95 \\
\hline England & 100 & 46 & 91 & 83 \\
\hline France & 99 & 92 & 98 & 96 \\
\hline Georgia & 100 & 98 & 99 & 100 \\
\hline Germany & 96 & 87 & 94 & 96 \\
\hline Hong Kong SAR & 98 & 98 & 99 & 97 \\
\hline Hungary & 100 & 90 & 99 & 98 \\
\hline Iceland & 99 & 76 & 90 & 91 \\
\hline Indonesia & 100 & 99 & 100 & 100 \\
\hline Iran, Islamic Rep. of & 100 & 99 & 100 & 100 \\
\hline Israel & 99 & 63 & 98 & 98 \\
\hline Italy & 100 & 97 & 100 & 100 \\
\hline Kuwait & 97 & 75 & 90 & 95 \\
\hline Latvia & 100 & 95 & 98 & 99 \\
\hline Lithuania & 100 & 98 & 100 & 99 \\
\hline${ }^{1}$ Luxembourg & 100 & 93 & 99 & 0 \\
\hline Macedonia, Rep. of & 98 & 97 & 95 & 84 \\
\hline Moldova, Rep. of & 100 & 97 & 96 & 97 \\
\hline Morocco & 100 & 98 & 99 & 74 \\
\hline Netherlands & 100 & 67 & 90 & 85 \\
\hline New Zealand & 99 & 65 & 96 & 96 \\
\hline Norway & 98 & 93 & 98 & 95 \\
\hline Poland & 100 & 98 & 100 & 100 \\
\hline Qatar & 99 & 72 & 81 & 92 \\
\hline Romania & 100 & 98 & 100 & 99 \\
\hline Russian Federation & 100 & 99 & 100 & 100 \\
\hline Scotland & 100 & 52 & 88 & 80 \\
\hline Singapore & 100 & 98 & 100 & 100 \\
\hline Slovak Republic & 100 & 97 & 99 & 100 \\
\hline Slovenia & 100 & 95 & 100 & 99 \\
\hline South Africa & 99 & 90 & 95 & 99 \\
\hline Spain & 100 & 62 & 96 & 91 \\
\hline Sweden & 100 & 94 & 95 & 99 \\
\hline Trinidad and Tobago & 99 & 89 & 97 & 98 \\
\hline 2 United States & 100 & 0 & 99 & 99 \\
\hline
\end{tabular}

1 Primary schools in Luxembourg do not have principals.

2 All countries except the United States administered the parents' questionnaire. In exhibits presenting data from this questionnaire, the United States has dashes (-). 


\begin{tabular}{|c|c|c|c|}
\hline Country & $\begin{array}{l}\text { Percent with } \\
\text { One Teacher }\end{array}$ & $\begin{array}{l}\text { Percent with } \\
\text { Two Teachers }\end{array}$ & $\begin{array}{l}\text { Percent with } \\
\text { Three Teachers }\end{array}$ \\
\hline Austria & 99 & 1 & 0 \\
\hline Belgium (Flemish) & 100 & 0 & 0 \\
\hline Belgium (French) & 100 & 0 & 0 \\
\hline Bulgaria & 100 & 0 & 0 \\
\hline Canada, Alberta & 95 & 5 & 0 \\
\hline Canada, British Columbia & 87 & 13 & 0 \\
\hline Canada, Nova Scotia & 92 & 8 & 0 \\
\hline Canada, Ontario & 100 & 0 & 0 \\
\hline Canada, Quebec & 100 & 0 & 0 \\
\hline Chinese Taipei & 100 & 0 & 0 \\
\hline Denmark & 96 & 4 & 0 \\
\hline England & 91 & 6 & 3 \\
\hline France & 100 & 0 & 0 \\
\hline Georgia & 99 & 1 & 0 \\
\hline Germany & 97 & 3 & 0 \\
\hline Hong Kong SAR & 100 & 0 & 0 \\
\hline Hungary & 100 & 0 & 0 \\
\hline Iceland & 92 & 8 & 0 \\
\hline Indonesia & 100 & 0 & 0 \\
\hline Iran, Islamic Rep. of & 100 & 0 & 0 \\
\hline Israel & 100 & 0 & 0 \\
\hline Italy & 100 & 0 & 0 \\
\hline Kuwait & 100 & 0 & 0 \\
\hline Latvia & 100 & 0 & 0 \\
\hline Lithuania & 100 & 0 & 0 \\
\hline Luxembourg & 90 & 8 & 2 \\
\hline Macedonia, Rep. of & 100 & 0 & 0 \\
\hline Moldova, Rep. of & 100 & 0 & 0 \\
\hline Morocco & 100 & 0 & 0 \\
\hline Netherlands & 99 & 1 & 0 \\
\hline New Zealand & 95 & 5 & 0 \\
\hline Norway & 100 & 0 & 0 \\
\hline Poland & 100 & 0 & 0 \\
\hline Qatar & 100 & 0 & 0 \\
\hline Romania & 100 & 0 & 0 \\
\hline Russian Federation & 100 & 0 & 0 \\
\hline Scotland & 79 & 21 & 1 \\
\hline Singapore & 100 & 0 & 0 \\
\hline Slovak Republic & 100 & 0 & 0 \\
\hline Slovenia & 100 & 0 & 0 \\
\hline South Africa & 100 & 0 & 0 \\
\hline Spain & 100 & 0 & 0 \\
\hline Sweden & 86 & 13 & 1 \\
\hline Trinidad and Tobago & 100 & 0 & 0 \\
\hline United States & 100 & 0 & 0 \\
\hline
\end{tabular}




\section{Data Collection}

Each participating country was responsible for carrying out all aspects of the data collection, using standardized procedures developed for the study. Training manuals were created for school coordinators and test administrators that explained procedures for receipt and distribution of materials, as well as for the activities related to the testing sessions. These manuals covered procedures for test security, standardized scripts to regulate directions and timing, rules for answering students' questions, and steps to ensure that identification on the test booklets and questionnaires corresponded to the information on the forms used to track students.

Each country was responsible for conducting quality control procedures and describing this effort in the online Survey Activities Report. In addition, the TIMSS \& PIRLS International Study Center considered it essential to independently monitor compliance with standardized procedures. ${ }^{14}$ To implement the independent monitoring program, the IEA Secretariat asked National Research Coordinators to nominate persons, unconnected with their national centers, to serve as quality control monitors (QCMs) for their countries. All countries and provinces participated in the program of quality control school visits.

The TIMSS \& PIRLS International Study Center developed manuals for the quality control monitors and, in a 2-day training session, staff briefed the monitors about PIRLS 2006, the responsibilities of the national centers in conducting the study, and their roles and responsibilities as quality control monitors. The training session, jointly conducted by the TIMSS \& PIRLS International Study Center and the IEA Secretariat, was attended by 42 quality control monitors. In countries where the data collection schedule made it impossible for one quality control monitor to visit all the sampled schools, monitors who attended the training session were asked to recruit other monitors as necessary, in order to allow for efficiency in the coverage of the territory and testing timetable.

In all, 103 quality control monitors and assistants participated in the program, visiting a sample of 15 schools in each country, where they

14 Steps taken to ensure high-quality data collection in PIRLS 2006 are described in detail in Johansone, I. \& Kennedy, A.M. (2007). Quality assurance in the PIRLS 2006 data collection. In M.O. Martin, I.V.S. Mullis, \& A.M. Kennedy (Eds.), PIRLS 2006 technical report. Chestnut Hill, MA: Boston College. 
observed testing sessions and interviewed school coordinators. Altogether, quality control monitors observed testing sessions and interviewed school coordinators in 669 schools from across all 45 PIRLS 2006 participants.

National Research Coordinators' comments in the Survey Activities Report indicate that, in general, national centers had prepared well for data collection and-despite the heavy demands of the schedule and shortages of resources-were able to conduct the data collection efficiently and professionally. Similarly, based on quality control monitors observations of the testing sessions, there is evidence that the PIRLS 2006 test was administered in compliance with international procedures-including the activities before the testing session, along with school-level activities related to receiving, distributing, and returning material from national centers.

\section{Scoring the Constructed-response Items}

Because almost two-thirds of the score points came from constructedresponse items, PIRLS 2006 implemented procedures for reliably evaluating student responses within and across countries. The TIMSS \& PIRLS International Study Center prepared detailed scoring guides containing the classification categories and explanations of how to implement the classifications, together with example student responses for the various categories. These scoring guides, along with training packets containing extensive examples of student responses for practice in applying the guides, were used as a basis for intensive training in scoring the constructedresponse items. The TIMSS \& PIRLS International Study Center conducted scoring training sessions for the PIRLS 2006 participants in conjunction with both the field test and the PIRLS 2006 assessment. The training sessions were designed for representatives from national centers, who would then be responsible for training personnel in their own countries to apply the scoring guides reliably.

To gather and document empirical information about the within-country agreement among scorers, PIRLS arranged to have systematic subsamples of at least 200 students' responses to each item scored independently by two 
readers. Exhibit A.11 shows the average range of the within-country exact percent of agreement between scorers on the free-response items. Scoring reliability within countries was high-the percentage of exact agreement, on average, across countries, was 93 percent.

PIRLS 2006 also took steps to ensure that those constructed-response items from the 2001 passages that were used in 2006 were scored in the same way in both assessments. In anticipation of this, countries that participated in PIRLS 2001 sent samples of scored student booklets from their 2001 assessment to the IEA Data Processing and Research Center (DPC), where they were digitally scanned and incorporated into custom-built presentation software for use in 2006. On average, the software contained about 5,000 student responses for each country. After being trained in using the scoring guides for these items, scorers scored half of the student responses, using the scoring software supplied by the IEA DPC. The software then produced reports on their scoring accuracy for these student responses. Scorers with less than 85 percent exact agreement with the scores assigned to the responses in 2001 were retrained before proceeding. Exhibit A.12 provides the average percentage agreement across items for the scores given in 2001 and in 2006 for each participant. Agreement between 2001 and 2006 was generally high90 percent exact agreement on average across countries. ${ }^{15}$

To monitor the consistency with which the scoring rubrics were applied across countries, PIRLS 2006 collected from the countries that administered PIRLS in English a sample of 200 student responses to 23 constructed-response questions from four of the assessment passages. This set of 4,600 student responses was then sent to each country having scorers proficient in English, to be scored independently by two of these scorers. Each of these responses was scored by 62 scorers from across the countries that participated. ${ }^{16}$ Making all possible comparisons among scorers gave 1,891 comparisons for each student response to each item, and 378,200 total comparisons when aggregated across all 200 student responses to that item. Agreement across countries was defined in terms of the percentage of these comparisons that were in exact agreement. Exhibit A.13 shows this percentage of exact agreement for each of the 23 items. As shown in this exhibit, the percentage of agreement averaged across the 23 items was 87 percent.

15 A number of participants were unable to complete the trend-scoring reliability task, because of software difficulties or because it was not possible to scan their 2001 student booklets.

16 Scorers proficient in English were available in 33 of the PIRLS 2006 countries. In some countries, more than two scorers shared the scoring effort. 
Exhibit A.11 PIRLS Within-country Constructed-response Scoring Reliability Data

PIRLS 2006

4th Grade
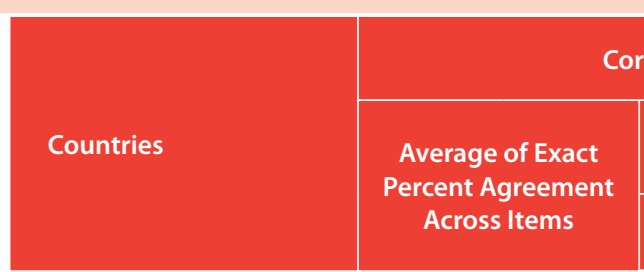

Correctness Score Agreement

Austria
Belgium (Flemish)
Belgium (French)

Bulgaria 98

Canada, Alberta

Canada, British Columbia

Canada, Nova Scotia

Canada, Ontario

Canada, Quebec

Chinese Taipei

Denmark

England

$95 \quad 80$
of Agreement

France 89

\begin{tabular}{l|r} 
Georgia & 85
\end{tabular}

\section{Germany}

\begin{tabular}{l|l} 
Hong Kong SAR & 96
\end{tabular}

Hungary 98

\begin{tabular}{l|r} 
Iceland $\quad 95$ \\
\hline
\end{tabular}

Indonesia

Iran, Islamic Rep. of 93

Israel

Italy $\quad 95$

Kuwait $\quad 86$

Latvia $\quad 90$

Lithuania

Luxembourg

Macedonia, Rep. of

Moldova, Rep. of

Morocco

Netherlands

New Zealand

Norway

Poland

Qatar

Romania

Russian Federation

Scotland

Singapore

Slovak Republic

Slovenia

South Africa

Spain

Sweden

Trinidad and Tobago

United States

\begin{tabular}{l|l}
90 & 73 \\
97 & 90
\end{tabular}

97

91

91

93

94

95

95

97

98

98

8

69

96

8

5

95

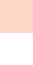

83
80

\begin{tabular}{l|l}
\hline 5 & 85 \\
86 & 80 \\
\hline
\end{tabular}

\begin{tabular}{|r}
80 \\
\hline
\end{tabular}

97
94

94
88
99

99
89

89
99

99
93
83

93
83

83
97

97
97

99
99

99
97

97
98

98
96

96
98
82

98
82

81
92

92
93

93
93

International Avg.

93

82

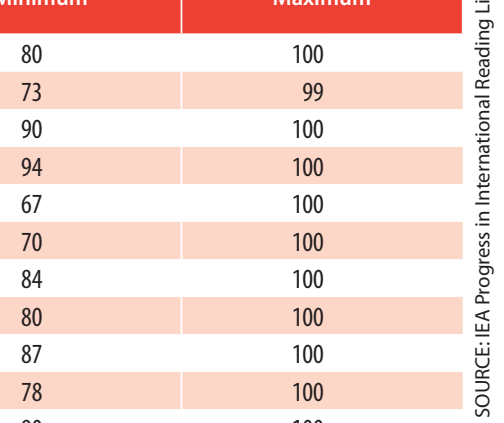

91

\begin{tabular}{|r|r}
\hline \\
\hline
\end{tabular}

$\begin{array}{r}78 \\ -\quad 97 \\ \hline\end{array}$

97
71

$\begin{array}{r}71 \\ \hline\end{array}$

93
80
66

66
93

93

96

\begin{tabular}{r|r}
96 \\
\hline
\end{tabular}

$\begin{array}{r}97 \\ \hline\end{array}$

\begin{tabular}{l|l}
96 & 100 \\
\hline 97 & 100 \\
\hline 89 & 100 \\
\hline 94 & 100 \\
\hline
\end{tabular}

$\begin{array}{ll}94 & 100 \\ 88 & 100\end{array}$

\begin{tabular}{rr}
88 & 100 \\
92 & 100 \\
63 & 92 \\
\hline
\end{tabular}

$\begin{array}{rr}92 & 100 \\ 63 & 92 \\ 61 & 96\end{array}$

\begin{tabular}{r|r}
\hline 61 & 96 \\
\hline 72 & 100 \\
\hline 71 & 100 \\
\hline 82 & 100 \\
\hline
\end{tabular}




\begin{tabular}{|c|c|}
\hline Countries & $\begin{array}{l}\text { Average Exact Percent } \\
\text { Agreement Across Items }\end{array}$ \\
\hline Bulgaria & - \\
\hline Canada, Ontario & - \\
\hline Canada, Quebec & - \\
\hline England & 89 \\
\hline France & 90 \\
\hline Germany & 88 \\
\hline Hong Kong SAR & 93 \\
\hline Hungary & 91 \\
\hline Iceland & - \\
\hline Iran, Islamic Rep. of & 92 \\
\hline Israel & 96 \\
\hline Italy & 91 \\
\hline Latvia & 84 \\
\hline Lithuania & 92 \\
\hline Macedonia, Rep. of & 81 \\
\hline Moldova, Rep. of & - \\
\hline Morocco & - \\
\hline Netherlands & 93 \\
\hline New Zealand & 90 \\
\hline Norway & 90 \\
\hline Romania & - \\
\hline Russian Federation & - \\
\hline Scotland & 88 \\
\hline Singapore & 88 \\
\hline Slovak Republic & 92 \\
\hline Slovenia & - \\
\hline Sweden & 89 \\
\hline United States & 93 \\
\hline International Avg. & 90 \\
\hline
\end{tabular}




\begin{tabular}{|c|c|c|c|}
\hline Purpose & Item Label ${ }^{1}$ & $\begin{array}{c}\text { Total Valid } \\
\text { Comparisons }^{2}\end{array}$ & $\begin{array}{c}\text { Exact Percent } \\
\text { Agreement }\end{array}$ \\
\hline \multirow{11}{*}{ 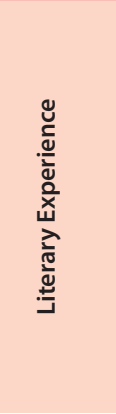 } & Flowers F06C & 377504 & $91 \%$ \\
\hline & Flowers F07C & 377957 & $80 \%$ \\
\hline & Flowers F08C & 375960 & $92 \%$ \\
\hline & Flowers F09C & 378078 & $93 \%$ \\
\hline & Flowers F10C & 376869 & $97 \%$ \\
\hline & Flowers F12C & 375684 & $63 \%$ \\
\hline & Unbelievable Night U05C & 377224 & $99 \%$ \\
\hline & Unbelievable Night U06C & 377385 & $93 \%$ \\
\hline & Unbelievable Night U08C & 378078 & $76 \%$ \\
\hline & Unbelievable Night U10C & 377453 & $96 \%$ \\
\hline & Unbelievable Night U12C & 377302 & $87 \%$ \\
\hline \multirow{12}{*}{ 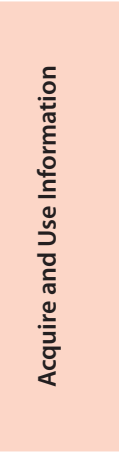 } & Antartica A01C & 378200 & $95 \%$ \\
\hline & Antartica $\mathrm{A} 03 \mathrm{C}$ & 378139 & $98 \%$ \\
\hline & Antartica A04C & 377542 & $89 \%$ \\
\hline & Antartica A07C & 378139 & $88 \%$ \\
\hline & Antartica A08C & 377722 & $80 \%$ \\
\hline & Antartica A09C & 377370 & $83 \%$ \\
\hline & Antartica A11C & 377363 & $81 \%$ \\
\hline & Day Hiking N02C & 377897 & $91 \%$ \\
\hline & Day Hiking N03C & 378139 & $94 \%$ \\
\hline & Day Hiking N08C & 376927 & $92 \%$ \\
\hline & Day Hiking N11C & 377773 & $77 \%$ \\
\hline & Day Hiking N12C & 330146 & $76 \%$ \\
\hline
\end{tabular}




\section{Test Reliability}

Exhibit A.14 displays the reading test reliability coefficient for each country. This coefficient is the median KR-2O reliability across the 12 test booklets and the PIRLs Reader. Reliabilities were generally high. Almost all countries had reliabilities between 0.8 and o.9, and eight countries-Bulgaria, England, Israel, Macedonia, New Zealand, Romania, South Africa, and Trinidad and Tobago-had reliabilities of 0.9 or greater. The median of the reliability coefficients across all countries was 0.88 .

\section{Data Processing}

To ensure the availability of comparable, high-quality data for analysis, PIRLS 2006 took rigorous quality control steps to create the international database. ${ }^{17}$ PIRLs prepared manuals and software for countries to use in creating and checking their data files, so that the information would be in a standardized international format before being forwarded to the IEA Data Processing and Research Center (DPC) in Hamburg for creation of the international database. Upon arrival at the IEA DPC, the data underwent an exhaustive quality-control process. This involved an iterative procedure of checking, editing, and rechecking designed to identify, document, and correct deviations from the international instruments, file structures, and coding schemes. The process also emphasized consistency of information within national data sets and appropriate linking among the student, parent, teacher, and school data files.

Throughout the process, the data were checked and double checked by the IEA DPC, the TIMSS \& PIRLS International Study Center, and the national centers. The national centers were contacted regularly, and given multiple opportunities to review the data for their countries. In conjunction with the IEA DPC, the TIMSS \& PIRLS International Study Center reviewed item statistics for each achievement item in each country to identify poorly performing items. ${ }^{18}$ In general, the items exhibited very good psychometric properties in all countries. However, as a result of the item review, one item was deleted from the achievement scaling for all countries because of a

\footnotetext{
17 These steps are detailed in Barth, J., \& Neuschmidt, O. (2007). Creating and checking the PIRLS 2006 database. In M.O. Martin, I.V.S. Mullis, \& A.M. Kennedy (Eds.), PIRLS 2006 technical report. Chestnut Hill, MA: Boston College.

18 See Martin, M.O., Kennedy, A.M. , \& Trong, K. L. (2004). Reviewing the PIRLS 2006 item statistics. In M.O. Martin, I.V.S. Mullis, \& A.M. Kennedy (Eds.), PIRLS 2006 technical report. Chestnut Hill, MA: Boston College.
} 


\begin{tabular}{|c|c|}
\hline Countries & $\begin{array}{l}\text { Reliability } \\
\text { Coefficient }\end{array}$ \\
\hline Austria & 0.86 \\
\hline Belgium (Flemish) & 0.83 \\
\hline Belgium (French) & 0.86 \\
\hline Bulgaria & 0.90 \\
\hline Canada, Alberta & 0.86 \\
\hline Canada, British Columbia & 0.86 \\
\hline Canada, Nova Scotia & 0.88 \\
\hline Canada, Ontario & 0.87 \\
\hline Canada, Quebec & 0.86 \\
\hline Chinese Taipei & 0.86 \\
\hline Denmark & 0.87 \\
\hline England & 0.91 \\
\hline France & 0.86 \\
\hline Georgia & 0.87 \\
\hline
\end{tabular}

Germany 0.86

\begin{tabular}{l|l} 
Hong Kong SAR & 0.82 \\
\hline
\end{tabular}

Hungary $\quad 0.88$

\begin{tabular}{l|l}
\hline Iceland & 0.88 \\
\hline
\end{tabular}

Indonesia $\quad 0.81$

\begin{tabular}{l|l}
\hline Iran, Islamic Rep. of & 0.88 \\
\hline
\end{tabular}

Israel 0.91

Italy 0.87

Kuwait $\quad 0.85$

\begin{tabular}{l|l}
\hline Latvia & 0.86 \\
\hline
\end{tabular}

Lithuania $\quad 0.83$

\begin{tabular}{l|l}
\hline Luxembourg & 0.88 \\
\hline
\end{tabular}

Macedonia, Rep. of $\quad 0.91$

\begin{tabular}{|l|l|}
\hline Moldova, Rep. of & 0.86 \\
\hline
\end{tabular}

Morocco $\quad 0.87$

\begin{tabular}{l|l} 
Netherlands & 0.81 \\
\hline
\end{tabular}

New Zealand $\quad 0.91$

\begin{tabular}{l|l} 
Norway & 0.86
\end{tabular}

Poland 0.89

Qatar 0.84

Romania $\quad 0.90$

\begin{tabular}{l|l} 
Russian Federation & 0.88
\end{tabular}

Scotland $\quad 0.89$

\begin{tabular}{l|r} 
Singapore & 0.89 \\
\hline
\end{tabular}

Slovak Republic $\quad 0.89$

\begin{tabular}{l|l} 
Slovenia & 0.88 \\
\hline
\end{tabular}

South Africa $\quad 0.92$

\begin{tabular}{l|l}
\hline Spain & 0.88 \\
\hline
\end{tabular}

Sweden $\quad 0.85$

\begin{tabular}{l|l}
\hline Trinidad and Tobago & 0.91 \\
\hline
\end{tabular}

United States 0.88

International Median $\quad 0.88$ 
problem with the scoring guide, and on a few occasions, items were deleted for individual countries because of translation errors.

\section{IRT Scaling and Data Analysis}

The primary approach to reporting the PIRLS 2006 achievement data was based on item response theory (IRT) scaling methods. ${ }^{19}$ Student reading achievement was summarized using a family of 2- and 3-parameter IRT models for dichotomously-scored items (right or wrong), and generalized partial credit models for items with 2 or 3 available score points. The IRT scaling method produces a score by averaging the responses of each student to the items that he or she took in a way that takes into account the difficulty and discriminating power of each item.

A notable feature of IRT scaling is that it is capable of estimating a student's score on an assessment even if the student has not responded to all of the items in the assessment pool. This characteristic of IRT scaling makes it particularly appropriate for PIRLS, where each individual student completed a single test booklet, comprising just two of the 10 passages in the PIRLS 2006 assessment (approximately 17 score points per passage). The PARSCALE ${ }^{20}$ program was used to estimate the IRT model parameters.

Although IRT methods are well suited to the PIRLs design, like other measurement approaches they provide the most reliable results when based on large numbers of items. Because individual students respond to relatively few items, particularly on the reading purposes and processes of comprehension scales, PIRLS uses a process known as "conditioning" to improve the reliability of the achievement measurement. The conditioning process combines students' responses to the items they were administered with information about the students' background characteristics to construct a distribution of achievement for each student that is conditional on the student's responses to the administered items and on the student's background characteristics.

To provide student scores that may be used in analyses, PIRLS uses the achievement distribution to predict or impute the achievement of each student conditional on his or her item responses and background

19 For a detailed description of the PIRLS 2006 scaling, see Foy, P., Galia, J., \& Li, Isaac. (2007). Scaling the PIRLS 2006 reading assessment data. In M.O. Martin, I.V.S. Mullis, \& A.M. Kennedy (Eds.), PIRLS 2006 technical report. Chestnut Hill, MA: Boston College.

20 Muraki, E., \& Bock, R.D. (1997). PARSCALE: IRT item analysis and test scoring for rating-scale data [Computer software and manual]. Chicago: Scientific Software. 
characteristics. These imputed scores, or "plausible values," are used as scale scores in analyses to create the exhibits in this report. To quantify any error in the imputation process, PIRLS generates five plausible values for each student and conducts all analyses five times. The average of the results of the five analyses is taken as the best estimate of the statistic in question, and the difference between them reflects the imputation error. PIRLS uses the MGROUP ${ }^{21}$ program developed by Educational Testing Service to implement the conditioning and generate plausible values.

The IRT analysis provides a common scale on which performance can be compared across countries. In addition to providing a basis for estimating mean achievement, scale scores permit estimates of how students within countries vary and provide information on percentiles of performance.

The PIRLS reading achievement scales were designed to reliably measure student achievement on the same scale for both the 2001 and 2006 assessments. The metric of the scales was established originally with the 2001 assessment. Treating all countries participating in PIRLS 2001 equally, the PIRLS scale average across those countries was set at 500, and the standard deviation was set at 100 . Since the countries varied in size, each country was weighted to contribute equally to the mean and standard deviation of the scale. The average and standard deviation of the scale scores are arbitrary and do not affect scale interpretation. To preserve the metric of the original 2001 scale, the 2006 assessment was first scaled using all of the items from both 2001 and 2006 and all students from countries that participated in both 2001 and 2006. Although the items from the four passages used in both assessments were the foundation for linking the two sets of assessment data, all items from 2001 and 2006 were included in this scaling. Having established the characteristics of the scale, scores were computed for students from countries that participated in 2006 but not in 2001.

Achievement scales were produced for each of the two reading purposes (reading for literary experience and reading for information) and for two processes of comprehension (retrieving and straightforward inferencing, and interpreting, integrating, and evaluating), as well as for reading overall. Exhibit A.15 presents the Pearson correlation coefficient indicating the linear 
Exhibit A.15 Correlation Between Two Scales for Purposes and Two Scales for Processes for Reading PIRLS 2006

\begin{tabular}{|c|c|c|}
\hline \multirow[b]{2}{*}{ Countries } & \multicolumn{2}{|c|}{ Pearson Correlation Coefficient } \\
\hline & $\begin{array}{l}\text { Between Reading } \\
\text { for Literary Experience } \\
\text { and Reading for } \\
\text { Information }\end{array}$ & $\begin{array}{l}\text { Between Retrieval } \\
\text { and Straightforward } \\
\text { Inferencing and } \\
\text { Interpreting, } \\
\text { Integrating, } \\
\text { and Evaluating }\end{array}$ \\
\hline Austria & 0.90 & 0.92 \\
\hline Belgium (Flemish) & 0.82 & 0.91 \\
\hline Belgium (French) & 0.88 & 0.92 \\
\hline Bulgaria & 0.83 & 0.92 \\
\hline Canada, Alberta & 0.81 & 0.92 \\
\hline Canada, British Columbia & 0.83 & 0.90 \\
\hline Canada, Nova Scotia & 0.86 & 0.93 \\
\hline Canada, Ontario & 0.83 & 0.91 \\
\hline Canada, Quebec & 0.81 & 0.88 \\
\hline Chinese Taipei & 0.85 & 0.91 \\
\hline Denmark & 0.87 & 0.92 \\
\hline England & 0.89 & 0.94 \\
\hline France & 0.86 & 0.91 \\
\hline Georgia & 0.81 & 0.91 \\
\hline Germany & 0.90 & 0.93 \\
\hline Hong Kong SAR & 0.77 & 0.87 \\
\hline Hungary & 0.87 & 0.92 \\
\hline Iceland & 0.86 & 0.92 \\
\hline Indonesia & 0.76 & 0.88 \\
\hline Iran, Islamic Rep. of & 0.88 & 0.93 \\
\hline Israel & 0.89 & 0.96 \\
\hline Italy & 0.82 & 0.89 \\
\hline Kuwait & 0.79 & + \\
\hline Latvia & 0.84 & 0.89 \\
\hline Lithuania & 0.85 & 0.90 \\
\hline Luxembourg & 0.90 & 0.92 \\
\hline Macedonia, Rep. of & 0.90 & 0.95 \\
\hline Moldova, Rep. of & 0.80 & 0.88 \\
\hline Morocco & 0.82 & + \\
\hline Netherlands & 0.79 & 0.89 \\
\hline New Zealand & 0.91 & 0.96 \\
\hline Norway & 0.82 & 0.90 \\
\hline Poland & 0.89 & 0.92 \\
\hline Qatar & 0.88 & + \\
\hline Romania & 0.88 & 0.95 \\
\hline Russian Federation & 0.88 & 0.93 \\
\hline Scotland & 0.89 & 0.93 \\
\hline Singapore & 0.90 & 0.96 \\
\hline Slovak Republic & 0.91 & 0.94 \\
\hline Slovenia & 0.88 & 0.94 \\
\hline South Africa & 0.93 & + \\
\hline Spain & 0.82 & 0.92 \\
\hline Sweden & 0.89 & 0.91 \\
\hline Trinidad and Tobago & 0.92 & 0.95 \\
\hline United States & 0.87 & 0.96 \\
\hline International Median & 0.88 & 0.92 \\
\hline
\end{tabular}


relationship between the two reading purposes in each of the PIRLS 2006 countries and between the two processes of comprehension. Across countries, the median correlation between reading for literary experience and reading for information was 0.88 , and the median correlation between the comprehension processes retrieving and simple inferencing and interpreting, integrating, and evaluating was 0.92 .

To facilitate comparisons of countries' relative performance on the two reading purposes (i.e., do students perform relatively better reading for literary experience or reading for information?) and on the two comprehension processes (again, do students perform relatively better on one process than the other?) PIRLS 2006 set all scales to have the same scale average and standard deviation -500 with a standard deviation of 10o. This means that any existing differences in the overall difficulty of the items comprising each of the scales are adjusted statistically to be equal in the interests of making relative comparisons. That is, the differences in performance among countries reflect differences in student achievement that would be expected on sets of items of equal difficulty.

To give an indication of the difficulty of the reading purpose and process scales, Exhibit A.16 presents the percentage of students responding correctly to each item, averaged across the items for each scale, for each participant. From this exhibit it may be seen that the items making up the literary and informational scales are similar in difficulty -55 percent correct, on average across all participants, for literary reading and 52 percent correct, on average, for informational reading. However, there is a much greater difference in average difficulty between the two scales for the comprehension processes. The average percent correct for the items on the retrieval and straightforward inferencing scale was 64 percent, compared with an average percent correct of just 44 percent for the interpreting, integrating, and evaluating items.

The items making up the interpreting, integrating, and evaluating scale were particularly difficult for students in Kuwait, Morocco, Qatar, and South Africa, where the average percentage of students answering the items correctly ranged from 11 to 14 percent. With average achievement as low as 


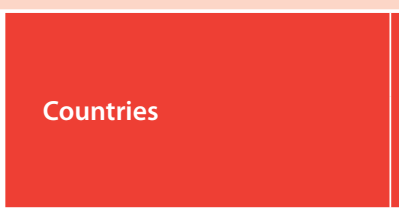

Austria

Belgium (Flemish)

Belgium (French)

Bulgaria

Canada, Alberta

Canada, British Columbia

Canada, Nova Scotia

Canada, Ontario

Canada, Quebec

Chinese Taipei

Denmark

England

France

Georgia

Germany

Hong Kong SAR

Hungary

Iceland

Indonesia

Iran, Islamic Rep. of

Israel

Italy

Kuwait

Latvia

Lithuania

Luxembourg

Macedonia, Rep. of

Moldova, Rep. of

Morocco

Netherlands

New Zealand

Norway

Poland

Qatar

Romania

Russian Federation

Scotland

Singapore

Slovak Republic

Slovenia

South Africa

Spain

Sweden

Trinidad and Tobago

United States

International Avg.

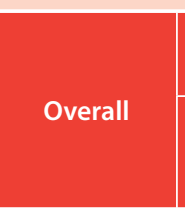

$61(0.5)$

$64(0.5)$

$51(0.7)$

64 (1.1)

$67(0.6)$

$67(0.6)$

$63(0.5)$

$66(0.7)$

$60(0.7)$

$61(0.5)$

$64(0.6)$

$62(0.6)$

$57(0.5)$

$45(0.8)$

$64(0.5)$

$69(0.6)$

$65(0.7)$

$54(0.3)$

$31(0.7)$

$35(0.6)$

$56(0.7)$

$65(0.7)$

$22(0.4)$

$63(0.6)$

$61(0.4)$

$66(0.2)$

$40(0.8)$

$52(0.8)$

$21(0.7)$

$64(0.4)$

$60(0.5)$

51 (0.6)

57 (0.6)

$24(0.2)$

50 (1.1)

$68(0.8)$

$59(0.7)$

$66(0.7)$

$60(0.7)$

$57(0.5)$

$21(0.9)$

$55(0.6)$

$64(0.5)$

$38(0.9)$

$62(0.8)$

$54(0.1)$

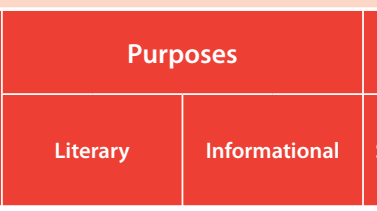

Processes

Retrieval and Interpreting,

Straightforward Integrating, Inferencing and Evaluating

$\begin{array}{llll}64(0.6) & 59(0.6) & 74(0.5) & 49(0.6)\end{array}$

\begin{tabular}{|l|l|l|l|}
\hline $66(0.6)$ & $62(0.5)$ & $74(0.4)$ & $54(0.5)$ \\
\hline
\end{tabular}

$53(0.7) \quad 49(0.7)$

$63(0.6)$

$65(1.1)$

$63(1.1)$

$70(0.6)$

$69(0.7)$

$72(0.9)$

$40(0.7)$

$66(0.6)$

$69(0.7)$

$62(0.8)$

$62(0.5)$

$66(0.6)$

$64(0.7)$

$58(0.5)$

$47(0.9)$

$67(0.5)$

$69(0.7)$

$69(0.8)$

$57(0.4)$

$29(0.7)$

$37(0.7)$

$58(0.8)$

$67(0.8)$

$22(0.4)$

$65(0.6)$

$65(0.5)$

$68(0.3)$

$68(0.3)$
$39(0.8)$

$52(0.8)$

$20(0.8)$

$20(0.8)$
$66(0.4)$

\section{$61(0.6)$}

$54(0.6)$

\section{$59(0.6)$}

$24(0.3)$

$52(1.2)$

\section{$70(0.8)$}

\section{$61(0.8)$}

$67(0.8)$

\section{$63(0.8)$}

$59(0.6)$

$59(0.6)$
$20(0.9)$

$58(0.7)$

\section{$66(0.6)$}

38 (1.0)

$38(1.0)$
$65(0.9)$

$55(0.1)$
$64(0.6)$

$64(0.7)$

$60(0.5)$

$63(0.8)$

$59(0.7)$

\section{$60(0.5)$}

$61(0.6)$

$60(0.6)$

$57(0.6)$

$42(0.8)$

\section{$61(0.6)$}

$68(0.6)$

$61(0.7)$

$51(0.4)$

$32(0.7)$

$33(0.6)$

$53(0.7)$

$63(0.7)$

$21(0.5)$

$60(0.6)$

$58(0.5)$

$64(0.3)$

$41(0.9)$

$53(0.8)$

$21(0.8)$

$21(0.8)$
$62(0.4)$

$62(0.4)$
$59(0.5)$

$48(0.7)$

$54(0.6)$

$23(0.2)$

$47(1.1)$

$66(0.8)$

$57(0.7)$

$66(0.7)$

$57(0.7)$

$75(0.6)$

$75(0.6)$

$71(0.5)$

$73(0.7)$

$71(0.6)$

$73(0.5)$

$74(0.5)$

$70(0.6)$

$69(0.5)$

$58(0.8)$

$76(0.5)$

$77(0.5)$

$73(0.7)$

$67(0.3)$

$42(0.8)$

$46(0.7)$

$65(0.7)$

$73(0.6)$

$30(0.5)$

$71(0.6)$

$71(0.4)$

$78(0.2)$

$50(0.8)$

$59(0.8)$

$30(0.9)$

$75(0.4)$

$68(0.5)$

$63(0.7)$

$67(0.6)$

$33(0.2)$

$60(1.2)$

$77(0.7)$

$69(0.6)$

$76(0.6)$

$70(0.7)$

$56(0.6)$

$21(0.8)$

$52(0.6)$

$62(0.6)$

$38(0.9)$

$60(0.8)$

$52(0.1)$

$64(0.1)$

$44(0.1)$ 
this, there is a danger that results may be distorted by a "floor" effect-that the achievement of low-performing students may be overestimated because there are not sufficient items matched to their ability levels. Even though the PIRLS scaling approach is very robust, there is concern that achievement results based on very low average performance may not be sufficiently accurate to report. Based on examinations of the data, PIRLS 2006 used a guideline of not reporting scaled achievement results for countries with an average percent correct of 20 percent or less on the set of items comprising the scale. ${ }^{22}$ Accordingly, results on the interpreting, integrating, and evaluating scale were not reported for Kuwait, Morocco, Qatar, and South Africa.

\section{Estimating Sampling Error}

Because the statistics presented in this report are estimates of national performance based on samples of students-rather than on the values that could be calculated if every student in every country had answered every question-it is important to have measures for the degree of uncertainty of the estimates. The jackknife procedure was used to estimate the standard error associated with each statistic presented in this report. ${ }^{23}$ As well as sampling error, the jackknife standard errors also include an error component due to variation between the five plausible values generated for each student. The use of confidence intervals (based on the standard errors) provides a way to make inferences about the population means and proportions in a manner that reflects the uncertainty associated with the sample estimates. An estimated sample statistic plus or minus 2 standard errors represents a 95 percent confidence interval for the corresponding population result.

\section{Reporting Student Achievement at the PIRLS 2006 International Benchmarks}

To provide richly descriptive information about what performance on the PIRLS reading scale means in terms of the reading skills that students have and the comprehension processes and strategies they can bring to bear, PIRLS identified four points on the scale for use as international benchmarks of

22 For further discussion, see Foy, P., Galia, J., \& Li, Isaac. (2007). Scaling the PIRLS 2006 reading assessment data. In M.O. Martin, I.V.S. Mullis, \& A.M. Kennedy (Eds.), PIRLS 2006 technical report. Chestnut Hill, MA: Boston College.

23 Procedures for computing jackknifed standard errors are presented in Kennedy, A.M. \& Trong, K. L. (2007). Reporting PIRLS 2006 student achievement in reading. In M.O. Martin, I.V.S. Mullis, \& A.M. Kennedy (Eds.), PIRLS 2006 technical report. Chestnut Hill, MA: Boston College. 
student achievement. Selected to represent the range of performance shown by students internationally, the advanced benchmark is 625 , the high benchmark is 550 , the intermediate benchmark is 475 , and the low benchmark is 400 .

It should be noted that the PIRLS 2006 international benchmarks were established using procedures different from those implemented in 2001. Unlike the PIRLS 2006 benchmarks, which are four fixed points evenly spaced on the scale $(625,550,475$, and 400), the PIRLS 2001 international benchmarks were identified on the basis of student achievement across the participating countries. The most challenging benchmark, the Top 10\% Benchmark, was defined as the $90^{\text {th }}$ percentile. Corresponding to a scale score of 615 , this was the point above which the top 10 percent of students scored, counting across all countries. Next most challenging, the Upper Quarter Benchmark was defined as the $75^{\text {th }}$ percentile and corresponded to a scale score of 570 , while the Median Benchmark, defined as the $50^{\text {th }}$ percentile, or median, corresponded to a scale score of 510, and the Lower Quarter Benchmark defined as the $25^{\text {th }}$ percentile, corresponded to a scale score of 435 .

Although the PIRLS 2001 approach to establishing benchmarks based on student achievement worked well for the first cycle of PIRLS, for measuring trends across successive cycles of PIRLS it has the disadvantage that, because the benchmarks must be recomputed with each new cycle of the study, benchmarks will change from cycle to cycle depending on the set of countries taking part. For example, if several new low-achieving countries joined a cycle, benchmarks based on percentiles of student achievement could decrease, perhaps giving the erroneous impression that standards had improved. To avoid misinterpretations based on movement in benchmarks between cycles, PIRLS 2006 adopted the fixed benchmark approach, instituted for TIMSs 2003, where the same four scale-score points $(625,550,475$, and 400 ), will be used as international benchmarks for all future cycles of PIRLS (i.e., in 2011, 2016, and so on).

In order to interpret the PIRLS scale scores and analyze achievement at the international benchmarks, PIRLS 2006 conducted a scale anchoring analysis to describe achievement of students at those four points on the scale. 
Scale anchoring is a way of describing students' performance at various points on a scale, in terms of the kind of reading they can do and the level of comprehension they exhibit. It involves a statistical component, in which items that discriminate between successive points on the scale are identified, and a judgmental component in which subject matter experts examine the items and generalize to students' knowledge and understandings. ${ }^{24}$ In PIRLS 2006, the Reading Development Group worked with the Reading Coordinator, the PIRLS Reading Consultant, and the TIMSS \& PIRLS International Study Center to describe student reading at the international benchmarks. The descriptions of the items developed as part of the scale anchoring analysis are found in Appendix E. 


Appendix B

Multiple Comparisons of Average Achievement in Reading Purposes and Processes of Comprehension 
Exhibit B.1 Multiple Comparisons of Average Reading Achievement for Literary Purposes

Instructions: Read across the row for a country to compare performance with the countries listed along the top of the chart. The symbols indicate whether the average achievement of the country in the row is significantly lower than that of the comparison country, significantly higher than that of the comparison country, or if there is no statistically significant difference between the average achievement of the two countries.
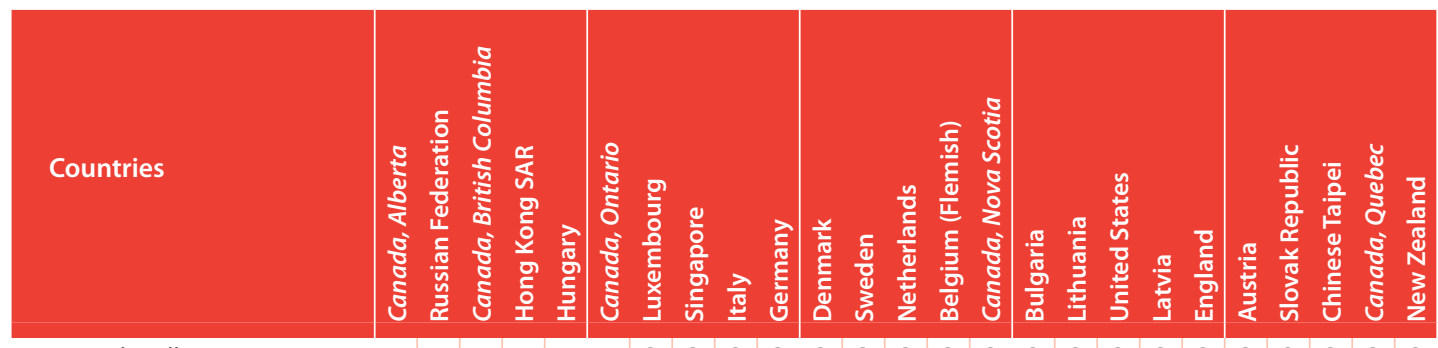

Canada, Alberta

Russian Federation

Canada, British Columbia

Hong Kong SAR

Hungary

Canada, Ontario

Luxembourg

Singapore

Italy

Germany

Denmark

Sweden

Netherlands

Belgium (Flemish)

Canada, Nova Scotia

Bulgaria

Lithuania

United States

Latvia

England

Austria

Slovak Republic

Chinese Taipei

Canada, Quebec

New Zealand

Scotland

Poland

Slovenia

Israel

Spain

France

Iceland

Norway

Belgium (French)

Romania

Moldova, Rep. of

Georgia

Macedonia, Rep. of

Trinidad and Tobago

Iran, Islamic Rep. of

Indonesia

Qatar

Kuwait

Morocco

South Africa \begin{tabular}{lllllllllllllllll|l|l|lll|l|l|l}
0 & 0 & 0 & 0 & 0 & 0 & 0 & 0 & 0 & 0 & 0 & 0 & 0 & 0 & 0 & 0 & 0 & 0 & 0 & 0 & 0 & 0 & 0 & 0 \\
\hline
\end{tabular} $\begin{array}{lllllllllllllllllllllll}0 & 0 & 0 & 0 & 0 & 0 & 0 & 0 & 0 & 0 & 0 & 0 & 0 & 0 & 0 & 0 & 0 & 0 & 0 & 0 & 0 & 0 & 0\end{array}$ $\begin{array}{llllllllllllllllllllll}0 & 0 & 0 & 0 & 0 & 0 & 0 & 0 & 0 & 0 & 0 & 0 & 0 & 0 & 0 & 0 & 0 & 0 & 0 & 0 & 0\end{array}$ $\begin{array}{lllllllllllllllllllll}0 & 0 & 0 & 0 & 0 & 0 & 0 & 0 & 0 & 0 & 0 & 0 & 0 & 0 & 0 & 0 & 0 & 0 & 0 & 0 & 0\end{array}$ $\begin{array}{llllllllllllllllllllll}0 & 0 & 0 & 0 & 0 & 0 & 0 & 0 & 0 & 0 & 0 & 0 & 0 & 0 & 0 & 0 & 0 & 0 & 0 & 0 & 0\end{array}$ $\begin{array}{llllllllllllllllllll}0 & 0 & 0 & 0 & 0 & 0 & 0 & 0 & 0 & 0 & 0 & 0 & 0 & 0 & 0 & 0 & 0 & 0 & 0 & 0\end{array}$ $\begin{array}{llllllllllllllllllllll}0 & 0 & 0 & 0 & 0 & 0 & 0 & 0 & 0 & 0 & 0 & 0 & 0 & 0 & 0 & 0 & 0 & 0 & 0 & 0 & 0\end{array}$ $\begin{array}{lllllllllllllllllllll}-1 & 0 & 0 & 0 & 0 & 0 & 0 & 0 & 0 & 0 & 0 & 0 & 0 & 0 & 0 & 0 & 0 & 0 & 0 \\ 0 & 0 & 0 & 0 & 0 & 0 & 0 & 0 & 0 & 0 & 0 & 0 & 0 & 0 & 0 & 0 & 0 & 0\end{array}$

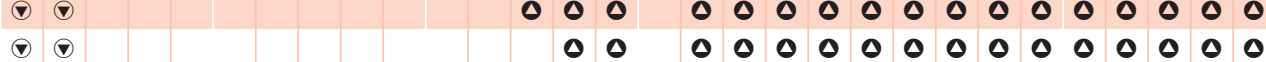

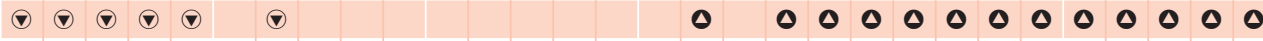

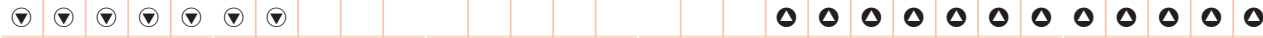

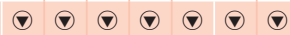

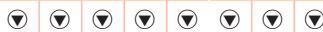
$\begin{array}{lllllllllllll}0 & 0 & 0 & 0 & 0 & 0 & 0 & 0 & 0 & 0 & 0 & 0\end{array}$ $\begin{array}{lllllllllll}0 & 0 & 0 & 0 & 0 & 0 & 0 & 0 & 0 & 0\end{array}$

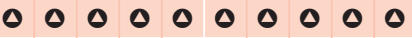
$\begin{array}{llllllllll}0 & 0 & 0 & 0 & 0 & 0 & 0 & 0 & 0\end{array}$ $\begin{array}{llllllll}0 & 0 & 0 & 0 & 0 & 0 & 0 & 0\end{array}$ $\begin{array}{llllllllll}0 & 0 & 0 & 0 & 0 & 0 & 0 & 0 & 0\end{array}$ $\begin{array}{llllllll}0 & 0 & 0 & 0 & 0 & 0 & 0 & 0\end{array}$ $\begin{array}{llllllll}0 & 0 & 0 & 0 & 0 & 0 & 0 & 0\end{array}$ $\begin{array}{lllllllll}0 & 0 & 0 & 0 & 0 & 0 & 0 & 0\end{array}$ $\begin{array}{lllllllll}0 & 0 & 0 & 0 & 0 & 0 & 0 & 0\end{array}$ 0000 000 000 00 000

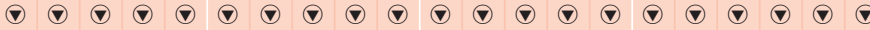

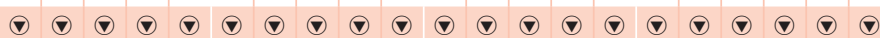

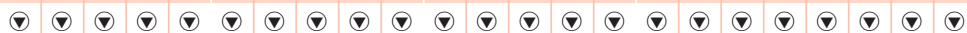

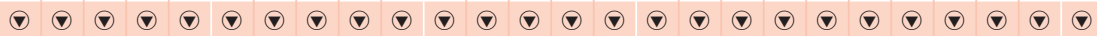

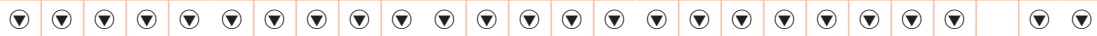

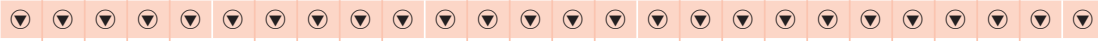

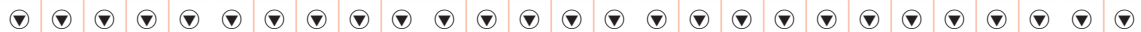

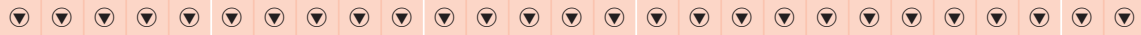

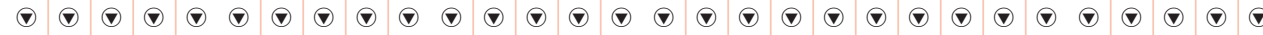

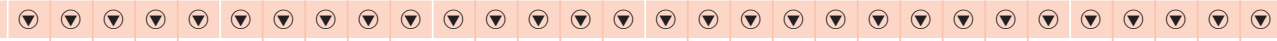

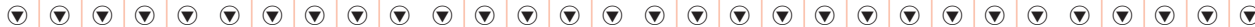

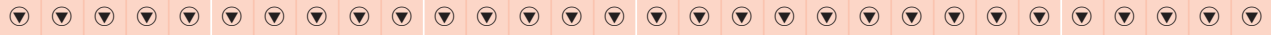

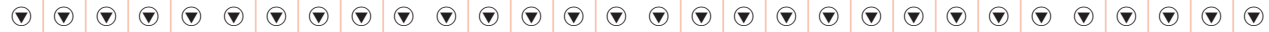

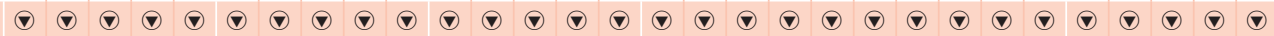

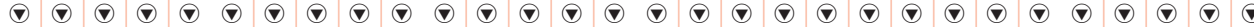

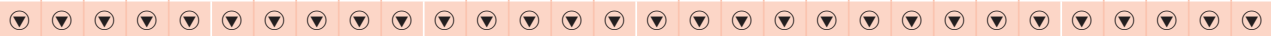

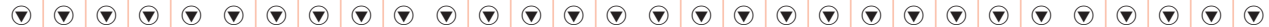

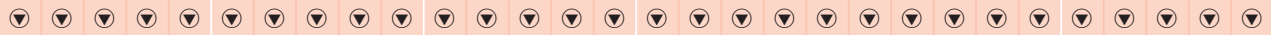

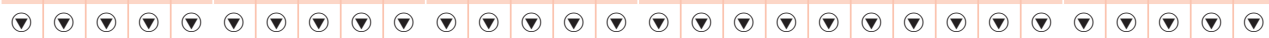

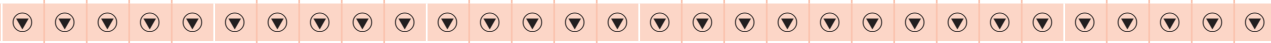

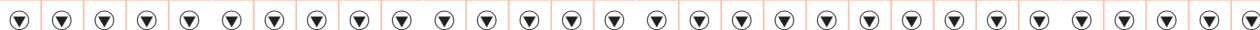




\section{Exhibit B.1 Multiple Comparisons of Average Reading Achievement for Literary Purposes} (Continued)

Instructions: Read across the row for a country to compare performance with the countries listed along the top of the chart. The symbols indicate whether the average achievement of the country in the row is significantly lower than that of the comparison country, significantly higher than that of the comparison country, or if there is no statistically significant difference between the average achievement of the two countries.

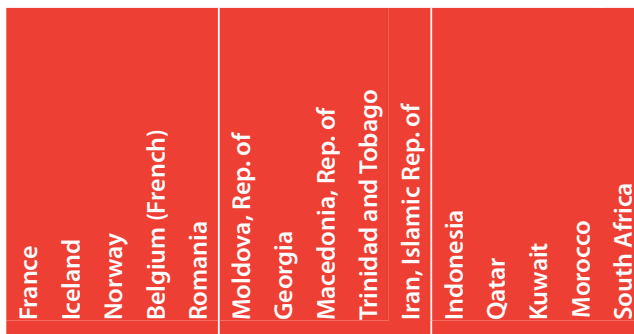

$\begin{array}{lllllllllllllllllllllll}0 & 0 & 0 & 0 & 0 & 0 & 0 & 0 & 0 & 0 & 0 & 0 & 0 & 0 & 0\end{array}$ $\begin{array}{llllllllllllllllllllllll}0 & 0 & 0 & 0 & 0 & 0 & 0 & 0 & 0 & 0 & 0 & 0 & 0 & 0 & 0\end{array}$

$\begin{array}{llllllllllllllll}0 & 0 & 0 & 0 & 0 & 0 & 0 & 0 & 0 & 0 & 0 & 0 & 0 & 0 & 0\end{array}$

$\begin{array}{lllllllllllllllllllllll}0 & 0 & 0 & 0 & 0 & 0 & 0 & 0 & 0 & 0 & 0 & 0 & 0 & 0 & 0\end{array}$

$\begin{array}{llllllllllllllllll}0 & 0 & 0 & 0 & 0 & 0 & 0 & 0 & 0 & 0 & 0 & 0 & 0 & 0 & 0\end{array}$

$\begin{array}{llllllllllllllllllllllllllllll}0 & 0 & 0 & 0 & 0 & 0 & 0 & 0 & 0 & 0 & 0 & 0 & 0 & 0\end{array}$

$\begin{array}{lllllllllllllllll}0 & 0 & 0 & 0 & 0 & 0 & 0 & 0 & 0 & 0 & 0 & 0 & 0 & 0 & 0\end{array}$

$\begin{array}{lllllllllllllll}0 & 0 & 0 & 0 & 0 & 0 & 0 & 0 & 0 & 0 & 0 & 0 & 0 & 0 & 0\end{array}$

$\begin{array}{llllllllllllllll}0 & 0 & 0 & 0 & 0 & 0 & 0 & 0 & 0 & 0 & 0 & 0 & 0 & 0 & 0\end{array}$

$\begin{array}{llllllllllllllll}0 & 0 & 0 & 0 & 0 & 0 & 0 & 0 & 0 & 0 & 0 & 0 & 0 & 0 & 0\end{array}$

$\begin{array}{llllllllllllllll}0 & 0 & 0 & 0 & 0 & 0 & 0 & 0 & 0 & 0 & 0 & 0 & 0 & 0 & 0\end{array}$

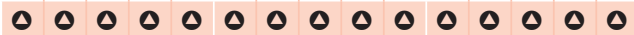

$\begin{array}{lllllllllllllllll}0 & 0 & 0 & 0 & 0 & 0 & 0 & 0 & 0 & 0 & 0 & 0 & 0 & 0 & 0\end{array}$

$\begin{array}{lllllllllllllll}0 & 0 & 0 & 0 & 0 & 0 & 0 & 0 & 0 & 0 & 0 & 0 & 0 & 0 & 0\end{array}$

$\begin{array}{llllllllllllllll}0 & 0 & 0 & 0 & 0 & 0 & 0 & 0 & 0 & 0 & 0 & 0 & 0 & 0 & 0\end{array}$

$\begin{array}{llllllllllllllll}0 & 0 & 0 & 0 & 0 & 0 & 0 & 0 & 0 & 0 & 0 & 0 & 0 & 0 & 0\end{array}$

$\begin{array}{llllllllllllllll}0 & 0 & 0 & 0 & 0 & 0 & 0 & 0 & 0 & 0 & 0 & 0 & 0 & 0 & 0\end{array}$

$\begin{array}{lllllllllllllll}0 & 0 & 0 & 0 & 0 & 0 & 0 & 0 & 0 & 0 & 0 & 0 & 0 & 0 & 0\end{array}$

$\begin{array}{lllllllllllllll}0 & 0 & 0 & 0 & 0 & 0 & 0 & 0 & 0 & 0 & 0 & 0 & 0 & 0 & 0\end{array}$

$\begin{array}{lllllllllllllll}0 & 0 & 0 & 0 & 0 & 0 & 0 & 0 & 0 & 0 & 0 & 0 & 0 & 0 & 0\end{array}$

$\begin{array}{llllllllllllllll}0 & 0 & 0 & 0 & 0 & 0 & 0 & 0 & 0 & 0 & 0 & 0 & 0 & 0 & 0\end{array}$

$\begin{array}{lllllllllllllll}0 & 0 & 0 & 0 & 0 & 0 & 0 & 0 & 0 & 0 & 0 & 0 & 0 & 0 & 0\end{array}$

$\begin{array}{llllllllllllllll}0 & 0 & 0 & 0 & 0 & 0 & 0 & 0 & 0 & 0 & 0 & 0 & 0 & 0 & 0\end{array}$

$\begin{array}{lllllllllllllll}0 & 0 & 0 & 0 & 0 & 0 & 0 & 0 & 0 & 0 & 0 & 0 & 0 & 0 & 0\end{array}$

$\begin{array}{llllllllllllllll}0 & 0 & 0 & 0 & 0 & 0 & 0 & 0 & 0 & 0 & 0 & 0 & 0 & 0 & 0\end{array}$

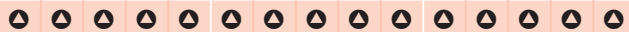

$\begin{array}{llllllllllllllll}0 & 0 & 0 & 0 & 0 & 0 & 0 & 0 & 0 & 0 & 0 & 0 & 0 & 0 & 0\end{array}$ $\begin{array}{llllllllllllll}0 & 0 & 0 & 0 & 0 & 0 & 0 & 0 & 0 & 0 & 0 & 0 & 0\end{array}$

$\begin{array}{lllllllllllll}0 & 0 & 0 & 0 & 0 & 0 & 0 & 0 & 0 & 0 & 0 & 0 & 0\end{array}$

$\begin{array}{llllllllllllll}0 & 0 & 0 & 0 & 0 & 0 & 0 & 0 & 0 & 0 & 0 & 0 & 0\end{array}$

$\begin{array}{llllllllllllll}0 & 0 & 0 & 0 & 0 & 0 & 0 & 0 & 0 & 0 & 0 & 0 & 0\end{array}$

$\begin{array}{llllllllllllll}0 & 0 & 0 & 0 & 0 & 0 & 0 & 0 & 0 & 0 & 0 & 0 & 0\end{array}$

$\left(\begin{array}{lllllllll|l|l|l}-1 & 0 & 0 & 0 & 0 & 0 & 0 & 0 & 0 & 0 & 0 & 0\end{array}\right.$

(-) $10 \quad 00000000$

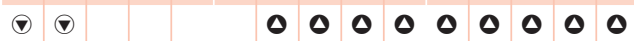

- (1) $10 \quad 000000000$

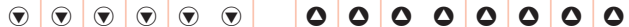

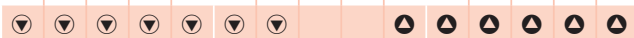

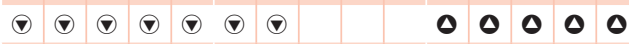

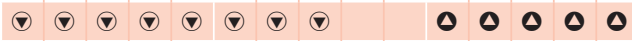

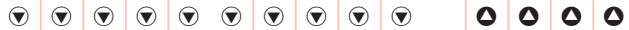

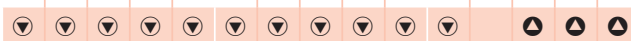

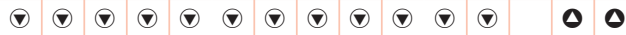

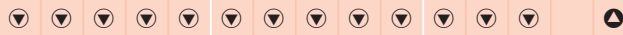

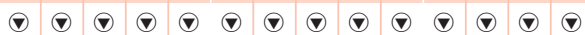

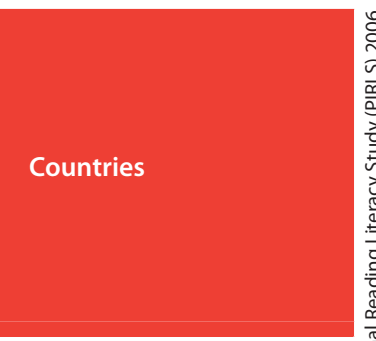

Canada, Alberta

Russian Federation

Canada, British Columbia

Hong Kong SAR

Hungary

Canada, Ontario

Luxembourg

Singapore

Italy

Germany

Denmark

Sweden

Netherlands

Belgium (Flemish)

Canada, Nova Scotia

Bulgaria

Lithuania

United States

Latvia

England

Austria

Slovak Republic

Chinese Taipei

Canada, Quebec

New Zealand

Scotland

Poland

Slovenia

Israel

Spain

France

Iceland

Norway

Belgium (French)

Romania

Moldova, Rep. of

Georgia

Macedonia, Rep. of

Trinidad and Tobago

Iran, Islamic Rep. of

Indonesia

Qatar

Kuwait

Morocco

South Africa
- Average achievement significantly higher than comparison country

$\checkmark$ Average achievement significantly lower than comparison country 


\section{Exhibit B.2 Multiple Comparisons of Average Reading Achievement for Informational Purposes}

Instructions: Read across the row for a country to compare performance with the countries listed along the top of the chart. The symbols indicate whether the average achievement of the country in the row is significantly lower than that of the comparison country, significantly higher than that of the comparison country, or if there is no statistically significant difference between the average achievement of the two countries.
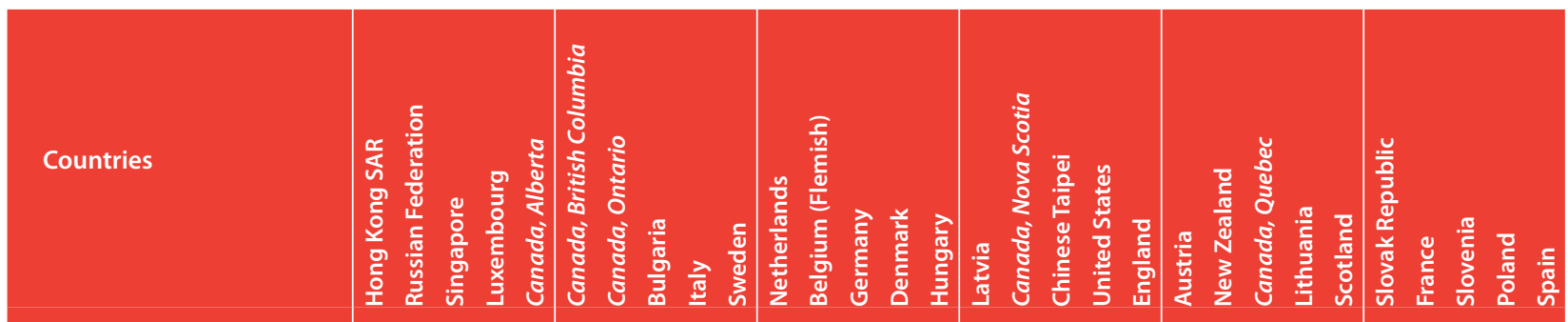

Hong Kong SAR

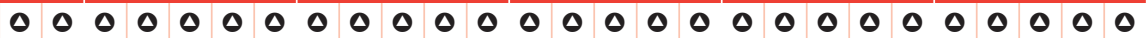

Russian Federation $\begin{array}{lllllllllllllllllllllllllll}0 & 0 & 0 & 0 & 0 & 0 & 0 & 0 & 0 & 0 & 0 & 0 & 0 & 0 & 0 & 0 & 0 & 0 & 0 & 0 & 0 & 0 & 0 & 0 & 0 & 0\end{array}$ Singapore

Luxembourg

Canada, Alberta

0

\begin{tabular}{lllllllllllllllllllllllllll}
0 & 0 & 0 & 0 & 0 & 0 & 0 & 0 & 0 & 0 & 0 & 0 & 0 & 0 & 0 & 0 & 0 & 0 & 0 & 0 & 0 & 0 & 0 & 0 & 0 \\
\hline
\end{tabular}

\section{Canada, British Columbia}

(1) (

- 0

- 0

Canada, Ontario

(-) $(\odot$

$\begin{array}{lllllllllllllllllllllll}0 & 0 & 0 & 0 & 0 & 0 & 0 & 0 & 0 & 0 & 0 & 0 & 0 & 0 & 0 & 0 & 0 & 0 & 0 & 0 & 0 \\ 0\end{array}$

Bulgaria

(४) ( $)$

$\begin{array}{llllllllllllllllll}0 & 0 & 0 & 0 & 0 & 0 & 0 & 0 & 0 & 0 & 0 & 0 & 0 & 0 & 0 & 0 & 0 & 0\end{array}$

Italy

(1) (1) (1)

$\begin{array}{llllllllllllllllll}0 & 0 & 0 & 0 & 0 & 0 & 0 & 0 & 0 & 0 & 0 & 0 & 0 & 0 & 0 & 0 & 0 & 0\end{array}$

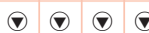

$\begin{array}{lllllllllllllll}0 & 0 & 0 & 0 & 0 & 0 & 0 & 0 & 0 & 0 & 0 & 0 & 0 & 0 & 0\end{array}$

Sweden

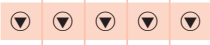

$\begin{array}{llllllllllllllll}0 & 0 & 0 & 0 & 0 & 0 & 0 & 0 & 0 & 0 & 0 & 0 & 0 & 0 & 0\end{array}$

Netherlands

$\nabla(\nabla) \odot$

○

$\begin{array}{lllllllllllllll}0 & 0 & 0 & 0 & 0 & 0 & 0 & 0 & 0 & 0 & 0 & 0 & 0 & 0 & 0\end{array}$

Belgium (Flemish)

(1) (1) (1) (1)

Germany

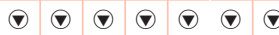

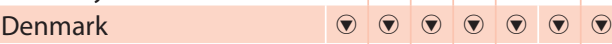

Hungary

(-) (1) (1) (1) (1) (

Latvia

(1) (1) (1) (1) (1) (1) (1) (1) (1) (1)

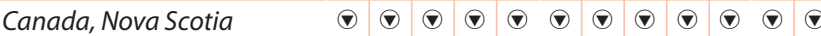

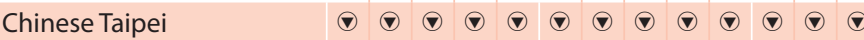

United States

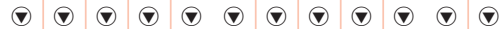

0

$\begin{array}{llllllllllllllll}0 & 0 & 0 & 0 & 0 & 0 & 0 & 0 & 0 & 0 & 0 & 0 & 0 & 0 & 0\end{array}$ $\begin{array}{llllllllllllllll}0 & 0 & 0 & 0 & 0 & 0 & 0 & 0 & 0 & 0 & 0 & 0 & 0 & 0 & 0\end{array}$ $\begin{array}{lllllllllllll}0 & 0 & 0 & 0 & 0 & 0 & 0 & 0 & 0 & 0 & 0 & 0\end{array}$ $\begin{array}{lllllllll}0 & 0 & 0 & 0 & 0 & 0 & 0 & 0 & 0\end{array}$ England

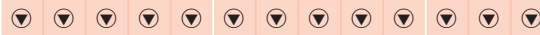

$\begin{array}{llllllllll}0 & 0 & 0 & 0 & 0 & 0 & 0 & 0\end{array}$ $\begin{array}{llllllll}0 & 0 & 0 & 0 & 0 & 0 & 0\end{array}$ Austria

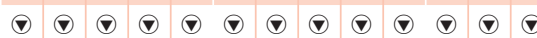
$\begin{array}{llllllll}0 & 0 & 0 & 0 & 0 & 0 & 0\end{array}$ - 00000

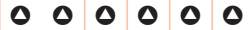

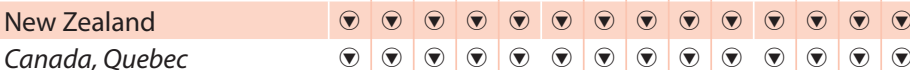

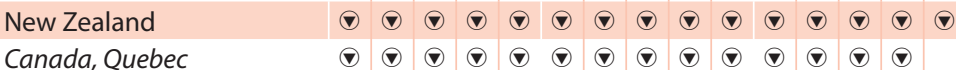
- 000000 $\begin{array}{llllllll}0 & 0 & 0 & 0 & 0 & 0 & 0\end{array}$

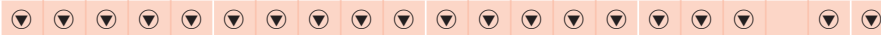
0000 Lithuania

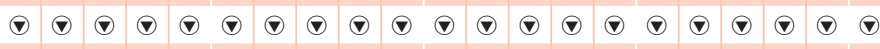
000 Scotland

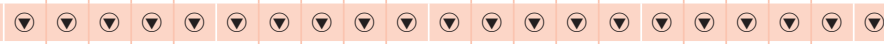
00

Slovak Republic

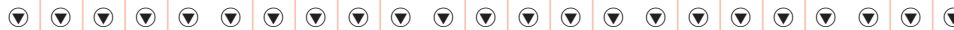
00 France

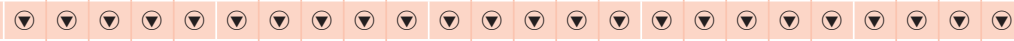

Slovenia

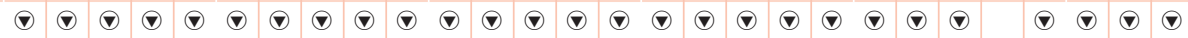

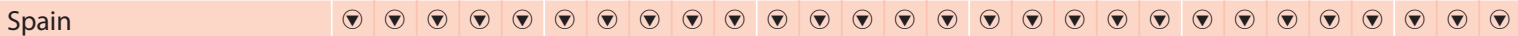

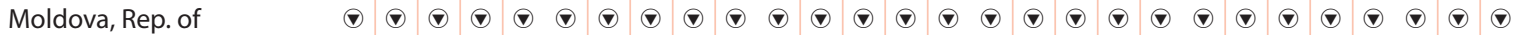

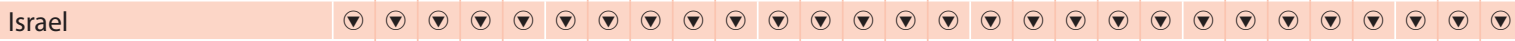

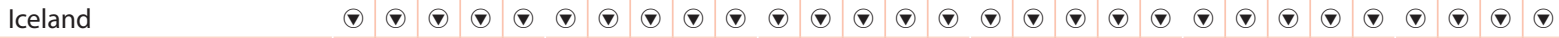

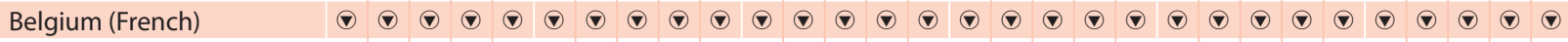

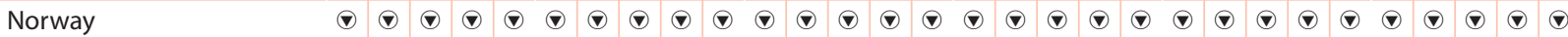

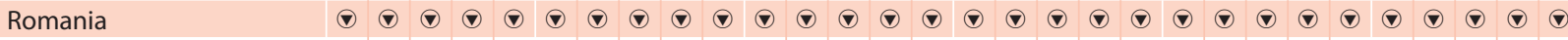

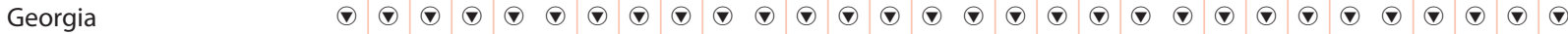
Macedonia, Rep. of

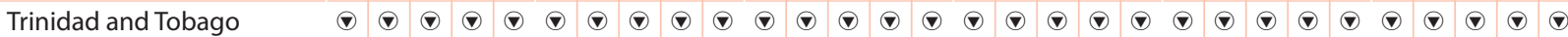
Iran, Islamic Rep. of $\quad$ (1)

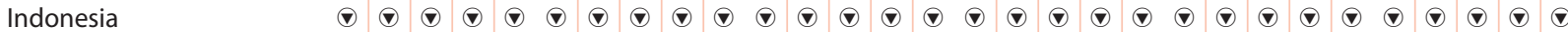

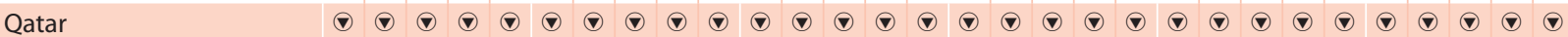
Morocco

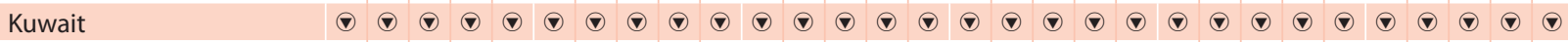
South Africa 


\section{Exhibit B.2 Multiple Comparisons of Average Reading Achievement for Informational Purposes} (Continued)

Instructions: Read across the row for a country to compare performance with the countries listed along the top of the chart. The symbols indicate whether the average achievement of the country in the row is significantly lower than that of the comparison country, significantly higher than that of the comparison country, or if there is no statistically significant difference between the average achievement of the two countries.

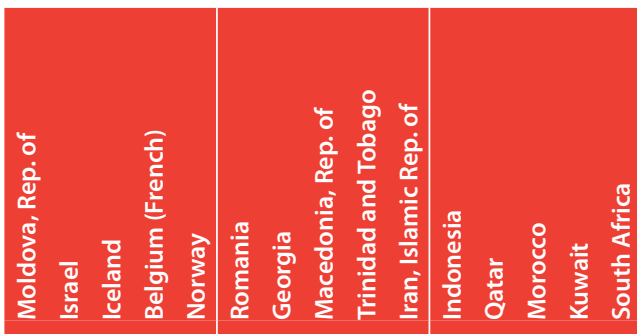

$\begin{array}{lllllllllllllll} & 0 & 0 & 0 & 0 & 0 & 0 & 0 & 0 & 0 & 0 & 0 & 0 & 0 & 0\end{array}$

000000000000000000

$\begin{array}{llllllllllllllll}0 & 0 & 0 & 0 & 0 & 0 & 0 & 0 & 0 & 0 & 0 & 0 & 0 & 0 & 0\end{array}$

- 0000000000

$\begin{array}{llllllllllllllll} & 0 & 0 & 0 & 0 & 0 & 0 & 0 & 0 & 0 & 0 & 0 & 0 & 0 & 0\end{array}$

$\begin{array}{lllllllllllllll}0 & 0 & 0 & 0 & 0 & 0 & 0 & 0 & 0 & 0 & 0 & 0 & 0 & 0\end{array}$

$\begin{array}{lllllllllllllllll}0 & 0 & 0 & 0 & 0 & 0 & 0 & 0 & 0 & 0 & 0 & 0 & 0 & 0 & 0\end{array}$

- 000000000

$\begin{array}{lllllllllllllll}0 & 0 & 0 & 0 & 0 & 0 & 0 & 0 & 0 & 0 & 0 & 0 & 0 & 0 & 0\end{array}$

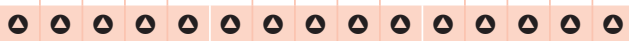

$\begin{array}{lllllllllllllllll} & - & 0 & 0 & 0 & 0 & 0 & 0 & 0 & 0 & 0 & 0 & 0 & 0 & 0 & 0\end{array}$

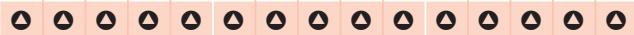

$\begin{array}{llllllllllllllll} & 0 & 0 & 0 & 0 & 0 & 0 & 0 & 0 & 0 & 0 & 0 & 0 & 0 & 0\end{array}$

$\begin{array}{lllllllllllllll}0 & 0 & 0 & 0 & 0 & 0 & 0 & 0 & 0 & 0 & 0 & 0 & 0 & 0\end{array}$

$\begin{array}{lllllllllllllllll}0 & 0 & 0 & 0 & 0 & 0 & 0 & 0 & 0 & 0 & 0 & 0 & 0 & 0 & 0\end{array}$

$\begin{array}{lllllllllllllll} & 0 & 0 & 0 & 0 & 0 & 0 & 0 & 0 & 0 & 0 & 0 & 0 & 0 & 0\end{array}$

$\begin{array}{llllllllllllllll}0 & 0 & 0 & 0 & 0 & 0 & 0 & 0 & 0 & 0 & 0 & 0 & 0 & 0 & 0\end{array}$

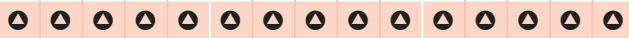

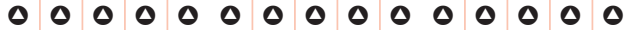

- 000000000000

$\begin{array}{lllllllllllllllll}0 & 0 & 0 & 0 & 0 & 0 & 0 & 0 & 0 & 0 & 0 & 0 & 0 & 0 & 0\end{array}$

- 000000000000

$\begin{array}{llllllllllllllll} & 0 & 0 & 0 & 0 & 0 & 0 & 0 & 0 & 0 & 0 & 0 & 0 & 0 & 0\end{array}$

$\begin{array}{lllllllllllllll} & 0 & 0 & 0 & 0 & 0 & 0 & 0 & 0 & 0 & 0 & 0 & 0 & 0 & 0\end{array}$

$\begin{array}{llllllllllllllll} & 0 & 0 & 0 & 0 & 0 & 0 & 0 & 0 & 0 & 0 & 0 & 0 & 0 & 0 & 0\end{array}$

$\begin{array}{lllllllllllllll} & 0 & 0 & 0 & 0 & 0 & 0 & 0 & 0 & 0 & 0 & 0 & 0 & 0 & 0\end{array}$

$\begin{array}{llllllllllllllll} & 0 & 0 & 0 & 0 & 0 & 0 & 0 & 0 & 0 & 0 & 0 & 0 & 0 & 0\end{array}$

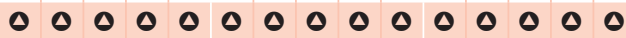

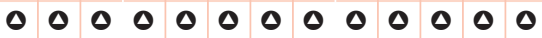

- 00000000

$\begin{array}{lllllllllllll}0 & 0 & 0 & 0 & 0 & 0 & 0 & 0 & 0 & 0 & 0 & 0\end{array}$

$\begin{array}{lllllllllllll}0 & 0 & 0 & 0 & 0 & 0 & 0 & 0 & 0 & 0 & 0\end{array}$

$\begin{array}{lllllllllllll} & \Delta & \Delta & 0 & 0 & 0 & 0 & 0 & 0 & 0 & 0 & 0\end{array}$

- 0000000

- 000000

- 000000

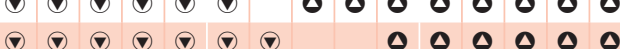

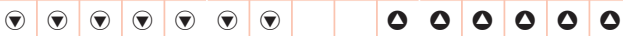

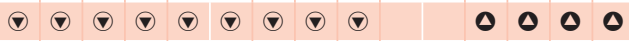

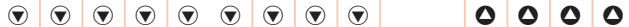

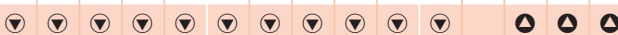

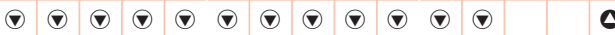

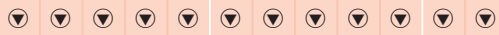

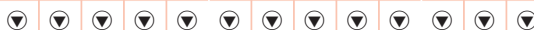

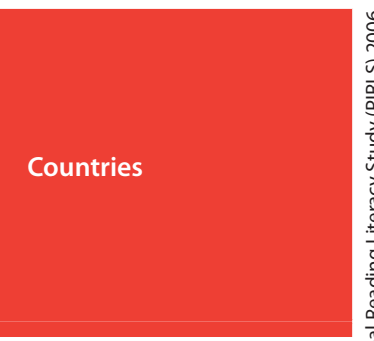

Hong Kong SAR

Russian Federation

Singapore

Luxembourg

Canada, Alberta

Canada, British Columbia

Canada, Ontario

Bulgaria

Italy

Sweden

Netherlands

Belgium (Flemish)

Germany

Denmark

Hungary

Latvia

Canada, Nova Scotia

Chinese Taipei

United States

England

Austria

New Zealand

Canada, Quebec

Lithuania

Scotland

Slovak Republic

France

Slovenia

Poland

Spain

Moldova, Rep. of

Israel

Iceland

Belgium (French)

Norway

Romania

Georgia

Macedonia, Rep. of

Trinidad and Tobago

Iran, Islamic Rep. of

Indonesia

Qatar

Morocco

Kuwait

South Africa
- Average achievement significantly higher than comparison country

๑ Average achievement significantly lower than comparison country 

Straightforward Inferencing Processes

Instructions: Read across the row for a country to compare performance with the countries listed along the top of the chart. The symbols indicate whether the average achievement of the country in the row is significantly lower than that of the comparison country, significantly higher than that of the comparison country, or if there is no statistically significant difference between the average achievement of the two countries.

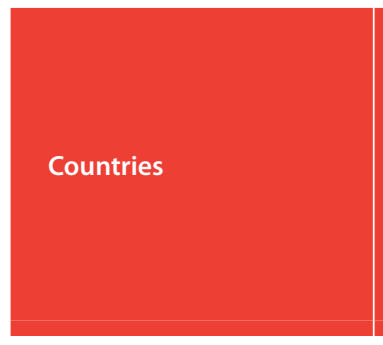

Luxembourg

Russian Federation

Singapore

Hong Kong SAR

Germany

Canada, Alberta

Netherlands

Denmark

Canada, British Columbia

Sweden

Belgium (Flemish)

Italy

Austria

Hungary

Canada, Ontario

Chinese Taipei

Bulgaria

Latvia

England

Canada, Nova Scotia

Canada, Quebec

United States

Lithuania

Slovak Republic

Scotland

New Zealand

France

Slovenia

Iceland

Poland

Spain

Israel

Norway

Belgium (French)

Romania

Moldova, Rep. of

Georgia

Macedonia, Rep. of

Trinidad and Tobago

Iran, Islamic Rep. of

Indonesia

Qatar

Kuwait

Morocco

South Africa
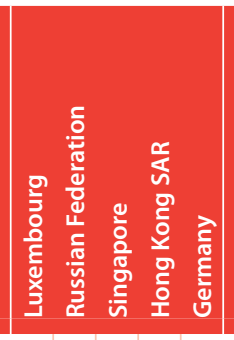

० ज

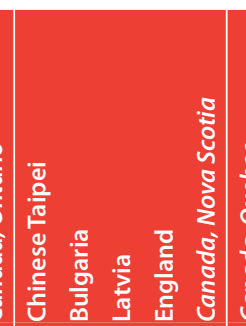

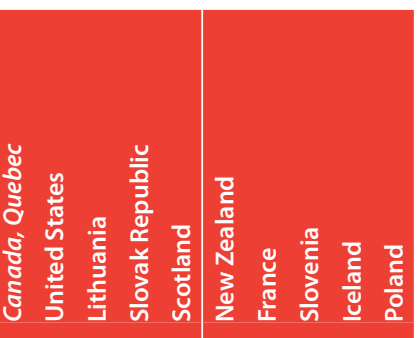

\begin{tabular}{lllllllllllllllllllllll|l|l|l}
0 & 0 & 0 & 0 & 0 & 0 & 0 & 0 & 0 & 0 & 0 & 0 & 0 & 0 & 0 & 0 & 0 & 0 & 0 & 0 & 0 & 0 & 0 & 0 & 0
\end{tabular} $\begin{array}{llllllllllllllllllllllllll}0 & 0 & 0 & 0 & 0 & 0 & 0 & 0 & 0 & 0 & 0 & 0 & 0 & 0 & 0 & 0 & 0 & 0 & 0 & 0 & 0 & 0 & 0 & 0 & 0\end{array}$ $\begin{array}{lllllllllllllllllllllllllll}0 & 0 & 0 & 0 & 0 & 0 & 0 & 0 & 0 & 0 & 0 & 0 & 0 & 0 & 0 & 0 & 0 & 0 & 0 & 0 & 0 & 0 & 0 & 0\end{array}$ $\begin{array}{lllllllllllllllllllll}0 & 0 & 0 & 0 & 0 & 0 & 0 & 0 & 0 & 0 & 0 & 0 & 0 & 0 & 0 & 0 & 0 & 0 & 0 & 0 & 0\end{array}$ \begin{tabular}{lllllllllllllllll|l|l|l}
0 & 0 & 0 & 0 & 0 & 0 & 0 & 0 & 0 & 0 & 0 & 0 & 0 & 0 & 0 & 0 & 0 & 0 & 0 & 0
\end{tabular}

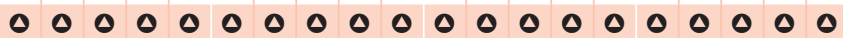
$\begin{array}{llllllllllllllllllllll}0 & 0 & 0 & 0 & 0 & 0 & 0 & 0 & 0 & 0 & 0 & 0 & 0 & 0 & 0 & 0 & 0 & 0 & 0 & 0\end{array}$ $\begin{array}{llllllllllllllll}0 & 0 & 0 & 0 & 0 & 0 & 0 & 0 & 0 & 0 & 0 & 0 & 0 & 0 & 0 & 0\end{array}$ $\begin{array}{llllllllllllllll}0 & 0 & 0 & 0 & 0 & 0 & 0 & 0 & 0 & 0 & 0 & 0 & 0 & 0 & 0\end{array}$ $\begin{array}{llllllllllllllll}0 & 0 & 0 & 0 & 0 & 0 & 0 & 0 & 0 & 0 & 0 & 0 & 0 & 0 & 0 & 0\end{array}$ $\begin{array}{llllllllllllll}0 & 0 & 0 & 0 & 0 & 0 & 0 & 0 & 0 & 0 & 0 & 0 & 0\end{array}$ $\begin{array}{llllllllllllll}0 & 0 & 0 & 0 & 0 & 0 & 0 & 0 & 0 & 0 & 0 & 0 & 0\end{array}$ $\begin{array}{llllllllllllll}0 & 0 & 0 & 0 & 0 & 0 & 0 & 0 & 0 & 0 & 0 & 0 & 0\end{array}$ $\begin{array}{llllllllllllll}0 & 0 & 0 & 0 & 0 & 0 & 0 & 0 & 0 & 0 & 0 & 0 & 0\end{array}$ $\begin{array}{llllllllllllll}0 & 0 & 0 & 0 & 0 & 0 & 0 & 0 & 0 & 0 & 0 & 0 & 0\end{array}$ $\begin{array}{llllllllllllll}0 & 0 & 0 & 0 & 0 & 0 & 0 & 0 & 0 & 0 & 0 & 0 & 0\end{array}$ $\begin{array}{llllllll}0 & 0 & 0 & 0 & 0 & 0\end{array}$ 000000 $\begin{array}{lllllll}0 & 0 & 0 & 0 & 0 & 0\end{array}$ $\begin{array}{lllllll}0 & 0 & 0 & 0 & 0 & 0\end{array}$ $\begin{array}{llllllll}0 & 0 & 0 & 0 & 0 & 0\end{array}$ 00000

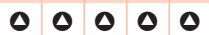
000 0 00 00

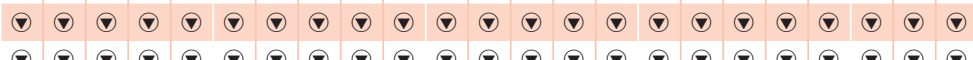

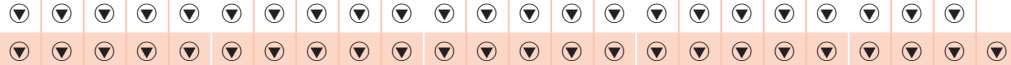

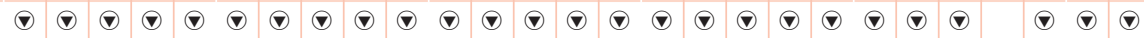

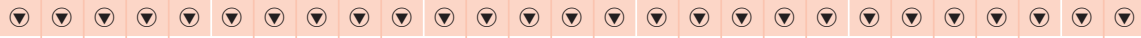

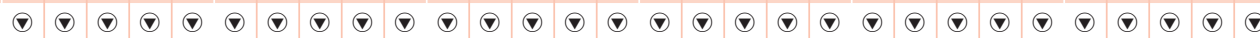

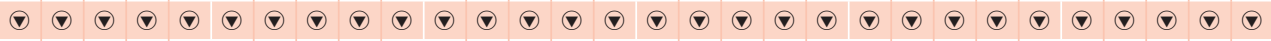

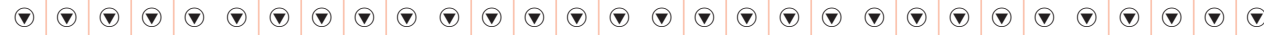

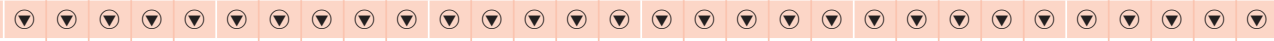

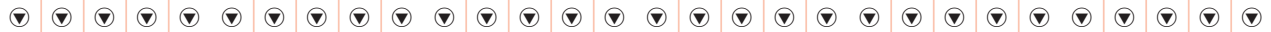

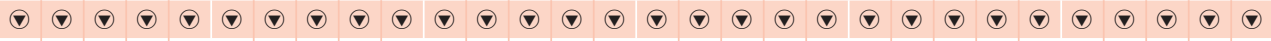

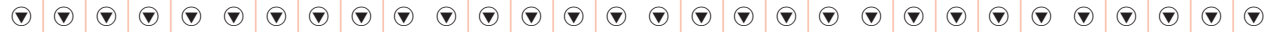

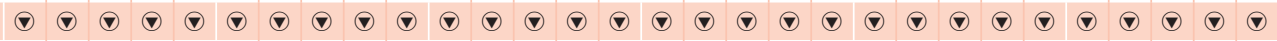

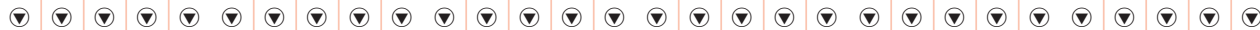

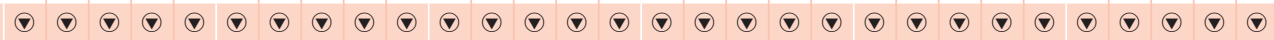

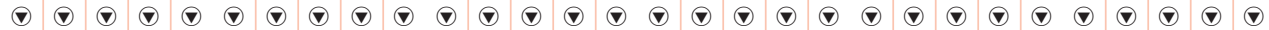

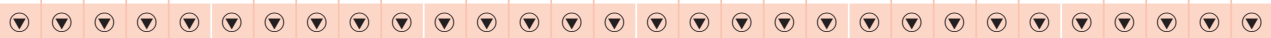

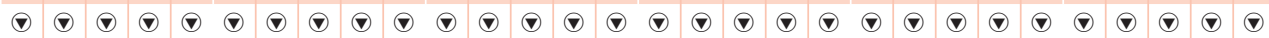

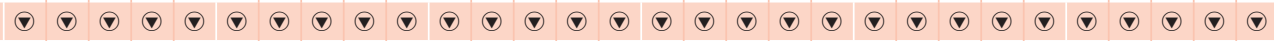

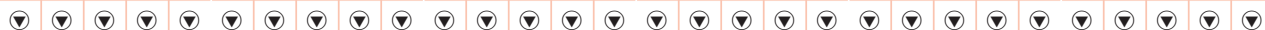




\section{Exhibit B.3 Multiple Comparisons of Average Reading Achievement for Retrieving and} Straightforward Inferencing Processes (Continued)

Instructions: Read across the row for a country to compare performance with the countries listed along the top of the chart. The symbols indicate whether the average achievement of the country in the row is significantly lower than that of the comparison country, significantly higher than that of the comparison country, or if there is no statistically significant difference between the average achievement of the two countries.

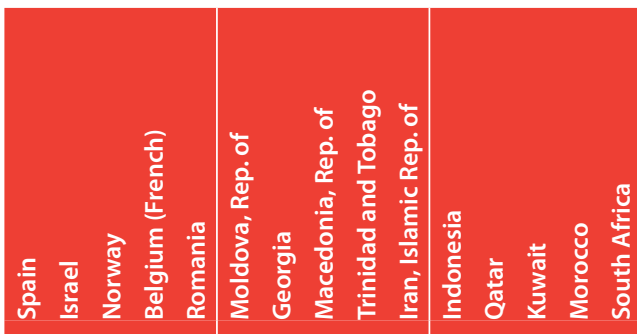

$\begin{array}{llllllllllllllll}0 & 0 & 0 & 0 & 0 & 0 & 0 & 0 & 0 & 0 & 0 & 0 & 0 & 0 & 0\end{array}$

$\begin{array}{llllllllllllllll}0 & 0 & 0 & 0 & 0 & 0 & 0 & 0 & 0 & 0 & 0 & 0 & 0 & 0 & 0\end{array}$

$\begin{array}{lllllllllllllllll}0 & 0 & 0 & 0 & 0 & 0 & 0 & 0 & 0 & 0 & 0 & 0 & 0 & 0 & 0\end{array}$

$\begin{array}{llllllllllllllll}0 & 0 & 0 & 0 & 0 & 0 & 0 & 0 & 0 & 0 & 0 & 0 & 0 & 0 & 0\end{array}$

$\begin{array}{llllllllllllllllllllll}0 & 0 & 0 & 0 & 0 & 0 & 0 & 0 & 0 & 0 & 0 & 0 & 0 & 0 & 0\end{array}$

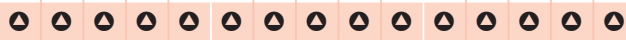

$\begin{array}{llllllllllllllll}0 & 0 & 0 & 0 & 0 & 0 & 0 & 0 & 0 & 0 & 0 & 0 & 0 & 0 & 0\end{array}$

$\begin{array}{lllllllllllllll}0 & 0 & 0 & 0 & 0 & 0 & 0 & 0 & 0 & 0 & 0 & 0 & 0 & 0 & 0\end{array}$

$\begin{array}{lllllllllllllllll}0 & 0 & 0 & 0 & 0 & 0 & 0 & 0 & 0 & 0 & 0 & 0 & 0 & 0 & 0\end{array}$

$\begin{array}{llllllllllllllll}0 & 0 & 0 & 0 & 0 & 0 & 0 & 0 & 0 & 0 & 0 & 0 & 0 & 0 & 0\end{array}$

$\begin{array}{llllllllllllllll}0 & 0 & 0 & 0 & 0 & 0 & 0 & 0 & 0 & 0 & 0 & 0 & 0 & 0 & 0\end{array}$

$\begin{array}{lllllllllllllll}0 & 0 & 0 & 0 & 0 & 0 & 0 & 0 & 0 & 0 & 0 & 0 & 0 & 0 & 0\end{array}$

$\begin{array}{llllllllllllllll}0 & 0 & 0 & 0 & 0 & 0 & 0 & 0 & 0 & 0 & 0 & 0 & 0 & 0 & 0\end{array}$

$\begin{array}{llllllllllllllll}0 & 0 & 0 & 0 & 0 & 0 & 0 & 0 & 0 & 0 & 0 & 0 & 0 & 0 & 0\end{array}$

$\begin{array}{llllllllllllllll}0 & 0 & 0 & 0 & 0 & 0 & 0 & 0 & 0 & 0 & 0 & 0 & 0 & 0 & 0\end{array}$

$\begin{array}{lllllllllllllll}0 & 0 & 0 & 0 & 0 & 0 & 0 & 0 & 0 & 0 & 0 & 0 & 0 & 0 & 0\end{array}$

$\begin{array}{lllllllllllllll}0 & 0 & 0 & 0 & 0 & 0 & 0 & 0 & 0 & 0 & 0 & 0 & 0 & 0 & 0\end{array}$

$\begin{array}{lllllllllllllll}0 & 0 & 0 & 0 & 0 & 0 & 0 & 0 & 0 & 0 & 0 & 0 & 0 & 0 & 0\end{array}$

$\begin{array}{llllllllllllllll}0 & 0 & 0 & 0 & 0 & 0 & 0 & 0 & 0 & 0 & 0 & 0 & 0 & 0 & 0\end{array}$

$\begin{array}{lllllllllllllll}0 & 0 & 0 & 0 & 0 & 0 & 0 & 0 & 0 & 0 & 0 & 0 & 0 & 0 & 0\end{array}$

$\begin{array}{llllllllllllllll}0 & 0 & 0 & 0 & 0 & 0 & 0 & 0 & 0 & 0 & 0 & 0 & 0 & 0 & 0\end{array}$

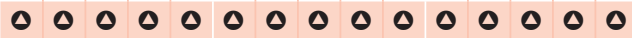

$\begin{array}{llllllllllllllll}0 & 0 & 0 & 0 & 0 & 0 & 0 & 0 & 0 & 0 & 0 & 0 & 0 & 0 & 0\end{array}$

$\begin{array}{lllllllllllllll}0 & 0 & 0 & 0 & 0 & 0 & 0 & 0 & 0 & 0 & 0 & 0 & 0 & 0 & 0\end{array}$

$\begin{array}{lllllllllllllll}0 & 0 & 0 & 0 & 0 & 0 & 0 & 0 & 0 & 0 & 0 & 0 & 0 & 0 & 0\end{array}$

$\begin{array}{lllllllllllllll}0 & 0 & 0 & 0 & 0 & 0 & 0 & 0 & 0 & 0 & 0 & 0 & 0 & 0 & 0\end{array}$

$\begin{array}{llllllllllllllll}0 & 0 & 0 & 0 & 0 & 0 & 0 & 0 & 0 & 0 & 0 & 0 & 0 & 0 & 0\end{array}$

$\begin{array}{llllllllllllllll}0 & 0 & 0 & 0 & 0 & 0 & 0 & 0 & 0 & 0 & 0 & 0 & 0 & 0 & 0\end{array}$

$\begin{array}{lllllllllllllll}0 & 0 & 0 & 0 & 0 & 0 & 0 & 0 & 0 & 0 & 0 & 0 & 0 & 0 & 0\end{array}$

$\begin{array}{lllllllllllllll}0 & 0 & 0 & 0 & 0 & 0 & 0 & 0 & 0 & 0 & 0 & 0 & 0 & 0 & 0\end{array}$

$\begin{array}{llllllllllll}0 & 0 & 0 & 0 & 0 & 0 & 0 & 0 & 0 & 0 & 0\end{array}$

$\begin{array}{lllllllllll}0 & 0 & 0 & 0 & 0 & 0 & 0 & 0 & 0 & 0 & 0\end{array}$

$\begin{array}{llllllllllll}0 & 0 & 0 & 0 & 0 & 0 & 0 & 0 & 0 & 0 & 0\end{array}$

$\begin{array}{llllllllllll}0 & 0 & 0 & 0 & 0 & 0 & 0 & 0 & 0 & 0 & 0\end{array}$

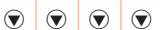

$\begin{array}{lllllllll}0 & 0 & 0 & 0 & 0 & 0 & 0 & 0\end{array}$

(1) (1) (1) 00000000

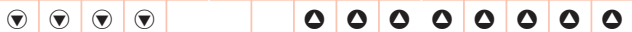

(-) (1) (1) (1) (1) 000000

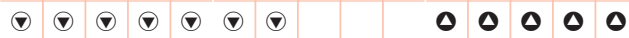

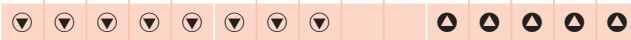

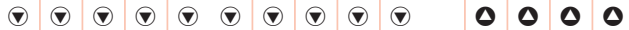

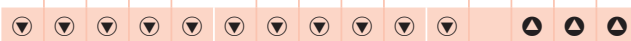

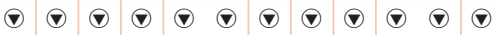

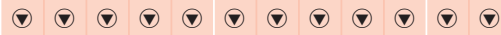

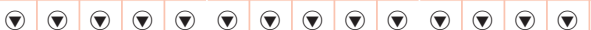

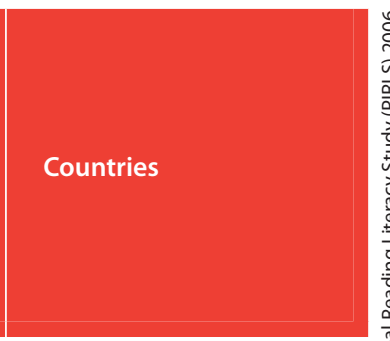

Luxembourg

Russian Federation

Singapore

Hong Kong SAR

Germany

Canada, Alberta

Netherlands

Denmark

Canada, British Columbia

Sweden

Belgium (Flemish)

Italy

Austria

Hungary

Canada, Ontario

Chinese Taipei

Bulgaria

Latvia

England

Canada, Nova Scotia

Canada, Quebec

United States

Lithuania

Slovak Republic

Scotland

New Zealand

France

Slovenia

Iceland

Poland

Spain

Israel

Norway

Belgium (French)

Romania

Moldova, Rep. of

Georgia

Macedonia, Rep. of

Trinidad and Tobago

Iran, Islamic Rep. of

Indonesia

Qatar

Kuwait

- Morocco

South Africa
- Average achievement significantly

higher than comparison country

$\checkmark$ Average achievement significantly

$\checkmark$ lower than comparison country 


\section{Exhibit B.4 Multiple Comparisons of Average Reading Achievement for Interpreting, Integrating,} and Evaluating Processes

Instructions: Read across the row for a country to compare performance with the countries listed along the top of the chart. The symbols indicate whether the average achievement of the country in the row is significantly lower than that of the comparison country, significantly higher than that of the comparison country, or if there is no statistically significant difference between the average achievement of the two countries.
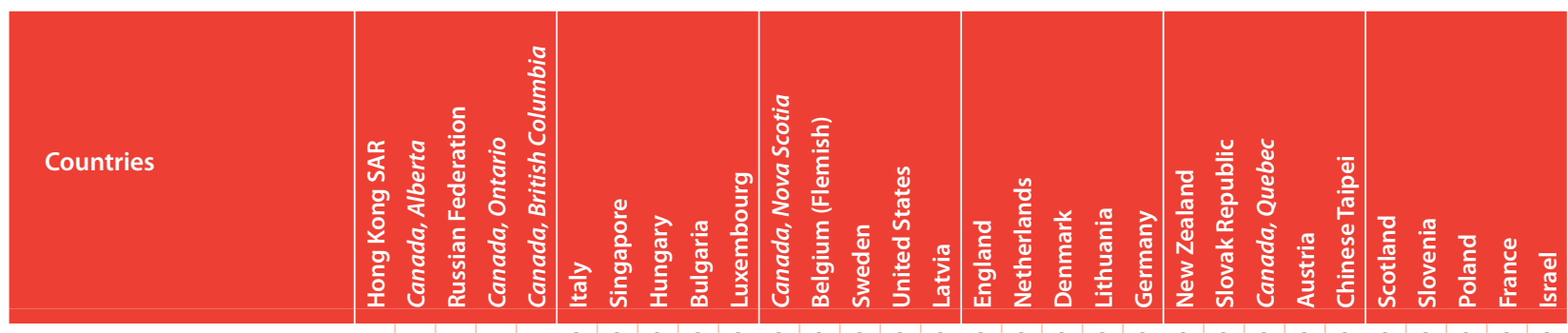

Hong Kong SAR

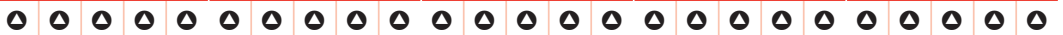

Canada, Alberta

$\begin{array}{llllllllllllllllllllllllll}0 & 0 & 0 & 0 & 0 & 0 & 0 & 0 & 0 & 0 & 0 & 0 & 0 & 0 & 0 & 0 & 0 & 0 & 0 & 0 & 0 & 0 & 0 & 0 & 0\end{array}$

Russian Federation

\begin{tabular}{llll|l|l|l|l|l|l|l|ll|l|l|l|l|l|l|l|l|l|l}
0 & 0 & 0 & 0 & 0 & 0 & 0 & 0 & 0 & 0 & 0 & 0 & 0 & 0 & 0 & 0 & 0 & 0 & 0 & 0 & 0 & 0 \\
\hline
\end{tabular}

Canada, Ontario

Canada, British Columbia

Italy

0

$\begin{array}{lllllllllllllllllllll}0 & 0 & 0 & 0 & 0 & 0 & 0 & 0 & 0 & 0 & 0 & 0 & 0 & 0 & 0 & 0 & 0 & 0 & 0 & 0 & 0\end{array}$

Singapore

$(\odot$

Hungary

(1) (1) (1) (1)

$\begin{array}{llllllllllllllllllllll}0 & 0 & 0 & 0 & 0 & 0 & 0 & 0 & 0 & 0 & 0 & 0 & 0 & 0 & 0 & 0 & 0 & 0 & 0 & 0 & 0\end{array}$

Bulgaria

$(\odot$

$\begin{array}{lllllllllllllllllllll}0 & 0 & 0 & 0 & 0 & 0 & 0 & 0 & 0 & 0 & 0 & 0 & 0 & 0 & 0 & 0 & 0 & 0 & 0 & 0 & 0\end{array}$

Luxembourg

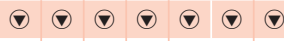

0

$\begin{array}{llllllllllllllll}0 & 0 & 0 & 0 & 0 & 0 & 0 & 0 & 0 & 0 & 0 & 0 & 0 & 0 & 0 & 0\end{array}$

Canada, Nova Scotia $\quad(\bullet)(\bullet)(\bullet)$

Belgium (Flemish) $\quad(\uparrow \uparrow \uparrow \uparrow \uparrow \uparrow$

Sweden

$(\bullet \odot \odot \odot \odot \odot \odot$

United States

(1) (1) (1) (1) (1)

Latvia

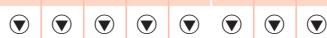

England

(1) (1) (1) (1) (1) (1) (1)

$\begin{array}{lllllllllllllll}0 & 0 & 0 & 0 & 0 & 0 & 0 & 0 & 0 & 0 & 0 & 0 & 0 & 0 & 0\end{array}$

Netherlands

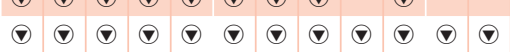

$\begin{array}{llllllllllllllll}0 & 0 & 0 & 0 & 0 & 0 & 0 & 0 & 0 & 0 & 0 & 0 & 0 & 0 & 0\end{array}$

Denmark

(1) (1) (1) (1) (1) (1) (1) (1) (1)

$\begin{array}{lllllllllllllll}0 & 0 & 0 & 0 & 0 & 0 & 0 & 0 & 0 & 0 & 0 & 0 & 0\end{array}$

Lithuania

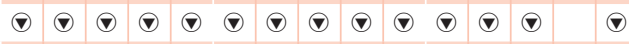

0

$\begin{array}{lllllllllllll}0 & 0 & 0 & 0 & 0 & 0 & 0 & 0 & 0 & 0 & 0 & 0\end{array}$ \begin{tabular}{lllllllll|l|l|l|l|l}
0 & 0 & 0 & 0 & 0 & 0 & 0 & 0 & 0 & 0 & 0 & 0
\end{tabular} $\begin{array}{lllllllllll}0 & 0 & 0 & 0 & 0 & 0 & 0 & 0 & 0 & 0\end{array}$

Germany

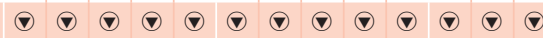

New Zealand

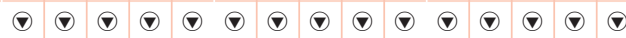

○

$\begin{array}{lllllllllll}0 & 0 & 0 & 0 & 0 & 0 & 0 & 0 & 0 & 0\end{array}$

Slovak Republic

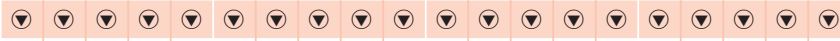

$\begin{array}{lllllllll}0 & 0 & 0 & 0 & 0 & 0 & 0 & 0\end{array}$

Canada, Quebec

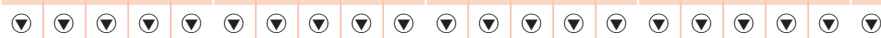

$\begin{array}{llllllllll}0 & 0 & 0 & 0 & 0 & 0 & 0 & 0 & 0\end{array}$

Austria

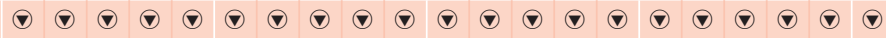

$\begin{array}{llllllllll}0 & 0 & 0 & 0 & 0 & 0 & 0 & 0\end{array}$

$\begin{array}{lllllllllll}0 & 0 & 0 & 0 & 0 & 0 & 0 & 0 & 0\end{array}$

$\begin{array}{llllllllll}0 & 0 & 0 & 0 & 0 & 0 & 0 & 0 & 0\end{array}$

$\begin{array}{lllllllll}0 & 0 & 0 & 0 & 0 & 0 & 0 & 0\end{array}$

Chinese Taipei

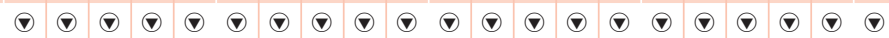

0000

Scotland

Slovenia

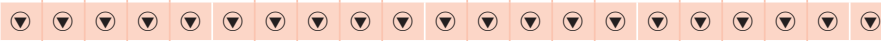

000

Poland

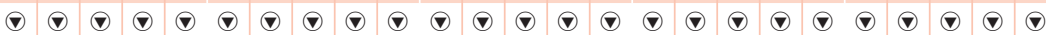

France

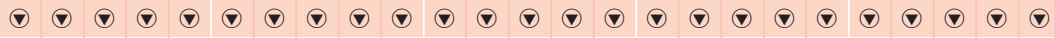

Israel

Moldova, Rep. of

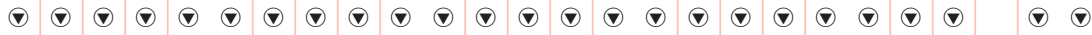

Spain

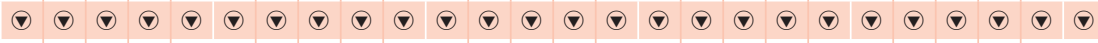

Iceland

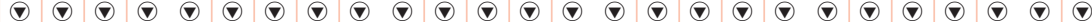

Belgium (French)

Norway

Romania

Georgia

Macedonia, Rep. of

Trinidad and Tobago

Iran, Islamic Rep. of

Indonesia

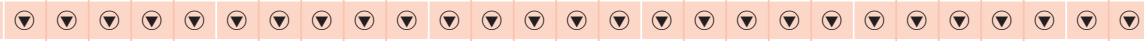

Qatar

Kuwait

South Africa

Morocco

- 00

000

- 0

Note: $5 \%$ of these comparisons would be statistically significant by chance alone.

A plus (+) sign indicates average achievement could not be accurately estimated on the

interpreting, integrating, and evaluating scale. 


\section{Exhibit B.4 Multiple Comparisons of Average Reading Achievement for Interpreting, Integrating, and Evaluating Processes (Continued)

Instructions: Read across the row for a country to compare performance with the countries listed along the top of the chart. The symbols indicate whether the average achievement of the country in the row is significantly lower than that of the comparison country, significantly higher than that of the comparison country, or if there is no statistically significant difference between the average achievement of the two countries.

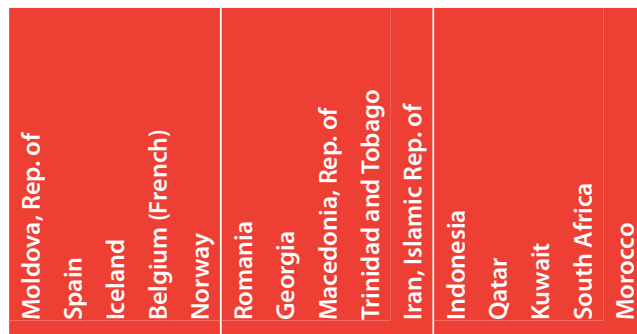

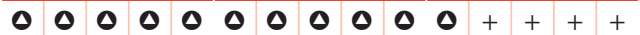

$\begin{array}{lllllllllllll}0 & 0 & 0 & 0 & 0 & 0 & 0 & 0 & 0 & 0 & 0\end{array}$

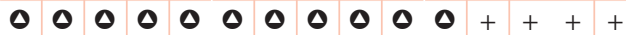

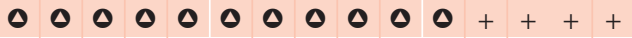

$\begin{array}{lllllllllllllll}0 & 0 & 0 & 0 & 0 & 0 & 0 & 0 & 0 & 0 & 0 & + & +++\end{array}$

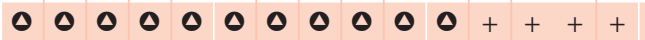

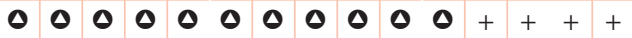

$\begin{array}{llllllllllll}0 & 0 & 0 & 0 & 0 & 0 & 0 & 0 & 0 & 0 & 0\end{array}$

$\begin{array}{llllllllllll}0 & 0 & 0 & 0 & 0 & 0 & 0 & 0 & 0 & 0 & 0\end{array}$

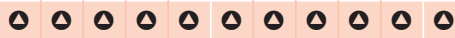

$\begin{array}{llllllllllllllllll}0 & 0 & 0 & 0 & 0 & 0 & 0 & 0 & 0 & 0 & 0\end{array}$

- 00000

$\begin{array}{lllllllllll}0 & 0 & 0 & 0 & 0 & 0 & 0 & 0 & 0 & 0 & 0\end{array}$

$\begin{array}{llllllllllll}0 & 0 & 0 & 0 & 0 & 0 & 0 & 0 & 0 & 0 & 0\end{array}$

$\begin{array}{llllllllllllllllll}0 & 0 & 0 & 0 & 0 & 0 & 0 & 0 & 0 & 0 & 0\end{array}$

$\begin{array}{llllllllllll}0 & 0 & 0 & 0 & 0 & 0 & 0 & 0 & 0 & 0 & 0\end{array}$

$\begin{array}{lllllllllll}0 & 0 & 0 & 0 & 0 & 0 & 0 & 0 & 0 & 0 & 0\end{array}$

$0 \quad 0 \quad 0 \quad 0 \quad 0 \quad 0 \quad 0 \quad 0 \quad 0 \quad 0 \quad 0$

$\begin{array}{lllllllllllllllllll}0 & 0 & 0 & 0 & 0 & 0 & 0 & 0 & 0 & 0 & 0\end{array}$

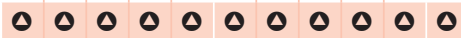

$\begin{array}{llllllllllll}0 & 0 & 0 & 0 & 0 & 0 & 0 & 0 & 0 & 0 & 0\end{array}$

00000000000

$\begin{array}{llllllllllll}0 & 0 & 0 & 0 & 0 & 0 & 0 & 0 & 0 & 0 & 0\end{array}$

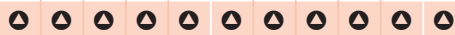

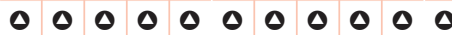

- 0000000

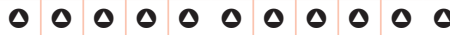

$\begin{array}{llllllllll}0 & 0 & 0 & 0 & 0 & 0 & 0 & 0 & 0\end{array}$

$\begin{array}{lllllllll}0 & 0 & 0 & 0 & 0 & 0 & 0 & 0 & 0\end{array}$

- 0000000

$\begin{array}{lllllllll}0 & 0 & 0 & 0 & 0 & 0 & 0 & 0 & 0\end{array}$

$\begin{array}{llllllllll}0 & 0 & 0 & 0 & 0 & 0 & 0 & 0 & 0\end{array}$

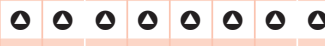

++++++++++++++ Morocco

Hong Kong SAR

Canada, Alberta

Russian Federation

Canada, Ontario

Canada, British Columbia

Italy

Singapore

Hungary

Bulgaria

Luxembourg

Canada, Nova Scotia

Belgium (Flemish)

Sweden

United States

Latvia

England

Netherlands

Denmark

Lithuania

Germany

New Zealand

Slovak Republic

Canada, Quebec

Austria

Chinese Taipei

Scotland

Slovenia

Poland

France

Israel

Moldova, Rep. of

Spain

Iceland

Belgium (French)

Norway

Romania

Georgia

Macedonia, Rep. of

Trinidad and Tobago

Iran, Islamic Rep. of

Indonesia

Qatar

Kuwait
- Average achievement significantly higher than comparison country

Average achievement significantly lower than comparison country 

Appendix C

Percentiles and Standard Deviations of Reading Achievement 
Exhibit C.1 Percentiles of Achievement in Reading

PIRLS 2006

4th Grade

5th percentile 25 th percentile 50 th percentile 75 th percentile 95 th percentile

Countries

$(327(3.5)$

Austria

Belgium (Flemish)

Belgium (French)

Canada, Alberta

Canada, British Columbia

Canada, Nova Scotia

Canada, Ontario

Canada, Quebec

Denmark

England

France

Georgia

Germany

Hong Kong SAR

Hungary

Iceland

Indonesia

Iran, Islamic Rep. of

Israe

Italy

Kuwait

Latvia

Lithuania

Luxembourg

Macedonia, Rep. of

Moldova, Rep. of

Morocco

Netherlands

New Zealand

Norway

Poland

Qatar

Romania

Russian Federation

Scotland

Singapore

Slovak Republic

Slovenia

South Africa

Spain

Sweden

Trinidad and Tobag

United States

$427(3.5)$

$451(3.7)$

$381(2.7)$

$397(10.0)$

$446(5.8)$

$439(5.5)$

$407(8.1)$

$433(4.7)$

$422(6.9)$

$420(3.9)$

$418(4.5)$

$383(8.0)$

$406(2.5)$

342 (5.1)

$430(4.9)$

$460(4.7)$

$427(6.4)$

$388(2.6)$

$271(7.5)$

$258(5.3)$

325 (11.0)

$435(5.3)$

$148(10.1)$

$433(9.2)$

440 (5.1)

$442(2.7)$

$272(8.4)$

$378(4.1)$

$144(9.6)$

457 (3.3)

$374(3.0)$

$378(3.8)$

$386(5.8)$

$198(2.9)$

317 (12.7)

$443(9.5)$

385 (5.5)

$420(5.8)$

$394(6.3)$

395 (3.7)

108 (3.4)

$390(4.1)$

437 (3.6)

$409(7.6)$

498 (4.1)

$512(1.8)$

455 (3.2)

498 (6.5)

$516(4.2)$

$513(4.8)$

495 (3.7)

$510(4.0)$

493 (3.4)

$497(3.0)$

505 (3.6)

$486(4.6)$

$478(2.4)$

$420(5.5)$

$508(3.0)$

$527(2.9)$

$507(5.0)$

$469(2.0)$

351 (4.6)

357 (4.7)

$453(5.6)$

507 (3.0)

251 (6.4)

501 (4.9)

$500(2.0)$

$514(1.8)$

$369(4.6)$

$457(4.2)$

$244(7.9)$

$513(1.8)$

$478(2.5)$

$457(5.5)$

470 (3.9)

284 (1.4)

$436(8.5)$

$523(4.4)$

480 (4.9)

$512(4.9)$

$488(4.0)$

$476(2.2)$

203 (3.6)

468 (3.4)

$512(3.4)$

$364(5.3)$

494 (3.5)

\begin{abstract}
$409(7.6)$
\end{abstract}

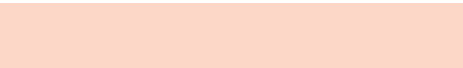
542

$542(2.6)$

$582(2.2) \quad 636(5.3)$

\begin{tabular}{l|l}
$585(1.9)$ & $636(4.6)$
\end{tabular}

$503(3.4)$

$553(4.6)$

547

$562(2.6)$

$561(2.8)$

$547(2.4)$

$557(3.5)$

$536(3.7)$

$540(1.7)$

$553(2.7)$

546 (2.9)

$546(2.9)$

$525(2.1)$
$475(3.4)$

$553(3.1)$

$567(1.6)$

$567(1.6)$
$555(3.1)$

$516(1.2)$

408 (4.1)

427 (3.6)

$427(3.6)$
$527(2.4)$

$554(3.3)$

331 (4.7)

$543(2.7)$

$543(2.7)$

$560(1.9)$

$448(6.0)$

$505(4.4)$

$321(8.3)$

$549(1.9)$

$549(1.9)$

$539(2.2)$

$503(3.2)$

$353(1.6)$

$353(1.6)$

$569(4.1)$

$532(4.1)$

$565(4.0)$

604

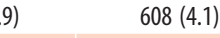

\begin{tabular}{l|l|}
\hline $604(3.4)$ & $673(6.0)$
\end{tabular}

$607(2.6) \quad 668(2.6)$

\begin{tabular}{l|l|}
\hline $606(2.8)$ & $668(4.1)$ \\
\hline
\end{tabular}

$594(2.8) \quad 658(3.8)$

\begin{tabular}{l|l}
$603(4.3)$ & $666(4.7)$ \\
\hline
\end{tabular}

$577(3.8) \quad 632(4.0)$

\begin{tabular}{l|l}
$579(2.0)$ & $633(4.7)$ \\
\hline
\end{tabular}

$594(1.8) \quad 649(2.9)$

\begin{tabular}{l|l}
$598(2.3)$ & $673(5.1)$ \\
\hline
\end{tabular}

$568(2.1) \quad 626(4.7)$

\begin{tabular}{|l|l|}
\hline $525(3.7)$ & $588(5.1)$ \\
\hline
\end{tabular}

\begin{tabular}{ll}
$593(2.3)$ & $588(5.1)$ \\
\hline $605(1.8)$ & $647(2.4)$
\end{tabular}

$605(1.8) \quad 647(2.4)$

\begin{tabular}{l|l}
$605(1.8)$ & $655(2.9)$ \\
\hline
\end{tabular}

$599(3.5) \quad 658(2.3)$

\begin{tabular}{l|l}
$558(2.4)$ & $615(2.3)$ \\
\hline
\end{tabular}

$460(4.6) \quad 529(3.2)$

\begin{tabular}{|l|l|}
\hline $489(4.6)$ & $567(2.5)$ \\
\hline
\end{tabular}

$\begin{array}{ll}489(4.6) & 567(2.5) \\ 582(2.8) & 653(5.2)\end{array}$

\begin{tabular}{l|l}
$582(2.8)$ & $653(5.2)$ \\
$599(4.3)$ & $658(3.3)$ \\
\hline
\end{tabular}

$411(4.4) \quad 510(2.5)$

\begin{tabular}{l|l}
$585(3.1)$ & $639(3.3)$ \\
\hline
\end{tabular}

$\begin{array}{ll}585(3.1) & 639(3.3) \\ 577(2.2) & 627(4.5)\end{array}$

\begin{tabular}{l|l}
$577(2.2)$ & $627(4.5)$ \\
$603(1.2)$ & $662(2.4)$ \\
\hline
\end{tabular}

$518(4.5)$

\begin{tabular}{|c|c|}
\hline$(4.5$ & $599(7.5)$ \\
\hline $547(2.2$ & $606(3.8)$ \\
\hline
\end{tabular}

\begin{tabular}{r|r}
$547(2.2)$ & $606(3.8)$ \\
\hline
\end{tabular}

$402(9.1) \quad 503(7.8)$

\begin{tabular}{l|l}
$584(1.8)$ & $631(2.1)$ \\
\hline
\end{tabular}

\begin{tabular}{l|l|}
\hline $592(2.1)$ & $664(4.0)$ \\
\hline $544(2.4)$ & $598(3.7)$
\end{tabular}

\begin{tabular}{l|l}
$544(2.4)$ & $598(3.7)$ \\
\hline $572(1.7)$ & $635(3.0)$ \\
\hline
\end{tabular}

$\begin{array}{ll}572(1.7) & 635(3.0) \\ 424(2.2) & 509(4.0)\end{array}$

\begin{tabular}{l|l}
$424(2.2)$ & $509(4.0)$ \\
\hline
\end{tabular}

$554(3.0) \quad 621(3.5)$

\begin{tabular}{l|l|}
$612(2.9)$ & $671(2.7)$ \\
\hline $581(3.8)$ & $651(8.4)$ \\
\hline
\end{tabular}

\begin{tabular}{l|l}
$612(2.8)$ & $651(8.4)$ \\
\hline $682(2.6)$ & $672(3.2)$ \\
\hline
\end{tabular}

\begin{tabular}{|l|l|l|}
\hline $565(4.0)$ & $612(2.8)$ & $672(3.2)$ \\
\hline $539(4.4)$ & $582(2.6)$ & $639(2.9)$ \\
\hline $527(1.9)$ & $571(1.7)$ & $629(2.6)$ \\
\hline
\end{tabular}

\begin{tabular}{|l|l|l|}
\hline $527(1.9)$ & $571(1.7)$ & $629(2.6)$ \\
\hline $283(4.8)$ & $384(9.2)$ & $562(13.2)$ \\
\hline $517(2.3)$ & $561(1.7)$ & $622(3.8)$ \\
\hline
\end{tabular}

\begin{tabular}{|l|l|l|}
\hline $517(2.3)$ & $561(1.7)$ & $622(3.8)$ \\
\hline $554(2.2)$ & $592(2.3)$ & $647(5.5)$ \\
\hline $443(6.0)$ & $510(4.1)$ & $595(6.6)$ \\
\hline
\end{tabular}

\begin{tabular}{|l|l|l|}
$554(2.2)$ & $592(2.3)$ & $647(5.5)$ \\
\hline $443(6.0)$ & $510(4.1)$ & $595(6.6)$ \\
\hline
\end{tabular}

\begin{tabular}{|l|l|l|}
\hline $443(6.0)$ & $510(4.1)$ & $595(6.6)$ \\
\hline $545(4.2)$ & $592(3.8)$ & $653(7.3)$
\end{tabular}

( ) Standard errors appear in parentheses. Because results are rounded to the nearest whole number, some totals may appear inconsistent. 
Exhibit C.2 Standard Deviations of Achievement in Reading

PIRLS 2006

4th Grade

\begin{tabular}{|c|c|c|c|c|c|c|}
\hline \multirow{2}{*}{ Countries } & \multicolumn{2}{|c|}{ Overall } & \multicolumn{2}{|c|}{ Girls } & \multicolumn{2}{|c|}{ Boys } \\
\hline & Mean & $\begin{array}{l}\text { Standard } \\
\text { Deviation }\end{array}$ & Mean & $\begin{array}{l}\text { Standard } \\
\text { Deviation }\end{array}$ & Mean & $\begin{array}{l}\text { Standard } \\
\text { Deviation }\end{array}$ \\
\hline Austria & $538(2.2)$ & $64(1.4)$ & $543(2.3)$ & $62(1.7)$ & $533(2.6)$ & $65(1.7)$ \\
\hline Belgium (Flemish) & $547(2.0)$ & $56(1.0)$ & $550(2.3)$ & $54(1.2)$ & $544(2.4)$ & $57(1.2)$ \\
\hline Belgium (French) & $500(2.6)$ & $69(1.3)$ & $502(2.8)$ & $67(1.5)$ & $497(2.9)$ & $70(1.6)$ \\
\hline Bulgaria & $547(4.4)$ & $83(2.4)$ & $558(4.4)$ & $80(2.7)$ & $537(5.0)$ & $84(3.1)$ \\
\hline Canada, Alberta & $560(2.4)$ & $68(1.2)$ & $564(2.4)$ & $67(1.6)$ & $556(2.7)$ & $67(1.3)$ \\
\hline Canada, British Columbia & $558(2.6)$ & $69(1.3)$ & $562(2.9)$ & $68(1.7)$ & $554(3.1)$ & 70 (1.6) \\
\hline Canada, Nova Scotia & $542(2.2)$ & $76(1.5)$ & $553(2.5)$ & $73(2.0)$ & $531(2.8)$ & $78(1.9)$ \\
\hline Canada, Ontario & $555(2.7)$ & $71(1.3)$ & $562(3.3)$ & $68(1.4)$ & $549(3.3)$ & $72(2.1)$ \\
\hline Canada, Quebec & $533(2.8)$ & $63(1.1)$ & $539(2.7)$ & $61(1.6)$ & $527(3.5)$ & $65(1.6)$ \\
\hline Chinese Taipei & $535(2.0)$ & $64(1.0)$ & $542(2.2)$ & $62(1.3)$ & $529(2.3)$ & $66(1.3)$ \\
\hline Denmark & $546(2.3)$ & $70(1.2)$ & $553(2.8)$ & $69(1.9)$ & $539(2.7)$ & $69(1.7)$ \\
\hline England & $539(2.6)$ & $87(1.6)$ & $549(3.0)$ & $87(2.1)$ & $530(2.8)$ & $86(1.8)$ \\
\hline France & $522(2.1)$ & $67(1.0)$ & $527(2.4)$ & $65(1.3)$ & $516(2.4)$ & $67(1.2)$ \\
\hline Georgia & $471(3.1)$ & $75(1.5)$ & $480(3.3)$ & $73(1.6)$ & $463(3.8)$ & $76(1.9)$ \\
\hline Germany & $548(2.2)$ & $67(1.2)$ & $551(2.5)$ & $66(1.4)$ & $544(2.5)$ & $68(1.8)$ \\
\hline Hong Kong SAR & $564(2.4)$ & $59(1.2)$ & $569(2.5)$ & $56(1.4)$ & $559(2.8)$ & $61(1.4)$ \\
\hline Hungary & $551(3.0)$ & $70(1.8)$ & $554(3.6)$ & $70(2.3)$ & $548(2.9)$ & $70(1.9)$ \\
\hline Iceland & $511(1.3)$ & $68(1.0)$ & $520(1.7)$ & $65(1.5)$ & $501(1.9)$ & $70(1.3)$ \\
\hline Indonesia & $405(4.1)$ & $79(2.1)$ & $415(4.2)$ & $76(2.1)$ & $395(4.6)$ & $80(2.6)$ \\
\hline Iran, Islamic Rep. of & $421(3.1)$ & $95(1.8)$ & $429(5.3)$ & $94(2.8)$ & $414(3.8)$ & $95(2.5)$ \\
\hline Israel & $512(3.3)$ & $99(2.6)$ & $520(4.1)$ & $96(3.0)$ & $506(3.7)$ & $101(2.8)$ \\
\hline Italy & $551(2.9)$ & $68(1.4)$ & $555(3.3)$ & $67(1.7)$ & $548(3.3)$ & $68(2.0)$ \\
\hline Kuwait & $330(4.2)$ & $\mathrm{h}(2.1)$ & $364(4.7)$ & $101(2.7)$ & $297(6.2)$ & $109(2.8)$ \\
\hline Latvia & $541(2.3)$ & $63(1.2)$ & $553(2.7)$ & $60(1.3)$ & $530(2.6)$ & $63(1.8)$ \\
\hline Lithuania & $537(1.6)$ & $57(1.1)$ & $546(2.0)$ & $56(1.3)$ & $528(2.0)$ & $56(1.4)$ \\
\hline Luxembourg & $557(1.1)$ & $66(0.7)$ & $559(1.3)$ & $66(0.9)$ & $556(1.6)$ & $67(1.0)$ \\
\hline Macedonia, Rep. of & $442(4.1)$ & $101(2.3)$ & $453(4.4)$ & $101(2.7)$ & $432(4.4)$ & $100(2.6)$ \\
\hline Moldova, Rep. of & $500(3.0)$ & $69(1.4)$ & $507(3.1)$ & $67(1.7)$ & $493(3.5)$ & $70(2.1)$ \\
\hline Morocco & $323(5.9)$ & $109(2.8)$ & $332(6.6)$ & $106(3.0)$ & $314(6.6)$ & h (3.8) \\
\hline Netherlands & $547(1.5)$ & $53(0.9)$ & $551(2.0)$ & $53(1.2)$ & $543(1.6)$ & $53(1.3)$ \\
\hline New Zealand & $532(2.0)$ & $87(1.3)$ & $544(2.2)$ & $81(1.5)$ & $520(2.9)$ & $90(1.8)$ \\
\hline Norway & $498(2.6)$ & $67(1.4)$ & $508(2.8)$ & $64(1.7)$ & $489(3.1)$ & $67(1.8)$ \\
\hline Poland & $519(2.4)$ & $75(1.3)$ & $528(2.6)$ & $74(1.6)$ & $511(2.7)$ & $76(1.6)$ \\
\hline Qatar & $353(1.1)$ & $96(0.9)$ & $372(1.7)$ & $90(1.1)$ & $335(1.7)$ & $97(1.3)$ \\
\hline Romania & $489(5.0)$ & $91(3.0)$ & $497(5.0)$ & $91(3.5)$ & $483(5.7)$ & $92(3.4)$ \\
\hline Russian Federation & $565(3.4)$ & $69(2.0)$ & $572(3.9)$ & $67(2.1)$ & $557(3.4)$ & $69(2.3)$ \\
\hline Scotland & $527(2.8)$ & $80(1.6)$ & $538(3.6)$ & $78(1.8)$ & $516(3.1)$ & $80(2.2)$ \\
\hline Singapore & $558(2.9)$ & $77(1.6)$ & $567(3.1)$ & $73(1.7)$ & $550(3.3)$ & $79(1.9)$ \\
\hline Slovak Republic & $531(2.8)$ & $74(2.1)$ & $537(2.7)$ & $73(2.1)$ & $525(3.3)$ & $75(2.7)$ \\
\hline Slovenia & $522(2.1)$ & $71(0.9)$ & $532(2.1)$ & $67(1.3)$ & $512(2.7)$ & $73(1.2)$ \\
\hline South Africa & $302(5.6)$ & $136(3.6)$ & $319(6.3)$ & $136(4.3)$ & $283(5.5)$ & $134(3.6)$ \\
\hline Spain & $513(2.5)$ & $71(1.6)$ & $515(2.6)$ & $71(1.9)$ & $511(3.1)$ & $71(2.3)$ \\
\hline Sweden & $549(2.3)$ & $64(1.3)$ & $559(2.6)$ & $62(1.5)$ & $541(2.6)$ & $64(1.7)$ \\
\hline Trinidad and Tobago & $436(4.9)$ & $103(2.6)$ & $451(4.9)$ & $98(3.2)$ & $420(6.0)$ & $106(2.9)$ \\
\hline United States & $540(3.5)$ & $74(1.3)$ & $545(3.3)$ & $71(1.5)$ & $535(4.4)$ & $77(1.7)$ \\
\hline
\end{tabular}

() Standard errors appear in parentheses. Because results are rounded to the nearest whole number, some totals may appear inconsistent. 
Exhibit C.3 Percentiles of Achievement in Literary Reading

PIRLS 2006

4th Grade

5th percentile 25 th percentile 50 th percentile 75 th percentile 95 th percentile

Countries

(2)

Austria

$427(4.1)$

Belgium (Flemish)

$445(4.0)$

Belgium (French)

$383(6.8)$

Bulgaria

$391(7.7)$

Canada, Alberta

$439(4.1)$

Canada, British Columbia

$436(3.0)$

Canada, Nova Scotia

$406(5.9)$

Canada, Ontario

$428(6.6)$

Canada, Quebec

$420(4.4)$

Chinese Taipe

$409(3.2)$

Denmark

England

$422(6.0)$

France

$382(5.5)$

Georgia

$402(4.0)$

Germany

$347(5.6)$

Hong Kong SAR

$431(5.4)$

Hungary

Iceland

$446(3.2)$

$435(4.2)$

Indonesia

$397(2.6)$

Iran, Islamic Rep. of

$265(10.3)$

Israe

$262(8.1)$

Italy

Kuwait

$34(13.5)$

$425(9.3)$

Latvia

$165(7.5)$

Lithuania

$430(8.2)$

Luxembourg

Macedonia, Rep. of

$441(4.1)$

$437(3.3)$

Moldova, Rep. of

$275(8.6)$

Morocco

$371(6.1)$

Netherlands

$28(10.8)$

New Zealand

$448(3.5)$

Norway

$373(3.5)$

Poland

$382(4.3)$

Qatar

$87(4.7)$

Romania

$201(5.7)$

Russian Federation

$323(10.4)$

$440(6.3)$

Scotland

$381(5.1)$

Singapore

Slovak Republic

Slovenia

South Africa

$408(5.8)$

$398(5.9)$

Spain

Sweden

$396(2.7)$

$107(3.7)$

$385(4.0)$

Trinidad and Tobag

United States

$437(4.4)$

$257(9.6)$

$403(6.6)$

$498(3.4)$

$508(2.2)$

$455(2.8)$

$492(4.6)$

$516(3.8)$

$512(3.7)$

$495(3.4)$

508 (3.4)

$541(2.9)$

$488(4.9)$

$488(3.1)$

$507(3.2)$

$483(2.3)$

546

$546(1.9)$

580

$580(2.8) \quad 633(3.5)$

\begin{tabular}{l|l}
$583(3.6)$ & $635(3.6)$
\end{tabular}

$548(4.3)$

$546(3.2) \quad 606(3.4)$

\begin{tabular}{l|l}
$599(5.4)$ & $670(4.8)$
\end{tabular}

$564(2.2)$

$562(3.1)$

609

\begin{tabular}{l|l|}
$609(2.7)$ & $674(4.5)$ \\
$608(3.9)$ & $674(4.3)$
\end{tabular}

547 (2.4)

5

$596(2.3)$

$662(3.6)$

$557(2.9)$

605 (3.4)

533 (3.4)

$533(3.4)$

574

$574(3.6)$
$577(2.0)$

\section{$594(1.6)$}

$554(2.0)$

\section{$474(3.1)$}

$544(3.7)$

$600(2.6)$

519 (2.4)

$510(1.6)$

479 (4.2)

$562(2.6)$

$530(4.2)$

$594(3.5)$

$555(3.0)$

\section{$517(4.3)$}

$560(2.5)$

$474(2.9)$

$560(2.7)$

594(

601

$\begin{array}{ll}604(2.5) & 657(3.4) \\ 660(3.6)\end{array}$

\begin{tabular}{ll}
$604(2.5)$ & $665(3.6)$ \\
$560(2.6)$ & $615(5.5)$ \\
\hline
\end{tabular}

$\begin{array}{llll}344(3.6) & 400(4.4) & 452(4.5) & 522(4.7)\end{array}$

\begin{tabular}{|l|l|l|l|}
\hline $360(3.8)$ & $432(4.1)$ & $496(3.0)$ & $574(5.2)$ \\
\hline $457(6.5)$ & $530(2.8)$ & $584(3.6)$ & $658(4.6)$ \\
\hline
\end{tabular}

\begin{tabular}{|l|l|l|l|}
\hline $504(3.2)$ & $554(3.5)$ & $603(3.6)$ & $669(6.9)$ \\
\hline
\end{tabular}

\begin{tabular}{llll}
$264(5.3)$ & $341(3.3)$ & $417(3.1)$ & $669(6.9)$ \\
\hline
\end{tabular}

\begin{tabular}{l|l|l|l|}
\hline $264(5.3)$ & $341(3.3)$ & $417(3.1)$ & $517(5.5)$ \\
\hline $499(3.5)$ & $543(1.8)$ & $582(2.7)$ & $638(3.2)$ \\
\hline
\end{tabular}

$\begin{array}{llll}504(2.1) & 543(2.7) & 582(3.3) & 635(3.8)\end{array}$

\begin{tabular}{|l|l|l|l|}
\hline $512(2.4)$ & $557(1.3)$ & $601(1.7)$ & $635(3.8)$ \\
\hline $368(4.7)$ & $443(3.8)$ & $511(5.4)$ & $590(3.4)$ \\
\hline
\end{tabular}

$\begin{array}{llll}368(4.7) & 443(3.8) & 511(5.4) & 590(3.4)\end{array}$

\begin{tabular}{|l|l|l|l|}
$368(4.7)$ & $443(3.8)$ & $511(5.4)$ & $590(3.4)$ \\
\hline $449(3.9)$ & $497(2.8)$ & $538(3.9)$ & $599(6.4)$ \\
\hline $234(5.6)$ & $316(6.8)$ & $402(8.4)$ & $507(7.1)$ \\
\hline
\end{tabular}

\begin{tabular}{|l|l|l|l|}
\hline $508(2.0)$ & $547(2.6)$ & $583(2.9)$ & $607(7.1)$ \\
\hline $473(2.8)$ & $635(3.4)$ \\
\hline
\end{tabular}

$\begin{array}{llll}473(2.8) & 534(3.0) & 588(3.1) & 658(2.3)\end{array}$

\begin{tabular}{|l|l|l|l|}
\hline $460(4.2)$ & $506(3.7)$ & $547(3.5)$ & $602(2.5)$ \\
\hline
\end{tabular}

$\begin{array}{llll}460(4.2) & 506(3.7) & 547(3.5) & 602(2.5) \\ 472(4.3) & 529(3.4) & 577(2.3) & 645(4.3)\end{array}$

\begin{tabular}{|l|l|l|l|}
\hline $472(4.3)$ & $529(3.4)$ & $577(2.3)$ & $645(4.3)$ \\
\hline $290(2.2)$ & $358(2.0)$ & $429(2.4)$ & $514(3.5)$ \\
\hline $440(7.2)$ & $501(5.4)$ & $557(3.9)$ & $627(3.3)$ \\
\hline
\end{tabular}

\begin{tabular}{|c|c|c|}
\hline $440(7.2)$ & $501(5.4)$ & $557(3.9)$ \\
\hline $517(3.7)$ & $565(4.0)$ & $608(2.7)$ \\
\hline
\end{tabular}

\begin{tabular}{|l|l|l|}
$517(3.7)$ & $565(4.0)$ & 557( \\
$478(4.0)$ & $532(3.7)$ & 582( \\
\hline
\end{tabular}

\begin{tabular}{|l|l|l|}
\hline $478(4.0)$ & $532(3.7)$ & 582 \\
\hline $502(5.1)$ & $557(3.5)$ & 608( \\
\hline $491(5.3)$ & $541(3.3)$ & 584 \\
\hline $476(3.9)$ & $525(2.2)$ & $567(1.5$ \\
\hline
\end{tabular}

\begin{tabular}{l|l|}
$608(2.7)$ & $670(5.9)$ \\
\hline $582(2.5)$ & $651(5.2)$ \\
\hline $608(3.0)$ & $673(4.7)$
\end{tabular}

$608(3.0) \quad 673(4.7)$

\begin{tabular}{l|l|}
$584(2.8)$ & $642(2.4)$ \\
$567(3.4)$ & $625(2.5)$
\end{tabular}

\begin{tabular}{|l|l|l|l|}
\hline $476(3.9)$ & $525(2.2)$ & $567(3.4)$ & $625(2.5)$ \\
\hline $203(4.0)$ & $280(4.5)$ & $381(8.5)$ & $555(10.9)$ \\
\hline $470(3.7)$ & $520(2.1)$ & $569(3.0)$ & $632(4.8)$ \\
\hline
\end{tabular}

\begin{tabular}{|l|l|l|l|}
\hline $470(3.7)$ & $520(2.1)$ & $569(3.0)$ & $632(4.8)$ \\
\hline $508(4.1)$ & $550(3.2)$ & $588(2.3)$ & $640(3.7)$ \\
\hline
\end{tabular}

\begin{tabular}{|l|l|l|l|}
\hline $508(4.1)$ & $550(3.2)$ & $588(2.3)$ & $640(3.7)$ \\
\hline $362(9.2)$ & $440(4.4)$ & $510(5.2)$ & $593(4.4)$ \\
\hline $492(5.3)$ & $546(3.3)$ & $595(3.2)$ & $660(5.4)$
\end{tabular}

( ) Standard errors appear in parentheses. Because results are rounded to the nearest whole number, some totals may appear inconsistent. 
Exhibit C.4 Standard Deviations of Achievement in Literary Reading

PIRLS 2006

4th Grade

\begin{tabular}{|c|c|c|c|c|c|c|}
\hline \multirow{2}{*}{ Countries } & \multicolumn{2}{|c|}{ Overall } & \multicolumn{2}{|c|}{ Girls } & \multicolumn{2}{|c|}{ Boys } \\
\hline & Mean & $\begin{array}{l}\text { Standard } \\
\text { Deviation }\end{array}$ & Mean & $\begin{array}{l}\text { Standard } \\
\text { Deviation }\end{array}$ & Mean & $\begin{array}{l}\text { Standard } \\
\text { Deviation }\end{array}$ \\
\hline Austria & $537(2.1)$ & $62(1.1)$ & $543(2.6)$ & $60(1.3)$ & $531(2.4)$ & $64(1.4)$ \\
\hline Belgium (Flemish) & $544(1.9)$ & $58(1.3)$ & $547(2.2)$ & $56(1.8)$ & $541(2.3)$ & $59(1.4)$ \\
\hline Belgium (French) & $499(2.4)$ & $67(1.2)$ & $504(2.6)$ & $66(1.4)$ & $495(2.8)$ & $68(1.5)$ \\
\hline Bulgaria & $542(4.5)$ & $84(3.0)$ & $553(4.6)$ & $80(2.8)$ & $532(5.4)$ & $86(4.1)$ \\
\hline Canada, Alberta & $561(2.7)$ & $71(1.8)$ & $567(2.9)$ & $70(2.3)$ & $556(3.0)$ & $71(1.7)$ \\
\hline Canada, British Columbia & $559(2.7)$ & $72(1.4)$ & $565(3.0)$ & $70(1.7)$ & $553(3.2)$ & $72(1.7)$ \\
\hline Canada, Nova Scotia & $543(2.4)$ & $77(1.4)$ & $552(3.4)$ & $74(1.7)$ & $534(2.6)$ & $79(1.9)$ \\
\hline Canada, Ontario & $555(3.0)$ & $74(1.2)$ & $562(3.5)$ & $72(1.8)$ & $549(3.3)$ & $76(2.0)$ \\
\hline Canada, Quebec & $529(2.8)$ & $64(1.4)$ & $536(3.1)$ & $61(2.2)$ & $523(3.4)$ & $66(1.6)$ \\
\hline Chinese Taipei & $530(2.0)$ & $69(1.3)$ & $538(2.2)$ & $67(1.7)$ & $523(2.2)$ & $71(1.4)$ \\
\hline Denmark & $547(2.6)$ & $68(1.7)$ & $554(3.0)$ & $68(2.2)$ & $541(3.1)$ & $68(1.8)$ \\
\hline England & $539(2.6)$ & $89(1.6)$ & $550(3.1)$ & $89(1.8)$ & $528(2.7)$ & $88(2.4)$ \\
\hline France & $516(2.4)$ & $66(1.1)$ & $523(2.6)$ & $64(1.3)$ & $510(2.7)$ & $67(1.3)$ \\
\hline Georgia & $476(3.2)$ & $75(1.6)$ & $484(3.7)$ & $74(1.4)$ & $470(3.6)$ & $76(2.4)$ \\
\hline Germany & $549(2.2)$ & $66(1.1)$ & $554(2.4)$ & $65(1.5)$ & $544(2.6)$ & $67(1.7)$ \\
\hline Hong Kong SAR & $557(2.6)$ & $64(1.3)$ & $564(2.6)$ & $61(1.4)$ & $551(3.3)$ & $66(1.6)$ \\
\hline Hungary & $557(2.9)$ & $70(1.9)$ & $560(3.6)$ & $70(2.4)$ & $553(2.9)$ & $70(2.1)$ \\
\hline Iceland & $514(1.7)$ & $66(1.0)$ & $525(2.4)$ & $63(1.4)$ & $504(1.9)$ & $67(1.3)$ \\
\hline Indonesia & $397(3.9)$ & $78(1.8)$ & $408(4.0)$ & $76(2.1)$ & $387(4.4)$ & $79(2.3)$ \\
\hline Iran, Islamic Rep. of & $426(3.1)$ & $96(2.0)$ & $432(5.3)$ & $97(2.6)$ & $421(4.0)$ & $96(2.7)$ \\
\hline Israel & $516(3.4)$ & $98(2.3)$ & $524(4.0)$ & $96(2.6)$ & $509(3.8)$ & $99(2.7)$ \\
\hline Italy & $551(3.3)$ & $74(1.7)$ & $556(3.6)$ & $72(1.8)$ & $548(3.6)$ & $75(2.4)$ \\
\hline Kuwait & $340(3.7)$ & $108(2.4)$ & $372(4.5)$ & $102(2.2)$ & $310(5.2)$ & $105(2.8)$ \\
\hline Latvia & $539(2.4)$ & $63(1.3)$ & $550(3.0)$ & $61(1.4)$ & $529(2.7)$ & $64(2.0)$ \\
\hline Lithuania & $542(1.9)$ & $58(1.0)$ & $550(2.4)$ & $58(1.3)$ & $533(2.0)$ & $58(1.2)$ \\
\hline Luxembourg & $555(1.0)$ & $68(0.8)$ & $557(1.4)$ & $68(1.0)$ & $552(1.4)$ & $69(1.0)$ \\
\hline Macedonia, Rep. of & $439(3.7)$ & $97(2.1)$ & $449(4.3)$ & $96(2.3)$ & $429(4.0)$ & $97(2.6)$ \\
\hline Moldova, Rep. of & $492(2.8)$ & $68(1.5)$ & $499(3.3)$ & $67(1.8)$ & $486(3.0)$ & $69(1.8)$ \\
\hline Morocco & $317(6.5)$ & $116(2.6)$ & $326(6.9)$ & $113(3.0)$ & $310(7.4)$ & $119(3.4)$ \\
\hline Netherlands & $545(1.8)$ & $57(1.3)$ & $548(2.2)$ & $57(1.6)$ & $541(2.3)$ & $56(1.6)$ \\
\hline New Zealand & $527(2.1)$ & $86(1.3)$ & $539(2.3)$ & $81(2.0)$ & $516(2.9)$ & $90(1.5)$ \\
\hline Norway & $501(2.5)$ & $67(1.4)$ & $512(2.8)$ & $64(1.7)$ & $491(2.7)$ & $67(1.9)$ \\
\hline Poland & $523(2.5)$ & $78(1.5)$ & $532(2.8)$ & $77(1.9)$ & $514(3.0)$ & $77(1.8)$ \\
\hline Qatar & $358(1.3)$ & $96(1.5)$ & $376(1.8)$ & $92(1.5)$ & $341(2.3)$ & $97(1.8)$ \\
\hline Romania & $493(4.8)$ & $91(3.3)$ & $501(4.9)$ & $90(3.7)$ & $485(5.6)$ & $91(3.6)$ \\
\hline Russian Federation & $561(3.3)$ & $69(2.0)$ & $568(3.8)$ & $68(2.1)$ & $554(3.3)$ & $70(2.3)$ \\
\hline Scotland & $527(2.6)$ & $81(1.7)$ & $538(3.4)$ & $79(2.1)$ & $515(3.0)$ & $82(2.1)$ \\
\hline Singapore & $552(2.9)$ & $80(2.0)$ & $560(3.2)$ & $77(1.9)$ & $544(3.4)$ & $82(2.4)$ \\
\hline Slovak Republic & $533(2.9)$ & $74(2.7)$ & $539(2.9)$ & $73(2.4)$ & $527(3.5)$ & $75(3.3)$ \\
\hline Slovenia & $519(2.0)$ & $69(1.2)$ & $529(2.3)$ & $66(1.6)$ & $511(2.6)$ & $71(1.4)$ \\
\hline South Africa & $299(5.2)$ & $135(3.5)$ & $318(6.0)$ & $134(3.9)$ & $281(5.3)$ & $132(3.8)$ \\
\hline Spain & $516(2.7)$ & $75(1.5)$ & $520(3.1)$ & $75(2.2)$ & $513(3.1)$ & $75(1.9)$ \\
\hline Sweden & $546(2.3)$ & $61(1.1)$ & $557(2.7)$ & $60(1.6)$ & $536(2.6)$ & $61(1.4)$ \\
\hline Trinidad and Tobago & $434(4.6)$ & $104(2.7)$ & $450(4.9)$ & $98(3.0)$ & $419(5.6)$ & $107(3.0)$ \\
\hline United States & $541(3.6)$ & $78(1.5)$ & $547(3.6)$ & $75(1.9)$ & $534(4.1)$ & $80(2.0)$ \\
\hline
\end{tabular}

() Standard errors appear in parentheses. Because results are rounded to the nearest whole number, some totals may appear inconsistent. 
Exhibit C.5 Percentiles of Achievement in Informational Reading

PIRLS 2006

4th Grade

5th percentile 25 th percentile 50 th percentile 75 th percentile 95 th percentile

Countries

(2)

Austria

$424(6.7)$

Belgium (Flemish)

$456(5.3)$

Belgium (French)

$383(4.5)$

Bulgaria

Canada, Alberto

$403(10.9)$

$495(2.5)$

Canada, British Columbia

$445(5.4)$

$437(6.0)$

Canada, Nova Scotia

$406(9.2)$

Canada, Ontario

$440(6.3)$

Canada, Quebec

$423(3.9)$

Chinese Taipe

$435(5.7)$

Denmark

$412(5.7)$

England

$387(5.3)$

France

$413(3.7)$

Georgia

$333(4.5)$

Germany

$428(4.0)$

Hong Kong SAR

$471(4.6)$

Hungary

Iceland

$419(4.2)$

Indonesia

$380(3.3)$

Iran, Islamic Rep. of

$275(5.2)$

Israe

$269(4.0)$

Italy

Kuwait

$321(5.3)$

439 (3.6)

Latvia

$131(6.8)$

Lithuania

$435(6.5)$

Luxembourg

Macedonia, Rep. of

$437(2.6)$

Moldova, Rep. of

448 (3.8)

Morocco

$276(5.9)$

Netherlands

$386(5.2)$

New Zealand

$64(9.3)$

Norway

Poland

$463(3.7)$

$512(2.7)$

540

$540(2.4)$

$\begin{array}{ll}581(2.0) & 637(3.0)\end{array}$

$549(2.8)$

$584(2.6)$

631 (1.9)

$453(4.2)$

$500(3.1)$

$500(5.5)$

$555(4.5)$

$545(2.7)$

$513(3.6)$

$605(3.3)$

606 (4.4)

\section{$510(3.7)$}

$557(3.5)$

$600(3.6)$

$678(3.5)$

$493(2.7)$

$555(3.3)$

509 (3.6)

$544(2.6)$

590

$600(2.5)$

$662(2.6)$

$662(4.7)$

$493(2.4)$

$554(3.6)$

597

\section{7 (3.6)}

653 (3.9)

$502(2.2)$

$536(3.4)$

$578(3.7)$

\section{8 (3.2)}

$542(1.9)$

$578(2.1)$

$592(2.3)$

659 (4.2)

$633(5.4)$

$628(2.2)$

\begin{tabular}{l|l}
$485(3.9)$ & $543(3.5)$ \\
\hline
\end{tabular}

$595(2.7)$

\section{$482(3.7)$}

20

$595(2.7)$

$520(4.1)$

$528(1.8)$
$469(3.5)$

$505(2.1)$

550 (2.6)

$520(4.1)$
$590(2.5)$

$649(3.1)$

$533(3.4)$

$572(2.1)$

607

607 (2.3)

$666(3.2)$

$666(3.2)$
$631(3.0)$

$586(6.3)$

$643(2.3)$

$654(2.2)$

$589(3.3) \quad 649(3.7)$

\begin{tabular}{|l|l|l|l|}
\hline $459(1.9)$ & $509(1.8)$ & $555(1.7)$ & $616(2.2)$ \\
\hline
\end{tabular}

$\begin{array}{llll}363(4.5) & 422(6.0) & 476(4.2) & 545(5.4)\end{array}$

\begin{tabular}{|l|l|l|l|}
\hline $357(4.2)$ & $422(3.6)$ & $485(4.3)$ & $564(5.1)$ \\
\hline $450(4.9)$ & $521(3.3)$ & $576(2.2)$ & $649(3.8)$ \\
\hline
\end{tabular}

\begin{tabular}{|l|l|l|l|}
\hline $508(2.6)$ & $552(3.1)$ & $593(3.6)$ & $651(6.7)$ \\
\hline
\end{tabular}

$\begin{array}{llll}243(5.2) & 326(5.0) & 412(6.3) & 518(6.9)\end{array}$

\begin{tabular}{|l|l|l|l|}
$243(5.2)$ & $326(5.0)$ & $412(6.3)$ & $518(6.9)$ \\
\hline $499(2.4)$ & $542(2.5)$ & $583(3.5)$ & $640(3.4)$ \\
\hline
\end{tabular}

$495(2.5) \quad 532(2.1)$

$\begin{array}{ll}495(2.5) & 532(2.1) \\ 514(3.2) & 559(1.7)\end{array}$

$640(3.4)$

$568(2.5) \quad 616(2.0)$

\begin{tabular}{l|l|l}
$514(3.2)$ & $559(1.7)$ & $601(1.4)$ \\
\hline
\end{tabular}

$658(3.3)$

$378(4.1) \quad 455(5.2)$

\begin{tabular}{l|l}
$378(4.1)$ & $455(5.2)$ \\
\hline $463(4.7)$ & $512(4.6)$ \\
\hline
\end{tabular}

$526(4.6)$

$463(4.7)$

$512(4.6)$

$526(4.6)$

$609(5.7)$

4097

$\begin{array}{ll}557(2.4) & 617(5.8) \\ 409(7.8) & 505(14.4)\end{array}$

\begin{tabular}{|l|l|l|l|}
\hline $515(2.9)$ & $549(2.4)$ & $582(1.9)$ & $627(5.1)$ \\
\hline
\end{tabular}

\begin{tabular}{|c|c|c|c|c|c|}
\hline New Zealand & $384(4.0)$ & $481(1.8)$ & $540(2.8)$ & $592(2.5)$ & $661(1.8)$ \\
\hline Norway & $375(4.9)$ & $450(4.9)$ & $499(3.1)$ & $542(3.8)$ & $599(4.4)$ \\
\hline Poland & $391(4.8)$ & $468(4.9)$ & $519(3.0)$ & $566(2.5)$ & $627(4.2)$ \\
\hline Qatar & $202(3.9)$ & $289(2.6)$ & $356(2.4)$ & $424(2.2)$ & $508(2.4)$ \\
\hline Romania & $323(13.7)$ & $435(7.0)$ & $497(6.4)$ & $548(3.7)$ & $616(5.6)$ \\
\hline Russian Federation & $448(6.1)$ & $522(4.1)$ & $568(3.1)$ & $609(2.6)$ & $667(4.2)$ \\
\hline Scotland & $393(6.0)$ & $478(2.9)$ & $531(2.8)$ & $580(2.9)$ & $649(3.6)$ \\
\hline Singapore & $437(6.2)$ & $520(3.5)$ & $569(3.1)$ & $613(3.8)$ & $668(3.1)$ \\
\hline Slovak Republic & $394(7.8)$ & $483(2.8)$ & $533(2.7)$ & $577(2.4)$ & $635(2.0)$ \\
\hline Slovenia & $399(5.7)$ & $477(3.3)$ & $528(1.8)$ & $572(2.7)$ & $632(2.2)$ \\
\hline South Africa & $126(5.2)$ & $220(3.8)$ & $298(4.3)$ & $395(8.5)$ & $568(10.0)$ \\
\hline Spain & $390(5.7)$ & $466(3.7)$ & $512(2.7)$ & $554(2.4)$ & $614(4.0)$ \\
\hline Sweden & $431(4.1)$ & $508(2.6)$ & $553(2.5)$ & $594(2.6)$ & $652(4.2)$ \\
\hline Trinidad and Tobago & $270(4.7)$ & $372(8.0)$ & $445(7.3)$ & $511(6.4)$ & $596(5.2)$ \\
\hline United States & $414(7.0)$ & $493(5.4)$ & $541(3.3)$ & $586(4.1)$ & $644(4.8)$ \\
\hline
\end{tabular}

( ) Standard errors appear in parentheses. Because results are rounded to the nearest whole number, some totals may appear inconsistent. 
Exhibit C.6 Standard Deviations of Achievement in Informational Reading

PIRLS 2006

4th Grade

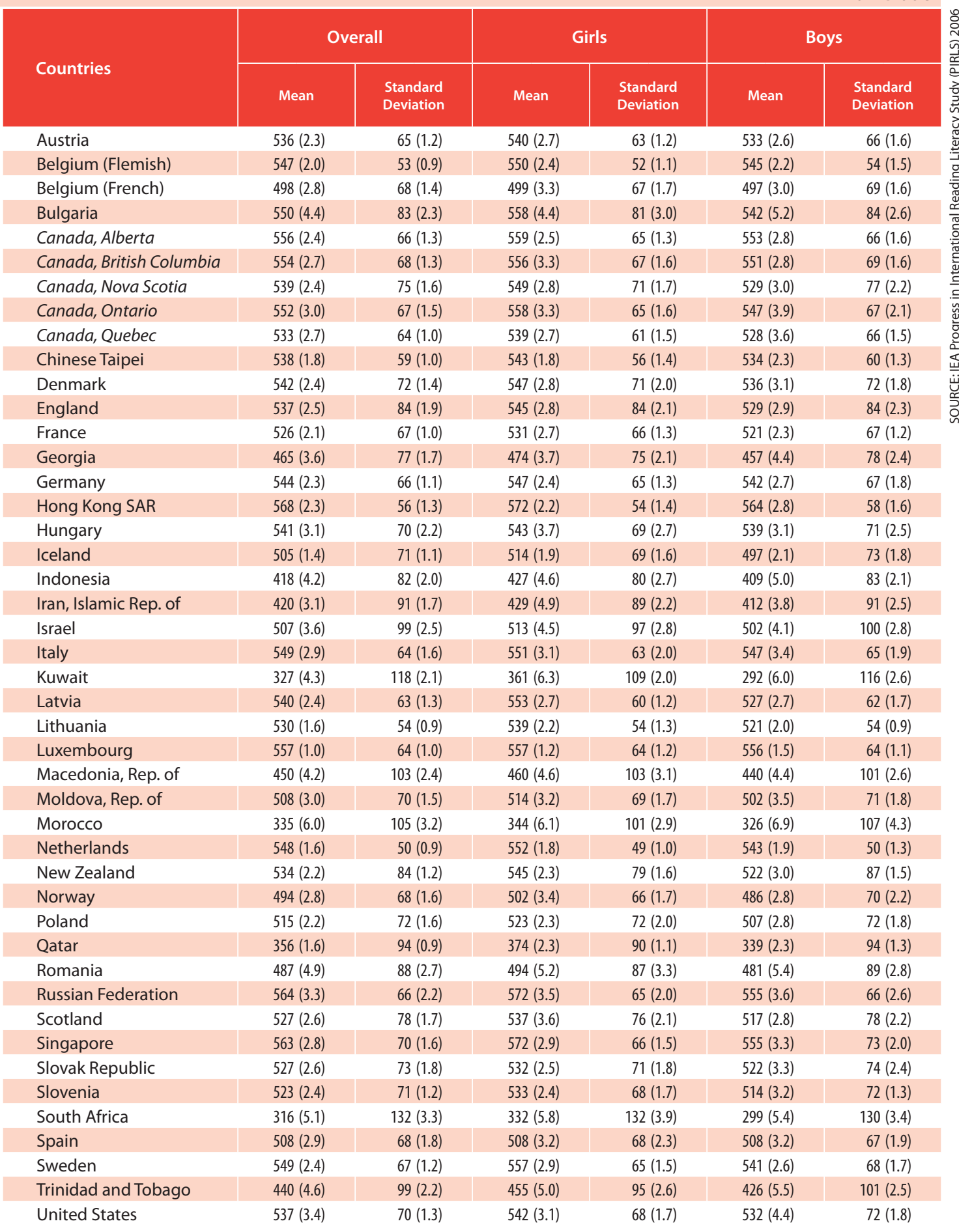

() Standard errors appear in parentheses. Because results are rounded to the nearest whole number, some totals may appear inconsistent. 
Exhibit C.7 Percentiles of Achievement in Reading for Retrieval and Straightforward Inferencing PIRLS 2006

4th Grade

\begin{tabular}{|c|c|c|c|c|c|}
\hline Countries & 5th percentile & 25th percentile & 50th percentile & 75th percentile & 95th percentile \\
\hline Austria & $428(3.7)$ & $502(1.8)$ & $548(2.0)$ & 589 (1.5) & 647 (2.7) \\
\hline Belgium (Flemish) & $444(3.4)$ & $507(2.2)$ & $547(1.9)$ & $585(2.4)$ & $638(3.1)$ \\
\hline Belgium (French) & $380(4.7)$ & $454(3.5)$ & $504(3.0)$ & $550(2.4)$ & $613(4.9)$ \\
\hline Bulgaria & $395(11.0)$ & $492(5.0)$ & $542(4.7)$ & $592(3.8)$ & $658(4.6)$ \\
\hline Canada, Alberta & $432(3.5)$ & $505(2.2)$ & $554(3.5)$ & $602(3.7)$ & $669(3.3)$ \\
\hline Canada, British Columbia & $429(4.4)$ & $504(3.4)$ & $553(2.8)$ & $599(2.6)$ & $664(4.9)$ \\
\hline Canada, Nova Scotia & $400(5.3)$ & $486(3.1)$ & $537(3.5)$ & $585(3.8)$ & $650(4.8)$ \\
\hline Canada, Ontario & $418(6.4)$ & $496(2.8)$ & $544(3.9)$ & $593(3.0)$ & $661(2.6)$ \\
\hline Canada, Quebec & $420(2.9)$ & $491(3.8)$ & $535(2.9)$ & $577(2.5)$ & $636(4.3)$ \\
\hline Chinese Taipei & $420(5.7)$ & $500(2.1)$ & 545 (1.9) & $587(1.9)$ & $645(3.3)$ \\
\hline Denmark & $409(6.8)$ & $504(3.6)$ & $557(3.6)$ & $605(3.0)$ & $669(4.0)$ \\
\hline England & $373(6.3)$ & $476(3.5)$ & $538(3.7)$ & $595(4.2)$ & $676(4.8)$ \\
\hline France & $407(4.7)$ & $479(2.8)$ & $526(2.0)$ & $570(2.5)$ & $628(3.0)$ \\
\hline Georgia & $353(5.0)$ & $429(3.9)$ & $482(3.9)$ & $530(3.1)$ & $594(5.6)$ \\
\hline Germany & $429(6.5)$ & $511(4.6)$ & $559(2.3)$ & $604(2.9)$ & $664(1.9)$ \\
\hline Hong Kong SAR & $455(3.7)$ & $521(3.1)$ & $561(2.6)$ & $598(1.6)$ & 649 (3.2) \\
\hline Hungary & $423(5.3)$ & $500(3.7)$ & $548(2.2)$ & $591(3.2)$ & $650(2.8)$ \\
\hline Iceland & $388(5.0)$ & $470(2.3)$ & $520(1.6)$ & $566(1.5)$ & $629(4.5)$ \\
\hline Indonesia & $278(8.7)$ & $358(4.4)$ & $412(4.5)$ & $464(3.8)$ & $532(2.7)$ \\
\hline Iran, Islamic Rep. of & $265(5.6)$ & $363(4.5)$ & $433(3.1)$ & 497 (3.1) & $578(4.3)$ \\
\hline Israel & $329(6.0)$ & $450(5.7)$ & $521(2.9)$ & $574(4.5)$ & $642(3.6)$ \\
\hline Italy & $425(6.6)$ & $498(3.9)$ & $546(3.6)$ & $592(3.6)$ & $655(4.4)$ \\
\hline Kuwait & $164(6.8)$ & $259(4.1)$ & $336(7.0)$ & $416(5.9)$ & $510(3.8)$ \\
\hline Latvia & $427(4.2)$ & $491(2.8)$ & $535(4.2)$ & $579(1.8)$ & $638(3.7)$ \\
\hline Lithuania & $427(3.4)$ & $492(2.3)$ & $534(2.5)$ & $572(2.5)$ & $626(4.5)$ \\
\hline Luxembourg & $440(2.9)$ & $518(2.4)$ & $567(2.0)$ & $615(2.1)$ & $681(2.9)$ \\
\hline Macedonia, Rep. of & $280(7.3)$ & $376(5.7)$ & $452(5.3)$ & $519(3.9)$ & $598(5.8)$ \\
\hline Moldova, Rep. of & $365(4.0)$ & $442(4.6)$ & $491(3.3)$ & $533(2.5)$ & $593(5.0)$ \\
\hline Morocco & $166(9.9)$ & $262(7.5)$ & $335(5.1)$ & $410(8.0)$ & $507(6.0)$ \\
\hline Netherlands & $448(3.8)$ & $511(2.2)$ & $553(2.3)$ & $592(2.4)$ & $649(3.0)$ \\
\hline New Zealand & $370(4.4)$ & $470(1.7)$ & $530(2.1)$ & $584(3.1)$ & $656(3.4)$ \\
\hline Norway & $375(4.0)$ & $457(3.1)$ & $506(2.8)$ & $552(3.1)$ & $613(3.9)$ \\
\hline Poland & $383(6.1)$ & $466(3.1)$ & $521(3.8)$ & $569(1.9)$ & $632(3.9)$ \\
\hline Qatar & $208(2.1)$ & $293(2.1)$ & $360(1.7)$ & $429(3.7)$ & $516(2.6)$ \\
\hline Romania & $322(13.4)$ & $437(6.4)$ & $498(5.8)$ & $551(5.3)$ & $619(3.8)$ \\
\hline Russian Federation & $439(6.7)$ & $518(3.7)$ & $566(4.0)$ & $610(3.4)$ & $672(5.8)$ \\
\hline Scotland & $377(8.9)$ & $475(5.6)$ & $529(2.7)$ & $579(2.7)$ & $651(6.0)$ \\
\hline Singapore & $408(4.7)$ & $508(4.1)$ & $566(4.2)$ & $619(3.1)$ & $687(3.3)$ \\
\hline Slovak Republic & $390(8.6)$ & $485(3.4)$ & $536(2.7)$ & $580(2.4)$ & $639(2.8)$ \\
\hline Slovenia & $391(4.9)$ & $473(3.0)$ & $524(2.5)$ & $569(2.2)$ & $629(4.0)$ \\
\hline South Africa & $121(3.8)$ & $212(4.1)$ & $287(4.9)$ & $385(8.4)$ & $559(14.6)$ \\
\hline Spain & $389(7.9)$ & $464(3.5)$ & $512(1.2)$ & $556(3.3)$ & $615(4.0)$ \\
\hline Sweden & $430(5.7)$ & $508(3.9)$ & $554(2.4)$ & $597(2.6)$ & $657(5.7)$ \\
\hline Trinidad and Tobago & $262(14.4)$ & $367(8.4)$ & $444(7.0)$ & $512(5.5)$ & $600(7.4)$ \\
\hline United States & $395(4.4)$ & $483(3.6)$ & $537(4.6)$ & $587(3.7)$ & $652(4.3)$ \\
\hline
\end{tabular}




\section{Exhibit C.8 Standard Deviations of Achievement in Reading for Retrieval and Straightforward Inferencing}

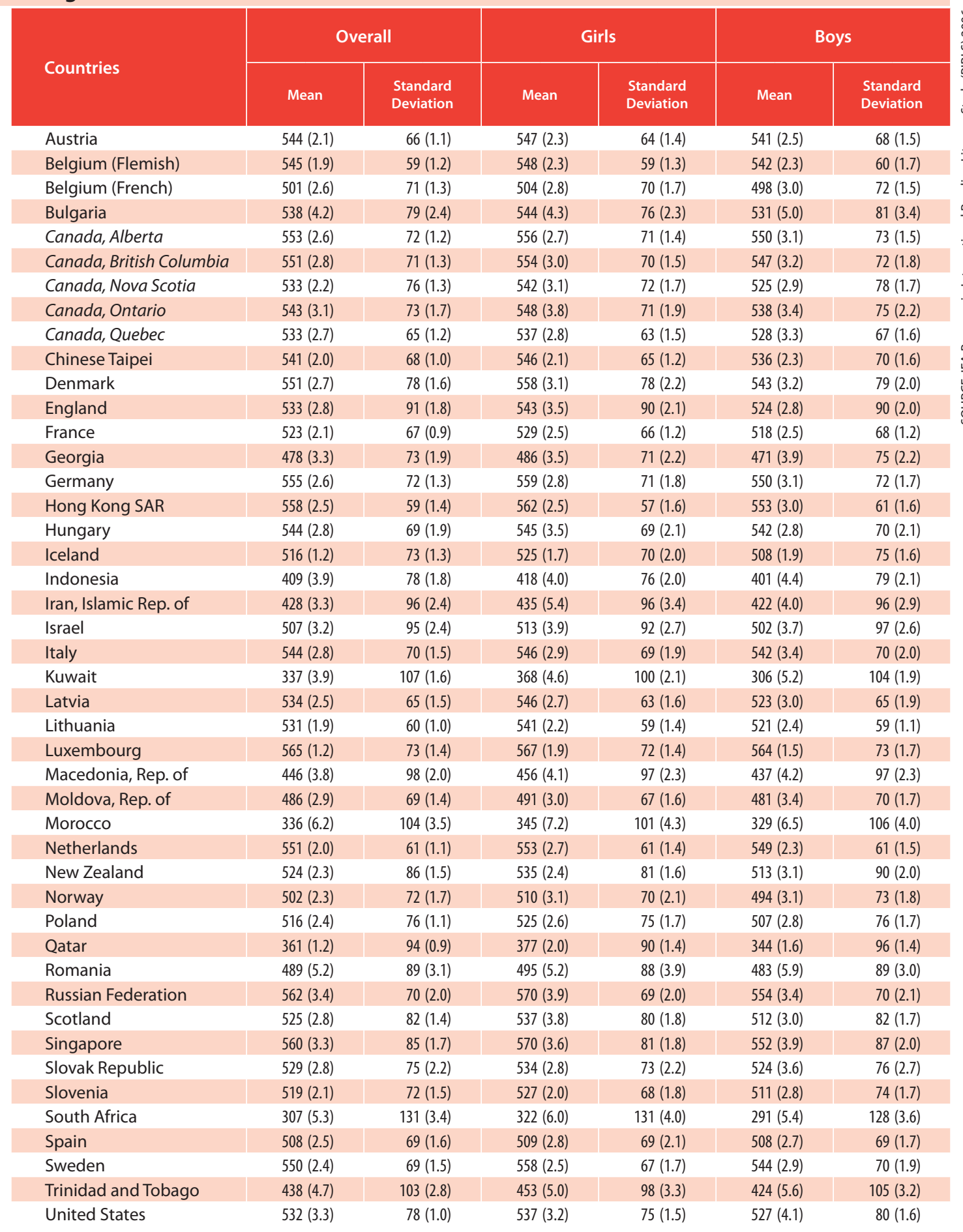


Exhibit C.9 Percentiles of Achievement in Reading for Interpreting, Integrating, and Evaluating PIRLS 2006 4th Grade

\begin{tabular}{|c|c|c|c|c|c|}
\hline Countries & 5th percentile & 25th percentile & 50th percentile & 75th percentile & 95th percentile \\
\hline Austria & $417(3.3)$ & $490(2.9)$ & $534(2.3)$ & $575(2.3)$ & $628(3.3)$ \\
\hline Belgium (Flemish) & $458(2.1)$ & $512(2.6)$ & $548(1.4)$ & $583(2.0)$ & $632(2.1)$ \\
\hline Belgium (French) & $383(4.6)$ & $452(2.7)$ & $499(2.8)$ & $544(2.4)$ & $604(3.6)$ \\
\hline Bulgaria & $397(12.0)$ & $504(7.5)$ & $559(3.6)$ & $610(5.0)$ & $679(6.9)$ \\
\hline Canada, Alberta & $454(3.0)$ & $523(3.2)$ & $566(2.7)$ & $608(4.0)$ & $665(4.1)$ \\
\hline Canada, British Columbia & $448(3.0)$ & $520(3.3)$ & $564(3.7)$ & $607(3.4)$ & $666(4.8)$ \\
\hline Canada, Nova Scotia & $421(6.9)$ & $504(2.0)$ & $552(1.7)$ & $598(2.7)$ & $657(2.3)$ \\
\hline Canada, Ontario & $450(5.1)$ & $522(2.7)$ & $565(3.8)$ & $607(3.8)$ & 664 (3.6) \\
\hline Canada, Quebec & $421(5.3)$ & $492(3.0)$ & $534(3.5)$ & $574(3.2)$ & $627(3.7)$ \\
\hline Chinese Taipei & $421(5.0)$ & $491(1.9)$ & $533(1.8)$ & $572(1.5)$ & $626(2.6)$ \\
\hline Denmark & $428(6.2)$ & $505(2.7)$ & $548(2.6)$ & $586(2.0)$ & $635(2.5)$ \\
\hline England & $399(8.8)$ & $492(2.4)$ & $548(2.4)$ & $598(2.9)$ & $670(4.7)$ \\
\hline France & $404(3.7)$ & $475(2.5)$ & $521(2.4)$ & $564(2.5)$ & $621(3.3)$ \\
\hline Georgia & $324(7.7)$ & $408(2.7)$ & $466(3.5)$ & $519(4.2)$ & $587(4.2)$ \\
\hline Germany & $427(3.8)$ & $503(2.3)$ & $545(2.7)$ & $584(2.7)$ & $636(2.9)$ \\
\hline Hong Kong SAR & $463(5.8)$ & $529(2.7)$ & $569(4.1)$ & $607(3.2)$ & $656(4.0)$ \\
\hline Hungary & $436(5.3)$ & $512(3.6)$ & $558(4.0)$ & $600(2.8)$ & $658(6.4)$ \\
\hline Iceland & $386(3.4)$ & $462(1.5)$ & $508(1.4)$ & $548(2.0)$ & $603(3.8)$ \\
\hline Indonesia & $268(7.7)$ & $350(6.2)$ & $407(5.3)$ & $460(4.0)$ & $531(3.8)$ \\
\hline Iran, Islamic Rep. of & $260(8.2)$ & $355(3.0)$ & $422(5.5)$ & $484(3.4)$ & $562(3.1)$ \\
\hline Israel & $331(13.7)$ & $461(6.9)$ & $531(3.5)$ & $584(2.7)$ & $654(4.5)$ \\
\hline Italy & $445(6.1)$ & $514(3.3)$ & $557(2.7)$ & $600(3.6)$ & $658(4.1)$ \\
\hline Kuwait & ++ & ++ & ++ & ++ & ++ \\
\hline Latvia & $446(4.0)$ & $508(3.3)$ & $547(3.0)$ & $586(1.5)$ & $637(2.6)$ \\
\hline Lithuania & $448(3.3)$ & $506(2.3)$ & $542(2.4)$ & $576(2.2)$ & $625(3.6)$ \\
\hline Luxembourg & $438(3.5)$ & $508(1.3)$ & $551(0.9)$ & $592(1.6)$ & $647(3.1)$ \\
\hline Macedonia, Rep. of & $262(7.0)$ & $363(3.4)$ & $444(4.0)$ & $517(5.4)$ & $601(5.3)$ \\
\hline Moldova, Rep. of & $397(5.8)$ & $473(4.5)$ & $520(3.2)$ & $561(2.6)$ & $620(3.6)$ \\
\hline Morocco & ++ & ++ & ++ & ++ & ++ \\
\hline Netherlands & $455(6.0)$ & $509(1.6)$ & $544(2.5)$ & $577(1.6)$ & $622(2.3)$ \\
\hline New Zealand & $392(3.3)$ & $487(2.2)$ & $545(3.0)$ & $595(2.9)$ & $661(2.7)$ \\
\hline Norway & $380(5.8)$ & $453(3.9)$ & $499(3.5)$ & $541(3.2)$ & $595(3.0)$ \\
\hline Poland & $394(7.7)$ & $474(3.6)$ & $527(1.7)$ & $573(2.2)$ & $633(2.0)$ \\
\hline Qatar & ++ & ++ & ++ & ++ & ++ \\
\hline Romania & $320(13.5)$ & $438(6.5)$ & $500(4.1)$ & $554(4.9)$ & $621(4.2)$ \\
\hline Russian Federation & $445(6.0)$ & $522(3.4)$ & $567(4.0)$ & $608(3.8)$ & $664(3.9)$ \\
\hline Scotland & $388(11.7)$ & $482(2.9)$ & $533(4.2)$ & $580(3.3)$ & $648(4.7)$ \\
\hline Singapore & $431(4.9)$ & $513(3.6)$ & $561(3.7)$ & $604(3.5)$ & $660(3.7)$ \\
\hline Slovak Republic & $400(5.5)$ & $489(3.8)$ & $539(3.7)$ & $580(2.9)$ & $635(3.1)$ \\
\hline Slovenia & $404(4.6)$ & $481(2.6)$ & $528(2.5)$ & $569(2.2)$ & $624(2.6)$ \\
\hline South Africa & ++ & ++ & ++ & ++ & ++ \\
\hline Spain & $389(7.3)$ & $471(3.5)$ & $520(3.3)$ & $564(3.1)$ & $625(3.1)$ \\
\hline Sweden & $437(4.8)$ & $508(2.2)$ & $551(2.7)$ & $589(2.8)$ & 641 (3.3) \\
\hline Trinidad and Tobago & $263(9.5)$ & $367(5.6)$ & $443(5.1)$ & $510(4.5)$ & $590(4.4)$ \\
\hline United States & $427(5.8)$ & $503(3.8)$ & $550(3.9)$ & $593(3.5)$ & 649 (3.1) \\
\hline
\end{tabular}


Exhibit C.10 Standard Deviations of Achievement in Reading for Interpreting, Integrating, and Evaluating

\begin{tabular}{|c|c|c|c|c|c|c|}
\hline Countries & Mean & $\begin{array}{l}\text { Standard } \\
\text { Deviation }\end{array}$ & Mean & $\begin{array}{l}\text { Standard } \\
\text { Deviation }\end{array}$ & Mean & $\begin{array}{l}\text { Standard } \\
\text { Deviation }\end{array}$ \\
\hline Austria & $530(2.2)$ & $64(1.1)$ & $536(2.7)$ & $62(1.4)$ & $524(2.4)$ & $66(1.5)$ \\
\hline Belgium (Flemish) & $547(1.8)$ & $53(0.9)$ & $550(2.4)$ & $52(1.1)$ & $544(2.0)$ & $54(1.1)$ \\
\hline Bulgaria & $553(4.4)$ & $84(2.8)$ & $565(4.4)$ & $80(2.6)$ & $540(5.1)$ & $85(3.6)$ \\
\hline Canada, Alberta & $564(2.3)$ & $64(1.3)$ & $570(2.5)$ & $64(1.7)$ & $558(2.8)$ & $64(1.4)$ \\
\hline Canada, British Columbia & $562(2.5)$ & $65(1.1)$ & $567(2.7)$ & $65(1.5)$ & $557(3.1)$ & $66(1.4)$ \\
\hline Canada, Nova Scotia & $548(2.0)$ & $71(1.5)$ & $559(2.2)$ & $68(1.7)$ & $537(2.6)$ & $73(1.8)$ \\
\hline Canada, Ontario & $563(2.9)$ & $64(0.9)$ & $569(3.2)$ & $62(1.2)$ & $556(3.1)$ & $66(1.5)$ \\
\hline England & $543(2.4)$ & $81(1.9)$ & $552(2.8)$ & $81(2.3)$ & $534(2.7)$ & $81(1.8)$ \\
\hline France & $518(2.3)$ & $66(1.0)$ & $523(2.6)$ & $65(1.5)$ & $513(2.5)$ & $67(1.0)$ \\
\hline Georgia & $461(3.5)$ & $80(2.0)$ & $471(4.1)$ & $78(2.7)$ & $453(4.1)$ & $82(2.4)$ \\
\hline Germany & $540(2.2)$ & $65(1.3)$ & $543(2.4)$ & $64(1.6)$ & $537(2.7)$ & $66(1.9)$ \\
\hline Hong Kong SAR & $566(2.4)$ & $59(1.5)$ & $572(2.6)$ & $56(1.4)$ & $559(2.8)$ & $61(1.8)$ \\
\hline Hungary & $554(3.0)$ & $68(1.8)$ & $557(3.6)$ & $67(2.1)$ & $551(3.0)$ & $67(2.0)$ \\
\hline Iceland & $503(1.3)$ & $66(1.1)$ & $514(1.9)$ & $62(1.4)$ & $493(1.7)$ & $67(1.5)$ \\
\hline Indonesia & $404(4.1)$ & $80(2.3)$ & $415(4.1)$ & $78(2.5)$ & $393(4.8)$ & $81(2.7)$ \\
\hline Iran, Islamic Rep. of & $418(3.3)$ & $93(2.1)$ & $425(5.5)$ & $93(2.8)$ & $412(4.2)$ & $92(2.8)$ \\
\hline Macedonia, Rep. of & $439(4.0)$ & $105(2.6)$ & $451(4.7)$ & $104(3.0)$ & $428(4.2)$ & $104(2.5)$ \\
\hline Moldova, Rep. of & $515(2.9)$ & $67(1.2)$ & $523(3.1)$ & $66(1.5)$ & $508(3.2)$ & $68(1.5)$ \\
\hline Morocco & ++ & ++ & ++ & ++ & ++ & ++ \\
\hline Netherlands & $542(1.5)$ & $51(1.1)$ & $547(2.0)$ & $50(1.4)$ & $538(1.8)$ & $51(1.5)$ \\
\hline New Zealand & $538(2.2)$ & $81(1.3)$ & $550(2.3)$ & $76(1.5)$ & $526(2.9)$ & $85(1.8)$ \\
\hline Norway & $495(2.4)$ & $66(1.5)$ & $505(2.5)$ & $64(1.8)$ & $485(2.9)$ & $66(1.7)$ \\
\hline Poland & $522(2.3)$ & $72(1.4)$ & $529(2.4)$ & $71(1.8)$ & $514(3.0)$ & $73(1.8)$ \\
\hline Qatar & ++ & ++ & ++ & ++ & ++ & ++ \\
\hline Romania & $490(5.3)$ & $91(3.4)$ & $498(5.6)$ & $90(4.2)$ & $482(5.9)$ & $91(3.4)$ \\
\hline Russian Federation & $563(3.2)$ & $66(1.9)$ & $569(3.8)$ & $65(2.1)$ & $555(3.2)$ & $67(2.0)$ \\
\hline Scotland & $528(2.6)$ & $77(1.2)$ & $538(3.3)$ & $75(1.7)$ & $519(2.9)$ & $77(1.7)$ \\
\hline Singapore & $556(2.7)$ & $69(1.5)$ & $564(2.8)$ & $66(1.7)$ & $548(3.2)$ & $72(1.7)$ \\
\hline Slovak Republic & $531(2.8)$ & $71(2.2)$ & $538(2.8)$ & $70(2.4)$ & $525(3.4)$ & $72(2.7)$ \\
\hline Slovenia & $523(2.0)$ & $66(1.0)$ & $534(2.1)$ & $62(1.3)$ & $514(2.4)$ & $68(1.3)$ \\
\hline South Africa & ++ & ++ & ++ & ++ & ++ & ++ \\
\hline Spain & $515(2.6)$ & $72(1.8)$ & $519(2.9)$ & $72(2.0)$ & $512(3.0)$ & $71(2.1)$ \\
\hline Sweden & $546(2.2)$ & $62(1.0)$ & $557(2.7)$ & $60(1.3)$ & $537(2.5)$ & $62(1.3)$ \\
\hline Trinidad and Tobago & $437(5.0)$ & $100(2.6)$ & $453(5.5)$ & $95(3.3)$ & $421(5.8)$ & $103(3.1)$ \\
\hline United States & $546(3.3)$ & $67(1.1)$ & $552(3.0)$ & $65(1.3)$ & $540(4.1)$ & $69(1.5)$ \\
\hline
\end{tabular}



Appendix D

Sample Passages, Questions, and Scoring Guides

Reading for Literary Experience

Little Lump of Clay

An Unbelievable Night

Reading to Acquire and Use Information

Antarctica: Land of Ice

Searching for Food 


\section{Antarctica: Land of Ice}

\section{Introducing Antarctica}

\section{What is Antarctica?}

Antarctica is a continent that is right at the south of the planet. (If you try to find it on a globe, you will see that it is at the bottom.)

It takes up one-tenth of the Earth's surface and is covered with a blanket of ice that can be as thick as 1,500 metres or more. The

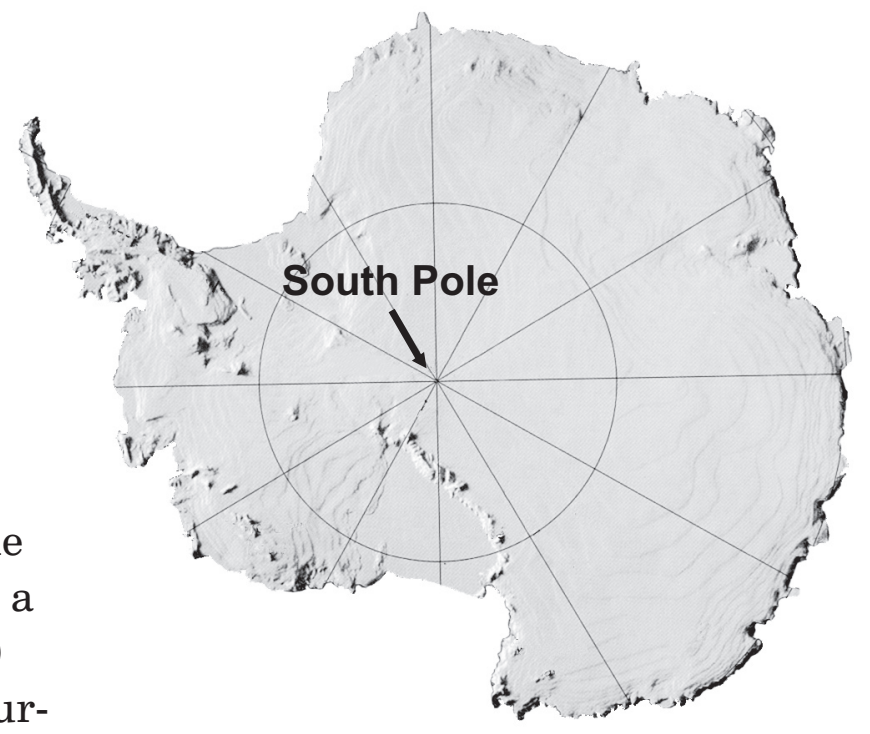

A Map of Antarctica South Pole is right in the middle of Antarctica.

Antarctica is the coldest continent, as well as the driest, the highest and the windiest. Very few people live there all year round. Scientists stay there for short periods, living in specially built

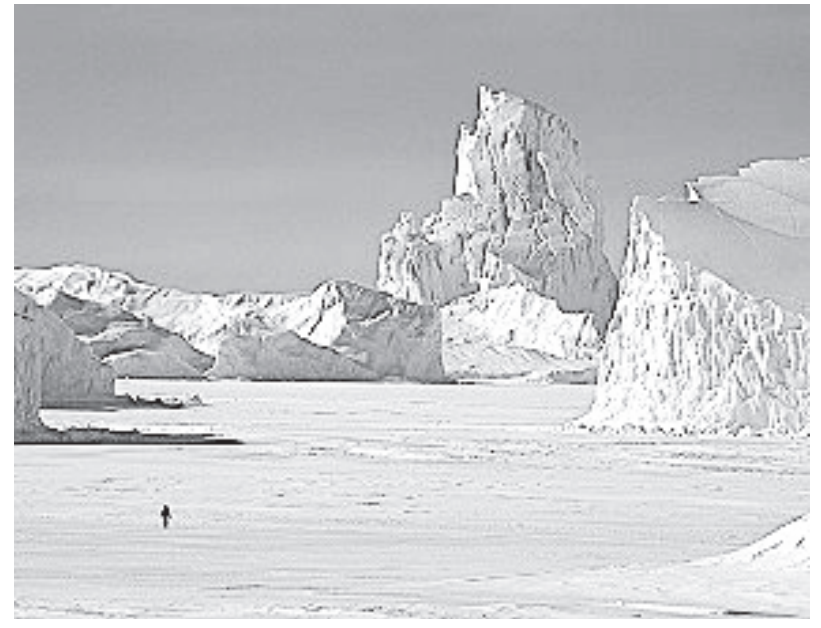
research stations.

Summer in Antarctica is between October and March. During this time there is non-stop daylight. In winter, April to September, the opposite happens and Antarctica is plunged into six months of constant darkness. 
In Antarctica, it is colder than you can possibly imagine, even in the summer! The South Pole is the coldest part of Antarctica. The average temperature for January, the middle of the summer, is minus 28 degrees Celsius (written as $-28^{\circ} \mathrm{C}$ ). Minus means colder than the freezing point, which is $0^{\circ} \mathrm{C}$.

In the winter, April to September, the average temperature at the South Pole can be as cold as $-89^{\circ} \mathrm{C}$. When it is

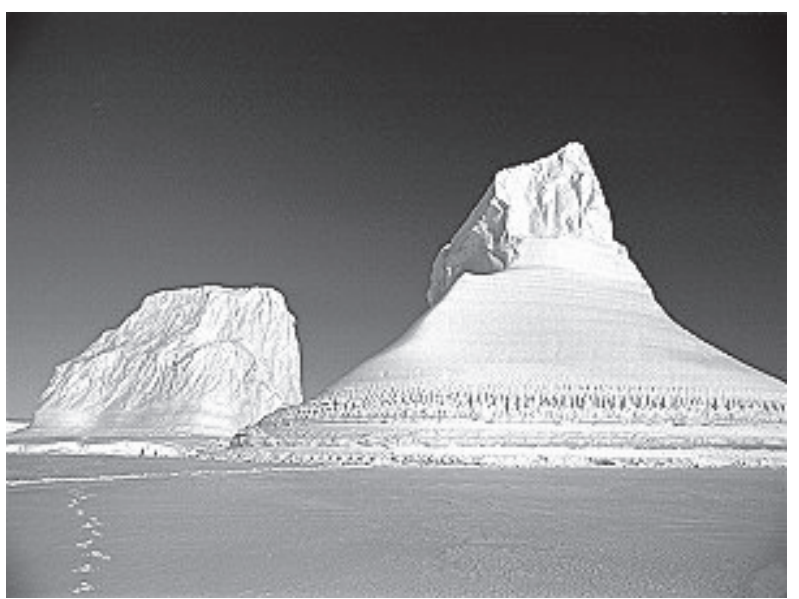
that cold, a mug of boiling water thrown in the air would freeze before it hit the ice. Sometimes the scientists have to use fridges to keep their samples warm!

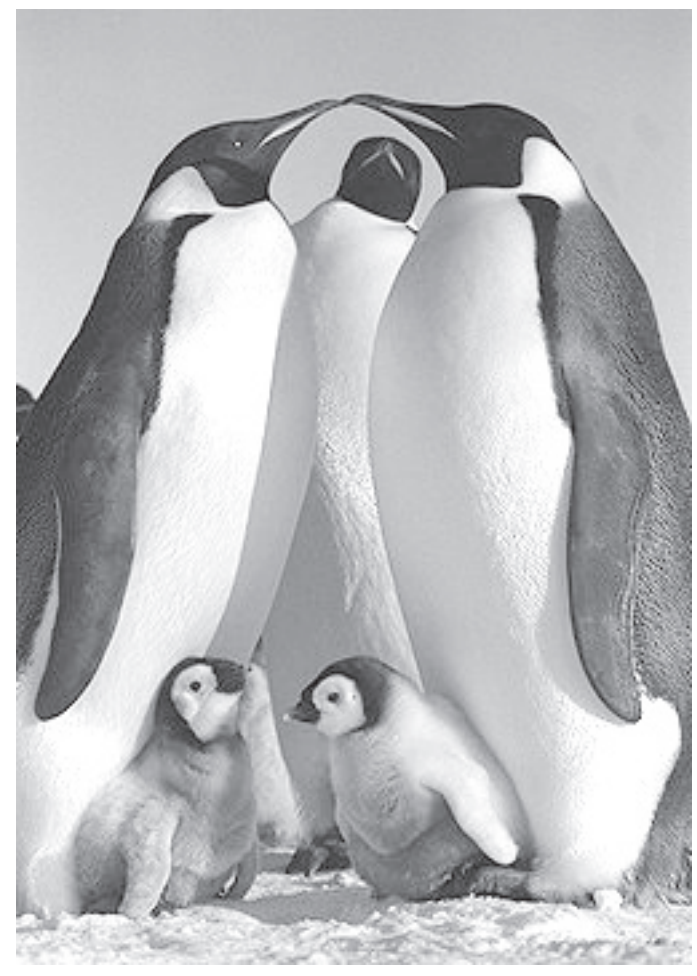

\section{Penguins in Antarctica}

There are more penguins in the Antarctic than any other bird.

They cannot fly but use their short wings as swimming flippers. They are superb swimmers. On land, they waddle upright or move in short hops.

Penguins have many feathers that overlap each other. These, together with woolly down feathers and a thick layer of fat, keep out the cold air, wind and water. For extra warmth, penguins huddle together in groups. 


\section{A Letter from Antarctica}

Sara Wheeler is one of the scientists working in Antarctica. By reading her letter to her nephew Daniel, you can learn more about her Antarctic experience.

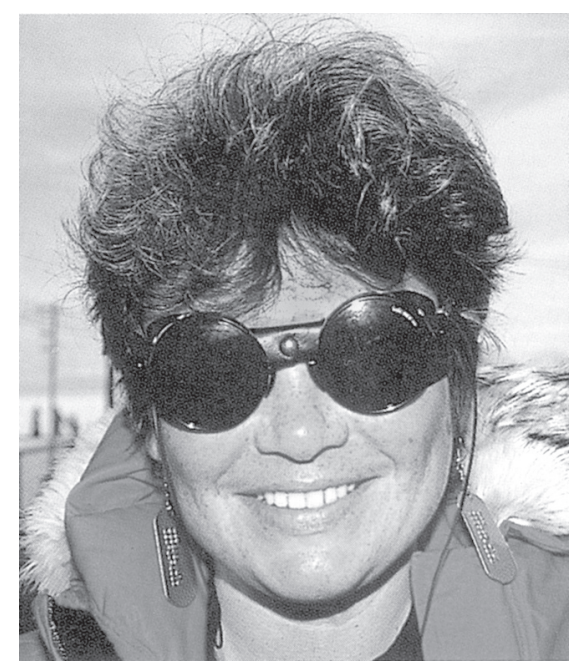

\section{Antarctica}

Friday, 9 December

Dear Daniel,

Here is the letter I promised to write to you from Antarctica, and a photograph. Imagine how excited I am to be here at last, following in the footsteps of so many famous explorers. It is very different from the world I am used to.

There is nothing fresh down here-and no supermarkets-so we have to eat a lot of dried, tinned or frozen food (it doesn't have to be put in the freezer-you can just leave it outside). We cook on small gas stoves, which take much longer than cookers at home. Yesterday I made noodles with tomato paste and vegetables out of a tin, followed by dried strawberries that tasted like cardboard.

I miss fresh apples and oranges-I wish you could send me some! Love from Sara 


\section{Questions Antarctica: Land of Ice}

1. Where can you find Antarctica on a globe?

(1)

2. Antarctica is the coldest place on Earth. What other records does it hold?

(A) driest and cloudiest

* (B) wettest and windiest

(C) windiest and driest

(D) cloudiest and highest

3. What is the coldest part of Antarctica? 
4. Think about what the article says about Antarctica. Give two reasons why most people who visit Antarctica choose not to go there between April and September.

(1) 1 .

2.

5. Why does the article tell you that 'a mug of boiling water thrown in the air would freeze before it hit the ice'?

A to tell you how hot the water is in Antarctica

(B) to show you what they drink in Antarctica

(C) to tell you about scientists' jobs in Antarctica

* (D) to show you how cold it is in Antarctica

6. According to the article, what do penguins use their wings for?

(A) flying

* (B) swimming

(C) keeping chicks warm

(D) walking upright 
7. Give three ways penguins are able to keep warm in Antarctica.
(1) 1 .
(1) 2 .
(1) 3 .

8. What are two things you learn about food in Antarctica from Sara's letter?

(1) 1 .

(1) 2 . 
9. Think about whether you would like to visit Antarctica. Use what you have read in both Introducing Antarctica and A Letter from Antarctica to explain why you would or would not like to visit.

2

10. Which section of the article tells you how thick the ice is in Antarctica?

* A What is Antarctica?

(B) The Weather in Antarctica

(C) Penguins in Antarctica

(D) A Letter from Antarctica 
11. In this article, there are two different ways of finding out about Antarctica:

- Introducing Antarctica

- A Letter from Antarctica

Which of these kinds of information do you find more interesting, and why?

(1) 


\section{Antarctica, Item 1}

\section{Where can you find Antarctica on a globe?}

Process: Focus on and retrieve explicitly stated information and ideas

\section{1 - Acceptable Response}

These responses identify the explicitly stated location of Antarctica. The response states that Antarctica is at the bottom of the globe, or describes it as being at the south of the planet.

\section{Antarctica, Item 3}

\section{What is the coldest part of Antarctica?}

Process: Focus on and retrieve explicitly stated information and ideas

\section{1 - Acceptable Response}

These responses identify the explicitly stated location of the coldest part of Antarctica. The response states that the South Pole is the coldest part. ("The middle part" is also acceptable.)

\section{Antarctica, Item 4}

\section{Think about what the article says about Antarctica. Give two reasons why most people who visit Antarctica choose not to go there between April and September.}

Process: Interpret and integrate ideas and information

\section{2 - Complete Comprehension}

These responses demonstrate complete comprehension by interpreting information about conditions in Antarctica during the winter. The response describes both of the winter conditions mentioned in the article: 1) the extreme cold, and 2) the constant darkness. (Note: it is not correct to just say that it is winter; it is necessary for the response to include the extreme cold or darkness of winter.)

\section{Example:}

It is plunged into six months of constant darkness. A mug of boiling water thrown in the air would freeze before it hit the ice.

\section{1 - Partial Comprehension}

These responses demonstrate partial comprehension by interpreting information about one condition in Antarctica during the winter. The response describes one of the winter conditions mentioned in the article: 1) the extreme cold OR 2) the constant darkness. (Note: it is not correct to just say that it is winter; it is necessary for the response to include the extreme cold or darkness of winter.)

\section{Example:}

It is very cold that time of year.

\section{Antarctica, Item 7}

\section{Give three ways penguins are able to keep} warm in Antarctica.

Process: Make straightforward inferences

\section{3 - Extensive Comprehension}

These responses demonstrate extensive comprehension by identifying most of the ideas in the article from which penguins' ability to stay warm can be inferred. The response describes at least three of the ways penguins are able to stay warm listed below.

\section{2 - Satisfactory Comprehension}

These responses demonstrate satisfactory comprehension by identifying some of the ideas in the article from which penguins' ability to stay warm can be inferred. The response describes two of the ways penguins are able to stay warm listed below.

\section{1 - Minimal Comprehension}

These responses demonstrate limited comprehension by identifying one idea in the 
article from which penguins' ability to stay warm can be inferred. The response describes only one of the ways penguins are able to stay warm listed below.

\section{Ideas from Article Explaining how Penguins Stay Warm}

They have many feathers which overlap each other.

They have woolly down feathers.

They have feathers (only counts as a separate idea if neither of the first two ideas about feathers is included in the response).

They have a thick layer of fat.

They huddle together in groups.

\section{Antarctica, Item 8}

\section{What are two things you learn about food in Antarctica from Sara's letter?}

Process: Focus on and retrieve explicitly stated information and ideas

\section{2 - Complete Comprehension}

These responses demonstrate complete comprehension by identifying two explicitly stated ideas related to food in Antarctica. The response identifies at least two of the ideas listed below.

\section{1 - Partial Comprehension}

These responses demonstrate partial comprehension by identifying one explicitly stated idea related to food in Antarctica. The response identifies only one of the ideas listed below.

\section{Ideas from Sara's Letter About Food in Antarctica}

There are no supermarkets.

There is a lot of dried, tinned, or frozen food lone or more of these adjectives is acceptable as an idea)/ Nothing is fresh.

Food doesn't have to be put in a freezer. /Food can be left outside.

They cook on gas stoves.

It takes longer to cook.
They eat noodles with tomato paste and vegetables.

Strawberries taste like cardboard.

They don't have apples and oranges.

Sara doesn't like the food in Antarctica. /It is not good.

\section{Antarctica, Item 9}

Think about whether you would like to visit Antarctica. Use what you have read in both Introducing Antarctica and A Letter from Antarctica to explain why you would or would not like to visit.

Process: Interpret and integrate ideas and information

\section{2 - Complete Comprehension}

These responses demonstrate complete comprehension by integrating information from across two different texts to fully support a personal opinion about text content. The response states or implies a personal opinion about visiting Antarctica and provides specific information from both texts - Introducing Antarctica and A Letter from Antarctica-to support the opinion. See chart below for appropriate ideas for each text.

\section{Example:}

No, because it is the coldest place on earth and there is nothing fresh to eat.

\section{1 - Partial Comprehension}

These responses demonstrate partial comprehension by supporting a personal opinion about text content with information from one text. The response states or implies a personal opinion about visiting Antarctica and provides specific information from one text-Introducing Antarctica OR A Letter from Antarctica-to support the opinion. See chart below for appropriate ideas for each text.

\section{Example:}

Yes, because many explorers have been there. 
Topics/Ideas from Each Text that May be Used to

Support Opinion

\section{Introducing Antarctica}

Extreme cold

Constant darkness

Penguins live there

Few people live there

Scientists stay there

\section{A Letter from Antarctica}

Food (freshness, tinned/dried, cooking, buying)

Cold

Famous explorers have gone there

\section{Antarctica, Item 11}

In this article, there are two different ways of finding out about Antarctica: Introducing Antarctica

\section{A Letter from Antarctica}

Which of these kinds of information do you find more interesting, and why?

Process: Examine and evaluate content, language, and textual elements

\section{1 - Acceptable Response}

These responses demonstrate understanding of the type of information presented in at least one of two texts. The response provides an opinion about which text is most interesting. In addition, it includes an explanation that accurately describes some element of the content, language, format, or tone of at least one of the texts.

Example:

Sara's letter because it makes you understand what it really feels like to be there. 


\section{The Little Lump of Clay}

by Diana Engel

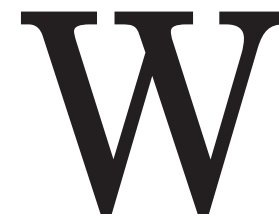

ay up high, in an old tower, there was a workshop. It was a pottery workshop, filled with barrels of colourful glazes, potter's wheels, kilns and, of course, clay. Near the window stood a big wooden bin with a heavy lid. There the clay was kept. Way at the bottom, squashed into the corner, was the oldest lump of clay. He barely remembered the last time he had been handled, a long time ago. Every day the heavy lid would open. Hands reached in, quickly grabbing bags or balls of clay. The little lump of clay could hear the cheerful sounds of people busy at their work.

"When will it be my turn?" he wondered. As each day passed in the darkness of the bin, the little lump of clay lost hope.

One day a large group of children came into the workshop with their teacher. Many hands reached into the bin. The little lump of clay was the last to be chosen, but he was out!

"Here's my big chance!" he thought, squinting in the light.

A boy put the clay on the potter's wheel, spinning it as fast as he could. "This is fun!" thought the little lump of clay. The boy tried pulling the clay up as the wheel went around. The little lump of clay felt the excitement of becoming something! After trying to make a bowl, the boy gave up. He pushed and pounded the clay into a neat ball.

"Time to clean up," said the teacher. The workshop was filled with the sounds of children sponging and wiping and washing and drying. Water dripped everywhere.

The boy plopped the lump of clay near the window and rushed to join his friends. After a while, the workshop emptied. The room was quiet and dark. The little lump of clay was terrified. Not only did he miss the moistness of the bin, he knew he was in danger.

"It's all over," he thought. "I'll just sit here and dry out until I'm as hard as a rock." 


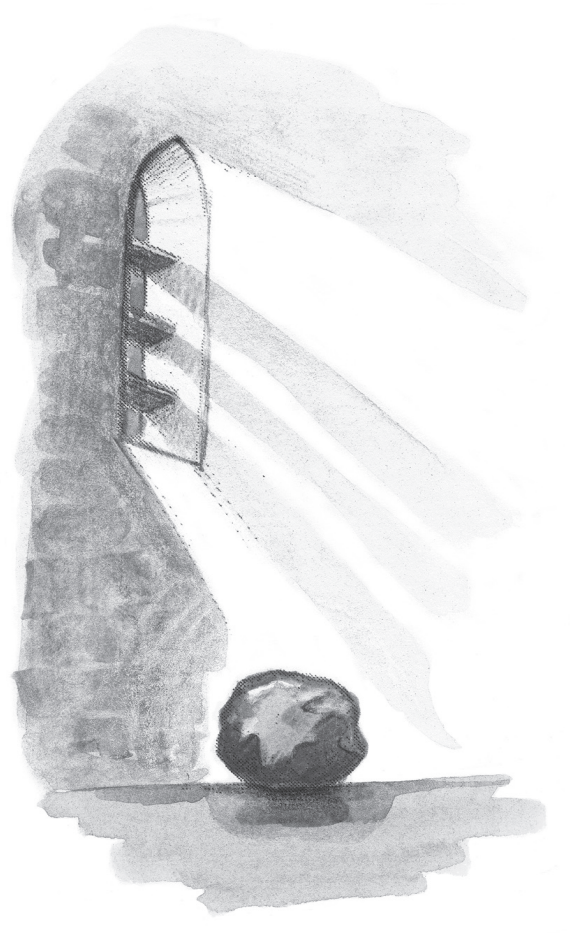

He sat by the open window, unable to move, feeling the moisture seep out of him. The sunlight beat down, the night breezes blew in, until he was rock hard. He was so hard he could hardly think. He only knew that he was filled with hopelessness.

But somewhere deep inside the little lump of clay, a tiny drop of moisture was left, and he refused to let it go.

"Rain," he thought.

"Water," he sighed.

"Please," he finally squeezed out of his dry hopeless self.

A passing cloud took pity on the little lump of clay, and a wonderful thing happened. Huge raindrops hammered through the open window, falling on the little lump of clay. All night it rained, and by morning he was as soft as his old self.

Voices drifted into the workshop.

"Oh no," said a woman. She was a potter who often used the workshop. "Someone has left the window open all weekend! We've got a mess to clean up. You can work with some clay while I find the towels," she said to her daughter.

The little girl saw the lump of clay sitting at the window.

"This looks like a perfect lump for me," she said.

Soon she was pressing and kneading the clay into pleasing shapes. To the little lump of clay, her fingers felt heavenly.

The girl thought as she worked, and her hands moved with purpose. The little lump of clay felt himself being gently pushed into a rounded, hollow shape. A few pinches, and he had a handle.

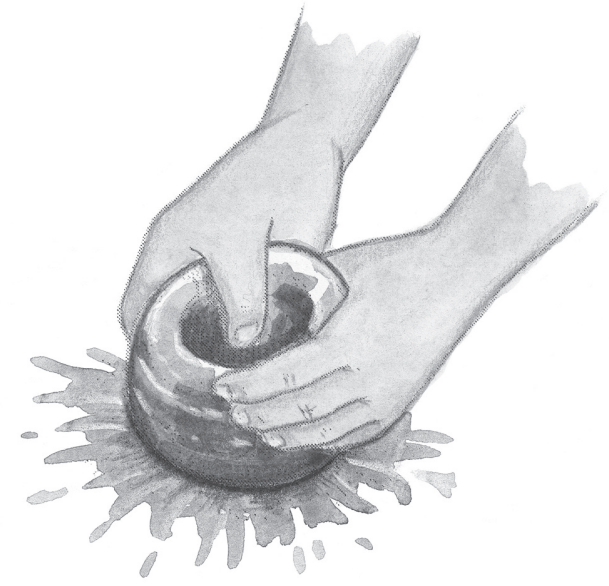


"Mommy, Mommy," called the girl, "I made a cup!"

"It's wonderful!" said her mother. "Put it on the shelf and it will be fired in the kiln. Then you can glaze it any colour you like."

Soon the little cup was ready to be taken to his new home. Now he lives on a shelf in the kitchen, next to the other cups and saucers and mugs. They are all very different and some are very beautiful.

"Breakfast!" calls the mother, setting the new cup on the table and filling him with hot chocolate.

The little girl holds him gently. How happy he feels with the smooth lines of his new shape. How well he does his job!

The little cup sits proudly. "At last—at last I am something."

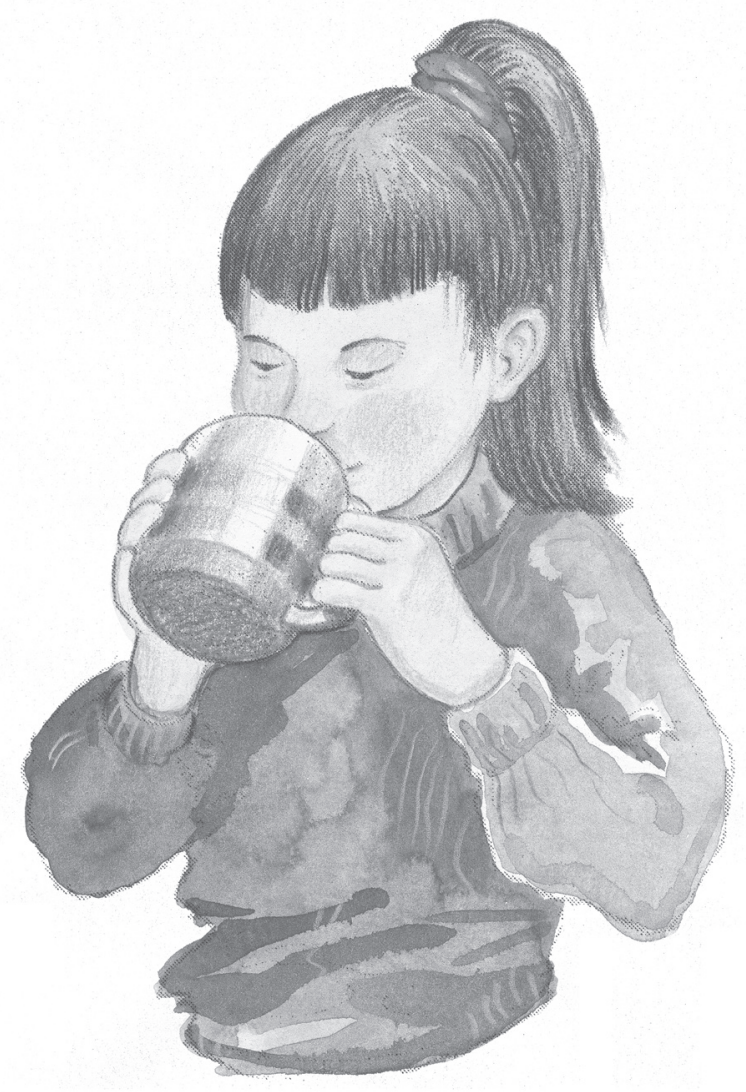




\section{Questions The Little Lump of Clay}

1. Number the sentences below in the order the events happened in the story. Number 1 has been done for you.

_ The rain made the lump of clay moist and soft.

_ A boy tried to make the lump of clay into a bowl.

_ A girl made the lump of clay into a cup.

_ The lump of clay dried out.

1 The lump of clay was in the bin.

2. Why was the lump of clay in the bin for such a long time?

(1)

3. At the beginning of the story, what did the lump of clay wish for?

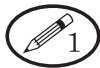


4. Why was the clay eventually taken out of the bin?

* A All the other lumps of clay were used.

B It was on top of the other lumps of clay.

(C) The boy chose that lump because he especially liked it.

D The teacher told the boy to use that lump.

5. What did the boy do that was careless?

A He left the clay on the potter's wheel.

B He was spinning the wheel as fast as he could.

* (C) He put the clay near the window.

D He pushed and pounded the clay.

6. The boy left the lump of clay in danger. What was the danger?

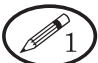


7. How did the lump of clay feel right after the boy left the pottery workshop?

* A satisfied

(B) scared

(C) angry

(D) proud

8. What wonderful thing happened after the lump of clay had been lying by the window for a long time? Why was this so wonderful for the lump of clay? 
9. Which words in the story show that the little girl knew what she wanted to make?

A 'her fingers felt heavenly.'

B 'The little girl saw the lump of clay.'

* (C) 'The little girl holds him gently.'

(D) 'her hands moved with purpose.'

10. Describe the different feelings the clay had at the beginning and the end of the story. Explain why his feelings changed.

(3) 
11. The little girl is an important person in this story. Explain why she was important to what happened.

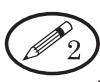

12. The author of the story writes about the lump of clay as if it were a person. What is the author trying to make you imagine?

A what it is like in the rain

* B how a lump of clay might feel

(C) what it is like to work with clay

D how it feels to make something

13. What is the main message of this story?

A People are easy to knead and shape like clay.

B There is a great deal of unhappiness in the world.

* (C) Everything is happiest when it finds a purpose.

D Pottery is the best way to do good in the world. 


\section{Clay, Item 1}

Number the sentences below in the order the events happened in the story. Number 1 has been done for you.

\section{_ The rain made the lump of clay moist} and soft.

A boy tried to make the lump of clay into a bowl.

A girl made the lump of clay into a cup. The lump of clay dried out.

1 The lump of clay was in the bin.

Process: Focus on and retrieve explicitly stated information and ideas

\section{1 - Acceptable Response}

These responses identify the appropriate order of story events. The response accurately numbers the sentences as shown below. In order to receive full credit, each sentence must have the appropriate number.

\section{Appropriate Ordering of Sentences}

4 The rain made the lump of clay moist and soft.

2 A boy tried to make the lump of clay into a bowl.

5 A girl made the lump of clay into a cup.

3 The lump of clay was dried out.

1 The lump of clay was in the bin.

\section{Clay, Item 2}

Why was the lump of clay in the bin for such a long time?

Process: Make straightforward inferences

\section{1 - Acceptable Response}

These responses provide an appropriate inference for the lump of clay's initial predicament. The response demonstrates understanding that the lump of clay was not as accessible as the other clay. It may focus on the fact that it was at the bottom or in a corner of the bin.

\section{Example:}

Because he was at the bottom.

Or, the response may focus on the fact that the other clay was always used first.

Example:

Because people used the other clay.

\section{Clay, Item 3}

At the beginning of the story, what did the lump of clay wish for?

Process: Make straightforward inferences

\section{1 - Acceptable Response}

These responses provide an appropriate inference for the lump of clay's feelings at the beginning of the story. The response demonstrates understanding that the lump of clay wanted to be used like the other clay in the bin (its short-term wish).

\section{Example:}

To be chosen.

Or, the response may focus on the outcomes of being used like the other clay (its long-term wish) and having a purpose or use, or having a sense of fulfillment.

\section{Example:}

To be made into an object and used a lot. 


\section{Clay, Item 6}

\section{The boy left the lump of clay in danger. What was the danger?}

Process: Interpret and integrate ideas and information

\section{1 - Acceptable Response}

These responses integrate ideas in the story to interpret the nature of the lump of clay's danger. The response demonstrates understanding that the lump of clay was in danger of drying out or becoming hard. (It is acceptable if the student's interpretation is that the clay is in danger of dying.)

Example:

The lump of clay's danger was that he might dry out.

\section{Clay, Item 8}

\section{What wonderful thing happened after the lump of clay had been lying by the window for a long time? Why was this so wonderful for the lump of clay?}

Process: Focus on and retrieve explicitly stated information and ideas

\section{2 - Complete Comprehension}

These responses demonstrate complete comprehension of events in the story related to the lump of clay's recovery. The response states that rain coming through the window was the wonderful thing that happened. In addition, the response explains that the rain made the clay wet again, or caused it not to become hard.

\section{Example:}

The wonderful thing was the rain because it moistened the clay.

\section{1 - Partial Comprehension}

These responses demonstrate partial comprehension of events in the story related to the lump of clay's recovery. The response describes the wonderful thing that happened as rain coming through the window, or as the clay becoming wet or soft again. However, the response does not make a connection between the rain and its physical effect on the clay.

\section{Example:}

It made it go really soft.

\section{Clay, Item 10}

Describe the different feelings the clay had at the beginning and the end of the story. Explain why his feelings changed.

Process: Interpret and integrate ideas and information

\section{3 - Extensive Comprehension}

These responses demonstrate extensive comprehension by integrating ideas from across the text to fully support an interpretation of why the clay's feelings changed during the story. The response provides an appropriate description of the clay's feelings at the beginning and at the end. It includes information from the story to explain why they changed. In the explanation the response demonstrates understanding of one of the following aspects of the clay's proud feelings about itself at the end of the story: fulfillment, usefulness, or beauty/aesthetics. See examples in chart below.

\section{Example:}

At the beginning the clay was sad. At the end he felt proud because he had become a cup.

\section{2 - Satisfactory Comprehension}

These responses demonstrate satisfactory comprehension by integrating ideas from across the text to support an interpretation of why the clay's feelings changed during the story. The response provides an appropriate description of the clay's feelings at the beginning and at the end. It includes information from the story to explain why they changed. However, the explanation for why they changed does not demonstrate understanding of one of the following aspects of the clay's proud feelings about itself at the end of the story: fulfillment, usefulness, or beauty/aesthetics. 


\section{Example:}

He was sad in the beginning. But he was happy in the end because of what the girl did.

Or, the response provides an appropriate explanation of his feelings at the beginning or the end (but not both) and in the explanation of that feeling demonstrates understanding of one of the following aspects of the clay's proud feelings about itself at the end: fulfillment, usefulness, or beauty/ aesthetics (see examples in chart below).

\section{Example:}

He is happy because he has been made into something, he is proud of his shape and he is proud of sitting on the shelf with all the other mugs.

\section{1 - Minimal Comprehension}

These responses demonstrate limited comprehension of how the clay's feelings changed during the story. The response provides an appropriate description of the clay's feelings at the beginning or at the end, or both, but does not include appropriate information from the story to explain why they changed.

\section{Example:}

He was sad in the beginning. But he was happy in the end.

Or, the response provides an explanation of the change that demonstrates feelings of fulfillment, usefulness, or aesthetics, but does not describe his feelings at the beginning or the end.

Example:

He became something useful.

\section{Explanations that Support the Clay's Feelings of Pride at End of Story: Examples}

The following examples represent some of the different ways students may support the clay's feelings of pride at the end of the story.

\section{Fulfillment}

The girl made him into something.

He had become something.

\section{Usefulness}

He had a job to do.
He became a cup.

People could use him.

He was something useful.

\section{Beauty/Aesthetic}

The girl made him beautiful.

He liked his new shape.

\section{Clay, Item 11}

\section{The little girl is an important person in this story. Explain why she was important to what happened.}

Process: Interpret and integrate ideas and information

\section{2 - Complete Comprehension}

These responses demonstrate complete comprehension of the story's supporting character by integrating ideas from across the text to interpret the character's significance to the story's outcome. The response explains the little girl's central role as the facilitator of the clay's change and addresses how her role contributes to the theme of fulfillment.

\section{Example:}

She made the clay into something beautiful.

\section{1 - Partial Comprehension}

These responses demonstrate partial comprehension of the supporting character's significance in the story. The response identifies the central action of the little girl as the facilitator of the clay's change but does not address the theme of fulfillment.

\section{Example:}

She was the one who made the clay into a cup. 


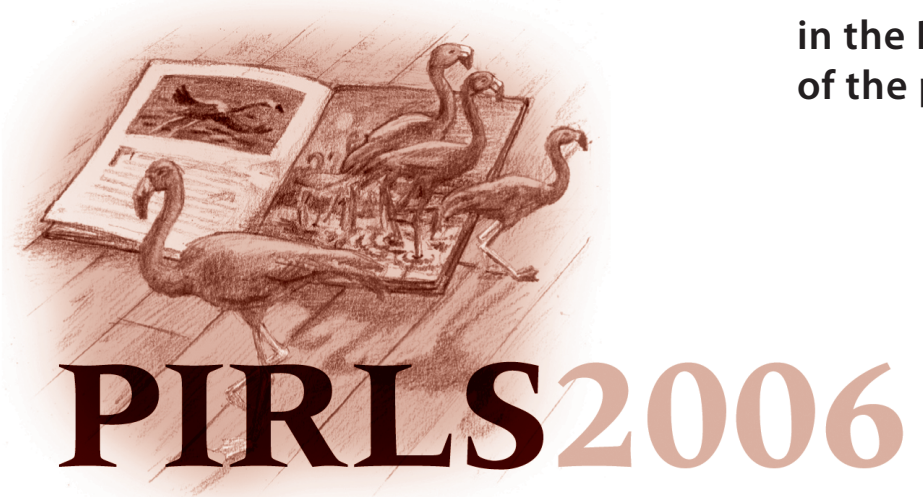

Text for "An Unbelievable Night" can be found in the PIRLS Reader Booklet, found in the back of the publication.
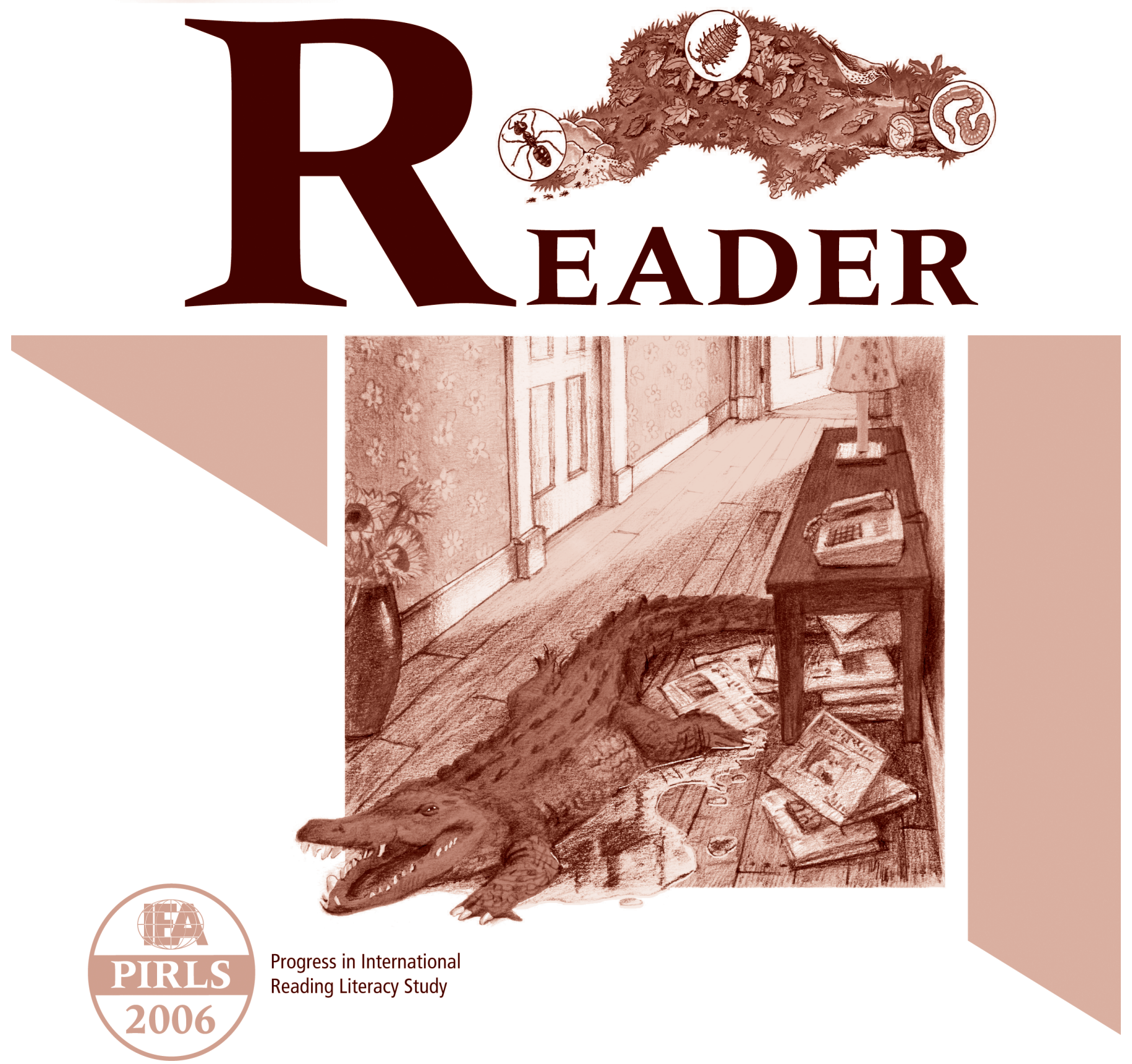

Progress in International

Reading Literacy Study 
1. What was the first sign that something unusual was happening?

A A pile of newspapers began to move.

(B) Anina saw the magazine cover.

(C) The door to her room was broken.

* D Anina heard a hissing sound.

2. Where did the crocodile come from?

(A) the bathroom

* (B) a magazine cover

(C) under the bed

(D) a nearby river

3. Which words tell you that Anina was frightened?

* A "frozen to the spot"

(B) "could not believe her eyes"

(C) "let her breath out"

D "sounded like a quiet hissing" 
4. Why did Anina think the crocodile was going to attack?

A It showed its long row of teeth.

(B) It let out a loud hissing sound.

(C) It started grunting and snorting.

* (D) It swung its tail back and forth.

5. Put the following sentences in the order in which they happened in the story.

The first one has been done for you.

Anina saw the crocodile.

The crocodile ate two flamingos.

Anina tried to explain the broken door to her parents.

1 Anina started to walk to the bathroom.

Anina ran to the bedroom and slammed the door.

6. Why did Anina call the flamingos?

(1) 
7. How did the bedroom door get broken?

* A The crocodile's tail pushed through it.

(B) The big vase cracked against it.

(C) The flamingo's sharp beak crashed into it.

D The bed smashed against it.

8. How did the magazine help Anina? Write two ways.

(1) 1 .

12 .

9. At the end of the story, how did Anina feel toward the flamingos?

(A) guilty

(B) cautious

* (C) grateful

(D) annoyed 
10. Name one thing Anina had difficulty explaining to her parents.

(1)

11. You learn what Anina was like from the things she did.

Describe what she was like and give two examples of what she did that show this. 
12. The author does not tell us whether Anina's adventure was all a dream.

Give one piece of evidence that it may have been a dream.

(1)

Give one piece of evidence that it may not have been a dream.

(1) 


\section{Unbelievable Night, Item 5}

Put the following sentences in the order in which they happened in the story. The first one has been done for you.

Anina sees the crocodile.

The crocodile ate two flamingos. Anina tried to explain to her parents why the door is broken.

1 Anina started to walk to the bathroom Anina ran to the bedroom and slammed the door.

Process: Make straightforward inferences

\section{1 - Acceptable Response}

The response accurately numbers the sentences as shown below. In order to receive full credit, each sentence must have the appropriate number.

\section{Appropriate Ordering of Sentences}

2 Anina sees the crocodile.

4 The crocodile ate two flamingos.

5 Anina tried to explain to her parents why the door is broken.

1 Anina started to walk to the bathroom.

3 Anina ran to the bedroom and slammed the door.

\section{Unbelievable Night, Item 6}

\section{Why did Anina call the flamingos?}

Process: Make straightforward inferences

\section{1 - Acceptable Response}

The response demonstrates an understanding that the flamingos were food to the crocodile.

Example:

\section{To feed the crocodile.}

Or, the response demonstrates a general understanding that Anina used the flamingos to help her keep safe from the crocodile.

Example:

So they would protect her from the crocodile.

\section{Unbelievable Night, Item 8}

How did the magazine help Anina? Write two ways.

Process: Interpret and integrate ideas and information

\section{2 - Complete Comprehension}

The response identifies two ways that Anina used the magazine to help her situation, either by teaching her about the animals from the magazine, helping her to get the animals out of her house, or feeding the crocodile. See the list below for appropriate ways that the magazine helped Anina.

\section{1 - Partial Comprehension}

The response identifies only one way the magazine helped her as listed below. The second way identified may be inaccurate or too vague.

\section{How the Magazine Helped Anina}

\section{Acceptable ideas:}

It told her that when crocodiles swing their tails/whip the water it means that they are going to attack.

It showed her where the crocodile had come from.

It provided the flamingoes. /It gave her something to feed to the crocodile.

It helped her to get rid of the crocodile/flamingoes (by sending them back on to the pages).

\section{Unbelievable Night, Item 10}

\section{Name one thing Anina had difficulty explaining to her parents.}

Process: Focus on and retrieve explicitly stated information

\section{1 - Acceptable Response}

The response identifies one of the things in the house that Anina might have had trouble explaining: the wet spot on the floor, the broken door, her mother's (missing) hat, the broken vase, or scattered sunflowers. 


\section{Unbelievable Night, Item 11}

You learn what Anina was like from the things she did. Describe what she was like and give two examples of what she did that show this.

Process: Interpret and integrate ideas and information

\section{3 - Extensive Comprehension}

The response provides at least one valid, appropriate description of what Anina was like (e.g., clever, fast thinker, innovative, creative, resourceful, brave, cautious, fearful, frightened, scared, appreciative, grateful, nice, good) with two things that she said or did in the story that support the description and illustrate her character.

\section{Example:}

She was brave to come out of her room and then put the magazine right under the crocodile's nose.

\section{2 - Satisfactory Comprehension}

The response provides at least one valid, appropriate description and only one supporting thing that she did.

\section{Example:}

She was clever because she made a plan to get rid of the crocodile.

\section{1 - Partial Comprehension}

The response provides an appropriate description with a reason that is vague or general.

\section{Example: \\ Anina was clever. She used the magazine.}

Or, the response provides at least one appropriate description without a reason.

\section{Example:}

Anina was a fast thinker.

Or, the response provides at least one appropriate reason without a description.

\section{Example:}

She let the flamingoes out of the magazine and she got the crocodile to go back to its home in the magazine.

\section{Unbelievable Night, Item 12}

The author does not tell us whether Anina's adventure was all a dream. Give one piece of evidence that it may have been a dream. Give one piece of evidence that it may not have been a dream.

Process: Examine and evaluate content, language, and textual elements

\section{2 - Complete Comprehension}

The response provides one piece of text-based evidence that Anina's adventure may have been a dream, and one piece of evidence that it may not have been a dream. See the list below for appropriate evidence for why it may or may not have been a dream.

\section{1 - Partial Comprehension}

The response provides one piece of text-based evidence that Anina's adventure may have been a dream, OR one piece of evidence that it may not have been a dream as listed below.

Evidence for Anina's Adventure Being a Dream/ Not a Dream

Acceptable evidence it may have been a dream:

It was nighttime and she was half awake.

There were (wild) animals in house.

Magazines can't come to life.

Acceptable evidence it may NOT have been a dream:

Her mother's hat was missing the next morning.

The door was cracked.

The carpet had a wet spot.

The vase was broken.

The sunflowers were scattered on the floor. 


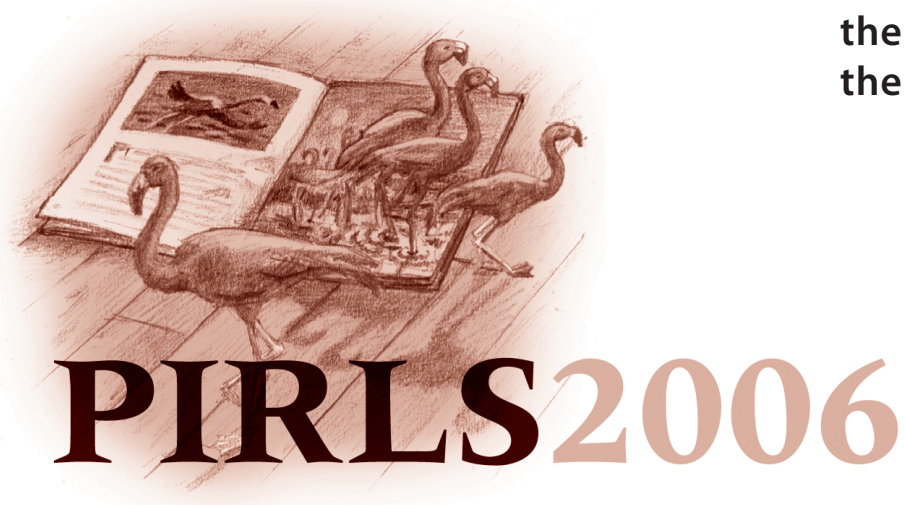

Text for "Searching for Food" can be found in the PIRLS Reader Booklet, found in the back of the publication.
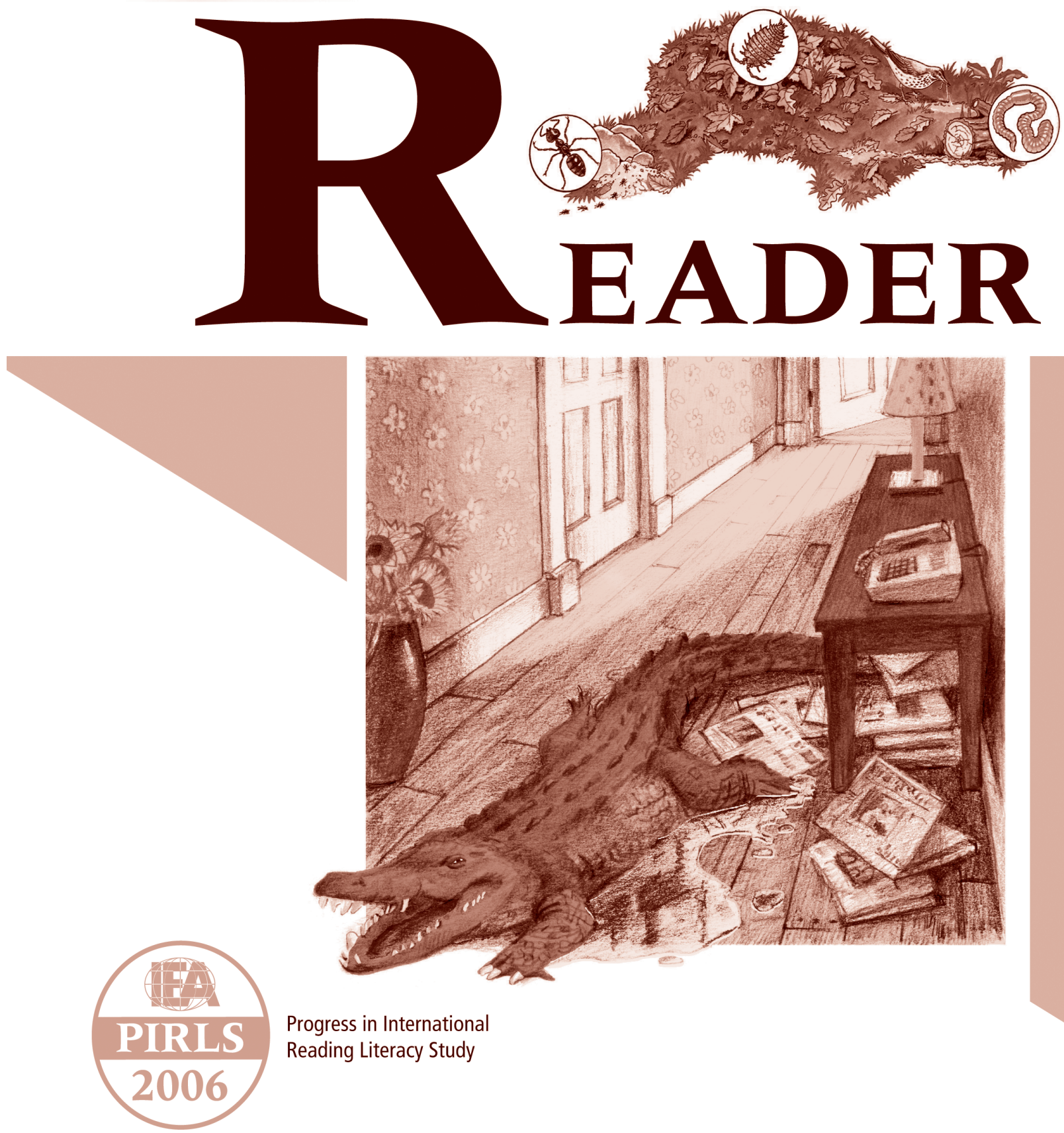

Progress in International

Reading Literacy Study 


\section{Questions: Searching for Food}

1. What is the main purpose of the article?

* A to describe different projects you can do

(B) to give information about ant trails

(C) to show what small creatures look like

(D) to explain what worms eat

2. What is one thing you should do to take care of the creatures?

(A) search for them under rocks and stones

(B) find out all about them

(C) collect as many as you can

* (D) put them back where you found them 


\section{Questions 3-5 are about the Ant Project}

3. Why do you put the apple by the ants' nest?
(A) to block the ants' trail
* (B) so the ants will make a trail
(C) to confuse the ants
(D) so the ants will scurry around

4. Once an ant finds some food, how do the other ants from the nest find it too?
(A) They watch the first ant and follow it.
(B) They run around until they find the food.
* (C) They sense the scent left by the first ant.
(D) They smell the food on the piece of paper.

5. Why do the ants scurry around after you've sprinkled the soil? 
6. How do pill bugs find the food?

(A) They walk down the passage.

* (B) They sense food with their antennae.

(C) They follow the scent trail.

(D) They see the food in the dark.

7. Look at the picture for Study Pill Bugs. How does the picture help you to know what to do in the experiment? 
8. Why do you need to let your pill bugs walk along the passage before putting the leaves in the box?

A To see if they can learn the maze.

* B To see what they do when there is no food.

(C) To see if the box is put together correctly.

D To see which ones turn which way.

9. In Step 3 of the pill bugs project, what do you think will happen if you move the damp leaves to the left corner of the box?

1

10. What is similar in the way ants and pill bugs find their food?

(1) 
11. Number the steps in the order you would follow to make a wormery. The first one has been done for you. put the bottle in the shoebox

1 poke holes in the top of the shoebox

_ drop in the worms add potato and onion fill the bottle with soil and sand

12. Explain why it is important to put layers of soil and sand in the bottle. 
13. Explain why putting the onion and potato on the surface of the soil is important to the wormery project.

(2)

14. Each project has What happens and Why in a separate box. What is the purpose of these boxes?

(A) to explain the steps of the project

B to tell you what you need for the project

(C) to tell you what to do when you have finished

* (D) to explain what you have seen

15. Which of the three projects did you find the most interesting? Use information from the text to explain your answer. 


\section{Searching for Food, Item 5}

\section{Why do the ants scurry around after} you've sprinkled the soil?

Process: Interpret and integrate ideas and information

\section{1 - Acceptable Response}

The response demonstrates understanding that the ants scurry because they have lost their trail (and therefore have to make a new one) or because they are looking for the food.

Example:

They have to make a new trail.

\section{Searching for Food, Item 7}

\section{Look at the picture for Study Pill Bugs.} How does the picture help you to know what to do in the experiment?

Process: Examine and evaluate content, language, and textual elements

\section{2 - Complete Comprehension}

The response provides an explanation of the necessity of the picture to know how to make the box, to know where to put things in the box, or to know what the box should look like.

\section{Example:}

It helps you to understand where you have to put the cardboard strips.

Or, the response shows understanding that it is the visual image of the box that makes it possible to make one the same way.

Example:

It shows what it is meant to look like.

\section{1 - Partial Comprehension}

The response describes the features of the picture without indicating how they are useful to doing the experiment.
Example:

It uses arrows and labels.

\section{Searching for Food, Item 9}

In Step 3 of the pill bugs project, what do you think will happen if you move the damp leaves to the left corner of the box?

Process: Interpret and integrate ideas and information

\section{1 - Acceptable Response}

The response provides the appropriate inference from the text that the pill bugs will (eventually) turn to the left toward the leaves. Note that it is appropriate to state that the pill bugs will turn to where the food is or will turn the other way from the original directions in the experiment without having to specifically mention the left corner.

Example:

They will sense the food and find it.

\section{Searching for Food, Item 10}

What is similar in the way ants and pill bugs find their food?

Process: Interpret and integrate ideas and information

\section{1 - Acceptable Response}

The response demonstrates understanding that ants and pill bugs find their food using their antennae or feelers to sense their food.

\section{Example:}

They use their feelers. 


\section{Searching for Food, Item 11}

Number the steps in the order you would follow to make a wormery.

The first one has been done for you.

_ put the bottle in the shoebox

1 poke holes in the top of the shoebox drop in the worms add potato and onion fill the bottle with soil and sand

Process: Make straightforward inferences

\section{1 - Acceptable Response}

The response accurately numbers the steps as shown below.

In order to receive full credit, each step must have the appropriate number.

\section{Appropriate Ordering of Steps \\ 5 put the bottle in the shoebox \\ 1 poke holes in the top of the shoebox \\ 4 drop in the worms \\ 3 add potato and onion \\ 2 fill the bottle with soil and sand}

\section{Searching for Food, Item 12}

\section{Explain why it is important to put layers} of soil and sand in the bottle.

Process: Interpret and integrate ideas and information

\section{1 - Acceptable Response}

The response demonstrates understanding that the effect of the tunneling (the mixing of the soil and sand) will be visible because of the layers.

\section{Example:}

To make it possible to see the effect of the worms tunnelling.

\section{Searching for Food, Item 13}

Explain why putting the onion and potato on the surface of the soil is important to the wormery project.

Process: Interpret and integrate ideas and information

\section{1 - Acceptable Response}

The response provides an appropriate explanation for putting the food on the surface in order for the worms to tunnel up to the top to eat (and tunnel down to avoid the light).

\section{Example:}

To make the worms go to the top.

\section{Searching for Food, Item 15}

Which of the three projects did you find the most interesting? Use information from the text to explain your answer.

Process: Interpret and integrate ideas and information

\section{2 - Complete Comprehension}

The response selects a project with specific information referring to the text, or may provide an inference clearly reflecting specific information in the text.

Example:

The ant project because I would like to see if ants would make a trail with food other than an apple.

\section{1 - Partial Comprehension}

The response selects a project and provides a general explanation that is related to the text, but could apply to any of the projects.

\section{Example:}

The pill bug project because it would be fun to find them. 


Appendix E

Item Descriptions Developed During the PIRLS 2006 Benchmarking 


\section{Low Benchmark Items (400)}

Literary

Credit Awarded

Constructed

Response

\begin{tabular}{lll}
\hline E01 & Locate and retrieve explicitly stated detail from the beginning of the text & \\
\hline E04 & Recognize a central story event & 1 of 2 \\
\hline F09 & Reproduce 1 (of 2) explicitly stated character actions \\
\hline U07 & Recognize a detail of a central event in the story \\
\hline U09 & Make a straightforward inference based on a specified part of the text
\end{tabular}

\section{Informational}

\begin{tabular}{lll}
\hline A01 & Locate and reproduce explicitly stated information from the beginning of the text & 1 of 1 \\
\hline A04 & Make 1 (of 2) straightforward inferences based on specific information & 1 of 2 \\
\hline A06 & $\begin{array}{l}\text { Locate and match explicitly stated information at the beginning of the section/ } \\
\text { subheading }\end{array}$ & 1 of 2 \\
\hline A08 & $\begin{array}{l}\text { Locate and reproduce 1 (of 2) pieces of explicitly stated information from a } \\
\text { paragraph in a specified part of the text }\end{array}$ & 1 of 2 \\
\hline K01 & $\begin{array}{l}\text { Locate and reproduce 1 (of 2) pieces of explicitly stated information from several } \\
\text { available in the same part at the beginning of the specified text }\end{array}$ \\
\hline L01 & $\begin{array}{l}\text { Locate explicitly stated information at the beginning of the text (continuous text } \\
\text { without subheadings) }\end{array}$ & \\
\hline
\end{tabular}




\section{Intermediate Benchmark Items (475)}

Literary

Credit Awarded

Constructed

Response

C03 Given a specified point in the story, infer the character's aims from narration/ 1 of 1 description of events and the character's reaction to the events

C05 Locate a relevant detail and make a straightforward inference to recognize a character's action

C07 Make a straightforward inference to identify the main character's feelings at a specified point in the story

C08 Reproduce an event by making connections between clearly related sentences 1 of 2

C10 Give a simple statement about a main character's feeling that is clearly suggested 1 of 3 at a specified point in the story

C11 Give the main character's importance to the plot 1 of 2

E02 Locate and make an inference about an embedded detail

E03 Locate a relevant detail and make a straightforward inference to recognize a character's action

E05 Locate a specified event and reproduces 1 (of 2) explicitly stated character actions, 1 of 2 out of five available

E06 Locate a specified episode and recognize a relevant action

E07 Give a simple explanation of a metaphor 1 of 2

F02 Combine, retrieve, and visualize concrete descriptive information and identifies matching picture

F03 Identify and infer reason for an action from a dialogue

F04 Retrieve a concrete, relevant, explicitly stated detail in the text

F10 Give a simple statement about a character's feeling that is clearly suggested at a 1 of 1 specified point in the story

U01 Make an inference to recognize a story event

U02 Make a straightforward inference to identify an important event in the story

U05 Sequence story events 1 of 1 


\section{Intermediate Benchmark Items (475) (Continued)}

\begin{tabular}{llc} 
U06 & Make an inference to explain character's action & $\begin{array}{c}\text { Credit Awarded } \\
\text { Constructed } \\
\text { Response }\end{array}$ \\
\hline U08 & Interpret to provide 1 (of 2) explanations of the purpose of a plot device & 1 of 1 \\
\hline U10 & Provide a relevant detail from the story & 1 of 1 \\
\hline U12 & $\begin{array}{l}\text { Make a connection with personal experience to interpret and provide a story detail } \\
\text { as evidence }\end{array}$ & 1 of 2 \\
\hline Y07 & \begin{tabular}{l} 
Recognize the motivation for a central story event \\
\hline Y09
\end{tabular} & $\begin{array}{l}\text { Locate a central event and make a straightforward inference to provide 1 (of 2) } \\
\text { character actions, out of several available }\end{array}$ \\
\hline
\end{tabular}

\section{Informational}

\begin{tabular}{lll}
\hline A03 & Locate and reproduce explicitly stated information from further in the text & 1 of 1 \\
\hline A05 & Recognize which piece of information an example illustrates & \\
\hline A07 & $\begin{array}{l}\text { Make 2 (of 3) straightforward inferences based on specific information in a single } \\
\text { paragraph }\end{array}$ & 2 of 3 \\
\hline A08 & $\begin{array}{l}\text { Locate and reproduce 2 pieces of information from a paragraph in a specified part } \\
\text { of the text }\end{array}$ & 2 of 2 \\
\hline A09 & $\begin{array}{l}\text { State a preference based on connecting text and personal experience and support } \\
\text { it with 1 (of 2) pieces of straightforward evidence }\end{array}$ & 1 of 2 \\
\hline K01 & $\begin{array}{l}\text { Locate and reproduce 2 pieces of explicitly stated information from a specified part } \\
\text { of the text }\end{array}$ & 2 of 2 \\
\hline K02 & $\begin{array}{l}\text { Make straightforward inference to locate and reproduce 3 pieces of explicitly stated } \\
\text { information from further in the text from several in same paragraph }\end{array}$ & 1 of 1 \\
\hline K12 & $\begin{array}{l}\text { Locate at least 2 text sections, categorize information, and reproduce specific } \\
\text { details to partially complete a table (3/6 entries) }\end{array}$ & 1 of 3 \\
\hline
\end{tabular}




\section{Intermediate Benchmark Items (475) (Continued)}

\begin{tabular}{llc}
\hline L03 & $\begin{array}{l}\text { Make a straightforward inference to locate and reproduce explicitly stated } \\
\text { information from further in the text (continuous text without subheadings) }\end{array}$ & $\begin{array}{c}\text { Credit Awarded } \\
\text { Constructed } \\
\text { Response }\end{array}$ \\
\hline L-4 & $\begin{array}{l}\text { Locate and reproduce explicitly stated text from further in the text (continuous text } \\
\text { without subheadings) }\end{array}$ & 1 of 3 \\
\hline N01 & $\begin{array}{l}\text { Recognize the main message of a brochure } \\
\text { N05 }\end{array}$ & $\begin{array}{l}\text { Locate a specified part of the text and recognize explicitly stated information } \\
\text { embedded in a list }\end{array}$ \\
\hline N06 & $\begin{array}{l}\text { Locate a specified part of the text and recognize explicitly stated information } \\
\text { embedded in a list }\end{array}$ & \\
\hline S02 & $\begin{array}{l}\text { Locate explicitly stated information at the beginning of the text at an unspecified } \\
\text { place }\end{array}$ & \\
\hline S03 & $\begin{array}{l}\text { Make an inference about the best explanation using several pieces of evidence in a } \\
\text { specified section of the text }\end{array}$ & \\
\hline S06 & $\begin{array}{l}\text { Locate a major section and recognize explicitly stated explanation embedded in a } \\
\text { fact box }\end{array}$ & \\
\hline S09 & Integrate information within the text to infer and provide an explanation & 1 of 1 \\
\hline
\end{tabular}




\section{High Benchmark Items (550)}

\section{Literary}

Credit Awarded

Constructed

Response

\begin{tabular}{|c|c|c|}
\hline $\mathrm{C01}$ & Establish the sequence of key (main) events & 1 of 1 \\
\hline $\mathrm{CO}$ & Make a straightforward inference to explain the cause of an event & 1 of 1 \\
\hline $\mathrm{CO4}$ & $\begin{array}{l}\text { Locate the relevant part of the text and make an inference to recognize the } \\
\text { explanation of an action }\end{array}$ & \\
\hline $\mathrm{CO6}$ & Make an inference and provides the immediate problem of the character & 1 of 1 \\
\hline C10 & $\begin{array}{l}\text { Contrast clearly suggested feelings of a character at the beginning and end of the } \\
\text { story and give an explanation for the change (plot-based) }\end{array}$ & 2 of 3 \\
\hline $\mathrm{C} 12$ & Recognize the idea of personification & \\
\hline $\mathrm{C} 13$ & Recognize main"message" stated in an abstract form & \\
\hline E05 & Locate a specified event and reproduces 2 explicitly stated character actions & 2 of 2 \\
\hline E09 & Make an inference to explain a character's action & 1 of 2 \\
\hline E10 & $\begin{array}{l}\text { Distinguish a relevant part of the text and evaluate the form of the language to } \\
\text { reproduce appropriate descriptive words }\end{array}$ & 1 of 1 \\
\hline E11 & Locate the relevant part of the text to interpret the significance of an event & \\
\hline E12 & Interpret a character's actions to provide either a description or example & 1 of 2 \\
\hline F01 & $\begin{array}{l}\text { Identify the narrator (in a first person story) from a range of clues in the first part of } \\
\text { the story and confirmed by the text and the pictures }\end{array}$ & \\
\hline F05 & Make an inference to recognize the main character's feelings & \\
\hline F06 & Make an inference to explain a character's reaction & 1 of 1 \\
\hline F07 & Give a simple statement about a character's action & 1 of 2 \\
\hline F08 & Infer the significance of a character's action from subsequent events & 1 of 1 \\
\hline F09 & $\begin{array}{l}\text { Reproduce explicitly stated actions of a character in relation to a particular goal } \\
\text { from two different parts of the text }\end{array}$ & 2 of 2 \\
\hline
\end{tabular}




\section{High Benchmark Items (550) (Continued)}

\section{Credit Awarded \\ Constructed \\ Response}

\begin{tabular}{|c|c|c|}
\hline F11 & Recognize the idea of ending the story with a joke & \\
\hline $\mathrm{F} 12$ & Distinguish a relevant detail of the text and interpret a feeling of the narrator & 1 of 2 \\
\hline F13 & $\begin{array}{l}\text { Recognize a conclusion to be drawn from the story based on an interpretation of } \\
\text { the whole text }\end{array}$ & \\
\hline U03 & Locate an embedded metaphorical phrase and infer its meaning & \\
\hline U04 & Locate the relevant part of the text and make a straightforward inference & \\
\hline U08 & Interpret to provide 2 explanations of the purpose of a plot device & 2 of 2 \\
\hline Y01 & $\begin{array}{l}\text { Follow a semantic chain to distinguish relevant details and recognize a connection } \\
\text { between appearance and imagery }\end{array}$ & \\
\hline Y02 & Interpret and generalize to recognize a summary of a character's attributes & \\
\hline Y04 & Locate and retrieve embedded detail & \\
\hline Y05 & Infer a character trait from an example by recognizing a synonym & \\
\hline Y06 & Locate and retrieve embedded detail & \\
\hline Y08 & Locate the relevant event in the story and make a straightforward inference & \\
\hline Y09 & $\begin{array}{l}\text { Locate a central event and make a straightforward inference to provide } 2 \\
\text { character actions }\end{array}$ & 2 of 2 \\
\hline Y11 & Locate a relevant part of the text and recognize the meaning of a metaphor & \\
\hline Y12 & Infer a character trait and support it with a specific action & 2 of 2 \\
\hline Y13 & Interpret and integrate story events and character actions to describe a character & 1 of 3 \\
\hline Y14 & $\begin{array}{l}\text { State a title preference based on evaluating story events and characters' actions } \\
\text { and supports it with } 1 \text { piece of plot-based evidence }\end{array}$ & 1 of 2 \\
\hline
\end{tabular}




\section{High Benchmark Items (550) (Continued)}

Informational

\begin{tabular}{|c|c|c|}
\hline & & $\begin{array}{c}\text { Credit Awarded } \\
\text { Constructed } \\
\text { Response }\end{array}$ \\
\hline $\mathrm{A} 02$ & Locate and distinguish relevant information & \\
\hline A07 & $\begin{array}{l}\text { Make } 3 \text { (of } 3 \text { ) straightforward inferences based on specific information available in } \\
\text { a single paragraph }\end{array}$ & 3 of 3 \\
\hline A10 & $\begin{array}{l}\text { Apply knowledge of headings to locate a specific piece of information and } \\
\text { recognize the section to which it belongs }\end{array}$ & \\
\hline A11 & Explain a preference based on evaluating features of 2 sections & 1 of 1 \\
\hline $\mathrm{KO3}$ & Recognize an explanation of a metaphor in a picture caption & \\
\hline K05 & $\begin{array}{l}\text { Make a straightforward inference to locate a text box and follow a reference chain } \\
\text { to reproduce a brief scientific explanation }\end{array}$ & 1 of 1 \\
\hline K06 & $\begin{array}{l}\text { Make an inference about the best explanation from difficult to locate } \\
\text { scientific information }\end{array}$ & \\
\hline K07 & $\begin{array}{l}\text { Locate } 1 \text { (of 2) specified text boxes and make an inference to reproduce a brief } \\
\text { scientific explanation }\end{array}$ & 1 of 2 \\
\hline K09 & Recognize the function of a presentational device & \\
\hline K11 & Integrate information within the text to infer and reformulate information & \\
\hline K12 & $\begin{array}{l}\text { Locate } 3 \text { sections, categorize information, and reproduce specific details to nearly } \\
\text { complete a table ( } 5 / 6 \text { entries) }\end{array}$ & 2 of 3 \\
\hline L05 & Recognize an explanation of an abstract metaphor & \\
\hline LO6 & Make an inference to provide textual support for an idea & 1 of 1 \\
\hline L08 & $\begin{array}{l}\text { Make a straightforward inference to identify and reproduce explicitly stated } \\
\text { information embedded in continuous text without subheads }\end{array}$ & 1 of 2 \\
\hline L10 & $\begin{array}{l}\text { Demonstrate interpretation of an abstract idea by providing an example from } \\
\text { continuous text }\end{array}$ & 1 of 2 \\
\hline$\underline{L} 11$ & Evaluate content and generalize to recognize the most appropriate title & \\
\hline L12 & Evaluate textual elements and content to provide author's point of view & 1 of 2 \\
\hline
\end{tabular}




\section{High Benchmark Items (550) (Continued)}

\section{Credit Awarded \\ Constructed \\ Response}

N02 Use subheadings to locate the appropriate section and reproduce 2 pieces of 1 of 1 explicitly stated information

N04 Apply knowledge of headings to locate a specific piece of information and recognize the section to which it belongs

N07 Locate information within a specified part of the text and make an inference about unfamiliar vocabulary

N08 Use information from a specified part of the text to infer and provide 1 of 1 an explanation

N09 Locate and distinguish relevant information from a table

N11 Locate a specified table of information and show understanding of 1 (of 2) uses of 1 of 2 the information

N12 Evaluate the content of 2 specified sections of non-textual information and give 1 of 2 1 (of 2) reasons for a preference

S01 Recognize main purpose of highly detailed 3-part text

SO4 Locate and understand scientific information to recognize an explanation

S05 Integrate information within the text to infer and provide an explanation 1 of 1

S10 Integrate information across 2 sections to identify a similarity 1 of 1

S15 Explain a preference based on evaluating 3 sections with scientific information 1 of 2 


\section{Advanced Benchmark Items (625)}

Literary

\section{Credit Awarded Constructed Response}

\begin{tabular}{lll}
\hline C08 & Describes cause-and-effect relationship & 2 of 2 \\
\hline C09 & Locate a descriptive phrase and recognize what it shows about the character & \\
& $\begin{array}{l}\text { Contrast clearly stated feelings at the beginning and end and give interpretation/ } \\
\text { larger significance of reasons for change (integrate and interpret ideas from across } \\
\text { the text) }\end{array}$ & 3 of 3 \\
\hline C11 & Describe abstract/larger significance function of character in developing theme & 2 of 2 \\
\hline E07 & Explain both parts of a metaphor in terms of the story & 2 of 2 \\
\hline E08 & Locate the relevant part of the text to interpret the significance of an event & \\
\hline E12 & Interpret a character's actions to provide a description with a supporting example & 2 of 2 \\
\hline F07 & Integrate ideas across text to interpret the character's feelings about the setting & 2 of 2 \\
\hline F12 & Interpret initial feelings and contrast with feeling at the end or supports feelings & 2 of 2 \\
\hline U11 & Interpret and integrate story events and character actions to describe a character & 2 of 3 \\
\hline U12 & $\begin{array}{l}\text { Make a connection with personal experience to evaluate the story from opposite } \\
\text { perspectives and support each with evidence }\end{array}$ & 2 of 2 \\
\hline Y03 & Locate and interpret figurative language to provide an explanation & 1 of 1 \\
\hline Y10 & $\begin{array}{l}\text { Interpret the motivation for a character's words by providing an example from } \\
\text { the story }\end{array}$ & 1 of 1 \\
\hline Y13 & $\begin{array}{l}\text { Interpret and integrate story events and character actions to describe a character } \\
\text { with a supporting detail from the text }\end{array}$ & 2 of 3 \\
\hline Y14 & $\begin{array}{l}\text { State a title preference based on evaluating story events and characters' actions } \\
\text { and explain the choice in terms of the significance or central role of the character }\end{array}$ & 2 of 2 \\
\hline
\end{tabular}




\section{Advanced Benchmark Items (625) (Continued)}

\section{Informational}

\begin{tabular}{|c|c|c|}
\hline & & $\begin{array}{l}\text { Credit Awarded } \\
\text { Constructed } \\
\text { Response }\end{array}$ \\
\hline $\mathrm{A} 04$ & Interpret information from 2 separate sections of text, further into the text & 2 of 2 \\
\hline A09 & $\begin{array}{l}\text { State a preference based on connecting text and personal experience and support } \\
\text { it with evidence from } 2 \text { different types of text }\end{array}$ & 2 of 2 \\
\hline K04 & $\begin{array}{l}\text { Locate and distinguish relevant scientific information embedded across several } \\
\text { text boxes }\end{array}$ & \\
\hline K07 & $\begin{array}{l}\text { Locate } 2 \text { specific text boxes and make } 2 \text { inferences to reproduce } 2 \text { explicitly stated } \\
\text { pieces of scientific information }\end{array}$ & 2 of 2 \\
\hline K08 & $\begin{array}{l}\text { Locate and distinguish relevant scientific information across several text boxes and } \\
\text { apply it to a given situation }\end{array}$ & \\
\hline K10 & Integrate scientific information from 3 text boxes to explain a sequence & 2 of 2 \\
\hline K12 & $\begin{array}{l}\text { Locate } 3 \text { text sections, distinguish, and categorize information and reproduce } \\
\text { specific details to fully complete a table }\end{array}$ & 3 of 3 \\
\hline L02 & $\begin{array}{l}\text { Distinguish and integrate relevant information across several parts of a text to } \\
\text { recognize a possible causal inference }\end{array}$ & \\
\hline L07 & $\begin{array}{l}\text { Integrate information across several parts of text to infer and recognize } \\
\text { an explanation }\end{array}$ & \\
\hline N03 & Locate, distinguish, and reproduce relevant information embedded in a leaflet & 1 of 2 \\
\hline N11 & $\begin{array}{l}\text { Locate a specified table of information and show an understanding of } 2 \text { uses } \\
\text { of information }\end{array}$ & 2 of 2 \\
\hline N12 & $\begin{array}{l}\text { Explain } 2 \text { reasons for a preference based on evaluating content of } 2 \text { specified } \\
\text { sections of non-textual information }\end{array}$ & 2 of 2 \\
\hline SO7 & Describe the function of a presentational device & 2 of 2 \\
\hline S11 & $\begin{array}{l}\text { Locate, distinguish, and take into account an entire section of text to reorder steps } \\
\text { of a procedural text with scientific information }\end{array}$ & 1 of 1 \\
\hline$\$ 12$ & $\begin{array}{l}\text { Integrates information across several parts of text to infer and provide } \\
\text { an explanation }\end{array}$ & 1 of 1 \\
\hline S14 & $\begin{array}{l}\text { Integrate across text to recognize the function of an organizational and } \\
\text { presentational device }\end{array}$ & \\
\hline S15 & $\begin{array}{l}\text { Provides specific information to explain a preference based on evaluating content } \\
\text { of } 3 \text { texts with scientific information }\end{array}$ & 2 of 2 \\
\hline
\end{tabular}



Appendix F

Iceland and Norway_Fifth Grade Reading Achievement 
Exhibit F.1 Iceland-Selected Reading Achievement Results for the Fifth Grade

Distribution of Reading Achievement - Fifth Grade

\begin{tabular}{|c|c|c|c|c|c|c|c|c|}
\hline Reading Achievement & Mean & $\begin{array}{c}\text { Years of } \\
\text { Formal } \\
\text { Schooling }\end{array}$ & $\begin{array}{c}\text { Average } \\
\text { Age }\end{array}$ & $\begin{array}{c}\text { 5th } \\
\text { Percentile } \\
\text { (Scale Score) }\end{array}$ & $\begin{array}{c}\text { 25th } \\
\text { Percentile } \\
\text { (Scale Score) }\end{array}$ & $\begin{array}{c}\text { 50th } \\
\text { Percentile } \\
\text { (Scale Score) }\end{array}$ & $\begin{array}{c}\text { 75th } \\
\text { Percentile } \\
\text { (Scale Score) }\end{array}$ & $\begin{array}{c}\text { 95th } \\
\text { Percentile } \\
\text { (Scale Score) }\end{array}$ \\
\hline Overall & $550(3.8)$ & 5 & 10.8 & $435(15.3)$ & $513(3.5)$ & $554(3.0)$ & $592(3.9)$ & $647(5.9)$ \\
\hline Literary & $551(3.0)$ & 5 & 10.8 & $434(9.8)$ & $508(4.5)$ & $555(4.0)$ & $594(4.6)$ & $656(6.4)$ \\
\hline Informational & $548(3.5)$ & 5 & 10.8 & $423(10.9)$ & $505(4.2)$ & $550(4.5)$ & $593(5.7)$ & $654(4.5)$ \\
\hline $\mathrm{RSI} I^{*}$ & $552(3.8)$ & 5 & 10.8 & $429(3.7)$ & $506(4.4)$ & $556(4.6)$ & $600(5.0)$ & $667(3.5)$ \\
\hline $\mathrm{IIE}^{* *}$ & $545(2.9)$ & 5 & 10.8 & $434(6.1)$ & $507(5.1)$ & $550(4.2)$ & $588(2.8)$ & $644(6.2)$ \\
\hline
\end{tabular}

Gender Differences in Reading Achievement - Fifth Grade

\begin{tabular}{|l|c|c|c|}
\hline Reading Achievement & Girls Mean & Boys Mean & Difference \\
\hline Overall & $560(3.9)$ & $539(4.9)$ & $21(4.4)$ \\
\hline Literary & $563(3.5)$ & $539(4.0)$ & $24(4.4)$ \\
\hline Informational & $556(3.0)$ & $537(5.2)$ & $18(4.6)$ \\
\hline $\mathrm{RSI}^{*}$ & $561(3.2)$ & $543(5.6)$ & $18(4.9)$ \\
\hline IIE* $^{* *}$ & $556(3.3)$ & $534(4.4)$ & $22(5.1)$ \\
\hline
\end{tabular}

Percentages of Students Reaching International Benchmarks in Reading

\begin{tabular}{|c|c|c|c|c|}
\hline Benchmark & Advanced & High & Intermediate & Low \\
\hline
\end{tabular}

Percent of Students

$10(2.1)$

$53(2.4)$

$87(1.6)$

$98(0.7)$

\begin{tabular}{|l|r|}
\hline \multicolumn{2}{|c|}{ Population Coverage and Sample Participation } \\
\hline School Population & 136 \\
\hline Student Population & 4174 \\
\hline National Desired Population & $100 \%$ \\
\hline Excluded Population (Schools) & $1.3 \%$ \\
\hline Excluded Population (Students) & $4.5 \%$ \\
\hline Excluded Population (Total) & $5.8 \%$ \\
\hline School Sample & 35 \\
\hline Student Sample & 1379 \\
\hline School Sample Participation (Before Replacement) & $100 \%$ \\
\hline School Sample Participation (After Replacement) & $100 \%$ \\
\hline Class Participation & $100 \%$ \\
\hline Student Participation & $88.3 \%$ \\
\hline Overall Participation (Before Replacement) & $88.3 \%$ \\
\hline Overall Participation (After Replacement) & $88.3 \%$ \\
\hline
\end{tabular}

* Retrieval and Straightforward Inferencing

* Interpreting, Integrating, and Evaluating

( ) Standard errors appear in parentheses. Because results are rounded to the nearest whole number, some totals may appear inconsistent. 


\section{Exhibit F.2 Norway-Selected Reading Achievement Results for the Fifth Grade}

Distribution of Reading Achievement - Fifth Grade

\begin{tabular}{|c|c|c|c|c|c|c|c|c|}
\hline Reading Achievement1 & Mean & $\begin{array}{l}\text { Years of } \\
\text { Formal } \\
\text { Schooling }\end{array}$ & $\begin{array}{c}\text { Average } \\
\text { Age }\end{array}$ & $\begin{array}{c}\text { 5th } \\
\text { Percentile } \\
\text { (Scale Score) }\end{array}$ & $\begin{array}{c}\text { 25th } \\
\text { Percentile } \\
\text { (Scale Score) }\end{array}$ & $\begin{array}{c}\text { 50th } \\
\text { Percentile } \\
\text { (Scale Score) }\end{array}$ & $\begin{array}{c}\text { 75th } \\
\text { Percentile } \\
\text { (Scale Score) }\end{array}$ & $\begin{array}{c}\text { 95th } \\
\text { Percentile } \\
\text { (Scale Score) }\end{array}$ \\
\hline Overall & $541(3.1)$ & 5 & 10.8 & $424(3.0)$ & $502(3.2)$ & $543(4.8)$ & $584(4.1)$ & $637(7.2)$ \\
\hline Literary & $540(3.3)$ & 5 & 10.8 & $418(5.9)$ & $497(4.1)$ & $543(3.8)$ & $583(2.4)$ & $648(6.4)$ \\
\hline Informational & $541(2.9)$ & 5 & 10.8 & $433(14.7)$ & $502(6.6)$ & $543(4.8)$ & $584(5.7)$ & 641 (14.1) \\
\hline $\mathrm{RSI}{ }^{*}$ & $541(3.2)$ & 5 & 10.8 & $417(9.5)$ & $497(5.4)$ & $543(4.4)$ & $587(4.5)$ & $653(10.1)$ \\
\hline$\| E^{* *}$ & $541(3.0)$ & 5 & 10.8 & $428(9.6)$ & $501(4.2)$ & $543(4.2)$ & $583(4.2)$ & $644(9.7)$ \\
\hline
\end{tabular}

Gender Differences in Reading Achievement - Fifth Grade

\begin{tabular}{|c|c|c|c|}
\hline Reading Achievement ${ }^{1}$ & Girls Mean & Boys Mean & Difference \\
\hline Overall & $548(4.6)$ & $533(3.3)$ & $15(5.1)$ \\
\hline Literary & $550(4.3)$ & $530(3.3)$ & $20(4.3)$ \\
\hline Informational & $547(3.8)$ & $536(3.1)$ & $11(3.9)$ \\
\hline $\mathrm{RSI}^{*}$ & $546(3.9)$ & $535(4.6)$ & $11(5.6)$ \\
\hline$\| E^{* *}$ & $550(3.8)$ & $532(3.6)$ & $18(4.4)$ \\
\hline
\end{tabular}

Percentages of Students Reaching International Benchmarks in Reading

\begin{tabular}{|l|c|c|c|c|}
\hline Benchmark & Advanced & High & Intermediate & Low \\
\hline Percent of Students & $8(1.9)$ & $46(2.1)$ & $85(1.3)$
\end{tabular}

Percent of Students

$8(1.9)$

$46(2.1)$

$85(1.3)$

$98(0.8)$

\begin{tabular}{|l|r|}
\hline \multicolumn{2}{|c|}{ Population Coverage and Sample Participation } \\
\hline School Population & 2413 \\
\hline Student Population & 61167 \\
\hline National Desired Population & $100 \%$ \\
\hline Excluded Population (Schools) & $1.0 \%$ \\
\hline Excluded Population (Students) & $2.7 \%$ \\
\hline Excluded Population (Total) & $3.7 \%$ \\
\hline School Sample & 66 \\
\hline Student Sample & 1808 \\
\hline School Sample Participation (Before Replacement) & $51 \%$ \\
\hline School Sample Participation (After Replacement) & $68 \%$ \\
\hline Class Participation & $99 \%$ \\
\hline Student Participation & $84.3 \%$ \\
\hline Overall Participation (Before Replacement) & $42.4 \%$ \\
\hline Overall Participation (After Replacement) & $56.9 \%$ \\
\hline
\end{tabular}

* Retrieval and Straightforward Inferencing

* Interpreting, Integrating, and Evaluating

1 Even though Norway worked very hard to meet the PIRLS sampling requirements, it did not meet the school participation rates as specified in the guidelines.

() Standard errors appear in parentheses. Because results are rounded to the nearest whole number, some totals may appear inconsistent. 

Appendix G

Organizations and Individuals Responsible for PIRLS 2006 


\section{Introduction}

PIRLS 2006 was a collaborative effort involving hundreds of individuals around the world. This appendix recognizes the individuals and organizations for their contributions. Given that work on PIRLS 2006 has spanned approximately five years and has involved so many people and organizations, this list may not include all who contributed. Any omission is inadvertent.

Of the first importance, PIRLS 2006 is deeply indebted to the students, parents, teachers, and school principals who contributed their time and effort to the study.

\section{Management and Coordination}

PIRLS is a major undertaking of IEA, and together with the Trends in International Mathematics and Science Study (TIMSs), comprises the core of IEA's regular cycles of studies. The PIRLS assessment at the fourth grade complements TIMss, which regularly assesses mathematics and science achievement at fourth and eighth grades.

The TIMSS \& PIRLS International Study Center at Boston College has responsibility for the overall direction and management of the TIMSS and PIRLS projects. Headed by Drs. Ina V.S. Mullis and Michael O. Martin, the study center is located in the Lynch School of Education. Dr. Ann M. Kennedy is the PIRLS Project Coordinator. In carrying out the project, the TIMSS \& PIRLS International Study Center worked closely with the IEA Secretariat in Amsterdam, which provided guidance overall and was responsible for verification of all translations produced by the participating countries. The IEA Data Processing and Research Center in Hamburg was responsible for processing and verifying the data submitted by the participants; Statistics Canada in Ottawa was responsible for school and student sampling activities; and Educational Testing Service (ETS) in Princeton, New Jersey consulted on psychometric methodology and provided software for scaling the achievement data.

The Project Management Team, comprised of study directors and representatives from the TIMSS \& PIRLS International Study Center, the IEA 
Secretariat, the IEA Data Processing and Research Center, Statistics Canada, and ETs met twice a year throughout the study to discuss the study's progress, procedures, and schedule. In addition, the study directors met with members of IEA's Technical Executive Group twice yearly to review technical issues.

Dr. Marian Sainsbury from the National Foundation for Educational Research in England (NFER) was the PIRLS 2006 Reading Coordinator and Dr. Patricia Donahue from ETs was a special reading assessment consultant. Together with the Reading Development Group, a panel of internationally recognized experts in reading research, instruction, and assessment, they provided excellent guidance throughout PIRLS 2006.

To work with the international team and coordinate within-country activities, each participating country designated an individual to be the PIRLS National Research Coordinator (NRC). The NRCs have the complicated and challenging task of implementing the PIRLS study in their countries in accordance with the PIRLS guidelines and procedures. The quality of the PIRLS assessment and data depends on the work of the NRCs and their colleagues in carrying out the very complex sampling, data collection, and scoring tasks involved. In addition, the Questionnaire Development Group, comprised of NRCs, provided advice on questionnaire development.

Continuing the tradition of truly exemplary work established in PIRLS 2001, the PIRLS 2006 NRCs (often the same NRCs as in 2001), performed their many tasks with dedication, competence, energy, and goodwill, and have been commended by the IEA Secretariat, the TIMSS \& PIRLS International Study Center, the IEA Data Processing and Research Center, and Statistics Canada for their commitment to the project and the high quality of their work.

\section{Funding}

A project of this magnitude requires considerable financial support. IEA's major funding partners for PIRLS included the World Bank, the U.s. Department of Education through the National Center for Education Statistics, and those countries that contributed by way of fees. The financial support provided by Boston College and NFER also is gratefully acknowledged. 


\section{IEA Secretariat}

Seamus Hegarty, IEA Chair

Hans Wagemaker, Executive Director

Barbara Malak, Manager, Membership Relations

Juriaan Hartenburg, Financial Manager

Suzanne Morony, Senior Manager Assistant

\section{TIMSS \& PIRLS International Study Center at Boston College}

Ina V.S. Mullis, Co-Director

Michael O. Martin, Co-Director

Pierre Foy, Director of Sampling and Data Analysis

Ann M. Kennedy, Coordinator of Project Development

and Operations, PIRLS Coordinator

Alka Arora, Timss Advanced 2008 Project Coordinator

Debra Berger, Production Editor

Marcie Bligh, Manager of Office Administration

Joann Cusack, Administrative Coordinator

Ebru Erberber, Timss Science Research Associate

Susan Farrell, Co-Manager of Publications

Joseph Galia, Senior Statistician/Programmer

Christine Hoage, Manager of Finance

Ieva Johansone, Survey Operations Coordinator

Isaac Li, Statistician/Programmer

Dana Milne, TIMss Graduate Assistant

Jennifer Moher, Data Graphics Specialist

Mario Pita, Co-Manager of Publications

Corinna Preuschoff, timss Research Associate

Ruthanne Ryan, Data Graphics Specialist

Feng Tian, Timss Graduate Assistant

Kathleen L. Trong, PIRLs Research Associate

\section{IEA Data Processing and Research Center}

Dirk Hastedt, Co-Director

Juliane Barth, Co-Manager, TIMss \& PIRLs Data Processing

Oliver Neuschmidt, Co-Manager, TIMss \& PIRLs Data Processing

Yasin Afana, Researcher

Milena Taneva, Researcher

Marta Kostek-Drosihn, Researcher

Sabine Meinck, Researcher

Olaf Zuehlke, Researcher

Christine Busch, Researcher

Alena Becker, Researcher

Simone Uecker, Researcher 
Michael Jung, Researcher

Tim Daniel, Researcher

Dirk Oehler, Researcher

Stephan Petzchen, Senior Programmer

Ralph Carstens, Programmer

Hauke Heyen, Programmer

Harpreet Singh Choudry, Programmer

\section{Statistics Canada}

Marc Joncas, Senior Methodologist

\section{Educational Testing Service}

Mathias von Davier, Senior Research Scientist

\section{Sampling Referee}

Keith Rust, Vice President and Associate Director of the Statistical Group, Westat, Inc.

\section{PIRLS 2006 Assessment Development}

\section{Reading Coordinator}

Marian Sainsbury, NFER

\section{Reading Assessment Consultant}

Patricia Donahue, ETs

\section{PIRLS 2006 Reading Development Group (RDG)}

Dominique Lafontaine, Service de Pedagogie Experimentale, Belgium

Jan Mejding, Danish University of Education, Denmark

Sue Horner, Qualifications and Curriculum Authority, England

Renate Valtin, Abteilung Grundschulpädagogik, Humboldt Universität, Germany

Galina Zuckerman, Psychological Institute, Russian Academy of Education, Russian Federation

Elizabeth Pang and Selene Tan, Ministry of Education HQ, Singapore

Karen Wixson, University of Michigan, United States

\section{PIRLS Questionnaire Development Group (QDG)}

Meng Hong Wei, The China National Institute of Education, China

Marc Colmant, Ministere de l'Education Nationale, France

Knut Schwippert, University of Hamburg, Institute for Comparative

\& Multicultural Education, Germany

Gabriella Pavan de Gregorio, Instituto Nazionale per la

Valuatazione del Sistema Dell'Istruzione, Italy

Bojana Naceva, Bureau for Development of Education, Republic of Macedonia

Mieke van Diepen, Expertisecentrum Nederlands, Netherlands

Ragnar Gees Solheim, National Center for Reading Education and Research, Norway

Larry Ogle, National Center for Education Statistics, United States 


\section{PIRLS 2006 National Research Coordinators (NRCs)}

\section{Austria}

Günter Haider

Birgit Suchań

Austrian IEA Research Centre,

Universität Salzburg

Belgium

Flemish

Jan Van Damme

Katholieke Universiteit Leuven

\section{French}

Annette Lafontaine

Université de Liège

\section{Bulgaria}

Tatyana Angelova

Felianka Kaftandjieva (through 2004)

University of Sofia

\section{Canada}

\section{Alberta}

Ping Yang

Learner Assessment Branch,

Alberta Education

\section{British Columbia}

Diane Lalancette

Exams \& Assessment Policy

\section{Nova Scotia}

Marthe Craig

Evaluation Coordinator, Evaluation Services

\section{Ontario}

Michael Kozlow

Francine Jaques (through 2004)

Education Quality and Accountability Office

\section{Québec}

Serge Baillargeon

Ministère de l'Éducation

\section{Chinese Taipei}

Hwawei Ko

Graduate Institute of Learning

and Instruction
National Central University

Denmark

Jan Mejding

The Danish University of Education

\section{England}

Liz Twist

National Foundation for Educational

Research in England and Wales

France

Marc Colmant

Ministère de l'Éducation Nationale

\section{Georgia}

Maia Miminoshvili

National Assessment and

Examinations Center

\section{Germany}

Wilfried Bos

Sabine Hornberg

Institut fuer Schulentwicklungsfurschung

University of Dortmund

\section{Hong Kong}

Tse Shek-Kam

The University of Hong Kong

\section{Hungary}

Ildiko Balazsi

Péter Balkányi

Annamária Szazó Rábai (through 2004)

National Institute of Public Education

Centre for Evaluation Studies

Iceland

Brynhildur Scheving Thorsteinsson

Institute for Educational Research

\section{Indonesia}

Burhanuddin Tola

Center for Educational Assessment

Bahrul Hayat (through 2004)

Ministry of National Education 
Iran, Islamic Republic of

Abdol'azim Karimi

Institute for Educational Research

Israel

Elite Olshtain

Hebrew University

Ruth Zuzovsky

Tel Aviv University

\section{Italy}

Silvana Serra

Gabriella Pavan de Gregorio (through 2005)

Instituto Nazionale per la Valuatazione

del Sistema Dell'Istruzione

\section{Kuwait}

Abdul Ghani Al-Bazzaz

Ministry of Education

\section{Latvia}

Antra Ozola

University of Latvia

Lithuania

Aiste Elijio

Ministry of Education and Science

\section{Luxembourg}

Pierre Reding

Martin Frieberg

Ministère de l'Éducation Nationale

\section{Macedonia, Republic of}

Tanja Andonova

Pedagogical Institute of Macedonia

Bojana Naceva (through 2006)

Bureau for Development of Education

\section{Moldova, Republic of}

Ilie Nasu

Ministry of Education and Science

\section{Morocco}

Mohammed Sassi

Departement de l'Évaluation Nationale

\section{Netherlands}

Andrea Netten

Mieke van Diepen (through 2004)

Expertisecentrum Nederlands

\section{New Zealand}

Megan Chamberlain

Ministry of Education

\section{Norway}

Ragnar Gees Solheim

Victor van Daal

Finn-Egil Toennessen (through 2005)

National Centre for Reading, Education

and Reading Research

University of Stavanger

\section{Poland}

Krzysztof Konarzewski

Institute of Psychology

Polish Academy of Science

\section{Qatar}

Abdessalem Buslama

Marcus Broer (through 2006)

Evaluation Institute

Supreme Education Council

Office of Student Assessment

\section{Romania}

Gabriela Noveanu

Institute for Educational Sciences

Evaluation and Forecasting Division

\section{Russian Federation}

Galina Kovalyova

The Russian Academy of Education 


\section{Scotland}

Fiona Fraser

Jo MacDonald (through 2003)

Scottish Office

Education Department

\section{Singapore}

Wong Look Kwang

New Yi Cheen (through 2005)

Research and Evaluation

Ministry of Education

\section{Slovak Republic}

Eva Obrancova

Zuzana Lukackova (through 2004)

SPU-National Institute for Education

\section{Slovenia}

Marjeta Doupona-Horvat

Educational Research Institute

\section{South Africa}

Sarah Howie

Elsie Venter

University of Pretoria

\section{Spain}

Mar Gonzalez Garcia

Flora Gil Traver (through 2005)

Instituto Nacional de Calidad y

Evaluacion del Sistema Educativo

\section{Sweden}

Bo Palaszewski

National Agency for Education

Caroline Liberg

Uppsala University

\section{Trinidad and Tobago}

Harrilal Seecharan

Mervyn Sambucharan

Division of Educational

Research and Evaluation

\section{United States}

Laurence Ogle

National Center for Education Statistics U.s. Department of Education 



\section{TIMSS \& PIRLS}

International Study Center

Lynch School of Education, Boston College

TYPOGRAPHY: Set in Meridien, Minion, and Myriad.

PHOTOGRAPH: Copyright (0) IEA 2007

COVER DESIGN: Susan L. Farrell

BOOK DESIGN: Mario A. Pita and Ruthanne Ryan

PRODUCTION COORdinator: Mario A. Pita

LAYOUT: Mario A. Pita and Ruthanne Ryan

BOOK PRODUCTION EDITOR: Debra R. Berger 

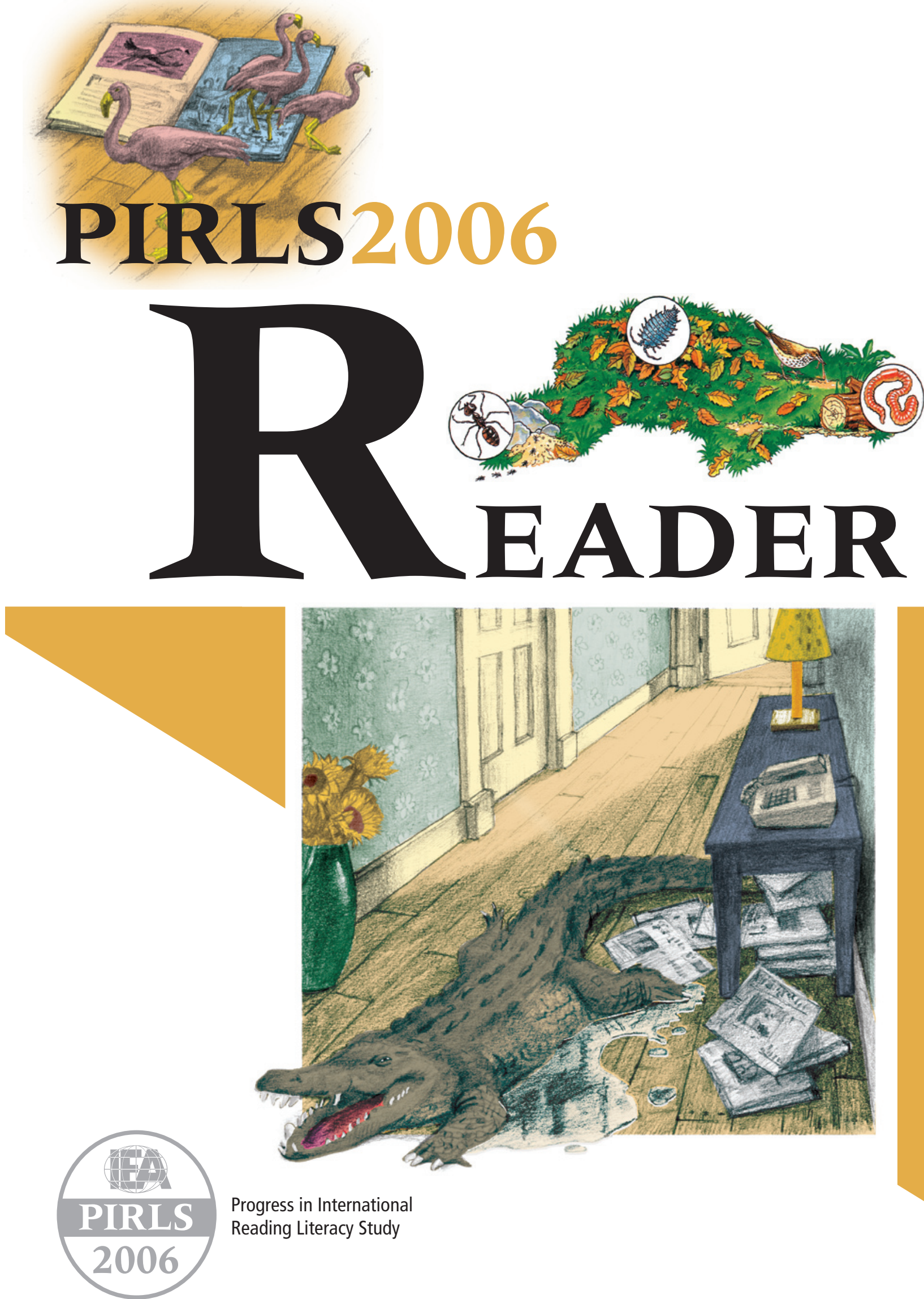

Progress in International

Reading Literacy Study 



\section{Contents}

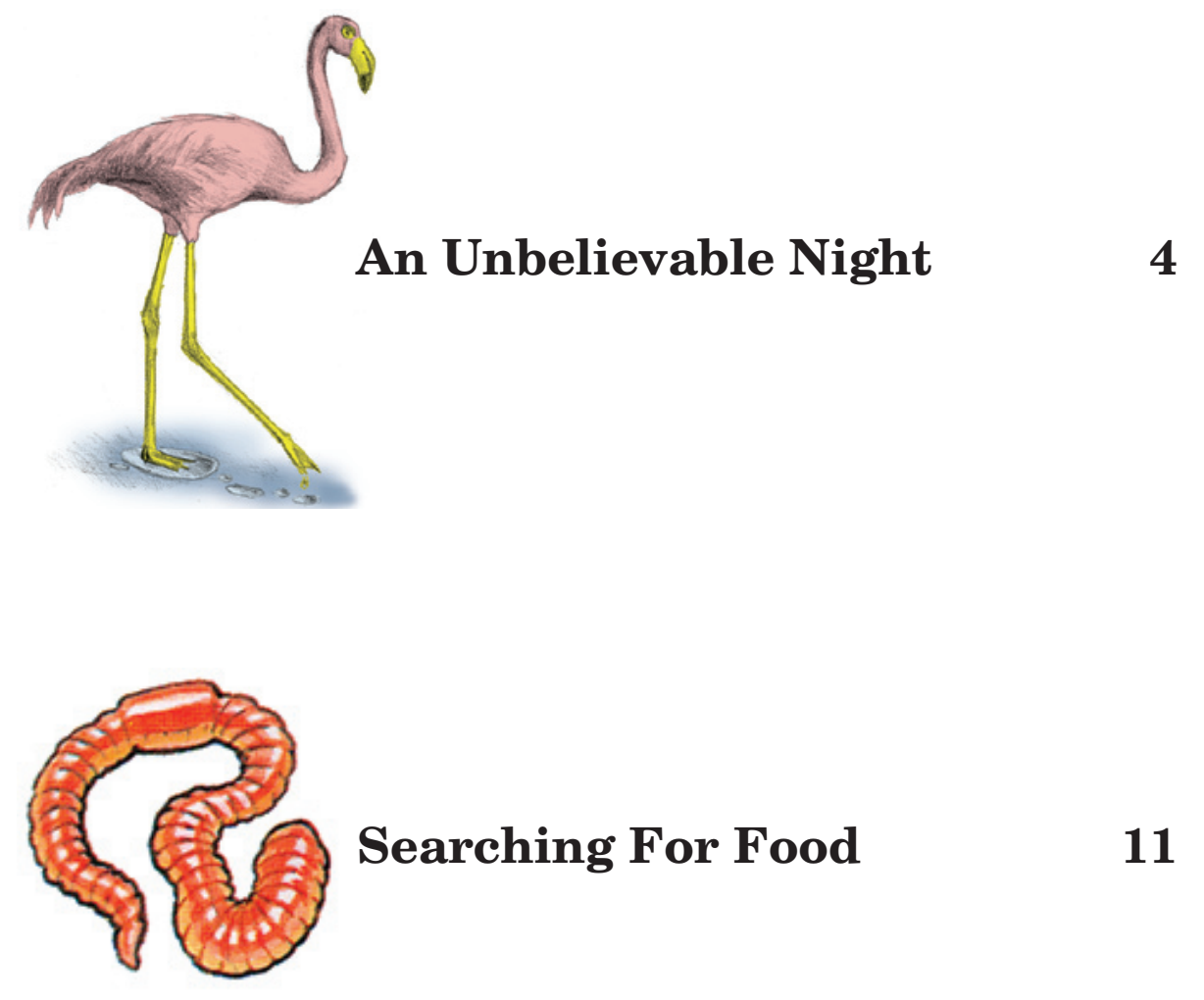




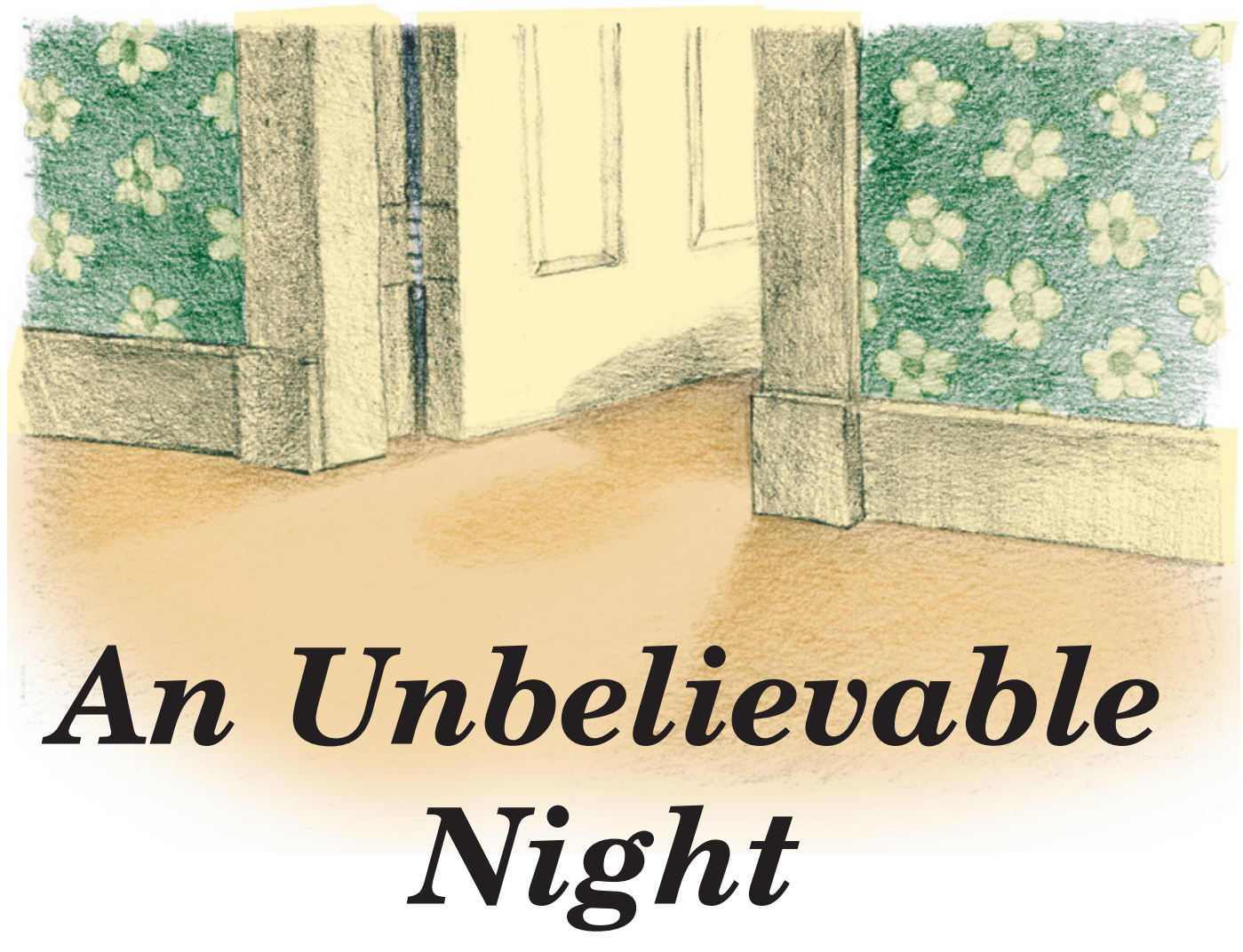

by Franz Hohler

nina was ten years old, so even half asleep she could find
her way from her room to the bathroom. The door to her
room was usually open a crack, and the nightlight in the hallway made it light enough to get to the bathroom past the telephone stand.

One night, as she passed the telephone stand on her way to the bathroom, Anina heard something that sounded like a quiet hissing. But, because she was half asleep, she didn't really pay any attention to it. Anyway, it came from pretty far away. Not until she was on her way back to her room did she see where it came from. Under the telephone stand there was a large pile of old newspapers and magazines, and this pile now began to move. That was where the noise was coming from. All of a sudden the pile started to fall over - right, left, forwards, backwards - then there were newspapers and magazines all over the floor. 
Anina could not believe her eyes as she watched a grunting and snorting crocodile come out from under the telephone stand.

Anina was frozen to the spot. Her eyes wide as saucers, she watched the crocodile crawl completely out of the newspapers and slowly look around the apartment. It seemed to have just come out of the water because its whole body was dripping wet. Wherever the crocodile stepped, the carpet under it became drenched.

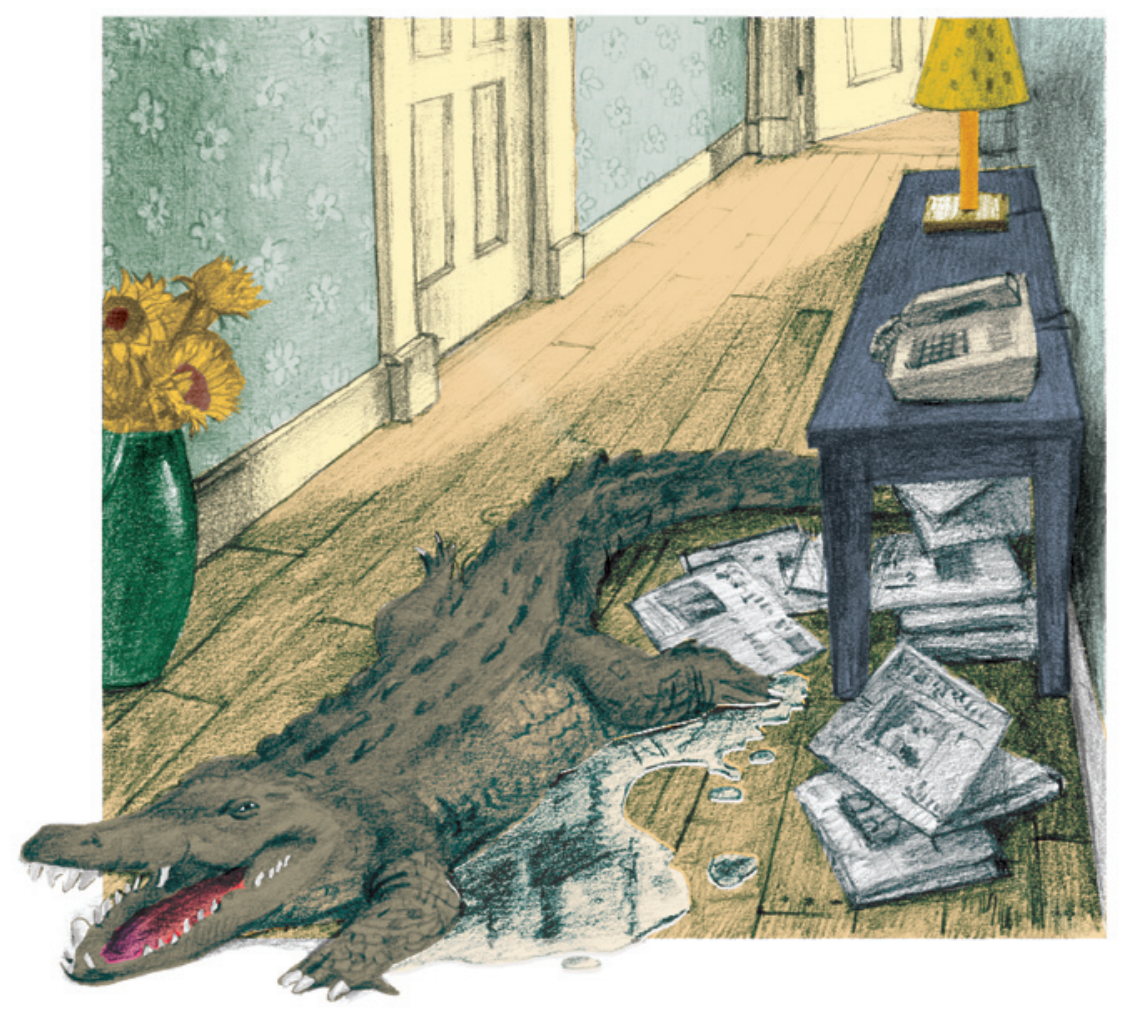


The crocodile moved its head back and forth letting out a loud hissing sound. Anina swallowed hard, looking at the crocodile's snout with its terribly long row of teeth. It swung its tail slowly back and forth. Anina had read about that in "Animal Magazine"- how the crocodile whips the water with its tail to chase away or attack its enemies.

Her gaze fell on the last issue of "Animal Magazine," which had fallen from the pile and was lying at her feet. She got another shock. The cover of the magazine used to have a picture of a big crocodile on a river bank. The river bank was now empty!

Anina bent down and picked up the magazine. At that moment the crocodile whipped his tail so hard that he cracked the big vase of sunflowers on the floor and the sunflowers scattered everywhere. With a quick jump Anina was in her bedroom. She slammed the door shut, grabbed her bed and pushed it up against the door. She had built a barricade that would keep her safe from the crocodile. Relieved, she let her breath out.

But then she hesitated. What if the beast was simply hungry? Maybe to make the crocodile go away you had to give it something to eat?

Anina looked again at the animal magazine. If the crocodile could crawl out of a picture then perhaps other animals could too. Anina hastily flipped through the magazine and stopped at a swarm of flamingos in a jungle swamp. Just right, she thought. They look like a birthday cake for crocodiles.

Suddenly there was a loud crack and the tip of the crocodile's tail pushed through the splintered door.

Quickly, Anina held the picture of the flamingos up to the hole in the door and called as loud as she could, "Get out of the swamp! Shoo! Shoo!" Then she threw the magazine through the hole into the hallway, clapped her hands and yelled and screamed. 
She could hardly believe what happened next. The entire hallway was suddenly filled with screeching flamingos wildly flapping their wings and running around all over the place on their long, skinny legs. Anina saw one bird with a sunflower in its beak and another grabbing her mother's hat from its hook. She also saw a flamingo disappear into the crocodile's mouth. With two quick bites he swallowed the flamingo and quickly followed it with another, the one with the sunflower in its beak.

After two portions of flamingo the crocodile seemed to have had enough and lay down contentedly in the middle of the hallway. When he had closed his eyes and no longer moved,

Anina quietly

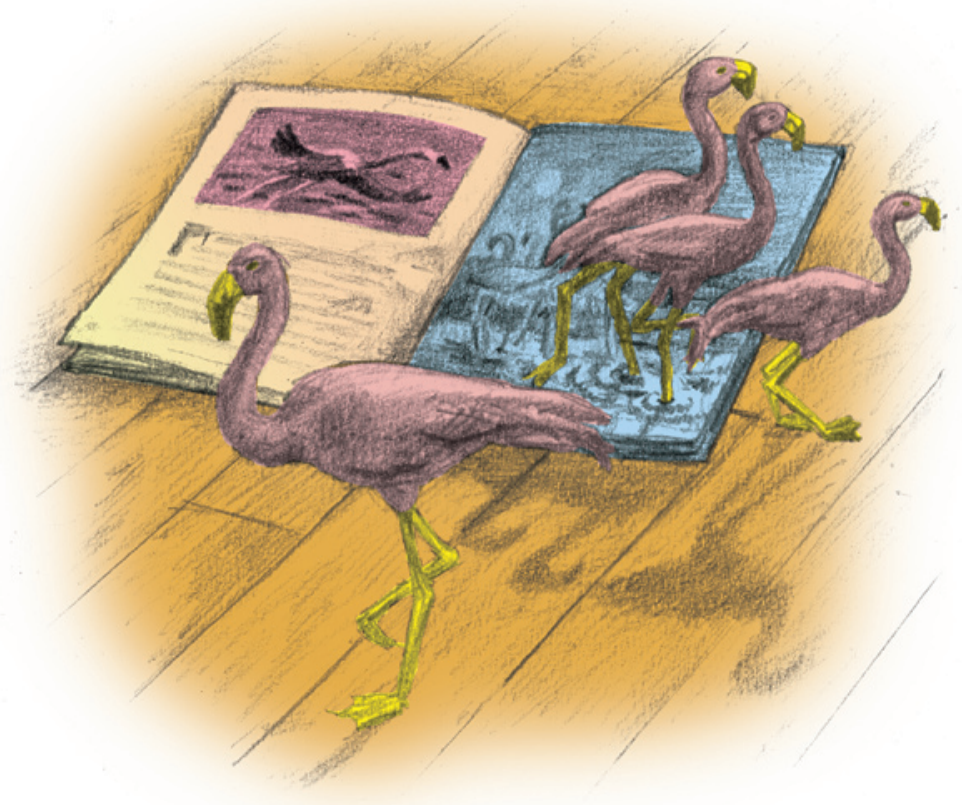
opened her door and slipped through it into the hallway. She placed the empty magazine cover in front of the crocodile's nose. "Please," she whispered, "please go back home." She crept back into the bedroom and looked through the hole in the door. She saw the crocodile back on the cover of the magazine.

She now went cautiously into the living room where the flamingos were crowded around the sofa and standing on the television. Anina opened the magazine to the page with the empty picture. "Thank you," she said, "thank you very much. You may now go back to your swamp." 
In the morning, it was very difficult for her to explain the giant wet spot on the floor and the broken door to her parents. They weren't convinced about the crocodile even though her mother's hat was nowhere to be found.

Adapted from Eine Wilde Nacht in Der Große Zwerg und Andere Geschichten by Franz Hohler. Published in 2003 by Deutscher Taschenbuch Verlag, Munchen, Germany. Illustrations copyright (C) 2003, IEA. An effort has been made to obtain copyright permission.

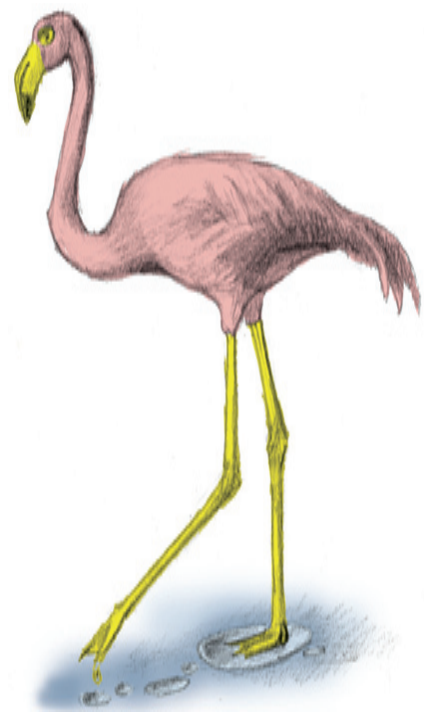




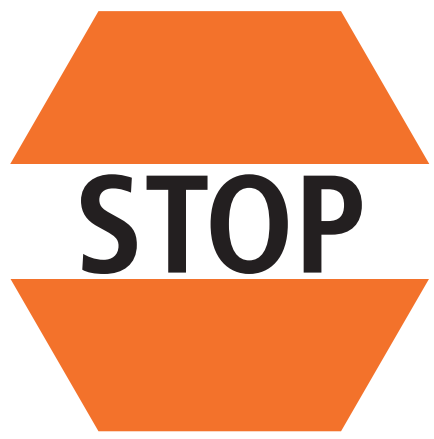

End of Part 1

Now go to your question booklet. 



\section{Searching for Food}

Here are three projects about the things small creatures eat and the ways they search for food. First you need to find actual ants, pill bugs, and worms. Treat them carefully and make sure you put them back where you found them after you have finished studying them.

- Follow an Ant Trail

- Study Pill Bugs

- Make a Wormery

\section{Where to find ants, pill bugs, and worms}

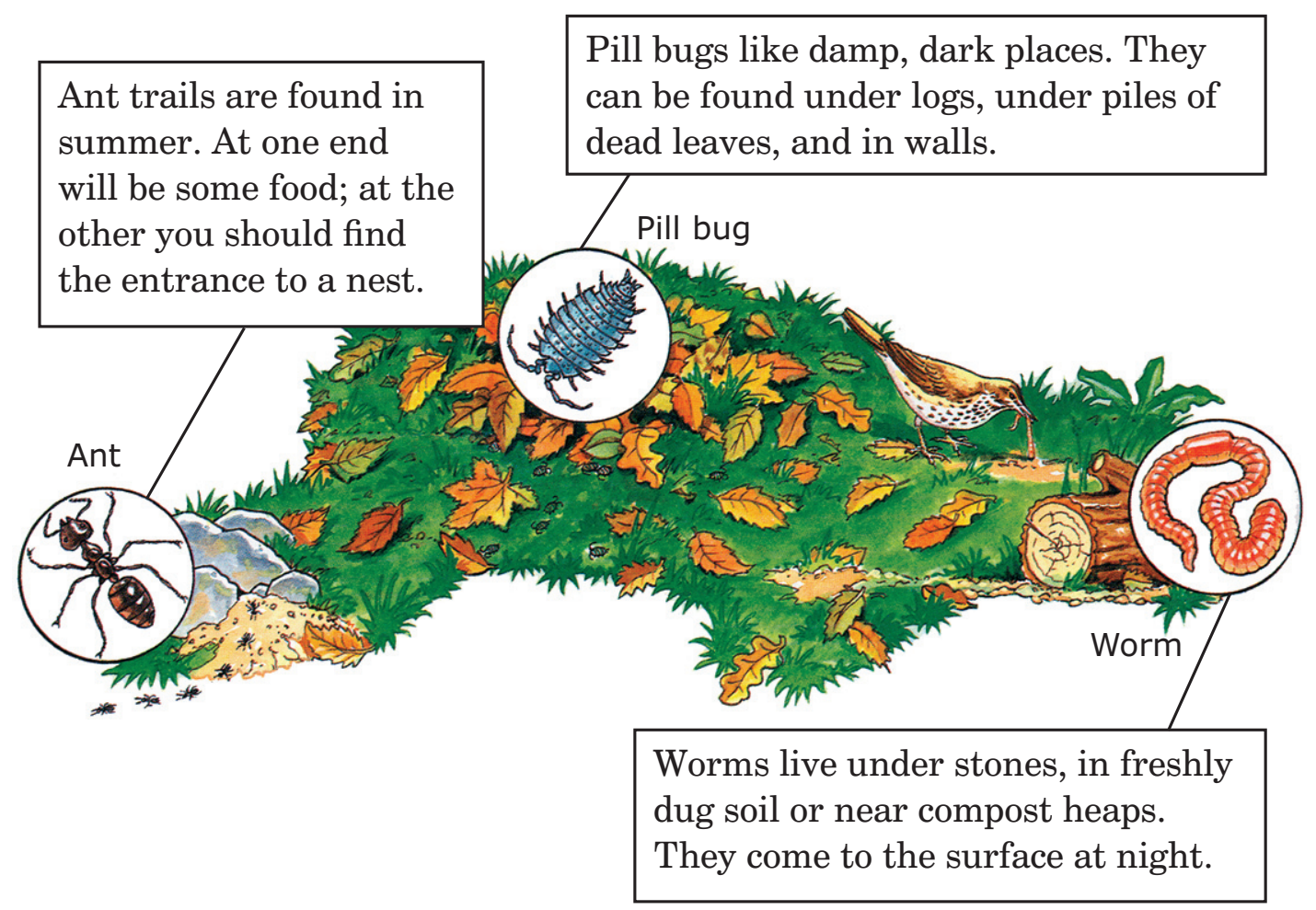




\section{-
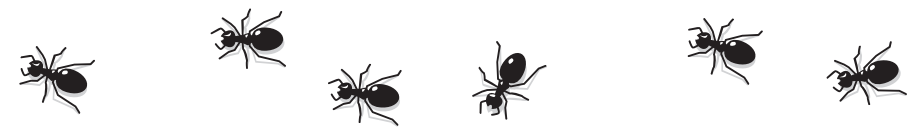

\section{Follow an Ant Trail}

Ants live together in nests. When an ant finds some food it makes a trail for others to follow. To do this experiment you will need to find an ants' nest. You will also need the following materials: a sheet of paper, a small piece of apple, a handful of soil.

1. Put the piece of apple on the sheet of paper and lay the paper close to an ants' nest. Wait for some ants to find the apple. They should all follow the same trail.

2. Move the apple. Do the ants go straight to it?

3. Now sprinkle soil on the paper to cover the trail. The ants should scurry around for a while. Do they make a new trail?

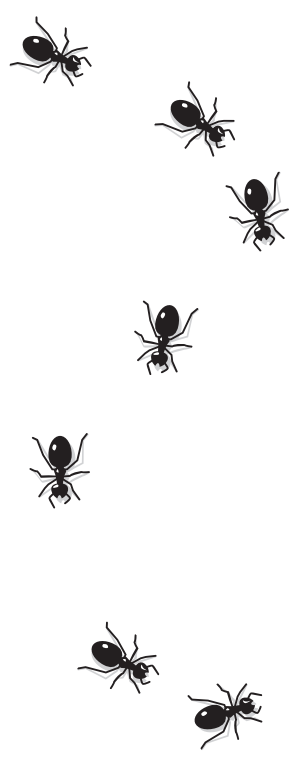

\section{What happens?}

Even after the food has moved, the ants still follow the old trail until a new one is laid.

\section{Why?}

Once an ant has found some food, it produces special chemicals that leave a scent trail. Other ants from the nest use their antennae, or feelers, to sense this scent. 


\section{Study Pill Bugs}

Pill bugs have sensitive antennae.

Make this box, then collect six pill bugs in a container. Watch how they find their way when you put them in a box. You will need: a small empty box with a lid, scissors, adhesive tape, and dead, damp leaves.

Cardboard strips - don't leave gaps at the bottom

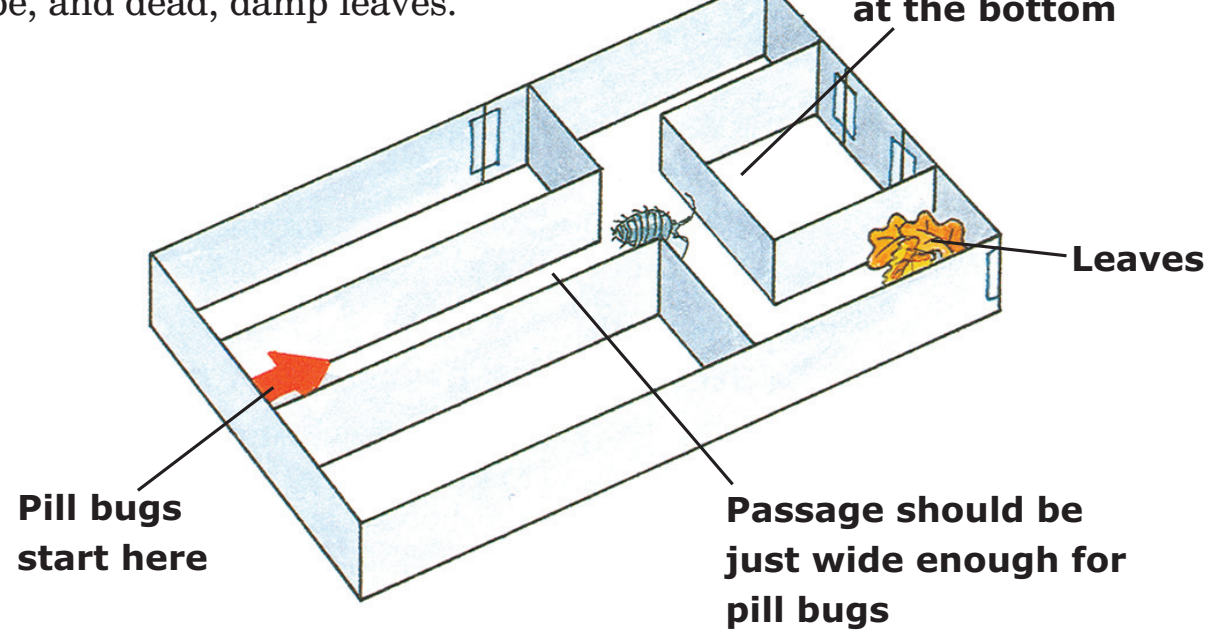

1. Use the lid to make three long strips for making the passages in the picture.

2. Let your pill bugs walk along the passage one at a time. When they reach the end of the passage, some will turn left and some will turn right.

3. Put damp leaves in the right hand side of the box. Now let the pill bugs walk through the box again. Which way do they go?

\section{What happens?}

The pill bugs will turn to the right toward the food.

\section{Why?}

The pill bugs can sense the food with their antennae. They use them to find the leaves. 


\section{Make a Wormery}

Worms are hard to study because they don't like the light. As soon as they sense it, they wriggle away, trying to find a dark place again. To see how worms live and feed, make a wormery like the one shown here. Then find two or three worms to put in it. It is important to remember

\section{You will need}

- Shoe box

- Adhesive tape

- Pen

- Scissors

- Large plastic bottle

- 1 mug of sand

- 3 mugs of damp, crumbly soil

- Small cubes of onion and potato not to pull on the worms or you may hurt them. They are covered with bristles that grip the soil tightly.

1. Tape one side of the shoe box lid to the box, so it opens like a door. Poke holes in the top of the box with the pen to let air and light into the wormery.

2. Cut the top off the bottle. Then fill it with loosely packed layers of soil and sand. Scatter potato and onion on the surface.

3. Gently drop in your worms, then stand the bottle in the box and close the door. Leave it outside in a cool, dry place for four days.

4. After four days, go back and look at the bottle. What is different about the sand and soil?

Don't forget: when you've finished with this project, put the worms back where you found them. 


\section{What happens?}

After four days, the layers of sand and soil will have been mixed together.

\section{Why?}

The worms mix the sand and soil coming to the surface to eat the food and then tunneling underground to get away from the light.

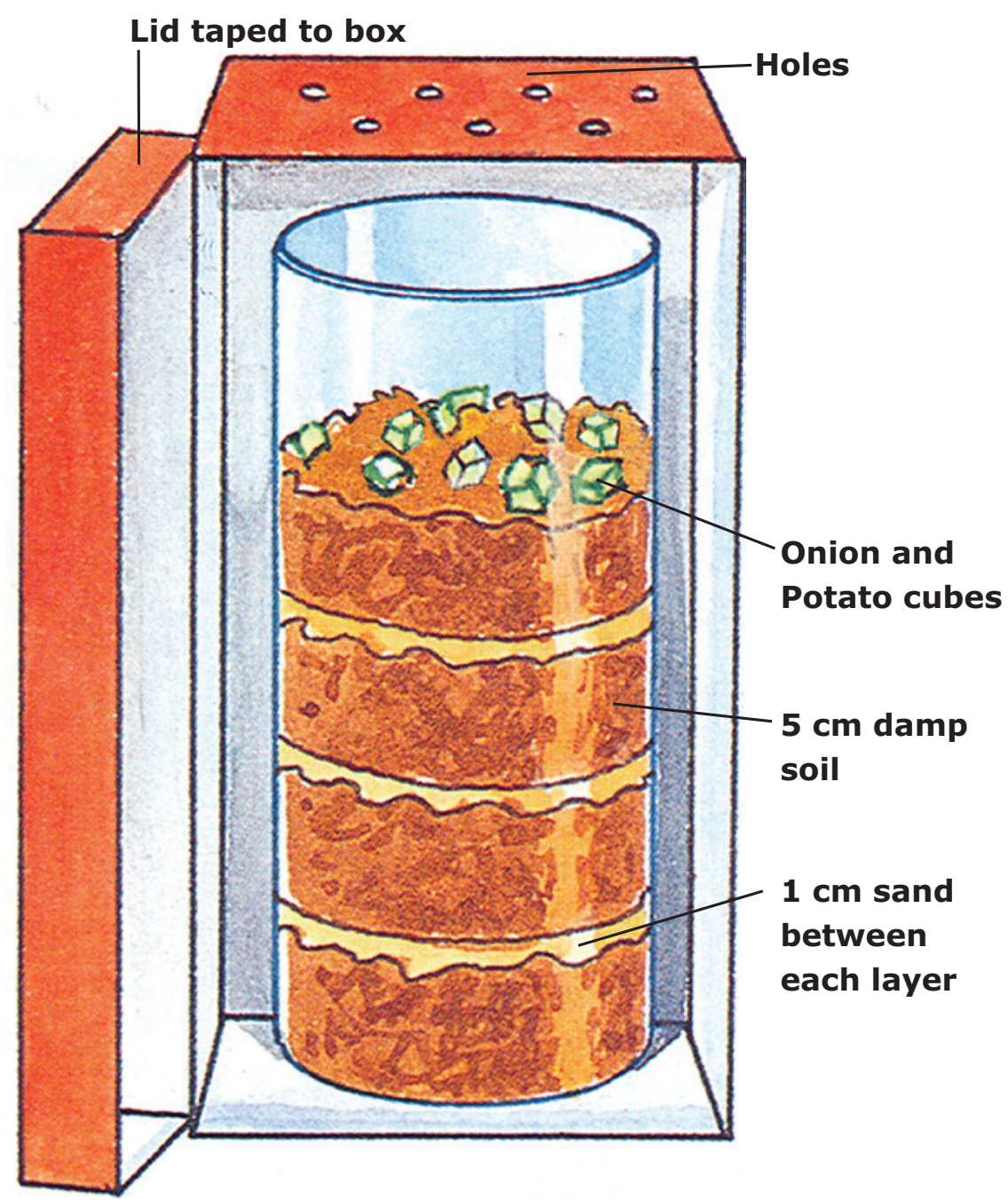

From Animal watching in the Usborne Big Book of Experiments published in 1996 by Usborne Publishing Ltd., London. An effort has been made to obtain copyright permission. 



\section{Stop}

End of Part 2.

Now go to your question booklet. 




\section{PIRLS2006}

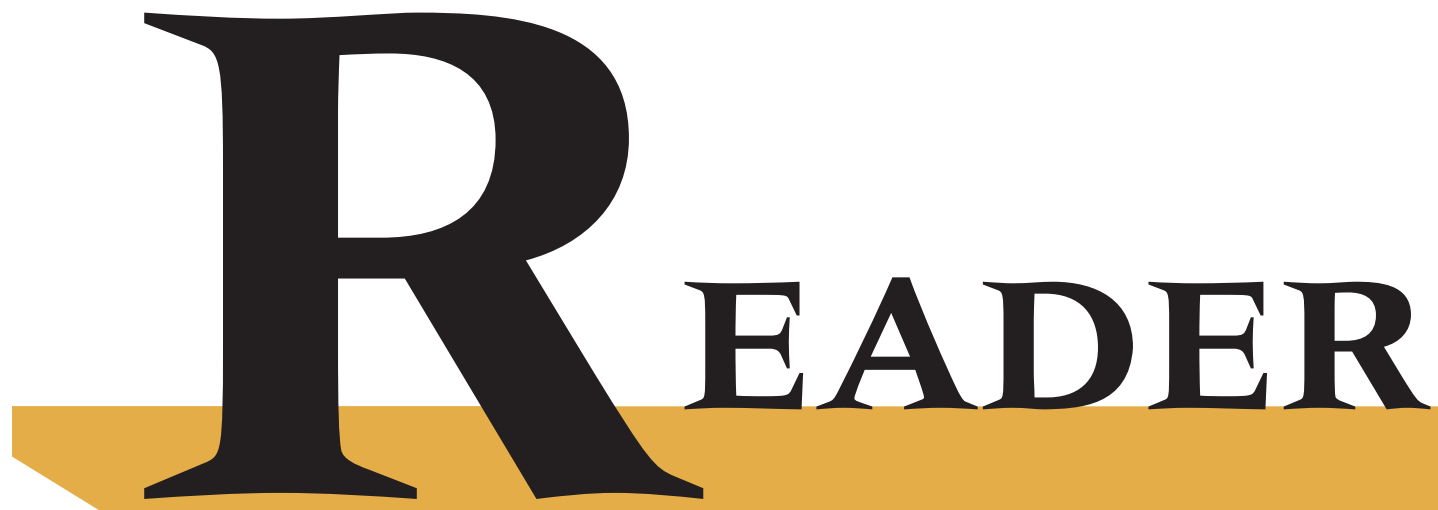





\section{TIMSS \& PIRLS}

International Study Center

Lynch School of Education, Boston College

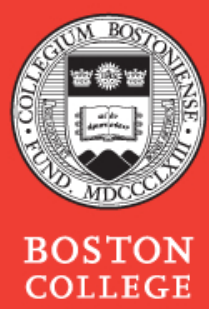

7. pirls.bc.edu

PIRLS Copyright $\odot 2007$ International Association for the 2006 Evaluation of Educational Achievement (IEA) 